OAK RIDGE NATIONAL LABORATORY

\author{
operated by \\ UNION CARBIDE CORPORATION \\ for the \\ U.S. ATOMIC ENERGY COMMISSION
}

INDEXES TO 563 UNCLASSIFIED DOCUMENTS

ON CIVIL DEFENSE

\author{
Joanne S. Levey \\ Ann S. Klein \\ Bobbie-Jean Hatcher \\ Editors
}




\section{DISCLAIMER}

This report was prepared as an account of work sponsored by an agency of the United States Government. Neither the United States Government nor any agency Thereof, nor any of their employees, makes any warranty, express or implied, or assumes any legal liability or responsibility for the accuracy, completeness, or usefulness of any information, apparatus, product, or process disclosed, or represents that its use would not infringe privately owned rights. Reference herein to any specific commercial product, process, or service by trade name, trademark, manufacturer, or otherwise does not necessarily constitute or imply its endorsement, recommendation, or favoring by the United States Government or any agency thereof. The views and opinions of authors expressed herein do not necessarily state or reflect those of the United States Government or any agency thereof. 


\section{DISCLAIMER}

Portions of this document may be illegible in electronic image products. Images are produced from the best available original document. 
Printed in the United States of America. Available from Clearinghouse for Federal Scientific and Technical Information, National Bureau of Standards,

U.S. Department of Commerce, Springfield, Virginia 22151

Price: Printed Copy $\$ 3.00$; Microfiche $\$ 0.65$

\section{LEGAL NOTICE}

This report was prepared as an account of Government sponsored work. Neither the United States, nor the Commission, nor any person acting on behalf of the Commission:

A. Makes any warranty or representation, expressed or implied, with respect to the accuracy, completeness, or usefulness of the information contained in this report, or that the use of any information, apparatus, method, or process disclosed in this report may not infringe privately owned rights; or

B. Assumes any liabilities with respect to the use of, or for damages resulting from the use of any information, apparatus, method, or process disclosed in this report.

As used in the above, "person acting on behalf of the Commission" includes any employee or contractor of the Commission, or employee of such contractor, to the extent that such employee or contractor of the Commission, or employee of such contractor prepares, disseminates, or provides access to, any information pursuant to his employment or contract with the Commission, or his employment with such contractor. 
Contract No. W-7405-eng-26

Director's Division

CIVIL DEFENSE RESEARCH PROJECT

INDEXES TO 563 UNCLASSIFIED DOCUMENTS

ON CTVTT, DFFERTSE

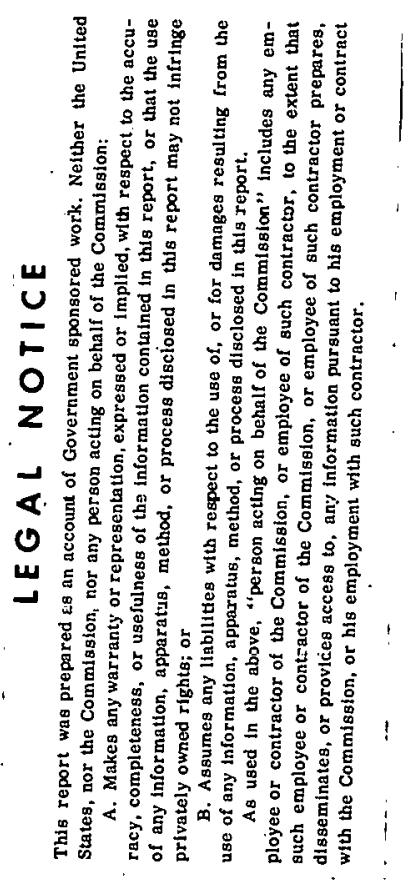

Prepared according to the terms of

Joanne S. Levey

Ann S. Klein

Bobbie-Jean Hatcher

Editors

Agreement No. OCD-PS-62-284 between the Office of Civil Defense and the U.S. Atomic Energy Commission, under Work Unit 4552B.

\title{
APRIL 1968
}

\author{
OAK RIDGE NATIONAL LABORATORY \\ Oak Ridge, Tennessee. \\ uperated by \\ UNION CARBIDE CORPORATION \\ for the \\ U.S. ATOMIC ENERGY COMMISSION
}


THIS PAGE

\section{WAS INTENTIONALLY LEFT BLANK}


TABLE OF CONTEINTS

$\underline{\text { Page }}$

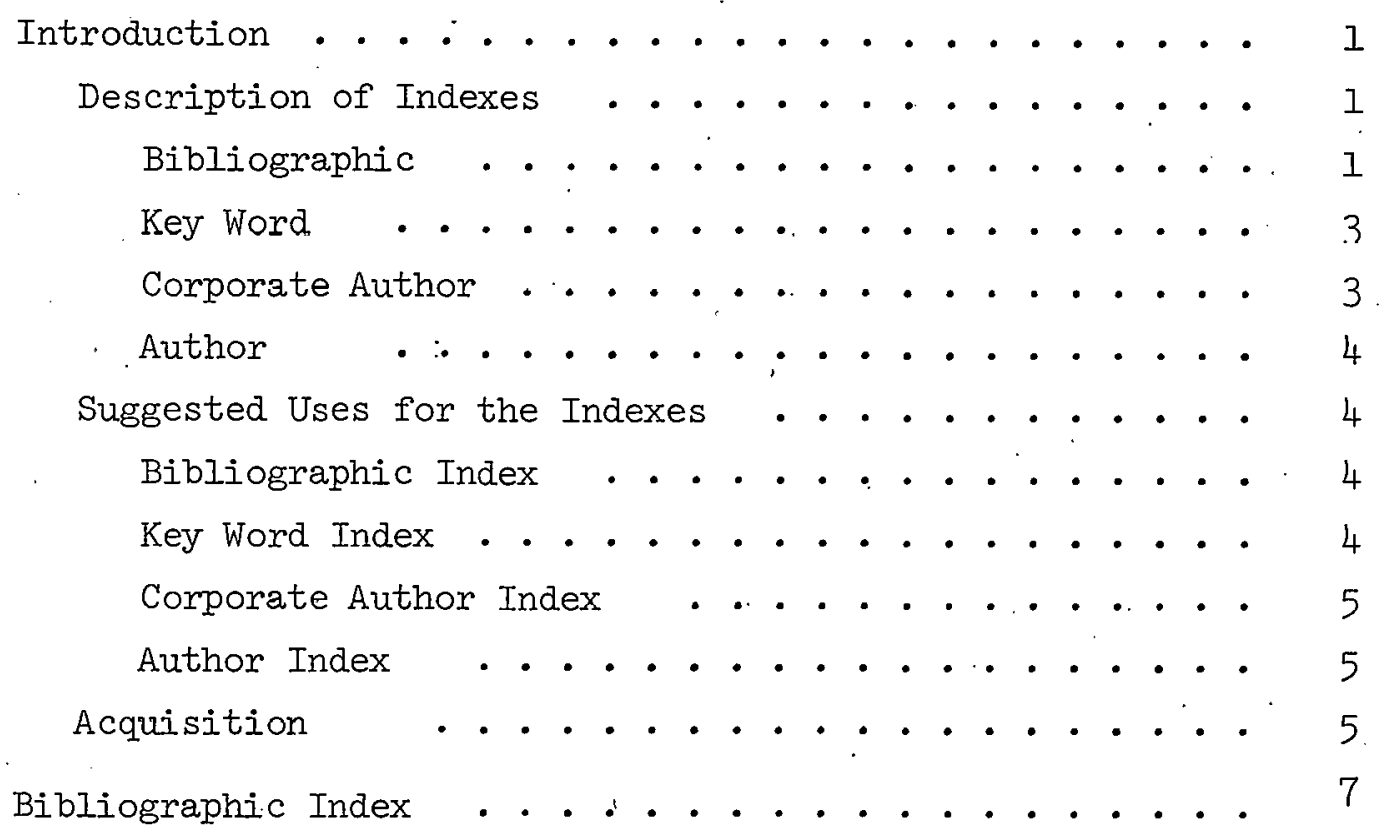

Key Word Index . . . . . . . . .... 75

Corporate Author Index . . . . . . . . . . . 159

Author Index . . . . . . . . . . . 167 
INDEXES TO 563 UNCLASSIFIED DOCUNENTS ON CIVIL DEFENSE

Joanne S. Levey, Ann S. Klein ${ }^{*}$, and Bobbie-Jean Hatcher, Editors

\section{INTRODUCTION}

Five hundred and sixty-three unclassified, unlimited-distribution documents $^{* *}$ from the Civil Defense Research Library are provided with four indexes: Bibliographic, Key Word, Corporate Author, and Author. They are described below.

\section{Description of Indexes}

\section{Bibliographic}

The Bibliographic Index lists the 563 documents according to the 22 subject fields and 188 groups provided by the cosATI ${ }^{1}$ Subject Category List (DOD--Modified). ${ }^{2}$ To begin to meet the special categorizing needs of the civil defense research literature, a twenty-third subject field and its five sublisted groups ${ }^{3}$ have been included.

In addition to the self-explanatory features (author, title, corporate author, etc.), each entry includes the following explanatory information.

*l'echnical Inf'ormation Division

** Indexes to the Civil Defense Research Library's classified and unclassified-limited-distribution documents will appear initially as ORNL-CD-3 and subsequently as ORNL-CD-odd number documents. They will carry the security classification CONFIDENTIAI

$I_{\text {Committee on Scientific and Technical Information of the Federal }}$ Council on Science and Technology

"CUSA'l'L Subject Category List (DOD--Modified), Defense Documentation Center, Defense Supply Agency, Alexandria, Virginia (1965)

3 Devised by Claire Nader, ORNL 


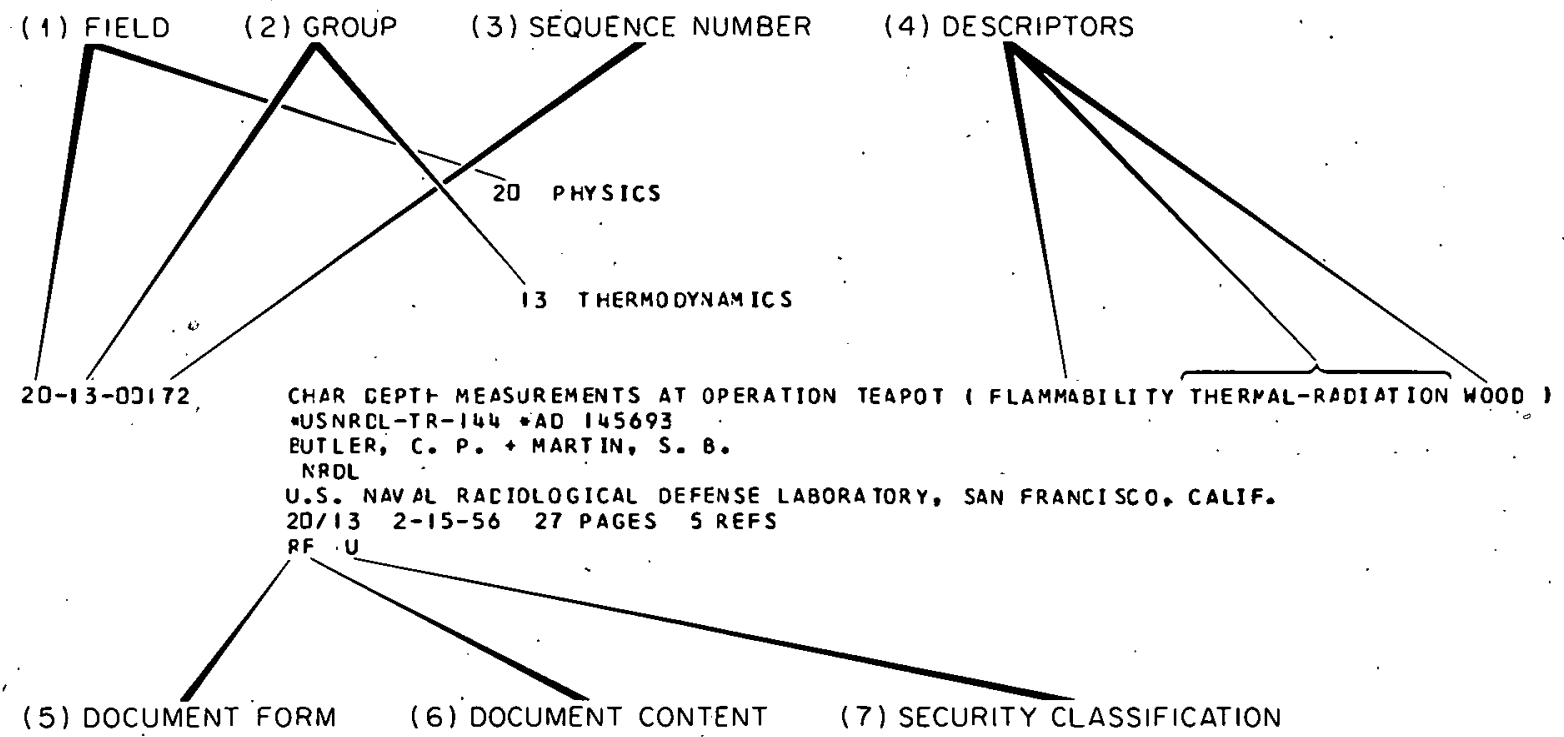
1 Field
2 Group
3 Arbitrary Sequence Number
4 Descriptors
5 Document Form ${ }^{4}$
B Book
$\bar{J}$ Journal
K Government Material
M Memorandum, Letters
P Patent
Q. Preprint
R Report
$X$ Other
Z Brochure

6 Document Content
A Bibliography
C Calculations

D Data Source, Handbook

E Research (Theoretical)

$\mathrm{F}$ Research (Experimental)

G General, Nontechnical

$\mathrm{H}$ Historical, Mainly

I Computers

L Social sciencè kesearch

IN News, Press Report

o other

S Symposium, Meeting Coverage

T. Topical Subject

U Progress Report

$V$ State-of-the-Art Review

$Y$ Trip Report

7 Security Classification

U Unclassified Unlimited

\footnotetext{
${ }^{4}$ Codes for Document Form and Document Content are adapted from the Document Types List of the Nuclear Fuel Technology Information Center (NUFTIC) at Oak Ridge National Laboratory
} 
For vocabulary control and compatibility with comparable systems, the descriptors added to the titles are selected to conform with those in three already existing thesauruses: Defense Documentation Center's Thesaurus of ASTIA Descriptors, ${ }^{5}$ Research Triangle Institute's Thesaurus of Civil Defense Descriptors, ${ }^{6}$ and the Engineers Joint Council's Thesaurus of Engineering Terms. 7

Key Word

The second index is a permuted Key Word Index (KWIC). Each significant word in the title of a document, together with each descriptive term by which the title has been extended, is printed in alphabetical order down the center of the page. Thus every title will appear in the index as many times as there are key words in the title.

When the title (with descriptors) is too long to fit on one line, the computer indicates the location of the omitted words by a slash (/). The user may quickly locate the complete title by checking the document number at the far right against that of the bibliographic entry in the first section.

Corporate Author

The third index lists alphabetically the names of the Corporate Authors which appear in the main bibliography. The user may link the corporate author, (or installation) with the entire bibliographic entry through the number at the right.

${ }^{5}$ Thesauru.s of ASTIA Descriptors, Second Edition, Armed Services Technical Information Agency, Arlington, Virginia (1962)

$6_{W}$. T. Herzog and J. E. Jenkins, A Thesaurus of Civil Defense Descriptors, Appendix $E$ of Final Report (Volume II) Civil Defense Information Systems Analysis, Research Triangle Institute, Durham, North Carolina

7 Thesaurus of Engineering Terms, Engineers Joint Council, New York (1964) 
Author

The fourth index lists the Authors whose names appear in the main bibliography. Again, as with the installations, the user may check the number at the right of each author against that of the bibliographic entry for a more complete description of the document. .

\section{Suggested Uses for the Indexes}

\section{Bibliographic Index}

1. To provide a rudimentary micro-abstract of the contents of the document through the use of descriptors attached to the title.

2. To indicate at'a glance the length and date of the document as $\dot{w e l l}$ as its form (report, journal, etc.) and nature (historical, bibliographical, etc.).

\section{Key Word Index}

1. To serve as a subject index to the 563 documents listed and.-when joined to subsequent indexes in the series -- to serve as a subject index to the entire ORNL civil defense research document collection.

2. To indicate the current research interests of the Civil Defense Research Project staff and -- over a period of time -- the scope of the Project's coverage. 8

3. To furnish descriptors which can serve as stepping stones to the collection of information in a given area. (For example, to help explore a subject as fully as possible, a selective list of the descriptors in the subject can be sent to an information center which specializes in this particular area, if such an information center exists.)

8 The index does not represent adequately. the research interests of the Project's'social scientists, who use books, monographs, and journals more frequently than documents. Also, the Civil Defense library occasionally receives a considerable number of documents in a research area as yet relatively unexplored by any member of. the staff. The descriptor Biological-Blast-Effects is an example of one such area. 
Corporate Author Index

To indicate the installations actively. engaged in research relevant to civil defense and to provide the nucleus for a referral-oriented information center on civil defense

Author Index

To indicate the individuals actively engaged in research relevant to civil defense

\section{Acquisition}

Since the ORNL Civil Defense Research Library is at present principally for the use of Project members, copies of the documents listed are not normally furnished to outside requestors. However, members of the library staff will gladly provide qualified users with information on how to obtain a document. 


\section{Bibliographic Index}

\section{AGRICULTURE}

$02-00-02103$

TRE AGRICLLTLRAL RESEARCH CENTER CF THE UNITEC STATES .DEPARTMENT OF AGRICULTURE AGR ICUL TURAL-ENG I NEER ING ANI NAL-HLSSANDRY CRCP-RESEARCH ENTOMOLOGY NUTR I TIOV WATER-CONSER VATION SOIL-CCNSERVATICA, \#AI $2-189$

L.S. DEPT. CF AGRICULTURE

U.S. DEPARTMENT OF AGRICULTURE

CZIOC 1966 C 37 PAGES D REFS

RE U

\section{Agricultural Chemistry}

$02-01-01615$

FARM CHEMICALS HANOBOOK I FERTILIZERS PESTICIDES EGUIPMENT AGRICULTURAL-CFEMISTRY I *A.L. 996

MEISTER PLBL CO

MEISTER PLELLISHING COMPANY, WILLCLGHBY, CHIC

QZIOI 1967 SSE PAGES

RC $L$

\section{Agricultural Economics}

$02-02-01616$

$02-02-[1617$

$02-02-01618$

$02-02-01619$

$02-02-01620$

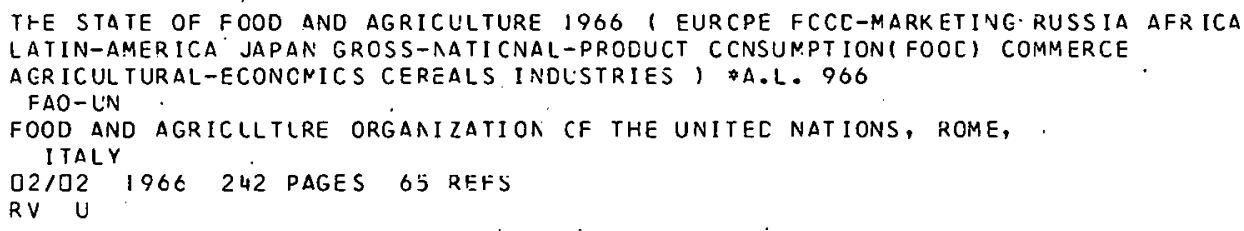

HANDBOOK OF AGRICLLTURAL CHARTS - 19661 POPULATICN AGRICULTURE URBAN-AREAS RURAL-AREAS COMMERCE MEAT RESOURCES CSREAL CCTTON VEGETAELES FRUITS FOODS, * AGR ICUL TLRE HANDBOOK NO. 335

L.5. DEPT. OF AGRICULTURE

U.S. DEPARTMENT OF AGRICULTURE, ECCNCNIC RESEARCH SERVICE $02 / 02$ 19E IS5 PAGES 157 FIGS

RC U

REgULATIONS GOVERIING THE REAT INSFECTICN OF THE UNITEC STATES DEPARTMEYT OF AGRICULTURE ( LEGISLATION) *C/MS-SRA-188

U.S. DEPT. OF AGRIC UL TURE

U.S. DEPAP.TMENT OF ACRICII ITIRF, CCASUMER ANC MARKETING SERVICE Q 2102 10- $\& 5 \quad 433$ PAGES [ REFS

RE $U$ 
$02-02-02109$

$02-02-02110$

$02-02-02111$

$02-02-02112$

$04-01-02113$

$04-02-02114$

$05-01-01621$

COMMODITY CREDIT CORPORATION WAREHCUSE RECEIFT WHEAT INVENTORY BY WAREHOUSE AVD LOCATION I AGRICULTURE STCRAGE SUPFLY-DEPOTS WAREFOUSES S.TCCKPILING ) *A.L. 1098 L.S. DEPT: OF AGRICULTURE U.S. DEPARTMENT OF AGRICULTURE 02102 10-14-66 141 PAGES

X $\mathrm{C}$

. URLGUAY LI VESTOCK AND MEAT INDUSTRY ( AGRICULTURE AGRICULTURAL-ECONOMICS I * FAS $M-1$ \& MORGAN, $Q$. M.

L.S. DEPT. OF AGRIC.ULTURE

U.S. DEPAR TMEN T OF AGRICULTURE, FCREIGN AGRICULTURAL SERV ICE Q $21025-67$ P PAGES 7 TABLES

RG. U

ARGENTINA LIVESTOCK ANO MEAT INDUSTRY I AGRICULTURE AGRICULTURAL-ECONOMICS 1 . *FAS $-M-182$

MORGAN, G. M.

U.S. DEPT. . OF AGRICULTURE

U.5. DEPARTMENI OF AGRICULTURE, FCREIGN AGRI CULTURAL SERVICE

$02 / 026-67$ IS PAGES 19 TABLES

$R G$ U

NEW ZEALAND - LI VESTOCK AND MEAT INDLSTRY ('AGRICULTURE AGRICUL TURAL-ECONOMICS ') *FAS-M- 184

GERRITY, M. V.

U.S. DEPT. OF AGRICULTURE

U.S. DEPARTMENT OF AGRICULTURE, FOREIGN AGRICULTURAL SERVICE

02/C2 5-67 1. PAGES I TABLES

RG U

\section{ATMOSPHERIC SCIENCES}

\section{Atmospheric Physics}

VALIDITY OF THE O2(-) NODEL FCR D REGICN RECCMBINATION 1 IONOSPHERE OXYGEN GAS-IONIZATION, AIRBURST ICNS POLAR-REGICNS ABSORPTION OZONE REACTION-KINETICS NUCLEAR-RADIATION COSMIC-RAYS ELECTRCMAGNETIC-WAVES PROPAGATION MODELSISIMULATIONS) I \#LMSC-2-12-66-6 \#AD 644354 KAUFMAN, A.

LOCKHEED MISSILES

LOCKHEED MISSILES AND SPACE COMPANY, PALO ALTO, CALIF.

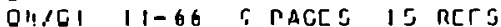
RF U

\subsection{Meteorology}

PROJECT SAND STORN - AN EXPERIMENTAL PRCGRAM IN ATMOSPHERIC DIFFUSION I ATMOSPHERE TRAN SPOR T-PROPERTIES EXPERINENTAL-CATA ATNCS PHERIC-MOT ION MET EOROLOGICAL-PARAMETERS ) $A A F C R L-65-6-49 * A D \quad 621658$

TAYLOR, J. H.

AF OFF AEROSPACE RES

AIR FORCE OFFICE CF AEROSFACE RESEARCH, RECFCRC, NASS $04 / 029-65$ I 16 PAGES 1.50 REFS

RF U

\section{BEHAVIORAL AND SOCIAL SCIENCES}

\section{Administration and Management}

SOME TRA INING IMPLICATIONS OF LARGE SHELTERS I SHELTER-MANAGEMENT MANAGEMENT-PLANNING PERSONNEL SHELTER-CCCUPANCY (S IMULAT ION) LEACERSHIP) * $\triangle I R-D-93 B I-9 / 66-F R$

BEND, E.

AMER INST RES

AMER ICAN INSTITUTES FOR RESEARCH; FITTSQURGH, PA. OE/U1.9-66 24 PAGES 5 REFS

RE U 
$05-01-01622$

$05-01-01623$

$05-01-01624$

$05-01-[1626$

$-$

$05-01-02115$

$05-01-02292$

$05-01-01625$

ALTERNATIVES IN WATER MANAGENENT ( RESCURCES (WATER) MANAGEMEVT-PLANNING DECISION-MAKING SOCIAL-SCIENCES SCIENTIFIC-RESEARCH UNITEC-STATES CISTRIBUTIOV; \#PL'BL. $14[8$

NAS-NRC

NATIONAL ACADEMY OF SCIENCES, NATICNAL RESEARCH COUNCIL,

WASHINGTON, D.C. $[S / G 1196052$ PAGES 3 REFS

RF U

A PROVISIONAL CONCEPT' OF ENERGENCY OPERATICNS UNCER NUCLEAR ATTACK 1 CIVIL-DEFENSE-OPERATING-PLANS FALLCUT-SHELTERS CCNSTRUCT ION-SL ANT ING OPERATIONS-RE SEARCH SHELTER-ALLOCATI CN COMMUNITY-SHELTER-PLANNING-PROGRAM MANAGEMENT-PLANNING , \#A.L. 1084

SIROPE, W. E.

$O C D$

OFFICE OF CIVIL DEFENSE

OS/OI 11-10-66 SE PAGES 6 FIGS

RE $U$

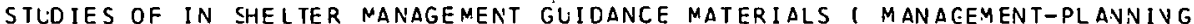
HAB I TABILI TY SHEL TER-OCC UPANCY INSTRUCTICN-MANUALS HANDQ.0OKS ) *AIR-D-93C1-9/66-FR BEND, E. + UNTER WAGNER, J. + NCINTYRE, F. F., JR.

AMLR INST RES

AMER ICAN INSTI TUTES, FOR RESEARCH, FITTSBURGH, PA. QE.

RF U

STATUS OF THE CIVIL DEFENSE PROJRAN I ORGANI ZATION CUAL-PURPOSE-SHELTERS FALLOUT-SHELTERS APPROPRIATIONS ADNINISTRATICN-ANC-MANAGEMENT, \#MP-46 OCD

OFFICE OF CIVIL DEFENSE, DEPARTMENT OF DEFENSE

$O S / O I$ I- 122 PAGES O REFS

RG U

AN EXPERIMENTAL STLDY OF INTEGRATEL GUICANCE FOR SHELTER MANAGEMENT I MANAGEMENT-PLANNING TRAINING SHELTER-OCCUPANCY EXPERIMENTAL-DATA । *AIR-DYSH $1121-916 t-F R$

SMITH, R. H. + BEND, E. + COLLINS, R. A.

JEFFREYS, F. 9 .

AMER INST RES

AMER ICAN INSTITUTES FOR RESFARCH, FITTSBURGH, PA. CSIOI 2-CE IET PAGES 7 REFS

RF U

SHELTER OCCUPANCY STUDIES 2. CONNLNITY FALLCUT SHELTERS HANDBOOK FOR UVTRAINED MANAGEMENT I SHELTER-MANAGENENT SHELTER-CCCUPANCY INSTRUCTION-MANUALS, $\$$ A.L. 1130 LNIV OF GEORGIA

UNIVERSITY OF GEORGIA, ATHENS, GA.

$05 / 01$ |2-3|-6t 145 PAGES TREFS

RC U

COST ANALYSIS FOR PLANNING PRGGRANNING BUDGETING COST BENEFIT STUCIES 1

COST-EFFEC TI VENES S MANAGENENT-ENGI AEERING MANAGEMENT-PL ANNING OPERAT IONS-RESEARCH MANAGEMENT-CONTROL-SYSTENS DECISICA-NAKING I \#AD 643472

MCCULLOUGH, J. D.

RAND

RAND CORRORATION, SANTA MGMIGA, GALIF.

QSE/OI 11-66 64 PAGES 18 FIGS 20 REFS

RF U

\section{Documentation and Information Technology}

$05-02-01627$

A DIRECTORY OF INFORMATION RESCURCES IN THE UNITEC STATES - PHYSICAL SCIENCES, B IOLOGICAL SCIENCES, ENGINEERING I.DATA, $\$$ A.L. 962 NAT REF CEN SCI TECH

NATIONAL REFERRAL CENTER FCR SCIENCE ANC TECHNOLOEY QITO2 1-CS 352 PACES $R C U$

$35-02-01628$ 
$05-02-0162.9$

$05-02-01630$

$05-02-02116$

$05-02-02117$

$05-02-02118$

$05-02 \div-2119$

$05-02-02120$

$05-02-02121$

$05-02-02122$

SPEC IAL I ZED SC IENCE INFORNATICN SERVICES IN THE UNITEC STATES - A DIRECTORY OF SELEC TED SPECIAL I ZED INFORMATICN SERVICES IN THE PHYSICAL AND BIOLOGICAL SCIENCES I RESOURCE SIINFORMA IION) DATA SCIENTIFIC-RESEARCH， \#NSF. 6I-t8

NAT SCI FOLND

NATIONAL SCIENCE FOUNDATICN, WASHINGTON, C.C. $05 / 02 \quad 11-61$ E2 8 PAGES

RD U

INFORMATION SYSTEN NETWORKS - LETS PROFIT FRCM WHAT WE KNOW I INFORMATION-RETRIEVAL DOCUMENTATION STA TE-OF-THE-ART-REVIEWS SYSTEMS-ENG INEER ING TECHN ICAL-INFORMA TION-CENTERS OATA-PROCESSING-SYSTEMS. MAN-MACHINE-SYSTEYS COMPUTERS ) $\$ \triangle F O S R-66-6873 * A D \quad 637488$

SWANSON, R. h.

AF OFF AEROSPACE RES

AIR FORCE OFFICE OF AEROSPACE RESEARCH, ARLINGTON, VA. CEIC2 6- GE 4 \& PAGE.S $217 \cdot$ RE.FS

RV U

DOCUMENT STORAGE AND RETRIEVAL TECHNIQUES - $\triangle P P E N C$ IX INDEXING TECHN IQUES, DESCR IPTION AND BACKGROUND I CCMNAND-AND-CCNTROL-SYSTEMS NAVAL -OP ERAT IONS

DATA-STORAGE-SYSTENS SUB JECT-INDEXING TEST-METHOCS INFORMATION-RETR IEVAL AUTOMATIC ECONOMICS, \#PRC-C-6.34A \#AD 414.713

BLACK, D. V.

PLANNING RESEARCH CORP

PLANNING RESEARCH CORPCRATICN, LCS ANGELES, CALIF.

$05 / 02$ 6-13-63 29 PAGES IO REFS

RF U

INDEX OF PLBLI SHED PAPERS I INDEXES REPCRTS COCUMENTATION PROFESS IONAL-PERSJNNEL SCIENTIFIC-RESEARCH ORGANIZATICNS, $\# A C .648767$

LAMBERT, C. G.

SYSTEM DEVELOPMENT CORP

SYSTEM DEVELOPMENT. CORPORATICN, SARTA NCNICA, CALIF:

$051022-15-67 \quad 234$ PAGES

RA U

FIVE YEAR S IN FOCLS - THE DOUGLAS DIRCRAFT COMPANY MECHANIZED INFORMATION SYSTEM I TECHN ICAL-INFORMA TION-CENTERS DOCLINENTTATION INFCRNATICN-RETRIEVAL AUTOMATION

L IBRAR IES AIRCRAF T-INDUSTRY SYSTENS-ENG INEER ING CATA-STORAGE-SYSTEMS COMPUITERS

DICTIONARIES, \#DCLGLAS PAPER-4J27 \#DD 646 D30

SMITH, F. R.

DOUGLAS AIRCRAFT

'DOUGLAS AIRCRAFT, INC., SANTA NONICA, CALIF.

CEIL2 10-7-66 22 PAGES 11 FIGS 4 REFS

$R G$ U

THE STATE OF THE ART OF CCORDINATE INDEXING I INCEXES INFORMATION-RETRIEVAL SUBJEC T-HEADINGS CLASSIFICATICN INFORMAT ION-THEORY COMPUTERS, ¥AC 275393 JASTFR, 1. .1. + MLRRAY, B. R. + TAL:BF, M.

DOCLIMENTATION INC

DOCUMENTATION INC CRPORATED, WASHINGTCN, D.C.

$05 / 02$ 2- 62 211 PAGES 212 REFS

R V U

DOD USER - NEEDS STUDY, PHASE II, FLCW OF.SCIENTIFIC AND TECHNICAL INFORMATION WITHIN THE DEFENSE INDUSTRY III. A. FREGUENCY CISTRIBUTIONS. AND CORRELATIOV 8. RELATIONSHIR AND COMPARI SCN I DEFAFTNENT-OF-CEFENSE DOCUMENTATION INFORMATION-RE TR I EVAL ENG INEERING-PERSCNNEL SCIENT IF IC-PERSOVNEL FL OW-CHARTING P SYCHOMETRICS TECHNICAL-INFORMATION-CENTERS JOB-ANALYSIS STATISTICAL-ANALYSIS MANAGEMEN T-ENG INEERING ) \#C6-2442/030(III) \#AC 649284

GOODMAN, A. F. + HCDGES, J. D., JR. + ANGALET, B. W.

MCCORD, R. B. + TAYLOR; C. C.

NORTH AMERICAN AVIATION

NORTH AMER ICAN A VI.ATION, INC., DCWNEY, CALIF.

. GE/O2 11-3E-6C.549 PAGES 3 FIGS 279 TAELES 8 REFS

RF U

THE ANALYSIS AND DESIGN OF INFORNATICN SYSTENS I TECHNICAL-INFORMAT ION-CENTERS SYSTEMS-ENG INEER ING DOCUNENTATICN CATA-STORAGE-SYSTEMS SUBJECT-IVDEXING INFORMATI ON-RETRIEVAL AUTCMATIC MANAGEMENT-ENGINEERING SYMPOSIA DATA-PROCESSING-SYSTEMS, $* S P-2655 * A D 645517$ BORKO, H.

SYSTEM DEVELOPMEAT CCRP

SYSTEM DEVELOPMENT CORPORATION, SANTA MONICA, CALIF. DS/O2 11-IC-66 21 PAGES 2 REFS

RF $L$

A BIBLIOGRAPHY ON INFORMATION SCIENCE ANC TECHNOLOGY FOR 1965 IV. 1 DOCUMENTATION 1 *TM-2625/GC4/OE \#AD 648562

NEELAND, $F$.

SYSTEM DE VELOPMENT CORP

SYSTEM DEVELOPNENT CORPORATION, SANTA NCNICA, CALIF.

$05 / 02$ 1-15-66 94 PAGES 406 PAGES

RA U 
$05-02-C 2123$

$05-02-02124$

$05-02-02125$

$05-03-01631$

$05-03-01632$

$05-03-01633$

$05-03-01634$

$05-03 \cdot 01635$

$05-03-01636$
A BIBLIOGRAPHY ON INF ORMATION SCIENCE ANC TECHNOLOGY FOR 1966 IV. 1 DOCUMEVTATION SCIENTIF IC-RESEARCH INFORNATICN-RETRIEVAL SCIENT IFIC-ORGANIZATIONS PERIDOICALS *TM-3008/CC4/DC *AD 649637

NEELAND, F

SYSTEM DE VE LOPMENT CORP

SYSTEM DEVELOPNENT CORPORATION, SAATA NONICA, CALIF.

05/02 1-16-67 93 PAGES 421 REFS

RA U

KEY WORDS AND THEIR USE CN OD FORN 1473 I REPORTS SUBJECT-IVDEXIVG

DEPAR TMENT-OF-DEFENSE DOCUNENTATIOA EFFECTIVENESS INFORMAT ION-RETRIEVAL, 316

RLDIN, I. S.

EAST COAST NICEP

EAST COAST NAVY INTERLABORATORY CCNMITTEE ON EDITING AND PUBL I SHING $0 E / 02$ 4-I-67 4 PAGES I REF

RD L

ANNUAL HISTORICAL SUMMARY - JULY-1,1965-JUNE-30,1966

TECHN ICAL-INFORMA TION-CENTERS ABSTRACTS COSTS DEPARTMENT-OF-OEFENSE

DATA-PROCE SSING-SYSTEMS DATA-STORAGE-SYSTENS INFORMAT ION-RETRIEVAL MICRJFICHE MANPOWER PRODUCTICN-CONTRCL HISTORY NILITARY-PUBLICATIONS REPRODUCT ION DOCUMENTATION, \#AD 645 5CD

REA, R. H

DDC

DEFENSE DOCUMENTATION CENTER, ALEXANDRIA, VA.

QSI02 ID-1-66 36 PAGES IO FIGS

RU U

\section{Economics}

UNIFORM SYSTEM OF ACCOUNTS FOR CLASS A AND B ELECTRIC UTILITIES I ECONOMICS ACCOUNTING REGULATIONS, \#A.L. IOO4

NAT ASSN RR UTIL COM

NATIONAL ASSOCIATION OF RAILROAD ANC UTILITIES COMMISSIONERS,

WASHINGTON, D.C.

$05 / 03$ I $95 E$ I 69 PAGES $C$ REFS

RD U

UNIFORM SYSTEM OF ACCOUNTS FDR CLASS A ANC B GAS UTILITIES I ECONOMICS $\triangle C C O U N T I N G$ REGULATIONS I *A.L. IOOS

NAT ASSN RR UTIL COM

NATIONAL ASSOCIATION OF RAILRCAD AND UTILITIES CCMMISSIONERS,

WA SHING TON, O.C.

CEI03 1958 212 PAGES 0 REFS

$C E / 03$
$R C U$

PROSPECTING IN ECONOMICS I SCIENTIFIC-RESEARCH RESCURCES MANPOWER INNOVATIOV PROJECTIONS MODELS, *A.L. 1975

FORD FOUNDATI CN

FORD FOUNDATION, NEH YORK, N.Y.

$0 \div 103$ 12-66 44 PAGES 13 REFS

RE $\mathrm{U}$

ECONOMIC. REPORT OF THE PRESIDENT - ANNUAL REPORT OF THE COUNCIL OF ECONOMIL ACVISERS I ECONOMICS UNI TED-STATES-GOVERNMENT POLITICAL-SCIENCE FOREIGN-POLICY I * A.L. 1061

L.S. EXEC OFF PRES

U.S. EXECLTIVE OFFICE OF THE PRESICENT

$0: 10 ? \quad 1-67 \quad 314$ PAGES 118 TABLES 0 RFFS

RD U

PLANNING IN A CAPITAI SIJRPLUS ECCNCNY - KUWAIT I MANAGEMENT-PLANNING OPERATIONS-RESEARCH ECONONICS DECISION-MAKING CONSERVAT ION DEPLET IUN PEIRULEUM MIDDLE-EAST, \$A.L. 973

LNIV OF COLORADC

UNIVERSITY OF COLCRADO, BCULEER, CCLC.

$051031966 \quad 16$ PAGES 45 REFS

RL $\quad U$

THE ALASKAN EARTHQUAKE - A CASE STLOY IN THE ECONCMICS OF CISASTLR I DAMAGE-A SSESSMENT DATA DECISI CN-NAKING RECOVERY HCUS ING FOCD FLOODS

CIVIL-DEFENSE-OPERATING-PLANS REPAIR CCNSTRUCT ION REAL-ESTATE REFUGEES BANKING INSURANCE LABDR LAh TRAFFIC I *IDA HG 65-4142

KUNREUTHER, H. C. + FIORE, E. S.

ICA

INSTITUTE FOR DEFENSE ANALYSES, WASHINGTCN, C.C.

Q5/03 2-66 174 PAGES 44 REFS

RF U 
$05-03-01638$

$.05-03-01639$

$05-03-01642$

$05-03-01643$

$05-03-01644$

$05-03-01645$

$05-03-01646$

$05-03-01647$

$05-03,01648$
U.S. SOURCES OF PETROLEUN ANO NATURAL GAS STATISTICS I RESOJRCES PETROLEUM-IVDUSTRY INDUSTRIES ECONOMICS, *A.L. 985

ROCQ, M. M.

SPECIAL LIBRARIES ASSN

SPECIAL LIBRARIES ASSOCIATION, NEW YCRK, N.Y.

$05 / 03$ 1961 94 PAGES 26 REFS

RD U

OCCUPATIONAL SKILLS AND CIVIL DEFENSE I INCUSTRIES RESOURCES( HUMAN) DISTRIBUTION VULNERAB ILITY LABCR ECONONICS ECCNCMIC-RECOVERY POSTATTACK OPERAT IOVS PJPULATION SLRVIVAL DAMAGE-A SSESSMENT CALIFCRAIA RECOVERY, \#SRI-MU-494:9-350-A

BROWN, S. L.

STANFORD RESEARCH INST

STANFORD RESEARCH INSTITUTE, MENLC PARK, CALIF.

$05 / 03$ 9-66 75 PAGES 7 TABLES 9 REFS

RF U

INTERNATIONAL CUSTCMS JOLRNAL - CANADA I LAW INTERNATIONAL-AFFAIRS TARIFFS

IMPOR T-DUTIES FOREIGN-TRADE CCNMERCE, \$PE-173 660

INTERNAT CUS TAR BUR

INTERNATIONAL CUSICMS TARIFFS BUREAU, BRUSSELS, BELGIUM

QS 103 10-66 187 PAGES 0 REFS

JC U

GAS FACTS - A STATISTICAL RECORD CF THE GAS UTILITY INDUSTRY IN 19651 UTILITIES RESER VES PRODUCTI CN TRANSNISSICN DISTRIBUT ION UNCERGROUNC-STORAGE SALES REVENUE

UTILIZATION FINANCING OPERATING-CCSTS CANADA CONSTRUCTION PRICES LABOR, $¥ 4 . L$. ICI7 AMER GAS ASSN

AMER ICAN GAS ASSOCIATIION, NEW YORK, N.Y.

$0 \leq / 031966253$ PAGES 225 TABLES 25 REFS

RE U

SOUTHERN PULPWCOD PRODUCTION, 1965 ( WOOC RESOURCES ECONOMICS WOOD-PULP

DISTRIBUTIION J \#PB 173659

WELCH, R. L.

SOUTHERN GOREST EXPER STA

SOUTHERN FOREST EXPERIMENT STATION, NEW ORLEANS, LA.?

DSIOZ 1966. 22 PAGES 7 FIGS 21 TABLES

RD U

NREC PROGRAMS FOR GAMING THE LCGISTICS OF NATIONAL SURV IVAL I SIMULATION DAMAGE-ASSESSMENT hARFARE RESOURCES INOUSTRIES VULNERABILITY ECONOMICS ;A.L. 1052 COKER, J. D. + GASKILL, I. E. + CLIVER, W. B.

NAT RES EVAL CENTER

NATIONAL RESOURCE EVALUATION CENTER

QS/O3 4-65 5I PAGES D REFS

RF U

IRON AND STEEL SCRAP CONSLNPTICN PRCBLENS I SALVAGE RESOURCES STOCKPILIVंG MOBILIZATION STRATEGIC-MATERIALS, $\$ A . L .9954$

DERR ICKSUN, G. F., EO.

L.S. DEPT. OF CONNERCE

U.S. DEPARTMENT ÓF COMMERCE, NATI CAAL BUREAU OF STANDAROS

[:S/C3 3-66 52 PÄGES. 41 REFS

RD U

PETROLEUM INDUSTRY OF GERMANY DURING THE WAR I MILITARY-STRATEGY ECONOMICS

PRODUCTION VULNERABILITY NANPChÉR RECOVERY. I \#RM-428 \#AC 210651

SKOGSTAD, A.

RAND

RAND CORPORATION, SÁNTA MCNICA, CALIF.

Q5/D? 4-26-50 42 PAGES 16 REFS

RF $\quad$ '

THE IMPORTANCE OF INDIVIDLAL INDUSTRIES FOR-CEFENSE PLANNING I SUPPLY-AND-DEMANO ECONOMICS RECOVERY POSTATTACK-CPERATIONS RESCURCES SURVIVAL INCUSTRIAL-PRODUCTION I \#P-2L.93 \#AD 641156

BEAR, D. V. T. + CLARK, P. G.

RAND

RAND CORPORATION, SANTA RCNICA, CALIF.

CEICZ 9-6L 22 PAGES 3 REFS

RG U

AN ANALYSIS OF MACHINE TOCL SUBSTITUTION POSSIBILITIES 1 INDUSTRIAL-EQUIPMENT ECONOMICS FEASIBILITY-STUDIES CLASSIFICATICN ) *RN-1512 *AC 87449

ROWE, A. J. + NARKCWITZ, H.

RAND

RAND CORPORATION, SANTA NCNICA, CALIF.

[SI $/ 03$ 6- $3[-55$. 95 PAGES 10 REFS

RD U . 
$05-03-01649$

$05-03-01650$

$05-03-01651$

$05-03-[1652$

$5-0 \geq-\tau 2126$

$05-03-02127$

$05-03-02128$

$05-0 \geq-02129$

$05-0 z-02130$
PROJECTIONS 1970 - INTERINOUSTRY RELATICNSHIPS, PCTENT I AL CEMAND, EMPLOYMENT I INDUSTRIES MANPOWER ECONCNICS GRCSS-NATI CNAL-PROCUCT SUPPLY-AN.C-DEMAND INDUSTRIAL-DROCUC TION SC I ENTI IFIC-ORGANIZAT IONS RESEARCH-PROGRAM-ADM IN ISTRATIDN OPERATIONS-RESEARCH PERSONNEL UNITED-STATES-GOVERNMENT COMMERCE CONSTRUCTION , * BULLLETIN NO. 1536

L.S. DEPT. OF LABCR

U.S. DEPARTMENT OF LABOR, BUREAU OF LABOR STATISTICS

DE 103 1966 155 PAGES 75 REFS

RD U

UNIFORM SYSTEM OF ACCOUNTS FOR CLASS A ANC B WATER UTILITIES I ECONOMICS REGULATIONS ACCOUNTING, \#A.L. 1003

NAT ASSN RR UTIL COM

NATIONAL ASSOCIATION OF RAILRCAD AND UTILITIES CCNMISSIONERS,

WASHINGTON, D. C

$05 / 03 \quad 1957 \quad 128$ PAGES D REFS

RO U

RECOVERY OF PE TROLEUM REFINERIES CCNTANINATEC EY FALLOUT I POSTATTACK-OPERATIOVS RADIOACTI VE-FALLOLT CONTANINATION INDUSTRIAL - PLANTS DECAY-RATES RESOURCESI SUR VIVAL) PROGRAMMING (COMPUTERS) REPAIR PERSCNNEL TRAINING COUNTERMEASURES, \#USNRDL-TR-656 \#AD 419334

MINVIELLE, L. + VAN HORN, K.

NRDL

U.S. NAVAL RADIOLGGICAL DEFENSE LABORATORY, SAN FRANCISCO, CALIF. $0 \leq 103$ 6-63 156 PAGES 18 REFS

RF U

ECONOMICS DEPARTMENT PUBLICATICNS, 1960-1965 - AN AUTHOR INDEX OF THE OPEN LITERATURE WITH ABSTRACTS ( BIBLI CGRAPHIES INDEXES), $\$ R M-2800-1$ (SUPP) $\$ A D 626930$ PORCH, H.

RAND

RAND CORPORATICN, SANTA NCNICA, CALIF:

$05 / 03 \quad 1-66^{\circ}$ I CO PAGES

RA U

INDUSTRIAL HARDENING CLASSIFICATICA - A NETHCDOLOCY FOR SIMPL IFYING THE EVALUATION OF HARDENING CCSTS II. APPENDIX B I INOUSTRIAL-EQUIPMENT INDUSTRIES FLOW-CHARTING SLRVIVAL RECOVERY ECONOMIC-RECCVERY PRODUCTS BLAST VULNERAEILITY AIR-BURST ECONOMICS EXPLOSION-EFFECTS PCSTATTACK-OFERATIONS, \#IDA/HG-66-5473

BICKLEY, L. J. + SACHS, A.

IDA.

INSTITUTE FOR DEFENSE ANALYSES, ARLINGTON, VA.

JSI03 IO- 66 145 PAGES IO8 FIGS

RF $U$

ECONOMIC VIABILITY AFTER THERNCNUCLEAR WAR - THE LIMITS OF FEASIBLE PRODUCTION ECONOMICS RECOVERY POSTATTACK-CPERATIONS SURVIVAL ECONOMIC-RECOVERY LABOR RESOUP.CES I $* R M-3436-P R$

WINTER, S. G., JR.

RAND

RAND CORPORATION, SANTA RCNICA, CALIF.

QS 103 9-63 224 PAGES 13 FIGS 25 TABLES 47 REFS

RF $L$

A SYSTEM ANALYSIS OF THE EFFECTS CF NUCLEAR ATTACK ON RAILROAD TRANSPORTATION IN THE CONTINENTAL UNITED STATES I SYSTEMS-ENGINEERING ECONOMICS RECOVERY

POSTATTACK-OPERATIONS DAMAGE-ASSESSNENT RACI DTICN-HAZARCS RADIOACTIVE-FALLOUT MODELS, \#SRI-IU-3C:84 \#PB 170595

DIXON, H. L. + HANEY, D. G. + JCNES, P. S.

STANFORO RESEARCH INST

STANFORD RE SEARCH INSTITUTE, NENLC PARK, CAL IF

$05 / 03$ 4-6E 254 PAGES 25 FIGS 58 TABLES

RF U

PARM SYSTEM MANUAL IV. RESOURCE CATA RCUTINE I CAMAGE-ASSESSMENT RESOURCES

DATA-PROCESSING, \#NREC- I 3

KOENIG, L. P. + ALLEN, J. T.

NAT RES EVAL CENTER

NATIONAL RESOURCE EVALUATIGN CENTER, WASHINGTOV, C.C.

$0 \leq / 03 \quad 10-62 \quad 180$ PAGES 30 FIGS

RO U

APPLICATIONS OF THE MANPOWER ANALYSIS SYSTEM I RESOURCES MODELS DAMAGE-ASSESSMENT POPULATION CASUALTIES PROGRAMMING (CCMPUTERS) GEOGRAPHY, \#NREC-30

M[CM, J. L.

NAT RES EVAL CENTER

NATIONAL RESOURCE EVALUATICN CENTER, WASHINGTON, C.C.

$0 \leq / 03 \quad 12-63$ 16 PAGES 3 FIGS 3 REFS

RI U 


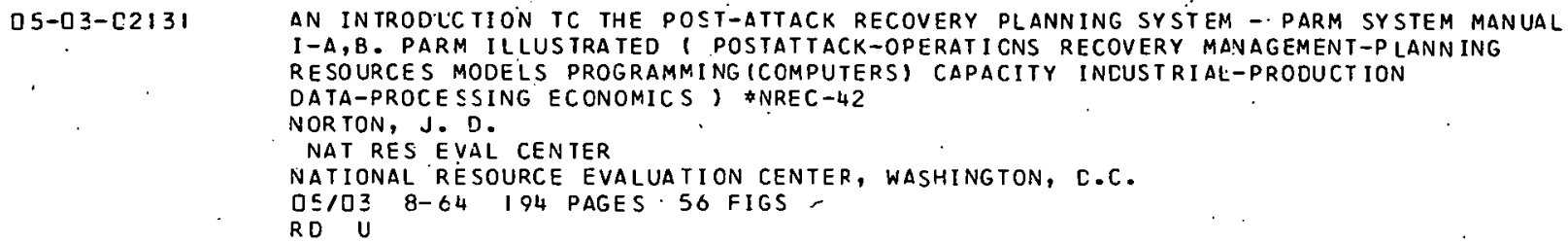

05-03-02132 RISK II ANALYSIS OF FOOD BALANCE I RESOURCES DAMAGE-ASSESSMENT VULNERABIL ITY POPULATION CASLALTIES FOOD-SUPPLIES GECGRAPHY, \#REC-29

GASKILL, I. E:

NAT RES EVAL CENTER

NATIONAL RESOURCE EVALUATION CENTER, WASHINGTON, [.C.

QST/O3 $11-63$ 14 PAGES 2 FIGS I TAELE

RF U

05-03-02133. COORDINATE CONVERSION FORNULAS FCR USE WITH WORLC WIDE RESOURCES GEODETIC PJSITION I GP I TO LNI IER SAL TRANSVERSE MERCATCR I UTM I ANC INVERSE ( MODELS GEOGRAPHY PROGRAMMING (COMPUTERS) MAPPING ) \$AREC-10

WILLIAMS, A. L. + MONCURE, P.

NAT RES EVAL CENTER

NATIONAL RESOURCE EVALUATION CENTER, WASHINGTON, [.C.

$05 / 03$ 6-62 81 PAGES 20 TABLES 14 REFS

$\mathrm{RC} \quad \mathrm{U}$

$05-03-02134$

WEAPOŃS-EFFEC TS WINO RADICACTIVE-FALLOUT CLINATOLOCY CASUALTIES F IRES

PROGRAMMING (CONPUTERS) BURST-CCNDITICNS BLAST YIELC GEOGRAPHY) \#NREC-2

LEWICKE, V. + GASKILL, I .. E.

NAT RES EVAL CENTER

NATIONAL RESOURCE EVALUATICN CENTER, WASHINGTON, C.C.

$05 / 03$ 8-61. 65 PAGES 8 FIGS 18 TABLES D REFS

R I U

05-02-02135 SURVIVAL I TEM ANALYSIS PRCGRAN I NLCLEAR-WARFARE CASUALTIES RESOURCES

DAMAGE-A SSE SSMENT FOOD LIGHT MEDICAL-SUPPLIES ELECTRIC-POWER SAN I TARY-EVG INEERING.

FUELS WATER-SUPPLIES MATHEMATICAL-ANALYSIS CLOTHING) ;NREC-4

KEEGAN, E. J.

NAT RES EVAL CENTER

NATIONAL RE SOURCE EVALUATICN CENTER, WASHINGTON, C.C.

I $5103 \quad 1.1-60$ (49 PAGES D REFS

RF U

05-02-02136 BIBLIOGRAPHY OF PLBLICATICNS I OANAGE-ASSESSNENT RESOURCES I \$NREC-121

NAT RES EVAL CEN IER

NATIONAL RESOURCE EVALUATION CENTER, WASHINGTON, C.C.

กSIח3 Q- G. 3I PAGFS

RA U

$05-03-02137$

RESOURCE DATA CATALOGUE I INFORMATION-SYSTEMS HOUSING RESOURCES MATHEMATICAL-MODELS COMPUTER S CASUALTIES DAMAGE-ASSE SSNENT WEAPONS-EFFECTS MANPOWER REFINERIES

VULNERAB ILITY MILITARY-FACILITIES ARNED-FORCES-SUPPLIES, COVERNMENT-FACILITIES

COMMUNICATIONS-FACILITIES TRANSPORTATI ON-PROPERT IES ELECTRIC-POWER-GENERATIJV

MILITARY-PER SONNEL POPULATION HOSPITALS SCHOOLS FCOD-SUPPLIES WATER-SUPOLIES

INDUSTRIAL-FLANTS FINANCE STOCKRILING STORAGE MANROHER, FNREC-134

NAT RES EVAL CENTER

NATIONAL RESOLRCE EVALUATICN CENTER, WASHINGTON, C.C.

$05103 \quad 5-63 \quad 275$ PAGES D REFS

RD U

05-03-02138 A LISTING OF TABLES AND PARAMETERS USED IN NREC CAMAGE ASSESSMENT PROGRAMS I VULNERABILITY, \#NREC-114111)

GASKILL, I. E. + HHITE, N. T. + BUYAS, G.

BARTYCZAK, J. A.

NAT RES EVAL CENTER

NATIONAL, RE SOURCE EVALUATICN CENTER, WASHINGTON, [.C.

- $05103 \quad 8-66$ 16 PAGES 52 REFS

RD. L

$55-03-02139$

ATTACK I - ATTACK PATTERN GENERATCR I SIMULATION CAMAGE-ASSESSMENT ATTACK-SCENAR IOS COMPUTERS HOUSING PROGRAMNING (CCRPUTERS) MILITARY-INSTALLATIONS POPULATION GOVERNMENT COMNUNICATICNS NILITARY-PERSONNEL HOSPITALS SCHOOL-QUILCINGS INDUSTRIAL-PROCUC TION, \#AREC-27

BRENER, H. A.

NAT RES EVAL CENTER

NATIONAL RESOURCE EVALUATICN CENTER, WASHINGTON, C.C. $05 / 039-63$ GL PAGES 5 FIG'S

R I U 
$05-03-02140$

$05-03-02141$

$05-03-02142$

$05-03-02143$

$05-] 2-[2144$

$05-0 \geq-0.2145$

$05-03-02146$

D5-03-02148

$05-03-02149$

$05-03-02150$
MAP IV - NREC SMALL SCALE MAPPING FROgRAM - NAPS CF CCNTINENTAL UNITED STATES I GEOGRAPHY RE SOLRCES DANAGE-ASSESSNENT WEAPCNS-EFFECTS NUCLEAR-WARFARE ATTACK-PATTERNS RAOI OACTIVE-FALLOUT, \#NREC- 12

ALLEN, J. T.

NAT RES EVAL CENTER

NATIONAL RESOURCE EVALUATICN CENTER, WASHINGTON, C.C. O5/O3 2-63 14 PAGES D REFS

$R F^{\circ} \quad{ }^{\circ}$

P IPEL INE NETHORK STUDY I DANAGE-ASSESSNENT MCDELS NUCLEAR-WARfARE PETROLEUM-PIPELINES GAS-PIPELINES TRANSPCRTATION， \#NREC- 28 NORTON, J. R.

NAT RES EVAL CENTER

NATIONAL RESOURCE EVALUATICN CENTER, WASHINGTON, C.C.

DSIO3 8-63 32 PAGES 3 FIGS 5 REFS

RF U

PARM SYSTEM MANUAL III-C. CAPACITY CCNCEPTS AND CATA I MORILIZATION MANAGEMENT-PLANNING RE SOURCES INDUSTRIAL-PROCUCTION INDUSTRIAL-PLANTS GEOGRAPHY * NREC -40

NORTON, J. O.

NAT RES EVAL CENTER

NATIONAL RESOURCE EVALUATION C.FNTFR, WASHINGTON, C.C. QS/O3 6-64 5E PAGES 23 REFS

RF $U$

A GENERAL NETWORK SIMULATION MGOEL I RESOURCES DISTRIBUTION-SERVICES SURPLUSES DATA-PROCESSING SHORTAGES WEAPCNS-EFFECTS， \#NREC-3I

CAGLEY, E. M.

NAT, RES EVAL CENTER

NATIONAL RESOURCE EVALUATICN CENTER, WASHINGTON, C.C. DSIO3 4-64 32 PAGES 4 FIGS J REFS

R I U

PARM PROJECT FINAL REPORT I DANAGE-ASSESSMENT RESOURCES MANAGEMEVT-PLANVING POSTATTACK-OPERATIONS RECCVERY DATA-PROCESSING INCUSTRI AL-PRODUCT.ION INVENTOR IES CAPACITY PROGR AMMING (COMPLTERS) ， NREC-44

NAT RES EVAL CEN TER

NATIONAL RESOURCE EVALUATICN CENTER, WASHINGTON, C.C. OE/03 8-64 2: PAGES O REFS RE 1

PARM SYSTEM MANUAL III-H. HOUSING, HOUSEHOLC SIZE CISTRIBUT ION, HOSPITALS, AVD AGRICULTURAL RESOLRCES ( CASUALTIES MEDICAL-PERSONNEL ) \#REC-39

NAT RES EVAL CENTER

VATIONAL RE SOURCE EVALUATION CENTER, WASHINGTCN, C.C. $0 S / 03$ S-64 4[ PAGES 2 TABLES 2 FIGS

RD $L$

STREAK IV - NREC RAPID DAMAGE ASSESSNENT PROGRAM I RESOURCES VULNERAGILITY PROGRAMMING (COMPUTERS) CASUALTIES FACILITIES PERS CNNEL ATTACK-PATTERNS WIND YIELO BIJRST-C.ONIITIONS BLAST RACIOACTIVE-FALLOUT FLOW-CHARTING I \#NREC-5

LAURE, L. G.

NAT RES EVAL CENTER

NATIONAL RESOURCE EVALUATICN CENTER, WASHINGTON, C.C. QE $103 \quad 3=64 \quad 116$ PAGES 17 FIGS 28 TABLES II RFFS

RI U

RESRURR.F DISTRIBUIION ( DAMAGE-ASSESSMENT RESOURCES VULNERABILITY PROGRAMMING (COMPUTERS) DATA-PRCCESSING-SYSTENS, \#NREC- 32

BORSODY, R. P. + MEEM, J. L.

NAT RES EVAL CENTER

NATIONAL RESOURCE EVALUATION CENTER, WASHINGTON, C.C. CE/G3 $3-6423$ PAGES O REFS

R I U

AN ANALYSIS OF THE RELIABILITY OF THE RISK I I COMPUTER STATISTICAL MOdEL - VAHICUS G? APPLICATION I RESOURCE $S$ VULNERAEILITY NUCLEAR-WARFARE WIND BLAST RADIOACTIVITY THEORY TESTS EXPERIMENTAL-DATA， \#AREC-22

NORSWORTHY, J. R. + NORTON, J. R.

NAT RES EVAL CENTER

NATIONAL RESOURCE EVALLAII IN CENTER, WASHINGTON, C.C. $0 S / 03 \quad 2-18-63$ 7t PAGES 12 TABLES 4 REFS

RI $\mathrm{I}$

PARM SYSTEM MANUAL $V$. FILE MAINTENANCE PRCCEDURES I RESOURCES DAMAGE-ASSESSMENT DATA-PROCESSING PROGRAMMING (CCNDUTERS) , \#NREC-14

PLGH, R. E. + LIPSNER, R. A.

NAT RES EVAL CENTER

NATIONAL RE SOURCE EVALUATICN CENTER, WASHINGTON, C.C.

05/ח3 9-63 160 PAGES 25 FIGS I RFFS

RC U 
$05-03-02151$

$05-03-02152$

$05-03-02153$

$05-03-02154$

$05-03-02155$

$05-03-02156$

$05-03-02157$

$05-03-02158$

$05-] 3-[2159$
PARM SYSTEM MANUAL III-B. PERSONAL CONSUMPT ION MOCEL I RECOVERY ECOVOMIC-RECOVERY DAMAGE-ASSESSMENT MODELS RESOURCES CAPACITY CECONTAMINATION REPAIR GOVERNMENT HOSP I TALS HOLSING AGRICULTURE CCNSTRUCTICN MANPOWER INOUSTRIAL-PROCUCTION COMMERCE TRANSPORTA TION, *NREC-15

SNYDER, E. M. + EDNONSTON, J. H.

NAT RES EVAL CENTER

NATIONAL RESOURCE EVALUATION CENTER, WASHINGTON; C.C. $05 / 03$ 1D- 63 230 PAGES 17 FIGS 18 TABLES 12 REFS

RD U

PARM SYSTEM MANUAL VI-2. PARM MAIN ROUTINE I RESOURCES CAPACITY DAMAGE-ASSESSMENT VULNERAB IL ITY EC ONOMIC-RECOVERY INCUSTRIAL-PRODUCTICN INVENT.ORIES BLAST, \#VREC- 38 WOOD, M. K. + ALLEN, J. T. + BERGSNAA, F. A.

EDMONSTON, J. H. + KOENIG, L. P. + PUGH, R. E.

NAT RES EVAL CENTER

NATIONAL RESOURCE EVALUATICN CENTER, WASHINGTON, C.C.

$05 / 03 \quad 3-64$ 4LO PAGES 75 FIGS

RD. U

PARM SYSTEM MANUAL III-O. NANPOWER ANC OCCUPATICNS I DAMAGE-ASSESSMENT RESOURCES VULNERAB ILITY MODELS NANUFACTURING NINING AGRICULTURE COMMERCE UT IL ITIES

TRANSPORTATION CONMUNICATIONS BANKING CONSTRUCTION MAINTENANCE REPAIR GOVERVMENT CASUALTIES CAPAC I TY, \#NREC-1?

BECKLES, M. P. + KAHLE, N. F. + KCENIG, L. P.

LIM, T. G. + RITZ, P. N. + WADDELL, R. M.

WOOO, M. K.

NAT RES EVAL CENTER

NATIONAL RE SOURCE EVALUATICN CENTEF, WASHINGTON, C.C.

$05 / 03 \cdot 12-63$ 230 PAGES 30 FIGS 5 REFS

RD $-U$

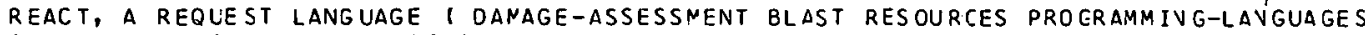
COMPUTER S CA SUALTIES RADI CACTIVE-FALLOUT. THERMAL-RACIAT ION I \#NREC-50

BACON, F. L. + MEEN, J. L.

NAT RES EVAL CENTER

NATIONAL RESOURCE EVALUATION CENTER, WASHINGTON, C.C.

$0 S / D 3$ 7-64 2[ PAGES 2 FIGS

RF U

READY I I RESOLRCES DAMAGE-ASSESSMENT LCGIC-CESIGR PRCGRAMMINGI COMPUTERS) MATHEMATICAL-MCDELS FLOW-CHARTING） \$NREC-24

ROWLAND, D. + CAGLEY, E.

NAT RES EVAL CENTER

NATIONAL RESOURCE EVALUATION CENTER, WASHINGTON, C.C. $05 / 03 \quad 7-31-63$ 1 20 PAGES 15 FIGS

R I U

TIME PHASED ACCESSIBILITY OF RESOURCES ( DAMAGE-ASSESSMENT WEAPONS-EFFECTS *NREC -25

R T Miv, M. H. + liLI UEK, U. A.

NAT RES EVAL CENTER

NATIONAL RESOURCE EVALUATION CENTER, WASHINGTON, C.C. $05 / 035-6 ? 31$ PAGES D REF S

R I U

ANNEX TO THIRD DRAFT OF READY I I RESOURCES ANALYSIS CATA-PROCESSING

DAMAGE-A SSESSMENT, \#NREC-24(1)

CAGLEY, E + DEVINF, P. + RCWLAND, D. + WILLIAMS, A.

NAT RES EVAL CENTER

NATIONAL RESOURCE EVALUATION CENTER, WASHINGTON, C.C.

$05 / 03$ 2-14-64 92 PAGES D REFS

RI $U$

ECONOMIC VIABILITY AFTER THERNCNUCLEAR WAR - THE LIMITS OF FEASIBLE PRODUCTION NUCL SAR-WARFARE SLRVIVAL ECONONICS PCPULATION AGRICULTURE INDUSTR IES

INUUS'TRIAL-PRODUCTION, $* R N-3436-P R * A D 426.996$

WINTER, S. G., JR .

RANU

RAND CORPORATION, SANTA NCNICA, CALIF.

Q $5 / 03$ 9- 63224 PAGES 13 FIGS 25 TABLES. 48 REFS

RF $U$

PARM SYSTEM MANUAL III-E. CAPACITY EXPANSION, RESTORATION AND CONVERSION 1

RESOURCES MANPOWER GE OGRAPHY INDUSTRIAL-PRODUCT I CN CAMAGE-ASSESSMENT, \#NREC- 35

FACCI, H. A. + LEWIS, R. A. + RITZ, P. M.

WADDEIII, R, M.

NAT RES EVAL CENTER

NATIONAL RESOURCE EVALUATION CENTER, WASHINGTON, C.C.

$0 S 1033-64$ 46 PAGES I REF

RF U 
$05-0 \geq-02160$

$05-0 \geq-02161$

$05-0 \geq-[2162$

$05-04-01658$

$05-04-01659$

$05-04-0166$

$05-04-01662$

$05-04-01063$

$05-04-01664$
DIMENSIONS OF SURVIVAL - POSTATTACK SURVIVAL CISPARITIES AND NATIONAL VIIARILITY 1 SOCIETAL-RECOVERY EC ONOMIC-RECCVERY RECOVERY ATTACK-SCENAR IOS WIND VUCLEAR-WARFARE RADIATION-INJURIES RADIOACTIVE-FALLOUT CASUALTIES CEMOGRAPHY AGE LABOR UREAN-AREAS MODELS RURAL-AREA S MANPOKER I \#RN-514D-TAB HANUN IAN, N.

RAND

RAND CORPORATION, SANTA NCNICA, CALIF.

DS/03 11-66 214 PAGES 23 FIGS IOU REFS

$R F U$.

REGIONALISM IN ASIA I ECONCMIC-CCNCITIONS POPULATION AGRICULTURE POL ITICAL-SC IENCE REGIONAL-PLANNING INDIA INTERNATICNAL-AFFAIRS COMNERCE , *A.L. 1152 PAUL, A.

ASIA FOUNDATION

ASIA FOUNDATION, SAN FRANCISCC, CALIF.

$05 / 03 \quad 196724$ PAGES D REFS

$X \in \quad U$

INDUSTRIAL HARDENING CLASSIFICATICA - A METHCCOLOCY FOR SIMPLIFYING THE EVALUATION OF HARDENING COSTS I. TEXT AND APPENDIX A I INDUSTRIAL-EQUIPMENT BLAST INDUSTRIES SURVIVAL RECOVERY ECONOMIC-RECOVERY PRCDUCTS VULNERABILITY EXPLOS ION-EFFECTS A IRBURST POSTA TTACK-OPERATICNS ECCACNICS, *IDA/FG-66-5472

RIC.KI.FY, L, J. + SACHS, A.

IDA

INSTITUTE FOR DEFENSE ANALYSES, ARLINGTON, VVA. $05 / 03$ 10-66 S9 PAGES I3 REFS

RF L

\section{History, Law, and Political Science}

UTILIZATION OF SOCIAL RESEARCH IN SHAPING POLICY CECISIONS 1 SOCIAL-SCIENCES MANAGEMENT-PLANNING UNITED-STATES-GOVERNMENT POLITICAL-SCIENCE ECONOMICS DECISION-MAKING LAK, \#P-2289 \#AD $63474 \%$

KEC SKEME TI, P.

RAND

KAND CORPORATION, SANTA NCNICA, CALIF.

$0 \subseteq 104$ 4-24-61 14 PAGES 5 RFFS

RL $U$

TOWARD A FLTLRE FCR PLANNING I MANAGEMENT-PLANNING CECISION-MAK ING

POL ITICAL-SC IENCE GOVERNMENT (FOREIGN) SOCIAL-COMNUNICAT ION LEADERSHIP ) *SP-2022

*AD $635 \quad 1$ C

$\triangle C E L$ SON, $M$.

SYSTEM DEVELOPMEAT CORP

SYSTEM DEVELOPMENT CORPORATION, SAMTA NCNICA, CALIF.

OE 104 6- $t E$ 17 PAGES 4 REFS

$R G \quad U$

THE OTHER WAR IN VIETNAM I TECHNICAL-ASSISTANCE FOREIGN-POLICY ECONOMIC-AID I *A.L. $1[82$

AGENCY INTERNAT DEVEL

$\triangle G E N C Y$ FOR INTERNATIONAL DEVELCPNEAT, WASHINETON, D.C.

$05 / 04 \quad 196654$ PAGES D REFS

KF U

STUDIES IN PROGRESS OR RECENTLY CCNPLETEC - ARMS CONTROL ANO CISARMAMEVT 1 CUMMUNIST-CHINA ECCNOMICS CONFLICT-RESOLUTION HISTORY INTERNATIONAL-AFFA IRS POL ITICAL-SCIENCE METHODOLOGY LAW ) \#ACL-6

U.S. DEPT. OF STATE

U.S. OEPARTMENT OF STATE, CFFICE CF EXTERNAL RESEARCH US/LI4 II-6.S 58 PAGES

RA 4

STUDIES IN PROGRE $: S$ UR RECENTLY CCNPLETED - ARMS CCNTROL AND CISARMAMENT B IBL IOGRAPHIES EC CNOMICS CCNMUNIST-CHINA CONFLICT-RESOLUT ION HISTORY LAW INTERNATIONAL-AFFAIRS POLITICAL-SCIENCE METHCDOLCGY I \#ACD-8

U.S. DEPT OF STATE

U.S. UEPAK IMEN I UF SIATE, OFFICE CF EXTERNAL RESEARCH $05,04 \quad 11-66 \quad 52$ PAGES

RA U

STLDIES IN PROGRESS OR RECENTLY CCNPLETEC - $\triangle R M S$ CCNTROL AND CISARMAMENT 1 COMMUNIST-CHINA ECONOMICS CCNFLICT-RESCLUTION HISTORY INT SRNAT IOVAL-AFFA IRS POLITICAL-SCIENCE METHODCLCGY LAW， \#ACL-7

U.S. DEPT. OF STATE

U.S. DEPARTMENT OF STATE, OFFICE CF EXTERNAL RESEARCH $0 S / 04$ 5-66 5L PAGE S 
05-04-01665

05-04-01671

05-04-01672

$05-04-02165$

$05-04-[2166$

$05-04-02167$

J5-04-02168

05-04-02169

$05-04-02170$

STUDIES IN PROGRESS OR RECENTLY CCNPLETED -. ARMS CONTROL ANO DISARMAMENT I B IBL IOGR APHIES EC CNOMICS CONFLICT-RESOLUTI ICN CONNUNIST-CHINA HISTORY LAW INTERNATIONAL-AFFAIRS ME THCDOLCGY FCLITICAL-SCIENCE, \#ACE-5

U.S. DEPT. OF STATE

U.S. DEPARTMENT OF STATE, OFFICE OF EXTERNAL RESEARCH

$05 / 04 \quad 11-65 \quad 51$ PAGES

RA U

U.S. CIVIL DEFENSE BEFORE 1950 - THE ROOTS OF PUBLIC LAW 920 I LEGISLATION SOCIAL-SC IENCES HISTORY CIVIL-DEFENSE-CPERAT ING-PL ANS CIVIL-DEFENSE-SYSTEMS MANAGEMENT-PLANNI NG DECI SI CN-MAKING CBJECTIVES PIIPI Tr.-OPINTON POI ITICAL-SCIENCF EXECUTIVE-BRANCH LOCAL-GOVERNMENTS STATE-GOVERNMENTS LEADERSHIP ATTITUDES )

* IDA /HQ- 65-3756

JORDAN, N .

IDA

INSTITUTE FOR DEFENSE ANALYSES, WASHINGTCN, C.C.

O5/0 4 5-66 196 PAGES 32 REFS

RH U

ON THE ESSENCE OF A NULCLEAR MISSILE WAR I RUSSIA POLITICAL-SCIENCE NUCLEAR-WARFARE BALLISTIC-MISSILES COLD-KAR FOREIGN-POLICY PROPAGANDA ATTITUDES COMMUNISM

* L T T - 65-104

RYBKIN, E.

RAND

RAND CORPORATION, . SANTA NCNICA, CALIF.

$05104 \quad 12-65$ 18 PAGES 13 REFS

RE $U$

BUDGET ESTIMATES FOR THE FINANCIAL YEAR 1967 AND INFORMATICN ANNEXES 1

UN ITED-NAYIONS COSTS INTERNATICNAL-AFFAIRS, \#UN-5-A/6305

LNITED NATIONS

UNITED NATIONS, NE.h YORK, N.Y.

$05 / 041966.212$ PAGES 157 TABLES

RO U

RESEARCH ON ACCEPTABILITY OF A NATIONAL SHELTER POLICY 1 'OPERATIONS-RESEARCH CIVIL-DEFENSE-SYSTEMS ATTITUOES FALLOUT-SHELTERS STRESS (PSYCHOLOGY)

THREAT-PERCEPTION BEHAVIOR. MODELS CCMMUNICATION CCGNITIVE-CECISION-MAKING INNOVATION, \#A.L. 1192

WITHEY, S. + BERLC, D.

LNIV OF MICHIGAN

UNIVERSITY OF MICHIGAN, ANN ARBOR, MICH.

$05 / 04$ IE-6I 20 PAGES O REFS

RL. $U$

VIETNAM - WHY I FCREIGN-PCLICY PCLITICAL-SCIENCE , \#A.L.. 1117

REPORTER MAGAZINE

REPORTER MAGAZINE COMPANY, NEW YCRK, N.Y.

CESTO4 1966 G3'PAGES U REFS

$\times T$ U

DEVELOPMENT OF UTILITY THEORY FOR ARMS CCNTRCL ANC CISARMAMENT I GAME-THEORY MATHEMATICAL-MODELS SIMULATION, \#FAR-396I(I)

ALMANN, R. J. + HARSANYI, J. C. + NASCHLER, N.

OBRIEN, G. + RADNER, R. + SHUBIK, N.

MATHEMATICA

MATHEMATICA, PRINCETON, .N. J.

$05 / 04$ 6-30-66 286 PAGES 36 REFS

$R L \cdot U$

DEVELOPMENT OF UTILITY THEORY FOR ARNS CCNTRCL ANC CISARMAMENT I GAME-THEORY SIMULATION, \#FAR-3961(1I)

ALIMANN, R. J. + HARSANYI, J. C. + MASCHLER; $\mathrm{N}$.

OBRIEN, G. + RADNER, R. + SHUSIK, N.

MATHEMATICA

MATHEMATICA, PRINCETON, N.J.

$05 / 04$ 6-30-66 21 PAGES 8 REFS

RL $\quad U$

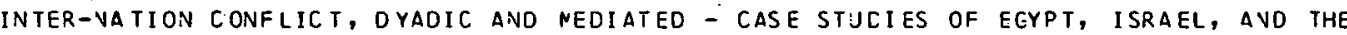
UNI TED NATIONS AT FIVE POINTS IN.TIME ( INTERNAT ICNAL-AFFAIRS POL IT ICAL-SCIENCE FOREIGN-POLICY THREAT-PERCEPTION'SUEZ-CRISIS ATTITUDES MICCLE-EAST METHODOLOGY ITEORY CON TENT-ANALYSI SICCNPUTERSI HISTORY I \#FAR-4D47

BRODY, R. A. + HOLSTI, O. R.

STANFORD LNIV.

STANFORD UNI VERSITY, STANFORD, CALIF.

CS 104 10-66 186 PAGES 20 TABLES 14 FIGS 120 REFS

RL $\quad U$ 
$05-04-0217$

$05-04-02172$

$05-04-02173$

$05-05-01673$

$05-09-[1675$

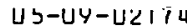

$05-09-\cap 2175$

$05-09-02176$

MODEL STUDY OF ESCALATION - VOLUME 3 I ARMS-CONTRCL BIBLIOGRAPHIES METHODOLDGY WAR-GAMES MODELS, \#FAR-3960

ACKOFF, R - L. + ALDERSON, $\mathrm{H}_{*}+$ + ANDERSON, J.

EMSHOFF, J.R. + GOMBERG, W. + MARTIN, N. W., JR.

NAGIN, R. P. + SISSON, R. L. + STEFHAN, P. D.

UNIV OF PENNSYLVANIA

UN IVERSITY OF PENNSYLVANIA, PHILADELPHIA, PA.

05104 3-1-66 14[ PAGES 34 REFS

RF U

SOLIDARITY IS THE BANNER - TWO RESCLUTIONS ON INTERNATIONAL AFFAIRS I ARMS-CONTROL COLD-WAR NSTO VIETNAM RED-CHINA FOREIGN-POLICY FOREIGN-AIC LABOR UNITED-NATIOVS WAGES, $\$$.L. IIS4

LNITED ALTO WORKERS

UNITED AUTO WORKERS, INTERNATIONAL AFFAIRS DEPARTNENT, WASHINGTON,

D.C.

T5/04 5-66 6I PAGES D REFS

$X \in \quad U$

SOVIET MILITARY TRANSLATICNS NUMBER 380 - TWENTY-FIFTH ANNIVERSARY OF THE BATTLE OF MOSCOW 1 L'SSR HISTORY CONNUNISN PRCPAGANCA, $J P R S-40,931$ TT-67-31.573

MALAKHOV, M. M.

JPRS

JOINT PUBLICATIONS RESEARCH SERVICES, WASHINGTON, D.C.

QS/O4 5-Q-67 5 PAGES C REFS

RE U

\section{Human Factors Engineering}

THE UTILIZATION OF HUMAN FACTORS INFORMATION BY CESIGNERS 1 HUMAV-EVG INEERIVG DECISION-MAKING DESIGN ENGINEERING-PERSONNEL SPECIFICATIONS HANDBOOKS TESTS, \#AD $642[57$

MEISTER, D. + FARK, U. E.

BUNKER RANO COQP

BUNKER-RAMO CORPORATION, CANCGA PARK, CALIF.

05/05 9-16-66 $9 E$ PAGES 10 FIGS 7 REFS

RF $U$

\section{Personnel Selection, Training, and Evaluation}

AN EVALUATION OF THE ROLE CF FEDERAL PERSONNEL IN RECRU IT ING SHELTER MAVACERS I SHEL TER-MANAGENENT UNI TED-STATES-GCVERNNENT (PERSONNEL)

ADMINISTRATION-AND-MANAGENENT URBAA-DREAS COSTS, \#AIR-C93E(1)-9/66-FR

JEFFREYS, F. B. + SMITH, R. W.

AMER INST RES

AMER ICAN INSTI TUTES FOR RESEARCH, FITTSBURGH, PA.

$05 / 09$ 9-66 38 PAGES 2 REFS

RF 1

STATE WORKMEN COMPENSATI OR LAHS, LCGISLATICA LAECR STATE-COVEONMENTS ACMIN I STRA TI CN-AND-MANAGENENT INSUFANCE, \#USCL-16I

U.S. DEPT. OF LABOR

U.S. OEPAR TMENT OF LABOR, BUREAU CF LABOR ST ANDARCS

DE/R9 9-6L 83 PAGES 17 TARLES

$R C U$

LAND TENURE AND REBELLION - A STATISTICAL ANALYSIS OF FACTORS AFFECTING GOVERNMENT CONTROL IN SOUTH VIETNAM ( POLITICAL-SCIENCE SOCICLCGY ECONOMICS) \#KM-5IEI-ARPA MITCHELL, E. J.

RANO

RAND CORPORATION, SANTA MCNILA, LALIF.

DEIO9 O-67 3I PAGES 7 FIGS I TABLE

RL U

SOVIET MILITARY IRANSLATICNS NUMBER 384 - CONBAT TRAINING METHODS AND THEIR SHORTCOMINGS DESCRIBED ( LSSR MILITARY-TRAINING ARMED-FORCES (FOREIGN) LEACERSHIP MIL I TARY-PER SONNEL OFFICER-PERSONNEL) \#JPRS -41,308 \#TT-67-31948 DAVIDENKO, H. V.

JPRS

JOINT PUBLICATIONS RESEARCH SERVICES, WASHINGTON, C. C.

DEIT9 6-8-67 5 PAGES 0 REFS

RE U 


\section{10. Psychology (Individual and Group Behavior)}

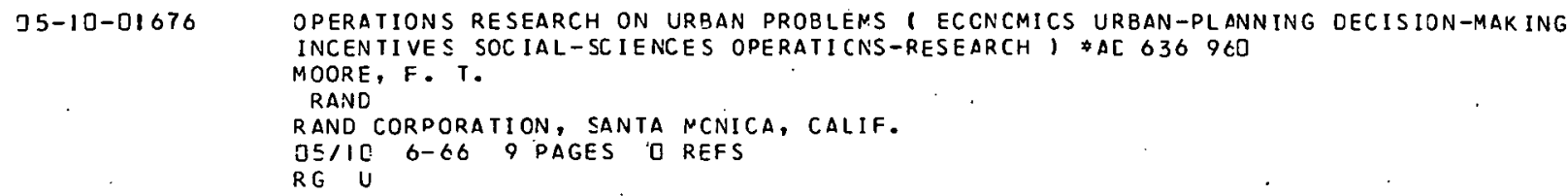

05-10-01677 CIVIL DEFENSE BIBLIOGRAPHY - A CCMFILATION OF REFERENCES REL-EVANT TO THE STUDY OF SOCIETAL RECOVERY FROM NUCLEAR ATTACK I STRESS (PSYCHOLOCY) GROUP-CYNAMICS SOC IAL-P SYCHOLOGY BEHAVI CR DISASTERS PUBLIC-CPINICN ATTITUCES METHODOLOGY WEAPONS-EFFECTS COMMUNICATION-SYSTEMS ECCNOMIC-RECOVERY MILITARY-STRATEGY, \#A.L. I 9.95

HLMAN SC IENCES RESEARCH

HLMAN SC IENCES RESEARCH, INC., MCLEAN, VA. $05 / 10 \quad 1-66 \quad 75$ PAGES

RA U

AMERICANS VIEWS ON CIVIL DEFENSE IN THE CCLC WAR CCNTEXT - 1966 I ATTITUDES PLBLIC-OPINION SURVEYS DI SARMANENT CIVIL-DEFENSE-SYSTEMS VIETNAM SHEL TER-ALLOCATION ; $\# 0 C D-O S-63-48$

NEHNEVAJSA, J.

UNIV OF PIITSBURGH

UNI VERSITY OF PITTSBURGH, PITTSBUREK, PA. OSIIO 12-66 I5O PAGES 94 TABLES D REFS

RL $\quad U$

THE CIVIL DEFENSE DEBATE \& CIVIL-DEFENSE-SYSTEMS ATTITUDES POL IT ICAL-SCIENCE PLBLIC-OPINION HI STORY COSTSIATTITUDES) INTERNAT ICNAL-AFFAIRS EFFECTIVEVESS, *A.L.
I [T3

WEAVER, L

MICHIGAN STATE LINIV

MICHIGAN STATE UNIVERSITY, EAST LANSING, MICF. $05 / 10196775$ PAGES 21 REFS

RG U

$35-10-01680$

RESEARCH DATA FRON SHELTER OCCUPANCY EXERCISES I HARITARILITY DATA-PROCE SSING-SYSTEMS TRAINING NETHOCGLOGY QUESTIONNAIRES SHELTER-MANAGEMENT A TTITUDE S , \#AIR-C 66-12/66-FR

COLLINS, R. A. + BEND, E.

AMER INST RES

AMERICAN INSTI TUTES FOR RESEARCH, FITTSEURGH, PA. $05 / 10.12-66 \quad 131$ PAGES 96 TABLES 2 REFS

RL $\quad U$

MENTAL HEALTH IMPL̈ICATIONS IN CIVILIAN ENERGENCIES I EMOT IONS ATT ITUUES FEA? STRE SS(P SYCHOL CGY) CA SUALTIES NOTIVAT TON PIIRLIC-INFORMATION TRAINING REACTION (PSYCHOLOGY) ADMINISTRATICA-AND-NANAGEMENT, *PHS-3II

ERICKSON, H. M. + NYSWANDER, D. B. + ZIMMERMAN, K. A. SKEELS, H. M.

l.S. DEPT. OF HEAL ED WEL

U.S. DEPARTMENT OF HEALTH, EDUCATICN AND WELFARE DS/1O 5-53 25 PAGES 90 REFS RE U

$05-10-01683$

PUBLIC RESPONSE TC THE NORTHEASTERN POWER BLACKOUT I BEHAVIOR ELECTR IC-POWER-PRCDUC TION (FAILURE) ATTITUDES PUELIC-OPINION PUBL IC-INFORMATION SLRVEYS DEMOGRAPHY COMMUNICATICN-SYSTEMS LEACERSHIP, \#A.L. 1055 NORC

NATIONAL OPINICN RESEARCH CENTER, CHICAGC, ILL. OSIIE IC- 66 107 PAGES 26 REFS

$R L \quad L$

$05-10-01684$

AN EXPERIMENTAL ANALYSIS CF SELECTED PROBLEMS OF L.ARGF. SHFITFR MANAGFMFNT, ENVIRONMENTAL THREAT, AND SMALL SHELTER HABITABILITY UNCER CONCIT IONS OF STRESS 1 SHEL TER-OCCUPANC Y FEASIB I LI TY-STUDIES UNCERW ATER(SHELTERS) LEADER SHIP CONFINED-ENVIRONMENTS STRESS(PSYCHCLOGY) SUPERVISICN ) \#AIR-D93A(1/2)-9/66-FR HALE, J. F. + NEAGLEY, D. E. + SNITH, R. W. DAVIS, R. L.

AMER IN ST RES

AMERICAN INSTI TUTES FOR RESEAREH, FITTSBURGH, PA. O5/10 9-6E 189 PAGES ID REFS

RF U.

SIMULMATICS CCRP

SIMULMATIC $\subseteq$ CORPORATION, NEW YORK, N.Y. - $05 / 10$ 12- 60 S2 PAGES D REFS

RL U 
$05-10-02177$

$05-10-02178$

$05-10-02179$

$05-10-02180$

$95-10-42181$

$05-10-02182$

$05-10-02183$

$05-10-[2184$

SHEL TER ASSIGNMENT CONCEPT - A STUCY IN PUBL IC ACCEPTANCE I SHELTER-ALLOCATION ATTITUDES CIVIL-DEFENSE-SYSTENS CCLD-WAR SURVEYS CULTURE DEMOGRAPHY SOCIOLOGY POPULATION DISARMANENT, *A.L. 1210

NEHNEVAJSA, J.

LNIV OF PI ITSBURGH

UNIVERSITY OF PITTSBURGH, PITTSBURGH, PA.

$05 / 10$ 4-67 87 PAGES 47 TABLES O REFS

RL $\quad U$

A METHOOOLOGICAL STUDY OF BEHAVICRAL ANC SEMANTIC CIFFERENTIAL SCALES RELEVANT TO INTERCUL TURAL NEGCTIATIONS 1 ATTITLDES BEHAVIOR NETHOCOLOEY SEMAVTICS

SOCIAL-SCIENCES PCLITICAL-SCIENCE CULTURE COCPERATION ) *FAR-4048

DAVIS, E. E.

LNIV OF ILLINCIS

UNIVERSI.TY OF ILLINOIS, URBANA, ILL.

QSIIO 6- EC 7[ PAGES 26 REFS

RL $\quad U$

SPECULATION ON THE STRUCTLRE OF INTERPERSONAL INTENTIONS I BEHAVIOR MOTIVATION CLLTURE RESPONSE REACTION(PSYCHOLCGY), \#FAR-4049

$O S G O O D, C$. E.

LNIV OF ILLINCIS

UNIVERSITY OF ILLINOIS, URBANA, ILL.

DS/ID 9- 6 C 27 PAGES 7 REFS

RI. II

CONSUMER BEHAVIOR AND WORKER PARTICIPATICN IN RECCVERY ACTIVITIES I

POSTATTACK-OPERATIONS SOCIETAL-RECCVERY ECCNCMIC-RECOVERY ATTITUDES MANPOWER MOTIVATION ECONOMICS SURVIVAL DISTRIBUTICN STRESS (PSYCHOLOEY) FOOC, $\# H S R-R Z-67 / 5-T I$

CHENAULT, h. h. + NORCLIE, P. G.

HUMAN SCIENCES RESEARCH

HLMAN SCIENCES RESEARCH, INC., MCLEAN, VA.

$0 S / I C 2-67$ 6S PAGES 28 REFS

RL U

ATTITLDES OF TROOPS IN THE TRCPICS I TROPICAL-REGICNS MIL ITARY-PERSOVNEL FOJD CLOTHING ENVIRONMENT EDUCATION ARNY-LCUIPNENT REACTIONIPSYCHOLOGY)

SOCIAL-PSYCHOL OGY ARMED-FORCES-OPERATIONS LOGISTICS AGING(PHYSIOLOEY)

* TR-67-57-PR \#AD 649540

LEE, R. J.

ROWLAND

ROWLAND AND CONPANY, HADDCNFIELD, $\mathrm{M} . J$.

DS 110 2-67 96 PAGES 47 REFS

$R F U$

MODEL STUDY OF ESCALATION II . SECTICNS 2-5 ( BEHAVIOR CONFLICT ARMS-COVTROL ARTIFICIAL-REALI TY HYPOTHESE S-FORNL'LATI ON CCNFLICT-RESOLUTION GAMES

MODEL SISIMLLATIONS) MATHENATICAL-ANALYS IS, \#FAR-3960III)

ACKOFF, R. L. + ALOERSON, W. + ANDERSON, J.

EMSHOFF, J. R. + GCMBERG, W. + MARTIN, M. W., JR.

NAGIN, R. P. + SISSON, R. L. + STEFHAN, P. D.

UNIV OF PENNSYL VANIA

UNIVERSITY OF PENNSYLVANIA, PHILADELFHIA, PA.

OSIIC 3-1-66 84 PAGES 13 REFS

RL $\quad U$

MODEL STUDY OF ESCALATION I SUMMARY I CONTLICT ARTITICIAL..RLALITY

CONFL ICT-RE SOLLTI CN COOPERATI I IN INTERNATICNAL-RELATIONS ARNS-CONTROL BEHAVIOR,

* FAR-3960(I)

$\triangle C K O F F, R_{-}$L. + ALDERSON, $W_{0}+$ ANDERSON, J

EMSHOFF, J. R. + GOMBERG, H. + MARIIN, M. W. , JR.

NAGIN, R. P. + SISSON, R. L. + STEFHAN, P. D.

UNIV OF PENNSYLVANIA

UNI VERSITY OF PENASYLVANIA, PHILACELPHIA, PA.

$05 / 10$ 3-1-66 40 PAGES II REFS

RL U

SHELTER OCCLPANCY. STUDIES I. FINAL REPCRT I FALLCUT-SHELTERS COVFIVED-ENVIZJVMENTS STOCKPIL ING SHELTER-MANAGENENT HANCBCOKS HAE ITAEIL ITY NUTRITION

SANI TARY-ENGINEER ING MEDICAL-SUPPLIES DEFECTIONS TRAINING SLEEP WATER FOO2

RECREATION EXERCI SE NOISE RELIGION TEMPERATURE-CCATROL VENTILATION, *A.L. 11 I

HAMMES, J. A. + AMEARN, T. R.

LNIV OF GEORGIA

UNIVERSITY OF GEORGIA, ATHENS, GA.

DSIIO I9E 376 PAGES 71 REFS

RF U 


\section{Sociology}

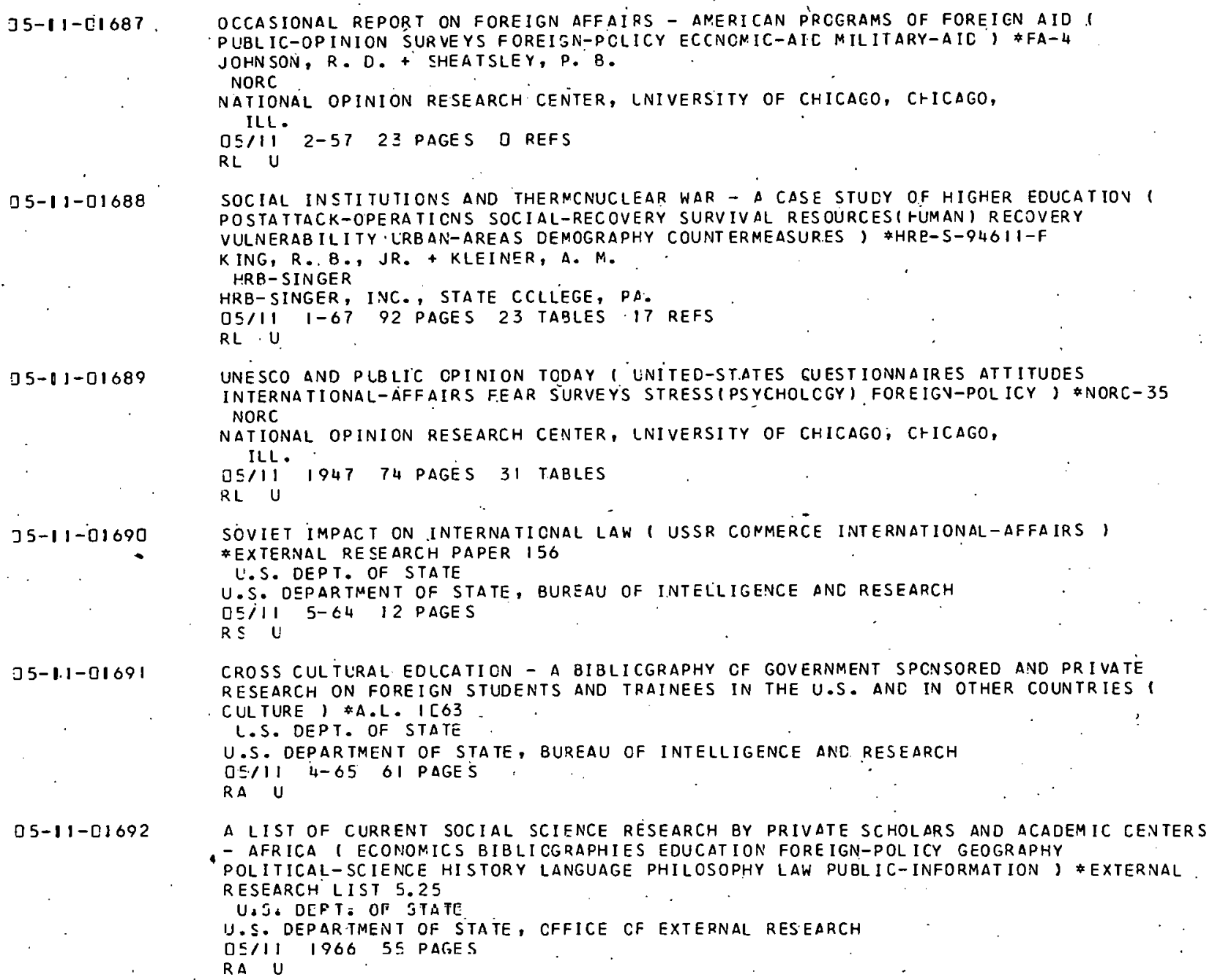

$05-11-01692$

A LIST OF CURRENT SOCIAL SCIENCE RESEARCH BY PRIVATE SCHOLARS AND ACADEMIC CENTERS - AFRICA I ECONOMICS BIBLICGRAPHIES EDUCATION FOREIGN-POL ICY GEOGRAPHY

POLITICAL-SCIENCE HISTORY LANGUAGE PHILOSOPHY LAW PUBLIC-INFORMATION ) *EXTERNAL RESEARCH LIST 5.25

U.J. DEFT: OF STATE

U.S. DEPARTMENT OF STATE, CFFICE CF EXTERNAL RESEARCH DSIII 1966 SS PASES

RA U

$05-11-0.1693$

A LIST OF CURRENT SOCIAL SCIENCE RESEARCH. BY PRIVATE SCHOLARS AND ACADEMIC CEVTERS - INTERNATIONAL AFFAIRS I BIBLIOGRAPHIES ATOMIC-ENERGY COMMUVICAT IONS LABOR COMMUNISM ECONOMICS AGRICULTURAL-ECONOMICS FINANCE INDUSTRIES COMMERCE EDLCATION POLITICAL-SCIENCE GE OGRAPHY LANGUAGE MILITARY-AFFAIRS PHILCSOPHY. LAW POPULATION PUBL IC-INFORMA TION, \#EXTERNAL RESEARCH LIST 7.25

l.S. DEPT. OF STATE

U.S. DEPARTMENT OF STATE, OFFICE OF EXTERNAL RESEARCH $05 / 111966^{\circ} 111$ PAGES RA U

$05-11-01694$

A LIST OF CURRENT SOCIAL SCIENCE RESEARCH BY. PRIVATE SCHOLARS AND ACADEMIC GENTERS - WESTERN EUROPE; GREAT BRITAIN ANC CANAOA ( BIGLIOGRAPHIES FRANCE GERMANY ITALY) * external research list 3.25

U.S. DEPT. OF STATE

U.S. DEPARTMENT OF STATE, CFFICE CF EXTERNAL RESEARCH

QSIII 1966 120 PAGES

RA U

05-11-01695 A LIST OF CURRENT SOCIAL SCIENCE RESEARCH BY PRIVATE SCHOLARS AND ACADEMIC CENTERS - MIDOLE EAST I BIBLIOGRAPHIES ECCNCMICS EDUCATICN POLITICAL -SCIENCE GEJ GRAPHY HISTORY LANGLAGE RELIGION, *EXTERAAL RESEARCH LIST 4.25 L.S. DEPT. GF STATE

U.S. DEPARTMENT OF STATE, OFFICE OF EXTERNAL RESEARCH $0 \leq 111$ I $966 \quad 2 E$ PAGES RA U 


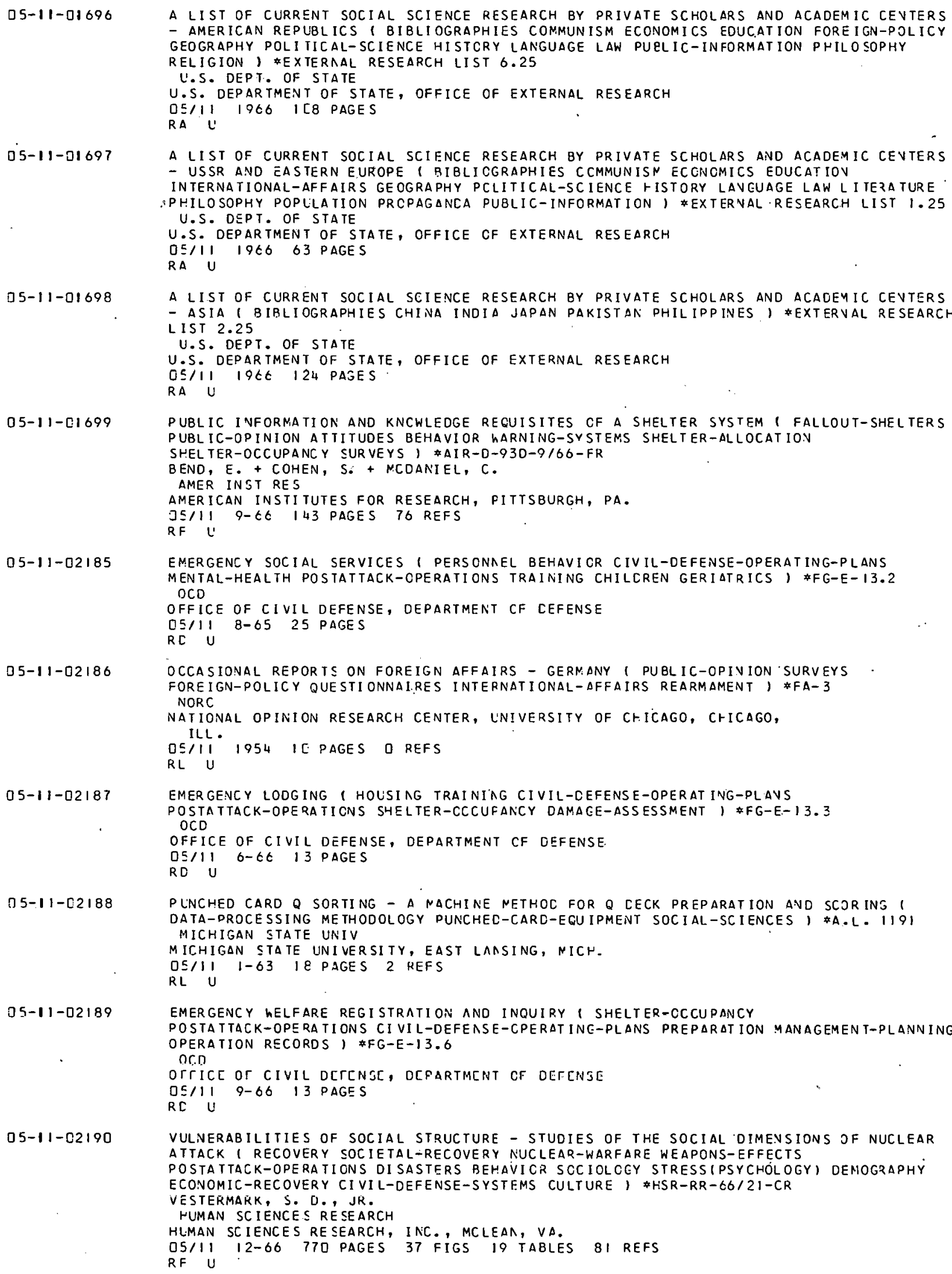


$05-11-02191$

$05-11-02192$

$05-11-02193$

05-11-02194

05-1.1-02195

$05-11-02196$

$05-11-02197$

J5-i1-02198

5 5-11-02199

THE FEASIBILITY OF DEVELOPING STANCARD CESCRIPTIONS OF POSTATTACK. SITUATIONS. SOC IETAL-RECOVERY NE THODOLOGY PROJECTIONS RECOVERY MANAGEMENT -PLANNING

POSTATTACK-OPERATIONS SOC.IAL-SYSTENS STANDARD-SITUAT.IONAL-CASES, \#HSR-RR-67/8-SD NORDLIE, P. G.

HLMAN SC IENCES RESEARCH

HUMAN SCIENCES RRE SEARCH, INC., MCLEAN, VA.

QS 111 6-67 36 PAGES 3 FIGS

RL $U$

THE FACTOR ANALYSIS OF Q DATA \& PUNCHED-CARDS STAT ISTICAL-ANALYSIS METHODOLOGY SOCIAL-SCIENCES CCRRELATICN VARIABLES） \$A.L. 1190

DANBURY, T.

MICHIGAN STATE UNIV

MICHIGAN STATE UNIVERSITY, EAST LANSING, MICH.

O5III 1953. 2 I PAGES 4 REFS

RF U

METHODOLOGY FOR ASSESSING TOTAL VULNERABILITY I SYSTEMS-ANALYSIS POLITICAL-SCIENCE ECONOMICS SOCIOLOGY SOCIETAL-RECOVERY DAMAGE-ASSESSMENT ATTACK-SCENARIOSI

*OCD-PS- 6L-2CI

DRESCH, F. W. + ELLIS, H.

STANFORD RESEARCH INST

STANFORD RESEARCH INSTITUTE, MENLC. PARK, CALIF.

$05 / 11$ 8- $t$ t 171 PAGES 2 FIGS 30 TABLES 81 REFS

RF U

NORC SOCIAL RESEARCH 1941-1964 - AN INVENTORY OF STUDIES AND PUBL ICATIOVS IN SOCIAL RESEARCH -I SOCIAL-SCIENCES SURVEYS BIBLICGRAPHIES ATTITULES GROUP-DYNAMICS

SOCIAL-P SYCHOLOGY PUBLIC-CPINICN NETHODCLOGY THECRY ECUCAT IOV HEALTH

WELFARE-SERVICES CCCUPATICNS PROFESSICNS COMMUNICAT ION-SYSTEYS TRAINING ECOVOMICS INTERNATIONAL-AFFAIRS POLITICAL-SCIENCE, \#A.L. 1 IOO

ALLSWANG, J. M. + ROVA, P.

NORC

NATIONAL OPINION RESEARCH CENTER, UNIVERSITY OF CFICAGO, CHICAGO,

ILL.

$05 / 11$ 1964 8E PAGES

RA U

CAN THE UNITED NATIONŚ ORGANI ZATICA PREVENT WARS I PEACE-KEEPING PUBL IC-OPIVION INTERNATIONAL-AFFAIRS SURVEYS ARNS-CCNTRCL, \#NORC-29

NORC

NATIONAL OPINION RESEARCH CENTER, UNIVERSITY OF CENVER, CENVER, COL'O.

QSIII 194620 PAGES 24 RQEFS

RL U

THE ONLY THING WE HAVE TO FEAR IS FEAR ITSEL'́ - WHAT THE PEOPLE KNOW AND THINK ABOUT THE BOMB AND ATONIC. ENERGY PUBLIC-CPINION ATT ITUDES SURVEYS QUESTIONNAIRES PEACE-KEEPING NUCLEAR-WARFARE ARMS-CONTROL， *A.L. I097

COPANCLLY, G. M.

NORC

NATIONAL OPINION RESEARCH CENTER, UNIVERSITY OF CENVER, DENVER,

COLO.

O5/II 6-56'75 PAGES 0 REFS

B IBL IOGRAPHY OF PLBLICATICNS, $1741-1960$ \& HEALTH PUEL IC-OPIN ION WELFARE-SERVICES SOCIAL PSYCHCLOGY AGING OCCUPATICNS PROFESSICNS MANPOWER L LBOR PCLITICAL-SCIENCE ELUCATION GROUP-DYNAMICS THEORY METHCDCLCGY, \#A.L. 1099

NORC

NATIONAL OPINION RESEARCH CENTER, LNIVERSITY OF CHICAGO, CHICAGO, ILL.

$05 / 11^{\circ} 12-66^{\prime} 39$ PAGES

RA' U

OCCASIONAL REPORT ON FOREIGN AFFAIFS - UNITEC NATICNS I INTERNAT IONAL-AFFAIRS PUBL IC-OPINION SURVEYS FOREIGN-PCLICY GUESTICNNAIRES PEACE-KEEPING USSR, NORC

NATIONAL OPINION RESEARCH CENTER, LNIVERSITY OF CHICAGO, CHICAGO,

ILL.
CISII 195312 PAGES D REFS
RL U

OCCASIONAL REPORT ON FOREIGN AFFAIRS - UNITEC NATICNS $($ INTERNATIONAL-AFFAIRS

SLRVEYS QLESIIONNAIRES PUBLIC-CPINICN FCREIGN-POLICY, \#FA-2

NORC

NATIONAL OPINION RESEARCH CENTER, LNIVERSITY OF CHICAGO, CHICAGO,

ILLIII 195412 PAGES 0 REFS

$R L$ U 
TABLES FOR YULES G ASSOCIATION CCEFFICIENT FCR PAIRS CF PERCENTAGES STATISTICAL-ANALYSIS SCCIAL-SCIENCES, \#A.L. 1396.

DAVIS, J.A. + GILNAN, R. + SCHICK, J.

NORC

NATIONAL OPINION RESEARCH CENTER, LNIVERSITY OF CHICAGO, CHICAGO,

ILL. 196521 PAGES 3 REFS

RD U

CIVIL DEFENSE IN POSTATTACK SOCIETY - A SUMMARY REPORT FROM A RESEARCH PROGRAM 1 POSTATTACK-OPERATIONS VULNERABILITY DISASTERS SOCIIETAL-RECCVERY RECOVERY SOCIOLOGY STRESS(PSYCHOLOGY), \#HSR-RR-67/2NE

NORDLIE, P. G. + VESTERMARK, S. C., JR.

HUMAN SCIENCE S RESEARCH

HUMAN SCIENCES RESEARCH, INC., MCLEAN, VA.

$0 \leq / 11 \quad 2-67$ 5E PAGES 26 REFS

$R L \quad U$

$05-11-02202$

VULNERABILITIES OF SOCIAL STRUCTURE - STUCIES OF THE SOCIAL DIMENSIONS OF NUCLEAR ATTACK I SOCIETAL-RECOVERY REC OVERY DENCGRAPHY ECCNCMICS PCL ITICAL-SCIEVCE STRESS(PSYCHOLCGY) ME THODCLOGY MANAGEMENT - PL ANNING, \#HSR-RR-66/21-CR SUMMAZY HEER, D. M. + SMELSER, N. J. + SWEARER, H. R .

VESTERMARK, 5. U., JR. + hINTER, S. G., JR.

HUMAN SCIENCES RESEARCH

HLMAN SCIENCES RE SEARCH, INC., MCLEAA, VA. $[5 / 11$ 12-66 40 PAGES 4 REFS

RL 4

$05-11-02203$

SPECIAL PROBLEMS OF CHILDREN IN CIVIL DEFENSE PLANNING I POSTATTACK-OPERATIOVS MANAGEMENT-PLANNING STRE SS (PSYCHCLCGY) SHELTERS SCHOOLS PUEL IC-IVFORMATION EOLCATION ORPHAN-CARE J

LL'TZ, F. h.

HLUMAN SCIENCES RESEARCH

HUMAN SC IENCES RE SEARCH, INC., MCLEAN, VA.

QE/II 2-67 49 PAGES 87 REFS

$\mathrm{RL} \quad$ II

\section{BIOLOGICAL AND MEDICAL SCIENCIES}

\section{Biology}

$06-03-02204$

BMD BIOMEDICAL COMPUTER PROGRANS I CCMPUTERS (PROGRAMMING) CATA-PROCESSIVG-SYSTEMS MULTIVARIATE-ANALYSIS STATISTICAL-ANALYSIS VARIANCE REGRESSION-ANALYSIS MEDICINE BIOLOGY, *A.L. I 123

DIXON, h, J. J

UNIV OF CALIF

UNIVERSITY OF CALIFORNIA, SCHCOL CF NECICINE, LOS ANCELES, CALIH.

DE/D3 9-1-65 619 PAGES 64 REFS

RI U

\section{Clinical Medicine}

$06-05-01701$

THE PATHOLOSY OF -ZINC DEFICIENCY ( NETABCLISN DEFICIENCY-CISEASES TISSUESIEIOLOGY) TESTES RATS MORPHCLOGY(BICLOSY) HISTCLCGY ESCPHAGUS SKIN I \#AC $63848 C$

FOLLIS, R. H.

ARMED FORCES INST PATH

ARMEO FORCES INSTITUTE DF PATHOLOGY, WASHINGTON, C.C.

DE/OS 1964 II PAGES 21 REFS

RF U

$06-05-[1 / 2$

PLAGUE IN THE LMI TED STATFS - AN ASSESSNENT CF ITS SIGNIFICAVCE AS A PRJBLEY FOLLOWING A THERMCNUCLEAR WAR I PASTEURELLA-PEST IS BIULOGICAL -WARFARE-ACENTS EPIDEMIOLOGY PLBLIC-HEALTH POSTATTACK-OPERATIONS INMUNOLOGY DISEASE-VECTORS CHEMOTHERAPY MEDICINE RODENTS.) $\#$ RN-4968-TAB MITCHELL, H. H.

RAND

RAND CORPORATICN, SANTA MCNICA, CALIF.

06105 6- 6 C 49 PAGES 15 REFS

RF U - 


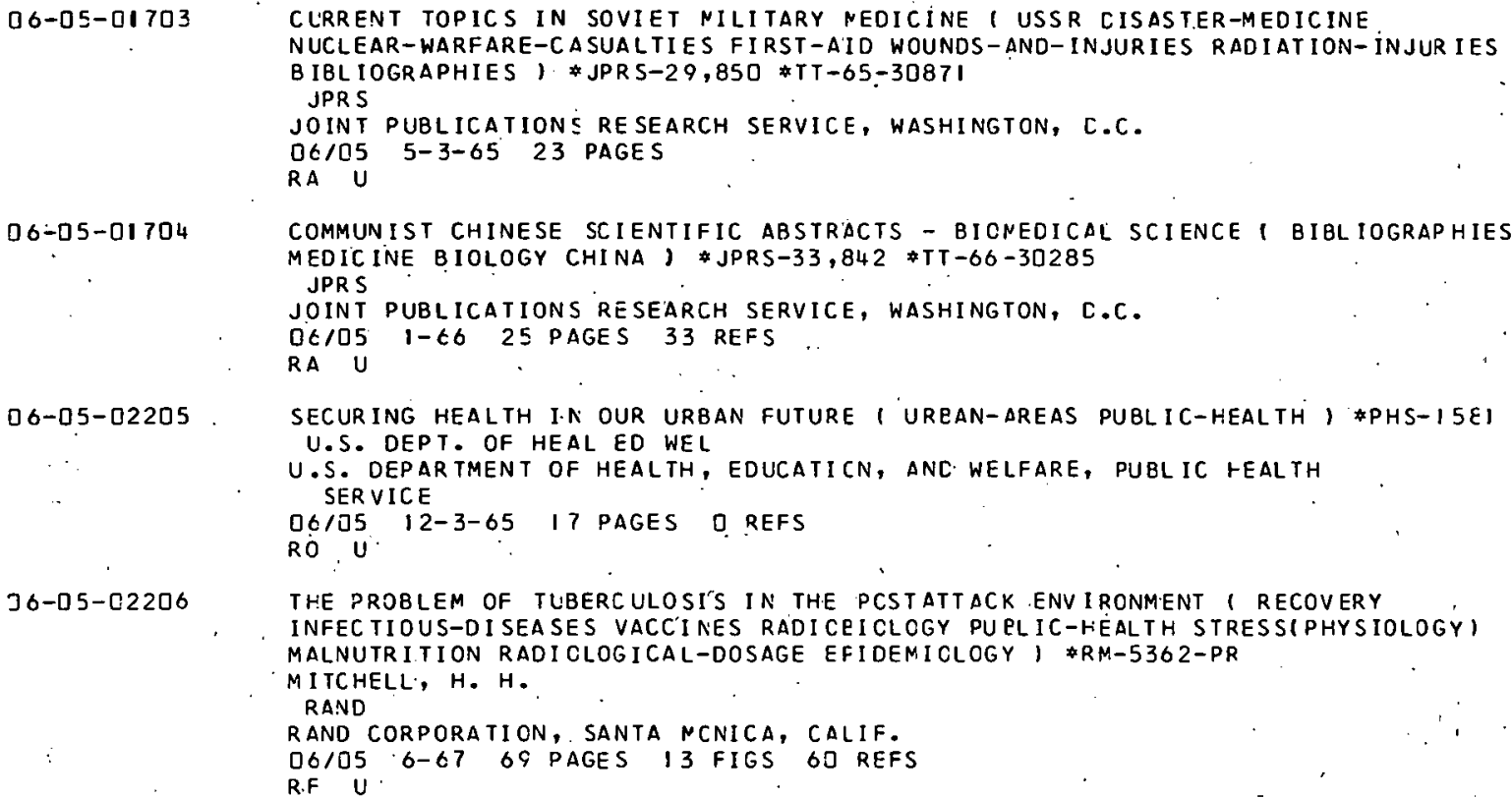

06-05-01704 COMMUNIST CHINESE SCIENTIFIC ABSTRACTS - BICNEDICAL SCIENCE I BIBLIOGRAPHIES MEDICINE BIOLOGY CHINA) \#JPRS-33,842 \#TT-66-30285

06-05-02205. SECURING HEALTH I.N OUR URBAN FUTURE（'UREAN-AREAS PUBLIC-HEALTH ) \#PHS-I5EI U.S. OEPT. OF HEAL ED WEL

U.S. DEPARTMENT OF HEALTH, EDUCATICN, ANC WELFARE, PUBL IC rEALTH SER VICE $06 / 05$ I2-3-65 I 7 PAGES 0 REFS RO U

$36-05-02206$

THE PROBLEM OF TUBERCULOSI'S IN THE PCSTATTACK .ENV IRONMENT I RECOVERY INFECTIOUS-DISEASES VACC'INES RADICEICLOGY PU RLIC-HEALTH STRESS(PHYSIOLOGY) MALNUTRIITION RADICLOGICAL-DOSAGE EFIDEMICLOGY, \$RM-5362-PR

MITCHELL, H. H.

RAND

RAND CORPORATION, SANTA MCNICA, CALIF.

06/05 6-67 69 PAGES I3 FIGS 6J REFS

R.F $U$

\section{Environmental Biology}

$06-06-01705$

$06-06-01706$

$06-06-01708$

$06-06-02207$

$06-06-[2208$
A REVISION OF THE BEE GENLS CALLICFSIS AND THE BICLOGY ANC ECOLOGY OF C. ANDRENIFORMI $\subseteq$ - HYMENOPTERA, ANDRENIDAE (BEE-POLLINATION) \$A.L.1046 SHINN, A. F.

LNIV. OF KANSAS

UNIVERSITY OF KANSAS, LAWRENCE, KAN.

DE/C6 1-6-67 I83 PAGES 156 FIGS 42 . REFS

RF . U

A. MATHEMATICAL MODEL OF FLASH BLINCNESS $($ VISION AVIATION-MEDICINE EYE) \$AD 627332 CZEH, R. S.

GENERAL EI,ER.TRIT.

GENERAL ELECTRIC COMPANY, OKLAHONA CITY, OKLA.

$06 / 06$ IM- 65 EQ PAGES

R I U

ECOSYSTEMS, SYSTENS ECOLOGY, ANO SYSTENS ECOLOGISTS 1 TRAINIVG COMPUTERS

TEST-EQUIPMENT TEST-METHCDS, \#ORNL-3957

VAN DYNE, G. M.

ORNL

OAK. RIDGE NATIONAL LABORATORY, OAK RIDGE, TENN

06106 6-66 4[ PAGES I2E REFS

RF U

RADIATION INDUCED VIABILITY MUTATICNS IN THE HONEY BEE ( HYMENOPTERA GENETICS, *NYO-2315-1

$L E E$, W. R.

UNIV OF NEW HAMP SHIRE

UNIVERSITY OF NEW HAMPSHIRE, DURHAN, N.H.

OC/O6 2-63 7 PAGES, 10 REFS

RF U

PARTIAL BODY RADIATION OF, GUEEN HCAEY BEES ( HYNENOPTERA) *TIC-20299

LEE, W. R.

LNIV OF NEW HAMPSHIRE

UNIVERSITY OF NEW HAMPSHIRE, DURHAN, N.H.

DEIDG 1958 O PAGES 2 REFS

R F U 


\section{Escape, Rescue, and Survival}

$06-07-01709$

$06-08-01710$

$06-08-01711$

$36-08-01712$

$06-08-01713$

J6-08-02209

$06-08-02210$

$36-08-0221$
ENGINEERING ANO RESC UE WORK IN A NUCLEAR STRIKE ZCNE \& CIVIL-DEFENSE-SYSTEMS USSR RECONNAI SSANCE INTERNATI ONAL-CIVIL-DEFENSE PCSTATTACK-OPERATIONS RESCUES

CONSTRUCTICN FIRES ROADS, *JPRS-27,419 \$TT-64-51669

VOLKOV, I. D. + ULANOVSKIY, R. Y. + USCV, N. A.

TSIVILEV, M. P.

JPR S

JOINT PUBLICATIONS RESEARCH SERVICE, WASHINGTON, C.C.

CE/J7 $11-17-64$ \$3 PAGES 42 FIGS

RD U

\section{Food}

ACCEPTABILITY OF SHELTER RATIONS IA COMBINATION WIIH ADJUNCTS I FOODIACCEPTABILITY) FALLOUT-SHELTERS CONFINED-ENVIRCNNENTS TASTE; \#SRI-4949-500 \$AD 638630 STONE, H. + OLIVER, S. M. + KLCEHN, J. M.

SINGLETON, R. C.

STANFORD RESEARCH INST SIANFORD RESEARCH INSTITUTE, MENLC PARK, CALIF. DE/L8 4-11-C6 22 PAGES 5 REFS RF U

POSTATTACK FOOD AVAILABILITY AND ACCESSIBILITY I POSTATTACK-OPERATIONS FOOD-DISPENSING DISTRIBUTI CN DAMAGE-ASSESSMENT CALIFORNIA URBAN-AREAS SUPPLY-DEPOTS I *SR I-MU- 5776 *USNRDL-TRC-67-3

BILLHEIMER, J. W.

STANFORD RESEARCH INST

STANFORD RESEARCH INSTI TUTE, NENLC PARK, CALIF. $06 / 58$ 11-67 145 PAGES 35 FIGS 100 REFS

RF U

BASIC COUR SE IN EMERGENCY MASS FEECING - HANCBOOK I KITCHEN-EQUIPMENT-AVD-SUPYLIES FOOD-DISPENSING FCCD STOCKPILING FALLOUT-SHELTERS SHELTER-OCCUPAVCY LOGISTICS WATER-SUPPLIES RATIONING SANITARY-ENGINEERING RACIOLOGICAL-CONTAMINATION(FOOD) INFANTS(DIET) STORAGE, , -15

OCD + AMER NAT RFD C.ROSS + U.S. DEPT. CF HEAL EL

hEL

OFFICE OF CIVIL DEFENSE, CEPARTMENT CF DEFENSE, AMERICAN NATIONAL

RED CROSS / U.S. DEPARTNENT OF HEALTH, EDUCATION ANC WELFARE

CEIOE 8- $t 6 \quad 182$ PAGES 25 RFFS

RD U

BASIC COUR SF IN ENERGENCY MASS FEECING - INSTRUCTORS GUIDE

KITCHEN-EQLIPMENT-AND-SUPPLIES STCCKPILING FCOD-CISPENS ING FOOD FALLOUT-SHELTERS LOGISTICS SHEL TER-OC.CUPANCY WATER-SUPPLIES ECUCATICN TRAINING INFANT-CIET) *OCD - IG-15.1 \#ART -2219

OCD + AMER NAT REO CROSS + U.S. DEPT. DF FEAL EC

WEL

OFFICE OF CIVIL DEFENSE, DEPARTMENT OF DEFENSE, AMERICAN NAT IONAL

RED CROSS, U.S. DEPARINENT OF HEALTH. EDUCATICN ANC. WELFARE

OCIOC O OE 56 DAGF $T$ REFS

RC $U$

FOOD STORAGE LIFE - A REPCRT BIBLICGRAPHY I REEF PRESERVATION SHLLF-L IFE BEVERAGLS BREAD CANNED CEREALS COFFEE CORN DEHYDRATED-FOODS EGGS FATS FROZEN-FOODS FRUITS IRRADIATED-FOOOS NEAT NILK PORK PCTATOES SEAFOOE VEGETABLES WHEAT, \#ARB-NO. Q76188 CCC

UEFENSE DOCUMENTATION CENTFR, CAMERCN, VA. TEL/OR 8-2-67 ISL PAGES

RA 1

EMERGENCY FEEDING I CIVIL-DEFENSE-CPERATING-PLANS FOOD FOOC-DISPENSING SHELTER-OCCUPANCY PCSTATTACK-OPERAT IOVS HOSPITALS TRAINING PERSUNNEL LOGISTICS * FG-E-I3.4 $O C D$

OFFICE OF CIVIL DEFENSE, DEPARTMENT OF DEFENSE DEIDE 6-66 IL PAGES

$R C$ U

ACCEPTABILITY GF SHELTER RATICNS IA COMBINAT ION WITH ACJUNCTS I FOOD FALLOUT-SHELTERS TASTE CIVIL-DEFENSE-SYSTEMS FRUITS CONDIMENTS SUCROSE FOOD-DISPENSING TESTS, *SRI 4949-500 \#AC 65I 944 STONE, H. + OLIVER, S. M. + SINGLETCR, R. C. STANFORD RESEARCH INST

STANFORD RESEARCH INSTITUTE, MENLC PARK, CALIF. DE 108 3-27-67 It PAGES 3 TABLES 3 REFS 
STORAGE STABILITY OF CIVIL DEFENSE SHELTER RATIONS I FOOD PRESERVATION FIBERBOARD PHYSICAL-PROPERTIES CONTAINERS TASTE FALLOUT-SHELTERS ) $\$ T R-67-25-C D \$ A D$ - 40823 WOODROOF, J. G. + CECIL, S. R. LNIV OF GEORG IA

UNI VER SI TY GF GEORGIA, ATHENS, GA.

D6/08 10-66'69 PAGES 26 TABLES

RF $U$

\section{Medical and Hospital Equipment and Súpplies}

$06-12-03714$

$06-13-01715$

06

$36-14-01716$

$06-16-01717$

$06-16-0.718$

$06-16-01719$

SOME PRELIMINA'RY CBSERVATICNS ON A HCSPITAL RESPONSE TO THE JACKSON, MISSISSIPPI TORNADO OF MARCH 3,1966 . I DI SASTER-NEDICINE MANAEEMENT-PLANNING FOSPITALS OPERATION CONTROL-SYSTEMS CONNUNI CATICNS-SYSTEMS SUPERVIS ION MEOICAL-PEZ SONVEL STORMS EMERGENCY-HEALTH-SERVICES, \#RESEARCH REPORT 17 KENNEDY, h. C.

OHIO 'STATE LNIY

OHIO STATE LNI VER SITYY, COLUMAUS, CHIC

$0 C / 12$ 2-1C-67 36 PAGES 3 REFS

RF U

\section{Microbiology}

IMMUNOFLUORE SCENCE, AN ANNOTATED BIBLIOGRAPHY - SUPPLEMENT, AUTHOR AND SUBJECT INDEXES I BACTERIA VIRUSES FUNGI PROTOZOA RICKETTSIA PHYSIOLOGY DIAGNOSIS ) \#MISC. PLB. Z, SLPPLENENT

SANBORN, h. R:

ARMY BIOL LAB

U.S. ARMY BIOLOGICAL LABCRATORIES, FCRT CETRICK, FREDERICK, MD. $06 / 13 \quad 12-65$ 37. PAGES D REFS

RA U

0614 Personnel Selection and Maintenance (Medical)

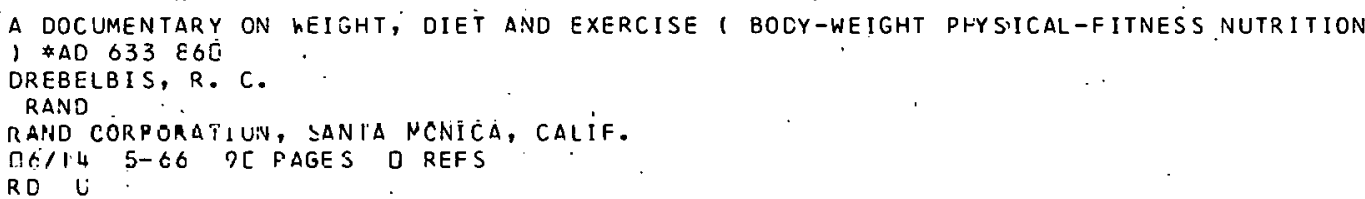

\section{Physiology}

VUTRITION IN THE POSTATTACK ENVIRCNMENT, I FCCD CIET POSTATTACK-OPERAT IOVS SURVIVAL STORACE (FDOD) PRODLCTIUN (FCCD) RESCURCES (FOOC) $\triangle$ GRICULTURE ) \#RM-5C52-TAB POERUND, R. S.

RAND

RAND CORPORATION, SANTA NCNICA, CALIF.

DE/16 12-66 64 PAGES 57 REFS

RF U .

SENSIBLE AND I.ATENT HEAT LCSSES FRCN CCCUPANTS OF SURVIVAL SHELTERS

SHELLTER-OCCUPANCY NETABOLI SM PHYSI CLCGY HEAT-EXCHANGE TEMPERATURE VENTILATION BODY-TEMPERATURE. COOLING， \#A.L. I QST

HLMPHREYS, C. M. + HENSCHEL, A. + LEE, C.' H. K:

L.S. DEPT. OF HEAL ED WEL

U.S. DEPARTMENT OF HEALTH, EDUCATICN ANC WELFARE, PUBLIC HEALTH SER VICE, WASHINGTON, D.C. DE II6 II- 65 28 PAGES İ FIGS 13 REFS

RF $\quad L$

SENSIBLE AND LATENT HEAT LOSSES FRCM OCCUPANTS OF SURVIVAL SHELTERS

HEAT-PRODUCTION(BIOLOGY) SLRVI VAL FALLCUT-SHELTERS VENT ILATION METABOL ISM BODY-TEMPERA TURE CLOTHING TEMPERATURE COOLINE PERSPIRAT ION EVAPORATION 1 \$AD 64846.7 HLMPHREYS, C. M. + HENSCHEL, A. + LEE, D. H. K.

U.S. DEPT. OF HEAL ED WEL

U.S. DEPAR TMENT OF HEALTH, EDUCATICN AND WELFARE, PUBlic reAlth SER VICE

. DE $/ 16 \quad 11-65$ 28 PAGES I 3 FIGS

RF U 
$06-16-01720$

$36-16-02213$

$06-18-01721$

$06-18-[.1722$

$06-18-04723$

$06-18-01724$

$06-18-01725$

$06-18-01726$
PROCEEDINGS OF THE HIGH LYSINE CCRA CONFERENCE I NUTRIT ION(HUMAN) NUTRITION(AVIMAL). PROTEINS GENETICS AGRICULTURAL-ECCNCMICS EIOCHEMISTRY， A.L.997

MERTZ, E. T. + NELSON, O. E. CORN REFINERS ASSN

CORN REFINERS ASSCCIATION, INC., WASHINGTCN, D.C. $06 / 16$ 6-66 I 86 PAGES 95 RFFS

$R \subseteq U$

WATER TRANSFER. THROUGH HLNAN SKIN I PERMEAEILITY TRANSPORT-PROPERTIES SORPTION SALTS HUMIOITY, \#AD 646848

BLETTNER, K. J. K. + ROBBINS, E. + CRICHLOW, J.

PITTS, M. + JONES, D. + RYAN, T. E. + ALLISON, P.

LNIV OF RASHINGTCN

UNIVER SI TY OF LA SHING TON, SEATTLE, WASH.

QK/16 1965 6 PAGES 4 REFS

RF U

\section{Radiobiology}

PATHOLOGY, CLINICAL PICTURE ANO THERAPY, IN AFFECTIONS WITH. TOXIC ANO RADIOLOGICAL AGENTS - USSR I RADI OLOGICAL-WARFARE-AGENTS CONTAMINAT ION RADIATION-INJURIES TOXICITY CYANO-ACIDS CARBCN-MCNOXICE VESICANTS PCISCNOUS-CASES LEWISITE

GAS-DETECTORS RADIATI ON-NCNITCRS DCSIMETERS PROTECTIVE-CLOTHING GAS-MASKS PHOSGENE INCENDIARY-GELS FLAME-THROWER-FUELS SMOKE-MUNITIONS FIRST-AID

NUCLEAR-WARFARE-CASUALTIES, \#JPRS 30,979 TT $65-31477$

BOGOANOV, N. A.

JPR S

JOINT PUBLICATIONS RESEARCH SERVICE, WASHINGTON, C.C.

QC/18 7-8-65 147 PAGES O REFS

RD U

HOW TO PURIFY ANO TO DISIAFECT WATER WITH THE SIMPLEST MEANS - USSR ,

WATER-SUPPLIES PURIFICATICN CCNTAMINATION RACIOLOGICAL-CONTAMINATION

CHEMICAL-WARFARE-AGENTS BIOLOGICAL-WARFARE-AGENTS, \#JPRS 34,578*TT. 66-31018

VEKHOV, S. P. + SCKOLOVA, N. R.

JPR S

JOINT PUBLICATIONS RESEARCH SERVICE, WASHINGTUN, L.C.

Q6/18 3-15-66 21 PAGES O REFS

RD U

INTRODUCTION TO LCNG TERN RIOLCGICAL EFFECTS OF NUCLEAR WAR I RADIOBIOLOGY

RADIATION-EFFECTS ECOLOGY RADIATION-DANAGE RADIOLCGICAL-CONTAMINATION IVSECTS

PLANTS(BOTANY) RADIOACTIVE-F.ALLOUT (S IMULATION) MOCELS (S IMULAT IONS) DECOVTAMINATION RECOVERY FOOD (CONTAMINATICN) WATER (CONTANINATION) RADIOLOGICAL-DOSAGE

RADIATION-INJURIES, \#SRI-NU-5779A

MILLER, C. F. + LARIVIERE, P. D.

- STANFORD RESEARCH INST

STANFORD RESEARCH INSTITUTE, MENLC PARK, CALIF.

חE/18 4-CE 156 PASES 95 REFS

RF U

EVALUATION OF FALLOUT CONTAMINATICA FRCM SURFACE RUNOFF ( WATER-SUPPLIES RADIOLOG ICAL-CONTAMINATION RADIOACTIVE-FALLOUT HYCROLOGY, \$A.L. IOS4 GRLNE, W. N. + ATLAS, H. S. + HANEL, G. J.

MERR IMACK COLLELE

MERR IMACK COLLEGE, NORTH ANDOVER, NASS.

$06 / 181966$ O3 PAGES 69 REFS

RF U

UPTAKE OF RADIONUCLIOES BY PLANTS I AGRICULTURE RACIOACT IVE-ISOTOPES

RADIOACTIVE-FALLOLT FCOD CCNTANINATICN MCDELSISIMULAT IONS) RADIOB.IOLOGY

RADIATION-HAZARDS SOIL'S PLANTSIBOTANY) RADIOLOGICAL-CONTAMINATION, *SRI-MU-5O95

SARTOR, J. D. + LANE, W. B. + ALLEN, J. J.

STANFORD RESEARCH INST

STANFORD RESEAREH INSTITUTE, NENLC PARK, CAL IF.

$06 / 18$ 12-66 246 PAGES 193 TABLES 26 REFS

RF U

RADIOACTIVE FALLOLT - PHYSICS, BICLOGICAL EFFECTS AND PROTECTIVE MEASURES I RADIATION-INJURIES USSR RADIATICN-HAZARLS RACIOLCGICAL-COSAGE COSIMETERS

RADIATION-EFFECTS CALCULATIONS DOSE-RATE SHIELDING RACIAT ION-MEASUREMEVT-SYSTEMS

RADIATION-MONITORS 1 *AEC-TR-6634

PETROV, R. V. + PRAVETSKII, V. N. + STEPANOV, Y. S.

SHALNOV, M. I .

$A E C$

U.S. ATOMIC ENERGY COMMISSION

DEIIR 1963 I 34 PAGES 43 FIGS 30 REFS

KL U 


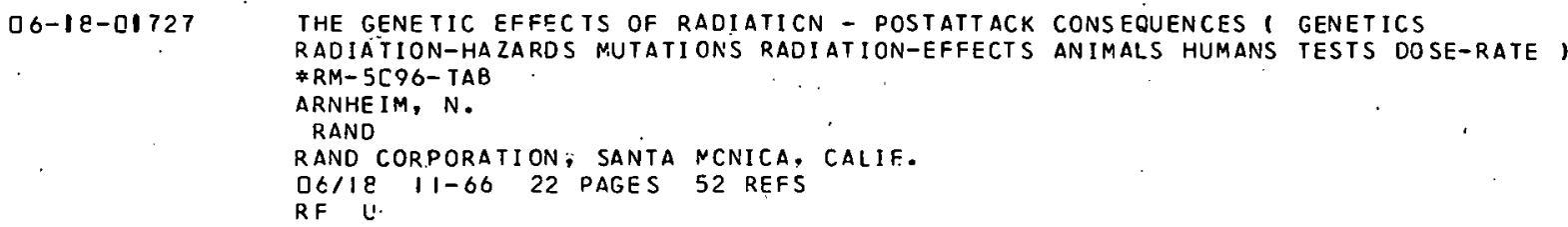

06-18-01728 INCORPORATION AND. ELIMINATION CF RADIOACTIVE MATERIALS BY THE ORGANISMI RADIOBIOLOGY , \#ORNL-TR-975

PRETRE, S.

FRANCE

FRANCE

OE 118 12-64 I PAGE. O REF

$20 \mathrm{U}$

06-18-CI729 DECONTAMINATION SCHEDULING PRCCEDURES FCR RACEF SYSTEMS I

RADIOLOGICAL-CONTAMINATION SHIELOING PROTECT ION-FACTOR RADIOLOGICAL-DOSAGE RECOVERY CALCULATIONS RADICACTIVE-FALLOUT MANPOWER-STUDIES TIME， \#USNRCL-TRC-42

LEE, H.

STANFORD RESEARCH INST

STANFORD RESEARCH INSTITUTE, NENL.C PARK, CALIF.

OCII\& 8- $-6 \quad 77$ PAGES 16 FIGS 2 REFS

RF U

$06-18-01.730$

A.TOMIC BOMB CASUALTY COMMISSICN ANAUAL REPORT JULY-1,1965-JUNE-30, 19661 RADIATION-EFFECTS NUCLEAR-WARFARE-CASUALTIES JAPAN HIROSHIMA DATA NAGASAKI

RADIATION-INJURIES RECORDS LIFE-SPAN -) $\triangle A R C C-65-66$

ATOMIC BOMB CASLALTY CON

ATOMIC BOMB CASUALTY COMNISSICN, HIROSHIMA AND NAGASAKI, JAPAN $0 C / 18$ 1966. 214 PAGES

RA U U

PROTECTING THE RURAL POPULATICN AGAINST RACICACT IVE CONTAMINATION I USSR CIVIL-DEFENSE-SYSTEMS SHELTERS INTERNATICNAL-CIVIL-DEFENSE

RADIOLOGICAL-CONTAMINATI ON RURAL-AREAS HARDENING PROTECTION-SYSTEMS I *JPRS-27,D3C * TT- $64-51286$

DLRIKOV, A.P. + CHUMAKOV, V. I.

JPRS

JOINT PUBLICATIONS RESEARCH SERVICE, WASHINGTON, C.C.

$C E / 18$ ID-22-64 59 PAGES 27 FIGS $\square$ REFS

RD U

LONG TERM RADIATICN DAMAGE - EVALUATION OF LIFE SPAN'STUDIES I RADIATION-INJURIES RADIOLOGICAL-DOSAGE EXPERIMENTAL-DATA AGING(PHYSIOLOGY) ANIMALS MICE RATS DOSE-RATE I $\$ R M-5083-T A B$

BROWN; B.

RANR:

RAND CORPORATICN, SANTA NCNICA, CALIF. DG/IR 12-66 TG PAGES TE REFS

$06-18-02214$

RADIOLOGICAL RECOVERY REQUIRENENTS, STRUCTURES, ANC OPERATIONS RESEA.CH I. INTRODUCTION AND SUMMARY I RADIOACTIVE-FALLOUT RACIOLOGICAL-CONTAMINATION DECONTAMINATION PROTECTI ON-FACTOR NCNTE-CARLC-METHCD PROGRAMMINGI COMPUTERS DOSE-RATE SHIELUING RECOVERY CALCULATICNS, \#R-OU-266(I)

BRYAN, F. A., JR.

RESEARCH TRIANGLE INST

RESEARCH TRIANGLE INSTITUTE, DURHAN, N.C.

DCIIE 5-67 IC PAGES 3 REFS

RF U

$06-18-0221.5$

RESEARCH REPORT - MEDICAL .OIVISICN I METHOCOLOGY RACIAT ION-IVJURIES

RADIATION-EFFECTS INSTRUMENTATION RADIOISOTCPES(MECICAL) RADIONUCLIDES VEOPLASIA, \#ORAU-1-

OAK RIDGE ASSOC LNI V

OAK RIDGE ASSOCIATED UNIVERSITIES, OAK RIDGE, TENN.

$06 / 18$ I2- $21-66 \cdot 190$ PAGES 93 FIGS 108 RFFS

RF U

$96-18-C 2216$

LIVESTOCK III ( DANAGE-ASSESSNENT AGRICULTURE COMPUTERS RACIOACTIVE

RADIOAGR I CULTURE, *NREC-139

SEWELL, C. H.

NAT RES EVAL CENTER

NATIONAL RESOURCE EVALUATICN CENTER, WASHINGTON, C.C.

$J 6 / 18$ 6-t3 it PAGES 4 FISS

RE $C^{\circ}$ 
$06-18-02217$

$06-18-02218$

$06-18-02219$

$06-18-C 2220$

$06-18-[222$

$06-18-02222$

$06-19-[11734$

96-19-01735

PARM SYSTEM MANUAL III-F. FALLOUT AND DECONTAMINAT.ION I RADIOACTIVE-FALLOUT RADIOLOGICAL-DOSE FARM-CRCPS RADIOLOGICAL-CCNTAMINATION RACIATION-FAZARDS SHIELDING RADIOAGR ICLLTURE, \$NREC-36

FACCI, H. A. + WOCD, M. K. + HUSBANDS, R. M.

NAT RES EVAL CENTER

NATIONAL RESOURCE EVALUATION CENTER, WASHINGTON, C.C.

$06 / 18$ 3-64 8[ PAGES 22 FIGS 9 FEFS

$R F \quad U$

RADIOLOGICAL RECOVERY REQLIRENENTS, STRUCTURES ANC OPERAT ICNS RESEARCH 1.

CALCULATIONAL TECHNIQUE FCR DETERNINING IMPCRTANCE OF LIMITED STRIP DECONTAYINATION PROCEDURES ( RADI CLOGICAL-CCNTAMINATION PROGRAMMING(COMPUTERS) RADIOACTIVE-FALLOUT SHIELDING WALLS CCBALT EXPERINENTAL-DATA THECRY CCSE-RATE, \$R-OU-266(I.) DOGGETT, h. O. + BRYAN, F. A., JR.

RESEARCH TRIANGLE INST

RESEARCH TRIANGLE INSTITUTE, DURHAN, N. C.

DE/18 5-67 222 PAGFS 8 REFS

R.F U

EXPER IMENTAL DETERMINATIOA OF NEUTRCN ENERGY SPECTRA IN CONCRETE DUCTS 1 SHEL TER.S ENTRANCEWAYS DCSE-RATE RADIATICN-MEASUREMENT-SYSTEMS PROTECTION-FACTOR MONTE-CARLO-METHOD GEOMETRY(DUCTS), \#NCEL-R-523

GARDNER, L. B. + NETTLER, A. J. + EURUICK, B.

NCEL

U.S. NAVAL CIVIL ENGINEERING LABCRATCRY, PORT HUENEME, CAL IF. Q6/18 5-67 84 PAGES 23 FIGS 27 TABLES 24 REFS

RF U

SLMMARY OF RAOIOLOGICAL TARGET ANALYSIS PROCEDURES FOR CECCVTAMINATION SCHEDULING I RADIOAC TI VE-FA LLOLT RADI OLOG I CAL-CCNTAMINAT I CN DOSE-RATE COUVTERMEASURES

FALLOUT-SHELTERS RADIOLOGICAL-DOSAGE MANPOWER， \$SRI-MU-5806 \#USNRCL-TRC-76

LEE, H.

STANFORD RESEARCH INST

STANFORD RE SEARCH INSTITUTE, NENLC PARK, CAL IF.

QE 118 4-67 44 PACES 11 FIGS 7 REFS

R.F U

RADIOLOGICAL RECOVERY REGUIREMENTS, STRUCTURES, ANC OPERATIOVS RESEARCH II . OAMAGED PF MULTIPLIER I RADIOACTIVE-FALLOUT PROTECTION-FACTOR DOSE-RATE BUILDINGS DECONTAMINATION BLAST SHIELDING THEORY MATHENATICAL-ANALYSIS

RADIOLOGILAL-LONTANINATION NDOELS hALLS, $* R-O U-266(11)$

JOHNSON, T.

RESEARCH TRIANGLE INST

RESEARCH TRIANGLE INSTITUTE, DURHAN, N.C.

$06 / 18$ 5- 67 43 PAGES 7 REFS

RF U

RADIOLOGICAL HEALTH AND CIVIL DEFENSE I HEALTH-PHYSICS RACIOACTIVITY DETECTION RADIOLOGICAL-DCSAGE EXPOSLRE RADICLCGICAL-CCNTAMINATION RACIATION-HAZARDS MECICINE RADIOISOTOPES RADIOACTIVE-hASTE CIVIL-DEFENSE-SYSTEMS DECONT AM INAT ION B IOLOGICAL-WARFARE CHFNIC.AL-WARFARE RESCUES, *A.L. I128

INIV DF FLORIDA

UNIVERSITY OF FLORIDA, GAINESVILLE, FLA.

DEIIR |1-5I IIO PAGES 130 REFS

R $\leq U$

\section{Stress Physiology}

HEAT TOLERANCE OF ELDERLY PERSCNS LIVING IN A SURTROPICAL CL IMATE I AGING(PHYSIOLOGY) AGE TROPICAL-REGIONS PERFORMANCE(HUMAN) STRESS(PHYSIOLOGY), *TR- 35

HENSCHEL, A. + COLE, M. + LYCZKOWSKY, O. + COYLE, T.

MARGOLIFS, L.

U.S. DEPT. OF HEAL EC WEL

U.S. DEPAR TMENT OF HEALTH, EDUCATICN ANC WELFARE

$06 / 19$ 2- 67 It PAGES 8 REFS

R.F II

SUPPLEMENT TO SHOCK AND VIBRATION PULLETIN I TOLERANCES (PHYS IOLOGY) MOTION-SICKNESS STRESS(PHYSIOLOGY) ADAPTATICN(PHYSIOLOGY) TUNEL,INE DCCELERATION-TOLERANCEI \#AD 94 697

U.S. DEP.T. OF DEFENSE

U.S. DEPARTMENT OF DEFENSE

DC/19 $7-55 \quad 47$ PAGES 23 REFS

$R \subseteq U$ 


\section{Weapon Effects}

$06-21-01737$

$06-.21-01738$

$06-21-01739$

$06-21-[1740$

$56-21-[1741$

$06-21-01742$

$06-21-02224$

$36-21-02225$

$06-21-[2226$

CATALOGUE OF PLBLICATIONS I DISASTER-MEDICINE EMERGENCY-HEALTH-SERVICES CANAOA MANUALS FILMS I *A.L. 1068

CANADA DEPI OF NATL HEAL

CANADA DEPARTMENT OF NATICNAL HEALTH ANC WELFARE, OTTAWA $O C / 21 \quad 1965$ 25. P.AGE'S

$\times 0 \mathrm{U}$

ADVANCED TREATMENT CENTRE - OPERATING MANUAL - EMERGENCY HEALTH SERVICES I DISASTER-MEDIC INE MEDICINE ADNINISTRATICN-AND-MANAGEMENT FIRST-AIC ORGAVIZATION TRAINING HOSPITAL S CASUALTIES PERSCNNEL NUCLEAR-WARFARE-CASUALTIES, \#A.L. IOT6.

CANADA DEPT OF NATL HEAL

CANAOA DEPARTMENT OF NATICNAL HEALTH ANC WELFARE, OTTAWA

$06 / 21 \quad 1965$ 3L PAGES 8 FIGS

RC U

HOSPITAL DISASTER PLANNING I DISASTER-NEDICINE EMERGENCY-HEALTH-SERVICES HOSPITALS CASUALTIES ADMINI STRATION-AND-MANAGENENT MEDICAL-SUPPLIES NUCLEAR-WARFARE-CASUALTIES STCCKPILING CANADA) $\$ \Delta . L .1069$

CANADA DEPT OF NATL HEAL

CANADA DEPARTMENT OF NATICNAL HEALTH AND WELFARE, OTTAWA $06 / 21 \quad 196497$ PAGES D REFS RD U

DISASTER MEDICAL CARE FILNS I DISASTER-NECICINE MECICINE MEDICAL-PERSONVEL HOSPITALS PUBLIC-HEALTH RESCUES NUCLEAR-WARFARE-CASUALTIES SCHOOLS SURVIVAL CASUALTIES FIRST-AID, \#A.L. 1077 AMER MED ASSN

AMER ICAN MEDICAL ASSOCIATION, CHICAGC, ILL.

$06 / 21 \quad 1965$ 2C PAGES

zO U

B IOLOGICAL EFFECTS OF BLAST ANO SHCCK I SHOCK-WAVES RIOLOEICAL-BLAST-EFFECTS SHOCK (PATHOLOGY) EXPLOSI-ON-EFFECTS TEST-NETHCOS, \$CASA-1777

RICHMOND, D. R. + WHITE, C. S.

LOVELACE

LOVELACE FOUNDATI CN, ALBUGUERGUE, M.N,

$06 / 21,4-66$ GI PAGES 38 REFS

RF U

SENSITIVITY ANALYSIS OF CÁSUALTY FLNCTICNS I CASUALTIES SYSTEMS-EVALUATION DAMAGE-AS SE SSMENT NUC LEAR-WARFARE-CASUALTIES MATHEMAT ICAL-MODELS METHODJLOGY POPULATION URBAN-AREAS DISTRIBUTICA, \#A.L. IOT8

PARSONS, E. A. + TITCHEN, R. S. + NCNULLAN, P. S.

CRUZE, A. M.

QESEARCH TRIANGLE INST

RESEARCA THIAIYGLE INSIIIUIE, UURHAN, N.C.

$06 / 21 \quad 19669$ PAGES 9 REFS

RF $U$

06-21-E2223. THE SCOPE OF BLAST AND SHCCK BIOLCGY ANC PROELEM AREAS IN RELATING PHYSICAL AND BIOLOGICAL PARAMETERS 1 BIOLOGICAL-BLAST-EFFECTS BLAST-BIOLOGY DISPLACEMENT BLAST-ENERGIZED-MISSILES BLAST-8IONEDICAL-CRITERIA） \#DASA-1856 WHITE, C. S.

LOVELACE

LOVELACE FOUNDATICN, ALBUGUERQUE, A. M.

D $6 / 21$ I I-66 28 PAGES 4 TABLES 55 REFS

RO U

B IBL IOGRAPHY OF PLBLI SHED PAPERS CF THE ATOMIC BONE CASUALTY COMYISSION I JAPAN NUCLEAR-WARFARE-CASUALTIES, $¥ A B C C$ TR D-66

ATOMIC BOMB CASUALTY CON

ATOMIC BOMB CASUALTY CCNNISSICN, HIRCSHINA ANC NACASAKI, JAPAN

$0 \in / 21 \quad 1966 \quad 5$ PAGES

$R A^{\prime} \cdot U^{\prime}$

DAPC46 TIME PHASED ¿ASUALTY ESTINATE I ANALYSIS NUCLEAR-WARFARE-CASUALTIES DAMAGF-ASSE SSMENT PATIENIS PRCGRANNING (CCMPUTERS) ) \#NREC-72

CAPOZZOLI, D.

NAT RES EVAL CENTER

NATIONAL RESOURCE EVALUATIICN CENTER, WASHINGTON, C.C.

$0 E / 2 \mid$ I-6I I T. PAGES O REF.S

RI 1

ROLE OF THE FEDERAL EMERGENCY HEALTH SERVICES DIVISION 1 CISASTER-MEDICINE

PUBL IC-HEALTH LAW ORGANI ZATI ON CIVIL-OEFENSE-SYSTEMS CASUALTIES CANADA

NL'CLEAR-WARFARE-CASUALTIES RADIATICN-INJURIES POSTATTACK-OPERATIONS

PLBLIC-INFORMATION MEDICAL-SUPPLIES, *A.L. 1119

CANADA DEPT OF NATL HEAL

CANADA DEPARTMENT OF NATICNAL HEALTH ANC WELFARE, CTTAWA

DE/21 1-64 9 PAGES 0 REFS

$\times 0$ U 
$06-21-[2227$

$06-21-02228$

$06-21-02229$

$36-21-[2230$

$06-21-0223$

$06-21-C 2232$

$06-21-0.2233$

$36-21-02234$

$06-21-02235$

ANALYSIS OF JAPANESE NUCLEAR CASUALTY CATA I NUCLEAR-WARFARE-CASUALTIES STATISTICAL-ANALYSIS WEAPCNS-EFFECTS HIRCSHINA NAGASAKI GRAPHS B IOLOGICAL-BLAST-EFFECTS BURNS RADIATICN-INJURIES SHIELCINC URBAV-AREAS CASUALTIES(PREDICTION), $\$ D C-F R-1045$

DAVIS, L. W. + BAKER, W. L. + SUNMERS, D. L.

CIKEWOOD

DIKEWOOD CORPORATION, ALBLQUERGUE, $A$. MEX.

$06 / 21$ 4-66 3[4 PAGES 69 FIGS IS TABLES, 11 REFS

RD U

SENSITIVITY OF MORTALITY ESTINATES TC UNCERTAINT IES IN SOME VUCLEAR DAMAGE

PARAMETERS I ATTACK-SCENARIOS NUCLEAR-WARFARE-CASUALTIES CASUALTIES CALCULATIONS WEAPONS-EFFECTS RADIATION-EFFECTS ECCLOGY WI ND RACIOACT IV ITY (CISTRIRUTION) RECOVERY JAPAN RADIOACTIVE-FALLCUT THERMAL-RACIATION EIOLOEICAL-BLAST-EFFECTS

DAMAGE-ASSESSMENT ) *RM-47D6-TAE

$\triangle R N S T E N, M . E$.

RAND

RAND CORPORATION, SANTA NCNICA, CALIF.

$06 / 21 \quad 11-66$ IIL PAGES 25 FIGS 83 REFS

RC U

SENSITIVITY OF MORTALITY ESTINATES TC UNCERTAINTIES IN SOME VUCLEAR DAMAGE:

PARAMETERS II I I NODELS DAMAGE-ASSESSMENT NUCLEAR-W ARFARE-CASUALTIES CA SLALTIES

MILITARY-STRATEGY WEAPONS-EFFECTS RADIATION-EFFECTS POPULATION PARTICLE-SIZE

SHELTERS DISTRIBUTION RADIOACTIVE-FALLCUT BICLOGICAL-COSAGE ATTACK-SCENARIOS WINDI \#RM-4707-TAB

ARNSTEN, M. E.

RAND

RAND CORPORATICN, SANTA NCNICA, CALIF.

$06 / 21 \quad 11-66 \quad 139$ PAGES .95 FIGS

RF U

AIR BLAST STLDIES WITH EIGHT SPECIES OF MAMMALS I RLAST-BIOLOGY 8 IOLOGICAL-BLAST-EFFECTS SHOCK-TUBES LUNG-DANAGE TOLERANCES(PHYSIOLOGY) ) *DASA-1854 RICHMOND, D. R. + DAMON, E. G. + BCWEN, I. G.

FLETCHER, E. R. + HHITE, C. S.

LOVELACE

LOVELACE FOUNDATICN, ALBUGUERQUE, R.N.

$06 / 21$ 8- 66 44 PAGES 21 FIGS 17 REFS

RF U

EFFECTS OF OVERPRESSURE ON THE EAR - A REVIEW I BLAST-BIOLOGY

B IOLOGICAL-8LA ST-EFFEC TS I \#DASA-1858

HIRSCH, F. G.

LOVELACE

LOVELACE FOUNDATICN, ALBUGUERGUE, N.N.

0 CI2। |1-t6 35 PAGES 6 FIGS 49 REFS

RF $U$

DASA AEC LOVELACE FOUNDATICN BLAST SIMULATION FACILITY 1 BIOLOGICAL-BLAST-EFFECTS BLAST-BIOLOGY SHOC.K-TUBES INSTRUMENTATION PRESSURE-GAGES-CALIBRATION

PRESSURE-TIME-PAT TERNS, \#DASA-1853

RICHMOND, D. R. + GAYLORD, C. S. + DAMCN, E. G.

TABORELLI, R. V.

LOVELACE

LOVELACE FOLNDATIGN, ALBUGUERLUU,

ES/21 Q-

RO $\mathrm{U}$

PROCEEDINGS OF MEOICAL C.TVII. DEFENSE. CCNFERENCE I CISASTER-MECICINE EMER GENCY-HEAL TH- SER VICES BI OLCSI CAL-WARFARE CHEMICAL-WARFARE PSYCHOLOGICAL-WARFARE ) $\#$ A.L. II 25

AMER MED ASSN

AMERICAN MEDICAL $\triangle S S O C I \triangle T I O N$, C.HICAGGC, ILL.

DEIZI 1454 SE PAGES D REFS

$R \leq U$

THE RELATIONSHIP Between SELECTEo elast waVe paraneters anc tre Response of mammals EXPOSED TO AIR BLAST I BLAST-BIOLCGY BICLOGICAL-PLAST-EFFECTS MONKEYS SWINE SHEEP LLNG-INJURIES EXPLCSI ONS TCLERANCES (PHYSIOLOGY) SHOCK-TUBES, \#DASA-1860 RIT.HMOND. D. R. + DAMON, E. G. + FLETCHER, E, R, BOWEN, I. G. + WHITE, C. S.

LOVELACE

LOVELACE FOUNDATICN, ALBUGUERGUE, $A . N$.

SE $21 \quad 11-6638$ PAGES 12 FIGS 20 REFS

RF IJ

B IOPHYSICAL MECHANISMS AND SCALING PROCEDURES APPLICABLE IN ASSESSING RESPOVSES OF THE THORAX ENERGI ZED BY AIR BLAST CVERPRESSURES OR BY NON-PEVETRATING MISSILES, B IOLOGICAL-BLA ST-EFFECTS NATHENATICAL-MOCELS EXPERIMENT AL-CATA LUNG-DAMAGE \#DASA- IOS?

BOWEN, I. G. + FLETCHER, E. R. + RICHMOND, D. R.

HIRSCH, F. G. + WHITE, C. S.

LOVELACE

LOVELACE FOUNDATICN, ALBUGUERGUE, $A . N$

$D E / 2 I$ II-6E 16 PACES 15 FIGS 26 P.EFS

RF U 


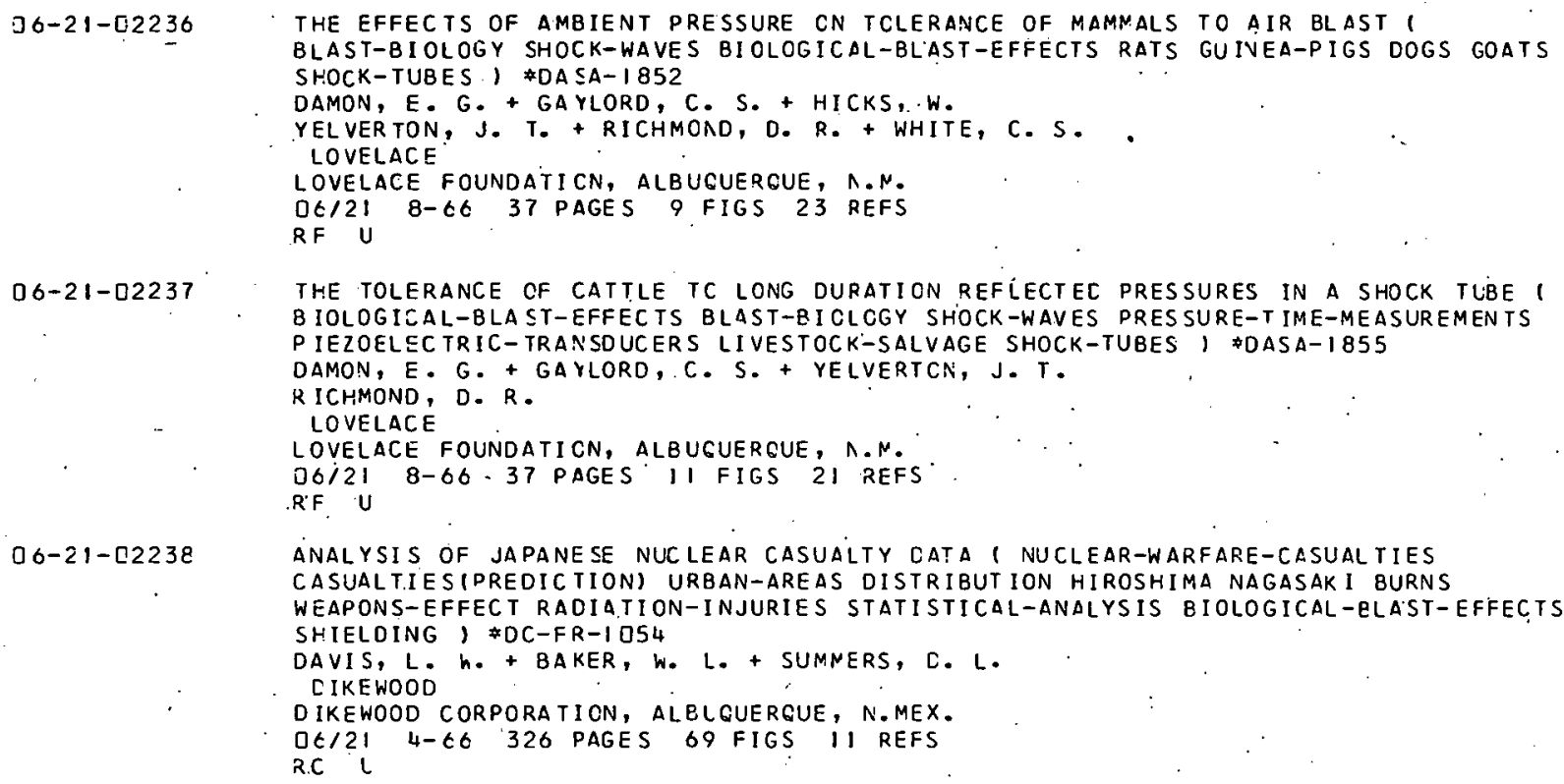

THE TOLERANCE OF CATTLE TC LONG DURATION REFLECTEC PRESSURES IN A SHOCK TLBE I B IOLOGICAL-BLAST-EFFECTS BLAST-BICLCGY SHOCK-WAVES PRES SURE-TIME-MEASUREMENTS PIEZOELECTRIC-TRANSDUCERS LIVESTOCK-SALVAGE SHOCK-TUBES, \#DASA-1855

ANALYSIS OF JAPANESE NUCLEAR CASUALTY CATA I NUCLEAR-WARFARE-CASUALTIES CASUALTIES (PREDIC TION) URBAN-AREAS DISTRIBUT ION HIROSHIMA NAGASAK I BURNS WEAPONS-EFFECT RADIATION-INJURIES STATISTICAL-ANALYSIS BIOLOGICAL-ELAST-EFFECTS SHIELDING， $\#$ DC-FR-I 054

DAVIS, L. h. + BAKER, W. L. + SUMNERS, C. L.

CIKEWOOD

DIKEWOOD CORPORATICN, ALELGUERGUE, N.MEX.

DE/21 4- 66 326 PAGES 69 FIGS II REFS

R.C $\mathrm{L}$

BLAST INDLCED TRANSLATIONAL EFFECTS I BLAST-EIOLCGY BIOLOGICAL-BLAST-EFFECTS EXPER IMENTAL-DATA THEORY NATHEMATICAL-MCCELS DISPLACEMENT ACCELERAT ION DECELERATION ) $\triangle D A S A-I E S 9$

FLETCHER, E. R . + BOWEN, I. G.

LOVELACE

LOVELACE FOLNDATICN, ALEUGUERGUE, . . N.

DE/2I II-C6 38 PAGES 14 FIGS 24 REFS

RF U

\section{EARTH SCIENCES AND OCEANOGRAPHY}

\section{6 - Geography}

UNIVERSAL TRANSVERSE MERCATOR GRIO I ARNY SIJRVEYING MAPPINE ENGIVEERING ARTILLERY GEODESIR.S I *TM. 5-24I-8.

ARMY, DEY'I OF

D.EPAR TMENT OF THE ARMY, WASHINGTCN, D.C.

$02 / 06$ 7-52 66 PAGES 25 FIGS 4 TABLES

RD U

\section{Geology and Mineralogy}

QLARTERLY OF THE CCLORADO SCHCCL CF NINES, 50111 CLASSIFICATION OF ROCKS 1 KOCK (GEOLOGY) MINERALS.TEXTURE PETRCGRAPHY I GNEOUS-ROCK SECIMENTARY-ROCK METAMORPHIC-ROCK , \#A.1.,1197

TRAVIS, R. B.

COLC SCH MINES RES FOLJN

COLORADO SCHOOL OF MINES RESEARCH FOUNDATICN, GOLLEE, COLO.

QR/O? 1-55 98 PAGES 4 FIGS 16 REFS

JD U

08-07-02242 MECHANICAL PROPERTIES OF ROCKS AT HIGH TENPERATURES. AND PRESSURES I ROCK-MECHAVICS LOADING (MECHANIC S) USSR EXCAVATICN SHEARING CUTTINE BENCING TORSION STRETCHIVG COMPRESSING TESTING-EQUI PNENT NARBLE DOLCMITE LIMESTONE ROCK-SALT ANHYOR ITE

SANDSTONES CLAY SILTSTONES GEOLOGY THERMAL-STRESS STRESSES STRAIN $)$ \#.L. 1159

BA IDYUK, . B. V.

CONSULTANTS BLREAL

CONSULTANTS BUREAL, NEK YCRK, N.Y.

QRIOT 1967 75 PAGES 54 FIGS I31 REFS

RF $U$ 
$08-07-02243$

$08-07-02244$

$08-07-02245$

$08-11-02246$

$08-1.1-02247$

QLARTERLY OF THE COLORADO SCHOCL GF MINES $51(3)$ SYMPOS IUM ON ROCK MECHANICS ( ROCK (GEOLOGY) LNDERGROUND-STRUCTURES FAILURE (MECHANICS) MINING-ENGINEERING STRESSES GRANITE OPENINGS BLOCK-CAVING-NINING BLASTING , *A.L.1200

COLO SCH MINES RES FOUN

COLORADO SCHODL OF MINES RESEARCH FOUNCATION, GOLCEN, COLO. DE/O7 7-56 239 PAGES 89 FIGS 12 ,TABLES 144 REFS

$\mathrm{J} \leq \mathrm{U}$

QUARTERLY OF THE COLORADO SCHOOL OF NINES $52(3)$ BEHAVIOR OF MATERIALS IN THE EARTHS CRUST - SECOND ANNUAL SYMPOSIUM ON ROCK MECHANICS ( ROCK (GEOLOGY)

FAIL URE (MECHANIC S) STRESSES THEORY DEFCRNATICN EXPERIMENT AL-DATA EEOLOGY

INSTRUMENTATION GEOLOGY OPENINGS UNDERGROUND-STRUCTURES, \$A.L.1198

COLO SCH MINES RES FOUN

COLORADO SCHOOL OF MINES RESEARCH FOUNDATION, GOLCEN, COLO.

DE/07 7-57 3[6 PAGES 91 FIGS 63 REFS

$J \subseteq U$

DESIGN OF UNDERGROUND OPENINGS IN CONPETENT ROCK I MINING-ENGINEERING ROCK(GEOLOGY) PILLARS STRESSES NATHEMATICAL-ANALYSIS ROCK-FORMATICNS STRAIN PHYSICAL-DROPEZTIES CALCULATIONS， \#BLLLETIN 587

OBERT, L. + DUVALL, W. I + + NERRILL, R. H.

U.S. DEPT. OF INTERIOR

U.S. DEPARTMENT OF THE INTERIOR, BLREAU CF MINES

DE/G7 1960 36 PAGES 27 FIGS 41 REFS

RF U

\section{Seismology}

PROCEEDINGS OF THE SECGND WORLD CCAFERENCE ON EART LGUAKE ENGINEER ING II. TOKYO AND KYOTO, JAPAN ( JAPAN SEISNOLOGY STRUCTURES SHOCK-WAVES ELASTICITY) \$A.L.IIQ5 VELETSOS, A. S. + NEWMARK, N. N.

JAPAN

JAPAN, SCIENCE COLNCIL OF JAPAN

$0 E / 117-6 C$ I P PAGES II FIGS 3 TABLES 6 REFS

$R \leq U$

QLARTERLY OF THE CCLORADO SCHOOL CF MINES 50(4) SEISMIC WAVE PROPAGATION AND PRESSURE MEASUREMENTS NEAR EXPLOSICNS 1 WAVEFORM-GENERATORS INSTRUM SNTAT ION TRAN SDUEERS OSCILLCSCOPES CAMERAS REFLECTICN REFRACTION WAVE-TRAVSMISSION THEJRY, \#A.L. 1199

PICKETT, G. R.

COLO SCH MINES RES FOUN

COLORADO SCHOOL OF MINES RESEARCH FCUNDATICN

QE/II $19 / 55 \quad 78$ FAGES 21 FIGS 5 TABLES 32 REFS

JF U

\section{Soil Mechanics}

$08-12-01745$

NLIC.L. EAR WAR AND SCIL EROSICN - SONE PROBLENS AND PROSPECTS 1 POSTATTACK-OPERATIONS SOIL-CONSERVATION SOIL-MECHANICS, \#RM-52:3-TAB

KATZ, Y. H.

RAND

RAND CORPORATION, SANTA NCNICA, CALIF.

DE/13 12-66 27 PAGES 3C REFS

RF $U$

$08-13-01746$

MOTION OF SUBSLRFACE SOIL INCLUSICNS SUEJECTED TO SURFACE BLAST LOADING - RESULTS OF SERIES III. TESTS I SHELTERS UNDERGROUND-STRUCTURES SOIL-MECHANICS SURFACE-BUR ST FAII, IJRF ( MEC.HANICS ) , *TR-508

WIIITC, C. $\mathrm{R}$.

NCEL

U.5. NAVAL CIVIL ENGINEERING LABORATORY, PORT HUENEME, CALIF.

$09 / 13 \quad 1-A 7$ 3T PAFIFS 5 REFS

R F U

$08-13-01747$

THEORETICAL GROUND SHOCK EFFECTS II. APPLICATION OF GENERAL IZED COORDINATES TO PLASTIC WAVE PROBLEMS I SCIL-NECHANICS MECHANICAL-WAVES PROPAGATION SHOCK-WAVES UNDERGROUND-EXPLOSIONS ELASTICITY EQUATICNS-CF-MOTICN PLASTICITY THEORYI \#DASA-1676-II \#AD 637197

BROOKS, J. A. + MCPHERSON, W. A. JR.

UNITED AIRCRAFT CSC

UNITED AIRCRAFT CORPORATE - SYSTEMS CENTER, EL SEGUNCC, CALIF. OEIIJ B-UU $T$ PAGLS 7 RELS

RE U 


\section{ELECTRONICS AND ELECTRICAL ENGINEERING}

\section{Components}

$09-01-01748$

$09-02-01750$

$09-02-01751$

$09-02-[2248$

$09-03-01752$

09-0 ב-01753

$09-03-01754$

SEMICONDUC TOR THERMOELECTRIC TRANSISTOR CCOLERS I SEMICONCUCTOR-DEVICES COOLING THERMOELEC TR IC ITY THERMAL-CONDLCTIVITY ELECTRICAL-CONCUCTANCE GERMANIUM, *RSIC-523 * AD $631 \quad 224$

GARACHUK, V. K. + NAYER, V. S.

REDSTONE SCI INFC CEN

REOSTONE SCIENTIFIC INFORNATICN CENTER, REDSTONE ARSENAL, ALA.

CG/JI 2- CE IC PAGES 3 REFS

RF $U$

\section{Computers}

RESEARCH AND TECHNOLOGY DIVISICN REPCRT ( CCNPUTERS PROGRAMMINGICOMPUTERS) PROGRAMMING-LANG UAGES EDUCATICN OPERATIONS-RESEARCH. TRA IN ING MATHEMATICS DATA-PROCESSING-SYS.TENS, \#TN-530-009-0D

BAUM, $C$.

SYSTEM DEVELOPMENT CORP

SYSTEM DEVELOPMENT CORPORATION, SANTA NONICA, CALIF.

QC/O2 I-C6 IRO PAGES 315 REFS

RV U

RESEARCH AND TECHNOLOGY DIVISICN REPORT FOR 1960 (PROGRAMMINGICOMPUTERS) DATA-PROCE SSING-SYSTEMS CCNPUTERS OATA-TRANSNISS ICN-SYSTEMS SIMUL AT ION INFORMATION-RE TRIEVAL GANE-THECRY EOUCATION TRAINING OPERATIONS-RESEARCH PROGRAMMING-LANGUAGES MATHEMATICS, \#TM-530/OID/OO

DRUKEY, D. L. + BARANCIK, B. O. + YARNCLD, K. W:

SCHWARTZ, J. I. + DOBBS, G. H. + BAUN, C.

SYSTEM DE VELOPMENT CORP

SYSTEM DEVELOPNENT CORPORATION, SANTA MONICA, CALIF.

QG/02 $1-67232$ PAGES 54 FIGS 417 REFS

RE U

TOWARD THE DEVELOPNENT OF A MACHINE WHICH CONPREFENCS 1 CIGITAL-COMPUTERS PROGRAMMING (EOMPUTERS) CONPUTERS SFECIAL-PURPOSE-COMPUTERS REAOING-MACHINES COMMUNICATION-THEORY DATA-STORAGE-SYSTENS , \#A.L. 1126

L INDSAY, R. K.

UNIV OF TEXAS

UNIVERSITY OF TEXAS, AUSTIN, TEXAS

OS/J2 5-61 93 PAGES 66 REFS

RG U

\section{Electronic and Electrical Engineering}

A OIRECT CLRRENT NOTOR WITH VC CCNTACTS ANC A TRANSISTOR COMMUITATOR I ELECTRIC-MOTORS RLSSIA CONNUTATORS SEMICCNDUCTOR-CEVICES TRANSISTORS CONTROL-SYSTEMS I \#FTD-TT-65-2035 \#AC 636610

LEBEDEV, N. I, + CVEHINNI KCV, I. Y.

AF FORE IGN 'TECH DIV

AIR FORCE FOREIGN TECHNOLCGY DIVISION, WRIGHT-PATTERSON AIR FORCE BASE, OHIO

CS/O3 3-21-66 IS PAGES 6 RFFS

RF $\quad$ L

UNDERGROUND DI STRIBUTICN I UNDERGRCUND-STRUCTURES

UNDERGROUND-RESIDENTIAL-DISTRIBUTICN-SYSTEN.S COSTS FAULT-LCCATION ELECTRIC-CABLES TRANSFORMERS HEAT-TRANSFER, \$31-C-35

IEEE

INSTITUTE OF ELECTRICAL AND ELECTRCNICS ENGINEERS, NEW YORK, N.Y. Q $/ 03 \quad 1966$ GIS PAGES 225 FIGS 55 REFS

RS $U$

- UNDERGROUND RESIDENTIAL DISTRIBUTICN I TRANSFORMERS

UNDERGROUNU-RE SIDENTI AL-DISTRIBUTI CN-'SYSTEMS UNDEREROUND-STRUCTURES DESIGN I

* T- IOC-S

IEEE

INSTI TUTE OF ELECTRICAL AND ELECTRCNICS ENGINEERS, NEW YORK, N.Y.

OQ $/ 031964143$ PAGES 9 REFS

$R S U$ 
$09-03-01755$

$09-03-02249$

$39-05-01756$

SEALED NICKEL CADNIUM BATTERIES FCR AIRCRAFT ELECTRICAL SYSTEMS I STORAGE-BATTERIES ALKALINE=CELLS AIRCRAFT-ECIIIPNENT-FOWER-SUPPLIES， \#AFAPL-COVF-66-6 $\$ 40634845$

MILLER, G. H.

AF AERO PROP LAB

$\triangle I R$ FORCE AERO PROPULSION LABORATCRY, WRIGHT-PATTERSON AIR FORCE

BASE, OHIO

I $10103 \quad 7-66 \quad 14$ PAGES 7 REFS

RF U

\section{MATERIALS}

\section{Wood and Paper Products}

$11-12-01766$

WOOD HANDBOOK I CCNSTRLCTICN DESIGN STRENGTH STRUCTURAL -PROPERTIES QUAL IIY FASTENER S GLUING PLYWOOD SANDWICH-CCNSTRUCTI CN FIRE-RES ISTANCE THERMAL-INSULATION PRESERVATION FINISHES PAINTS, HANDBOOK NG. 72

U.S. DEPT. OF AGRICULTURE

IJ.5. DFPARTMFNT OF AGRICLLTURE, FOREST SERVICE

$11 / 12$ 1 255 528 PAGES 35 REFS

RD U

AL

INSTITUTE OF ELECTRICAL AND ELECTRCNICS ENGINEERS, NEW YORK, N.Y.

\section{Subsystems}

AIR FORCE FOREIGN TECHNOLOGY DIVISION, WRIGHT-PATTERSON AIR FORCE

BASE, OHIO

DAGES D REFS

WATT THERMOELECTRIC PCWER SYSTEN FCR EUCY APPLICATIONS I THERMOELECTRICITY TATORS POWER-SUPPLIES ELECTRIC-POWER-PRCCUCTION I \#AD 636436

WESTINGHOLSE ELECTRIC CORPORATION, LIMA, OHIC

$1[/ 02$ 19E6 4 PAGES D REFS

\section{Energy Storage}




\section{MATHEMATICAL SCIENCES}

\section{Mathematics and Statistics}

$12-01-02250$

$|2-01-0225|$

LOGIC, ME THODOLOGY AND PHILOSOPHY CF SCIENCE - PROCEECINGS OF THE 1964

INTERNATIONAL CONGRESS 1 SYMDCSIA MATHENATICAL-LOCIC MATHEMATICS METAMATHEMATICS PFYSICS LANGLAGE PHYSIOLOGY MECHANICS, *AD 646739

BAR-HILLEL, Y.

HEBREW UNIV

HEBREW UN I VERSITY, JERUSALEN, ISRAEL

I $2 / 01$ 4-65 452 PAGES

RS U

THE CHI-SOLARE TEST OF GOCDNESS CF FIT FCR A.RIVARIATE NORMAL CISTR IBUTION 1 STATISTICAL-TE STS STATISTICAL-DISTRIEUTICNS PROBAEILITY-CENSITY-FUNCTIONS RANDOM-VARIAELES hEAPON-SYSTEMS ANALYSIS-OF-VARIANCE， \#TM-K-77/66 \#AD 64E 141 BATES, C. B.

NAVAL WEAPONS LAB

U.S. NAVAL WEAPONS LABORATORY, DAHLGPEN, VA.

$12 / 01$ 2-3-67 3I PAGES 5 FIGS 2 TABLES 18 REFS

RF U

\section{Operations Research}

\footnotetext{
12-02-01767 CIVIL DEFENSE RESEARCH ANALYSIS 1 SYSTENS-ENEINEERING

$12-02-01768$ P. ESEARCH-PROGRAM- ADMINI STRATICN OPERATICNS-RESEARCF CIVIL-CEFENSE-SYSTEMS METHODOLOGY, *A.L. IOI3

METHODOLOGY I
DEVANEY, J. F. OCD

OFFICE OF CIVIL DEFENSE

$12 / \mathrm{L} 212-15-66 \quad 128$ PAGES 63 FIGS 5 REFS

RO U

CUTTHROAT GAMES ( OPERATICNS-RESEARCH GAME-THEORY ) RAC-P-2J \#AD 638 220

JORDAN, S. L.

RESEARCH ANALYSI $\subseteq$ CORP

RESEARCH ANALYSIS CORPORATION, MCLEAN, VA.

$12 / 02 \cdot 8-6621$ PAGES ID REFS

RF U
}

\section{MECHANICAL, INDUSTRIAL, CIVIt, AND MARINE ENGINEERING}

\section{Air Conditioning, Heating, Lighting, and Ventilating}

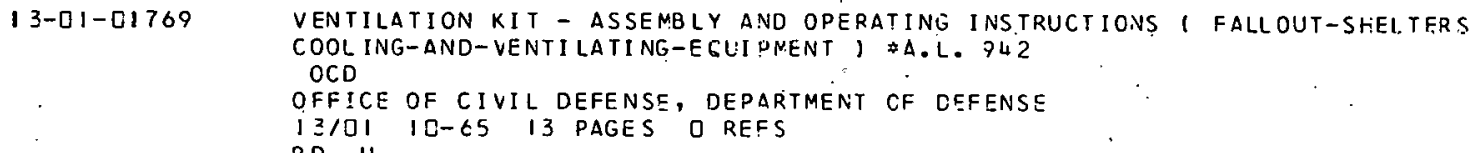

IZ-Q1-G1771 NATURAL VENTILATICN TEST CF AN ABCVEGROUNC FALLOUT SHELTER IV FVANSTON, ILLINOIS I ENVIRONMENTAL-CONTROL EXPERI NENTAL-CATA HABITABILITY FANS PORTABLE INSTRUMEVTATION PUNKAH-PUMPS, \#GARD-REPORT 1263-51

MEIER, H. A. + MADSON, C. A.

GEN AM TRANSPORT CORP

GENERAL AMERICAN TRANSPORTATICN CCRPCRATION, NILES, ILL.

$1 \Xi / 01 \quad 1-66 \quad 112$. PAGES 33 FIGS 7 REFS

RF $U$

13-01-01773 THE STUDY OF A SHELTER COCLING SYSTEN USING METHANOL AS THE HEAT SINK AVO AS THE FLEL. I FUELS COOLING-AND-VENTILATIAG-EGUIPMENT FALLOUT-SHELTERS BLAST-SHELTERS EVAPORATORS COSTS ENVIRONMENTAL-CONTROL, *AC 637953 HLMMELL, J. D.' + BECK, W. D.

BATTELLE MEMORIAL INST

BATTELLE MEMORIAL INSTITUTE, CCLUNEUS, OHIO

$1 \geq 101 \cdot 6-6617$ PAGES O REFS

RF U 


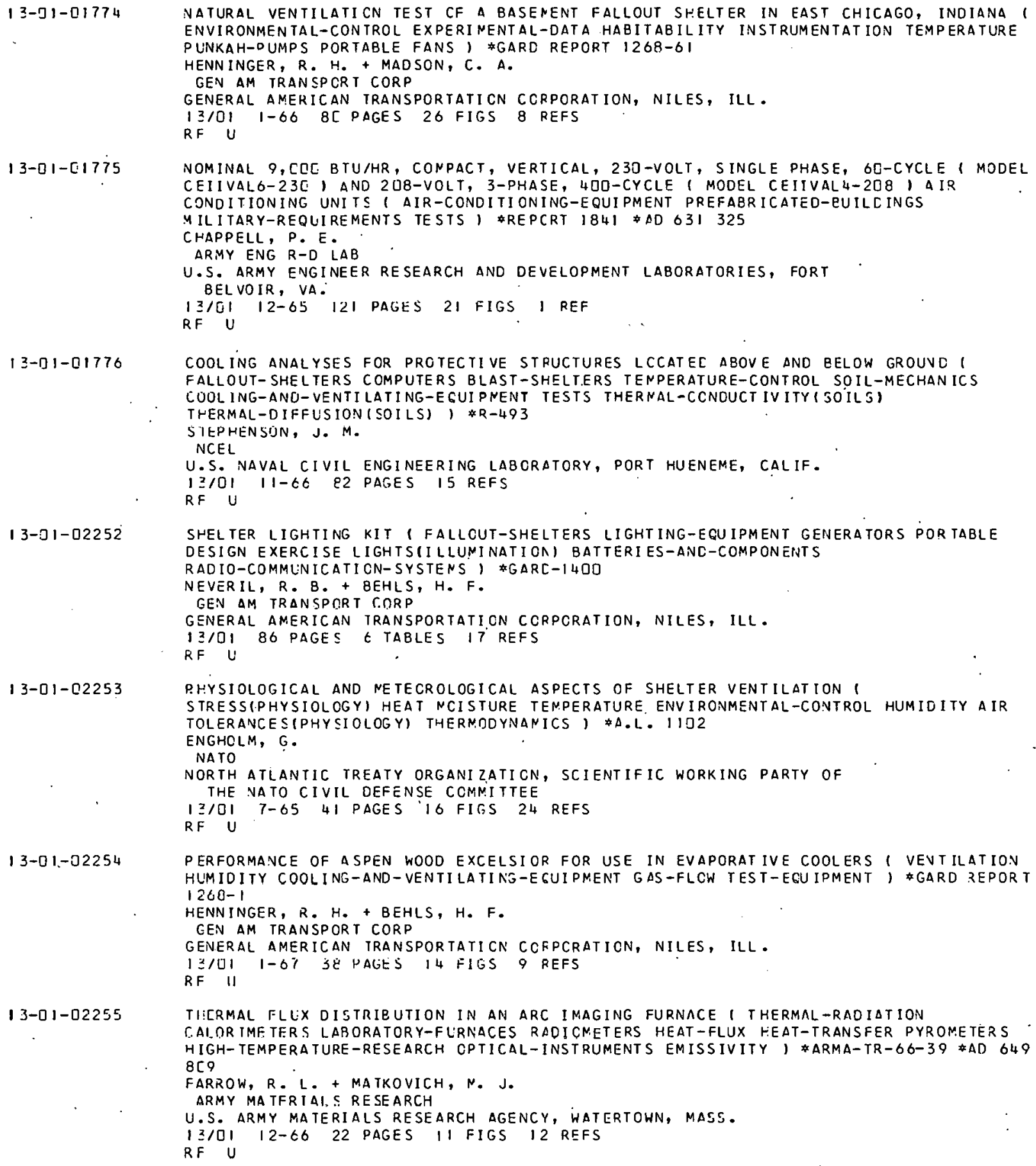

\section{02 Civil Engineering}

13-02-01777 A SIMPLE HEAT ENGINE OF PCSSIBLE UTILITY IN PRIMITIVE ENVIRONMENTS I HEAT-ENGINES DESERTS FEASIBILITY-STLDIES SOLAR-RADIATION EGYPT ENGINES-ANO-MOTORS

IRRIGATION-SYSTENS ) $\$$ P-3367*AD 638687

MLRROW, R. B.

RANO

RAND CORPORAIION, SANTA NCNICA, CALIF.

$13 / 02$ 8-66 31 PAGES 3 REFS

RF U 
$13-02-01778$

$13-02-01779$

$13-02-[1780$

$1.3-02-0178$

$13-02-01782$

$13-02-01783$

$13-02-01784$

$13-02-01785$

$13-02-01.786$

PURLIC HEALTH SER VICE DRINKING WATER STANDARCS I WATER-SUPPLIES REGULATIONS RAOIOACTIVITY CONTROL, \#PHS-956

L..S. DEPT. OF HEAL ED WEL

U.S. DEPARTMENT OF HEALTH, EDUCATICN $\triangle N D$ WELFARE, PUBL IC rEALTF SER VICE

$13 / 021962$ OI PAGES 7 REFS

$\mathrm{RC} U$

CONTROL OF SLRFACE STORM WATER BY STCRM. CRAINS ANC CRAINAGE CHANVELS II . PLANS, PROFILES, CROSS SECTIONS, AND GENERAL LOCATICN OF RIGHTS OF WAY TO BE ACCUIRED I FLOODS CALIFORNIA; $*$ A. L. 987

HEDGER, H. E.

LA COUN FLOOD CON DIS

LOS ANGELES COLNTY FLOOD CONTRCL CISTRICT, LCS ANGELES, CALIF.

13102 I I SE IEO PAGES I80 FIGS

RO U

POSTATTACK SANITATION, WASTE OISPCSAL, PEST ANC VECTOR CONTROL, $\triangle N C$ THE EFFECTS OF FALLOUT IN WASTE WATER AND SEWER SYSTEMS 1 PCSTATTACK-OPSRATIONS

RADIOAC TIVE-FALLOLT WASTE S (SANITARY-ENG INEERING) WASTES (INCUSTR IAL) GARBAGE

SAN I TARY-ENG INEER ING SEWAGE DISEASE-VECTCRS CISEASES (CONTROL) DRA INAGE

COUNTERMEA SURES URBAN-AREAS SAN-JCSE CALIFORNIA RECOVERY PEST-CONTROL）

* USNR DL - TRC -79

HARMON, J. A. + LEACH, J. N.

ENG INEER ING-SCIENCE

ENG INEER ING-SC IENCE, INC., ARCADI A, CALIF。

$13 / 02 \quad 1-67 \quad 92$ PAGES 36 REFS

RF U

CONTROL OF SURFACE STORM WATER BY STCRM CRAINS ANC CRAINAGE CHANNELS II. PLANS, PROFILES, CROSS SECTIONS, AND GENERAL LCCATICN OF RIGHTS OF WAY TO BE ACOUIRED CIVIL-ENGINEERING FLOODS , *A.L: 972

$S \triangle I, S B I J R Y, M: E$.

LA COUN FLOOD CON DIS

LOS ANGELES COLNTY FLOOD CONTRCL DISTRICT, LCS ANGELES, CALIF.

$13102 \quad 1964$ I 65 PAGES 165 FIGS

RO. U

CONTROL OF SURFACE STORM MATER BY STCRM CRAINS ANC CRAINAGE CHANNELS I. GENERAL DESCR IPTION OF WORK PROPOSED AND NAP SHCWING GENERAL LOCATION OF PROJECTS 1 FLODDS FLOOD-CONTROL CALIFORNIA FINANCING CCNSTRUCT ION I \$A.L. IDEO HEDGER, H. E.

LA COUN FLOOD COA DIS

LOS ANGELLES COLNTY FLOCD CONTRCL DISTRICT, LCS ANGELES, CALIF.

I $3 / 021952$ III PAGES O REFS

RF U

CONTROL OF SURFACE STORM LATER BY STCRN CRAINS ANC CRAINAGE CHANNELS - I958 PROGRAM I. GEVERÁL DESCRIPTI ON OF WORK, PRCPCSEC ANC MA.P SHONING CENERAL LOCATION OF PROJECTS I FLOODS FLOOD-CCNTROI. C.AIIIFORNTA) \#A.L. IOBI HECGER, H. E.

LA COUN FLOOD COA DIS

LOS ANGELES COLNTY FLOOD CONTRCL DISTRICT, LCS ANEELES, CALIF.

$13 / 02 \quad 1958 \quad 237$ PAGES O REFS

RF L

CONTROL OF-SURFACE STORM WATER BY STCRN CRAIAS ANC CRAINAGE CHANNELS - IGGL PROGRAM

I. GENEKAL UESCRIPTION OF WCRK PRCPCSEC ANC MAP SHOWING GENERAL LOCATION OF PROJECTS (.FLDOOS FLOOD-CCNTROL CALIFORNIA CCNSTRUCTION COSTS I \#A.L. IOT9

SAL SBIJRY, M. E.

LA COUN FLOOD CON DIS

LOS ANGELES COLNTY FLOCD CONTROL DISTRICT, LCS ANGELES, CALIF.

$13 / 02 \quad 1964$ I 89 PAGES 0 REFS

RF $\cdot U$

PHASE II I SLBSLRFACE INVESTIGATICN - ŃATICNAL CAPITAL RAPIC TRANSIT SYSTEM PORTION OF CONNEC TICLT AVENUE ROUTE RETWEEA COLUMBIA ROAD AND VAN NESS STREET I

TRANSPORTATION SUBHAYS TESTS BORING WASHINGT CN CONSTRUCTION SETTLEMENT(STRUCTURAL) CONSOLIDATION DEC CNPOSITICN SHEAR-STRENGTH, \#A.L. IOZ2

MLESER RLTLEDGE

MUESER, RLTLEDGE, WENTWORTH AND JOFNSTCN, NEW YORK, N.Y.

$13 / 02 \quad I C-19-66 \quad 9.7$ PAGES 63 .FIGS

RF L

EXTENSIVE STREET BLILDING PROGRAN IN HANBURG 1 GERMANY ROALS CONSTRUCTIJN URBAN-AREAS TUNNELS(TRANSPORTATI CN) VENTILAT ION TRAFFIC I \$ORNL-TR-1596

SILL, O.

GERMANY

IIIDRANY 196624 PAGES I $^{\prime} 3$ REFS

RF U 
$13-02-01787$

$13-02-01788$

$13-02-01789$

13-02-02256

$13-0 z-02257$

$13-02-02258$

$13-02-02259$

$13-06-02260$

$13-11-01792$

REPOR T ON HARD ROCK TUNNELLING INVESTIGATICN ( EXCAVATION TUNNELSITRANSPORTATION) BLASTING COSTS DRILLING NCRTHEAST-CCRRIDOR FEASIBILITY-STUCIES, 1 AP I7D 511 HIR SCHFELO, R. C.

MIT

MASSACHUSETTS INSTITUTE OF TECHNCLCGY, CAMBRIDGE, MASS.

12102 10-15-65 56 PAGES IOFIGS 47 REFS

RF U

CIVIL OEFENSE ASPECTS OF NUNICIPAL WATER SYSTEMS 1 CIVIL-CEFENSE-OPERATING-PLANS UIILITIES DATA WATER-SUPPLIES L'RBAN-AREAS POSTATTACK-CPERATIONS MANAGEMENT-PLANNING RECOVERY VLLLNERABILITY RECCRDS DISTRIBUTION(WATER) SURVEYS, \$A.L. 969 HARMON, J. A.

ENG INEER ING - SCIENCE

ENGINEER ING-SCIENCE, INC. , ARCADIA, CALIF.

$1 \geq / 02 \quad 11-66$ 4C PAGES 6 REFS

RF U

SOL ID WASTES RESEARCH NEEDS I SCIENTIFIC-RES EARCH SANITARY-ENGINEEER ING PUBL IC-HEALTH DISPOSAL COSTS WASTES (SANITARY-ENGINEERING) STORAGE . EARBAGE PROJECTIONS, *A.L. 981

BLGHER, R. D.

AMER PUBLIC WORKS ASSN

AMERICAN PLBLIC WCRKS ASSCCIATION, CHILAGU, ILL.

13102 5- 62 8[ PAGES 14 REFS

RV U

URBAN FORM AS A PASSIVE DEFENSE VARIABLE I CCSTS UREAN-AREAS CONFIGURATION URBAN-PLANNING EFFICIENCY SELECTIOA POPULATICN DENSITY CISTRIRUTION ECONOMICS FEASIBILITY-STLDIES MANAGENENT-PLANNING CIVIL-CEFENSE-SYSTEMS, \#PRC-C- 1341 \$AD E49 TC

PLANNING RESEARCH CORP

PLANNING RESEARCH CORPORATION, LCS ANGELES, CALIF. $12 / 02 \quad 1-16-67$ 39 PAGES 4 FIGS 3 REFS

RF $U$

A NEW ERA FOR AMERICAS HATERS I WATER-POLLUTICN WATER-SUPPLIES I \#C.WA-1

U.S. DEPI. OF INTERIOR

U.S. DEPARTMENT OF THE INTERICR

$131023-6724$ PAGES 13 REFS

$x ?:$

INVESTIGATION OF ALTERNATIVE AQUEDLCT SYSTEMS TO SERVE SOUTHERN CALIFORNIA FEATHE? RIVER AND DELTA DIVERSION FRCJECTS I WATER-SUPPLIES URBAN-AREAS ECOVOMICS COSTS, $\approx$ QLLLETIN NO. 78

CALIFORNIA, STATE OF

STATE OF CALIFORNIA, DEPARTMENT CF WATER RESOURCES

$1 \geq / J 2$ 12-59 179 PAGES 33 FIGS 39 TABLES

R V U

DESIGV AND CONSTRLCTION OF SANITARY AND STORN SEWERS I SANITARY-SEWERS 1 \#WPCF-9 WAIER PULLUTION CON FED

WATER POLLUTION CONTROL TEDERATICN, WASHINGTCN, . . C.

$12 / 621967283$ PAGES IO2 FIGS 21 TABLES 119 REFS

RC U

\section{Ground Transportation Equipment}

EFFECTS OF NUCLEAR ATTACK CN FREIGHT TRANSPORTAT ICN SYSTEMS - INTERACTIONS AND COMPARISONS AMONG MODES I CARGO POSTATTACK-OPERATICNS VULNERABILITY ROADS RAILROADS CARGO-VEHICLES AIRCRAFT WEAPONS-EFFECTS DANAGE-ASSESSNENT TRUCKS, DIXON, H. L. + TEBBEN, T. H.

STANFORD RESEARCH INST

STANFORD RESEARCH INSTITUTE, MENLC PARK, CALIF.

$13 / 06 \quad 3-67$ 5I DAGES 7 FIGS 21 REFS

$R F \quad U$

\section{Pumps, Filters, Pipes, Tubing and Valves}

\footnotetext{
DEVELOPMENT OF U-FOAM AIR FILTERS I VENTILATION FILTERS (FLUID) CHEMICAL-PROPERTIES CONSTRUC TION MECHANICAL-PROPERTIES THERNAL-RESISTANCE COSTS ACICT-RESISTANCE PRESSLRE-DROP I \#AEET-244

KHAN, A. A. + AMALRAJ, R, V. + THCNAS, K. T. IND IA

INRIA. ATOMIS. ENERGY ESTABLISHNENT, TRONEAY, BOMBAY II/1 IO66 III PAGES D REFS

RF U
} 


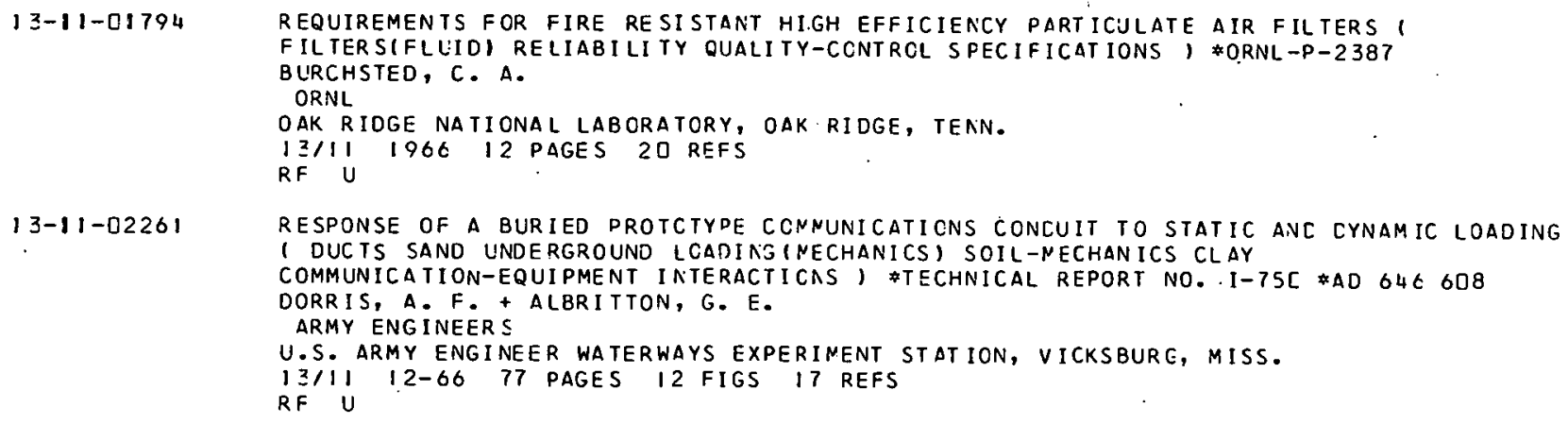

\section{Safety Engineering}

,3-12-01795

$13-82-01796$

$13-12-01797$

$13-12-01798$

$13-12-0.1799$

$13-12-01800$

$13-12-0180$
THERMAL STRESSES IN A SENI INFINITE SOLIC BY A FINITE DIFFERENCE TECHNIQUE I THERMAL-RADIATION WEAPONS-EFFECTS NUCLEAR-WEAPONS, APR-4 HEILFERTY, R. J. + KOCH, J. E. + CCHEN, M. L. NAVAL APP SCIENCE LAR

U.S. NAVAL APPLIED SCIENCE LABCRATCRY, BROCKLYN, N.Y. $13 / 12$ 1-3-67 36 PAGES I REF RF U

WINDOW AND WINDOW SCREENS AS MCDIFIERS CF THERMAL RADIATION RELEASED IN NUCLEAR DETONATIONS I GLASS ATTENLLTICN UREAN-AREAS IGNITION IA.L. 97 I BRACCIAVENTI, J.

NAVAL APP SCIENCE LAB

U.S. NAVAL APPLIED SCIENCE LABCRATCRY, BROOKLYN, N.Y.

12112 Q- $66 \quad 17$ PAGES 5 REFS

RF

EQUATIONS AND COMPLTER PRCGRAM TO CALCULATE THE THERMAL HISTORY OF A DUAL LAYERED PLATE SUBJECT TO THE THERNAL PLLLSE CF A N'UCLEAR WEAPON ( CALCULAT IONS

PROGRAMMING (CONPUTERS) THERNAL-RADI ATI ON GEONETRY MATHEMAT ICAL-MODELS.HEAT-TRANSFER WEAPONS-EFFECTS SHIP-STRUCTURAL-CCNPONENTS NAVAL-RESEARCH I*A.L.984 HEILFERTY, R. J. + KOCH, J. E. + CCHEN, M. L.

NAVAL APP SCIENCE LAB

U.S. NAVAL APPLIED SCIENCE LABCRATCRY, BROOKLYN, N.Y.

$13 / 12 \quad 12-66 \quad 78$ PAGES D REFS

RF U

PROJECT FIRE SCAN - THE EVALUATICN OF AN AIREORNE INFRAREC MAPPER AS A TCOL FOR DETECTING AND MEASLRING FIRES I INFRAREC-DETECTORS INFRAREL-TRACK ING FOREST-FIRES PATROL-PLANES AIRCRAFT, \&INT-25

WILSON, R. A. I NCSTE, N. V.

U.S. DEPT. OF AGRICULTURE

U.S. DEPAR TMENT OF AGRICULTURE, FOREST SERVICE

$13 / 12$ 6- 66 62 PAGES 12 REFS

RF U

FIRE BIBLIOGRAPHY - SELECTED URBAN AND MIXED URBAN RURAL FIRES.1940-19641 B IBL IOGRAPHIES URBAN-AREAS RURAL-AREAS FIRE-SAFETY, STOREY, T. G. + DIETERICH, J. H. + NCORE, W. R.

JAY, J. W.

L.S D DEPT = OF AGRICULTURE

U.S. DEPARTMENT OF AǴRICULTURE, FCREST SERVICE

$13 / 125-65$ 46 PAGES 4 REFS

RA U

MASS FIRE LIFE HA ZARD I NLCLEAR-EXPLOSIONS FIRES CASUALTIES SIMIII. A.TION FIRE-SAFETY TOXICITY HEAT CARBON-MONOXIDE CARBCN-DIOXICE AIR SMOKES WIND SHELTERS GERMAVY MICE ) $\#$ NRDL. TRC -63

PRYOR, A. J. + YUILL, C. H.

SOUTHWEST RESEARCH INST

SOUTHWEST RESEARCH INSTITLTE, SAN ANTONIC, TEXAS

$13 / 12$ 9-66 I Q0 PAGES 386 REFS

R $\mathrm{U}$

PARAMETER S GOVERNING URBAN VULNERABILITY TO FIRE FRCM NUCLEAR BURSTS 1

BLRST-CONDITIONS LRBAN-AREAS FIRES NUCLEAR-EXPLCSICN-CAMACE, \#USNRDL-TR-IC4D

RENNER, R. H. + MARTIN, S. B. + JCNES, R. E.

NRDL

U.S. NAVAL RADIOLCGICAL DEFENSE LABORATORY, SAN FRANCISCO, CAL IF.

I $I / 12$ 6- 3 C-66 335 PAGES 173 REFS

RF. U 
$13-12-01802$

$13-12-01803$

$13-12-01804$

$13-12-01805$

$13-12-01806$

$13-12-01808$

$13-12-01809$

$13-1 \hat{z}-01810$

$13-12-01807$
TEMPERATURES ATTAINED IN WOCD EXPCSED TO HIGH INTENSITY THERMAL RADIATION I CALOR IMETERS IGNITION HIGH-TEMPERATURE-RESEARCH RADIOMETERS PYROLYSIS SOLAR-FURNACES COMBUSTION DECCNPCSITION I \#AC 147715

GARDON, R.

MI T

MASSACHUSETTS INSTITUTE OF TECHNCLCGY, CANBRIDGE, MASS.

$13 / 12$ 4-5 35 PAGES 55 FIGS 15 REFS

RF U

DESIGN AND CONSTRLCTION OF A CRASH DUMNY FOR TESTING SHOULCER HARNESS AVD SAFETY BELTS ( TEST-EQUIPNENT TEST-DUNMY (ANTHRCFOMCRPHIC) SAFETY-FARNESS SAFETY-DEVICES.)

*A.L. 983

SWEAR INGEN, J. J.

CIVIL AERO ADNIN AERO CEN

C IVIL AERONALTICS ADMINISTRATICN AERCNAUTICAL CENTER, OKLAFOMA

CITY, OKLA.

$13 / 124-51$ I 4 PAGES IOFIGS 4 REFS

RF U

ENGINEER ING AND RESC UE OPERATICNS AT THE SITE OF AN ATOMIC STRIKE I RESCUES

C IVIL-DEFENSE-PER SONNEL USSR INTERAATI CNAL-C IVIL-CEFENSE TRAINING MANUAL $S$

NUCLEAR-EXPLOSION-DAMAGE NUCLEAR-WARFARE SHOCK-WAVES THERMAL-RADIAT ION

RADIOLOG ICAL-CONTAMINATI ON FIRES CCNSTRUCTICN POSTATTACK-OPERATIONS REPAIR

MILI TARY-ENGINEER ING RECONNAISSANCE , JPRS-31,014 \$T-65-

VOLKOV, I. D. + ULANOVSKIY, B. Y. + USOV, N. A.

TSIVILEV, M.P.

JPR S

JOINT. PUELICATIONS RE SEARCH SERVICE, WASHINGTON, [.C.

13112 7-9-65 191 PAGES 43 FIGS 25 REFS

Q 1

FIRESTORM ANALYSI $\leq($ FIRES WIND METEOROLOGY COMPUTERS (PROGRAMMING) $)$ *DC-TN-2046-1 LOMMASSON, T. E. + KELLER, J. A. + KIRKPATRICK, R. G.

OIKEWOOD

DIKEWDOD C. ORPORATION, ALBUQUERGUE, N.MEX.

$13 / 12$ 2-1-67 III PAGES 63 FIGS

RF U

DFFFNDING THE LNI TED STATES FRCM NLCLEAR FIRE I FIRES FIRE-PROTECTION

RESOURCES (VULNERABILITY) FALLOUT-SHELTERS EQUIPMENT URBAN-AREAS RURAL-AREAS

MANAGEMENT-PLANNING COST-EFFECTIVEAESS TRAINING FIRE-STORMS CONFLAGRATIONS RESCUES

VULNERAB IL I TY CIVIL-DEFENSE-OPERATING-PLANS NAPPING (INFRARED-EQUIPMENT), \#A.L. 95 I

MOORE, W. R. + JAY, J. W. + DIETERICH, J. H.

U.S. DEPT. OF AGRICULTURE

U.S. DEPARTMENT OF AGRICULTURE, FOREST SERVICE

$13 / 12$ 5- 6 C 268 PAGES 163 REFS

RV U

SHEL TER HABITABILITY IN EXISTING. BUILDINGS UNDER FIRE EXPOSURE I FALLOUT-SHEL TERS FIRE-PROTECTION SNCKE CARBON-NCNCXIDE OXYGEN-SUPPLY GAS-DETECTORS FIRE-DETECTORS

CARBON-MONOXIDE-INDICATORS TENPERATURE WIND-PRESSURE PRESSURE HEAT-TRANSMISSION

TESTSIFIRESI SLRVEYSIDATA-COLLECTICN), *NRDL-TRC-16 \$AD 637806 WATERMAN, T. E.

IITR I I

ILLINOIS INSTITUTE OF TECHNOLOGY RESEARCH INSTITIITF, C.HIC.ACO, II.I. $13 / 12$ 6-tC 92 PAGES IOREFS

Kr $U$

ANTHROPOMORPHIC TEST DUMMY I TEST-EQUIPMENT CESIGN SAFETY-CEVICES

TEST-OUMMY (ANTHROPUMURHHIC) CCNSTRLCIICN, $\#$ A.L. 982

ALDER SON RE SEARCH LABS

ALDER SON RESEARCH LABCRATCRIES, INC., NEW YORK, N.Y.

$1 \Xi / 12195216$ PAGES D REFS

RF ij

THE CONFLAGRATION POTENTIAL IN SAN JCSE AND ALBUGUERQUE I AERIAL-PHOTOGRAPHS URBAN-AREAS FIRES MAPS TEST-METHODS VULNERABILITY CLASS IFICATION BUILOIVGS, *REPORT NO. O5t9

COHN, B. M.

GAGE BABCOCK

GAGE-BABLULK ANU AS SOLIIATES, INC., CHICAGO, ILL.

$13 / 12$ 10-66 30 PAGES 0 REFS

RF U IO

TEMPERATURES IN A LARGE NATURAL FUEL FIRE I FIRES TEST-METHODS MOOELS(SIMULATIONSI

TEST-EQUIPMENT, \#PSW-9ت

PFILPOT, C. W.

PACIFIC $S h$

PACIFIC SOLTHWEST FOREST AND RANGE EXPERINENT STATION, BERKELEY, CAL IF.

$13 / 12$ i965 14 PAGES 14 REFS

RF U 
1 3-1 $2-c 2262$

$13-12-02263$

$13-12-02264$

$13-12-02265$

$13-12-02266$

$13-12-[2267$

$13-12-02268$

$13-12-02269$

$\mid 3-13-01811$

A STUDY OF hORLD hAR II GERNAN FIRE FATALITIES I FIRES URBAN-AREAS DAMAGE-ASSESSMENT CASUALTIES POPULATION FIRE-STORMS I \#DC-TN-1.0SO-3.

KELL.ER, J. A.

DIKE WOOD

DIKEWOOD CORPORATION, ALGLGUERGUE, N. MEX.

13112 4-66 2[ PAGES 9 TABLES IO REFS

RF U

OPERATION RISTER - THERMAL RADIATICN EFFECTS ON PAINTS, PLASTICS, AND COATED

FABR ICS $\$ T-407$

MILLER, H.

$\triangle R M E D$ FORCES SWP

ARMED FORCES SPECIAL WEAPCNS PROJECT, WASHINETON, C.C.

$13 / 12 \quad 7-52$ J 44 PAGES IL FIGS 23 TABLES

RF

PRACTICAL EXPERIENCES OF FIRE PRCTECTICN SERVICES - A CRITICAL PRESENTATION DF THE ORGANIZATION, TECHNOLOGY, AND TACTICS OF FIRE PROTECTION AS EMPLOYED BY A. C.IVIL DEFENSE ORGANI ZATICN DURING WORLD hAR II - GERMANY - I I FIRES FIRESTORMS

THERMAL-RADIATION INCENDI ARY-BOMBS (WEAPONS-EFFECTS) URBAN-DREAS

FIRE-FIGHTING-EQLIPMENT HANBURG PHCTCGRAPHS ＊A.L. 1122(II)

BRUN SWIG, H.

IOA

INSTITUTE FOR DEFENSE ANALYSES, ARLINGTON, VA.

$13 / 12$ 6- 66 2 96 PAGES 5E0 FIGS O REFS

RO U

PRACTICAL EXPERIENCES OF FIRE PROTECTICN SERVICES - A CRITICAL PRESENTATION OF THE ORGANIZATION, TECHNOLOGY, ANO TACTICS CF FIRE PROTECTION AS EMPLOYED BYY A CIVIL DEFENSE ORGANIZATION DURING WORLD WAR II - GERMANY I I FIRES FIRESTORMS FAMBURG FIRE-FIGHTING-EQUIPMENT URBAN-AREAS THERNAL-RADIATION, \#A.L. II22(I) BRUNSHIG, H.

IDA

INSTITUTE FOR DEFENSE ANALYSES, ARLINGTCN, VA.

$12 / 12$ 6-66 449 PAGES 140 REFS

RV

FLAME I - FIRE SPREAD SIMLLLATION MCDEL ( FIRES PRCGRAMMING(COMPUTERS) DATA-PROCE SSING URBAN-AREAS MATHENATICAL-MODELS GECGRAPHY ; \#NREC-2I NAT RES EVAL CENTER

NATIONAL RESOURCE EVALUATI ON CENTER; WASHINGTON; C.C.

$13 / 12 \quad 1-64 \quad 156$ PAGES 17 FIGS OREFS

R I U

PRELIMINARY AERIAL INFRARED FIRE- NAPPING SYSTEMS ANALYS IS I.MICHIEAN

ATIACK-SCENARI OS AIRCRAFT NUCLEAR-hEAPONS-EFFECTS MILIT ARY-IVTELL IGENCE

SYSTEMS-ANALYSIS POST-ATTACK-OPERAIICNS DAMAGE-ASSESSMENT FIVE-CITY-STUDIFS )

* $\$ 771,1-F$

SHEGDER, R.:D. I DLSUAY, G. L.

HRB - SINGER

HRB-SINGER, INC., STATE CCLLEGE, PA.

$13 / 12$ 4-67 $9 E$ PAGES I3 FIGS IS TABLES 21 REFS

RF U

SHADOW GEOMETRY OF BUILDINNG IN UREAN AREAS I FIRES THERMAL-RACIATION SWECEN

*A.L. IIIR

ONNERMARK, B. + GCTHFRSKJCLK, L.

SWEDEN

SWEDEN

IIII2 7 PAGES 6 FIGS 0 REFS

RF U

DEVELOPMENT OF STANDARD FIRE TEST RATING SYSTEMS FCR SHELTER COMPONENTS 1 FALLOLT-SHELTERS FIRE-SAFETY FIRES STRUCTURES SMOKES EU ILCINGS CONSTRUCTION RLAST RADIOACTIVE-FALLOLT HEAT-TRANSFER EXPOSURE TCXICITY CONSTRUCTION-MATERIALS,

* IITRI-NG[OGI *AD $Z 50323$

LABES, W. G. +. hATERMAN, T. E. + VARLEY, R. R.

IITR I

ILLINOIS INSTITUTE OF TECHNOLOGY RESEARCH INSTITUTE, CHICACO, ILL.

$13 / 12$ I2-66 164 PAGES 12 FIGS 42 REFS

RF U

\section{Structural Engineering}

AN INVESTIGATION CF MININAL EgUIPNENT NEEDS IN PERSONNEL SHELTERS FALLOUT-SHELTERS PHYSIOLOGY EXPLOSION-EFFECTS SANITARY - ENG IVEERING DESIGN COOL ING-AND-VENTILATING-EGUIPNENT HAZARCS CCSTS I \#AD 631442

HAVERS, J. A. +. MCNK, C. B., JR. + KCELLER, E. H, IITR I

ILLINOIS INSTI TUTE OF TECHNOLOGY RESEARCH INSTITUTE, CHICACO, ILL.

$13 / 13$ 6-65 5 PAGES O REFS

RF U 
$13-13-01812$

$13-13-01813$

$13-13-01814$

$13-13-01815$

$13-13-01816$

$13-13-01818$

$13-13-01819$

$\mid 3-13-01821$

$13-13-01822$
TRANSIENT LOADING TESTS ON A RIGID CIRCULAR FOOTING I FOUNCATIONS(STRUCTURES) SOIL-MECHANICS LOADING (MECHANICS) DYNAMICS EXPERIMENT AL-CATA COMPRESS IVE-PRJPERTIES THEORY, \#AD 63I 369

DRNEVICH, V. P. + HALL, J. R., J JR. + RICHART, F. E.

UNIV OF MICHIGAN

UNIVERSITY OF MICHIGAN, ANN ARBOR, NICH.

$12 / 13$ 2-66 67 PAGES 9 REFS

$R F U$

VENTILATION TESIS OF FALLOUT SHELTER SPACES IN NEW YORK CITY ANO VICINITY FALLOUT-SHEL TERS LRBAN-AREAS COOLI AG-AND-VENTILAT ING-EQU IPMEVT BU ILDIVGS UNDERGROUND-STRUC TLRES MCDELS(SINULATICNS) , *AD 631475

GUY B. PANERO, INC

GLYY B. PANERO, INC., NEW YORK CITY, N.Y.

$13 / 13$ 2-66 3 PAGES I REF.

RF $U$

VENTILATION TESTS OF FALLOUT SHELTER SPACES IN NEW YORK CITY ANC VICINITY 1 URBAN-AREA S UNDERGROUND-STRUCTURES BUILOINGS MOCELSISIMULATIONS I HEAT-EXCHAVGERS TEMPERA TURE HEAT-TRANSFER COCLING-AND-VENT ILATING-EQU IPMENT I *AD $6 \geq 142 E$ TEMPERA TURE HEAT TRAN
GUY B. PANERO, INC

GLY B. PANERO, INC., NEW YCRK CITY, N.Y.

IZ/13 2-66 IIZ PAGES IS REFS

RF $\quad$ '

DESIGN MODIFICATICNS AND 1962 COST ANALYSIS FOR A STANOARDIZED SERIES OF FALLOUT SHEL TER S I LNDERGROUND-STRUCTURES CCNSTRUCTI CN URPAN-AREAS PROTECTION-FACTOR BLAST-SHELTER S COSTS, *USNRDL-TR-582

PORTEOUS, L. G. MIRDL

U.S. NAVAL RADIOLCGICAL DEFENSE LAEORATCRY, SAN FRANCISCO, CALIF. $13 / 13$ 9-17-62 13 . PAGES 12 REFS

RF U

BLILDING CCDE OF THE DISTRICT CF CCLUMEIA I RUILCING-COCES REGULATIONS WASHINGTON BUILDINGS STRUCTURES, \$A.L. 995

DISTRIC T OF COLUNBIA

DISTR ICT OF COLUMBIA, WASHINGTCN, C.C.

$13 / 13 \quad 1-1-61 \quad 447$ PAGES

RE U

13-12-01817 MANUAL FOR THE BUILDING CCDE OF THE DISTRICT OF COLUMEIA I WASHINGTON BLILDING-CODES REGLLATIONS STRUCTURES BUILCINGS, $\$$.L. IOCI

DISTRICT OF COLUNBIA

OISTRICT OF COLUMBIA, WASHINGTCN, C.C.

$13 / 13 \quad 1-1-61$ 44[ PAGES

RE $\mathrm{C}$

BLNKER TYPE FALLOLT SHELTER FOR BEEF CATTLE I BOVINES SHIELDING CONSTRUCTION VENTILATION, $\$ M I S C$. PÜB. NO. 947

U.S. DEPT. OF AGRICULTURE

U.S. DEPAK IMENT OF DGRITIII.TURF

$13 / 13 \quad 1-64$ 2 PAGES O REFS

xO U

AN INVESTIGATION CF MINIMAL EQUI PNENT NEEOS IN PERSONNEL SFELTERS 1

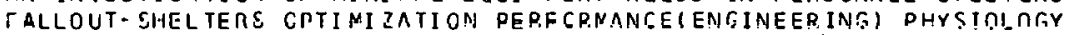

COOLING-AND-VENTI LATI ON-EGUI PNENT SHELTER-OCCUPANCY SYSTEMS-ENG INEER ING

SAN I TAKY-ENG INEER ING UESIGN TEMPERATURE-CONTROL ENVIRONMENTAL-CONTROL COSTS

LIGIITING-EQUIPMENT FIRES, \$AD 631424

HAVERS, J. A. + MCNK, C. B., JR. + KCELLER, E. H.

IITR I

ILLINOIS INSTI TUTE OF TECHNOLOGY RESEARCH INSTITUTE, CHICAGO, ILL.

$1 ? / 13$ 6- 65 4 46 PAGES OC FICS 126 REFS

$R F U$

AN INVESTIGATION OF SCHOOL DESIGNS TC RESIST INTEERATED NUCLEAR WEAPONS EFFECTS I STRUCTURES FALLOUT-SHELTERS BLAST-SHELTERS THERMAL-RACIAT ION CONSTRUCTION MATERIALS FIRE-SAFETY COSTS, *TR-ICD

BRLCE, R. N. + BRANCH, J. E. + GRCNBECK, N. P.

HANLEY, J. T. I MCCAHILL, D. F.

TULANE UNIV

TLLANE UNIVERSITY, NEW ORLEANS, LA.

$13 / 13$ 10-.65 199 PAGES 25 REFS

RF U

EXPER IMENTAL EVALLATION OF TECHNIGUES FOR IMPROVING FALLOUT PROTECTION IN HOME BASEMENTS I SHELTERS FALLCUT-SHELTERS NEVADA WEAPCNS-TESTS SIMULATION RADIOACTI VE-FALLOLT PRCTECTION-FACTCR, $\#$ CEX -65.5

SLMMERS, R. L. + EURSON, Z. G.

CETO

CIVIL EFFECTS TEST OPERATICNS, USAEC

I $3 / 13$ 12-66 112 PAGES 65 FIGS 23 REFS

RF U 
1 3-13-01823

/

$13-13-01825$

$13-13-01826$

$13-13-0182.7$

$1 \cdot 3-13-01828$

$13-13-01829$

$13-13-01830$

$|3-13-0183|$

$13-13-01832$
STUDY OF SHOCK I.SOLATION FOR HARDEAED STRUCTURES I SHCCK-ARSOREERS VIBRATION-I SOLATORS I MPAC T-SHOCK NUCLEAR-EXPLOS I CN-CAMAGE SPR INGS PER SOVNEL DESIGN INSTRUMENTATION , \#AJA-6424 \#AD 639303

JACOBSEN, L. S. + AGBABIAA, M. S.- + KARAGOZIAN, J.

MALTHAN, J. A. + BAREIS, D. W. + BRANDT, R. J.

AGBABIAN-JACOBSEN ASSOC

$\triangle G B A B I A N-J A C O B$ SEN ASSOCIATES, LOS ANGELES, CALIF.

$13 / 13$ 6-66 724 PAGES 300 FIGS 79 REFS

R.V U

THE ELASTIC RESPONSE OF BLRIED CYLINDERS, CRITICAL LITERATURE REVIEW AND PILOT

STUDY ( SHELTERS LNDERGROLNO-STRUCTURES ELASTICITY TEST DYNAMICS LOADING(MECHANICS)

STRAIN(MECHANICS) THEORY, \#TECHNICAL REPORT NO: I-720

ALBRITTON, G. E. + KIRKLAND, J. L. + KENNECY, T. E.

DORR IS, A. F.

ARMY ENG INEERS

U.S. ARMY ENGINEER WATERWAYS EXPERIMENT STATION, VICKSBURE, MISS.

I $3 / 13$ 4-66 ICI PAGES 23 FIGS 23 REFS.

$R F \cdot U$

SIMPLE SHELTERS FCR PROTECTION AGAINST WEAPON'S OF MASS CESTRUCTION I USSR

INTERNATIONAL-CI VIL-DEFENSE CIVIL-CEFENSE-SYSTEMS HASTY-SHELTERS. CONSTRUCTIOV

VUCLEAR-EXPLOSION-DAMAGE EXPLOSION-EFFECTS RURAL-DREAS PROTECT ION-FACTO?

DECONTAMINATION WATER-SUPPLIES, \#JPRS-30,003

GORCHAKOV, A. D. + ZHUKOV, Y. A. + KCSHELEV, L. I .

ROSSAL, N. A.. + KHOMKO, A. A.

JPRS

JOINT PUBLICATIONS RE SEARCH SERVICE, WASHINGTON, C.C.

$13 / 13$ S-11-65 I[4 PAGES 39 FIGS

RC U

INVESTIGATIONS ON THE PROBLEM OF THE INTERRELATICNSHIP OF. PRESSURE STREVGTH, SIZE AND COST OF MULTIPLRPOSE BUILOINGS FCR A FEDERAL TRANSPORTAT ION ACMINISTRATION

SHEL TER S DLAL-PURPOSE-SHELTERS. CCSTS GERNANY CONSTRUCTION FARCENING DESIG:I

DYNAMIC-PRESSURE SHIELDING CALCULATICNS GARAGES, \#ORNL-TR-1646

GIRNAU; G. + BEHRENDT, G. + ZIMMERNANN, K.

GERMANY

GERMANY, STLDIENGESELLSCHAFT FUR UNTERIRCISCHE

VERKEHR SANLAGEN EV., DUSSELDCRF

$13 / 13$ 6-6.5 62 PAGES, 14 TABLES 10 REFS

RF U

METHOD OF SHELTER COST ANALYSIS ( COSTS NETHCDOLOGY SHELTERS COMPUTERS(PROGRAMMING) BUILDINGS CONSTRUCTION, \#AD 646847

BARK SDALE, R. H., JR. + WADE, S. R.

FAYES SEAY MATTERN

HAYES, SEAY, MATTERN, AND NATTERN, RCANOKE, VA.

$13 / 13$ 2-67 154 PAGES 44 RFFS

RF $U$

UN THE DESIGN OF RISK ORIENTED, LCh COST FALLOUT SHELTER SYSTEMS

MANAGEMENT-PLANNI NG SURVI VAL URBAN-AREAS DANAGE-LIMITING-SYSTEMS EVACUATION

VULNERAB ILITY MARNING-SYSTEMS HASTY-SHELTERS THREAT-EVALUATION, \$HI-486/Z-RR

ROCKETT, F. C. + BROWN, W. M.

HUDSON INSTITLTE

HLCSON INSTITUTE, CROTCN-CN-HUDSCN, N.Y.

$13 / 133-7-67$ 3i PAGES 34 REFS

RF U

STATIC ANO DYNAMIC TESTS CF BURIEO UNREINFORCED CCNCRETE ARCHES

UNDERGROUNO-STRUC TLRES DYMAMICS LOADING (NECHANICS) SHELTERS) \#MISC. PAPER VO. I-809

FLATHAU, i. . J. + NEYER, G. D.

$\triangle R M Y$ ENG INEERS

U:S. ARMY ENGINEER WATERWAYS EXPERINENT STATION, VICKSBURG, MISS.

1. ZII3 4- CE IE PAGES 8 FIGS 9 REFS

RF U

PROTECTIVE CONSTRLCTION INSTITUTE STUDIES I SHELTERS FALLOUT-SHELTERS

UNDERGROUNO-STRUC TLRES COLORADO DUAL-PURPOSE-SHELTERS TRANSPORTATION UTILITIES

URBAN-PLANNING . UTILI TY-TUNNELS CCNNUNI CATI CN-SYSTEMS STRESS(PSYCHOLOGY)

PLBL IC-INFORMA TION MOVEMENT, \$A.L. 1371

VETTER, G. H.

UNIV OF COLORADO

UNIVERSITY OF COLORADO, BCULDER, CCLC.

$1 \geq / 13196626$ PAGES 20 FISS

RF U

PRELIMINARY TESTS ON A SHALLOW UNREINFORCED CCNCRETE SHELL I

SHELLSISTRLC TURAL-FORMS) NEMBRANES STRESSES LOACING(MECHANICS) FAILURE(YECHAVICS)

DESIGN COSTS MODELSISINULATICNS) STRUCTURAL-DNALYS IS, \#TR-5IC

$A L L G 000, J$. R. + RAIL, R. D. + CHIL, R. H.

NCEL

U.S. NAVAL CIVIL ENG INEERING LABCRATCRY, -PORT HU'ENEME, CAL IF.

$13 / 13 \quad 1-67 \quad 71$ PAGES 48 FIGS 10 REFS.

RF $U$ 
$13-13-01833$

1 3-13-01834

13-13-01835

$13-13-01837$

$13-13-01838$

13-13-01839

$13-12-01840$

$13-13-01841$

$13-13-02270$

$|3-13-0227|$

COMPUTER ANALYSIS OF PROTECTIVE STRUCTURES FCR PROTECTION FROM NUCLEAR BLAST I SHELTERS SHOCK-WAVES BLAST-SHELTERS. UNDERGROUND-STRUCTURES OYNAMICS MATHEMATICAL-ANAL YSIS DI FFERENTIAL-EGUATIONS COMPUTERS(PROGRAMMING), \#A.L. 1085 T. Y. LIN

T. Y. LIN AND ASSOCIATES, VAN NUYS, CALIF.

$1 \geq 113$ 6-65 66 PAGES 16 FIGS 15 REFS

RF U

ALTERNATIVE APPROACHES TO FINANCING A NATIONWIDE SHELTER PROGRAM I FALLOUT-SHELTERS FEDERAL-BUDGETS COSTS CI VIL-DEFENSE-SYSTEMS NANAGEMENT-PL ANN ING STATE-GOVERVMEVTS LOCAL-GOVERNMENTS, \$SRI-4536-110 \$AD 635930 HARVEY, E. C.

STANFORD RESEARCH INST

STANFORD RESEARCH INSTITLLTE, NENLC PARK, CALIF. $1 \geq 113$ 4-66 93 PAGES I4 REFS

RF U

ENTRANCEWAYS ANO EXITS FOR BLAST RESISTANT FULLY BURIED PERSONNEL SHELTERS I BLAST-SHEL TERS DESIGN COSTS SINGLE-PURPOSE-SHELTERS DOORS UNDERGROUND-STRUCTURES STRUC TURAL-PROPERTIES STEEL REINFCRCED-CCNCRETE SHELTER-COORS TRAFFIC STRUC TURAL-SHELLS ) \#M6D64(3) \#AD 636048

STEVENSON, J. D. + HAVERS, J. A.

IIIRI

ILLINOIS INSTI TUTE OF TECHNOLOGY RESEARCH INSTITUTE, CHICACO, ILL. IIIIS

RF U

YIELDING MEMBRANE ELEMENTS IN PRCTECTIVE CCNSTRUCT ION I BLAST-SHELTERS UNDERGROUND-STRUC TLRES DYAAMICS SHELLS (STRUCTURAL-FORMS) BEAMS(STRUCTURAL) ANALYSIS, SLABS (NONMETALLIC) FFASIPILITY-STUCIES COSTS DESIGN LOADINGIMECHANICS SOIL(MECHANICS), \#AD 625782

HARRENSTEIN, H. + GUNDERSCA, R. + HANSCN, $\dot{\text { S. }}$

BLRNS, J. + SALMONS, J. + NEILSCN, D.

LNIV OF ARIZONA

UNIVERSITY OF ARI ZONA, TUCSCN, ARI 2 .

$1 \geq 113$ 5-65 121 PAGES 36 REFS

$R F U$

AREA WIDE SHFI TER SYSTEMS I FALLCUT-SHELTERS COSTS BLAST-SHELTERS

MANAGEMENT-PLANNING SI TE-SELECTION NATIONAL-FALLUUI-SHELTERR-SURVEY POPULATION RESOURCES UROANMAREAS BUILDINGS SAA-INSE VENTILATICN, \#SRI-MU-4536-1IO\#AD 63593 CONDIT, R. I .

STANFORD RESEARCH INST

STANFORD RESEARCH INSTITUTE, MENLO PARK, CALIF.

$13 / 13 \quad 12-65 \quad 34.6$ PAGES 62 FIGS 12 REFS

RF $U$

SHEL TER DESIGN AND ANALYSIS I. FALLCUT PROTECTION I FALLOUT-SHEL TERS NUCLEAR-WEAPONS SHIELOING DOSE-RATE CALCULAT IONS SLANTING, *TR-20(I) OCD

DFFICE OF CIVIL DEFENSE, DEPARTMENT CF DEFENSE

$13 / 13$ 5-64 214 PAGES 81 FIGS 3 REFS

RF $U$

STUOY TO DETERMINE THE OPTIMUM SECTICN CF REINFORCEL CONCRETE BEAMS SUBJECTED TO BLAST LUAUS ( OESIGN BEAMS (STRUCTURAL) LCACING(MECHANICS) PROGRAMMING (COMPUTERS) \#An $\sin 284$

SWIHART, G. R。

NCE

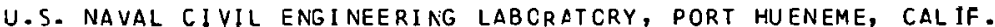
$13 / 13 \quad 2-59 \quad 35$ PAGES D REFS

RF U

DYNAMIC SHEAR STRENGTH OF REINFORCED CONCRETE BEAMS II I TEST-EQUIPMEVT BEAMSISTRUCTURAL) LOADING (MECHANICS) STATICS, \#TR-502 *DASA-13.012 SEABOLO, R. H.

NCEL

U.S. NAVAL CIVIL ENGINFF, RING LABCRATCRY, PORT HUENEME, CAL IF.

$13 / 13 \quad 1-67 \quad 87$ PAGES 7 REFS

RF U

CONSTRUC IION COST TRENDS $($ UNITED-STATES BUREAU-OF-RECLAMATION , \#A.L. 1 IST(4)

L.S. DEPT. OF INTERIOR

U.S. DEPARIMENT OF INTERICR, BUREAL CF RECLANATICN

$13 / 134-67$ 4 PAGES D REFS

RE $\mathrm{U}$

STUDY OF THE PROPCSED EAST WEST FREEWAY TUNNEL WEST ORANGE, NEW JERSEY, AS A CIVIL DEFENSE PLBLIC SHELIER FACILITY I TRANSPCRTATION FEASIBILITY-STUDIES

DLAL-PURPO SE-SHELTERS STOCKPILING ENTRANCEWAYS COSTS WATER-SUPPLIES

ENVIRONMENTAL-CONTROL VENTILATION ELECTRIC-POWER-PRODUCTION HEATING COOL ING

LIGHTING WASTES(SANI TARY-ENGINEERING) I AA.L. 112

BRONSON, G. + KILLAM, E. T. + $\triangle R C H I B A L D, G_{0}$ R.

CHARLES A. MAGUIRE ASSOC

CHARLES A. MAGLIRE AND ASSOCINTES, BOSTRN, MASS.

$1 \geq 1133-63$ IS9 PAGES 38 FIGS 14 REFS.

RF U 
TIME COMPRESSION PCTENTIAL OF AN ENERGENCY BLAST SHELTER PROGRAM I URBAV-AREAS HASTY-SHEL TERS DESIGN MATERIALS EQUIPMENT LABOR MOBILIZATION MANAGEMENT-PLANNING CALCULATION, \#HI-774-RR

BROWN, W. M. + CANDELA, B. + CANDLIN, 5.

KRUPKA, R. A. + PANERO, R. B.

HUDSON INSTITLTE

HUDSON INSTITUTE, INC., CROTON-CN-HUDSON, N. Y.

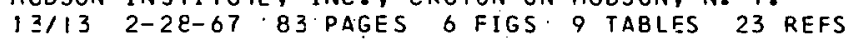

RF $U$

QLARTERLY OF THE CCLORADO SCHOOL OF MINES $46(1)$ AN INTRODUCTION. TO THE DESIGN OF UNDERGROUND OPENINGS FOR. DEFENSE I SHELTERS ELAST STRESSES UNCERGROUND-STRUCTURES ROCK (GEOLOGY) HEA TING MINING-ENGINEERING LCACING(MECHANICS) COOL ING

FAILURE (MECHANICS) SHOCK-hAVES VENTILATICN , *A.L. 1205

LIVINGSTCN, C. W.

COLO SCH MINES RES FOUN

COLORADO SCHOOL OF MINES RESEARCH FCUNCATICN, GOLLEN, COLO.

$13 / 13$ 1-51 3[4 FAGES 118 FIGS. 22 TARLES 149 REFS

JF U

13-13-02274 METHODS AND COSTS OF CONSTRUCTING THE UNDERGROUNC FACILITY OF NORTH AMERICAN AIR DEFENSE COMMAND AT CHEYENAE NCUNTAIN, EL PAST COUNTY, COLORADO I COVSTRUCTION UNDERGROUND-STRUC TL'RE'S BLASTING EXCAVAT ICN REINFORCED-CONCRETE ROCK-STABILIZATION SHOTCRETE ROCK-BOLTINGS CHAIN-LINK-FABRIC EPCXY-RESINS CEMENT-GROUT .) \$IC-8294 HOWES, M. H.

L.S. DEPT. OF INTERI OR

U.S. DEPARTMENT OF THE I.NTERICR, EUREAU OF MINES

$13 / 13196.609$ PAGES 52 FIGS D REFS

RF $U$

13-12-22275 QLARTERLY OF THE CCLORADO SCHCCL OF NINES 62121 TUNNEL MECHANICS I

UNDERGROUNO-STRUC TURES ROCK(GEOLOGY) STRESSES ELASTICITY THEORY GEOLOGY

INSTRUMENTATION, DEFORMATI CN LOADING(NECHANICS) CONSTRUCTION STATISTICAL-ANALYGIS, *A.L. 1206

$A B E L, J . F$, JR.

COLO SCH MINES RES FOLN

COLORADO SCHOOL OF MINES RESEARCH FCUNDATICN, GOLLEN, COLO.

$13 / 134-6788$ PAGES 34 FIGS 15 TABLES 27 REFS

JF $U$

13-13-02276 VATIONAL DIREC TORY OF ARCHI TECTURAL, ENGINEERING ANC CONSULTING FIRMS WITH

CERTIFIED.FALLOUT SHELTER ANALYSTS ; RACIOACTIVE-FALLOUT BUILOINGS CONSTRLCTION

CESIGN SHIELDING CONSTRUCTION-SLANTING , \#FG-F-I.3

OCD

OFFICE OF CIVIL DEFENSE, hASHINGTCA, C.C.

$13 / 13$ 6-67 145 PAGES

RO U

BUILDING MATERIALS AS CONMONLY IISFR IN FXISTING, IIBPAM -BIIILEIMES IN THE UAITLD STATES $I$ CONSTRUC TION URBAN-AREAS RACICACTIVE-FALLOUT FALLOUT-SHELTERS SHIFIITIVG, STRUL IUKAL-HRUPERTIFS I \$AP-8.5?।

CANNON, E. W.

LNIV OF CALIF

UNIVERSITY OF CALIFORNIA, INSTITUTE CF ENGINEERINE RESEARCH

$13 / 13 \cdot 1-8-5842$ PAGES 4 TABLES 27 REFS

RE U

PROTECTIVE SHELTER SYSTEMS - AN ANNCTATEC BI LLIOGRAPHY OF U.S. NAVAL CIVIL ENGINEER ING LABORATORY PUBLICATICNS I FALLOUT-SHELTERS BLAST-SHELTERS B IOLOGICAL-WARFARE-AGENTS CHEMICAL-WARFARE-AGENTS， \#NCEL-TN-562 SEABOLD, R. H.

NCEL

U.S. NAVAL CIVIL ENGINEERING LABCRATCRY, PORT HUENEME, CAL IF. $13 / 132-28-64$ 5[ PAGES

RA U

13-13-02279 A STUDY OF THE RF SPONSE OF BACKPACXING MATERIAL ENCASING A TUNNEL. LINER SLBJECTED TO A DYNAMIC DISTLRBANCE \& UNDERGRCUND-STRUCTURES CEFORMATION CONSTRUC TION-MATERIALS MA THENATICAL-NODELS CIFFERENT IAL-EQUAT IONS EQUATION S-OF-MOTICN LOADING (MECHANICS) SHOCK -WAVES NUCLEAR-EXPLOSION-DAMAGE ) \#AD 647 OC6 6

NOSSEIR, S. + PERRY, E. S. + THOMPSCN, J. N.

UNIV OF TEXAS

UNIVERSITY. OF TEXAS, AUSTIN, TEX.

$13 / 13$ 12-60 67 PAGES 24 FIGS 2 TABLES 7 REFS

RF U

13-13-02280 TEST AND EVALUATICN OF CCNPUTER ANALYSIS PROGRAMS FOR SHELTERS IV BUILDINGS DYNAMICS COMPUTER-PROGRANS SHCCK-WAVES ELAST SHIELCING STRUCTURAL-ANALYSIS RADIOACTIVE-FALLOLT FIRE-SAFETY FALLOUT-SHELTERS, *A.L. 1207

WEISSMAN, S. + DINAPOLI, P. + COHEA, E.

AMMANN AND WHITNCY

AMMANN AND hHI TNEY, NEW YCRK, N. Y. $13 / 13$ 5-67 86 PAGES 12 FIGS 18 TABLES 9 REFS

RF U 
$13-13-02281$

$13-1 \geq-c 2282$

$13-13-02283$

$13-1 \leq-02284$

1]-1:-0250

$13-12-02286$

$13-13-02287$

$13-13-02280$

1 3-1 3-02289

$13-13-02290$

$13-13-02291$

$\triangle$ MODEL BLILLING CODE ARTICLE CN FALLOUT SHELTERS WITH RECOMMENDATIONS FOR INCLUSION OF REQUIREMENTS FOR FALLCUT SHELTER CONSTRUCT ION IV FOUR NATIONAL MODEL BUILDING CODES I CONSTRUCTION DUAL-PURPOSE-SHELTERS ) *TR-Z6

CRAVES-HILL

GRAVES-HILL AND ASSOCIATES, ARCHITECTS, LEXINGTON, KY. I $3 / 13$ 10-7-65 44 PAGES

RO U

PARM SYSTEM MANUAL III-K. CONSTRUCTION IN THE PRCTCIYPE MOOEL I REPAIR RECOVERY DATA-PROCESSING NAINTENANCE PCSTATTACK-OPERATIONS, \#NREC- 24

RITZ, P. M. + HADUELL, R. M.

NAT RES EVAL CENTER

NATIONAL RESOURCE EVALUATICN CENTER, WASHINGTON, C.C.

I $21133-64$ 5L PAGE S I REF

RI U

CONSTRIT: TION AND ENGINEERING dATA - WATER CCNVEYANCE TUNNELS - gRAVITY AND PRESSLRE $($ IINTTED-STATE $\$$ BLREAU-OF-RECLAMATICN) $\# A . L$. 1158

U.S. DEPT. OF INTERIOR

U.S. DEPARTMENT OF INTERICR, BUREAU CF RECLAMATION

$13 / 13 \quad 1-61$ I27 PAGES 6 FIGS O REFS

RC L

CONSTRUCTION COST TRENDS（UNITED-STATES BUREAU-OF-RECLAMATION） \#A.L. IIS7(2)

U.S. DEPT. OF INIERIOR

U.S. DEPAR TMEN T OF INTERICR, BUREAU CF RECLANATICN

$1 ? I I 3 \quad 4-65$ P PAGES D REFS

RD U

GONETRUGTIOM EOST TRFNOS I IINITFM-STATES BUREAU-CG-RECLAMATION , \#A.L. II57(3)

U.S. DEPT. OF INTERIOR

U.S. DEPARTMENT OF INTERICR, BUREAU CF RECLANATION

$13 / 13 \quad 1-67 \quad 12$ PAGES $D$ REFS

$R \mathrm{C} U$

FALLOUT SHELTERS - CODES AND STANDARES I SHIELDINE RACIAT ION-EFFECTS

RADIOACTI VE-FALLOLT CONSTRUCTICN CCNSTRUCT ION-SLANT ING OESIGN, ATR-30

OCD

OFFICE OF CIVIL DEFENSF, DEPARTMENT CF CEFENSE

$12113 \quad 12-66$ \& PAGES 13 FIRS

RG $U$

THE SECOND ORDER ELASTIC ANALYSIS CF PLANE RIGID FRAMES I STRUCTURES

STRUC TURAL-PROPERTIES STEEL CCMPUTER-PROGRAMS ELASTICITY LOADING(MECHANICS), \#FEL-297.17 \#AC 645586

HARRISON, H. B.

LEHIGH UNIV

LEHIGH UNI VERSITY, BETHLEHEM, PA.

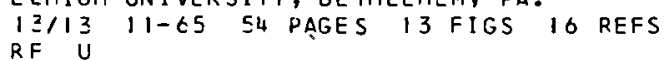

CONSTRUCTION COST TRENOS ( UNITFN-STATES BUREAU-OF-RECLAMATION ) \#A.L. 115711 )

U.S. DEPT. OF INTERIOR

U.S. DEPARTMENT OF INTERICR, BUREAU CF RECLANATICN

$13 / 13 \quad 1-65$ II PAGES O REFS

RO U

EFFECTS ON LONG TIME LOADS ON PRESTRESSEC CONCRETE REAMS I BEAMSISTRUCTURAL)

CONSTRUE TION-MATERIALS LUAU-DISTRI EUTICN CEFLECT ICN ST CLL RODS CRACKS

STRAIN(MECHANICS) LOADING (NECHANICS) , *NCEL-TR-518 \#AD 650329

BRECKENR IDGE, R. A. + VALENT, P. J. + BUGG, S. L.

NCEL

U.S. NAVAL CIVIL ENGINEERING LABCRATCRY, PORT HUENEME, CALIF.

$13 / 13$ 3-67 43 PAGES 32 FISS 7 REFS

RF $U$

RESPONSE OF DEEP REINFORCED CCNCRETE SLAES. ( TESTS. LOADING(MECHANICS)

UNDERGROUND-STRUC TLRES SHELTERS PERFORNANCEIENGINEERING) STATICS STRUCTURAL-PARTS, *WL-TDR-64-54 \#AD 613545

GREGORY, R. K. + DEHART, R. C. + ALSTIN, W. J.

. SOUTHWEST RESFARC.H INST

SOUTHWEST RESEARCH INSTI TLTE, SAN ANTONIO, TEXAS

$12 / 13$ 2-65 ISO PAGES 76 FIGS 14 REFS

RF U

SIMULATION OF THE RESPONSE OF EQUIPMENT LOCATED IN UNCERGRCUND INSTALLATIONS TO BLAST IVDLCED GROLND MOTICNS I UNDERGRCUNO-STRUCTURES EXPLOS ION-EFFECTS

SHOCK (MECHANICS) VLLNERABILI TY NUCLEAR-EXPLCSIONS SHOCK -WAVES LOAOINGIMECHANICS)

SLSPENSION-DEVICES STRUCTURES VIBRATION-ISOLATORS MACHINES VIBRATION FREQUENCY

PNEUMATIC-DEVICES FEASIBILITY-STUDIES CIVIL-ENGINEERING, \$DASA-1549 \$AD 608531

RUZICKA, J. E. + CALCATERRA, P. C.

BARRY CONTROLS

BARRY CONTROLS, WATERTOWN, MASS.

$13 / 13$ 10-64 187 PAGES 63 FIGS 13 TABLES 5 REFS

RF U 


\section{METHODS AND EQUIPMENT}

\section{Laboratories, Test Facilities, and Test Equipment}

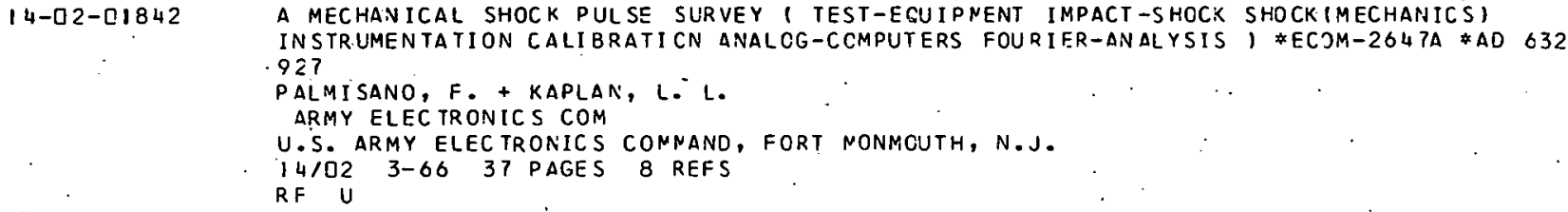

\section{MILITARY SCIENCES}

\section{Chemical, Biological, and Radiological Warfare}

$15-02-01843$

$15-02-01849$

$15-02-01850$

$15-02-0185$

$15-02-01852$

$15-02-01853$

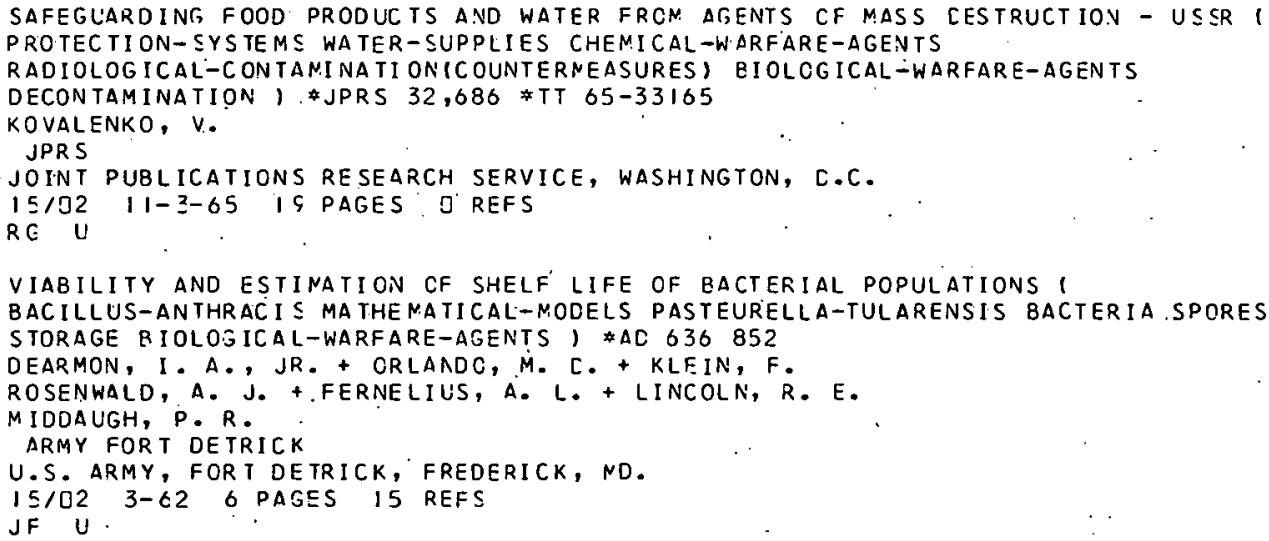

T.HE PATHOGENESIS OF PSITTACOSIS - SERIAL STULIES CN RFESUS MONKEYS EXPOSED TO A SMALL PARTICLE AERCSCL OF THE BORG STRAIN I EIOLCEICAL-NARFARE-AGENTS MIYAGAWANELLA-PSITTACI AERCSOLS, 1 \#DO 636814

AIR FILTRATION OF MICROBIAL PARTICLES I BICLCGICAL-WARFARE-AGENTS AEROBIOLOGY BACTERIA GAS-FILTERS MICRCCRTANISNS AIR-FOLLUTION FILTERS. I *A0 637407 UECKER, H: M. + GLCHANAN, L. N. + HALL, L. B. GOODARD, K. R

AMER PUB HEAL TH ASSN

AMERICAN PUBLIC HEALTH ASSOCIATICN, NEW YORK, N.Y.

$15 / 0219637$ PAGES O REFS

RF $U$

PATHOGENESIS OF CCCCIDIOIDES INMITIS IN NONKEYS 1 BIOLOGICAL-WARFARE-AGENTS PATHOLOGY LUNGS SFORES.) *AD 636815

CONVERSE, J. L. + LOWE, E. P. + CASTLEGERRY, M. W.

BLUNDELL, G. P. + BESEMER, A. F.

ARMY FORT DETRICK

U.S. $\triangle R M Y$, FORT DETRICK, FREDERICK, ND.

$15 / 02 \quad 11-61$ \& PAGES 16 REFS

$J F \cdot U$

IMMUNOLOGICAL STUDIES OF ANTHRAX II. LEVELS OF INNUNITY AGAINST BACILLUS AVTHRACIS OBTAINED WITH PROTEC TIVE ANTIGEN AND I.TVE VACCINE I SPORES

B IOLOGICAL-WARFARE-AGENTS ANTIGENS-ANO-ANTIBCDIES RESISTANCEIBIOLOGICAL, 1 \#DD 636 893 .

KLEIN, F. + DEARMCN, K. A., JR. + LINCCLN, R. E.

MAHLANOT, B. G. +. FERNELILS, A. L.

ARMY CHEMICAL CORPS

U.S. ARMY CHEMICAL CORPS, FORT DETRICK, FRECERICK, MD.

$1 \leq / 0219625$ PAGES 20 REFS.

$J F$ U 
$15-02-01854$

$15-02-01855$

$15-02-01857$

$15-0\{-01858$

$|5-02-0186|$

$15-02-01863$

$15-02-01869$

15-02-01875

$15-02-01881$

PATHOGENESIS AS RELATEO TC PHYSI CLCGICAL STATE OF ANTIRAX SPORE ANC CELLI B IOLOGICAL-WARFARE-AGENTS BACILLUS-ANTHRACIS DIAGNOSIS PATHOLOGY HISTOLOGY INFECTIONS INMLNITY, \#AD 636813

LINCOLN, R. E. + RHIAN, M. A. + KLEIN, F.

FERNELIUS, $\triangle$.

ARMY FORT DETRICK

U.S. ARMY, FORT DETRICK, FREDERICK, ND.

I $5102 \quad 1957$ 1 8 PAGES 24 REF 5

RF U

AIR RORNE IINFECTIVITY OF THE VARIOLA VACCINIA GRCUP OF POXVIRUSES FOR THE CYNOMOLGUS MONKEY, MACACA IRUS I BICLOGICAL-WARFARE-AGENTS POX-VIRUSES MONKEYS IMMUNITY INFECIIONS, \#AD 636905

HAHON, N. + MCGAVRAN, N. H.

ARMY CHEMICAL CORPS

U.5. ARMY CHEMICAL CORPS, FORT DETRICK, FREDERICK, MD.

$15 / 02 \quad 1961 \quad 5$ PAGES 9 REFS

JF U

STUDIES ON IMMLNIIY TO TOXINS OF CLOSTRIDIUM BOTULINUM VIII. IMMUNOLOGICAL RESPONSE OF MAN TC DURIFIED BIVALEAT AB BOTULINUM TOXOID

BIOLOGICAL-WARFARE-AGENTS TOXIC-TOLERANCES TCXINS-AND-ANTITOXINS $1 * A 0636854$

FIOCX, M. A. + DEVINE, L. F. I GEARINGER, N. F.

DUFF, J. T. + WRIGHT, G. G. + KADULL, P. J。

ARMY CHEMICAL CORPS

U.S. ARMY CHEMICAL CORPS, FCRT DETRICK, FRECERICK, MD.

$15102 \quad 19617$ PAGES 6 REFS

JF $U$

EFFECT OF AFROSOI. AGE ON THE INFECTIVITY OF AIRBORNE PASTEURELLA TULAREVSIS FOR MACACA MULATTA AND MAN I BIOLCGICAL-WARFARE-AGENTS EACTERIAL-AEROSOLS EXPOSURE

VACCINES DOSAGE INMUNITY NONKEYS MICE GUINEA-PIGS HUMANS ) \$AD 635744

SAWYER, W. D. + JEMSKI, J. V. + HOGGE, A. L., JR.

EIGELSBACH, H. T. + WOLFE, E. K. + DANGERFIEĹ, H. G.

GOCHENOUR, W. S., JR. + CRCZIER, D.

ARMY BIOL LAB

U.S. ARMY BIOLOGICAL LABORATORIES, FURT UETRICK, FREDERICK, MD.

$15 / 02 \quad 2-66 \quad 5$ PAGES 19 REFS

RF U

PROCEEDINGS OF A CCNFERENCE ON BOTLLINUM TOXIN I PIOLOGICAL-WARFARE-AGEVTS

CLOSTRIDIUM-BOTILINUM TOXINS-AND-ANTITOXINS PHARNCCOLOGY MUSCLES MONKEYS CHICKENS

DOSAGE, *EASP-IDC-I*AD 637265

HASSETT, C. C.

ARMY EDGE WOOD AR SENAL

U.S. ARMY EDGE hOOD ARSENAL, ND.

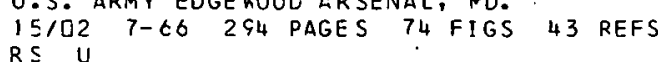

SOME CHEMICAL AND PHYSICAL PROPERTIES OF PARALYTIC SHELLFISH POISONS RELATED TO

TOXICITY I PELECYPODA TOXINS-AND-ANTITOXINS BIOLCGICAL-WARFARE-AGENTS PARALYSIS FOOD-POISONING MUSSEL-POISCNS,

SCHANTZ, E. J.

ARMY CHEMICAL CORPS

U.S. ARMY CHEMICAL CORPS, FORT DETRICK, FREDERICK, MD.

$15 / 02 \quad 1961 \quad 9$ PAGES 24 REFS

JF IJ

DISEASE STATLS REPORTS - CI PHTHERIA, PERTUSS IS, TETANUS, POL IOMYELITIS, SMALLPOX, MEASLES, INFLUENZA I DEMOGRAPHY INNUNOLOGY BIOLOGICAL-WARFARE-AGENTS CASUALTIES I *A.L. 1042

L.S. DEPT. OF HEAL ED WEL

U.S. DEPARTMEVT OF HEALTH, EDUCATICN ANC WELFARE, PUBLIC HEALTH SER VICE

$15 / 02$ 10-66 35 PAGES 18 HIGS 13 REFS

RD U

SELECTIVE MEDIA FCR THE ISOLATION CF PASTEURELLA PEST IS I EIOLOGICAL-WARFARE-AGENTS CLLLTURE-MEDIA GROHTH, \#AD 638440

KNISELY, R. F. + ShANEY, L. M. + FRIEDLANDER, H.

ARMY BIOL LAB

U.S. ARMY EIOLCGICAL LABORATORIES, FCRT DETRICK, FREDER ICK, MD.

$15 / 02 \quad 3-64 \quad 6$ PAGES 12 REFS

RF U

FINAL REPORT ON ATTENUATICN ANU UISPERSICN OF SOUNC IN AN AEROSOL 1 ACOUSTIC-PROPERTIES GAS-FICW PROPAGATICN BIOLOGICAL-WARFARE-AGENTS

SOUND-TRANSMISSION, \#NONR-562(37)/3 \#AC 641013

DOBBINS, R. A.

BROWN UNIV

HROW'N UN IVEKSIT'Y, PROVIDERCE; R. I .

$15 / 02$ 10-66.7 PAGES 5 REFS

R F U 
$15-02-01891$

$15-02-01892$

$15-02-01894$

$15-03-01897$

$15-03-01907$

$15-03-01908$

$15-03-01913$

$15-03-019 i 7$

PLAQUE FORMATION BY' PSITTACOSIS VIRUS 1 BIOLCGICAL-WARFARE-AGENTS.

MIYAGAWANELLA-PSI TTACI CULTURE-MEDIA) \$DD 638494

KOZIKOWSKI, E. H. + HAHON, N.

ARMY BIOL LAB

U:S. ARMY BIOLOGICAL LABORATORIES, FCRT CETRICK, FREDERICK, MD.

$15 / 02$ 3- 642 PAGES D REFS

RF U

TEST NEW ELECTRIC INCINERATOR DESIGN FCR STERILIZINE LABORATORY AIR B IOLOGICAL-WARFARE-AGENTS SPORES NEDICAL-EQUIPMENT ELECTRICAL-EQUIPMENT

LABORATORY-EQUIP.MENT DECONTAMINATICN , $\$ D C 638410$

HARR IS, G. J. + GREMILLION, G. G. + TOWSCN, P. H.

ARMY EIOL LAB

U.S. ARMY. BIOLOGICAL LABCRATORIES, FCRT CETRICK, FREDERICK, MO.

$15 / 02 \quad 19642$ PAGES 8 REFS

$J F \quad U^{\circ}$

COMBINED TREATMENT OF EXPERINENTAL MELICIDOSIS. WITH ANTIBICT ICS ANC SULFADIMESIN I B IOLO'GICAL-WARFARE-AGENTS EFFECTIVENESS NICE CHEMOTIERAPEUTIC-AGENTS INFECTION.S

S.TREP TOMYCINS CHLCRTETRACYCLINE CHLCRAMPHENICOL USSR I \#AC 63819

KHUNOANOV, L. E. + KUPTSEVICH, E. I \& + DEMIDCVA, E. K.

SMIRNOVA, L. A. + SHKURKC, E. D.

NAVAL MEDICAL SCHCOL

U.S. NAVAL MEDICAL SCHOOL, BETHESDA, ML.

$15 / 0219617$ PAGES G REFS

RF U

\section{Defense}

CRISIS CIVIL DEFENSE AND DETERRENCE I VULNERABILITY MIL ITARY-STRATEGY INTERNATIIONAL-CIVIL-DEFENSE RUSSIA DAMAGE-LINITINE-SYSTEMS DISPERSAL CHINA EVACUATION. URBAN-AREAS POPULATION, \#II-777-RR

ROCKETT, F. C.

HUDSON INSTITLTE

HUDSON IN STITUTE, CROTON-CN-HUOSCN, N.Y.

$15 / 03$ I2-66 84. PAGES 111 REFS

RF U

.PROTECTION OF FOOD AND AGRICULTURE AGAINST NUCLEAR ATTACK I RURAL-AREAS

RADIOACTIVE-FALLOLT SHIE LDING RADIATICN-HAZAROS CECONTAMINAT ION-RACIATION-EFFECTSI

* AGR ICUL TLRE HANDECOK 234

U.S. DEPT. OF AGRICULTURE

U.S. DEPARTMENT OF AGRICULTURE, AGRICULTURAL RESEARCH SERVICE

$1 \subseteq / 03 \quad 1962 \quad 41$ PAGES 12 REFS

RE U

WHY COUPLE BMD TO FALLOUT SHELTERS I URBAN-AREAS ANT IMISSILE-DEFENSE SYSTEMS

M IL ITARY- STRATEGY, \#HI-72?-P

BRENNAN, D. G.

HUDSON INSTITLTE

HUDSON INSTITUTE, HARMON-CN-HUDSCN, N.Y.

$15 / 03$ 8-2-66 IO PAGES O REFS

RE U

ANNUAL STATISTICAL REPORT I FINANCING SHELTERS WARNING-SYSTEMS

CIVIL-DEFENSE-DPERATING-PLANS POPULATION COMMAND-ANC-CONTROL-SYSTEMS TABLES RADIATION-MEA SLRENENT-SYSTEMS, *A.L. 1094

OCD

OFFICE OF CIVIL DEFENSE, DEPARTMENT OF CEFENSE

$15 / 03$ 6-30-66 75 PAGES 67 TARLES

RV U

SOVIET MILITARY TRANSLATI CNS NUMBER 198 - CIVIL CEFENSE I RJSSIA TRAINIVG DISPER SAL CIVIL-DEFENSE-PER SCNNE L (TRAININ'G) NANAGENENT-PLANNING， $J$ JRS 30, 320

JPR S

JOINT PUBLICATIONS RESEARCH SERVICE, WASHINGTON, C.C.

$15 / 03$ 5-28-.65 4[ PAGES D REFS

$R G \quad U$

SOVIET MILITARY TRANSLATICNS NUMBER 341 I USSR CIVIL-CEFENSE-OPERAT ING-PLANS TRAINING EDUCA TION INTERNATI ONAL-CIVIL-DEFENSE RESCUE(TRAININE) REPAIR(TRAIVING) PROTECTIVE-CLOTHING PROPAGANDA CCNNUNISM PROTECTIVE-MASKS EIOLOGICAL-WARFARE-AGENTS MANAGEMENT-PLANNING, JPRS-38,291*TT-66-34717

JPR S

JO.INT PUBLICATIONS RESEARCH SERVICE, WASHINGTON, C.C.

I 5/03 I D-24-6't 53 PAGES 0 REF.S

RG 
$15-02-01918$

$15-03-01922$

$15-03-01928$

$15-03-01930$

$15-02-01933$

$15-03-01934$

$15-02-01935$

$15-03-01937$

$15-53-01938$
DEFENSE AGAINST WEAPONS OF MASS DESTRUCTION IN THE RURAL AREA I USSR CIVIL-DEFENSE-SYSTEMS RE SCUES INTERNAT I ONAL-CIVIL-CEFENSE CHEMICAL-WARFARE-AGENTS B IOLOGICAL-WARFARE-AGENTS NUCLEAR-hEAPONS FIRST-AID WARNINE-SYSTEMS RADIOLOGICAL-CONTAMINATI ON VETERINARY-MECICINE PRCTECTIVE-CLOTHING । \#PRS-32,877 * TT-65-33455

OLOVYANISHNIKOV, N. P.

JPRS

JOINT PIJBLICATIONS RESEARCH SERVICE, WASHINGTON, C.C.

$15 / 03$ 11-16-65 42 PAGES 38 FIGS D REFS

RO U

CRISIS CIVIL DEFENSE AND DETERRENCE I VULNERABILITY INT ERNAT IONAL-C IV IL-DEFEVSE EARLY-WARNING-SYSTEMS USSR NOVEMENT POPULATICN RES OURCES EVACUATION URBAN-AREAS DAMAGE-LIMITING-SYSTEMS DENOGRAPHY TRAFFIC ANTIMISSILE-DEFENSE-SYSTEMS UNITED-STATES SURVIVAL EUROPE CONNUNICATIONS CHINA SHELTERS I *HI-777-RR(CRAFT) ROCKETT, F. C.

HISDSON INSTITLTE

HLISSN INSTITUTE, CROTON-CN-HUDSCN, N.Y.

$15 / 03$ 12-20-66 76 PAGES 21 FIGS 113 REFS

RF U

SOVIET MILITARY TRANSLATI CNS NUMBER 178 \& USSR INTERNAT IONAL-C IV IL-DEFEVSE CIVIL-DEFENSE-SYSTEMS RURAL-AREAS FUBLIC-INFORMATICN(RADIO) TRAINING TELEVISION) * JPR S-29, 1.95

JPRS

JOINT PUBLICATIONS RESEARCH SFRVICE, WASHINGTON, C.C.

$15 / 03$ 3-19-65 31 PAGES O REFS

$R G \quad U$

CIVIL DEFENSE SHELTER REPRESENTATICN FGR PGPULATICN DAMAGE CAL CULAT ION - $1970-1925$ ( CALCULATIONS DANAGE-ASSESSMENT SHELTERS SHELTER-ALLOCAT ION DATA WARNIVG-SYSTEMS * TECHNICAL NOTE 89

WARMACK, $S .5$.

STANFORD RESEARCH INST

STANFORD RESEARCH INSTITUTE, HIINTSVILLE, ALA.

$15 / 0.3 \quad 11-6532$ PAGES 12 TAELES

RF U

EXTENSION OF THE GENERAI. SF, SSITIVITY ANALYSIS II. TECHNICAL APPENCIXES CIVIL-DEFENSE-SYSTEMS WEAPONS-EFFECTS EXPLOS ION-ËFFECTS BLAST-SHELTERS

RADIOACTIVE-FALLOLT NUCLEAR-WARFARE-CASUALTIES SYSTEMS-EVALUATION CASUALTIES

STATISTICAL-ANALYSIS MATHEMATICAL-NODELS) *RTI-OU-230-2(II)

CRUZE, A. M. + READ, C.

RESEARCH TQIANGLE INST

RESEARCH TRIANGLE INSTITUTE, DURHAN, N.C.

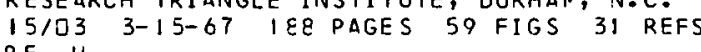

RF 0

EVACUATION DF THE URBAN PCPULATICN AS A MEASURE OF PROTECT IOY AGA INST NUCLEAR WEAPONS I USSR SHELTERS INTERNATIONAL-CIVIL-CEFENSE CIVIL-CEFENSE-SYSTEMS URBAN-AREAS, \#.JPRS-32,120 TT-65-32613

TSTVII.EV, M. P. + PAVLIY, Y. G.

JPR S

JOINT PUBLICATIONS RESEARCH SERVICE, WASHINGTON, C.C.

$15 / 03$ 9-23-65 25 PAGES 7 FIGS

RG U

EXTENSION OF THE GENERAL SENSITIVITY ANALYSIS I. METHOOCOLOGY

CIVIL-DEFENSE-SYSTEMS METEOROLOGY SYSTEMS-FV.ALUATICN OPERATIONS-RESEARCH

MATHEMATICS WEAPONS-EFFECTS RADI CACTIVE-FALLCUT FALLOUT-SHELTERS

MODEL SISIMULATIONS) STATISTICAL-ANALYSIS， \#RTI-OU-230-2(I)

MCMULLAN, D. S. + CRUZE, A. M.

RESEARCH TRIANGLE INST

RESEARCH TRIANGLE INSTITUTE, DURHAN, N.C.

15/03 3-15-67 OL PAGES 17 FIGS 22 REFS

RF $U$

SOVIET MILITARY TRANSLATI CNS NUMBER 170 ( USSR CIVIL-CEFENSE-SYSTEMS

INTERNATIONAL-CIVIL-DEFENSE SHELTERS EVACUAT ION WARNING-SYSTEMS I \#JPRS- 28,352

IVANOV, N. N. + LEBEDEVA, Y. A. + NOSKALEV, V. D.

JPRS

JOINT PUBLICATIONS RESEARCH SERVICE, WASHINGTON, C.C.

$15 / 33 \quad 7-64$ 22 PAGES D REFS

$R \in U$

EXIENSION OF THE GENERAL SENSITIVITY ANALYSIS III. THE ANCET COMPUTER PROGRAM I SYSTEMS-EVALUATION CIVIL-DEFENSE-SYSTEMS WEAPONS-EFFECTS MODELS EXPLOSION-EFFECTS RADIOACTIVE-FALLOLT BLAST-SHELTERS MATHEMATICS PROGRAMMINGICOMPUTERSI CASUALTIES VUCLEAR-WARFARE-CASUALTIES, \#RTI-CU-230-2 (I II)

CRLZE, A. M. + WILKERSON, D. B. + hCCDSICE, M. B.

RËSEARCH TRIANGLE INST

RESEARCH TRIANGLE INSTITUTE, DURHAN, N.C.

ISI03 3-15-67 222 PAGES 22 FIGS 


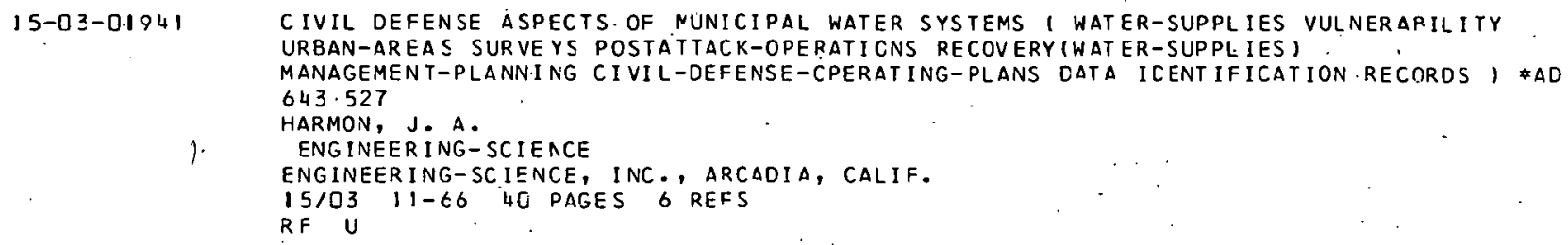

15-0Z-01942 EFFECT OF POPULATICN MOBILITYY ON THE LCCATION OF. COMMUNAL SHELTERS 1 WARNING-SYSTEMS URBAN-AREAS MOVENEAT, \#0RO-SP-30 \#AC 204090

ALEXANDER, M. N. + FEREBEE, J. B. + GRIN, D. J.

LEBOW, L. S. + SENTURIA, S: D. + SINGLETERRY, A. M.

DPERATIONS RE SEARCH

OPERATIONS RESEARCH, SILVER SPRING, ND.

$15 / 03 \quad 10-57 \quad 37$ PAGES 9 REFS

RF, U

15-03-01945 MOVEMENT TO SHELTER IN A NAJOR CITY I SHELTERS CIVIL-CEFENSE-OPERATING-PLANS URBAN-AREAS MANAGEMENT-PLANNING, $\gg$ A.L. 974

MCGILLIVRAY, P. C.

DETROIT OCD

DETROIT OFFICE OF CIVIL DEFENSE:

$15 / D 3 \quad 12-66,10$ PAGES D REFS

RF U

15-03-01946 CODE OF FEDERAL'REGULATI ONIS - NATICNAL CEFENSE I ARMEC-FORCES(UNITED-STATES)

MIL I TARY-GOVERNMENT EMERGENCY-PLANA ING IGCVERNMENT-AGENC IES) LEGISLATION

CIVIL-DEFENSE (GOVERNMENT-AGENCIES) AIR-FCRCE ARMY NAVY GOVERNMENT-AGENCIES 1 \#.L. $9 \varepsilon t$

GSA

GENERAL SERVICES ADMINISTRATICN, WASHINGTON, D.C.

$15 / 031960521$ PAGES

$K C \mathrm{U}$

15-03-01949 NLCLEAR ATTACK HA ZARD IN CONTINENTAL UNITEC STATES - 1963 A. BACKGROUND AND PROCEDURES FOR APPLICATIONS ( ATTACK-SCENARICS VULNERABILITYIANALYSIS) SURVIVAL DAMAGE-LIMITING-SYSTEMS DAMAGE-ASSESSNENT RE COVERY THREAT-EVALUAT IOV

POSTA TTACK-OPERATICNS RE SCURCES NARAGENENT - PLANNING CIVIL-CEFENSE-SYSTEMS MODELS RADIOLOG ICAL-MONI TORING LCG ISTICS NANPOWER S HEL.TERS PROGRAMM ING (COMPUTERS)

DISPERSAL INDUSTRIES STOCKPILING' ,

L.S. DEPT. OF DEFENSE + U.S. EXEC CFF PRES

U.S. DEPARTMENT OF DEFENSE, i.S. EXECUTIVE CPFFICE OF PRESIDEVT,

OFFICE OF EMERGENCY PLANNING

$15 / 03,19.6376$ PAGES 8.REFS

$R \in U$

15-03-C1952 RE-USABILITY OF BLILDINGS AFTER A WARFARF I FIRFS NIISI EAB=EYRLOSIOMI OAMAGE-A SSE SSMENT FIRE-SAFETY PROGRAMMING (COMPUTERS) CEBR IS BL-AST IGNITION) *AD 025867

TROXELL., G. E. + DEGENKOLB, J. G. + EENUSKA., K. L.

LOYA, B. R.

T. Y.. LIN

T. Y. LIN AND ASSCCIATES, VAN NUYS, CALIF.

I $5 / 03 \quad 1-64$ TL PAGES 6. REFS

$R F U$

15-03-01953 AN APPROACH TO DEFINING PCSTATTACK RECOVERY MANAGEMENT CONCEPTS ANC TECHNIQUES 1 COUVTERMEA SLRES PCSTATTACK-OPERATICNS MANAGENENT-PLANNING SUPVIVAL

RADIOLOG ICAL-WARFARE (RECCVERY) ECCNONIC-RECCVERY ECOLOGY STRATEGIC-MATERIALS HARDEVING SOC I TTAL-RECOVERY FOOD (STCCKPILING) STOCKPILING INDUSTRIAL-PLANTS

UTIL ITIES (HÁRDENING) RESOLRCES DECCNTAMINATI CN PEST-CONTROL MORTUARY-SER VICES

PUBLIC-HEALTH INDLSTRIES SANITATION REPAIR MANPOWER , \#SRI-MU-4536

CLARK, D. E., JR. + MILLER, C. F. + HOPKINS, G. D.

STANFORD RESEARCH INST

STANFORD RE SEARC.H INSTITUTE, NENLC PARK, CALIF.

$15 / 03 \quad 11-66$ 61. PAGES 42 REFS

$R E \cdot U$

15-0Z-02147. WORLD WIDE CIVIL DEFENSE I CIVIL'DEFENSE-SYSTEMS INTERNAT ICNAL-CIVIL-CEFENSE WESTERV-ELROPE NATO JREAT-BRITAIN FRANCE GERNANY RENELUX NORNAY DENMARK GREECE SWEDEN SWITZERLAND IRELAND USSR INCIA AUSTRALIA CANADA, \#I-272-C

SWEDEN SWI TZERLAN

HUDSON INSTITLTE

HUDSON IN STITLTE, HARMON-CN-HUUSCN, N.Y.

$15 / 03$ 7-26-63 185 PAGES 185 REFS

RF $U$ 
$15-03-02293$

$15-03-02294$

$15-03-02295$

$15-03-02296$

$15-03-02297$

$15-03-02298$

$15-0]-[2299$
AN APPRJACH TO DEFINING PCSTATTACK RECOVERY NANAGEMENT CONCEPTS ANC TECHN IQUES 1 COUV TERMEA SURE S NLCLEAR-E XPLOSION-CANAGE MANAGEMENT-ENG INEER ING MANPOWER-STUD IES COST-EFFEC TIVENESS ENVIRONMENT MANAGEMENT-PL ANNINE SURVIVAL CIVIL-CEFENSE-SYSTEMS MANAGEMENT-CONTROL-SYSTEMS, *AD 646627

CLARK, D. E., JR. + MILLER, C. F. + HOPKINS, G. C.

STANFORD RESEARCH INST

STANFORD RESEARCH INSTITUTE, NENLC PARK, CALIF.

$15 / 03 \quad 11-66$ CI PAGES 42 REFS

$R G$ U

EXERPTS FROM CONGRESSIONAL TESTINCAY CN CIVIL DEFENSE AND RELATED CEFENSIVE SYSTEMS 1 DEFENSE-SYSTEMS APPROPRIATICNS ANTIMISSILE-DEFENSE-SYSTENS UNITED-STATES USSR COMMUN IST-CHINA THREA T-E VALUATI ON NILITARY-FACILIT IES (PROTECT ION) POPULA TIOV DETERRENCE DAMAGE-LIMITING-SYSTEMS COSTS FALLOUT-SHELTERS PUBLIC-INFORMATION SHEL TER-MANAGEMENT, *MP-47

$O C D$

OFFICE OF CIVIL DEFENSE, DEPARTMENT CF CEFENSE

$15 / J 3 \quad 5-67$ I 35 PAGES 2 TABLES

KO

CRISES CIVIL DEFENSE AND DETERRENCE ( USSR MCVEMENT INTERNAT IONAL-CIVIL-DEFEVSE COMMUN IST-CHINA INCUSTRIES TRANSPGRTATION EV ACUAT ION CISPERSAL URBAN-AREAS FOOD SHEL TERS FALLOL'T-SHELTERS EUROPE SLRVIVAL RECOVERY VULNERARIL ITY MIL ITARY-STRATEGY THREAT-EVALLIATION, $\$ H 1-777 / 2$-PR

ROCKETT, F. C.

HUD SON INSTITLTE

HUDSON INSTI TUTE, CROTON-CN-HUDSON, N.Y.

$15 / 33$ 4-14-67 \&[ PAGES 16 FIGS 97 REFS

RF U

CIVIL DEFENSE HANDBOOK - USSR I RURAL-AREAS ROMBS GUICED-MISS ILES NUCLEAR-WEAPONS CONTAMINATION FOOD CHEMICAL-WARFARE-AGENTS BIOLOGICAL-WARFARE-AGENTS

CIVIL-DEFENSE-OPERATI NG-PLANS CI VIL-DEFENSE-SYSTENS FIRE-FIGHT ING-AND-EQUIPMENT DECONTAMINATION RESCUES DISI NFECTI CN WATER-SUPPLIES COOPERAT ION DISASTEZ-MEDICINE EMERGENCY-HEALTH-SERVICES REPAIR， *JPRS-17,889 *0TS-63-21232

YFGOROY, P. T, + SHLYAKHOV, I. A. + DOLBNIN, T . V.

MORDVINOV, V. $s$.

JPR S

JOINT PUBLICATIONS RESEARC.H SFRVICE, WASHINGTON, C.C.

$15 / 03 \quad 1962 \quad 363$ PAGES 24 REFS

Re 4

PSYCHOLOGICAL, ENGINEERING, AND PHYSIOLOGICAL EVALUATION OF SHELTER EQUIPMEVT AND PROCEDURES II. LABORATORY STUDIES I FALLOUT -SHELTERS PHYS IOLOGY

SYSTEMS-ENG INEER ING COOL ING-AND-VENT ILAT ING-EOUIONENT SHELTER-MANAGEMENT

SHEL TER-OCCUPANCY PSYCHOLCGY PERF CRNANCE (HUMAN) INSTRUCT ION-MANUALS WATER-SUPPLIES ) *GARD-1292(II)

HALE, J. F. + BEHLS, H. F.

GEN AM TRANSPORT CORP

GENERAL AMERICAN TRANSPORTATICN CCRP., NILES, ILL.

ISIOZ 2-6? 210 PAGES TL FIGS 4 REFS

RF IS

PSYCHOLOGICAL, ENGINEERING, AND PHYSIOLCGICAL EVALUATION OF SHELTER EQUIPMENT AND PROCECURES III. HABI TABILITY STUNIFS I FALLCUT-SHELTERS SYSTEMS-ENGINEER ING COOL ING-AND-VENTI LATING-EGUI PNENT SHELTER-MANAGEMENT SHELTER-OCCUPAVCY PHYSIOLOGY PEP.FORMANIF (HIIMANI INSTRUITTI DN-MANUALS PSYCHCLOGY STRESS (PHYSIOLOGY) WATER-SUPPLIES FOOD BEDDING TOILET-FACILITIES) $* G A R D-1292($ III)

SMITH, R. h. + MADSON, C. A.

GEN AM TRANSPORT CORP

GENERAL AMERICA.V TRANSPORTATICN CORP, NILES, ILL. ISIO3 2-67 212 PAGES 37 FIGS 17 REFS

RF U

REVIEh: DF FEUERALLY SPONSCRED RESEARCH RELAT CD TO EMERGENCY PLANN ING SCIENTIFIC-RESEARCH BLAST FI QES UNITED-STATES-GOVERNMENT

OFFICE-OF-EMERGENCY-PLANNING SHELTERS ELECTRCMAGNETIC-PULSE RADIATION SHIELDING RADIOACTIVE-FALLOLT RADI OLOGICAL-DCSAGE STORNS CECCNTAMINATION EARTHQUAKES VOLCANOES LANDSLIDES ELECTRIC-POWER-PRCCUCTICN PETROLEUM RACIAT IDN-EFFEC TS MAVPJWER EMERGENCY-HEAL TH-SERVICES RADIOBIOLOGY FLOOCS DISASTER-MEDICINE TSUVAMIS WATER-SUPPLIES LOGISTICS TRANSPORTATIGN COMMMUNICATION-SYSTEMS, \#A.L. III $\triangle$ YRES, R. L.

NAS-NRC

VATIONAL ACADEMY CF SCIENCES, WASHINGTON, C.C. $15 / 03$ 4-67 111. PAGES D REFS

RV U 
PSYCHOLOGICAL, ENGINEERING, AND PHYSIOLOGICAL EVALUAT ION OF SHELTER EQUIPMEVT AVD P.ROCEDURES I - SUNMARY AND :REVIEW I. FALLCUT-SHELTERS SYSTEMS-ENGINEER IVG COOL ING-AND-VENTI LATING-EGUI PNENT SHELTER-MANAGEMENT SHELTER-OCCUPANC.Y DHYSIOLOGY PERFORMANCE (HUNAN) INSTRUCTI ON-MANUALS PSYCHCLOGY STRESSIPHYSIOLOGY) FOOD WATER-SUPPLIES BEDOING TOILET-FACILITIES । \#GARO-12921 I).

MEIER, H. A. + ENGHOLN, G.

GEN AM TRANSPORT' CORP

GENERAL AMERICAN TRANSPORTATICN CCRP., NILES, ILL.

IS/O3 2-67 26 PAGES 5 REFS

$15-0 \geq-c 230$

KINSHIP AND VOLUNTARY ORGANIZATICN IN PCST TRERMCNUCLEAR ATTACK SOCIETY - SOME EXPLORATORY STLOIES ( POSTATTACK-OFERATICNS STRESS (PSYCHOLOSY) URBA.Y-AREAS SOCIOLOGY SOCIETAL-RECOVERY METHODCLCGY STRESS (PSYCHOLOGY) I \#HSR-RR-6S/1-CR GREER, S. A. +. WINCH, R. F.

HLIMAN SC IENCE S RESEARCH

HUMAN SC IENCES RESEARCH, INC., MCLEAN, VA.

$15 / 03$ 9-65 348. PAGES O. REFS

RF $U$

$15-03-52302$

SOVIET MILITARY TRANSLATICNS NUMBER 385 I. USSR CIVIL-CEFENSE-SYSTEMS

INTERNATIONAL-CIVIL-DEFENSE TRAINING RESCUES PUBLIC-INFORMAT ION REPAIR FILMS

PROPAGANDA RURAL-AREAS CIVIL-DEFENSE-OPERATING-PLANS INDUSTR IESICIVIL-DEFENSE)

PUBL IC-HEALTH TRANSPORTATION COMNUNICATICN, *JPRS-41,417 \#TT-67-32051

JPR S

JOINT PUBLICATIONS RESEARCH SERVICES, WASHINGTON, C.C.

$15 / 03$ 6-15-67 12 PAGES 0 REFS

RE $U$

$15-03-02303$

TECHNIQUES OF SYSTEMS ANALYSIS I NILITARY-STRATEGY MANAGEMENT-PL ANN IVG OPERATIONS-KE SEARCH MODE LS THREAT-EVALUATION DEFENSE-SYSTEMS DESIGN USSR MIL I TARY-INTELLIGENCE , \#RN-1829-1 \#AD 133012

KAHN, H. + MANN, I

RAND

RAND CORPORATION, SANTA MCNICA, CALIF.

$15 / 03$ 12-3-56 I6I PAGES 86 FISS

RF $U$

15-03-[2304 CIVIL DEFENSE NOTICN PICTLRE CATALCGUE ( PUPLIC-INFORMATION CIVIL-DEFENSE-SYSTEMS I $\$ M P-6$

OCD

OFFICE OF CIVIL DEFENSE, DEPARTMENTT CF CEFENSE

$15103 \quad 9-66$ 3E PAGES

x० 4

$15-03-02305$

CIVIL DEFENSE IN THE UNI TED KINGOCN I GREAT-ERITAIN INTERNAT IONAL-CIVIL-CEFEVSE CIVIL-DEFENSE-SYSTEMS EARLY-WARNING-SYSTENS NAT IONAL-CEFENSE RECRUITING TRAINING WARFARE CIVIL-DEFENSE-PERSONNEL CASUALTIES MCBILIZATION ARMED-FORCES-OPERATIONS LOGISTICS COSTS CIVILIAN-PERSCNNFL RESEARCH-PROGRAM-ACMINISTRATINN WAB=DOTENTIAL BLUGETS, \$SRI-IM-4559 \#AD 602 I3.

DIXON, H. + MOLL, K.

STANFORD RESEARCH INST:

STANFORD RESEARCH INSTITUTE, MENLO PARK, CALIF.

$15 / 03$ 3-64 62 PAGES 4 FIGS 8 REFS

$R E U$

\section{Logistics}

$15-05-02306$

EMERGENCY CLOTHING I DISTRIBUTICN LOGISTICS CIVIL-CEFENSE-CPERATING-PLANS. CLEANING REPAIR TRAINING， \#FG-E-13.5

CCD

OFFICE OF CIVIL DEFENSE, DEPARTMENT CF CEFENSE

$15 / 05$ 9-65 I 4 PAGES 3 FIGS

RO U

\section{Nuclear Warfare}

15-06-01955 NATO-CONFERENCE ON DAMAGE ASSESSNENT - SUMNARY REPORT I UNITEC-STATES CIVIL-DEFENSE- SYSTEMS CALCULA.TION'S RESCURCES MANAGEMENT-PLANVING COMPUTER VLLNERAB ILITY, $* A . L . \quad 1053$

NATO

NORTH ATLANTIC TREATY ORGANIZATICN, PARIS, FRANCE

I $5 / 06 \quad 1964127$ PAGES 42 FIGS D REFS

RF $\cdot \mathrm{U}$ 
$15-06-01957$

$15-06-01958$

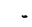

$15-06-01959$

$15-0 t-C 1962$

$15-06-01963$

$15-06-01964$

$15-06-01.965$

$15-06-02307$

A SUMMARY OF AIRBLAST EFFECTS ON HASTY FIELD FORTIFICATIONS ; MATHEMATICAL-PREDICTION SHELTERS AIR-BURST VULNERAEILITY SOIL-MECHANICS WEATHERING MIL I TARY-PER SONNEL (VULNERABILITY) HOLES BLAST MILITARY-ENEIVEERING) \$REPORT-I859 MEDD ING, R. S.

ARMY ENG R-D LAB

U.S. ARMY ENGINEER RESEARCH ANO DEVELOPMENT LABORATORIES, FORT BEL VOIR, VA. $15 / 06$ 5- 66 5 57 PAGES 38 REFS RF U

SOME IMPLICATIONS ON UNCERTAINTIES IN THE HIROSHINA EXPLOSION I AIR-BURST YIELD WIND NUCLEAR-WARFARE-CASUALTIES TARGETS SCALE(RATIO), \$RM-4725-TAB

GOELLER, B. F. +. SPECHT, R. D.

RAND

RAND CORPORATION, SANTA MCNICA, CALIF.

15106 II- 60 34 PAGES IT REFS

RF U

STROP - A STRATEGIC PLANNING NODEL I GAME-THEORY NUCLEAR-WARFARE MODEL-THEORY

STRATEGIC-WARFARE MILITARY-STRATEGY OPTIMIZATION, \$RM-4817-PR $\$ 40637464$

DALKEY, N. C.

RAND

RAND CORPORATION, SANTA NCNICA, CALIF.

I $5 / 06$ 7-66 3E PAGES 5 REFS

RI U

ON DAMAGE ASSESSMENT MODELS I RADI CACTIVE-FALLCUT NUCLEAR-EXPLOSION-DAMAGE POPULATI ON (DIS TR I BUT I ON) GEOGRAPHY INDUS TRIES NUCLEAR-WARFARE-CASUALTIES DEMOGRAPHY BURST-CONDITIONS RADIATI ON-INJURIES YIELD RACIOLOGICAL-COSAGE WIND FIRES SIMULATION BLAST STATE-OF-THE-ART-REVIEWS) \$HI-593-RP.

AYRES, R. L:

HUDSON INSTITLTE

HUDSON INSTITUTE, CROTON-CN-HUDSON, N.Y.

15106 12-3E-66 55 PAGES 24 REFS

RV U

THERMAL HARDENING CONSIDERATIONS PERTAINING TO RES ICENT IAL AREAS 1

NLCLEAR-EXPLOSIONS FIRES AIRBURST STRUCTURES VULNERABIL ITY RECOVERY DAMAGE

RECLAMATION URBAN-AREAS MCDELS(SINULATIONS) GRAPHICS TABLES MICHIGAN)

* USNRDL-TR-984 \#AD $63525 \mathrm{C}$

PARKER, W. J.

NRDL

U.S. NAVAL RAOIOLCGICAL DEFENSE LAEORATORY, SAN FRANCISCO, CALIF.

I $5 / 06$ 7-6-66 60 PAGES 29 REFS

RF U

ON THE ANGLE AND ENERGY DISTRIBUTICN OF PHOTCNS PROCUCEC EY A REAL AND TWO SIMULATED FALLCUT FIELDS I RAOIOACTIVITY RADIOACTIVE-FALLOUT CALCULATIOVS ARMED-FORCES-OPERATIONS RADIOBIOLCGY GECNETRY RACIAT ION-HAZARCS RACIOLOGICAL-DOSE

SIMULATION, \#AFRRI-SP66-I

GARRETT, C. W.

ARMED FORCES RADICDIO RES

ARMED FORCES RADI CBI OLOGY RESEARCH INSTITUTE, BETHESDA, MC.

$15 / 06 \quad 7-66 \quad 36$ PAGES 5 REFS

Q. $\mathrm{U}$

ItE HUSIAIIALK PÜULATION OF THE URITLD STATCS I CAMACE-ASSESSMENT VUCLEAR-EXPLOSION-DAMAGE AGE NCOELS(SINULATICNS) SURVIVAL [EMOGRAPHY RECOVERY ATTACK-SCENARI OS FERTILITY URBAN-AREAS RURAL-ARLAS VULNERAEILITY NUCLEAR-WARFARE-CASUALTIES SEX RACE MANAGEMENT-PLANNING SOCIAL-SCIENCES, ARM- $5115-T A B$

LOWRY, I. S.

RAND

RAND CORPURAIIUN, SANTA NCNICA, CALIF.

\begin{tabular}{lll}
$15 / 06$ & $12-66$ & 139 \\
\hline & PAGES 28 TABLES & OD REFS
\end{tabular}

RF U

POSTATTACK RECOVERY OF DANAGED URBAN AREAS 1 BLAST CAMAGE-ASSESSMENT VULNERABILITY FIRES REPAIR RADI CACTIVE-FALLCUT RADIOLOGICAL-CONTAMINATION RESOURCES PJPLLATION

FACILITIES DECONTAMINATION MATHENATICAL-ANALYSIS RACIAT ION-HAZARDSI

\& SRI-MU-4 $44 Y-1 \leq C$

LARIVIERE, P. D. + LEE, H.

STANFORD RESEARCH INST

STANFORD RESEARCH INSTITUTE, NENLC PARK, CALIF.

$15 / J 6 \quad 11-66$ G 3 PAGES 18 FIGS 32 REFS

R $\mathrm{U}$ 


\section{Operations, Strategy, and Tactics}

THE SEVSITIVITY OF MORTALITY ESTINATES TO VARIATICNS IN AGEREGATE POPULATION REPRE SE.V TA TIONS I NUE LEAR-WARFARE-CASUALTIES (MATHEMAT ICAL-PRECICT IDV)

MIL I TARY-STRATEG Y DAMAGE-ASSESSMENT THREAT-EVALUAT ION DISTRIBUTION(POPULATION) MODELSISIMULATIONS), *RM-5I $11-T A B$ GOELLER, B. F.

RAND'

RAND CORPORATION, SANTA MCNICA, CALIF.

$15 / 07$ 12-66 78 PAGES 15 REFS

R F U

$15-07-01966$

$15-07-02309$

$15-07-02310$

$16-01-01973$

$15-07-02 \cdot 308$
$16-01-01972$ THE CHANGING STRA TEGIC MILITARY BALANCE - USA VS USSR I MILITARY-STRATEGY
ANTIMISSILE-DEFENSE-SYSTENS SPACE-hEAPCNS GUIDED-NISS ILE-DEFENSE-SYSTEMS SUBMARINE-LAUNCHED-BALLI STIC-NISSILES ANTISUEMARINE-DEFENSE-SYSTEMS, U.S. CONGRESS HOLSE

U.S. CONGRESS HOUSE OF REPRESENTATIVES, WASHINGTON, D. C.

$15 / 07$ 7-67 94 PAGES 9 FIGS 7 TABLES

K V U

SOVIET MILITARY TRANSLATICNS NUMBER 382 ( USSR UNITED-STATES

ANTIMISSILE-DEFENSE-SYSTENS MCRALE FIRE-FIGHTING(MANUALS) WARFARE(TRAININE)

MILITARY-PSYCHOLOGY, *JPRS-41, J62 $¥ T T-67-31703$

JPRS

JOINT PUBLICATIONS RESEARCH SERVICES, WASHINGTON, D.C.

$15 / 07$ 5-17-67 2\& PAGES 6 REFS

RG U

SOVIET MILITARY TRANSLATICNS NUMBER 386 I USSR ARMEC-FORCES-OPERATIONS

ARMED-FJRCES (FOREIGN) WARFARE NORALE MILITARY-PSYCHOLOGY MIL ITARY-PER SOVNEL WEAPONS ARMAMENT MILITARY-STRATEGY) \#JPRS-41,406*TT-67-32099

JPRS

JOINT PURLICATIONS RESEARCH SERVICES, WASHINGTON, C.C.

$15 / 07$ 6-2L-67 36 PAGES 5 REFS

$R G U$

\section{MISSILE TECHNOLOGY}

\section{Missile Launching and Ground Support}

SOVIET LITERATLRE ON PROTECTIVE STRUCTURE ANC COMPONENTS $\because$ GUIDED-MISSILES RAILROADS BRIDGES GROUND-SUPPORT-EGUIPMENT RAILROAD-TRACKS ROADS USSR UPDCRGROUPND-STRLETLRE 3, \&AD OJI OUI

I. IBRARY OF CONGRESS

L IBRARY OF CONGRESS, AEROSHACE TECHNCLOGY CIVISION, WASHINGTON, D.C.

$16 / 01 \quad 11-20-64 \quad 12$ PAGES 15 FIGS 12 REFS

RA U

PROTECTIVE STRLCTLRES AND COMPCNENTS - SURVEYS OF CHINESE COMMUNIST SCIENTIFIC AND TECHNICAL LITERATIRF I GUIDED-NISSILES $\triangle B S T R A C T S$ SPACECRAFT TRANSPORTATION LAUNCHING-SITES METEOROLOGY RAILRCADS SHIPS HOISTS CHINA, \#ATD-66-I34 *AD O45 029 NYBERG, P. R.

LIBRARY OF CONGRESS

LIBRARY OF CONGRESS, AERCSPACE TECHNCLCGY CIVISION, WASHINGTON,

D.C.

$16 / 91 \quad 11-8-6622$ PAGES 10 FIGS 41 REFS

RA U

\section{NAVIGATION, COMMUNICATIONS, DETECTION AND COUNTERMEASURES}

\subsection{Communications}

$17-02-01978$

CODE OF FEDERAL REgULATICNS - TELECCNMUNICAT ION 1 COMMUNICATION-SYSTEMS ! \#A.L. 96I GSA

GENERAL SERVICES ADMINISTRATICN

$17 / 02 \quad 1-1-66 \quad 543$ PAGES

RD U 
$17-02-02311$

$17-02-02312$

$17-02-02313$

$17-09-01988$

$18-02-01991$

$18-02-01995$

$18-03-02001$
COMPENDIUM OF PROTECTIVE CONSTRUCTION - VOLUNE I I COMMANC-AND-CONTROL-SYSTEMS B IBL IOGRAPHIES DESIGN HARDENING BLAST AIR-BURST VIBRATION-ISOLATORS SHOCK-WAVES SHOCK (MECHANIC S) ATTENUATION SHELTERS WEAPONS-EFFECTS SURVIVAL (EQUIPMEVT) STRUC TURES COMMUNICATIONS-SYSTEMS RADIATION GEOLOGY HUMAN-ENGINEERING ) *SR-45 *NP-1 I 768-VOL-1

GUNTHER, h. D. + CHASE, R. T. + BECARD, N. A.

MITRE

MITRE CORPORATION, BEDFORD, MASS.

$17 / 02 \quad 1-62 \quad 170$ PAGES

RA U

CIVIL DEFENSE COMMLNICATICNS REQUIRENENTS AT THE LOCAL, STATE, ANC REgIONAL LEVELS 1 COMMUNICATIONS-SYSTEMS COMMAND-AND-CONTROL-SYSTEMS CECIS ION-MAK ING CIVIL-DEFEN SE-OPERATING-PLANS ATTACK-SCENARI CS LOCAL-GOVERNMENTS STATE-GOVERVMENTS RESOURCES I *SRI-NL-4949-120

THAYER, S. B. + SELF, C. R. + BURCC, R. A.

TIFFANY, $\mathrm{H}$. D.

STANFORO RESEARCH INST

STANFORD RESEARCH INSTITUTE, NENLO PARK, CALIF.

$17 / 027-66$ 1 74 PAGES 54 TABLES 35 REFS

RF

COMMUNICATIONS FOLIPMENTS AND SYSTENS TC SUPPORT INTRASTATE CIVIL CEFENSE OPERATIONS - CIRCA 1970 I RADIO-CCNMUNICATION-SYSTEMS COMMUNICATION-SYSTEMS WIRE-COMMLNICA TI ON-SYSTEMS MODELS CCSTS COMMAND-ANC-CONTROL-SYSTEMS

PERFORMANCE (ENGINEERING) ； *SRI-MU-4949-121.

CONE, D. R. + BAER, J. A. + SHAPIRC, E. B.

STANFORD RE SEARCH INST

STANFORD RESEARCH INSTITUTE, MENLC PARK, CALIF.

$17 / 02 \quad 12-65$ \&3 PAGES 7 FIGS 47 REFS

RF U

\section{Radar Defection}

RADAR MASKING BY EARTHS TERRAIN I SITE-SELECTION RACAR-INTERFERENCE RADAR-SIGNALS MAPS ELECTROMAGNETIC-COMPATIBILITY PROPAGATICN， \$P-253 $* A[633540$

MAR TIN, J.J.

IDA

INSTI TUTE FOR DEFENSE ANALYSES, WASHINGTCN, C.C.

$17 / 09$ 3- 6 C 34 PAGES 4 REFS

RF U

\section{NUCLEAR SCIENCE AND TECHNOLOGY}

\section{Isotopes}

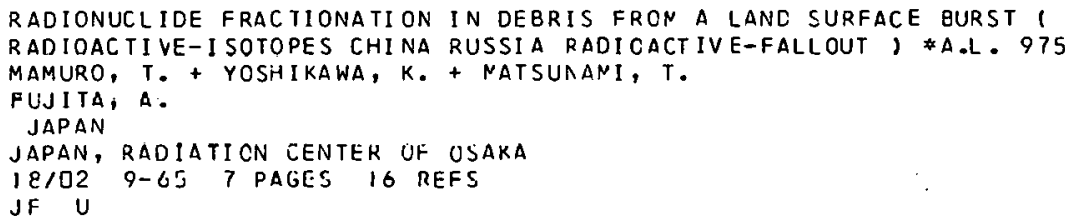

RADIONUCLIDE FRACTIONATION IN DEBRIS FRON A LANC SURFACE BURST 1 RADIOACTIVE-I SOTOPES CHINA RUSSIA RADICACTIVE-FALLOUT) *A.L.975 MAMURO, T. + YOSHIKAWA, K. + NATSUAANI, T. FUJITA, A. JAPAN

JAPAN, RADIATICN CENTER OF USAKA

$18 / 02$ Q-6S 7 PAGES 16 REFS

JF U

\section{Nuclear Explosions}

COMBINED EFFECTS OF NUCLEAR NEAPONS CN NFSS TYPE STRUCTURES 1

NATIONAL-FALLOLT-SHELTER-SURVEY(NFSS) FALLOUT-SHELTERS PROTECTION-FACTOR

CALCULATIONS MODELSISIMULATIONS) LCACING (MECHANICS) BUILCINGS FAILURE(MECHANICS) EXPERIMENTAL-DATA BLAST FIRES, \#URS-658-3

WIEHLL, L. K. + OLRBIN, W. L.

URS COKP

UNITED RE SEARCH SERVICES CORPORATICN, BURLINGAME, CALIF. I $2 / 03$ 9-66 ILO PAGES 32 FIGS 45 REFS

R F

.

BLAST EFFECTS CN BUILOINGS AND STRUCTURES - CPERATION OF SIX-FOOT AND TWO-FUOT SHOCK TUBES - EXPERIMENTAL OSSERVATIONS OF INTERICR PRESSURES IN HOLLOW MODELS ( SHOCK-WAVES EXPERIMENTAL-DATA TEST-NETHCCS PRESSURE ATTENUATORS, \#ARF MOO9 \#AD I3I $84 \mathrm{C}$

ARMOUR

ARMOUR RESEARCH FOLNDATION, CHICAGC, ILL.

$18 / 033-5626$ PAGES I8 FISS 9 REFS

RF U 


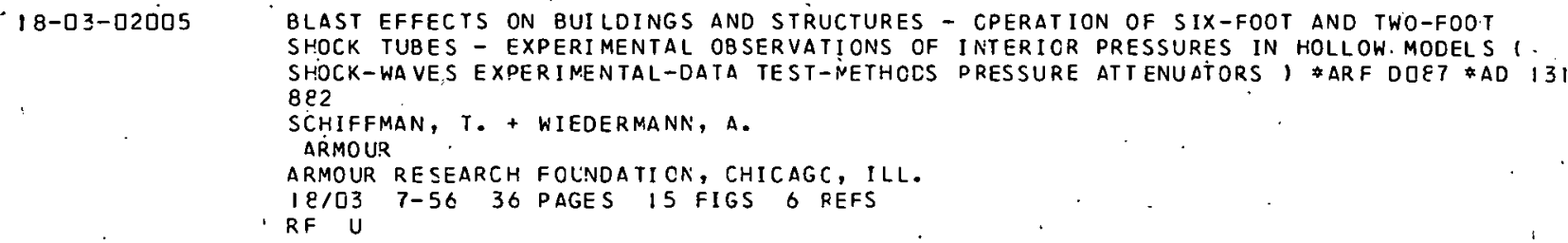

18-03-02007 MATER IALS'FOR LUSE IN MITIGATING BLAST LOAOS CN DEEPLY BURIED PROTECTIVE STRUCTURES ( CONCRETE LNDERGROUND-STRUCTURES EXPANCED-PLASTICS GLASS FOVEYCOME-CORES LOADING (MECHANICS) SHOCK(NECHANICS), \#AC 634691

$18-03-[2008$

MOTION OF SUESLRFACE SOIL INCLUSICAS SUBJECTED TO SURFACE ELAST LOADING - RESULTS OF SERIES III TESTS. ( SOIL-MECHANICS SHOCK-WAVES FAILURE(MECHANICS) UNDERGROUND-STRUC TURES NUCLEAR-EXPLOSIONS, \#TR 508 \#ASA-13.018

- WHITE, C. R.

NCEL

U.S. NAVAL CIVIL ENG I NEERING LABORATCRY, PORT HUENEME, CAL IF. $18 / 03 \cdot 1-67^{\circ} 3$ C DAGES 5 REFS

RF U

$18-03-[2017$

MATERIALS FOR LSE IN MITIGATING BLAST LCADS CN DEEPLY BURIED DROTECTIVE STRUETURES 1. UNDERGROUD-STRUCTURES CONSTRUCTICN LOAOING (MECHANICS) SHCCK(MECHANICS) FEASIBILITY-STLOIES STRE SSES ELASTICITY. CONCRETE HCNEYCOME-CORES EXPANDED-PLASTICS $D E S I G N$, $\angle A D 634$ 691

HOFF, G+C.

ARMY ENGINEERS

U.S. ARMY ENGINEER WATERWAYS EXPERINENT STATION, CORPS OF ENGINEER $S, V I C K S B U R G$, MISS. $1 E / 031966$ IS PAGES 24 REFS R F U

B IBL IOGRAPHY OF OPEN AND LINCLASSIFIED LITERATURE PERTAINING TO THE EFFECTS OF HIGH ALTITUDE NLCLEAR EXPLOSIONS I. AND II. ( UNITED-STATES USSR, \#DASA-1795 ROSEN, D. J. + KRALS, A. F. + GOLDELATT, S. L. NUE RES ASSOC

NUCLEAR RESEARCH ASSOCIATES, INC., LCNG ISLAND CITY, N.Y. $18 / 03$ 5-15-6' 4 [2 PAGES 726 REFS RA U

PLOWSHARE - NUCLEAR EXPLOSIONS - PEACEFUL APPLICATICNS INTERRUPTED BY.A DLD I CRATERING । \#SC-RR-67-3

VORTMAN, L. J.

SANDIA CORP

SANDIA CORPORATION, ALBUQLEROUE, N.NEX.

$19 / 03$ 2-67 34, PAGES 10 FIGS 10 REFS

RF

$18-03-02315$

EFFECT OF WATER 'CCNTENT, YIELD, NECIUM, ANC CEPTH OF EURST ON CAVITY RADIII CRATERING EXPLCSI CN-EFFEC TS NUCLEAR-EXPLOSIONS EQUATIONS TUFF ALLUVIUM SALT LRANITE MATHEMATICAL-ANALYSIS, \#LCRL-50203

HIGGINS, G. H. + BLTKOVICH, T. R.

LNIV OF CALIF

UNI VERSITY OF CALIFORNIA, LAWRENCE RADIATION LABORÁTORY,

LIVERMORE, CALIF.

IE/03 2-21-67 24 PAGES, D REFS

RF U

$18-03-02316$

A REAPORAISAL OF THE APPLICATICN CF STATIC EQUILIBRIUM THEORY TO DCCOUPLING CRATERING EXPLOSICN-EFFECTS NUCLEAR-EXPLOSIONS SEISMOLOGY GRANITE

MATHEMATICAL-ANAL YSIS LNDERGROUND-EXPLCSICNS, \#UCRL-50182

KNOX, J. B.. + HEARST, J. R.

UNIV OF CALIF

UNIVERSITY OF CALIFORNIA, LAWRENCE RADIATICN LABCRATORY,

LIV́RRMORE, CALIF.

$18 / 03 \quad 1-19-67$ IE PAGES II FIGS

RF $U$

$18-03-0231^{-17}$

PLOWSHARE - NUCLEAR EXPLOSIONS - PEACEFUL APPLICATICNS - A STUCY OF THE MECHANICS OF SLIDE DAMS WITH SAND MCDELS ( EXPERIMENTAL-DATA ANGLES (CEOMETRY) GEOMETRY REVETMENTS EFFEC'TI VENESS SLCPES, \$SC-RR-67-24 YOUNG, G. A.

MICHIGAN TECH UNIV + SANDIA CORP

MICHIGAN TECHNOLOGICAL UNIVERSITY, HOUGHTON, MICH, I SANDIA CORPORATION, ALBLQUERQUE, N. NEX.

$18 / 034-67$ I [6 PAGES 60 FIGS 4 REFS

RF $U$ 
$18-03-02318$

$18-03-02319$

$18-04-02026$

$18-05-[.2027$

$18-05-02320$

$10-06-02028$

$18-06-02029$

$18-06-02030$

OPERATION TEAPOT - DISTRIBUTICN ANC DENSITY. CF MISSILES FROM NUCLEAR EXPLOSIONS SECONDARY-MISSILES BLAST-ENERGI ZED-NISSILES VELOCITY GLASS STONE BLAST EXPER IMENTAL-DATA INSTRUNENTATICN THEORY, \#WT-I168

BOWEN, I. G. + STREHLER, A. F. + WETHERBE, M. B.

LOVELACE

LOVELACE FOUNDATICN, ALBUGUERQUE, $\mathrm{A} . \mathrm{N}$

$18 / 03$ 3-56 113 PAGES 73 FIGS 12 REFS

RF U

EARTH VIBRATIONS FROM A NLCLEAR EXFLCSION IN A SALT COME - PROJECT DRIBBLE SALMON EVENT I SEISMIC-WAVES EXPLOSI CN-EFFECTS VELOCITY ACCELERAT ION MOTION PRJPAGATIOV EARTHQUAKES, \#VUF-3014 \#AO 649701

MICKEY, W. V. + LCWRIE, L. M. + SHLGART, T. R.

U.S. DEPT. OF CONMERCE

U.S. DEPAR TMENT OF COMNERCE, CCAST AND GEOCETIC SURVEY, ROCKVILLE, MD.

18103 3-17-67 157 PAGES 26 FIGS 69 REFS

RF U

\section{Nuclear Instrumentation}

INSTALLATION AND TESTING CF AN AUTCMATIC REMOTE RACIOLOGICAL MONITORING SYSTEM I INSTRUMENTATION RADIATION-MONITORS DETECTICN TESTS RADIO-TELEMETRY

RADIATION-MEASLRENENT-SYSTENS I *TR-LG.0201.003

HARR IES, h. S. + NETZLER, R. E. + LAWSCN, M. E., JR.

GAUTNEY AND JONE $\&$ COM

GAUTNEY AND JONES COMMLNICATICNS, INC., WASHINGTCN, D.C.

I YIULI I-6I 165 HALES 38 PIG3 1 Q REFJ

RF U

\section{Nuclear Power Plants}

CIVILIAN NLCLEAR POWER - THE 1967 SUPPLEMENT TO TFE 1962 REPORT TO THESPRESIDENT NLCLEAR-REACTORS NLCLEAR-POWER-PLAATS ELECTRIC-POWER-PRODUCTION ECONOMICSI 1 A.L. I $[58$

AEC

U.S. ATOMIC ENERGY COMMISSION

$18 / 05$ 2- 67 56 PAGES D REFS

RF U

NLCLEAR REACTORS BLILT, BEING BUILT, OR PLANNED IN THE UNITED STATES AS OF OEC.31,1966 ( NUCLEAR-POWER-PLANTS) \$TIL-820D(15TH-REV)

$\triangle E C$

U.S. ATOMIC ENERGY COMMISSION, DIVISICN OF TECHNICAL INFORMATION $1 E / 05$ I2- $31-6 E$ 24 PAGES O REFS

RD U

\section{Radiation Shielding and Protection}

PR.OTECTION $\triangle G A I N S T$ NUCLEAR WEAPCN FALLCUT IN EMERGENCY HEALTH SERVICES UNITS I SHIELDING PATIENTS RADIOACTIVE-FALLCUT GAMMA-RAYS RADIATION-MONITORS RADIATION-MEASLRENENT-SYSTENS PERSCNNEL COSINETERS COSE-RATE

RADIOLOGICAL-CONTANINATION RADIATICN-HAZARDS RADIOGRAPHIC-FILM(PROTECTION) 1 *A.L. $1 \subset 45$

PACE, F. C.

CANADA DEPT OF NATL HEAL

CANADA DEPARTMENT OF NATI CNAL HEALTH AND WELFARE, OTTAWA

18106 2-65 17 PAGES 13 REFS

P. G U

SHIELDING IV - CI VIL DEFENSE I RADICACTIVE-FALLOUT ATTENUATION UNDERGROUND-STRUCTLRES DOSE-QATE PHOTONS SCATTERING ALBEDO GAMMA-RAYS

ANGLE-OF-INCIDENCE RADIATION(COPALT) BLAST-SHELTERS CYLINCERS(STEEL)) *A.L. 976

AMER VUC SOC

AMER ICAN NLCLEAR SCCIETY

I $2 / 06 \quad 1965$ P PAGES 31 REFS

JF U

AN EXPERIMENTAL EVALUATION OF ROCF REDUCTICN FACTCRS I SFIELDING

RADIOACTIVE-FALLOLT DOSE-RATES RACICLOGICAL-COSAGE ATTENUATION SIMULATION(MODELS) I * PSDC - TR - 16

MCDONNEL; $C$. + VFIIFTRI, .1.

PSDC

PROIECTIVE STRLCTLRES DEVELCDNENT CENTER, FORT BELVOIR, VA.

$18 / 06$ 5-66 73 PAGES 12 REFS

RF.U 
DESIGN OF ROOOF WASHDOWN SYSTENS (FINAL REPORT) I CCNSTRUCTION-MATER IALS RADIDACTIVE-FALLOLT SHIELDING BUILCINGS SHELTERS COSTS PARTICLES, \#USNRDL-TR-IOEL HEISKELL, R. H.

NRDL

U.S. NAVAL RADIOLOGICAL DEFENSE. LaBORATORY, SAN FRANCISCO, CALIF..

1 \& 106 1-27-65 5E PAGES 10 FIGS 13 TABLES 18 REFS

RF $\cdot U$

\section{Radioactive Wastes and Fission Products}

$18-07-02033$

$18-07-02034$

1 8-08-02035

1 8-08-02037

$18-08-02038$

$18-08-02039$

i 8-08-02322

THE TRANSPORTATION OF HIGHLY RADICACTIVE NATERIALS I CONTAINERS RAOIÖACTIVE-WASTES WASTES(INDLSTRIAL) SHIPPING-CCNTAIAERS, \$NYC-977.4

THOMP SON: J. T.

JOHNS HOPKINS UNIV.

JOHNS HOPKINS LNI VERSITY, BALTIMCRE, MD.

$18 / 07$ 1D-63 26 PAGES - 9 FIGS 7 REFS

RF U

A STUDY OF THE POSSIBLE CCNSEQUENCES ANC COSTS OF ACCIDENTS IN THE TRANSPORTATION OF HIGH LEVEL RADICACTIVE NATERIALS I UREAN-AREAS CECONTAMINATIOV

RADIOACTIVE- hASTE S SHIPPING-CCNTAINERS, \#NYC-9772

MORGAN, J. M., JR. + KNAPP, J. W. + THCMPSCN, J:T

JOHNS HOPK'INS UNIV

JOHNS HOPKINS LNI VERSITY, BALTIMCRE, MC.

$12 / 07$ 8-61. 116 PAGES 34 TABLES I6 FIGS 108 REFS

RF U

\section{Radioactivity}

SLRVEILLANCE NETWCRKS I RADI OLCGICAL-MONITORING RACIOACTIVE-FALLOUT

RADIOACTIVE-ISOTOPES AIR NILK ATMCSPHERIC-PRECIPITATION WATER-SUPPLIES FOOD UNITED-STATES CANADA, MEXICC I TID-23286

$A L L E N, R$, E.

$A E C$

U.S. ATOMIC ENERGY COMMISSION, DI.VISION OF OPERATIONAL SAFETY, WASHINGTON, D.C.

$18 / 08$ 6-66 97 PAGES 58 FIGS

RF U

A CALCULATIONAL MCDEL FOR CONDENSED STATE DIFFUSICN CONTROLLEC FISSION PRODUCT ABSORPTION DLRING FALLOUT FGRNATICA I RACIOACTIVE-FALLOUT RADIOACTIVE-DECAY PARTICLE-SIZE HENRYS-LAW HALF-LIFE DISTRIBUT ION CCMPUTERS (PROGRAMMIVG).YIELD) * GA-7598

KORTS, R. F. + NORNAN. .I. H.

GENERAL DYNAMICS

GENERAL DYNAMICS, GENERAL ATONICS [IVISICN, SAN CIEGO, CALIF.

$18 / 08$ I-IE-67 9I PAGES ID FIGS, 5 REFS

R I U

JPERATION CENI ZA ARENA - TECHNIQUES FOR THE NEASUREMENT OF DEPOSITIOV AVD REDISTRIBUTION OF FALLOUT AROUND STRUCTURES I. RACIOACTIVE-FALLOUT PARTICLES DEBR.IS VOLCANOES COSTA-RICA WIND INSTRUNENTATION, \#SRI-NU-577Y(B)

CLARK, D. E., JR. + SARTOR, J. D.

STANFORD RESEARCH INST

STANFORD RESEARCH INSTITUTE, NENI.C PARK, CALIF.

$18 / 08$ 12-66 22 PAGES 20 FIGS 14 TABLES 3 REFS

RF U

AN ANALYSIS OF THE FALLOUT PREDICTION NCICELS I. ANALYS IS, COMPARISON, AND CLASSIFICATION OF NODELS I RADIOACTIVE-FALLOUT MATHEMAT ICAL-PREOICTION YIELD GEOMETRY DISTRIBUTION PARTICLE-SIZE WIND) \#USNRCL-TRC-68 POLAN, M.

NRDL

U. 5 . NAVAL RADIOLCGICAL DEFENSE LAECRATORY, SAN FRANCISCO, CALIF.

$18 / 08 \cdot 12-66 \quad 157$ PAGES SO REFS

RS U

DUSTY II I - AN IMPROVED MATHFNATICAL METHCE CF LCCATING FALLOUT ON A SPHERICAL EARTH ( WIND YIELLD RADIOACTIVE-FALLCUT PRCGRAMMING(COMPUTERS) BURST-COVDITIOV MATHEMATICAL-ANALYSIS,). \$NREC-9

BREWER, H. + CAGLEY, E. + MARTIN, R. + KUSE, E.

ROWLAND, D.

-NAT RES EVAL CENTER

NATIONAL RE SOURCE EVALUATICN CENTER, WASHINGTON, C.C.

$18 / 083-6352$ PAGES 28 FIr,S 2 REFS

RC U 


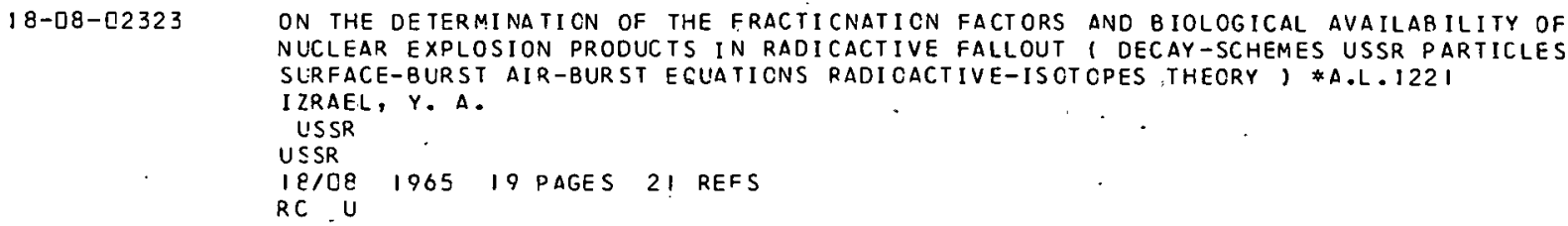

ON THE DE TERMINATICN OF THE FRACTICNATICN FACTORS AND BIOLOGICAL AVAILABILITY OF NUCLEAR EXPLOSION PRODUCTS IN RADICACTIVE FALLOUT I DECAY-SCHEMES USSR PARTICLES SLRFACE-BURST AIR-BURST EGUATICNS RADICACTIVE-ISOTCPES,THEORY, *A.L.122I I ZRAEL, Y. A.

USSR

USSR

IE/O\& 1965 I9 PAGES 2 ! REFS

$R C$ U

$18-08-02324$

$18-08-02325$

$18-09-02040$

$19-91-02042$

$19-04-02043$

$19-04-02044$

SEDAN PROJECT E2. REC AERORADIOACTIVITY SURVEY \& WEAPONS-EFFECTS RADIOACT IVE-FALLOUT DISTR IBUTION DOSE-RATE GANMA-RAYS INSTRUNENT AT ION EXPERIMENTAL-CATA, CETO

CIVIL EFFECTS TEST OPERATIONS / USAEC

1 IIOE 5- 67 I 6 PAGES I FIG 1 TAELE 7 REFS

RF U

HENRYS LAY CONSTANTS FOR DISSOLUTICN OF FISSION PROCUCTS IN A SILICATE FALLOUT PARTICLE MATRIX I RADI OACTIVE-DECAY RADIOACT IVE-FALLOUT THERMODYNAMICS SOLUBILITY TEMPERATURE DITFUSIVITY HALF-LIFE YIELD, \$GA-7Q58 NORMAN, J. H.

GENERAL DYNAMICS

GENERAL DYNAMICS, GENERAL ATOMICS DIVISICN, SAN CIEGO, CALIF. $18 / 08$ 1?-2G-6G 3\& PAGES 5J REFS

RF U

\section{Reactor Engineering and Operation}

PREL IMINARY CONCEPTS OF SUBSURFACE REACTOR CCNTAINMENT STRUCTURES TO WITHSTAND DIFFERENTIAL EARTH DI SPLACEMENT I REACTOR-FEAS IBILITY-STUCICS

REAC TOR-SAFETY-SYSIEMS SOIL-MECHANICS CYNAMICS GROUND-MOT ION UNDERWATER DESIGN COSTS LOADING (MECHANICS) CYLINDERS, SD-7I-66(R)-5872

BECHTEL CORP + CRNL

BECHTEL CORPORATICN / CAK RIDGE NATICNAL LABCRATORY

$18 / 09$ 8-66 S3 PAGES 19 FIGS IT REFS

RF $U$

\section{ORDNANCE}

\section{$19 \cdot 01$ Ammunition, Explosives, and Pyrotechnics}

FINAL RESLLIS OF SMOKE SCREEN SYSTEN DEVELOPNENT (

THERMAL-RADIATION (COUNTERNEASURES) LIQUID-FUELS SMOKE-GENERATORS

FEASIBILITY-STUDIES DESIGN THEORY EXPERIMENT AL-CATA MATERIALS PARTICAL-SIZE COSTS

OPTICAL-PROPERIIIES COST-EFFECTIVENESS) \#URS $643-9$ \#NRDL-TRC-49

STAACKMANN, M. + EAKER, R. L. + CCLVIN, C. E.

GOUDALE, T. C. + HAWKINS, N. B.

URS CORP

UNITED RESEARCH SFRVICES CORPORATICN, BURLINGAME, CALIF.

IGICI O- CO IH2 PAGES 34 REFS

R F U

\section{Explosions, Ballistics, and Armor}

THE SLRFACE WAVES FROM SHALLOW UNDERWATER EXPLOSICNS - FORMULAE ANC PROGRAM SPECIFICATIONS I HYDRODYNANICS WATER-WAVES MATHEMATICAL-PREDICTION THEORY CRATER ING PROPAGATION, \#NCRE/R-525 \#AD 637289 HICKS, A. N.

NAVAL CONST RES ESTAB

NAVAL CONSTRLCTIOA RE SEARCH ESTABLISHMENT, CUNFERNLINE, FIFE

$19 / 64$ 6-66 3[ PAGES 9 FIGS II REFS

RE U

THEORETICAL GROUND SHCCK EFFECTS I. GRCUND NOTICN IN ELASTIC HALF SPACES IVDUCED BY AIR BLAST ( ELASTICITY SHOCK-WAVES NECHANICAL-WAVES LOACING(MECHANICS) SURFACE-BURST ACCOLSTICS STRESSES, \#DASA-1676-I \#AC 633618 GAN 5, Q. F. + BROOKS, J. A.

LNITED AIRCRAFT CSC

UNITED AIRCRAFT CCRPORATE SYSTEMS .CENTER, EL SEGUNCO, CALIF. $19 / 04 \quad 20 / 11 \quad 12-65 \quad 125$ PAGES 12 REFS

RF U 
MECHANISMS IN THE GENERATION CF AIRBLASTS BY UNDERWATER EXPLOSIONS I SHOCK-WAVES CALCULATIONS MODELS I. *NOLTR-66-88

MALME, C. I . + CARBONELL, J. R. + CYER, I.

BOLT BERANEK, AND NE WMAN

BOLT BERANEK AND NEWMAN, INC. , CANERIDGE, MASS.

1 I /O4 9.-23-66 156 PAGES 62 FIGS 15 REFS

RF U

\section{Guns}

$19-06-02.327$

$19-\mathrm{Dt}-\mathrm{C} 2328$

$19-06-02329$

$20-01-02047$

$20-01-02048$

$20-0 \geq-02330$

CONCEPT AND FEASIBILITY STUDIES CF NUZZLE BRAKE ELAST SUPPRESSION CEVICES FOR IISMM AND I $55 M M$ HOWITZERS, SHOCK-WAVES ATTENUATION DESIGN GUN-BARREL-ATTACHMENTS

INHIBITION PRESSURE STRESSES PROJECTILES INTERIOR-BALLISTICS GAS-FLOW LEVIN, S. + KAFADAR, A. D.

ORDNANCE ENG ASSCC

ORDNANCE ENGINEERING ASSOCIATES, INंC., DES PLAINES, ILL.

$19 / 36,2-26-64$ 99 PAGES 31 FIGS 13 REFS

R.F U

MLZZLE BRAKES II THEORY ( GUN-AUXILIARY-EGUIPMENT RECOIL-MECHAVISMS

INTER IOR-BALLISTICS, \$AD 111482

HAMMER, E. h.. JR.

FRANKLIN INSTITUTE

FRANKLIN INSTITUTE, LABORATCRIES FCR RESEARCF ANL CEVELOPMENT

I $9 / 06$ 6-49 374 PAGES 50 FIGS

RE U

CONTRIBUTION TO THE ANALYSIS CF NUZZLL BRAKE DESIGN I ART ILLERY COMPUTERS

GUN-BARREL-ATTACHNENTS INTERI CR-BALLISTICS, BIBLICGRAPHIES CIGITAL-COMPUTERS EXPLOSIONS GAS-DI. SCHARGES HEAT IGNITERS PROJECT ILES MATHEMAT ICAL-ANALYSIS PROGRAMMING (CONPUTERS) THEORY PROPELLANTS ） \$RIA-62-1794\$AD.276 I54

SCHLENKER, G.

ROCK ISLANO ARSENAL

ROCK ISLAAND AR SENAL, ROCK I SLAND, ILL.

19106 5-62 144 PAGES 25 REFS

RE U,

\section{PHYSICS}

\section{0 ol Acoustics}

THE RESPONSE OF SOILS TO DYNAMIC L,CADINGS - WAVE VELOCITIES THROUCH FARTIALLY SATURATED SAND - CLAY MI XTURFS I SCIL-NECHANICS ACCOUST ICS MOISTURE. MEEHANICAL - WA VES CIVIL-ÉNGINEERING. PROPAGATICN, $R 66-13 * \triangle D 638.027$ BALAKRISHNA RAO, H. A.

$M I$ T

MASSACHUSETTS INSTITUTE OF TECHNCLCGY, CANERICGE, MASS -

$2 C / 01$ 3-66 61 PAGES 8 REFS

RF U

INTERACTIION BE TWEEN AIR FLOW ANO AIKBCRNE SCUNC IN A CUCT I ACOUSTICS GAS-FLOW WEST-GERMANY DLCTS LAMINAR-BOUNDARY-LAYER STABILITY MEASUREMENT, \#AMRL-TR-65-53 * AD 640825

MECHEL, F. P. + MERTENS, P. A. + SCHILZ, W. N. ,

GERMANY

GERMANY

2 LIO1 9-65' 58 PAGES 34 FIGS 26 REFS

RF U

\section{Electricity and Magnetism}

RESEARCH ON ELECTROMAGNETICS FCR PROJECT DEFENDER I PLASMA-PHYSICS

ELECTROMAGNETIC-PROPERTIES FILMS FLUID-MECHANICS SCIENTIFIC-RESEARCH ABLÁTION

ANTENNAS PROPAGATION SHOCK-WAVES KINETIC-THECRY ELECTRON-CENSITY CERENKJV-RADIATION

MICROWAVE $S$ OXIDES. PLASTICS) \#PIBNRI-1295.3-66 *AC 6112271

HUTTER, R. G. E.

POLY INST BROOKLYN

POLYYECHNIC INSTITLTE. OF BRCOKLYN, BRCCKLYN, N.Y.

- 2 [ /03 9-3L-66 288 PAGES 125 FIGS 105 REFS

RF U 


\section{Fluid Mechanics}

$20-04-[2050$

$20-04-02051$

$20-04-02053$

$20-04-02055$

$20-04-c 233$

$20-04-02332$

$20-04-02333$

$20-05-02056^{\prime}$

SHOCK WAVE ATTENUATION FORMULAS FCR STRAIGHT TUNNELS - A COMPARISON I SHOCK-TUBES CALCULATIONS EXPERIMENTAL-DATA EXPLOSION-EFFECTS， \$A.L. 984

ERIKSSON, $S$.

SWEDEN

SWEDEN

2 CIO4 196223 PAGES 14 FIGS 3 REFS

RF U

NOMOGRAM FOR CALCLLATING ATTENUATICN OF AIR SHOCK WAVES IN TUNNELS I PRJPAGATION SWEDEN, \#REPORT NC. $103-24$ \#AEC-TR-5025

ERIKSSON, $\subseteq$.

SWEDEN

SWEDEN

2Г. 104 4-|4-6I IS PAGES 5 FIGS 3 REFS

RC U

SHOCK TUBES - A BIBLIOGRAPHY $\#$ NLN-1189

MELNITK, A. M.

MON SANTO

MONSANTO COMPANY, NOIIND LARORATORY, NIAMISBURG, OHIO

$2 C / 043-64$ 55 PAGES 667 REFS

RA U

THE PROPAGATION OF AIR SHCCK WAVES IN A TUEE I SHOCK-TUBES EXPERIMENTAL-DATA PRESSURE-GAGES I *CRNL-TR-1598

LRANSIKUM, S.

SWEDEN

SWEDEN

$2[/ 04$ 10-1C-5C IC8 PAGES 18 FIGS 12 REFS

RF $U$

FLOW CONTROL BY GENERATION OF STANCING VCRTICES ANC THE CUSP EFFECT I HYDRODYNAMICS DESIGN CUSPS FLUID-FLOW, *AD 107323

RINGLEB, F. O.

PRINCETUN UNIY

PRINCETON LNI VERSITY, PRINCETON, N.J.

2C104. 7-27-55 73 PAGES 13 REFS

RF $U$

NECESSARY CONDITICNS FCR SIMILAR. SCLUTICNS OF PRORLEMS OF TURPULENT GAS DYNAMICS GAS-FLOW EQUATIONS-OF-STATE MATHENATICAL-ANALYSIS FLUID-FLOW SHEAR-STRESSES, * L'CRL-50211

CROWLEY, B. K.

UNIV OF CALIF

UNIVERSITY OF CALIFORNIA, LAWRENCE RADIATICN LABCRATORY,

LIVERMORE, C, $\triangle 1$. T.F.

$2[/ D 4$ 1-31-67 26 PAGES O FIGS 0 REFS

RF $U$

SHOCK PROPAGATION IN NONHCNOGENEOUS ATMOSPHERES ANC MODIFIED SACHS SCALING I EXPONENTIAL IDEAL GAS ATMCSPHERE I SHOCK-WAVES FLUIC-CYNAMIC-PROPERTIES HYCR.ODYMAMISS, \$NOI.TR $67-2 \pi$

LLTZKY, M. + LEHTC, D.

NAVAL ORONANEE LAB

U.S. NAVAL OP.DNANCE LABORATORY, WHITE OAK, ML,

2 C:104 6- $19-67$ 17 PAGES 2 FIGS 4 REFS

RF $U$

RELATIVE MOTION AND COAGULATICN CF PARTICLES IN A TURBULENT. GAS I TURBULENCE FLUID-DYNAMICS PARTICLE-SI ZE-DISTRIBUTION AEROSOLS REYNOLCS-VUMBER BROWNIAN-MOVEMENT EQUATI ONS, \#NROL-TR-67-49

HUEBSCH, I. 0 .

NRUL

U.S. NAVAL RADIOLCGICAL DEFENSE LAEORATORY, SAN FRANCISCO, CALIF. 2C/O4 5-4-67 113 PAGES 9 FIGS 5 TABLES 32 REFS RF $U$

\section{Masers and Lasers}

INVESTIGATIONS OF GIANT PULSING METHODS IN RUBY LASERS ( PUMPING(OPTICAL) CAVITY-RESONATORS OPTICS CRYSTALS NIRRCRS, *AD 637825

DEAN, D. R. + COLLINS, R. J.

LNIV UF MINNE SUTA

UNIVERSITY OF MINNESOTA, NINNEAPOLIS, MINN.

$2[105$ 8-te $7 E$ PAGES 21 REFS

RF U 
LASER CAVITIES I . RESEARCH ON OPEA RESONATORS II. BEHAVIOR OF THE OUTPUIT FROM A MANY ELEMENT LASER I LASERS OPTICAL-EQUIPMENT-COMPCNENTS OPTICS , \#AD 638193 CHECCACCI, P. F. + SCHEGGI, A. M. + PRATESI, R.

RONCHI, L.

UNIV OF FLORENCE

UNIVERSITY OF FLORENCE, FLORENCE, ITALY

$2\left[/ 05^{\circ} 4-3[-66\right.$ 6I PAGES 46 REFS

$20-05-\square 2060$

RF $U$

20-05-02060

INVESTIGATION OF THE MECHANISMS ASSOCIATED WITH GAS BREAKDOWN UNDER INTENSE OPTICAL ILLUMINATION I GAS-IONI ZATION BEANS (ELECTROMAGNETIC) LASERS HEL IUM-GROUP-GASES PLASMA-MEDILM. LUMINE SCENCE ARGON NECN, \#UACRL E920272-6*AD 627. 233 HAUGHT, A. F. + SNITH, D. C.

- UNITED AIRCRAFT RES LABS

UNITED AIRCRAFT RESE'ARCH LABORATORIES, EAST HARTIFORC, CONN.

$2[\% 95 \quad 1-66 \quad 34$ PAGES 6 REFS

$20-05-02062$

NRL PROGRESS IN HIGH POWER LASER RESEARCH I LASERS SCIENTIFIC-RESEARCH GLIASS OPTICAL-PROPER TIES RUBY CRYOGENICS GASES PUMPING (CPTICAL) FLASH-LAMPS LIQUIDS SPECTROSCOPY, *NRL-6444\#AD 637616 NAVAL RESEARCH LAB

U.S: NAVAL RESEARCH LABORATORY, WASHINGTCN, C.C. 2CIOS O-66 44 PAGES 26 REFS

$20-05-0.2064$

STUDIES ON THE INTERACTI ON OF LIGHT AND PART ICLE REAMS I MAGNETO-OPTIC-EFFECT MAGNETIC-FIELDS LASERS HYSTERESIS, \#AFOSR-66-1090 \#AC 635016

WANIEK, R. W. + JARMUZ, P. J. + CHENG?. H.

GIVENS, R. T. + GILSOORF, H. J. + LEE, S. W.

ADVANCED KINETICS

ADVANCED KINETICS, INC., CCSTA MESA; CALIF.

REIOS 3-15-66, 2I PAGES 13 REFS

\section{Optics}

$20-0.6-02066$

$20-0.6-0.2067$

STUDY OF LIGHT ATTENUATION UNDER SUBTRCPICAL CLIMATIC CONOITIONS I

L IGHT-TRAN SMI SSION COLORS OPTICAL-INAGES OPT. ICAL-PHENOMENA METEOROLOGY FLORIDA, * 209-2

HISER, H. W. + ROBERT, R. W. + CCURTRIGHT, C. L.

UNIV DF MIAMI

UNIVERSITY OF MIANI, MIANI, FLA.

$2 C / 06$ 12-66 326 PAGES 312 FISS 6 REFS

RF U,

THE MEASUREMENT OF EVERAL CPTICAL NCNLINEARITIES USING FOCUSED gAUSSIAV LASER BEAMS I CRYSTALS CPTICAL-PHENCNENA HARNCNIC-GENERATORS REFRACTION LASERS NONL INEAR-SYSTEMS AMMONI UN-CONPOUNCS FCCUSINE PHOSPHATES CALCITE POLARIZATION, *ML-1405 \#AFOSR-66-9490*AD 637500 B JORKHOLM, J. E.

STANFORD UNIV

STANFORD LNIVERSI IY, STANFORO, C.ALIF. $2[106.1-66$ 1 29 PAGES 38 REFS

$R F$ U

\section{Particle Physics}

$20-08-[2068$

A STUDY OF'THE BASIC MECHANISMS OF DUST EROSION I PARTICLE-SIZ.E EXPER IMENTAL-DATA GAS-TURB INES VELOCITY, \#AD 420083 WOOD, C. O .

SOUTHWE ST RE SEARCH INST

SOUTHWEST RESEARCH INSTITUTE, SAN ANTONIC, TEXAS

2C/O8 9-63 48 PAGES 27 FIGS 9 REFS

$20-08-02070$

RF U

FLNOAMENTAL NUCLEAR. ENERGY RESEARCH - 19601 BIOLOGY MEDICINE ECOLOGY RADIATION-EFFECTS GENETICS PHYSICS SCIENTIFIC-RESEARCH METALLURGY MATERIALS CHEMISTRY MA THEMATICS COMPUTERS NUCLEAR-REACTORS HEAT-TRANSFER NUCLEAR-REAC TOR-MATER I ALS FLUID-DYMANIC-PRCPERT IES ENERGY-CONVERS ION REACTOR-SAFE YY-SYSTEMS RADI CACTIVE-WASTE, \$A.L. ID83

$\triangle E C$

U.S. ATOMIC ENERGY.COMMISSION

2C108 12-66 359 PAGES 24I FIGS 
$20-08-02334$

$20-09-02335$

$20-11-02074$

$20-11-02075$

$20-11-02076$

$20-11-02077$

$20 \div 11-02078$

$20-11-02079$

$20-11-02080$
ENERGY AND ANGULAR DISTRIBUTI CN OF NEUTRONS. AND GAMMA RAYS - OPERATION BREN 1 ION I IING-RADIATION-SCATTERING） $\$ C E X-62.12$

THORNGATE, J. H. + AUXIER, J. A. + HAYWCCD, F. F.

HELF, S.

CETO

CIVIL EFFECTS TESI OPERATICNS / USAEC

2L/OE 2-67 91 PAGES 71 FIGS I TABLE 23 REFS

RF U

\section{Plasma Physics}

PLASMA STREAMING INTO a MAGNETIC FIELD I GAS-FLOW PLASMA-MEDIUM PLASMA-OSCILLATIONS STABILITY BOUNDARY-VALUE-PROBLEMS , \#UCLA-R-IO $\# A C 648083$

OSSAKOW, S. L

UNIV OF CALIF

UNIVERSITY OF CALIFORNIA, LOS ANGELES, CALIF.

$2[/ 09 \quad 11-66$ 65 PAGES I5 REFS

RF U

\section{Solid Mechanics}

THE SHOEK AND VIBRATION BULLETIN I ROCKETS OYNAMICS STRUCTURAL-ANALYSIS ELASTICITY BEAMSISTRUCTURAL) SHOCK(MECHANICS) LAUNCHING (SIMULATION) ACOUSTICS

VIBRATION-I SOLATORS, *BULLETIN $35(3)$

NAVAL RESEARCH LAB

U. S. NAVAL RESEARCH LABORATORY, WASHINGTCN, [.C.

2 CIII. 1-66 279 PAGES 206 FIGS 168 REFS

RS U

TIIE SHOCK AND VIBRATION BLLLFTIN I INSTRUMENTATION ACCELEROMETERS

SPEC TRUM-ANALYZER S TRANSDUCERS MATHENATICAL-ANALYSIS STRUCTURES DYNAMICS,

*BULLETIN $35(4)$ \#AD 63) 233

NAVAL RESEARCH LAB

U.S. NAVAI RESEARCH LABORATORY; WASHINGTCN, C.C.

$2[$ i) 2-6E 238 PAGES 233 FIGS 74 REFS

R S

THE SHOCK AND VIBRATION BULLETIN I TRANSPORTATION VIBRATION-ISOLATORS

AIR-TRAN SPORTA TI ON RAILROADS ARMED-FORCES-TRANSPORT ATION SFIPPING(MAR INE) DAMPING

SPACE-FLIGHT ) \#BLLLETIN $35(5)$ \#D 63I 234

NAVAL RESEARCH LAB

U.S. NAVAL RESEARCH LABORATORY, WASHINGTCN, C.C.

2 [II 2-66 328 PAGES 286 FIGS 135 REFS

$R \subseteq U$

THE SHOCK AND VIBRATION BULLETIN I IMPACT-TESTING SHOCK(MECHANICS), \$BULLETIN $35(6)$ NAVAL RESEARCH LAB

U.S. NAVAL RESEARCH LABORATORY, WASHINGTCN, C.C.

$2[111$ 4-6E 371 PACES 402 FIGS 131 REFS

$R S U$

STUDY OF GROUND MOTIONS FOR SIMULATICN BY SHCCK TESTING MACHINE I NUCLEAR-WEAPONS SHOCK - WA VES MECHANICAL-WAVES SHOCK (MECHANICS) CES IGN TESTS MOCELS(SIMULATIOVS)

SOIL-MECHANICS IMPACT-TESTS, \$CR-05.JOI

JACOBSEN, L. S. + KARAGOZIAN, J. + MALTHAN, J. A.

AGBABIAN-JACOBSEN ASSOC + NCEL

AGBAB IAN-JACOB SEN ASSOCI AIES, LOS $\triangle N G E L E S$, CALIF, I U S N NAVAL

CIVIL ENGINEERING LABORATORY, PORT HIJENËME, CALIF. $2[11, ; 1-65132$ PAGES 7 REFS

RF U

STUDIES ON LONGITLOINAL ANO BENDING WAVES IN LONG ELASTIC RODS 1 MECHANICAL-WAVES STRESSES PROPAGATION ELASTICITY THEORY, \#DRL-376 \#AD 631648

PLAS\$, H. J., JR . + STEYER, C. C.

LNIV OF TEXAS

UNIVERSITY OF TEXAS, DEFENSE RESEARCH LABORATORY, AUSTIN, TEXAS

2 CIII 4-24-56 I3C PAGES 40 FIGS 18 REFS

ACCELERATION WAVES IN NONLINEAR MATERIALS $I$ VISCOELASTICITY MECHANICAL-WAVES PROPAGATION MA THENATICAL-ANALYSIS ELASTICITY SHOCK-WAVES CONTINUUM-MECHANICS LINEAR-SYSTEMS FUNCTIONS, \#AD 631419

COLEMAN, B. D. + GLRTIN, N. E.

BROWN UNIV

BROWN UNIVERSITY, PROVIDENCE, R.I .

$2[11112-65$ 18 PAGES 17 REFS 


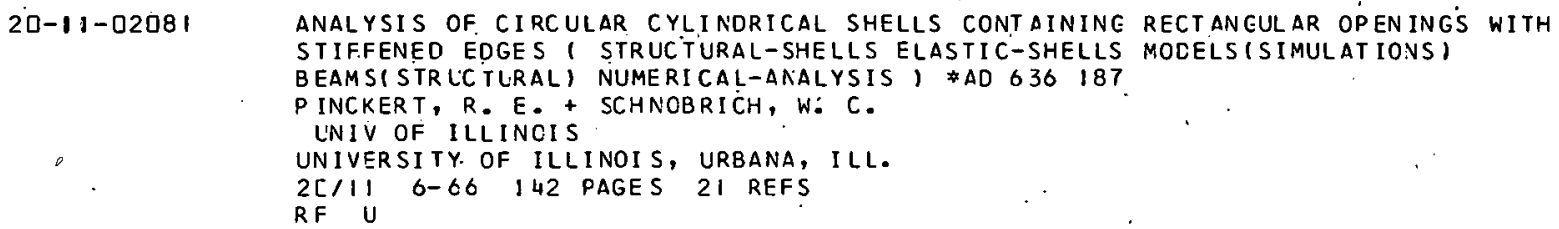

$20-11-02082$

ON LONGI TUDINAL AND TRANSVERSE WAVES IN ELASTIC. ROCS 1 MECHANICAL-WAVES ELASTICITY PROPAGATION STRAINIMECHANICS) EQUATICNS-OF-NCTION STRESSES, \#CRL-362 \#AD 631816

$20-11-02083$

DAMAGES RESULTING FROM LABCRATORY VIBRATION AND HIGH IMPACT SHOCK TESTS I NAVAL-RESEARCH DESIGN ELECTRONIC-E GUIPMENT RELIABILITY(ELECTRON.IC) NAVAL-VESSELS SHOCK (MECHANICS), \#AD 37880

WOODWARD, $K$. E.

NAVAL RESEARCH LAB

U.S. NAVAL RESEARCH LABORATORY, WASHINGTCN, C.C.

2 C/11 9-53 36 PAGES 14 REFS

RF U

$20-11-02084$

ANALYSIS OF STRESS DISTRIBUTICN BENEATH EMBANKMENTS ( STRESSES SOIL-MECHANICS SETTLEMENT (STRLCTLRAL) HIGH-SPEED-GRCUND-TRANSPORT MATHEMATICAL-ANALYSIS CONSOLIDATION. DEFORMATION TRANSPCRTATION ELASTICITY NORTHEAST-CORRIDOR COMPUTER SIPROGRAMNING) PLASTICITY, \$R66-53

LAMBE, T. W. + HIRSCHFELD, R. C. + CHRISTIAN, J. T. MIT

MASSACHUSETTS IN'STITUTE OF.TECHNCLCGY, CAMBRIDGE, MASS.

$2[11], 11-1-6652$ PAGES 20.FIGS 23 REFS

RF $U$

$20-11-02086$

A RHEOLOGIC INVESTIGATION OF THE DYNAMIC RESPONSE SPECTRA CF COHESIVE SOILS DYNAMICS SOIL-MECHANICS THEORY EXPERIMENTAL-CATA STRAIN(MECHANICS) STRESS LOADING (MECHANIC S). SHOCK-WAVES VISCOELASTICITY BLAST, \#CONTRACT REPORT NO. 3-148 K INDNER, . R . L.

ARMY ENGINEER

U.S. ARMY ENGINEER WATERWAYS EXPERINENT STATION, VICKSBURG, MISS.

$2[111.11-65$ IOO PAGES 45 FIGS II REFS

RF W

$20-11-02087$

ARTIAL BIBLIOGRAPHY ON SLBJECTS RELATED TO ACT IVE VIBRATICN ISOLATION. AND ACTIVE VEHICLE SLSPENSIONS, VIBRATICN-ISCLATCRS.CALCULAT IONS MATHEMATICAL-MODELS HAROWARE DESIGN, \#DSR 76IC9-2 \#PB 173649

PALL; I. L. + BENDER, E. K.

MIT

MASSACHUSETTS INSTITUTE OF TECHNOLCGY, CANBRIDGE, MASS. $2\left[I^{\prime} I 1 \quad 11-1-6631\right.$ PAGES 300 REFS

RA U

A THEORETICAL AND EXPERI MENTAL INVESTIGATION OF A DYNAMICALLY LOADED RING WITH RADIAL ELASTIC SUPPORT I RINGS VIBRATION FREGUENCY BENDING ELASTICITY LOADING (MECHÁNICS) IMPACT, *TNB-7D4*AD 650178

WENK, E., JR.

NAVY TAYLOR MODEL BASIN,

U.S. NAVY, DAVID TAYLOR MCDEL BASIN, WASHINGTON, C.C. $2\left[1117-5 C^{-76}\right.$ PAGES 30 FIGS 43 REFS

RE U

$20-11-02337$

DYNAMIC SHEAR RESISTANCE OF THIN WEBBED REINFORCEC CONCRETE BEAMS BEAMS (STR LC TURAL). DYNAMICS SHEAR-STRESSES WE SS (STRUCTURAL) DESIGN STRUCTURES LOADING (MECHANICS) SHOCK-WAVES MATHEMATICAL-ANALYS IS） \#NCEL-R-534 FUSS; 0.5 NCEL

-U.S. NAVAL CIVIL ENGINEERING LABCRATCRY, PORT HUENEME, CALIF. $2[/ 11$ 6-67 79 PAGES 39 FIGS 7 TAELES 19 REFS RF U

$20-11-02338$

AN EXPERIMENTAL INVESTIGATION OF FRANGIBLE PLATE FRAGMENTATION ( LOADING (MECHANICSI CRACK ING (FRACTLRING) FRACTURE (MECHANICS) CRACK-PRCPAGAT ION IMPACT-TESTS DYNAMICS BLAST SHOCK-WA VES ELASTICITY STRESS NECHANICAL-ANALYSIS, \#M6095

LIBER, T. + BARNETT, R. L. I ITR I

ILLINOIS INSTITUTE OF TECHNOLOGY RESEARCH INSTITUTE, CHICACO $2[1 / 1$ ID-66 99 PAGES 9 REFS 39 FIGS 15 TABLES R F U 
$20-11-02339$

$20-11-02340$

$20-11-[2342$

$20-11-02343$

$20-11-02344$

$20-11-02345$

$20-11-02346$

$20-11-02347$

$20-1 !-0234$

FLEXURAL MECHANICS OF REINFORCED CCNCRETE - PROCEECINGS OF THE INTERNATIONAL SYMPOSIUM ( LOADING (MECHANICS) STRLCTURES NONLINEAR-ANALYSIS BEAMS(STRUCTLRAL) FRAMES LINEAR-STRLCTURES) *ASCE-1965-50*ACI-SP-12

AMER SOC CIVIL ENG

AMER ICAN SOCIETY OF CIVIL ENGINEERS

2 CIII 1965 6CI PAGES 301 FIGS 403 REFS

RS U

DEFLECTIONS OF REINFORCED CONCRETE FLOOR SLAES I STRUCTURES DESIGN THEORY BEAMS (STRUCTURAL FRAMES LCADING (MECHANICS) CCLUMNS (STRUCTURAL) DEFORMATION MATHEMATICAL-MODELS MATHENATICAL-ANALYSIS，

VANDERBILT, M. D. + SOZEN, N. A. + SIESS, C. P. L'NIV OF ILLINOIS

UNIVERSITY OF ILLINOIS, URBANA, ILL.

2L/1I 4-64 287 PAGES 113 FIGS 47 REFS

R F U

STATIC. AND DYNAMIC BEARING TESTS CA A STRIP FOOT ING IN SATURATEU SAVD I

LOADING(MECHANICS) SOIL-MECHANICS CYNAMICS SHELTERS UNDERGRUUNÜSTRUCTURES SAND

SHOCK - WAVES BLAST FOOTINGS FOUNDATICNS VCID-RATIO ARCHES, \#NCEL-R-536 \#DASA-13. [II8 NCEL

U.S. NAVAL CIVIL ENGINEERING LABCRATORY, PORT HUENEME, CALIF.

$2[111606725$ PAGES 4 TABLES 16 FIGS 9 REFS

RF

SHOCK SPECTRA FOR A GENERAL FORCING FUNCTION ( SHOCK(MECHANICS) VIBRATION ELASTICITY VELOCITY DEGREES-OF-FREEDOM MATHENAT ICAL-ANALYSIS ACCELERATION D I SPLACEMENT , \#UCRL-729I

TODARO, A. F.

UNIV OF CALIF

UNIVERSITY OF CALIFORNIA, LAWRENCE RADIATICN LABORATORY,

LIVERMORE, CALIF.

$2[/ 1$ I 3-25-63 II PAGES 5 FIGS

RF L

VIBRATION ISOLATION THROUGH PNEUMATIC SPRING AND DAMPING I SHOCK(MECHANICSI

VIBRATION-I SOLATORS SIMULATION MATHEMATICAL-ANALYS IS SHOCK-WAVES ANALOG-COMPUTERS,

* CONF- 65I [:16-1

HALL, R. L.

EDGERTON GERMESHAUSEN

ECGERTON, GERMESHAUSEN, ANO GRIER, INC., LAS VEgAS, NEV.

2 III I IES 23 PAGES II FISS 4 REFS

RF U

LARGE DEFLECTIONS OF CLAMPED RECTANGULAR PLATES I PANELSISTRUCTURAL I

RECTANGULAR-BOCIES STRESSES THRUST THERMAL-STRESSES LOACING(MECHANICS)

SIRAIN (MECHANICS) BUCKLING(MECHANICS) MATHEMATICAL-ANALYSIS, \#SRSM-7-168*AD 649

833

NOLTE, L. J.

HUGHES AIRCRAFT

HLGHES AIRCRAFT CCMPANY, CULVER CITY, CALIF.

$2[1119-1-5757$ PAGES 14 FIGS 4 REFS

RF U

VIBRATION OF MLLTI-LAYERED ANISOTRCPIC CYLINDRICAL SHELLS I STRUCTURAL-SHELLS

'CYLINDR ICAL-BODIE S COMPOSITE-MATERIALS, EGUATIONS-OF-MOTION, STRESSES

STIFFENED-CYLINDERS.STRAIN(NECHANICS); \#WVT -67I7 4 AD 649662

K. LNUKKASSFRII., V. X.

ARMY WEAPONS COMNAND

U.S. ARMY WEAPONS COMMAND, WATERVLIET, N.Y.

$2[11,2-67$ PAGES 7 FIGS 6 TABLES 6 REFS

FORCED MOTION OF SHALLOW SPHERICAL SHEIIL, SPHERES STRUCTURAL-SHELLS VIBRATION LOADING(MECHANICS) SHEAR-STRESSES CEFORMATION ROTAT ION INERT IA

MATHEMATICAL-ANALYSIS， *REPORT 21 \&D 653221

R.EI SMANN, H. + R.III KOWSKI, P. M.

STATE UNIV OF NEK YORK

STATE UNIVERSI TY OF NEK YCRK, BUFFALC, N.Y.

$2[/ 11$ 4-67 82 PAGES 79 PAGES 18 FIGS 12 REFS

RF U

AN EXPERIMENTAL INVESTIGATION OF THE YIELD STRENGTH OF A MACHINED RING STIFFENED CYLINDR ICAL SHELL I MODEL BR-7N, UNDER HYORCST ATIC PRESSURE \& ST IFFENED-CYLINDERS TENSILE-PROPERTIES PLASTICITY STRUCTURAL-SHELLS RINGS WELDING STRESSES DEFLEC TION ROLL ING (ME TALLURG Y) ELASTICITY, \#CTMB-I 225 \#AD 650540

LUNCHICK, M. E. + OVERBY, J. A.

NAVY TAYLOR MODEL BASIN

U.S. NAVY, DAVID TAYLOR NCOEL BASIA, WASHINGTON, C.C.

2[III II-58 2I PAGES II FIGS IO RFFS.

RF

.




\section{Thermodynamics}

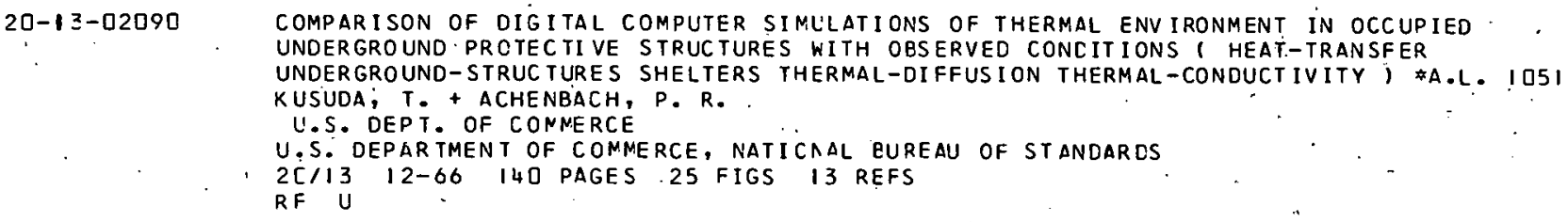

\section{Wave. Propagation}

electromagnetic 'Pllse propagaticn in à cisturbed TerRestrial waveguide- 1 NUCLEAR-EXPLOSIONS I ONOSPHERE ATNCSPHERE-MODELS WAVE-TRANSMISSION LOW-FREQUENCY VERY-LOW-FREQUENC Y EXTREMELY-LCW-FREGUENCY PHASE TRANS FORMAT IONS (MATHEMAT ICS)

ELECTRON-DENSI TY , *IER-22-ITSA-22 \#AD 648002

JOHLER, J. R.

INST ENVIRON RES

INSTITUTES FOR ENVIRONMENTAL RESEARCH, BCULDER, COLO. $2[114 \quad 12-66 \quad 77$ PAGES 57 FIGS 10 REFS

$R F$ U

$20-14-02349$

ELECTROMAGNETIC PLLSE PROPAGATION IN THE NORMAL TERRESTRIAL WAVEGUIDE ENVIRONMENT VUCLEAR-EXPLOSIONS WA VE-TRANSMISSI CN ATMOS PHERE LOW-FREQUENCY PHASE.

VERY-LOW-EREQUENC Y, EXTRENELY-LOW-FREQUENCY INT EGRAL-TRANSFORMS ATMOSPHERE-MODELS TRANSFORMATIONS(MATHEMATICS) , \#IER-2I-ITSA-2I *AC 648 DOI

JOHLER, UJ. $\dot{R}$.

INST ENVIRON RES

INSTITUTES FOR ENVIRONMENTAL RESEARCH, BCULDER, CCLO.

$20114 \quad 12=66 \quad 44$ PAGES 25 FIGS 13 REFS

RF U

HEAT TRANSFER ASPECTS OF THE ATMOSFHERIC RE-ENTRY OF LONG RANGE BALLISTIC MISSILES 1 ATMOSPHERIC-ENTRY REENTRY-VEHICLES DESIGN DERODYNAMIC-HEATING HEATING COOL ING-SYSTEMS FLUID-DYNAMIC-PROPERTIES DRAG SURFACE-TEMPERATURES BOUNDARY-LAYER. LAMINAR-BOLNDARY-LAYER I \#R-273*AC 80305

GAZLEY, C., JR.

RAND

RAND CORPORATION, SANTA MCNICA, CALIF.

22/02, 8-1-54. IDS PAGES 43 FIGS 20 REFS

RF U

SYSTEMS AND PROGRAMS - MANAGEMENT, DEVELOPMENT, TECHNICAL AND SCIENTIFIC. SUPPDRT, PLANNING, STUDIES AND ANALYSES ( CCMMUNICATICN-SDTELLITES COMMUNICATION-EQUIPMENT SATELLITESIARTIFICIAL) SPACECRAFT CEFENSE-SYSTEMS COST-EFFECT IVENESS VULNERABILITY DATA-PROCESSING-SYSTEN.S, \#A.L. 1127

SYSTEM SCIENCES CORP

SYSTEM SCIENCES CCRPORATICN, FALLS CHURCH, VA.

$22 / 0219.67$ 16.PAGES D REFS

RG.U

\section{URBAN AFFAIRS}

\section{Transportation}

STUDIES IN TRA VEL DEMAND ( MODELS (SIMULATIONS) TRANSPORTATION AIR-TRANSPORTA.TION BUS-TRAN SPORTA TION! RAIL-TRANSPORTATION HIGHWAY-TRANSPORTATION, *PB 173499 MATHEMA TICA

MATHEMATICA, PRINCETON, N. J.

$23 / 02 \cdot 10-65$ 191, PAGES 211 REFS.

RF U

$23-02-02092$

KEYSTONE CORRIDOR TRANSPORTATICN STUDY I PENNSYLVANIA HIGF-SPEEO-GROUND-TRANSPORT RESEARCH-PROGRAM-ADMINISTRATICN, A.L. 1062

CARNEGIE INST OF TECH.

CARNEGIE INSTI TUTE OF TECHNOLOGY, PITTSBURGH, PA. $23 / 02 \cdot 7-65$ 5[ PAGES 27 REFS

RF U 
$23-02-02093$

$23-02-02094$

$23-02-02095$

$23-02-[2096$

$23-02-02097$

$23-02-02098$

$23-02-02099$

$23-02-02100$

$23-02-02101$

$23-02-[2352$

$23-02-02353$
B IBL IOGRAPHY OF HIGH SPEED GROUND TRANSPORT I TRANSPORTAT IOV BIBL IOGRAPHIES URBAN-AREAS EXCAVATION, \#PB 170581

MIT

MASSACHUSETTS INSTITUTE OF TECHNCLCGY, CAMBRIDGE, MASS .

$23 / 02$ 10-15-65 78 PAGES 943 REFS

RA U

A SYSTEMS ANALYSI $\subseteq$ OF SHORT HAUL AIR TRANSPORTATICN I PASSENGER-VEHICLES NOR THEAST-CORR IDOR COSTS LRBAN-AREAS MANAGEMENT-PLANNING DES IGN 1 \#PB 169521 MIT

MASSACHUSETTS INSTITUTE OF TECHNOLCGY, CAMBRIDGE, MASS.

$23 / 02$ 8-65.477 PAGES 133 FIGS 67 REFS

RF U

SELECTED IMPAC IS CF RAILRCAD MERGERS ( TRANS PORTAT ION LEGISLATION COMPETITION EMPLOYMENT TAXES SERVICES MANAGENENT-PLANNING COSTS ILLINOIS OHIO INDIANA MICHIGAN 1 *SRI-II-4895

BRANDES, E. M. + BROWN, R. C. + JCAES, P. S.

STANFORD RESEARCH INST

STANFORD RESEARCH INSTITUTE, NENLC PARK, CALIF.

$2 \Xi 102$ 5-64 2C3 PAGES 23 TABLES 6 FIGS

RF U

UNIVERSAL DRAG LAh I TRANSPORTATICA PRCPULSICN HIGH-SPEED-GROUND-TRANSPJRT PERFORMANCE (ENGINEERING) VEHICLES CESIGN, *LSK-76108-2 *PE 173647 GOUSE, S. W. JR. + SWARDEN, N.

MIT

MASSACHUSETTS INSTITUTE OF TECHNCLCGY, CAMBRIDGE, MASS.

23/02 12-6-66 66 PAGES 21 FIGS 67 REFS

R F U

MOVING SIDEWALKS AND THE PROBLEM CF SHCRT RANGE MASS TRANSPORTATION I URBAN-AREAS MOVING-BELTWAYS TRAFFIC DESIGN COSTS NEW-YORK CHICAGO WASHINGTON PHILADELPHIA BOSTON LONOON, *HI-82O-D

AYRES, R. L. + KRLPKA, R. A.

HIJDSON INSTITITF

HUDSON INSTITUTE, CROTCN-CN-HUDSON, N.Y.

$23 / 02196764$ PAGES 27 FIGS 3 REFS

RF $U$

EXPRESSWAY PLANS I TRANSPCRTATION URBAN-AREAS NEW-YORK MANAGEMENT-PLANNING URBAN-PLANNING, $\&$ RPN-73-74

REGIONAL PLAN ASSN

REGIONAL PLAN ASSCCIATION, NEW YORK, N.Y.

$23 / \mathrm{2} 25-6419$ PAGES 8 REFS

JE U

RAIL TRANSIT PLANS I TRANSPORTATION NEW-YORK URBAN-AREAS PCPULATION RAILROADS

SLBBWYS COSTS MANAGEMENT-PLANNI NG URBAN-PLANNING, \#RPN-71-7.2

REG IONAL PLAN ASSN

REGIONAL PLAN ASSCCIATION, NEW YORK, N.Y.

$23 / 02$ 12-63 15 PAGES I3 REFS

$J G U$

HIGH SPEED RAILROADS I TRANSPORTATION URBANURBAN-PLANNING WASHINGTON BOSTON NEW-YCRK I \$RPN-79

REGIONAL PLAN ASSN

REGIONAL PLAN ASSCCIATION, NEW YORK, N.Y.

$2 \Xi / 02$ 8-65 IS PAGES O REFS

JE U

AERODYNAMIC DRAG CN VEHICLES IN ENCLOSED GUI CEWAYS ( TUNNELS(TRANSPORTATION) TRAN SPORTATION URBAN-AREAS MODELS (SIMULAT IONS) PASSENGER-VEHICLES LAMINAR-FLOW TLRBULENT-FLOW, *DSR-76108-1 $\$ P 8-173646$

GROUSE, S. W., JR. + NhUDE, J.

MIT

MASSACHUSETTS INSTITUTE OF TECHNCLCGY, CAMERIDGE, MASS .

$23 / 02 \quad 12-66$ 5\% PAGES 21 FIGS 19 KLFS

RF U

NEW TRANSPORT TECHNOLOGY FCR THE BIG CITY I URBAN-AREAS TRANSPORTATION COSTS HIGH-SPEED-GROUND - TRANSPORT, \#A.L. 1211

ROSS, H. R.

STANFORD RESEARCH INST

STANFORD RESEARCH INSTITUTE, NENLO PARK, CALIF.

$23 / 021-23-675$ PAGES 5 FIGS 3 TABLES

$J R$ U

NATIONAL CAPITAL TRANSPORTATICN AGENCY RAPID TRANSIT SYSTEM - MANUAL OF DESIGN

CR, ITER,IA I IJRBAN-ARFACI $\$ \Delta .1 .1193$

DE LEUH CATIIER

DE LEUW, CATHER AND COMPANY, WASHINGTCN, D.C.

$2 \geq 102$ 211 PAGES 36 FIGS

RO U 
SLMMARY OF RE SEARCH AT MIT ON HIGH SPEED GROUND TRANSPORT. 1 TRANSPORTATION NETWORKS TERMINALS 'SCHEDUL ING. SWI TCHING PRO'FULSION-SYSTEMS AERODYNAMICS DRAG I *PB I73 658 SEIFERT, W. W. + HANSEN, R. J. MI T

MASSACHUSETTS INSTITUTE CF TECHNOLCGY, CAMBRIDGE, MASSS. $23 / 02 \quad 12-66 \quad 46$ PAGES 18 FIGS

INTER IM REPORT. ON TRANSFER SYSTEN AND FARE STRUCTURE FOR TIE OFFICE OF TRANSPORTATION ADNINISTRATION, OFFICE OF THE MAYOR OF THE CITY OF NEW YORK AND FOR NEW YORK CITY IRANSIT AUTHORITY ( L'REAN-AREAS, \#A.L. II56 EBS MANAGEMENT CCN

EBS MANAGEMENT CONSULTANTS, INC., NEW YORK, N.Y.

$23 / 02 \cdot 1-67$ 131 PAGES I 5 FIGS 133 REFS

RU $U$

$23-02-02356$

$23-02-02357$

INNOVATIONS'IN TRANSIT DEVICES I RAPID-TRANSIT-SYSTEMS TRANSPORTATION URBAN-AREAS RAILROADS MONORAILS MOVI NG-BELTWAYS HISTCRY , *A.L. 1129

GOULD, J.H.

KAISER

KAISER ENGINEERS, OAKLAND, CALIF.

$22102 \quad 2-67 \quad 34$ PAGES 30 FIGS

RG U

TRANSPORTATION AND RELATED PROBLENS CF THE DCWNTOWN MANHATTAN AREA BETWEEN THE HOLLAND TUNNEL AND THE MANHATTAN AND WILLIAMSBURG ERICGES AND ALTERNATIVE SOLUTIONS THEREFORE I LRBAN-AREAS NEW-YORK ) *A.L. II5S

EBS MANAGEMENT CCN

EBS MANAGEMENT CONSULTANTS, INC., NEW YORK, N.Y.

$23 / 02 \cdot 1966$ 3[ PAGES 6 FIGS $]$ REFS

$\mathrm{RT} \cdot \mathrm{U}$

\section{Urban Renewal}

$23-03-02102$

SCIENCE AND THE CITY ( URBAN-AREAS PUBLIC-HEALTH URBAN-PLANNING POPULATION

UTILITY-TUNNELS, $\$$ HUD-MP-39

TORREY, $V$.

U.S. DEPT. HOL'S LR DEVEL

U.S. DEPARTMENT OF HOUSING AND URBAN DEVELOPNENT

$23 / 03$ 1967. 43 PAGES II FIGS

RG U

\section{04 Demography}

$23-04-02103$

A STUDY OF THE DENOGRAPHY OF NUCLEAR WAR - EMPIRICAL GUICELINES FOR THE SELECTION OF DEMOGRAPHIC VARIABLES AND AREAL UNITS FOR STUCYING POSTATTACK SOCIETY I POPULATION DISTRIBUTION URBAN-AREAS SURVIVAL AGE POSTATTACK-OPERATIONS

URBAN-PLANNING ME IHODOLOGY SEX) \#HSR-RR-66/14-PR-APP-I

HADOEN, J. K.. + BCRGATTA; E. F.

HLMAN SC IENCES RESEARCH

HUMAN SC IENCES RE SEARCH, INC., MC.I.FAN, VA.

$23 / 04$ 5-6E IL6 PAGES 67 REFS

RF U

$23-04-02104$

A STUDY OF THE DENOGRAPHY OF NUCLEAR WAR I SURVIVAL NUCLEAR-WARFARE POPULATION METHODOL OGY MANAGEMENT-PLANNING POSTATTACK-OPERAT ICNS SEX AGE URBAN-AREAS

DECIISION-MAKING, $\$ H S R-R R-66 / 14-P R$

PENDLETON, h. h.

HUMAN SC I ENCES RE SEARCH

HUMAN SCIENCES RE SEARCH, INC., MCLEAN, VA.

$23 / 04$ 5- 66 241 PAGES 16 FIGS 27 TABLES IDS REFS.

RF U

$23-04-02358$

POPULATION ESTIMATES - PRCJECTIONS OF THE POPULATICN OF THE UNITED STATES BY AGE, SEX, AND COLOR TO 1990 WITH EXTENSIONS OF TOTAL PCPULATION TO 20151 DEMOGRAPHY TABLES, $P-25-359$

U'.S. DEPT. OF CONNERCE

U.S. DEPARTMENT OF COMMERCE, BUREAU OF THE CENSUS

$23 / 04$ 2-2C-67 22 PAGES 6 TABLES 7 REFS

RD U 


\section{Urban Planning}

$23-06-02105$

$23-0 t-02106$

$23-06-02107$
PROCEEDINGS OF THE MID AMERICA CCNFERENCE CN URBAN CES IGN I UREAN-AREAS URBAN-PLANNING URBAN-RENE hAL NANAGENENT-PLANNING INTERGOVERNMENTAL-RELATIONS, * A.L. IOOC

MIDWEST RESEARCH INST

MIDWEST RESEARCH INSTI TUTE, KANSAS CITY, MO.

$23 / 06$ 3-66 IIS PAGES 9 FIGS 0 REFS

RS U

IMPLEMENTATION OF AN ORDERED SPRAWL URBAN CONFIGURATION 1 URBAN-AREAS

DECENTRALIZATION TAXES VULNERABILITY.URBAN-PLANNING LANC-USE POPULATION

TRANSPOR TATION UTILITIES FISCAL-AND-NONETARY ECONCNICS, \$PRC C-I219

BLUNT, K. R. + LIFTER, J. J. + VANCAAM, P.

PLANNING RESEARCH CORP

PLANNING RESEARCH CORPORATION, LOS ANGELES, CALIF.

$23 / 06^{\circ} 1-16-67^{\circ} 221$ PAGES 69 REFS

RF U

URBAN FORM AS A PASSIVE DEFENSE VARIABLE I ECONOMICS URBAN-AREAS DECENTRALIZATION VULNERAB ILITY LAND-USE URBAN-PLANNING POPULATION POLITICAL-SCIENCE, PLANNING RE SEARCH CORP

PLANNING RESEARCH CORPORATION, LOS ANGELES, CALI-F.

$23 / 06$ 1-16-67 4[ PAGES O REFS

$R \mathrm{~F} U$ 


\section{Key Word Index}

IVIII. IMMUNOLOGICAL RESPONSE OF NAN TO PURIFIED BIVALENT AR BOTULINUM TOXOID 1 BIOLOGICAL-WARFARE-AGENTS/ IS-0?-UIB5T ITIC-PROPERTIES FILMS FLUID-MECHANICS SCIENTIFIC-RESEARCH ABLATION ANTENNAS PROPAGATION SHOCK-WAVES KINET/ 20 -D3-D2330 $S$ I ENVIRONMENTAL-CONTROL/ NATURAL VENTILATION TEST OF AN ABCVEGROUND FALLOUT SHELTER IV EVANSTON, ILLINOI I3-DI -OI77I IFOR CONDENSED STATE DIFFLSION CONTROLLEO FI SSICN FRODUCT ABSORPTICN OJRING FALLOUT FORYATIOV I RADIOACTI I IB-O8-O2O37 IPIERE OXYGEY GAS-IONIZATION, AIRBLRST I ONS POLAR-PEGIONS ABSORPT ICN OZ ONE REACTION-KIVETICS NUELEAR-RADI/ O4-DI -O2I I 3 MEDIC INE BIOLOGY CHINA, \#J' COMMLAIST CHINESE SCIENTIFIC ABSTRACTS - BIOMEOICAL SCIENCE I BIBLIOGRAPHIES O6-05 -0I7O4 /1960-1965 - AN AUTHOR INDEX OF THE OPEN LITERATURE HITH ABSTRACTS 1 BIBLIOGRAPHIES INDEXES I *RM-2BDQ- I/ O5-03-0I652 I JULY-1, 1965-JUNE-30, 1966 I TECHNICAL-INFORMATICN-CENTERS ABSTRACTS COSTS CEPARTMENT-OF-DEFEVSE DATA-PROC I O5-02-O2I25 IST SCIEVTIFIC AND TECHVICAL LITERATURE I GUIDED-MISSILES ABSTRACTS SPACECRAFT TRANSPORTATIJV LAUNCHING-SI I6-OI -QI973 1 CURRENT SOCIAL SCIENCE RESEARCH BY PRI VATE SCHOLARS ANC ACADEMIC CENTERS - AFRICA I ECONOMICS BIBLIOGRAI O5-II -0I692 I CURRENT SOCIAL SCIENCE RESEARCH BY PRIVATE SCHOLARS AND ACADEMIC CENTERS - AMERICAN REPUBLICS I BIBLIOG/ O5-II-OIO96 1 CURRENT SOCIAL SCIENCE RESEARCH BY PRIVATE SCHOLARS AND ACADEMIC CENTERS - ASIA I BIBI IOGRAPHIES CHINA, OS-II-OIOQ8 1 CURRENT SOCIAL SC IENCE RESEARCH BY PRIVATE SCHCLARS ANC ACADEMIC CENTERS - INTERNATIOYAL AFFAIRS I BIBL, O5-II -DI693 1 CURRENT SOCIAL SCIENCE RESEARCH BY PRIVATE SCHOLARS ANC AC DDEMIC CENTERS - MIODLE EASTI BIBLIOGRAPHIESI OS- 11 -DIG95 / CURRENT SOCIAL SCIENCE RESEARCH BY PRIVATE SCHOLARS AND ACADEMIC CENTERS - USSR AND EASTERN EUROPE I BI/ Q5-II -0I69? / CURRENT SOCIAL SCIENCE RESEARCH BY PRIVAIE SCHOLARS AND ACADEMIC CENTERS - WESTERN EUROPE, GREAT BRITAI/ OS-1I-OIG94 EXPER IMEVTAL-DATA THEORY MATHEMA TICAL-MODELS DISPLACEMENT ACCELERAT ION DECELERAT ION, \#DASA-I859 /EFFEC TS O6-2I-D2239 ST ICITY VELOCITY DEGREES-OF-FREED ON. MATHENATICAL-AMALYSIS ACCELERAT ION DISPLACEMENT, \#UCRL-729I, /TION ELA $20-11-02342$ I SALMON EVENT I SEISMIC-WAVES EXPLOSI CN-EFFECTS VELOCITY ACCELERATION MOTION PROPAGATION EARTHQUAKES I *I I8-D3-O23I9 OELASTICITY MECHANICAL-WAVES PROPAGATION NATHEMATICAL-AN/ ACCELERATION WAVES IN NONLINEAR MATERIALS I VISC 20-II-D2OBO CKNESS STRESSIPHYSIOLOGY) ADAPTATION(PHYSIOLOGY) TLMBLING ACCELERATION-TOLERANCE 1 *AC 9469711 MOTION-SI D6-19-0I735 THEMA/ THE SHOCK AND VIBRATION BLLLETIN I INSTRUNENTATICN ACCELEROMETERS SPECTRUM-AVALYZERS TRAVSDUCERS MA $20-1 I$-D2O75 RATIONS-RESEARCH CIVIL-DEFENSE-5YSTEMS ATTIT/ RESEARCH ON ACCEPTAEILITY OF A NATIONAL SHELTER POLICY I OPE O5-O $4-02166$ WITH ADJUNCTS I FOODIACCEPIABILITY) FALLOUT-SHELTERS CON/ ACCEPTABILITY OF SHELTER RATIONS IN COMBINATION DO-OB-OI7ID HIIH ADJUNCTS FOOD FALLOLI-SHEL IERS TASTE CIVIL-CEFENS/ ACCEPT AEILITY OF SHELTER RATIONS IV COMBINATION D6-OB-O22II DEFENSE-SI SHEL TER ASSIGNMENT CONCEPT - STUDY IN PUBLIC ACCEPI ANCE I SHELTER-ALLOCATION ATTITUDES CIVIL- O5-ID-O2I77 NSING, DISTRIBUTION DAMAI POSTATTACK FOOD AVAILABILITY AND ACCESSIBILITY I POSTATTACK-OPERATIOVS FOOD-DISPE DO-OB-DI7II EAPONS-EFFECTS, \#NREC-25 TINE PHASED ACCESSIBILITY OF RESOURCES I DAMAGE-ASSESSMENT W O5-D3-D2I S6 DIOACT/ A STUDY OF THE POSSIBLE CCNSE QUENCES AND CCSTS OF ACCIDENTS IN THE TRANSPORTATION OF HIGH LEVEL RA I8-OT-02034 FOR CLASS A AND B WATER UTILITIES I ECONONICS REGULATIONS ACCOUNTING I \#A.L. IOD3, $10 R M$ SYSTEM OF ACCOUNTS O5-D3-DIO5D

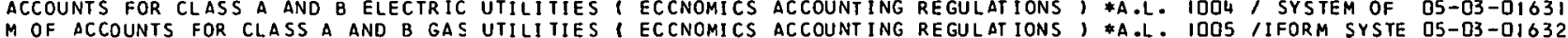
ECONOMICS ACCOUNTING REGULATIONS, \#A.I UNIFORN SYSTEM OF ACCOUNTS FOR CLASS A AVD B ELECTRIL UTILITIES I OS-03-DI63I MICS ACCOUNTING REGULATIONS, \#A.L. IO/ UNIFORM SYSTEM OF ACCOUNTS FOR CLASS A AND B GAS UTILITIES I ECONO OS-O3-DIO32 NOMICS REGULATIONS ACCOUNTING, \$A.L. / UNIFORM SYSTEM OF ACCOUNTS FOR CLASS A AND B WATER UTILITIES I ECO OS-Q3-DIGSD IART I ALLY SATURATED SAVD - CLAY MIXTURES I SOIL-MECHANICS ACCOUSTICS MOISTURE MECHAVICAL-WAVES CIVIL-ENGI/ $20-0 I-02 D 47$

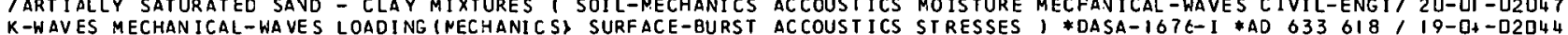

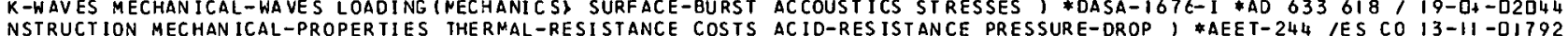
NSTRUCTION MECHANICAL-PROPERTIES THERPAL-RESISTANCE COSTS ACID-RESISTANCE PRESSURE-DROP \$AEET-244 /ES CO I3-II-DI792 IT ON ATTENUATION AND DISPERSION CF SOUND IN AN AEROSOL
IERACTION BETWEEN AIR FLOW AND AIRBORNE SOUND IN A DUCT I ACCUSTICS GAS-FLOW WEST-GERMAVY DUCTS LAMINAR-B/ $20-02$-OIBOL I EEAMSI STRUCTURAL) SHOCK (MECHANICS) LAUNCHING (SI MULATICN) ACCUSTICS VIBRATION-ISOLATORS, *BULLETIN 35 (3) 20-1I-02074 OSS SECTIONS, AND GENERAL LOCATIOA OF RIGHTS OF WAY TO BE ACQUIREC ( CIVIL-ENGINEERIVG FLODDS I *A.L. 972 I3-0?-OI78I OSS SECTIONS, AND GENERAL LOCATION OF RIGHTS OF WAY TO BE ACGUIREC I FLOODS CALIFORNIA I \$A.L. 987 /ES, CR I3-02-OI779 IHY ON SUBJECTS RELATED TO ACTIVE VIBRATION I SOLATION AND ACTIVE VEHICLE SUSPENSIONS I VIBRATION-ISOLATOR/ 20-II-O2OB7 SPENSIONS $1 /$ PARTIAI BIRI IOGRAPHY ON SUBJECTS RELPTEO TC ACTIVE VIBRATION ISOLATIOV AVD ACTIVE VEHICLE SU $20-1 I-02087$ IOL ER ANCES(PHYSIOLOGY) MOTION-SICKNESS STRESS (PHYSICLOGY) ADAPTAT ICN(PHYS IOLOGY) TUMBLIVG ACCELERATION-TOI O6-I I-OI735 ENS/ ACCEPTABILITY OF SHELTER RATICNS IN COMBINATICN WITH ADJUNCTS I FOOD FALLOUT-SHELTERS TASTE CIVIL-DEF D6-O3-D22II CON/ ACCEPTABILITY OF SHELTER RATIONS IN COMBINATICN WITH ADJUNCTS I FOOD(ACCEPTABILITY) FALLOUT-SHELTERS D6-OB-DI7ID ION DUAL-PURPOSE-SHEL TER S FALLOUT-SHELTERS APPROPRIATIONS ADMINISTRATION-AND-MANAGEMENT I *MP-46 /RGANIZAT O5-OI-OI 625

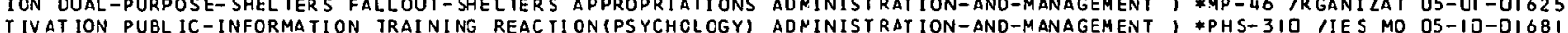
- EMER GENCY HEAL TH SER VICES I DI ASTER-NEDICINE MEDICINE ACNINISTRATION-AND-MANAGEMENT FIRST-AID ORGANI I DS-2I - I738 7- EMERGENCY HEAL TH SERVICES T OI SASTER-NEDICINE MEDICINE ACMINISIRATION-AND-MANAGEMENT FIRST-AID ORGANI ZI D6-2I-DI738 / COMPENSATION LAWS 1 LEGI SLATION LABOR STATE-GOVERNMENTS ADMINISTRATION-AND-MANAGEMENT INSURANCE \#USDLI DS-OP-O2I74 /-MF. ICINF. EMER GENCY-HEAL TH-SERVICES HOSPI TALS CASUALTIES ADMINISTRAT ION-AND-MANAGEMENT MEDICAL-SUPPLIES I D6-2I - I 739

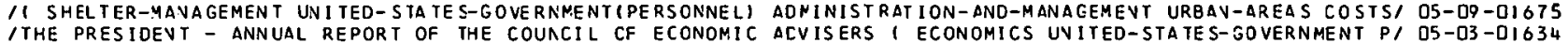
$Y$ I BIOLOGICAL-BLAST-EFFECTS BLAST-BIOLOGY SHOCK-TL/ DASA AEC LOVELACE FOUNDATION BLAST SIMULATION FACILIT O6-2I -O2232 MICH. I GAN ATTACK-SCENARIOS AIRCRAF T NUCLEAR-W/ PRELININARY AERIAL INFRARED FIRE MAPPING SYSTEMS ANALYSIS I I3-I?-D2267 I HE CONFLAGRATION POTENTIAL IN SAN JOSE AND ALBUQUERQUE 1 AERIAL-PHOTOGRAPFS URBAN-AREAS FIRES MAPS TEST-I I3-I?-QIBO9 IATION OF MICRORIAL. PARTICLES I BIOLOGICAL-WARFARE-AGENTS AEROBIOLOGY BACTERIA GAS-FILTERS MICROORGANISMSI IS-D2-OIB5I YS I TUNNELSITRANSPORTATION) TRANSPORTATION URBAN-AREAS I AEROOYNAMIC DRAG ON VEHICLES IN EVCLOSED GUIDEWA $23-02-D 2 I U 1$ ITIC MISSILES I A TMOSPHERIC-ENTRY REENTRY-VEHICLES DESIGN AEROOYNAMIC-HEAT ING HEATING CJOLING-SYSTEMS FLU/ 22-OZ-O235O ETWORKS TERMINALS SCHEDUL ING SHITCHING PRCPULSION-SYSTEMS AERODYNAMICS DRAG 1 \$PB I73 658 12ANSPORTATION N $23-02-02354$ ACT IVE-FALOUT DISTRIBUTION DOSE-RAI SEDAN PROJECT 62.80C AERORADIOACTIVITY SURVEY I WEAPONS-EFFECTS RADIO I8-O3-D2324 ACT IINAL REPORT ON ATTENUATION ANO DISPERSI ON OF SOUNO IN AN AEROSOL ACOUSTIC-PROPERTIES GAS-FLOW PROPAGATI IS-O2-OI88 URELL TULARENSIS FOR MACACA MLLA ITA AND NAN TI EFFECT CF AEROSOL AGE ON THE INFECTIVITY OF AIRBORNE PASTE I5-D2-DI858 IAL STUDIES ON RHESUS MONKEYS EXPCSED TO A SNALL PDRTICLE AEROSOL OF THE BORG STRAIV I BIOLOGILAL-WARFARE/ IS-O2-DI85O STRAIN I BIOLOGICAL-WARFARE-AGENTS MIYAGAWANELLA-PSITTACI AEROSOLS I *AD 636 8I4 /CLE AEROSOL OF THE BORG $15-02-01850$ /S I TURBULENCE FLUID-DYNAMICS PARTICLE-SI ZE-DISTRIBUTI ON AEROSOLS REYNOLDS-NUMBER BROWVIAN-MOVEMENT EQUAI 20-O4-O2333
LIC-OPINION SURVEYS FOREIGN/ OCSA SIONAL REPORT ON FOREIGN AFFAIRS - AMERICAN PROGRAMS OF FOZEIGN AID I PUB O5-II -DI GB7 GN-POLICY QUEST IONNAIRES I/ OCCASIONAL REPCRTS ON FOREIGN AFFAIRS - GERMANY I PUBLIC-OPINIOV SUR VEYS FOREI DS-II -UZIBO PUBL IC-OPINION SLRVEYS FOR/ OCCASIONAL REPORT ON FOREIGN AFFAIRS - UNITED NATIONS I INTERNATIONAL-AFFAIRS O5-II -02I 98 SURVEYS QUESTIONNAIRES PUB/ OCCASIONAL REPORT ON FOREIGN AFFAIRS - UNITED NATIONS I INTERNATIONAL-AFFAIRS DS-II - D2I 99 IICARITY IS THE BANNER - TWO RESOLLTIONS GN INTERNATIONAL AFFAIRS I ARMS-CONTROL COLD-WAR NATO VIETNAM RE / O5-D4-02I 72 /BY PRIVATE SCHOLARS AND ACADENIC CENTERS - INTERNATIONAL AFFAIRS 1 BIBL IOGRAPHIES ATOMIC-EVERGY COMMUNIC/ O5-II -DI693 USSR I RADIOL/ PATHOLOGY, CLINICAL PICTURE AND THERAPY IN AFFECTIONS WITH TOXIC AND RADIOLOGICAL AGENTS - DG-IS-DI72I IENCE RESEARCH BY PRIVATE SCHOLARS AND ACADEMIC CENTERS - AFRICA I ECONOMICS BIBLIOGRAPHIES EDUCATION FORI OS-II-OIG92 IENCE RESEARCH BY PRIVATE SCHOLARS AND ACADEMIC CENTERS - AFRICA I ECONOMICS BIBLIOGRAPHIES EDUCATION FOR/ OS-II-OI692
IFOOC AND AGR ICUL TURE 1966 (ELROPE FOOD-MARKETING RUSSIA AFRICA LATIN-AMERICA JAPAN GRJSS-VATIONAL-PRODU/ O2-OZ-OIOI6 
IND/ REGIONAL ISM IN ASIA I ECONOMIC-CONDITIONS POPULATION AGRICULTURE POL ITICAL-SCIEVCE REGIOVAL-PLANNING O5-O3-O2IGI LUT FOOD CONTAMINAT/ UPTAKE OF RADIONUCLIDES BY PLANTS। AGRICULTURE RADIOACTIVE-ISOTOPES RADIOACTIVE-FAL D6-IB-OI725 IOUSE RECEIPT WHEAT INVENTORY BY hAREHOUSE AND LOCATION 1 AGRICULTURE STORAGE SUPPLY-DEPOTS WAREHOUSES STI O2-02-O2IOQ T RE/ HANOBOOK OF AGR ICULTLRAL CHARTS - 1966 I POPULATION AGRICULTURE URBAN-AREAS RURAL-AREAS COMMERCE MEA O2-D? -DIOI9 AFR ICA LATIN-AMER ICA JAPAN GROSS-N/ THE STATE OF FCOD AND AGRICULTURE 1966 I EUROPE FOOD-MARKETING RUSSIA O2-02 -0IOIO IW ELECTR IC. INC INERATOR DESIGN FOR STERILIZING LABCRATORY AIR 1 BIOLOGICAL-WARFARE-AGENTS SPORES MEDICAL-1 I5-O2-OI892 IT WAVE PARAMETER AND THE RE SPONSE OF MAMMALS EXPCSED TO AIR BLAST B BLAST-BIOLOGY BIOLOGICAL-BLAST-EFFE I OG-21-D2234 IE EFFECTS OF AMBIENT PRESSURE ON TOLERANCE OF MANRALS TC AIR BLAST 1 BLAST-BIOLOGY SHOCK-WAVES BIOLOGICA I O6-2I-D2236 IECTS I. GROUND MOTION IN ELASTIC HALF SPACES INOUCED BY AIR BLAST 1 ELASTICITY SHJCK-WAVES MECHANICAL-W/ 19-0+-02044 ICABLE IN ASSESS ING RESPONSES OF THE THORAX ENERGIZED BY AIR BLAST OVERPRESSURES OR BY NON-PENETRATING M/ D6-2I -D2235 I BLAST-BIOLOGY BIOLOGICAL-BLAST-EFFECTS SHOCK-TUBES LUN/ AIR BLAST STUDIES WITH EIGHT SPECIES OF MAMMALS OG-2I-O2230 1 BLAST-BIOLOGY B IOLOGICAL-BLAST-EFFEC TS SHOCK-TUBES LUN/ AIR BLAST STUDIES WITH EIGHT SPECIES OF MAMMALS O6-2I-D2230 OUP OF POXVIRUSES FOR THE CYNOMOLGLS MONKEY, MACACA IRUS, AIR BORNE INFECT IVITY OF THE VAR IJLA VACCINIA GR I5-02-DI855 1 AND 208-VOLT, 3-PHA SE, 4CD-C YCLE I MODEL CEIIVAL4-2J8 I AIR CONCIT ION ING UVITS I AIR-CONDITIOVING-EQUIP' I3-OI -0I775

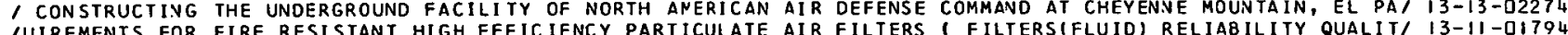
IUIREMENTS FOR FIRE RESISTANT HIGH EFFICIENCY PARTICULATE AIR FILTERS I FILTERSIFLUID) RELIABILITY QUALIT/ I3- II -OI794 L-PROPERT IES CONSTRUCTION MECHANIC/ DEVELOPMENT OF U-FOAN AIR FILTERS I VENTILATION FILTERSIFLUIDI CHEMICA I3-II-DI792 AL-WARFARE-AGENTS AEROBIOLOGY BAC IERIA GAS-FILTERS NICRC/ AIR FILTRATION OF YICROBIAL PARTICLES I BIOLOGIC IS-O2-OI85I S GAS-FLOW WEST-GERMANY DUCTS LAMINA' INTERACTION PETWEEN AIR FLOW AND AIREORNE SOUND IV A OUCT I ACOUSTIC 2O-OI-O2OL8 IICAL-MON I TOR ING RADIOACTIVE-FALLOUT RADI OACTIVE-ISOTOPES AIR MILK ATMOSPHER IC-PRECIPITATION WATER-SUPPLI/ I8-O3-O2035 NTAL-CATA PRESSURE-GAGES, *ORNL-IR-I/ THE PROPAGATION OF AIR SHOCK WAVES IN A TUBE 1 SHOCK-TUBES EXPERIME 2 ID-O4-O2OS3 1 *REPORT NO. IO' NOMOGRAM FOR CALCULATING ATTENUATICN OF AIR SHOCK WAVES IV TUNYELS 1 PROPAGATION SHEDEN $20-0+$-O2OSI IFIRE-SAFETY TOXICITY HEAT CARBON-NONOXIDE CARBON-CIOXIDE AIR SMOKES WIND SHELTERS GERMANY MICE I \#NROL T/ I3-I?-OIBOD IHEAT MOISTURE TEMPERATURE ENVIROAMENTAL-CONTROL HUMIDITY AIR TOLERANCES (PHYSIOLOGY) THERMODYNAMICS ) \$A.' I3-OI -O2253 T-CORRIDOR COSTS URBAN-1 A SYSTEMS ANALYSIS OF SHORT HAUL AIR TRANSPORTAT ION I PASSENGER-VEHICLES NORTHEAS $23-02-02094$ RECOVERY ECONOMIC-RECOVERY PRODLCTS BLAST VULNERABILITY AIR-BURST ECONOMICS EXPLOSION-EFFECTS POSTATTACI O5-03-D2I26 TREOVERY ECONOMIC-RECOVERY PRODCCTS BLASY SULNERABILITY AIR-BURST ECONOMICS EXPLOSION-EFFECTS POSTATTAC IIVE FALLOUT DECAY-SCHEMES USSR PAR TICLES SURFACE-BURST AIR-BURST EQUAT IONS RADIOACTIVE-ISOTJPES THEORYI I8-O3-O232 IND-CONTROL-SYSTEMS BIBLIOGRAPHIES DESIGN HARDENING BLAST AIR-BURST VIBRATION-ISOLATORS SHOCK-WAVES SHOCKI I7-02-D23I 1 FIELD FORTIFICATIONS I MATHE MATICAL-PREDICTICN SHELTERS AIR-BURST VULNERABILITY SOIL-MECHANICS WEATHERI I5-OS- II95 IPLICATIONS ON UNCERTAINTIES IN THE HIROSHIMA EXPLCSION I AIR-BURST YIELD WINO NUCLEAR-WARFARE-CASUALTIES/ 15 -O6-DI958 1OD-CYCLE ( MODEL CEIIVAL4-2C8, AIR CONDI TI ONING UNITS I AIR-CONCIT ION ING-EQUIPMENT PREFABRICATED-BUILOI/ I3-CI-OI775 /AGENCIES) LEGI SLATION CIVIL-DEFEN SE (GOVERNMENT-AGENCIESI AIR-FORCE ARMY NAVY GOVERVMEVT-AGEVCIES I \#A.L.' I5-O3 -DI946 RE-AGENTS AEROB IOLOGY BACTERIA GAS-FILTERS MICROORGANISNS AIR-POLLUTION FILIERS I \#AD 637 407 /GICAL-WARFA I5-O2-OI85I

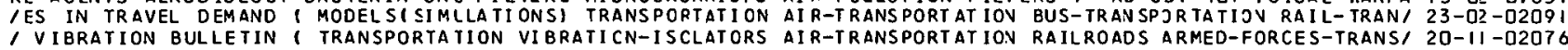
MATHEMAT ICAL-PREDICTION SHELTERS AIR-BURST/ A SUNNARY OF AIRBLAST EFFECTS ON HASTY FIELD FORTIFICATIONS I IS-DG-OI957 CALCULAT IONS MOOELS, \# I MECHANI SMS IN THE GENERATICN OF AIRBLASTS BY UNCERHATER EXPLOS IONS 1 SHOCK-WAVES I9-OH-O2326 ANC MEASURING / PROJECT FIRE SCAN - THE EVALUATICN OF AN AIRBORNE INFRAREC MAPPER AS A TOOL FOR DE TECTING I3-I?-DI798

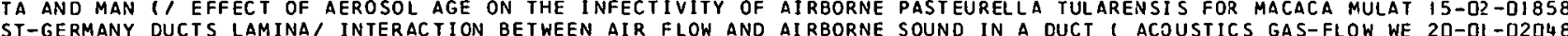
IREGION RECOMBINATION I IONOSPHERE OXYGEN GAS-IONIZATION, AIRBURST IONS POLAR-REGIONS ABSORPTION OZONE REI OL-OI -O2II IONOM IC-RECOVERY PRODUCTS VULNERABILI

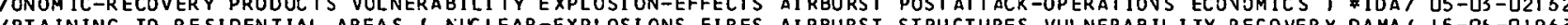
/RTAINING TO RESIDENTIAL AREAS I NLCLEAR-EXPLOSIONS FIRES AIRBURST STRUCTURES VULNERABILITY RECOVERY DAMA IS-OS-OI963

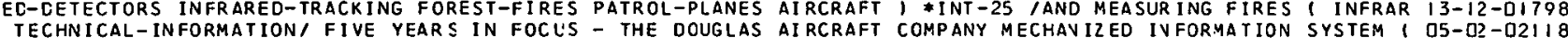

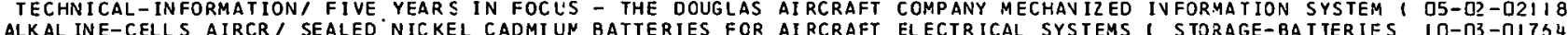
ALKAL INE-CELLS AIRCR / SEALED NICKEL CADMIUN BATTERIES FOR AIRCRAFT ELECTRICAL SYSTEMS I STORAGE-BATTERIES IO-O3-OI7S4
IIRE MAPP ING SYSTEMS ANALYSIS I MICHIGAN ATTACK-SCENARI OS AIRCRAFT NUCLEAR-WEAPONS-EFFECTS MILITARY-INTEL/ I3-I?-02267 IRE MAPP ING SYSTEMS ANALYSIS 1 MICHIGAN ATTACK-SCENARI OS AIRCRAFT NUCLEAR-WEAPONS-EFFECTS MILI TARY-INTELI I3-I? -02267
/-OPERATIONS VULNERABILIYY ROADS RAILROADS CARGO-VEHICLES AIRCRAFT WEAPONS-EFFECTS DAMAGE-ASSESSMENT TRUC, I3-OS-O226D IFT ELECTRICAL SYSTEMS I STORAGE-BATTERIES ALKALINE-CELLS AI RCRAFT-EQUIPMENT-POWER-SUPPLIES, \$AFAPL-CONF, IO-D3-OI7GL I COCUMENTATION IN FORMATION-RE TRIE VAL AUTONATICN LI ERARIES AIRCRAFT-INCUSTRY SYSTEMS-ENGINEERIVG DATA-STOR/ O5-02 - Q2II 8 CS OF DISASTER I DAMAGE-ASSESSMEN D DATA DECISICN-MAKI THE ALASKAN EARTHQUAKE - A CASE STUDY IN THE ECONOMI O5-O3-OIG36 ITION UNDERGROUND-STRUCTURES DOSE-RATE PHOTCNS SCATTERING ALBEDO GAMMA-RAYS ANGLE-OF-INCIDENCE RADIATIONI/ I8-0S-02029 ES MAPS TEST/ THE CONFLAGRATION PCTENTIAL IN SAN JCSE AND AL BUOUERQUE I AERIAL-PHOTOGRAPHS URBAV-AREAS FIR I3-I2-DIBO /RIES FOR AIRCRAFT ELECTRICAL SYSTEMS I STORAGE-BATTERIES ALKALINE-CELLS AIRCRAFT-EQUIPYENT-POWER-SUPPLIE, IO-D3-OI 76L IRING EXPLOSION-EFFECTS NUCLEAR-EXPLOSIONS EQUATIONS TUFF ALLUVIUM SALT GRAVITE MATHEMATICAL-AVALYSIS, * I8-O3-D23I5 IWER ELECTRIC-POWER-TRANSMISSION IRANSMISSION-LINES COSTS ALTERNATE-CURRENT ECONOMICS DIRECT-CURREN T STATI DQ-D3-OZ2Z SHEL ELERRIC SHELTER PROGRAM T FALLOUT-SHE LTERS FEDERAL-BUDGETS COST/ ALTERNAT IVE APPROACHES TO FINANCING A NATIONWIDE I3-I3-01834
ALIFORNIA - FEATHER RIVER AND DEL TA DIV/ INVESTIGATION OF ALTERNATIVE AQUECUCT SYSTEMS TO SERVE SOUTHERN C I3-O2-O2258

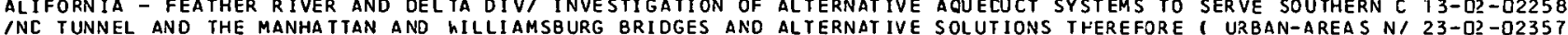
INC TUNNEL AND THE MANHA TIAN AND hILLIAMSBURG BRIDGES AND ALTERNAT IVE SOLUTIONS THEREFORE I URBAN-AREAS N/ $23-02$-02357
ER) MANAGEMEN T-PLANNING DECI SION-NAKING SCCIAL-SCIENCES, ALTERNAT IVES IN WATER MANAGEMEN I I RESOURCES (WAT DS-OI -0IG22 ER) MANAGEMEN T-PLANNING DECISION-NAKING SCCIAL-SCIENCES I ALTERNAT IVES IN WATER MANAGEMENT 1 RE SOURCES (WAT DS-OI -0IG22
INCLASSIFIED LI TERATURE PERTAINING TO THE EFFECTS CF HIGH ALTITUDE NUCLEAR EXPLOSIONS I. AND II. I UNITE/ I8-O3-02DI8 INCLASSIFIED LI TERATURE PERTAINING TO THE EFFECTS CF HIGH ALTITUDE NUCLEAR EXPLOSIONS I O AND II. I UNITE I $18-03$-02DI8

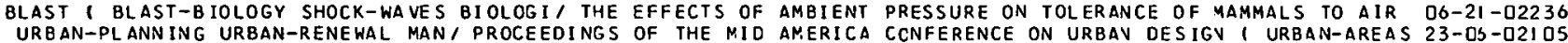
URBAN-PLANNING URBAN-RENEWAL MAN / PROCEEDINGS OF THE MID AMERICA CCNFERENCE ON URBAV DESIGV I URBAN-AREAS 23 - OS-O2I OS
, COSTS OF CONSTRLCTING THE LNDERGROUND FACILITY CF NORTH AMERICAN AIR DEFENSE COMMAND AT CHEYENNE MOUNTA I I3-I3-O2274 N SURVEYS FOREIGN/ OCCASIONAL REPCRT ON FCREIGN AFFAIRS - AMERICAN PROGRAMS OF FOREIGN AID 1 PUBLIC-OPINIO OS-II-OIGB IENCE RESEARCH BY PRIVATE SCHOLARS AND ACADEMIC CENTERS - AMERICAN REPUBLICS I BIBL IOGRAPHIES COMMUNISM I DS-II-DIGQ6

CONTEXT - 19661 ATTITUDES PUBLIC-OPINI ON SURVEYS DISARI AI I HCWA-I

A NEW ERA FOR HARMONIC-GENERA TORS REFRACTION LASERS NCNLINEAR-SYSTEMS ON-ISOLATORS SIMULATION MATHEMATICAL-ANALYSIS SHOCK-WAVES IRING AND CONSULTING FIRMS WITH CERTIFIED FALLOUT SHELTER IEXIENSION OF THE GENERAL SENSI TI VI TY ANALYSIS I I I. THE
HE B IOLOGY AND ECOLOGY OF C. ANDRENIFORMIS - HYMENCPTERA, HE B IOLOGY AND ECOLOGY OF C. ANDRENIFORMIS - HYMENCPTERA,
IE BEE GENUS CALL IOPSIS AND THE BIOLOGY AND ECOLOGY OF C D BY A REAL ANO TWO SIMULATED FALLOUT FIELDS I RAI ON THE ITRUCTURES DOSE-RATE PHOTONS SCATTERING ALBEDO GANMA-RAYS IANICS OF SL IDE DAMS WITH SAND MODELS I EXPERINENT AL-DATA - OPERATION BREN I IONIZING-RADI ATION-SCATTER' ENERGY AND TTES CEPARTMENT OF AGR ICULTURE ( AGR I CULTLRAL-ENG INEERING I GENETICS RAOIATION-HAZARDS MUTATIONS RADIATION-EFFECTS S RADIOLOGICAL-DO SAGE EXPERIMENTAL-DATA AGING (PHYSIOLOGY) LS CYLINDRICAL-BODIES COMPOSI/ VIBRATION CF NULTI-LAYERED YSIS DATA-PROCESSING DAMAGE-ASSE SSMENT) \#NREC-24(1)

IET ESTIMATES FOR THE FINANCIAL YEAR 1967 AND INFORMATION R/ SOVIET MIL ITARY TRANSLATIONS NLMBER 380 - TWENTY-FIFTH /RT IES FILMS FLUID-MECHANICS SCIENTIFIC-RESEARCH AELATION ICIES OF ANTHRAX II. LEVELS OF I NNUNITY AGAINST BACILLUS ANTHRACIS OBTA INED WITH PROTEC/ IMMUNOLOGICAL STUOIES OF
TS BAC/ PATHOGENESIS AS RELATED TO PHYSIOLOGICAL STATE OF TS BAC/ PATHOGENESIS AS RELATED TO PHYSI OLOGICAL STATE OF
GN SAFETY-DEVICES TEST-OUMMY (ANTHROPOMORPHIC) CONSTRUCTI/ GN SAFETY - DEVICES TEST-DUMMY (ANTHROPOMORPHIC) CONSTRUCT I
E-AG/ COMBINED TREATMENT OF EXPERINENTAL NELI OIDOSIS WITH AMERICAS WATERS I WATER -POLLUT ION HATER-SLPPLIES 13-03-02257 AMMONIUM-COMPOUNDS FOCUSING PHOSPHATES CALCI TE, 20-DS-02067 ANALOG-COMPUT ERS , *CONF-651016-1 /NICS) VIBRATI 20-11-02343 ANCET COMPUTER PROGRAM ( SYSTEMS-EVALUA TION CIVI 15-03-01938 ANCRENIDAE , BEE-POLL IVATION, *A I QH6 /AND T DO-DS-DI705 ANDRENI FORMIS - HYMENOP TERA, ANDREN IDAE ( BEE-P) D6-05-01705 ANGLE ANC ENERGY DISTRIBUTION OF PHOTONS PRODUCE 15-06-01964 ANGLE-OF-INCI DENCE RADIAT IOV(COBALT) BLAST-SHEL, 18-05-02029 ANGLES ( $C E O M E T R Y$ ) GEOMETRY REVETMEVTS EFFECTIVEN/ 18-03-02317 ANGULAR DISTRIBUTION OF NEUTRONS AND GAMMA RAYS 20-03-02334 ANHYDRITE SANDSTONES CLAY SILTSTONES GEOLOGY TH/ 08-07-02242 AN IMAL-HUS BANORY CROP-RESEARCH EN TOMOLOGY NUTRI/ 02-00-02108 AN IMALS HWMANS TESTS DOSE-RATE, *RM-5C96-TAB /S O6-19-01727 ANISOTROPIC CYL. INDRICAL SHELLS I STRULTURAL-SHEL 20-11-02345 ANNEX TO THIRD DRAFT OF READY I I RESOURCES ANAL D5-03-02157 ANNEXES I UNITED-NATIONS COSTS INTERNATIONAL-AF, 05-04-02165 ANNIVERSARY OF THE RATTLE OF MOSCOW I USSR HISTO D5-04-02173 ANTENNAS PROPAGATION SHOCK-HAVES KIVET IC-THEORY 20-03-02330 ANTHRAC IS OBT AINED WITH PROTEC TIVE AVTISEN AND , 15-0? ANTHRAX II. LEVELS OF IMMUNITY AGAINST 8 ACILLUS 15-02-01853 ANTHRAX SPORE ANC CELL 1 BIOLOGICAL-WARFARE-AGEN $15-02-01854$ ANTHROPOMORPHIC TEST DUMMY I TEST-EQUIPMENT DESI $13-12-01808$ 
IIGEN AND LIVE VACCINE (SPORES BIOLOGICAL-WARFARE-AGENTS ANTIGENS-AND-ANT IBODIES RESISTANCE(BIOLOGICAL) 1 (5-02-OI853 7FI-729/ WHY COUPLE BMO TO FALLOUT SHELTERS 1 URBAN-AREAS ANTIMISS ILE-DEFENSE SYSTEMS MILITARY-STRA TEGY 1 15-03-01907 IET MIL ITARY TRAVSLATIONS NUMBER 382 I USSR UNITED-STATES ANTIMISS ILE-DEFENSE-SYSTEMS YJRALE FIRE-FIGHTIN/ 15-07-023]9 ILITARY BALANCE - USA VS USSR I MILITARY-STRATEGY EOMBERS ANTIMISSILE-DEFENSE-SYSTEMS SPACE-WEAPONS GUIDE/ I5-07-D2308 IN URBAN-AREAS DAMAGE-LIMITING-SYSTEMS DEMOGRAPHY TRAFFIC ANTIMISSILE-DEFENSE-SYSTEMS UNITED-STATES SURVI/ 15 -03-01922 ILATEC DEFENS IVE SYSTEMS I DEFENSE-SYSTENS APPROPRIATIONS ANTIMISS ILE-DEFENSE-SYSTEMS UNITED-STATES USSR I I5-D3-D2294 ILE-DEFEVSE-SYSTEMS SUBMAR INE-LALNCHED-BALLISTIC-NISSILES ANTISUBMARINE-DEFEYSE-SYSTEYS, * A.L. 12 C8 /I SS I5-D7-D2308 ICTION SURVEY I ATTITUDES FALLOUT-SHELTERS PUBLIC-CPINION ANXIETY CIVIL-OEFEVSE-SYSTEMS SLRVEYS, \$A.L. 11 O5-13-01685 ENERG IZED 1 BIOPHYSICAL MECHANISMS AND SCALING PROCEDURES APPLICABLE IN ASSESS ING RESPONSES OF THE THORAX D6-21-02235 GRAM I FALLOUT-SHEL TERS FEDERAL-BLOGETS COST/ ALTERNATIVE APPROACHES TO FINANCING A NATIONHIOE SHEL TER PRO $13-13$-QI834 IAM I ORGANIZATION DUAL-PURPOSE-SHELTERS FALLOUT-SHELTERS APPROPRIATIONS ACYINISTRATIOV-AND-4AVAGEMENT, 1 O5-0I-OIO25 I OEFENSE AND RELATED DEFENSIVE SYSTEMS I DEFENSE-SYSTEMS APPROPRIAT IONS ANT IMISS ILE-DEFENSE-SYS TEMS UNI T/ I5-03-02294 FEATHER RIVER AND DELTA DIV/ INVESTIGATION OF ALTERNATIVE AQUEDUCT SYSTEMS TO SERVE SOUTHERN CALIFORNIA - 13 -02 -02258 TERS LABORATORY-FURNACES THERMAL FLUX DISTRIBUTION' IN AN ARC IMAGING FURNACE T THERMAL-RADIATION CALORIME STAT IC AVD DYVAMIC TESTS OF BLRIED UNRE INFORCED CCNCRETE ARCHES I UNCERGROUVD-STRUCTURES DYVAMICS LOADING $13-13-01830$ AND OYNAMTCS LOADING $13-13-01830$ WITH CERTIFIEO FALLOUT SHELTER ANA NATI ONAL DIRECTORY OF ARCHITECTURAL, ENG INEER ING AND CONSULTING FIRMS $13-13$-02276
CEFENSE AGAINST WEAPDNS OF MASS CESTRUC TION IN THE RURAL AREA I USSR CIVIL-DEFENSE-SYSIEMS RESCUES INTERN IS-D3-DIOII

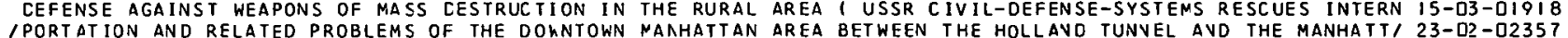
/PORTAT ION AND RELATED PROBLEMS OF THE DONNTOWN MANHATTAN AREA BETWEEN THE HOLLAVD TUNVEL AND THE MANHATT/23-02-02357

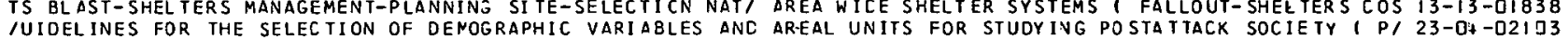
IUIDEL INES FOR THE SELEC TION OF OENOGRAPHIC VARIABLES ANC AREAL UNIS FOR STUDYING PSSTATACK SOCIETY 1 P/ $23-04-02103$
RES REPAIR RADIOACT/ POSTATTACK RECOVERY OF DAMAGEC URBAN AREAS 1 BLAST DAMAGE-ASSESSMENT VULNERABILITY FI I5-0S-02307 RES REE

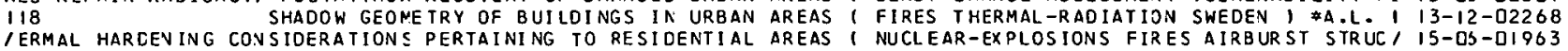
TERS I T THE SCOPE OF BLAST ANO SHOCK BI OLCGY AND FROBLEM AREAS IN RELATINE PHYSICAL AVD BIOLOGICAL PARAME D6-21-02223 TION AND REDISTRIBUTION OF FALLOUT AROU/ CPERATION CENIZA ARENA - TECHNIOUES FOR THE MEASUREMENT OF DEPOSI $18-03$-D2038

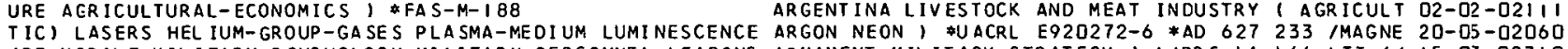
IRE MORALE MIL I TARY-P SYCHOLOGY MILITARY-PERSONNEL WEAPCNS ARMAMENT MILIIARY-STRATEGY 1 *JPRS-41,460 *TY-61 15-07-02310 RFARE MOR/ SOVIET MILITARY TRANSLATIONS NUMBER 386 I USSR ARMED-FORCES-OPERATIONS ARMED-FORCES(FORE IGN) WA IS-DT-D23ID RFAR ARMY-EQUIPMEN T REACTION (PSYCHCLOGY) SOCIAL-PSYCHOLOGY ARMED-FORCES-OPERATIONS LOGISTICS AGING (PHYSIOLI OS-IJ-02IBI ION ARMY-EQUIPMEN T REACTION IPSYCHCLOGY) SOCIAL-PSYCHOLOGY ARNED-FORCES-OPERAT IONS LOGISTICS AGINGIPHYSIOLI DS-1J-02181

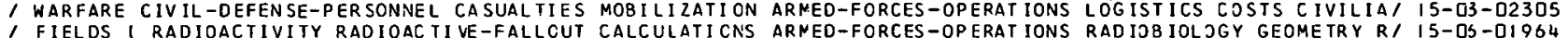

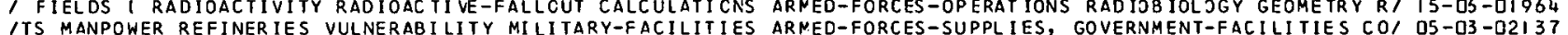

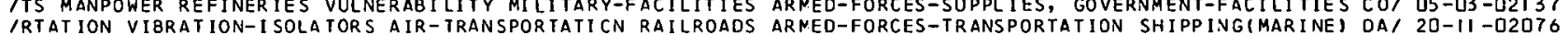

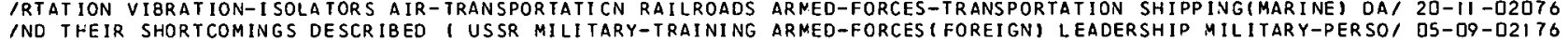
IND THEIR SHORTCOMINGS DESCRIBEO (USSR MILI TARY-TRAINING ARMED-FORCES (FOREIGN) LEADERSHIP MILITARY-PERSO/ OS-D9-D2176 IY TRANSLAT IONS VUMBER 386 ( USSR ARMEO-FORCES-OPERATI CNS ARNED-FORCES ( FOREIGN) WARFARE MORALE MILI TARY-P/ $15-07$ - D23ID EMERGEN I CODE OF FEDERAL REGLLATICNS - NATIONAL DEFENSE ( ARNED-FORCES (UN ITED-STATES) MILITA2Y-GOVERNMENT IS-03-0I946

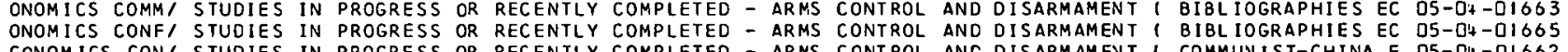
CONOMICS CON/ STUDIES IN PROGRESS OR RECENTLY COMPLETEO - ARMS CONTROL ANC OISARMAMENT 1 COMMUNIST-CHINAE O5-DH-DI662 CONOMICS CON/ STUDIES IN PROGRESS OR RECENTLY COMPLETEO - ARMS CONTROL AND DISARMAMENT! COMMUVIST-CHINAE OS-DH-DI664 MAT ICAL-MODELS SIMULAT/ DEVELOPMENT OF UTILITY THECRY FOR ARMS CONTROL AND OISARMAMENT 1 GAME-THEORY MATHE O5-Dt-02168 ATION I FAR-396IIII) DEVELOPMENT OF UTILITY THECRY FOR ARMS CONTROL AND DISARMAMENT I GAME-THEORY SIMUL OS-D4-D2169 UCES SURVEYS QUESTIONNAIRES PEACE-XEEPING NUCLEAR-RARFARE ARNS-CONTROL, A.L. 1097, PUBLIC-OPINION ATIIT O5-II-O2I96

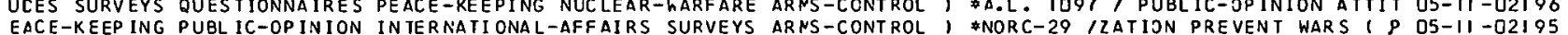
EACE-KEEP ING PUBL IC-OPINION IN IERNAII ONAL-AFFAIRS SURVEYS ARNS-CONTROL ' \#NORC-29 12ATION PREVENT HARS IOP O5-11-02195 TIUCY OF ESCALATION II. SEC TIONS 2-5 T BEHAVICR CCNFLICT ARMS-CONTROL ART IF ICIAL-REALITY HYPOTHESE S-FORM/ O5-1]-02182 $Y$ CONFL ICT-RE SOLUTION COOPERATION INTERNATIONAL-RELATI ONS ARMS-CONTROL BEHAVIOR I \#AR-3960III /IAL-REALIT O5-IJ-02183
5 MODELS, \#FAR-3/ MOOEL STUOY OF ESCALATION - VOLUME 3 , ARMS-CONTROL 8IBLIOGRAPHIES METHODOLOGY WAR-GAME O5-D4-O2171 1 THE BANNER - TWO RE SOLUTIONS ON INTERNATI ONAL AFFAIRS I ARMS-CONTROL COLC-WAR NATO VIETNAM RED-CHINA FO/ O5-O4-02172

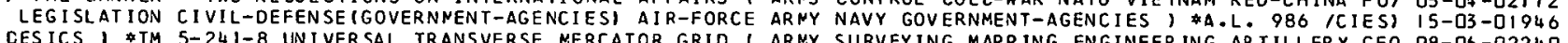
CESICS I \#TM 5-241-8 UNIVER SAL TRANSVERSE MERCATOR GRID I ARMY SURVEYING MAPP ING ENGINEER ING ARTILLERY GEO D8-DS-O224D

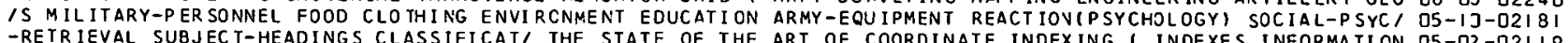
FOR INCLUSION OF REQUIREMENTS FORI A MODEL BUILOING CODE ARTICLE ON FALATE INDEXING I INDEXES INFORMAT ON INTE/ MOOEL STUDY OF ESCALA TION I. SUNMARY I CCNFLICT ARTIFICIAL-REAL ITY CONFL ICT-RESOLUTIDN COOPERATI DS-IJ-0228 IAT ION II. SECTIONS 2-5 I BEHAVICR CONFLICT ARMS-CONTROL ARTIFICIAL-REAL ITY HYPOTHESES-FOR YULATION CONFL/ O5-IJ-02182 IO/ CONTRIBUTION TO THE ANALYSIS CF MUZZLE BRAKE DESIGN I ARTILLERY COMPUTERS GUN-BARREL-ATIACHMENTS INTER I9-DS-D2320 SVERSE MERCATOR GRID I ARMY SURVEYING MAPPING ENGINEERING ARTILLERY GEODUTERS GUN-BARREL-ATTACHMENTS INTER 19-0S-D2329

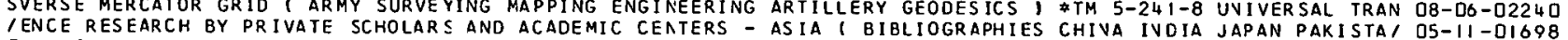

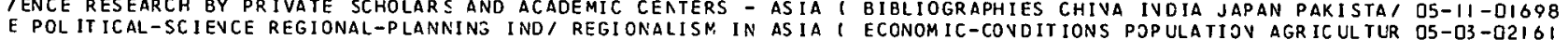

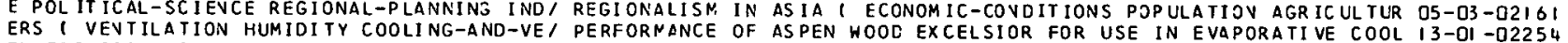

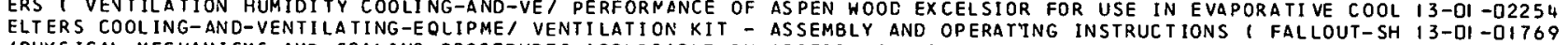
IPHYS ICAL MECHANISMS AND SCALING PROCEDURES APPLICABLE IN ASSESS ING RES PONSES OF THE THORAX ENERGIZED QY 1 O6-21-D2235 PUL 1 IICAL-SC IEVCE ECONOMIC SOC IOLOGY SI ME THODOLCGY FOR ASSESS ING TOT AL VULNERABILITY I SYSTEMS-ANALYSIS D5-II-D2193

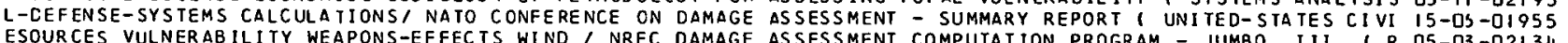
ESOUR CES VULNER AB ILI TY WEAPONS-EFFEC TS HIND I NREC DAMAGE ASSESSMENT COMPUTATION PROGRAM - JUMBO III. I R O5-D3-02134 EXPLOSION-CAMAGE POPULATION IDISTR IBUTIONI GEOG/ ON DAMAGE ASSESSMENT MODELS I RADIOACTIVE-FALLOUT NUCLEAR- IS-DS-DI 962 OWING A THERMONUCLEAR W/ PLAGUE IN THE UNI TED STATES - AN ASSESS NENT OF ITS SIGNIFICANCE AS A PROBLEM FOLL OL-05-OITO2 GRAMM INGI COMPUTERS) CASUAL/ STREAK IV - NREC RAPID DAMAGE ASSESSMENT PROGRAM ( RESOURCES VULNERABILITY PRO 05-03-D2I46 1) A LISTING OF TABLES AND PARAME TERS USED IN NREC DAMAGE ASSESSMENT PROGRAMS I VULNERABILITY, $\$$ NREEC-1141 O5-03-02138 E I SHELTER-ALLOCATION ATTITUDES CIVIL-DEFENSE-S/ SHELTER ASSIGNMENT CONCEPT - A STUDY IN PUBLIC ACCEPTANC D5-IJ-D2177 ISTATISTICAL-ANALYSIS SOCIAL-SC IENC I TABLES FOR YULES O ASSOCIATION COEFFICIENT FOR PAIRS JF PERCENTAGES DS- $11-02200$ IRES ANO MODIFIED SACHS SCALING I. EXPONENTIAL I DEAL GAS AT MOS PHERE ( SHOCK-WAVES FLUID-DYVAMIC-PROPERTI/ $20-D ;-02332$ /GUIDE EVVIRONMEN T I NUCLEAR-E XPLCSI ONS WAVE-TRANSNISSION AT MOSPHERE LOH-FREQUENCY PHASE VEZY-LOW-FREQUEN/ $20-14-02349$ /ORM - AN EXPER IMENTAL PROGRAM IN AIMOSPHERIC UIFIUSION 1 AT MOS PHERE TR ANSPORT-PROPERTIES EXPERIMENTAL-DA/ O4-OL-D2IIL

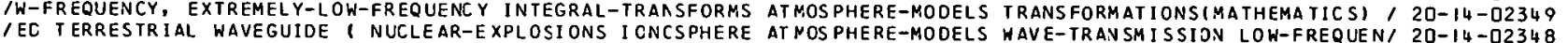
ENT IAL IDEAL GAS ATM/ SHOCK PROPAGATION IN NONHONCGENEOUS AT MOSPHERES AND MODDIFIED SACHS SCALIVG IVER EXPON $20-0+-02332$ PERTIES SPROJECT SAND STORM - AN EXPERI MENTAL PROGRAM IN AT MOSPHER IC DIFFUS ION I ATMOSPHERE TRANSPORT-PRO OU-D2 - D21I4 SILES I ATMOSPHER IC-ENTRY R I HEAT TRANSFER ASPECTS OF THE AT POS PHERIC RE-ENTRY OF LONG RANGE BALLISTIC MIS $22-02-02350$ 1 ATMOSPHERIC RE-ENTRY OF LONG RANGE BALLISTIC MISSILES I AT MOSPHERIC-ENTRY REENTRY-VEHICLES OESIGN AEROD, $22-02-02350$

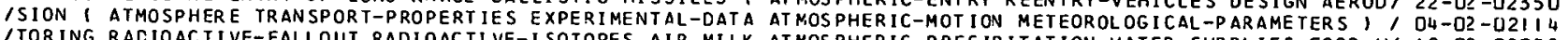
TOR ING RACIOACTIVE-FALLOUT RADIOACTI VE-I SOTOPES AIR MILK ATMOSPHERIC-PRECIPITATION WATER-SUPPLIES FO0D UI I8-D9-D2035 WARFARE-CASUALTI/ BIBLIOGRAPHY OF PUBLI SHED PAPERS OF THE ATCMIC BOMB CASUALTY COMMIISSION 1 JAPAN NLCLEAR- DO-21-D2224 LY-1, 1965-JUNE-30, 1966 I RADIA TION-EFFEC TS NUCLEAR-WARFA/ AT OMIC BOMB CASUALTY COMMISSION ANNUAL REPORT JU O6-13-01730 ISELF - WHAT THE PEOPLE KNOW AND THINK ABCUT THE 8CMB AND AT CMIC ENERGY I PUBL IC-OP IN IOV ATTI TUDES SURVEY/ D5- II-O2196

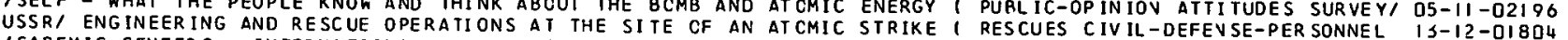

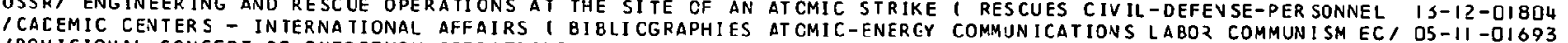

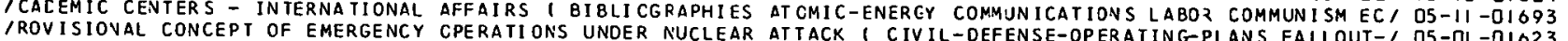

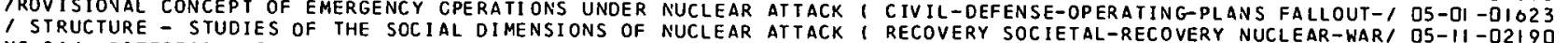
NG RAI PROTECTION DF OF THE SOCIAL DIMENSIONS OF NUCLEAR ATTACK I RECOVERY SOCIETAL-RECOVERY NUCLEAR-WAR/ OS-11-02I90 NG RA/ PROTECTION OF FODD AND AGR ICUL TURE AGAINST NUCLEAR ATTACK I RURAL-AREAS RADIOACTIVE-FALLOUT SHIELOI I5-D3-OIB87
I STRUCTURE - STUDIES OF THE SOCIAL DIMENSIONS OF NUCLEAR ATTACK $I$ SOCIETAL-RECOVERY RECOVEZY DEMOGRAPHY, O5- $11-02202$ 
I RELEVAVT TO THE STUDY OF SOCIETAL RECOVERY FRON NUCLEAR ATTACK I STRESSIPSYCHOLOGY) GRDLP-DYNAMICS SOCI/ DS-ID-DI677 3 A. BACKGROUND AND PROCEDURES FOR APPLICATICNS/ NUCLEAR AT TACK HAZARD IN CONTINENTAL UNITED STATES - I96 I5-O3-DI949 CAMAGE-ASSESSMENT ATTACK-SCENARIOS COMPUTERS HOUSING PR/ ATTACK I - ATTACK PATTERN GENERATOR I SIMULATION OS-D3-D2139 NTAL UN ITED OMP SYSTEM ANALYSIS OF THE EFFECTS OF NUCLEAR ATTACK ON RAILROAD TRAVSPORTATION IV THE CONTINE D5-D3 - D2I28 ESSMENT ATTACK-SCENARIOS COMPUTERS HOUSING PRI ATTACK I - ATTACK PATTERN GEVERATOR I SIMULATION DAMAGE-ASS O5-O3-D2I3O KINSHIP AND VOLUNTARY ORGANI IATICN IN POST THERMONUCLEAR ATTACK SOCIETY - SOME EXPLORATORY STUDIES I POST IS-O3 - 2139

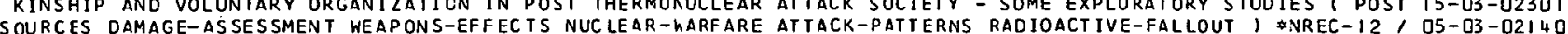

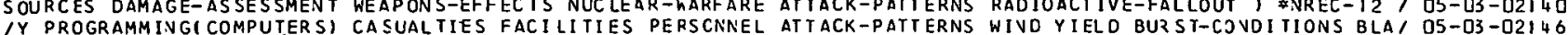
IY PROGRAMMING(COMPUTERS) CASUALTIES FACILITIES PERSCNNEL ATTACK-PATTERNS WIVD YIELD BURST-COVDITIONS BLA/ O5-D3-02I 46
E ECONOMICS SOCIOLOGY SOCIETAL-RECCVERY DAMAGE-ASSESSMENT ATTACK-SCENAR IOS, \$0CD-PS-64-2LI/ITICAL-SCIENC O5- 11 -D2193

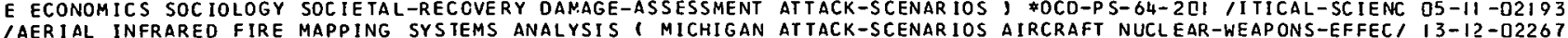

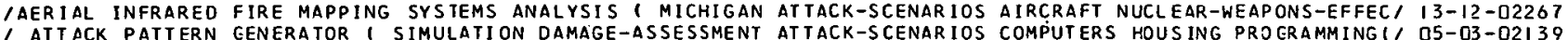
I ATT ACK PATTERN CENERATOR I SIMULATION DAMAGE-ASSESSMENT ATTACK-SCENARIOS COMPUTERS HOUS ING PROCRAMMING I/ O5-03-02I39
I AGE AGE MODELSISIMULATIONS) SLRVIVAL DENCGRAPHY RECOVERY ATTACK-SCENAR IOS FERT ILITY URBAN-AZEAS RURAL-AR/ IS-OS-DI965

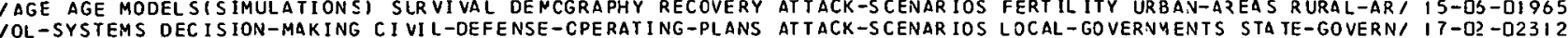
TIES TO UNCERTAINTIES IN SOME NUCLEAR DAMAGE PARAMETERS I ATTACK-SCENARIOS VUCLEAR-WARFARE-CASUALTIES CASI D6-2I -O2228 $1-1963$ A. BACK GROUND AND PROCEDURES FOR APPLICATICNS 1 ATTACK-SCENAR IOS VULNERABIL ITY (ANALYSIS) SURVIVI IS-D3-OI949 ELTERS DISTRIBUTION RADIOACTIVE-FALLOUT BIOLOGICAL-DOSAGE AT TACK-SCENAR IOS WINL 1 WRM-47O7-TAB 1 LE-SIZE SH OS-2I-O2229 IVIABIL ITY I SOCIETAL-RECOVERY ECCNOMIC-RECOVERY RECCVERY AT TACK-SCENAR IOS NIND VUCLEAR-WARFARE RADIATION/ OS-D3-G2ISO L RCOUSTIC-PROPERTIES GAS-FLOW PROPAGA / FINAL REPORT ON ATTENUATION AND CISPERSION OF SOUND IN AN AEROSO I5-O2-DI8BI ISION DEVICES FOR IO5MM AND $155 M M$ HOWITZERS I SHOCK-WAVES ATTENUATICN DESIGN GUN-BARREL-ATTACHMENTS INHIB/IO-OG-D2327 MPARISON I SHOCK-TUBES CALCULA TIONS EXPERI NEN/ SHCCK WAVE ATTENUAT ION FORMULAS FOR STRAIGHT TUVVELS - A CO $20-0+-D 2 O 5 D$

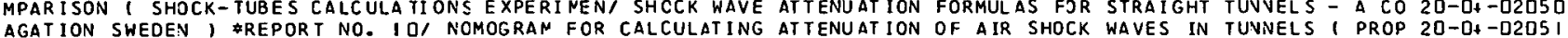

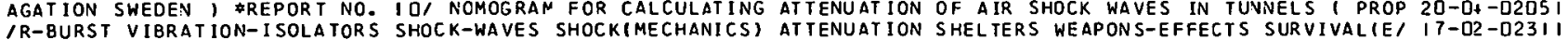
IR-BURST VIBRAT ION-I SOLA TORS SHOCK-WAVES SHOCK (MECHANICS) ATTENUATION SHEL TERS WEAPONS-EFFECTS SURVIVAL (E) I7-02-D23II
ELOING RADIOACT IVE-FALLOUT DOSE-RATES RADIOLOGICAL-DOSAGE ATTENUATION 5 IMULATIONIMODELS),

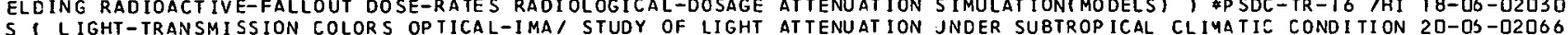
5 I LIGHT-TRANSMISSION COLORS OPTICAL-IMA I STUDY OF LIGHT AT TENUAT ION JNDER SUBTROPICAL CLIMATIC CONDITION $20-03-02066$
TONS / SHIELDING IV - CIVIL DEFENSE I RADIOACTIVE-FALLOUT AT TENUAT ION JNDERGROUND-STRUCTURES DOSE-RATE PHO IB-DS-O2O29

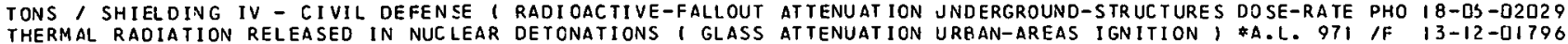
ELS 1 SHOCK-WAVES EXPERIMENTAL-DATA TEST-NETHODS PRESSURE ATTENUATORS; \$ARF DO87 \$AD $131882 /$ HOLLOW MOD I8-D3-D2OO5 ELS 1 SHOCK-WAVES EXPER IMENTAL-DATA TEST-NETHODS PRESSURE ATTENUATORS; \#ARF MO69 \$AD $131840 /$ HOLLOW MOD $18-03-02001$ MS TRAINING METHODOLOGY QLESTIONNAIRES SHELTER-MANAGEMENT ATTITUCES, \#AIR-D66-12/66-FR/-PBJCESSING-SYSTE D5-IJ-OI6BD IVE-BRANCH LOCAL-GOVERNMENTS STATE-GOVERNMENTS LEACERSHIP ATTITUDES / *IDA/HQ-65-3756/ICAL-SCIENCE EXECUT Q5-O'4-DI67I IERENTIAL SCALES RELEVANT TO INTERCULTURAL NEGOTIATIONS I ATTITUDES BEHAVIOR METHODOLOGY SEMAVTICS SOCIALI D5-IJ-O2I 78 IES OF A SHELTER SYSTEM I FALLOUT-SHELTERS PUBLIC-CPINI ON AT TITUOES BEHAVICR WARNING-SYSTEMS SHELTER-ALLOI O5-II-DIG 99 /CEPT - A STUDY IN PUBLIC ACCEPTANCE I SHELTER-ALLCCATI ON ATTITUCES CIVIL-CEFENSE-SYSTEYS COLD-WAR SURVEYI O5-IJ-D2I 77 ARE BALLISTIC-MISSILES COLD-WAR FCREIGN-PCLICY PROFAGANCA ATTITUDES COMMUNISY 1 *LT-65-IO4 /E NUCLEAR-WARF O5-D $\$-01672$ INSE TO THE RHODE ISLAND HOME FALLOUT PROTECTICN SURVEY I ATTITUDES FALLOUT-SHELTERS PUBLIC-OPINION ANXIEI O5-I]-0I685 IELTER POL ICY I OPERATIONS-RESEARCH CIVIL-DEFENSE-SYSTEMS ATTIIUDES FALLOUT-SHELTERS STRESSIPSYCHOLOGY) T/ OS-O4-O2I 66 IL FEALTH IMPL ICATIONS IN CIVILIAN EMERGENCIES I ENOTIONS ATTITUCES FEAR STRESS(PSYCHOLOGY) EASUALTIES MO/ O5-IJ-DI6BI /SOCIAL RESEARCH S SOCIAL-SCIENCES SURVEYS BIBLICGRAPHIES ATTITUCES GROUP-CYVAMICS SOCIAL-PSYCHOLOGY PUBL/ O5-II -D2I 94 1 AND PUBL IC OP IN ION TODAY I UNI TED-STATES QUESTICNNAIRES ATTITUOES INTERNATIONAL-AFFAIRS FEAR SURVEYS ST/ O5-II -OI 899 IOSTATTACK-OPERATIONS SOCIETAL-RECOVERY ECONOMIC-RECOVERY ATTITUDES MANPOWER MOTIVATION ECOVOMICS SURVIVA I O5-IJ-O2I8O IMICS SOCIAL-PSYCHOLOGY BEHAVIOR DISASTERS PUBLIC-CPINION ATTITUOES MET HOCOLOGY WEAPONS-EFFECTS COMMUNICA' OS-IO-OI677 ICAL-SCIENCE FOREIGN-POLICY THREA I-PERCEPII ON SUEZ-CRISIS ATTITUDES MIDCLE-EAST METHODOLOGY THEORY CONTEN/ D5-O+-O2I 70 GIONS MIL I TARY-PER SONNEL FOOD CLOTHING ENVIRONNENT ECUCA/ ATTITUDES OF TROOPS IN THE TROPICS 1 TROPICAL-RE D5-IJ-O2I8I RY COST/ THE CIVIL DEFENSE DEBATE I CIVIL-DEFENSE-SYSTEMS ATTITUDES POLITICAL-SCIENCE PUBLIC-OPINION HISTO DS-IJ-DIG79 IR RI AC.K NIT I BEHAVIOR ELECTRIC-POKER-PRODUCTION (FAILURE) ATTITUDES PUBLIC-OPINION PUBLIC-IVFJRMATION SUR/ OS-1J-DI 683 THINK ABOUT THE 8OMB AND ATOMIC ENERGY \& PUBLIC-CPINION AT TITUDES PUBLIC-OPINION SURVEYS DISARMAMENT CI/ D5-IJ-DI678 RWAY CENMARK GREECE SWEDEN SWITZERLAND IRELAND USSR INDIA AUSTRALIA CANADA, \#HI-272-DIRES PEACE-KEEPING I OS-II -D2I96 IUNOFLUORESCENCE, AN ANNOTATED BIBLIOGRAPHY - SUPPLEMENT, AUTHOR ANO SUBJECT INDEXESI BACTERIA VIRUSES FI DG-I3-DITIS IS 1 B/ ECONOMICS DEPARTMENT PLBLICATIONS, 1960-1965 - AN AUTHOR INCEX OF THE OPEN LITERATURE WITH ABSTRAC OS-O3-OIO52 YCR OF THE.CITY OF NEW YORK AND FOR NEW YORK CITY TRANSIT AUTHORITY I URBAN-AREAS, \#A.L. II56/. OF THE MA $23-02-02355$ STEMS SUBJECT-INDEXING TEST-ME THODS INFORNATICN-RETRIEVAL AUTOMATIC ECONOMICS I PRC-D-634A $\$$ AD 4147 II IY O5-02-O2II6

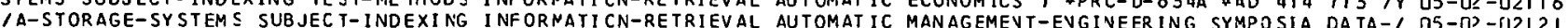
I INSTRUMENTATION RADIATI/ INSTALLATION AND IESTING OF AN AUTOMATIC REMOTE RADIOLOGICAL MONITORING SYSTEM I8-D $\$$-D2O26

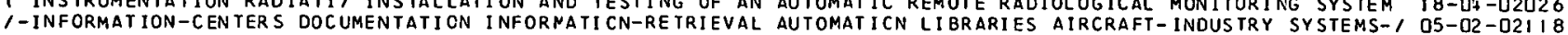

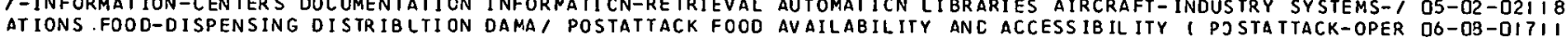

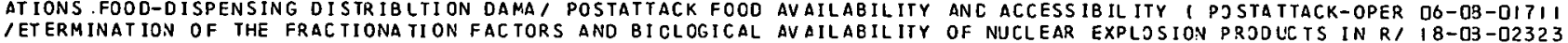
IETERMINATION OF THE FRACTIONATION FACTORS AND BICLOGICAL AVAILABILITY OF NUCLEAR EXPLOSION PRJDLCTS IN R/ I8-03-D2323
IONAL CAPITAL RAPID TRANSIT SYSTEN PORTION OF CONNECTICUT. AVENUE ROUTE BETWEEN COLUMBIA ROAD AND VAN NFSS/ I3-D? -OITBS

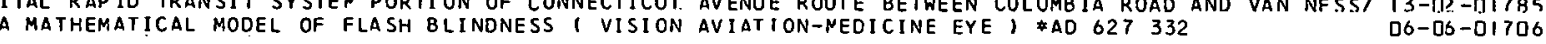

/PLIFYING THE EVALUATION OF HAROENING COSTS II* AFPENDIX B I INDUSTRIAL-EGJIPMENT IVDUSTRIES FLOW-CHARTI/ 05-03-02I26 LAT IONS, \#A.' UN IFORM SYSTEM OF ACCOUNTS FOR CLASS A AND B ELECTRIC UT IL ITIES 1 ECONOYICS AZEOUNTING REGL OS-D3-DIG3I NS, \#A.L. 10\% UN IFORM SYSTEM OF ACCOUNTS FOR CLASS A ANC 8 GAS UTILITIES I ECONOMICS ACCOUVTING REGULATIO O5-03-DI632 TING, \#A.L.' UNIFORM SYSTEM OF ACCOUNTS FOR CLASS A ANO B WATER UTILITIES I ECONOMICS REGULATIONS ACCOUN DS-O3-DI65O III. A. FREOUENCY DISTR IBUTIONS AND CORRELATION B. RELATICNSHIP AND COMPARISON 1 OEPAR TMENT-OF-I OS-D2-02I 20 ITE OF ANTHRAX SPORE AND CELL I BIOLOGICAL-WARFARE-AGENTS BACILLUS ANTHRACIS O8TAINED WITP PROTECTIVE ANTI I5-Q2 -OI853 1 AND ESTIMATION OF SHELF LIFE OF BAC TERIAL POPULATIONS 1 BACILLUS-ANTHRACIS MATHEMATICAL-MODELS PASTEURE, I5-O2-OIB49

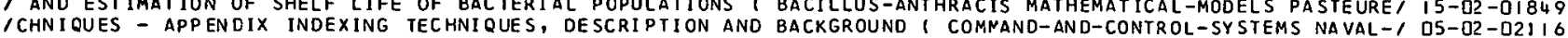
IAR ATTACK HAZARD IN CONTINENTAL LNITED STATES - 1963 A. BACKGROUNC AND PROCEDURES FOR APPLICATIONS I AT/ I5-O3-DI949 IAR ATTACK HAZARD IN CONTINENTAL LNITED STATES - 1963 A. BACKGROUNC AND PROCEDURES FOR APPLICATIONS I AT/ I5-O3-DI 949
JECTED TO A DYVAMIC DISTURBAN/ A STUDY OF THE RESPCNSE OF EACKPACKING MATERIAL ENCASIVG A TINNNFI I INER SIJR I3-I3-D2279 JECTED TO A DYVAMIC DISTURBAN/ A STUDY OF THE RESPCNSE OF EACKPACKING MATERIAL ENCASIVG A TINNFI I INER SIIR I3-I3-D2279
IROBIAL PARTICLES I BIOLOGICAL-WARFARE-AGENTS AEROBIOLOGY BACTERIA GAS-FILTERS MICROORGANISMS AIR-POLLUTI/ I5-O2-DIB5 I IROB I AL PARTICLES I B IOL OGICAL-WARFARE-AGENTS AEROBIOLOGY BACTERIA GAS-FILTERS MICROORGANISMS AIR-POLLUTI/ I5-02 - QI85I
ILUS-ANTHRACIS MATHEMATICAL-MODELS PASTEURELLA-TULARENSIS BACTERIA SPORES STORAGE BIOLOGICAL-WARFARE-AGEN/ IS-O2-OI84 9 ILUS-ANTHRACIS MATHEMATICAL-MODELS PASTEURELLA-TULARENSIS BACTERIA SPORES STORAGE BIOLOGICAL-WARFARE-AGEN/ IS-02-OI84 9
BIBLIOGRAPHY - SUPPLEMENT, AUTHOR AND SLBJECT INCEXES 1 BACTERIA VIRJSES FUNGI PROTOZJA RICKETISIIA PHYS/ O6- 13 -DI7I 5

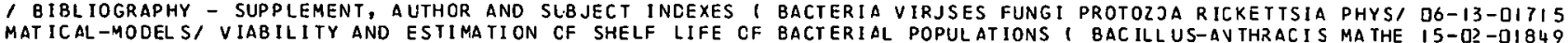
MAT ICAL-MODEL SI VIABILITY AND ESIIMATION CF SHELF LIFE CF BACTERIAL POPUL ATIONS I BAC ILLUS-AVTHRACIS MATHE IS-O2-DI849 IS FOR MACACA MULATTA AND MAN I BIOLOGICAL-WARFARE-AGENTS BACTERIAL-AEROSOLS EXPOSURE VACCIVES DOSAGE IMM/ I5-02-OI858
S ANT IMISSILE-DEFENSE-SY/ THE CHANGING STRATEGIC MILITARY BALANCE - USA VS USSR I MIL ITARY-STRATEGY BOMBER I5-D?-O2308 ITY POPULATION CA SUALIIES FOOD-I RISK II ANALYSIS CF FOOC BALANCE I RESOURCES DAYAGE-ASSESSMENT VULNERABIL O5-D3-02I 32 IANSFER ASPECTS OF THE ATMOSPHERIC RE-ENTRY OF LCNG RANGE BALLISTIC MISSILES ITMOSPHER IC-EYTRY REENTRY-I 22 -D2-C235 IR MISSILE WAR ( RUSSIA POLITICAL-SCIENCE NUCLEAR-hARFARE BALLISTIC-MISSILES COLD-WAR FJREIGV-POLICY PROP, O5-D $4-0 I G 72$ ICULTURE COMMERCE UTILITIES TRANSPORTATI ON COMMUNICATIONS BANKING CONSTRUCTION MAINTENAVCE REPAIR GOVERNMI O5-QZ3-D2I53 IOPERAT ING-PLANS REPAIR CONSTRUETICN REAL-ESTATE REFUGEES BANKING INSURANCE AABOR LAW TZAFFIC, $\$ 10 A$ HO OI O5-D3-DIO36 $S 1$ ARMS-CONTROL COLD-WAR NATO VIETNAM/ SCLIDARITY IS THE BANNER - TWO RESOLUTIONS ON INTERNATIONAL AFFAIR OS-OH-O2I 2 A ENVIRONMENTAL-CONTROL I NA TURAL VENTILATICN TEST OF A BASEMENT FALLOUT SHELTER IV EAST CHICAGO, INDIAN I3-OI-OI774 IN OF TECHNIQUES FOR IMPROVING FALLOUT PRCTECTION IN HCNE BASEMENTS I SHELTERS FALLOUT-SHEL TERS NEVADA WE I I3-I3-DI822 AGE-BATTERIES ALKAL INE-CELLS AIRCR/ SEALED NICKEL CADMIUM BATTERIES FOR AIRCRAFT ELECTRICAL SYSIEMS I STOR ID-O3-DI764 AGE-BATTER IES ALKALINE-CELLS A IRCRI SEALED NICKEL CADMIUM BATTERIES FOR AIRCRAFT ELECTRICAL SYSTEMS I STOR ID-O3-DI764
IGENERATORS PORTABLE DESIGN EXERCISE LIGHTS (ILLUMINATION) BATTERIES-AND-COMPONENTS RADIO-COMMUNICATION-SYI I 3-OI-D2252

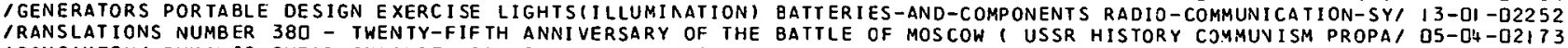
ACINGIMECH/ DYVAMIC SHEAR STRENGTH OF REINFORCED CCNCRETE BEAMS II. 1 TEST-EQUIPMEVT BEAMSISTZUCTURAL) LO I3-I3-DI84I 
LOAD/ EFFECTS ON LONG TIME LOADS ON PRESTRESSED CCNCRETE BEAMS I BEAMS ISTRUCTURALI CONSTRUCTION-MA TERIALS I3-I3-02289 IAMIC SHEAR RESISTANCE OF THIN WEBBED REINFORCED CCNCRETE BEAMS $($ BEAMS (STRUCTURAL) DYNAMICS SHEAR-STRESS/ 20-II -D2337 IERAL OPT ICAL NONLINEARITIES USING FOCUSED GAUSSIAN LASER BEAMS I CRYSTALS OPTICAL-PHENDMENA HARMONIC-GEN/ 20-D6-O206? ERS HYSI STUDIES ON THE INTERACTION OF LIGHT AND PARTICLE BEAMS I MAGNETO-OPT IC-EFFECT YAGNETIC-FIELOS LAS 20-DS-D2064 1 TO CETERMIVE THE OPTIMUM SEC IION OF REIAFORCED CCNCRETE BEAMS SUEJECTED TO BLAST LOADS 1 DESIGN BEAMSIS/ I3-I3-DIBLO ICOWN UNDER IN TEN SE OPIICAL. ILLUMINATI ON I GAS-I ONIZATION BEAMS (ELECTROMAGNET IC) LASERS HELIUM-GROUP-GASEI 20-05-0206O I INFORCED CONCRETE FLOOR SLABS I STRUCTURES DESIGN THEORY BEAMS (STRUCTURAL FRAMES LOADING (MECHANICS) COLU/ 20-II-0234D CUNDERGROUND-STRUCTURES VDYNAMICS SHELLSISIRUCTURAL-FORMS) BEAMS (STRUCTURAL) ANALYSIS SLABS(VOYMETALLIC) F I I3-I3-OI83?

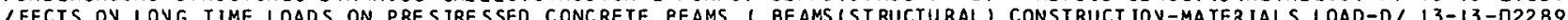
IAR RESISTAYCE OF THIN WEBBED REIAFORCED CCNCRETE PEAMS I BEAMS ISTRUCTURAL, DYNAMICS SHEAR-STRESSES WEBSI, 20-II -O2337 IOS IUM I LOADINGIMECHANICS) STRUC TURES NONLINEAR-ANALYS IS BEAMS (STRUCTURAL) FRAMES LINEAR-SIRUCTURES I *A, 20-II -O2339 INFORCED CONCRETE BEAMS SUBJEC TED TO BLAST LOADS I DESIGN BEAMS(STRUCTURAL) LOADINGIMECHANICS) PROGRAMMIN/ I3-I3-DIB4O /ENETH OF REINFORCED CONCRETE BEAMS 11. I TEST-EQLIPMENT BEAMS (STRUCTURAL, LOADINGIMECHANICS) STATICS ), I3-I3-OI84 I IS I STRUCTURAL-SHELLS ELASTIC-SHELLS MOOELSISIMULOTIONS) BEAMS (STRUCTURAL I NUMERICAL-AVALYSIS I *AD 636 / $20-I I-0208$ I /LLET IN ( ROCKETS DYNAMICS STRUCTLRAL-ANALYSIS ELASTICITY BEAMS (STRUCTURAL) SHOCK IMECHAYICS) LAUNCHING (SI/ 2O-II -02O74 ND $($ LOADINGIMECHANICSI SOIL-MECHANIC/ STATIC ANO CYNAMIC BEARING TESTS ON A STRIP FOOTING IN SATURATED SA 20-II-D234I MANUALS PSYCHOLOGY STRESSIPHYSIOLOGY) FOOD WATER-SLPPLIES BEODING TOILET-FACILITIES) \#GARD-I292II) /TION- IS-03-023OD MANUALS PSYCHOL OGY STRESS(PHYSIOLCGY) HA TER-SUPPLIES FCCD BECOINE TOILET-FACILITIES, \#GARD-12921LII) $10 N-15-03-02298$

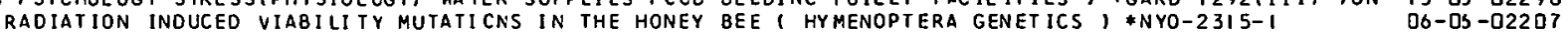
OF C. AYORENIFORMIS - HYNENOP TERA, AN/ A REVISION OF THE BEE GENUS CALLIOPSIS ANO THE BIOLOGY AND ECOLOGY O6-DS-DI7OS

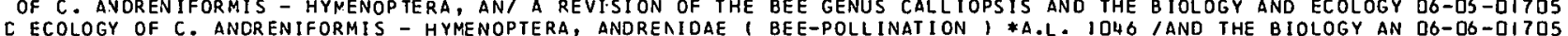

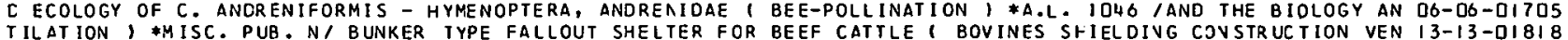

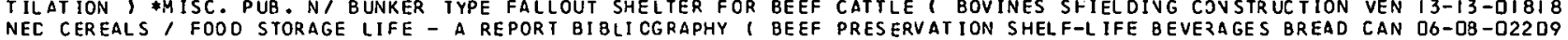

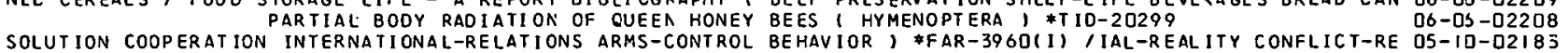

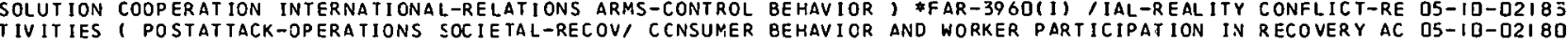
ALT F POSTATTACK-OP/ EMERGENCY SOCIAL SERVICES I PERSCNNEL BEHAVIOR CIVIL-DEFENSE-OPERATING-PLAVS MENTAL-HE QS-II-D2IBS Y HYPOTHESI MODEL STUDY OF ESCALATION I I SECTIONS 2-S I BEFAVIOR CONFLICT ARMS-CONTROL ARTIFICIAL-REALIT OS-IJ-O2IB2 ICK ( STRESSIPSYCHOLOGY) GROUP-DYNAMICS SCCIAL-PSYCHOLOGY BEHAVIOR DISASTERS PUBL IC-OPIVION ATTITUDES MET/ OS-IJ-DIO77 TUD/ PUBL IC RESPONSE TO THE NORTHEASTERN POWER 8LACKOUT I BEHAVIOR ELECTRIC-POWER-PRODUCTION(FAILURE) ATTI O5-ID-0I 683

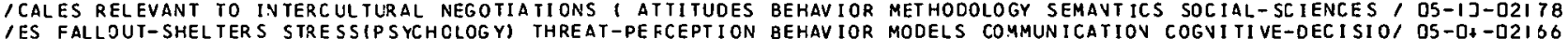
IECULATION ON THE STRUCTURE OF INTERPERSONAL INTENTIONS 1 BEHAVIOR MOTIVATION CUL TURE RESPONSE REACIIONIP/ OS-IJ-O2I79 NO ANNUI OUARTERLY OF THE COLORADO SCHOOL OF MINES 5213) BEHAVIOR OF MATERIALS IN THE EARTHS CRUST - SECO D8-D7-D2244 1 LI LASER CAVITIES I. RESEARCH ON OPEN RESCNATORS II. BEHAVIOR OF THE OUTPUT FRDM A MANY ELEMENT LASER 20-O5-O2O5 7 I-WARFARE WEAPDNS-EFFEC TS POSTATTACK-OPERATIONS DISASTERS BEHAVIOR SOCIOLOGY STRESSIPSYCHOL DGYI DEMOGRAPHI O5-II -O21 OO IELTER SYSTEM I FALLOUT-SHELTERS PUBLIC-OPINICN ATTITUDES BEHAVIOR WARN ING-SYSTEMS SHELTER-ALLOCATION SHE/ OS-II-DIG9Q VANT TO INTERCULTURAL NEGOTIAT/ A METHODOLOGICAL STUDY OF BEHAVIORAL AND SEMANTIC DIFFERENTIAL SCALES RELE O5-IO-O2I7B ICF A CRAST DUMMY FOR TESTING SHOLLDER HARNESS AND SAFETY BELTS I TEST-ECUIPMENT TEST-DUMMYIAVTHROPOMORPH/ I3-12-DIBD3 I WITH RADIAL ELASTIC SUPPORT I RINGS VIBRATICN FRECUENCY BENDING ELAST ICITY LOADIVGIMECHANICS) IMPACT, / $20-1 I$-O2336 INICS LOADING (MECHANICS) USSR EXCAVATI ON SHEARING CUTTING BENOING TORSION STRETCHING COUPRESSING TESTING-/ D8-OT-O2242 WAVES STRESSES PROPAGATION EI STUDIES ON LONGITUOINAL AND BENDING WAVES IN LONG ELASTIC RODS I MECHANICAL- 20-11 -O2O79 TTLEMENT (STRUCTURAL) HIG/ ANALYSI S OF STRESS DISTRIBUTION BENEATH EMBANKMENTS I STRESSES SOIL-MECHANICS SE 2D-II-O2O84 EN/ COST ANALYSIS FOR PLANNING PROGRAMNING BUDGETING CCST BENEFIT STIJIES 1 COST-EFFECTIVEVESS MANAGEMENT- DS-OI -D2292 ICEFENSE WESTERN-EUROPE NATO GREA T-BRITAIA FRANCE GERMANY BENELUX NORWAY DENMARK GREECE SWEDEN SWITZERLAN/ I5-D3-D2I 47 IE - A REPORT BIBLIOGRAPHY I BEEF PRESERVATION SHELF-LIFE BEVERAGES BREAD CANNED CEREALS COFFEE CORN DEHYI DG-O3-O22D9 SE - D REPORT B BLIOGRAPHY B

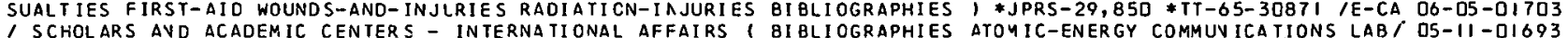
IUBL ICAT IONS IN SOCIAL RESEARCH I SOCIAL-SCIENCES SURVEYS BI BL IOGRAPHIES ATT ITUDES GROUP-DYNAMICS SOCIAL-I O5-II-O2I 4 ISEARCH BY PRIVATE SCHOLARS ANO ACADEMIC CENTERS - ASIA I BIPLIOGRAPHIES CFINA INDIA JAPAN PAKISTAN PHILI/ DS-II-DIO98 IATE SCHOLARS AND ACADEMIC CENTERS - ARERICAN REPUBLICS 1 BI QLIOGRAPHIES COMMUNISM ECONOMICS EDUCATION FOI Q5-II -DIG96 /CHOL ARS AND ACADEMIC CENTERS - USSR AND EASTERN EUROPE 1 BI BL IOGRAPHIES COMMUNISM ECONOMICS EDUCATION IN/ OS-II -DI697 IVE CONSTRUCTION - VOLUME I I COMNAND-AND-CONTROL-SYSTEMS BIBLIOGRAPHIES DESIGN HARDENIVG BLAST AIR-BURSTI I7-O2-O23II IERY COMPUTERS GUN-BARREL-ATTACHMENTS INTERIOR-BALLISTICS BI RLIOGRAPHIES OIGITAL-COMPUTERS EXPLOSIONS GASI I9-DS-O2329 IS OR RECENTLY COMPLETED - ARMS CONTROL AND OISARNANENT 1 BI QLIOGRAPHIES ECOVOMICS COMMUNIST-EHINA CONFLI I OS-DH -OI O63 /S OR RECENTLY COMPLETED - ARMS CCNTROL AND DISARMAMENT $/$ BIBL IOGRAPHIES ECONOMICS CONFL ICT-RESOLUTION CO/ O5-DA-OI66S IIVATE SCHOLARS AND ACADEMIC CENTERS - $\triangle F R I C A$ I ECCNOMICS BIBLIOGRAPHIES ECUCAT ION FOREIGN-POLICY GEOGRAPI O5-II -OIOQ2 IMIC CENTERS - WESTERN I AN AUTHOR INDEX OF THE OPEN LITERATURE HITH ABSTRACTS ( BIBLIOGRAPHIES INDEXES, *RM-28CD-IISUPP) \$AD 6/ O5-D3-DIG52

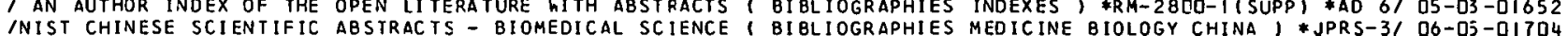

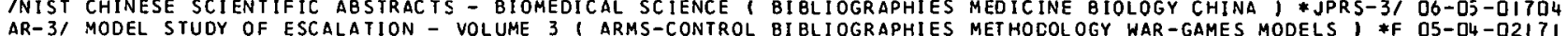

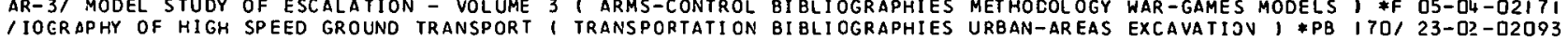
1 - SELECTED URBAN AVD MIXED LRBAN RURAL FIRES 1740-1964/ BI LLIOGRAPHIES URBAV-AREAS RUZAL-AREAS FIRE-SAF, I3-I?-DI799 ANT TO THE STUDY OF SOCIETAL RECOVERY FRON/ CIVIL DEFENSE BIBLIOGRAPHY - A COMPILATION OF REFERENCES RELEV OS-IJ-DIGT7 RAL FIRES 1940-19641 BIBLIOGRAPHIES URBAN-AREAS RLI FIRE BIBLIOGRAPHY - SELECTED URBAN AND MIXED URBAN RU I3- 12 -OI799 CEXES I BACTER IA VIRUSE/ IMMUNOFLLORE SCENCE, AN ANNOTATED BIBLICGRAPHY - SUPPLEMENT, AUTHOR AVD SUB JECT IN D6-I3 - OI7IS RAGES BREAD CANNED CEREALS / FOOD STORAGE LIFE - A REPCRT BIELIOGRAPHY I BEEF PRESERVATION SHELF-LIFE BE VE DG-DB-D22D9 SHOCK TURES - $\triangle$ BIBLIOGRAPHY *MLM-1100 RESEARCH ON FOREIGN STUDEN/ CROSS CULTURAL EDUCATION - A BIBLIOGRAPHY OF GOVERNMENT SPONSORED AND PRIVATE O5-II -OIGOI ANSPORTATION BIBL IOGRAPHIES URBAN-AREAS EXCAVATION) *PB/ BIBLIOGRAPHY OF HIGH SPEED GROUND TRAVSPORT I IR 23-02-O2D93 PERTAINING TO THE EFFEC TS OF HIGHALTITLDE NUCLEAR EXPL/ BI BLIOGRAPHY OF OPEN AVO UVCLASSIFIED LITERATURE I8-O3-02DI8 RESOURCES, NREC- 121 BI QL IOGRAPHY OF PUBLICATIONS 1 DAMAGE-ASSESSMENT OS-O3-D2136 PUBLIC-OPINION WELFARE-SERVICES SOCIAL-PSYCHOLOG AGING/ BIELIOGRAPHY OF PURLICATIONS, $1941-19601$ HEALTH O5-11-02197 OMATORY PUBLIC/ PRO TECTIVE SHELTER SYSTENS - AN ANAOTATED BIBLIOGRAPHY OF U.S. NAVAL CIVIL EVGIVEERING LAB I3-I3-D2278

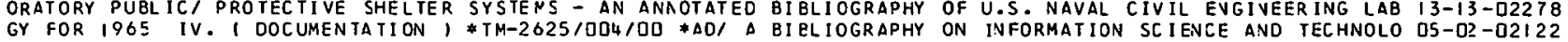

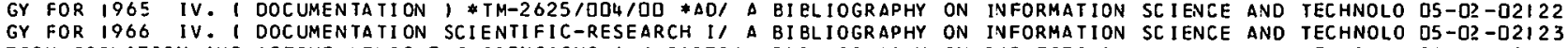
TION ISOLATION ANO ACTIVE VEHICLE SUSPENSIONS 1 ' PARTIAL BI BLI OGRAPHY ON SUBJECTS RELATED TO ACTIVE VIBRA 2D-II-O2DB7 -SPEEC-GROUVD-TRANSPORT TINEW TRANSPORT TECHNOLOGY FOR THE BIG CITY 1 URBAN-AREAS TRAVSPJR TATION COSIS HIGH $23-02-02352$ UTRITION(ANIMAL) PROTEINS GENE IICS AGRICULTURAL-ECCNOMICS BIOCHEMISTRY, \#A.L. 997 IE 1 NUTRITIONIHUMAN) N O6-I6-DI720 LUF ON THE DETERM INATION OF THE FRACTIONATION FACTCRS ANO BI CLOGICAL AVAILABILITY OF NUCLEAZ EXPLOSION PRO I8-DB-O2323 VES B IOLOGICAL-BLAST-EFFECTS SHOCKIPATHOLCGYI EXPLCSICN-; BIOLOGICAL EFFECTS AND PRDTECTIVE YEASURES I RAD D6-I9-0I726 RADIATION-EFFECTS ECOLOGY RADI INTRODUCTION TO LONG TERM BIOLOGICAL EFFECTS OF NUCLEAR HAR 1 RADIOBIOLOGY OO-IS-OIT23 ISHOCK BIOLOGY ANC PROBLEM AREAS IN RELA ING PHYSICAL AND BI CLOGICAL PARAMETERS I BIOLOGICAL-BLAST-EFFECT/ OL-21-O IZ23 ITEC SPEC IAL IZED INFORMATION SER VICES IN THE PHYSICAL AND BICLOGICAL SCIENCES I RESOURCESIIVFJRMATIONI DA, OS-OQ -OIG29 ITION RESOURCES IN THE UNI TED STA TES - PHYSICAL SCIENCES, BI OLOGICAL SCIENCES, EVGIVEERING I DATA I $\$$ I DA. OS-O? -OI 299

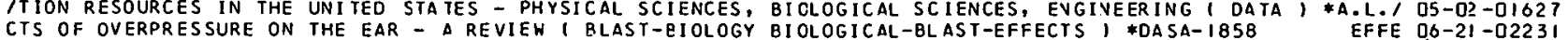
IM AREAS IN RELATING PHYSICAL AND BIOLOGICAL PARAMETERS B BI CLOGICAL-BLAST-EFFECTS BLAST-BIOLOGY DISPLACEI OO-21 -O2231 IM AREAS IN RELATING PHYSICAL AND BIOLOGICAL PARAMETERS
CASA AEC LOVELACE FOUNDATION BLAST SIMULATION FACILITY 1 BI CLOGICAL-BLAST-EFFECTS BLAST-BIDLOGY DISPLACE/ O6-2I -O2223 CASA AEC LOVELACE FOUNDATION BLAST SIMULATION FACILITY 1 BICLOGICAL-BLAST-EFFECTS BLAST-BIJLOGY SHOCK-TUB O6-2I-O2232
IE TO LOVG DURATION REFLECTED PRESSURES IN A SHOCK TUBE /TICAL-ANALYSIS WEAPONS-EFFECTS HIROSHIMA NAGASAKI GRAPHS BIOLOGICAL-BLAST-EFFECTS BURNS RADIATION-INJURI/ D6-2I-D2227 
ION) RECOVERY JAPAN RADIOACTIVE-FALLOUT THERMAL-RADIATION BI CLOGICAL-BLAST-EFFECTS DAMAGE-ASSESSMENT I *R/ O6-2I -02228 $Y$ MA' BLAST INDUCED TRAVSLATIONAL EFFECTS I BLAST-BIOLOGY BI CLOGICAL-BLAST-EFFECTS EXPER IMEVTAL-OATA THEOR DG-2I -O2239 IAIR BLAST OVERPRESSURES OR BY NON-PENETRATING MISSILES I BI OLOGICAL-BL AST-EFFECTS MATHEMATIEAL-MODELS EX/ O6-2I -02235 IRESPONSE QF MAMMALS EXPOSED TO AIR BLAST I BLAST-EIOLOGY BIOLOGICAL-BLAST-EFFECTS MONKEYS SWINE SHEEP LU/ O6-2I -D2234 I ANCE OF MAMMALS TO AIR BLAST I BLAST-BI OLOGY SHOCK-WAVES BI OLOGICAL-BLAST-EFFECTS RATS GUINEA-PIGS DOGS I O6-2I-D2236 IS WEAPONS-EFFECT RADIATION-INJURIES STATISTICAL-ANALYSIS BI CLOGICAL-BLAST-EFFECTS SHIELDING, \$DC-FR-IOS, O6-2I-02238 IST STUDIES WITH EIGHT SPECIES OF MAMMALS I BLAST-QIOLOGY BICLOGICAL-BLAST-EFFECTS SHOC $-T U B E S$ LLNG-DAMAG I O6-21-O223O ION-1 BIOLOGICAL EFFECTS OF BLAST ANO SHCCK
PARTICLE-SIZE SHELTERS DISTRIBUTION RADIOACTIVE-FALLOUT BIOLOGICAL-DOSAGE ATTACK-SCENAR IOS WIND, $\$ R M-4 /$ D6-2I -O2229 PARTICLE-SI ZE SHEL TER S DISTRIBUTION RADI OACTIVE-FALLOUT BI OLOGICAL-DOSAGE ATTACK-SCENAR IOS WIND I \$RM-4/ D6-21 -02229
/CONFERENCE DISASTER-MEDICINE ENERGENC Y-HEALTH-SERVICES BI CLOGICAL-WARFARE CHEMICAL-WARFARE PSYCHOLOGIC/ O6-2I -D2233

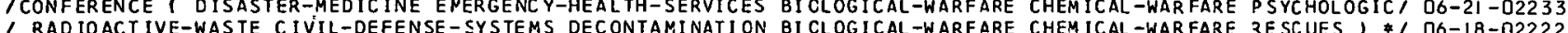

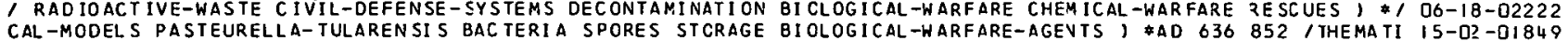

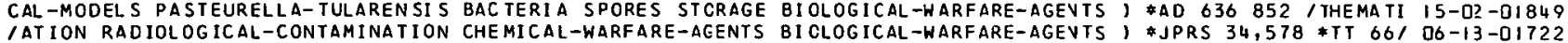
AS-FILTERS MICRO/ AIR FILTRATION OF MICROBIAL PARTICLES I BICLOGICAL-WARFARE-AGENTS AEROBIOLOGY BACTERIAG I5-D2-DI85I ITA INED WITH PROTECTIVE ANTIGEN AND LIVE VACCINE I SPORES BI CLOGICAL-WARFARE-AGENTS ANTIGENS-AVD-ANTIBODI/ I5-02-DI853 ILATEC TO PHYSIOLOGICAL STATE OF ANTHRAX SPORE AND CELL ( BI CLOGICAL-WARFARE-AGEVTS BACILLUS-AVTHRACIS DI/ I5-O?-DI854 IORNE PASTEURELLA TULARENSIS FOR MACACA MULATTA ANC MAN I BIOLOGICAL-WARFARE-AGEVTS BACTERIAL-AEROSOLS EXI IS-D?-DIB58 IIS, SMALLPOX, MEASLES, INFLUENZA I DEMOGRAPHY IMMUNOLOGY BI OLOGICAL-WARFARE-AGENTS CASUALTIES I \#A.L. IQ/ IS-DZ-DI869 /ABORATORY PUBL ICATIONS I FALLOUT-SHELTERS BLAST-SHELTERS BI OLOGICAL-WARFARE-AGENTS CHEYICAL-WARFARE-AGEN/ I3-I3-O2278 ICLEAR-WEAPONS CONTAMINATION FOOD CHEMICAL-WARFARE-AGENTS BI CLOGICAL-WARFARE-AGENTS CIVIL-DEFENSE-OPERATI/ I5-03-D22 96 TOXINS-I PROCEEDINGS OF A CONFERENCE ON BCTULINUN TOXIN 1 BIOLOGICAL-WARFARE-AGENTS CLOSTRIDIUM-BOTLLINUM IS-D2-DI86I IELECTIVE MEDIA FOR THE ISOLATION OF PASTEURELLA PESTIS I BICLOGICAL-WARFARE-AGEVTS CULTURE-MEDIA GROHTH I I5-OZ -OIBT /ELECTIVE MEDIA FOR THE ISOLATION OF PASTEURELLA PESTIS ( BI CLOGICAL-WARFARE-AGENTS CULTURE-MEDIA GROWTH I IS-O2 -OI875 /RFARE-AGENTS RADIOLOGICAL-CONTAMINATION ICOUNTERMEASURESI BIOLOGICAL-WARFARE-AGENTS OECONTAMIVATION I *JPI I5-O2-OI843 /RIMENTAL MEL IO IDOSIS WITH ANTIBIOTICS AND SULFADINESIN
IOBLEM FOLLOWING A THERMONLCLEAR WAR I PASTEURELLA-PESTIS BI OLOGICAL-WARFARE-AGEVTS EPIOEMIOLOGY PUBLIC-H/ OG-OS-DI7O2

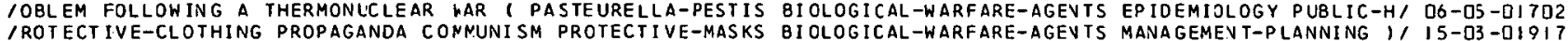
/ROTECT IVE-CLOTHING PROPAGANDA CONMUNI SM PROTECTIVE-MASKS BI OLOGICAL-WARFARE-AGEVTS MANAGEMEVT-PLANNING I/ I5-03 - I I I 7
IEXPOSED TO A SMALL PARTICLE AEROSOL OF THE BORG SYRAIN 1 BIOLOGICAL-WARFARE-AGENTS MIYAGAWANELLA-PSITTAC/ I5-O2-OI85D CULTURE-MEOIA / PLAQUE FORMA TION BY PSI TTACOSIS VIRUS I BI CLOGICAL-WARFARE-AGENTS MIYAGAWANELLA-PSITTACI IS-OZ-DIB9I I CUES INTERNATIONAL-CIVIL-DEFENSE CHEMICAL-WARFARE-AGENTS BI CLOGICAL-WARFARE-AGEVTS VUCLEAR-WEAPONS FIRST/ I5-O3-OI9I 8 IS RELATED TO TOXICITY 1 PELEC YPODA TOXINS-AND-ANTITOXINS B I OLOGICAL-WARFARE-AGEVTS PARALYSIS FOOD-POI SONI IS-OZ -OI863 1 \#ADI PATHOGENESIS OF COCCIDIOIDES IMMITIS IN MONKEYS I BIOLOG ICAL-WARFARE-AGENTS PATHOLOGY LUNGS SPORES IS-O2-OI852 作

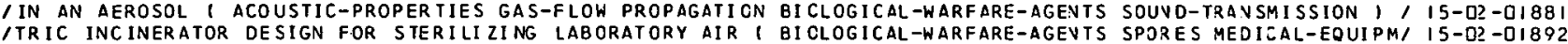
ISPONSE OF MAN TO PUR IFIED BIVALENT AB BOTULINUM TCXOID I BIOLOGICAL-WARFARE-AGEVTS TOXIC-TJLERANCES TOXI/ IS-O2-OIBST TAT IST ICAL-ANALYSIS VARIANCE REGRESSION-ANALYSIS MEDICINE BIOLOGY, \#A-L II23, MS MULTIVARIATE-ANALYSIS S OO-O3-D22OL

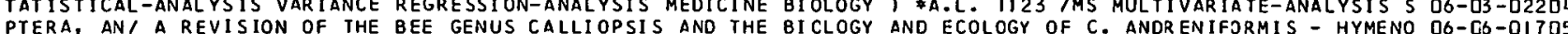
NC BIOLOGICAL PARAMEIERS, THE SCOPE OF BLAST ANC SHOCK BI CLOGY AND PROBLEY AREAS IV RELATIVG PHYSICAL A DG-2I -D2223

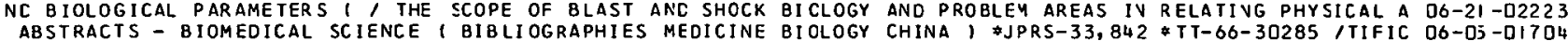

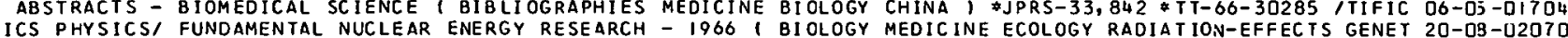
ICS PHYSICS/ FUNDAMENTAL NUCLEAR ENERGY RESEARCH - 1966 I BI OLOGY MEDICINE ECOLOGY RADIAT ION-EFFECTS GENET 20-OB-U207D
MING) DATA-PROCESSING-SYSIEMS MUL TIVARIA IE-ANALYSIS I BMD BICMEDICAL COMPUTER PROGRAMS 1 COMPUTERSIPROGRAM D6-D3-O22DI

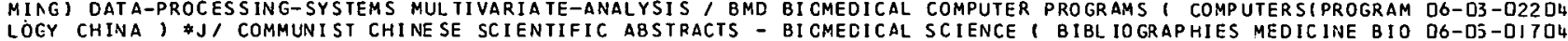

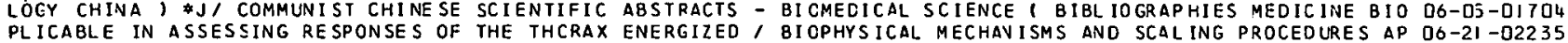
ITUL INUM VIII. IMMUNOLOGICAL RE SPONSE OF MAN TO PURIFIEO BIVALENT AB BOTULINUM TOXOID 1 BIOLOGICAL-WARFA/ IS-OZ-OIBS7 S STATISTIC/ THE CHI-SQUARE TEST OF GOODNESS OF FIT FOR A BIVARIATE NORMAL DISTRIBUTION I STATISTICAL-TEST I2-OI-O225I ILURE, ATTITUD/ PUBL IC RESPONSE TO THE NORTHEASTERA POWER BLACKOUT, BEHAVIOR ELECTRIC-POWEZ-PRJDUCTIONIFA O5-IJ-DIGB3 CS) STRESS LOADINGIMECHANICS) SHOCK-WAVES VISCOELASTICITY BLAST, \#CONTRACT REPORT NO. $3-148$ /RAINIMECHANI 20-II-O2O86 IL ITY ECONOMIC-RECOVERY INDUSTRIAL-PRODUC IION INVENTORIES BLAST; \#NRC-38/ITY DAMAGE-ASSESSMENT VULNERAB OS-O3-O2IS2 IVE PARAMETERS AND THE RE SPONSE OF MAMMALS EXPOSED TO AIR BLAST 1 BLAST-8IOLOGY BIOLOGICAL-BLAST-EFFECTS I DG-2I-D2234

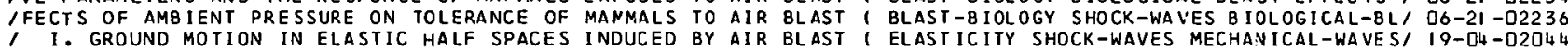
ISIS OF PROTECTIVE STRUCTURES FOR PROTECTION FRON NUCLEAR BLAST I SHELTERS SHOCK-WAVES BLAST-SHELTERS UND/ I3-I3-DI833 IMAND-AND-CONTROL-SYSTEMS BIBLIOGRAPHIES DESIGN HARDENING BLAST AIR-BURST VIBRATION-ISOLATOZS SHOCK-WAVESI I7-ODZ-D23II IMAND-AND-CONTROL-SYSTEMS BIBLIOGRAPHIES DESIGN HARDENING BLAST AIR-BURST VIBRATION-ISOLATORS SHOCK-WAVESI I7-02-023II
FFECTS SHOCK(PATHOLOGY) EXPLOSION-1 BIOLOGICAL EFFECTS OF BLAST AND SHOCK I SHOCK-WAVES 8 IOLOGICAL-BLAST-E O6-2I-OI74 1 ATING PHYSICAL AND BIOLOGICAL PARAMETERS I I THE SCOPE OF BLAST AND SHOCK BIOLOGY AND PROBLEM AREAS IN REL DO-2I-O2223 AT ING PHYSICAL AND B IOLOGICAL PARAMETERS I THE SCOPE OF BLAST AND SHOCK BIOLOGY AND PROBLEM AREAS IN REL O6-21 - 2223
IR RACIOACT/ POSTATTACK RECOVERY OF DAMAGED URBAN AREAS I BLAST DAMAGE-ASSES5MENT VULNERABILITY FIRES REPA I5-OG-D23O IR RACIOACT' POSTATTACK RECOVERY OF DAMAGED URBAN AREAS 1 BLAST DAMAGE-ASSESSMENT VULNERABILITY FIRES REPA I5-OG-D23O7
ATION OF SIX-FOOT AND TWO-FOOT SHOCK TUBES - EXPERINENTA/ BLAST EFFECTS ON BUILDINGS AND STZUCTURES - OPER IB-D3-D2ODI ATION OF SIX-FOOT AND TWO-FOOT SHOCK TUBES - EXPERINENTA/ BLAST EFFECTS ON BUILDINGS AVD STZUCTURES - OPER IB-D3-D2DOI
ATION OF SIX-FOOT AND TWO-FOOT SHCCK TUBES - EXPERIMENTAI BLAST EFFECTS ON BUILDINGS AVD STRUCTURES - OPER I8-OS-O2OOS TY-MISSILES BLAST-ENERGIZED-MISSILES VELOCITY GLASS STONE BLAST EXPERIMENTAL-DATA INSTRUMENTATION THEORY , IB-D3-O23I8 MECHANICS) BUILDINGS FAILURE (MECHANICS) EXPERIMENTAL-DATA BLAST FIRES) \#URS-658-3/(SIYULATIONS) LOADINGI I B-O3-QI995 1 ARCH REL ATED TO EMERGENCY PLANNING I, SCIENTIFIC-RESEARCH BLAST FIRES INITED-STATES-GOVERNMEVT OFFICE-OF-, I5-O3-O22 99 IYNAMICS SHEL TERS UNDERGROUND-STRLCTURES SAND SHOCK-WAVES BLAST FOOTINGS FOUVDATIONS VOID-RATIO ARCHES I I $20-11$-O234I MAGE-ASSESSMENT FIRE- SAFE TY PROGRAMMING (COMPUTERS) DEBRIS BLAST IGNITION, *AD 625 867 /LEAR-EXPLOSIONS DA I5-O3-OI952 INSE OF EQUIPMENT LOCATED IN UNDERGROUND INSTALLATIONS TO BLAST INDUCED GROUND MOTIONS 1 UNDERGROUND-STRU/ I3-I3-O229I OGY B IOLOGICAL-BL AST-EFFEC TS EXPERIMENTAL-DATA THECRY MA/ BLAST INCUCED TRANSLATIONAL EFFECTS I BLAST-BIOL D6-2I-D2239 IING COSTS I TEXT AND APPENDIX A I INDUSTRIAL-EOUIPMENT BLAST INDUSTRIES SURVIVAL RECJ VERY EZONOMIC-REC I D5-O3-O2IS2 IOT ION OF SUB SURFACE SOIL INCLUSICNS SUB JECTED TO SURFACE BLAST LOADING - RESULTS OF SERIES III TESTS 1 SI OB-I3-QITLO IOTION OF SUBSURFACE SOIL INCLUSIONS SUB JECTED TO SURFACE BLAST LOADING - RESULTS OF SERIES III IESTS I S, IB-D3-D2OOB IOT ION OF SUBSURFACE SOIL INCLUSIONS SUB JECTED TO SURFACE BLAST LOADING - RESULTS OF SER IES III TESTS I S/ I8-D3-D2OD8
PT IMUM SECTION OF REINFORCED CONCRE TE BEAMS SUB JECTED TG BLAST LCACS I DESIGN BEAMSI STRUCTURALI) LOADINGI/ I3-I3-OIBLO

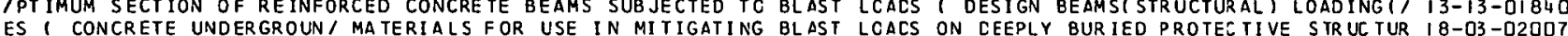
ES 1 CONCRETE UNDERGROUN/ MA TERIALS FOR USE IN MITIGATING BLAST LCACS ON CEEPLY BUR IED PROTECTIVE STRUC TUR I8-03-D2OD7

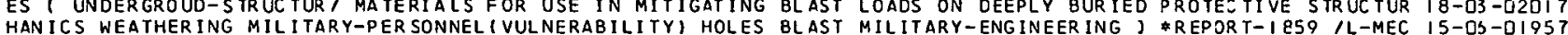
HAN ICS WEATHER ING MILITARY-PER SONNEL TVULNERABILITY) HOLES BLAST MILITARY-ENGINEER ING J *REPORT-I 859 IL-MEC I5-OS-QI957
ILE IN ASSESSING RESPONSES OF THE THORAX ENERGIZED BY AIR BLAST OVERPRESSURES OR BY NON-PENETRATING MISSI/ O6-21-O2235 IES PERSONNEL ATTACK-PATTERNS WIND YIELD BURST-CCNDITICNS BLAST RACIOACTIVE-FALLOUT FLOW-CHAZTIVG, \#REC, O5-O3-O2ILO IRE-SAFETY FIRES STR UCTURES SMOKES 8 UI LDINGS CCNST FUCTION BLAST RADIOACTIVE-FALLOUT HEAT-TRAVSFER EXPOSURI I3-I?-O22SQ IPL ICATION I RESOURCES VULNERABILITY NUCLEAR-WARFARE WIND BLAST RADIOACTIVITY THEORY TESTS EXPERIMENTAL-D/ O5-03-O2IL9 I BLAST-SHELTERS DESIGN COSTSI ENTRANCEWAYS AND EXITS FOR BLAST RESISTANT FULLY BURIED PERSONNEL SHELTERS I $3-I 3-D I B 35$ RS DESIGN MAT/ TIME COMPRESSION POTENTIAL OF AN EMERGENCY BLAST RESOURCES PRTGRAMMIVG-LANGIIATFS C.OMPIITFRS 05-03-02I54 IRS IN BUILDINGS I DYNAMICS CONPUIER-PROGRAMS SHOCK-WAVES BLAST SHIELCING STRUCTURAL-ANALYSIS RADIOAC TIVEI I3-I3-D22BO IUT PROTECTION-FACTOR DOSE-RATE BLILOINGS DECCNTANINATION BLAST SHIELOING THEORY MATHEMATICAL-AVALYSIS RAI DO-IS-D22BO /CTUREIMECHAN ICSI CRACK-PROPAGATI CN IMPACT-TESTS DYNAMICS BLAST SHOCK-WAVES ELASTICITY STRESS MECHANICAL-I $20-I 1$-O2338 ECTS BLAST-BIOLOGY SHOCK-TU/ DASA AEC LOVELACE FOUNDATION BLAST SIMULATION FACILITY I BIOLOGICAL-BLAST-EFF O6-2I-O2232 -INJURIES YIELD RADIOLOGICAL-DOSAGE WIND FIRES SIMULATION BLAST STATE-OF-TrE-ART-REVIEWS I *HI-693-RR /ION I5-OS-DI962 IHE DES IGN OF UNDERGROUND OPENINGS FOR DEFENSE ( SHELTERS BLAST STRESSES UNDERGROUND-STRUCTURES ROCKIGEOLI I3-I3-D2273 AST-BIOLOGY BIOLOGICAL-BLAST-EFFECTS SHOCK-TUBES LUN/ AIR BLAST STUCIES WITH EIGHT SPECIES JF MAMMALS I BL O6-2I -O223D WITZERS / CONCEPT AND FEASIBILITY STUDIES OF MUZZLE BRAKE BLAST SUPPRESSION DEVICES FOR IOSMM AND IS5MM HO IQ-OS-D2327 1 OW-CFART ING SURVIVAL RECOVERY ECONOMIC-RECOVERY PROOUCTS BLAST VULNERABILIIY AIR-BURST ECOVDMICSEXPLOSI/ O5-C3-D2I26 S EXPOSED TO AIR BLAST' THE RELATIONSHIP BETHEEN SELECTED BLAST WAVE PARAMETERS AND THE RESPOVSE OF MAMMAL O6-2I -O223 4

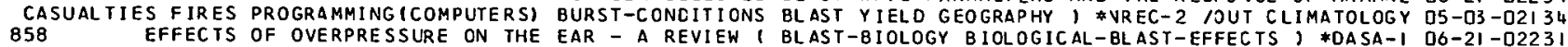
TAL-DATA TIEORY MAI BLAST INDUCED TRANSLATIONAL EFFECTS 1 BLAST-BIOLOGY BIOLOGICAL-BLAST-EFFECTS EXPERIMEN D6-2I-O2239 IETERS AND THE RESPONSE OF MAMMALSEXPOSEO TO AIR BLAST I BLAST-BIOLOGY BIOLOGICAL-BLAST-EFFELTS MONKEYS, O6-2I-D2234 
ES LUN/ AIR BLAST STUDIES hITH EIGHT SPECIES OF MANMALS 1 BLAST-BIOLOGY BIOLOGICAL-8LAST-EFFECTS SHOCK-TUB O6-21-02230 ICAL AND BIOLOGICAL PARAMETERS 1 BIOLOGICAL-BLAST-EFFECTS BLAST-BIOLOGY DISPLACEMENT BLAST-ENERGIZED-MISS/ D6-21-O2223 IION BLAST SIMULATION FACILITY I BIOLOGICAL-BLAST-EFFECTS BLAST-BIOLOGY SHOCK-TUBES INSTRUMENTATION PRESSI O6-21-02232 I AMBIENT PRESSURE ON TOLERANCE OF MAMMALS TO AIR BLAST 1 BLAST-BIOLOGY SHOCR-WAVES BIOLOGICAL-BLAST-EFFE I OG-2I-02236 /TED PRESSURES IN A SHOCK TLBE I BIOLOGICAL-BLAST-EFFECTS BLAST-BIOLOGY ShOCS-WAVES PRESSURE-TIME-MEASURE/ D6-2I-D2237 FECTS BLAST-8 IOLOGY DISPLACEMENT BLAST-ENERGIZED-NISSILES BLAST-BIOMEDICAL-CRITER IA I' \#DASA-I856 /BLAST-EF D6-21-02223
IRS I BIOLOGICAL-BLAST-EFFECTS BLAST-BIOLOGY DISPLACEMENT BLAST-ENERGIZED-MISSILES BLAST-BIOMEDICAL-CRITE/ D6-21-D2223 IRS 1 BIOLOGICAL-BLAST-EFFECTS BLAST-BI OLOGY DISPLACEMENT BLAST-ENERGIZED-MISSILES BLAST-BIOMEOICAL-CRITEI D6-2I-D2223
IOF MISSILES FROM NUCLEAR EXPLOSIONS ISECONDARY-NISSILES BLAST-ENERGIZED-MISSILES VELOCIIY GLASS STONE B/ I8-D3-D23I8 L ENEINEER IVG UND-STRUCTURES CONSTRUC TION URBAN-AREAS PRCTECTION-FACTOR BLAST-SHELTERS COSTS, \$USVRDL-TR-582, UNDERGRO I3-I3-DI8I5 NG ALBEDO

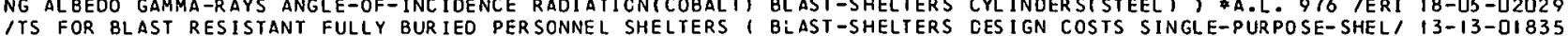
/FUELS COOL IVG-AND-VENTILATING-EQLIPMENT FALLOUT-SHELTERS BLAST-SHELTERS EVAPORATORS COSTS EVVIRONMENTAL-/ I3-DI -0I773 N NAT/ AREA WIDE SHELTER SYSTEMS I FALLOUT-SHELTERS COSTS BLAST-SHELTERS MAVAGEMENT-PLAVNIVG SITE-SELECTIO I3-I3-DI838 IONS-EFFECTS MODELS EXPLOSION-EFFECTS RADIOACTIVE-FALLOUT BLAST-SHELTERS MATHEMATICS PRJGRAMMINGICOMPUTER/ I5-03-OI938 / CIVIL-DEFENSE-SYSTEMS WEAPONS-EFFECTS EXPLOSION-EFFECTS BLAST-SHELTERS RADIOACTIVE-FALLOUT VUCLEAR-WARF/ I5-O3-DI933 /ATED ABOVE AND BELOW GROUNO I FALLOUT-SHELTERS CCNPUTERS BLAST-SHELTERS TEMPERATURE-CONTROL SOIL-MECHANI/ I3-DI - OI776 IEO NUCLEAR WEAPONS EFFECTS I STRLCTURES FALLOUT-SHELTERS OLAST -SHELTERS THERMAL-RAOIATION IFOR PROTECTION FROM NUCLEAR BLAST I SHELTERS SHOCK-WAVES BLAST -SHELTERS UNDERGROUND-STRUCTURES DYNAMICS / I3 YIELDING MEMBRANE ELEMENTS IN PROTECTIVE CONSTRUCTION I BLAST-SHELTERS UNDERGROUND-STRUCTURES DYNAMICS S I3-I3-DIB37 ENG INEER ING STRESSES GRANITE OPENINGS BLOCK-CAVING-NINING OLASTING, \$A UL I2DO IFAIIUREIMECHAVICSI MINING- O8-OT-O22 3

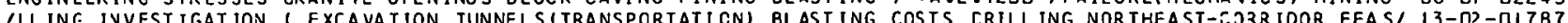
1I COUNTY, COLORADO I CONSTRUC II ON UNDERGRCUND-STRUCTURES BLASTINE EXCAVATIOV REINFORCED-COYCRETE ROCK-ST/ I3-13-D2274 27332 A NATHEMATICAL MODEL OF FLASH BLINDNESS I VISION AVIATION-MEDICINE EYEI IAD 6 DG-OS-DITO6 E(MECHANICS) MINING-ENGINEERING STRESSES GRANITE OPENINGS BLOCK-CAVING-MINING BLASTING ) \#A.L. I200 /FAILUR O8-O7-02243 GRAMM ING) CATA-PROCESSING-SYSTEMS MULTIVARIATE-ANALYSIS, BMC BIONECICAL COMPUTER PROGRAMS I CJMPUTERSIPRO O6-O3-O22OL E-DEFENSE SYSTEMS MILITARY-STRATEGY, \#HI-729, WHY CCUPLE BMC TO FALLOUT SHELTERS: URBAN-AZEAS ANTIMISSIL I5-D3-DI9O7 $1+T$ TO-20299

/IOLOEY) SURVIVAL FALLOUT-SHELTERS VENTILATION METABOLISM BOOY-TEN.PERATURE CLOTHING TEMPERATURE COOLING PI DG-IO-DITIO TABOL ISM PFYSIOLOEY REAT-EXCHANGE TEMPERATURE VENTILATICN BOCY-TEMPERATURE COOLIVG I \#A.L. IO5T /UPANCY ME O6-IS-DITI8 860 A DOCUMENTARY ON WEIGHT, OIET AND EXERCISE I BODY-WEIGHT PHYSICAL-FITNESS VUTRITION I *AD 633 O6-IH-DI7IO IS FEAR ITSELF - hHAT THE PEOPLE KNOW AND THINK ABCUT THE BONB ANC ATOMIC ENERGY I PUBLIC-OPINION ATTITUDI O5-II - I I OS -CASUALTI/ BIBL IOGRAPHY OF PUBLI SHED PAPERS OF THE ATOMIC BOMB CASUALTY COMMISS ION I JAPAN NUCLEAR-WARFARE DG-2I -D2224 65-JUNE-30, 1966 I RADIATION-EFFEC TS NUCLEAR-WARFA/ ATOMIC BONB CASUALTY COMMISSION ANNUAL REPORT JULY-I, IO D6-IB-DI73D ITEEIC MII ITARY BALANCE - LSA VS LSSR I MILI TARY-STRATEGY BONBERS ANT IMISS ILE-DEFENSE-SYSTEMS SPACE-WEAPO/ I5-OT-D23 O ION FOOD CHEI CIVIL DEFENSE HANDBCCK - USSR I RURAL-AREAS BONBS GUICED-MISSILES VUCLEAR-WEAPONS CONTAMINAT IS-D3-D22 IHESUS MONKEYS EXPOSED TO A SMALL PARTICLE AEROSOL OF THE BORG STRAIN ( BIOLOGICAL-WARFARE-AGENTS MIYAGAW/ I5-O2-DIBSO 1 ROAC AND VAN NESS STREET I TRANSPORTATION SUBWAYS TESTS BORING WASHINGTON CONSTRUCTIOV SETTLEMENTISTRUC, I $3-$ D2-DITBS OF POXVIRUSES FOR THE CYNOMOLGLS MONKEY, NACACA IRUS/ AIR BORNE INFECTIVITY OF THE VARIJLA VACCINIA GROUP I5-O2-OI855 FIC DES IGN COSTS NEW-YORK CHICAGO hASHINGTON PHILACELPHIA BOSTON LCNCON, \$HI-820-0/MJVING-BELTWAYS TRAF $23-02-02097$ URBAN-AREAS MANAGEMENT-PLANNING URBAN-PLANNING WASHINGTON BOSTON NEW-YORK, 1 RPN-79 /ADS 1 TRANSPOR TATION $23-02-02 I D D$ O PURIFIED / STUDIES ON IMMUNITY TO TOXINS OF CLOSTRIDIUM BOTULINUM VIII. IMMUNOLOGICAL RESPONSE OF MAN T IS-O2-OIBS7 TRICIUM-BOTUL INUM TOXINS- 1 PROCEEDINGS OF A CCNFERENCE ON BOTULINUM TOXIN 1 BIOLOGICAL-WARFARE-AGENTS CLOS I5-02-DIB6I 1. IMMUVOLOGICAL RESPONSE OF NAN TO PURIFIED BIVALENT AB BOTULINUM TOXOIC I BIOLOGICAL-WARFARE-AGENTS TO/ I5-O2-OI857 ISTEMS FLUID-DYNAMIC-PROPERTIES DRAG SURFACE-TEMPERATURES BOUNOARY-LAYER LAMINAR-BOUNDARY-LAYER I \$R-273 $/ 22-02-02350$ C CAS-FLOW PLASMA-MEDIUM PLASMA-OSCILLATIONS STABILITY BOUNDARY-VALUE-PROBLEMS \#UCLA-R-IO \$AD O48 DB $20-09-02335$ SC. PUB. N/ BUNKER TYPE FALLOUT SHELTER FCR BEEF CATTLE ( BOVINES SHIELDINE CONSTRUCTIOV VEVTILATION I \$MI I3-I3-OIBIB IH OF A MACHINED RING STIFFENED CYLINDRICAL SHELL I MODEL BR-7M I UNDER HYCROSTATIC PRESSURE I STIFFENED- 2 . 20-11-02347 IACFMENTS IVIER IOI CONTR IBUTION TO THE ANALYSIS OF MUZZLE BRAKE DESIGN I ART ILLERY COMPUTERS GUN-BARREL-AT I Q-DS-O2329 OIL-MECHAN ISMS IN TERIOR-BALLISTICS) \$AD 11142 MUZZLE BRAKES II. THEORY G GUN-AUXIL IARY-EOUIPMENT REC I9-OS-D2328 IRT BIBL IOGRAPHY I BEEF PRESERVATICN SHELF-LIFE BEVERAGES BREAD CANNED CEREALS COFFEE CORN DEHYDRATED-FOO/ OO-O3-O22D9 AS-I/ INVESTIGATION OF THE MECHANISMS ASSOCIATED WITH GAS BREAKDOWN UNDER INTENSE OPTICAL ILLUMINATION 1 G 20-05-02060 IULAR DISTRIBUT ION OF NEUTRONS AND GAMMA RAYS - OPERATION BREN ( IONIZING-RADIATION-SCATTER IVG I *CEX-62. $20-08-02334$ IEN THE HOLLAND TLNNEL AND THE MANHATTAN AND WILLIAMSBURG BRIDGES AND ALTERVATIVE SOLUTIONS THEREFORE I U/ $23-02-02357$ IIVE STRUCTURE AND COMPONENTS ( GLIDED-MISSILES RAILROADS BRIDGES GROUNO-SUPPORT-EQUIPMENT RAILROAD-TRACK/ IO-OI -OI9T2 ITE SCHOLARS AND ACADEMIC CENTERS - WESIERN EUROPE, GREAT BRITAIN AND CANACA 1 BIBL IOGRAPHIES FRANCE GERM/ O5-II-DI694 AMICS PARTICLE-SIZE-DISTRIBUTION AEROSOLS REYNOLOS-NUMBER BRCWNIAN-MOVEMENT EQUAT IONS ) \#NRDL-TR-67-49 /YN 20-O4-02333 E, 60-CYCLE ( MODEL CEIIVAL6-230 I AND 208 / NCNINAL 9,DOD) BUCKL INGIMECHANICSI MATHEMATICAL-AI

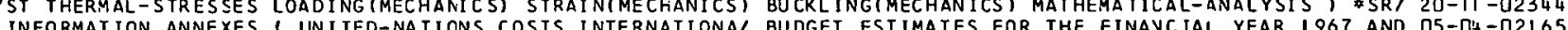
INFORMATION ANNEXES 1 UNI TEO-NATIONS COSTS INTERNATIONA/ BUDGET ESTIMATES FOR THE FINAVCIAL YEAR I 967 AND O5-D 4 -021 65 ESS MANAGEMEVT-EV/ COST ANALYSIS FOR PLANNING PROGRAMMING BUCGETING COST BENEFIT STUDIES 1 COST-EFFEC TIVEN O5-DI -D2292

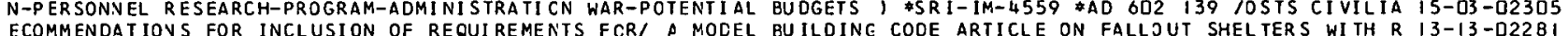
ECOMMENDATIONS FOR INCLUSION OF REQUIREMENTS FCRI D MODEL BUILDINE CODE ARTICLE ON FALLOUT SHELTERS WITH R I3-13-D22BI DING-CODES REGULATIONS WASHING TON BUILDINGS STRUCTURES I/ BUILDING CODE OF THE DISTRICT OF COLUMBIA I BUIL I3-I3-DI8I6 INGTON BUILDING-COOES REGULATIONS STRUCTU/ MANUAL FOR THE BUILOING CODE OF THE DISTRICT OF COLUMBIA I WASH I3-I3-OI8I7
FOR FALLOUT SHELTER CONSTRUC TION IN FOUR NATIONAL MODEL BUILDING CODES IONSTRUCTION DUAL-PURPOSE-SHEL/ I3-I3-O228I URBAN BUILCINGS IN THE UNITED STA TES I CONSTRUCTICA URBA/ BUILDING MATERIALS AS COMMONLY USED IN EXISTING I3-I3-O2277 TRUCT ION URBAN-AREAS TUNNELSITRANSPORTAI EXTENSIVE STREET BUILDING PROGRAM IN HAMBURG 1 GERMANY ROADS CONS 13 -0? - DI786 IE BUILDING CODE OF THE DISTRICT OF COLUMBIA I WASHINGTON BUILDING-CODES REGULATIONS STRUCTURES BUILDINGSI I3-I3-OIBI 7 STRUCTURES / BUILOING CODE OF THE DISTRICT OF COLLMBIA 1 BUILOING-CODES REGULATIONS WASHINGTON BUILDINGS I3-I3-DI8I 6 LUMEIA ' WASHINGTON BUILDING-CODES REGULAIIONS STRUCTURES EUILDINES, \#A.L. IODI IOE OF THE DISTRICT OF CO I3-I3-OIBI IEVALUAT ION OF COMPUTER ANALYSIS PROGRAMS FOR SHELTERS IN BUILOINGS I DYNAMICS COMPUTER-PROGRAMS SHOCK-WAI I3-I3-O22BO FIRES VUCLEAR-EXPLOS $15-13-02280$ IONS CAMAGE-ASSESSMENT FIRE-SAFETY PROGR/ RE-USABILITY OF BUILDINGS AFTER A WARFARE I FIRES VUCLEAR-EXPLOS IS-O3-OI9S2
AND TWO-FOOT SHOCK TUBES - EXPERIMENTAI BLAST EFFECTS ON BUILDINGS AND STRUCTURES - OPERATION OF SIX-FOOT IB-D3-O2DOI AND TWO-FOOT SHOCK TUBES - EXPERIMENTA' BLAST EFFECTS ON BUILDINGS AND STRUCTURES - OPERATION OF SIX-FOOT IB-D3-O2DOI
AND TWO-FOOT SHDCK TUBES - EXPERIMENTA' BLAST EFFECTS ON BUILDINGS AND STRUCTURES - OPERATION OF SIX-FOOT IB-D3-O2OOS

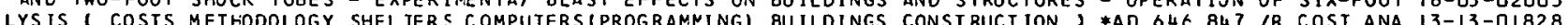
IS I FALLOUT-SHELTER S FIRE-SAFETY FIRES STRUCTURES SNOKES BUILOINGS CONSTRUCTION BLAST RADIDACTIVE-FALLOU/ I3-I2-D22 S9 ICERT IFIED FALLOUT SHELTER ANALYSTS I RADIOACTIVE-FALLOUT BUILDINGS CONSTRUCTION DESIGN SHIELDING CONSTRU/ I3-I3-O2276 IIPLIER I RADIOACTIVE-FALLOUT PROTECTION-FACTOR DOSE-RATE BUILDINGS DECONTAMINATION BLAST SHIELDING THEOR/ D6-IB-D222I ICTOR CAL CULATIOVS MODEL SISIMULATIONS) LOADING (MECHANICSI BUILDINGS FAILUREIMECHANICSI EXPEZIMEVTAL-DATA I I8-03-OI995 /SHIP OF PRESSURE STRENGTH, SI ZE AND COST OF NULTIFURPOSE BUILCINES FOR A FEDERAL TRANSPORTATION ADMINIST/ I3-I3-OIB27 BA' BUILDING MATERIALS AS COMMCNLY USED IN EXISTING URBAN BUILDINGS IN THE UVITED STATES 1 CONSTRUCTION UR I3-I3-D2277 ON SWEDEN, \#A.L. III8 SHADOW GEOMETRY OF BUILDINGS IN URBAN AREAS I FIRES THERMAL-RADIATI I3-12-02268 IK CITY ANC VIC IN ITY URBAN-AREAS UNDERGROUNO-STRLCTURES BUILDINGS MODELS (SIMULATIOVS) HEAT-EXCHANGERS T/ I3-I3-0I8I 4 ATIONS WASHINGTON BUILDINGS STRUCTURES I WA PL TRICT ONSTRUCTION-MA TERIALS RADI TACTE RE TRICT OF COLUMB IA I BUILOING-CODES REGULATIONS WASHINGTON BUILDINGS STRUCTURES I*A.L. 995 TODE OF THE DIS I3-13-0I8I6
FIRE-PROTECTION SMOKE, SHELTER HABITABILITY IN EXISTING BUILDINGS UNDER FIRE EXPOSURE I FALLOUT-SHELTERS I3-I?-OI8O7

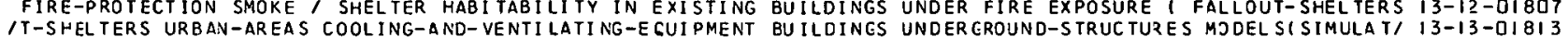
TES AS OF DEC 3 I IOSO NUCLEAR-PCHER-P/ RUCLEAR REACTORS BUILT, $.31,1966$ ( NUCLEAR-POWER-P/ NUCLEAR REACTCRS BUILT, BEING BUILT, OR PLANNEC IN THE UNITED STATES AS OF DEC I8-O5-D2320 
LLETIN $35(6)$

THE SHOCK AND VIERATION BULLETIN I IMPACT-TESTING SHOCKIMECHANICSI) \#BU 20-11-02077 UM-ANALYZERS TRAN SOUCERS MATHEMA / THE SHOCK AND VIERATION BULLET IN 1 INSTRUMENTAT ION ACCELEZOMETERS SPECTR $20-11$-O2O7S ELAST ICITY BEAMSI STRUCTURAL) SHO/ THE SHOCK AND VIERATION BULLET IN I ROCKETS DYNAMICS STRUCTURAL-ANALYSIS 2O-II -D2OTL S STRESSIPHYSIOLOGY) A/ SUPPLENENT TO SHOCK AND VIERATION BULLETIN 1 TOLERANCESIPHYSIOLOGY) MOTION-SICKNES D6-I7-OI735 R-TRANSPORTATION RAILROADS ARMED/ THE SHOCK ANO VIERATION BULLETIN I TRANSPORTATION VIBRATION-ISOLATORS AI 2O-II-O2OT6 VINES SHIELDING CONSTRUCTION VENTILATION, \#MISC. PUB. N/ BUNKER TYPE FALLOUT SHELTER FJR BEEF CATTLE I BO I3-I3-OIBI8 RATORS POWER-SU/ ICO WATT THERMOELEC TRIC POWER SYSTEN FCR BU OY APPLICAT IONS I THERMOELECTRICITY BUOYS GENE ID-O2-DI76I IC POWER SYSTEM FOR BUDY APPLICATIONS I THERMOELECTRICITY BUOYS GENERATORS POWER-SUPPLIES ELECTR IC-POWER-I IO-OZ-DI7SI CONSTRUCTION COST TRENDS 1 UNITED-STATES BUREAU-OF-RECLAMATION, $\$ A . L$. II57(1), I3-13-02288 CONSTRUCTION COST TRENDS ( UNITED-STATES BUREAU-OF-RECLAMATION) \$A.L. 115712 ( 13 (3-13-02284 CONSTRUCTION COST TRENDS I UNITED-STATES BUREAU-OF-RECLAMATION; *A.L. $1157131,13-13-02284$ CONSTRUCTION COST TRENDS 1 UNITED-STATES BUREAU-OF-RECLAMATION, \#A.L. $1157(4), 13-13-02285$ CONVEYANCE TUNNELS - GRAVITY AND PRESSURE I UNITED-STATES BUREAU-OF-RECLAMATION, \$A.L. IIS8 /ATA - WATER I3-I3-02283 PILOT STUOY I SHELTERS UNDERGRO/ THE ELASTIC RESPCNSE OF BURIEO CYLINDERS, CRITICAL LITERATURE REVIEW ANO I3-I3-OIB25 N COSTSI ENTRANCEWAYS AND EXITS FOR BLAST RESI STANT FULLY BURIED PERSONNEL SHELTERS I BLAST-SHELTERS DESIG I3-I3-OI835 UN/ MATER IALS FOR LSE IN MITIGATING BLAST LOADS CN DEEPLY BURIED PROTECT IVE STRUCTURES 1 COVCRETE UNDERGRO I8-DS-Q2DO7 UR/ MATER IALS FOR USE IN MITIGATING BLAST LOADS ON DEEPLY BURIED PROTECTIVE STRUCTURES I UNDERGROUD-STRUCT I8-O3-O2OI 7 C AND DYNAMIC LOADING I DUCTS SAND UNDERGR/ RESPONSE OF A BURIED PROTOTYPE COMMUNICATIONS CONDUIT TO STATI I3-II -D22 OI D-STRUCTURES OYNAMICS LOADIN/ STA IIC AND DYNAMIC TESTS OF BURIED UNREINFORCED COVCRETE ARCHES I UNDERGROUN I3-I3-0I83D IFECTS HIROSHIMA NAGASAKI GRAPHS BIOLOGICAL-BLAST-EFFECTS BURNS RADIATION-INJURIES SHIELDING URBAN-AREAS / D6-2I - O2227 /(PREDICTION) URBAN-AREAS DISTRIBLTION HIROSHIMA NAGASAKI BURNS WEAPONS-EFFECT RADIATIOV-INJURIES STATIST/ DO-2I -D2238 RADIONUCLIDE FRACTIONATION IN OEBRIS FROM A LAND SURFACE BURST I RADIOACTIVE-ISOTOPES CHINA RUSSIA RADIOA I8-D?-OIQ9I CTS/ EFFECT OF WATER CONTENT, YIELD, MEDIUN, AND DEPTH OF BURST ON CAVITY RADII I CRATERING EXPLOSION-EFFE IB-O3-D23I5 H 1 WIND YIELD RADIOACTIVE-FALLOUT PROGRANMING ICONFUTERSI BURST-CONDITION MATHEMATICAL-AVALYSIS I \#NREC- 9 I8-O8-O2322 H W IND YIELD RADIOACTIVE-FALLOUT PROGRANMINGICONFUTERSI BURST-CONOIT ION MATREMAT ICAL-AVALYSIS I \$NREC- 9 I 8-OB- 2322

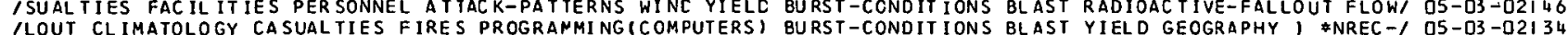

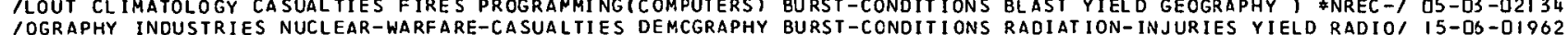
IOGRAPHY INDUSTRIES NUCLEAR-WARFARE-CASUALTIES DEMCGRAPHY BURST-CONDITIONS RADIATION-INJURIES YIELD RADIOI I5-OG-OI962
IERNING URBAN VULNERABILITY TO FIRE FROM NUCLEAR BURSTS I BURST-CCNDIT IONS IJRBAN-AREAS FIRES VUCLEAR-EXPL/ I3-I?-OI8OI IERNING URBAN VULNERABILITY TO FIRE FROM NUCLEAR BURSTS 1 BURST-CCNDITIONS IJRBAN-AREAS FIRES VUCLEAR-EXPLI I3-I?-OI8OI
IETERS GOVERVING URBAN VULNERABILITY TO FIRE FRON NUCLEAR BURSTS 1 BURST-CONDITIONS URBAN-AREAS FIRES NUC, I3-I?-OI8OI I MODEL S (SIMULA TIONS) TRANSPORTATI ON AIR-TRANSPORTATI ON BUS-TRANSPORTAT ION RAIL-TRANSPORTATION HIGHWAY-/ $23-02$-O2OSI :STICS, AND COATED FABRICS \#WT-407 OPERATION BUSTER - THERMAL RADIATION EFFECTS ON PAINTS, PPL I3-12-O22G3

$C$

THE BEE GENUS CALL IOPSIS AND THE BIOLOGY AND ECOLOGY OF C. ANDRENIFORMIS - HYMENOPTERA, AVDREVIDAE I BE, DG-DG-DITOS S I STORAGE-BATTERIES ALKALINE-CELLS AIRCR/ SEALED NICKEL CACMIUM RATTERIES FOR AIRCRAFT ELEC TZICAL SYSTEM ID-O3 - IISL INONL INEAR-SYSTEMS AMMONIUM-COMPOLNDS FOCUSING PHOSPHATES CALCITE POLARIZATION, \$ML-14D5 \$AFOSR-66-0490, $20-05-02067$ PLATE SUBJECT TO THE TI EQUATIONS AND COMPUTER PROGRAM TO CALCULATE THE THERMAL HISTORY OF A DUAL LAYEREO I3-I?-UIT9? NNELS I PROPAGATION SWEOEN, \#REPORT NO. IO/ NOMOGRAM FOR CALCULATING ATTENJATION OF AIR SHOCK WAVES IN TU $20-04-02051$ N CONTROLLED FISS ION PRODUCT ABSORPTION DLRING FALLOUT/ A CALCULAT IONAL MOCEL FOR CONDEVSED STATE DIFFLSIO I8-O8-O2D37 IERY REQUIREMENTS, STRUC TLRES AND OPERATICNS RESEARCH I. CALCULAT IONAL TECHVIQUE FOR DETERMINIVG IMPORTA/ DO-I3-O22IB /-EQU IPMENT IMPAC T-SHOCK SHOCK (MECHANICS) INSTRUMENTATION CALIBRATION ANALOG-COMPUTERS FOURIER-ANALYSIS I/ I4-D2-DIB 2 OWING GENERAL LOCATION OF PROJECTS I FLOODS FLOOD-CONTROL CALIFORNIA, *A.L. IDBI /ORK PROPJSED ANO MAP SH I3-D2-OIT83 GENERAL LOCATION OF RIGHTS OF hAY TO BE ACQUIRED I FLOODS CALIFORNIA; $\$$.L. 9B7/ES, CROSS SECTIONS, AND I3-O2-DI779 / GAT ION OF AL TERNATIVE AQLEDUC T SYSTEMS TC SERVE SCUTHERN CALIFORNIA - FEATHER RIVER AVD DEL TA DIVERSION I I3-O2-O2258 OWING GENERAL LOCATION OF PROJECTS I FLOODS FLOOD-CONTROL CALIFORNIA CONSTRUCTION COSTS I. *A.L. IOT9 IP SH I3-02-OI784 OWING GENERAL LOCATION OF PROJECTS I FLOODS FLOOD-CONTROL CALIFORNIA FINANCING CONSTRUCTION; $\$$ A.L. IOBD, I3-O2-OI782 STATTACK-OPERATIONS POPULATION SURVI VAL DAMAGE-ASSESSMENT CALIFORNIA RECOVERY, \$SRI-MU-4949-350-A /ERY PO OS-03-DI639 STATTACK OPARATON ISICONTROL DRA INAGE COUNTERMEASURES URBAN-AREAS SAN-JOSE CALIFORNIA RECOVERY PEST-CONTROL I \#USNRDL-TRC-I I3-O2-DI7BO IPERATIONS FOOD-DISPENSING DISTRIBUTION DAMAGE-ASSESSMENT CALIFORNIA URBAN-AREAS SUPPLY-DEPOTS I \#SRI-MU-I O6-OB-DI7II
RENIFORMIS - HYMENOP TERA, AN/ A REVISION CF THE BEE GENUS CALLIOPS IS AND THE BIOLOGY AVD ECOLOGY OF C. AND O6-OS-OITOS RENIFORMIS - HYMENOP TERA, AN/ A REVISION CF THE BEE GENUS CALLIOPS IS AND THE BIOLOGY AVD ECOLOGY OF C. AND OO-OS-DI7OS IED IN WOOD EXPOSED TO HIGH INTENSITY THERMAL RADIATION I CALORIMETERS IGN ITION HIGH-TEYPERATURE-RE SEARCH' I3-I?-OI8O2 ISTRIBUTION IN AN ARC IMAGING FURNACE T THERMAL-RACIATION CALORIMETERS LABORATORY-FURNACES RADIOMETERS HEI I3-DI - D225S I CRM-GENERATORS INSTRUMENTATION IRANSDUCERS OSCI LLCSCOPES CAMERAS REFLECT ION REFRACTION WAVE-TRANSMISSION/ D8-II-O2247 T MEDICAL-SUPPLIES NUCLEAR-WARFARE-CA SUALTIES STOCKPILING CANADA *A.L. JO69 /DM IN ISTRATIOV-AVD-MANAGEMEN D6-2I -QI739 RK GREECE SWEDEN SWITZERLAND IRELAND USSR INDIA AUSTRALIA CANADA, \$HI-272-D /GERMANY BENELUX NORWAY DENMA IS-O3 -O2I 47 IAND ACADEMIC CENTERS - HESTERN ELROPE, GREAT BRITAIN AND CANADA I BIBL IOGRAPHIES FRANCE GERMANY ITALY I I O5-II-OIG94 T-DUT IES FOREIGN-TRADE C' INTERNA IIONAL CUSTONS JOLRNAL - CANADA I LAW INTERNATIONAL-AFFAIRS TARIFFS IMPOR O5-O3-OI G4I CANADIAN WHEAT MARKE TING ( FOOD-MARKETING CANADA AGRICJLTURAL-ECONOYICS, $*$ FA S-4-14L ORAGE SALES REVENLE UTILI ZATION FINANCING CPERATING-COSTS CANADA CONSTRUCTIOV PRICES LABOR I *A.L. IDI7 II OS-03-OI642 UBL ICATIONS 1 DISASTER-MEDICINE ENERGENCY-HEALTH-SERVICES CANADA MANUALS FILMS I \#A.L. IO68 CATALOGUE OF P O6-2I -DIT37 MOSPHERIC-PRECIPITATION HATER-SUPPLIES FOOD UNITED-STATES IHEALTH LAW ORGAN IZATION CIVIL-DEFENSE

CANADA MEXICO, \#IID-23286 /ISOTOPES AIR MILK AT 18-03-02035 CANADI NUCLEAR-W

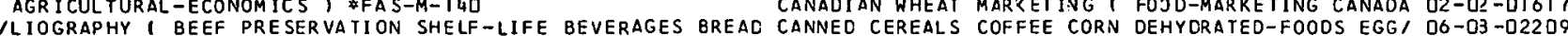

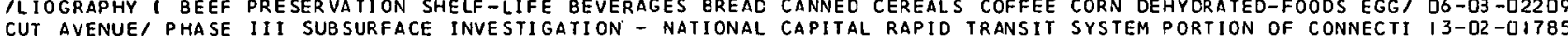
CUT AVENUE/ PHASE II I SUBSURFACE INVESTIGATION - NATIONAL CAPITAL RAPID TRANSIT SYSTEM PORTION OF CONNECTI I3-02-OI785 ANNING OPERAT IONS-RESEARCH ECONOMICS DECISI PLANNING IN A CAPITAL SURPLUS ECONOMY - KUWAIT 1 MANAGEMENT-PL O5-O3-OI 63S EM - MANUAL OF DESIGN CRITERIA T LRBAN-AREAS I NATIONAL CAPITAL TRANS PORTATION AGENCY RAPID TRANSIT SYST 23 -Q2 - D2353
IIES SIMULATION FIRE-SAFETY TOXICITY HEAT CARBON-MCNOXIDE CARBON-CIOXIDE AIR SMOKES WIVD SHEL TERS GERMANYI I3-I?-OIBDD IIES SIMULATION FIRE-SAFETY TOXICITY HEAT CARBON-MCNOXIDE CARBON-CIOXIDE AIR SMOKES WIVD SHEL TERS GERMANYI I3-I?-OI8DD INS FIRES CASUALTIES SIMULATION FIRE-SAFETY TOXICITY HEAT CARBON-MONOXIDE CARBON-DIOXIDE AIR SMOKES WIND I I3-I2-DI8OD
IR FIRE EXPOSURE I FALLOUT-SHELTERS FIRE-PROTECTION SMOKE CARBON-NONOXIDE OXYGEN-SUPPLY GAS-DETECTORS FIR/ I3-IZ-DIBO? ITS CONTAMINATION RADIATION-IN JURIES TOXICITY CYANC-ACIDS CARBON-MONOXICE VESICANTS POISONOUS-GASES LEWISI OG-IB-DI72I /RBON-MOVOXIDE OXYGEN-SUPPLY GAS-DETEC TORS FIRE-DETECTORS CARBON-MONOXIDE-INDICATORS TEYPERATURE WIND-PREI I3-I?-OI8D PARATION AND SCOR ING I DATA-PROCESSING ME THODOLO/ PUNCHED CARD O SORTING - A MACHINE METHOD FOR Q DECK PRE OS-II -O2IBQ PERSONNEL HOSPITALS PUBLIC-HEALTH RESCU/ DISASTER MEDICAL CARE FILMS I DISASTER-MEDICINE MEDICINE MEOICAL- D6-21 - IITLD IION SYSTEMS - INTERACTIONS AND COMPARI SONS AMONG NODES 1 CARGO PCSTATTACK-OPERAT IOVS VULNERABILITY ROADSI I3-0S-O226O /ARGO POSTATTACK-OPERATIONS VULNERABILITY ROADS RAILROADS CARGO-VEHICLES AIRCRAFT WEAPONS-EFFECTS DAMAGE-I I3-DS-O226D FLUENZA 1 CEMOGRAPHY IMMUNOLOGY B IOLOGICAL-WARFARE-AGENTS CASUALTIES \$A.L. IO42 1, SMALLPOX, MEASLES, IN I5-OQ-QI869 / I DISASTER-MEDICINE EMERGENC Y-HEALTH-SERVICES HCSPITALS CASUALTIES ADMINISTRATION-AND-MANAGEMENT MEDICA $/$ DO-2I-DI739

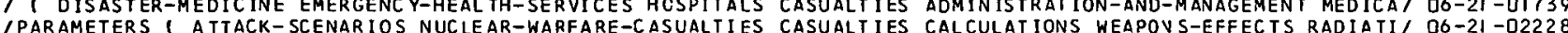
IPARAMETERS
IINE PUBL IC-HEALTH LAW ORGANI ZATI CN CIVIL-DEFENSE-SYSTEMS CASUALTIES CANADA NUCLEAR-WARFARE-CASUALTIES RA/ O6-2I - O222 6

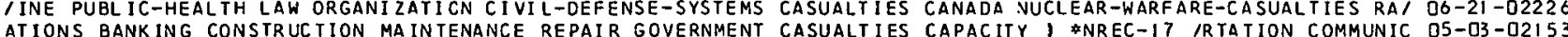
ATIONS BANKING CONSTRUCTION MAINTENANCE REPAIR GOVERNMENT CASUALTIES CAPACITY I \#NREC-17 /RTATION COMMUNIC O5-O3-O2153

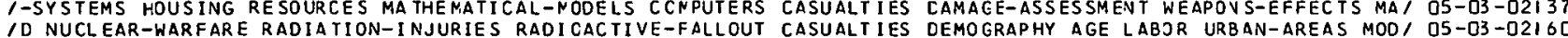
ID NUCL EAR-WARFARE RADIATION-INJURIES RADICACTIVE-FALLOUT CASUALT IES DEMOGRAPHY AGE LABDR URBAN-AREAS MOD I O5-O3-D2I 60 IPROGRAM I RESOURCES VULNERABILITY PROGRANMINGICOMFUTERSI CASUALTIES FACILITIES PER SONNEL ATIACK-PATIERNSI O5-O3-O2I 46
IITY WEAPONS-EFFECTS WIND RADIOAC TIVE-FALLOUT CLIMATOLOGY CASUALTIES FIRES PROGRAMMINGICOMPUTERSI BLRST-CI D5-O3-D2I 34 IITY WEAPONS-EFFECTS WIND RADI OAC TIVE-FALLOUT CLIMATOLOGY CASUALTIES FIRES PROGRAMMINGICOMPUTERSI BLRST-C / O5-D3-D2I 34 EALTH RESCUES VUCLEAR-WARFARE-CASLALTIES SCHOOLS SLRVIVAL CASUALTIES FIRST-AID 1 \#A.L. ID77 /TALS PUBLIC-H O6-2I-DI74 CE I RESOURCES DAMAGE-ASSESSMENT VLLNERABILITY PC FULATI ON CASUALT IES FOOD-SUPPL IES GEOGRAPHY I \#NREC-29 /N O5-D3-02I 32 I I MODELS DAMAGE-ASSESSMENT NUCLEAR-WARFARE-CASUALTIES CASUALTIES MILITARY-STRATEGY WEAPOYS-EFFECTS RAI DG-2I-D2229 /ENSE RECRUIT ING TRA INING WARFARE CI VIL-DEFENSE-PERSONNEL CASUALTIES MOBIL IZATION ARMED-FORCES-OPERATIONS/ I5-03-023OS / EMERGENCIES I EMOTIONS ATTITUDES FEAR STRESSIPSYCHOLOGY) CASUALTIES MOT IVAT ION PUBLIC-INFOZMATION TRAINI/ O5-IJ-DIGB /ALLOUT BLAST-SHELTERS MA THEMATICS PROGRANMING (COMPUTERS) CASUALTIES NUCLEAR-WARFARE-CASUALTIES I \$RTI-OU/ I5-03-DI938 
IANC-MANAGEMENT FIRST-AID ORGANI ZATION TRAINING HOSPITALS CASUALTIES PERSONNEL NUCLEAR-WARFARE-CASUALTIES/ O6-2I -0I738 I AN FIRE FATAL ITIES I FIRES URBAN-AREAS DAMAGE-ASSESSMENT CASUALTIES POPULAT ION FIRE-STORMS I \&DC-TN- IOSO/ I3-12-O2262 /S SYSTEM I RESOURCES MODELS DAMAGE-A SSE SSNENT PCPULATICN CASUALT IES PROGRAMMINGI COMPUTER SI GEOGRAPHY I \$ O5-03-02130 T MEDIC/ SURVIVAL ITEM ANALYSIS PROGRAM NUCLEAR-WARFARE CASUALTIES RESOURCES DAMAGE-ASSESSMENT FOOO LIGH O5-D3 - D2I 35 CARBON-1 NASS FIRE LIFE HAZARD I NUCLEAR-EXPLOSIONS FIRES CASUALT IES SIMULATION FIRE-SAFETY TOXICITY HEAT I3-I -OIBOO TVE-FALLOUT VUCLEAR-WARFARE-C YSTEMS-EVALUAT I CN CASUAL NUCLEAR-WAR/ SENSITIVITY ANALYSIS OF CASUALTY FUNCTICNS I CASUALTIES SYSTEMS-EVALUATION DAMAGE-ASSE SSMENT D6-2I -DIT4 2

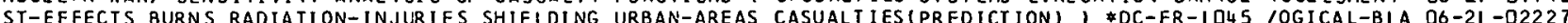
IANESE NUCLEAR CA SUALTY DATA I NUCLEAR-WARFARE-CASUALTIES CASUALTIES (PREDICT ION) URQAN-AREAS DISTRIBUTIONI O6-2I -02238 ALT II BIBL IOGRAPHY OF PUBL ISHED PAPERS OF THE AIONIC BOMB CASUALTY COMMISS ION I JAPAV VUCLEAZ-WARFARE-CASU DO-2I -02224 ALT NE-30, 1966 I RADIATION-EFFECTS NLCLEAR-WARFAI ATOMIC BCMB CASUALTY COMMISS ION ANVUAL REPORT JULYLTIES(PREDICTION) URBAN-ARE I ANALYSIS OF JAPANESE NUCLEAR CASUALTY DATA 1 NUCLEAR-WARFAZE-CASUALTIES CASUA D6-21 -D223 STICAL-ANALYSIS WEAPONS-EFF/ ANALYSIS OF JAPANESE NUCLEAR CASUALTY DATA I NUCLEAR-WARFARE-CASUALTIES STATI DO-2I-0222 UALTIES DAMAGE-ASSESSMENT PATIENTS PRI DAPO46 TIME PHASED CASUALTY ESTIMATE I ANALYSIS VUCLEAR-WARFARE-CAS O6-2I -0222 ON CAMAGE-ASSESSMENT NUCLEAR-WAR I SENSITIVITY ANALYSIS CF CASUALTY FUNCTIONS \& CASUALTIES SYSTEMS-EVALUAII O6-2I -OI74 2 S MATPEMAIICAL-MODELS COMPLITERS CIASUALTIESI RESOURCE DATA TEMS I \$MP-6 CIVIL DEFENSE MOTION PICTURE ERGENCY - HEALTH-SERVICES CANADA MANUALS FILMS ) \#A.L. 1068 ION I *MISC. PUB. N' BUNKER TYPE FALLOUT SHELTER FCR BEEF SHOCK TUBE I B IOLOGICAL-BLAST-EFFEC TS / THE TCLERANCE OF IT OF WATER CONTENT, YIELD, MEDIUN, AND DEPTH OF BLRST ON I GIANT PULSING METHODS IN RUBY LASERS ( PUMPING (OFTICAL) C CEI IVAL 6-230, ANO 208-VOLT, 3-PHA SE, 40D-CYCLE! NODEL
/ PACT, VERTICAL, $30-$ VOLT, SINGLE PHASE, OD-CYCLE, MOOEL /PACT, VERTICAL, 230-VOLT, SINGLE PHASE, 6D-CYCLE I MOOEL IS AS RELATED TO PHYSIOLOGICAL STATE OF ANTHRAX SPCRE AND FORMERS COILS CIR / CONTROL DEVICE FOR ELECTROLUMINESCENCE OF LTURE 1 AGRICUL TURAL-ENGINEERI / THE AGRICULTURAL RESEARCH ISOCIAL SCIENCE RESEARCH BY PRIVATE SCHOLARS AND ACADEMIC ISOCIAL SCIENCE RESEARCH BY PRIVATE SCHOLARS AND ACAOENIC ISOCIAL SCIEVCE RESEARCH BY PRIVATE SCHOLARS AND ACADEMIC /SOCIAL SCIEVCE RESEARCH BY PRIVATE SCHOLARS AND ACACEMIC /SOCIAL SCIENCE RESEARCH BY PRIVATE SCHOLARS AND ACAOEMIC ISOCIAL SCIENCE RESEARCH BY PRIVAIE SCHOLARS AND ACADEMIC ISOCIAL SCIEVCE RESEARCH BY PRIVATE SCHOLARS AND ACADENIC VICES 1 DISASTER-MEDICINE MEOICINE AD / ADVANCED TREATMENT I ICUL TURE URBAN-AREAS RURAL-AREAS COMMERCE MEAT RESOURCES IHY I BEEF PRESER VATION SHELF-LIFE BEVERAGES BREAD CANNED 1 OF PRCH ION SHOCK-WAVES KINE TIC - THE ORY ELECTRCN-CENSITY IRCED-CONCRETE ROCK-SIABILIZATION SHOTCRE TE ROCK-BCLTINGS ITROL OF SURFACE STORM WATER BY STORM DRAINS AND DRAINAGE ITROL OF SURFACE STORM WATER BY STORM DRAINS AND DRAINAGE ITROL OF SURFACE STORM WATER BY SIORM DRAINS AND CRAINAGE ITROL OF SURFACE STORM WATER BY STORM DRAINS AND CRAINAGE IAR EXPLOSIONS - PEACEFUL APPLICATIONS - CRATERS FROM ROW AS RURAL-AREAS COMMERCE MEAT REI HANOBOOK OF AGRICLLTURAL ELLFISH POISONS RELATED TO TOXICI IY I PELECYPODA TC/ SOME
IPMENT OF U-FOAM AIR FILTERS VENTI LATI ON FILTERS (FLUID) /PMENT OF U-FOAM AIR FILTERS I VENTILATI ON FILTERS (FLUIO) / ER-MEDICINE EMER GENCY-HEALTH-SER VICES BIOLOGICAL-WARFARE CIVIL-DEFENSE-SY STEMS DECON TAMINAJION BI OLOGICAL-hARFARE FALLOUT-SHELTER S BLAST-SHELTER S BIOLOGICAL-WARFARE-AGENTS IES PUR IFICATION CONTAMINATION RADIOLOGICAL-CCNTAMINAT ION /IVIL-DEFENSE-SYS TEMS RESCUES INTERNA TIONAL-CIVIL-DEFENSE IS CESTRUCIIION - USSR I PROTEC TION-SYSTEMS WATER-SUPPLIES PMENT AGR ICUL TURAL-CHEMISTRY I \#A OLO 996

FARM IENET ICS PHYSICS SCIENTIFIC-RESEARCH METALLURGY MATERIALS /FADIMESIN I BIOL OGICAL-WARFARE-AGENTS EFFECTIVENESS MICE C-HEALTH POSTATTACK-OPERATIONS IMNUNOLOG Y DI SEASE-VECTORS /GROUND FACIL ITY OF NORTH AMERICAN AIR DEFENSE CCNHAND AT TE NORMAL OISTR IBLTION I STATI STICAL-TESTS STATISTICI THE
IRBAN-AREAS MOVING-BEL TWAYS TRAFFIC DESIGN COSTS NEW-YORX IRBAN-AREAS MOVING-8ELTWAYS TRAFFIC DESIGN COSTS NEW-YORK IL VENT ILATION TEST OF A BASEMENT FALLOUT SHELTER IN EAST UL INUM TOXIVS-AND-ANTITJXINS PHARNOCOLOGY NUSCLES NONKEYS RAT ING-PLANS MENTAL-HEALTH POSTAT TACK-OPERATICNS TRAINING OPERATIONS MANAGEMENT-PLANNING STRES/ SPECIAL PROBLEMS OF TS - EIOMECICAL SCIENCE I BIBLIOGRAPHIES NEDICINE BIOLOGY ITE SCHOLARS AND ACADEMIC CENTERS - ASIA B BIBLIOGRAPHIES $N$ DEBRIS FROM A LAND SURFACE BLP.ST I RADI CACTIVE-ISOTOPES ENSE-SYSTEMS UNITED-STATES SUR VI VAL EUROPE COMNUNICATIONS ATURE I PROTECTIVE STRUC TURES AND COMPONENTS - SURVEYS OF ATURE I PROTECTIVE STRUCTURES AND COMPONENTS - SURVEYS OF E $($ B IBL IOGRAPHIES MEDICINE BIOLOGY CHINA ' J' CCNMUNIST ISS MICE CHEMOTHERAPEUTIC-AGENTS INFECTIONS STREPTCMYCINS /SYSTEMS TO SUPPORT INTRASTATE CIVIL CEFENSE OPERATIONS ' 1 LIGHTING-EQUIPMENT CONTROL-SYSTEMS TRANSFORMERS COILS AR OPENIVGS WITH STIFFEVED EDGES I STRUC TURA' ANALYSIS CF
MECHANICS LOADINGIMEC/ TRANSIENT LOADING TESTS ON A RIGIC BAN-AREAS MANAGEMENT-PLAN/ MOVEMENT TO SHELTER IN A MAJOR CIRCULAR FOOT ING 1 FOUVDATIOVSISTRUCTURES) SOIL- I3-I3-DI8I 2 POPULATION UTILITY-TUNVELS, *HUD-MP-39 SCIENCE AND THE CITY 1 URBAN-AREAS PUBLIC-HEALTH URBAV-PLANNING 23-D3-D2ID2 EL-GROUND-TRANSPORT/ NEW TRANSPORT TECHNOLOGY FOR THE BIG CITY I URBAN-AREAS TRAVSPJRTATION COSTS HIGH-SPE 23-DZ-D2352 I VENTILATIOV TESTS OF FALLOUT SHELTER SPACES IN NEW YORK CITY ANC VICINITY I FALLOUT-SHELTERS URBAN-AREAS I3-13-DIBI 3 I VENTILATION TESTS OF FALLOUT SHELTER SPACES IN NEW YORK CITY ANC VICINITY I URBAN-AREAS UNDERGROUND-STRU I3-I3-DIBI 4 IRANSPORTATION ADMINISTRATION, OFFICE OF THE MAYOR OF THE CITY OF NEW YORK AND FOR NEW YORK CITY TRANSIT I $23-02-02355$
IRAT ICE OF THE MAYOR OF THE CIIY, OFFICE OF THE MAYOR OF THE CITY OF NEW YORK AND FOR NEW YORK CITY TRANSIT 23 -02-O2355

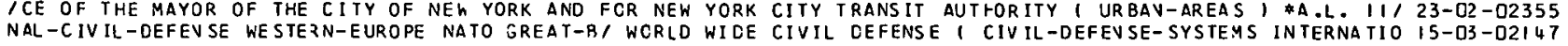


ECT ION RADIOLOGICAL-DOSAGE EXPOS/ RADIOLOGICAL HEALTH AND CIVIL DEFENSE I rEALTH-PHYSICS RADIOACTIVITY DET D6-I3-OZZ22 RIBUT ION VULNERABILITY LABOR ECO/ OCCUPATIONAL SKILLS AND CIVIL DEFENSE I INDUSTRIES RESOURCESIHUMANI DIST OS-D3-DIG3 UNCERGROUNC-STRUCTURES DOSE-RA TE PHOTONS I SHIELDING IV - CIVIL DEFENSE I RADIOACTIVE-FALLOUT ATTENUATION I8-DO-D2O2 CEFENSE-PERSON $/$ SOVIET MILITARY TRANSLATI CNS NUMBER I88 - CIVIL CEFENSE I RUSS IA TRAINIVG DISPER SAL CIVIL- I5-O3-OIQI3 ERNAT IONAL-CIVIL-DEFENSE COMMUNIST-CHINA INDUSTRI/ CRISES CIVIL DEFENSE ANC DETERRENCE I USSR MOVEMENT INT I5-O3-D2295 ERNAT IONAL-CIVIL-DEFENSE EARLY-WARNING-SYSTEMS USI CRISIS CIVIL DEFENSE ANC DETERRENCE 1 VULNERABILITY INT IS-O3-DI922 ITARY-STRATEGY INTERNATIONAL-CIVIL-DEFENSE RUSSIA/ CRISIS CIVIL DEFENSE ANC DETERRENCE I VULVERABILITY MIL IS-D3-DI896 FENSE-SYSTEMS AP I EXERPTS FROM CONGRESSIONAL TESTIMONY ON CIVIL DEFENSE ANC REL ATEC DEFENSIVE SYSTEMS I DE I5-O3-TI229L I CIVIL-DEFENSE-OPERATING-PLANS L.TILITIES DATA WATER-SU/ CIVIL CEFENSE ASPECTS OF MUNICIPAL WATER SYSTEMS I3-O?-OIT8 I C AW LAW 9201 LEGISLATION SOCIAL-SCIENCES HISTORY CIVILI U.S. CIVIL DEFENSE BEFORE 1950 - THE ROOTS OFF PUBLIC D5-OH -DIO7I FERENCES RELEVANT TO THE STUDY OF SOCIETAL RECOVERY FROM/ CIVIL DEFENSE BIBL IOGRAPHY - A COYP ILATION OF RE O5-IO-OIG77
LOCAL. STATE, AND REGIONAL LEVELSI COMNUNICATICNS-SYST/ CIVIL DEFENSE COMMUNICATIONS REOUIREMENTS AT THE I7-D?-O23I LOCAL. STATE, AND REGIONAL LEVELS I COMMUNICATICNS-SYST/ CIVIL DEFENSE COMMUNICATIONS REQUIREMENTS AT THE I7-D?-Q23I 2 RGENCY-HEAL TH-SER VICES 8 IOLOGICALI PROCEEDINGS OF NECICAL CIVIL DEFENSE CONFERENCE I DISASTER-MEDICINE ENE DS-2I -O2233 ITUDES POLIIICAL-SCIENCE PUBLIC-OPINION HISTORY COST/ THE CIVIL DEFENSE DEBATE I CIVIL-DEFEVSE-SYSTEMS ATT D5-IJ-OIOT9
S GUI DED-MISSILES NUCLEAR- HEAPONS CONTAMINATICN FOCD CHE/ CIVIL DEFENSE HANDBOOK - USSR I RURAL-AREAS BOMB IS-O3-O2296 REPORT FROM A RESEARCH PROGRAM I POSTATTACK-OPERATIONS V/ CIVIL DEFENSE IN POSTATTACK SJCIETY - A SLMMARY OS-II-D22JI TIITUCES PUBLIC-OPINION SLRVEYS DISAR/ ANERICANS VIEWS ON CIVIL DEFENSE IN THE COLD WAR CONTEXT - I966 I A O5-IJ-0IG78 AIN INTERNATIONAL-CIVIL-DEFENSE CIVIL-DEFENSE-SYSTEMS EA/ CIVIL DEFENSE IN THE UVITED KINGDOM I GREAT-BRIT IS-O3-O23OS INFORMATION CIVIL-DEFENSE-SYSTEMS, *MP-6 CIVIL DEFENSE MOTION PICTURE CATALOGUE I PUBLIC- I5-03-O23D4 IMUNICATIONS EQUIPMENTS AND SYSTENS TO SUPPORT INTRASTATE CIVIL DEFENSE OPERATIONS-CIRCA 19701 RADIO-CI I7-G?-D23I3 IHNOL OGY, AND TACTICS OF FIRE PROTECTION AS ENPLOYED 8Y A CIVIL DEFENSE ORGAVIZATIOV DUR ING WORLD WAR II $13-1$ ? -O2264 ANAGEMENT-PLANNING STRESI SPECIAL PROBLEMS OF CHILOREN IN CIVIL DEFENSE PLANNING POSTATIACK-OPERATIONS M DS-II-D22O

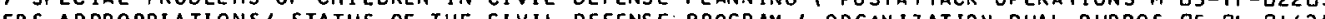
作 /C EAST WEST FREEWAY TUNNEL WE ST CRANGE, NEW JERSEY, AS A CIVIL CEFENSE PUEL IC SHEL TER FACILITY 1 TRANSPO/ $13-13-02271$ ERING RESEARCH-PROGRAM-AOMINI STRATION OPERATICNS-RESEARC/ CIVIL DEFENSE RESEARCH ANALYSIS ISYSTEMS-ENGINE I2-0? -0I76 7 N FIBERBOARD PHYSICAL-PROPERTIES COI STORAGE STABILITY OF CIVIL DEFENSE SHELTER RATIONS $/$ FOOD PRESERVATIO D6-O3-02212 ION DAMAGE CALCULATION - 1970-1985 I CALCULATIONS DAMAGE/ CIVIL DEFENSE SHELTER REPRESEVTATION FOR POPULAT IS-D3-OIO3D IFELTER SYSTEMS - AN ANNOTA TED BIBLIOGRAPHY OF U. S. NAVAL CIVIL ENGINEERINE LABORATORY PUBLICATIONS I FALI I3-I3-O2278 IIONS-SYSTEMS COMMAND-AND-CONTROL-SYSTEMS DECISI ON-MAKING CIVIL-DEFENSE-OPERATING-PLAVS ATTACK-SCENARIOS 1 I 7 -O2 -02312 I OF PUBLIC LAW 9201 LEGISLATION SOCIAL-SCIENCES HISTORY CIVIL-DEFENSE-OPERAT ING-PLANS CIVIL-DEFENSE-SYS/ OS-DI-DI67/ IN FOOD CHEMICAL-WARFARE-AGENTS BIOLOGICAL-WARFARE-AGENTS CIVIL-DEFENSE-OPERATING-PLANS CIVIL-DEFENSE-SYSI I5-03-02296 AININC, ,FG/ EMERGENCY CLOTHING (DISTRIBUTICN LOGISTICS CIVIL-DEFENSE-OPERAT ING-PLANS CLEAVIVG REPAIR TR IS-O5-023D6 1-OPERAT IONS RECOVER Y (WA TER-SUPPLIES) MANAGENENT-PLANING CIVIL-CEFENSE-OPERATING-PLANS DATA IDENIIFICATI I IS-D3-OI9'+I IL CONCEPT OF EMERGENCY OPERATIONS UNDER NUCLEAR ATTACK 1 CIVIL-DEFENSE-DPERATING-PLANS FALLOUT-SHELTERS I OS-DI -OIG23 NG SHELTER-OCCUPANCY POSTATTAC T-OPER/ EMERGENCY FEEDING I CIVIL-DEFENSE-OPERATING-PLANS FOOD FOOD-DISPENSI O6-OB-O22ID IS PUEL IC-INFORMATION REPAIR FILMS PROPAGANDA RURAL-AREAS CIVIL-DEFENSE-OPERAT ING-PLANS INDUSTRIESICIVIL-I I5-G3-02302 IRAINING FIRE-STORMS CONFLAGRA TIONS RESC UES VULNERABILITY CIVIL-CEFENSE-OPERATING-PLANS MAPP IVGIINFRARED-I I3-I?-DIBO6 ATTACK-OP/ EMERGENCY SOCIAL SERVICES I PERSONNEL BEHAVIOR CIVIL-CEFENSE-OPERATING-PLANS MENTAL-HEALTH POST OS-II -D2IR5

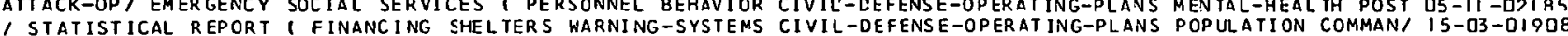

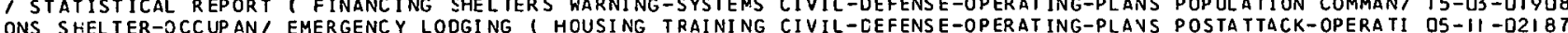
ONS SFELTER-OCCUPAN EMERGENCY LODGING I HOUSING TRAINING CIVIL-CEFENSE-OPERATING-PLAVS POSTATTACK-OPERATI D5-II -D2IB ION AND INQUIR T SHELTER-OCCUPANCY POSTATIACK-OPERATIONS CIVIL-DEFENSE-OPERATING-PLANS PREPARATION MANAGI O5-II -D2I B ISSMENT DATA DECISION-MAKING RECOVERY HOUSING FOOD FLOOOS CIVIL-DEFENSE-OPERATING-PLANS REPAIR CONSTRUCTI/ D5-D3-OI636
INTERNAT/ SOVIET MILITARY TRANSLATIONS NLMBER 341 I USSR CIVIL-DEFENSE-OPERATING-PLANS TRAIVIVG EDUCATION I5-O3-DI9I 7 MENT-PLAV/ MOVEMENT TO SHELTER IN A MAJOR CITY I SHELTERS CIVIL-DEFENSE-OPERATING-PLAVS URBAN-AREAS MANAGE I5-D3-OIQUS ER-SU/ CIVIL DEFENSE ASPECTS OF MLNICIPAL WATER SYSTEMS 1 CIVIL-DEFENSE-OPERATING-PLANS UTILITIES DATA WAT I3-0?-QI7B8 IING-SYSTEMS NATIONAL-DEFENSE RECRUITING TRAINING WARFARE CIVIL-DEFENSE-PERSONNEL CASUAL TIES MOBILI ZATION/ I5-D3-023OS I CUE OPERATIONS AT THE SITE. OF AN ATOMIC STRIKE I RESCUES CIVIL-DEFENSE-PERSONNEL USSR INTERVATIONAL-CIVI I I3-I?-OIBDL IS NUMBER 188 - CIVIL DEFENSE (RLSSIA TRAINING DISPERSAL CIVIL-CEFENSE-PERSONNEL (TRAIVING) MAVAGEMENT-PL) I5-03-DI9I3 VIL DEFENSE MOTION PICTURE CATALOGUE ( PUBLIC-INFORMATION CIVIL-DEFENSE-SYSTEMS ; \#MP-6 IBUT I ON ECONOMICS FEASIBILITY-STUDIES MANAGEMENT-PLANNING CIVIL-DEFENSESYSTEMS ITS E PUBLIC-OP INION HISTORY COST/ THE CIVIL DEFENSE DEBATE I CIVIL-CEFENSE-SYSTEMS ATTITUDES POLITICAL-SCIENC DS-IJ-DI679 E PUBLIC-OPINION HISTORY COST

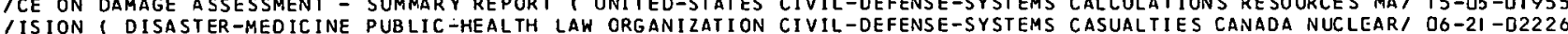
IISION I DISASTER-MEO ICINE PUBLIC-HEALTH LAW ORGANIZATION CIVIL-DEFENSE-SYSTEMS CASUALTIES CANADA NUCLEARI O6-2I -D2226

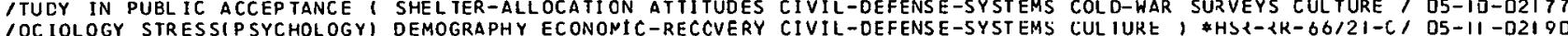
/OC IOLOGY STRESSIPSYCHOLOGYI DEMOGRAPHY ECONONIC-RECCVERY CIVIL-DEFENSE-SYSTEMS CUL IURE I \#HSR-2K-66/2I-C DS-II-D2I 9D

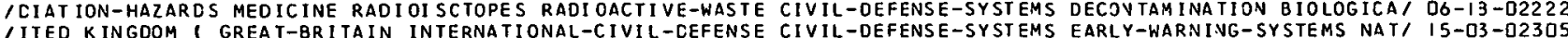

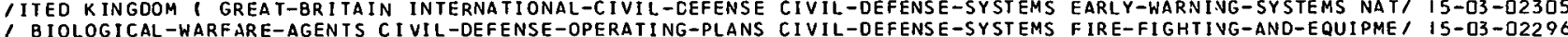
1 BIOLOGICAL-WARFARE-AGENTS CI VIL-DEFENSE-OPERATING-PLANS CIVIL-DEFENSE-SYSTEMS FIRE-FIGHTIVG-AND-EQUIPME I I5-03-O2296

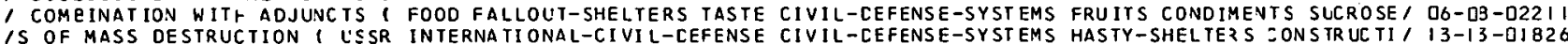

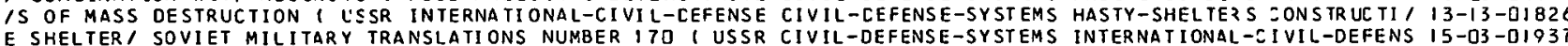
E WESTERN-EUROPE NATO GREAT-B / WORLD WIDE CIVIL OEFENSE 1 CIVIL-DEFENSE-SYSTEMS INTERNATIONAL-CIVIL-DEFENS I5-O3-D2IL E TRA ININ/ SOVIET MIL ITARY TRANSLATIONS NUMBER 385 I USSR CIVIL-DEFENSE-SYSTEMS INTERNATIONAL-CIVIL-DEFENS I5-D3-D23D2 IT-EFFECT IVENESS ENVIRONMENT MANAGEMENT-PLANNING SURVIVAL CIVIL-CEFENSE-SYSTEMS MANAGEMENT-COVTROL-SYSIEMI IS-US-UZ2Y3 ION SOCIAL-SCIENCES HISTORY CI VIL-DEFENSE-OPERATING-PLANS CIVIL-DEFENSE-SYSTEMS YANAGEMEN I-PLANNING UECISI O5-OH-OIG 7 I ISHEL TER PROGRAM I FALLOUT-SHELTERS FEDERAL-BUDGETS COSTS CIVIL-DEFENSE-SYSTEMS MANAGEMENT-PLANNING STATE I I3-I3-QI834

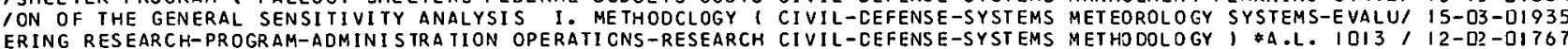

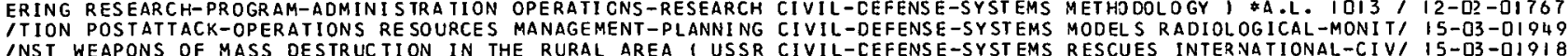
INST WEAPONS OF MASS DESTRUCTI ON IN THE RURAL AREA 1 USSR CIVIL-CEFENSE-SYST EMS RESCUES INTERNATIONAL-CIVI IS-D3-0I I 8
IANSLATIONS NUMBER I 78 I USSR INTERNATIONAL-CIVI L-CEFENSE CIVIL-DEFENSE-SYSTEMS RURAL-AZEAS PUBLIC-INFORMI IS-D3-DI928

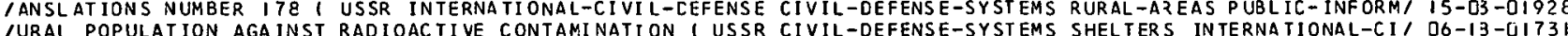
IURAL POPULATION AGA INST RADIOACTIVE CONTAMINATI ON I USSR CIVIL-DEFENSE-SYSTEMS SHELTERS INTERNATIONAL-CI D6-I3-UIT3I

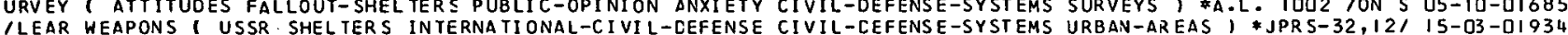

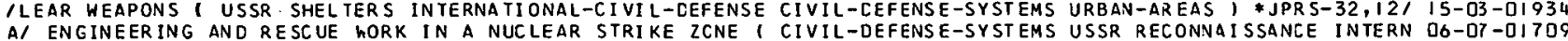
IXT - 1966 I ATTI TUDES PUBLIC-OPINION SURVEYS DISARMAMENT CIVIL-DEFENSE-SYSTEMS VIETVAM SHELTER-ALLOCATIO/ O5-IJ-DIG78 IGENERAL SENSITIVITY ANALYSIS II. TECHNICAL APPENDIXES I CIVIL-DEFENSE-SYSTEMS WEAPONS-EFFEC TS EXPLOSION/ IS-O3-OI933 I IS III. THE ANCET COMPUTER PROGRAM ( SYSTEMS-EVALUATI ON CIVIL-OEFENSE-SYSTEMS WEAPONS-EFFECTS MOOELS EX/ IS-D3-DI938 /MENT EMER GEVCY-PLANN ING (GOVERNMENT-AGENCIES) LEGI SLATI ON CIVIL-CEFENSEIGOVERNMEVT-AGEVCIES) AIR-FORCE AR / I5-O3-DI 946 VIBRATION FREQUENCY PNEUMATIC-DEVICES FEASIBILITY-STUDIES CIVIL-ENGINEERING 1 \$DASA-IS49 \#AD 608 531 /NES I3-13-02291 5. AND GENERAL LOCATION OF RIGHTS OF WAY TO BE ACQUIRED I CIVIL-ENGINEERING FLOODS I \$A.L. 972 1 SS SECTION I3-O2-OI78I TRESSIPSYCHOLOGY) TRESS PPYCHOLOGY CA SUALTI MENTAL HEALTH INPLICATICNS IN CIVILIAN EMERGENCIES I EMOTIOVS ATTITUDES FEAR S OS-IJ-OI6B THE 1962 REPORT TO THE PRESIDENT ( NUC LEAR-REACTORS NUCL/ CIVILIAN NJCLEAR POWER - THE I 967 SUPPLEMENT TO 18 -05-02027
IIES MOBILIZATION ARMED-FORCES-OPERATIONS LOGISTICS COSTS CIVILIAN-PERSONNEL RESEARCH-PROGRAM-ADMINISTRAT/ IS-D3-D23IJ5 IIES MOBILIIZATION ARMED-FORCES-OPERATIONS LOGISTICS COSTS CIVILIAN-PERSONNEL RESEARCH-PROGRAM-ADMINISTRAT/ I5-D3-D23DS RECTANGULAR-BODIES STRESSES THRUST I LARGE DEFLECTIONS OF CLAMPED RECTANGULAR PLATES IPANELSISTRUC TURALI 20-1I-O23L4

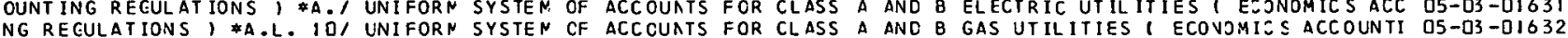
TIONS ACCOUNTING, \#A.L. I UNIFORN SYSTEM OF ACC NUNTS FOR CLASS A AND B WATER UTILITIES 1 ECONOMICS REGULA OS-O3-DI65O TIES I INDUSTRIAL-EQUIPMENT ECONONICS FEASIBILITY-STUDIES CLASSIFICATION, \$RM-ISI2 \$AD $87449 /$ POSSIBILI O5-O3-DIG48 
HE EVALUATION OF HARDEVING COSTS I/ INDUSTRIAL HARDENING CLASSIFICATION - A METHOCOLOGY FDR SIMPLIFYING T O5-03-D2I 26 INDUSTRIAL HARDENING CLASS IFICAT ION - A METHODOLOGY FOZ SIMPLIFYING T O5-03-02132 OGRAPHS URBAN AREAS FIRES MAPS TEST-METHODS VULNERABILITY CLASSIFICATION BUILOINGSI \$REPORT NO. OSO9 /HOT I3-I2-DIBO9 INOEXING I INOEXES INFORMAIION-RE IRIEVAL SUB JECT-HEADINGS CLASSIFICATION INFORMATION-THEORY COMPUTERS I *I O5-O2-O2IIO I FALLOUT PREDICT ION MODELS I. ANALYSIS, COMPARISCN, AND CLASSIFICATION OF MODELS I RADIOACTIVE-FALLOUT I I8-08-D2O39 TEXTUR/ QUARTERLY OF THE COLORADO SCHOOL OF NINES SJ(I) CLASS IFICATION OF ROCKS ( ROC< (GEJLOGY) MINERALS OB-OF-O224I ICUCTS SAND UNDERGROUND LOADING (MECHANICS) SOIL-MECHANICS CLAY COMMUNICAT IOV-EQUIPMEVT INTERACTIONS ) *TEI I3-II-O22SI INGS - WAVE VELOCITIES THROUGH PARTIALLY SATURATED SAND - CLAY MIXTURES 1 SOIL-MECHANICS ACCOUSTICS MOISTI 20-DI -O2O4 7 IMARBLE DOLOMITE LIMESTONE ROCK-SALT ANHYDRI TE SANDSTONES CLAY SILTSTONES GEOLOGY THERMAL-STRESS STRESSESI D8-OZ-O224 2

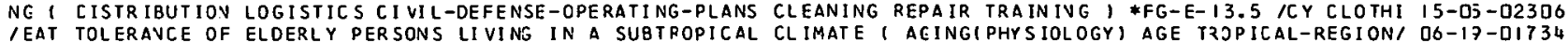
OPT ICAL-IMA/ STUDY OF LIGHT AT TENLATI ON UNDER SUETROPICAL CLIMAT IC CONDITIONS I LIGHT-TRANSMISSION COLORS 2O-DB-D2D66 OPT ICAL-IMAI STUDY OF LIGHT AT TENLATION UNDER SUETROPICAL CLIMAT IC CONDII IONS I LIGHT-TRANSMISSION COLORS 2O-DS-D2D66
IS VULNERABIL ITY WEAPONS-EFFEC TS WIND RADIOACTIVE-FALLOUT CLIMATOLOGY CASUALTIES FIRES PROGRAMMINGICOMPUT, OS-O3-O2I 44 IS VULNERABILITY WEAPONS-EFFEC TS WIND RADIOACTIVE-FALLOUT CLIMATOLOGY CASUALTIES FIRES PROGRAMMINGICOMPUT, OS-O3-O2134
TOXIC AND RADIOLOGICAL AGENTS - USSR I RADIOL/ PATHOLOGY, CLINICAL PICTURE AYD THERAPY IN AFFECTIONS WITH DG-IB-OI72I TOXIC AND RADIOLOGICAL AGENTS - USSR I RADIOLI PATHOLOGY, CLINICAL PICTURE AVD THERAPY IN AFFECTIONS WITH D6-IB-OI72I

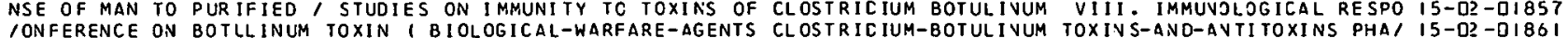
RY-ENGINEER ING FUELS WATER-SUPPLIES MATHENATICAL-ANALYSIS CLOTHING) 1 NREC-4 /PPLIES ELECTRIC-POWER SANITA OS-O3-O2I35 OPERATING-PLANS CLEANIVG REPAIR TRAINING, \#FG/ EMERGENCY CLOTHING 1 DISTRIBUTION LOGISTICS CIVIL-DEFENSE- I5-DS-D23O6 IN THE TROPICS I TROPICAL-REGIONS MILITARY-PERSCNNEL FCOD CLCTHING ENVIRONMEVT EDUCATIOV ARYY-EZUIPMENT R/ DS-IJ-D2IBI IFALLOUT-SHEL TERS VENIILATION ME TABOLI SM BODY-TEMPERATURE CLOTHING TEMPERATURE CDOLIVG PERSPIZATION EVAPO/ DG-IS-DI 7 I 9 REULENCE FLUID-DYNAMICS PARYICLE-SIZ/ RELATIVE MOTION ANO COAGULATION OF PARTICLES IN A TURBULENT GAS 1 TU $20-04-02333$ STER - THERMAL RADIATION EFFEC TS ON PAINTS, PLASTICS, AND COATED FABRICS $4 W T-407$ INA IOGRAMMIVG(COMPUTERS) RADIOACTIVE-FALLOUT SHIELOING WALLS COBALT EXPERIMENTAL-DATA THEORY DJSE-RATE I \#R-/ O6-IB-O22I 8 FARE-AGEVIS PATHOLOGY LUNGS SPORE $\$$, \$AD/ PATHOGENESIS OF COCCIDIOICES IMMITIS IV MOVKEYS 1 BIOLOGICAL-WAR IS-02-0I852 FARE ARMEC-FORCES IUNI TED-STATESI MILI IARY-GOVERNMENT ENERGEN/ CODE OF FEDERAL REGULAT IONS - NATIOVAL DEFENSE 1 IS-O3-OI946 ARMEC-FORCESIUNITED-STATESI MILI IARY-GOVERNMENT ENERGEN/ CODE OF FEDERAL REGULATIONS - NATIOVAL DEFENSE I I5-O3-OI946
COMMUNICATIOV-SYSTEMS,

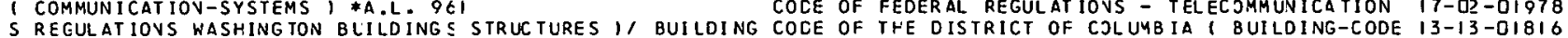
S RECULAT IOVS WASHING ION BLILDINGS STRUC TURES I/ BUILDING COCE OF THE DISTRICT OF COLUMBIA 1 8UILDING-CODE I3-13-OI8I6 ILDING-CODES REGULATIONS STRUC TU/ NANUAL FOR THE BUILOING COCE OF THE DISTRICT OF COLUMBIA 1 WASHINGTON BU I3-I3-DI8I7
IOUT SHELTER CONSTRUCTION IN FOUR NATIONAL MODEL BUILDING CODES / CONSTRUCTION DUAL-PURPOSE-SHELTERS I \$T, I3-I3-D228I IOUT SHELTER CONSTRUCTION IN FOUR NATIONAL MODEL BUILDING CODES I CONSTRUCTION DUAL-PURPOSE-SHELTERS I \$T I I-I3-O228I
5 RAD S RADIOACTIVE-FALLOUT CONSTRUCTION COI FALLOUT SHELTERS - CODES AND STANDARDS I SHIELDIVG RADIATION-EFFECT I3-I3-O2286 AL-ANALYS IS SOC IAL-SC IENC I TABLES FOR YULES O ASSCCIATION COEFFICIENT FOR PAIRS OF PERCENTAGES I STATISTIC OS-II-D22OO
IF PRESERVATION SHELF-LIFE BEVERAGES BREAD CANNED CEREALS COFFEE CORN DEHYCRATED-FOJDS EGGS FATS FROZEN-FI DO-D3-O22D9 /CHOL OGY) THREAT-PERCEPTION BEHAVIOR MODELS COMMUNICATION COGNITIVE-DECISION-MAKING INNOVATION I \$A.L. II/ D5-OH-D2I OO /EOLOGIC INVESTIGATION OF THE OYNAMIC RESPONSE SPECTRA OF COHESIVE SOILS I DYNAMICS SOIL -MECHANICS THEORY/ $20-11$-O2O86 I CELLS I LIGHTIVE-EOUIPMENT CONTROL-SYSTEMS TRANSFORMERS COILS CIRCUITS USSR PATENTS I \#FTD-TT- $65-1169$ * O9-05-DI756 ON SURVEYS DISAR/ AMERICANS VIEWS ON CIVIL DEFENSE IN THE COLD WAR CONTEXT - I966 1 ATTITUDES PUBLIC-OPINI O5-IJ-OIG78 ISIA POLITICAL-SCIENCE NUCLEAR-WARFARE BALLISTIC-MISSILES COLD-WAR FOREIGN-POLICY PROPAGANDA ATTITUDES CO/ DS-O+-DIOT2 ITWO RESOLUT IONS ON INTERNATIONAL AFFAIRS I ARMS-CONTROL COLD-WAR NATO VIETNAM RED-CHIVA FJREIGN-POLICY / O5-O4-O2172
INCE I SHELTER-ALLOCATION ATTI TUDES CIVIL-DEFENSE-SYSTEMS COLD-WAR SURVEYS CULTURE DEMOGRAPHY SOCIOLOGY PI O5-IJ-O2I77 $/$ OF THE POPULATION OF THE UNI TED STATES BY AGE, SEX, AND COLOR TO 1990 WITH EXTENSIONS OF TOTAL POPULATI/ $23-04-02358$ IIR DEFEVSE COMMAND AT CHEYENNE MOUNTAIN, EL PAST COUNTY, COLORADO I CONSTRUCTIOV UNDERGROUVD-STRUC TURES, I3-I3-O2274 IUDIES I SHEL TERS FALLOUT-SHEL TERS UNDERGROUND-STRUCTURES COLORACO CJAL -PURPJSE-SHELTERS TRAYSPORTA TION UI I3-I3-OIB3I TO THE DESIGN OF LNDERGROUND OPENINGS FI GUARTERLY OF THE COLORACO SCHOOL OF MINES 4 TII AV INTRODUCTION I3-13-D2273

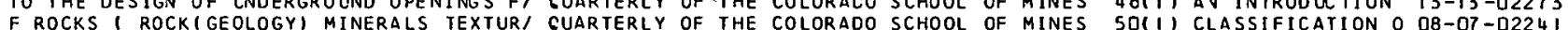
PAGATION AND PRESSURE MEASUREMENIS NEAR/ CUARTERLY OF THE COLORADO SCHOOL OF MINES SOI 4 , SEISYIC WAVE PRO D8- 11 -O224 7 K MECFANICS ( ROCKIGEOLOGY) UNDERGROUND/ CUARTERLY OF THE COLORADO SCHOOL OF MINES 5 I( 3 ) SYYPOSIUM ON ROC O8-C7-O2243 RIALS IN THE EARTHS CRUST - SECOND ANNU/ GUARTERLY OF THE COLORACO SCHOOL OF MINES $52(3)$ BEHAVIOR OF MATE OQ-07-O2244 ( UNDERGROUND-STRUCTURES ROCK (GEOLOGY), QUARTERLY OF THE COLORADO SCHOOL OF MINES O2(2) TUNNEL MECHANICS I3-I3-D2275 ICER SUBTROPICAL CLIMATIC CONDITIONS I LIGHT-TRANSNISSION COLORS OPTICAL-IMAGES OPTICAL-PHEVOMEVA METEOROI 20-D6-O2O66 BUILCINGS STRUCTURES // 8 UILDING CODE OF THE DISTRICT OF COLUMBIA I EJ ILCING-CODES REGULATIONS WASHINGTON I3-I3-OIBI 6 STRUCTU/ MANUAL FOR THE BLILDING CODE OF THE DISTRICT OF COLUMBIA 1 WASHINGTON BUILDIVG-CODES REGULATIONS I3-I3-DIBI 7 IANSIT SYSTEM PORTION OF CONNECTICUT AVENUE ROUTE BETWEEN COLUMBIA ROAD ANC VAN VESS STREET I TRANSPORTAT/ I3-O?-OITBS ICESIGN THEORY BEAMS (STRUC TURAL FRAMES LOADING (MECHANICS) COLUMNSISTRUCTURALI DEFORMATION MATHEMATICAL-MOI 2O-II-O23UO

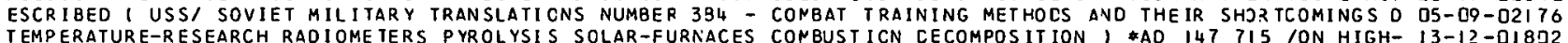

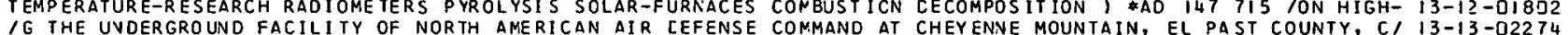
IG IHE UVDERGROUND FACILITY OF NORTH AMERICAN AIR CEFENSE COMMAND AT CHEYENNE MOUNTAIN, EL PAST COUNTY, C/ $13-13$-O2274 N HAR, COMPENOIUM OF PROTECTIVE CONSTRUC TION- VOLUME I I COMMAND-AND-CONTROL-SYSTEMS BIBLIOGRAPHIES DESIG I7-02-023II
ICAL. STATE, AVD REGIONAL LEVELS I COMMUNICATIONS-SYSTEMS COMMAND-AND-CONTROL-SYSTEMS DECISIOY-MAKING CIIVI I7-02-O23II

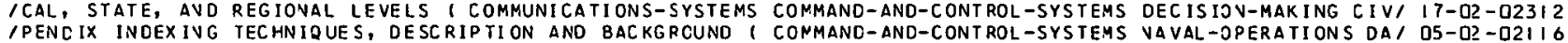

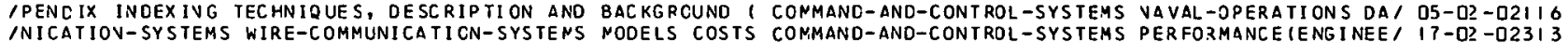
INICATION-SYSTEMS WIRE-COMMUNICATICN-SYSTENS NODELS COSTS COMMAND-AND-CONTRDL-SYSTEMS PER FORMANCE IENG I NEE I I7-02-023I 3

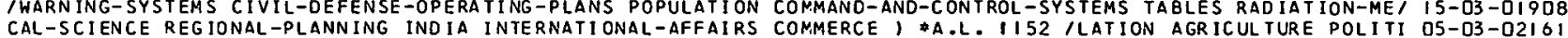

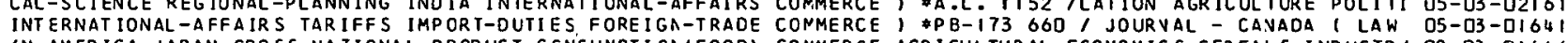
IN-AMERICA JAPAN GROSS-NA TIONAL-PRODUCT CCNSUMPTICAIFOODI CONMERCE AGRICULTURAL-ECONOMICS CEREALS INDUSTRI O2-D? -OIOI 6 ON OPERATIONS-RESEARCH PER SONNEL LNI IED-STATES-GOVERNMENT COMMERCE CONSTRUCT ION, 1 BULLETIN NO. I536 /RATI O5-03-OI649
INISM ECONOMICS AGRICULTURAL-ECONOMICS FINANCE INDLSTRIES COMMERCE EDUCATION POLITICAL-SCIENCE GEOGRAPHY, OS-III-DIO93 RCH PAPER 156 SOVIET IMPACT ON INTERNATIONAL LAW USSR COMMERCE INTERNATIONAL-AFFA IRS I *EXIERNAL RESEA O5- II - IOS RCH PAPER 156 SOVIET IMPAC T ON INTERNA TI ONAL LAW USSR COMMERCE INTERNATIONAL-AFFAIRS I *EXIERNAL RE SEA O5- II -DI 69D

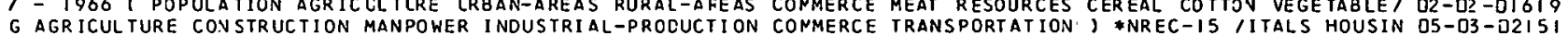

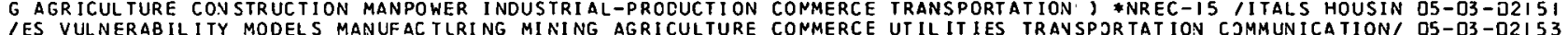
IES VULNERABIL ITY MODELS MANUFAC TLRING MINING AGRICULTURE CONMERCE UTILITIES TRAVSPJRTATION COMMUNICATION/ OS-D3-O2I53

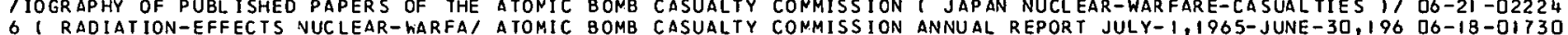
6 I RADIATION-EFFECTS NUCLEAR-WARFA' ATOMIC BOMB CASUALTY CONMISSION ANNUAL REPORT JULY- 1 , $1965-J U N E-30,196$ D6-18-OI730 HEAT INVENTORY BY WAREHOUSE AND LOCATION I AGRICULTURE S/ CONMODITY CREDIT CORPORATION HAREHOUSE RECEIPT W O2-O2-D2ID9
UNIT ED STATES I CONSTRUC TION LRBA/ BUILOING MATERIALS AS CONMONLY USED IN EXISTING URBAN BUILDINGS IN THE I3-I3-D2277 MOVEMEN/ EFFECT OF POPULAIION MOBILITY ON THE LOCATION OF CONMUNAL SHELTERS I WARNIVG-SYSTEMS URBAN-AREAS I5-D3-DIOU 2 NS INCUSTRIES(CIVIL-DEFENSE) PUBLIC-HEALTH TRANSPORTATION CONMUNICATION, *JPRS-41, 417*TT-67-32C5I/G-PLA 15-D3-02302 /ERS STRESSIPSYCHOLOGY) THREAT-PERCEPTION BEHAVIOR MODELS CONMUNICAT ION COGNITIVE-DEC ISION-MAKING INNOVAT/ O5-O4-D2I 66 I SANC UVDERGROUVD LOADING (MECHANICS) SOIL-MECHANICS CLAY COMMUNICATION-EQUIPMENT IVTERACTIOVS I *TECHNICI $13-11$-O226I /LANN ING, STUDIES AND ANALYSES ( COMMUNICATION-SATELLITES CONMUNICATION-EOUIPMENT SATELLITESIARTIFICIAL) , 22-D?-D235। IANC SCIENTIFIC SLPPORT, PLANNING, STUDIES AND ANALYSES I CONMUNICATION-SATELLITES COMMUNICATION-EQUIPMEN/ 22-Q2 -O235I MEOICINE TSUNAMIS WATER-SUPPLIES LOGISTICS TRANSPORTATION COMMUNICATION-SYSTEMS, $\$ A . L$. IIL IDS DISASTER- IS-03-02299 COCC OT FCDCRAL REGULATIONS. TCLLCOMHUMICATIOA I C IERS PUBL IC-OPINION AITITLOES ME THODOLOGY WEAPCNS-EFFECTS UCES PUBL IC-OPINION PUBLIC-INF ORMATI ON SURVEYS DEMCGRAPHY I CUCATION HEALTH WELFARE-SERVICES OCCUPATIONS PROFESSIONS ICUCATION HEALTH WELFARE-SERVICES OCCUPATIONS PROFESSIONS
ISE OPERATIONS - CIRCA 197 ( RADIO-COMMUNICATION-SYSTEMS (RS) COMPUTERS SPECIAL-PURPOSE-CONPUTERS READING-MACHINES IING MINING AGR ICUL TURE COMMERCE LTILITIES TRANSPORTATION INT IM ISSILE-DEFEN SE-SYSTEMS UNITED-STATES SURVIVAL EUROPE DINE 1 DUCTS SAND UNDERGR / RESPONSE OF A BURIED PRCTOTYPE INTRASTATE CIVIL DEFENSE OPERATICNS - CIRCA 19701 RADI/

COMMUN ICAT IOH.SYSTCHS, \$A.L. 261 $17 \cdot 02 \cdot 01770$ CONMUNICATION-SYSTEMS ECOVOMIC-RECOVERY MILITAR/ 05-13-D1677 COMMUNICAT ION-SYSTEMS STRESSIPSYCHDLOGY) PUBLIC, 13-13-01831 COMMUNICATION-SYSTEMS TRAINIVG ECOYOMICS INTERNI O5-11-02194 COMMUNICAT ION-SYSTEMS WIRE-COYMUNICATION-SYSTEM/17-02-02313 COMMJNICATION-THEORY DATA-STORAGE-SYSTEMS, *A., 09-02-02248 COMMUNICAT IONS BAVKING COVSTRUCTION MAINTENANCE/ 05-03-02153 COMMUNICAT IONS CHINA SHELTERS, *HI-777-RRIDRAF/ 15-03-01922 CONMUNICAT IONS CONDUIT TO STATIC AVD DYNAMIC LOA $13-11-02261$
CONMNICAT IONS ECUIPMEVTS AND SYSTEMS TO SUPPORT $17-02-02313$ 
IS - INTERNATIONAL AFFAIRS I BIBLIOGRAPHIES ATOMIC-ENERGY COMMUNICATIONS LABOR COMMUNISM ECONOMICS AGRICUI OS-II -0IG93 作 ANC REGIONAL LEVELS I COMMUNICATIONS-SYST/ CIVIL CEFENSE CONMUNICATIONS REQJIREMENTS AT THE LOCAL, STATE, I T-O2-O23I -FAC IL ITIES ARMED-FORCES-SUPPLIES, GOVERNMENT-FACILITIES CONMJNICATIONS-FACIL ITIES TRAVSPOZTATION-PROPER I OS-03-D2I 37 I REQUIREMENTS AT THE LOCAL, STATE, AND REGI ONAL LEVELS I COMMUNICATIONS-SYSTEMS COMMAVD-ANO-CONTROL-SYST/ I7-CU?-O23I 2 ( SHELTERS WEAPONS-EFFECTS SURVIVALIEQUIPNENT) STRUCTURES COMMUNICATIONS-SYSTEMS RADIATION GEOLOGY HUMAN-I IT-D2-O23.I I I NANAGEMENT-PLANNING HOSPITALS OPERATION CONTROL-SYSTEMS CONMUNICATIONS-SYSTEMS SUPERVISIOV MEDICAL-PERSI O6-I2-OI TI 4 TIC-MISSILES COLD-WAR FOREIGN-POLICY PROPAGANDA ATTITUDES CONMUNISM, *LT-65-104/E VUCLEAR-WARFARE BALLIS OS-0H-OIG72 IAIRS 1 8IBL IOGRAPHIES ATOMIC-ENERGY COMNUNICATIONS LABOR COMMUNISM ECONOMICS AGR ICUL TURAL-ESOVOMICS FINAI OS-II -DI693 10 ACADEMIC CENTERS - AMERICAN REPUBLICS 1 BIBLIOGRAPHIES COMMUNISM ECONOMICS EDUCATION FOREIGN-POLICY GE/ OS-II -0I696 IDEMIC CENTERS - USSR AND EASTERN EUROPE I BIBLIOGRAPHIES CONMUNISM ECONOMICS EDUCATION INTERNATIONAL-AFF I O5-II-OIOO 7 DFIFT DO 31 *TT-67-31571 05-04-02173 IRA IN ING) REPAIR ( TRA INING) PRO TEC TIVE-CLOTHING PRCFAGANOA COMMUNISM PROTECTIVE-MASKS BIJLOGIEAL-WARFARE-A I I5-O3-OI9I 7

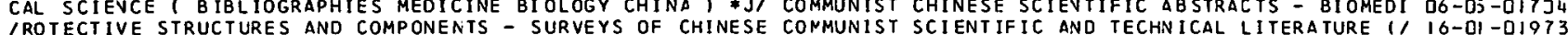

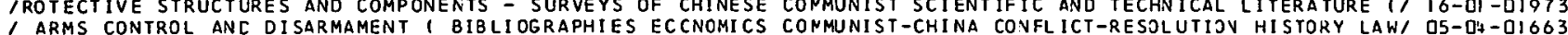
/ ARMS CONTROL ANC DISARMAMENT I BIBLIOGRAPHIES ECCNOMICS CONMUNIST-CHINA CONFLICT-RESOLUTIOV HISTORY LAW/ OS-D'4-QIO63
IS OR RECENTLY COMPLETED - ARMS CONTROL AND DISARNANENT I COMMJNIST-CHINA ECONOMICS CONFLICT-ZESOLUTION H/ OS-CI-DIOS2

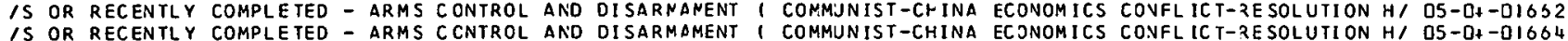

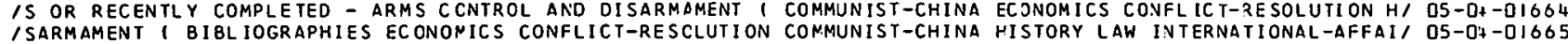
1 C CETERRENCE ( USSR MOVEMENT INTERNATIONAL-CIVIL-DEFENSE CONMUNIST-CHINA INDUSTRIES TRANSPJRTATION EVACU/ IS-D3-02295 IPRIATIOVS AVTIMISSILE-DEFENSE-SYSTEMS UNI TED-STATES USSR CONMJNIST-CHINA THREAT-EVALUATIOV Y ILITARY-FACI/ IS-03-02294 C MANAGEMENT I SHELTER-MAN/ SHELTER OCCUPANCY STUDIES 2 . COMMUNITY FALLOUT SHELTERS HAVDBOJK FOR UNTRAINE OS-OI-D2II 5 OUT PROTECTION SURVEY I ATTITUDES/ AN EVALUATION STUDY OF CONMUNITY RESPONSE TO THE RHODE ISLAND HOME FALL OS-IJ-DIG85 ITRUCTION-SLANTING OPERATIONS-RE SEARCH SHELTER-ALLCCATION CONMUNITY-SHELTER-PLANNING-PRJGRAM MAVAGEMENT-P/ OS-DI-DIG 3 A CIRECT CURRENT MOTOR WITH NO CONTACTS AND A TRANSISTOR CONMJTATOR I ELECTRIC-MOTORS RUSSIA COMMUTATORS D9-D3-DIT52 ICTS AND A TRAVSISTOR COMMUTATOR I ELECTRIC-MOTORS RUSSIA COMMUTATORS SEMICOVDUCTOR-DEVICES TRAVSISTORS CI OQ-O3-DIT52 CLE I MOQEL CEI IVAL G-230, AND $20 E /$ NOMINAL 9, ODD ETU/HR, COMPACT, VERT ICAL, $230-V O L T$, SINGLE PHASE, OD-CY I3-DI -CI775 1 COMMAND-AND-CONTROL-SYSTEMS BIBLIOGRAPHIES DESIGN HAR CONPENDIUM OF PROTECTIVE CONSTRUCTIOV - VOLUME I I7-C2-023I I

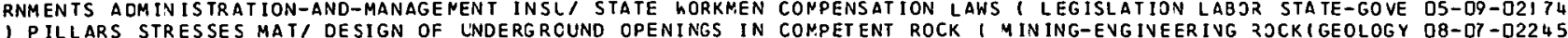
I P ILLARS STRESSES MAT/ DESIGN OF LNDERG RCUND OPENINGS IN COMPETENT ROCK I M INING-EVG IVEERIVG ZJCKIGEOLOGY D8-D7 -D224S IIMPACTS OF RA ILROAD MERGERS ( TRANSPORTATION LEGISLATION COMPET IT ION EMPLOYMENT TAXES SERVICES MANAGEMEN/ 23 -D?-02095
OF SOCIETAL RECOVERY FROM/ CIVIL DEFENSE BIBLIOGRAPHY - A COMPILATION OF REFERENCES RELEVANT TO THE STUDY O5-IJ-DI677 OF SOCIETAL RECOVERY FROM/ CIVIL DEFENSE BIBLIOGRAPHY - A COMPILATION OF REFERENCES RELEVANT TO THE STUDY O5-IJ-DI677
IFIC AND TECHNICAL LI IERATURE, PROTECTI VE STRUC TURES AND CONPONENTS - SURVEYS OF CHINESE COYMUVIST SCIENT IO-DI -OI973 ILOPMENT OF STANDARD FIRE TEST RATING SYSTEMS FOR SHELTER CONPONENTS I FALLOUT-SHELTERS FIRE-SAFETY FIRESI I3-I 2-O22S9 ROUND-SUPP/ SOVIET LITERATLRE ON PROTECTI VE STRUCTURE AND COMPONENTS 1 GUICED-MISSILES RAILZJADS BRIDGES G I6-OI-DIQ72 IYL IN OR ICAL SHELLS I STRUC TURAL-SHELLS CYLINDRICAL-BODIES COMPOSITE-MATER IALS, EOUATIONS-OF-MOTION, STRES/ 2O-II -O2345

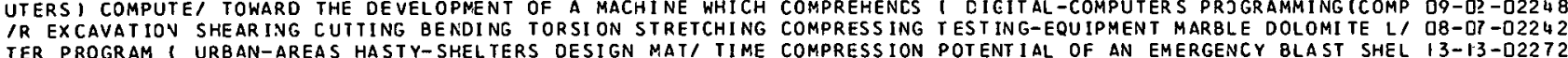
L-MECIANICS LOADINGIMECHANICSI DYNAMICS EXPERINENTAL-DATA CONPRESSIVE-PROPERTIES THEORY I *AD 63I 369 /SOI I3-13-0I8I2 LNERABIL ITY WEAPONS-EFFECTS WIND, NREC DANAGE ASSESSMENT COMPUTAT ION PROGRAY - JUMBO III. 1 RESOURCES VU D5-O3-02134 ROTECTION FROM NUCLEAR BLAST I SHELTERS SHOCK-WAVES BLAS/ COMPUTER ANALYSIS OF PROTECTIVE STRUCTURES FOR P I3-I3-OIB33 INGS I DYNAMICS COMPUTER-PROGRAMSI TEST AND EVALUATION OF COMPUTER ANALYSIS PROGRAMS FOR SHELTERS IN BUILD I3-I3-02280 ISION OF THE GENERAL SENSITIVI TY ANALYSIS III. THE ANCET CONPUTER PROGRAM I SYSTEMS-EVALUATIOV CIVIL-DEFI IS-D3-OIQ38 Y OF D DUAL LAYEREO PLATE SUBJEC T TO THE T/ ECUATI CNS AND COMPUTER PROGRAM TO CAL CULATE THE THERMAL HISTOR I 3-I? -0I797

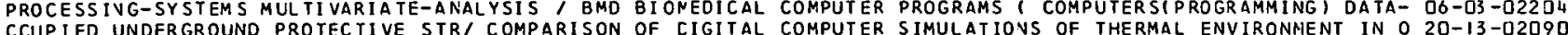
CCUPIED UNDERGROUND PROTECTIVE STRI COMPARISON OF CIGITAL COMPUTER SIMULATIONS OF THERMAL ENVIRONAENT IN O 20-13 -02090

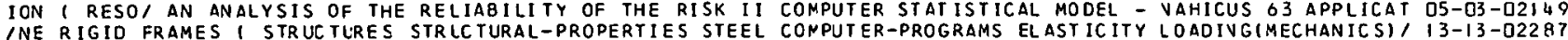

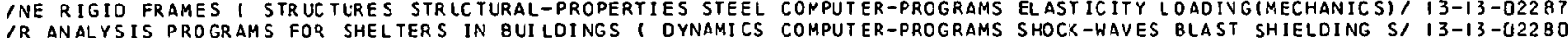
IR ANALYSIS PROGRAMS FOQ SHELTERS IN BUI LOINGS I OYNAMICS COMPUTER-PROGRAMS SHOCK-WAVES BLAST SHIELDING SI I3-13-U22BO

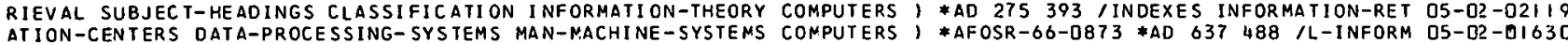
CTURES LOCATED ABOVE AVD BELOW GROUND I FALLOUT-SHELTERS CONPUTERS BLAST-SHELTERS TEMPERATURE-CONTROL SO/ I3-DI - OI776 INFORMATION-SYSTEMS HOUSING RE SOURCES MATHENATICAL-MODELS COMPUTERS CASUALTIES DAMAGE-ASSESSMEVT WEAPONS- 1 OS-O3 -02I 37 I DAMAGE-ASSESSMENT BLAST RESOURCES PROGRAMMING-LANGUAGES COMPUTERS CASUALTIES RADIOACTIVE-FALLOUT THERMAI O5-O3-D2I54 IOR 1966 ( PROGRAMMINGICOMPUTERS) DATA-PRCCESSING-SYSTEMS CONPUTERS DATA-TRANSMISSION-SYSTEMS SIMULATION, Q9-O2-OITSI

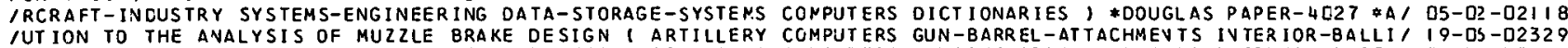
IENERATOR I SIMULATION DAMAGE-ASSESSMENT ATTACK-SCENARIOS COMPUTERS HOUSINE PROGRAMMINGICOMPUTERSI MILITAI DS-U3-O2I39 IFIC-RESEARCH ME TALLURGY MATERIALS CHEMISTRY MATHEMATICS COMPUTERS NUCLEAR-REACTORS HEAT-TRANSFER NUCLEAI 20-O3-D2D7O IIFIC-RESEARCH ME TALLURGY MA TERIALS CHEMISTRY MATHEMATICS COMPUTERS NUCLEAR-REACTORS HEAT-TRANSFER NUCLEA 20-D3 -D2D70 GUAGES EDUCAT I/ RESEARCH AND TECHNOLOGY DIVI SICN REPORT I CONPUTERS PROGRANMINGICOMPUTER I PR GGRAMMING-LAN O9-02 -OI75D
30

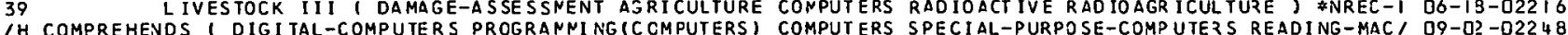

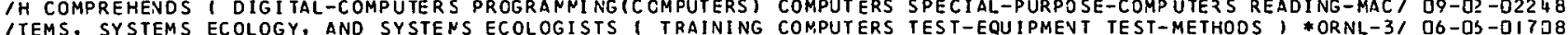
ITEMS, SYSTEMS ECOLOGY, AND SYSTENS ECOLOGISTS I TRAINING COMPUTERS TEST-EQUIPMENT TEST-METHODS I *ORNL-3/ D6-DS-DI7D8

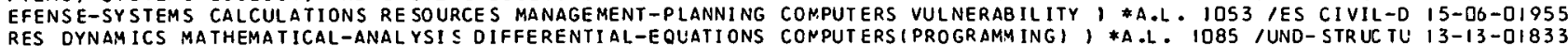

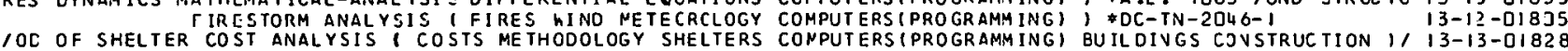

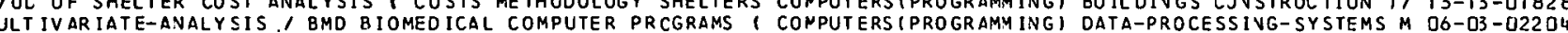
DEFORMATION TRAN SPOR TATION ELASTICITY NORTHEAST-CCRRIDOR CONPUTERS (PROGRAMMING) PLASTICITY, *R 66-53/ION 2D-III-0209L IVE-DECAY PARTICLE-SIZE HENRYS-LAh HALF-LIFE DISTRIBUTION COMPUTERS (PROGRANMING) YIELD, \$GA-7598 /ADIOACT IB-D3-D2D37 OSIUM I LOADINGIMECHANI' FLEXURAL MECHANICS OF REINFORCED CONCRETE - PROCEEDINGS OF THE INTERVATIONAL SYMP $20-11$-O2339 S LOACIN/ STATIC AND OYNAMIC TESTS OF BURIED UNREIAFCRCED CONCRETE ARCHES 1 UNDERGROUNO- STRUETURES DYNAMIC I3-I3-OIB30 TURAL) LOADIVGIMECH/ DYNAMIC SHEAR STRENGTH OF REIAFORCED CONCRETE BEAMS II. 1 TEST-EQUIPMEVT BEAMSISTRUC I3-I3-QIB4I MATERIALS LOAD/ EFFECTS ON LONG TIME LOADS ON PRESTRESSED CONCRETE BEAMS ( BEAMSISTRUCTURAL) CONSTRUCTION- I3-I3-O22B9 R-STR/ DYNAMIC SHEAR RESISTANCE OF THIN WEBBED REINFDRCED CONCRETE BEAMS I BEAMSISTRUCTURAL) DYVAMICS SHEA $20-1$ I -O2337

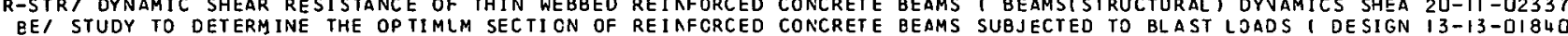
EE/ STUDY TO DETERMINE THE OP TIMLM SECTICN OF REIIFCRCED CONCRETE BEAMS SUBJECTED TO BLAST LOADS I DESIGN I3-I3-DI84O

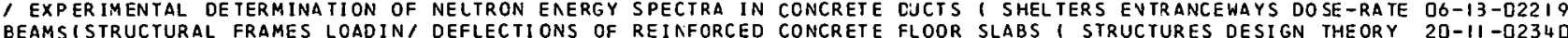
/SHOCKIMECHANICS) FEASIBILITY-STUDIES STRESSES ELASIICITY CONCRETE HONEYCOMB-CORES EXPAVDED-PLASTICS DESI/ IB-D3-O2OI 7 NES STRESSES/ PRELIMINARY TESTS ON A SHALLOW UNREI AFORCED CONCRETE SHELL I SHELLS (STRUCTURAL-FJRMS) MEMBRA I3-I3-OIB32 ROUND-STRUCTURES SHELTERS PE/ RESPONSE OF DEEP REIAFORCED CONCRETE SLABS 1 TESTS LOADIVG(MECHAVICSI UNDERG I3-I3-02290 IING BLAST LOADS ON DEEPLY BURIED PROTECTIVE STRUCTURES ( CONCRETE UNDERGROUND-STRUCTURES EXPANDED-PLASTI I I 8 -O3-O2OJ DUCT ABSORPTION DURING FALLOUT/ A CALCULATIONAL MOCEL FOR CONDENSEO STATE CIFFUSION CONTROLLED FISSION PRO I8-OB-D2O37 /FOOD FALLOUT-SHELTERS TASTE CIVIL-DEFENSE-SYSTEMS FRUITS CONDIMENTS SUCROSE FOOD-CISPEVSIVG TESTS I *SRI I D6-OB-O22II

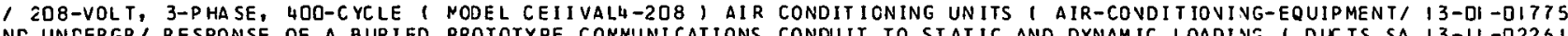
NC UNCERGR/ RESPONSE OF A BURIED PROTOTYPE CONMUNICATIONS CONDUIT TO STATIC AND OYNAMIC LOADING 1 DUCTS SA I $3-1 \mathrm{I}$-D22SI SERVICES BIOLOGICALI PROCEEDINGS OF MEDICAL CIVIL DEFENSE CONFERENCE 1 OISASTER-MEDICINE EMERGENCY-HEALTH- DO-2I -O2233

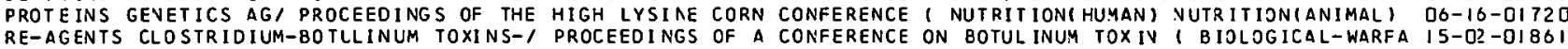
I UN ITED-STATES CIVIL-DEFENSE-SYSTEMS CALCULATIONS/ NATO CONFERENCE ON DAMAGE ASSESSMEVT - SUMMARY REPORT IS-DS-DI955

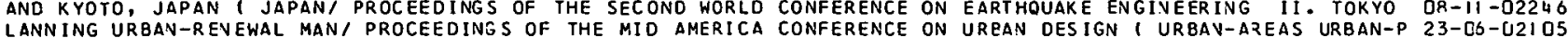


ES VULNERABIL I/ IMPLEMENTATION OF AN ORDERED SPRAWL URBAN CONFIGURATION I URBAN-AREAS DECENTRALIZATION TAX $23-06-02$ I O6 IN FORM AS A PASSIVE DEFENSE VARIABLE I CCSTS URBAA-AREAS CONFIGURAI ION URBAV-PLANNIVG EFFICIEVCY SELECTI I3-D?-D2256 IER OCCUPANCY STUDIES I. FINAL REPORT, FALLOUT-SHELTERS CONFINEC-ENVIRONMEVTS STOCKPILING SHELTER-MANAG/ OS-IJ-02I84 /ANCY FEASIBILI TY-STUDIES UNDERWA TER (SHELTERS) LEACERSHIP CONFINEC-ENVIRONMEVTS STRESS(PSYCHOLOGY) SUPERV/ O5-1)-OI684 IION WITH ADJUVCTS I FODD(ACCEPTABILI TY) FALLOUT-SHELTERS CONFINED-ENVIRONMEVTS TASTE I \#SRI-4949-5CD \$AD/ O6-C8-OI7IO QUE I AER IAL-PHOTOGRAPHS LRBAN-AREAS FIRES NAPS TEST/ THE CONFLAGRAT ION POTEVTIAL IV SAV JOSE AND ALBUQUER I3-I?-DIBOQ IAGEMENT-PLANNING COST-EFFECTI VENESS TRAINING FIRE-STORMS CONFLAGRATIONS RESCUES VULVERABILITY $=$ IVIL-DEFE/ I3-I?-OI8DO S/ MODEL STUDY OF ESCALATION II. SECTIONS 2-5, BEHAVIOR CONFLICT ARMS-CONTROL ARTIFICIAL-REALITY HYPOTHE OS-IJ-D2IB2 COOPERATION INTE/ MODEL STUDY OF ESCALATICN I. SUNMARY I CONFLICT ARTIFICIAL-REALITY CJNFLICT-RESOLUTION OS-ID-D2IB3 ECYPT, ISRAEL, AND THE UNITED NATIONS AT FI/ INTER-NATION CONFLICT, GYACIC ANC MEDIATED - CASE STUDIES OF OS-OI-O217O I ARMS CONTROL AND DISARMAMENT I BIBLIOGRAPHIES ECCNOMICS CONFLICT-RESOLUTIOV COMMUVIST-CHIVA HISTORY LAWI DS-O+-OIGG5 ? IT ARMS-CONTROL ARTIFICIAL-REALITY HYPOTHESES-FORMULATION CONFLICT-RESOLUTIOY GAMES MODELSISIMULATIONSI MI OS-IJ-D2IB2

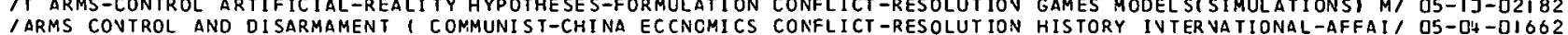

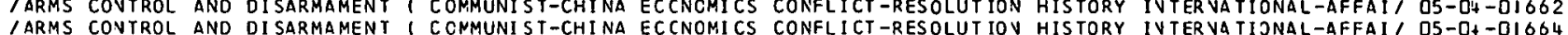

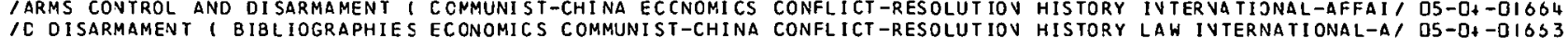
IC DISARMAMENT I BIBLIOGRAPHIES ECONOMICS COMMUNIST-CHINA CONFLICT-RESOLUTIOV HISTORY LAW IVTERNATIONAL-A/ D5-D\$-DI6S3
ISOPHY OF SCIENCE - PROCEEDINGS OF THE 1964 INIERNATIONAL CONGRESS SYMPOSIA MATHEMATICAL-LOGIC MATHEMAT/ I2-OI-D225D

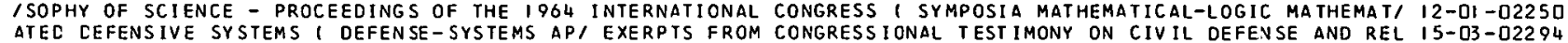

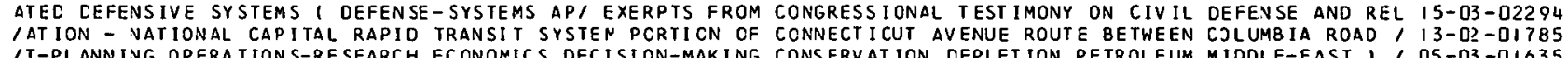
IT-PL ANNING OPERATIONS-RE SEARCH ECONOMICS DECISION-MAKING CONSERVATION DEPLETION PETROLEUH MIODLE-EAST, $/$ O5-D3 -DI635 TS BOR IVG WASHINGTON CONSTRUC TION SE TTLEMENT (STRUCTURALI CONSOL IDATION DECOMPOSIT ION SHEAR-STRENGTH I *AT I3-O2 - DIT85 A SILICATE FALLOUT PARTICLE MATRIX I RADIOAC/ HENRYS LAW CONSTANTS FOR DISSOLUTION OF FISSIJY PRODUCTS IN IB-08-02325 MER ICAV AIR OEFEN SE COMMAND AT CHEY/ METHCDS AND CCSTS OF CONSTRUCT ING THE UVDERGROUVO FACILITY OF NORTH A I3-I3-D2274 N OF PROJECTS \& FLOODS FLOOD-CONTROL CALIFORNIA FINANCING CONSTRUCTION, \$A.L. IO8D $10 W I N G$ GENERAL LOCATIO I3-D?-OI7B2 UIPMENT DESIGN SAFETY-DEVICES TESI-DUMMYIANTHROPOMCRPHICI CONSTRUCTION) COMP TEST-DUMMYIANTHROPOMCRPHIC) CONSTRUCTION, \#A.L. 982 /C TEST DUMMY I TEST-EQ 13-12-01808 STS METHOCOLOGY SHEL TERS COMPUTER S (PROGRANMING) BUILDINGS CONSTRUCTION, \$AD $646847 / R$ COST AVALYSIS 1 CO $13-13-01828$ STEMS BIBL IOGRAPHIES DESIGN HAR/ COMPCNDIUM OF PROTECTIVE CONSTRUCTION - VOLIMMF I COMMAND-AND-CONTROL-SY I7-D?-D23II URES OYNAMICS 5 I YIELDING MEMBRANE ELEMENTS IN PROTECTIVE CONSTRUCTION ANCE OYNAMICS SI IT-S INDARLSERS FIRE-SAFETY FIRES STRLCTURES SNOKES BUILOINGS CONSTRUCTION GLAST RADIOACTIVE-FALLJUT HEAT-TRA I I $3-I$ - - 22ZS TNDARDS T SHIEL DING RADIATION-EFFECTS RADICACTIVE-FALLOUT OF-RECLAMATION， *A.L. I I $157(4)$

OF-RECLAMATION, \#A.L. 115712

OF-RECLAMATION, \#A.L. $1157(3)$

CONSTRUCTION COST TRENOS, UNITED-STATES BUREAU- 13-13-02270

CONSTRUCT ION COST TRENDS UNITED-STATES BUREAU $13-13-02284$ CONSTRUCTION COST TRENDS ( UNITED-STATES BUREAU- 13-13-02285 CONSTRUCTION COST TRENDS I UNITED-STATES BUREAU- 13-13-02288 AL LOCATION OF PROJECIS I FLOODS FLOOD-CONTROL CALIFGRNIA CONSTRUCTION COSTS, \#A.L. IO79/P SHOWING GENER I3-O2-DI784 IFALLOUT SHEL TER ANALYSTS I RADI OACTIVE-FALLOUT BUILDINGS CONSTRUCTION DESIGV SHIELDING CONSTRUETION-SLAN/ I3-I3-O2276 ES QUAL ITY FASTENERS GLUING PL YWOOD SAND/ WOOD HANCBOOK I CONSTRUCTION DESIG SHIELDING CONSTRULTION-SLAN/ I3-13-O2276 LTER CONSTRUCTION IN FOUR NATIONAL MODEL BUILOING CODES I CONSTRUCTION DUAL-PURPOSE-SHELTERS, $\$$ TR-36/SHE $13-13-0228$ I INTERNAT IONAL-CIVIL-DEFENSE POSTA ITACK-OPERATIONS RESCUES CONSTRUCTION FIRES ROADS, \$JPRS-27, 419 \% TT-64-/ O6-O7-DI7O9 1 ISTRATION 1 SHEL TER S DUAL-PURPOSE-SHELIERS COSTS GERMANY CONSTRUCTION HARCEVING DESIGV DYNAMIE-PRESSURE $/$ I $3-13-0 I 827$ IATIONS FOR INCLUSION OF REDUIREMENTS FOR FALLOUT SHELTER CONSTRUCTION IN FOUR NATIONAL MODEL BUILDING CO/ I3-I3 -O22BI OVERY DATA-PROCESSING MAINTEN/ PARM SYSTEN MANUAL III-K. CONSTRUCTION IN THE PROTOTYPE MODEL I REPAIR REC I3-I3-D2282 T-SHELTERS UNDERGROUND-STRUCTURES COLORADC DU/ PROTECTIVE CONSTRUCTION INSTITUTE STUDIES 1 SHELTERS FALLOU I3-I3-OIB3I IPLY BURIED PROTECTIVE STRLCTURES ( UNDERGROUD-STRUCTURES CONSTRUCTION LOACIVGIMECHAVICS) SHOCK (MECHANICS/ IB-O3-O2OI 7 / COMMERCE UTILITIES TRANSPORTATION COMMUNICATIONS BANKING CONSTRUCTION MAINTENANCE REPAIR GJVERNMENT CASU/ O5-03-D2I 53 IMINATION REPAIR GOVERNMENT HOSPI TALS HOUSING AGRICULTURE CONSTRUCTION MANPOWER INDUSTRIAL-PRODUCTION COM/ O5-03-D2I5I /CTURES FALLOUT-SFELTERS BLAST-SHELTERS THERMAL-RACIATION CONSTRUCTION MATERIALS FIRE-SAFETY COSTS, *TR-/ I3-I3-OI82I /FILTERS I VENTILATION FIL TER S(FLLID) CHENICAL-PRCFERTIES CONSTRUCTION MECFAVICAL-PROPERTIES THERMAL-RESI/ I3-II-DI 72 IIONAL-CIVIL-DEFENSE CIVIL-DEFENSE-SYSTENS HASTY-SHELTERS CONSTRUCT ION NUCLEAR-EXPLOSIOY-DAMAGE EXPLOSIONI I3- 3 -OIB26 ER HARNESS AND SAFETY BELTS I TEST EQ TARY-SEWERS, *WPCF-9 WWAES THERMAL-RADIATION RADIOLOGICAL-CONIAMINATICA FIRES CONSTRUCTION POSTATTACX-OPERAT IONS REPAIR MILITI I3-I?-OIBO ALES REVEVUE UT ILA TION FINANCING OPERATING-CCSTS CANADA CONSTRUCT ION PRICES IHOUSING FOOD FLOODS CIVIL-DEFENSE-OPERATING-PLANS REPAIR CONSTRUCTION REAL-ESTATE REFUGEES BANKING INSUR/ O5-O3-DIG36 / STREET I TRANSPORTATION SUBWAYS 'TESTS BORING WASHINGTON CONSTRUCTION SETTLEMENT (STRUCTURAL) CONSOLIDATI/ I3-O2-DI7B5 RY GEOLOGY INSTRUMENTATION DEFORMATION LOADING (MECHANICS) CONSTRUCTION STATISTICAL-AVALYSIS I \$A.L. I2O6 /O I3-I3-02275 /COMMAVD AT CHEYENNE MOUNTAIN, EL PAST COUNTY, CCLCRADC 1 CONSTRUCTION UNDERGROUVD-STRUCTURES BLASTING EX/ I3-13-02274 IIZED SER IES OF FALLOUT SHELTERS I UNDERGROUND-STRUCTURES CONSTRUCTION URBAN-AREAS PROTECTION-FACTOR BLASI I3-I3-OI8IS 1 USED IN EXISTING URBAN BUILDINGS IN THE UNITED STATES 1 CONSTRUCTION URBAN-AREAS RADIOACTIVE-FALLOUT FA/ I3-I3-O227 7 INS IVE STREET BUILDING PROGRAM IN HAMBURG I GERMANY ROADS CONSTRUCTION URBAN-AREAS TUNVELSITRAVSPORTATION/ I3-C2 -DI786 TYPE FALLOUT SHELTER FDR BEEF CA TILE, BCVINES SHIELDING CONSTRUCTION VENTILATION, \#MISC. PU8. NO. 947, I3-13-0I8I8 /LAST RADIOACTIVE-FALLOUT HEAT-TRANSFER EXPOSURE TCXICIITY CONSTRUCTION-MATERIALS, *IITRI-N6D6I *AD O5O 3/ I3-I?-D2269 T LOACS ON PRESTRESSED CONCRETE BEAMS ( BEAMS (STRUCTURAL) CCNSTRUCTION-MATERIALS LOAD-DISTR IBUTION DEFLECI I3-13-02289

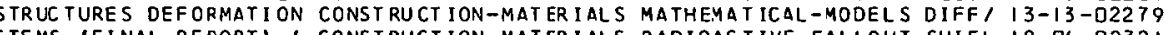

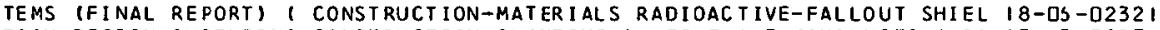
DIOACTIVE-FALLOUT BUILDINGS CONSTRLCTION DESIGN SHIELDING CONSTRUCTION-SLANTING I *FG-F-I.3 /ANALYSTS I RA I3-I3-02276 ELCINE RADIAT ION-EFFECTS RADIOAC TIVE-FALLOUT CONSTRUCTION CONSTRUCTION-SLANT ING DESIGN I \$T3-39 /RDS I SHI I3-I3-02286 ATTACK I CIVIL-DEFENSE-OPERA TING-PLANS FALLCUT-SHELTERS CONSTRUCTION-SLANT ING JPERATIOVS-RESEARCH SHELT/ O5-OI -OIG 23 -ANAI NATIONAL DIRECTORY OF ARCHI TECTURAL, ENGINEERING AND CONSULTING FIRMS WITH CERTIFIED FALLOUT SHELTER I3-I3-O2276 COVERY ACTIVITIES I POSTATTACK-OPERATIONS SOCIETAL-RECOVI CONSUMER BEHAVIOR AND WORKER PARTICIPATION IN RE OS-IJ-O2IBO RICULTURAL STATISTICS 1966 ( AGRICULTURE COSTS PRODUCTION CONSUMPTION FACILITIES, \#A.L. IOI8 AMACE-ASSESSMENT MOD/ PARM SYSTEM NANIIAI III -B. PERSCNAL CONSUMPTION MODEL I RECOVERY ECONOYIL-RECOVERY D OS-D3-D2I5I LING MOBILIZATION STRATEGIC-MATERIA/ IRON AND STEEL SCRAP CONSUMPTION PROBLEYS I SALVAGE RESOURCES STOCKPI US-US-UIOUS IRUSSIA AFRICA LATIN-AMERICA JAPAN GROSS-NATICNAL-PRODUCT CONSUMPTION(FOODI COMMERCE AGR ICULTURAL-ECONOMI/ O2-D2-DI6I6 MOTORS RUSSIA COMMUTATORS/ A DIRECT CURRENT MOTOR WITH NO CONTACTS AND A TRANSISTOR COMMUTATJR I ELECTRIC- D9-D3-OI752 SH/ THE TRAYSPOR TATION OF HIGHLY RADIOACTI VE RATERIALS I CONTAINERS RACIOACTIVE-WASTES WASTES(INDUSTRIAL) I8-OP-D2O33 ITIONS ( FOOD PRESER VATION FIBERB CARD PHYSICAL-PROFERTIES CONTAINERS TASTE FALLOUT-SHELTERS ) *TR-67-25-CI D6-03-022I 2 EARTH DISPLA/ PRELIMINARY CONCEPIS OF SUBSURFACE REACTOR CONTAINMENT STRUCTURES TO WITHSTAND OIFFERENTIAL I8-OF-O2O4O RADIOACTIVE-FALLOUT CON RECOVERY OF PETROLEUM REFINERIES CONIAMINATED BY FALLOUT 1 POSTATTACK-OPERATIONS OS-O3-DIO5I ERS, PROTECTING IHE RURAL POPLIAIION AGAINST RADICACTIVE COATAMINATION I USSR CIVII-DEFENSE-SYSTEMS SHELT DG-IS-OI73I USSR I RURAL-AREAS BOMBS GUIDED-NISSI ES RACIOLOGICAL-CONTAMINATION RAOI I EVALUATION OF FALLOUT CONTAMINAT ION FROM SURFACE RUVOFF 1 WA TER-SUPPLI OO- 3 -DIT24 IC BY FALLOUT I POSTATTACK-OPERATIONS RADIOACTIVE-FALLOUT CONTAMINATION INCUSTRIAL-PLANTS DECAY-RATES RESI O5-D3-DIOSI IGR I CULTURE RACIOACTIVE-I SOTOPES RADIOAC II VE-FALLOLT FOOD CONTAMINAT ION MOCELSISIMULATIONS) RADIOBIOLOGY, OG-I8-DI725 IRACIOLOGICAL AGENTS - USSR I RADIOLOGICAL-WARFARE-AGENTS CONTAMINATION RACIATION-IVJURIES TOXIEITY CYANO/ DO-IS-DIT2I I THE SIMPLEST MEANS - USSR I WATER-SUPPLIES PURIFICATION CONTAMINATION RACIOLOGICAL-COVTAMINATION CHEMIC/ OG-IJ-DIT22 TION SUEZ-CRISIS ATTI TUDES MIDDLE-EAST ME IHODOLOGY THEORY CONTENT-ANALYSIS (COMPUTERS) HISTORY, 1 FAR-4O47 OS-D4-O2I 7O S DISAR' AMER ICANS VIEWS ON CIVIL DEFENSE IN THE CCLD WAR CONTEXT- 1966 I ATTITUDES PUBL IC-JPINION SURVEY DS-IJ-OIOT8 DAMAI MAP IV - NREC SMALL SCALE MAPPING PRCGRAN - NAPS OF CONI INENTAL UNIIED STATES I GEOGRAPHY RESOURCES DS-D3-O2I 40 
/ECTS OF NUCLEAR ATTACK ON RAILROAD TRANSPORTATION IN THE CONTINENTAL UNITED STATES 1 SYSTEMS-ENGINEERING/ O5-03-02I 28 /PROPAGAT ION MATHEMATICAL-ANALYSIS ELASTICITY SHOCK-WAVES CONTINUUM-MECHANICS LINEAR-SYSTEMS FUNCTIONS I $120-11$-O2OBO ATER STAVDARDS I WATER-SUPPLIES REGULAYI ONS RADIOACTI CS COMM/ STUDIES IN PROGRESS OR RECENTLY CCMPLETED - ARMS CONTROL AND DISARMAMENT I BIBL IOGRAPHIES ECONOMI DS-0+ -OI663 CS CONFI STUDIES IN PROGRESS OR RECENTLY CCMPLETEC - ARMS CONTROL AND DISARMAMENT 1 BIBLIOGRAPHIES ECONOMI OS-D4 -OI G6S ICS CON/ STUDIES IN PROGRESS OR RECENTLY CCMPLETED - ARMS CONTROL AND DISARMAMENT 1 COMMUNIST-CHINA ECONOM O5-D4-DI662 ICS CON/ STUDIES IN PROGRESS OR RECENTLY COMPLETED - ARMS CONTROL AND DISARMAMENT COMYUNIST-CHINA ECONOM OS-OH-DI 664 AL-MODELS SIMULAT/ DEVELOPMENT OF LTILITY THEORY FCR ARMS CONTROL AND DISARMAMENT I GAYE-THEJRY MATHEMATIC OS-D - -D2I GB ' *FAR-396IIII) DEVELOPMENT OF UTILITY THEORY FCR ARMS CONTROL ANO DISARMAMENT I GAME-THEORY SIMLLATION DS-DH-O2IS9 HE CUSP EFFECT I HYDRODYNAMICS DESIGN CUSPS FLUID-FI FLOW CONTROL BY GENERATION OF STANDING VORTICES AND T 20-DH-D2O5S GHT ING-EOUIPMENT CONTROL-SYSTEMS TRANSFORMERS COILS CIR/ CONTROL DEVICE FOR ELECTROLUMINESCEVCE CELLS I L O9-O5-OI756 - A STAT ISTICAL ANALYSIS OF FAC TCRS AFFECTING GOVERANENT CONTROL IN SOUT VIETNAM I POL ITICAL-SCIENCE SOI DS-DF-D2I 75 NC DRAINAGE CHANNELS II. PLANS, PROFILES, CROSS SECTION/ CONTROL OF SURFACE STORM WATER BY STORM DRAINS A I3-D?-DI7R ND DRAINAGE CHANNEL I I PENA ND DRAINAGE CHANNELS I GENERAL DESCRIPTION OF WORK PRO/ CONTROL OF SURFACE STORM WATER BY STORM ORAINS A I3-O2-OI782 NC CRAINAGE CHANNELS - 1958 PROGRAM I. GENERAL DESCRIPT/ CONTROL CF SURFACE STORM WATER BY STORM DRAINS A I3-02-DI783
NC CRAINAGE CHANNELS - 1964 PROGRAN I. GENERAL DESCRIPT/ CONTROL OF SURFACE STORM WATEZ BY STORM DRAINS A I3-O2-DI7BL NC CRAINAGE CHANNELS - 1964 PROGRAN I. GENERAL DESCRIPI' CONIROL OF SURFACE STORM WATEZ BY STORM DRAINS A $13-02$-DI7B4

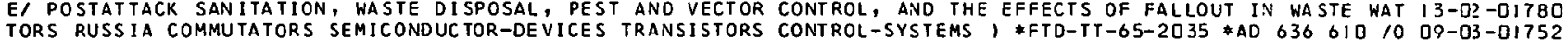

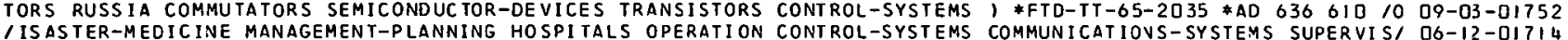

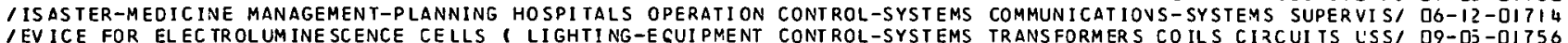
IEV ICE FOR ELEC TROLUMINE SCENCE CELLS I LIGHTING-E CUIPMENT CONTROL-SYSTEMS TRANSFORMERS COILS CIRCUITS LSSI OQ-DJ-DI756
LOUT/ A CALCULATIONAL MODEL FOR CCNDENSED STATE DIFFUSION CONTROLLED FISSIOV PRODUCT ABSORPTION DURING FAL I8-03-02037 YYSTEM MANUAL III-E. CAPACITY EXPANSION, RESTORATION AND CONVERSION I RESOURCES MANPOWER GEOGRAPHY INDUSI O5-O3-D2ISQ URCES GEODETIC POSITION I GP, TO UNIVERSAL T/ COORDINATE CONVERSION FORMULAS FOR USE WITH WORLD WIOE RESO O5-O3-O2I33 EC-STATES BURE/ CONSTRUETION AND ENGINEERING DATA - WATER CONVEYANCE TUNNELS - GRAVITY AND PZESSURE I UNIT I3-I3-D2283 ECTRICITY THERMA I SEMICONDLCTOR THERMOELECTRIC TRANSISTOR COCLERS 1 SEMICONDJCTOR-DEVICES.COOLING THERMOEL DQ-OI -OI748 GY HEAT-EXCHANGE TEMPERATURE VENTILATION BODY-TEMPERATURE COOLING, EC ABOVE AND BELOW GROUND I FALLOLT-SHELTERS COMPUTERS B/ COCLING ANALYSES FOR PROTECTIVE STRUCTURES LOCAT I3-II-GIT IKI /K( GEOLOGY) HEATING MINING-ENG INEERI NG LOADI NG (MECHANICS) COCLING FAILJRE(MECHANICS) SH)CK-WAVES VENTILATI $13-13$-O2273 /AL-CONTROL VENTILATION ELECTRIC-PCWER-PRCOUCTION HEAT ING COOLING LIGHT ING WASTESISAVITARY-EVGINEERING) I/ I3-I3-O227I TILAT ION METABOLI SM BOOY-TEMPERATLRE CLOTHING TEMPERATURE COOLING PERSPIRATION EVAPORATION I *AD 648 467 / D6-1S-OI7I9 NC AS THE FUEL I FUELS COOLING-AN THE STLOY OF A SHELTER COCLING SYSTEM USIVG METHAVOL AS THE HEAT SINK A $13-0 I$-QI773

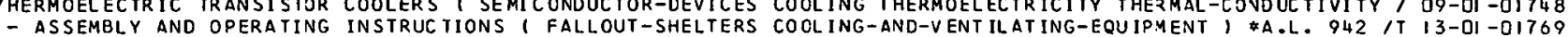
LSISIMULATIONS) HEAT-EXCHANGERS TEMPERATURE HEAT-TRANSFER COCLING-AND-VENT ILATING-EQUIPYENT, $\$$ ADD G3I 420 I3-13-DI8I4 IEH YORK CITY AND VICINITY I FALLCUT-SHELTERS URBAN-AREAS COOLING-AND-VENTILATING-EQUIPMENT BUILDINGS UND/ I3-I3-OIBI3 I USING METHANOL AS THE HEAT SINK AND AS THE FUEL I FUELS COOLING-AND-VENTILATING-EQUIPMENT FALLOUT-SHELTI I3-OI-DI773 IOR FOR USE IN EVAPORATIVE COOLERS I VENTILATION HUMIOITY COOLING-ANO-VENT ILATING-EQUIPYENT GAS-FLOW' TESTI I3-DI -D22S4 IPHYS IOLOGY EXPLOSION-EFFEC TS SANI TARY-ENGINEERING DESIGN COCLING-AND-VENT ILAT ING-EQUIPYENT HAZARDS COSTSI I3-I3-OI8I I ITUDIES ( FALLOUT-SHELTERS PHYSI OLOGY SYSTEMS-ENGINEERING COCLING-AND-VENT ILATING-EQUIPMENT SHEL TER-MANAG I5-O3-O2297 IITABILITY STUDIES I FALLOUT-SHEL TERS SYSTEMS-ENGI NEERING COOL ING-AND-VENTILAT ING-EQUIPMENT SHELTER-MANAG I IS-D3-D2298 IUMMARY AND REVIEW 1 FALLOUT-SHELTERS SYSTEMS-ENGINEERING COOLING-AND-VENT ILATING-EQUIPYENT SHELTER-MANAGI IS-O3-O23OD

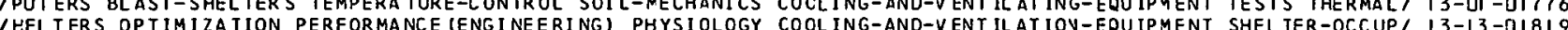
HEL TERS OP IIMIZA TION PERFORMANCE (ENG I NEERING) PHYSI OLOGY COOLING-ANO-VENT ILATIOV-EQUIPMENT SHEL TER-OCCUP I I3-I3-OI8I 9 INTRY REENTRY-VEHICLES DESIGN AERCDYNAMIC-HEATING HEATING COOLING-SYSTEMS FLUID-DYNAMIC-PROPERTIES ORAG S/ 22-02-D2350
OLOGY SEMANTICS SOCIAL-SCIENCES PCLITICAL-SCIENCE CULTURE COOPERATION, \$FAR-4D48 /TITUDES BEHAVIOR METHOD O5-IJ-D2I78

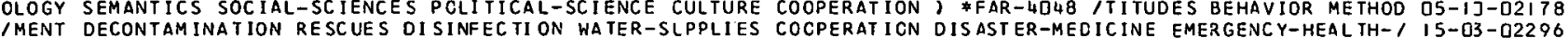

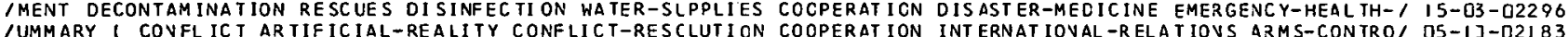
DWIDE RESOURCES GEODETIC POSI TIOA I GP, TO UNIVERSAL T/ COORDINATE CONVERSION FORMULAS FOR USE WITH WORL D5-D3-D2I3 3 D WIDE RESOURCES GEODETIC POSI TI ON I GP, TO UNIVERSAL T/ COORDINATE CONVERS ION FORMULAS FOR USE WITH WORL DS-D3-D2I33
VAL SUBJECT-HEADINGS CLASSIFICAT/ THE STATE OF THE ART OF COORDINATE INDEXING I INDEXES INFORMATION-RETRIE OS-O2-O2II ICAL GROUNC SHOCK EFFECTS II. APPLICATION OF GENERALIZED COORDINATES TO PLASTIC WAVE PROBLEMS 1 SOIL-MEC, D8-13-QIT4 7 MAL) PROTEIVS GENETICS AG I PROCEEDINGS OF THE HIGH LYSINE CORN CONFERENCE ( NUTRITIOVIHUMAN) NUTRITION(ANI OG-IS-DIT2D IRVAT ION SHELF-LIFE BEVERAGES BREAD CANNEO CEREALS COFFEE CORN DEFYDRATED-FOODS EGGS FATS FZJZEN-FOODS FR/ D6-03-O22J9 WARE HOUSE AND LOCATION I AGRICUL TURE SI COMMODITY CREDIT CORPORAT ION WAREYOUSE RECEIPT WHEAT INVENTORY BY D2 -OZ -D2IO9 H-SPEEO-GROUND-TR ANSPORT RESEARCH-PROGRAM-ADMIN/ KEYSTONE CORRIDOR TRANSPORTATION STUDY 1 PENNSYLVANIA HIG 23 -G2-O2092 IONS ABSORPTION OZONE REACTION-KINETICS NLCLEAR-RACIATION COSMIC-RAYS ELECTROMAGNETIC-WAVES PROPAGATION MI OL-OI -O21 I 3 TERS (PROGRAMM ING) BUILOINGS CONSTRUC TI METHOD OF SHELTER COST ANALYSIS 1 COSTS METHODOLOGY SHELTERS COMPU I3-I3-OIB28 UT SHELTERS I UNDERGROUND- $/$ DESIGN MODIFICATIONS AND 1962 COST ANALYSIS FOR A STANOARDIZED SERIES OF FALLC I3-I3-DIBIS COST BENEFIT STUDIES I COST-EFFECTIVENESS MANAGEMENT-EN/ COST ANALYSIS FOR PLANNING PROGRAMMING BUDGETING O5-DI-D2292 MENT-EN/ COST ANALYSIS FOR PLANNING PROGRAMMING BUCGETING COST BENEFIT STUCIES 1 COST-EFFECTIVEVESS MANAGE D5-II-D2292 NE SURVIVAL URBAN-AR/ ON THE DESIGN OF RISK ORIENTED, LOW COST FALLOUT SHELTER SYSTEMS I MAVAGEMENT-PLANNI I3-I3-OIB29 NE SURV IVAL URBAN-AR I ON THE DESIGN OF RISK ORIENTED, LOW COST FALLOUT SHELTER SYSTEMS 1 MAVAGEMENT-PLANNI I3-I3-OIB29
OF THE INTERRELATIONSHIP OF PRESSURE STRENGTH, SIZE AND COST OF MULTIPURPJSE BUILDINGS FOR A FEDERAL TR/ I3-I3-OI827

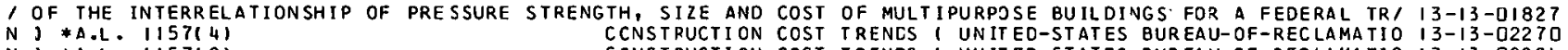

N $* A . L \cdot 1157(2)$

N *A.L. $1157(3)$

CCNSTPUCTION COST. TRENOS I UN IT ED-STATES BUR EAU-OF-RECLAMATIO 13-13-02284

C.ONSTRIICTION COST TRENDS IUNITED-STATES BUREAU-OF-RECLAMAIIO I3-13-02285

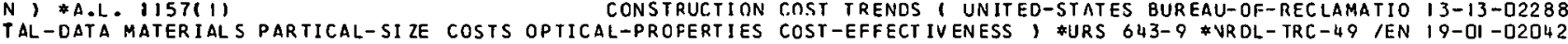
/EXPLOSION-DAMAGE MANAGEMENT-ENGINEERING NANPOWER-STUDIES COST-EFFECTIVENESS ENVIROVMENT MAVAGEMENT-PLANN/ I5-D3-02293 1 PR PLANNING PROGRAMMING BUDGE IING COST BENEFIT STUDIES 1 COST-EFFECTIVENESS MANAGEMENT-ENGIVEERING MANAG/ O5-0I-O2292 IRS EOUIPMENT URBAN-AREAS RURAL-AREAS MANAGEMENT-PLANNING COST-EFFECTIVENESS TRAINING FIRE-STORMS CONFLAGI I3-I2-OIBDG IIPMENT SATELL I TESIARTIFICIAL) SPACECRAFT DEFENSE-SYSTEMS COST-EFFECT IVENESS VULNERAB IL ITY DATA-PROCESSIN/ 22 -D2 - O235I IUCTURES I RADIOACTIVE-FALLOUT PARTICLES DEBRIS VOLCANOES COSTA-RICA WIND IVSTRUMEVTATION , *SZI-MU-57T9I/ I8-D3-02O38

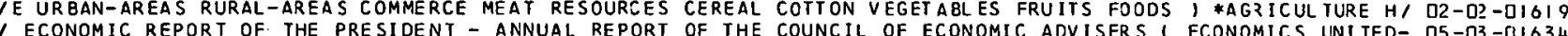
RCES (HUMAN) RECOVERY VULNERABILE UY USAN-AREAS DEMCGRAPHY COUNCIL OF ECONOMIC ADVISERS 1 ECONOMICS UNITED- D5-03-DIO3L

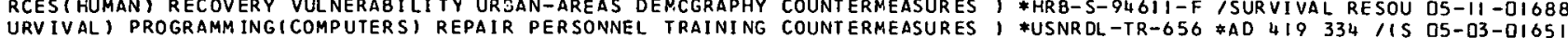
IRAC IOACT IVE-FALLOUT RADIOLOGICAL-CONTAMINATIIN NOSE-RATE COUNTERMEASURES FALLOUT-SIIELTERS PADIOLOGICAL-OI UG-IS-UZ2ZO

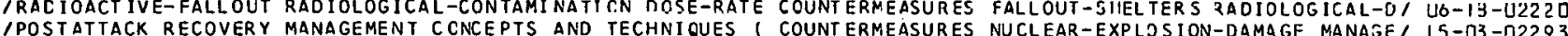

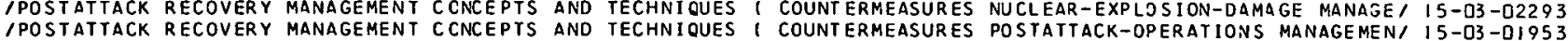

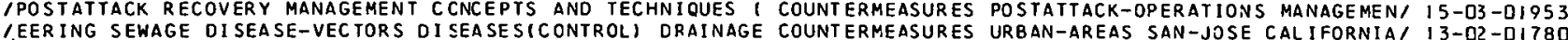

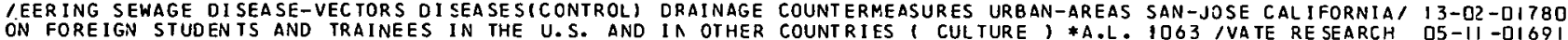

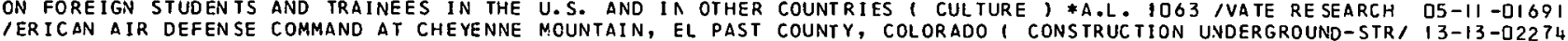
IER ICAN AIR DEFEN SE COMMAND AT CHEYENNE MOUNTAIN, EL PAST COUNTY, COLORADO I CONSTRUCTION UNDERGROUND-STR, I3-13-02274
IMISS ILE-DEFENSE SYSTEMS MILITARY-STRATEGY, $\$$ HI-729, WHY COUPLE, BMD TO FALLOUT SHELTERS I URBAN-AREAS ANT I5-O3-UI9O7

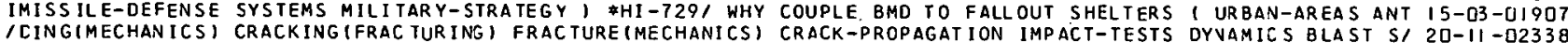

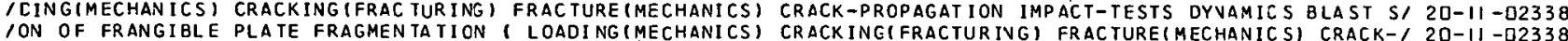
ION OF FRANGIBLE PLATE FRAGMENTATION I LOADING (MECHANICS) CRACKING(FRACTURING) FRACTURE(MECHANICS) CRACK-I $20-1$ - - 2338 IUCT ION-MATERIALS LOAD-DISTRIBUTICN DEFLECTION STEEL RODS CRACKS STRAIN(MECHANICS) LOADINGIMECHANICS), * I3-I3-D2289 ETY BELTS 1 TEST-EQUIPMENT IOESIGN AND CONSTRUCTICN OF A CRASH DUMHY FOR TLSTING SHOULDER HARVESS AND. SAF IS-I?-UIBO3 IENT, YIELD, MEDIUM, AND DEPTH OF BURST ON CAVITY RADII I CRATERING EXPLOSIOV-EFFECTS VUCLEAZ-EXPLOSIONS, I8-D3-O23IS IAPPL ICATION OF STATIC EQUILIBRIUN THEORY TO DECOUFLING ( CRATERING EXPLOSION-EFFECTS NUCLEAR-EXPLOSIONS, I8-O3-O23IS HYCRODYNAMICS PLOWSHARE - NUCLEAR EXPLOSIONS - PEACEFUL APPLICATIONS - CRATERS FROM ROW CHARGES IVTERRUPTED BY A DUD I IB-O3-O23I4 
TORY BY WAREHOUSE AND LOCATION I AGRICULTLRE S/ CCNMODITY CREDIT CORPORAT IOV WAREHOUSE $2 E C E I P T$ WHEAT INVEN D2 -0? - D2I D9 ENT INTERNATIONAL-CIVIL-DEFENSE CCMMUNIST-CHINA INCUSTRI/ CRISES CIVIL DEFEVSE AND DETERRENCE I USSR MOVEM IS-D3-D2295 ITY MILII ARY-STRATEGY INTERNATIONAL-CIVIL-DEFENSE RUSSIA' CRISIS CIVIL DEFENSE AND DETERRENCE I VULNERABIL IS-D3-DI896 ITY INTERNAT IONAL-CIVIL-DEFENSE EARLY-WARNING - SYSTENS USI CRIS IS CIVIL DEFENSE AVD DETERRENCE I VULNERABIL I5-D3-DI 222 SPORTATION AGEVCY RAPID TRANSIT SYSTEM - NANUAL CF DESIGN CRITERIA ( URBAN-AREAS, \#A.L. II93 /APITAL IRAN $23-02$-Q2353 LTERS UNDERGRO / THE ELASTIC RE SPONSE OF BURIEO CYLINOERS, CRITICAL LITERATURE REVIEW AND PILOT STUDY I SHE I3-I3-OI825 OLI PRACTICAL EXPERIENCES OF FIRE PROTECTION SERVICES - A CRITICAL PRESENTATION OF THE ORGAVIZATION, TECHN I3- I2 -D2264 OLI PRACT ICAL EXPER IENCES OF FIRE PROTECTION SERVICES - A CRITICAL PRESENTATION OF THE JRGAVIZATION, TECHN I3- I? -O226S I AGR ICUL TURE I AGR ICUL TURAL-ENGI NEERI NG ANI MAL-HUSBANORY CRCP-RESEARCH ENTOYOLOGY VUTRITIOV WATER-CONSER/ O2-OJ-O2I D8 ERNMENT SPONSORED AND PRIVATE RE SEARCH ON FOREIGN STUDEN/ CROSS CULTURAL ECUCATION - A BIBLIOGRAPHY OF GOV OS-II -DIGQI ISTORM ORAINS AND DRAINAGE CHANNELS II. PLANS, PRCFILES, CROSS SECTIONS, AND GEVERAL LOCATION OF RIGHTS I I3-D? -OI779 ISTORM DRAINS AND ORAIVAGE CHANNELS I1. PLANS, PRCFILES, CROSS SECTIONS, AND GENERAL LDCATIJN OF RIGHTS, I3-02-0I78I IHOOL OF MINES 52(3) 8EHAVIOR OF MATERIALS IN THE EARTHS CRUST - SECOND ANVUAL SYMPOSIUM ON ROCK MECHANI/ O8-D7-D224 4 ILASERS SCIENTIFIC-RESEARCH GLASS OPTICAL-PROPERTIES RUBY CRYOGENICS GASES PUMPIVG(JPTICAL) FLASH-LAMPS L/ 20-0 -02062 /LASERS SCIENTIFIC-RE SEARCH GLASS OPTICAL-PROPERTIES RUBY CRYOGENICS GASES PUMPIVG(JPTICAL) FLA SH-LAMPS L/ 20-OJ -02062 N RUBY LASERS ( PUMPINGIOPTICAL) CAVITY-RESONATORS OPTICS CRYSTALS MIRRORS \#AD 637825 NULSING ME THODS I $20-D 5-02056$ IICAL NONLINEARITIES USING FOCUSED GAUSSIAN LASER BEAMS I CRYSTALS OPT ICAL-PHENOMENA HARMONIC-GENERATORS I 20-DS - D2D6 7 作 TUDENTS ANC TRA INEES IN THE L.S. AND IN OTHER COUNTRIES I CULTURE 1 \#A.L. 1063 IVATE RESEARCH OV FOREIGN S OS-II -OIO9I OLOGY) DEMOGRAPHY ECONOMIC-RECOVERY CIVIL-DEFENSE-SYSTEMS CULTURE I \#HSR-RR-66/21-CR ICIOLOGY STRESSIPSYCH OS-II -D2I 9D R MET FODOLOGY SEMANTICS SOCIAL-SC IENCES PCLITICAL-SCIENCE CULTURE COOPERATION, *FAR-4D48 /TITUDES BEHAVIO DS-1D-D2I78 IOCATION ATTITUDES CIVIL-DEFENSE-SYSTENS COLD-WAR SURVEYS CULTURE DEMOGRAPHY SOCIOLOGY POPULATION DISARMAI D5-IJ-O2I77 IUCTURE OF INTERPER SONAL INIENTIONS I BEHAVIOR MOTIVATION CULTURE RESPONSE REACTION(PSYCHOLOGY) I \&FAR-4O/ O5-IJ-O2I 79 VIRUS 1 BIOLOGICAL-WARFARE-AGENTSMI YAGAMANELLA-PSITTACI CULTURE-MEDIA, \#AD O38 $404 / T I O N$ SY PSITTACOSIS I5-O2-DI89I OLAT ION OF PASTEURELLA PESTIS 1 B IOLOGICAL-WARFARE-AGENTS CULTURE-MEOIA GRONTH 1 \$AD O38 440 /A FOR THE IS I5-OZ-DI875 COMMUTATOR I ELECTRIC-MOTORS RLSSIA COMMUTATORS/ A DIRECT CURRENT MOTOR WITH NO CONTACTS AND A TRANSISTOR OQ-D3 -DI752 ARS AND ACADEMIC CENTERS - AFRICA I ECONONICS, A LIST OF CURRENT SOCIAL SCIENCE RESEARCH BY PRIVATE SCHOL Q5-II - DIG92 ARS AND ACADEMIC CENTERS - INTERNATIONAL AFFAI/ A LIST OF CURRENT SOCIAL SCIENCE RESEARCH BY PRIVATE SCHOL O5-II -DI693 ARS AND ACADEMIC CENTERS - WESTERN EUROPE, GREI A LIST CF CURRENT SOCIAL SCIENCE RESEARCH BY PRIVATE SCHOL DS-II -DIOOU ARS AND ACADEMIC CENTERS - MIDDLE EAST I ARS AND ACADEMIC CEN TERS - MIODLE EAST BIBLI/ A LIST OF CURRENT SOCINL SCIENT.F RESEARCH BY PRIVATE SCHOL DS-III -DIOQS ARS AND ACADEMIC CENTERS - AMERICAN REPUBLICS / A LIST OF CURRENT SOCIAL SCIENCE RESEARCH BY PRIVATE SCHOL O5- II -UIBYO ARS AND ACADEMIC CENTERS - USSR AND EASTERN EUI A LIST OF CURRENT SOCIAL SCIENCE RESEARCH BY PRIVATE SCHOL Q5-II-DIO97 ARS AND ACACEMIC CENTERS - ASIA 1 BIBLIOGRAPHI/ A LIST OF CURRENT SCCIAL SCIENCE RESEARCH BY PRIVATE SCHOL Q5-II-OI698 R OISASTER-YEDICINE NUCLEAR-hARFARE-CASUALTIES FIRST-AID/ CURRENT TOPICS IN SOVIET MILITARY MEDICINE I USS DG-DS-OI7J3 1 FLOW CONTROL BY GENERATION OF STANDING VORTICES ANO THE CUSP EFFECT I HYCRODYNAMICS DESIGN CUSPS FLUID-F $20-0$ '-D2O55 NCING VORTICES AND THE CUSP EFFECT ( HYDRODYNAMICS DESIGN CUSPS FLUID-FLOW) *AD IO7 323 /EVERATION OF STA $20-0$ + -02055 AIRS TARIFFS IMPORT-DUTIES FOREIGN-TRADE C/ INTERNATIONAL CUSTOMS JOURNAL - CANADA I LAW INTERNATIONAL-AFF O5-O3-DI G4I

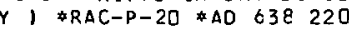
/CK-MECHANICS LOADING IMECHANICSI) LSSR EXCAVATION SHEARING CUTI ING BENDING TORSIOV STRETCHING COMPRESSING / D8-D7-D2242 IWARFARE-AGENTS CONTAMINATION RADIATION-INJURIES TCXICITY CYANO-ACIDS CARBOY-MONOXIOE VESICANTS POISONOUS/ D6-IS-OIT2I GROUND-MOIION UNDERWATER DESIGN COSTS LOADINGIMECHANICS) CYLINDERS *SD-7I-66(R))-5872 /ECHANICS DYNAMICS I8-09-D2040 STUCY I STELTERS LNDERGRO/ THE ELASTIC RESPONSE OF BURIED CYLINCERS, CRITICAL LITERATURE REVIEW AND PILOT $13-13-D I 825$ THYS ANGLE-

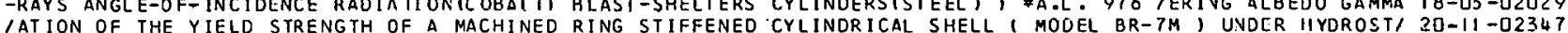
AL AL-80CIES COMPOSI/ VIBRATION OF MLLTI-LAYERED ANISCTROPIC CYLINDRICAL SHELLS I STRUCTURAL-SHELLS CYLINDRIC 20-1I-O2345 GS WITH STIFFENED EOGES I STRUCTURA ANALYSIS OF CIRCULAR CYLINDRICAL SHELLS CONTAIVING RECTAVGULAR OPENIN 2O-II -D2D8I

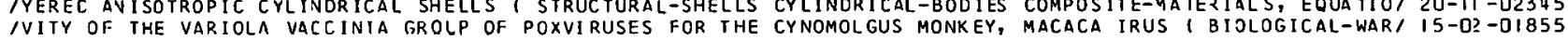

D

ONIZATION, AIRBURST IONS/ VALIOITY OF THE 02 (-) MOOEL FOR D REGION RECOMBINATION I IONOSPHERE OXYGEN GAS-1 O4-OI -02II3 SPORT ATION UT IL IT IES FISCAL-AND-MCNE TARY ECONOMICS, NL-USE URBAN-PLANNING POPULATI ON POLITICAL-SCIENCE, \#PRC D-1341/-AREAS CECENTRALIZATIJN VULVERABILITY LA 23-DS-D2ID7 TION-INJURIES RADIOLOGILAL-DOSAGE EXI LONG TERM RACIATION DAMAGE - EVALUATION OF LIFE SPAN STUDIES I RADIA OO-I3-OI733 ES CIVIL-DEFENSE-SYSTEMS CALCULATIONSI NATO CONFERENCE ON DAMAGE ASSESSMENT - SUMMARY REPORT 1 UNITED-STAT I5-OS-OI955 11. I RESOURCES VULNERABILIIY WEAPONS-EFFECTS WIND / NREC DAMAGE ASSESSMENT COMPUTATION PROGZAM - JUMBO I OS-O3-02I34 UCLEAR-EXPLOS ION-DAMAGE POPULATIONIDISTRIBUTICN) GEOG/ ON DANAGE ASSESSMENT MODELS I RADIOACTIVE-FALLOUT N I5-DS-OIOS2 ITY PROGRAMMING (COMPUTERS) CASLAL/ STREAK IV - NREC RAPID DAMAGE ASSESSMENT PROGRAM 1 RESOUREES VULNERABIL DS-D3-D2I 46 EC- $114(1)$ A LISIING UR TABLES AND PARAMETERS USED IN NREC DAMAGE ASSESSMFNT PROGRAMS I VULNERABILITYI \#NR O5-O3-O2I 38 MAGE/ CIVIL DEFENGE SHELTER REPRESENTATION FRR POPLLATION DAMAGE CALCULATICN - 1970-1985 OF MORTAL ITY ESTIMATES TO UNCER IAINIIES IN SOME NUCLEAR DANAGE PARAMETERS II. 1 MOCELS OAYAGE-ASSE SSME $/$ DO-2I -D2229

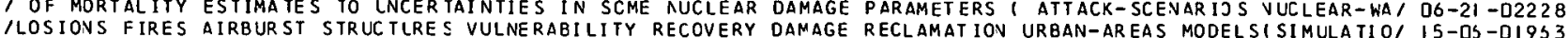

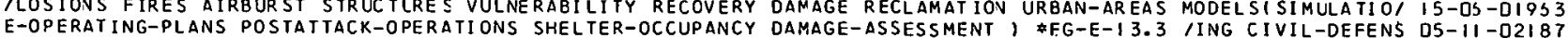
E-OPERATING-PLANS POSTATTACK-OPERATIONS SHELTER-OCCUPANCY DAMAGE-ASSESSMENT, \$FG-E- I3.3 /ING CIVIL-DEFENS D5- II-O2I87
IRC CRAFT OF READY I ( RESOURCES ANALYSIS DATA-PROCESSING DANAGE-ASSESSMENT, \$NREC-24II)

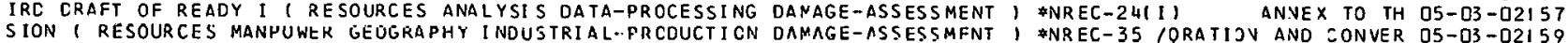

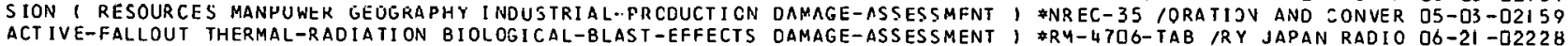
IVE RADIOAGRICULTURE, *NREC-I 39 LIVESTOCK III I DAMAGE-ASSESSMENT AGRICULTURE COMPUTERS RADIOACT DG-I9-O22I6 1 POL ITICAL-SCIENCE ECONOMICS SOCIOLOGY SOCIETAL-RECOVERY DANAGE-ASSESSMENT ATTACK-SCENARIOS, $\$ 0 C D-P S-64$, OS- II-O2I93 SING PR/ ATTACK I - ATTACK PATTERN GENERAIOR I SINULATION DANAGE-ASSESSMENT ATTACK-SCEVAR IDS EJMPUTERS HOU O5-O3-O2I 39 NGUAGES COMPUTERS CA SUALTIES/ REACT, A RECUEST LANGUAGE I OAMAGE-ASSESSMENT BLAST RESOUZCES PROGRAMMING-LA DS-D3-CIII 4 INOMIC-RECOVERY POSTATTACK-OPERATICNS POPULATICN SURVIVAL DAMAGE-ASSESSMENT CALIFOR.NIA RECOVERY I \$SRI-MU/ OS-D3-DIO39 IITY I POSTATTACK-OPERATIONS FOOD-DI SPENSING DISTRIBUTION DANAGE-ASSESSMENT CALIFORNIA URBAV-AREAS SUPPLYI OB-O -OIIII I WORLD WAR II GERMAN FIRE FAIALI IIES I FIRES URBAA-AREAS DA MAGE-ASSESSMENT CASUALTIFS POPULATION FIRE-STI I3-I?-D2262 IEART TOUAKE - A CASE STUDY IN THE EC ONOMICS OF DISASTER I DAMAGE-ASSESMENT CASUALT IFS POPULATION FIRE-STI I3-I?-D2262 ATI RECOVERY/ 05-03-0I636

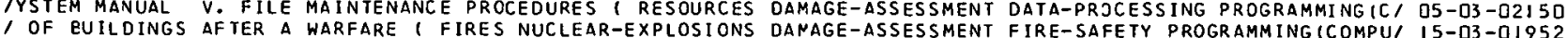

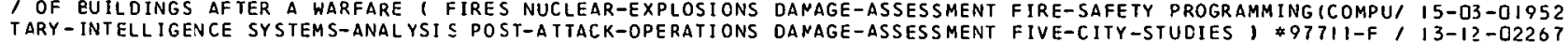

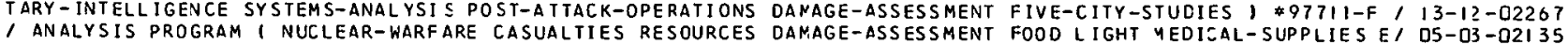
ANALYSIS PROGRAM I NUCLEAR-WARFARE CASUALTIES RESOURCES DAMAGE-ASSESSMENT FOOD LIGHT YEDIIAL-SUPPLIES E/ DS-D3-O2I 35
TERS) MATHEMATICAL-MODELS FLOW-CHART/ READY I I RESOURCES DAMAGE-ASSESSMENT LOGIC-DESIGV PRJGRAMMINGICOMPU OS-O3-D2I5S TERS ) MATHEMATICAL-MODELS FLOW-CHART/ READY I I RESOURCES DAMAGE-ASSESSMENT LOGIC-DESIGV PRJGRAMMINGICOMPU OS-03-D2I 5S
UM-PIPEL INES GAS-PIPELINES TRAN/ PIPELINE NETWORK STUDY 1 DAMAGE-ASSESSMENT MODELS NUCLEAR-HARFARE PETROLE O5-D3-D2I 1 I / PERSONAL CONSUMPIION MOOEL I RECOVERY ECONOMIC-RECOVERY DAMAGE-ASSESSMENT MODELS RESOURCES CAPACITY DEC I OS-D3-O2I II OCELSIS/ THE POSTATTACK POPULATION OF THE UNI TED STATES 1 DAMAGE-ASSESSMENT VUCLEAR-EXPLOSIJV-DAMAGE AGE M IS-0S-DI965 ITAINTIES IN SOME NUCLEAR DAMAGE PARAMETERS II. 1 MODELS DAMAGE-ASSESSMENT NUCLEAR-WARFARE-CASUALTIES CAI O6-21-O2229 1 IS OF CASUAL TY FUNCTIONS 1 CASUALIIES SYSTEMS-EVALUATION DANAGE-ASSESSMENT VUCLEAR-WARFARE-CASUALTIES MAI O6-2I -OI? 42 / CASUALTY ESTIMATE I ANALYSIS NUCLEAR-WARFARE-CASUALTIES DAMAGE-DSSESSMENT PATIENTS PRJGRAYMIVG ICOMPUTER/ O6-2I -D2225 ITIONS OF THE MANPOWER ANALYSIS SYSTEM I RESOURCES MODELS DA:IAGE-ASSESSMENT POPULATION CASUALIIES PROGRAMI OS-D3-D2I /EMS-ENGINEER ING ECONOMICS RECOVERY POSTATTACK-OPERATIONS DAMAGE-ASSESSMENT RADIATION-HAZARDS RADIOACTIVE/ O5-O3-D2I 8 IVULNERAB IL ITYI ANALYSISI SUR VIVAL DAMAGE-LINITING-SYSTENS DANAGE-ASSESSMENT RECOVERY THREAT-EVALUATION POI IS-C3 STR REC-I3 PARM SYSTEM MANUAL IV. RESOURCE DATA ROUTINE REC- 13 POSTATTACK-OPERAT IONS RECOVEI PARN PROJECT FINAL REPORT
MI PARM SYSTLM MANUAL III O. MANPOWER AND OC.IIPATICNS

DANAGE-ASSESS MENT RECOVERY THR EAT-EVALUAT
DAMAGE-ASSESSMENT RESOURCES, INREC-III $05-03-02136$ DAMAGE-ASSESSMENT RESOURCES DATA-PROSESSING, \#N O5-03-02129 DAMAGE-ASSESSMENT RESOURCES MANAGEMENT-PLANNING O5-03-02144
DANAGE-ASSESSMENT RESOURCES VULNERABILITY MODELS $05-03-02153$ 
MMING (COMPUTERS) DATA-PROCESSING / RE SOURCE DISTRIBUTION I DAMAGE-ASSESSMENT RESOURCES VUL NERABILITY PROGRA O5-03-D2I 48 IPOPULATION DAMAGE CALCULATION - 1970-1985 I CALCULATIONS DAMAGE-ASSESSMENT SHELTERS SHEL TER-ALLOCATION D/ I5-03-DI930 IRE-CASUALTIESIMA THEMATICAL-PREDICTIONI MILITARY-STRATEGY DAMAGE-ASSESSMENT THREAT-EVALUATION DISTRIBUTIO/ IS-O7-OI966 $Y$ ROACS RAILROAOS CARGO-VEHICLES AIRCRAFT WEAPONS-EFFECTS DAMAGE-ASSESSMENT IRUCKS I \#SR I-MU-4949-150 /LIT I3-06-02260 ITEM MANUAL VI-2. PARM MAIN ROUTINE I RE SOURCES CAPACITY DANAGE-ASSESSMENT VULNERABILITY ECOVJMIC-RECOVE I QS-03-02IS2 ITEM MANUAL VI - 2. PARM MA IN ROUTINE T RE SOURCES CAPACITY DANAGE-ASSESSMENT VULNERABILITY ELJ VJMIC-RECOVE I OS-O3 -02I 52 IOACT P PSTATTACK RECOVERY OF DAMAGED URBAN AREAS I BLAST DAMAGE-ASSESSMENT VULNERABILITY FIRES REPAIR RAD IS-OS -O23OT LTIES FOOD-' RISK II ANALYSIS OF FOOD BALANCE I RESOURCES DAMAGE-ASSESSMENT VULNERABILITY POPULATION CASUA OS-03 -02I32 TUS ING RESOURCES TIME PHASED ACCESSIBILI TY OF RE SOLRCES I DANAGE-ASSESSMENT WEAPONS-EFFECTS I \$VREC-25 IUS ING RESOURCES MATHEMATICAL-MODELS COMPUTERS CASUALTIES DAMAGE-ASSESSMENT WEAPONS-EFFEC IS MAVPOWER REFI/ DS-O3-D2I 37 1 MAPS OF CONTINENTAL UNI TED STATES I GEOGRAPHY RESOURCES DAMAGE-ASSESSMENT WEAPONS-EFFECTS NUCLEAR-WARFA/ OS-D3-O2I 40 ION MIL ITARY-FACILITIESIPROTECTION) POPULATION DETERRENCE DANAGE-LIMITING-SYSTEMS COSTS FALLOUT-SHELTERS I IS-O3-D229L IIONS I ATTACK-SCENARIOS VULNERABILI TY(ANALYSIS) SLRVIVAL DANAGE-LIMITING-SYSTEMS DAMAGE-ASSESSMENT RECOVI IS-D3-DI94 9 ISSR MOVEMENT POPULATION RE SOURCES EVACUATION URBAN-AREAS DAMAGE-LIMITING-SYSTEMS DEMOGRAPHY TRAFFIC ANTI, IS-03-0I922 IITY MIL ITARY-STRATEGY INTERNATIONAL-CIVIL-DEFENSE RUSS I D DAMAGE-LIMITING-SYSTEMS DISPER SAL CHINA EVACUATI I5-D3-OI8QS IELTER SYSTEMS 1 MANAGEMENT-PLANNING SURVIVAL URBAN-AREAS DAMAGE-LIMITING-SYSTEMS EVACUATION VULNERABILIT/ I3-I3-OIB29 I REQUIREMENTS, STRUCTURES, AND CPERATI ONS RESEARCH II. DANAGED PF MULTIPLIER I RADIOACTIVE-FALLOUT PRO/ O6-IB-0222I HIGH IMPACT SHOCK TESTS I NAVAL-RESEARCH DESIGN ELECTRON/ DAMAGES RESULTINE FROM LABDRATORY VIBRATION AND IS-DS-O23O IMULATI IN RA ROACS ARMED-

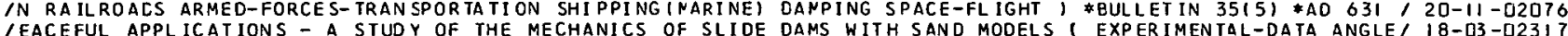
IEACEFUL APPL ICATIONS - A STUDY OF THE MECHANICS OF SLIDE DAMS WITH SAND MODELS I EXPERIMENTAL-DATA ANGLE' I8-03-0231 7
NUCLEAR-WARFARE-CASUALTIES DAMAGE-ASSESSMENT PATIENTS PR/ DAPD46 TIME PHASED CASUALTY ESTIMATE 1 ANALYSIS D6-21 -D2225 NUCLEAR -WARFARE-CASUALTIES DAMAGE-ASSE SSMENT PATIENTS PR/ DAPI46 TIME PHASEO CASUAL TY ESTIMATE ( ANALYSIS D6-21 -D2225

CILITY I B IOL OG ICAL-BLAST-EFFECTS BLAST-BI OLOGY SHCCK-TU/ DASA AEC LOVELACE FOUNOAT ION BLAST SIMULATION FA DO-2I -02232
IV. RESOURCE DATA ROUTINE I DAMAGE-ASSESSMENT RESOURCES DATA-PROCESSING I \$NREC-I3 PARY SYSTEM MANUAL OS-O3-CI2I29 ANAEX TO THIRD DRAFT OF READY I RESOURCES ANALYSIS DATA-PROCESS ING CAYAGE-ASSESSYENT, \#NREC-24(I) OS-O3-D2IS7 ELS PROGRAMMING (COMPUIERSI CAPACITY INDUSTRIAL-PROCUCTION DATA-PROCESSING ECONOMICS 1 *NREC-42 $10 U R C E S$ MOD OS-O3-02I 31 IURCES MANAGEMENT-PLANVING POSTATTACK-OPERATICNS RECOVERY DATA-PROCESSING INDUSTRIAL-PRJDUCTIOV INVENTORI/ O5-O3-O2ILL /K. CONSTRUCTIOV IN THE PROTOTYPE MODEL I REPAIR RECOVERY DATA-PROCESSING MAINTENANCE PJSTATTACK-OPERATIO/ I3-I3-U22B2 1. A MACHIVE METHOD FOR Q DECK PREPARATICN AND SCCRING I DATA-PROCESS ING METHODOLOGY PUNCHED-CARD-EQUIPM/ OS-II -D2I SB

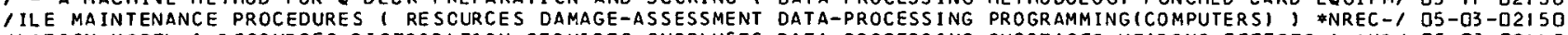
ILAT I ON MOCEL ( RESOURCES DISTRIBLTION SERVICES SURPLUSES DATA-PROCESSING SHORTAGES WEAPONS-EFFECTS 1 FNRI QS-O3-O2I 33 IE SPREAD SIMULAT ION MODEL I FIRES PROGRANMI NG (CONFUTERS) DATA-PRCCESS ING URBAN-AREAS MATHEYATICAL-MODELSI I3-I?-D2266 ACECRAFT DEFENSE-SYSTEMS COST-EFFECTI VENESS VULNEROBILITY DATA-PROCESSING-SYSTEMS 1 \#A.L. 1127 TFICIALI SP 22-02 -0235 ASSES SMENT RE SOURCES VULNERABI LITY PROGRANMING (COMPUTERS) DATA-PROCESSING-SYSTEMS, *NREC-32 $10 N$ I DAMAGE- OS-03-02I 48

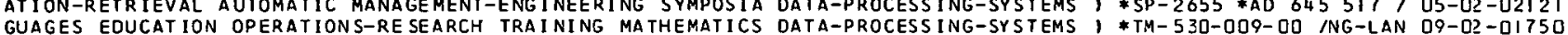

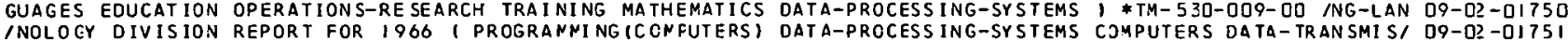
INOLOGY DIVISION REPOR T FOR 1966 ( PROGRANMING (CONFUTERS) DATA-PROCESS ING-SYSTEMS COMPUTERS DATA-TRANSMIS/ D9-0? -0I75I
INFORMATION-CEN TERS ABSTRACTS COSTS DEPARTMENT-OF-CEFENSE DATA-PROCESSING-SYSTEMS DATA-STORAGE-SYSTEMS IN/ OS-D?-D2I 25

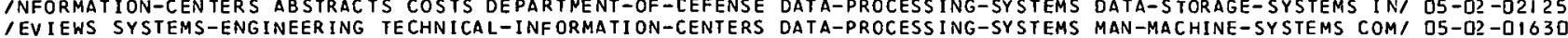

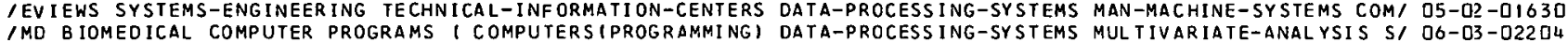
IRCH CATA FROM SHELTER OCCLPANCY EXERCISES I HABITABILITY DATA-PRCCESS ING-SYSTEMS TRAIVIVG YE THODOLOGY QU/ D5-IJ-OI GBD L-PURPOSE-COMPUTERS READING-MACHI AES COMNUNICATICN-THEORY CATA-STORAGE-SYSTEMS I \#A.L. II26 /PUTERS SPECIA O9-O2-Q2248 ITOMATION LIBRARIES AIRCRAFT-INDUSTRY SYSTENS-ENGINEERING DATA-STORAGE-SYSTEMS COMPUTERS DICTIONARIES, */ OS-OZ -O2IIB IACTS COSTS DEPAR TMENT-OF-DEFENSE DATA-PRCCESSING-SYSTEMS DATA-STORAGE-SYSTEMS INFORMATION-RETR IEVAL MICR/ O5-D2-O2I 25 1 AL - INFORMAT ION-CENTERS SYSTEM S-ENGINEERI NG DOCUMENTATION DATA-STCRAGE-SYSTEYS SUBJECT-IVDEXING INFORMA TII OS-OD -O2I 21 1 ACK GROUND 1 COMMAND-AND-CONTROL-SYSTEMS AAVAL-OPERATIONS DATA-ST ORAGE-SYSTEMS SUBJECT-INDEXING TEST-METHI OS-D? -O2IIG NT-OF-DEFENSE DOCUMENTATION E/ KEY WORDS ANO THEIR USE ON DD FORM 14731 REPORTS SUBJECT-INOEXING DEPARTME D5-D?-02I24 AL-SCIENCE PUBL IC-OP INION HISTORY COST/ THE CIVIL DEFENSE DEBATE I CIVIL-DEFENSE-SYSTEYS ATTITUDES POLITIC O5-IT-OILTO IONS CAMAGE-ASSESSMENT FIRE-SAFE IONS CAMAGE-ASSES SMENT FIRE-SAFE TY PROGRANMING (CONFUTERS) DE ERIS ELAST IGN IT ION 1 \#AD O25 867 /LEAR-EXPLOS IS-03-DI952 SOTOPES CHINA RUSSIA RADIO/ RADI ONUCLIDE FRACTICNATION IN DEBRIS FROM A LAND SURFACE BURST 2 RADIOACTIVE-I I8-O2 -QI99I

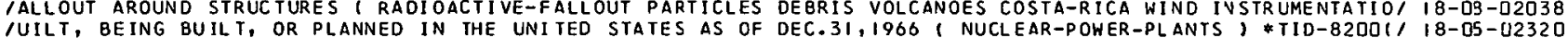
IUILT, BEING BUILT, OR PLANNED IN THE UNI TED STATES AS OF DEC.31, 1966 ( NUCLEAR-POWER-PLANTS I *TID-8200I/ I8-05-U2320
IONS RADIOACTIVE-FALLOUT CONTAMINATION I NDUSTRIAL-PLANTS DECAY-RATES RESOURCESISURVIVAL) PZJGRAMMING ICOM/ O5-O3-OI65I

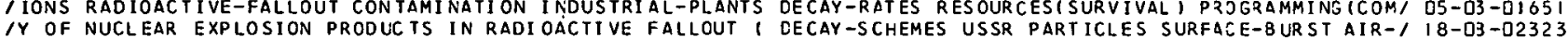

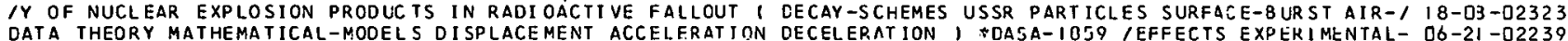

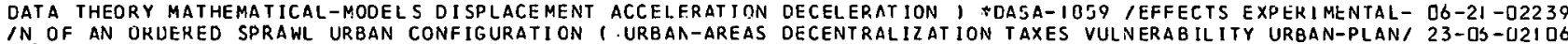
IRM AS A PASSIVE DEFENSE VARIABLE I ECONONICS URBAN-AREAS DECENTRALIZATION VJLNERABIL ITY LAVD-USE URBAN-P/ $23-0 S-02107$ T-PLANN IVG OPERAT ION S-RE SEARCH MANAGEMENT-CONTROL-SYSTENS DECISION-MAK ING, *AD 642 472 /NEER IVG MANAGEMEN DS-DI-02292 GEMENT-PLANNING POSTATTACK-OPERATIONS SEX AGE URBAN-AREAS DECISION-MAKING, *HSR-RR-66/IL-PR 1ODOLOGY MANA $23-0 *-02104$ IELS I COMMUNICATIONS-SYSTEMS COMNAND-AND-CONTROL-SYSTEMS DECISION-MAKING CIVIL-DEFENSE-OPERATING-PLANS AI I7-D2-D23I 2 IWAIT I MANAGEMENT-PLANNING OPERATIONS-RESEARCH ECCNOMICS OECISION-MAKING CONSERVATION DEPLETIOV PETROLEU/ O5-O3-OIG35 INS RESEARCH ON URBAN PROBLEMS I ECONOMICS URBAN-PLANNING DECIS ION-MAK ING IVCENTIVES SOCIAL-SEIENCES OPER/ $55-1]-81676$ NING UN ITED-STA TE S-GOVERNMENT POL

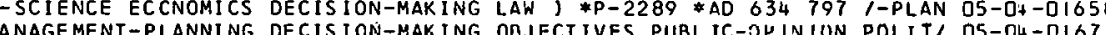
/PERATING-PLANS CIVIL-DEFENSE-SYSTEMS MANAGEMENT-PI.ANNING DECISION-MAKING ODJECTIVES PUBL IC-OPINIUN POLIT/ OS-D4-DI67I EIEN)/ TOWARO A FUTURE FOR PLANNING I MANAGENENT-PLANNING OECIS ION-MAK ING POL ITICAL-SCIENCE GJVERNMENT(FOR OS-C4-DI659
IDY IN THE ECONOMICS OF DISASTER IDAMAGE-ASSESSMENT DATA DECIS ION-MAKING RECOVERY HOUSIVG FJJD FLOODS CI/ DS-D3-DIG36

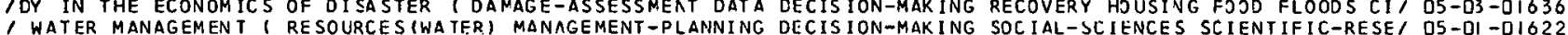

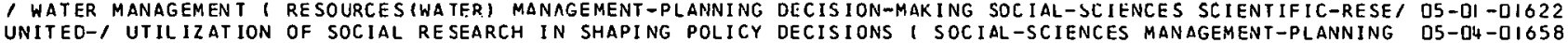
ETHODOL O/ PUNCHED CARD O SORTING - A MACHINE NETHOD FOR O DECK PREPARAT ION AVC SCORIVG 1 DATA-PZOCESSING M O5-II-D2IB8 -RESEARCH RADIOME TER S PYROLYSIS SOLAR-FURAACES CONBUSTION DECOMPOS II ION 1 *AD 1477 IS $10 \mathrm{~V}$ HIGH-TEMPERATURE I3-I? -DIBJ2 HINGTON COVSTRUCTION SETTLEMENT(STRUCTURAL) CONSOLIDATION DECOMPOSITION SHEAR-STRENGTH) \#A.L. IOT2 /G WAS I3-D2-DI785 DICAL-EQU IPMENT ELECTRICAL-EQUIPMENT LABORATORY-EQUIPMENT DECONTAMINATION, \$AD $638410 /-A G E N T S$ SPORES ME I5-D2-0I892

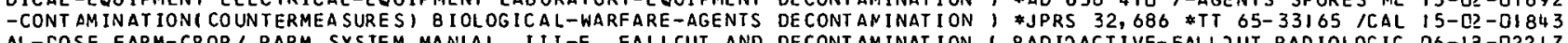
AL-COSE FARM-CROP / PARM SYSTEM MANLAL III-F. FALLCUT AND DECONTAMINAT ION I RADIJACTIVE-FALLJUT RADIOLOGIC O6-13-D22I 7 INE RADIOISOTOPES RADIOACIIVF-WASTE CIVI L-DEFENSE-SYSTEMS CECONTAMINATION BIOLOGICAL-WARFARE CHEMICAL-WAR/ DG-I3-O2222 IADIOACTIVE-FALLOUT PROTECTION-FACTOR DOSE-RATE BUILDINGS DECONTAMINATION ELAST SHIELOIVG THEORY MATHEMAT/ O6-I3-02221 ISHIELDING RADIOACTIVE-FALLOUT RADIOLOGICAL-DOSAGE STORMS DECONTANINATION EARTHQUAKES VOLCAVJES LANDSLIDEI I5-O3-U2299 1 IIOLOGICAL-CONTAMINATION RE SOLRCES POPULATION FACILITIES DECONTAMINATION MATHEMATICAL-ANALYSIS RADIATION/ IS-OS-D23O7 IKP ILING INDUSTRIAL-PLANTS UTILITIES (HARDENING) RESOURCES DECONTANINAT ION PEST-CONTROL MORTUARY-SERVICES , IS-OS -D23O7
IS-D3-OI953 I AL TECHN IQUE FOR DETERMINING IMPCRTANCE CF LIMITEC STRIP DECONTANINATION PROCEDURES I RADIOLOGICAL-CONTA/ OO-1S-D22I8

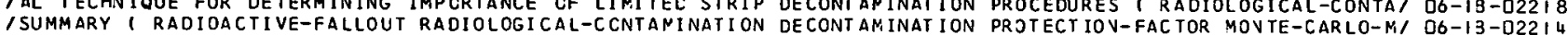

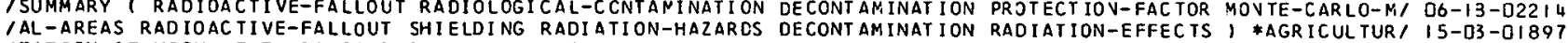
TTATION OF HIGH LEVEL RADIOACIIVE MATERIALS I URBAA-AREAS DECONT ANINATION RADIOACTIVE-WASTES SHIPPING-CON/ I8-DT-O2O34 (ANY) RADIOACTIVE-FALLOUT(SIMULATION) MODELS (SIMULATIONS) DECONT AMINATION RECOVERY FOOD(CONTAMINATION) WA/ TB-IR-DIT23 IMIC-RECOVERY DAMAGE-ASSESSMEN T MRDELS RESOURCES CATACITY DECONTAMINAT ION KEPAIR GOVERVYENT HOSPITALS HOU/ OS-03-02I I 1-PLANS CIVIL-DEFENSE-SYSTEMS FIRE-FIGHTING-AND-EQUIPMENT DECONTAMINATION RESCUES DISIVFECTION WATER-SUPP/ I5-03-D2296 I SUMMARY OF RAD IOLOGICAL TARGET ANALYSIS PROCEDURES FOR DECONTAMINATI ON SCHEDUL ING 1 RADIOACTIVE-FALLOUT O6-I8-O222O SYSTEMS I RAOIOLOG ICAL-CONTAMINATION SHIELDING PROTECTIO/ DECONT ANINAT ION SCHECUL ING PROCEDURES FOR RADEF O6-IS-OI729 IAISAL OF THE APPLICATION OF STATIC EQUI LIBRIUM THEORY TO OECOUPLING ( CRATERING EXPLOSION-EFFECTS NUCLEA/ IB-D3-O23IG 
ECHAN ICS) UNDERGROUND-STRUCTURES SHELTERS PE/ RESPCNSE OF DEEP REINFORCED CONCRETE SLABS I TESTS LOADINGIM I3-I3-02290 NCERGROUN/ MATERIALS FOR USE IN MITIGATING BLAST LCADS ON DEEPLY EURIED PROTECTIVE STRUCTURES I CONCRETE U I8-03-O2OD7 -STRUCTUR / MATERIALS FOR USE IN MITIGATING BLAST LCADS ON DEEPLY EURIED PROIECTIVE STRUCTURES I UNDERGROUD I 8-D3-D2OI 7 ITABILITY NUTRITION SANI TARY-ENGINEERING NEDICAL-SUPPLIES DEFECT IONS TRAINIVG SLEEP WATER FOJD RECREATION/ DS-1J-O2IB4
TIES FILMS FLUI/ RESEARCH ON ELEC TROMAGNETICS FOR PROJECT DEFENDER I PLASMA-PHYSICS ELEC TROMAGNETIC-PROPER 20-D3-O2330 TIES FILMS FLUI / RESEARCH ON ELEC TROMAGNETICS FOR PROJECT DEFENDER I PLASMA-PHYSICS ELECTROMAGNETIC-PROPER 20-03-O2330 FIRES FIRE-PROTECTION RESOURCESIVLLNERAB ILITY) FALLOUT-S/ DEFENDING THE UNITED STATES FROM VUCLEAR FIRE I I3-I2-DI8OG OVERNMENT EMERGEN / CODE OF FEDERAL REGULATIONS - NATICNAL OEFENSE I ARMED-FORCESIUNITEO-STATESI MILITARY-G I5-O3-DI946 RACIOLOGICAL-DOSAGE EXPOSI RADIOLOGICAL HEALTH ANO CIVIL DEFENSE HEALTH-PHYSICS RADIOACTIVITY DEIECTION DS-I3-D2222 ON VULNERABILITY LABOR ECO/ OCCUPATIONAL SKILLS ANC CIVIL DEFENSE ( INDUSTRIES RESOURCESIHUMAN) DISTRIBUTI O5-O3-DIO3O ROUNC-STRUCTURES COSE-RATE PHOTONS I SHIELDING IV - CIVIL DEFENSE I RADIOACT IVE-FALLOUT ATTEVUATION UNDERG IO-OS-DIOZ E-PERSON 7 SOVIET MILITARY TRAN SLA IIONS NUNBER 188 - CIVIL DEFENSE 1 RUSSIA TRAINING DISPERSAL CIVIL-DEFENS IS-D3-DIQI 3 IN INTRODUCTION TO THE DESIGN OF LNDERGRCUND OPENINGS FOR DEFENSE I SHELTERS BLAST STRESSES UNDERGROUND-S/ $13-13-$ O2273
HE RURAL AREA I USSR CIVIL-DEFENSE-SYSTEMS RESCUES INTER/ DEFENSE AGAINST WEAPONS OF MASS DESTRUCTION IN T IS-D3-DI9IB ONAL-CIVIL-DEFENSE COMMUNIST-CHINA INDUSTRI/ CRISES CIVIL DEFENSE AND DETERRENCE I USSR MOVEMEVT INIERNATI I5-D3-O229S ONAL-CIVIL-DEFEVSE EARLY-WARNING- SYSTEMS LSI CRISIS CIVIL DEFENSE AND DETERRENCE I VULVERABILITY IN TERNATI IS-O3-OIQ22 STRATEGY INTERNAT IONAL-CI VIL-DEFENSE RUSSIA/ CRISIS CIVIL CEFENSE AND DETERRENCE I VULVERABILITY MILITARY- IS-03-OI896 SYSTEMS AP/ EXERPTS FROM CONGRESSIONAL TESTIMCNY ON CIVIL DEFENSE AND RELATED DEFENSIVE SYSTEMS I DEFENSE- IS-03-O2294 IL-CEFENSE-OPERAT ING-PLANS UTILITIES DATA WATER-SU/ CIVIL DEFENSE ASPECTS OF MUNICIPAL WATER SYSTEMS I CIV I3-02-0I788 ER-SUPPLIES VULNERAB ILITY URBAN-AREAS SURVEYS POST/ CIVIL. DEFENSE ASPECTS OF MUNICIPAL WATER SYSTEMS I WAT I5-D3-DI94I O 1 LEGISLATION SOCIAL-SCIENCES HISTORY CIVIL/ U.S. CIVIL DEFENSE BEFORE 1950 - THE ROOTS OF PUBLIC LAW 92 05-04-DI67I ES RELEVANT TO THE STUDY OF SOCIE IAL RECOVERY FRON/ CIVIL DEFENSE BIBLIOGRAPHY - A COMPILATIDN OF REFERENC OS-IJ-OIGT7 /STRUCTIVG THE UNDERGROUND FACILI TY OF NORTH ANERICAN AIR DEFENSE COMMAND AT CHEYENVE MJUNTAIV, EL PAST C I I3-I3-O2274 STATE, AND REGIONAL LEVELS I CONMUNICATIONS-SYST/ CIVIL DEFENSE COMMUNICATIONS REOUIREMENTS AT THE LOCAL IT-OZ -O23I - STATE, ANO REGIONAL LEVELS I CONMUNICATIONS-SYST/ CIVIL OEFENSE COMMUNICATIONS REQUIREMENTS AT THE LOCAL I7-O2-O23I2 作 POLIYICAL-SCIENCE PUBLIC-OPINION HISTORY COST' THE CIVIL DEFENSE DEBATE I CIVIL-DEFENSE-SYSTEMS ATYITUDES OS-IJ-DIOTO EC-MISSILES NUCLEAR-WEAPONS CONTANINATION FOOD CHE/ CIVIL OEFENSE HANCBOOK - USSR I RURAL-AREAS BOMBS GUID I5-O3-D2296

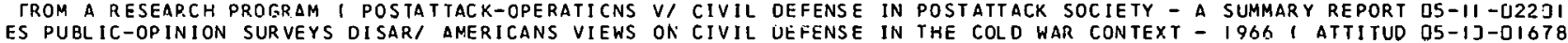
TERNATIONAL-CIVIL-DEFENSE CIVIL-DEFENSE-SYSTEMS EA/ CIVIL DEFENSE IN THE UNITED KINGDOM I GREAT-BRITAIN IN I5-D3-02305 I FLOW OF SCIEVIIFIC AVD TECHNICAL INFORNAIION WITHIN THE DEFENSE INDUSTRY III. A. FREZUEVCY DISTRIBUTIOI QS-D?-O2IZD ATION CIVIL-DEFEN SE-SYSTEMS, MPP-6 CIVIL CEFENSE MOTION PICTURE CATALOGUE 1 PUBLIC-INFORM IS-D3-D23J4 ITIONS EQUIPMENTS ANO SYSTEMS TO SUPPORT INTRASTATE CIVIL DEFENSE OPERATIONS - CIRCA 1970 ( RADIO-COMMUNI I I-O2-D23I3 IY, AND TACTICS OF FIRE PROTECTION AS EMPLOYED BY A CIVIL DEFENSE ORGANIZATION DURING WDRLD WAR II - GERMI I3-12-O22 O4 IY, AND TACTICS DF FIRE PROTECTION AS EMPLOYED BY A CIVIL. DEFENSE ORGANIZATION DURIVG WJRLD WAR II - GERMI I3-I -02255 ENT-PLANVING STRES/ SPECIAL PROBLEMS OF CHILDREN IA CIVIL DEFENSE PLANN ING I POSTATTACK-OPEZATIONS MANAGEM D5-II-D22 J3 ECOVERY POST/ THE IMPORTANCE OF INDIVIDUAL INDUSTRIES FOR DEFENSE PLANNING I SUPPLY-AND-DEMAND ECONOMICS R OS-03-OIG47

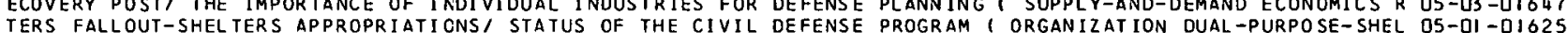
TERS FALL OUT-SHEL TERS APPROPRIATICNS/ STATUS OF THE CIVIL DEFENSE PROGRAM I ORGANIZATION DUAL -PURPOSE-SHEL OS-OI -DI625
I WEST FREEWAY TUNNEL WEST ORANGE, NEW JERSEY, AS A CIVIL DEFENSE PUBLIC SFELTER FACILITY 1 TRAVSPORTATIO/ I3-I3-CO227I RESEAPCH-PROGRAM-ADMINISTRATION OPERA TIONS-RESEARC/ CIVIL DEFENSE RESEARCH AVALYSISI SYSIEYS-EVGINEERING I2-OZ-OI7G 7 RBOARD PHYSICAL-PROPERTIES CO/ STCRAGE STABILITY UI LIVIL DEFENSE SHELTER RATIONS 1 FOOD PRFSERVATION FIBE DO-D3-O22II MAGE CALCULATION - 141U-1985 / CALCULATIONS DAMAGE/ CIVIL DEFFNSE SHELTER REPRESENTATION FOR POPULATION DA IS-O3-DI930 ON UREAN-PLANNING EFFICIENCY SEL/ CRBAN FORM AS A FASSIVE DEFENSE VARIABLE I COSTS URBAN-ARLAS CONFIGURATI I3-02-02256 ALIZATIOV VUL NERABILITY LAND-USE / LRBAN FCRN AS A PASSIVE DEFENSE VARIABLE I ECOVOMICS URBAV-AREAS DECENTR 23 -0S-O2I O7 /ST IMONY ON CIVIL DEFENSE ANO RELATED DEFENSIVE SYSTEMS I DEFENSE-SYSTEMS APPROPRIATIONS ANTIMISSILE-DEFE I IS-O3-O2294 IOMMUNICATION-EQUIPMENT SATELLITESIARTIFICIAL) SPACECRAFT DEFENSE-SYSTEMS COST-EFFECTIVENESS VULNERABILIT/ 22 -O2-O235I INT - PLANN ING OPERATIONS-RESEARCH NODELS THREAT-EVALUATION DEFENSE-SYSTEMS CESIGN USSR MILITARY-INTELLIGEN/ I5-03-D23O3 /ROM CONGRESSIOVAL TESTIMONY ON CIVIL DEFENSE AND RELATED DEFENSIVE SYSTEMS I OEFENSE-SYSTEYS APPROPRIATI I IS-O3-D2294 MORPHOLOG THE PATHOLOGY OF ZINC DEFICIENCY ( METABOLISM DEFICIENCY-DISEASES TISSUES (BIOLOGY) TESTES RATS DG-OS-DI7OI ANC TECHNIQUES \& COUNTERMEASURES POSTATT I AN APPROACH TO DEFINING POSTATTACK RECOVERY MANAGEMENT CONCEPTS I5-O3 -QI S5 3 ANC TECHNIQUES 1 COUNTERMEA SURES NUCLEAR/ AN APPRCACH TO DEFINING POSTATTACK RECOVERY YANAGEMENT CONCEPTS IS-O3-O2293 ANC TECHNIQUES 1 COUNTERMEA SURES NUCLEAR/ AN APPRCACH TO DEFINING POSTATTACK RECOVERY YANAGEMENT CONCEP TS I5-03-D2293

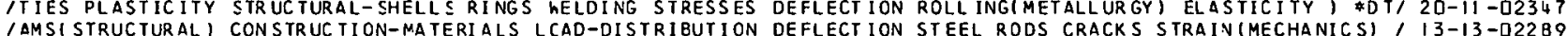
LSISTRUCTURAL, RECTANGULAR-BODIES STRESSES THRUST, LARGE DEFLECTIONS OF CLAMPED RECTANGULAR PLATES I PANE $20-11$ - D2344 STRUCTURES DESIGN THEORY BEAMSISTRUC TURAL FRAMES LOADINI DEFLECT IONS OF REINFORCEO CONCRETE FLOOR SLABS I $20-11-0234$ IJECTED TO A DYNAMIC DISTURBANCE I UNDERGROUND-STRUCTURES DEFORMAT ION CONSTRUCT ION-4ATER IALS MATHEMATICAL, I3-I3-O2279 I ANICS I ROCK (GEOLOGY) FAILURE (MECHANICS) STRESSES THEORY CEFORMAT ION EXPERIMENTAL-DATA GEOLOGY INSTRUMEN/ DB-D7-D2244 /LOGY, STRESSES ELASTICITY THEORY GEOLOGY INSTRUMENTATION DEFORMAT ION LOADING(MECHANICS) CONSTRUCTION STA, I3-I3-O2275 /STRUCTURAL FRAMES LOADING (MECHANICS) COLUMNS (STRUCTURAL) DEFORMAT ION MATHEMAT ICAL-MODEL S MATHEMATICAL-AN/ 20-II -O234O / TURAL-SHELLS VIBRATION LOADING (MECHANICS) SHEAR-STRESSES DE FORMATICN ROTATION INERTIA MATHEMATICAL-ANALYI $20-11$-O2346 /EED- GROUND-TRANSPOR T MA THEMATICAL-ANALYSIS CCNSCLICATION DEFORMATION TRANSPJRTATIOV ELASTICITY NOR THEAST/ $20-1 I$-D2D8 4 IUNCT ION I SHOCKIMECHANICSI VIBRAIION ELASTICITY VELOCITY DEGREES-OF-FREEDOM MATHEMATICAL-AYALYSIS ACCELE/ $20-11$-D234 2 ION SHELF-LIFE BEVERAGES BREAD CANNED CEREALS COFFEE CORN OEHYORATED-FOODS EGGS FATS FROZEN-FOODS FRUITS, O6-DB-O22O9 ISYSTEMS TO SERVE SOUTHERN CALIFORNIA - FEATHER RIVER AND DELTA DIVERS ION PROJECTS I WATER-SUPPLIES URBAN/ I3-02-Q2258

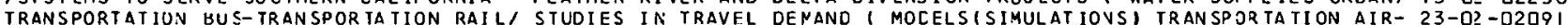
TRANSPORTATIUN BUS-TRANSPORTA TION RAILI STUDIES IN TRAVEL DENAND I MOCELSISIMULAT IOVS) TRANSPJRTATION AIR- 23 -0? - D2O9I 7ROJECTIONS 1970 - INTER INDUSTRY RELATI ONSHIPS, POTENT IAL DEMAND, EMPLOYMENT 1 IVDUSIRIES MANPOWER ECONOMI OS-D3 - DI6UQ I NUCLEAR WAR - EMPIRICAL GUIDELINES FOR THE SELECTION OF DEMOGRAPHIC VARIABLES AND AREAL UNITS FOR STUDY/ $23-04-02 I 03$

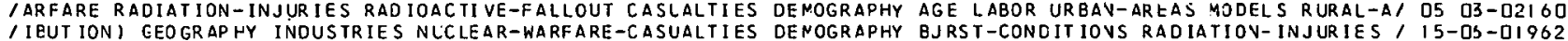

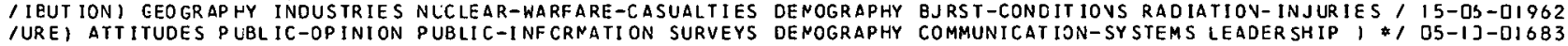
VIVAL RESOURCESIHUMAN) RECOVERY VLLNERABILITY URBAN-AREAS DEMOGRAPHY COUNTERYEASURES, \#HRB-S-946II-F /SUR OS-II-DIG8B IERAT IONS DISASTERS BEHAVIOR SOC I OLOGY STRESSIPSYCHOLOGY) OEMOGRAPHY ECONOMIC-RECOVERY CIVIL-DEFENSE-SYSI/ O5-II -O2I 9D IIMENSIONS UF NUCLEAR ATTACK I SOCIETAL-RECOVERY RFCCVERY DENOGRAPHY ECONOMICS POLITICAL-SCIENCE STRESSIP/ OS-II-O22O2 1, TETANUS, POL, IOMYELITIS, SMALLPUX, MEA SLES, I NF LUCNZA I DENOGRAPHY IMMIINOLOGY B IULJGICAL-WARFARE-AGLNTSI I5-D2 -OI868 FOR THE SELECTION OF DEMOGRAPHIC VARIABL/ A STUDY OF THE GEMOGRAPHY OF NUCLEAR WAR - EYPIRICAL GUIDELINES $23-01$ - O2IJ3 FARE POPULATION METHODOLOGY MANAGENENT-PL/ A STUDY OF THE DEMOGRAPHY OF NUCLEAR WAR I SURVIVAL NUCLEAR-WAR 23 -DI-O2ID 4 IUCLEAR-EXPLOSION-DAMAGE AGE MODELSISIMULATIONS) SLRVIVAL DEMOGRAPHY RECOVERY ATTACK-SCENARIOS FERTILITY, I5-OS-OIOG5 IATI ITUDES CIVIL-DEFENSE-SYSTENS COLD-WAR SURVEYS CULTURE DENOGRAPHY SOCIOLOGY POPULATION DISARMAMENT, IAT ITUDES CIVIL-DEFENSE-SYSTENS COLD-WAR SURVEYS CULTURE DENOGRAPHY SOCIOLOGY POPULATION DISARMAMENT I \#/ O5-1J-02I77
LOR TO 1990 WITH EXTENSIONS OF TOTAL POPULATICN TO 2015 IS DEMOGRAPHY TABLES I P-25-359/Y AGE, SEX, AND CO $23-0 .+02358$ LOR TO 1990 WITH EXTENSIONS OF TOTAL POPULATICN TO 20151 DEMOGRAPHY TABLES 1 P-25-359 /Y AGE, SEX, AND CO $23-0+4-02358$
IRESOURCES EVACUATION URBAN-AREAS DAMAGE-LIMI TING-SYSTEMS DEMOGRAPHY TRAFFIC ANTIMISSILE-DEFENSE-SYSTEMS, I5-O3-OIS22 /RESOURCES EVACUATION URBAN-AREAS DAMAGE-LIMITING-SYSTEMS DEMOGRAPHY TRAFF IC ANTIMISS ILE-DEFENSE-SYSTEMS 1 I5-O3-OI922
/-EUROPE NATO GREAT-BRITAIN FRANCE GERMANY BENELUX NORWAY DENMARK GREECE SWEDEN SWITZRLAND IRELAND USSR , I5-O3-D2I 47 7-EUROPE NATO GREAT-BRITAIN FRANCE GERMANY BENELUX NORWAY DENMARK GREECE SWEDEN SWITZERLAND IRELAND USSR I I5-O3-D2I 47
IIGURATION URBAN-PLANNING EFFICIENCY SELECTION POPULAT ION DENSITY DISTRIBUTION ECONOMICS FEASIBILITY-STUD/ I3-D2-D2256 /IGURATION URBAN-PLANNING EFFICIENCY SELECTION POPULAT ION DENSITY DISTRIBUTION ECONOMICS FEASIBILITY-STUD/ I3-D2-D2256
CONDARY-YISSILES 8LA / OPERATION TEAPOT - OISTRIBUTION AND DENSITY OF MISSILES FROM VUCLEAR EXPLOSIONS I SE I8-03-D23II8 CONDARY-YISSILES 8LA / OPERATION TEAPOT - OISTRIBUTION AND DENSITY OF MISSILES FROM VUCLEAR EXPLOSIONS I SE I8-O3-023I8
RI/ TIE AGRICUL TURAL RESEARCH CENTER OF THE UNITED STATES DEPARTMENT OF AGRICULTURE I AGR ICUL TUZAL-ENGINEE O2-OJ-D2IIOB ITIONS GOVERVING THE MEAT INSPECIION OF THE UNITED STATES DEPARIMENT OF AGRICULTURE I LEGISLATIONI NCEX OF THE OPEN LITERATURE WITH ABSTRACTS I B/ ECCNOMICS DEPARTMENT PUBL ICATIONS, $1960-1965$ - $A N$ AUTHOR I OS-03-DIO52 $1-30,1906$ ( TECHNICAL-INFORMATION-CENTERS ABSTRACTS COSTS DEPARTMENT-OF-DEFENSE DATA-PROCESSING-SYSTEMS D/ DS-02-O2I 25 I ANC THEIR USE ON DD FORM 1473 I REPORTS SUB JECT-INOEXING DEPARTMENT-OF-DEFEVSE DOCUMEVTATIJV EFFECTIVENE, OS-D?-O2I /BUT IONS AND CORRELATION B. RELATIONSHIP AND CCMPARISON I CEPARTMENT-OF-DEFEVSE DOCUMENTATIJN INFORMATION/ D5-O?-D2I 20 PERAT IONS-RESEARCH ECONOMICS DECI ION-MAKING CONSERVATION DEPLETION PETROLEUM MIODLE-EAST, \$A.L. 973 IG O O5-03-DIO35 IERATION CENIZA ARENA - TECHNIQUES FOR THE MEASURENENT OF DEPOSITION ANO REOISTRIBUTION OF FALLOUT AROUND/ I8-OB-O2O38 SION. CTIECTS/ EFFECT OF WATER T.NN IENT, YIELD, MEDILI, AND DEPTH OF BURST ON CAVITY RADII I CRATERING EXPLO IB-O3-O23IS 
IBER 284 - COMBAT TRAINING ME THODS AND THEIR SHORTCCNINGS DESCRIBEC I.JSSR MIL ITARY-TRAINING ARMED-FORCESI OS-07-02I76 -RECOVERY METHODO / THE FEASIBILITY OF DEVELOPING STANDARO DESCRIPTIONS OF POSTATTACK SITUATIONS I SOCIETAL QS-II -0219I COSSIBLE UTILITY IN PRIMITI VE ENVIRONMENTS I HEAT-ENGINES DESERTS FEASIBILITY-STUDIES SOLAR-RADIATION EGYI I3-O?-OI777 SIGN ENGI THE UTILIZATION OF HUMAN FAC TORS INFORMATION BY DESIGNERS 1 HUMAN-ENGIVEERING DECISIJV-MAKING DE D5-O5-OIG73 ECTS I STRUETURES FALOUT-SHEI AN INVESTIGATICN OF SCHOOL DESIGNS TO RESIST INTEGRATED VUCLEAZ WEAPONS EFE I3-13-OIB2I SAFEGUARDING FOOD. PRODUCTS AND WATER FROM AGENTS CF MASS DESTRUCTION - USSR I PROTECTIDN-SYSTEMS WATER-SU IS-O2-DI843 SAFE SE-SYSTEMS RESCUES INTER/ DEFENSE AGAINST WEAPONS CF MASS DESTRUCTION IN THE RURAL AREA I USSZCIVIL-DEFEN IS-D3-DIQI8 EVALUAT ION OF AN A IRBORNE INFRARED MAPPER AS A TCOL FOR DETECT ING AND MEASUR ING FIRES I IVFRARED-DETECI/I $3-1 ?-D I 798$ , HEALTH AND CIVIL DEFENSE, HEALTH-PHYSICS RADIOACTIVITY DETECT ION RADIOLOGICAL-OOSAGE EXPJSURE RADIOLOG/ DG-I3-O2222 IL MONITORING SYSIEM I INSTRUMENTATI ON RADIATION-NCNITORS DETECTICN TESTS RADIO-TELEMETRY RADIATION-MEASU/, I8-D'-O2O26 RETE BEAMS SUBJECTED TO BLAST LOADS I DESIGN BE/ STUDY TO DETERMINE THE OPIIMUM SECTIOV OF REINFORCED CONC I3-13-DI84D EFENSE COMMUVIST-CHINA INDUSTRI/ CRI SES CI VIL DEFENSE AND DETERRENCE I USSR YOVEMENT INTERNATIONAL-CIVIL-D I5-O3-D2295 EFENSE EARLY-WARN ING-SYSTEMS US/ CRISIS CIVIL DEFENSE AND DETERRENCE I VULNERABIL ITY IVTERNATIONAL-CIVIL-D I5-O3-DI922 ERNAT IONAL-CIVIL-DEFENSE RUSSIA/ CRISIS CIVIL DEFEASE AND DETERRENCE 1 VULNERABILITY MILITARY-STRATEGY INT I5-03-DI896 IAT-EVALUATION MILITARY-FACILITIES(PROTECYION) POPULATION DETERRENCE DAMAGE-LIMITING-SYSTEMS COSTS FALLOU/ IS-O3-O2294 INS AS MDDIFIERS OF THERMAL RADIA ION RELEASED IN NUCLEAR DETONAT IONS 1 GLASS ATTENUATION URBAV-AREAS IGNI I3-I?-OI796 INS AS MODIF.IERS OF THERMAL RADIATION RELEASED IN NUCLEAR DETONAT IONS I GLASS ATTENUATIJN URBAV-AREAS IGN/ I3-I2-OI796 ITUAT IONS I SOC IE TAL-RECOVERY ME THODO/. THE FEASIBILITY CF OEVELOPING ST ANDARD.DESCR IPTIJNS JF POSTATTACK S OS-II -O2I 1 I EQUIPMENT CONTROL-SYSTEMS TRANSF ORMERS COILS CIR/ CONTROL DEVICE FOR ELECTROLUMIVESCENCE CELLS RBAN-AREAS RAILROADS MONORAILS MO INNOVATIONS IN TRANSIT DEVICES T RAPID-TRANSIT-SYSTEMS TRAVSPORTATION /ND FEASIBILITY STUDIES OF MUZZLE BRAKE BLAST SUPPRESSION DEVICES FOR IDSMM AND IS5MM HOWITZERS 1 SHOCK-W/ IQ-DS-O2327

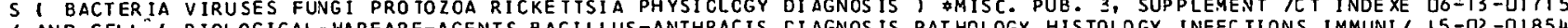

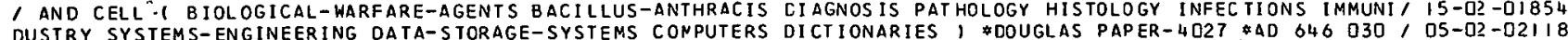

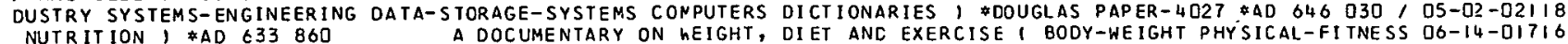
, PROCUCT/ VUTR ITION IN THE POSTATTACK ENVIRONMENT I FOOD DIET PCSTATIACK -OPERATIONS SURVIVAL STORAGEIFOOD DG-IS-OITI 7 -E/ THERMAL STRESSES IN A SEMI INFINI TE SCLID BY A FINITE CIFFERENCE TECHNIQUE I THERMAL-RADIATION MEAPONS I3-I? -OI79S IF SUBSURFACE REACTOR CONTAINMENT STRUCTURES TO WITHSTAND DIFFERENTIAL EARTH DISPLACEMEVT I REAETOR-FEASI/ I8-DF-O2DLD GOTIAT/ A METHODOLOGICAL STUDY OF BEHAVIORAL AND SEMANTIC DIFFERENTIAL SCALES RELEVANT TO IVTERCULTURAL NE OS-IJ-O2IT8 IRS UNDERGROUND-S TRUCTURES DYNAMIC'S MATHENATICAL-AMALYSIS OI FFERENTIAL-EQUAT IONS COMPUTERS(PR.JGRAMMING) I/ I3-I3-OIB33 IS DEFORMATION CONSTRUCTION-MATERIALS MATHEMATICAL-MODELS DIFFERENTIAL-EQUATIONS EQUATIONS-OF-MOTION LOADI I3-I3-O227O IJECT SAND STORM - AN EXPERIMENTAL PROGRAN IN ATMOSPHERIC DIFFUSION I ATMOSPHERE TRANSPORT-PROPERTIES EXP/ O4-D?-O2IIL CURING FALLOUT/ A CALCULATIONAL MODEL FOR CONDENSEC STATE DIFFUSION CONTROLLED FISSION PRODUSTABSORPTION IB-OS-O2O37

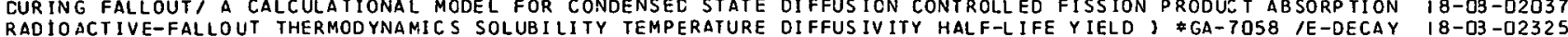
RADIOACTIVE-FALLOUT THERMODYNAMICS SOLUBILITY TEMPERATURE DIFFUS IVITY HALF-LIFE YIELD, \#GA-7058 /E-DECAY I8-D3-02325
ENT IN OCCUPIED UNDERGROUND PROTECTIVE STR/ COMPARISON OF DIGITAL COMPUTER SIMULATIONS OF THERMAL ENVIRONM 2O-I3-O2O9D

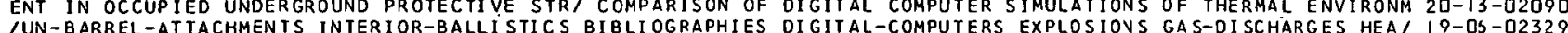
/UN-BARREL-AT TACHMENTS INTERIOR-BALLISTICS BIBLI OGRAPHIES DIGITAL-COMPUTERS EXPLOSIOVS GAS-DISCHARGES HEA/ I9-0S-02329
TOWARD THE DEVELOPMENT OF A MACHINE WHICH COMPREHENDS 1 DI GITAL-COMP:JTERS PROGRAMMIVGICOMPUTERSI COMPUTE O9-D2-O2248 ' TOWARD THE DEVELOPMENT OF A MACKINE WHICH COMPREHENDS
INERABILITIIES OF SOCIAL STRUCTURE - STUDIES OF THE SOCIAL DIMENSIONS OF NUCLEAR ATTACK INERABILITIES OF SOCIAL STRUCTURE - STUDIES OF THE SOCIAL DIMENSIONS OF NUCLEAR ATTACK I SOCIETAL-RECOVERI DS-II-D22O2 PARITIES AND NATIONAL VIABILITY I SOCIETAL-RECOVERY ECON/ DINENSIONS OF SURVIVAL - POSTATTACK SURVIVAL DIS OS-O3-O2ISO MALLPOX, MEASLES, INFLUENZA 1 O' DISEASE STATUS REFORTS - DI PHTHERIA, PERTUSSIS, TETANUS, PJLIOMYELITIS, S I5-DZ-OI8S9 IION TRANSMISSIOŃ-LINES COSTS AL TERNATE-CURRENT ECCNOMICS DIRECT-CURRENT STATE-OF-THE-ART IVDUSTRIAL-RESE / O9-O3-Q2249 ULT ING FIRMS WI TH CERTIFIED FALLOLT SHELTER ANAT NATIONAL DIRECTORY OF ARCHITECTURAL, ENGINEERING AND CONS I3-I3-D2276 STATES - PHYSICAL SCIENCES, BIOLOGICAL SCIENCES, ENGI/ A DIRECTORY OF INFORMATION RESOURCES IV THE UNITED OS-D?-OIG27 STATES - SOCIAL SC IENCES TIN A DIRECTORY OF INFORMATION RESOURCES IN -THE UNITED Q5-D2-DI628 IED SCIENCE INFORMATION SERVICES IN THE UNITED STATES - A DI RECT ORY OF SELECTED SPECIAL IZED INFORMATION S/ OS-D? -DI 62 9 COLD-WAR SUR VEYS CUL. YURE DEMOGRAPHY SOCICLOGY POPULATION DISARMAMENT, \#A.L. I2IO IIES IN PROGRESS OR RECENTLY COMPLETED - ARMS CONTROL ANO OI SARMANENT IIES IN PROGRESS OR RECENTLY COMPLETED - ARMS CONTROL AND DISARMAMENT

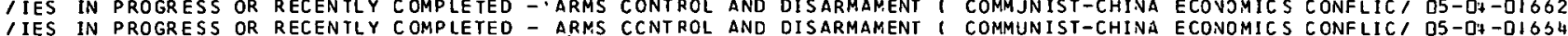
IES IN PROGRESS OR RECENTLY COMPLETED - ARMS CCNTROL AND DISARMAMENT
MULAT/ DEVELOPMENT OF UTILITY THE ORY FOR ARMS CONTROL AND DISARMAMENT MULAT/ DEVELOPMENT OF UTILITY THE GRY FOR ARMS CONTROL AND DISARMAMENT
(II) IDEVELOPMENT OF UTILITY THEORY FOR ARMS CONTROL AND DISARMAMENT ILC WAR CONTEXT - 1966 A ATITLDES PUBLIC-OPINICN SURVEYS DISARMAMENT CIVIL-DEFEVSE-SYSTEMS VIETNAM SHELT/ OS-IJ-DI678 IHE ALASKAN EAR THQUAKE - A CASE STUDY IN THE ECONONICS OF DISASTER I DAMAGE-ASSESSMEVT DATA DECISION-MAKI/ O5-D3-DI636 MEDICINE MEDICAL-PER SONNEL HOSPITALS PUBLIC-HEALTH RESCU/ DISASTER MEDICAL CARE FILMS I DISASTER-MEDICINE D6-2I-DI74D HEALTH-SERVICES HOSP ITALS CASUALTIES ADMINISTRA/ HCSPITAL DI.SASTER PLANNING I DISASTER-YEDICINE EMERGENCY- DO-21-DI739 OGICAL/ PROCEEDINES OF MEDICAL CIVIL DEFENSE CONFERENCE I DISASTER-NECICINE EMERGENCY-HEALTH-SERVICES BIOL O6-2I -D2233 CA MANUALS FILMS ; *A.L. 1068 CATALOGUE OF PUBLICATICNS 1 DISASTER-MEOICINE EMERGENCY-HEALTH-SERVICES CANA D6-2I -DI73 7 ITALS CASUALTIES ADMINISTRA/ HOSPITAL DI SASTER PLANNING I DISASTER-MEDICINE EMERGENCY-HEALTH-SER VICES HOSP Q6-2I -DI739 IMINATION RESCUES DISINFECTION WATER-SUPPLIES COOPERATION DISASTER-MEDICINE EMERGENCY-HEALTH-SERVICES REP, I5-03-D2296 IE TO THE JACKSON, MISSISSIPPI TORNADO OF MARCH $3,1966,1$ DISASTER-MEDICINE MANAGEMEVT-PLANVING HOSPI TALSI DO-I?-DI7II 1 CENTRE - OPERATING MANUAL - EMERGENCY HEALTH SERVICES 1 DISASTER-MEDICINE MEDICINE ADMINISTRATION-AND-M/ O6-I II-DI7IL PITALS PUBL IC-HEALTH RESCU/ DISASTER MEDICAL CARE FILMS I DISASTER-MEOICINE MEDIC INE MEDICAL-PERSONNEL. HOS Q6-2I-DI74D ST-AIC/ CURRENT TOPICS IN SOVIET NILI TARY MEDICINE I USSR DISASTER-MECICINE NUCLEAR-WARFARE-CASUALTIES FIR D6-OS-OI7O3

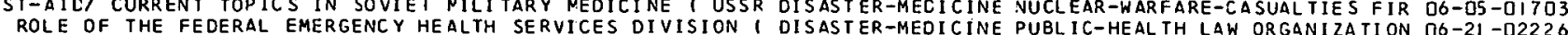
IS MANPOWER EMERGENCY-HEALTH-SERVICES RADIOBIOLOGY FLOODS DISASTER-MEDICINE TSUNAMIS WATER-SUPPLIES LOGIS/ I5-D3-O2299 IS MANPOWER EMERGENCY-HEALTH-SER IICES RADIOBIOLOGY FLOODS DISASTER-MEDICINE TSUNAMIS WAIER-SUPPL IES LOGIS/ I5-O3-O2299
IRY NUCLEAR-WARFARE WEAPONS-EFFEC TS POSTATTACK-OPERATIONS DISASTERS BEHAVIOR SOCIOLOGY STRESS(PSYHOLOGY)/ OS-II -O2I 90

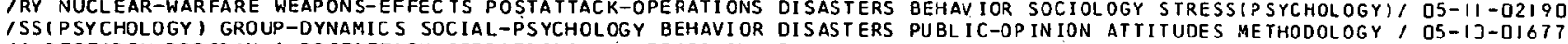
IA RESEARCH PROGRAM I POSTATTACK-CPERATIONS VULNERABILITY CISASTERS SOCIETAL-RECOVERY RECOVEZY SOCIOLOGY, OS-II -O22JI TETANUS, POLIOMYELITIS, SMALLPOX, MEASLES, INFLUENZA I DI DISEASE STATUS REPORTS - DIPHTHERIA, PERTUSSIS, IS-DZ-OI869 IOEMIOLOGY PUBL IC-HEALTH POSTA TTACK-OPERATIONS IMMUNOLOGY DISEASE-VECTORS CHEMOTHERAPY YECICINE RODENTS I/ O6-OS-DITO2 1) WASTES (INDUSTR IAL) GARBAGE SANI TARY-ENGINEERING SEWAGE DISEASE-VECTORS CISEASES(CONTROL) DRAINAGE COUN/ I 3-OZ-DITBO IIAL) GARBAGE SAN ITARY-ENG INEERING SE WAGE DI SEASE-VECTORS DISEASES (CONT ROL) DRAIVAGE COUN TEZMEA SURES URBAI I 3 -O2 -OI 7BO WATER-SUPPLIES PUR IFICAIION CONTAN/ HOW TO PURIFY ANO TO DISINFECT WATER WITH THE SIMPLEST MEANS - USSR I OG-I3-DI722 ITEMS FIRE-FIGHTING-AND-EQUIPMENT DECONTAMINATION RESCUES DISINFECTION WATER-SUPPLIES COOPERATION DISASTEI IS-O3-D2296

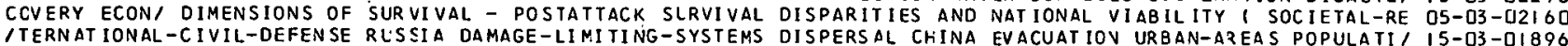
IRANSLATIONS NUMBER 188 - CIVIL DEFENSE (RUSSIA TRAINING DISPERSAL CIVIL-CEFENSE-PERSONNELITRAINING) MAN/ IS-03-DISI3 TORING LOGISTICS MANPOWER SHEL TERS PROGRANMING ICOMPUTERS) OISPERSAL INDUSTRIES STOCKPILING I. A.L. 97D INI 15 -D3-DI949 INSE COMMUNIST-CHINA INDUSTRIES TRANSPORTATION EVACUATION DISPERSAL URBAN-AREAS FOOD SHEL TERS FALLOUT-SHE IS-D3-O2295

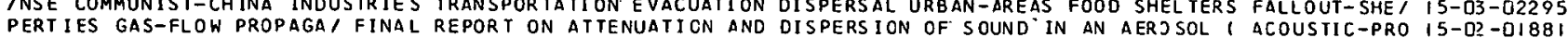

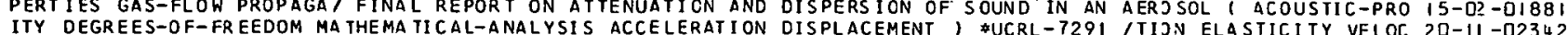
ITY DEGREES-OF-FREEDOM MA THEMA TICAL-ANALYSIS ACCELERATION DISPLACEMENT, \#UCRL-7291 /TIJN ELASTICITY VELOC $20-11-02342$
IR CONTAINMENT STRUCTURES TO WITHSTAND DIFFERENTIAL EARTH DISPLACEMENT, REACTOR-FEASIBILITY-STUDIES REAC, I8-DF-O2O4D

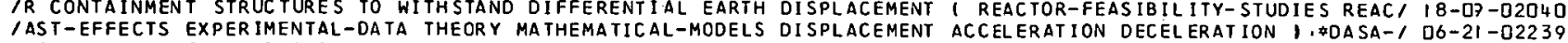
IICAL PARAMETERS 1 B IOLOGICAL-BLAST-EFFEC TS BLAST-8I OLOGY DISPLACEMENT BL AST-ENERGIZEO-MISSILES BLA ST-BIO/ O6-2I -O2223 (1 SCIENT IFIC-RESEARCH SANI TARY-ENGINEERI NG PUBLIC-REALTH DISPOSAL COSTS WASTES (SANITARY-ENGIVEERING) STOI I3-0? - II7B9 TS OF FALLOUT IN WASTE WATE' POSTATTACK SANITATION, WASTE DISPOSAL, PEST AND VECTOR CONTROL, AVD THE EFFEC I3-0?-OI7SO LLOUT PARTICLE MATRIX I RADIOAC/ HENRYS LAW CONSTANTS FOR DISSOLUTION OF FISSION PRODUCTS IN A SILICATE FA I8-DS-O2325 (N) MIL IT ARY-STRATEGY DAMAGE-A SSE SSMENT THREAT-E VALUATION DISTRIBUTIONI POPULAT ION) MODEL SISIMULAYIONS),, I 5-OT-DI 96O AT IONS MANAGEMENT-PLANNING RECOVERY VULNERABI LITY RECORDS DISTRIBUTION(WATER) SURVEYS, \$A.L. 969 /CK-OPER I3-O? -DITB8 $S$ WASHINGTON BUILDINGS STRUCTURES// BUILDING CODE OF THE DISTRICT OF COLUMBIA 1 -BUILOING-CODES REGULATION I3-I3-OIBIG 
REGULATIONS STRUCTU/ MANUAL FOR THE BUILDING CODE OF THE DISTRICT OF COLUMBIA I WASHINGTON BUILDING-CODES I3-I3-OIZI 7 1 MATERIAL ENCASING A TUNNEL LINER SUB JECTED TO A DYNANIC DISTURBANCE I UNCERGROUND-STRUCTUZES DEFORMAIIOI I3- 33 - $227 \%$ IONS IONOSPHERE A I ELEC TROMAGNETIC PULSE PROPAGATICN IN A DISTJRBEO TERRESTRIAL WAVEGUIDE 1 VUCLEAR-EXPLOS $20-1+$-D2348 IS TO SERVE SOUTHERN CALIFORNIA - FEATHER RIVER ANC DELTA DIVERSION PROJECTS I WATER-SUPPLIES URBAN-AREASI I3-0? -02258 NDIX INDEXING TECHNIQUES, DESCRIPTION AND BACKGROUND I C/ DOCUMENT STORAGE AND RETRIEVAL IECHNIQUES - APPE QS-02-Q2IIG WEIGHT PHYSICAL-FITNESS NUTRIIION, \#AD 633860 A DOCUMENT ARY ON WEIGHT, DIET AVOEXERCISE I BODY- O6-I4-OI7IG UCT ION-COVTROL HISTORY MILITARY-PLBLICATICNS REPRCOUCTICN DOCUMENT AT ION, \#AD 645 5OD /FICHE MAVPOWER PROD O5-D2-O2I 25 PHY ON IVFORMATION SCIENCE AND TECHNOLOGY FOR 1965 IV. I DOCUMENTATION, ITEMS I TECHNICAL-INFORMATION-CENTERS SYSTEMS-ENGINEERING DOCUMENTATION DATA-STORAGE-SYSTEMS SUBJECT-INDE/ O5-02 - 2 I 2 I INIZED. IVFORMATION SYSTEM I TECHNICAL-INFORNATICN-CENTERS DOCUMENT AT ION INFORMATIOV-RETZIEVAL AUTOMATION I DS-0? -02II 8 IN B. RELATIONSHIP AND COMPARI SON (DEPARTMENT-OF-CEFENSE COCUMENT AT ION INFORMATION-RETZ IEVAL ENGINEERING/ D5-O? -O2I 20 RESEARCH ORG/ INDEX OF PUBLI SHED PAPERS I INOEXES REPORTS DOCUMENTATION PROFESSIONAL-PER SONNEL SCIENTIFIC- DS-D?-D2I I 7 IHY ON INFORMAT ION SCIENCE AND TECHNOLOGY FOR 1966 IV. ( DOCUMENTATION SCIENT IFIC-RESEARCH INFTRMAIION-R, O5-D2-O2I 23 - LETS PROFIT FROM WHAT WE KNOW I INFORMATI CN-RETRIEVAL DOCUMENT AT ION STATE-DF-THE-ART-REVIEWS SYSTEMS- I O5-OZ-OIG3D IFIC AND TECHNICAL INFORMATION WI THIN THE DEFENSE INDUST/ DOC USER - NEEDS STUDY, PHASE II, FLOW OF SCIENT OS-O? -O2120 OGY SHOCK-WAVES B IOLOGICAL-BLAST-EFFECTS RATS GUINEA-PIGS DOGS GOATS SHOCK-TUBES I \#DASA- 1852 / BLAST-BIOL O6-2I -02236 / TORSION STRETCHING COMPRESSING TESTING-EQUIPMENT MARBLE DOLOMITE LIMESTONE ROCK-SALT ANHYDRITE SANDSTON/ D8-O7-O224 2 VES / EARTR VIBRATIONS FROM A NUCLEAR EXPLOSION IN A SALT DONE - PROJECT CRIBBLE SALMOV EVEVT I SEISMIC-WA I8-O3-O23I 9 /RS B BLAST-SHELTERS DESIGN COSTS SINGLE-PURPOSE-SHELTERS DOORS UNCERGROUNL-STRUCTURES STRUITUZAL-PROPERT/ I3-I3-0I835 $X I N S$ - AND-ANTITOXINS PHARMOCOLOGY NUSCLES NONKEYS CHICKENS COSAGE / \#EASP-IDO-I \#AD 63? $265 / M-B 0 T U L I N U M$ TO I5-D? -DI86I IICAL-WARFARE-AGENTS BAC TERIAL-AEROSOLS EXPOSURE VACCINES DOSAGE IMMUNITY MONKEYS MICE GUINEA-PIGS HUMANS/ I5-D?-OI858 E-FALLOUT SHIELDING WALLS COBALT EXPERIMENTAL-DATA THEORY DOSE-RATE। \#R-OU-266(I)/(COYPUTERS) RADIOACTIV O6-IB-O22I8 ACE EXPER IMEVTAL-DATA AGING (PHYSIOLOSY) ANIMALS MICE RATS DOSE-RATE) *RM-5083-TAB /URIES RADIOLOGICAL-DOS O6-13-OI733 - HAZARDS MUTATIOYS RADIATION-EFFECTS ANI MALS HUMANS TESTS DOSE-RATE, \#RM-S096-TAB /S I GENETICS RADIATION DO-I3-DI727 IED PF MULTIPL T PROTECT ION I. FALLOUT-SHELTERS NLCLEAR-WEAPONS SHIELDING DOSE-RATE CALCULAT IONS SLANTIVG, \#TR-2OIII ILOU I3-I3-DIB3O IECUL ING I RADIOACTIVE-FALLOUT RADIOLOGICAL-CGNTANINATI ON DOSE-RATE COJNTERMEASURES FALLOUT-SHELTER S RADI I D6-IB-O222D IURVEY I WEAPONS-EFFECTS RADIOACTIVE-FALLCUT DISTRIBUT ION DOSE-RATE GAMMA-RAYS INSTRUMEVTATION EXPERIMENT/ IB-O3-O2324 $1 /$ RACIOACTIVE-FALLOUT ATIENUATION UNDERGROUND-STRLCTURES DOSE-RATE PHOTONS SCATTERING ALBEDO GAMMA-RAYS / I8-DG-D2O29 IENERGY SPECTRA IN CONCRETE DUCTS I SHELTERS ENTRANCEWAYS DOSE-RATE RADIAT IOV-MEASUREMEVT-SYSTEMS PROTECT/ DG-I9-O22I 9 IITORS RADIATION-MEA SUREMENT-SYSTEMS PERSCNNEL OOSIMETERS DOSE-RATE RADIOLOGICAL-COVTAMINATION RADIATION-I I8-OS-D2O28 /LOGICAL-DOSAGE DOSIMETERS RADIATION-EFFECIS CALCULATIONS DOSE-RATE SHIELDING RADIATION-MEASUREMENT-SYSTE/ D6-IS-DI726 ITECT ION-FACTOR MONTE-CARLO-ME THOD PROGRANMING (CONFUTERS) OOSE-RATE SHIELCING RECOVERY CALCULATIONS ) \#R-I O6-18-O22 I 4 IF ROOF REDUCTION FACTOZS I SHIELDING RAOIOACTIVE-FALLOUT DOSE-RATES RACIOLOGICAL-DOSAGE ATTEVUATION SIMU/ I 8-OS-D2O3O ICIAT ION-MONITORS RADIATION-MEASUREMENT-SYSTEMS PERSONNEL DOSIMETERS DOSE-RATE RADIDLOGICAL-CONTAMINATION/ I8-0S-D2028 IOISONOUS-GASES LEWISITE GAS-DETECTORS RADIATION-MCNITORS DOSIMETERS PRDTECTIVE-CLOTHING GAS-MASKS PHOSGEI OG-I3-DI72I 1 TION-INJURIES USSR RADIATION-HAZAROS RADIOLOGICAL-DOSAGE DOSIMETERS RADIATION-EFFECTS CALCULATIONS DOSE- 1 O6-IB-DI726 SYSIEM I TECHNICAL-INFORMATION I FIVE YEARS IN FOCUS - THE DOUGLAS AIRCRAFT COMPAVY MECHAYIZED IVFORMATION OS-OP -D2II EL ANO THE MAI TRANSPOR TA TON AND RELATED PROBLEMS OF THE OOWTA SSING DAMAGE-ASSESSMENT, \$NREC-24III ANNEX TC THIRD DRAFT OF READY I I RESOURCES ANALYSIS DATA-PROCE D5-D3-D2I57 NALS SCHEDULING SWITCHINC PROPULSION-SYSTEMS AER DOYNAMICS DRAG, \$PB 173 GSB /RANSPORTATION VETWORKS TERMI $23-02-02354$ GROUN C-TRANSPORT PERFORMANCE (ENGINEERING) VEHI/ UNIVERSAL DRAG LAW I TRANSPORTATION PRJPULSIOV HIGH-SPELD- 23-0?-02096 (TRAN SPORTATION) TRAN SPOR TATION URBAN-AREAS I AEROCYNAMIC ORAG ON VEHICLES IV ENCLOSED GUIDEWAYS I TUNNELS 23 -D2-O2I QI IHEAT ING HEATING COOLING-SYSTEMS FLUID-D YNAMIC-PROPERTIES DRAG SURFACE-TEMPERATURES BOUVDARY-LAYER LAMINA/ 22 -02-02350 $K$ PRO/ CONTROL OF SURFACE STORM WATER BY STORM DRAINS AND DRAINAGE CHANNELS I. GENERAL DESCRIPTION OF WOR I3-O2-UI782 CTION' CONTROL OF SURFACE STORM WATER BY STORM DRAINS AND DRAINAGE CHANNELS II. PLAVS, PROFILES, CROSS SE I3-DZ-DIT79 CTION/ CONTROL OF SURFACE STORM WATER BY STORM DRAINS AND ORAINAGE CHANNELS II. PLANS, PRJFILES, CROSS SE I3-O2-OI7BI CRIPT/ CONIROL OF SURFACE STORM WATER BY STORM DRAINS AND DRAINAGE CHANNELS - 1958 PROGRAM I. GENERAL DES I3-02 -DI783 CRIPT/ CONTROL OF SURFACE STORM WATER BY STORM DRAINS AND DRAINAGE CHANNELS - 1964 PROGRAM I IARY - ENGINEER ING SEWAGE DI SEA SE-VEC TORS DI SEASE SICCNTROLI DRAINAGE COUNT ERPEASURES URBAY-AREAS SAN-JOSE C 7 I 3 - Q? - DI 780 TION OF WORK PRO/ CONTROL OF SLRFACE STORN WATER BY STORM CRAINS ANC CRAINAGE CHANNELS I GEVERAL OESCRIP I3-OZ -DI7B2 S, CROSS SECTION/ CONTROL OF SLRFACE STORN WATER BY STORM DRAINS ANC CRAINAGE CHANNELS II. PLANS, PROFILE I3-02 -OI779 S, CROSS SECTION/ CONTROL OF SURFACE STORN WATER BY STORM DRAINS AND DRAINAGE CHANNELS II. PLANS, PROFILE I3-02 - DI78I GENERAL DESCR IPT/ CONTROL OF SLRFACE STORN WATER BY STORM DRAINS ANC CRAINAGE CHANNELS - 1904 PZOGRAM I. 3 - O?-DI784 IAT IONS FROM A NUCLEAR EXPLOSION IN A SALI DONE - FRCJECT ORIBBLE SALMON EVEVT I SEISMIC-WAVES EXPLOSION-I I8-O3-J23IQ ITION I EXCAVATION TUNNELSITRANSPCRTATIONI BLASTINE COSTS DRILLING NORTHEAST-CORRIDOR FEASIBILITY-STUDIES/ I3-D2-JI787 TIIONS RADIOACTIVITY CONTROL I \#PHS/ PUBLIC HEALTH SERVICE DRINKING WATER STANDARDS I WATER-SUPPLIES REGULA I3-D2-DI778 10 COMPUTER PROGRAM TO CALCULATE THE THERNAL HISTORY OF A DUAL LAYEREC PLATE SUBJECT TO THE THERMAL PULSE $/$ I3-I?-OI797 TION IN FOUR NATIONAL MODEL BUILDING CODES I CONSTRUCTION DUAL-PURPOSE-SHELTERS ) \$TR-36 /SHELTER CONSTRUC I3-I3-D228I IS FOR A FEOERAL 'TRANSPORTATION ADMINISTRATION I SHELTERS DUAL-PURPOSE-SHELTERS COSTS GERMAVY CONSTRUCTIO/ I3-13-OIB2 7 TIONS/ STATUS OF THE CIVIL DEFENSE PROGRAN 1 ORGANIZATION DUAL-PURPOSE-SHELTERS FALLOUT-SHELTEZS APPROPRIA OS-OI-DIG 5 IIC SHELTER FAC IL ITY I TRANSPORTATION FEASIBILITY-STUDIES DUAL-PURPOSE-SHELTERS STOCKPIL ING ENTRANCEWAYS, I3-13-O227I IHELTERS FALLOUT-SHELTERS UNDERGRCUND-STRUCTURES CCLORADO DUAL-PURPOSE-SHELTERS TRANSPORTATION UTILITIES I I3-I3-OIB3I INAI INTERACT INA INTERACTION BETWEEN AIR FLOW AND AIRRORNE SOUND IN A DUCT I ACOJST ICS GAS-FLOW WEST-GERYAVY DUCTS LAM 20-DI -O2DU8

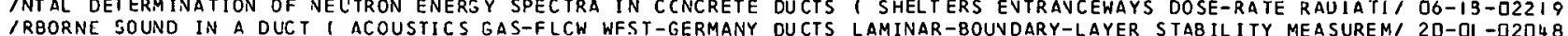
IRBORNE SOUND IN A DUCT I ACOUSTICS GAS-FLCW WFST-GERMANY DUCTS LAMINAR-8OUNDARY-LAYER STAB ILITY MEASUREMI 2D-OI - D2048 IE COMMUNICATIONS CONDUI TO STATIC AND DYNAMIC LOAOING 1 DUCTS SANO UNDERGROUND LOADINGIMECHANICSI SOIL-I I3-II -O226I T-DUMMY (ANTHROPOMORPHIC) CONSTRUCTI\% ANTHROPOMORPHIC TEST OUMMY 1 IEST-EQU IPYENT DESIGN SAFETY-DEVICES TES I3-I?-DIBD8 LTS I TEST-EQUIPMENT / DESIGN AND CONSTRUCTION OF A CRASH OUMMY FOR TESTINE SHOULDER HARNESS ANO SAFETY BE I3-I?-QI8J3 IOLOGICAL-BLAST-EFFECTS I THE TOLERANCE OF CATTLE TO LONG DURATION REFLECTED PRESSURES IN A SHOCK TUBE I B D6-2I-D2237 AS-TURBINES VELOCITY, $/$ STUNY OF THF BASIC MECHANISMS OF DUST EROSION I PARTICLE-SIZE EXPERIMENTAL-DATA G 20-03-02068 OCAT ING FALLOUT ON A SPHERICAL EARTH I HIND YIELD RADION/ DUSTY III - DN IMPROVED MATHEYAIILAL METHOD OF I I 8-DS-D2322 AEL, AND THE UVITED NATIONS AT FI/ INTER-AATICN CCNFLICT, OYADIC ANC MECIATED - CASE STUDIES OF EGYPT, ISR DS-D+-O2I 70 RATED SAND I LOADING(MECHANICSI SCIL-MECHANIC/ STATIC AND DYNAMIC BEARING TESTS ON A STRIP FOOTING IN SATU $20-1 . I-0234 I$ /KPACKING MATER IAL ENCASING A TUNNEL LINER SUBJECTED TO A DYNAMIC DISTURBANCE I UNDERGROUND-STRUCTURES DE/ I3-I3-O2279 1 A BURIED PROTOTYPE COMMUNICATIONS CONDUIT TO STATIC AND DYNAMIC LOADING DUCTS SANC UNDERGROUND LOADIN/ I3-II-D226I ALLY SATURATED SAND - CLAY MIXI THE RESPCNSE OF SCIIS TC DYNAMIC LOACINGS - WAVE VELOCITIES THROUGH PARTI 2O-OI - D2OL? ALICS SOIL-MECHANICS TH/ A RHEOLOGIC INVESTIGATION OF THE CYNAMIC LOCINGS - WAVE VELOCITIES THROUGH PARTI $20-0 I$-D2O4? AMC CONCRETE BEAMS ( BEAMS (STRUCTURAL) DYNAMICS SHEAR-STRI DYNAMIC SHEAR RESISTANCE OF THIN WEBBED REINFORC 20-II -D2DB6 AMS II. ( TEST-EQUIPMENT BEAMSISTRUCTURAL) LOADING IMECHI DYNAMIC SHEAR STRENGTH OF REIVFORCED CONCRETE BE I3-I3-DIBLI

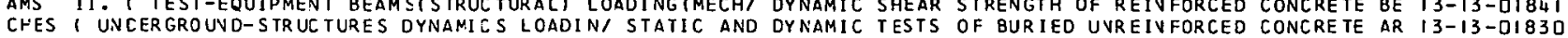
IOSE-SHEL TERS COSTS GERMANY CONSTRUCTION HAROENI AG DESIGN CYNAMIC-PRESSURE SHIELDING CALCULAIIONS GARAGES/ I3-I3-QI827 ORT $1 /$ A THEORETICAL AND EXPERIMENTAL. INVESTIGATICN OF A DYNAMICALLY LOADED RING WITH ZADIAL ELASTIC SUPP $20-I I$-O2336 UM-ANALYZERS TRAN SOUCERS MATHEMATICAL-ANALYSIS STRUCTURES DYNAMICS, *BULLETIN $35(4)$ \&AD 631233 /S SPECTR $20-11-02075$ ITIONS FOR SIMILAR SOLUTIONS OF PROBLEMS CF TURBULENT GAS DYNAMICS GAS-FLON EQUATIONS-OF-STATE MATHEMAT/ $20-04-02331$ /RING I FRACTURE (MECHANICS) CRACK-PROPAGATICN INPACT-TESTS DYNAMICS RLAST SHOCK-WAVES ELASTICITY STRESS ME / 20-II -02338 IF COMPUTER ANALYSIS PROGRAMS FOR SHELTERS IN BUILIINGS 1 DYNAMICS COMPUTER-PROGRAMS SHJCK-WAVES BLAST SH I I3-I3 -O22BD /OUNDATIONSISTRUC TURES) SOIL-MECHANICS LOADING (MECHANICS) DYNAMICS EXPERIMENTAL-DATA COMPRESSIVE-PROPERTI/ I3-I3-OI8I 2 IEAS IEIL ITY-STUDIES REACTOR-SAFETY-SYSTEMS SOIL-MECHANICS DYNAMICS GROUND-MOTION UNDERWATER DESIGN COSTS / I8-09-O2OUD 
( STUDY ( SHEL TER S UNOERGROUND-STRUC TURE S ELASTICITY TEST DYNAMICS LOADING(MECHAVICS) STRAIV(MELHANICS) T/ $13-13$-DI825 /ELTERS SHOCK-WAVES BLAST-SHEL TERS UNDERGROUND-STRUCTURES DYNAMICS MATHEMATICAL-ANALYSIS DIFFERENTIAL-EQU/ I3-13-01833 IHIN WEBBEC REINFORCED CONCRETE BEAMS ( BEAMS (STRUC TURAL) DYNAMICS SHEAR-STRESSES WEBSISTRUCTURAL) DESIGN $20-11-02337$ IIVE CONSTRUCTION I BLAST-SHEL TER S UNDERGROUND-STRUCTURES DYNAMICS SHELLS (STRUCTURAL-FORMSI BEAMSISTRUCTU/ I3-13-DI837 ING IN SATURATED SAND I LOADING IMECHANICSI SOIL-NECHANICS DYNAMICS SHELTERS JNDER GRJUVD-STRUC TUZES SAND S/ $20-11$-0234I TTION OF THE DYNAMIC RESPONSE SPECTRA OF COHESIVE SOILS I DYNAMICS SOIL -MECHANICS THEORY EXPERIMENTAL-DATI $20-11$-D2DBG

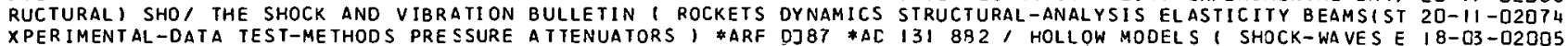

EFFECTS ) \#DASA-1E58 EFFECTS OF OVERPRESSURE ON THE EAR - A REVIEW I BLAST-BIJLOGY BIJLOGICAL-BLAST- D6-2I -0223I IRITA IN IVIERNATIONAL-CIVIL-DEFENSE CI VI L-DEFENSE-SYSTEMS EARLY-WARNING-SYSTEMS VATIONAL-DEFEVSE RECRUITI, IS-D3-O23J5 IC DETERRENCE 1 VULNERAB ILITY INTERNATIONAL-CIVIL-CEFENSE EARLY-WARNING-SYSTEMS USSR MOVEMENT POPULATION I I5-D3-DI922 ID MATHEMATICAL METHOD OF LOCATING FALLOUT ON A SPHERICAL EARTH I WIND YIELO RAOIOACTIVE-FALLOUT PROGRAMM I I -DS -OI922

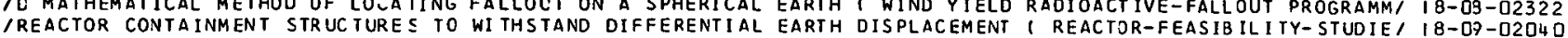

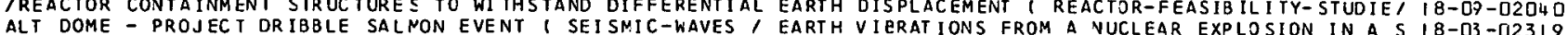
ALT DOME - PROJEC T DR IBBLE SALNON EVENT I SEISMIC-WAVES ' EARTH VIERAT IONS FROM A NUCLEAR EXPLOSION IN A S I8-D3-O23I SASTER T DAMAGE-A SSESSMENT DATA DECISION-MAK/ THE ALASKAN EARTHOUAKE - A CASE STUDY IN THE ELONOMICS OF DI O5-O3-DIG36 AN JAPAN PROCEEDINGS OF THE SECOND WORLD CCNFERENCE ON EARTHQUAKE ENGINEERING II. TOKYO AND KYOTO, JAP D8-II-O2246 XPLOS ION-EFFECTS VELOCITY ACCELERATION MOTION PRCPAGATICN EARTHQUAKES, \$VUF-3014 \$AD 649 7DI $1 M I C-W A V E S E$ I 8-03-023I9 /CT IVE-FALLOUT RADIOLOGICAL-DOSAGE STORMS DECCATANINATION EART HQUAKES VOL CAVJES LAVDSLIDES ELEETRIC-POHERI IS-03-O22 99 /RACO SCHOOL OF MINES S2(3) BEHAVIOR OF NATERIALS IN THE EARTHS CRUST - SECOND ANNUAL SYMPOSIUM ON ROCK , OB-OT-D2244 CE RADAR-SIGNALS MAPS ELECTROMAGNETIC-C/ RADAR MASKING BY EARTHS TERRAIN. I SITE-SELECTION RADAR-INTERFEREN I7-09-DIOB8 /SEARCH BY PR IVATE SCHOLARS AND ACAOEMIC CENTERS - MIDDLE EAST 1 BIBLIOGRAPHIES ECONOMICS EDUCATION POLIT/ O5-I1-OI695 IATURAL VENTILATION TEST OF A BASEMENT FALLOUT SHELTER IN EAST CHICAGO, INCIANA I EVVIRJNMEVTAL-CONTROL E, I3-DI -OI7TL - AS A CIVIL DEFENSE PUBLIC SHELTEI STUOY OF THE PROPOSEC EAST WEST FREEWAY TUNNEL WEST ORAVGE, NEW JERSEY I3-I3-D2271 IARCH OY PRIVATE SCHOLARS AND ACADEMIC CENTERS - USSR AND EASTERN EUROPE 1 BIBL IOGRAPHIES COMMUNISM ECONO/ O5-II -DIG 7 EST-METHOOS, * ECOSYSTEMS, SYSTEMS ECOLOGY, ANO SYSTEMS ECCLOGISTS I TRAINING COMPUTERS TEST-EQUIPMENT T OG-DG-OI7DB IREVISION OF THE BEE GENUS CALLIOPSIS ANO THE BIOLCGY AND ECCLOGY OF C. ANCRENIFJRMIS - HYMEVOPTERA, ANDR, O6-0S-OI7O5 , EFFECTS OF NUCLEAR WAR I RADIOBIOLOGY RADIATION-EFFECTS ECOLOGY RADIATION-DAMAGE RADIOLOGICAL-CONTAMINA/ DO-13-OI723

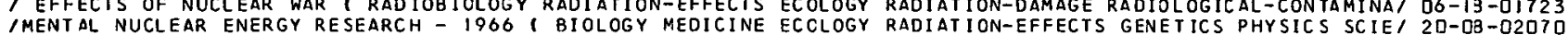

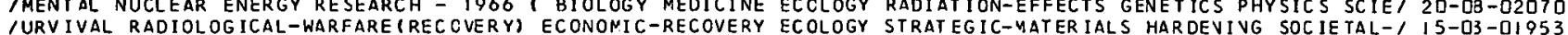

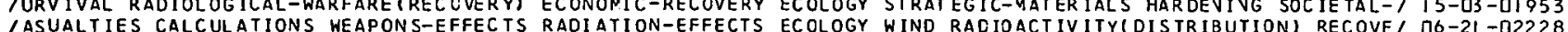
/ASUALTIES CALCULATIONS WEAPONS-EFFECTS RADIATION-EFFECTS ECOLOGY WIND RACIOACTIVITYIDISTRIBUTION) RECOVE/ Q6-2I -D2228
TERS TEST-EQUIPMENT TEST-METHODS, \# ECOSYSTEMS, SYSTEMS ECCLOGY, AND SYSTEMS ECOLOGISTS 1 TRAINING COMPU D6-OS-DI7O8 IEPORT OF THE PRESIDENT - ANNUAL REPORT OF THE COUNCIL OF ECCNOMIC AOVISERS I ECONOMICS UNITED-STATES-GOVI DS-O3-OIO34 OF THE COUNCIL OF ECONOMIC ADVI SERS I ECCNONICS UNITEO-/ EC CNOMIC REPORT OF THE PRESIDENT- AVVUAL REPORT OS-O3-OIO34
IMITS OF FEASIBLE PRODUCTION I ECONOMICS RECOVERY POST/ ECONOMIC VIABILITY AFTER THER YONUELEAR WAR - THE OS-D3-O2I 7 LIMITS OF FEASIBLE PRODUCTION I NUCLEAR-WARFARE SURVIVAI ECONOMIC VIABILITY AFTER THERMONUCLEAR WAR - THE OS-D3 -02I27 THER WAR IN VIETNAM I TECHNICAL-ASSI STANCE FOREIGN-POLICY ECCNOMIC-AIC; $\$ A . L$. 1082 MS OF FOREIGN A IO 1 PUBLIC-OPINION SURVEYS FOREIGN-PCLICY ECONOMIC-AIC MILITARY-AID) ICAL-SCIENCE REGIONAL-PLANNING IND/REGI ONALISM IN ASIA 1 ECONOMIC-CONDIT IOVS POPULATIOV AGRISULTURE POLIT OS-O3-O2ISI IERY ACTIVITIES I POSTATTACK-OPERATIONS SOCIETAL-RECOVERY ECONOMIC-RECOVERY ATTITUDES MANPOWER MOTIVATION/ O5-IJ-O2IBO ISASTERS BEHAVIOR SOCIOLOGY STRE SS(PSYCHOLOGY) DEMCGRAPHY ECONOMIC-RECOVERY CIVIL-CEFENSE-SYSTEMS CULTURE/ OS-II-O2I 9D /TEM MANUAL III-B. PER SONAL CONSIMPTION NODEL I RECOVERY ECCNOMIC-RECOVERY DAMACE-ASSESSMEVT MDDELS RESO/ OS-03-02I5I IAGEMENT-PLANNING SUR VIVAL RADIOLOGICAL-WARFARE (RECOVERY) ECONOMIC-RECOVERY ECOLJGY STRATEGIL-MATERIALS H/ IS-D3-DIO53 IINE I RESOURCES CAPACITY DAMAGE-ASSESSMENT VULNERABILITY EC CNOMIC-RECOVERY INDUSTRIAL-PRODUCTION I NVENTO/ OS-D3-D2I52 INE I RESOURCES CAPACITY DAMAGE-ASSE SSMENT VULNERABILITY EC CNOMIC-RECOVERY INDUSTRIAL-PRODUCTION INVENTO/ OS-03-02I52
CTION I ECONOMICS RECOVERY POSIATTACK-OPERATICNS SURVIVAL ECONOMIC-RECOVERY LABOR RESOURCES I *RM- $3436-P R$ OS-O3-02127 CTION I ECONOMICS RECOVERY POSTATTACK-OPERATICNS SURVIVAL ECONOMIC-RECOVERY LABOR RESOURCES I *RM- $3436-P R$ OS-03-021 27

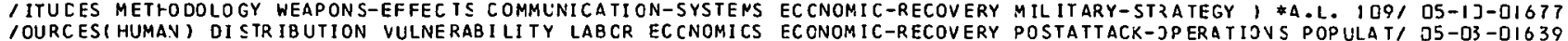
IOURCES (HUMAN) DISTR IBUTION VULNERABI LI TY LABCR EC CNOMICS ECONOMIC-RECOVERY POSTATTACK-JPERATIOVS POPULAT/ D5-D3-DI639
IIAL-EQUIPMENT INDUSTRIES FLOW-CHARTING SURVIVAL RECOVERY ECONOMIC-RECOVERY PRODUCTS BLAST VULNERABILIIY, OS-D3-O2I 26 /IAL-EQUIPMENT INDUSTRIES FLOW-CHARTING SURVIVAL RECOVERY ECONOMIC-RECOVERY PRODUCTS BLAST VULNERABILIIY / OS-D3-O2I 26
INDUSTRIAL-EQUIPMENT BLAST INOUSTRIES SLRVIVAL RECOVERY ECCNOMIC-RECOVERY PRODUCTS VULNERABILITY EXPLOS/ OS-D3-O2IG2 I INDUSTRIAL-EQUIPMENT BLAST INOUSTRIES SLRVIVAL RECOVERY ECCNOMIC-RECOVERY PRODUCTS VULNERABILITY EXPLOS/ OS-O3-O2I 62
/L CISPAR ITIES AND NATIONAL VIABILITY I SOCIETAL-RECOVERY ECCNOMIC-RECOVERY RECOVERY ATTACK-SCEVARIOS WIN/ O5-O3-02I OO AGE-ASSESSMEVT WARFARE RESOURCES INDUSTRIES VULNERABILITY ECCNOMICS, *A.L. IO52 /URVIVALI SIMULATION DAM DS-D3-DIGL4 R-REACTORS NUCLEAR-POWER-PLANTS ELECTRIC-POWER-PROCUCTION ECONOMICS; \#A.L. IOS8 10 THE PRESIDENT, NUCLEA I8-0J-O2O27 GAS STATISTICS 1 RESOURCES PETROLEUM-INOUSTRY INDUSTRIES ECONOMICS, *A.L. 985, OF PETROLEUM AND NATURAI. T5-U3-0IG38 RABIL ITY EXPLOSION-EFFECTS AIRBIRST POSTATTACK-DPERATIONS ECCNOMICS, \#IUA/HQ-66-5472/VERY PRJOUCTS VULNE DS-03-02I62

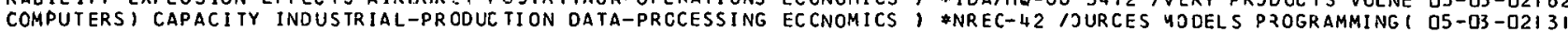
E POPULATION TRAVSPORTATION LTILIIIES FI SCAL-AND-MCNETARY ECONOMICS, \#PRC D- $1219 /$ URBAN-PLANNING LAND-US $23-0 S-I 2 I O 6$ ECT-INDEXING TEST-METHODS INFORMATION-RETRIEVAL AUTOMATIC ECONOMICS NT CONTROL IN SOUTH VIETNAM I POLIIICAL-SCIENCE SOCIOLOGY ECONOMICS, \#RM-5IBI-ARPA IRS AFFECTIVG GOVERNME OS-D2-02II6 ER INNOVATION PRO JECTIONS MODELS, *A.L., PROSPECTING IN ECONOMICS 1 SCIENTIFIC-RESEARCH RESOURCES MANPOW OS-D3-DIO33

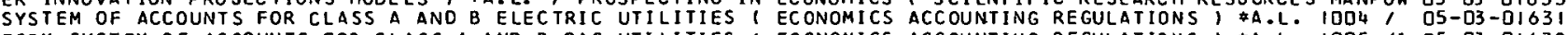
FORM SYSTEM OF ACCOUNTS FOR CLASS A AND B GAS UTILITIES I ECCNOMICS ACCOUNTIVG REGULATIONS I \#A.L. IOOS II O5-O3-OIO32 /LIOGRAPHIES ATOMIC-ENERGY COMMUNICATIONS LABCR CCNMUNISM ECONOMICS AGRICULT JRAL-ECOVOYICS FIVAVCE INDUST/ DS-II -DIG 3 /ARCH BY PRIVATE SCHOLARS AND ACADEMIC CENTERS - AFRICA I ECONOMICS BIBLIOGRAPHIES EDUCATIOV FOREIGN-POLI/ DS-II - IIR92 IOMPLETED - ARMS CONTROL AND DISARMAMENT I BIBLIOCRAPHICS ECONOMICS COMMUNISI-CHINA CONFLICT-RESOLUTION H/ O5-DU-DI663 IOMPLETED - ARMS CONTROL AND DISARMAMENT 1 BIBLIOGRAPHIES ECCNOMICS CONFL ICT-RESOLUTION COMMUVIST-CHINA H/ D5-O4-OI 665 IMPLETED - ARMS CONTROL AND DI SARNAMENTI CONNUNIST-CHINA ECONOMICS CONFL ICT-RESOLUTIOV HISTORY IVTERNATI/ OS-D+-OIOS2 IMPLETED - ARMS CONTROL AND DI SARNAMENT I COMMUNIST-CHINA ECONOMICS CONFL ICT-RESOLUTION HISTJRY INTERNATI/ O5-OH-OIOS4 AND DELTA DIVERSION PROJECTS I WATER-SUPPLIES URBAN-AREAS ECONOMICS COSTS 1 \#ULLETIN NO. 78 IATHER RIVER I3-D2-O2258 IONOMY - KUWAIT I MANAGEMENT-PLANNING OPERATICNS-RESEARCH ECCNOMICS DECISICN-MAKING COVSERVATIOV DEPLETIOI O5-03-OIG35

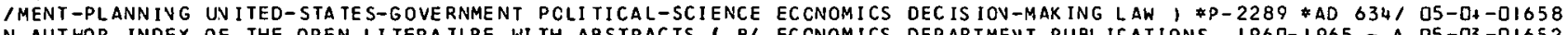
N AUTHOR INDEX OF THE OPEN LI TERATLRE WI TH ABSTRACTS I E/ ECCNOMICS DEPARTMEVT PUBL ICATIONS, $1960-1965$ - A DS-03-DI652 ISTRIES RESOURCES I HUMAN) DISTR IBUTION VULNERABILITY LABOR ECCNOMICS ECONOMIC-RECOVERY POSTATTACK-OPERATIO/ O5-D3 - 22 49 CENTERS - AMERICAN REPUBLICS I BI LIOGRAPHIES CCNMUNISN ECCNONICS ECJCATIOY FOREIGV-PJL ICY GEDGRAPHY POI O5-II -OIOQO C /ERS - USSR AVD EASTERN ELROPE I BIBLIOGRAPHIES CCNMUNISM ECONOMICS EDUCAT IOV INTERVATIJVAL-AFFAIRS GEOGR/ O5-1I-UI697 ILARS AND ACADEMIC CENTERS - MIDOLE EAST I BIBLIOGRAPHIES ECONOMICS EDUCATIOV POLITICAL-SCIENCE GEOGRAPHY/ Q5-11 -DI695 IECONOMIC-RECOVERY PRODUCTS BLAST VULNERABILITY AIR-BURST ECONOMICS EXPLOS ION-EFFECTS POSTATIACK-OPERATIO/ D5-O3-O2I26 IE TOOL SUBST ITUTION POSSIBILITIES I INDUSTRIAL-EQLIPMENT ECCNOMICS FEASIBIL ITY-STUDIES CLASSIFICATION $/$ I O5-O3-OI 648

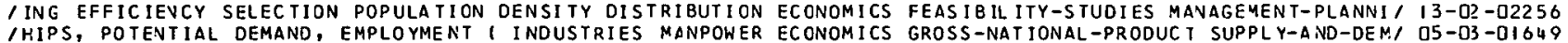

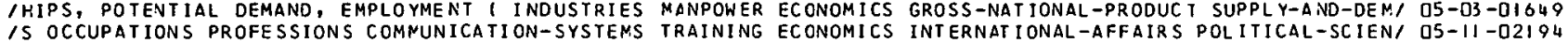
ECISION-MAK/ THE ALASKAN EARTHOUAKE - A CASE STUDY IN THE ECONOMICS OF DISASTER I DAMAGE-ASSESSMENT DATA D DS-O3-DIG36 IF NUCLEAR ATTACK I SOCIETAL-REC O VERY RECOVERY OEMCGRAPHY ECCNOMICS POL IT ICAL-SCIENCE STRESSIPSYCHOLOGY) I O5-II-D22D2 ILIMITS OF FEASIBLE PRODUCTION I NUCLEAR-WARFARE SURVIVAL ECONOMICS POPULATION AGRICULTURE IVDUSTRIES IND/ D5-D3-D2I58 IM INDUSTRY OF GERMANY DURING THE WAR I MILITARY-STRATEGY ECONOMICS PRODUCTION VULNERABILITY MANPOWER RECI DS-O3-DIG46 IN IN THE CONTINENTAL UNITED STATES I SYSTEMS-ENGINEERING ECONOMICS RECOVERY POSTATTACK-OPERATIJNS DAMAGEI O5-D3-O2I 88 IDUAL INDUSTR IES FOR DEFENSE PLANNING I SLPPLY-AND-DEMAND ECCNOMICS RECOVERY POSTATTACK-OPE $2 A T I O N S$ RESOUR I D5-O3-DIOL 7 1 THERMOVUCLEAR WAR - THE LIMITS OF FEASIBLE PRODUCTION 1 ECONOMICS RECOVERY POSTATTACK-OPEZATIONS SURVIVI O5-O3-O2I27 RM SYSTEM OF ACCOUNTS FOR CLASS A AND B WATER UTILITIES I ECONOMICS REGULATIONS ACCOUNTING \$A.L. IOD3 IO O5-OB-DIO5O RM SYSTEM OF ACCOUNTS FOR CLASS A AND B WATER UTILITIES I ECONOMICS REGULATIONS ACCOUNTING $\$$ \&A.L. IOD3 10 OS-O3-D I650
ITOTAL VULNERABILITY I SYSTEMS-ANALYSIS POLITICAL-SCIENCE FCONOMICS SOCIOLOGY SOCIETAL-RECOVERY DAMAGE-AS/ O5-II-D2I93 
/RECOVERY ECONOMIC-RECOVERY ATTITLDES MANPOWER MOTIVATION ECCNOMICS SURVIVAL OISTRIBUTION STRESSIPSYCHOLO/ O5-IJ-O2I8D INT - ANVUAL REPORT OF THE COUNC IL OF ECONGMIC ADVISERS I ECONOMICS UNITEC-STATES-GOVERVMENT POLITICAL-SC I O5-D3-OI634 LITY LAND-USE/ URBAN FORM AS A PASSIVE DEFENSE VARIABLE I ECONOMICS URBAN-AREAS DECEVTRALIZATION VULNERABI 23 -OS-D2IO? VES SOCIAL-SCIEN/ OPERATIONS RESEARCH ON URBAN PROBLEMS, EC ONOMICS URBAN-PLANNIVG DECISION-MAKING INCENTI D5-IJ-OIGTO

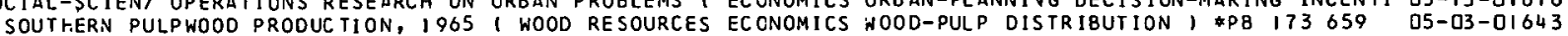
S-RESEARCH ECOVOMICS DECIS/ PLANNING IN A CAPITAL SURPLUS ECCNOMY - KUNAIT 1 MANAGEYENT-PLAVYING OPERATION O5-D3-DI635 STS I TRA INIVG COMPUTERS TEST-EQUIPMENT TEST-METHOCS I \#/ ECOSYSTEMS, SYSTEMS ECOLOGY, AND SYSTEMS ECOLOGI DG-OS-QI7DB /AL SHELLS CONTAINING RECTANGULAR OPENINGS WITH STIFFENED EDGES I STRUCTURAL-SHELLS ELASTIC-SHELLS MODELS/ $20-1 /$-O2DB I ED AND PR IVATE RESEARCH ON FOREIGN STUDEN/ CROSS CULTURAL EDUCATION - A BIBL IOGRAPHY OF GOVERNMENT SPONSOR O5-II -DI OQI ITITUTIOVS AVD THERMONUCLEAR WAR - A CASE STUDY OF HIGHER EDUCATICN I POSTATTACK-OPERATIONS SOCIAL-RECOVE I O5-1I -OI688 /CAL-REGIONS MIL I TARY-PER SONNEL FCOD CLOTHING ENVIRCNMENT EDUCATION ARMY-EOUIPMEVT REACTIONIPSYCHOLOGY) S/ O5-II]-O2IBI IAND ACADEMIC CEN TERS - AFRICA I ECONOMICS BIBLIOGRAPHIES EOUCATION FOREIGN-POLICY GEOGRAPHY POLITICAL-SC/ O5-1I -DI692 / AMERICAN REPUBL ICS I BIBLIOGRAPHIES COMMUNISM ECCNOMICS EDUCATION FOREIGN-POLICY GEOGRAPHY POLITICAL-SC/ O5-II -OI696 IOCCUPATIONS PROFESSIONS MANPOWER LABOR PCLITICAL-SCIENCE EDUCATICN GROUP-CYNAMICS THEORY METHODOLOGY I $\$$ I OS- 11 - 22197 AMICS SOC IAL-PSYCHOLOGY PLBLIC-OPINI ON ME THODCLOGY THECRY EDUCAT ION HEALTF WELFARE-SERVICES OCCUPATIONS P/ O5-II -D2I 44 TOND

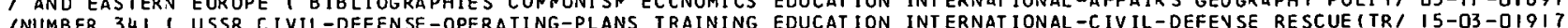
CI COMPUTERS PROGRAMMING (COMPUTERS) PROGRAMMING-LANGUAGES EDUCAT ION OPERATIONS-RESEARCH TRAIN ING MATHEMAT/ DQ-O2 - OI 75O NE STRESS (PSYCHOL OGY) SHEL TERS SCHOOLS PUBLIC-INF ORMATION EDUCAT ICN ORPHAN-CARE) \#HSR-2R-67/3-CR /-PLANNI O5-II -O22J3

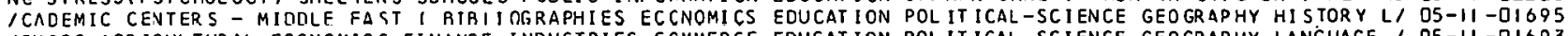
IOMICS AGR ICULTURAL-ECONOMICS FINANCE INDUSTRIES CCMMERCE EDUCAT ION POL. IT ICAL-SCIENCE GEOGRAPHY LANGUAGE / OS-II -DI693 ILOUT -SHELTERS LOGISTICS SHELTER-OCCUPANCY WATER-SLPPLIES EDUCATION TRAINING INFANT-DIET I *OCD-IG- I5. I \#/ O6-O3-OI7I 3 IION-SYSTEMS SIMULATION INFORMATION-RETRIEVAL GAME-THEORY EDUCATICN TRAINING OPERATIONS-RESEARCH PROGRAMM/ D9-OZ-OI75I IC-OP INION HISTORY COSTSIATTITLDES) INTERAATICNAL-AFFAIRS EFFECTIVENESS 1 \#A.L. IO73 /LITICAL-SLIENCE PUBL OS-IJ-OI679 TRTS SUBJECT-INDEXING DEPARTMENT-CF-DEFENSE DOCUMENTATION EFFECT IVENESS INFORMATION-RETZ IEVAL J \$AD 650 $3 /$ O5-0? -D2I 24 IRTS 1 EXPERIMEVTAL-DATA ANGLES(GEOME TRY) GEOMETRY REVETMENTS EFFECTIVENESS SLOPES, \$SC-RR-67-24 /SAND MODELS I8-03-023I 7 IERAGES BREAD CANNED CEREALS COFFEE CORN DEHYDRATEC-FOODS EGGS FATS FROZEN-FOODS FRUITS IRRADIATED-FOODS, O6-O3-022D9 SHEAT-ENGINES DESERTS FEASIBILITY-STUDIES SOLAR-RADIATION EGYPT ENGINES-ANC-YOTORS IRRIGAIION-SYSTEMS I $\$ 1$ I3-D2-OI777 IR-NATION CONFL ICT, DYADIC AND MEDIATED - CASE STUDIES OF EGYPT, ISRAEL, AND THE UN ITED NATIONS AT FIVE PI OS-DI -D217O INORT AYERICAN A IR DEFENSE CONMAND AT CHEYENNE MOUNTAIN, EL PAST COUNTY, COLORADO I COVSTRUCTION UNDERGR, I3-13-02274 RES STRUCTURAL-PROPERTIES STEEL CONPUTE/ THE SECOND ORDER ELASTIC ANALYSIS OF PLANE RIGID FRAMES I STRUCTU I3-I3-02287 IC/ TREORETICAL GROUND SHOCK EFFECTS I. GROUND MOTION IN ELASTIC HALF SPACES INDUCED BY AIR 8LAST I ELAST I9-D4-O2OL4 ITERATURE REVIEW AND PILOT STUDY I SHELTERS UNDERGRO/ THE ELASTIC RESPONSE OF BURIED CYLINDERS, CRITICAL L I3-I3-OI825 GATION STRAIVIME/ ON LONGITUDINAL ANO TRANSVERSE WAVES IN ELASTIC RODS 1 MECHANICAL-WAVES ELASTICITY PROPA $20-I I$-02082 TION E/ STUDIES ON LONGITUDINAL AND BENDING WAVES IN LONG ELASTIC ROOS 1 MECHANICAL-WAVES STRESSES PROPAGA 20-II -O2079 IL INVESTIEATION OF A DYNAMICALLY LOADED RING WITH RADIAL ELASTIC SUPPORT I RINGS VIBRATION FRE2UENCY BEN/ $20-11$-D2336 INGUL AR OPENINGS WITH STIFFENED EOGES I SIRUC TURAL-SHELLS ELASTIC-SHELLS MODELSISIMULATIONS) BEAMS(STRUC T/ 20-II-02OBI NC KYOTO, JAPAN I JAPAN SEISMOLOGY STRUCTLRES SHCCK-WAVES ELASTICITY, *A.L. II95 /EVGIVEERIVG II. TOKYO A O8-1I -02246 LLS RINGS WELDING STRESSES DEFLECTION ROLLING (METALLURGY) ELASTICITY, \#DTMB-1225 *AD 650 540/UCTURAL-SHE 20-1I -02347 /IBRATION BULLETIN I ROCKETS DYNAMICS STRUCTURAL-ANALYSIS ELASTICITY BEAMSISTRUCTURAL) SHOCK(MECHANICS) L/ 2D-II-02D74 IMFCHANICSI SHDCK (MFCHANICS) FEA SIBILI TY-STUDIES STRESSES ELASTICITY CONCRETE HONEYCOMB-CORES EXPANDED-PL, I8-D3-O2OI 7 ICAL-WAVES PROPAGATION SHOCK-WAVE S UNDERGROUND-E XPLOSI CNS EL ASTICITY EOUAT IOVS-OF-MOT IOY PLASTICITY THEOR/ DB-13-DI747 STTRUCTURES STRUC TURAL-PROPERTIES STEEL CCMPUTER-PROGRAMS ELAST ICITY LOADINGIMECHANICSI 1 \&FEL-297-17 $\$$

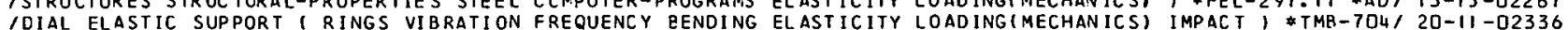
IAT ICAL-ANALYSIS CON SOLIDATION DEFORMATI ON TRANSPORTATION ELASTICITY NORTHEAST-CORR IDOR COMPUIER SIPROGRAMI $20-11$-O2D84 1 AND TRANSVERSE WAVES IN ELASTIC RODS I NECHANICAL-WAVES ELASTICITY PROPACATION STRAIVIMECHAVICSI EQUATI/ 20-II-O2OB2 IICITY MECHAVICAL-WAVES PROPAGATI CN MATHENATICAL-ANALYS IS ELASTICITY SHOCK-WAVES COVTIVUUM-MECHANICS LINE/ $20-I I$-D2OBD IUND MOTION IN ELASTIC HALF SPACES INOUCED BY AIR ELAST ( ELASTICITY SHOCK-WAVES MECHANICAL-WAVES LOADING/ I9-OH-O2O44 CRACK-PROPAGATION JMPACT-TESTS DYNAMICS BLAST SHOCK-WAVES ELASTICITY STRESS MECHANICAL-ANALYSIS, \#MGO9S, 20-II-02338 /REV IEW ANC PILOT STUDY ( SHEL TER S UNDERGROUND-STRUCTURES ELASTICITY TEST CYNAMICS LOADINC(MECHANICS) STR/ I3-I3-DIB25 LONG ELASTIC RODS 1 MECHANICAL-WA VES STRESSES PRCPAGATION ELASTICITY THEORY I \#DRL-376 \$AD O3I O48 IES IN 20-11-O2079 7ECHANICS I UNDER GROUND-STRUC TURES ROCK(GEOLOGY) STRESSES ELASTICITY THEORY GEOLOGY INSTRUMEVTATION DEFOR/ I3-I3 -D2275 AGINGIPHYSIOLOGY) RATORY AIR I BIOIOGICAL-WARFARE-AGENTS SPCRES NI TEST NEW ELECTRIC INCINERATOR DESIGV FJR STERILIZING LABO IS-O2-DI892 RATORY AIR I BIOLOGICAL-WARFARE-AGENTS SPCRES NI TEST NEW ELECTRIC INCINERATOR DESIGV FJR STERILIIING LABO IS-O2 -DI892 TENT IAL-DISTR IBUT ION-SYSTEMS COSTS. NND-RESIDENTIAL-DISTR IBUTION-SYSTEMS COSIS FAULT-LCCATION ELECTRIC-CABLES TRANSFORMERS HEAT-TRAVSFER I *3I O9-O3-DI753 IENT MDTOR WITH NO CONTAC TS AND TR ANSISTOR COMMUTATOR I ELECTRIC-MOTORS RUSSIA COMMUTATORS SEMICONDUC TOI OO-D3-DI752 IGROUND POWER TRANSMISSION ANDLA I RESOURCES DAMAGE-ASSESSMENT FOOD ITIES COMMUVICATIONS-FACILITIES TRANSPORTATI ON-PROFERTIES ELECTRIC-POWER-GEVERATION MILITARY-PER SONNEL PO/ O5-D3-D2I 37 TIONS THERMOELECTRICITY BUOYS GENERATORS POWER-SUPPLIES ELECTRIC-POWER-PRODUCTION, \$AD 636 436/APPLICA ID-D2-OI76I ITO THE PRESIDENT I NUCLEAR-REAC TCRS NUCLEAR-POWER-PLANTS ELECTRIC-POWER-PRODUCTION ECOVOMICS, \$A.L. IO5/ I8-05-02027 IS COSTS WATER-SUPPLIES ENVIRONMENTAL-CONTROL VENTILATION ELECTRIC-POWER-PRODUCTION HEATING CODLING LIGHI/ I3-I3-O227I I STORMS DECONTAMINATION EAR THOUAKES VOLCANOES LANCSLIDES ELECIRIC-POWER-PRODUCTION PETROLEUM ZADIATION-EI I5-O3-O2299 IC RESPOVSE TO THE NOR THEASTERN PChER BLACKOUT I BEHAVIOR ELECTRIC-POWER-PRODUCTIONIFAILUREI ATTITUDES PU/ OS-IJ-OIG83 /ANSMISSION I ELECTRIC-UTILITIES LTILITIES ELECTRIC-POWER ELECTRIC-POWER-TRANSMISSION TRANSMISSION-LINES / D9-O3-02249 TRIC-POWER-TRANSM ISSION/ UNDERGROLND POWER TRANSMISSICN\&. ELECIRIC-UTILITIES UT ILITIES ELECTR IC-POWER ELEC O9-03-02249 CELLS AIRCR/ SEALED NICKEL CADMIUN BATTERIES FCR AIRCRAFT ELECTRICAL SYSTEMS I STDRAGE-BATTERIES ALKALINE- IO-O3-DI764 R-OEVICES COOL ING THERMOELECTRICITY THERNAL-CONDUCTIVITY ELECTRICAL-CONDUCTANCE GERMAVIUM I *2SIC-523 *A/ 09-DI -DI748 IAIR ONTROL-SYSTEMS TRANSFORMERS TERRESTR IAL WAVEGUIDE I NUCLEAR-EXPLOSI ONS I CNOSPHERE AI FLFITRONAGNET IC PJLSE PROPAGATION IV A DISTURBED 2D-I7-O23 HB TERRESTRIAL WAVEGUIDE ENVIRONMENT I NUCLEAR-EXPLOSIONS W/ ELECTROMAGNET IC PULSE PROPAGATION IV THE NORMAL $20-I+-D 2349$ IN ( SITE-SELECTION RADAR-INTERFERENCE RADAR-SIGNALS MAPS ELECTROMAGNET IC-COMPATIBILITY PROPAGATION ) \$P-I, I 7 -DF-DI988 IN ELECTROMAGNETICS FOR PROJEC T DEFENDER I PLASMA-PHYSICS ELECTRONAGNET IC-PROPERT IES FILMS FLUID-MECHANIC/ 20-03-D2330 /-STATES-GOVERNMENT OFFICE-OF-EMERGENCY-PLANNINS SHELTERS ELECTRGNAGNET IC-PULSE RACIATIJN SHIELDING RADIO/ IS-D3-O2299 ION OZONE REACT ION-KINETICS NUCLEAR-RADIATION COSNIC-RAYS ELECT ROMAGNET IC-WAVES PROPAGATION YODELSISIMULA/ O4-OI -O2II 3 HYSICS ELECTROMAGNETIC-PROPERTIES FILMS FLUI' RESEARCH ON ELECTROMAGNET ICS FOR PROJECT DEFEVDER I PLASMA-P $20-D 3$-02330 XIREMELY-LOW-FREQUENCY PHASE TRANSFORMATIONS(MATHENATICS) ELECTRON-OENSITY) \#IER-22-ITSA-22 \$AD 648 DO2 / 20-14-02348 I ABLATION ANTENNAS PROPAGATION SHOCK-WAVES KINETIC-THEORY ELECTRON-CENS ITY CERENKOV-RADIATIJV MICROWAVES / 20 -03-O2330 ITION AVD HIGH IMPAC T SHOCK TE STS I NAVAL-RESEARCH DESIGN ELECIRONIC-EQJIPMEVT REL IAB IL ITYIELE:TRONIC) NA/ $20-11$-02083 NISM I RADIOBIOLOGY, \$ORNL-TR-975 INCORPORATION ANC EL IMINATION OF RADIOACTIVE MATERIALS 8 Y IHE ORGA O6-I3-DI728 (STRUCTURAL) HIGI ANALYSIS OF STRESS DISIRIBUTICN BENEATH EMQANKMENTS I STRESSES SOIL-MECHANICS SEITLEMENT 20-II -D2OB4 CHOLOGY) CASUAL II MENTAL HEAL TH IMPLICATIONS IN CIVILIAN EMERGENCIES I EMOT IONS ATTITUDES FEAR STRESSIPSY OS-IJ-OIG8I SIY L-DEFENSE-OPERATING-PLANS CLEANING REPAIR TRAINING, \#FG/ EMERGENCY CLOTHING I DISTRIBUTION LOGISTICS CIVI I5-OJ-O23J6 5 FOOD FOOD-DISPENSING SHELTER-OCCUPANCY POSTATTACT-OPER/ EMERGENCY FEEDING I CIVIL-DEFENSE-OPERATING-PLAN DG-O3-D22ID OICINE AD/ ADVANCEO TREATMENT CENTRE - OPERATING MANUAL - EMERGENCY HEALTH SERVICES 1 DISASIER-MEDICINE ME O6-21-DIT38 CICINE PUBLIC-MEALTH LAW ORGANIZATIO/ ROLE OF THE FEDERAL EMERGENCY HEALTH SERVICES DIVISIOV 1 DISASTER-ME D6-2I-O2226 ENTS RADIOA/ PROTECTION AGAINSI NLCLEAR WEAPON FALLOUT IN EMERGENCY HEALTH SERVICES UNITS 1 SHIELDING PATI IB-DS-O2O2A 
SE-OPERATING-PLANS POSTATTACK-OPERATIONS SHELTER-OCCUPAN/ EMERGENCY LODGINE I HOUSIVG TRAINING CIVIL-DEFEN Q5-II -O2IB 7 PMENT -AND-SUPPL IES FOOD-DISPENSING FOOD, BASIC COURSE IN EMERGENCY MASS FEEDING - HANDBOOK 1 KITCHEN-EQUI D6-OZ-DI7I 2 CHEN-EOUIPMENT-ANC-SUPPL IES STOCKPILING / BASIC COLRSE IN EMERGENCY MASS FEEDING - INSTRUCTORS GUIDE I KIT DO-DB-DITI3 C IRES U/ REVIEW OF FEDERALLY SPONSCRED RE SEARCH RELATED TO EMERGENCY PLANN ING I SCIEVTIFIC-RESEARCH BLAST F I5-03-O2299 IRES U/ REVIEW OF FEDERALLY SPONSCRED RE SEARCH RELATED IVIL-CEFENSE-OPERATING-PLANS MENTAL-HEALTH POSTATTACK-OP/ EMERGENCY SOCIAL SERVICES I PER SONNEL BEHAVIOR C OS-II -02I85 MMUN ICAT IONS-SYSTEMS SUPER VISION MEDICAL-PERSCNNEL STORHS EMERGENCY-HEALTH-SERVICES, IRESEAREH REPORT IT DG-I?-OI7I 4 OF MEDICAL CIVIL DEFENSE CONFERENCE I DISASTER-MEOICINE EMERGENCY-HEALTH-SERVICES BIDLOGICAL-WARFARE CH/ O6-2I-D2233 *A.L. IO68 CATALOGUE OF PUBLICATIONS I DISASTER-MEDICINE EMERGENCY-HEALTH-SERVICES CANADA MANUALS FILMS I DS-2I -DI737 DMINISTRA/ HOSPITAL DISASTER PLANNING ( DISASTER-MEDICINE EMERGENCY-HEALTH-SERVICES HOSPITALS CASUALTIES A D6-21-OI739 IIC-POWER-PRODUCT ION PETROLEUM RADIATION-EFFECTS MANPOWER EMERGENCY-HEALTH-5ERVICES RADIOBIOLOGY FLOODS O/ I5-O3-O2299 1 IS INFECT IOV WA TER-SUPPLIES COOPERATI ON DISASTER-MEDICINE EMERGENCY-HEALTF-SERVICES REPAIR I * JPRS- I7, 889/ I5-O3-O2296

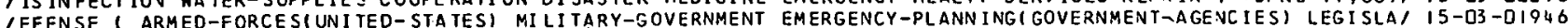

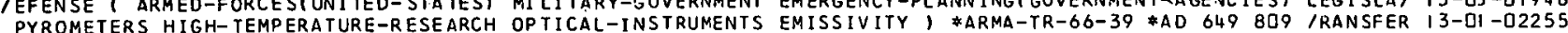
LTI/ MENTAL HEALTH IMPLICATIONS IN CIVILIAN EMERGENCIES I EMOTIONS ATTITUDES FEAR STRESS (PSYCHOLOGY) CASUA OS-ID-DIOBI

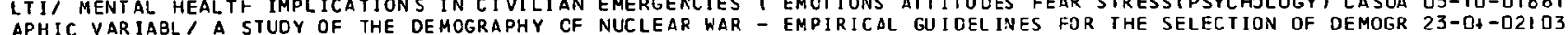

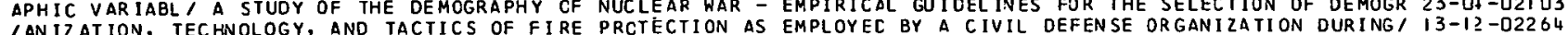

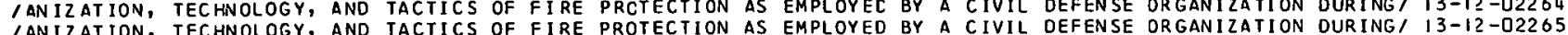
IANIZATION, TECHNOLOGY, AND TACTICS OF FIRE PROTECTION AS EMPLOYED BY A CIVIL OEFENSE ORGANIZATION DURING/ $13-12-02265$ INS 1970 - INTERINDUSTRY RELATIONSHIPS, POTENTIAL DEMAND, EMPLOYMENT I INCUSTRIES MANPOWER ECONOMICS GROS/ DS-03-01649 /AILROAD MER GERS I TRANSPORTATION LEGISLATION COMPETITION EMPLOYHENT TAXES SERVICES YAVAGEMEVT-PLANNING C I 23-02-O2095 ISTURBAN' A STUDY OF THE RE SPONSE OF BACKPACKING NATERIAL ENCAS INE A TJNEL LINER SUBJECTED TO A DYNAMIC D I3-I3-O2279 NSPORTATION URBAN-AREAS, AEROOYNAMIC DRAG ON VEHICLES IN ENCLOSEC GUIDEWAYS I TUNNEL SITRANSPORTATIONI TRA $23-02$-D2IOI /OCEDURES APPLICABLE IN ASSESSING RESPONSES OF THE THORAX ENERGIZED BY AIR BLAST OVERPRESSURES OR BY NON-I O6-21-O2235
IWHAT THE PEOPLE KNOW AND THINK ABOUT THE BOMB AND ATOMIC ENERGY ( PJBLIC-CPINION ATTITUDES SUZVEYS QUESTI O5-II -O2I 96 GAMMA RAYS - OPERATION BREN I IONI ZI NG-RADIATICN-SCATTER/ ENERGY ANC ANGULAR DISTRIBUTIJN OF NEUTRONS AND $20-03$-O2334 L AND TWO SIMULATED FALLOUT FIELDS RAUN THE ANGLE ANO ENERGY DISTRIBUTION OF PHOTONS PRODUCED BY A REA IS-DS-QISO4 ANCEWAYS DOSE-RATE/ EXPERIMENTAL DETERMINATION OF NEUTRON ENERGY SPECTRA IN CONCRETE DUCTS 1 SHELTERS ENTR OS-I3-O22I 9 ANSFER NUCLEAR-REACTOR-MATERIALS FLUID-DYNAMIC-PROPERTIES ENERGY-CONVERSION REACTOR-SAFETY-SYSTEMS RADIOAI 2D-O3-O2O7D FNTS NEAT-ENG INES DESERTS FEASIBILITY-ST/ SIMPLE HEAT ENGINE OF POSSIBLE UTILITY IV PRIMITIVE ENVIRONM I3-OP - DI?7? PROCEEDINGS OF THE SECOND

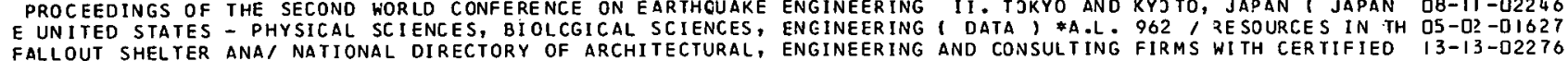
FALLOUT SHELTER ANA/ NATIONAL DIRECTORY OF ARCHITECTURAL, ENGINEERING AND CONSULTING FIRMS WITH CERTIFIEO I3-13-D2276
AN ATOMIC STRIKE I RESCUES CIVIL-DEFENSE-PERSONNEL USSR/ ENGINEERING AND RESCUE OPERATIONS AT THE SITE OF I3-I2-DI8D4 AN ATOMIC STRIKE ( RESCUES CIVIL-DEFENSE-PERSONNEL USSR/ ENGINEERING AND RESCUE OPERATIONS AT THE SITE OF I3-I2-DI8D4 ZONE I CIVIL-DEFENSE-SYSTEMS USSR REC ONNAISSANCE INTERNA/ ENGINEERING AND RESCUE WORK IV A VUCLEAR STRIKE D6-O7-DI7D9 IVERSAL TRAVSVERSE MERCA TOR GRID I ARMY SLRVEYING NAPPING ENGINEERING ART ILLERY GEODESICS I *TM 5-24I-8 UN O8-0S-O2240 AVITY AND PRESSURE I UNITED-STATES BURE/ CONSTRUCTION AND ENGINEERING DATA - WATER CONVEYANCE TUNNELS - GR I3-13-O2283
SYSTEMS - AN ANNOTATED BIBLIOGRAPHY OF U.S. NAVAL CIVIL ENGINEERING LABORATORY PUBL ICAT IOVS I FALLOUT-S/ I3-I3-D2278 SYSTEMS - AN ANNOTATED BIBLIOGRAPHY OF U.S. NAVAL CIVIL ENGINEERING LABORATORY PUBL ICAT IOVS I FALLOUT-SI I3-I3-D2278
LTER EQUIPMENT AND PROCEDURES II. LABORA/ PSYCHOLCGICAL, ENGINEERING, ANC PHYSIOLOGICAL EVALUATION OF SHE IS-D3-O2297 LTER EQUIPMEVT AND PROCEDLRES III. HABIT/ PSYCHOLCGICAL, ENGINEERING, AND PHYSIOLOGICAL EVALUATION OF SHE I5-03-O2298 LTER EQUIPMEYT AND PROCEDURES I. SUMMARY, PSYCHOLCGICAL, ENGINEERING, AND PHYSIOLOGICAL EVALUATION OF SHE IS-O3-D23JO IEPARTMENT-OF-DEFENSE DOCUMENTATION INFORNATION-RETRIEVAL ENGINEERING-PERSONVEL SCIENTIFIC-PER SONNEL FLOW/ O5-02-O2I 20 1 BY CESI GNERS 1 HUMAN-ENGINEERING DECISI ON-MAKING DESIGN ENGINEERING-PERSONNEL SPECIFICATIJVS HANDBOOKS / O5-OS-DIG 73 INGINES DESERTS FEASIBILITY-STLOIES SOLAR-RADIATION EGYPT ENGINES-AND-MOTORS IRRIGATIOV-SYSTEMS I $\$$ P- $3367 /$ I3-D2-Q I777 1 AGR ICUL TURAL-ENGINEER ING ANI MAL-HUSBANDRY CROP-RESEARCH ENTOMOLOGY NUTRITION WATER-COVSER VATION SOIL-CO/ O2-OJ-D2IJ8 BUR IED PERSONNEL SHELTERS 1 BLAST-SHELTERS DESIGN COSTS/ ENTRANCEWAYS AND EXITS FOR BLAST RESISTANT FULLY I3-13-DI835 ION FEASIBILITY-STUDIES DUAL-PURPOSE-SHELTERS STOCKPILING ENTRANCEWAYS COSTS WATER-SUPPLIES ENVIRONMENTAL/ I3-13-0227I IN OF NEUTRON ENERGY SPEC IRA IN CONCRETE DUCTS I SHELTERS ENTRANCEWAYS DOSE-RATE RADIATION-MEASUREMENT-SYI O6-IS-D22I 9 RVIVAL STORAGEIFOOD) PRODLCT, NUTRITION IN THE PCSTATTACK ENVIRONMENT, FOOD DIET POSTATTACK-OPERATIONS SU O6-IS-OI7I 7 RVIVAL STRAELAOA NES RADIOBR THE PROBLEM OF TUBERCLLOSIS IN THE POSTATTACK ENVIRONMENT RECOVERY INFECTIOUS-DISEASES VACCI DG-OS-D22O6 NES RADIOB/ TRE PROBLEM OF TUBERCLLOSIS IN THE POSTATTACK ENVIRONMENT I RECOVERY INFECTIOUS-DISEASES VACCI OO-OS-D22OS TRI COMPARISON OF DIGITAL COMPLTER SIMULATIONS OF THERMAL ENVIRONHENT IN OCCUPIED UVOERGROUND PROTECTIVE S 2O-I3-O2OOO INAGEMENT-ENG INEER ING MANPOWER-STLOIES COST-EFFECTIVENESS ENVIRONMENT MANAGEMENT-PLAVNIVG SURVIVAL CIVIL-I I S-O3-O22 33 /ALYS IS OF SELECTEO PROBLEMS OF LARGE -SHELTER MANAGEMENT, ENVIRONMENTAL THREAT, AND SMALL SHELTER HABITAB/ D5-IJ-QIO84 UIPMENT FALLOUT-SHELTERS BLAST-SHELTERS E VAPORATORS COSTS ENVIRONNENT AL-CONTROL 1 \%AD $637953 / T I L A T I N G-E Q$ I 3 -CI -DI 773 IF AN ABOVEGRDUND FALLOUT SHELTER IN ENANPERATURE-CONTROL ENVIRONMENTAL-CONTROL COSTS LIGHTIVG-EQUIPMENT 1 I3- I3-DI8I9 IOF A BASEMENT FALLOUT SHELTER IN EAST CHICAGO, ILLDIANA IENVIRONMENTAL-CONTROL EXPER IMENTAL-DATA HABITAB I I3-OI -DI77I INTILATION ( STRESS(PHYSIOLOGY) HEAT MOI STURE TEMPERATURE ENVIRONMENTAL-CONTROL HUMIOITY AIZ TOLERANCESIPI I3-OI -D2253 IE-SHELTERS STOCKPILING ENTRANCEWAYS COSTS WATER-SUPPLIES ENVIRONMENTAL-CONTROL VENTILATION ELECTRIC-POWEI I3-13-O227I ST/ A SIMPLE HEAT ENGINE OF POSSIBLE UTILITY IN PRIMITIVE ENVIRONMENTS I HEAT-ENGINES DESERTS FEASIBILITY- I3-O2-OI777 EALTH STRESSIPHYSIOLOGY) MALNUTRITION RADIOLOGICAL-DOSAGE EPIDEMIOLOGY, \$RM-5362-PR /ADIOBIOLOGY PUBLIC-H D6-DJ-D2206 ICLEAR WAR I PASTEURELLA-PESTIS BIOLOGICAL-WARFARE-AGENTS EPIDEMIOLOGY PUBLIC-HEALTH POSTATTACK-OPERATION/ DG-OJ-DITO2 K-STABILIZATION SHOTCRETE ROCK-BOLTINGS CHAIN-LINK-FABRIC EPOXY-RESINS CEMENT-GROUT, \$IC-8294 /NCRETE ROC $13-13-02274$

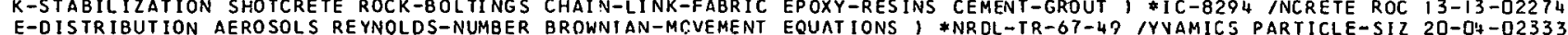
E-DISTRIBUTION AEROSOLS REYNOLDS-NUMBER BROWNIAN-MCVEMENT EQUAT IONS I *NRDL-TR-67-49 /YVAMICS PARTICLE-SIZ 20-O4-02333
THERMAL HISTORY OF A DUAL LAYERED PLATE SLBJECT TO THE T/ EOUAT IONS AND COMPUTER PRDGRAY TO CALCULATE THE I3-I?-DI797

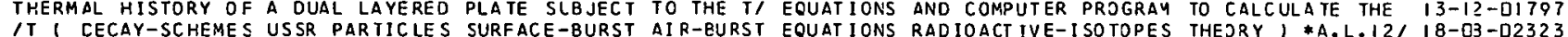
$1 T$
1

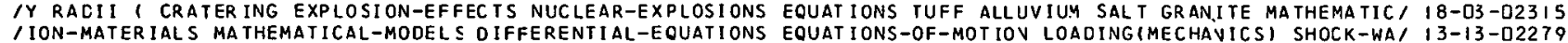

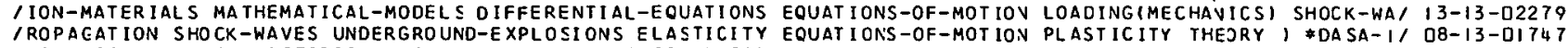

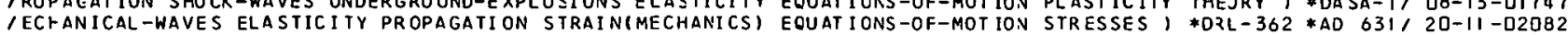
ITRUCTURAL-SHELLS CYLINDR ICAL-BODIES COMPCSITE-MATERIALS, EOUAT IONS-OF-MOT IOV, STRESSES STIFFEVED-CYLINDE, $20-11$ - D2345 ILUTIONS OF PROBLEMS OF TURBULENT GAS DYNAMICS 1 GAS-FLOW EQUAT IONS-OF-STATE MATHEMATICAL-AVALYSIS FLUID-, $20-0 \%$-U233I LOSION-EFFECT/A REAPPRAISAL OF THE APPLICATICN OF STATIC EQUILIBRIUM THEORY TO DECOUPLING I CRATERING EXP I8-03-U23IO FARM CHEMICALS HANDBOOK I FERTILI ZERS PESTICIOES EQUIPMENT AGRICULTURAL-CHEMISTRY I *A.L. 996 IAL, ENGINEERING, AND PHYSIOLOGICAL EVALUATI ON OF SHELTER EQUIPMENT AND PROCEDURES I. SUMMAZY AND REVIEW/ IS-D3-O23JO /AL. ENIINEERING, AND PHYSIOLOGICAL EVALUATION OF SHELTER EQUIPMENT AND PROCEOURES II. LABORATORY STUDIE / I5-O3-O2297 IAL, ENGINEER ING, AND PHYSIOLOGICAL EVALUATION OF SHELTER EQUIPMENT AND PROCEDURES III. HABITABILITY STU/ IS-03-O2298
IER PROGRAM I URBAN-AREAS HASTY-SHEL TERS OESIGN MATERIALS EQUIPMENT LABOR MOBIL IZATION YANAGEMENT-PLANNIN/ I3-I3-O2272 /ER PROGRAM URBAN-AREAS HASTY-SHEL TERS DESIGN MATERIALS EQUIPMENT LABOR MOBIL IZATION YANAGEMENT-PLANNIN/ I3-I3-02272
0 BLAST INDUCED GROUND MOT/ SIMULATI ON OF THE RESPCNSE OF EOUIPMENT LOCATEC IN UVDERGRJUVD IVSTALLATIONS T I3-13-D229I SHELTERS PHYSIOLOGY EXPLOSIO/ AN INVESTIGATION OF NINIMAL EOUIPMENT NEEDS IV PERSONVEL SHELTERS IFALLOUT- I3-I3-OIBII

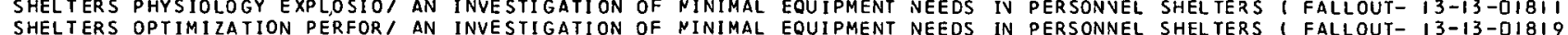
SHELTERS OPTIMIZATION PERFOR/ AN INVESTIGATION OF NINIMAL EQUIPMENT NEEOS IN PERSONNEL SHELTERS I FALLOUT- I3-13-OI8I9
IIRE-PROTECTION RESOURCESIVULNERABILI TYI FALLOUT-SHELTERS EQUIPMENT URBAN-AREAS RURAL-AREAS MAVAGEMENT-PL/ I3-I2-DI8O6

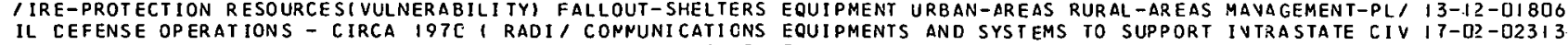
SUPPL IES, *CWA-1 A NEW ERA FOR AMERICAS WATERS I WATER-PJLLUTION WATER- I3-02-0225? CK-OPERATIONS SOIL-CONSERVATION SOI' NUCLEAR WAR AND SOIL EROSION - SOME PROBLEMS AND PROSPECTS 1 POSTATTA D8-13-UI745 REINES VELOCITY A STUDY OF THE BASIC MECHANISMS CF DUST EROSION 1 PARTICLE-SIZE EXPERIMENTAL-DATA GAS-TU 20-0B-O2068 LITY CONFL ICT-RESOLUTION COOPERATION INTE/ MODEL STUDY OF ES CALATION I SUMYARY I CONFL ICT ARTIFICIAL-REA O5-IJ-O2I 83 ARMS-CONIROL ARTIFICIAL-REALITY, FPOTHE ISSUES(BIOLOGY) TESTES RATS MORPHOLOGY(BIOLOGY) HISTOLOGY ESOPHAGUS SKIH, *AD G38 480, ICIEVCY-OISEASES T O6-05-OITRI 
CAL-SCIENCE NUCLEAR-WARFARE BALLISTIC-MISSILES CO/ ON THE ESSENCE OF A NUCLEAR MISSILE WAR 1 2USSIA POLITI 05-04-01672 AMAGE-ASSESSMEYT PATIENTS PR/ DAPC46 TIME PHASED CASUALTY ESTIMATE I ANALYSIS NUCLEAR-WARFAZE- -ASUALTIES D D6-21-D2225 UNITED STATES BY AGE, SEX, AND CCLOR TO 1990 \% POPULATION ESTIMATES - PROJECTIONS OF THE POPULATION OF THE $23-0+-02358$ ATION ANMEXES I UNITED-NATIONS COSTS INTERNATIONA/ BUDGET ESTIMAIES FOR THE FINANCIAL YEAR 1907 AND INF0RM D5-OH-02165 E PARAMETERS ( ATTACK-SCENARIOSI SENSI IIVITY OF MORTALITY ESTIMATES TO UNCERTAINT IES IN SOME VUCLEAR DAMAG O6-21-D2228 E PARAMETERS II. I MODELS DAMA/ SENSITIVITY CF MORTALITY ESTIMATES TO UNCERTAINTIES IV SOME VUELEAR DAMAG DO-2I-D2229 REPRESENTATIONS $i$ NUCLEAR-h/ THE SENSITIVITY GF MCRTALITY ESTIMATES TO VARIATIONS IV AGGREGATE POPULATION IS-07-01956 $S 1$ BACILLUS-ANTHRAC IS MATHEMATICAL-MODELS/ VIABILITY AND ESTIMATION OF SHELF LIFE OF BAC TERIAL POPULATION $15-02-01849$ IPRIVATE SCHOLARS AND ACADEMIC CENTERS - USSR ANO EASTERN EUROPE I BIBLIOGRAPHIES COMMUVISM ECOVOMICS EDU/ D5-II-DIS97 IAFFIC AVT IMISSILE-DEFEVSE-SYSTEMS UNI TED-STATES SURVIVAL EUROPE COMMUNICATIONS CHINA SHELTERS, *H1-777-1 15-03-01922 A JAPAN GROSS-N/ THE STA TE OF FOOD AND AGRICULTURE 1966 I EUROPE FOOD-MARKET ING RUSSIA AFRISA LATIN-AMERIC O2-02-01616 IION DISPERSAL URBAN-AREAS FOOD SHELTERS FALLCUT-SHELTERS EUROPE SURVIVAL RECOVERY VULNERABILITY MILITARYI $15-03-02295$ /EARCF BY PRIVATE SCHOLARS AND ACADEMIC CENTERS - WESTERN EUROPE, GREAT BRITAIN AND CANADA 1 BIBLIOGRAPHI/ O5-11-01694 I-CIV IL-DEFEVSE COMMUN IS T-CHINA INDUSTRIES TRANSPORTATION EVACUAT ICN DISPERSAL URBAV-AREAS FJ JD SHELTERS 1 I5-D3-D2295 OF PROTECTIOV AGA INST VUCLEAR hEAPONS I USSR SHELTERS IN/ EVACUATION OF THE JRBAV POPULATIOV AS A MEA SURE $15-03-01934$

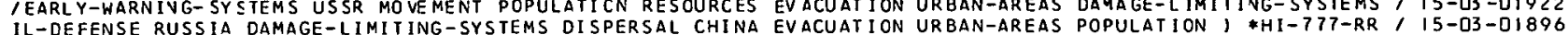

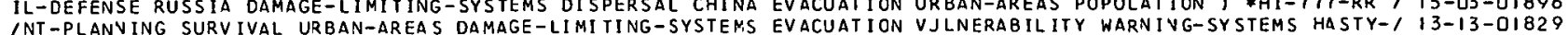

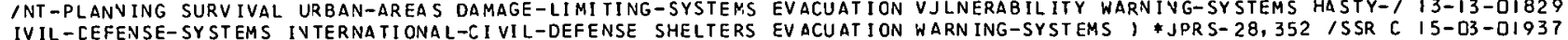

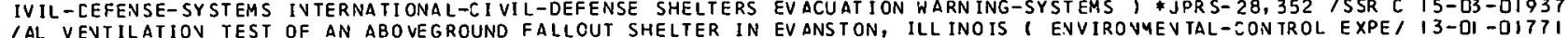
IAL VEVTILATIOV TEST OF AN ABOVEGROUND FALLOUT SHELTER IN EV ANSTON, ILL INO IS I ENVIROVMEVTAL-LONTROL EXPE/ I3-DI - DI77I

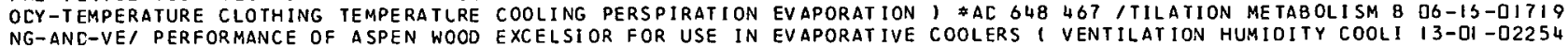
IND-VENT ILATING-EQUIPMENT FALLOUT-SHELTERS BLAST-SHELTERS EVAPORATORS COSTS ENVIRONMENTAL-CJVTROL, 1 *AD $6113-01-01773$ JUCLEAR EXPLOSIOV IN A SALT DOME - PROJECT DRIBBLE SALMON EVENT I SEISMIC-WAVES EXPLJSIJV-EFFECIS VELOCIT/ $18-03$-O23I9 UND TRANSPORT I TRANSPORTATION BIBLIOGRAPHIES URBAN-AREAS EXCAVATION) \#PB 170 58I/APHY OF HIGH SPEED GRO 23-02-D2093 / COLORADO 1 CONSTRUCTION UNDERGROUND-STRUCTURES 8LASTING EXCAVATION REINFORCED-CONCRETE ROCK-STABILI ZATI/ 13-13-D2274 IS ANC PRESSURES (ROCK-MECHANICS LOADING (MECHANICS) USSR EXCAVATION SHEARING CUTTING BENDIVG TORSION STR/ O8-07-02242 $S$ DRIL-IV/ REPORT ON HARD ROCK TUNNELLING INVESTIGATION ( EXCAVATION TUNNELSITRANSPORTATION) BLASTING COST 13-D2-D1787 LAT ION HUMICITY COOL ING-AND-VE, PERF ORMANCE OF ASPEN WOOD EXCELSIOR FOR USE IN EVAPORATIVE CJOLERS I VENTI $13-01-02254$ IIS ION-MAK IVG OBJECTIVES PLBLIC-OPINI ON PCLITICAL-SCIENCE EXECUT IVE-BRANCH LOCAL-GOVERVMENTS STA TE-GOVERN/ O5-D - 01671

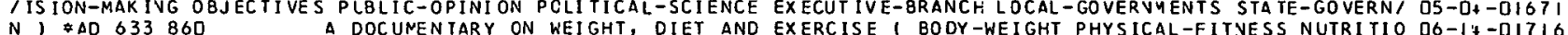

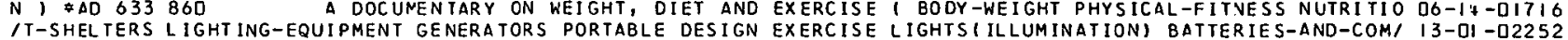
/T-SHEL TERS LIGHT ING-EQUIPMENT GENERATORS PORTABLE DESIGN EXERCISE LIGHTS IILLUMINATION) BATTERIES-AND-COM/ I3-OI-D2252
/SUPPLIES CEFECTIONS TRAINING SLEEP WATER FOOD RECREATION EXERCISE NOISE RELIGIOV TEMPERATURE-CONTROL VEN/ O5-IJ-O2184 TRAINING METHODOLO/ RESEARCH DATA FROM SHELTER OCCUPANCY EXERC ISES I HABITABIL ITY DATA-PROCESSING-SYSTEMS O5-IJ-01680 FENSE ANO RELATED DEFENSIVE SYSTERS I DEFENSE-SYSTEMS AP/ EXERPTS FROM CONGRESSIONAL TESTIMJVY ON CIVIL DE I5-03-02294 -SHELTERS FIRE-PROTEC TION SMOKE I SHELTER HABITABILITY IN EXISTING BUILDINGS UNDER FIRE EXPOSURE 1 FALLOUT $13-12-01807$ COMMCNLY USED IN EXISTING URBAN BUILOINGS IN THE UVITED STATES $1,13-13-02277$ LITY-STUDIES STRESSES ELASTICI TY CONERETE HONEYCOMP-CORES EXPANDEC-PLASTICS DESIGN I *AD 634 691 / FEASIB1 18 -03-D2017 1 PROTECTIVE STRUCTURES 1 CONCRETE UNDERGROUNO-STRUCTURES EXPANDEC-PLASTICS GLASS HOVEYCOMB-CORES LOADING) $18-03-02007$ S MANPOHER GEOGRAPHY/ PARM SYSTEM MANUAL III-E. CAPACITY EXPANSION, RESTORATION AND COVVERSIOV 1 RESOURCE DS-O3-O2IS9 ICAL PRESENTATION OF THE ORGANIZATION, TECHNOL; PRACTICAL EXPERIENCES OF FIRE PROTECT IOV SERVILES-A CRIT 13-1?-02264 ICAL PRESENTATION OF THE ORGANIZATION, TECHNOL/ PRACTICAL EXPERIENCES OF FIRE PROTECTIOV SEZVICES - A CRIT I3-12-022B5 ICAL PRESENTATIDV OF THE ORGANIZATION, TECHNOL' PRACTICAL EXPERIENCES OF FIRE PROTECTIOY SERVICES - A CRIT 13-12-02255
IORGANIZATION IN POST THERMONUCLEAR ATTACK SOCIETY - SOME EXPLORATORY STUCIES I POSTATTACK-JPERATIONS STR, I5-D3-D23DI

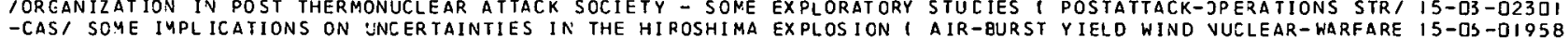
N EVENT I SEISM IC-WAVES, EARTH VIBRATIONS FRON A NUCLEAR EXPLOSION IN A SALT DOME-PROJECT DRIBBLE SALMO I8-03-023I9

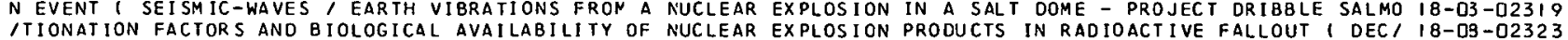

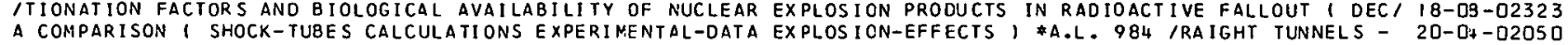

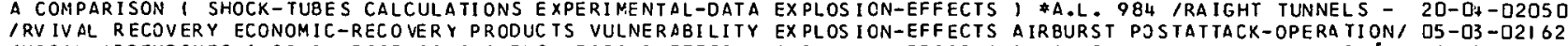
INICAL APPENDIXES 1 CIVIL-DEFENSE-SYSTEMS WEAPONS-EFFECTS EXPLOSION-EFFECTS BLAST-SHELTERS RADIOACTIVE-FA/ 15-O3-01933 1. MEDIUM, AND DEPTH OF BURST ON CAVI TY RADII 1 CRATERING EXPLOSION-EFFECTS VUCLEAR-EXPLOSIONS EQUA TIONS 1 18-D3-023I5 IN OF STAT IC EQUIL IBR IUM THEORY TO DECOUPLING I CRATERING EXPLOS 1 ON-EFFECTS NUCLEAR-EXPLOSIJVS SEISMOLOGYI 18 -03-O23IS IECOVERY PRODUC TS BLAST VULNERABILITY AIR-BURST ECCNCMICS EX PLOS ION-EFFECTS POSTATTACK-JPERATIOVS I *IDA /I O5-D3-02126 TEMS HASTY-SHELTERS CONSTRUC TYS TEMS WEAPONS-EFFECTS MODELS EXPLOS ION-EFFECTS RADIOACTIVE-FALLJUT BLA ST-SHE/ $15-03$-DI938 /EEDS IN PERSONNEL SHELTERS I FALLOUT-SHELTERS PHYSIOLOGY EXPLOSION-EFFECTS SANITARY-ENGINEER ING DESIGN C/ I3-13-DIBII ITO BLAST INDUCEO GROUND MOTIONS I UNDERGROUND-STRUCTURES EXPLOS ICN-EFFECTS SHOCK IMECHAVICSI VULNERAB ILIT/ $13-13-02291$ $K$ I SHOCK-WAVES B IOLOGICAL-ELAST-EFFEC TS SHOCK(PATHOLOGY) EXPLOSION-EFFECTS TEST-METHODS I \#DASA-1777 /HOC DO-21-OI74I

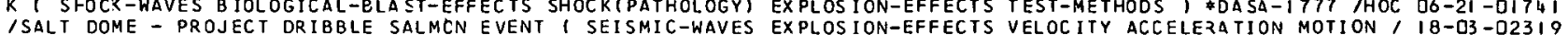

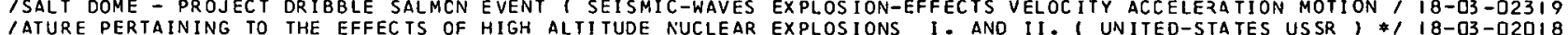

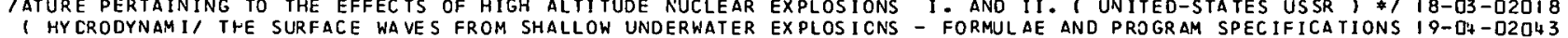
THE MECHANICS OF SLIDE DAMS WI TH SAN' PLOWSHARE - NUCLEAR EXPLOS ICNS - PEACEFUL APPLICATIONS - A STLDY OF IB-DS-D23I7 $M$ ROW CHARGES INTERR UPTED BY A DUD (/ PLOWSHARE - NUCLEAR EXPLOSIONS - PEACEFUL APPLICATIONS - CRATERS FRO 18 -03-02314 IAPOT - DISTRIBUTION AND DENSITY OF MISSILES FROM NUCLEAR EXPLOSIONS I SECONDARY-MISSILES BLAST-ENERGIZED/ I8-03-023IB 1 MECHANISMS IN THE GENERATION OF AIRBLASTS BY UNDERWATER EXPLOS ICNS 1 SHOCK -WAVES CALCULATIONS MODELS 1 * 19 -O4-O2326 I SEISMIC WAVE PROPAGATION AND PRE SSURE MEASUREMENTS NEAR EXPLOSICNS 1 WAVEFORM-GENERATJRS IVSTRUMENTATIO/ O8-11-O2247 INTS INTER IOR-BALLISTICS BIBLI OGRAPHIES DIGI TAL-CONPUTERS EXPLOS IONS GAS-CISCHARGES HEAT IGVI TER S PROJECT/ $19-0 S-D 2329$ IOLOGICAL-8LAST-EFFEC TS MONKEYS SHINE SHEEP LUNG-INJURIES EXPLOSIONS TOLERAVCES(PHYSIOLJGY) SHOCK-TUBES $1 /$ O6-21-02234 IONHOMOGENEOUS ATMOSPHERES AND MODIFIED SACHS SCALING I. EXPONENT IAL IDEAL GAS ATMOSPHERE I SHOCK-WAVES $120-04-02332$

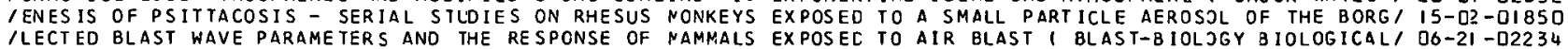

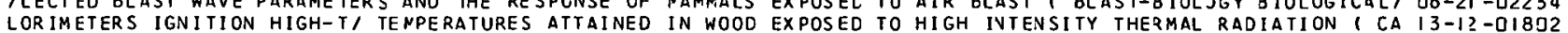
LOR TMERS E S THETER HAB TABILITY IN EXISTING BUILDINGS UNDER FIRE EXPOSURE Y FALLOUT-SHELTERS FIRE-PROTECTION SMOK 13-12-DI8DT

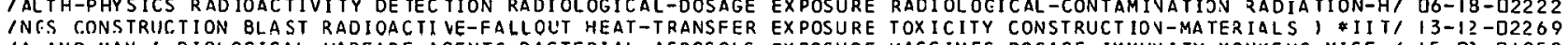
IA AND MAV 1 8IOLOGICAL-WARFARE-AGENTS 8ACTERIAL-AEROSOLS EXPOSURE VACCINES DOSAGE IMMUVITY MOVKEYS MICE $115-02-01858$

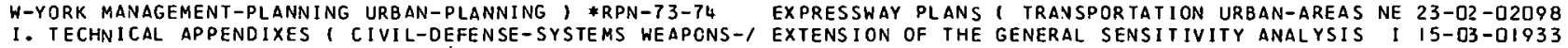
- METHODOLOGY I CIVIL-DEFENSE-SYSIEMS ME TEOROLOSY SYSTENI EXTENSICN OF THE GENERAL SEVSITIVITY ANALYSIS I I5-03-OI935 II. THE AVCET COMPUTER PROGRAM I SYSTENS-EVALUATI ON CIVI/ EXTENS ION OF THE GENERAL SEVSITIVITY ANALYSIS I I5-O3 - DI938 IOF THE UNITEO STATES BY AGE, SEX, ANO COLOR TO 1990 WITH EXTENSIONS OF TOTAL POPULATION TO 20151 DEMOGR/ $23-04-02358$ ERMANY ROADS CONSTRUCTION URBAN-AREAS TUNNELSITRANSPORTA/ EXTENS IVE STREET BJILDING PROGRAM IN HAMBURG 1 G $13-02-01786$ ISS ION ATMOSPHERE LOW-FREQUENC Y PHASE VERY-LOW-FREGUENCY, EXTREMELY-LON-FREOJENCY IVTEGRAL-TZAVSFORMS ATM/ 20-1'4-D2349 IODELS WAVE-TRAVSMISSION LOW-FREQLENE Y VERY-LOW-FRECUENCY EXTREMELY-LON-FREQJENCY PHASE TRAVSFJRMATIONSIM/ $20-1+-02348$ TICAL MODEL OF FLASH BLINDNESS I VISI ON AVIATION-MEDICINE EYE, $\$$ AD 627332 .

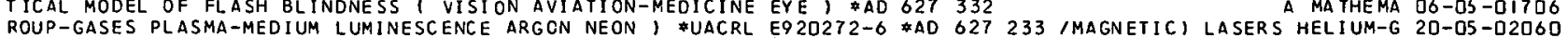


THERMAL RACIATION EFFECTS ON PAINTS, PLASTICS, AND COATED FABRICS \#WT-4Q7 7 OPERATIOV BUSTER - $13-1$ ?-02263 INCUSTRY IN 1965 I UTILITIES RESERVES PRODUCTICN TRAI GAS FACTS - A STATISTICAL RECORD JF THE GAS UTILITY O5-D3-DIGL LTERS UNDERGROUND-STRUCTURES SOIL-NECHANICS SURFACE-BURST FAILURE(MECHANICS) \$TR-508 /ES III TESTS ( SHE D8- 33 -UI746 (ISTRUCTURAL-FORMS) MEMBRANES STRESSES LOAOING(MECHANICS) FAILURE(MECHANICS) DESIGN COSTS MODELSISIMULATI/ I3-I3-0I832 ILATIONS MODELS(SIMULATIONS) LOADING (MECHANICS) BUILDINGS FAILUREIMECHANICS) EXPERIMENTAL-DATA BLAST FIRE/ I8-D3-DI995 ION ROCK MECHAN ICS 1 ROCK (GEOLOGY) UNDERGROUND-STRLCTURES FAILURE(MECHANICS) MINING-ENGINEERIVG STRESSES / O8-D7-D2243 YY) HEATING MIN ING-ENGINEERING LOADING (MECHANICS) COOLING FAILUREIMECHANICS) SHOCK-WAVES VEVTILATION I \#A/ I3-13-02273 IECOND ANNUAL SYMPOSIUM ON ROCK MECHANICS I ROCK(GEOLOGY) FAILURE(MECHANICS) STRESSES THEORY DEFORMATION 1 O8-OT-D2244 /RESULTS OF SER IES III TESTS I SOIL-MECHANICS S4OCK-WAVES FAILURE(MECTANICS) UNDERGRJUND-STRUCTURES NUCLEI I8-D3-O2ODB TIVE MEASURES I RADIATION-INJURIES USSR RADI/ RADICACTIVE FALLOUT - PHYSICS, BIOLOGICAL EFFECTS AND PROTEC O6-I3-DIT26 IVAILABIL ITY OF NUCLEAR EXPLOSION PRODUCTS IN RADICACTIVE FALLOUT I DECAY-SCHEMES USSR PARTICLES SURFACE- I IB-DS-O2323 OUT CON/ RECOVERY OF PETROLELM REFINERIES CONTAMINATEO BY FALLOUT 1 POSTATTACK-OPERATIOVS RADIOACTIVE-FALL O5-D3-DIOSI T RADIOLOGICAL-DOSE FARM-CROP/ PARM SYSTEN MANUAL III-F. FALLOUT AND DECONTAMINATIOV I RADIOACTIVE-FALLOU O6-IS-O22I 7 / FOR THE MEA SUREMENT OF DEPOSITI CN AND REDISTRIBUTION OF FALLOUT AROUND STRUCTURES I RADIOACTIVE-FALLOUT/ IB-O3-D2O38
R-SUPPLIES RADIOLOGICAL-CONTAMINATION RADI/ EVALUATION OF FALLOUT CONTAMINATION FROM SURFACE RUNOFF I WATE DG-IS-OI724 IRIBUTION OF PHOTONS PRODUCED BY A REAL AND THO SINULATED FALLOUT FIELDS I RADIOACTIVITY RADIOACTIVE-FALL, IS-OS-OIGG4 IRIBUTION ION CONTROLLED FISSION PRODUCT ABSORPIICN OURING FALLOUT FORMAIION I RADIOACTIVE-FALLOUT RADIOAC, IB-O3-O2D3? IE CIFFUSION CONTROLLED FISSION PRODUC T ABSORPTI CN OURING FALLOUT FORMAIION I RADIOACTIVE-FALLOUT RADIOAC I I8-O3-O2D3? ELDING PATIENTS RADIOA/ PROTEC TION AGAINST NUCLEAR WEAPON FALLOUT IN EMERGEVCY HEALTH SERVICES UNITS I SHI I8-OS-D2O28 ITE DISPOSAL, PEST AND VECTOR CONTROL, AND THE EFFECTS OF FALLOUT IN WASTE WATER AND SEWER SYSTEMS I POST' I3-OZ-UI78D 1 DUSTY III - AN IMPROVED MA THEMAIICAL ME THOD OF LCCATING FALLOUT CN A SPHERICAL EARTH 1 HIVD YIELO RADIOA I8-CB-O2322

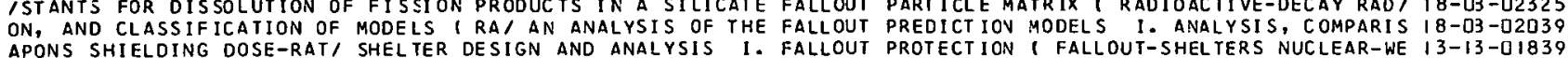
APONS SHIELDING DOSE-RAT/ SHEL TER DESIGN AND ANALYSIS I. FALLOUT PROTECT ION I FALLOUT-SHELTERS NUCLEAR-WE I3-13-0I839
FALL EXPERIMENTAL EVALUATION OF TECHNIQUES FOR IMPROVING FALLOUT PROTECT ION IN HOME BASEMEVTS I SHELTERS I3-I3-OIB22 FALL' EXPERIMENTAL EVALUATION OF TECHNIQUES FOR IMPROVING FALLOUT PROTECT ION IN HOME BASEMEVTS I SHELTERS I3- I3-OI822
ION STUDY OF COMMUNITY RESPONSE TO THE RHODE ISLAND HCNE FALLOUT PROTECT ION SURVEY, ATTITUDES FALLOUT-S/ OSS-IJ-OIOBS IOCTURALY OF COMMUN IS WITH RECOMMENDATIONS FOR INCLUSION OF REQUIREMENTS FOR FALLOUT SHELTER COVSTRUCTION IN FOUR NATIONAL MI I3-I3-O22BI ING CONSTRUCTION VENTILATION, \#MISC. PUB. N/ BUNKER TYPE FALLOUT SHELTER FOR BEEF CATTLE 1 BOVINES SHIELD I3-I3-OIBIB ONMENTAL-CONTROL / NATURAL VENTILATION TEST OF A BASEMENT FALLOUT SHELTER IN EAST CHICAGO, IVDIANA I ENVIR I3-OI-OITTL ENTAL-COVTROL/ NA TURAL VENTILATION TEST OF AN ABOVEGROUND FALLOUT STELTER IV EVAVSTOV, ILLIVJIS I ENVIRONM I3-OI - DITZI NITY I FALLOUT-SHELTERS URBAN-AREASI VENTILATION TESTS OF FALLOUT SHELTER SPACES IN NEW YORK IITY AND VICI $13-13-01813$ NITY NIYY

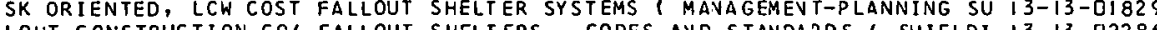
NG RADIATION-EFFECTS RADIOACTIVE-FALLOUT CONSTRUCTION CO/ FALLOUT SHELTERS - CODES AVD STAVDARDS 1 SHIELDI I3-13-O2286 IIONS AND 1962 COST ANALYSIS FOR A STANDARDI ZED SERIES OF FALLOUT SHELTERS I UNOERGROUVD-STRUCTURES CONSTI I3-I3-DI8IS SE SYSTEMS MILITARY-STRATEGY I AHI-7297 WHY COUPLE BMD TO FALLOUT SHELTERS I URBAN-AREAS ANTIMISSILE-DEFEN I5-D3-OI9D7

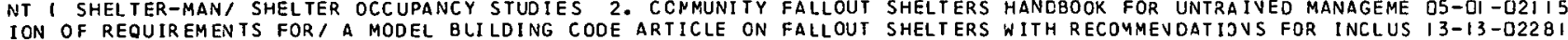
LDING STRUCTURAL-ANALYSIS RADIOAC TIVE-FALLOUT FIRE-SAFETY FALLOUT-SHELTERS, \#A.L. 1207 /-WAVES BLAST SHIE I3-13-02280 SERVATION FIBERBOARD PHYSICAL-PROPERTIES CONTAINERS TASTE FALLOUT-5HELTERS, \$TR-67-25-CD *4D 640 823 /PRE 06-08-022I2 IVIL CEFENSE PROGRAM I ORGANIZATION DUAL-PURPOSE-SHELTERS FALLOUT-SHELTERS APPROPRIATIJVS ADMIVISTRATION- 1 O5-OI -DIO 25 IFU.S. VAVAL CIVIL ENGINEERING LABORATORY PUBLICATIONS I FALLOUT-SHELTERS BLAST-SHELTERS BIOLOGICAL-WARFI I3-I3-O2278 INO AS THE FUEL I FUELS COOLING-AND-VENTILATING-EQUIPMENT FALLOUT-SHELTERS BLAST-SHELTERS EVAPORATORS COS/ I3-JI-DI773 10 RESIST INTEGRATED NUCLEAR WEAPONS EFFECTS I STRUCTURES FALLOUT-SHELTERS BLAST-SHELTERS THERMAL-RADIATI/ I3-I3-DI82 I IR PROTECTIVE STRUCTURES LOCATED ABOVE AND BELOW GROUND 1 FALLOUT-SHELTERS COMPUTERS BLAST-SHELTERS TEMPEI I3-DI-DI7T6 NG SHELTER-1 SHEL TER OCCUPANCY STLDIES I. FINAL REPORT I FALLOUT-SHELTERS CONFINED-ENVIRONMENTS STOCKPILI OS-II-O2IB4 ITIONS IN COMBINATION WITH ADJUNC TS I FOOD (ACCEPTAEILITY) FALLOUT-SHELTERS CONFINED-ENVIRONMENTS TASTE ' ${ }^{\prime}$ OG-DS-DI7I O IONS UNDER NUCLEAR ATTACK I CI VIL-DEFENSE-OPERATING-PLANS FALLOUT-SHELTERS CONSTRUCTIOV-SLAVTIVG OPERATIOI OS-DI -UIG23
VENTILATIOV KIT - A SSEMBLY AND OPERATING INSTRUCTIONS I FALLOUT-SHELTERS COOL IVG-AVD-VENTILATING-EQUIPME I3-OI -OITSO I VENTILATION KIT - ASSEMBLY AND OPERATI NG INSTRUCTIONS 1 FALLOUT-SHELTERS COOL IVG-AVD-VENTILATING-EQUIPME I3-OI -OI7S9 -PLANNING SITE- SELEC TION NAT/ AREA WIDE SHELTER SYSTEMS I FALLOUT-SHELTERS COSTS BLAST-SHEL TERS MANAGEMENT I3-I3-CI838 IAR FIRE I FIRES FIRE-PROTECTION RESOURCES (VULNERAEILITY) FALLOUT-SHELTERS EQUIPMENT URBAN-AREAS RURAL-AR/ I3- I2-OI8O6
IPORTATION EVACUATION DISPERSAL URBAN-AREAS FOOD SHELTERS FALLOUT-SHELTERS EJ FOPE SURVIVAL RECOVERY VULNEI IS-O3-O2295

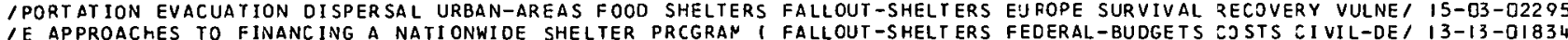
\begin{tabular}{l} 
IE APPROACMES TO FINANCING A NATIONWIOE SHELTER PRCGRAN \\
IHABITABILITY IN EXISTIING BUILOINGS UNDER FIRE EXPCSURE \\
\hline
\end{tabular} IANDARD FIRE TEST RATING SYSTEMS FOR SHELTER COMPONENTS 1 FALI OUT-SHELTERS FIRE-SAFETY FIRES STRUCTURES S/ I3-I?-O2269 ORTABLE DESIGN EXERCISE LIGHTSIILI SHELTER LIGHTING KIT I FALLOUT-SHELTERS LIGHTING-EQUIPMEVT GENERATORS P I3-OI -O22S2 1 -EQU IPMENT-AND-SUPPL IES STOCKPI LING FOOD-DI SPENSIAG FOON FAII OIIT-SHELYERS LOGISTICS SHELTER-OCEUPANCY HA/ DG-O3-OI7I IRESEARCH MATHEMATICS WEAPONS-EFFECTS RADICACTIVE-FALLOUT FALLOUT-SHELTERS YDOELSISIMULATIOVS) STATISTICAI I5-O3-OI935 IMPROVING FALLOUT PROTECTION IN HCME BASENENTS I SHELTERS FALLOUT-SHELTERS VEVADA WEAPOVS-TESTS SIMULATIO/ I3-I3-OI822 RAT/ SHELTER DESIGN AND ANALYSIS I. FALLOUT PROTECTION I FALLOUT-SHELTERS NUCLEAR-WEAPONS SHIELOING DOSE- I3-I3-QIB39 IATION OF MINIMAL EQUIPMENT NEEOS IN PER SONNEL SHELTERS 1 FALLOUT-SHELTERS OPTIMIZATION PERFJRMANCE IENGIN/ I3-I3-OIBI9 IAT ION OF MIVIMAL EQUIPMENT NEEDS IN PERSCNNEL SHELTERS 1 FALLOUT-SHELTERS PHYSIOLOGY EXPLOSIOV-EFFECTS S/ I3-I3-OIBII IELTER EQUIPMENT AND PROCEDURES II. LABCRATORY STUDIES 1 FALLOUT-SHELTERS PHYSIOLOGY SYSTEMS-ENGINEERING I5-O3-O2297 /ELTER EQUIPMENT AND PROCEDURES II. LABCRATORY STUDIES
TYPE STRUCTURES I NATIONAL-FALLCUT-SHELTER-SURVEYINFSS) FALLOUT-SHELTERS PROTECTION-FACTOR CALCULATIONS/ I8-OS-DIO95

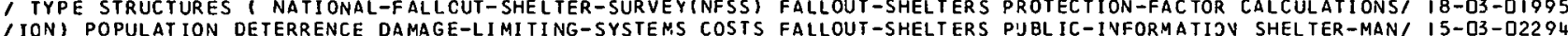
IIONI POPULATION DETERRENCE DAMAGE-LIMITING-SYSTENS COSTS FALLOUT-SHELTERS PJBLIC-INFORMATIJV SHELTER-MAN/ I5-03-O2294

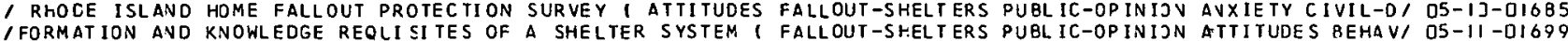
/FORMAT ION AND KNOWLEDGE REQLI SI TES OF A SHELTER SYSTEM I FALLOUT-SHELTERS PUBL. IC-OPINIJN ATTITUDES BEHAV/ O5-II-DI699
IOUT RADIOLOGICAL-CONTAMINATION DCSE-RATE COUNTERMEASURES FALLOUT-SHELTERS RADIOLOGICAL-DOSAGE MANPOWER I/ D6-I3-OO222O /-EQUIPMENT-AND-SUPPLIES FOOD-DI SPENSING FOOD STOCKPILING FALLOUT-SHELTERS SHELTER-OCCUPANCY LOGISTICS WA, D6-D3-DI7I2 /ED STATES I CONSTRUCTION URBAN-AREAS RADIOACTIVE-FALLOUT FALLOUT-SHELTERS SHIELDING STRUCTURAL-PROPERTIE/ I3-I3-O2277 ICY 1 OPERATIONS-RESEARCH CIVIL-DEFENSE-SYSTENS ATTITUDES FALLOUT-SHELTERS STRESSIPSYCHOLOGY) THREAT-PERC, O5-D+-D2I 66 IER EQUIPMENT AND PROCEDURES III. HABITABILITY STUCIES 1 FALLOUT-SHELTERS SYSTEMS-EVGIVEER ING COOLING-AN/ I5-O3-U2298 IY OF SHELTER RATIONS IN COMBINA I. SUMMARY AND REVIEW I FALLOUT-SHELTERS SYSTEMSEENGINEERING COOLING-ANI IS-O3-D23OIS CU I PROTECTIVE CONSTRUCTION INSTITUTE STUDIES I SHELTERS FALLOUT-SHELTERS UVDERGROUVD-STRUCTURES COLORADO I3-I3-DI83I IF FALLOUT SHELTER SPACES IN NEW YORK CITY AND VICINITY I FALLOUT-SHELTERS URBAN-AREAS COOLING-AND-VENTIL/ I3-I3-OIBI3 IOF SURVIVAL SHELTERS ( HEAT-PRODLCTION (BIOLOGY) SURVIVAL FALLOUT-SHELTERS VENTILATION METABOLISM BODY-TE I OO-IG-OI7IS ABILITY IASTRUMENTATION TEMPERATURE PUNKAH-PUMPS PORTABLE FANS, \#GARO REPORT I268-6I/RIMEVTAL-DATA HABIT I3-DI-DIT74 ABILITY INSTRUMENTATION TEMPERATURE PUNKAH-PUMPS PORTABLE FANS \$GARO REPORT I268-6I /RIMEVTAL-DATA HABIT I3-OI-DI774

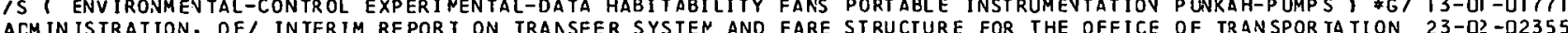

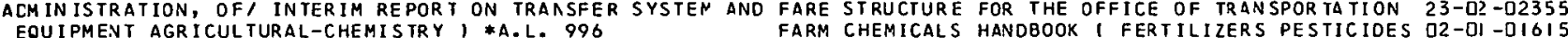
EQUIPMENT AGR ICUL TURAL-CHEMISTRY I *A.L. 996
DECONTAMINATION I RADIOACTIVE-FALLOUT RADIOLOGICAL-DOSE FARM-CROPS RADIOLOGICAL-CONTAYINATION RADIATIONI O6-IS-O22I I DECONTAMINATION I RADIOACTI VE-FALLOUT RADIOLOGICAL-DOSE FARM-CROPS RADIOLOGICAL-CONTAYINATION RADIATION/ O6-I9-O22I7
INSTRUCTION DESIGN STRENGIH STRUC TLRAL-PRCPERTIES GUALITY FASTENERS GLJING PLYWODD SANDWICH-CONSTRUCTION I II-I?-OI766 INSTRUCTION DESIGN STRENGTH STRUC TLRAL-PRCPERTIES GUALITY FASTENERS GLJING PLYWODD SANDWICH-CONSTRUCTION I II-I2-OI766
CASUALTIES POPULATI/ A STLDY OF hORLD WAR II GERNAN FIRE FATALITIES I FIRES URBAN-AREAS DAMAGE-ASSESSMENT I3-I?-D22SS2 IS BREAD CANNED CEREALS COFFEE CORN DEHYDRATED-FOOCS EGGS FATS FROZEN-FOODS FRUITS IRRADIATED-FOODS MEAT, OO-D3-D22O9 ITURES UNDERGROUND-RESIDENTIAL-DI STRIBUTION-SYSTEMS COSTS FAULT-LOCATION ELECTRIC-CABLES TRANSFORMERS HEA/ Q9-03-0I753 /UNO-STRUCTURES ELEC TRIC-CABLES DESIGN POWER-TRANSFORMERS FAULT-LOCAT ION L IGHTNING-ARRESTERS TELEPHONE-EQ/ O9-D3-DI755 HINK ABOUT THE BOMB AND ATOMIC, THE ONLY THING WE HAVE IC FEAR IS FEAR ITSELF - HHAT THE PEJPLE KNOW AND T O5-II -D2I 96 UT THE BOMB AND ATOMIC' THE ONLY THING WE HAVE TO FEAR IS FEAR ITSELF - WHAT THE PEJPLE KNOW AVD THINK A8O O5-II -D2I96 IMPL ICATIONS IN CIVILIAN EMERGENCIES ( EMCTIONS ATTITUDES FEAR STRESS(PSYCHOLOGY) CASUALTIES MOTIVATION PI DS-IJ-DIG8I OF POSTATTACK SITUATIONS I SOCIETAL-RECOVERY METHCDO/ THE FEASIBILITY OF CEVELOPING STAVDARD DESCRIPTIONS D5-II-G2I 1 
IONOMIC VIABILITY AFTER THERMONUCLEAR WAR - THE LIMITS OF FEASIBLE PRODUCT IOV 1 ECOVOMICS RECOVERY POSTAT/ 05-D3-D2I Z7 IONOMIC VIABILITY AFTER THERMONUCLEAR WAR - THE LINITS OF FEASIBLE PRODUCTION I VUCLEAR-WARFARE SURVIVAL I OS-03-02I58 IERNATIVE AOUEDUC T SYSTEMS TO SER VE SOUTHERN CALIFCRNIA - FEATHER RIVER ANC OELTA DIVERSION PRJ JECTS I WAI I3-O2-O2258 ASTER-MEDICIVE PUBL IC-HEAL TH LAW CRSANI ZATIO/ ROLE OF THE FEDERAL EMERGENCY HEALTH SERVICES DIVISION I DIS DG-2I-02226 I SHELTER-MANAGEMEN T UNI TEI AN EVALUATI ON OF THE ROLE OF FECERAL PERSONNEL IN RECRUITIVG SHELTER MANAGERS DS-DF-DI6 75 ORCESIUNITED-STATESI MILI ICATION-SYSTEMS, $\$$ A.L. 961 CODE OF FEDERAL REGULATIONS - VATIONAL DEFENSE I ARMED-F 15-03-01946 I STRENGTH, SIZE AND COST OF MLLTIPURPOSE BUILDINGS FOR A FEDERAL TRANSPORTATION ADMIVISTRATIOV 1 SHELTER/ I3-I3-QIB27 I INANCING A VATIONWIDE SHELTER PROGRAM I FALLCUT-SHELTERS FECERAL-8UCGETS COSTS CIVIL-DEFENSE-SYSTEMS MAN/ I3-I3-OIB34 $Y$ PLANNING 1 SCIENTIFIC-RESEARCH BLAST FIRES U/ REVIEW OF FEDERALLY SPONSORED RESEARCH PELATED TO EMERGENC I5-03-02299 IES FOOD-DISPENSING FOOD / BASIC COURSE IN EMERGENCY MASS FEEDING - HANDBOOK 1 KIICHEN-EQUIPMENT-AND-SUPPL D6-OS-DI 7 I 2 ANC-SUPPL IES STOCKPILING / BASIC COURSE IN EMEROENCY MASS FEEDING - INSTRUCTORS GUIDE 1 KITCHEV-EQUIPMENT- O6-OB-OI7I 3 C-CISPENSING SHEL TER-OCCUPANCY POSTATTACT-OPER/ ENERGENCY FEEOING 1 CIVIL-CEFENSE-OPERATING-PLAVS FOOD FOO DO-D3-O22ID PECOVERY ATTACK-SCENARIOS FERTILITY JRBAN-AREAS RURAL-AZEAS VULMERABILITYI I5-0S-019S5

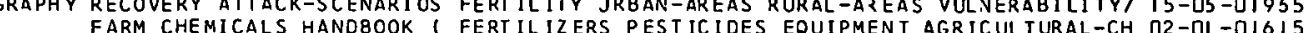

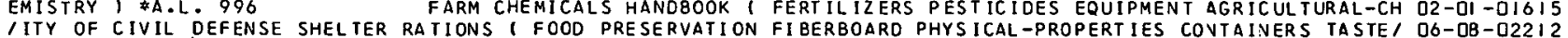

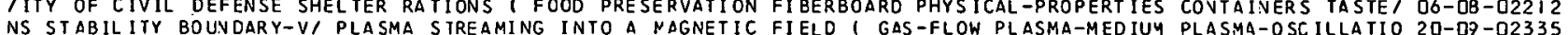

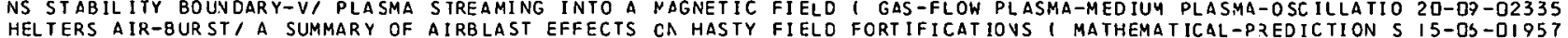
$I$ OF PHOTONS PROOUCED BY A REAL AND TWO SIMULATED FALLOUT FIELOS I RADIOACTIVITY RADIOACTIVE-FALLOUT CALC/ IS-OS-DIOG 4 SSESSMENT DATA-PROCESSING PROGRAM, PARM SYSTFM MANUAI. V. FILE MAINTENANCE PROCEDURES 1 RESJURCES DAMAGE-A OS-O3-O2I5O ISAST ER-METIICINE FMFRGFNCY-HEALTH-SERVICES CANADA NANUALS FILMS, A.L. IOGS CATALOGUE OF PUBLICA.TIONS I D O6-2I -0I737 NNEL TOSPITALS PUBLIC-HEALTH RESCL/ OISASTER NEDICAL CARE FILMS I CISASTER-MEDICINE MEOICINE MEDICAL-PERSU O6-2I -DITHO IECT DEFENDER I PLASMA-PHYSICS ELECTROMAGNETIC-PROPERTIES FILMS FLUID-MECHAVICS SCIEVTIFIC-2ESEARCH ABLAT/ $20-03$-O2330 /CIVIL-DEFENSE TRAINING RESCUES PLBLIC-INFORMATION REPAIR FILMS PROPAGANDA RURAL-AREAS CIVIL-DEFENSE-OPER/ I5-D3-D2302 8 IOLOCY BACTERIA EAS-FILTERS MICROORGANI SNS AIR-POLLUTION FILTERS, *AD 637 407 /GICAL-WARFAZE-AGENTS AERO IS-OZ-DI85I IMENTS FOR. FIRE RFSISTANT HIGH EFFICIENCY PARTICULATE AIR FILTERS ( FILTERSIFLUID) RELIABILITY QUALITY-CO/ I3-II-DI794 OPERTIES CONSTRUCTION MECHANIC/ DEVELOPMENT OF U-FCAM AIR FILTERS ( VENTILATION FILTERSIFLUID) CHEMILAL-PK I3-II-OIT92 MECIANIC, CEVELOPMENT OF L-FOAM AIR FILTERS I VENTILATION FILTERS (FLUIC) CHEM ICAL-PROPERTIES COVSTRUCTION I3-II-OI792

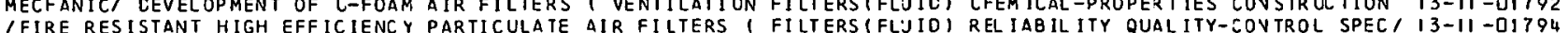

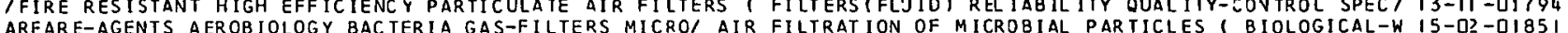
ICAT IONS ABOR COMMUN ISM ECONOMICS AGRICULTURAL-ECCNOMICS FINANCE INDUSTRIES COMMERCE EDUCATION POLITICAL/ OS-III-DI693 IS SCHOOLS FOOD-SUPPLIES WATER-SLPPLIES INDUSTRIAL-PLANTS FINANCE STOCKPILING STORAGE MANPOWER I *NREC-I3/ OS-D3-O2I37

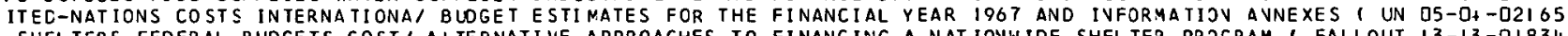
-SHEL TERS FEDERAL-BUDGETS COST/ ALTERNATI VE APPROACHES TO FINANCING A NAT IOVWIDE SHEL TER PROGRAM I FALLOUT I3-I3-OI834 AL LOCATION OF PROJECIS I FLOOOS FLOOD-CONTROL CALIFORNIA FINANCING CONSTRUCTION, \#A.L. IDBD $10 W I N G$ GENER I3-D2-QI782 ISTRIEUTION UNDERGROUND-STORAGE SALES RE VENUE UTILIZATION FINANCING OPERATING-COSTS CAVADA CONSTRUCIION P/ OS-O3-OIGU2 -OPERATIVG-PLANS POPULATION I ANNLAL STATISTICAL REPORT I FINANCING SHELTERS WARVIVG-SYSTEMS CIVIL-DEFENSE IS-O3-OI 9DB STRUCTIOV FIRE-RESISTANCE THERMAL-INSULATI ON PRESERVATION FINISHES PAINTS, \#HANDBODK NO. 72 /SANDWICH-ZON II-1?-OI7SO WEAPONS-EI THERMAL STRESSES IN A SEMI INFINITE SOLID BY A FINITE DIFFERENCE TECHNIQUE I THERMAL-RADIATION I3-12-DI79S LITY) FALLOUT-S/ DEFENDING THE UNITED STATES FRON AUCLEAR FIRE I FIRES FIRE-PROIECTION RESOURCESIVULNERABI I3-I?-OIBO6 ST-EQUIPMENT, \#P SW/ TEMPERATURES IN A LARGE NATURAL FUEL FIRE I FIRES TEST-METHJDS MODELS(SIMULATIONSI TE I3-I?-OIBID AN RURAL FIRES 1940-1964 (BIBLIOGRAPHIES URBAN-AREAS RII/ FIRF BIBLIOGRAPHY - SELECTED URBAV AVD MIXED URB I3-I?-DI799 SMOKE / SHEL TER HABITABILITY IN EXISTING BUILDINGS UNDER FIRE EXPOSURE I FALLOUT-SHELIERS FIRE-PROIEC TION I3-I?-DI8OI SMENT CASUAL IES POPULATI N-AREAS FIREI PARAMETERS GOVERNING URBAN VULNERABILITY TO FIRE FRCM NUCLEAR BURSTS 1 BURST-CDYDITIONS URBA I3-I?-DI8DI ALTIES SIMULATION FIRE-SAFETY TOXICITY HEAT CARBCN-I MASS FIRE LIFE TAZARD I VUCLEAR-EXOLOSIOVS FIRES CASU I3-I?-DI8OO SCENARIOS AIRCRAFI NUCLEAR-W/ PRELIMINARY AERIAL INFRARED FIRE MAPPING SYSTEMS ANALYSIS 1 MICHIGAN ATTACK- I3-I?-O2267 IENTATION OF THE ORGANIZATION, TECHNOLOGY, AND TACTICS OF FIRE PROTECTION AS EMPLOYED BY A CIVIL DEFENSE I I3-I2-D2264 /ENTATION OF THE ORGANIZATION, TECHNOLOGY, AND TACTICS OF FIRE PRCTECTION AS EMPLOYED BY A CIVIL DEFENSE, I3-I?-O2265 ON OF THE ORGAVIZATION, TECHNOLI PRACTICAL EXPERIEACES OF FIRE PROTECTION SERVICES - A CRITICAL PRESENTATI I3-I?-O22G4 ON OF THE ORGANIZATION, TECHNOL/ PRACTICAL EXPERIENCES OF FIRE PROTECTION SERVICES - A CRITIEAL PRESENTATI I3-I?-O226S ILTERS ( FILTERS(FLUID) RELIABILITY QUA' REQUIREMENTS FOR FIRE RESISTANT HIGH EFFICIENCY PARTICULATE AIR F I3-II -OI794 EC MAPPER AS A TOOL FOR DETECTING AND MEASURING / PROJECT FIRE SCAN - THE EVALUAT IOV OF $\triangle N$ AIRBJRNE INFRAR I3-I? - OI798 (COMPUTERS) DATA-PROCESSING URBAN-AREAS MATHEN/ FLANE I - FIRE SPREAD SIMULATION MODEL I FIZES PROGRAMMING I3-I?-O22SO 1 FALLOUT-SHELTER S FIRE-SAFETY F/ DEVELOPNENT OF STANDARD FIRE TEST RAT ING SYSTEMS FOR SHELTER COMPONENTS I3-I?-D2269 IECTION SMOKE CARBON-MONOXIDE OXYGEN-SUPPLY GAS-DETECTORS FIRE-DETECTORS CARBON-MONOXIDE-INDICATORS TEMPE/ I3-I2-DI8D 7 /ECTION SMOKE CARBON-MONOXIDE OXYGEN-SUPPLY GAS-DETECTORS FIRE-DETECTORS CARBON-MONOXIDE-INDICATORS TEMPE/ I3-I2-DI8J7
IENTS CIVIL-DEFEN SE-OPERATING-PLANS C IVIL-DEFENSE-SYSTEMS FIRE-FIGHTING-ANE-EQUIPMEVI DECONTAMINATION RES/ I5-O3-O2296 /ENTS CIVIL-DEFEN SE-OPERATING-PLANS CIVIL-DEFENSE-SYSTEMS FIRE-FIGHTING-ANC-EQUIPMEVT DECONTAMINATION RES/ I5-O3-02296

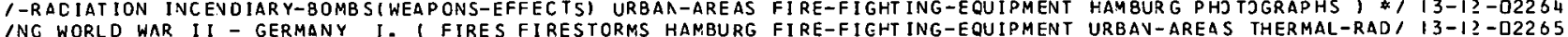
ING WORLD WAR I I - GERMANY I. I FIRES FIRESTORMS HAMBURG FIRE-FIGHT ING-EQUIPMENT URBAV-AREAS THERMAL-RAD/ I3-I?-D2265

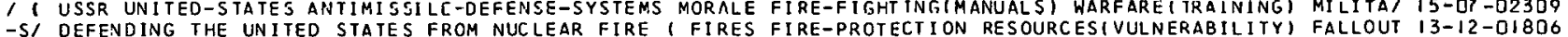

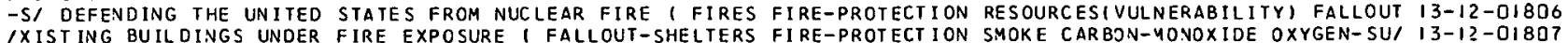
IXIST ING BUILDINGS UNDER FIRE EXPOSURE I FALLOUT-SHELTERS FIRE-PROTECTION SMOKE CARBON-YONOXIDE OXYGEN-SU/ I3-I?-OI8D7 IS QUAL ITY FASTEVERS GLUING PL YWOOD SANDWICH-CCNSTRUCTI ON FIRE-RES IST ANCE THERMAL-IVSULATIOV PQESER VATION/ II-I? -OI 756

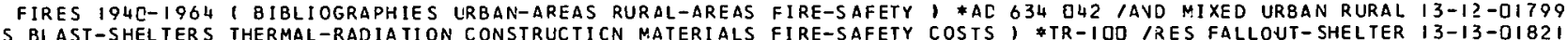
I DLAST SHIELOINC ETRUCTURAL-AMALYIS RAOIOACTIVE-FAII.QUT FIRE-SAFETY FALLOJT-SHELTERS I \#A.L. 1207 1-WAVE I3-13-0228D IRAT ING SYSTEMS FOR SHEL TER COMPONENTS (FALLCUT-SHELTERS FIRE-SAFETY FIRES STRUCTURES SMOKES BUILOINGS LI I $3-1$ ?-U22OG IR A WARFARE I TIRCS NUCLLAR-EXPLOSI ONS DANAGE-ASSFSSMFNT FIRE-SAFETY PROGRAMMINGICOYPUTERSI DEBRIS BLAST/ I5-O3 -DIOS2 I HAZARO I NUCLEAR-EXPLOSIONS FIRES CASUALTIES SIMULATION FIRE-SAFETY TOXICITY HEAT CARBON-MONOXIDE CARBO/ IS-IZ-UIUUU FIRES URBAN-AREAS DAMAGE-ASSESSMENT CASUALIFS POPULATION FIRE-STORMS, \#DC-TN-IO50-3, FIRE FATALITIES I I3-I2-02262

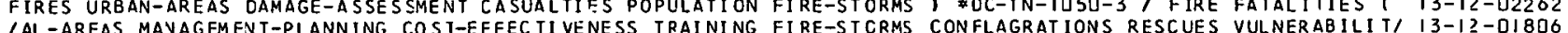
/AL-AREAS MAVAGEMENT-PLANNING COSI-EFFECTIVENESS TRAINING FIRE-ST CRMS CONFLAGRATIONS RESCUES VULNERABILIT/ I3-I?-DI806

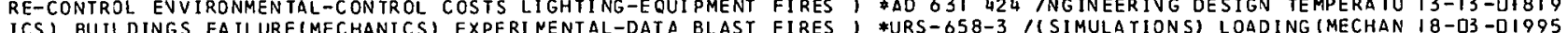
ICS) BUILDINGS FAILURE(MECHANICS) EXPERI NENTAL-DATA BLAST FIRES I *URS- $558-3$ /1SIMULATIONS) LOADING (MECHAN $18-03-01995$ INC INTRARED MAPPER AS A TOOL FOR DETECTINC, AND MEASURING FIRES 1 INFRARED-DETECTORS INFRARED-TRACKING FOI I3-I2-QIT98 IONS PERTAINING TO RESIDENTIAL AREAS I NUCLEAR-EXPLOSIONS FIRES AIRBJRST STRUCTURES VULVERABILITY RECUVEKI IS-OS-OIY3 3 FIAT CARBOV-I MASS FIRE LIFE HAZARD I NUCLEAR-EXPLOSICNS FIRES CASUALTIES SIMULATIOV FIRE-SAFETY TOXICITY I3-I?-OIBOD ISHOCK-WAVES THERMAL-RADIATION RADIOLOGICAL-CCNTAMINATION FIRES CONSTRUCT ION POSTATTACK-OPEZATIONS REPAIR/ I3-I? -OI8J4 ALLOUT-S/ DEFENDING THE UNIIED STATES FRON NUCLEAR FIRE I FIRES FIRE-PROTECT ION RESOURCESIVULNERABILITYIF I3-I?-OIBQ6 IDEFENSE ORGANIZATION DUR ING WORLD WAR 1 I - GERMANY I - I FIRES FIRESTORMS HAMBURG FIRE-FIGHTIVG-EQUIPMEN/ I3-I?-O2265 IFENSE OR CANIZATION DURING WORLO WAR II - GERMANY - I I FIRES FIRESTORMS THERMAL-RADIATIOV IVCENDIARY-B/ I3-I? -O2264 I AN JOSE AND ALBUQUERQUE I AERIAL-PHOTOGRAPHS URBAN-AREAS FIRES MAPS TEST-METHODS VULNEZABILITY CLASSIFIC/ I3-I?-DIBJ9 FIRE FROM NUCLEAR BURSTS / SAFETY PROGR/ RE-USABILITY OF BUILOINGS AFTER A WARFARE I FIRES NUCLEAR-EXPLOSIONS DAMAGE-ASSESSMENI FIRE- I5-O3-OIOS2 1-EFFECTS WIVD RADIOACTIVE-FALLOUI 1-EFFECTS WIVD RAOIOACTIVE-FALLOUT CLIMATCLOG CASUALTIES FIRES PRCGRAMMINE( COMPUTERS AN-AREAS MATHEM FLAME I - FIRE SPREAD SINULATON NCDEL T FIRES PROERAMMINCI COMPUTERS DATA-PROCESSING URB I /AGED URBAN AREAS I BLAST DAMAGE-ASSE SSMENT VULNERABILITY FIRES REPAIR RADIOACTIVE-FALLJUT RADIOLOGICAL-C7 IS-OS -023日7 -CIVIL-DEFENSE POSTATIACK-OPERATIONS RESCLES CONSTRUCTION FIRES ROACS 1 \#JPRS-27, 419 \#TI-64-51669 1 ATIONAL O6-07-OI7O9 IITIONS RADIATIOV-INJURIES VIELD RADI OLOGICAL-DOSAGE WIND FIRES SIMULATION BLAST STATE-2F-THE-ART-REVIEWS/ IS-OS-OI962
IMS FOR SHELTER COMPONENTS I FALLOUT-SHELTERS FIRE-SAFETY FIRES STRUCTURES SMOKES BUILDINGS COVSTRUCTION I I3-I?-O2269 PMENT, \$PSW/ TEMPERA TURES IN A LARGE NATLRAL FUEL FIRE (FIRES TEST-METHOCS MODELS(SIMULATIOVS) TEST-EQUI I3-I?-OIBIO SHADOW GEOMETRY OF BUILDINGS IN URBAN DREAS I FIRES THERMAL-RACIATION SWEDEN, \$A.L. IIIB, I3-I?-02268 IELATEO TO EMERGENCY PLANNING I SCIENTIFIC-RESEARCH BLAST FIRES UNITED-STATES-GOVERVMEVT OFFICE-OF-EMERGE/ I5-D3-O2299 OPIII ATI/ A STUDY OF WORLD WAR II GERMAN FIRE FATALITIES I FIRES URBAN-AREAS DAMAGE-ASSESSMEVT CASUALTIES P I3-I2-D2262 
$\because C C-T N-2046-1$

FIRESTORM ANALYSIS ( FIRES WIND METEOROLOGY COMPUTER S(PRJGRAMMING) I 13-1?-01805 FIRE BIBL IOGRAPHY - SELECTED LRBAN
UTERS (PROGRAMMING), \$DC-TN-2O46-1 IE ORGANILATION DURING WORLO WAR II - GERNANY I. I FIRES FIR

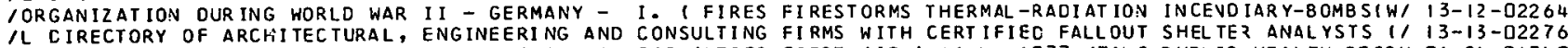
ES NUCLEAR-WARFARE-CASUALIIES SCHCOLS SURVIVAL CASUALTIES FIRST-AID, \#A.L. IOT7 ITALS PUBLIC-HEALTH RESCU O6-2I -0174O IGENE INCENDIARY-GELS FLAME-THROWER-FUELS SMOKE-MUNITIONS FIRST-AID NUCLEAR-WARFARE-CASUALTIES I \&JPRS 3D/ D6-I3-DI72I ICISASTER-MEDICINE MEOICINE ADMINISTRATI ON-AND-MANAGEMENT FIRST-AID ORGANIZATION TRAINIVG HOSPITALS CASUA/ D6-21-OI738 IWARFARE-AGENTS B IOLOGICAL-WARFARE-AGENTS NUCLEAR-hEAPONS FIRST-AID WARNING-SYSTEMS RADIOLOGICAL-CONTAMIN/ IS-D3-OI9I8 ICINE I USSR DISASTER-MEDICINE NUCLEAR-WARFARE-C ASUALTIES FIRST-AID WOJNDS-AVD-IVJURIES RADIATION-INJURIE/ DG-DJ-OI7I3 BAN-PLANNING LAND-USE POPULATION TRANSPORTATICN UTILITIES FISCAL-AND-MONETARY ECONOMICS 1 \#PRC D-I2I9 $/$ UR $23-0 S-02 I 06$ IULAT IONAL MODEL FOR CONDENSED STATE DIFFUSION CONTROLLED FISSION PRODUCT ABSORPTION DUR ING FALLOUT FORMA/ I8-DB-D2O37 MATRIX I RADIOAC/ HENRYS LAW CONSTANTS FOR DISSOLUTION OF FISSION PRODUCTS IN A SILICATE FALLOUT PARTICLE I8-OB-02325 TICAL-TESTS STATISTIC/ THE CHI-SQLARE TEST OF GOODNESS OF FIT FOR A BIVARIATE NORMAL DISTRIBUTION I STATIS I2-OI-O22SI SYSTEMS-ANALYSIS POST-ATTACK-OPERATI ONS DANAGE-ASSESSMENT FIVE-CITY-STUDIES, \$97TII-F/TARY-INTELLIGENCE I3-1?-022 S7 ROGRAMMINGICOMPUTERSI DATA-PROCESSING URBAN-AREAS NATHEM/ FLAME I - FIRE SPREAD SIMULATION MODEL I FIRES P I3-I2-02266 ROGRAMMING (COMPUTERSI DATA-PROCE SSING URBAN-AREAS NATHEMI FLAME I - FIRE SPREAD SIMULATION MODEL

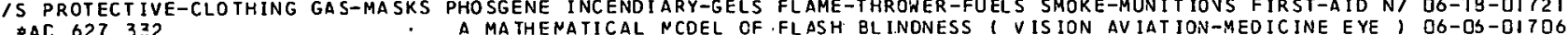

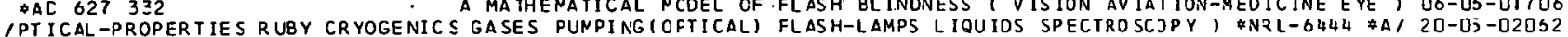

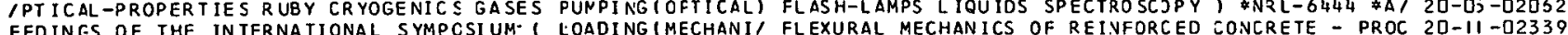
EEDINES OF THE IN TERNATIONAL SYMPCSIUM- I LOADING IMECHANI/ FLEXURAL MECHANICS OF REINFORCED CONCRETE - PROC 2D-II -02339 SED AND MAP SHOWING GENERAL LOCATION OF PROJECTS IEC AND MAP SHOWING GENERAL LOCATION OF PROJECTS I FLOODS FLCOD-CCNTROL CALIFORNIA CONSTRUCTIOV COSTS I \#/ I3-O2-DI7B4 IED ANO MAP SHOWING GENERAL LOCATION OF PROJECTS I FLOODS. FLCOD-CONTROL CALIFORNIA FINAVCING CONSTRLCTION/ I3-O2-OI782 ATION OF RIGHTS OF WAY TO BE ACQUIRED I CIVIL-ENGINEERING FL COOS 1 A.L. 972, /SS SECT IOVS, AND GENERAL LOC I3-02-OI78I S. AND GENERAL LOCATION OF RIGHTS OF WAY TO BE ACGUIRED I FLCODS CAL.IFORNIA, \$A.L. 987 /ES, CZOSS SECTION I3-D2-OI779 IGE-ASSESSMENT DATA DECISION-MAKING RECOVERY HCUSING FOOD FLOODS CIVIL-DEFENSE-OPERATING-PLANS REPA IR CON/ OS-O3 -DIO36 /-EFFECTS MANPOWER EMERGENCY-HEAL TH-SERVICES RADIOBIOLOGY FLOODS CISASTER-MEOICIVE TSUNAMIS WATER-SUPPLIE I I5-O3-O2299 $K$ PROPOSED AND MAP SHOWING GENERAL LOCATION OF PROJECTS I FLOODS FLOOD-CONTROL CALIFORNIA I \$A.L. IOBI IOR I3-D2-OI783 1 PROPOSEO AND MAP SHOWING GENERAL LOCATICN OF PROJECTS 1 'FLCODS FLOOD-CONTROL CALIFORNIA CONSTRUCTION CO/ I3-O? -OI784 1 PROPOSED ANO MAP SHOWING GENERAL LOCATION OF PROJECTS I FLOODS FLOOD-CONTROL CALIFDRNIA FINANCING CONSTI I3-O? -DI7B2 UCTURAL FRAMES LOADIN/ DEFLECTIONS OF REINFORCED CCNCRETE FLOOR SLABS I STRUCTURES DESIGN THEORY BEAMSISTR $20-11$-D234D SSION COLORS OP TICAL-IMAGES OP TICAL-PHENONENA METECROLOGY FLORIDA, \$8209-2, IC CONDITIONS I LIGHT-TRANSMI 20-06-02066 S-FLOW WEST-GERMANY DUC TS LAMINAI INTERACTION BETWEEN AIR FLOW ANC AIRBORNE SOUND IV A DUCT I ACOUSTICS GA $20-0 I-O 2 O 48$ AND THE CUSP EFFECT I HYDRODYNAMICS DESIGA CUSPS FLUID-FI FLOW CONTROL.BY GEVERATIOV OF SIAVDIVG VORTICES $20-D T-D 2 O 55$ HIN THE DEFENSE INDUST, DOD USER - NEEDS STUDY, PHASE II, FLOW OF SCIENTIFIC AND TECHNICAL INFORMATION WIT OS-D?-02I2D T LOG IC-DESIGN PROGRAMMING (COMPUTERS) MATHEMATICAL-MODELS FIOW-CHARTING) RNS WIND YIELD BURST-CONDITIONS BLAST RADIOACTIVE-FALLOIT FLCW-CHARTING, \#NREC-S, PERSONNELATTACK-PATTE OS-D3-O2I 46 I ION-RETR IEVAL ENGINEER ING-PER SONNEL SCIENTIFIC-PE.RSCNNEL FLOW-CHART ING PSYCHOMETRICS TECHNICAL-INFORMATI $/$ Q5-0?-02I 20 1 COSTS II: APPENDIX B I INDUSTRIAL-EQUIPMENT INDUSTRIES FLOW-CHARTING SURVIVAL RECOVERY ECONOMIC-RECOVE, O5-03-O2I26 IICLES DESIGN AERODYNAMIC-HEATING HEATING COOLING-SYSTEMS FLUID-DYNAMIC-PROPERT IES DRAG SURFACE-TEMPERATU/ 22 - D2-D235D INUCLEAR-REACTORS HEAT-TRANSFER NLCLEAR-REAC TOR-MATERIALS FLUID-DYNAMIC-PROPERT IES EVERGY-COVVER SION REACI 2 O-OB-O2OTO IAL ING I . EXPONENTIAL IDEAL GAS ATMOSPHERE I SHOCK-WAVES FLUID-CYNAMIC-PROP.ERTIES HYDRJOYNAMICS I *NOLTR/ $20-0+-02332$ /COAGULATION OF PARTICLES IN A TURBULENT GAS I TUREULENCE FLUID-DYNAMICS PARIICLE-SIZE-DISTRIBUTION AEROS/ $20-0+02333$ VORTICES AND THE CUSP EFFECT I HYDRODYNAMICS DESIGN CUSPS FLUID-FLOW, AMICS I GAS-FLOW EQUATIONS-OF-STA ME MATHEMATICAL-ANALYSIS FLUIO-FLOW SHEAR-STRESSES I \&UCRL-5D2II IGAS DYN $20-04-0233$ AMENDER I PLASMA-PHYSICS ELEC TROMAGNE IIC-PROPERTIES FILMS FLUID-MECHANICS SCIENTIFIC-RESEARCH ABLATION ANI 2D-O3-D2330

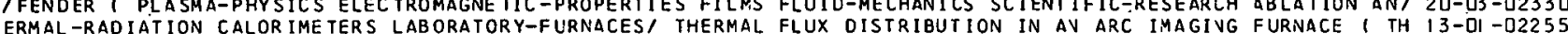
ERMAL-RADIATION CALORIME TERS LAB ORATORY-FURNACES/ THERMAL FLUX DISTRIBUTION IN AV ARC IMAGIVG FURNACE 1 TH 13 -OI -D2255 INFORMATION SYSTEM I TECHNICAL-INFORMATION/ FIVE YEARS IN FOCUS - THE DOUGLAS AIRCRAFT COMPANY MECHANI ZED OS-0Z-O2II

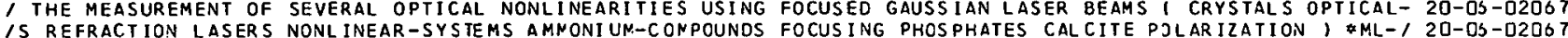

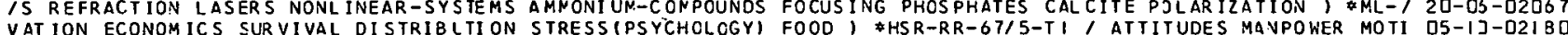
VAT ION ECONOMICS SURVIVAL DISTRIBLTION STRESS (PSYCHOLCGYI FOOD, \#HSR-RR-67/5-TII ATTITUDES MAVPOWER MOTI O5-IJ-D2IBD RAL-AREAS RADIOAC TIVE-FALLOUT SHIELOING RAI PROTECTION OF FOOD ANL AGRICULTURE AGAINST VUCLEAR ATTACK I RU IS-O3-OI897 G RUSSIA AFRICA LATIN-AMERICA JAPAN GROSS-N/ THE STATE OF FOOD AND AGRICULTURE 1966.1 EUROPE FOOD-MARKETIN D2-O2-OI6I6 -OPERATIONS FOOD-DISPENSING DISTRIBUTION DAMA/ POSTATTACK FOOD AVAILABILITY AND ACCESSIBILITY I POSTATTACK DG-DS-OITII RAB IL ITY POPULATION CASUALIIES FOOD- 1 RI SK II ANALYSIS OF FOOD BALANCE 1 RESOURCES DAMAGE-ASSESSMENT VULNE O5-O3-O2132 ION-MANUALS PSYCHOLOGY STRESS (PHYSI OLOGY) WATER-SUPPLIES FOOD BEDDING TOILET-FACIL ITIES I \$SARD- 1292 (IIII I5-03-D2298 /REAS BOMBS GUIDED-MISSILES NUCLEAR-WEAPONS R.CNTAMINATION FOOD CHEMICAL -WARFARE-AGCNTS BIOLOGICAL-WARFAREI IS-DS-O2ZYO IOPS IN THE TROPICS I TROP.ICAL-REGIONS MILITARY-PERSONNEL FOOD CLOTHING ENVIRONMENT EOUCATION ARMY-EQUIPM/. Q5-IO-O2I8I IS I AGR ICULTURE RADIOACTIVE-I SOTOPES RADIOACTIVE-FALLOUT FOCD CONTAMINATION MOOELS(SIMULATIDVS) RADIOBIOI OS-IB-OIT25 (FOOD) PRODUCT/ VUTRITION IN THE POSTATTACK ENVIRONMENT I FOOD DIET POSTATTACK -OPERATIOVS SUR VIVAL STORAGE DG-IS-OITI 7 TTCHEN-EQUIPMENT-AND-SUPPLIES STOCKPILING FOOD-DISPENSING FOOD FALLOUT-SHELTERS LOGISTICS SHELTER-OCCUPANI O6-O3-OI7I

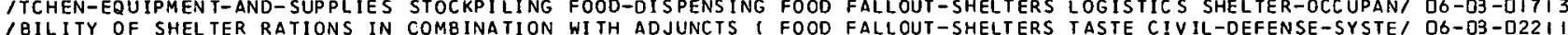

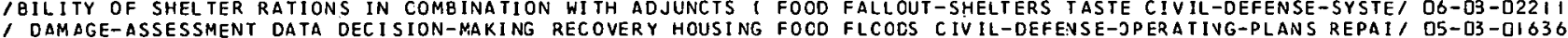

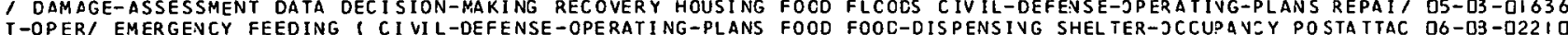

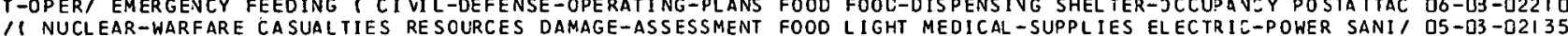

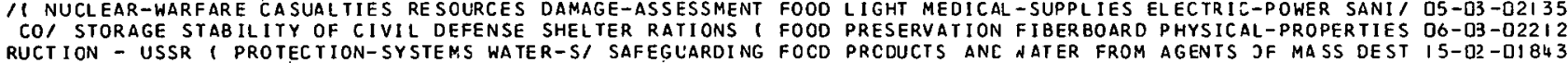
INEER ING MEDICAL-SUPPLIES DEFECTICNS TRAINING SLEEF WATER FOCO RECREAT ION EXERCISE VOISE RELIGION TEMPERA I DS-IJ-D2IB4 ICUSTRIES TRANSPORTATION EVACUATION OISPERSAL URBAN-AREAS FOOD SHELTERS FALL OUT-SHELTERS EURJPE SUR VIVAL, I5-D3-O2295 I ANDBOOK I KITCHEN-EQUIPMENT-AND-SUPPLIES FOOD-DISPENSING FOOD STOCKPIL ING FALLOUT-SHELTERS SHELTER-OCCUP/ DG-D3-OITI 2 PRESERVATION SHELF-LIFE BEVERAGES BREAD CANNED CEREALS I FOCD STORAGE LIFE - A REPORT BIBLIOGRAPHY I BEEF O6-OS-O2209 SOTOP ES AIR MILK ATMOSPHERIC-PREC IPI TA TI ON WATER-SUPPLIES FOOD UNITED-STATES CANACA YEXICO I \#TIO-23286 II I8-0B-O2035 IHUMAN) INSTRUCTION-MANUALS PSYCHOLOSY STRESS (PHYSIOLOGY) FOOD WATER-SJPPLIES BEDOING. TJ ILET-FACILITIES II I5-CI3-O23OD $1 D$ AVAILABILITY AND ACCESSIBILITY I POSTATTACK-OPERATIONS FOOD-D̃ISPENSING CISIRIBUTION DAMAGE-ASSESSMENT I DG-O3-OI7II ICTORS GUIDE I KITCHEN-EQUIPMENT-AND-SUPPLIES STOCKPILING FOOD-DISPENSING FOOD FALLOUT-SHELTERS LOGISTICSI O6-OB-OI7I 3 ICTORS GUIDE TITCHEN-EQUIPMENT-AND-SUPPLIES STOCKPILING FOOD-DISPENSING FOOD FALLOUT-SHELTERS LOGISTICSI O6-O3-OITI

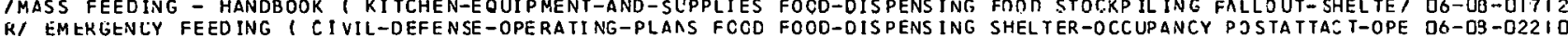
R/ EMERGENCY FEEDING ( CIVIL-DEFENSE-OPERATING-PLANS FCCD FOOD-OISPENS ING SHELTER-OCCUPANCY P SSTATTAET-0PE D6-O3-O22ID

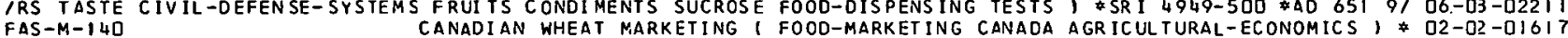

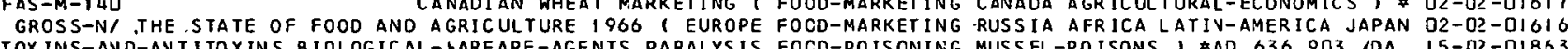

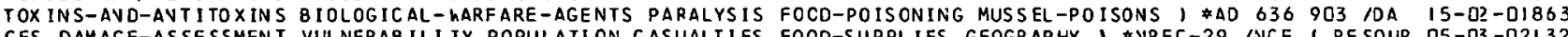
CES DAMAGE-ASSESSMENT VULNERABILI TY POPULATI ON CASUALTIES FOOD-SUPPLIES. GEOGRAPHY, \#VREC-29 /NCE I RESOUR QS-03-02I32 INERATION MIL ITARY-PER SONNEL POPULATION HOSPITALS SCHOOLS FOOD-SUPPLIES WATER-SUPPLIES INDUSTRIAL-PLANTS, DS-O3-O2I 37 $1 B I L I T Y$ OF SHEL TER RATIONS IN COMBINA TION WI TH AD JUNCTS 1 FOOD(ACCEPTABIL ITY) FALLOUT-SHELTER S CONFINED-E I D6-OB-OITIO /SIMULATION) MODELSISIMULATIONS) DECONTAMINATION RECOVERY FOOD (CONTAMINATION) WATER (CONTAMIVATION) RADIOL, O6-I3-DIT23 1. ECOLOGY STRATEGIC-MATERIALS HARDENING SCCIETAL-RECOVERY FOONISTOCKPIL-INGI STOCKPILING INDUSTRIAL-PLANTSI IS-OB-UIY53 5 COMMERCE MEAT RESOURCES CEREAL COTTON VEGE TABLES FRUITS FOCOS, \#AGRICULTURE HANDBOOK NO. 325 IURAL-AREA O2-0?-DI6I9 LOADINGIMEC/ TRANSIENT LOADING TESTS ON A RIGID CIRCULAR FOOTING 1 FOJNDATIONSISTRUCTUZES) SOIL-MECHANICS I3-I3-OI.8I 2 OIL-MECHANIC/ STATIC AND DYNAMIC BEARING TESTS ON A STRIP FOOT ING IN SATURATED SAND 1 LOADINGIMECHANICSI.S $20-1 I$-O234 1 IS SHELTERS UNDER GROUND-STRUCTURES SAND SHOCK-WAVES BLAST FOOTINGS FOUNDATIONS VOIC-RATIO ARCHES, \$NCEL- $120-11$ - U234

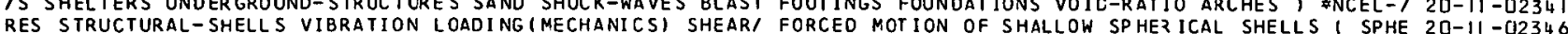

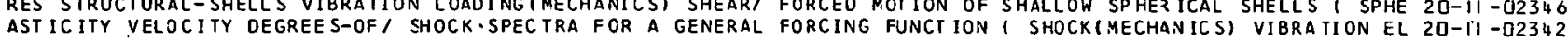


IC 1 PUBLIC-OPINION SURVEYS FOREIGN/ OCCASIONAL REPORT ON FOREIGN AFFAIRS - AMERICAN PRJGRAMS OF FOREIGN A D5-II -DIGB? YS FOREIGN-POLICY OUESTIONNAIRES I/ OCCASIONAL REPCRTS ON FOREIGN AFFAIRS - GERMANY I PUBLIC-OPINION SURVE D5-II-O2I86 - AFFAIRS PUBL IC-OPINION SURVEYS FOR/ OCCA SI ONAL REFORT ON FOREIGN AFFAIRS - JNITED VATIJNS I IVTERNATIONAL O5-II -O2I 98 -AFFAIRS SURVEYS QUESTIONNAIRE S PLB/ OCCASIONAL REFORT ON FOREIGN AFFAIRS - JNITED VATIJVS I IVTERNATIONAL OS-II -O2I Q9 IASIONAL REPORT ON FOREIGN AFFAIRS - AMERICAN PROGRAMS OF FOREIGN AID I PUBL. IC-OPINION SURVEYS FOREIGN-PO/ O5-II -OIGB /IOGRAPHY OF GOVERNMENT SPONSORED AND PRIVATE RESEARCH ON FOREIGN STUDENTS AND TRAINEES IN THE U.S. AND I/ OS-II -DIGQ IS-CONTROL COLD-WAR NATO VIETNAM RED-CHINA FOREIGN-POLICY FOREIGN-AID LABOR JNITEO-VATIONS WAGES, \#A.L. I, O5-O4-O2I T2 RS 1 ECONOMICS UVITED-STA IES-GOVERNMENT PCLITICAL-SCIENCE FOREIGN-POLICY, \$A.L. 106I, OF EZJVOMIC ADVISE O5-D3-DIO34 TERNATIOVAL-AFFAIRS SUR VEYS QUESTIONNAIRES PUBLIC-CPINION FOREIGN-POLICY, IFA-2 RRS-UNITED NATIONS I IN OS-II -D2IOQ DES INTERNATIONAL-AFFAIRS FEAR SURVEYS STRESS (PSYCFOLOGY) FOREIGN-POLICY, *VORC-35 /OUESIIONNAIRES ATTITU OS-II -DIG89 THE OTHER WAR IN VIETNAN I TECHNICAL-ASSISTANCE FOREIGN-POLICY ECONOMIC-AID, *A.L IOB2 FOREIGN AID ( PUBLIC-OPINI ON SURVEYS FOREIGN-POLICY ECONOMIC-AID MILITARY-AID IFA- \#F IL AFFAIRS I ARMS-CONTROL COLD-WAR NATO VIETNAN REC-CHINA FOREIGN-POLICY FOREIGN-AID LABOR UVITED-NATIONSI DS-O I-D2I 72 IIC CENTERS - AFR ICA I ECONOMICS BIBLI OGRAPHIES EDUCATION FOREIGN-POL ICY GEOGRAPHY POLITICAL-SCIENCE HIST/ OS-II -DIOQ I

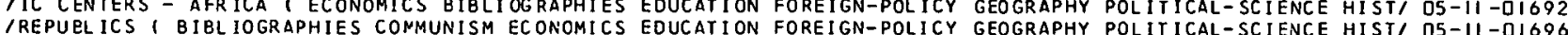

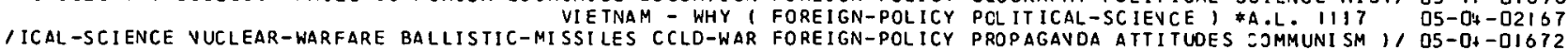
ITS ON FOREIGN AFFAIRS - GERMANY I PUBLIC-OPINION SURVEYS FOREIGN-POLICY OUESTIOVNAIRES INTERNATIONAL-AFF/ OS-D $\$$-DI 672 ID NATIONS I INTERNATIONAL-AFFAIRS PUSLIC-OPINION SURVEYS FOREIGN-POLICY OUESTIONNAIRES PEACE-KEEPING UFSI O5-II -02I86 /POINTS IN TIME I INTERNATIONAL-AFFAIRS POLITICAL-SCIENCE FOREIGN-POLICY THREAT-PERCEPTION SUEZ-CRISIS AT/ O5-OU-D2I7O CANACA I LAW INTERNATIONAL-ATFAIRS TARIFFS IMPORT-DUTIFS FOREIGN-TRACE COMMERCE I *PB- I7Z 660//JOURNAL - O5-D3-0I64I ND MEASUR ING FIRES I INFRARED-DETECTORS I AFRARED-TRACKING FOREST-FIRES PATROL-PLANES AIRCRAFT, *INT-25 IA I3-1:-UI/YB - AREAS CONFIGURATION URBAN-PLANNING EFFICIENCY SEL/ URBAN FORM AS A PASSIVE DEFEVSE VARIABLE I COSTS URBAN I 3 -D2 - D225 REAN-AREAS DECEVTRAL IZATION VULNERABILITY LANO-USEI URBAN FORM AS A PASSIVE DEFEVSE VARIABLE I ECONOMILS U 23 -DS-O2I O7 OF-DEFENSE DOCUMENTATION E/ KEY WCRDS AND THEIR USE ON DD FORM 14731 REPORTS SUBJECT-IVDEXING DEPARTMENT- OS-D2-D2I 24 1/ I HE SURFACE WAVES FROM SIIALLOW UMDERWATEP. EX.PLOSINNS - FORMULAE AND PROGRAM SPECIFICATIOVS I HYDRODYNAM I9-O4 - D2O 43 HOCK - TUBES CALCULATIONS EXPERIMEN I SHOCK KAVE ATTENUATION FORMULAS FOR STRAIGHT TUNVELS - A COMPARISUN I 3 ZO-OH-O2OSO TIC POSITION, GP, TO UNIVERSAL I/ COORDINATE CCNVERS ION FORMULAS FOR USE NITH WORLD WIDE 2 SSOURCES GEODE DS-D3 -D2I 33 5 AIR-BURST, A SUMMARY OF AIRBLAST EFFECTS ON HASTY FIELD FORT IFICATIONS I MATHEMATICAL-PREDICTION SHELTER IS-DS-DIOS? L-BLAST-EFFECTS BLAST-BIOLOGY SHOCK-TUI OASA AEC LCVELACE FOUNDATICN BLAST SIMULATIONFACIITY I BIOLOGICA OO-21-O2232 IS UNCERGROUND-STRUCTURES SAND SHOCK-WAVES BLAST FCOTINGS FOUNDAT IONS VOID-RATIOARCHES I \#VCEL-R-536 \$DAI 20-II-D234 I EC/ TRANSIEVT LOADING TESIS ON A RIGID CIRCULAR FOCTING 1 FOUNDATIONSISTRUCTJRES) SJIL-MECHAVIES LOADING IM I3-I3-OIBI KIMECHANICS) INSTRUMENTATION CALIBRATION ANALOG-CONPUTERS FOURIER-ANALYSIS, *ECOM-2647A *AD 632 927 /SHOC I4-0?-0I842 Y OF NUCLEAR EXPLOSION PRODU/ ON THE DETERMINATION OF THE FRACTIONATION FACTORS ANO BIOLOGICAL AVAILABILIT I8-D3-O232 T I RADIOACTIVE-ISOTOPES CHINA RUSSIA RADIO/ RADIONUCLIDE FRACTIONATION IN DEBRIS FROM LAVD SURFACE BURS I B-O?-DIO9I ( FRAGMEVTATIOV 1 LOADING (MECHANICS) CRACKING (FRACTURING) FRACTURE(MECHANICS) CRACK-PROPAGATION IMPAC T-TE / $20-11$-O2338 TUR INGI' AN EXPER IMENTAL. INVESIIGATI ON OF FRANGIBLE PLATE FRAGMENT AT ION 1 LOADING(MECHAVICS) CRACKING (FRAC 20-II -D2338 COMPUTE/ THE SECOND ORDER ELASTIC ANALYSIS OF PLANE RIGID FRAMES 1 STRUCTURES STRUCTURAL-PROPERTIES STEEL I3-I3-D2287 /CHANICS) STRUCTURES NONLINEAR-ANALYSIS BEAMS (STRUCTURALI FRAMES LINEAR-STRUCTURES I \#ASCE-1965-50 *ACI-SI 20-11-D2339

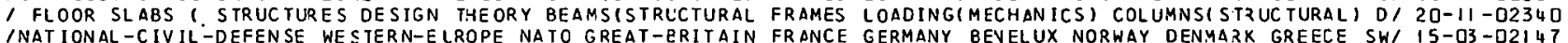

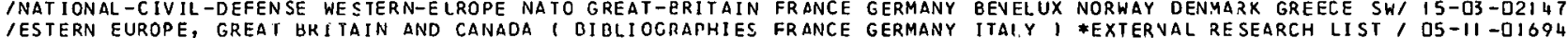

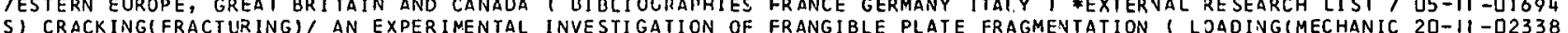
S) CRACK ING(FRACTURING)' AN EXPER IMENTAL INVESTIGATION OF FRANGIBLE PLATE FRAGMENTATION $($ LJADINGIMECHANIC 20- II - D2338 U COMPARISOVS AMONG MODES I/EFFECTS OF NUCLEAR DTTAC.K GN FREIGHT TRANSPORTATION SYSTEMS - IVTERACTIONS AN I3-DS-D22 OD IOADEC RIVG WITH RADIAL ELASTIC SLPPORT I RINGS VIERATION FREOUENCY BENDING ELASTICITY LOADINGIMECHANICSI/ $20-11$-O2336 ICHNICAL INFORMATION WITHIN THE DEFENSE INDUSTRY III. A. FREOUENCY DISTRIBUTIONS AND CORRELATION B. RELA' O5-D2-O2I2O IEVICES STRUCTURES VIBRATION-I SOLATORS MACHINES VIBRATION FREQUENCY PNEUMATIC-DEVICES FEASIBILITY-STUDIESI I3-13-Q229I IAC CANNED CEREALS COFFEE CORN DEHYDRATEO-FOODS EGGS FATS FRCZEN-FOODS FRUITS IRRADIATED-FOJDS MEAT MILK I D6-03-O22OQ INCTS I FOOD FALL OUT-SHELTERS TASTE CIVIL-DEFENSE-SYSTEMS FRUITS CONDIMENTS SUCROSE FODD-DISPENSING IESTSI O6-D3-D22II AL-AREAS COMMERCE MEAT RESOURCES CEREAL COTTON VEGETABLES FRUITS FOODS, WAGRICULTURE HANDBOOK NO. 325 /UR Q2-0?-OI6I9 IEALS COFFEE CORN DEHYDRATED-FOODSEGGS FATS FROZEN-FOODS FRUITS IRRADIATEC-FOODS MEAT MILK PORK POTATOESI D6-OB-O22O9 $100 L$ ING SYSTEM USING METHANOL. AS THE HEAT SINK ANO AS THE FUEL 1 FUELS COOLING-AVD-VEVTILATIVG-EQUIPMENT, I3-OI -OI773 5) TEST-EQUIPMENT, *PSWI TEMPERATURES IN A LARGE AATURAL FUEL FIRE I FIRES TEST-METHODS MODELSISIMULATION I3-I?-OIBIO ISYSTEM USING METHANOL AS THE HEAT SINK AND AS THE FUEL I FUELS COOL ING-ANC-VENTILATING-EQUIPMENT FALLOUTI I3-OI -OI773 7SYSTEM USING METHANOL AS THE HEAT SINK AND AS THE FUEL I FUELS COOL ING-ANC-VENTILATING-EQUIPMENT FALLOUT/ I3-DI - II773 IGHT MEDICAL-SUPPLIES ELECTRIC-PONER SANI TARY-ENG INEERING FUELS WATER-SUPPLIES MATHEMATICAL-ANALYSIS CLOT/ OS-D3-D2I 35
CESIGN COSTS/ EN TRANCEWAYS AND EXITS FOR BLAST RESISTANT FULLY BURIED PERSONNEL SHEL TERS I BLAST-SHELTERS I3-I3-OI835 CESIEN COSTS I EN TRANCEWAYS AND EXITS FOR BLAST RESISTANT FULLY BURIED PERSONNEL SHELTERS 1 BLAST-SHELTERS I3-I3-DI835 VELOCITY DEGREES-OF' SHOCK SPEC TRA FOR A GENERAL FORCING FUNCTION I SHOCK (MECHANICS) VI8RATIOV ELASTICITY $20-11$-Q2342

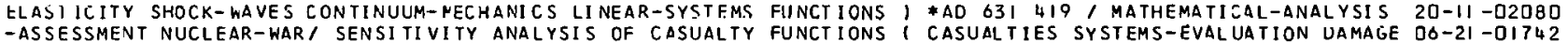

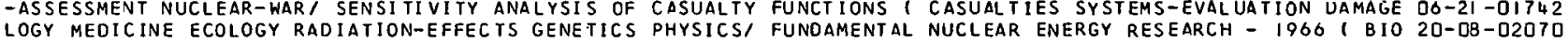
IUPPL EMENT, AUTHOR AND SUBJECT INDEXES I BACTERIA VIRUSES FUNGI PROTOZOA RICKETTSIA PHYSIOLOGY DIAGNOGIS I D6-I3-OITIS ORY-FURNACES/ THERMAL FLUX DISTRIBLTION IN AN ARC IMAGING FURNACE 1 THERMAL-RADIATIOV CALORIMETERS LABORAT I3-DI -O225S

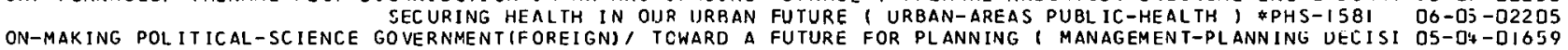

CUTTHROAT GAMES I OPERATICNS-RESEARCH GAME-THEORY） *RAC-P-20 *AD 638 220

ITA-TRANSMISSION-SYSTEMS SIMULATI CN INFORNATI CN-RETRIEVAL GANE-THEORY EDUCAT ION TRAIV IVG OPEZATIONS-RE SEA I O9-0? -DI75I IENT OF UT ILITY THEORY FOR ARMS CCNTROL AND DISARMAMENT I GAME-THEORY MAT HEYATICAL- YODELS SIMULATION I GF/ OS-D+ -D2ISB IC-WARFARE MILITARY/ STROP - A STRATEGIC PLANNING NODEL GAME-THEORY NUCLEAR-WARFARE MODEL-THEORY STRATEG I5-DS-DIQ59 MENT OT UT IL ITY THEORY FOR ARMS CCNTROI AND DISARMAMENT! GAME-THEORY SIMULATION, *FAR-396IIII)

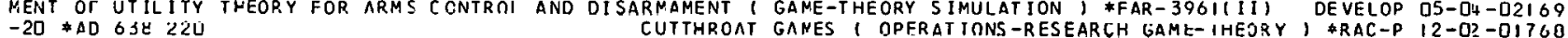

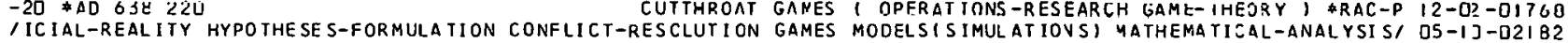
LATION DAMAGE-ASSESSMENT WARFARE RESOU' NREC PROGRAMS FOR GAMING THE LOGISTICS OF NATIONAL SURVIVAL I SIMU O5-O3-OIG 44 -SCATTER/ ENERGY AND ANGULAR OISTRIBUTION OF NEUTRCNS AND GAMMA RAYS - OPERATION BREN I IONIZING-RADIATION 20-O3-O2334 / CERGROUVD-STRUCTURES DOSE-RATE PHOTONS SCATTERING ALBEDO GANMA-RAYS ANGLE-OF-INCIDEVCE RADIATIJNICOBALTI/ I8-05-O2D29 / APONS-EFFECTS RADIOACTI VE-FALLOUT DISTRIBUTICN DCSE-RATE GAMMA-RAYS INSTRUYENTAT ION EXJERIMEVTAL-DATA, / I8-D3-O2324 I SERVICES UNITS I SHIELDING PATIENTS RADIOACTIVE-FALLOUT GAMMA-RAYS RAOIATION-MONITORS RADIATION-MEASURE/ I8-OS-D2O28 HAROENING DESIGN OYNAMIC-PRESSURE SHIELDING CALCULATIONS GARAGES, \#ORNL-TR-1646/TS GERMANY CONSTRUCTION I3-I3-OIB27 EALTH DISPOSAL COSTS WASTESISANITARY-ENGINEERINSI STORAGE GARBAGE PROJECT IONS I *A.L. 98I IEERIVG PUBLIC-H I3-D?-OI TB9 IATIVE MOT TON AND TENT GAS I TURBULENCE FLUID-DYNAMICS PARTIC

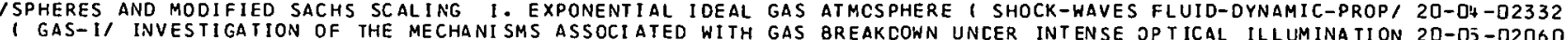
IONOITIOVS FOR SIMILAR SOLUTIONS OF PROBLENS OF TURBULENT GAS DYNAMICS UNCER INTENSE OPTICAL ILLUMINATION 20-DJ-O206D ITY INDUSTRY IN 1965 I UTILITIES RESERVES PRODUCTICN TRAI GAS FACTS - A STATISTICAL RECORD JF THE GAS UTIL O5-O3-OIO4
ITY IST ITY INDUSTRY IN 1965 I UTILITIES RESERVES PRODUCTICN TRA' GAS FACTS - A STATISTICAL RECORD OF THE GAS UTIL OS-O3-OI642
DUSTRIES ECOYOMICS/ U.S. SOURCES CF PETROLEUM AND NATURAL GAS STATISTICS I RESOURCES PETROLEUM-INDUSTRY IN O5-D3-DIG38 1 *A.L. IO/ UNIFORM SYSTEM OF ACCCUNTS FCR CLASS A AND B GAS UTILITIESI ECONOMICS ACCJUNTING REGULATIONS OS-O3-DIG32 S PROOUCTION TRA/ GAS FACTS - A STATISTICAL RECORD OF THE GAS UTILIIY INDUSTRY IN I965 1 UTILITIES RESERVE DS-O3-DIO42 ITERS FIRE-PROTECTION SMOKE CARBON-MONOXIDE OXYGEN-SUPPLY GAS-DETECTORS FIRE-DETECTORS CARBDN-MONOXIDE-INI I3-12-DIBD7 
IR-BALL ISTICS BIBLIOGRAPHIES DIGI TAL-COMPUTERS EXPLOSIONS GAS-DISCHARGES HEAT IGVITERS PROJECTILES MATHEM/ I9-DS-O2329 /RTICLES I BIOLOGICAL-WARFARE-AGENTS AEROBIOLOGY BACTERIA GAS-FILTERS MICROORGANISMS AIR-POLLUTION FILTER/ IS-D2-OI85I IBITION PRESSURE STRESSES PROJECTILES INTERIOR-BALLISTICS GAS-FLOW) *AD 60I 728 /N-BARREL-ATTACHMENTS INH IQ-0S-D2327 IIMIL AR SOLUTIONS OF PROBLEMS OF TLRBULENT GAS DYNAMICS 1 GAS-FLOW EQUATIONS-OF-STATE MATHEMATICAL-ANALYS/ 20-D+-O233I LITY BOUVDARY-V/ PLASMA STREAMING INTO A NAGNETIC FIELD I GAS-FLOW PLASMA-MEOIUM PLASMA-OSCILLATIONS STABI 20-O7-O2335 1 DISPERSION OF SOUND IN AN AEROSOL I ACOUSTIC-PROPERTIES GAS-FLOW PROPAGATION BIOLOGICAL-WARFARE-AGENTS / IS-0?-DI8BI I VENT ILATION HUMIDITY COOLING-AND-VENTILATING-EQUIPMENT GAS-FLOW TEST -EQUIPMENT I \#GARD REPORT I268-1 /S I3-OI -G2254 IETWEEN AIR FLOW ANO AIRBORNE SOUND IN A DUCT ( ACCUSTICS GAS-FLOW WEST-GERMANY DUCTS LAMINAR-BOUNDARY-LA' 20-OI -O2O4B I WITF GAS BREAKDOWN UNDER INIENSE OPTICAL I LLUMINATION 1 GAS-IONIZATION BEAYSIEL ECTROMAGNETIC) LASERS HE $/ 20-03$-O2OGO (1-) MODEL FOR D REGION RECOMBINATION I I CNOSPHERE OXYGEN GAS-IONIZATION, AIRBURST IONS POLAR-REGIONS ABSI OL -DI -D2II 3 /ECTORS RADIATION-MONITORS DOSIME TERS PROTECTIVE-CLOTHING GAS-MASKS PHOSGENE INCENDIARY-GELS FLAME-THROWEI O6-13-OI72I AGE-ASSESSMENT MDDELS NUCLEAR- LARFARE PE TROLEUM-PIPELINES GAS-PIPELINES TRANSPORTATION I \#NZEC-28 IY 1 DAM OS-D3-02I 4

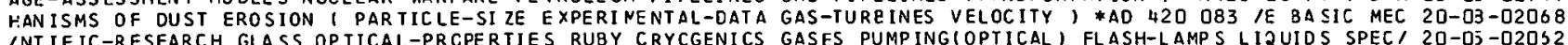
INT IFIC-RESEARCH GLASS OPTICAL-PRCPERTIES RUBY CRYCGENICS GASES PUMPINGIOPTICAL) FLASH-LAMPS LI2UIDS SPECI $20-05$-02032 /SUREMENT OF SEVERAL OPTICAL NONL INEARITIES USING FOCUSED GAUSSIAN LASER BEAMS I CRYSTALS OPTICAL-PHENOME/ 2D-DS -02067 1 I THEORETICAL. EROUND SHOCK EFFECTS II A APPLICATION OF GENERALIZED COORCINATES TO PLASTIC WAVE PROBLEMS O8- I3-OI747

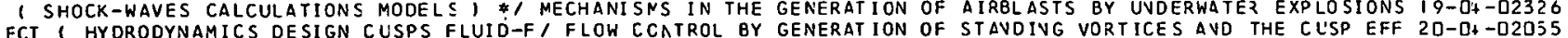

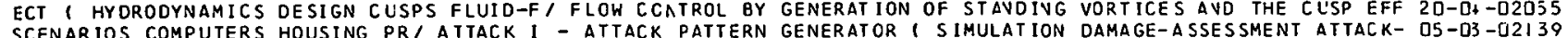
SCENARIOS COMPUTERS HOUSING PR/ ATTACK I - ATTACK PATTERN GENERATOR I SIMULATION DAMAGE-ASSESSMENT ATTACK- OS-D3 -OII39
SELTER LIGHTING KIT I FALLOUT-SHELTERS LIGHTING-EQUIPMENT GENERATORS PORTARLE DES IGN EXERCISE LIGHTSIILLU/ I3-OI-O2252 IELTER LIGHTING K IT I FALLOUT-SHELTERS LIGHTING-EQUIPMENT GENERATORS PORTARLE DES IGN EXERCISE LIGHTSIILLU/ I3-OI -O2252
IR SYSTEM FOR BUOY APPLICATIONS I THERMOELECTRICITY BUOYS GENERATCRS POWER-SJPPLIES ELEC TRIC-POWER-PRODUC/ IO-OZ-UI76I

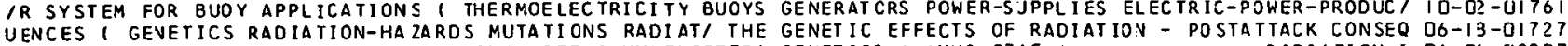
NCUCED VIABIL ITY MUTATIONS IN THE HONEY BEE I HYNENOPTERA GENETICS, \#NYO-23I5-I I CONFERENCE I NUTRITION (HUMAN) NUTRI TI ON (ANINAL) PROTEINS GENETICS AGRICULTURAL-ECONOMICS BIJCHEMISTRY I $/$ D6-I6-DI72O /ARCH - 1966 I BIOLOGY MEDICINE ECOLOGY RADIATION-EFFECTS GENET ICS PHYS ICS SCIENTIFIC-RESEARCH METALLURGY/ 20-O3-D2O7D /GENETIC EFFECTS OF RADIATION - POSTATTACK CONSEQUENCES I GENETICS RADIATIOV-HAZARDS MUTATIOVS RADIATION-I OG-IS-DI727 C. ANCREN IFORMIS - HYMENOPTERA, ANI A REVISION OF THE BEE GENUS CALLIOPSIS AND THE BIOLJGY AND ECOLOGY OF OO-OS-ODITO5 CATOR GRID 1 ARMY SUR VEYING MAPPING ENGINEERING ARTILLERY GECDESICS, \#M 5-24I-8 UVIVEZSAL TRAVSVERSE MER D8-OS-O224D ITE COVVERSION FORMULAS FOR USE WITH WORLD WIDE RESOURCES GEODET IC POSITION I GP, TO UVIVERSAL TRANSVERSI O5-03-O2I33

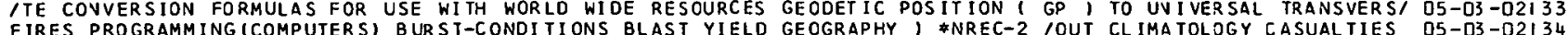
FIRES PROGRAMMING (COMPUTERS) B UR ST-CONDI TIONS BLAST YIELD GEOGRAPHY
OMPUT ERS) DATA-PROCESSING URBAN-AREAS MATHEMATICAL-MODELS GEOGRAPHY

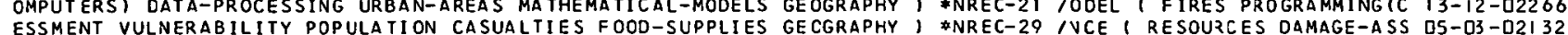
ESSMENT VULNERABILITY POPULATION CASUALTIES FOOD-SUPPLIES GECGRAPHY I \#NREC-29 /VCE I RESOURCES DAMAGE-ASS D5-03-D2I 32 E-ASSESSMENT POPULATION CASUALTIES PROGRANNING (CONFUTERS) GEOGRAPHY， \$NREC-30 /4 ( RESJURCES MODELS DAMAG Q5-03-O2I30 LANN ING RESOURCES INDUSTR IAL-PRODLCTI ON INDUSTRI AL-PLANTS GEOGRAPHY I \#NREC-40 I MOBILIZATIJN MANAGEMENT-P D5-O3-U2I 42 IT I BIBL IOGRAPHIES ECONOMICS EDUCATION POLITICAL-SCIENCE GEOGRAPHY HISTORY LANGUAGE RELIGION I \#EXTERNAL/ D5- II-OIG95 IPANS ION, RESTORATION AND CONVERSION I RE SOURCES NANPOWER GECGRAPHY INDUSTRIAL-PROCUCTIJN DAMAGE-ASSESSME I O5-O3-D2I59 IALLOUT VUCLEAR-EXPLOSION-DAMAGE POPULATI CNIOISTRIBUTION) GEOGRAPHY INDUSTRIES NUCLEAR-WARFAZE--ZASUALTIES/ I5-OS-DIGS? I FINANCE INDUSTR IES COMMERCE FRUT.ATION PCLI ICAL-SCIENCE GEOGRAPHY LANGUAGE MIL ITARY-AFFAIZS PHILOSOPHY I O5-II -DIO 93 ILIOGRAPHIES COMMUNISM ECONOMICS EDUCATI ON FOREIGN-POLICY GEOGRAPHY POL IT ICAL-SCIENCE HISTORY LANGUAGE LAI OS- II-DIG96 IHIES COMMUNISM ECONOMICS EDUCATICN INTERNATICNAL-AFFAIRS GECGRAPHY POL II ICAL-SCIENCE HISTOZY LANGUAGE LAI O5- II-DI 697 IRICA I ECONOMICS BIBLIOGRAPHIES EOUCATI ON FOREIGN-PCLICY GECGRAPHY POL IT ICAL-SCIENCE HISTORY LANGUAGE PH/ DS-II -DI692

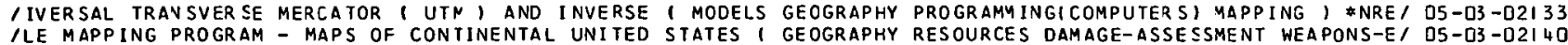
/LIEQUIPMENT) STR UCTURES COMMUNICATIONS-SYSTEMS RADIATION GEOLOGY HUMAN-ENGINEERING I \#SR-45 * VP- II T68-VO, IT-O2-U23II

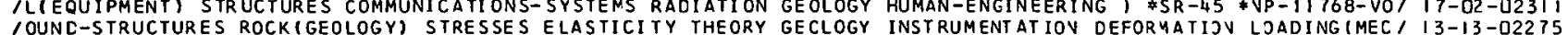
/MECHAVICS) STRESSES THEORY DEFORMATI ON EXPERINENTAL-DAT A GEOLOGY INSTRUMENTAT IOV GEOLOGY ODENINGS LNDERG O OB-OT-D22LL IRY DEFORMATION EXPERIMENTAL-DATA GE OLOGY INSTRUMENTATION GEOLOGY OPENINGS UVDERGROUND-STRUCTURES, *A.L. I O8-O7 -D2244 ILIMESTONE ROCK-SALT ANHYORITE SANDSTONES CLAY SILTSTONES GEOLOGY THERMAL-STRESS STRESSES STRAIV, FA.L., OB-OT-O2242 ICELS I RACIOACTIVE-FALLOUT MA THENATICAL-PREDICTION YIELD GECMETRY OISTRIBUT ION PARTICLE-SIZE HIND I USN/ IB-OB-O2O39 1 I CAL CULATIOVS PROGRAMMING (COMPLTERS) THERMAL-RADIATION GE CMETRY MATHEMAT ICAL-MODELS HEAT-TRANSFER WEAPI I3-I? -DI7O7

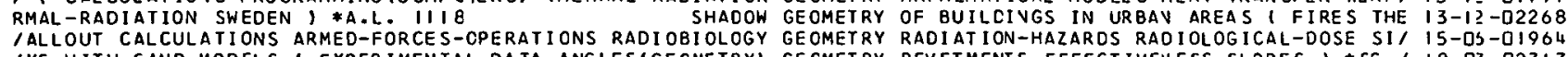
INS WITH SAND MODELS I EXPER IMENTAL-DATA ANGLES (GECMETRY) GECMETRY REVETMENTS EFFECTIVEVESS SLOPES I *SC- I I8-O3-023I? -MEASUREMENT-SYSTEMS PRO TECTION-FACTOR MONTE-CARLO-METHOD GECMETRY (CJCTS), INCEL-R-523 /SE-2ATE RADIATION O6-13-022I 9 ANS MENTAL-HEAL TH POSTA TIACK-OPERATI ONS TRAINING CHILDREN GERIATRICS, \#FG-E-13.2 /IL-OEFENSE-OPERA TING-PL O5-11-U2IB5 E-ASSESSMENT CASUALTIES POPULATI A STUDY OF WORLD WAR II GERMAN FIRE FATALITIES I FIRES URDAN-AREAS DAMAG I3-IZ-U2262 MOLLECTRICITY THERMAL-CUNUULTI TIT ELECTRICAL-CONDUCTANCE GERMANIUM, \#RSIC-523 *AD 631 224 / COOLING THER D9-O1-O1748 IED BY A CIVIL DEFENSE ORGANI ZATICN DURING WORLD HAR II - GERMANY I. I IIRES FIRESTJRMS HAYBUZG FIRE-FIGI I3-I?-D2265 IED BY A CIVIL DEFENSE ORGANIZATI ON DURI NG WORLD WAR II - GERMANY - I. I FIRES FIRESTJZMS THEZMAL-RADIAT' I3-I?-Q2264 QUESTIONVAIRES I/ OCCASIONAL REPORTS ON FOREIGN AFFAIRS - GERMANY I PUBLIC-OPINION SURVEYS FOREIGN-POLICY D5-II -D2IR6 IL-CIVIL-DEFENSE WESTERN-EUROPE NATO GREAT-BRITAIN FRANCE GERMANY BENELUX NORWAY DENMARK GREECE SWEOEN SW' IS-03-O2IL 7

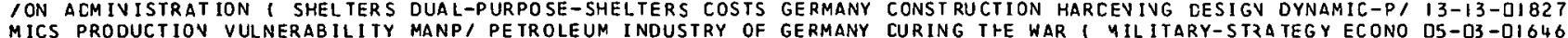
MICS PRODUCTIOV VULNERAB ILI TY MANP/ PETROLEUM INDUSTRY OF GERMANY CURING THE WAR I YILITARY-STZATEGY ECONO D5-D3-DI646
EUROPE, GREAT BR ITAIN AND CANADA I BIBLI CGRAPHIES FRANCE GERMANY ITALY, \#EXTERNAL RESEARCH LIST 3.25 IRN O5-II -OIO94

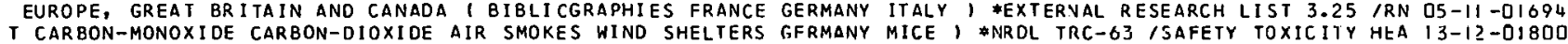
T CARBON-MONOXIDE CARBON-DIOXIDE AIR SMOKES WIND SHELTERS GFRMANY MICE I \#NRL TRC-63 $15 A F E T Y$ TOXICIIY HEA I3-I2-OI8OD
RANSPORTA' EXTENSIVE SIREET BUILDING PROGRAM IN HANBURG 1 GERMANY ROADS CONSTRUCTION URBAN-AREAS TUNNELSIT IS-UZ-OI786 PT ICAL) CAVITY-RE SONATORS UPTICS CRYST, INVESTIGATIONS OF GI ANT PULSING METHOOS IN RUBY LASERS 1 PUMPING IO $20-05-020 S 6$ IS OF THERMAL RADIATION RELEASED IN NUCLEAR DETONATIONS I GLASS ATTENUATION URBAV-AREAS IGNITION, \#A.L. , I3-I?-OIT96 IURES I CONCRETE UNDERGROUND-STRUCTURES EXPANDED-PIASTICS GLASS HONEYCOMB-CORES LOADINGIMECHANILSI SHOCKI/ I8-D3-D2OD7 IN HIGH PUWER LASER RESEARCH I LASERS SCIENTIFIC-RESEARCH GLASS OPTICAL-PRCPERTIES RUBY CRYJGEVICS GASES 1 2D-05-D2062 IS 1 SECONCARY-MISSILES BLAST-ENERGI ZED-MISSILES VELOCITY GLASS STONE BLAST EXPER IMEVTAL-DATA IVSTRUMENTA I IB-O3-O23I 8 I DES IGN STRENGTH STRUC TURAL-PROPERTIES QUALITY FASTENERS GLUING PLYHOOD SAVDWICH-CONSTZUCTION FIRE-RESISI II-IZ-OI7O6 HOCK-WAVES BIOLOGICAL-BLAST-EFFECTS RATS GUINEA-PIGS DOGS GOATS SHOCK-TUBES, \$DASA- $1852,13 L A S T-B I O L O G Y$ S O6-21 -02236 ION I STATISTICAL-TESTS STATISTIC, THE CHI-SOUARE TEST OF GOODNESS OF FIT FOR A 8 IVAR IATE NORMAL DISTRIBUT I2-DI -D225I ES DEPARTMENT OF AGR ICULTURE I LEGISLAII ON / / REGULATI ONS GOVERNING THE MEAT INSPECTION OF THE UNITED STAT O2-D2-OIOZO ES DEPARTMENT OF AGR ICUL TURE I LEGISLATI ON I REGULATIONS GOVERNING THE MEAT INSPECTION OF THE UNITED STAT O2-Q2 - DI O2O AR BURSTS I BUR ST-CONDITIONS URBAN-AREAS FIRE/ PARAMETERS GOVERNING URBAN V'JLNERABILITY TO FIRE FROM NUCLE I3-I?-OIBDI ON COMMUVICATIONS BANKING CONSTRUCTI ON MAINTENANCE REPAIR GOVERNMENT CASUALTIES CAPACITY 1 QVREL- 17 IRTATI O5-03-O2I53

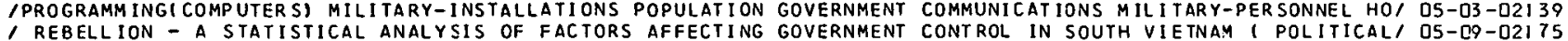

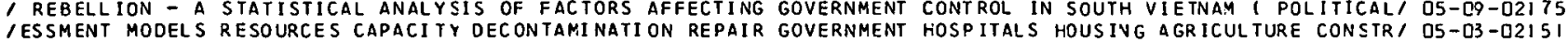
EIGN STUDEN/ CROSS CULTURAL EDUCATION - A BIBLIOGRAPHY OF GOVERNMENT SPONSORED AVD PRIVATE RESEAREH ON FOR OS-II -DIG9I ON CIVIL-DEFENSE (GOVERNMENT-AGENCIES) AIR-FORCE ARMY NAVY GOVERNMENT-AGENCIES, \#A.L. 986 /CIES) LEGISLATI I5-03-0I946 /VULNERABILITY MILITARY-FACILI IIES ARMED-FORCES-SUPPLIES, GOVERNMENT-FACILITIES COMMUNICATIONS-FACILITIES/ OS-03-O2I 37 1 I MANAGEMENT-PL ANN ING DECISI ON-NAKING POLI TICAL-SCIENCE GOVERNMENT (FORE I EN) SOCIAL-COMMUNICATION LEADER/ O5-D+ - DI 599 IAS FOR USE WITH WORLD WIDE RE SOURCES GEOOETIC POSITION ( GP ) TO UNIVERSAL TRANSVERSE YERCATOR I UTM I A I OS-03-O2I 33 N-EFFECTS NUCLEAR-EXPLOSIONS EQUATIONS TUFF ALLUVIUM SALT GRANITE MATHEMAT ICAL-ANALYSIS I \#UCRL-5O2O3 ISIO I8-03-TO3I5 IRATERING EXPLOSION-EFFEC TS NUCLEAR-EXPLOSIONS SEISMCLOGY GR ANITC MATMEMAT ICAL-AVALYSIS UNDERGROUND-EXPLO/ I 8-D3 - D23I 6 /TRUC TUKES FAILURE(MECHANICS) MINING-ENGINEERING STRESSES GRANITE OPENINGS BLOCK-CAVING-MINING BLASTING /, OB-D7-02243 IOVERY DAMAGE RECLAMATION URBAN-AREAS MODELS (SIMULATIONS) GRAPHICS TABLES MICHIGAN, 1 STATIST ICAL-AVALYSIS WEAPONS-EFFECTS HIROSHIMA NAGASAKI GRAPHS BIOLOGICAL-BLAST-EFFECTS BURVS RADIATION/ OL-2I -02227

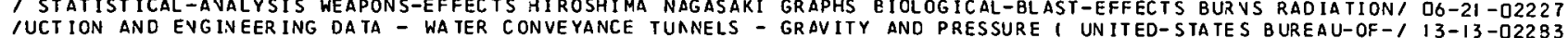

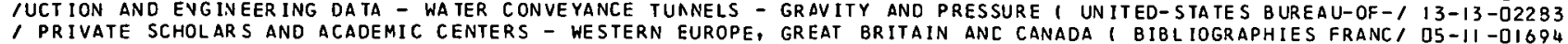
1-SYSTEMS INTERNATIONAL-CIVIL-DEFENSE WE STERN-EUROPE NATO GREAT-BRITAIN FRANCE GERMANY BENELUX NOR WAY DEN/ IS-D3-D2I 47 
DEFENSE-SYSTEMS EA/ CIVIL DEFENSE IN THE UNITED KINGDOM I GREAT-BRITAIN INTERNATIONAL-CIVIL-DEFENSE CIVIL- I5-D3-O23O5 INATO GREAT-BR I TA IN FRANCE GERMANY BENELUX NORWAY DEMMARK GREECE SWEDEN SWIIZ ERL AND IREL AND USSZ INDIA AUI I5-03-D2I 47 ERY GEODESICS, TM 5-241-8 UNIVERSAL TRANSVERSE MERCATOR GRID I ARMY SURVEY ING MAPPIVG ENGINEERING ARTILL O8-OS-D224D / EUROPE FOOD-MARKETING RUSSIA AFRICA LATIN-AMERICA JAPAN GROSS-NATIONAL-PRODUCT CONSUMPTIOV(FOOD) COMMERI O2-D?-DIOI 6 INT IAL DEMAND, EMPLOYMENT I INDUSTRIES MANPOWER ECCNOMICS GROSS-NATIONAL-PRODUCT SUPPLY-AND-DEMAND INDUST/ OS-D3-OIGL 9 IALYSES FOR PRD TECTIVE STRUC TURES LOCATED ABOVE AND BELOW GRCUND I FALL OUT -SHELTERS COMPUTEZS BLAST-SHELTI I3-OI -OI776 AIR BLAST I ELASTIC/ THEORETICAL GROUND SHCCK EFFECTS I. GROUNC MOTION IN ELASTIC HALF SPACES INDUCED BY I9-D $\$$-O2OL4 INT LOCATED IN UNDERGROUND INSTALLATI ONS TO BLAST INDUCED GROUND MOT IONS 1 UVOERGROUND-STRUCTURES EXPLOSI I I 3 - I3-O229I ACHINE I NUCLEAR-WEAPONS SHOCK-WAVES MECHANICAL/ STUDY OF GROUND MOTIONS FOR SIMULATION BY SHOCK TESTING M $20-11-$-U2O78 C HALF SPACES INDUCED BY AIR BLAST / ELASTIC/ THEORETICAL GROUND SHOCK EFFECTS I. GROUVD MOTIOV IN ELASTI I9-O+ -D2OL 4 IZEC COORDIVATES TO PLASTIC WA VE PROBLEMS 1 , THECRETICAL GRCUND SHOCK EFFECIS II. APPLICATION OF GENERAL DB- $13-0 I 747$ URBAN-AREAS EXCAVATION, \#PB/ BIBLI OGRAPHY OF HIGH SPEED GROUND TRANSPORT I TRAVSPJRTATION BIBLIOGRAPHIES 23 -0? -O2OQ3 NALS SCHEDULING/ SUMMARY OF RE SEARCH AT MIT ON HIGH SPEED GROUND TRANSPORT I TRAYSPORTATION NETWORKS TERMI $23-$ OP -D2354

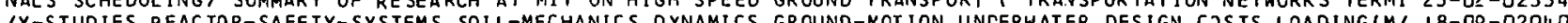
TY-STUOIES REACTOR-SAFETY-SYSTEMS SOIL-MECHANICS OYNAMICS GROUNO-NOTION UNCERWATER DESIGN CJSIS LOADINGIMI I8-O9-O2OLO SIES RAILROADS RRIDGES GRCUND-SUPPORT-ECJIPMEVT RAIL POAD-TRACKS ROADS / 16-0I -01972 /CIETAL RECOVERY FROM NUCLEAR ATTACK I STRESS (PSYCHOLOGY) GROUP-DYNAMICS SOCIAL-PSYCHOLJGY BEHAVIOR DISAS/ OS-1J-0I677 IEARCH I SOCIAL-SCIENCES SURVEYS BIBLIOGRAPHIES ATTITUDES GROUP-DYNAMICS SOCIAL-PSYCHOLOGY PUBLIC-OPINION/ OS- II -O2I 94
NS PROFESSIOVS MANPOWER LABOR POLITICAL-SCIENCE EOLCATION GROUP-OYNAMICS THEORYMETHODOLOGY,

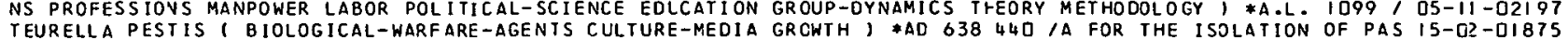
NNING TRAINING SHELT/ AN EXPERIMENTAL STUDY OF INTEGRATED GUIDANCE FOR SHELTER MANAGEMEVT I MAVAGEMENT-PLA OS-OI -OIG26 ILITY SHEL TER-OCCLPANCYI STUDIES CF IN SHELTER MANAGEMENT GUIDANCE MATERIALS 1 MANAGEMEVT-PLANVING HABITAB O5-OI -DIG 24 NG 1 BASIC COUR SE IN EMERGENCY MASS FEEDING - INSTRUCTORS GUIDE I KITCHEN-EDJIPMENT-AND-SUPPL IES STOCKPILI O6-OS-DI7I 3 IRATEGY BOMBERS ANTIMISSILE-DEFEN SE-SYSTENS SPACE-WEAPONS GU IDED-MISS ILE-DEFENSE-SY STEMS SUBMARINE-LAUNCHI IS-O7-D23 J8 / CHINESE COMMUNIST SCIENTIFIC AND TECHNICAL LITERATURE 1 GU IDED-MISS ILES ABSTRACTS SPACECRAFT TRANSPORTA/ I6-OI - DI973 OC CHE/ CIVIL DEFENSE HANOBOOK - LSSR I RLRAL-AREAS BOMBS GUIDED-NISS ILES NUCLEAR-WEAPOVS COVIAMINATION FO I5-O3-O2296
IVIET LITERATURE ON PROTEC TIVE STRLCTURE AND COMPCAENTS ( GU IDED-MISSILES RAILROADS BRIDGES GRJUND-SUPPOR/ I6-OI -OI972 ABLI A STUDY OF THE DEMOGRAPHY OF NUC LEAR WAR - EMFIRICAL GUIDELINES FOR THE SELECTION JF OEMOGRAPHIC VARI $23-0+-02 I J 3$ ON URBAN-AREAS, AERODYNAMIC ORAG ON VEHICLES IN ENCLOSED GU IDEWAYS I TUNNELSITRANSPORTATIOY TRANSPORTATI 23-O2-O2I DI I BLAST-BRL L-AEROSOLS EXPOSURE VACCINES DOSAGE I MMUNI TY NCNKEYS MICE GUINEA-PIGS HUMANS I $\$ A D$ G35 744 IGEVTS BAC TERIA I5-O2-DI85B OR-BALLISTICS, \#AD III 482 MUZZLE BRAKES II. THEORY I GUN-AUXILIARY-EQUIPMENT RECOIL-MESHAVISMS INTERI I 9-OS-D232B $15 M M$ AND $155 M M$ HOWITZERS I SHOCK- WAVES ATTENUATION DESIGN GUN-BARREL-AT TACHMENTS INHIBITION PRESSURE STREI I9-OS-D2327 IHE ANALYSIS OF MUZZLE BRAKE DESIGN I ARTILLERY CONPUTERS GUN-BARREL-ATTACHMENTS INTERIOR-BALLISTICS BIBL/ IO-OS-D2329

H

THOCOLO/ RESEARCH DATA FROM SHELTER OCCUPANCY EXERCISES I HABITABILITY DATA-PROCESSIVG-SYSTEMS TRAINING ME DS-IJ-OIGBO ISTON, ILLIVOIS I ENVIRONMENTAL-CONTROL EXPERIMENTAL-DATA HAEITABILITY FANS PORTABLE IVSTRUMEVTATION PUNK/ I3-QI -QIT7I POSURE I FALLOUT-SHELTERS FIRE-PRCTECTION SMOKE I SHELTER HABITABILIIY IN EXISTIVG BUILDINGS UVDER FIRE EX I3-I? -OIBD? 1 ICAGO, INDIANA I ENVIRONMENTAL-CONTROL EXPERIMENTAL-DATA HABITABILITY INSTRUMENTATION TEMPERATURE PUNKAH/ I3-DI - I T74 IED-ENVIRONMENTS STOCKPILING SHEL TER-MANAGEMENT HANDBOOKS HABITAEILITY NUTRITION SAVITAZY-EVGIVEERING MED/ OS-IO-O2I BL ILTER MANAGEMENT GUIDANCE MATERIALS ( MANAGENENT-PLANNING HABITABILITY SHELTER-OCCUPAVCY INSTRUETION-MANU/ DS-DI -OIG24
ICAL EVALUATION OF SHEL TER EOUIPMENT AND PROCEDURES III. HABITABILITY STUCIES I FALLOUT-SHELTEZS SYSTEMS/ IS-O3-O2298 ILTER MANAGEMENT, ENVIRONMENTAL THREAT, AND SMALL SHELTER HABITABILITY UNDER CONDITIONS OF STRESS I SHELTI OS-IJ-OIOB IETICAL GROUND SHOCK EFFECTS I. GROUND MOTION IN ELASTIC HALF SPACES INDUCED BY AIR BLAST I ELASTICITY SI I9-D4-O2O44 IET ICAL GROUND SHOCK EFFECTS I. GROUND MOTION IN ELASTIC HALF SPACES INDUCED BY AIR BLAST I ELASTICITY SI I9-D4-D2O44
ICT IVE-FALLOUT RADIOACTIVE-DECAY PARTICLE-SI ZE HENRYS-LAW HALF-LIFE DISTRIEJTION COMPUTERSIPRJGRAMMING) YI I8-OB-OO2O3? ICT IVE-FALLOUT RADIOACTIVE-DECAY PARTICLE-SI ZE HENRYS-LAW HALF-LIFE DISTRIEUTION COMPUTER S(PR JGRAMMING) YY I8-O3-O2O37
FALLOUT THERMOOYNAMICS SOLUBILITY TEMPERATURE DIFFUSIVITY HALF-LIFE YIELD I *GA-7OS8 /E-DECAY RADIOACTIVE- I 8-O3-D2325 FALLOUT THERMOOYNAMICS SOLUBILITY TEMPERATURE DIFFUSIVITY HALF-LIFE YIELD I *GA-7D58 IE.-DECAY RADIOACTIVE- I8-O3 -D2325 TUNNELSITRANSPOR TA' EXTENSIVE STREET BUILDING PROGRAM IN HAMBURG I GERMANY ROADS CONSTRUCTION URBAN-AREAS I3-O2 - QI7B6
IION CURING WORLD WAR II - GERMANY I. I FIRES FIRESTORMS HAMBURG FIRE-FIGHTING-EQUIPMEVT URBAN-AREAS THEI I3-I2-O2265 OMBS (WEAPONS-EFFECTS) URBAN-AREAS FIRE-FIGHTING-EQUIPMENT HANBURG PHOTOGRAPHS, *A.L. II22(II) /CENOIARY-B I3- I?-O2264 LES NUCLEAR-WEAPONS COVTAMINATION FOOD CHE/ CIVIL CEFENSE HANDBOOK - USSR I RURAL-AREAS BOMBS GUIDED-MISSI IS-O3-02296 AL-PROPERTIES QUALITY FASTENERS GLUING PLYWOOD SANCI WOOD HANDBOOK 1 CONSTRUCTION DESIGV STRENGTH STRUCTUR II-I?-DI7SG FARM CHEMICALS HANDBOOK
CULTURAL-CHEMISTRY, FERTILIZERS PESTICIDES EQUIPMENT AGRI O2-OI -DIOIS
ISPENSING FOOD I BASIC COURSE IN EMERGENCY MASS FEEDING - HANDBOOK 1 KITCHEN-EQUIPMEVT-AND-SUPPLIES FOOD-D O6-OB-OI7I 2 SHELTER OCCUPANCY STUDIES 2. CONMUNI TY FALLOUT SHELTERS HANDBOOK FOR UNTRAINED MAVAGEYENT I SHEL TER-MANA OS-DI - O2I IS ION AGR ICULTURE URBAN-AREAS RURAL-AREAS COMMERCE MEAT REI HANDBOOK NO. 325 /URAL-AREAS COMMEREE MEAT RE SOU O2-D? - II6I 9 -HAZAROS DECONTAMINATION RADIAIION-EFFECTS, \#ASRICULTURE HANDBOOK 33 IACTIVE-FALLOUT SHIELDIVE RADIATION I5-D3-DIOIO ANNINC IERS CONFINED-ENVIRONMENTS STOCKPILING SHELTER-MANAGEMENT HANDBOOKS HAB ITARIL ITY NUTR ITIDN SAVITARY-ENGIN/ OS-IJ-O2I

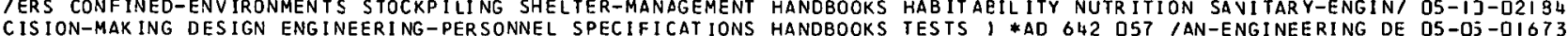

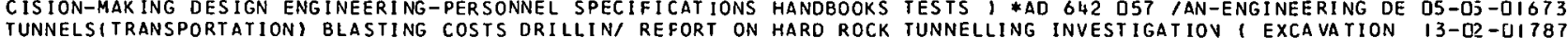
ISOLATORS IMPACT-SHOCK NUCL/ SIUDY OF SHOCK ISOLATION FOR HARDENEC SIRJCTURES ISHOCK-ABSORBERS VIBRATION- I3-13-DI823

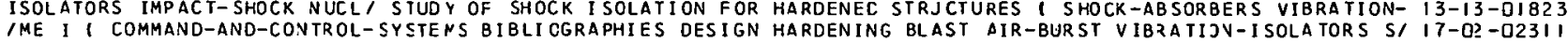
PLIFYING THE EVALUATION OF HARDENING COSTS I/ INDUSTRIAL HARDENING CLASSIFICATION - A YETHDDOLOGY FOR SIN O5-D3-02I26 PLIFYING THE EVALUATION OF HARDENING COSTS I/ INOUSTRIAL HARDENING CLASSIFICATION -. A METHODOLOGY FOR SIM OS-D3-02IG2 AL AREAS I NUCLEAR-EXPLOSIONS FIRES AIRBURST STR/ THERMAL HAROENING CONSIDERATIOVS PERTAINIVG TO RESIDENTI I5-OG-OIQS3 I CATION - A METHDDOLOGY FOR SIMPLIFYING THE EVALUATICN OF HARDENING COSTS I. TEXT AVD APPEVDIX A I INDUS/ O5-D3-O2IS2 ICATION - A YETHODOLOGY FOR SIMPLIFYING THE EVALUATION OF HARDENING COSTS II APPEVDIX B I INDUSIRIAL-EQ/ OS-O3 -02I 26 INAL-CIVIL-DEFENSE RADIOLOGICAL-CONTAMINATION RURAL-AREAS HARDENING PROTECTION-SYSTEMS I $\$$ SRS-27, D3O CALI TS-IB-DI73 NS

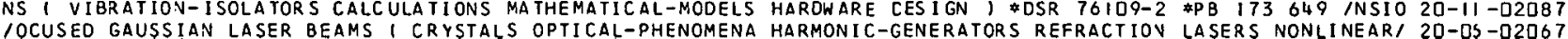

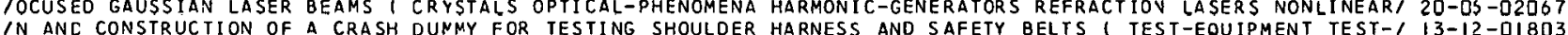
IN ANC CONSTRUCTION OF A CRASH DUNMY FOR TESTING SHOULDER HARNESS AND SAFETY BELTS 1 TEST-EQUIPMENT TEST-I I3-I2-DI8D3
TION SHELTERS A IR-8UR ST/ A SUMMARY OF AIRBLAST EFFECTS ON HASTY FIELD FORTIFICAT IONS I YATHEYATICAL-PREDIC I5-DS-OIO5? TION SHELTERS AIR-8URST A SUMMARY OF AIRBLAST EFFECTS ON HASTY FIELD FORTIFICATIONS I YATHEYATICAL-PREDIC I5-DS-OI957 I USSR INTERNATIONAL-CI VIL-DEFENSE CI VIL-DEFENSE-SYSTEMS HASTY-SHELTERS COVSTRUCTIOV VUCLEAZ-EXPLOSION-D/ $13-13-0 I 826$
ITIAL OF AN EMERGENCY BLAST SHELTER PROGRAM I URBAN-AREAS HASTY-SHELTERS DES IGN YATER IALS EQUIPMENT LABOR/ I3-I3-D2272 LIMITING-SYSTEMS EVACUATION VULNERABILITY WARNING-SYSTEMS HASTY-SHELTERS THREAT-EVALUATION I 4 HI-486/3-RR I3-13-DIB29 THEAST-CORRIDOR COSTS URBAN- 1 A SYSTEMS ANALYSIS OF SHORT HAUL AIR TRANSPORTATION 1 PASSENGER-VEHICLES NOR $23-02-02094$ ULAT ION FIRE-SAFETY TOXICITY HEAT CARBON-1 MASS FIRE LIFE HAZARD? NJCLEAR-EXPLOS IOVS FIRES CASUALTIES SIM I3-I?-OIBOD ACK GROUND AND PROCEDURES FOR APPLICATIONS/ NUCLEAR ATTACK HAZARD IN CONTINENTAL UNITED STATES - 1963 A. 8 I5-D3-DI949 TARY-ENGINEER ING DESIGN COOLING-AND-VENTILATING-EQUIPMENT HAZARDS COSTS, \#AD $631442 / L O S I O N-E F F E C T S$ SANI I3-13-0I8II CTIVITY DETECTION RADIOLOGICAL-DOSAGE EXPOS/ RAOIOLOGICAL HEALTH AND CIVIL DEFENSE I HEAL TH-PHYSICS RADIOA OG-I8-O22?? OT IONS ATTITUDES FEAR STRESSIPSYCHOLOGY CASUALYI/ MENTAL HEALTH IMPLICATIONS IN CIVILIAN EMERGENCIES I EN O5-IJ-OIOSI HEALTH 1 PHS- I58I SECURING HEALTH IN OUR URBAN FUTURE I URBAV-AREAS PUBLIC- OO-O5-U22J5 YCHOLOGY AGIVG/ BIBL 1OGRAPHY OF PLBLICATICNS, 1941-19601 HEALIH PUBLIC-OPINION WELFARE-SERVICES SOCIAL-PS OS-1I-O2197

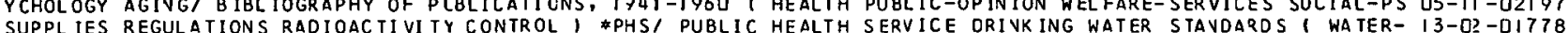
SUPPL IES REGULATIONS RADIOACTIVITY CONTROL 1 \#PHS/ PUBLIC HEALTH SERVICE DRIVK ING WATER STAVDAQDS 1 WA TER- I3-O2-DI778
ADVANCED TREATMENT CENTRE - OPERATING MANUAL - EMERGENCY HEALTH SERVICES I DISASTER-MEDICINE MEOICINE ADM O6-2I -DI738 LIC-HEALTH LAW ORGANIZATIO/ ROLE OF THE FEDERAL EMERGENCY HEALTH SERVICES CIVISION 1 DISASTER-MEDICINE PUB DG-2I-D2226 A' PROTECTIOV AGA INST NUCLEAR WEAPON FALLOUT IN EMERGENCY HEALTH SERVICES UVITS 1 SHIELDING PATIENTS RADIO IB-OS-O2O2B /L-PSYCHDLOGY PUBLIC-OPINION METHODOLOGY THEORY EDUCATION HEALTH WELFARE-SERVICES OCCUPATIOVS PROFESSIONS/ O5-II-D2I 44
CAL-DOSAGE EXPOS/ RADIOLOGICAL HEALTH AND CIVIL DEFENSE 1 HEALIH-PHYSICS RADIOACTIVITY DETECTION RADIOLOGI D6-I3-O2222 
/LOSIONS FIRES CASUALTIES SIMULATION FIRE-SAFETY TCXICITY HEAT CARBON-MONOXIDE CARBON-DIOXIDE AIR SMOKES / I3-I2-0IBOO IRONMENTS I HEAT-ENGINES DESER TS FEASIBILIIY-ST/ A SIMPLE HEAT ENGINE OF POSSIBLE UT ILITY IV PRIMIIIVE ENV I3-O? -OI77? /BL IOGRAP HIES DIG ITAL-COMPLTER S EXPLOSI ONS GAS-DISCHARGES HEAT IGNITERS PROJECTILES MATHEMATICAL-ANALYSI S/ I9-0S-O2329 (SHELTER-OCCUPANCY METABOLISM PHYSI/ SENSIBLE AND LATENT HEAT LOSSES FROM OCCUPANTS OF SLRVIVAL SHELTERS D6-1S-DI7IB ( HEAT-PROCUCTION (BIOLOGY) SURVIVAL', SENSIBLE AND LATENT HEAT LOSSES FROM OCCUPANTS OF SURVIVAL SHELTERS DO-16-DI7/9 IICAL ASPECTS OF SHELTER VENTILATION (STRESS (PHYSIOLOGY) HEAT MOISTURE TEMPERATURE ENVIRONYEVTAL-CONTROL, I3-OI-O2253 ' STUCY OF A SHEL TER COOLING SYSTEM USING METHANOL AS THE HEAT SINK AND AS THE FUEL 1 FUELS ZOJLING-ANO-VI I3-DI -QI773 BTROPICAL CLIMATE I AGING (PHYSIOLCGY) AGE IROPICAL-REGIO/ HEAT TOLERANCE OF ELDERLY PERSONS LIVING IN A SU O6-I I-DI734 $Y$ OF LONG RANGE BALLISTIC MISSILES I ATMOSPHERIC-ENTRY R/ HEAT TRANSFER ASPECTS OF THE ATMOSPHER IC RE-ENTR 22-D2-02350 IT ENGINE OF POSSIBLE UTILITY IN PRIMI TI VE ENVIRONNENTS I HEAT-ENGINES DESERTS FEAS IBILITY-STUDIES SOLAR- 13 I 3 -D? -DI777

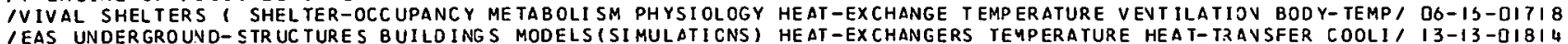
/L-RACIATION CALORIMETERS LABORATCRY-FURNACES RADICMETERS HEAT-FLUX HEAT-TRAVSFER PYROMETERS HIGH-TEMPERA/ I3-OI -02255 IL-RACIATION CALORIMETERS LABORATCRY-FURNACES RADICMETERS HEAT-FLUX HEAT-TRAVSFER PYROMETERS HIGH-TEMPERA' I3-OI -D2255
/LATENT HEAT LOSSES FROM OCCUPANTS OF SURVIVAL SHELTERS ( HEAT-PRODUCTION (BIOLOGY) SURVIVAL FALLOUT-SHELT/ D6-I6-0I7IQ /LATENT HEAT LOSSES FROM OCCUPANTS OF SUR VIVAL SHELTERS I HEAT-PRODUCTION (BIOLOGY) SURVIVAL FALLOUT-SHELT/ O6-16-0I7I9
SYSTEMS COSTS FAULT-LOCATION ELEC TRIC-CABLES TRANSFORMERS HEAT-TRANSFER I \$3I-C-35/IDEVTIAL-DISTRIBUTION- O9-O3-OI753 SYSTEMS COSTS FAULT-LOCATION ELEC TRIC-CABLES TRANSFORMERS HEAT-TRANSFER 1 \#3I-C-35 /IDEVTIAL-DISTRIBUTION- O9-O3-OI753 IUIL.D INGS MODEL SI SIMULATIONS) HEA T-EXCHANGERS TEMPERATURE HEAT-TRANSFER COOL ING-AND-VEVTILATING-EQUIPMENT/ I3- 13 -018I 4 I SMOKES BUILDINGS CONSTRUCTION BLAST RADIOACTIVE-FALLOUT HEAT-TRANSFER EXPOSURE TOXICITY CONSTRUCTION-MA/ I3-I?-D2269 ITER IALS CHEMISTRY MATHEMATICS CONPUTERS NUCLEAR-REACTORS HEAT-TRANSFER NUCLEAR-REACTOR-MATERIALS FLUID-D/ 2D-D3-D2O70 IN CALORIMETERS LABORATORY-FURNACES RADI OMETERS HEAT-FLUX HEAT-TRANSFER PYROMETERS HIGH-TEMPERATURE-RESEA I 3 -DI -O2255 /RGROUND PROTECTIVE STRUC TLRES WI TH OBSERVED CONDITICNS I HEAT-TRANSFER UNEERGROUND-STRUCTURES SHEL TERS T/ $20-13$-02O9O IOMPUTERS) THERMAL-RADIATION GEOMETRY MATHEMATICAL-MOOELS HEAT-TRANSFER WEAPONS-EFFECTS SHIP-STZ UCTURAL-C/ I3-I?-DI797 /N-MONOXIDE-INDICATORS TEMPERA TURE WIND-PRESSURE PRESSURE HEAT-TRANSMISSION TESTSIFIRES) SURVEYSIDATA-COL/ I3-I?-OI8O7 /IRONMENTAL-COVTROL VENTILATION ELEC TRIC-POWER-PRODUCTION HEAT ING COOL ING LIGHTIVG WASTESISAVITARY-ENGINE I I3-I3-D227 IPHER IC-ENTRY REENTRY-VEHICLES DE SIGN AERCDYNAMIC-HEATING HEAT ING COOLING-SYSTEMS FLUID-DYNAMIC-PROPERTIE/ 22 -02-D2350 ITERS BLAST STRESSES UNDERGROUND-STRUC TURES ROCK (GEOLOGY) HEAT ING MINING-ENG INEER ING LOADINGIMECHANICS) C I I3- $13-02273$ IUMINATION I GAS- IONIZATION BEAMS (ELECTRONAGNETIC) LASERS HELIUM-GROJP-GASES PLASMA-MEDIUM LUMINESCENCE A' $20-05-02060$ PRODUCTS IN A SIL ICATE FALLOLT PARTICLE MATRIX I RADIOAC/ HENRYS LAW CONSTAVTS FJR DISSJLUTION OF FISSION I8-03-O232S
ION I RADIOACTIVE-FALLOUT RADI OACTIVE-DECAY PARTICLE-SIZE HENRYS-LAW HALF-LIFE DISTRIBUTION COMPUTERSIPROI I8-O3-O2O3? ION I RADIOACTIVE-FALLOUT RADI OACTIVE-DECAY PARTICLE-SIIE HENRYS-LAW HALF-LIFE DISTRIBUT ION COMPUTERSIPRO/ I8-O3-O2037
IANC UNCLASSI IFIED LITERATURE PERTAINING TO THE EFFECTS OF HIGH ALTITUDE NUCLEAR EXPLOSIONS I. AND. II. I I I8-OB-D20I8.

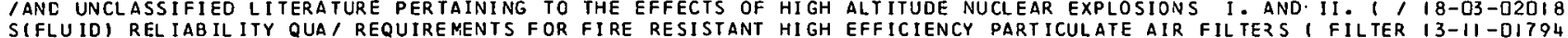

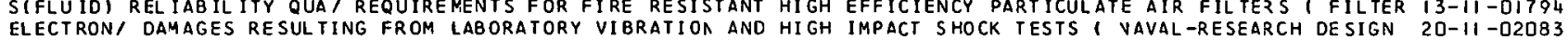
ELECTRON/ DAMAGES RESULIING FROM LABORA IN WOODEXPCSED TO HIGH INTENSIIY THERMAL RADIATION ICALORIMETERS I3-I?-OIBO2 IGNIIION HIGH-T' TEMPERATURES ATTAINED IN WOOD EXPCSED TO HIGH INTENSIT THERMAL RAOIATION I CALORIMETERS I3-I?-OI8O2
IEQUENCES AND COSTS OF ACCIOENTS IN THE TRANSPORTATION OF HIGH LEVEL RADIOACTIVE MATERIALS I URBAN-AREAS, I8-D7-O2O34 UTRIT ION (ANIMAL) PROTEINS GENE TICS AG / PROCEEDINGS OF THE HIGH LYSINE CORN CONFERENCE I NUTRITIONIHUMAN) N D6-IG-DIT2O SEARCF GLASS OPTICAL-PROPERTIES RLBY CRY/ NRL PROGRESS IN HIGH POWER LASER RESEARCH I LASERS SCIENTIFIC-RE $20-03-02062$ LIOGR APHIES URBAN-AREAS EXCAVATION, \$PB/ BIBLIOGRAPHY OF HIGH SPEEO GROUNC TRANSPORT I TRANSPORTATION BIB 23-O? -O2OQ3 WORKS TERMINALS SCHEDULINGI SUMMARY OF RESEARCH AT MIT ON HIGH SPEED GROUND TRANSPORT I TRANSPORTATION NET 23 -0?-02354 S MANAGEMENT-PLANNING URBAN-PLANNING WASHINGTON BOSTON N/ HIGH SPEED RAILROADS I TRANSPORTATION URBAN-AREA 23 -02-02I OO LOADINGIMECHAN ICS) US/ MECHANICAL PROPERTIES OF RCCKS AT HIGH TENPERATURES ANO PRESSURES 1 RJCK-MECHANICS O8-D7-O2242 OLOGY FOR THE BIG CITY ( URBAN-AREAS TRANSPORTATION COSTS HIGH-SPEEO-GROUNC-TRANSPORTI \#A.L. 1211 I TECHN $23-02-02352$ INKMENTS I STRESSES SOIL-MECHANICS SETTLENENT (STRUCTURAL) HIGH-SPEED-GROUNC-TRANSPORT MATHEMATICAL-ANALYSI 20-II -020B4 ING) VEHI UNIVER SAL DRAG LAW ( TRANSPORTATION PROPULSION HIGH-SPEED-GROUNL-IRANSPORT PERFORMANCEIENGINEER 23-0? -O2O96 IN/ KEYSTONE CORR IDOR TRANSPOR TATION STUDY I PENNSYLVANIA HIGH-SPEED-GROUNC-TRANSPORT RESEAZCH-PROGRAM-ADM 23-02-O2OQ2 I-FURNACES RADIOMETERS HEAT-FLLX HEAT-TRANSFER PYRCMETERS HIGH-TENPERATURE-RESEARCH OPTICAL-IVSTRUMENTS EI I3-OI -02255 IHIGH INTENSITY THERMAL RAOIATION I CALORINETERS IGNITION HIGH-TEMPERATURE-RESEARCH RADIOMETERS PYR OLYSI S/ I3-I? -OIBO2 IIAL INSTITUTIONS AND THERMONUCLEAR WAR - A CASE STUDY OF HIGHER EDUCATION I POSTATTACK-OPERATIONS SOCIAL/ DS-II-OIOB8 AIR-TRANSPORTATION BUS-TRANSPORTA TION RAIL-TRANSPORTAIION HIGHWAY-TRANS PORTATION 1 \#P B 173 499 /POR TATION $23-0 ?$ - D2091 1966 I RADIAT ION-EFFECTS NUCLEAR- WARFARE-CASUALTIES JAPAN HIROSHIMA CAIA NAGASAKI RAOIATION-IVJURIES RECOI D6-I3-0IT3O AR-WARFARE-CAS/ SOME IMPLICATIONS ON UNCERTAINTIES IN THE HIROSHIMA EXPLOSIOV I AIR-BURST YIELD WIND NUCLE I5-OS-0I958 /SUALTIES CASUALTIESIPREDICTION) LRBAN-AREAS DISTRIBUTION HIROSHIMA NAGASAKI BURVS WEAPONS-EFFEST RADIATI/ DO-2I -D223B I-WARFARE-CASUALTIES STATISTICAL-ANALYSIS WEAPONS-EFFECTS HIROSHIMA NAGASAKI GRAPHS BIOLOGICAL-8LASI-EFFE/ D6-2I -O2227 DISEASES TISSUESIBIOLOGY) TESTES RATS MORPHOLOGY (BIOLOGY) HISTOLOGY ESOPHAEJS SKIN, \$AD G38 $480 / I C I E N C Y-$ DG-05-DI7DI CAL-W ARFARE-AGENTS BACILLUS-AN THRACIS DI AGNOSIS PATHOLOGY HISTOLOGY INFECT IOVS IMMUVITY 1 \#AD 636 813 IOGI I5-D? -OI854 TECHN ICAL-INFORMA TION-CENTERS ABSTRACTS COSTS DEP/ ANNUAL HISTORICAL SUMMARY - JULY-1, $1965-J U N E-30,19661$ O5-02-02125 I COLE-EAST METHODOLOGY THEORY CONTENT-ANALYSI SICOMPUTERS) HISTORY, *FAR-4O47 IION SUEZ-CRISISATTITUDES M OS-OH-02I7O ITE RODTS OF PUBL IC LAW 92C I LEGISLATION SOCIAL-SCIENCES HISTORY CIVIL-OEFEVSE-OPERATIVG-PLAVS CIVIL-DEF, OS-DI-OIOTI

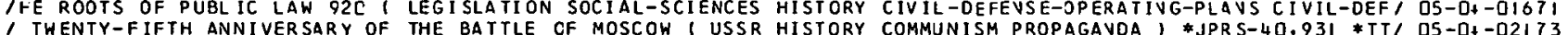

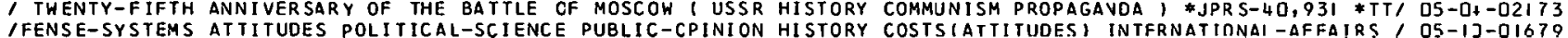

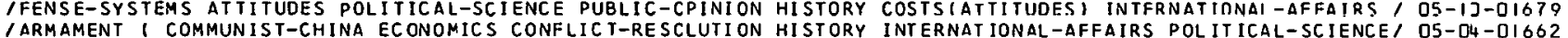
IARMAMENT, COMMUNIST-CHINA ECONONICS CONFLICT-RESCLUTION HISIORY INIERNAT IOVAL-AFFAIRS POLITILAL-SCIENCE, O5-D4-DIOL

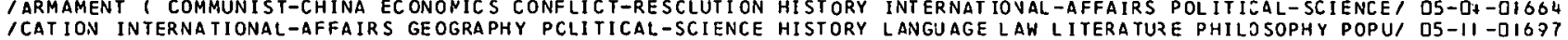
ICAT ION INTERNA TIONAL-AFFAIRS GEOGRAPHY PCLITICAL-SCIENCE HISTORY LANGUAGE LAW LITERATUZE PHILOSOPHY POPU/ O5-II -DI697
IICS EDUCATION FOREIGN-POLICY GEOGRAPHY PCLITICAL-SCIENCE HISTORY LANGUAGE LAW PUBLIC-IVFORMATION PHILOSO/ O5- II -DI696 IIES EDUCATION FOREIGN-POLICY GEOGRAPHY POLITICAL-SCIENCE HISTORY LANGUAGE PHILOSOPHY LAW PUBLIC-INFORMAT/ O5-II-OIG92 / CRAPFIES ECONOMICS EDUCATION POLITICAL-SCIENCE GECGRAPHY HISTORY LANGJAGE RELIGION, \#EXTERVAL RESEARCH / D5-II-QI G95 1 IOGR APHIES ECONOMICS CONFLICT-RE SOLUTION CONNUNIST-CHINA HISTORY LAW INTERNAT IOVAL-AFFAIRS METHODOLOGY PI O5-D -OIOS5 IIOGR APHIES ECONOMICS COMMUNIST-CHINA CONFLICT-RESCLUTION HISTORY LAW INTERVATIONAL-AFFAIRS POLITICAL-SCI/ OS-OH-OIGS 3 IRMAT ION-RETR IEVAL MICROFICHE MANPOWER PRODUCTION-CONTROL HISTORY MILITARY-PUBLICAT.IONS REPRODUCTION DOCUI OS-OZ-O2I 25 RANSPORTAT IOV LAUNCHING-SITES ME TEOROLOGY RAI THE THERMAL HISTORY MIL IL-MECHAVICS WEATHER ING MILITARY-PERSONNEL IVULNERAEILITY) HOLES BLAST MIL ITARY-EVGIVEERING I \&REPORT-I859 I5-DS-DI957 IATED PROBLEMS OF THE DOWNTOWN MANHATTAN AREA BETWEEN THE HOLLAND TUNNEL AND THE MANHATTAN AND WILLIAMSBU/ 23 - D2 -D2357 IATED PROBLEMS OF THE DOWNTOWN MANHATTAN AREA BETWEEN THE HOLLAND TUNNEL AND THE MANHATTAN AND WILLIAMSBU/ 23 - Q2 - 2357
IBES - EXPERIMENTAL OBSERVATIONS OF INTERIOR PRESSLRES IN HOLLOW NODELS I SHOCK-WAVES EXPER IMEVTAL-DATA T/ I8-O3-O2ODI /BES - EXPERIMENTAL OBSER VATIONS OF INTERIOR PRESSLRES IN HOLLOW NODELS I SHOCK-WAVES EXPER IMEVTAL-DATA T/ I8-O3-O2ODI IUAT ION OF TECHNIQUES FOR IMPROVING FALLOUT PRCTECTION IN HOME BASEMENTS I SHELTERS FALLOUT-SHEL TERS NEVAI I3-I3-DI822 IUATION OF TECHNIQUES FOR IMPROVING FALLOUT PRCTECTION IN HOME BASEMENTS I SHELTERS FALLOUT-SHEL IER S NEVAI I3-I3-DI822 /ALUATION STUDY OF COMMUNITY RESPCNSE TO THE RHODE ISLAND HOME FALLOUT PROTECTION SURVEY I ATTITUDES FALL/ O5-1J-DI685
RADIATION INDUCED VIABILITY NUTATIONS IN THE HONEY BEF HYMFNOPTERA GFNFTIRS I \$YYO-23I5-I O6-D6-D2207

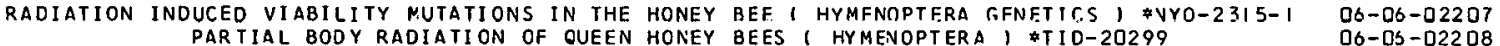
I HANICS) FEASIBIL ITY-STUDIES STRE SSES ELASTICITY CCNCRETE HONEYCOMB-CORES EXPANOED-PLASTICS DESIGN, *AD, I8-O3-D2OI 7 I CONCRETE UNDERGROUND-STRUCTURES EXPANDED-PLASTICS GLASS HONEYCOMB-CORES LOADINGIMECHAVICS) SHOCK(MECHAN/ I8-O3-D20J7 MERGENCY-HEAL TH-SERVICES HOSPI TALS CASUALTIES ADMINISTRAI HOSPITAL DISASTER PLANNING 1 OISASTER-MEDICINE E D6-2I -DI739 RNACO OF MARCH 3, $196 /$ SOME PRELI YINARY OBSERVATIONS ON A HOSPITAL RESPONSE TO THE JACKSON, 4 ISSISSIPPI TO O6-I?-DI7I 4 IR PL ANNING $I$ DISASTER-MEDICINE ENERGENCY-HEALTH-SERVICES HOSPIIALS CASUALTIES ADMIVISTRATION-AND-MANAGEMI O6-21 -OI739 IISTRATION-AND-MANAGEMENT FIRST-AIO ORGANIZATION TRAINING HOSPITALS CASUALTIES PERSONNEL NUCLEAR-WARFARE-/ OO-2I -OI738 IELS RESOURCES CAPACITY DECONTAMINATION REPAIR GOVERNMENT HOSPITALS HOUSINE AGRICULTURE CONSTRUCTION MANP, OS-O3-O2I5I IOF MARCH 3, 1966,1 DISASTER-MEDICINE MANAGEMENT-PLANNING HOSPITALS OPERAT IOV COVTROL-SYSTEMS COMMUNICATI/ O6-I?-DI7I 4 IARE FILMS; DISASTER-MEDICINE MEDICINE MEDICAL-PERSONNEL HOSPITALS PUBLIC-HEALTH RESCUES NULLEAR-WARFARE/ DO-2I-DI74G IARE FILMS

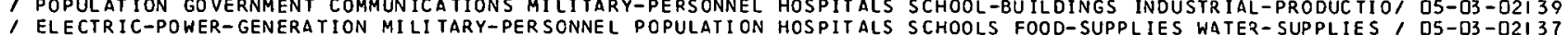
/ ELECTRIC-POWER-GENERATION MILI TARY-PER SONNEL POPULATION HOSPITALS SCHOOLS FOOD-SUPPLIES WATER-SUPPLIES / OS-D3-D2I37

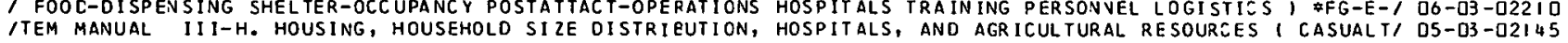

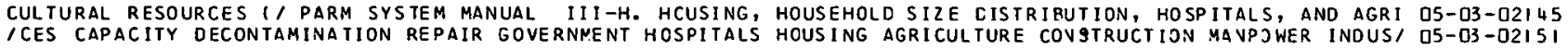


/SASTER I DAMAGE-ASSESSMENT DATA DECISION-NAKING RECOVERY HOUS ING FOOD FLOODS CIVIL-DEFENSE-JPERATING-PLA/ O5-03-0I636 I SIMULAT ION DAMAGE-ASSESSMENT ATTACK-SCENARIOS CONPUIERS HOUS ING PROGRAMMIVGICOMPUTERSI MILITARY-INSTALL CASUALTIES/ RESOURCE DATA CATALOGLE I INFORMATION-SYSTEMS HOUSING RESOURCES MATHEMATICAL-MODELS COMPUTERS DS-O3-O2I 37 OST ATTACK-OPERATIONS SHEL TER-OCCUPAN I EMERGENCY LOCGING 1 HOUSING TRAINING CIVIL-OEFENSE-OPERATING-PLANS P O5-1I -O2IB 7 AND AGR ICUL TURAL RESOURCES I/ PARM SYSTEN NANUAL III-H. HOUS ING, HOUS EHOLO SIZE DISTR IBUTIOV, HOSPI TALS, OS-O3 -02I 45 /2ZLE BRAKE BLAST SUPPRESSION DEVICES FOR 105 MN ANC I55MM HOWITZERS 1 SHOCK-NAVES ATTENUATIDN DESIGN GUN-/ I9-DS-D2327 STATE REFUGEES BANKING INSURANCE LABOR LAW TRAFFIC, \#IDA HQ 65-4/42/ING-PLANS REPAIR CONSTRUCTION REAL-E D5-D3-0I636 NGINEER ING DECISION-MAKING DESIGN ENG/ THE UTILI ZATICN OF HUNAN FACTORS INFORMATION BY DESIGVERS I HUMAN-E OS-O5-OIGT3 ORPT ION SALTS HUM IDITY, \$AD 646 , WATER TRANSFER THROUGH H'J MAN SKIN I PERMEABIL ITY TRAVSPOZT-P ZOPERTIES S O6-IS-O22I 3 MENT, STRUCTURES COMMUNICATIONS-SYSTEMS RADIATION GEOLOGY HUMAN-ENGINEERINE 1 *SR-45 *VP- II768-VOL- 1 /QUIP I $7-0 ?-02311$ I UTILIZATION OF HUMAN FACTORS INFORMATION BY DESIGNERS 1 HUMAN-ENGINEERING DECISION-MAKING DESIGN ENGINE/ OS-D 5 -DIG73 XPOSURE VACCINES COSAGE IMMUNI TY NONKEYS MICE GUINEA-PIGS HUNANS, \$AD 635744 /GENTS BACTERIAL-AEROSOLS E I5-O2-0I858 ICS RADIATION-HAZARDS MUTATIONS RADIATION-EFFECTS ANIMALS HUNANS TESTS DOSE-RATE, $\$$ RM-5D96-TAB /S I GENET O6-IS-OI727 N SK IN I PERMEABIL ITY TRAN SPOR T-PROPER TIES SORPT IOA SALTS HUMIDITY,

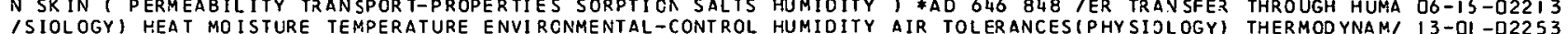

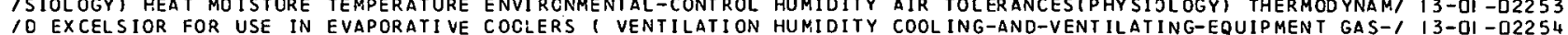
IO EXCELSIOR FOR USE IN EVAPORATI VE COCLERS I VENTILATI ON HUNIDITY COOLING-AND-VENTILATING-EQUIPMENT GAS-I I3-OI -02254

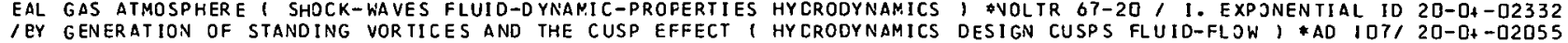
IWATER EXPLOSIONS - FORMULAE AND PROGRAM SPECIFICATIONS 1 HYCRODYNAMICS WATER-WAVES MATHEMATICAL-PREDICTI/ I9-0 +-O2O 43 K-SUPPLIES RADIOLOGICAL-CONTAMINATION RADIONCTIVE-FALLOUIT HY RROL.OGY, \#A.L, IOS4 $10 M$ SURFACE RUNOFF I WATE O6-IJ-DI724 IC RINC STIFFENED CYLINDR ICAL SHELL I MDNFI BR-7M, UNDER HYOROSTATIC PRESSURE I STIFFEVED-EYLINDERS TENSI $20-I I-O 2347$ PARTIAL BODY RADIATION OF QUEEN HONEY BEES, HYMENOPIERA, \$TIO-20299

RACIATION INDUCEC VIABILITY MUTATIONS IN THE HONEY BEE ( HYNENOPTERA GENETICS, \#YOO-23I5-1, O6-OS-O22O7 IIOPS IS AND THE BIOLOGY AND ECOLOGY OF C. ANDRENIFCRNIS - HYMENOPTERA, ANCREVIOAE 1 BEE-POLLIVATION, \$A. I O6-DS-OI7OS 2-5 1 BEHAVIOR CONFLIC T ARMS-CONTROL ARTIFICIAL-REALITY HYPOTHESES-FORMULAT ION COVFL ICT-RESOLUTION GAME/ O5-IJ-O2I 22 TICLC BEAMS I MAGNETO-OPTIC-EFFER.T MAGNFTIS.FIFI.DS LASERS HYSTERESIS, \$AFOSR-66-1090 \$AD 635 OI6/AND PAR 20-D5-02064

IST-ATTACK RECOVERY PLANNING SYSTEM - PARN SYSTEM NANUAL CIAN ICS I STRA IVIMECHANIC SI THEORY - TECHNICAL REPCRT NO. MUN ICAT STRA IVIMECHANICSI THEORY) TECHNICAL REPCRT NO. MUN ICATION-EQUIPMENT INTERAC TI ONS, * TECHNICAL REPCRT NO.
5 DYNAMICS LOADINGIMECHANICS) SHELTERS, \#MISC. PAPER NO. IS ATMOSPHERES AND MODIFIED SACHS SCALING I. EXPONENTIAL ION OF ROCKS 1 ROCK (GEOLOGY) MINERALS TEXTURE PETRCGRAPHY IRAPIIES OIGITAL-COMPUTERS EXPLOSIONS GAS-OI SCHARGES HEAT ED IN NUCLEAR DETONATIONS I GLASS ATTENUATION URBAN-AREAS SSESSMENT FIRE-SAFETY PROGRAMMING (COMPUTERS) DEBRIS BLAST IPOSEL TO HIGH INTENSITY THERMAL RADIATION 1 CALORIMETERS ITION TEST OF AN ABOVEGROUNO FALLOUT SHELTER IN EVANSTON, ITION EMPLOYMENT TAXES SCR VICES MANACEMENT-PLANNING COSTS IISMS ASSOCIATED WITH GAS BREAKDOHN UNDER INTENSE CPTICAL ICOVERY PLANNING SYSTEM - PARM SYSTEM MANUAL I A, P. PARM LABORATORY - FURNACES/ THERMAL FLUX DISTRIBUTICN IN AN ARC ATHOLOGY LUNGS SPORES, \#AD/ PATHOGENESIS OF COCCIDIOIDES CILLUS-AVTHRACIS OIAGNOSIS PATHOLOGY HISTCLOGY INFECTIONS H PROTECI IMMUNOLOGICAL STUDIES OF ANTHRAX II LEVELS OF IRFARE-AGENTS BACTERIAL-AEROSOLS EXPOSURE VACCINES DOSAGE I. IMMUNOLOGICAL RESPONSE OF MAN IO PURIFIED I STUDIES ON IES ON IMMUNITY TO TOXINS OF CLOSTRIDIUM BOTULINUM VIII. IMMUN ITY AGAINST BACILLUS ANTHRACIS OBTAINEO WITH PROTEC, IPOL IOMYEL ITIS, SMALLPOX, MEASLES, INFLUENZA I DEMCGRAPHY I-AGENTS EPIDEM IOLOGY PUBLIC-HEAL IH POSTATTACK-OPERATICNS VIBRATION FREOUENCY BENDING ELASTICI.TY LOADING IMECHANICS) RNAT IONAL-AFFAIRS, \#EXTERNAL RESEARCH PAPER 156 SOVIET RON/ DAMAGES RESULTING FROM LABORATORY VIBRATION AND HIGH IARLENEU STRUCTURES 1 SHOCK-AB SORBERS VIBRATICN-ISCLATORS LIBRATI/ A MECHAN ICAL SHOCK PULSE SUR VEY I TEST-E QUIPMENT THE SHOCK AND VIBRATI ON BULLET IN 1
ECHANICS) DESIGN TESTS MODELSISIMLLATIONSI SOIL-MECHANICS ACK INGI FRACTUR IVG) FRAC TURE (MECHANI I ONS) SOIL-MECHANICS ISLAT ION COMPETITION EMPLOYMEN T TAXES SER IICES SELECTED URATION ( URBAN-ARLAS DECENTRALI ZATI ON TAXES VULNFRABILI) ATI ITUDES FEAR STRESS (PSYCHOLOGY) CASUALTI/ MENTAL HEALTH ENT MANAGEMENT-PLANN ING PERSONNEL SHELTER-/ SOME TRATNING
XPLOSION I AIR-BURST YIELD WIND NLCLEAR-WARFARE-CAS/ SONE XPLOS ION 1 AIR-BURST YIELD WIND NLCLEAR-WARFARE-CAS/ SONE
IOMS JOURNAL - CANADA LAh INTERNATIONAL-AFFAIRS TARIFFS IOMS JOURNAL - CANADA I LAW INTERNATIONAL-AFFAIRS TARIFFS
11 - GERMANY - I I IRES FIRESTORMS THERMAL-RADIATIION I I I - GERMANY - I I FIRES FIRESTORMS THERMAL-RADIATI ON
/NITORS DOSIMETERS PROTECTIVE-CLOTHING GAS-MASKS PHOSGENE IREAN PROBLEMS I ECONOMICS URBAN-PLANNING DECISI ON-NAKING $R /$ BIOLOGICAL-WARFARE-AGENTS SPORES M/ TEST NFW ELECTRIC IODE ARTICLE OV FALLOUI SHELTERS WITH RECOMMENDATICNS FOR RESUL TS OF SERIES III TESTS I MOTION OF SUBSURFACE SOIL MENTATIOV PROFE SS IONAL-PER SONNEL SCIENTIF IC-RESEARCH ORG/ ECONOMICS DEPARTMENT PUBLICATIONS, 1960-1965 - AN AUTHOR EX OF THE OPEN LITERATURE WITH ABSTRACTS 1 BIBLIOGRAPHIES 1 ANNOTATED BIBLIOGRAPHY - SUPPLENENT, AUTHOR AND SUBJECT LASSIFICAT, THE STATE OF THE.ART OF COORDINATE INDEXING NNEL SCIENTIFIC-RESEARCH ORG/ INDEX OF PUBLISHED PAPERS - -EADINGS CLASSIFICAT/ THE STATE CF THE ART OF COCRDINATE - FEADINGS CLASSIFICAT' THE STA TE CF THE ART OF COCRDINATE C/ COCUMENT STORAGE AND RETR IEVAL TECHNI QUES - APPENDIX
LUX NORWAY DENMARK GREECE SWEDEN ShI TZERLAND IRELAND USSR IUX NORWAY DENMARK GREECE SWEDEN ShI TERLAND IRELAND USSR
IPULATION AGR ICULL TURE POL I ITICAL-SCIENCE REGI ONAL-PLANNING 1 OL ARS AVD ACADEM IC CENTERS - ASIA I BIBLIOGRAPHIES CHINA ITION TEST OF A BASEMENT FALLOLT SHELTER IN EAST CHICAGO, NT IAXES SERVICES MANAGEMENT-PLANNING COSTS I LLINOIS OHIO
PLY-AND-DEMAND ECONOMICS RECOVERY POST/ THE IMPORTANCE OF OGY FOR SIMPL IFYING THE EVALUATION OF HARDENING COSTS nGY FNR SIMPI IFYING THE EVALUATION OF HARDENING CCSTS

-A,B. PARM ILLUSTRATED \& POSTATTACK-JHERATIONS/ O5-03-02131 -72J /TURES ELAST ICITY TEST DYNAYICS LOADING IME 13-13-01825 $1-753$ \#DD 646 608 /NICS) SO IL-MECHAVICS CLAY COM 13-11-02261 -809 10 CONCRETE ARCHES I UNDERGROUND-STRUCTURE 13-13-01830 IDEAL GAS ATMOSPHERE I SHOCK-WAVES FLUID-DYNAMI/ 20-04-02332 IGNEOUS-ROCK SECIYENTARY-ROCK METAYORPHIC-ROCK， O8-DT-02241 GNITERS PROJECTILES MATHEYATICAL-AVALYSIS PROG/ 19-05-02329 GNITION, \#AD 625867 /LEAR-EXPLOSIONS DAMAGE-A 15-03-01052 IGNIT ION HIGH-T EMPERATURE-RESEARCH RADIOMETERS, 13-1?-0I802 IGNTION HIG-TEMPERATURE-RESEARCH RADIOMETERS 13-12-01802 ILLINOIS IENVIRCNMENTAL-CONTROL EXPERIMENTAL-D/ 13-0I-0J771
IIIIINOIS OHIO INCIANA MICHIGAV, \$SRI-II-4895/T 23-02-02095

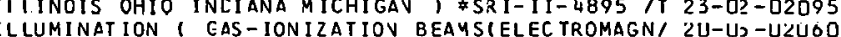
ILLUSTRATED ( POSTATTACK-OPERATIOVS RECOVERY MA/ O5-03-02131 MAGING FURNACE I THERMAL-RADIATION CALOR IMETERS 13-DI-D2255 MMITIS IN MONKEYS 1 BIOLOG ICAL-WAR FARE-AGENTS P 15-02-01852 IMMUNITY ' \#AD 636 813 10GICAL-WARFARE-AGENTS BA 15-02-01854 IMMUNIIY AGAINST BACILLUS ANTHRACIS DBTAINED WIT I5-0?-01853 MMUNITY INFECT IONS, *AD 636905 /US MONKEY, MA 15-02-01855 MMUNITY MONKEYS MICE GUINEA-P IGS HUMANS, *AD, I5-02-01858 MMUNITY TO TOXINS OF CLOSTRIDIUM BOTULINUM VII 15-D?-0I857 MMUNOLOGICAL RESPONSE OF MAN TO PURIFIED BI VAL/ 15-0?-01857 IMMUNOLOGICAL STUDIES OF ANTHRAX II - LEVELS OF 15-02-01853 IMMUNOLOGY CISEASE-VECTORS CHEMOTHERAPY MEDICIN/ 06-05-01702 MPACT, \#IMB-704 *AD 650,78/SUPPORT/RINGS 20-11-02336 MPACT ON INTERNATIONAL LAW I USSR COMMERCE INTE 05-11-01690 MPACT SHOCK TESTS I NAVAL-RESEARCH DESIGN ELECT 20-11-02083 IMPAC.T -SHOCK NUCLEAR-EXPLOS ION-DAMAGE SPR INGS P/ 13-13-01823
IMPACT -S HOCK SHOC (MECHAN ICS) INSTRUMENTA TION CA $14-[12-01842$ MPACT -TESTING SHOCK (MECHAN ICS), *BULLETIN 3516 20-11-02077 IMPACT-TESTS, \#CR-65.001/HAVICAL-WAVES SHOCK IM 20-11-02078 IMPACT-TESTS OYNAMICS BLAST SHOCK-WAVES ELASTIC/ 20-11-02338 IMPACTS OF RAILROAD MER GERS I TRAVSPJR TA TION LEG $23-02-02095$ MPLICATIONS IN CIVILIAN EMERGENCIES I EMUIIUNS 05-IJ-0IO8I IMPLICAT IONS OF LARGE SHELTERS I SHELTER-MANAGEM 05-0I-01621 MPLICATIONS ON UNCERTAIVTIES IN THE HIROSHIMA E 15-03-01958 MPLORT - CUT IES FOREIGN-TRADE COMMEREE, $\$ P B-173,05-03-01641$

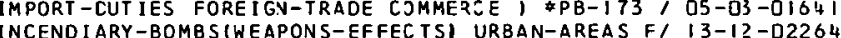
INCENDI ARY-GELS FLAME-THROWER-FUELS SMOKE-MUNIT/ 06-19-01721 NCENT IVES SOCIAL-SCIEVCES OPERATIJVS-RE SEARCH，05-1]-01676 NCINERATOR DES IGV FOR STER IL IZING LABORA TORY AI I5-0?-0I892 INCLUS ION OF REQUIREMENTS FOR FALLOUT SHELTER C/ 13-13-0228I
INCLUSIONS SUBJECTED TO SURFACE BLAST LOAOING - 08-13-01746 NCLUS ICNS SUBJECTED TO SURFACE BLAST LOADING - 18-03-02008 NCEX OF PUBL ISHED PAPERS IVDEXES REPORTS DOCU 05-02-02117 INDEX OF THE OPEN LITERATURE WITH ABSTRACTS INCEXES ) \$RM-2800-1(SUPP) *AD 626 930/THOR IND O5-03-01652 INCEXES 1 BACTERIA VIRUSES FUVGI PZOTOZOA RICKE/ D6-13-017I5 RORMAT IOV-RETRIEVAL SUBJECT-HEADINGS C 05-D2-021 NEXES REPORTS COCUMEVTATION PROFESSIONAL-PERSO 05-02-021 7 NCEXINE 1 INDEXES INFORMATION-RETRIEVAL SUBJECT 05-0?-02119 NDEX ING TECHNIQUES, DESCRIPTION AVD BACKGROUND DS-02-02116 INDIA INTERNAT IONAL-AFFAIRS CJMMERCE, \#A.L. $11 /$ 05-03-02161
INOIA NCIA JAPAN PAK ISTAN PHIL IPPIVES / *EXIERNAL RE/ 05-11-01698 INDI ANA I ENV IRONMENTAL-COVTROL EXPERIMENTAL-DA/ I3-OI-D1774 NCIANA MICHIGAN, \#SRI-11-4895 /TITION EMPLOYME 23-02-02095 INDIVIDUAL INDUSTRIES FOR DEFENSE PLANNING I SUP D5-03-0164 INCUSTRIAL HARDENIVG CLASSIFICATIJV - A METHODOL D5-D3-D2I 62 
/ EVALUATION OF HARDENING COSTS I. TEXT AND APPENCIX A I INDUSTRIAL-EQUIPMEVT BLAST INDUSTRIES SURVIVAL $/$ DS-03-02IS2 AN ANALYSIS OF MACHINE TOOL SLBBSTITUTION POSSIBILITIES I INDUSTRIAL-EQUIPMENT ECONOMICS FEASIBILITY-STUDI Q5-D3-DIG48 IYING THE EVALUATION OF HARDENING COSTS 11. APPENCIX B I INDUSTRIAL-EOUIPMENT INCUSTRIES FLOW-CHARTING SI O5-G3-O2I26 POSTTT IOPULATIJN HOSP ITALS SCHOOLS FOOD-SUPPLIES WATER-SUPPLIES INDUSTRIAL-PLANTS FINAVCE STOCKPILIVG STORAGE M/ OS-D3-OLII 7 ATION MANAGEMENT-PLANNING RE SOURCES INDUSTRIAL-PRODUCTION INDUSTRIAL I AREENING SOCIETAL-RECOVERY SOURCES INDUSTRIAL-PRODUCTION INDUSTRIAL-PLANTS GEOGRAPHY FNREC-4O MUN ICAT IONS MIL ITARY-PER SONNEL HOSPI TALS SCHOOL-BUILDINGS INDUSTRIAL-PRODUCTION, 4 VREC- 27 , ONOMICS RECOVERY POSTATTACK-OPERATIONS RESCURCES SURVIVAL INDUSTRIAL-PRODUCI ION, \$P-2093 $\$ 40 \% 41156,1$ EC O5-03-DI647 FARE SURVIVAL ECONOMICS POPULAIION AGRICULTURE INDUSTRIES INDUSTRIAL-PRODUCTION, $\$$ RM-3436-PR \$AD 426 906 DS-03-02158 IMENT HOSPITALS HOUSING AGRICULTURE CONSTRUCTION MANPOWER INDUSTRIAL-PRODUCT ION COMMERCE TRANSPORTATION I/ OS-O3-O2ISI IESTORATION AND CONVERSION I RESOLRCES MANPOWER GECGRAPHY INCUSTRIAL-PROOUCT ION DAMAGE-ASSESSMEVT, \&NREC, D5-03-D2I SO IESTN ING RESOURCES MODELS PROGRAMNING (CONPUTERS) CAPACITY INCUSTRIAL-PRODUCTION DATA-PRJCESSIVG ECONOMICSI OS-D3-D2I 31 CIS AND DATA I ITS AND DATA I MOBILIZATION MANAGEMENT-PLANNING RESOURCES INDUSTRIAL-PRODUCTION INDUSTRIAL-PLANTS GEOGRAPI DS-D3 -U2I 42

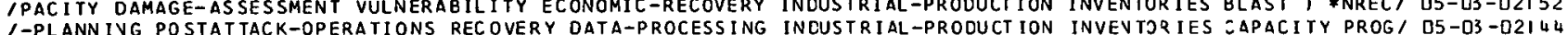

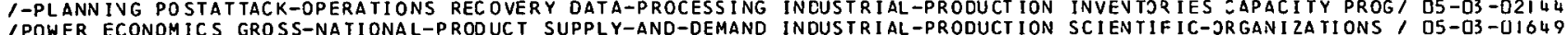
IPOWER ECONOMICS GROSS-NATIONAL-PRODUCT SUPPLY-AND-DEMAND INDUSTRIAL-PRODUCTION SCIENTIFIC-JRGANIZATIONS $/$ O5-O3-UIO4 9 TERNATE-CURRENT ECONOMICS DIRECT-CLRRENT STATE-OF-THE-ART INDUSTRIAL-RESEARCH ) \#A.L. 1105 ILINES COSTS AL O9-O3-O2249 CONSUMPT ION(FOOD) COMMERCE AGRICULTURAL-ECONONICS CEREALS INCUSTRIES 1 \#A.L. 966 / GROSS-NATIJVAL-PRODUCT D2-02-DI6I6 /LABOR COMMUNISM ECONOMICS AGRICULTURAL-ECONOMICS FINANCE INCUSTRIES COMMERCE EDUCATION POLITIZAL-SCIENCEI DS-II -QI693 AND NATURAL GAS SIATISTICS I RESOLRCES PETROLEUM-INDUSTRY INDUSTRIES ECONOMICS, IA.L. 985, OF PETROLEUM D5-O3-DI638 IF HARDEN ING COSTS II. APPENDIX B I INDUSTRIAL-EQLIPMENT INDUSTRIES FL OW-CHART ING SURVIVAL RECOVERY ECONI DS-D3-D2I 26 AND ECONOMICS RECOVERY POST/ THE IMPORTANCE OF INDIVIDUAL INDJSTRIES FOR CEFENSE PLAVNIVG 1 SUPPLY-AND-DEM O5-O3-OIG4 7 IUCLEAR-WARFARE SLRVIVAL ECONONICS POPULATION AGRICULTURE INCUSTRIES INDUSTRIAL-PROOUCTION I \$RM-3436-PR I D5-03-02I 58 IER INDUSTRY RELATIONSHIPS, POTENTIAL DEMAND, EMPLOYMENT I INDUSTRIES MANPOWER ECONOMICS GROSS-NATIONAL-PR/ OS-DS-OIOL 9 ILEAR-EXPLOSION-DAMAGE POPULATIONIDISIRIBLTION) GECGRAPHY INDUSTRIES NUCLEAR-WARFARE-CASUALTIES DEMOGRAPH/ IS-OS-DI962 BIL ITY LABOR ECO/ OCCUPATIONAL SKILLS AND CIVIL DEFENSE I INCUSTRIES RESOURCES(HUMAV) DISTRIBUTION VULNERA D5-D3-DIG39 BIL IT INTAM INATION PEST-CONTROL MOR TLARY-SERVICES PUBLIC-HEALTH INCJSTRIES SANITATION REPAIR YANPJWER I \# SRI-MU/ IS-O3-OI953

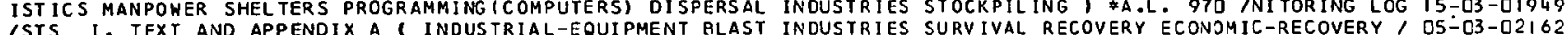

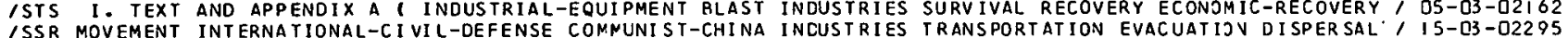
ISSR MOVEMENT INT ERNA TIONAL-C I VIL-DEFENSE COMNUNIST-CHINA INCUSTRIES TRANSPORTATION EVACUATIJY DISPER SAL' I I S-D3-D2295 SURV IVAL I SIMULATION DAMAGE-A SSE SSMENT WARFARE RESOURCES INCUSTRIES VJLNERABILITY ECOVJMICS $)$ \$A.L. 1052 O5-03-0I644 ILMS PROPAGANDA RURAL-AREAS CI IVIL-DEFENSE-OPERATING-PLANS INDUSTRIES (CIVIL-OEFENSE) PUBL IC-HEALTH TRANSPO/ I5-03-U2302 I SCIENTIFIC AND TECHNICAL INFORMATION WITHIN THE CEFENSE \#FAS $-M-186$

\#FAS $-M-188$
\#FAS $-M-184$

URLGUAY LI VESTOCK AND MEAT IN

INDUSTRY III. A. FREQUENCY DISTRIBUTIONS AND C/

$05-02-02120$

ARGENTINA LI VESTOCK AND MEAT

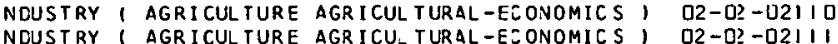

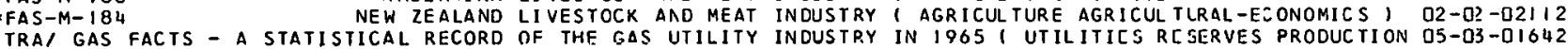
TATEG ECONOMICS PRODUCTION VULNERABILITY MANPI PETROLEUM INOUSTRY OF GERMANY DUR ING THE WAR I MILITARY-ST O5-O3 - I IOL IN LOADING(MECHAN ICS) SHEAR-STRES SES DEFORMATION RCTATION INERTIA MATHEMATICAL-AVALYSIS I \#REPJRT 2 I \$AD , $20-11-02346$ STICS SHELTER-OCCUPANCY WATER-SUPPLIES EDUCATION TRAINING INFANT-CIET, *0CD-IG-15.I*ARC-22I9/LTERS LOGI D6-D3-O17I3 ING SANITARY-ENGINEER ING RADIOLOG ICAL-CONTAMINATI ONIFOOD) INFANTSICIET) STORAGE, *H- IS /R-SUPPLIES RATION DG-O3-DI7I2 ING SANITARY-ENGINEER ING RADIOLOG ICAL-CONTAMINATI CNIFOOD) B IOLOGICAL-WAR FARE-AGENTS POX-VIRUSES NONKEYS I MUNITY
E-AGENTS BACILLUS-ANTHRACIS DIAGNOSIS PATHOLOGY HISTOLOGY E-AGENTS BACILLUS-ANTHRACIS DIAGNOSIS PATHOLOGY HI STOLOGY
/ARFARE-AGENTS EFFECTIVENESS MICE CHEMOTHERAPEUTIC-AGENTS /ARFARE-AGENTS EFFECTIVENESS MICE CHE MOTHERAPEUTI I -AGENTS
IOF TUBERCULOSIS IN THE POSTATTACK ENVIRONMENT I RECOVERY IOF TUBERCULOSIS IN THE POSTATTACK ENVIRONMENT I RECOVERY OR MACACA MULATTA AND MAN (' EFFECT OF AEROSOL AGE ON THE
VIRUSES FOR THE CYNOMOLGUS MONKEY, MACACA IRUS, AIR BORNE 1 THERMAL-RADIATION WEAP ONS-E / THERMAL STRESSES IN A SEMI IA, PERTUSSIS, TE TANUS, POLIOMYELITIS, SNALLPOX, MEASLES, R SYSTEM I FALLOUT-SHELTERS PUBLIC-OPINI ON ATTITU/ PUBLIC NAT IONA' BUDGEY ESTIMATES FOR THE FINANCIAL YEAR 1967 AND IS ION-MAK ING DESIGN ENGI THE UTILIZATION CF HUMAN FACTORS SICAL SCIENCES, B IOLOGICAL SCIENCES, ENGI/ A DIRECTORY OF IAL SCIENCES 1 DATA 1 \#A.L. 963 A DIRECTORY OF INFANTSICIET) STORAGE, *H- IS /R-SUPPLIES RATION D6-03-01712 INFECT IONS IMMUNITY, \#AD 636813 /JGICAL-WARFAR 15-02-D1854 INFECT IONS SIREPTOUYCIVS CHLOR TETRACYCLINE CHLO/ $15-02-01894$ INFECT IOUS-DISEASES VACCIVES RADIOBIOLOGY PUBLI/ O6-05-02236 NFECTIVITY OF AIRBORNE PASTEURELLA TULARENSIS F 15-02-DI858 INFECTIVITY OF THE VARIOLA VACC INIA GROUP OF POX I 5-02-0I855 INFINITE SOLID BY A FIVITE OIFFEREVEE TECHNIQUE 13-12-01795 INFLUENZA I DEMOGRAPHY IMMUVOLOGY 3 I OLOGICAL-WAI I 5-02-01869 INFORMATION AND KVOWLEDGE REQUI SITES OF A SHELTE 05-11-01699 INFORMATION ANNEXES I UNITED-VATIJYS COSTS INTER 05-04-02165 INFORMAT ION BY CES IGNERS I HUMAN-EVGIVEER ING DEC 05-05-D1673 NFORMAT ION RESOURCES IN THE UVITED STATES - PHY D5-02-D1627 NFORMATION RESOURCES IN THE UNITED STATES - PHY D5-02-01627

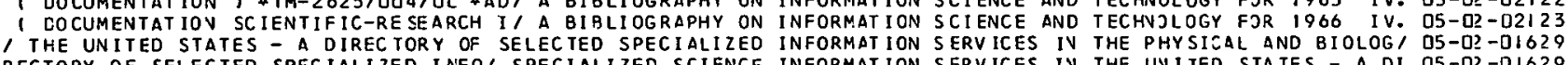

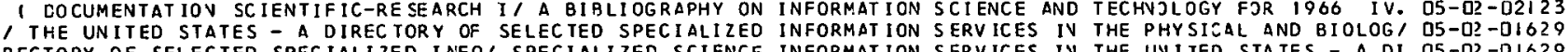
RECTORY OF SELECTED SPECIIALIZED INFO SPECIALIZED SCIENCE INFORMATION SERVICES IV THE UVITED STATES - A DI OS-O2-DI629 RECTORY OF SELECTED SPECIALI ZED INFOI SPECIALIZED SCIENCE INFORMAT ION SERVIEM I TECHNICAL-INFORMATION-CENTI O5-D2 -D2II 8 IYEARS IN FOCUS - THE DOUGLAS AIRCRAF T CONPANY MECHANIZED INFORMAT ION SYST TM
HAT WE KNOW I INFORMATION-RETR IE VAL DOCUMENTATION STATE-/ INFORMATION SYSTEM NETWORRS - LETS PROFIT FROM W OS-D2-UIG3O

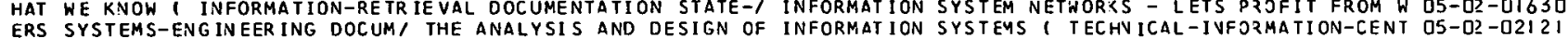

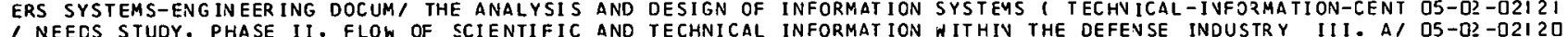
' NEECS STUDY, PHASE I I, FLOW OF SCIENTIFIC AND TECHNICAL INFORMAT ION NITHIV THE DEFENSE INDUSTRY III. AI D5-02-O2I 20 NCEXING DEPARTMENT-OF-DEFENSE DOCLMENTATION EFFECTIVENESS INFORMATION-RETRIEVAL I \#AD 650 316 /S SUBJECT-I OS-D?-02124 /TIONS DATA-STORAGE-SYSTEMS SUBJECT-INOEXING TEST-NETHODS INFORMATION-RETRIEVAL AUTOMATIC ECOVOMICS I \#PR/ O5-D2-D2II 6 /RING DOCUMEVTATION DATA-STORAGE- SYSTEMS SUBJECT-INDEXING INFORMATION-RETRIEVAL AUTOMATIC MAVAGEMENT-ENGI/ O5-Q2-02I 21 IION SYSTEM I TECHNICAL-INFORMATI CN-CENTERS DOCUNEATATION INFORMAT ION-RETR IEVAL AUTOMATION LIBZARIES AIRCI OS -O2 - D2III 8 IMATION SYSTEM NETWORKS - LETS PRCFIT FRON WHAT WE KNOW 1 INFORMAT ION-RETRIEVAL DOCUMENTATION STATE-OF-THI OS-DS -DIG3O IHIP AND COMPAR ISON ( DEPARTMENT-OF-DEFENSE DOCUMENTATION INFORMATION-RFTRIFVAL ENGINEER ING-PERSONNEL SCI/ O5-D2 -O2I 20 1G-SYSTEMS COMPUTERS DATA-TRAN SMI SSION-SYSTEMS SI MLLATI ON INFORMATION-RETRIEVAL GAME-THEORY EDUCATION TRAI QQ9-Q2-OI 75I 1-OF-CEFENSE DATA-PROCESSING-SYSTEMS DATA-STORAGE-SYSTEMS INFORMATION-RETR IEVAL MICROFICHE MANPDWER PRODU/ O5-O2-D2I 25 INOLOEY FOR 1966 IV. I DOCUMENTATION SCIENTIFIC-RESEARCH INFORMAT ION-RETRIEVAL SCIEVTIFIC-JRGANIZATIONS I OS-D? -O2I 23 AT/ THE STATE OF THE ART OF COORDINATE INDEXING 1 INDEXES INFORMATION-RETRIEVAL SUBJECT-HEAOINGS CLASSIFIC O5-02-D2II 9 AL-MOCELS COMPUTERS CASUALTIES/ RESOURCE DATA CATALOGUE 1 INFORMATION-SYSTEMS HOUSING RESOURCES MATHEMATIC O5-03-02I 37 XES INFORMATION-RETR IEVAL SUBJECT-HEADINGS CLASSIFICATICN INFORMATION-THEORY COMPUTERS I \#AD 275 393 /INDE O5-D2-D21 I9 N AT TACK-SCENAR IOS AIRCRAFT NUCLEAR-W' PRELIMINARY AERIAL INFRAREC FIRE MAPPING SYSTEMS ANALYSIS I MICHIGA I3-I?-O2267 URING I PROJECT FIRE SCAN - THE EVALUATI ON OF AN AIRBORNE INFRAREC MAPPER AS A TDOL FOR DETECTING AND MEAS I3-I?-DI798
IREO MAPPER AS A IOOL FOR DETECTING AND MEASURING FIRES I INFRAREC-CETECTORS INFRARED-TRACKIVG FOREST-FIR/ I3-I2-DI798 /REO MAPPER AS A IOOL FOR DETECYING AND MEASURING FIRES I INFRAREC-CETECTORS INFRARED-TRACKIVG FOREST-FIR/ I3-I2-DI798 $1 L$ FOR DETECTING AND MEASURING FIRES I INFRARED-DETECTORS INFRAREC-TRACKING FOREST-FIRES PATROL-PLANES AII I3-I2-OI798 / I SHOCK-WAVES ATTENUATION DESIGN GUN-BARREL-ATTACHMENTS INHIBITION PRES SURE STRESSES PROJECTILES INTERI/ I9-CS-O2327

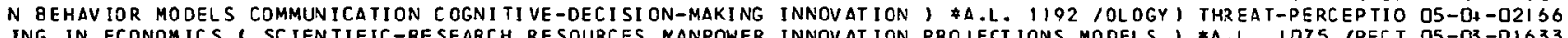
ING IN ECONOMICS I SCIENTIFIC-RE SEARCH RE SOURCES NANPOWER INNOVAT ION PROJECT IONS MODELS, $\$$ AA.L. IOT5 /PECT O5-03-DI633 YSTEMS TRANSPOR TA TION URBAN-AREAS RAI LROAOS MCNORAILS MO/ INNOVATIONS IN IRAVSIT DEVICES 1 RAPID-TRANSI T-S 23 -02-O2356 5 CIVIL-DEFENSE-OPERA/ EMERGENCY LELFARE REGISTRATION AND INQUIRY I SHELTER-OCCUPANCY POSTATTACK-OPERATION O5-11-02I89 /ECTS ECOLOGY RADIATION-DAMAGE RADIOLOGICAL-CONTAMINATION INSECTS PLANTSI EOTANY) RADIOACTIVE-FALLOUTISIMU/ D6-IB-DI723 RICULTURE I LEGISLAIION I/ REGLLAIIONS GOVERNING THE MEAT INSPECTION OF THE UNITED STATES DEPAZTMENT OF AG O2-0?-DIG?O RADIOLOGICAL MONI TORING SYSTEM I INSTRUMENTATION RADIAT I/ INSTAI,LATION AND TESTIVG OF AV AUTOMATIC REMOTE I8-O+-D2D26
111 IUN OF THE RESPONSE OF EOUIPMENT LOCATED IN UNDERGROUND INSTALLATIONS TO BLAST INOUCED GROUND MOTIONS I/ I3-13-0229I DERGROUND-STRUCTURES COLORADO DUI PROTECTIVE CONSTRUCTION INSTITUTE STUDIES I SHELTERS FALLJUT-SHELTERS UN I3-I3-DIB3I Y OF IIGHER EDUCATION I POSTATTACK-OPERATIONS SOC/ SOCI IAL INSTITUTIONS AND THERMONUCLEAR WAR - A CA SE STUD O5-II -DI688 Y OF FIGHER EDUCATION I POSTATTACK-OPERATIONS SOC/ SOCIAL INSTITUTIONS ANO THERMONUCLEAR WAR - A CA SE STUD O5-II -DI688

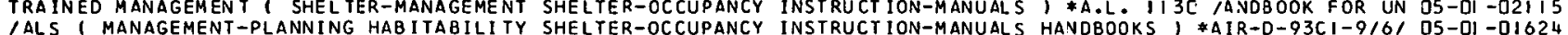
INAGEMENT SHELTER-OCCUPANCY PHYSIOLOGY PERFORMANCE (HUMAN) INSTRUCTION-MANUALS PSYCHOLOGY STRESSIPHYSIOLOG, IS-O3-D2298 
INAGEMENT SHELTER-OCCUPANCY PHYSIOLOSY PERFORMANCE (HUMAN) INSTRUCT ION-MANUALS PSYCHOLOGY STRESSIPHYSIOLOG I S-O3 -D23OO ILAT ING-EQUIP TER -OCCLPANCY PSYCHCLOGY PERFORNANCE (HUMAN) INSTRUCT ION-MANUAL WATER-SUPPL IES 1 *GARO-12927 I5-O3 - 2297 ILATING-EQUIPME , VENTILATION KIT - ASSEMBLY AND OPERATING INSTRUCTIONS I FALLOUT-SHELTERS COOLING-AND-VENT I3-OI -DI769
ES STOCKPILING, BASIC COURSE IN EMERGENCY MASS FEEDING - INSTRUCTORS GUICE I KITCHEN-EQUIPYENT-AND-SUPPLI OS-DS-DI7I3

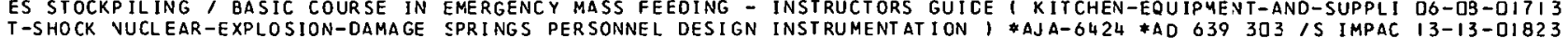

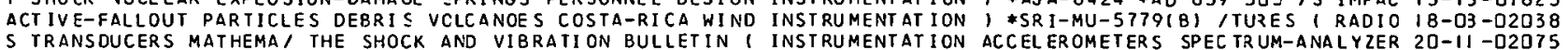
5 TRANSDUCERS MATHEMA I THE SHOCK AND VIBRATION BULLETIN I INSTRUMENTATION ACCELEROMETERS SPEC TRUM-ANALYZER 2O-II -D2O75
ISE SURVEY I TEST-EQUIPMENT IMPACT-SHOCK SHOCKIMECHANICS) INSTRUMENTATION CAL IBRATION AVALOG-COMPUTERS FO/ IL-D2-DI842 /UCTURES ROCK (GEOLOGY) STRESSES ELASTICITY THEORY GEOLOGY INSTRUMENTAIION CEFORMATION LOADIVG(MECHANICS), I3-I3-D2275 CTS RADIOACTIVE-FALLOUT DISTRIBUTION DOSE-RATE GANNA-RAYS INSTRUMENTATION EXPERIMENTAL-DATA I \&CEX-62.8OC I 8-D3-D2324 (S) STRESSES THEORY DEFORMA TION EXPERI NENTAL-DATA GEOLOGY INSTRUMENTAT ION GEOLOGY OPEVIVGS UVDERGROUND-ST/ O8-D7-O2244 IITY I BIOLOGICAL-BLAST-EFFECTS BLAST-BI OLOGY SHOCK-TUBES INSTRUMENTATION PRESSURE-GAGES-CALIBRATION PRES/ D6-2I -D2232 ITAL-CONTROL EXPERIMENTAL-DATA HABITABILITY FANS PORTABLE INSTRUMENTATION PUNKAH-PUMPS, \#GARD REPORT I26, I3-DI-DI77I 1 OF AN AUTOMAT IC REMOTE RADIOLOG ICAL MONITORING SYSTEM I INSTRUMENTAIION RADIATION-MONITORS DETECTION TEI IB -D I O2O26 IISION I METMATC REMOTE RADIOLOGICAL MONITORING SYSTEM I INSTRUMENTAT ION RADIATION-MONITORS DETECTION TE I I8-O+-O2O26 FIT

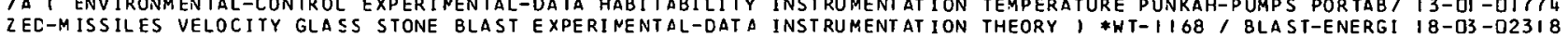

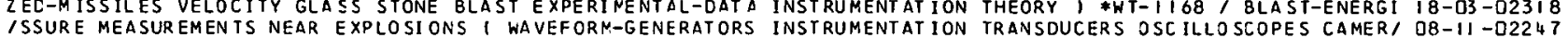
ION LABOR STATE-GOVERNMENTS ADMINISTRATI ON-AND-MANAGEMENT INSURANCE, \#USOL-16I /PENSATION LAWS, LEGISLAT O5-OZ-O2I 74 NG-PLANS REPAIR CONSTRUCTION REAL-ESTATE REFUGEES EANKING INSURANCE LABOR LAN TRAFFIC J IDA HP O5- $4 I 42$ II O5-O3-OIO36 IOUENCY PHASE VERY-LOW-FREQLENCY, EXTREMELY-LCW-FREGUENCY INTEGRAL-TRANSFORMS ATYOSPHERE-MODELS TRANSFORM/ 2D-I +-O234 9 AGEMENT-PLANN ING TRA INING SHELT/ AN EXPERINENTAL STUDY OF INTEGRATED GU IDANCE FOR SHELTER MAVAGEMENT 1 MAN QS-DI -OIG26 FALLOUT-SHEI AV INVESTIGATION OF SCHOOL DESIGNS TO RESIST INTEGRATED NUCLEAR WEAPONS EFFECTS 1 STRUCTURES I $3-13$-DI82I ION OF THE MECHAN ISMS ASSOCIATED WITH GAS BREAKDOWN UNDER INTENSE OPI ICAL ILLUMINATION 1 GAS- IOVIZATION B/ 2O-DS-O2O6O ION HIGH-T/ TEMPERATURES ATIAINED IN WOOD EXPOSED TO HIGH INTENSITY THERMAL RADIATIOV I CAL.JPIYETERS IGNIT I3-1? -DI8D2 E REACTION/ SPECULATION ON THE STRUCTURE CF INTERPERSONAL INTENTIONS I BEHAVIOR MOTIVATION ZULTURE RESPONS OS-IJ-O2I 79 IN A CUCT I ACOUSTIC S GAS-FLOW WEST-GERMANY DUCTS LAMINAI INTE

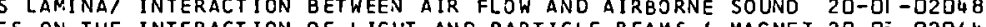

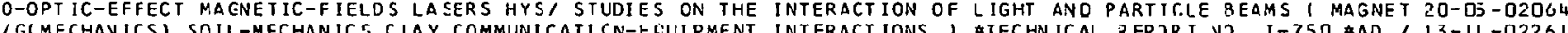

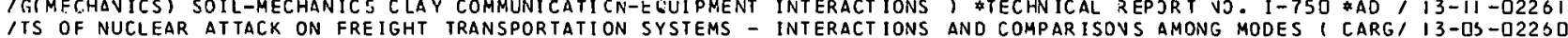
TS OF NUCLEAR ATTACK ON FREIGHT TRANSPORTATION SYSTEMS - INTERACT IONS AND COMPARISOVS AMONG MODES 1 CARG I I3-OS-O226D

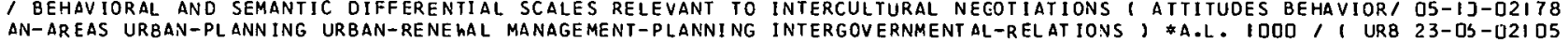
MPLOYMENT 1 INDUSTRIES MANPOWER ECONO/ PRCJECTIONS 1970 - INTERINCUSTRY RELATIONSHIPS, POIEVIIAL DEMANO, E OS-O3-OIO49 1 AND TWJ-FOOT SHOCK TUBES - EXPERIMENTAL OBSERVATIONS OF INTERIOR PRESSURES IN HOLLOW MODELS 1 SHOCK-WAV/ I8-O3-O2OOI 1 AND TWO-FOOT SHOCK TUBES - EXPERIMENTAL OBSERVATIONS OF INTERIOR PRES SURES IN HOLLOW MODELS I SHOCK-WAVI I8-O3-O2DDS S II. THEORY ( GUN-AUXILIARY-EQUIPMENT RECOIL-MECHANISMS INTERIOR-8ALLISTICS, \$AC 1 II 482 IRAKE DES IGN I AR TILLERY COMPUTER S GUN-BARREL-ATTACHNENTS INTERIOR-BALL IST ICS BIBL IOGRAPHIES DIGITAL-COMPI I9-OS-O2329 RREL-ATTACHMENTS INHIBITION PRESSLRE STRESSES PROJECTILES INTERIOR-BALL ISTICS GAS-FLOW, TO VIETNAM/ SOL IOARITY IS THE RANNER - TWO RESOLUTIONS ON INTERNATIONAL AFFAIRS 1 ARMSIENCE RESEARCH BY PRIVATE SCHOLARS AND ACADEMIC CENTERS - INTERNATIONAL AFFAIRS 1 BIBLIOGRAPHIES ATOMIC-E/ O5-II-OIG93 ILOEY AND PHILOSOPHY OF SCIENCE - PROCEEDINGS OF THE 1964 INTERNAT IONAL CONGRESS, SYMPJSIA MATHEMAIICAL-/ I2-DI -D225O ERNAT IONAL-AFFA IRS TARIFFS IMPORT-DUTIES FORFION-TRADE C/ INTERNAT IONAL CUSTOMS JOURNAL - LAVADA I LAW INT OS-O3-DIOUI ERNAI IONAL-AFFA IRS TARIFFS IMPORT-DUTIES FORFIISN-TRADE C/ INTERNAT IONAL CUSTOMS JOURNAL - LAVADA I LAW INT OS-O3 -OIG4 I
AFFAIRS, \#EXTERNAL RESEARCH PAPER IS6 SOVIET IMPACT ON INTERNATIONAL LAH I USSR COMMERCE INTERNATIONAL- O5-II -DIOSD

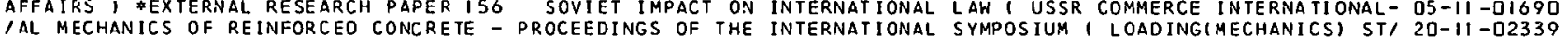

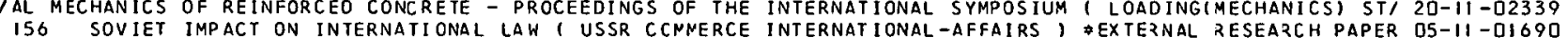

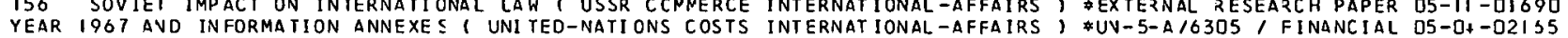

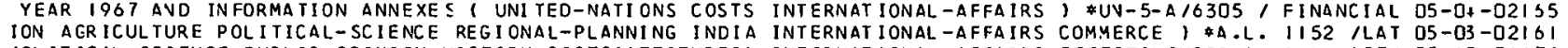
IOL I ICAL-SCIENCE PUBL IC-OPINI ON HISTORY COSTS (ATIITUDES) INTERNAT IONAL-AFFAIRS EFFECTIVENESS ) *A.L. IDT/ O5-IJ-QIOTO IC OPINION TODAY ( UNITED-STATES QUESTIONNAIRES ATTITUDES INTERNATIONAL-AFFAIRS FEAR SUPVEYS STRESSIPSYCH/ OS-II - DIGBQ IRN EURDPE 1 BIBL IOGRAPHIES CONMUNISM ECONOMICS EDUCATI ON INTERNAT IONAL-AFFAIRS GEOGRAPHY PJLITICAL-SCIEN/ O5-II-DIOQ7 /CONOMICS CONFL IC T-RE SOLUTION COMNLNIST-CHINA HISTCRY LAW INTERNAT IONAL-AFFAIRS METHODOLOGY POLITICAL-SCI/ OS-DH -OIOS5 IONS PROFESSIONS COMMUNICATION-SYSTEMS TRAINING ECCNOMICS INTERNAT IONAL-AFFAIRS POL ITICAL-SCIENCE, \#A.L., OS-II -02I 4 I ISRAEL, AND THE UNITED NATIONS AT FIVE POINTS IN TIME 1 INTERNATIONAL-AFFAIRS POLITICAL-SCIEVCE FOREIGN/ O5-C4-O2I TO 1 COMMUN IST-CHINA ECOVOMICS CONFLIC T-RE SOLUTION HISTORY INTERNAT IONAL-AFFAIRS POL IT ICAL-SCIEVCE METHODO/ OS-OH-DIOS2 I CONOMICS COMMUVIST-CHINA CONFLICT-RE SOLUTION HISTCRY LAW INTERNAT IONAL-AFFAIRS POL ITICAL-SIIEVCE METHODO/ OS-OH-DIOS3 1 COMMUNIST-CHINA ECONOMICS CONFLICT-RESOLUTION HISTORY INTERNAT IONAL-AFFAIRS POLITICAL-SCIENCE METHODOI DS-D +-DIOG 4

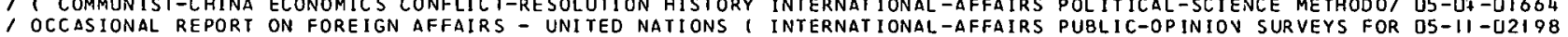

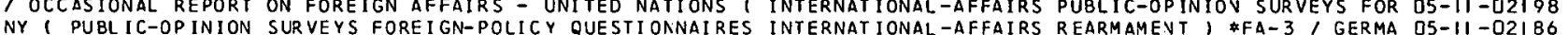

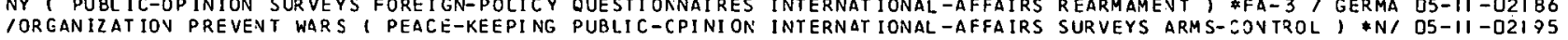

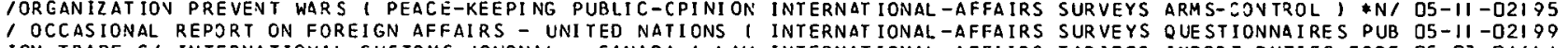
IGN-TRADE C/ INTERNATIONAL CUSTOMS JOURNAL - CANADA I LAW INTERNATIONAL-AFFAIRS TARIFFS IMPORT-DUTIES FORE QS-O3-OIG I IN IN THE RURAL AREA I USSR CIVIL-DEFENSE-SYSTEMS RESCUES INTERNAT IONAL-C IVIL-DEFENSE CHEMICAL-WARFARE-AG/ IS-O3-OIOI 8 IOR PROTECTION AGAIVST WEAPONS OF MASS DESTRUCTION I USSR INTERNATIONAL-CIVIL-DEFENSE CIVIL-DEFENSE-SYSTE/ I3-I3-DIB26 IRE OF PROTECTION AGAINST NUCLEAR WEAPONS I USSR SHELTERS INTERNAT IONAL-C IVIL-DEFENSE CIVIL-DEFENSE-SYSTE/ I5-D3-QI934 S RURAL-A/ SOVIET MILITARY TRANSLATIONS NUMBER I78)/ USSR INTERNATIONAL-CIVIL-DEFENSE CIVIL-DEFENSE-SYSTEM IS-O3-DIQ28 5 EA/ CIVIL DEFENSE IN THE UNI TED KINGDOM I GREAT-8RITAIN INTERNAT IONAL-CIVIL-DEFENSE CIVIL-DEFENSE-SYSTEM IS-D3-023O5 STRI' CRISES CIVIL DEFENSE AND DE TERRENCE I USSR MCVEMENT INTERNAT IONAL-C IVIL-DEFENSE COMMUVIST-CHINA INDU IS-O3-D229S DE ITIVE CONTAMINATION I USSR CIVIL-DEFENSE-SYSTEMS SHELTERS INTERNAT IONAL-CIVIL-DEFENSE RADIOLOGICAL-CONTAMI OG-IS-OIT3I

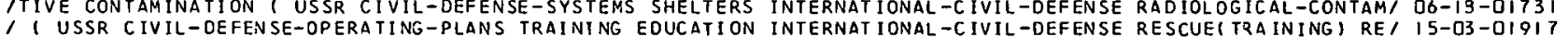
ICEFENSE AND DETERRENCE I VULNERABILI TY MILI TARY-STRATEGY INTERNAT IONAL-C IVIL-DEFENSE RUSSIA DAMAGE-LIMIT/ IS-D3-OIB96 /CEFENSE AND DETERRENCE 1 VULNERABILI TY MILI TARY-STRATEGY INTERNAT IONAL -C IVIL-DEFENSE RUSSIA DAMAGE-LIMIT/ I5-D3-OI896

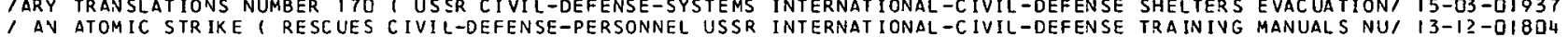
I ARY TRAVSLATIONS NUMRER 385 I IIS SR IIVIL-DEFENSE-SYSTEMS INTERNAT IONAL-C IVIL-UEFENSE TRAINIVG RESC UES PUI IS-D3-U23O2 GREAT-B/ WORLD WIDE CIVIL DEFENSE I CIVIL-DEFENSE-SYSTEMS INTERNATIONAL-C IVIL-DEFENSE WESTERV-EUROPE NATO I5-D3-D2I 7 IFLICT ARTIFICIAL-REALITY CONFLIC T-RE SOLUTION COOPERATION INTERNAT IONAL-RELATIONS ARMS-CONTZJL BEHAVIOR I/ GS-1J-D2I83 ULTURE RESPOVSE REACTIOV/ SPECLLATION ON THE STRUCIURE OF INTERPERSONAL INTEVT IOVS 1 BEHAVIJP YOTIVATION C OS-IJ-O2I 79 OSIONS - PEACEFUL APPLICATIONS - CRATERS FROMLEM OF THE INTERRELATIONSHIP OF PRESSURE STREVGTH, SIZE AND I3-13-OI827 I RACI/ COMMUNICATIONS EQUIPMENTS AND SYSTEMS TO SUPPORT INTRASTATE CIVIL OEFENSE OPERATIOVS - CIRCA IOTO I7-OZ-O23I 3 ENT VULNERABIL I TY ECONOMIC-RECOVERY INDUSTRI AL-PROOUCTION INVENT CRIES BLAST, \#NRE-38/ITY DAMAGE-ASSESSM OS-O3-O2IS2 /PERATIOVS RECOVERY OATA-PROCE SSI NG I NDUSTRI AL-PROCUCTION INVENTORIES CAPACIIY PROGRAMYINGI COMPUTER SI I \#/ O5-D3-O2I 44 E SI COMMODITY CREDIT CORPORATION WAREHOUSE RECEIPT WHEAT INVENTORY BY WAREHJUSE AND LOCATION I AGRICULTUR O2-O ?-O2IJQ E ST COMMODITY CREDIT CORPORATION WAREHOUSE RECEIPT WHEAT INVENTORY BY WAREHJUSE AND LOCATION ( AGRICULTUR O2-02 -02IJ9 RESEARCH

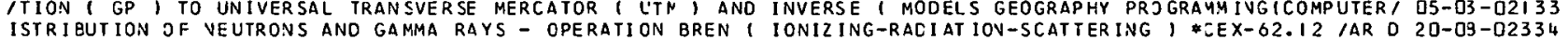
IN A DISTURBED TERRESTRIAL WAVEGUIDE I NUCLEAR-EXPLOSIONS IONOSPHERE ATMOSPHERE-YODELS WAVE-TRAYSMISSION I $20-1+-02348$ VALICITY OF THE Q2I-) MODEL FOR D REGION RECOMBINATION $($ IONOSPHERE OXYGEN GAS-IONIZATION, AIRBURST 1 ONS OL-DI -D2II 3 ICOMBINATION 1 IONOSPHERE OXYGEN GAS-IONIZATICN, AIRBURST IONS POLAR-REGIONS ABSORPTION OZOVE REACTION-KI/ OL-OI-OZII 3

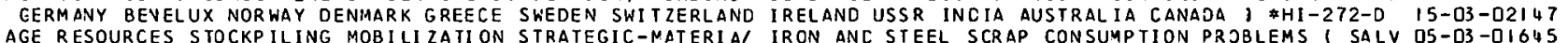


LFFEE CORN DEHYDRATED-FOODS EGGS FATS FROZEN-FOODS FRUITS IRRADIATED-FOODS MEAT MILK PORK POTATOES SEAFOOI D6-O3-U2209 SIBIL ITY-STUDIES SOLAR-RADIAIION EGYPT ENGINES-AND-MOTORS IRRIGATION-SYSTENS, \$P-3367 \$AD 638 687/TS FEA 13-02-UI777 IA GROUP OF POXVIRUSES FOR THE C YNOMOLSUS MONKEY, MACACA IRUS I BIOLOGICAL-NARFARE-AGEVTS PJX-VIRUSES MO/ I5-Q?-DI85S SI AN EVALUATIOV STUDY OF COMMLNI TY RESPONSE TO THE RHODE IS LAND HOME FALLOUT PRJTECTIOV SURVEY I ATIITUDE O5-I]-DI695 S/ AN EVALUATION STUDY OF COMMLNI TY RESPONSE TO THE RHODE ISLAND HOME FALLOUT PRJTECTIDV SURVEY I ATIITUDE OS-IJ-DI635 IN CONFLICT, DYADIC AND MEOIATED - CASE STUDIES OF EGYPT, ISRAEL; AND THE UNITED NATIONS AT FIVE POINTS I/ D5-D\$-D2I70 GREAT BRITAIN AND CANADA I BIBLI OGRAPHIES FRANCE GERMANY ITALY FEXTERNAL RESEARCH LIST 3.25 /RN EUROPE, O5- II -QI694
ES RESOURCES DAMAGE-ASSESSMENT FOOD LIGHT MEDIC/ SLRVIVAL ITEM ANALYS IS PROGRAM I NUCLEAR-WARFARE CASUALTI DS-O3-O2I35 E BOMB AVD ATOMIC, THE ONLY THING HE HAVE TO FEAR IS FEAR ITSELF - WHAT THE PEOPLE KVOW AND THIVK ABOUT TH OS-II-D2I 96

IE PREL IMINARY OB SER VATIONS ON A HOSPITAL RESPONSE TO THE JACKSON, MISS ISSIPPI TORNADO JF MAREH 3 , 1966 I/ O6-I?-OI7I 4 INFERENCE ON EARTHQUAKE ENGINEERING II. TOKYO AND KYOTO, JAPAN I JAPAN SEISMOLOGY STRUCTURES SHOCK-WAVESI D8-II -02246 11966 (EUROPE FOOD-MARKETING RUSSIA AFRICA LATIN-AMERICA JAPAN GROSS-NATIONAL-PROCUCT CONSUMPTION(FOOD) $/$ O2-02-DIOI6 IE-30, 1966 I RADIATION-EFFEC TS NUCLEAR-WARFARE-CASUALTIES JAPAN HIROSHIMA CATA NAGASAKI RADIATION-INJURIE/ D6-I3-OI730 IUBLISHED PAPERS OF THE ATOMIC BOMB CASUALTY COMMISSION ( JAPAN NUCLEAR-WARFARE-CASUALTIES I FABCC TR O-6/ O6-21-U2224 IANC ACADEMIC CENTERS - ASIA I BIBLIOGRAPHIES CHINA INDIA JAPAN PAKISTAN PHIL IPPINES I \#EXTERVAL RESEARCHI DS-II -DI698 IFFECTS ECOLOGY WIND RADIOACTI VI TYIDISTRIBUTICN) RECOVERY JAPAN RAOIOACTIVE-FALLOUT THERMAL-2ADIATION RIOI OG-2I -J2228 1 ON EARTHQUAKE ENGINEERING I1. TOKYO AND KYOTO, JAPAN 1 JAPAN SEISMOL OGY STRUCTURES SHOCK-WAVES ELASTIC/ OB-1I - Q22LO - CASUALTIES STATISTICAL-ANALYSIS MEAPONS-EFF/ ANALYSIS OF JAPANESE NJCLEAR CASUALTY DATA I VUCLEAR-WARFARE O6-2I-D222 7 -CASUALTIES CASUALTIES(PREDICTION) URBAN-ARE/ ANALYSIS OF JAPANESE NJCLEAR CASUALTY DATA I VULLEAR-WARFARE DG-2I -O2238 /F. THE PROPOSED EAST WEST FREE HAY TUNNEL WEST ORANGE, NEW JERSEY, AS A CIVIL DEFENSE PUBL IC SHELTER FACILI I $3-13$-D22 7 I /LOW-CHARTING P SYCHOMETRICS TECHNICAL-INFORMATICN-CENTERS JOB-ANALYSIS STATISTICAL-ANALYSIS MANAGEMENT-EN/ OS-02-O2I 20 AREAS FIRES MAPS TEST/ THE CONFLAGRATION POTENTIAL IN SAN JOSE AND ALBUQUERQJE 1 AERIAL-PHOTJGRAPHS URBAN- I 3-I2-OI8O9 IFFS IMPORT-DUTIES FOREIGN-TRADE C/ INTERNATIONAL CUSTOMS JOURNAL - CANADA I LAW INTERVATIOVAL-AFFAIRS TAR O5-D3-QIOLI LEAR-WARFA' ATOMIC BOMB CASUALTY COMMISSICN ANNUAL REFORT JULY-1, 1965-JUNE-30, 19661 RADIATIOV-EFFECTS NUC O6-13-DI730

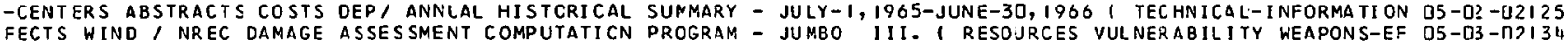

K

S SUBJECT-INDEXING DEPARTMENT-OF-DEFENSE DOCUMENTATICN E/ KEY WORDS AND THEIR USE ON DD FORY I473 I REPORT D5-O2-D2I 44 VANIA HIGH-SPEED-GROUND-TRANSPORT RE SEARCH-PROGRAN-ADMIN/ KEYSTONE CORRIDOR TRANSPORTATION STUDY I PENNSYL $23-0 ?$-O2O92 ITIFIC-RESEARCH ABLATION ANTENNAS PROPAGATION SHOCK-WAVES KINET IC-THEORY ELECTROV-DEVSITY CEZENKOV-RAOIAT/ 20 -03-02330 NSE CIVIL-DEFENSE-SYSTEMS EA/CIVIL DEFENSE IN THE UNITED KINGDOM I GREAT-BRITAIN INTERNATIONAL-CIVIL-DEFE I5-O3-O23OS ONUCLEAR ATYACK SOCIETY - SOME EXPLORATORY STUDIES I POSI KINSHIP AND VOLUNTARY ORGAVIZATIOV IV POST THERM IS-D3-O23OI

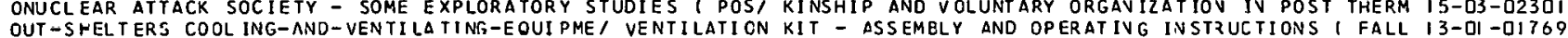

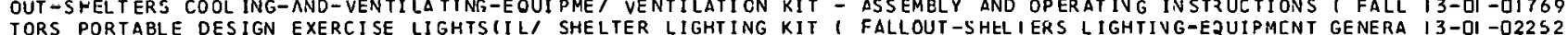

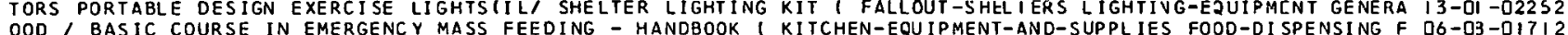
OOD $/$ BASIC COURSE IN EMERGENCY MASS FEEDING - HANDBOOK 1 KITCHEN-EQUIPMENT-AND-SUPPL IES FOOD-DISPENSING F O6-O3-0I7 I 2
IC COURSE IN EMERGEVCY MASS FEEDING - INSTRUCTORS GUIDE 1 KITCHEN-EQUIPMENT-AND-SUPPL IES STOCKPILING FOOD/ D6-UB-DI7I 3

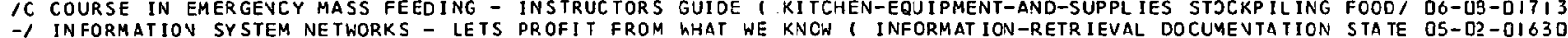
IY THING WE HAVE TO FEAR IS FEAR ITSELF - WHAT THE PECPLE KNOW ANC THINK AROUT THE BOMB AND ATOMIC ENERGYI DS-II-O2I 76 UT-SHEL TERS PUBLIC-OPINION ATTITU/ PUBLIC INFORMATION AND KNOWLEOGE REQUISITES OF A SHEL TER SYSTEM I FALLO DS-II -OIG99 ECONOMICS DECISI PLANNING IN A CAPITAL SURPLUS ECCNOMY - KUWAIT I MANAGEMENT-PLANNING JPERATIONS-RESEARCH O5-03-OIO3S IORLD CONFERENCE ON EARTHQUAKE ENGINEERING I1. TOKYO AND KYOTO, JAPAN I JAPAN SEISMOLOGY STRUCTURFS SHOC/ D8-II-O2246

TION FINANCIVG OPERATING-COSTS CANADA CONSTRUCTION PRICES LAECR, *A.L. IQI7 /TORAGE SALES $2 E$ VEVUE UTILIZA O5-O3-QI642 IAL AFFAIRS 1 B IBLTOGRAPHIES A TOMIC-ENERG Y CCMMUNI CATIONS LABOR COMMUNISM ECJNOMICS AGRICULTURAL-ECONOMIC/ O5-II -DI693 / I INDUSTRIES RESOURCES(HUMAN) DISTRIBUTION VULNERABILITY LABOR ECONOMICS ECONOMIC-RECOVERY POSTATTACK-DP/ O5-03-DI639 EPAIR CONSTRUCTION REAL-ESTATE REFLGEES BANKING INSURANCE LABOR LAW TRAFFIC, \$IDA HQ 65-4I42, /ING-PLANS R DS-03-0IO36 1 I URBAN-AREAS HASTY-SHELTER S DESIGN MA TERIALS EQUI PMENT LAROR MCBILIZ AT ION MANAGEMENT-PLAVNING CALCILATI I3-I3-D2272 ISOCIAL -P SYCHOLOGY AGING OCCUPATI ONS PROFESSIOA' MANPOWER RECOVERY POSTATTACK-OPERATIONS TUR RECOVERY POSTATTACK-OPERATIONS SURVI VAL ECONOMIC-RECOVERY LABOR RESOURCES 1 *RM-3436-PR /TIOV 1 ECONOMICS O5-03-02127 EMENT INSII STATE WORKMEN COMPENSATION LAWS 1 LEGISLATION LABOR STATE-GOVERNMENTS ADMINISTRATION-AND-MANAG O5-DZ-O2I 74

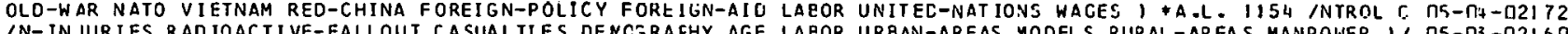
/N-INJURIES RAD IOACTI IE-FALLOUT CASUALTIES DENCSRAFHY AGE LAEOR UREAN-AREAS MODELS RURAL-AREAS MANPOWER I/ O5-D3 -02I 60 /ARE-AGEVTS SPORES MEDICAL-EQUIPMENT ELECTRICAL-EQUIPMENT LABORATORY-EQUIPMEVT DECOVTAMINATIOV I \#AD 638 I IS-OZ -UI892 I AN ARC IMAGING FURNACE I THERMAL-RADIATION CALORIMETERS LABORATORY-FURNACES RADIOMETERS HEAT-FLUX HEAT-I I3-DI -02255 NAMIC-PROPERT IES DRAG SURFACE-TEMPERA TURES BOUNDARY-LAYER LANINAR-EOUNDARY-LAYER, *R-273 *AD 80305 /D-DY $22-02-02350$ ' SOUND IN A DUCT ' ACOUSTICS GAS-FLOW WEST-GERMANY DUCTS LANINAR-BOUNDARY-LAYER STAB ILITY YEASUREMENT, I $20-0 I-02048$ IAT ION URBAN-AREA S MODELSISIMULATIONS) PASSENGER-VEHICLES LAMINAR-FLOW TURBJLENT-FLOW, \$DS?-76IO8-I *PB-/ $23-0$ ? -02IOI RUSSIA RADIO/ RADIONUCLIDE FRACTIONATION IN DEBRIS FROM A LAND SURFACE BURST I RADIOACTIVE-ISOTOPES CHINA I8-D?-OI99I IS OF FACTORS AFFECTING GOVERNMENI CONTROL IN SOUTH VIET/ LAND TENURE AND REBELLION - A STATISTICAL ANALYS OS-07-02I75 IREAS DECENTRAL IZATION TAXES VLLNERABILITY URBAN-PLANNING LAND-USE POPJLATIOY TRANSPJRTATIOV UTILITIES FI/ $23-0 S-02 I$ OG IE I ECOVOMICS URBAN-AREAS DECENTRALI ZATI CN VULNERABILITY LAND-USE URBAN-PLAVVING POPULATIOV PJLITICAL-SC, 23 -OS-O2I T IICAL-DOSAGE STORMS DECUNIAMINATICN EARTHCUAKES VOLCANOES LANDSLIDES ELECTRIS-POWFR-PRODUCTION PETROLEUM I I5-O3-O2209 GRAMM ING LANGUAGES COMPUTERS CASUALTIESI REACT A REOUEST LANGUAGE LAH LITERATURE PHILOSOPHY PJPULATION PI 05-03-02154 DAH PUTERTURE PHILOSOPHY PJPULATION P/ 05-11-01697 IAT ION FJREIGN-POLICY GEOGRAPHY POLI TICAL-SCIENCE HISTORY LANGUAGE LAW PUBLIC-INFORMATIJV PHILOSOPHY RELI/ O5-II -DI696 NNDUSTRIES COMMERCE EOUCATION POLITICAL-SCIENCE GECGRAPHY LANGUAGE MILITARY-AFFAIRS PHILOSOPHY LAW POPULAI D5-II -DI673 INTION F

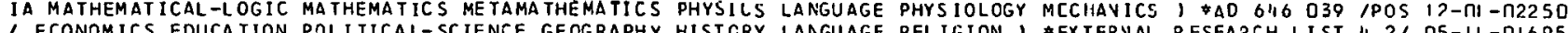
I ECONOMICS EDUCA TION POLI TICAL-SCIENCE GEOGRAPHY HISTCRY LANGUAGE RELIGION I *EXTERVAL RESEARCH LIST 4 . $2 /$ OS-II -DI695 ISONATORS II BEHAVIOR OF IHE OUTPUT FRON A MANY CLCMENT LASER I LASERS OPTICAI-FQIUIPMENT-ZJMPONENTS OPT/ 20-OJ-D2O57 IOF SEVERAL OPTICAL NONLINEARI IIES USING FOCUSED GAUSSIAN LASER BEAMS I CRYSTALS OPTICAL-PHENOMENA HARMON/ $20-0 S-02067$ II. BEHAVIOR OF THE OUTPUT FROM A MANY ELEMENT LASER I L/ LASER CAVITIES I. RESEARCH OV OPEV RESONATORS 2D-O5-D2OS 7 $S$ OPT ICAL -PROPERTIES RUBY CRY/ NRL PROGRESS IN HIGH POWER LASER RESEARCH I LASERS SCIEVTIFIC-RESEARCH GLAS $20-05-020 S 2$ CS CRYSTI INVESTIGATIONS OF GIANT PULSING NETHODS IN RUBY LASERS I PJMPINGIOPTICALI CAVITY-ZE SONATORS OPTI 2O-OJ-D2OSG ICAL ILLUMINATION I GAS-IONIZATION BEAMS IELECTROMAGNETIC) LASERS HELIUM-GROUP-GASES PLASMA-MEDIUM LUMINESI 20-DS-D2O6O

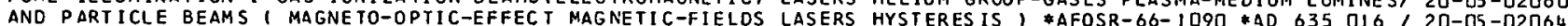
/RYSTALS OPTICAL-PHENOMENA HARMONIC-GENERATORS REFRACTION LASERS NONLINEAR-SYSTEMS AMMOVIUM-ZJMPOUNDS FOC/ $20-0 S-02057$ 1 II. BEHAVIOR OF THE OUTPUT FRON A MANY ELEMENT LASER I LASERS OPT ICAL-EQUIPMENT-COMPONENTS OPIICS, *A/ 20-D5-U2O57 IES RUBY CRY N NRL PROGRESS IN HIGH POWER LASER RESEARCH I LASERS SCIENTIFIC-RESEARCH CLASS OPTICAL-PROPERT 2O-OS-O2OS2 ELTERS I SHELTER-OCCUPANCY METABOLISM PHYSI/ SENSIELE ANO LATCNT HEAT LOSSES FROM OCCUPANTS JF SURVIVAL SH O6-1S-DI7I8 ELTERS I HEAT-PRODUCTION (BIOLOGY) SURVIVAL, SENSIRLE AND LATENT HEAT LOSSES FROM OCCUPANTS OF SURVIVAL SH D6-1S-OI7I8 IOTS I HEAT-PRODUC TION (BIOLOGY) SURVIVAL SENSIELE AND LATENT HEAT LOSSES FROM OCCUPANTS JF SURVIVAL SH D6-IS-DI7I9

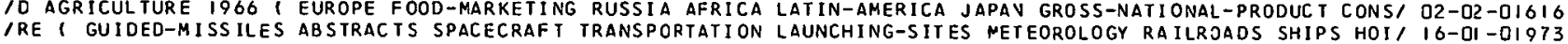
/L-ANALYSIS ELASTICITY BEAMSISTRUCTURAL) SHOCK(MECHANICS) LAUNCHING(SIMULATION) ACOUSTICS VIBRAIION-ISOLA/ 2D-II -D2O74 STORY INTERVATIONAL-AFFAIRS POLITICAL-SCIENCE METHCDOLOGY LAW, ACC-6 /A ECONOMICS CONFLICT-RESOLUTION HI O5-O+-OIGS2 
STORY INTERNATIONAL-AFFAIRS POLITICAL-SCIENCE METHCOOLOGY LAW, \#ACD-7 /A ECONOMICS CONFLICT-RESOLUTION HI O5-OH-DIO64 ES-GOVERNMENT POL ITICAL-SCIENCE ECONOMICS DECISION-MAKING LAW ) \$P-2289 \$AC 634797 1-PLANNIVG UNITED-STAT 05-D4-01658 C-TRANSPORT PER FORMANCE (ENGINEERING) VEHI / UNI VERSAL ORAG LAW I TRANSPORTATION PROPULSIJN HIGH-SPEEU-GROUN $23-0 ?-02096$ ERNAL RESEARCH PAPER I56 SOVIET IMPACT CN INTERNATIONAL LAW I USSR COMMERCE INTERVATIJNAL-AFFAIRS I *EXT O5-II -DI69O $S$ IN A SILICATE FALLOUT PARTICLE NATRIX I RADIOAC/ HENRYS LAW CONSTANTS FOR OISSOLUTIOV OF FISSION PRODUCT IB-O3-D2325 IES ECONOMICS CONFL ICT-RESOLUTION COMMUNI ST-CHINA HISTORY LAW INTERNAT IONAL-AFFAIRS METHODOLOGY POLITICALI OS-DU-DI G6S IES ECONOMICS COMMUNIST-CHINA CONFLIC T-RESOLUTION HISTORY LAW INTERNATIONAL-AFFAIRS POLITICAL - SLIENCE MET/ OS-D $+-D I O 63$ FOREIEV-TRADE C/ INTERNATIONAL CUSTOMS JOURNAL - CANADA I LAW INTERNAT IONAL-AFFAIRS TARIFFS IMPORT-DUTIES DS-O3-DIG 4 I INAL-AFFAIRS GEOGRAPHY POLITICAL-SCIENCE HISTORY LANGUAGE LAW LITERATURE PHILOSOPHY POPULATIJN PROPAGANDA/ OS-II -DIGQ7 IALTH SERVICES DIVISION I DISASTER-MEDICINE PUBLIC-HEALTH LAW ORGANIZATION CIVIL-DEFENSE-SYSTEMS CASUALTI/ O6-2I -O2226 IL-SCIENCE GEOGRAPHY LANGUAGE MILITARY-AFFAIRS PHILOSOPHY LAW POPULATION PUBLIC-INFORMATION I *EXTERNAL RI D5-II - DIGQ3 1 GEOGRAPHY POL IT ICAL-SCIENCE HISTORY LANGUAGE PHI LOSOPHY LAW PUBLIC-INFORMATION I *EXTERNAL RESEARCH LIS/ D5-II -0I692 IEIGN-POL ICY GEOGRAPHY POLITICAL-SCIENCE HISTORY LANGUAGE LAW PUBLIC-INFORMATION PHILOSJPHY RELIGION I *E/ D5-II -DIG96 CONSTRUCTION REAL-ESTATE REFUGEES BANKING INSURANCE LABOR LAW TRAFFIC, \#ICA HQ 65-4I42 /ING-PLANS REPAIR D5-03-DI636 VIL/ U.S. CIVIL DEFENSE BEFORE I 95C - THE ROOTS OF PUBLIC LAW 920 I LEGISLATION SOCIAL-SCIEVCES HISTORY CI OS-O4-DIO7I ISTRATIOV-AVD-MAVAGEMENT INSLI STATE WORKNEN COMPENSATION LAWS I LEGISLAT IOV LABDR STATE-GOVERVMENTS ADMIN O5-O9-D2I74 IPUTER PROGRAM TO CALCULATE THE THERMAL HISTORY OF A DUAL LAYERED PLATE SUBJECT TO THE THERMAL PULSE OF A' I3-I?-OI797 BLIC-INFORMATION SURVEYS DEMOGRAPHY COMMUNICATICN-SYSTEMS LEADERSHIP, *A.L. IO55 /TUDES PUBLIC-OPINION PU D5-IJ-01683 NAGEMENT-PLANNING PERSONNEL SHELTER-OCCUPANCY(SIMULATION) LEADERSHIP

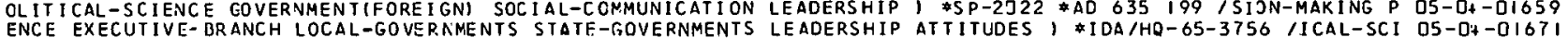

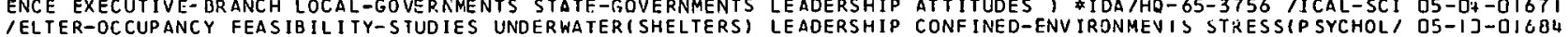
IDESCRIBED ( USSR MILITARY-TRAINING ARMED-FORCES (FCREIGN) LEADERSHIP MILITARY-PERSONVEL OFFIIEZ-PER SONNEL/ OS-OP-D2I T6 SPECT ION OF THE UNITED STATES DEPARTMENT CF AGRICULTURE I LEGISLATION ICAMS-SRA-I88 IVERNING THE MEAT IN O2-O2 - IIG20 (ITARY-GOVERNMENT EMERGENCY-PLANNING (GOVERNMENT-AGENCIES) LEGISLAIION CIVIL-DEFENSE (GOVERNMENT-AGENCIES) I IS-D3-QI946 $S$, SELECTED IMPACTS OF RAILROAD NERGERS I TRANSPORTATION LEGISLATION COMPET ITION EMPLOYMENT TAXES SERVICE 23 -02-O2O95 ON-AN C-MANAGEMENT INSU/ STATE hOR KMEN CONPENSATION LAWS I LEGISLATION LABOR STATE-GOVERVMEVTS ADMINISTRATI O5-D7-D2I T4 IIVIL DEFENSE BEFORE 1950 - THE ROOTS OF PUBLIC LAW 920 I LEGISLATION SOCIAL-SCIENCES HIS IOZY EIVIL-DEFEN/ OS-OH-DI67I INICATIONS REQUIREMENTS AT THE LOCAL, STATE, AND REGIONAL LEVELS 1 COMMUNICATIONS-SYSTEMS COMMAVD-AND-CON/ I7-D?-O23I2 TAINEC WIT PROIEC/ IMMUNOLOGICAL STUDIES OF ANTHRAX II. LEVELS OF IMMUNITY AGAINST BAC ILLUS AYTHRACIS OB IS-D?-DIB53 TAINEC WIT PROTEC I MMUNOLOGICAL STUDIES OF ANTHRAX II. LEVELS OF IMMUNITY AGAINST BACILLUS AVTHRACIS OB I5-O2 - DI853

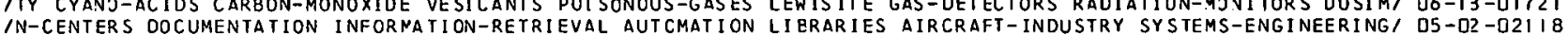

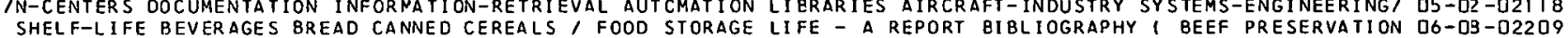

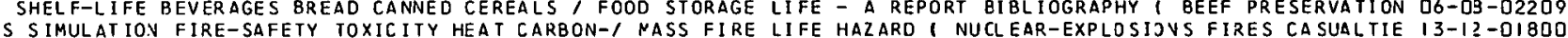
S SIMULATION FIRE-SAFETY TOXICITY HEAT CARBON-I NASS FIRE LIFE HAZARD I NUCLEAR-EXPLOSIJVS FIRES CASUALTIE I3-I?-OI8OD
IS MATHEYATICAL-MODELS/ VIABILITY AND ESIINATION OF SHELF LIFE OF BACTERIAL POPULATIOYS I BACILLLS-ANTHRAC IS-D?-OIB49 IS MATHEMATICAL-MODELS/ VIABILITY AND ESTINATION OF SHELF LIFE OF BACTERIAL POPULATIDVS $184 E$ ILLLS-ANTHRAC IS-D2 -DI849
CAL-DOSAGE EX/ LONG TERM RADIATIOA DAMAGE - EVALUATION OF LIFE SPAN STUDIES I RADIATION-INJUR IES RADIOLOGI OG-I3-DI733 JAPAN HIROSHIMA DATA NAGASAKI RACIATION-INJURIES RECOROS LIFE-SPAN, *ABCC-65-66/LEAR-WARFARE-CASUALTIES D6-IS-0I730 MAGNETIC-FIELDS LASERS HYS/ STLDIES ON THE INTERACTION OF LIGHT AND PARTICLE BEAMS 1 MAGNETO-JPTIC-EFFECT $20-03-02064$ CITIONS 1 LIGHT-TRANSMISSION COLORS OPTICAL-IMA/ STUDY OF LIGHT ATTENUATION JNDER SUBTRJPICAL ZLIMATIC ZON 20 -OS-O2OS6 ILEAR-WARFARE CASLALTIES RESOURCES DANAGE-ASSESSMENT FOOD LI GHT MEDICAL-SUPPL IES ELECTR IC-POWER SANITARY-I OS-D3 - D2I 35 IIGHT AIIENUATION UNDER SUBTROPICAL CLIMATIC CONOITIONS I LI GHT-TRANSMISSION COLORS OPTICAL-IMAGES OPTICA/ 2O-OS-O2O6G NT GENERATORS PORTABLE DESIGN EXERCISE LIGHTSIIL/ SHEITER LIGHTING KIT I FALLOUT-SHELTERS LIGHTING-EQUIPME I3-0I -O2252 $1 O L$ VENTILATIOV ELECTRIC-POWER-PRODUCTION HEATING COOLING LIGHTING WASTES (SAVITARY-EVGIVEERIVG), \#A.L. I' I3-13-O22?I NG DESIGV TEMPERA TURE-CONTROL ENVIRONMENTAL-CCNTROL COSTS LIGHTING-EQUIPMENT FIRES 1 \#AD 63I 424 /NGINEERI I3-13-0I8I9 ERCISE LIGHTSIIL/ SHELTER LIGHIING KIT I FALLOUT-SHELTERS LIGHTING-EQUIPMENT GENERATORS PORTABLE DESIGN EX I3-OI -D2252

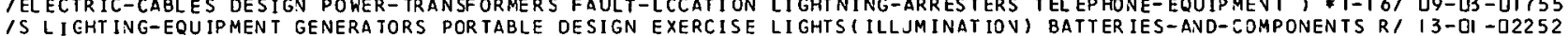
/STRETCHING COMPRESSING TESTING-ECUIPMENT MARBLE OCLOMITE LIMESTONE ROCK-SALT ANHYORITE SANDSTONES CLAY.SI OB-OT-D224 2 11. CALCULATIONAL TECHNIQUE FOR DETERMINING IMPORTANCE OF LIMITED STRIP DECONTAMINATION PROCEDURES I RADI/ O6-I3-O22I8 RY POST/ ECOVOMIC VIABILITY AFTER THERMONUCLEAR WAR - THE LIMITS OF FEASIBLE PRODUCTION 1 ECJVJMICS RECOVE OS-O3-O2I 7 SURVIVA/ ECONOMIC VIABILITY AF TER THERMONLCLEAR WAR - THE LIMITS OF FEASIBLE PRODUCTIOV I VULLEAR-WARFARE OS-D3-D2ISB S) STRUCTURES NONLINEAR-ANALYSIS BEAMSISTRUCTURAL) FRAMES LINEAR-STRUCTURES, \$ASCE-1965-50 \$ACI-SP-I2 /IC 20-II-O2339 TICAL-ANALYSIS ELASIICITY SHOCK-WAVES CONTINUUM-MECHANICS LINEAR-SYSTEMS FUNCTIOVS 1 \$AD 63141919 MATHEMA $20-11$-O2080 IF THE RESPONSE OF BACKPACKING MA TERI AL ENCASING A TUNNEL LINER SUBJECT ED TO A DYNAMTC DISTURBANCE ERT IES RUBY CRYOEN MAGE ASSESSMENT PROGRAMS I VULAR.ABITY, - SURVEYS OF CHINESE COMMUNIST SCIENTIFIC AND TECHNICAL LITERATURE I GUICED-MISSILES ABSTRACTS SPACECRAI IO-DI -OIS73 - SURVEYS OF CHINESE COMMUNIST SCIENTIFIC AND TECHNICAL LITERATURE
$S$ I GUIDEC-MISS ILES RAILROADS BRI OGES GROLND-SUPP/ SOVIET LITERATURE ON PROTECTIVE STRUC TURE AND COMPONENT IO-OI - QIST2 ITUCE NUCLEAR EXPLS RAILROADS BRIOGES GROLND-SUPP) SOVIET LITERATURE ON PROTECTIVE STRUCTURE AND COMPONENT I6-OI - QI972 ITUCE NUCLEAR EXPL BIBLIOGRAPHY CF OPEN AND UNCLASSIFIED LITERATURE PERTAINING TO THE EFFECTS OF HIGH ALT I8-03-O2OI 8 IAFFAIRS GEOGRAPHY POLIIICAL-SCIENCE HISTORY LANGUAGE LAW LITERATURE PHILOSOPHY POPULATION PROPAGANDA PUB/ O5-1I -01697
ERGRO/ THE ELASTIC RESPONSE OF BURIED CYLINDERS, CRITICAL LITERATURE REVIEW AND PILOT STUDY I SHELTERS UND I3- I3-OIB25 ERGRO/ THE ELASTIC RESPONSE OF BURIED CYLINDERS, CRITICAL LITERATURE REVIEW AND PILOT STUDY I SHELTERS UND I3-13-OI825
INT PUBLICATIONS, $1960-1965$ - AN ALTHOR INDEX OF THE OPEN LITERATURE WITH ABSTRACTS 1 BIBLIOGRAPHIES INDE/ OS-O3-DIOS2 1 BACILLUS AVTHRACIS OBTAINED HI TH PRDTER,TIVF ANTIGEN AND LIVE VACCINE I SPORES BIOLOGICAL-WARFARE-AGENTSI IS-D?-OIBS3 LTURAL-ECONOMICS, $F A S-M-186$ LTURAL-ECONOMICS, \&FAS-M-108 LTURAL-ECONOMICS, \#FAS-M-184 MPUTERS RADIOACTIVE RADIOAGRICLLTLRE, \#NREC-I3 URUGUAY LIVESTOCK AND MEAT INDUSTRY I AGR ILULTURE AGRICU D2-02-021 IO NEW ZEALAND LIVESTOCK ANO MEAT INDUSTRY AGR ICUL TURE AGRICU 02-02-02112 GY) AEE TROPICAL-REGIO/ MEAT TOLERANCE OF ELOERLY PERSONS LIVING IN A SUBTROPICAL CLIMATE I AGINGIPHYSIOLO D6-I7-OI734 IONCRETE BEAMS I BEAMSISTRUCTURAL) CONSTRUCTICN-MATERIALS LOAD-DISTRIBUTION DEFLECTION STEEL ROOS CRACKS / I3-I3-O2289 IORET ICAL AND EXPER IMENTAL INVESTIGATION OF A DYNANTCAI I.Y LOADED RING NITH RACIAL ELASTIC SUPPORT I RINGS/ $20-11$-02336 IOF SUBSURFACE SOIL INCLUSIONS SUB JECTED TO SURFACE BLAST LOADING - RESULTS OF SERIFS III TESTS I SHELTER/ O8-I3-OI74O IOF SUBSURFACE SO IL INCLUSIONS SUB JECTED TO SURFACE BLAST LOADING - RESULTS JF SERIES III TESIS I SOIL-ME/ I8-O3-02OJ8 ID PROTOTYPE COMMUNICATIONS CONDUIT TO STATIC AND CYNAMIC LOADING 1 OUCTS SAVD UNOERGROUND LOADINGIMECHAN/ I3- II -O226I DAT IONS (STRUCTURES) SOIL-MECHANICS LOADINGIMEC/ TRANSIENT LOADING TESTS ON A RIGID CIRCULAR FOOTING I FOUN I3-I3-OIBI 2 STRU CTURAL-PROPERTIES STEEL COMP (TER-PROGRAMS ELASTICITY LOADING(MECHANICS), *FEL-297.17*40 645 586 /ES I3-13-02287 STR IBUTION DEFLEC TION STEEL RODS CRACKS STRAIN(MECHANICS) LOAOING(MECHANICS)

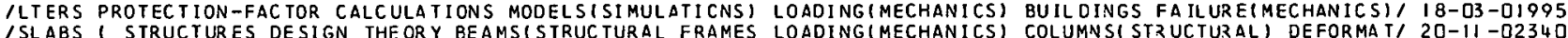
IES ELASTICITY THEORY GEOLOGY INSTRUMENTATION DEFORMATION LOADINGIMECHANICSI CONSTRUCTION STATISTICAL-ANA/ I3-I3-O2275 IOUND-STRUCTURES ROCK(GEOLOGY) HEAIING MINING-ENGINEERING LOAOING(MECHANICS) COOL ING FAILURE(MECHANICS) S/ I3- 3 - D2273 IIMENTAL INVESTIGATION OF FRANGIBLE PLATE FRAGMENTATICN ( LOADING (MECHANICS) CRACKIVG(FRACTUR IVG) FRACTUR/ $20-1$ I -U2338 IMECHANICS DYNAMICS GROUND-MOTION UNDERWATER DESIGN COSTS LOADING(MECHANICS) CYLINDERS) \$SD-7I-66(R)-587/ I8-DZ-D204D IIRCULAR FODTING I FOUNDATIONS (STRUCTURES) SOIL-MECHANICS LOADING (MECHANICS) DYNAMICS EXPER IMENTAL-DATA CI I3-I3-OIBI IRETE SHEL I SHELLSISTRUC TURAL-FORMS) MENBRANES STRESSES LOADING(MECHANICS) FAILURE(MECHAN ICS) DESIGN COI I3-I3-DIB32

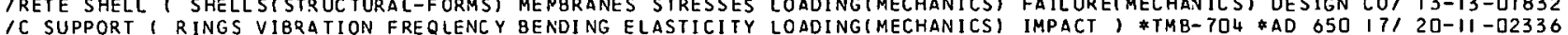

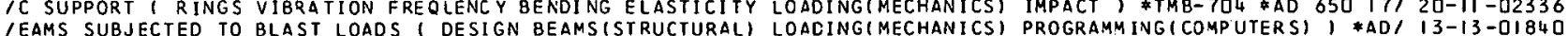
IEAMS SUBJECTED TO BLAST LOADS I DESIGN 8EAMS (STRUCTURAL) LOACING(MECHANICS) PROGRAMMING (COMPUTERS) I *ADI I3-I3-OI840
IW SP TERICAL SHELLS I SPHERES STRLCTURAL-SHELLS VIBRATION LOADINGIMECHANICS) SHEAR-STRESSES DEFORMATION RI 2O-II -O2346 IWORCED CONCRETE ARCHES I UNDERGROUND-STRUCTURES DYNAMICS LOADING(MECHANICS) SHELTERS 1 IMISC. PAPER NO. $/$ I3-I3-0I830 INAM ICS SHEAR-STRESSES WEBSISTRUC TLRAL) DESIGN STRUCTURES LOADING(MECHANICS) SHOCK-NAVES MATHEYATICAL-ANA/ 20-II-02337 IAT ICAL-YODELS DIFFERENTIAI-FQLATIONS EQUATIONS-CF-MCTION LOADINGIMECHANICS) SHOCK-WAVES NULLEAR-EXPLOSIO/ I3-I3-02279 
/ HANICS THEORY EXPERIMENTAL-DATA STRAIN(MECHANICS) STRESS LOAOING(MECHANICS) SHOCK-WAVES VISLOELASTICITY / 20-II -02096 IROUND-STRUCTURES EXPANDED-PLASTICS GLASS HONEYCOMB-CORES LOADING (MECHANICS) SHOCK(MECHANICS) ) \#AD 634 6/ I8-D3-O2OD7 IOTECTIVE STRUCTURES I UNDERGROUD-STRUCTURES CONSTRUCTION LOADING(MECHANICS) SHOCK(MECHANICS) FEASIBILITY/ I8-O3-020I 7 TT TO STATIC AND DYNAMIC LOADING ( DUCTS SAND UNDERGROUND LCADINGIMECHANICS) SOIL-MECHAYICS CLAY COMMUNIC, I3-II -O22GI IMIC BEARING ANESTS ON A STRIP FOOTING IN SATURATED SAND ( LOADINGIMECHANICS) SOIL-MECHAVICS DYVAMICS SHELI, $20-11$-O23 4 I

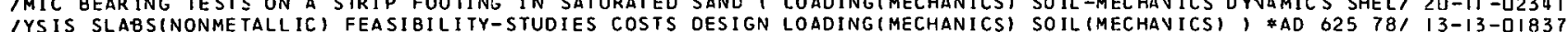
IYSIS SLABSINONMETALLIC) FEASIBILITY-STUDIES COSTS OESIGN LOADING(MECHANICS) SOIL (MECHAVICS) I \$AD O25 78, I3-13-0I837 IC CONCRETE BEAMS II. I TEST-EQUIPMENT BEAMS(STRUCTURALI LOADING(MECHANICS) STATICS I \#TR-502 *0ASA-13.0/ I3-13-0184I /RAL I REC.TANGUL. AR-BODIES STRESSES THRUST THERMAL-STRESSES LOADING(MECHANICS) STRAIN(YECHANIES) BUCKLING (M/ 20-II -02344 /SHEL TERS UNDER GR OUND-STRLCCTLRES ELASTICI TY TEST OYNAMICS LOADINGIMECHANICS) STRAIV(MECHANIZS) THEORY I \#/ I3-I3-01825 / CONCRETE - PROCEED INGS OF THE INTERNATI ONAL SYMPCSIUM I LOADING(MECHANICS) STRUCTURES NONLINEAR-ANALYSII $20-11$-O2339 10 BY AIR BLAST ' ELASTICITY SHOCK-WAVES MECHANICAL-WAVES LOADINGIMECHANICS) SURFACE-BURST ACCDUSTICS STR/ I9-04-02044 (MECHANICS) VULNERAB ILI TY NUCLEAR-E XPLOSI ONS SHOCK-WAVES LOAOING (MECHANICS) SUSPENSION-DEVICES STR UC TURE I I3-I3-D229I RS PE RESPONSE OF DEEP REINFORCED CONCRETE SLABS I TESTS LOADINGIMECHANICS) UNDERGRJUYD-STZUETURES SHELTE I3-I3-D2290 IOCKS AT HIGH TEMPERA TURES AND PRESSURES (ROCK-MECHANICS LDADINGIMECHANICS) USSR EXCAVATIOV SHEARING CUT/ DB-O7-D2242 URATEC SAND - CLAY MIXT/ THE RESPONSE OF SOILS TO OYNAMIC LOADINGS - WAVE VELOCITIES THROUGH PARTIALLY SAT $20-D I$-O2OL 7 I SECTION OF REINFORCED CONCRETE BEAMS SUB JECTED TC BLAST LOADS I DESIGN BEAMSISTRUCTURAL) LOADINGIMECHAN/ I3-I3-DI8LO ONCRETE UNDERGROUN/ MATERIALS FOR LSE IN NITIGATING BLAST LOADS ON CEEPLY EJRIED PRJTECTIVE STZUCTURES I C IB-O3-D2OD7

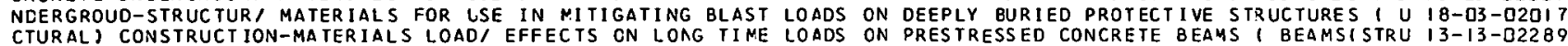

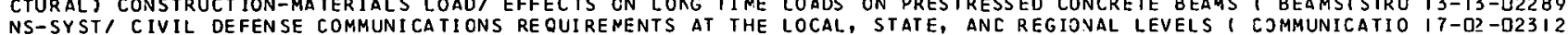

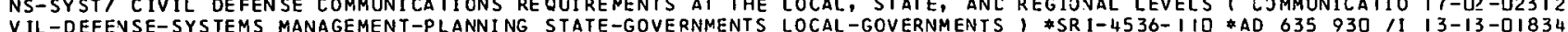

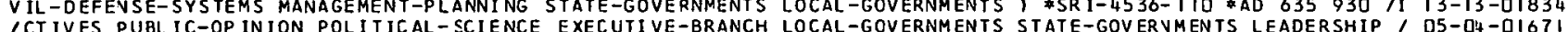
ICT IVES PUBL IC-OP INION POLITICAL-SCIENCE EXECUTIVE-BRANCH LOCAL-GOVERNMENTS STATE-GOVERVMENTS LEADERSHIP 1 D5-O4-DI67I

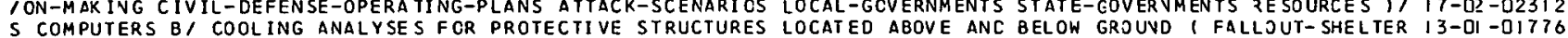
5 COMPUTERS B / COOLING ANALYSES FGR PROTECTIVE STRUCTURES LOCATED ABOVE ANC BELOW GROUVD I FALLOUT- SHELTER I3-DI -DI776
DUCED GROUNO MOT/ SIMULATION OF THE RESPONSE OF EQUIPMENT LOCATED IN UNDERGROUND INSTALLATIOVS TO BLAST IN I3-I3-OO229I LC RACIOA/ DUSTY III - AN IMPROVED MATHEMATICAL METHOD OF LOCATING FALLOUT ON A SPHERICAL EARTH I WIND YIE I8-O3-02322 IAT ION WAREHOUSE RECEIPT WHEAT INVENTORY BY WAREHOUSE ANO LOCATION 1 AGRICUL TURE STORAGE SUPPLY-DEPOTS WA / O2-D?-O2IDQ URBAN-AREAS MOVEMEN/ EFFECT OF POPLLATION NOBILITY CN THE LOCATION OF COMMUVAL SHELTERS I WAZVIVG-SYSTEMS IS-D3-DIQ42 IRAL DESCRIPTION OF WORK PROPOSED AND MAP SHOWING GENERAL LOCATION OF PROJECTS 1 FLOODS FLOOD-CONIROL CAL/ I3-D?-DI782 IRAL CESCRIPTION OF WORK PROPOSED AND MAP SHOWING GENERAL LOCATION OF PROJECTS I FLOODS FLOOD-CONTROL CAL/ I3-O2-UI7B3 1 ANNELS II. PLANS, PROFILES, CROSS SECTIONS, AND GENERAL LOCATION OF RIGLTS OF WAY TO BE ACOUIRED, CIVI/ I3-OZ -OI78I

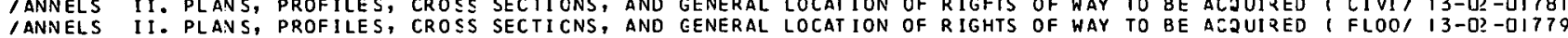
NG-PLANS POSTATTACX-OPERATIONS SHELTER-OCCUPAN/ EMERGENCY LODGING, HOUSING TRAINING CIVIL-DEFENSE-OPERATI OS-II-O2IB?

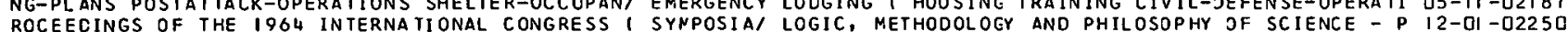

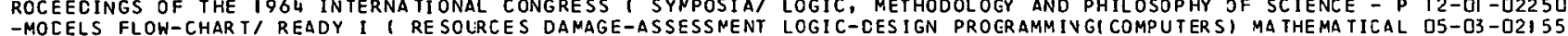

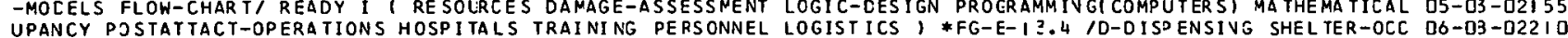
IONIPSYCHOLOGY) SOCIAL-PSYCHOLOGY ARMED-FORCES-OPERATIONS IOSISTICS AGING(PHYSIOLOGY), *TR-67-57-PR \$AD, OS-IJ-U21BI KEHAIR TRAINING, \#FG/ EMERGENCY CLOTHING 1 DISTRIBUTION LOGISTICS CIVIL-CEFENSE-OPERATING-PLANS CLEANING IS-O5-O23D6 IERSONNEL CASUALTIES MOBILIZATION ARMED-FORCES-OPERATICNS LOGISTICS COSTS CIVILIAN-PERSJNNEL RESEARCH-PRO/ IS-D3-O2305 I ING CIVIL-DEFENSE-SYSTEMS MODELS RADIOLOGICAL-MONITCRING LOGIST ICS MANPOWER SHEL TERS PZDGRAYMIVGICOMPUTE/ IS-D3-DIQ49 GE-ASSESSMENT WARFARE RESOU/ NREC PROGRAMS FOR GAMING THE LOGISTICS OF NAT IOVAL SURVIVAL 1 SIMULATION DAMA D5-D3-OIG4L IPPLIES STOCKPILING FOOD-DISPENSING FOOD FALLOUT-SHELTERS LOGISTICS SHELTER-OCCUPANCY WATER-SUPPLIES EDUC/ D6-O3-OI7I3 IEIOL OGY FLOODS DISASTER-MEOICINE TSUNAMI S WATER-SLPPLIES LOGISTICS TRANSPCRTATION COMMUNICATIOV-SYSTEMS / I5-03-O2299 ISINE FOJD STOCKP ILING FALLOLT-SHELTERS SHELTER-OCCUPANCY LOGISTICS WATER-SJPPL IES RATIJVIVG SAVITARY-ENG / DG-DB-DI7I 2 IGN COSTS NEW-YORK CHICAGO WASHINGTON PHILADELPHIA 8OSTON LONOON I *HI-820-D/ MOVIVG-8EL TWAYS TRAFFIC DES 23-0?-U2077 ODS I MECHANICAL-WAVES STRESSES PROPAGATION E/ STUCIES ON LONGITUDINAL AND BENDING WAVES IN LONG ELASTIC R $20-1 I-02079$ 51 MECHAN ICAL - WAVES ELASTICITY PROPAGATICN STRAINIME/ ON LONGITUDINAL AND TRANSVERSE WAVES IV ELASTIC ROD $20-I I$-O2OB2 T-PROCUCT ION(BIOLOGY) SURVIVAL, SENSIBLE AND LATENT HEAT LOSSES FROM OCCUPAVTS JF SURVIVAL SHELTERS I HEA D6-IS-DI7I LTER-OCCUPANCY METABOLISM PHYSI, SENSIBLE AND LATEAT HEAT LOSSES FROM OCCUPAVTS OF SURVIVAL SHELTERS I SHE DG-IS-DI7IB B IOLOGICAL-BLAST-EFFECTS BLAST-BI CLOGY SHCCK-TU/ DASA AEC LOVELACE FOUNDATION BLAST SIMULATION FACILITY I DO-2I -02232 ANNING SURVIVAL URBAN-AR/ ON THE DESIGN OF RISK ORIENTED, LOW COST FALLOUT SHELTER SYSTEMS I MAVAGEMENT-PL I3-I3-OIB29 IONMENT I NUCLEAR-EXPLOSIONS WAVE-TRANSMI SSI ON ATRCSPHERE LOW-FREGUENCY PHASE VERY-LOW-FREQUEVCY, EXTREME $/ 20-I+-02349$ IXPLOSIOVS IONO SP HERE ATMOSPHERE-MODELS WAVE-TRANSNISSI ON LOW-FREOUENCY VERY-LOW-FREQUEVCY EXTZEMELY-LOW- $20-1+$-02348 (ELECTROMAGNETIC) LASERS HELIUM-GROUP-GASES PLASMA-MEDIUM LUMINESCENCE ARGON NEON 1 \#UACRL E920272-6 *AO $/ 20-05-02060$ GICAL-BLAST-EFFECTS MATHEMATICAL-NODELS EXPERIMENTAL-DATA LUNG-DANAGE, \#DASA-1857 IATING MISSILES I BIOLO O6-21-D2235 MALS I BLAST-BIOLOGY B IOLOGICAL-BLAST-EFFECTS SHOCK-TUBES LUNG-DANAGE TOLERANCES (PHYSIOLOGY) I \$OASA-I 854 OSO-21-D2230 IAST-BIOLOGY BIOLOGICAL-BLAST-EFFECTS MONKEYS SWINF. SHEEP LUNG-INJURIES EXFLOSIONS TOLERANCES(PHYSIULULYII D6-2I -O2234 ION(ANIMAL) PROTEINS GENETICS AG, PROCEEDINGS OF THE HIGH LYSINE CORN CONFERENCE ( VUTRITION(HUMAN) NUTRIT O6-IS-OI72O

VACCINIA GROUP OF POXVIRUSES FOR THE CYNOMOLGUS MONKEY, MACACA IRUS I BIOLOGICAL-WARFARE-AGEVTS POX-VIRI I5-0?-OI85S S I STUDY OF GROUND MOTIONS FOR SIMLLATI ON BY SHOCK TESTING MACHINE I NUCLEAR-NEAPONS SHOCK-WAVES MECHANICAL $20-11$-D2O78 G DAIA-PROCESSING METHODOLO PUNCHED CARD O SORT ING - A MACHINE METHOD FOR O DECK PREPARATION AND SCORIN OS-II - I2IBB G 1 DATA-PROCESSING METHODOLO PUNCHED CARD O SORTING - A MACHINE METHOD FOR Q DECK PREPARATION AND SCORIN QS-II-D2IB8 RIAL-EQUIPMENT ECONOMICS FEASIBILITY-STUD/ AN ANALYSIS OF MACHINE TOOL SUBST ITUTION POSSIBILITIES I INDUST DS-O3-DI648
OGRAMMING (COMPUTERS) COMPUTE/ TOWARD THE DEVELOPMENT OF A MACHINE WHICH COMPREHENDS I DIGITAL-COMPUTERS PR D9-D2-O22 48 OGRAMMING(COMPUTERS) COMPUTE/ TOHARD THE DEVELOPMENT OF A MACHINE WHICH COMPREHENDS I DIGITAL-COMPUTERS PR D9-O2-02248
AN EXPER IMEVTAL INVESTIGATION OF THE YIELD STRENGTH OF A MACHINEC RING STIFFENED CYL IVDR ICAL SHELL I MODE 2O-II-O234 7 /ANICSI SUSPENSION-DEVICES STRUCTLRES VIBRATICN-ISCLATORS MACHINES VIBRATION FREQUEVCY PNEUMATIC-DEVICES / I3-I3-O229I SCILLATIONS STABILITY BOUNDARY-V/ PLASMA STREAMING INTO A MAGNETIC FIELD I GAS-FL OW PLASMA-MEDIUM PLASMA-O $20-03-02335$ ICT ION OF LIGHT AND PARTICLE BEAMS I MAGNETO-OPTIC-EFFECT MAGNET IC-FIELOS LASERS HYSTERESIS I *AFOSR-66-I/ 20-D5-D20S4 STUDIES ON THE INTERAC TION OF LIGHT AND PARTICLF PFAMS 1 MAGNET O-OPT IC-EFFECT MAGNET IC-FIELDS LASERS HYSI $20-$ OS-O2OG4 ENT VULNERABILITY ECONOMI/ PARM SYSTEM MANUAL VI-2. PARM MAIN ROUT INE 1 RES DURCES CAPACIIY DAMAGE-ASSESSM O5-D3 - D2IS2 IN THE PROTOTYPE MODEL 1 REPAIR REC OVERY DATA-PROCESSING MAINT ENANCE POSTATIACK-OPERATIONS I \#NREC-34 $10 N$ I3- 3 - D2282 MENT DATA-PROCESSING PROGRAM/ PARM SYSTEM MANUAL V. FILE MAINTENANCE PROCEDJRES I RESOURCES DAMAGE-ASSESS DS-O3-O2I5O IT IES TRANSPORTATION COMMUNICATIONS BANKING CONSTRUCTION MAINTENANCE REPAIR GOVERNMEVT CASUALTIES CAPACI/ D5-O3-O2IS3 IS VACCIVES RAD IOBIOLOGY PLBLIC-HEALTH STRESS (PHYSICLOGY) MALNJTRITION RACIOLOGICAL-DOSAGE EP IDEMIOLOSY I) DG-D -O22DG SHOCK-TUBES LUN/ AIR BLAST STLDIES WI TH EIGHT SPECIES OF MAMMALS I BLAST-BIDLOGY BIOLOGICAL-BLAST-EFFECTS DG-2I-D2230 SHOCK-TUBES LUN/ AIR BLAST STLDIES WI TH EIGHT SPECIES OF MAMMALS I BLAST-BIOLOGY BIOLOGICAL-BLA ST-EFFECTS DG-2I -D2230
ITWEEN SELECTED BLAST WAVE PARAME TERS AND THE RESPCNSE OF MANMALS EXPOSEO TO AIR BLAST I BLAST-BIOLOGY BII DO-2I-D2234

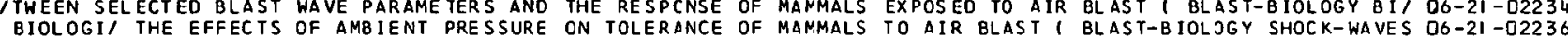
BIOLOGI/ THE EFFECTS OF AMBIENT PRESSURE ON TOLERANCE OF MAMMALS TO AIR BLAST I BLAST-B IOLJGY SHOCK-WAVES O6-2I-D2236
IF AIRBORNE PASTEURELLA TULARENSIS FOR MACACA MULATTA AND MAN I BIOLOGICAL-WARFARE-AGEVIS BACTERIAL-AEROSI I5-D2-DIB58 IF AIRBORNE PASTEURELLA TULARENSIS FOR MACACA MULATTA AND MAN I BIOLOGICAL-WARFARE-AGEVTS BACTERIAL-AEROSI I5-02-DI858 ING TECHY ICAL-INFORMATION-CENTERS DATA-PRCCESSING-SYSTEMS MAN-MACHINE-SYSTEMS COMPUTERS, \$AFOSZ-66-0873, O5-D?-DI63O ING TECHV ICAL-INFORMATION-CENTERS DATA-PRCCESSING-SYSTEMS MAN-MACHINE-SYSTEMS COMPUTERS 1 *AF OSR-66-0873 I O5-OZ-DI63O
AN EXPERIMEVTAL STUDY OF INTEGRATED GUIDANCE FOR SHELTER MANAGEMENT I MANAGEMENT-PLANVING TRAIVING SHELTE DS-UI -UIGZ6

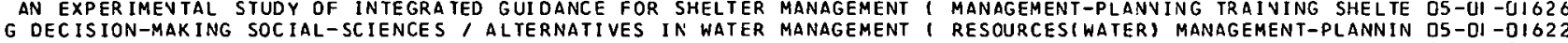
IES 2. COMMUN ITY FALLOUT SHEL TER S HANDBOOK FOR UNTRAINED MANAGEMENT I SHELTER-MANAGEMEVT SHELTER-OCCUPAN/ O5-OI-O2IIS URES POSTATT/ AN APPROACH TO DEFINING POSTATTACK RECOVERY MANAGEMENT CONCEPTS AND TECHVIQUES I ZOUNTERMEAS IS-O3-DI953 URES NUCLEAR I AN APPROACH TO DEFINING POSTATTACK RECOVERY MANAGEMENT CONCEPTS AND TECHVIOLES 1 IOUNTERMEAS I5-O3-O2293 ING HABITABIL ITY SHEL TER-OCCUPANCY/ STUDIES OF IN SHELTER MANAGEMENT GUIOANCE MATERIALS I MANAGEMENT-PLANN DS-DI - II62 4 ¿ SUPPORT, PLANNING, STUDIES AND / SYSTEMS AND PROGRAMS - MANAGEMENT, DEVELOPMENT, TECHVICAL AVD SCIENTIFI 22-02-0235I 
/ERIMENTAL ANALYSIS OF SELECTED PROBLEMS OF LARGE SHELTER MANAGEMENT, ENV IRONMENTAL THREAT, AVD SMALL SHE / DS-IJ-OI684

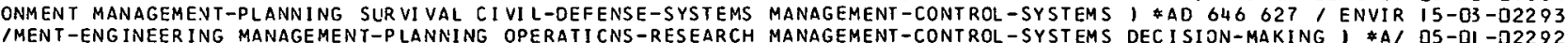

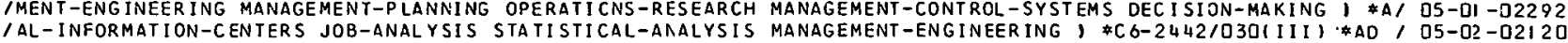
IMING BUDGET ING COST BENEFIT STUDIES 1 COST-EFFECTIVENESS MANAGEMENT-ENGINEERING MANAGEYENT-PLANNING OPER/ D5-DI - D2292 IND TECHVIQUES 1 COUNTERMEA SLRES NLLLEAR-EXPLOSI ON-DAMAGE MANAGEMENT-ENGINEER ING MAVPOWER-STUDIES COST-EF/ IS-O3-O2293 /SYSTEMS SUBJECT-INDEXING INFORMATION-RETRIEVAL AUTOMAT IC MANAGEMENT-ENGINEER ING SYMPOSIA OATA-PROCESSING/ OS-D2-D2I 2 I RCH SHELTER-ALLOCATION COMMUNI TY-SHELTER-PLANNING-PROGRAM MANAGEMENT-PLANNING, \#A.L. IO84 /ERATIONS-RESEA OS-DI -0IG23 CITY (SHELTERS C IVIL-DEFENSE-OPERATING-PLANS URBAN-AREAS MANAGEMENT-PLANNING) \$A.L. $974 /$ TER IN A MAJOR I5-D3-OI945 CONOMICS POL ITICAL-SCIENCE STRESS (PSYCHOLCGY) NETHCDCLOGY MANAGEMENT-PLANN ING, *FSR-RR-6O/2I-C S SUMMARY, OS-II -O22J2

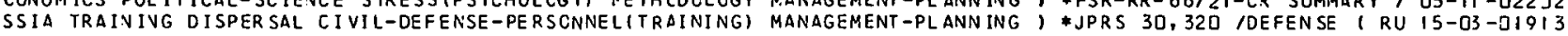
ANDA COMMUNISM PROTECTIVE-MASKS BIOLOGICAL-WARFARE-AGENTS MANAGEMENT-PLANNING, *JPRS-38, 291 10EFENSE ( RU I5-03-D1913

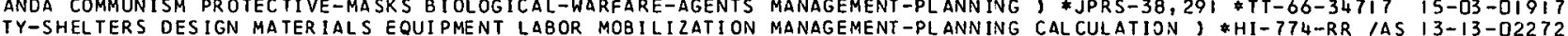
TY-SHELTERS DESIGN MATER IALS EQUIPMENT LABOR MOBILIZATION MANAGEMENT-PLANNING CALCULATIJN 3 \#HI-774-RR /AS I3-I3-02272 IS SURVEYS POETATTACK-OPERATIONS RECOVERY TATER-SUPPLIES) MANAGEMENT - PL ANNING CIVIL-DEFENSE-JPERATING-PLA I IS-D3-DI94I IAT ION DEVSITY DISTRIBUTION ECONONICS FEASIBILITY-STUDIES MANAGEMENT-PLANNIVG CIVIL-DEFENSE-SYSTEMS I \$PR/ I3-U?-022S6 / COVERY THREAT-EVALUATION POSTATTACK-OPERATIONS RESOURCES MANAGEMENT-PLANNING CIVIL-DEFEN SE-SYSTEMS MODELI IS-O3 -DI949 IITED-STATES CIVIL-DEFENSE-SYSTEMS CALCULATIONS RESOURCES MANAGEMENT-PLANNING COMPUTERS VULVERABILITY, \#/ IS-OS-OIQ55 /LITYI FALLOUT-SHEL TER S EQUIPMENT LRBAN-AREAS RURAL-AREAS MANAGENENT-PLANNING COST-EFFECTIVEVESS TRAINING/ I3-I?-DI8D6 TATION LEGISLATION COMPE IITION ENPLOYMENT TAXES SERVICES MANAGEMENT-PLANNING COSIS ILLINOIS OHIO INDIANA/ $23-0 ?-02095$ DIN IENCE GOVERNMENTI FOREIGN), TOWARD A FUTURE FOR PLANNING I MANAGEMENT-PLANNING DFCIS ION-YAKIVG POLITICAL-SC O5-DH-QIG59 CES I ALTERVATIVES IN WA TER MANAGEMENT I RESOURCES (WATER) MANAGEMENT-PLANNING DECISIDN-MAKIVG SOCIAL-SCIEN O5-DI -UIG2\% 1 PASSENGER-VEHICLES NOR THEAST-CORRIDOR COSTS URBAN-AREAS MANAGEMENT-PLANNING DESIGV \$PB 169521 /ATION $23-0 ?-02094$ CYI STUDIES OF IN SHELTER MANAGEMENT GUI IMISS ISS IPP I TORNADO OF MARCH 3,1960 I DISASIER-NEDICINE MANAGEMENT -PLANN ING HOSPITALS OPEPAIIDN CONTROLI O6-I?-OITI 4

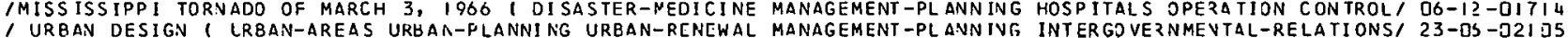

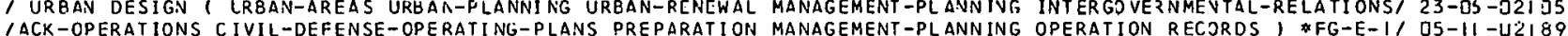

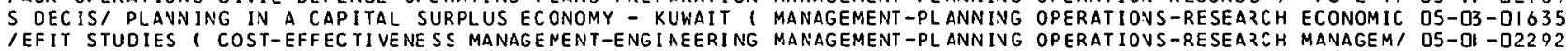
HREAT/ TECHVIQUES OF SYSTEMS ANALYSIS 1 MILITARY-STRATEGY MANAGEMENT-PLANN IVG OPERATIOVS-RESEARCH MODELS T I5-D3-D23J3 INING IMPLICATIONS OF LARGE SHELTERS I SHELTER-MANAGEMENT MANAGEMENT-PLANNING PER SOVVEL SHELTER-OCCUPANCYI OS-DI -OIG2I 1 PARM PROJECT FINAL REPORT 1 DAMAGE-ASSESSMENT RESOURCES MANAGEMENT-PLANN ING POSTATTACK-OPERATIONS RECOVE D5-D3-O2I 44 IAR WAR 1 SURVIVAL NUCLEAR-WARFARE POPULATION ME THCOOLOGY MANAGENENT -PL ANN ING POSTATTAC<-OPEZATIONS SEX AI 23 - OI -O2I O4 IONS 1 SOCIETAL-RECOVERY ME THODOLOGY PROJECTICNS RECOVERY MANAGEMENT-PLANNING POSTATTACK-OPEZATIONS SOCIAI OS-1I -O2I 91 IES DATA WATER-SUPPLIES URBAN-AREAS POSTATTACK-OPERATIONS MANAGEMENT-PLANNIVG RECOVERY VULNERABILITY RECO/ I3-D? -OI788 IMANUAL III-C. CAPACITY CONCEPTS AND DATA I MOBILIZATION MANAGEMENT-PLANNING RESOURCES INDUSTRIAL-PRODUC I O5-O3-O2I 22 I-A, P PARM I CUSTRATED I POSTATTACK-OPERATIONS RECOVERY MANAGEMENT-PLANNING RESOURCES MODEIS PROGRAMMIN OS I

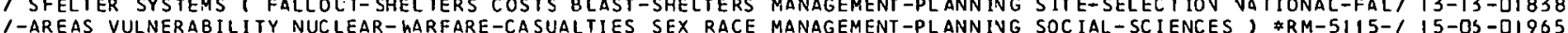

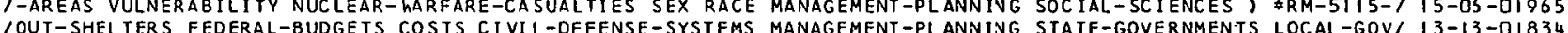
IOUT-SHEL TERS FEDERAL-BUDGETS COSTS CIVIL-OEFENSE-SYSTEMS MANAGEMENT -PLANNING STATE-GOVERNMENTS LOCAL-GOV/ I3- 13 -OI834
IILOREN IN

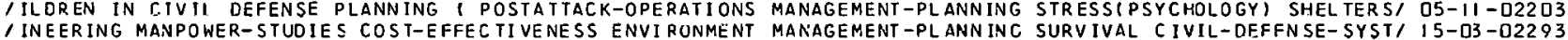

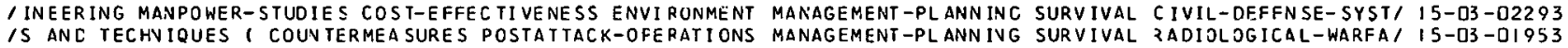
IS ANC TECHVIQUES I COUN TERMEA SURES POSTATTACK-OFERATIONS MANAGEMENT-PLANN IVG SURVIVAL ZADIOLOGICAL-WARFA/ IS-D3-DI953
IIGN OF RISK OR IENTED, LOW COST FALLOUT SHELTER SYSTEMS I MANAGEMENT -PLANNIVG SURVIVAL URBAV-AREAS DAMAGE/ I3-I3-DIB29 IAL STUOY OF INTEGRATED GUIDANCE FUR SHELTER MANAGEMENT, M MANAGEMENT-PLANNING TRAINING SHELTER-OCCUPANCY I OS-DI-OIG26

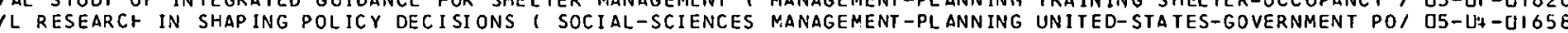
N NEW-YORK URBAN-AREAS POPLATION RAILROADS SUBWAYS COSTS MANAGEMENT-PLANNIVG URBAN-PLAVNING, \&RPN-7I-72 $23-0 ?-02099$ EXPRESSWAY PLANS 1 TRANSPOR TATION URBAN-AREAS NEW-YORK MANAGEMENT-PLANN ING URBAN-PLANN ING ) \$RPN-73-74 $23-02-02098$ STON N/ HIGH SPEED RAILROADS I TRANSPORTATION URBAN-AREAS MANAGEMENT-PLANNING URBAN-PLANN ING WASHINGTON BO 23 -D2 - O2IOD

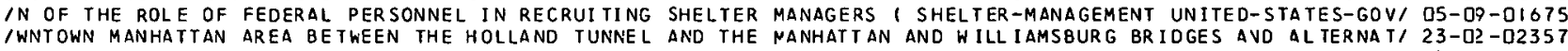
E MA/ TRANSPORTATION AND RELATED PROBLEMS CF THE DCWNTOWN MANHATTAN AREA BETWEEV THE HOLLAND TUNNEL AND TH $23-0 ?$ - 2357 ER-SUPPLIES INDUS TR IAL-PLANTS FINANCE STOCKPILING STORAGE MANPOWER, \$NREC-I34, SCHOOLS FOOD-SUPPLIES WAT O5-03-02I37 LIIES DEMOGRAPHY AGE LABOR URBAN TUARY-SERVICES PUBLIC-HEALTH INDUSTRIES SANI TATION REPAIR NANPOWER,

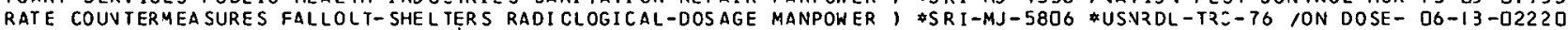
GE-ASSESSMENT POP LLATION CASUALTIES I APPLICATIONS OF THE MANPOWER ANALYSIS SYSTEM 1 RESOLRCES MODELS DAMA DS-D3-O2I 30 OURCES VULNERAB IL ITY MODELS M/ PARM SYSTEN MANUAL III-O. MANPOWER AND OCCUPATIONS 1 DAMAGE-ASSESSMENT RES OS-D3-D2I 3 IRELATIONSTIPS, POTENTIAL OEMAND, EMPLOYMENT I INDLSTRICS MANPOWER ECONOMICS GROSS-NATIONAL-PRJDUCT SUPPL/ O5-O3-OIG49 /ES ELECTR IC-PUWER-PRODUCTION PE TROLEUM RADIATION-EFFECTS MANPOWER EMERGENCY-HEAL TH-SERVICES RAOIOBIOLOGY/ IS-O3-UZZYG

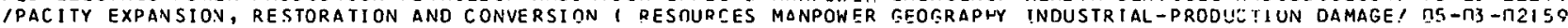

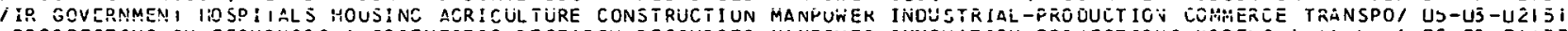
PROSPECTING IN ECONOMICS I SCIENTIFIC-RESEARCH RESOURCES MANPOWER INNOVATION PROJECTIONS MJDELS, \#A.L. 1 OS-D3-DIG33 ISERV ICES SOCIAL-PSYCHOLOGY AGING OCCUPATIONS PROFESSIONS MANPOWER LABOR POL ITICAL-SCIEVCE EDULATION GROU/ O5-II -O2I 7 IOPERAT

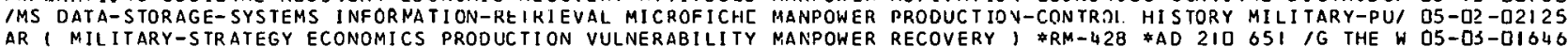

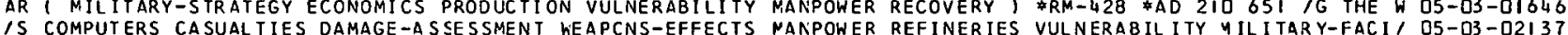

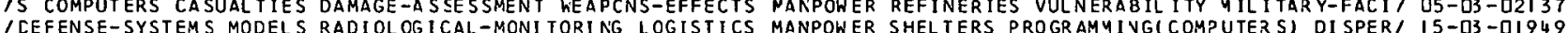

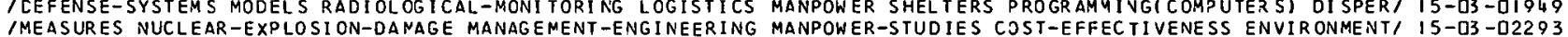
IMEASURES NUCLEAR-EXPLOSI ON-DANAGE MANAGEMENT-ENGINEERING MANPOWER-STUDIES COST-EFFECTIVENESS ENVIRONMENT/ I5-D3-O2293

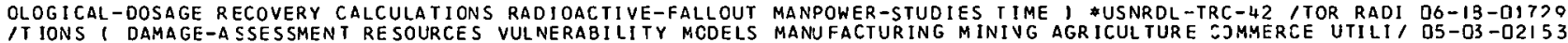
OF T.ONTINENTAL UNITED STATES I GEOGRAPHY RESOURCES DAMAI MAP IV - NREC SMALL SCALE MAPPING PROGRAM - MAPS OS-O3-O2ILD IGE CHANNELS I. GENERAL DESCRIPTION UF WORK PROPOSED AND MAP SHOWING GENERAL I OCATION OF PROJECTS I FLOO/ I3-O2-DITB2 1958 PROGRAM I. GENERAL DESCR IPTION OF WCRK PROPOSED AND NAP SHOWING GENERAL LOCATION JF PZJJECTS 1 FLOOI I3-UL-UI783 1964 PROGRAM I. GEVERAL DESCRIPTION OF hCRK PROPOSED ANO MAP SHOWING GENERAL LOCATION JF PZJJEETS 1 FLOOI I3-0? -QI784 IOJECT FIRE SCAN - THE EVALUATION OF AN AIRBORNE INFRAREO MAPPER AS A TOOL FJR DETECTING AND MEASURING FI/ I3-I? -OI798 M) AND INVERSE 1 MODELS GEOGRAPHY PROGRAMMINGICOMPUTERSI MAPPING \#NREC-IO /SAL TRANSVERSE MERCATOR I UT OS-03-02I33 241-8 UNIVERSAL TRANSVERSE MERCATOR GRID I ARMY SURVEYING MAPPING ENGINEERING ARTILLERY GEODESICS I \$TM 5- D8-03-02240 TES 1 GEOGRAPHY RESOURCES DAMA / MAP IV - NREC SMALL SCALE MAPPING PROGRAM - YAPS OF COVTINEVTAL UNITED STA D5-O3 -D2I 4 Q RIOS AIRCRAFT VUCLEAR-W/ PRELIMINARY AERIAL INFRARED FIRE MAPPING SYSTEMS AVALYSIS 1 MICHIGAV ATIACK-SCENA I3-I?-O22S7 TIONS RESCUES VULNERABILI IY CI VIL-DEFENSE-OPERAT ING-PLANS MAPPINGIINFRARED-EQUIPMENT) 3 " A.L. 95I /NFLAGRA I3-12-0I8J6 IERRAIN SOURCES DAMA MAP IV - VREC SMALL SCALE MAPPING PRCGRAN - MAPS OF CONT INENTAL UNITED STATES I GEOGRAPHY RE O5-D3-O2I 40 /E ANC ALBUQUERQUE I AER IAL-PHOTOGRAPHS URBAN-AREAS FIRES MAPS TEST-METHOOS VULNERABILITY CLASSIFICATION I I3-I?-OI8O9
IBENDING TORSION STRETCHING CONPRESSING TESTING-EQUIPMENT MARBLE COLOMITE LIYESTONE ROCK-SALT ANHYORITE S/ OB-OF -D2242 /HOSPITAL RESPONSE TO THE JACKSON, MISSISSIPPI TORNADO OF MARCH 3, 1966 I CISASTER-MEDICINE MANAGEMENT-PL/ O6-12-DI7I 4 CONOMICS, $\$$ FAS-M-140 CANADIAA WHEAT MARKET ING I FOOD-MARKET ING CANACA AGRICULTURAL-E O2-O2-OIGI7 -INTERFERENCE RADAR-SIGVALS MAPS ELECTROMAGNETIC-CI RADAR MASKING EY EARTHS TERRAIN I SITE-SELECTION RADAR I7-OF-DI9B8 ER-S/ SAFEGUARDING FOOD PRODUC TS AND WATER FROM AGENTS OF MASS OESTRUCTION - USSR I PROTECTION-SYSTEMS WAT I5-O2-OI843 NSE CI/ SIMPLE SHELTERS FOR PROTECTION AGAINST WEAPONS OF MASS DESTRUCTION I USSR IVTERNATIONAL-CIVIL-DEFE I3-I3-DI826 DEFENSE-SYSTEMS RESCUES INTER/ DEFENSE AGAINST WEAFONS OF MASS DESTRUCTION IN THE RURAL AREA 1 USSR CIVIL- I5-O3-OI9I 8 SUPPL IES FOOD-DISPENSING FOOD, BASIC COURSE IN EMERGENCY MASS FEECING - HANDBOOL I KITCHEV-EZUIPMENT-AND- DO-OS-OI7I 2
MENT-AND-SUPPLIES STUCKHILING, BASIC COURSE IN EMERGENCY MASS FEECINE - INSTRUCTORS GUIDE 1 XITCHEN-EQUIP QG-D3. DI7I3 
CASUALTIES SIMULATION FIRE-SAFETY TOXICI IYY HEAT CARBON-1 MASS FIRE LIFE HAZARD I NUCLEAR-EXPLOSIONS FIRES $13-12-01800$ 5 TRAFFI MOVIVG SIDEWALKS AND THE PROBLEN OF SHORT RANGE MASS TRANSPORTATIOY I URBAV-AREAS MOVING-BEL TWAY $23-02$ - 02097 ERICAL EARTH I WIND YIELD RADIOA/ DUSTY III - AN INPROVED MATHEMATICAL METHOD OF LOCATIVG FALLOUT ON A SPH $18-03-02322$ VIATION-MEDICINE EYE, FAD $627 \quad 332$ A MATHEMAT ICAL MOOEL OF FLASH BL INDVESS, VISION A 00-0S-01706 NICS) COLUMVSISTRUCTURAL) DEFORMATION MATHEMATICAL-MCDELS MATHEMATICAL-ANALYS IS, \$AD 401800 /ADING(MECHA $20-11$-O2340 FORMULATION CONFL ICT-RE SOLLTION GAMES MODELS (SIMULATIONS) MATHEMAT ICAL-ANALYS IS ( UCTURAL) DESIGN STRUC TURES LOAOING (MECHANICS) SHOCK-WAVES MATHEMAT ICAL-AN ALYSIS, *VCEL-R-534 IES WEBSISTR $20-11$-02337 ACIOACTIVE-FALL OUT PROGRAMMING (CONPUTERS) BURST-CONDITI ON MATHEMATICAL-ANALYS IS, *NREC-9, 1 WIND YIELD R IB-03-02322 NGI MECHAN ICS) SHEAR-STRE SSE S DEFORMA I ON ROTATI ON INERT I A MATHEMAT ICAL-ANALY IS LOADING(MECHAN IC S) STRAIN (MECHANICS) BUCKLING (MECHANICS) MATHEMAT ICAL-ANALYS IS ; \$5R S.M-7-168 *4D $64983320-11-02344$ S NUCLEAR-EXPLOSIONS EQUATIONS TUFF ALLUVIUM SALT GRANITE MATHEMAT ICAL-ANALYSIS " \#UCRL-5L.203 $/ 510 N-E F F E C T 18-03-02315$ ELEC TR IC-POWER SANI TARY-ENGINEER ING FUELS WATER-SLPPLIES MATHEMATICAL-ANALYS IS CLOTHING IAN ICS SETTLEMEVT ISTRUC TURAL) HIGH-SPEED-GROUND-TRANSPORT MATHEMAT ICAL-AN ALYS IS CON SOL IDATIJY DEFDRMATION/ $20-11$-O2084 IOCK -WAVES BLAS T- SHEL TER S LNDERGRCUND-SIRUCTURES DYNAMI IS MATHEMAT ICAL-AN ALYS IS DIFFEREYTIAL-EDUATIONS CO/ $13-13-01833$

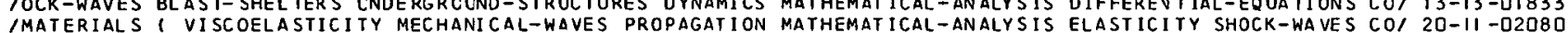

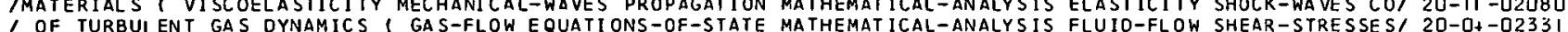
OF SRBLEN GAS DYNAMICS GA S-FLOW EUAT ONS-OF-STATE MATHEMAT ICAL-ANALYSIS FLUIO-FL OW SHEAR-STRESSESI $20-0+-02331$ TTERS EXPLOS IONS GAS-DISCHARGES HEAT IGNI TERS PROJECTILES MATHEMAT ICAL-ANALYS IS PROGR AMY ING(COMPUTERS) TH/ 19-0S-O2329

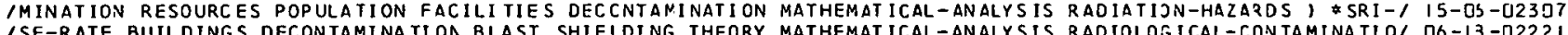
ISE-RATE BUILDINGS DECONTAMINATION BLAST SHIELDING THEORY MATHEMATICAL-ANALYSIS RADIOLOGICAL-CONTAMINATIOI O6-13-D2221

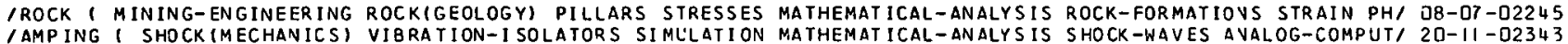
JUMENTAT ION ACC EL EROMETER S SPEC TRLM-ANALYZERS TRANSDUCERS MATHEMAT ICAL-ANALYS IS STRUCTUZES DYVAMIES I \$8U/ $20-11-02075$ 1 EXPLOSION-EFFEC TS NUCLEAR-E XPLOSIONS SEE SMOLOGY GRANITE MATHEMAT ICAL-ANALYSIS UNDER GRJ UND-E XPLOSI ONS 1118 -03-O2316 IROCEEDINGS OF THE 1964 INTERNATI ONAL CONGRESS I SYMPOSIA MATHEMAT ICAL-LOGIC MATHEMATICS METAMATHEMATICS 1 12-0I-02250 ALT IES SYSTEMS-EVALUATION CA SUALTIES STATISTICAL-ANALYSIS MATHEMATICAL-MOOELS 1 *RTI-OU-230-21III 1 RE-CASU 15 -03-01933 IE CATA CATAL OGUE I INFORMATION-SYSTEMS HOUSI NG RESOURCES MATHEMAT ICAL-MOOELS COMPUTERS CASUALTIES OAMAGE/ O5-03-02137 INDER EROUND-STR UC TURES DEFORMA TI ON CONSTRUCTI CN-MATERIALS MATHEMAT ICAL-MODELS DIFFERENTIAL-EOUATIONS EQUA/ I3-13-02279 7 IOLOGY BIOLOGICAL-8LAST-EFFEC TS EXPERIMENTAL-DATA THEORY MATHEMAT ICAL-MODEL S DISPLACEMENT ACCELERATION D/ O6-21-D2239 TR OGY BIOLOGICAL-8LAST-EFFEC TS EXPERIMENTAL-DATA THEORY MATHEMAT ICAL-MOOELS DISPLACEMENT ACCELERA TION D/ DO-21-02239

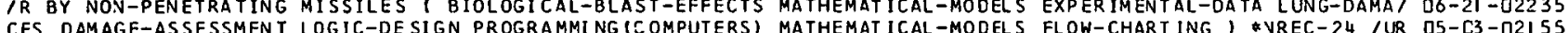
CES DAMAGE-ASSESSMENT LOGIC-DE SIGN PROGRAMMI NG (COMPUTERS) MATHEMAT ICAL-MODELS FLOW-CHART ING I \&VEC-24 IUR O5-C3-02155

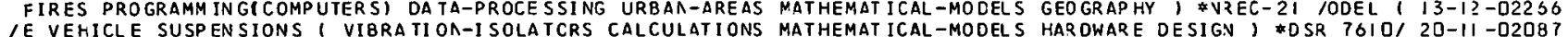

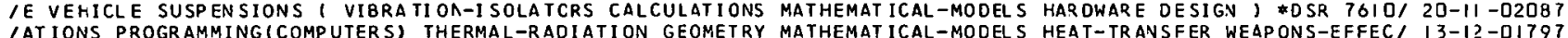

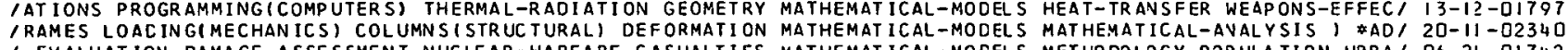
1-EVALUAT ION DAMAGE-ASSE SSMENT NUCLEAR-WARFARE-C ASUALTIES MATHEMAT ICAL-MOCELS METHODOLOGY POPULATION URBA/ O6-21-01742

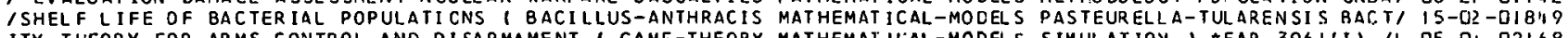

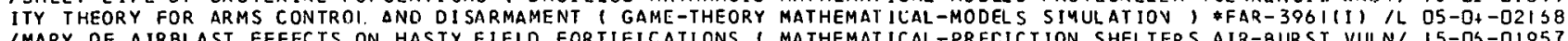
IMARY OF AIRBLAST EFFECTS ON HASTY FIELD FORTIFICATIONS I MATHEMATICAL-PRECICTION SHELTERS AIR-BURST VULN/ 15-0S-O1957 IE ANC PROGRAM SPECIF ICATIONS 1 HYDRODYNANICS WATER-WAVES MATHEMATICAL-PRECICT ION THEORY CRATER ING PROPAG/ 19 -O4-O2D43 YISON, AVD CLASSIFICA IION OF MODELS I RADI OACIIVE-FALLOUT MATHEMAT ICAL-PRECICTIOV YIELD GEOME TZY OISTRIBU/ 18-03-D2039 CAT ION TRAINING OPERA TIONS-RE SEARCH PROGRAMMING-LANGUAGES MATHEMAT ICS I *TM-530/010/00 /AL GAME-THEORY EDU D9-C?-Q175! /YSICS SCIENTIFIC-RESEARCH METALLLRGY MATERIALS CHEMISTRY MATHEMATICS COMPUTERS NUCLEAR-REACTORS HEAT-TRA/ $20-03-02070$ CRAMM ING-LANGUAGES EDUCATION OPERATIONS-RESEARCH TRAINING MATHEMATICS DAT A-PROCESSING-SYSTEMS, *TM-530-0, 09-02-01750

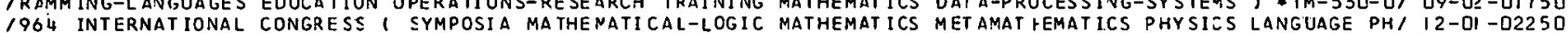

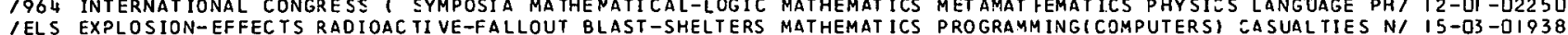

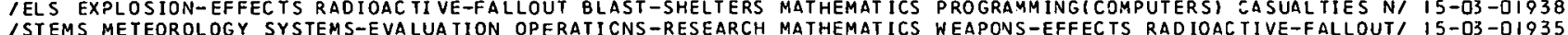
ISTEMS METEOROLOGY SYSTEMS-EVALUA TION OPERATICNS-RESEARCH MATHEMATICS WEAPONS-EFFEC TS RAD IOAC TIVE-FALLOUT, $15-03$-DI935
IUTION OF FISSION PROOUC TS IN A SILICATE FALLOUT PARTICLE MATRIX I RADIOACTIVE-DECAY RAD IOACTIVE-FALLOUT, I8-D3-O2325

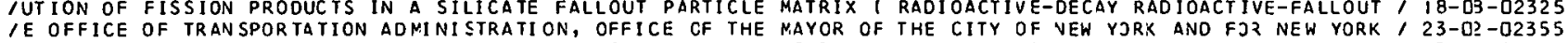
IOIPHTHER IA, PERTUSSIS, TE TANUS, POLI OMYELITIS, SMALLPGX, MEASLES, INFLUENZA I DEMOGZAPHY IMMUNOLOSY BIOL/ I5-D2-018S9 USSR SHELTERS IN/ EVACUATION OF THE URBAN POPULATIICN AS A MEASURE OF PROTECTION AGAINST NUCLEAR WEAPONS 1 15-03-OI934 IIVE FALLOUT - PHYSICS, BIOLOG ICAL EFFEC TS AND PROTECTIVE MEASURES ( RADI ATION-INJURIES USSR 2ADIATION-HA/ O6-13-01726 1 AN AIRBORNE INFRAR ED MAPPER AS A TOOL FCR DETECTING AND MEASURING FIRES I INFRARED-DETECTJ3S INFRARED-T/ I3-1?-01798 CS, \#FAS-M-186 CS $\quad$ FAS $M-188$

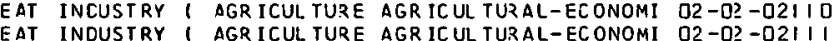
NEW 2EALAND LIVESTCCK AND MEAT INOUSTRY I AGR ICUL TURE AGR ICUL YURAL-ECONOMI D2-02-021 I 2 TTED-FOODS EGGS FATS FRD IEN-FOODSGULATI ONS GOVERNING THE MEAT INSPECTI ON CF THE UNITED STATES DEPARTMENT D2-02-DIO20

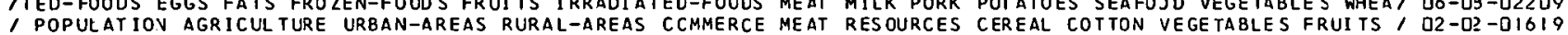
RES AND PRESSURES I ROCK-MECHANICS LOADING IMECHANICSA) US/ MECHANIICAL PROPERT IES OF ROCKS AT HIGH TEMPERATU DQ-07-02242

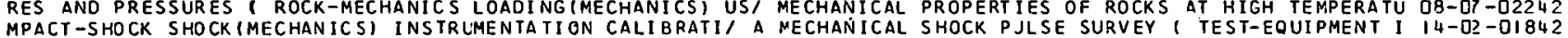

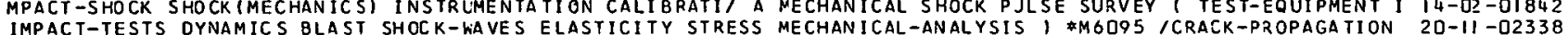
IMPACT-TESTS OYNAMICS $8 L A$ ST SHOC K-KAVES ELASTICITY STRESS MECHANICAL-ANALYSIS TM

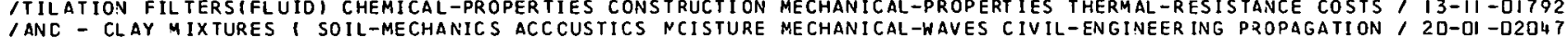

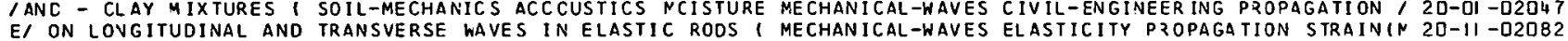
/ALF SPACES INOUCED BY AIR BLAST (ELASTICITY SHOCK-WAVES MECHANICAL-WAVES LOADING(MECHANICS) SURFACE-BUR/ I9-DH-D2044 /ELERATION WAVES IN NONL INEAR MATERIALS, VI SCOELASTICITY MECHANICAL-WAVES PROPAGATIOV YATHEYATICAL-ANALYI 20-11-O2080 IEC COORDINATES TO PLASIIC HAVE PROBLEMS I SOIL-NECHANICS MECHANICAL-HAVES PROPAGATION SHOCK-WAVES LNDERG/ O8-13-01747 IN BY SHOCK TESTING MACHINE ( NUC LEAR-WEAPONS SHOCK-WAVES MECHANICAL-WAVES SHOCK (MECHAVICS) DESIGN TESTS $120-11$-02078 ION LONGITUDINAL AND BENDING WAVES IN LONG ELASTIC RODS 1 MECHANICAL-HAVES STRESSES PROPAGATIOV ELASTICIT/ $20-11-02079$ C MAT HEMAT ICS METAMA THEMATICS PHYSICS LANGUAGE PHYSIOLOGY MECHANICS) *AD 646 O39 1 POSIA MATHEMATICAL-LOGI I2-0I-O2250 ILS IN THE EARTHS CRUST - SECOND ANNUAL SYMPOSIUM CN ROCK MECHANICS 1 ROCK (GEOLOGY) FAIL URE(MECHANICS) ST/ D8-OT-D2244 IOF THE COLORADO SCHOOL OF MINES 51 (3) SYMPOSIUM CN ROCK MECHANICS ( ROCK (GEOLOGY) UVDER GRJUND-STRLC TURE/ O8-D7-D2243

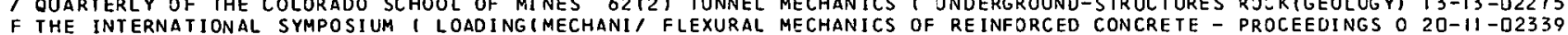

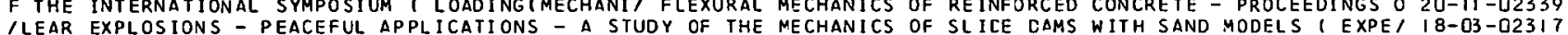
/LEAR EXPLOSIONS - PEACEFUL APPLICATIONS - A STUDY OF THE MECHANICS OF SLICE CAMS WIIT SAND MODELS 1 EXPE/ I8-03-02317

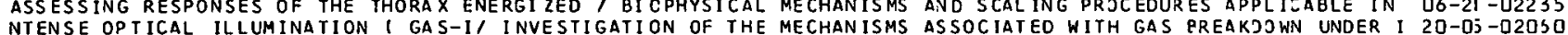
NTENSE OPTICAL ILLUMINATION GAS-1 INVESTIGATION OF THE MECHAN ISMS AS SOC IATEO WITH GAS PREAKJOWN UNDER I 20-DJ-D20SO

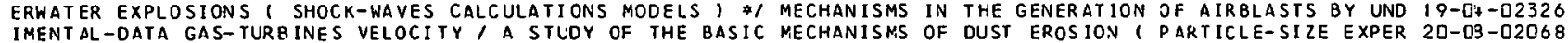

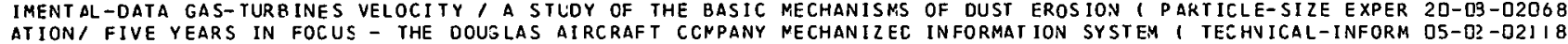
BIOLOGICAL-WARFARE-AGENTS CULTLRE-NEDIA GROWTH/ SELECTIVE MECIA FOR THE ISOLATIOV OF PASTEUZELLA PESTIS 1 I5-0?-OIBTS E UNITEO NATIONS AT FII INTER-NATION CONFLICT, DYACIC AND MEDIATED - CASE STUDIES OF EGYPT, ISRAEL, AND TH O5-Di-O21 PO MEDICAL-PERSONNEL HOSPITALS PUBLIC-HEALTH RESCU/ DISASTER MEDICAL CARE FILMS I DISASTER-MEDICINE MEDICINE DO-21-DI740 CINE EMERGENCY-HEALTH-SER VICES 81 OLOGICAL/ PROCEEDINGS OF MEOICAL CIVIL DEFEVSE CONFEREVCE 1 DISASTER-MEDI D6-21-02233 S RADIATION-EFFEC IS INSTR UMENTATI CN RA / RESEARCH REPORT - MECICAL CIVIS ION I METHODJLOGY RADIATION-INJURIE DG-I3-022 IS ILIZ ING LABORATORY AIR I BIOLOGICAL-WARFARE-AGENTS SPORES MEOICAL-EQUIPMENT ELLCTRICAL-EQUIPMENT LABORATO/ $15-02-01892$

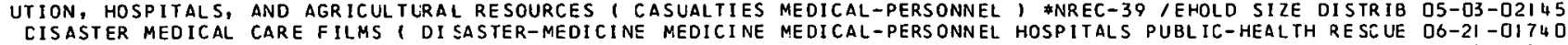
IAT ION CONTROL-SYSTEMS COMMUNICA TIONS-SYSTENS SUPERVISION MECICAL-PERSONNEL STORMS EYERGENCY-HEALTH-SERVI / OO-I?-DITIL

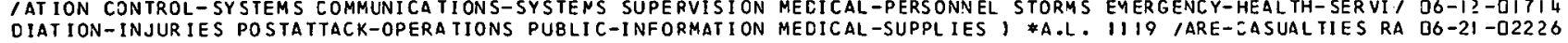


INT HANDBOOKS HABITABILITY NUTRI TION SANI TARY-ENGI IEERING MEOICAL-SUPPLIES DEFECT IOVS TZAIVIVG SLEEP WATE I OS-IJ-O2I84 I ARFARE CASUALTIES RE SOURCES DAMAGE-A SSE SSNENT FOOC LIGHT MECICAL-SUPPL IES EL ECTR IC-POWER SAVITARY-ENG INE I DS-D3 -D2I 35 IVICES HOSPITALS CASUALTIES ADMINISTRATI ON-AND-MANAGEMENT MEDICAL-SUPPL IES VUCLEAR-WARFARE-CA SUALTIES STOI O6-2I -DIT3Q E-CASUALTIES FIRST-AID/ CURRENT TCPICS IN SOVIET MILITARY MEDICINE 1 USSR CISASTER-MEDICINE VUCLEAR-WARFAR OG-OS-DI7O3 16 MANUAL - EMERGENCY HFAL TH SER VICES I DISASTER-NEDICINE MECICINE AOMINISTRATIOV-AVD-MANAGEMEVT FIRST-AI/ DG-2I - DI738

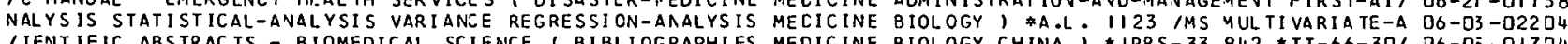
ICS TF RESCUI DISASTER MEDICAL CARE FILMS I DISASTER-NEDICINE MEDICINE MEDICAL-PERSOVNEL HDSP ITALS PUBLIC-HEAL DO THE IGE EXPOSURE RADIOLOGICAL-CONTAMI NATION RAOI ATION-HAZARCS MECICINE RACIOISOTJPES RADIOACTIVE-WASTE CIVIL-I D6-I3-O22 22

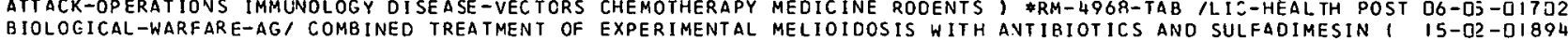
B IOLOCICAL-WARFARE-AG I COMBINED TREA TMENT OF EXPERIMENTAL MELIOIDOSIS WITH ANTIBIOTICS AND SULFADIMESIN I IS-O2-DIB94
LAST-SHELTERS UNDERGROUVD-SIRUCTURES DYNANICS SI YIELDING NENBRANE ELEMENTS IN PROTECTIVE CONSTRUCTION I B I3-I3-DIB37 IW UNREIVFORCEO COVCRETE SHELL I SHELLS(STRUCTURAL-FORMS) MEMBRANES STRESSES LOADING(MECHAVIES) FAILURE (M) I3-I3-OI832 ES I EMOT IONS ATT ITUDES FEAR STRE SSIPSYCHCLOSY) CASUALTI/ MENT AL HEALTH IMPL ICATIONS IV CIVILIAN EMERGENCI OS-IJ-DIGBI IVICES 1 PERSONNEL BEHAVIOR CIVIL-DEFENSE-OPERATING-PLANS MENTAL-HEALTH POSTATTACK-OPERATIONS TRAINING CH/ O5-II-O2IB5 /SOURCES GEODETIC POSITION, GP; TO UNI VERSAL TRAASVERSE MERCATOR ( UTM, ANC IVVERSE 1 MODELS GEOGRAPHY/ O5-O3-O2I33 NG ARTILLERY GEODESICS, *IM 5-24I-8 UNI VERSAL TRAASVERSE NERCATOR GRID I ARYY SURVEYIVG MAPPIVG ENGINEERI D8-OS-O2240 EMPLOYMEVT TAXES SER VICES, SELECTED IMPACTS CF RAILROAD MERGERS I TRANSPORTATION LEGISLATIOV COMPETITION $23-0 ?$-O2OS5 IRODUCTION (BIOLOGY) SURVIVAL FALLCUT-SHELTERS VENTILATION METABOLISM BODY-TEMPERATURE CLOTHING TEMPERATUR/ O6-IS-OI7IO TESTES RAIS MORPHOLOG/ THE PATHOLCGY OF ZINC DEFICIENCY I METABOLISM DEFICIENCY-DISEASES TISSUESIBIOLOGYI D6-OS-DITOI FROM OCCUPANTS OF SURVIVAL SHEL TERS, SHELTER-CCCUPANCY METABOL ISM PHYS IOLOGY HEAT-EXCHAVGE TEMPERATURE, O6-IS-DI7I 8

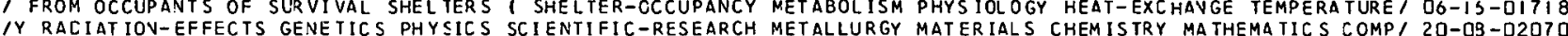
IIONAL CONGRESS I SYMPOSIA MA THEMATICAL-LCGIC MATHEMATICS METAMATHEMAT ICS PHYSICS LAYGUAGE PHYSIOLOGY MEC/ I2-DI - D225O

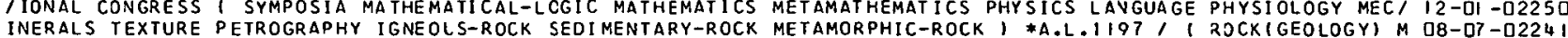

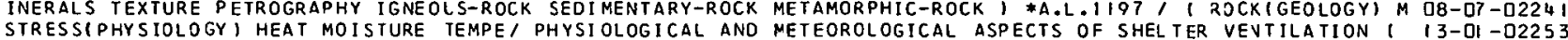
IRANSPORT-PROPERT IES EXPERIMEN TAL-DATA ATNOSPHERIC-MCTION METEOROLOGICAL-PARAMETERS I *AFCRL-65-6-49 *AD I D4-D? -O2III4 - 1 FIRESTORN ANALYSIS I FIRES WINO METEOROLOGY COMPUTERSIPROGRAM4ING) I *DC-TN-2046 13-I?-OI8JS IGHT-TRANSMISSION COLOKS UPIICAL-IMAGES OPTICAL-PIIENOMENA METEOROLOGY FLORIDA, \$82T9-2 /IC CONDITIONS I L 20-DS-D2066 IILES ABSTRACTS SPACECRAFT TRANSPORTATION LAUNCHING-SITES METEOROLOGY RAILROADS SHIPS HJISTS CHINA, *ATD/ IO-OI-DI973 IITIVITY ANALYSIS I. ME THODOLOGY I CIVIL-DEFENSE-SYSTEMS METEOROLOGY SYSTEYS-EVALUATIOV OPEZATIONS-RESEA/ IS-O3-DI935 S COOL ING-AN/ THE STUDY OF A SHELIER COOLING SYSTEN USING METHANOL AS THE TEAT SINK AVD AS THE FUEL I FUEL I 3 -OI -QIT73 OIFFERENTIAL SCALES RELEVANT TO INTERCULTURAL NEGOTIAT/ A METHOOOLOGICAL STUDY OF BEHAVIORAL AND SEMANTIC O5-IJ-02I 78 -ACMINISTRATION OPERATIONS-RESEARCH CIVIL-DEFENSE-SYSTEMS METHODOLOGY, \#A-L. IOI 3 /ERIVGRESEARCH-PROGRAM I2-OD-OI767 R LABOR POLITICAL - SC IEVCE EDUCATION CROUP-DYNAMICS THEORY MFTHODOLOGY) \#A. IDOQ INS PROFESSIJNS MANPOWE O5-II - O2I UTION HISTORY LAW INTER NA TIONAL-AFFAIRS PCLITICAL-SCIENCE METHODOLOGY; \#ACD-8 IUNIST-CHINA GOVFLICT-RE SOL US-OH-DIO63 SYSTEM/ EXTENSION OF THE GENERAL SENSITIVITY ANALYSIS J. METHODOLOGY, CIVIL-DEFENSE-SYSTEMS METEOROLOGY IS-D3-DIO35 SYSTEM EXTENSION OF THE GENERAL SENSITIVITY ANALYSIS J. METHODOLOGY I CIVIL-DEFENSE-SYSTEMS METEOROLOGY IS-O3 -DIQ35
NGS OF THE 1964 INTERNATIONAL CONGRESS 1 SYMPOSIAI LOGIC, METHODOLOGY AND PHILOSOPHY OF SCIEVCE - PROCEEDI I2-OI -O2250 NGS OF THE 1964 INTERNATIONAL CONGRESS I SYMPOSIA/ LOGIC, METHODOLOGY AND PHILOSOPHY OF SCIEVCE - PROCEEDI I2-OI -02250
SYSTEMS-ANALYSIS POLITICAL-SCIENCE ECONOMICS SOCIOLOGY S/ METHODOLOGY FOR ASSESSING TOTAL VULVEZABILITY 1 O5-II-O2I93 RCENING COSTS I/ INDUSTRIAL HAROENING CLASSIFICATION - A METHODOLOGY FOR SIYPLIFYIVG THE EVALUATION OF HA OS-D3-O2I 26 RCENING COSIS $1 /$ INDUSTRIAL HARDENING CLASSIFICATION - A METHODOI DGY FDR SIMPLIFYIVG THE EVALUATION OF HA DS-O3 -O2I 22 ESOLUTION HISTORY INTERNATIONAL-AFFAIRS POLITICAL-SCIENCE METHODOLOGY LAW I *ACD-6 /A ECONOMICS CONFLICT-R O5-D\#-OIGG2 ESOLUTIOV HISTORY INTERNATIONAL-AFFAIRS POLITICAL-SCIENCE METHODOLOGY LAW, *ACD-7 IA ECONOMICS CONFLICT-R D5-O4-OI664 / EMOGRAPHY ECONOMICS POLITICAL-SCIENCE STRESS IPSYCHOLOGY) METHODOLOGY MANACEMENT-PLAVNIVG, \&HSP-RR-66/2I/ O5-II-D22O2 PHY OF VUCLEAR WAR I SUR VIVAL NUCLEAR-WARFARE FOPULATION METHODOLOGY MANAGEMENT-PLAVVIVG PJSTATTACK-OPER/ $23-0+021$ I OLUT ION COMMUNIST-CHINA HISTORY LAW INTERNATICNAL-AFFAIRS METHOOOLOGY POLITICAL-SCIENCE, $\$ A C O-5$ ILICT-RES DS-DI-OIG6S ISSESSMENT NUCL.EAR-WARFARE-CASUALTIES MATHEMATICAL-MODELS METHODOLOGY POPULATION URBAN-AREAS OISTRIBUTION/. D6-2I -OIT42 /ESCR IPTIONS OF POSTATTACK SITLATIONS I SCCIETAL-RECOVERY METHODOLOGY PROJECTIONS RECOVERY YAVAGEMENT-PLA/ O5-II -O2I 1 IFOD FOR Q DECK PREPARATION AND SCORING I DATA-PROCESSING METHODOLOCY PUNCFED-CARD-EQUIPMENT SOEIAL-SCIEN/ D5-II-D2IB8 IXERCISFS OEC INSTRUMENTAT INSTRUMENTATION RA' RESEARCH REPORT - MEDICAL DIVISION I METHODOLOGY RADIATION-INJURIES RADIATION-EFFECTS D6-IS-D22IS N-AREAS SURVIVAL AGE POSTATTACK-OPERA TIONS URBAN-PLANNING METHODOLOGY SEMANTICS SOCIAL-SCIEVCES POLITICALI OS-ID-02I T8 DINGS COVSTRUCTI METHOD OF SHELER CINGS COVSTRUCTI/ METHOD OF SHELTER COST ANALYSIS I COSTS METHODOLOGY SHELTERS CDMPUTERSIPRJGRAMMINGI BUIL I3-13-DIB28
ANALYSIS OF D DATA I PUNCHED-CARDS STATISTICAL-ANALYSIS METHODOLOGY SOCIAL-SCIENCES CORRELATION VARIABL/ O5- II -O2I92 I ANALYSIS OF O DATA I PUNCHED-CARDS STATISTICAL-ANALYSIS METHODOLOGY SOCIAL-SCIENCES CORRELATION VARIABL/ O5- II-O2192
/RESS (PSYCHOLOGY) URBAN-AREAS SOCIOLOGY SOCIETAL-RECOVERY METHODCLOGY STRESS (PSYCHOLOGY), ILICY THREAT-PERCEPTION SUEZ-CRISIS ATTI TLDES MIODLE-EAST METHODOLOGY THEORY CONTENT-AVALYSISICOMPUTERS) I OS-DH-U2I7D ITTITUDES GROUP-DYNAMICS SOCIAL-PSYCHOLOGY PUBLIC-CPINION METHODOLOGY THEORY EDUCATION HEALTH WELFARE-SER/ Q5-II -O2I 44 DY OF ESCALATION - VOLUME 3 ( ARMS-CONTROL BIBLIOGRAPHIES METHODOLOGY WAR-GAYES YODELS, *FAR-3960 /EL STU O5-0+-O2I7I IL-PSYCHOLOGY BEHAVIOR DISASTERS PUBLIC-OPINION ATTITUDES METHODOLOGY WEAPONS-EFFECTS COMMUVICATION-SYSTEI OS-IJ-OIGT7 IC-PRECIPITATION WATER-SUHPLIES FCOD UNI TED-STATCS CANACA MEXICO, \#TID-232RA /ISOTOPES AIR MILK ATMOSPHER IB-D3-D2D35 -MONOXIDE CARBON-DIOXIDE AIR SMOKES WIND SHELTERS GERMANY MICE I \#NRDL TRC-63 /SAFETY TJXICITY HEAT CARBON I3-I? -DIBDO IC SULFADIMESIV I 8 IOLOGICAL-WARFARE-AGENTS EFFECTIVENESS MICE CHEMOTHERAPEUTIC-AGEVTS INFECTIONS STREPTO/ IS-D2-OI894 TER IAL-AEROSOLS EXPOSLRE VACCINES DOSAGE IMMUNITY NONKEYS MICE GUINEA-PIGS HUMANS, \$AD 635744 IGENTS BAC I5-D2-0I858 OGICAL-DOSAGE EXPERIMENTAL-DATA AGING (PHYSIOLOGY) ANIMALS MICE RATS DOSE-RATE) \#RM-5083-TAB /URIES RADIOL D6-13-0I733 SERVICES MAVAGPMENT-PLANNING COSIS (PHYSIOLOGY) ANIMALS MICE RATS DOSE-RATE I \#RM-5083-TAB /URIES RADIOL D6-13-0I733 SERVICES MAVAGEMENT-PLANNING COSTS ILLINOIS OHIO INDIANA MICHIGAN, \#SRI-II-4895 /TITION EYPLJYMENT TAXES $23-02-02095$ CLAMATIOV URBAV-AREAS MODELSISIMULATIONSI GRAPHICS TABLES MICHIGAN I \#SNRCL-TR-984 \#AD 635 250 TDAMAGE RE I5-OS-OI953 LIMINARY AER IAL. INFRARED FIRE MAPPING SYSTEMS ANALYSIS I MICHIGAN ATTACK-SCENAR IOS AIRCRAFT VUCLEAR-WEAP/ I3-I? -U2267
AEROPIOLOGY BACTERIA GAS-FILTERS MICRO/ AIR FILTRATION OF MICROBIAL PARTICLES I 8IOLOGICAL-WARFARE-AGENTS IS-D? - IBSI AEROP IOLOGY BACTERIA GAS-FILTERS MICRO/ AIR FILTRATION OF MICROBIAL PARTICLES I 8 IOLOGICAL-WARFARE-AGENTS I5-D2 - II85I

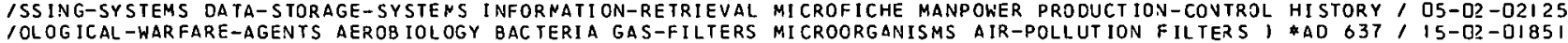
IWAVES K INETIC-THEORY ELECTRON-DENSI TY CERENKOV-RACIATION MICROWAVES OXIDES PLASTICS I *P IBYRI- I 295 .3-66, $20-03$-02330 REAS URBAV-PL AVVING URBAN-RENE WAL NAN/ PRCCEEDINGS OF THE MIC AMERICA CONFEREVCE ON URBAN DESIGV I URBAN-A $23-0 S$-O2IJ5 IENCE RESEARCH BY PRIVATE SCHOLARS AND ACADEMIC CENTERS - MIOOLE EAST I BIBLIOGRAPHIES ECONOMICS EDUCATIO/ OS-II-DIGQS CONOMICS DECISIOV-MAKINC CONSERVATION DEPLETICN PFTROLEUM MI DDLE-EAST) *A.L. 973 /G OPERATIJNS-RESEARCH E OS-D3-DIG35 IE FOREIGN-POL ICY THREAT-PERCEPTI ON SUEZ-CRISIS ATTITUDES MI DOLE-EAST METHODOLOGY THEORY COVTEVI-ANALYSI SI OS-O GY BOMBERS AVTIMISSILE-DEFENSE-SY/ THE CHANGING STRATEGIC MILITARY BALANCE - USA VS USSZ I MILITARY-STRATE I5-OT-023J8 AR-WARFARE-CASUALTIES FIRST-AID/ CURRENT TOPICS IN SOVIET MILITARY MEDICINE I USSR DISASTER-MEDICINE NUCLE O6-DJ-OI7D3
FENSE-SYSTEMS INTERNATIONAL-CIVIL-OEFENSE SHELTER/ SOVIET MILITARY TRANSLATIONS NUMBER ITDI USSR CIVIL-DE I5-O3-OI937

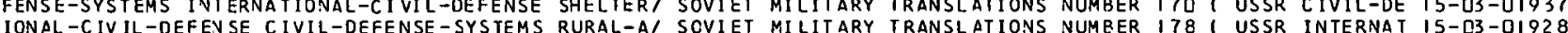

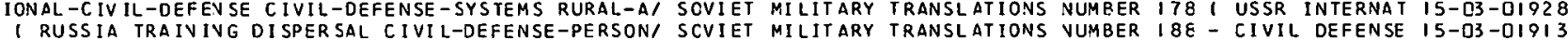

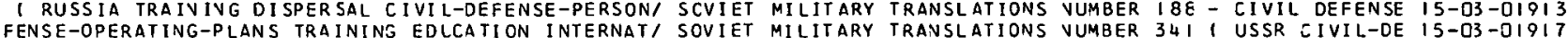
FENSE-OPERATING-PLANS TRAINING EDLCATION INTERNAT/ SOVIET MILITARY TRANSLATIONS VUMBER 34 I 1 USSR EIVIL-DE $15-03-01917$
ANNIVERSARY OF THE BATTLE OF MOSCOL USSR HISTOR/ SOVIET MILITARY TRANSLATIONS NUMBER $380-$ TWENTY-FIFTH D5-D4-D2I73

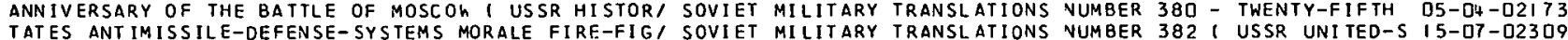
TATES ANT IMISSILE-DEFENSE-SYSTEMS MORALE FIRE-FIG/ SOVIET MILITARY TRANSLATIONS NUMBER 382 I USSR UNITED-S I5-O7-02309
NG METHODS AVD THEIR SHOR TCOMINGS DE SCRIBED I USS/ SCVIET MILITARY TRANSLATIONS VUMBER $384-$ COMBAT TRAINI OS-OP-O2I76 FENSE-SYSTEMS INTERNATIONAL-CIVIL-DEFENSE TRAININ/ SOVIET MILITARY TRAVSLATIONS VUMBER 385 I USSR OIVIL-DE IS-D3-O23O2 RCES-OPERATIONS ARMED-FORCESIFOREIGN) WARFARE MOR/ SOVIET MILITARY TRANSLATIONS VUMBER 386 ( USSR ARMED-FO IS-OT-023ID 1 COMMERCE EDUCATION POL ITICAL-SCIENCE GEOGRAPHY LANGUAGE MILITARY-AFFAIRS PHILOSOPHY LAW PJPULATION PUBL/ OS-II -DIG93 AIC I PUBLIC-DPINION SURVEYS FOREIGN-POLICY ECONCNIC-AID MILITARY-AIC) *FA-4 /ERICAV PROGRAMS OF FOREIGN O5-II-OIOBT WEAT FER IVG MILITARY-PER SONNEL (VULNERABILITY) HOLES BLAST MILITARY-ENGINEERIVG) \$REPORT- 1859 /L-MECHANICS I5-DS-OI957 IMINATIOV FIRES CONSTRUITION POSTATTACK-OPERATIONS REPAIR MILITARY-ENGINEERIVG RECONVAISSANCE , \#JPRS-3I, I3-I?-OI8I4 
ISSMENT WEAPONS-EFFECTS MANPOWER REFI NERIES VULNERABILITY MILITARY-FACILITIES ARMED-FORCES-SUPPLIES, GOVE I OS-C3-U2I 37 IEMS UNITED-STATES USSR COMMUNIST-CHINA THREAT-EVALUATI ON MILITARY-FACILIT IES (PRJTECTIOV) PJPULATION DETE' IS-O3-O22Q4 ILATIONS - NATIONAL DEFENSE ( ARMED-FORCES IUNITED-STATES) MILITARY-GOVERNMENT EMERGENCY-PLAVVING (GOVERNMEI I 5-03-OI946 ITTACK-SCENARIOS COMPUTERS HOUSING PROGRANMING (COMPUTERS) MILITARY-INSTALLAT IONS POPULAT ION GOVERNMENT COI OS-O3-D2139 ARCH MODELS THREA T-EVALUA TION DEFENSE-SYSTEMS DESIGN USSR MILITARY-INTELLICEVCE, \$RM-1829-1 \$AD I33 DI2, I5-03-D2333 ICHIGAN ATTACK-SCENAR IOS AIRCRAFT NUCLEAR-WEAPONS-EFFECTS MILITARY-INTELL IGEVCE SYSTEMS-ANALYSIS POST-ATT' I 3 - I? - D2267 CAI ATT ITUDES OF TROOPS IN THE TROPICS 1 TROPICAL-REGIONS MILITARY-PERSONNEL FOOD CLOTHING EVVIRONMENT EDU OS-IJ-D2IBI IITARY-INSTALLATIONS POPULATION GOVERNMENT COMMUNICATIONS MILITARY-PERS ONNEL HOSPITALS SCHOJL-BUILDINGS I $/$ O5-O3-D2I 39 , USSR MIL ITARY-TRAINING ARMED-FORCE S (FOREIGN) LEACERSHIP MILITARY-PERSONNEL OFFICER-PER SONVEL I JPR S- 4 I / OS-D7-O2I 76 ITIES TRANSPORTATION-PROPERTIES ELECTRIC-POWER-GENERATION MILITARY-PERSONNEL POPULATIOV HOSPITALS SCHOOLSI O5-D3-O2137 IARMED-FORCES(FOREIGN) WARFARE MORALE MILITARY-PSYCHOLOGY MILITARY-PERSONNEL WEAPONS ARMAMEVT MILITARY-STI IS-O7-O23IO IARMEO-FORCES (FOREIGN) WARFARE MORALE MILITARY-PSYCHOLOGY MILITARY-PERSONNEL WEAPONS ARMAMEVT MILITARY-ST/ IS-O7-O23IO
/ELTERS AIR-BURST VULNERABILITY SOIL-MECHANICS WEATHERING MILITARY-PERS ONNEL. (VULNERAB IL ITY) HOLES BLAST MI IS-DS-DI95?

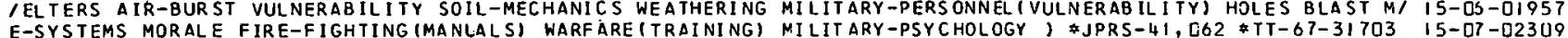

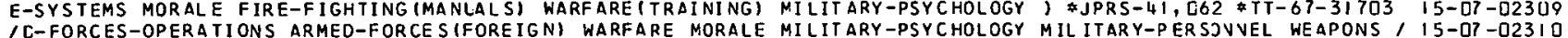

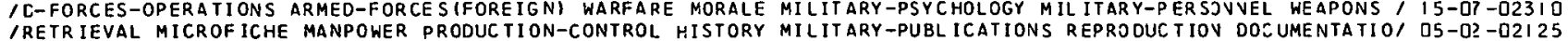
IRETR IEVAL MICROF ICHE MANPOWER PRODUCTION-CONTROL HISTORY MILITARY-PUBL ICATIONS REPRODUCTIOV DOCUMENTATIO/ OS-02 -O2I 25
IITS AIR-CONDIT IONING-EQUIPMENT PREFABRICATED-BUILDINGS MILITARY-REQUIREMENTS IESTS,

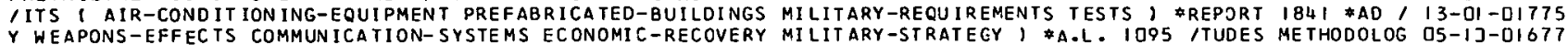

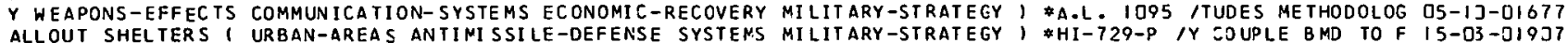
E MIL ITARY-P SYCHOLOGY MILITARY-PERSONNEL WEAPONS ARMAMENT MILITARY-STRATEGY, \$JPRS-41,466 TT-67-32099/L I5-07-023ID 1 THE CHANGING STRATEGIC MILITARY BALANCE - USA VS USSR I MILITARY-STRATEGY BOMBERS ANTIMISSILE-DEFENSE-SY IS-DT-O23OB INS ( NUCLEAR-WARFARE-CASUAL TIESIMATHEMATICAL-PREDICTION) MILITARY-STRATEGY DAMAGE-ASSESSMEVT THREA T-EVALI I 5-O7-DI966 LITY MANP / PETROLEUM INDUSTRY OF GERMANY DURING THE WAR I MILITARY-STRATECY ECONOMICS PRODUCTIOV VULNERABI OS-D3-DIOU 6 SSIA/ CRISIS CIVIL DEFENSE AND DE TERRENCE 1 VULNERABILITY MILITARY-STRATEGY INTERNATIOVAL-CIVIL-DEFENSE RU IS-D3-DI896 -RESEARCH MODELS THREAT/ TECHNIQUES OF SYSTEMS ANALYSIS I MILITARY-STRATEGY YANAGEMEVT-PLANNING OPERATIONS IS-D3-D23J3 IME-THEORY NUCLEAR-WARFARE MODEL-THEORY STRATEGIC-hARFARE MILITARY-STRATEGY OPTIMIZATIOVI \#RM-48I7-PR \$A/ I5-OS-OISS9 IME-THEORY NUCLEAR-WARFARE MODEL-THEORY STRATEGIC-hARFARE MILITARY-STRATEGY OPT IMIZAT IOV

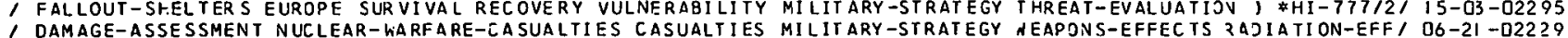
\begin{tabular}{l} 
DAMAGE-ASSESSMENT NUCLEAR-WARFARE-CASUALTIES CASUALTIES MILITARY-STRATEGY NEAPDNS-EFFECTS 3 AJIATION-EFF/ O6-2I -D22? 9 \\
\hline
\end{tabular} 7TRAINING METHODS AND THEIR SHORTCOMINGS DESCRIBED US USR MILITARY-TRAINING ARMEO-FORCES (FORE IGN) LEADERS/ O5-07-02176

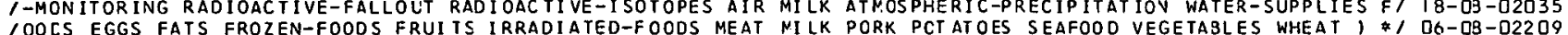
IOOCS EGGS FATS FROZEN-FOODS FRUITS IRRADIATED-FOODS MEAT MILK PORK PCT ATOES SEAFOOD VEGETASLES WHEAT I * 1 D6-O3-D2209 ( OF MINES SO( I) CLASSIFICATION OF ROCKS I ROCK (GEOLOGY) MINERALS TEXTURE PETROGRAPHY IGNEJUS-2OCK SEDIM/ D8-O7 -O224
DERGROUND OPEN INGS FI QUARTERLY OF THE COLORADO SCHOOL OF MINES $46(1)$ AN IVTRODUCTION TO THE DESIGN OF UN I3-I3-O22?3 OGY) MINERALS TEXTUR/ QUARTERLY OF THE COLORADO SCHOOL OF MINES SO(1) CLASSIFICATION OF ROCKS 1 ROCKIGEOL D8-O7-O224I RE MEASUREMENTS NEAR/ QUARTERLY OF THE COLORADO SCHOOL OF MINES 5D 141 SEISMIC WAVE PROPAGATIJV AVD PRESSU D8-1I-O224 7 GEOLOGY) UNDERGROUND / OUAR TERLY OF THE C OLORADO SCHOOL OF MINES 5I(3) SYMPOS IUM ON ROCK MEZHAVICS I ROEK( D8-D7-D2243 CRUST - SECOND ANNU/ QUAR TERLY OF THE COLORADO SCHOOL OF MINES 52131 OEHAVIOR OF YATERIALS IN THE EARTHS O8-O7-O2244 FALLOUT-SHELTERS PHYSIOLOGY EXPLOSIO/ AN INVESTIGATION OF MINIMAL EQUIPMENT NEEOS IV PERSONVEL SHEL TERS I I3-I3-DI8II FALLOUT-SHELTERS OPTIMIZATION PERFOR/ AN INVESTIGATION OF MINIMAL EOJIPMENT VEEDS IV PERSONVEL SHELTERS I I3-I3-DIBIQ

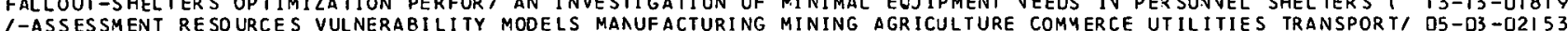
IST STRESSES UNDERGROUND-STRUC TURES ROCK (GEOLOGY) HEATING MINING-ENGINEER ING LOADINGIMECHANICS) COOLING FI I3-13-O2273

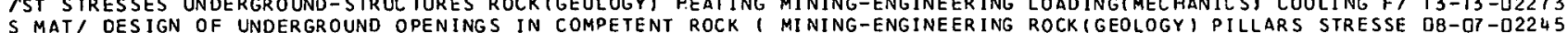

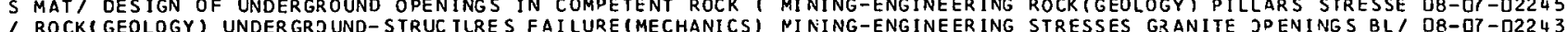
SERS I PUMPING OP IICAL) CAVI TY-RE SONA TORS OPTICS CRYSTALS MIRRORS, \$AD 637825 /ULSING METHJOS IN RUBY LA $20-03-02056$ SERS ( PUMPINGTOPIICALI CAVITY-RE SONA TORS OPTICS CRYSTALS MIRRORS \#AD 637825 IULSING METHJOS IN RUBY LA $20-03-02056$ ARFARE BALLISTIC-MISSILES CO/ ON THE ESSENCE OF A NUCLEAR MISSILE WAR I RUSSIA POLITICAL-SCIENCE NUCLEAR-W Q5-04-DI672
IECTS OF THE ATMOSPHERIC RE-ENTRY OF LONG RANGE BALLISTIC MISSILES I ATMOSPHERIC-ENTRY REENTRY-VEHICLES D/ 22-D2-U2350 IERGIZED BY AIR BLAST OVERPRES SURES OR BY NON-PENETRATING MISSILES 1 BI OLOEICAL-BLAST-EFFECTS MATHEMATIZA/ OG-2I -O2235 SILES BLA/ OPERATION TEAPOT - DISTRIBUTI ON AND DENSITY OF MISSILES FROM NUCLEAR EXPLOSIJVS 1 SECONDARY-MIS I8-O3-O23I8 INARY OBSERVATIONS ON A HOSPITAL RESPONSE TO THE JACKSON, MISSISSIPPI TORNADO OF MARCH 3 ,. I966 I DISASTER/ D6-I?-OI7IL ION NETWORKS TERMINALS SCHEDULING/ SUMMARY OF RESEARCH AT MIT ON HIGH SPEEC GROUND TRANSPORT I TRANSPORTAT $23-02-02354$ VE STRUCTURES / CONCRETE UNDERGROLN/ MATERIALS FOR USE IN MITIGATING BLAST LOADS ON DEEPLY BURIED PROTECTI IB-O3-O2OD? VE STRUCTURES I UNDERGROUD-STR UCTLR/ MATERIALS FOR USE IN MITIGATING BL AST LOADS ON DEEPLY BURIEO PROTECTI I8-D3-D2DI 7 ES URBAN-AREAS RU/ FIRE BIBLIOGRAPHY - SELECTED UREAN AND MIXED URBAN RURAL FIRES 194D-I9E4 I BIBLIOGRAPHI I3-I?-OI799 I WAVE VELOCITIES THROUGH PARTIALLY SATURATED SANO - CLAY MIXTURES ( SOIL-MECHANICS ACCOUSTICS MOISTURE MI $20-$ OI -D2O4? LE AEROSOL OF THE BORG STRAIN I BIOLOGICAL-WARFARE-AGENIS MIYAGAWANELLA-PSITIACI AEROSOLS, *AD G36 814 IC IS-02-DI8SD /RMAT ION BY PSITTACOSIS VIRUS, B IOLOGICAL-WARFARE-AGENTS MIYAGAWANELLA-PSITTACI CULTURE-MEOIA, \$AD 638 , I5-D?-DI89I WARN ING SYSTEMS URBAN-AREAS MO BEMEN EFFECT WARN ING-SYSTEMS URBAN-AREAS MOVEMEN EFFECT OF POPIIATI ON MOPILITY ON THE LOCAT ION OF CJMMUVAL SHEL TLRS I IS-03-0IQ42 IAN AREAS HASTY-SHELTERS DESIGN

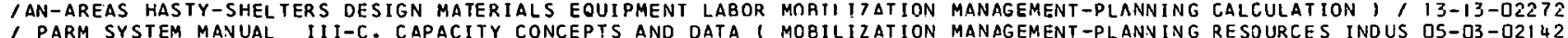
' PARM SYSTEM MANUAL III-C. CAPACITY CONCEPTS AND DATA 1 MOBILIZATION MANAGEMENT-PLAN. ING RESOURCES INDUS Q5-03-02I 42
CRAP CONSUMPTION PROBLEMS I SALVAGE RESOURCES STOCKPILING MOEILIZATION STRATEGIC-MATERIALS

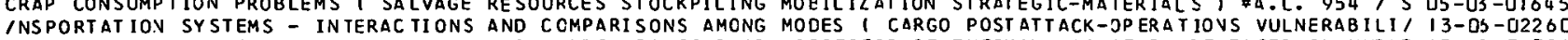
AR CETONATIOVS 1 GLASS ATTE/ WINDOW AND WINDOW SCREENS AS MOCIFIERS OF THERMAL RADIATIOV RELEASED IN NUELE I3-I?-DI796 ITURATED SAVD - CLAY MIXTURES I SCIL-MECHANICS ACCCUSTICS MOISTURE MECHANICAL-WAVES CIVIL-EVGINEERING PROI 20-OI -020L 7 IASPECTS OF SHELTER VENTILATION ( STRESS (PHYSIOLOGY) HEAT MOISTURE TEMPERATURE ENVIRONMENTAL-CONTROL HUMI/ I3-OI-O2253 IALLATION AND TESTING OF AN AUTOMATIC REMOTE RADIOLOGICAL MONITORING SYSTEM I INSTRUMEVTATION RADIATION-M/ I8-DH-D2OZG I VAR IOLA VACCINIA GROUP OF POXVIRUSES FOR THE CYNCNOLGUS MONKEY, MACACA IRJS I BIOLOGICAL-WARFARE-AGENTS/ I5-D?-OIB5S NGS SPORES, *AD/ PATHOGENESIS OF COCCIDICIDES IMMITIS IN MONKEYS 1 BIOLOGICAL-WARFARE-AGENTS PATHOLOGY LU IS-Q2-OI852

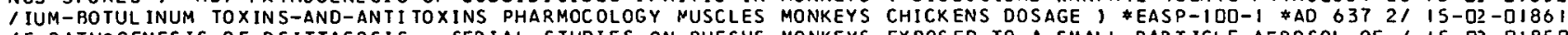
IE PATHOGENES IS OF PSITTACOSIS - SERIAL STUDIES ON RHESUS MONKEYS EXPOSED TO A SMALL PARTICLE AEROSOL OF / IS-D?-OI85O NKEY, MACACA IRUS I BIOLOGICAL-WARFARE-AGENTS POX-VIRUSES NOKKEYS IMMUNITY IVFECTIOVS, *AD 636905 /US MO I5-D?-0IB55 NKEY, MACACA IRUS I BIOLOGICAL-WARFARE-AGENTS POX-VIRUSES NOKKEYS IMMUNITY IVFECT IOVS ENTS BACTER IAL-AERO SOLS EXPOSURE VACCINES DOSAGE I NMUNITY MONKEYS MICE GUINEA-PIGS HUMAVS I 40 O35 744 IG IS-O2-0I858 IEO TO AIR BLAST I BLAST-BIOLOGY BIOLOGICAL-BLAST-EFFECTS MONKEYS SWINE SHEEP LUVG-IVJUR IES EXPLOSIONS TOI O6-2I-02234 APID-TRANSIT-SYSTEMS TRANSPORTATICN URBAN-AREAS RAILROADS MONORAILS MOVING-BELTWAYS HISTORY 1 *A.L. II29 $123-02$-02356 IOSE-RATE RADIATION-MEA SUREMENT-SYSTEMS PROTECTI ON-FACTOR NONTE-CARLO-MET HOD GEOMETRY (DUCTS)

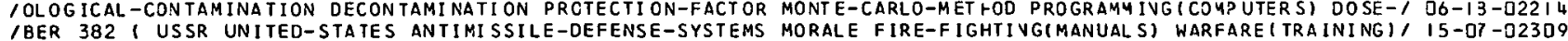

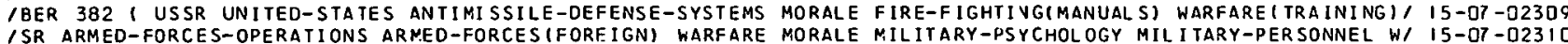

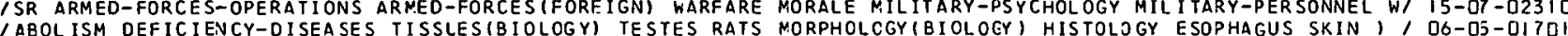
LEAR DAMAGE PARAMEY OISEASES TISSLES LEAR DAMAGE PARAMETERS II. I MODELS DAMA' SENSITIVITY OF MORTALITY EST IMATES TO UNCERTAIVTIES IN SOME NUC O6-21-D2229 OPULATION REPRESENTATIONS I NUCLEAR-W/ THE SENSITIVITY OF MORTALITY ESTIMATES TO VARIATIONS IN AGGREGATE P I5-OF-OIQ66 ILITIES (HARDENING) RESOURCES DECONTAMINATION PEST-CONTROL MORIUARY-SERVICES PUBLIC-HEALTH IVDUSTRIES SANI/ IS-O3-OI953 IS NUMBER 380 - TWENTY-FIFTH ANNI VERSARY OF THE BATTLE OF NOSCON 1 USSR HISTJRY COMMUVISM PZJPAGANDA I \$J/ OS-O+-02I73 NT GAS, TURBULENCE FLUID-DYNAMICS PARTICLE-SIZ RELATIVE MOTION AND COAGULATION OF PARTICLES IV A TURBULE $20-0$ - D2333 ST I ELASTIC/ THEORETICAL GROUND SHOCK EFFECTS I. GROUND MOTION IN ELASTIC HALF SPACES INDUCED BY AIR BLA I9-OI-O2O4 4

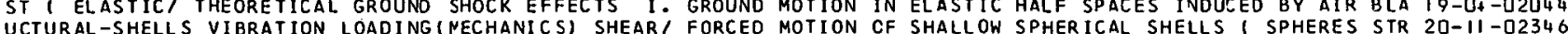
UCTURAL-SHELLS VIBRATION LOADING (NECHANICS) SHEAR/ FORCEO MOTION CF SHALLOW SPHER ICAL SHELLS I SPHERES STR 20-II-O2346
OSURFACE BLAST LOADING - RESULTS OF SERIES III TESTS /, MOTION OF SUBSURFACE SOIL INCLUSIJYS SUBJECTED T D8-I3-OIT46 O SURFACE BLAST LOADING - RE SULTS OF SERIES III TESTS I MOTION OF SUBSURFACE SOIL INCLUSIJVS SUBJEC TED T OB-13-OI746 O SURFACE BLAST LOADING - RE SULTS OF SERIES III TESTS I I MOTION OF SUBSURFACE SOIL IVCLUSIJVS SUBJEE TED T I 8-03-D2DJ8
VIL-DEFEVSE-SYSTEMS, \#MP-6
CIVIL CEFENSE MOTION PICTURE CATALOGUE I PUBLIC-INFORMATION CI IS-O3-D23J4 VIL-DEFEVSE-SYSTEMS I \#MP-6 CIVIL CEFENSE MOTION PICTURE CATALOGUE I PUBLIC-INFORMATION CI I5-O3-023J4
1 SEISMIC-WAVES EXPLOSION-EFFECTS VELOCITY ACCELERATION MOTION PROPAGATION EARTHQUAKES, \$VUF-30I4 \#AD, IB-O3-D23I TTO SHOCK AND VIBRATION BULLETIN (TOLERANCES (PHYSIOLOGY) MOTION-SICKNESS STRESS (PHYSIOLOGY) ADAPTATION(P) OG-IQ-DIT35 
ITED IN UNOERGROUND INSTALLATIONS TO BLAST INOUCED GROUNO MOTIONS 1 JNDERGROUND-STRUCTURES EXPLOSION-EFFE/ I3-13-O2291 1 NUCLEAR-WEAPONS SHOCK-WAVES MECHANICAL I STUDY OF GROUND MOTIONS FOR SIMULATION BY SHOCK TESTING MACHINE $20-11$-O2OTB 1 ON THE STRUCTURE OF INTERPER SONAL. INTENIIONS, BEHAVIOR NOTIVATION CULTURE RESPONSE REACTIOVIPSYCHOLOGY, DS-IJ-O2I79 IS SOCIETAL-RECOVERY ECONOMIC-RECCVERY ATTITUDES MANPOWER MOTIVATION ECONOMICS SURVIVAL DISTRIBUTION STREI O5-IJ-D2IBD I EMOTALS OR EMOTIONS ATTITUOES FEAR SIRESSIPSYCHOLOGY) CASUALTIES MOTIVATION PUBL IC-INFORMATION TRAINING REAC YIONI OS-IJ-OIOBI OR ELECTRIC-MOTORS RUSSIA COMMUTATORS/ A DIRECT CURRENT MOTOR WITH NO CONTACTS AND A TRANSISTOR COMMUTAT O9-D3-OI752 ICIL ITY OF NORTH AMER ICAN AIR DEFENSE COMNANO AT CHEYENNE MOUNTAIN, EL PAST COUNTY, COLJRADJ I ZONSTRUCTI/ I3-I3-D2274 MMUN ICATION-SYSTEMS STRESS(PSYCHOLOGY) PUBLIC-INFORMATION MOVEMENT, *A.L. IOTI /ANVING UTILITY-TUNNELS CO I 3-I3-DIB3I CATION OF COMMUNAL SHELTERS 1 KARNING-SYSTEMS URBAN-AREAS MOVEMENT, *0RO-SP-30*AD 204 090/1TY ON THE LO I5-D3-0I942 HINA INDUSTRI/ CRISES CIVIL OEFENSE AND DETERRENCE I USSR MOVEMENT INTERNATIONAL-CIVIL-DEFEVSE COMMUNIST-C IS-O3-O2295 IY INTERNATIONAL-CIVIL-DEFENSE EARLY-WARNING-SYSTENS USSR MOVEMENT POPJLAT ION RESOURCES EVACUATION URBAN-I IS-D3-DI922 IVIL-CEFENSE-OPERATING-PLANS URBAN-AREAS NANAGEMENT-PLAN/ MOVEMENT TO SHELTER IN A YAJOP CITY I SHELTERS C IS-03-OIQ45

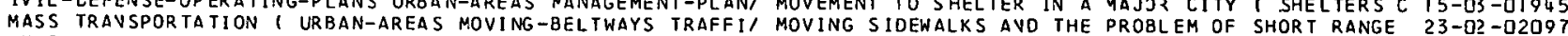
IT-SYSTEMS TRANSPORTATION URBAN-AREAS RA-BELTWAYS TRAFFI' MOVING SIOEWALKS AVO THE PROBLEM OF SHORT RANGE 23 -02 -O2O97 IPRORLEM OF SHORT RANGE MASS TRANSPORAITA XPER IMENTAL-DATA TEST-METHODS PRE SORE XNFECTIVITY DF ARE MNFECTIVITY OF AIRBORNE PASTEURELLA TULARENSIS FOR MACACA MULATTA AND MAN I BIOLOGICAL-WARFARE-AGENTS BACI I5-02-OI858 TRUCTURAL-SHELLS CYLINDRICAL-BODIES COMPOSI/ VIBRAIION OF MULTI-LAYERED ANISOTROPIC CYLINDRICAL SHELLS I S $20-11-02345$ INTS, STRUCTURES, AND OPERATIONS RESEARCH II. DANAGED PF MULTIPLIER I RADIOACT IVE-FALLOUT PRJTECTION-FACI DG-IB-D222I /INT ERRELATIONSHIP OF PRESSURE STRENG TH, SI ZE AND COST OF NILTIPURPOSE BU ILDINGS FOR A FEDEZAL TRANSPORTA/ I3-I3-DIBZ 7 /ROGRAMS I COMPUTERSIPP.OGRAMMING) DATA-PRCCESSING-SYSTEMS MULTIVARIATE-ANALYSIS STATISTICAL-ANALYSIS VARI/ D6-D3-O22J4 G-PLANS UTILITIES DATA WATER-SU/ CIVIL DEFENSE ASPECTS OF MUNICIPAL WATER SYSIEMS 1 CIVIL-DEFENSE-OPERAIIN I3-0Z-OI7B8 BILITY URBAN-AREAS SURVEYS POST/ CIVIL DEFENSE ASPECTS OF MUNICIPAL WATER SYSTEMS I WATER-SUPPLIES VULNERA I5-O3-DI9L 1 ICLOSTRIDIUM-80 TUL IVUM TOXINS-AND-ANTI TOXINS PHARNCCOLOGY MUSCLES MONKEYS CHICKEVS DOSAGE I \#EASP-IDO-I \#/ I5-0?-DI86I TOX INS BIOLOGICAL-WARFARE-AGENTS PARALYSIS FOOD-POISONING MUSSEL-POISONS, $\$ A D$ O36 903 IDA TOXIVS-AND-ANTI I5-OP-DI8S3 $51+N Y 2315-1$ RADIATION INIICED VIABILITY MUTATIONS IN THE HDNEY BEE 1 HYMENOPTERA GENETIC DG-0S-D22OT IN - POSTATTACK CONSEQUENCES I GENETICS RAOIATION-HAZAROS MUTATIONS RADIATION-EFFECTS ANIMALS HUMANS IESTI DO-IS-QIT2 7 ANC ISSMM HOWITZERS, CONCEPT AND FEASIBILITY STUDIES OF MUZZLE BRAKE BLAST SUPPRESSION CEVICES FOR IOSMM I9-DS-O2327 RREL-ATTACHMENTS INTERIO/ CONTRIBLTION TO THE ANALYSIS OF MUZZLE ERAKE CESIGV I ARTILLERY CJMPUTERS GUN-BA I Q-0S-D2329 ENT RLCOIL-MECHANISMS INTERIOR-BALLISTICS, \$AN 111482 MUZZLE BRAKES II. THEORY I GUN-AUXILIARY-EQUIPM I9-OS-O2328

( $\triangle S U A L T I E S(P R E O I C T I O N)$ URBAIN-AREAS DISTRIBUTICN HIROSHIMA NAGASAKI BURNS WEAPONS-EFFECT RADIATION-INJURIEI D6-2I -02238 I ASUALTIES STATISTICAL-ANALYSIS WEAPONS-EFFECTS HIROSHIMA NAGASAKI GRAPHS EIOLOGICAL-BLAST-EFFECTS BURNS I O6-2I -D2227 I-EFFECTS NUCLEAR-WARFARE-CASUALTIES JAPAN HIROSHINA DATA NAGASAKI RADIATION-INJURIES RECORDS LIFE-SPAN I/ DG-I3-DI73D 1-EFLIABILITY OF THE RISK II COMPLTER STATISTICAL NODEL - NAHICUS O3 APPL ICATION I RESOURCES VULNERABILIT/ OS-O3-O2I49 RELIABILITY OF THE RISK II COMPLTER STATISTICAL NODEL - NAHICUS 63 APPL ICATION 1 RESOURCES VULNERABILIT/ QS-03-02149

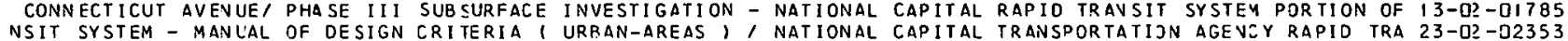
NSIT SYSTEM - MANCAL OF DESIGN CRITERIA I URRAN-AREAS ' NATIONAL CAPITAL TRANSPORTATIJN AGEVLY RAPID TRA 23 -0? -O2353
ILITARY-GOVERNMENT EMERGEN/ CODE OF FEDEP.AL REGULATIONS - NATIONAL DEFENSE I ARMED-FORCES(UNITED-STATES) M I5-O3-DIQ46 ANC CONSULTING FIRMS WITH CERTIFIED FALLOUT SHELTER ANA/ NATIONAL DIRECTORY OF ARCHITECTURAL, ENGINEERING I3-I3-O2276 IOF REQUIREMENTS FOR FALLOUT SHELTER CONSTRUCTION IN FOUR NATIONAL MODEL BUILDING CODES I CJVSTRUETION DU/ I3-I3-O228I VIL-DEFEVSE-SYSTEMS ATIIT/ RESEARCH ON ACCEPTABILITY OF A NATIONAL SHELTER PJLICY I JPEZATIJVS-RESEARCH CI DS-D+ -02I G6 WARFARE RESOU/ NREC PRUGRAMS FOR GAMING THE LOGISTICS OF NATIONAL SURVIVAL. 1 SIMULATIOV DAMAGE-ASSESSMENT O5-03-0IGLL INSIONS OF SURVIVAL - POSTATTACK SURVIVAL DISPARITIES AND NATIONAL VIABILITY, SOCIETAL-RECJVERY ECONOMIC, DS-O3-D2I GD IIVIL-DEFENSE CIVIL-DEFENSE-SYSTENS EARLY-WARNING-SYSTEMS NATI ONAL-DEFENSE RECRUITIVG TRA IVIVG WARFARE CIII I S-O3-O23OS 1 COSTS BLAST-SHELTER S MANAGEMENT-PLANNI NG SI TE-SELECTION NAT IONAL-FALLOUT-SHELTER-SURVEY PJPULATION RE SOI I $3-13$-OI838 INED EFFECTS OF NLCLEAR WEAPONS ON NFSS TYPE STRUCTURES I NAT IONAL-FALLOUT-SHELTER-SURVEY(NFSS) FALLOUT-S7 I $8-03-D I Q 95$ URVEYS FOR/ OCCASIONAL REPORT ON FOREIGN AFFAIRS - UNITED NATIONS I INTERNATIONAL-AFFAIRS PUBLIC-OPINION S OS-II -D2198 NAIRES PUB DCCASIONAL REPORT ON FOREIGN AFFAIRS - UNITED NATIONS INTERNAIIONAL-AFFAIRS PUBLIC-OPINION S OS-11 - D2I 98

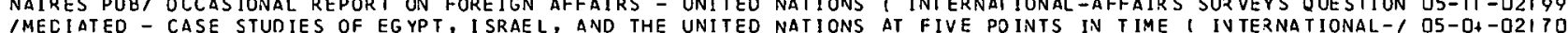
GMECIATED - CASE STUDIES OF EG YPT, ISRAEL, AND THE UNITED NAIIONS AT FIVE POINTS IN TIME I IVTERNATIONAL-I OS-DH-02ITD G PUBLIC-OPIVIJN INTERNATIONAL-AFFAIRS SUI CAN THE UNITEO NATIONS ORGANIZATION PREVEVT WARS

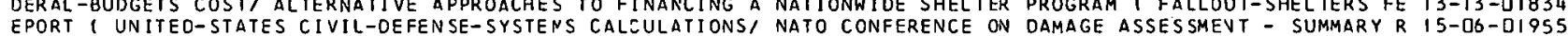
EPORT I UNITED-STATES CIVIL-OEFENSE-SYSTENS CALCULATIONS/ NATO CONFERENCE ON DAMAGE ASSESSMEVT - SUMMARY R IS-OS-OI9S5
IFENSE-SYSTEYS IVTERNATIONAL-CIVIL-DEFENSE WESTERN-EUROPE NATO GREAT-BR IT IV FRAVCE GERMANY BEVELUX NORWA / I5-D3-D2I 47 IFENSE-SYSTEYS IVTERNATIONAL-CIVIL-OEFENSE WESTERN-EUROPE NATO GREAT-BRITAIV FRANCE GERYANY BEVELUX NORWA/ I5-O3-D2I 47
ILUTIONS ON INTERNATIONAL AFFAIRS I. ARNS-CCNTROL CCLD-WAR NATO VIETNAM REC-CHINA FOREIGV-POLICY FOREIGN-A/ D5-O+-D2I72 MULATIONS) TEST-EQUIPMENT, \#PSWI TEMPERATURES IN A LARGE NATURAL FUEL FIRE I FIRES TEST-METHODS MODELSISI I3-I?-0IBID USTRY INDUSTRIES ECONUMILS/ U.S. SOURCES OF PETROLEUM AND NATURAL GAS STATISTICS 1 RESOURCES PETROLEUM-IND DS-D3-OIG38 HELTER IV EAST CHICAGO, INOIANA I ENVIRONNENTAL-CONTROL, NATURAL VENTILATIOV TEST OF A BASEMEVT FALLOUT S I3-OI -OI7TL UT SHELTER IV EVANSTON, ILLINOIS I ENVIRCNMENTAL-CCNTROLI NATURAL VENTILAYIDV TEST OF AV ABJVEGROUND FALLO I3-OI -DI7ZI ITIVE SHELTER SYSTEMS - AN ANNOTATEO BIBLIOGRAPHY CF U.S. NAVAL CIVIL ENGINEER ING LABORATORY PUBLICATIONSI I3-I3-O2278 ICESCRIPT ION AND 8ACKGROUND I COMNAND-AND-CONTROL-SYSTEMS NAVAL-OPERATIONS OATA-STORAGE-SYSTEMS SUBJECT-I/ OS-D2-O2II6 HEAT-TRANSFER WEAPON 5-EFFEC IS SHIP-STRUCTURAL-COMPONENTS NAVAL-RESEARCH I *A.L. 984 1 YATHEYATICAL-MODELS I3-I?-OI797 I FROM LABORATORY VIBRATION AND HIGH IMPACT SHOCK IESTS I NAVAL-RESEARCH CESICN ELECTROVIC-EOUIPMENT RELII $20-11$-O2OB3 EARCH DES IGV ELEC TRONIC-EQUIPMENT RELIABILITYIELECTRONIC) NAVAL-VESSELS SHOC (MEC,HAVICS), \$AD 37880 IRES $20-11$-D2083 SLAT ION CIVIL-DEFENSE (GOVERNMENT-AGENCIES) AIR-FORCE ARMY NAVY GOVERNMENT-AGENC IES, \$ PUBL IC-HEALTH DISPOSAL COSTS WAST/ SOLID WASTES RESEARCH NEEDS I SCIENTIFIC-RESEARCH SAN ITARY-ENGINEERING I3-OZ-OI789 PTIMIZATION PERFJR/ AN INVESIIGATION OF MINIMAL EQUIPMENT NEEDS IN PERSONNEL SHELTERS 1 FALLOUT-SHELTERS O I3-I3-DIBI9 HYSIOLOGY EXPLOSIO/ AN INVESIIGATION OF NINIMAL EGUIPMENT NEEOS IN PERSONNEL SHELTERS I FALLOUT-SHELTERS P $13-13-018 I I$ CHINICAL INFORMATION WITHIN THE DEFENSE INDUST/ DOD USER - NEEDS STUDY, PHASE II, FLOW DF SCIENTIFIC AND TE D5-D?-O2I2D ID SEMANTIC DIFFERENTIAL SCALES RELEVANT TO INTERCLLTURAL NEGOTIATIONS I ATTITUDES BEHAVIOR METHODOLOGY S/ DS-IJ-D2I78 ASERS HEL IUM-GROUP-GASES PLASMA-MEDI UM LUNINE SCENCE ARGON NECN, \#UACRL E920272-6 \$AD 627233/MAGNETIC) L 20-D5-02060 ECTS INSTRUMENTATION RADIOISOTUPE \& (MEOICAL) RADICNUCLIDES NCONLASIA, \#ORAU-I-I /ON-IV.IUR IES 3ADIATION-EFF DG-I3-O22IS IF CONNECTICUT AVENUE ROUTE BETWEEN COLUNBIA ROAD AND VAN NESS STREET I TRAVSPORTATION SUBWAYS TESTS BORII I3-O2-DI7B5 N SERVICES SURPLUSES DATA-PROCESSING SHORTAGES/ A GENERAL NETWORK SIMULATION MODEL 1 RESOURCES DISTRIBUTIO OS-03-D2I 33 -WARFARE PETROLEUM-PIPELINES GAS-PIPELINES TRAN/ PIPELINE NETWORK STUDY 1 DAMAGE-ASSESSYENT YODELS NUCLEAR OS-D3-D2I 1 MAI IUN-RETRIEVAL DOCUMEVTATION STATE-I INFORMATI DN SYSTEM NETWORKS - LETS PROFIT FROM WHAT WE KVOW I INFOR OS-O2 - OI 330 MAI IUN-RETRIEVAL DOCUMEVTATION STATE-I INFORMATION SYSTEM NETWORKS - LETS PROFIT FROM WHAT WE KVOW 1 INFOR OS-OZ-DIO3O ALLOT RADIOACTIVE-I SOTOPES AIR MILK ATMOSPI SURVEILLANCE NETWORKS I RADIOLOGICAL-MOVITJRING RAUIUACTIVE-F I8-D3 - D2O3S ERS ENTRANCEWAYS DOSE-RATE EXPERIMENTAL DETERMINATION OF NEUTRON ENERGY SPECTRA IN CONCRETE DUCTS 1 SHELT O6-I8-O2219

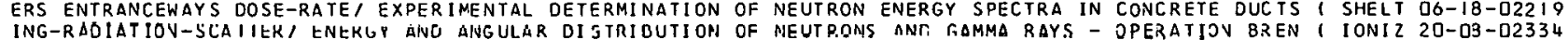
IPROTECTION IN HOME BASEMENTS I SHELTERS FALLOUT-SHELTERS NEVADA WEAPONS-TESIS SIMULATIJN RADIJACTI VE-FAL/ I3-IS-UISL2 BRIDGES ANO ALTERNATIVE SOLUTIONS THEREF ORE I URBAN-AREAS NEW-YORK, *A.L. IISS /NHATTAV AND WILLIAMSBURG 23 -02-D2357 REAS MANAGEMENT-PLANNING URBAN-PLANNING WASHINGTON BOSTON NEW-YORK, *RPN-79/ADS I TRANSPORTATION URBAN-A $23-02-02100$ IAT ION I URBAN-AREAS MOVING-BELTWAYS TRAFFIC DESIGA COSTS NEW-YORK CHICAGO WASHINGTON PHILADELPHIA BOSTON/23-02-02OQ7 PN-73-74 EXPRE SSWAY PLANS I TRANSPORTATION URBAN-AREAS NEW-YORK MANAGEMENT-PLANNIVG URBAV-PLANNING I *R 23-D2-D2O98 5 COSTS MANAGEMENT-P/ RAIL TRANSIT PLANS I TRANSPORTATION NEW-YORK URBAN-AREAS POPULATION RAILROADS SUBWAY 23 -02 -O2099 SURVEY(NFSS) FALL / COMBINED EFFECTS OF NUCLEAR WEAPONS ON NFSS TYPE STRUCTURES 1 NATIONAL-FALLOUT-SHELTER- I8-03-0I995 SYSTEMS I STORAGE-BATIERIES ALKALINE-CELLS AIRCR/ SEALED NICKEL CADMIJM BATTERIES FOR AIRCRAFT ELECTRICAL ID-O3-DI764 ICEFECTIONS TRAINING SLEEP WATER FCOD RECREATION EXERCISE NOISE RELIGION TEMPERATURE-CONTROL VENTILATION TLS-IJ-D2IBL

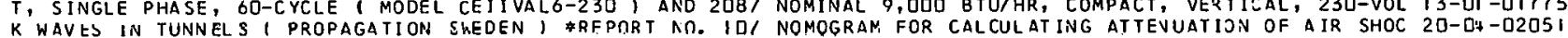


IOF THE THORAX ENERGIZED BY AIR BLAST OVERPRESSURES CR BY NON-PENETRATING MISS ILES I BIJLOGICAL-BLAST-EFF/ D6-2I-O2235 ALING I. EXPONENTIAL IDEAL GAS ATN' SHOCK PROPAGATION IN NONHOMOGENEOUS ATMOSPHERES AVD MODIFIED SACHS SC $20-0+-{ }^{2} 332$ -WAVES PROPAGATION MATHEMATICAL-AN/ ACCELERATION WAVES IN NONL INEAR MATERIALS I VISCOELASTICITY MECHANICAL 20-II-O2OBO / INTERNATIONAL SYMPOSIUM I LOAOING(MECHANICS) STRLCTURES NONL INEAR-ANALYSIS BEAMSISTRUCTURAL) FRAMES LIN/ $20-11$-D2339 I OPT ICAL-PHENOMENA HARMONIC-GENERATORS REFRACTI ON LASERS NONLINEAR-SYSTEMS AMMOV IUM-COMPOUNOS FOCUSING PI $20-0 S-D 2 D S 7$ $S$ I CRYSTALS OPTICAL- 1 THE MEA SURENENT OF SEVERAL CPTICAL NONLINEARITIES USIVG FOCUSED GAUSSIAV LASER BEAM $20-03-32067$ STUDIES AND PUBL ICATIONS IN SOCIAL RESEARCH I SOCIAL-SC/ NORC SOCIAL RESEARCH 1941-I964- AN IVVENTORY OF OS-II-D2I 94 C/ THE CHI-SOUARE TEST OF GOODNESS OF FIT FOR A BIVARIATE NORMAL DISTRIBUTION I STATISTICAL-TESTS STATISTI I2-OI-D22SI AR-EXPLOSIONS WI ELECTROMAGNETIC PULSE PROPAGATION IN THE NORMAL TERRESTRIAL WAVEGUIDE EVVIZJVMENT I NUCLE $20-14-02349$ ICS AND COSTS OF CONSTRUC TING THE UNDERGRCUND FACILITY OF NORTH AMERICAN AIR DEFENSE CJMMAND AT CHEYENNE, I3-I3-D2274

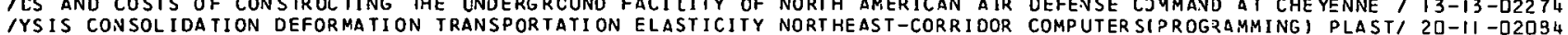

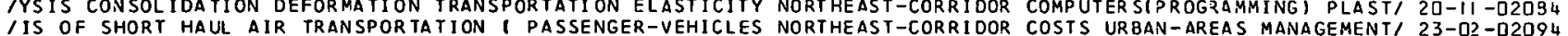
ICAVATION TUNNELSITRANSPORTATION) BLASTING COSTS DRILLING NORTHEAST-CORRIDCR FEAS IBILITY-STUDIES, \$PB 17 , I3-0?-OI78? POWER-PRD DUCT IWESTERN-EUROPE NATO GREAT-BRI TAIN FRANCE GERMANY EENELUX NORWAY CENMARK GREECE SWEDEY SWITZERLAND IRELANI I5-I]-OI 833 BO III. I RESOURCES VULNERABILITY WEAPONS-EFFECTS WIND, NREC DAMAGE ASSESSMENT COMPUTATIOY PROGRAM - JUM OS-03-02134 1 \#NREC- 114111 A LISTING OF TABLES AND PARAMETERS LSED IN NREC DAMAGE ASSESSMENT PROGRAMS 1 VULVERABILITY O5-03-02I38 AL SURVIVAL I SIMULATION DAMAGE-ASSESSMENT WARFARE RESOU/ NREC PRCGRAMS FOR GAMIVG THE LOGISTICS OF VATION DS-D3-QIG44 VULNERAB IL ITY PROGRAMMING (COMPUTERS) CASUAL/ STREAK IV - NREC RAPIC CAMAGE ASSESSMEVT PROGPAM 1 RE SOUREES OS-O3 -02146 NENTAL UVITED STATES I GEOGRAPHY RESOURCES DAMAI MAP IV - NREC SMALL SCALE YAPPIVG PROGRAM - MAPS OF CONTI OS-D3-O2ILO RS SCIENTIFIC-RESEARCH GLASS OPTICAL-PROPERTIES RUBY CRY/ NRL PROGRESS IN HIGH POWER LASER RESEARCH I LASE 20-05-D2O62 ALLO/ A PROVISIONAL CONEEPT OF EMERGENCY CPERATIONS UNDER NJCLEAR ATIACK 1 CIVIL-DEFENSE-OPERATING-PLANS F OS-OI-OIG 33 IF SOCIAL STRUCTURE - STUDIES OF THE SOCIAL DIMENSIONS OF NUCLEAR ATTACK I RECOVERY SOCIETAL-RECOVERY NUC, D5-II -O2IQO SHIELDING RA' PROTECTION OF FOOD AND AGRICULTURE AGAINST NUCLEAR ATIACK I RJRAL-AREAS RADIOACTIVE-FALLOUT I5-O3-DI897
IF SOCIAL STRUCTURE - STUDIES OF THE SOCIAL DINENSIONS OF NUCLEAR ATTACK I SOCIETAL-RECJVERYRESOVERY DEM/ O5-II-O22O2 IF SOCIAL STRUC TURE - STUDIES OF THE SOCIAL DINENSIONS OF NUCLEAR ATTACK
IFERENCES RELEVAVT TO THE STUDY OF SOCIETAL RECOVERY FROM NUCLEAR ATIACK I STRESSIPSYCHOLOGY) GROUP-DYNAM/ DS-IJ-DIOT7 ES - 1963 A. BACKGROUND AND PROCEDURES FOR APPLICATIONS/ NUCLEAR ATTACK HA2ARD IN CONIINENTAL UNITED STAT I5-03-OIO49 INTERACTIONS AND COMPAR ISONS AMONG MODES II EFFECTS OF NUCLEAR ATTACK ON FREIGHT TRAVSPO2TATION SYSTEMS I3-OS-O226O CONT INENTAL UNITED, A SYSTEM ANALYSIS OF THE EFFECTS OF NUCLEAR ATIACK ON RAILROAD TRANSPJPTATION IN THE DS-D3-O2I 28 IER ANALYSIS OF PROTECTIVE STRLCTLRES FOR PROTECTICN FROM NUCLEAR BLAST, SHELTERS SHOC. TAVES BLAST-SHEL/ I3-I3-0I833 RE/ PARAMETERS GOVERNING URBAN VULNERABILITY TO FIRE FROM NUCLEAR BURSTS I BURST-CONDITIONS URBAN-AREAS FI I3-I?-OIBII ES STATISTICAL-ANALYSIS WEAPONS-EFF/ ANALYSIS OF JAPANESE NUCLEAR CASUALTY OATA I NUCLEAR-WARFARE-CASUALTI DG-2I -0222 7 ES CASUALTIES (PREDICTION) URBAN-ARE/ ANALYSIS OF JAPANESE NUCLEAR CASUALTY DATA I VUCLEAR-WARFARE-EASUALTI D6-2I -O223 8 ISITIVITY OF MORTAL ITY ESTIMATES TO UNCERTAINTIES IN SOME NJCLEAR CAMAGE PARAMETERS II. 1 MJDELS DAMAGE- 1 D6-21 -02229 ISITIVITY OF MORTALITY ESTIMATES TO UNCERTAINTIES IN SOME NUCLEAR OAMAGE PARAMETERS I ATTACK-SCENARIOS NU/ O6-21-O2228 IOW SCREENS AS MODIFIERS OF THERMAL RADIATION RELEASED IN NUCLEAR DETONAT IONS I GLASS ATTENUATION URBAN-A/ I3-I2-OI796 E ECOLOGY RADIATION-EFFECTS GENETICS PHYSICS/ FUNDANENTAL NUCLEAR ENERGY RESEARCH - 19661 BIOLJGY MEDICIN $20-09-02070$ LE SALMOV EVENT I SEISMIC- MAVES / EARTH VIBRATIONS FROM A N'J CLEAR EXPLOSION IN A SALT DJME - PZJJECT DRIBB I8-O3-O23I9 ITHE FRACTIONATION FACTORS AND BICLOSICAL AVAILABILITY OF NUCLEAR EXPLOSION PRODUCTS IN RADIJACTIVE FALLOI I8-O3-D2323 IEO LITERATURE PERTAINING TO THE EFFECTS OF HIGH ALTITUDE NUCLEAR EXPLOSIONS I. AND II. 1 UNITED-STATES, I8-03-D20I8 TUCY OF THE MECHANICS OF SLIOE DANS WITH SAN/ PLOWSHARE - NUCLEAR EXPLOSIONS - PEACEFUL APPLICATIONS - A S $18-03$-O23I 7 TERS FROM ROW CHARGES INTERRLP TED BY A DUD / PLOWSHARE - NJ CLEAR EXPLOS IONS - PEACEFUL APPLILATIONS - CRA I8-D3 -023IL IAT ION TEAPOT - DISTRIBUTION AND DENSITY CF MISSILES FROM NUCLEAR EXPLOSIONS I SECONDARY-MISSILES BLAST-EI I8-O3-D23IB ULNERABIL ITY) FALLOUT-S/ DEFENOING THE UNITED STATES FROM NUCLEAR FIRE ( FIRES FIRE-PROTECTION RESOURCES (V I $3-1$-OI8D6 UCLEAR-WARFARE BALLISTIC-MISSILES CO/ ON THE ESSENCE OF A NUCLEAR MISSILE WAR I RUSSIAPOLITICAL-SCIENCE N O5-OU-DIGT2 REPORT TO THE PRESIDENT I NUCLEAR-REACTORS NUCLI CIVILIAN NJ CLEAR PONER - THE 1967 SUPPLEMEVT TO THE I962 I8-OJ-02027 IN THE UNITED STATES AS OF DEC.31,1966 I NUCLEAR-PCWER-P/ NUCLEAR REACTORS BUILT, BEING BUILT, OR PLANNED I8-05-02320 RECONNAISSANCE INTERNA/ ENGINEERING AND RESCUE WORK IN A NUCLEAR STRIKE ZONE I CIVIL-DEFENSE-SYSTEMS USSR D6-O?-DITD9 TION OF DEMOGRAPHIC VARIABL/ A STLOY OF THE DEMOGRAPHY OF NUCLEAR WAR - EMPIRICAL GUIDEL INES FJR THE SELEC 23-DH-O2ID3 LOGY RAD/ INTRODUCTION TO LONG TERM BIOLOGICAL EFFECTS OF NUCLEAR WAR I RACIOBIOLOCY RADIATIJV-EFFECTS ECO OG-IB-OI723 N METHODOLOGY MAVAGEMENT-PL, A STLOY OF THE DEMOGRAPHY OF NUCLEAR WAR I SURV IVAL NUCLEAZ-WAZFAZE POPULATIO $23-0+-021$ J4 PROSPECTS 1 POSTATTACK-OPERATIONS SOIL-CCNSERVATICN SOI/ NUCLEAR WAR AND SOIL EROSION - SOME PROBLEMS AND D8-13-OI745
IF A DUAL LAYERED PLATE SUBJECT TG THE THERMAL PULSE OF A NUCLEAR WEAPON 1 CALCULATIONS PROGRAMMINGICOMPU/ I3-I?-OI797 CES UNITS I SHIELDING PATIENTS RADIOA/ PROTECTION AGAINST NUCLEAR WEAPON FALLOUT IN EMER GENCY HEALTH SERVI I8-06-D2O28 1 THE URBAN POPULATION AS A MEASURE OF PROTECTION AGAINST NUCLEAR WEAPONS 1 USSR SHELTERS IVTEZVATIONAL1. TN INVESTIGATION OF SCHOOL DESIGNS TO RESIST INTEGRATED NUCLEAR WEAPONS EFFECTS STR STCTURES FALLOUT-SHE I3- 13 -OI82I AN INVESTIGATION OF SCHOOL DESIGNS TO RESIST INTEGRATED NUCLEAR WEAPONS EFFECTS 1 STRUCTURES FALLOUT-SHE $13-13$-OI82I
AL-FALLOUT-SHEL TER-SURVEYINFSSI FALL/ COMBINED EFFECTS OF NUCLEAR WEAPONS ON NFSS TYPL STRUCTURES I NATION I8-US-UIOQ5 AL-FALLOUT-SHEL TER-SURVEYTNFSS FALL/ COMBINED EFFECIS OF NUCLEAR WEAPONS ON NFSE TYPE 3 TRUCTURES 1 NATION 18 -US-UIO95 AIIONS EQUAT IONS-OF-MOTION LOADING IMECHANICSI SHOCK-WAVES NUCLEAR-EXPLOSION-DAMAGE 1 \$AD O47 OD6 /TIAL-EQU $13-13-02279$ FROM NUCLEAR BUR STS I BUR ST-CONDITI ONS URBAN-AREAS FIRES NUCLEAR-EXPLOSION-DAMAGE 1 \#USNRDL-TR-IOLL IFIRE I3-I?-OI8DI
ITACK POPULATION OF THE UNITED STATES 1 DAMAGE-ASSESSMENT NUCLEAR-EXPLOS ION-DAMAGE AGE YODELSISIMULATIONS/ I5-DS-OI965 IEFEN SE CIVIL-DEFENSE-SYSTEMS HASTY-SHELTERS CONSTRUCTION NUCLEAR-EXPLOSION-DAMAGE EXPLOSION-EFFECIS RURAI I3-I3-DIB2O IERY MANAGEMENT CONCEPTS AND TECHNIOUES I COUNTERMEASURES NUCLEAR-EXPLOSION-DAMAGE MANAGEMEVT-EVGINEERING 15 -D3-02293 / SONNEL USSR INTERNATIONAL-CIVIL-DEFENSE IRAINING NANUALS NUCLEAR-EXPLOSION-DAMAGE NUCLEAR-WARFARE SHOCK- I I3-I?-OI8D4 , EEOG/ ON DAMAGE ASSESSMENT MODELS I RAOIOACTIVE-FALLOUT NUCLEAR-EXPLOSION-DAMAGE POPULATIJVIDISTRIBUTION IS-DS-DI9G2 ITURES I SHOCK-AB SORBERS VIBRA TI ON-I SOLATCRS IMPACT-SHOCK NUCLEAR-EXPLOSION-DAMAGE SPRIVGS PER SONNEL DESI/ I3-I3-DIB23 ICS SHOCK-WAVES FAILUREIMECHANICSI UNDERGROUND-STRUCTURES NUCLEAR-EXPLOSIONS I \#TR 508 \#DASA-13.0I8 /ECHAN I8-03-02008 PROGR/ RE-USABILITY OF BUILOINGS AFTER A WARFARE I FIRES NUCLEAR-EXPLOSIONS DAMAGE-ASSESSMEVT FIRE-SAFETY IS-O3 - OI 952 IRDEN ING CONSIOERATIONS PERTAINING TO RE SIDENTIAL AREAS NUCLEAR-EXPLOS IONS FIRES AIRBUR ST SLLUC UMRES VUI IS-O3 -D23IS IRDENING CONSIOERATIONS PERTAINING TO RESIOENTIAL AREAS I NUCLEAR-EXPLOSIONS FIRES AIRBUR ST STRUCTURES VU/ IS-OS -DI9S3

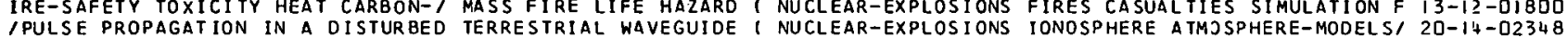
/PULSE PROPAGATION IN A DISTURBED TERRESTRIAL WAVEGUIDE I NUCLEAR-EXPLOSIONS IONOSPHERE ATMJSPHERE-MODELS/ $20-14$-02348
/ERIUM THEORY TO DECOUPL ING I CRA TERING EXPLOSION-EFFECTS NUCLEAR-EXPLOSIONS SEISMOLOGY GRAVITE MATHEMATI/ I8-03-O2316 IER IUM THEORY TO DECOUPL ING I CRA TERING EXPLOSION-EFFECTS NUCLEAR-EXPLOS IONS SE ISMOLOGY GRAVITE MATHEMATI I $18-03$-O2316
/UCTURES EXPLOSION-EFFEC TS SHOCK (NECHANICS) VULNERABILITY NUCLEAR-EXPLOS IONS SHOCK-WAVES LOADIVG IMECHANIC, I 3 - I3-D2291 IGATION IN THE NORMAL TERRESTRIAL WAVEGUIDE ENVIRONMENT 1 NUCLEAR-EXPLOSIONS WAVE-TRANSMISSION ATMOSPHEREI $20-1+$-O2349 UILT, OR PLANNED IN THE UNITED STATES AS OF DEC.31,1966 ( NUCLEAR-POWER-PLANTS, \#TID-82ODII5TH-REVI /NG B I8-0J-02320 IT TO THE 1962 REPORT TO THE PRESIDENT I NUCLEAR-REACTORS NUCLEAR-PONER-PLANTS ELECTRIC-POWER-PRODUCTION I I8-O5-D2O27 IST IONS POLAR-REGIONS ABSORPTION OZONE REACTION-KINETICS NUCLEAR-RADIATION COSMIC-RAYS ELECTZJMAGNETIC-WI OL-OI -D2II 3 ITRY MATHEMATICS COMPUTERS NUC LEAR-REACTORS HEAT-TRANSFER NUCLEAR-REACTOR-MATERIALS FLUID-DYVAMIC-PROPERT/ $20-03$-02DTO /RCH METALLURGY MATER IALS CHEMISTRY MATHENATICS CONPUTERS NUCLEAR-REACTORS HEAT-TRANSFER NUCLEAR-REACTOR-/ 20-D3-02070 IHE 1967 SUPPLEMENT TO THE 1962 REPORT TO THE PRESIDENT 1 NUCLEAR-REACTORS NUCLEAR-POWER-PLAVTS ELECTRIC-/ I8-05-O2027 IC-OP INIOV ATTITUDES SUR VEYS QLESTIONNAIRES PEACE-KEEPING NUCLEAR-WARFARE ARMS-CONTROL 1 \#A.L. 1 [O97 1 PUBL OS-11-O2196 I GEOGR APHY RESOURCES DAMAGE-A SSESSMENT WEAPONS-EFFECTS NUCLEAR-WARFARE ATTACK-PATTERVS RADIOACTI VE-FALI DS-D3-D2I 40 SENCE OF A NUCLEAR MISSILE WAR $?$ RUSSIA POLITICAL-SCIENCE NUCLEAR-WARFARE EALLISTIC-MISS ILES COLD-WAR FOR/ OS-D + - 1672 SSMENTY A 作 S TRAN/ PIPELINE NETWORK STUDY I DAMAGE-ASSESSMENT MODELS NUCLEAR-WARFARE PETROLEUM-PIPEL INES GAS-PIPELINE DS-03-D2I 4 I T-PL A STUDY OF THE DEMOGRAPHY OF NUCLEAR WAR I SLRVIVAL NUCLEAR-WARFARE POPULAT ION METHODJLOGY MANAGEMEN 23 -DH-D2I O4 /ECOVERY ECONOM IC-RECOVERY RECOVERY A TTACK-SCENARICS WIND NUCLEAR-WARFARE RADIATION-IVJUR IES RAOIOACTI VE-I OS-03-O2ISD 1-CIVIL-DEFENSE TRAINING MANUALS NUCLEAR-EXPLOSI ON-DAMAGE NUCLEAR-WARFARE SHOCK-HAVES THERMAL-RADIA IION RI I3-I?-DI8J4 / THERMONUCLEAR WAR - THE LIMI TS OF FEASIBLE PRODUCTION I NUCLEAR-WARFARE SURVIVAL ECONOMICS POPULATION A/ OS-03-O2IS8
/IMENSIONS OF VUCLEAR ATTACK I RECOVERY SOCIETAL-RECOVERY NUCLEAR-WARFARE WEAPONS-EFFECTS PJSTATTACK-OPER/ O5- 11 -D2I OD IMODEL - NAHICUS 63 APPLICATION I RE SOURCES VULNERABILITY NUCLEAR-WARFARE WIVD BLAST RADIOACTIVITY THEORYI O5-O3-D2I 49 -AID ORGANIZATION TRAINING HOSPITALS CASUALTIES PERSONNEL NUCLEAR-WARFARE-CASUALTIES, \$A.L. IO76, IT FIRST DS-2I-DI738 
HED PAPERS OF THE ATOMIC BOMB CASLALTY CONMISSION 1 JAPAN NUCLEAR-WARFARE-CASUALTIES I *ABCC IR D-60 /BLIS O6-2I - B2224 ICIARY-GELS FLAME-THROWER-FUELS SMOKE-MUNITIONS FIRST-AID NUCLEAR-WARFARE-CASUALTIES, \#JPRS 30, 979 *TT G, O6-I8-DI72I ST-SHEL TERS YATHEMATICS PROGRAMMING (COMPUIERS) CASUALTIES NUCLEAR-WARFARE-CASUALTIES I *RTI-JU-230-21III) I5-D3-DI938 IIES IN SOME NUCLEAR DAMAGE PARAMETERS I ATTACK-SCENARIOS NUCLEAR-WARFARE-CASUALTIES CASUALTIES CALCULATI/ OO-2I -Q2228 7UCLEAR DAMAGE PARAMETERS II. I MODELS DAMAGE-ASSESSMENT NUCLEAR-WARFARE-CASUALTIES CASUALTIES MILITARY- 1 D6-2I -0222 9 I URBAN-ARE/ AYALYSIS OF JAPANESE NUILEAR CASUALTY DATA I NUCLEAR-WARFARE-CASUALTIES CASUALTIESIPREOICTION DG-2I -O2238 IENTS PRI DAPD46 TIME PHASED CASLALTY ESTIMATE I ANALYSIS NUCLEAR-WARFARE-CASUALTIES DAYAGE-ASSESSMENT PAT D6-2I -D2225 IION-CAMAGE POPULATIONIDI STRIBUTI CNI GEOGRAPHY INDUSTRIES NUCLEAR-WARFARE-CASUALTIES DEYOGRAPHY BUR ST-CONI IS-DS-DIOS2 IICS IN SOVIET MILITARY MEDICINE ( USSR DISASTER-MEDICINE NUCLEAR-WARFARE-CASUALTIES FIRST-AID WOUNDS-AND/ OS-05-DI7J3 INUAL REPORT JULY-1 $1905-J U N E-30,1966$ I RADIATION-EFFECTS NUCLEAR-WARFARE-CASUALTIES JAPAN HIROSHIMA DATA I OS- IS-OI73O

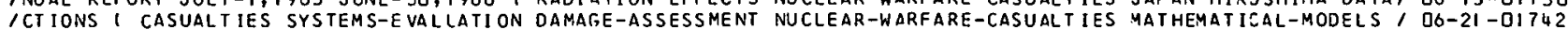
LLAW ORGANIZATION CIVIL-DEFENSE-SYSTEMS CASUALTIES CANACA NUCLEAR-WARFARE-CASUALTIES RADIATION-INJURIES PI J6-2I -O22 ?6 ICICINE MEDICAL-PER SONNEL HOSPITALS PUBLIC-HEALTH RESCUES NUCLEAR-WARFARE-CASUALTIES SCHOOLS SUR VIVAL CASI O6-2I -OIT4O ICENARIOS FERTILITY URBAN-AREAS RLRAL-AREAS VULNERABILITY NUCLEAR-WARFARE-CASUALTIES SEX RACE MANAGEMENT- 1 IS-DS-QI96S WEAPONS-EFF' AVAL YSIS OF JAPANESE NUCLEAR CASUALTY DATA I NUCLEAR-WARFARE-CASUALTIES STATISTICAL-ANALYSIS DO-21-D222 7 I ASUALTIES AOMINISTRATION-AND-MANAGEMENT NEOICAL-SUPPLIES NUCLEAR-WARFARE-CASUALTIES STJCKPILING CANADA I/ O6-2I -OI739 ICTS EXPLOSION-EFFECTS BLAST-SHELTERS RADIOACTIVE-FALLOUT NUCLEAR-WARFARE-CASUALTIES SYSTEMS-EVALUATION C/ IS-O3-OI93 3 IINT IES IN THE HIROSHIMA EXPLOSI ON I AIR-BURST YIELD WIND NUCLEAR-WARFARE-CASUALTIES TARGETS SCALEIRATIOI/ IS-DS-OI958 TO VARIATIONS IN AGGREGATE POPULATI ON REPRESENTATIONS I NUCLEAR-WARFARE-CASUALTIESIMATHEMAT ICAL-PREDIC T/ IS-OT-OIOGO CIFFERENCE TECHNIOUE I THERMAL-RADIATION WEAPONS-EFFECTS NUCLEAR-WEAPONS I CIFFERENCE TECHN IOUE ITHERMAL-RADIATION WEAPONS-EFFECTS NUCLEAR-WEAPONS I *PR-4 /IVITE SOLID BY A FINITE I3-I?-DI795 ENSE HANDBOOK - USSR I RURAL-AREAS BOMBS GUIDED-MISSILES NUCLFAR-WEAPONS CONTAMINATION FOOD CHEMICAL-WAR I5-03-02296 IEFENSE CHEMICAL-WARFARE-AGENTS B IOLOGICAL-WARFARE-AGENTS NUCLEAR-WEAPONS FIRST-AIC WARVING-SYSTEMS RADIO/ IS-O3-DI9II IN ANC AVALYSIS I. FALLOUT. PROTECIION I FALLOUT-SHELTERS NUCLEAR-WEAPONS SHIELDING DOSE-RATE EALCULATION/ I3-I3-OI839 IGROUND MOTIONS FOR SIMULATION BY SHOCK TESTING MACHINE I NUCLEAR-WEAPONS SHOCK-WAVES MECHAVICAL-WAVES SHI 20-II -D2O78 ING SYSTEMS ANALYSIS 1 MICHIGAN ATIACK-SCENARIOS AIRCRAFT NUCLEAR-WEAPONS-EFFECTS MILITARY-IVTELLIGENCE SI I3-12-O2267 ELLS ELASTIC-SHELLS MODELS(SIMLLATIONS) BEAMSISTRUCTURAL) NUNERICAL-ANALYSIS, \#AD 636187, STZUCTURAL-SH $20-11$-D2O8I WEIGFT, DIET AND EXERCISE ( BODY-LEIGHT PHYSICAL-FITNESS NUIRITION I \#AD G33 860 A IET POSTATTACK-OPERATIONS SURVIVAL STORAGE (FOOD) PRODUCT' NUTRITION IN THE PJSTATTACK ENVIRJNMENT I FOOD D OG-1S-OI7I 7 ITS STOCKPILING SHELTER-MANAGEMENT HANDBOOKS HABITABILITY NUTRITION SANITARY-ENGINEER ING MEDICAL-SUPPLIES/ OS-IJ-D2I84 IAL-ENGINEERING ANIMAL-HUSBANDRY CROP-RE SEARCH ENTCMOLOGY NUTRITICN WATER-COVSERVATION SOIL-COVSERVATION 1 Q2-OJ-D2I O8 TICS AG/ PROCEEDINGS OF THE HIGH LYSINE CORN CONFERENCE I NUTRITIONIHUMAN) VUTRITIOVIAVIMAL) PROTEINS GENE TG-IS-DI720

\section{0}

/ IVIL-DEFENSE-SYSTEMS MANAGEMENT-PLANNING DECISION-MAKING ORJECTIVES PUBL IC-OPINION POLITICAL-SCIENCE EXE/ DS-O'H-OIGTI PRCGRAMS OF FOREIGN AID I PUBLIC-OPINION SURVEYS FCREIGN/ OCCASIONAL REPORT ON FOREIGN AFFAIRS - AMERICAN O5-II-OIG87 TIONS 1 INTERNATIONAL-AFFAIRS PUBLIC-OPINION SURVEYS FCR/ OCCASIONAL REPORT ON FOREIGN AFFAIRS - UNITED NA O5-II -D2I98 TIONS I INTERNATIONAL-AFFAIRS SUR VEYS QUESTIONNAIRES PUB/ OCCASIONAL REPORT ON FJREIGN AFFAIRS - UNITED NA DS-II -OZIQQ 1 PUBLIC-OPINION SURVEYS FOREIGN-POLICY OUESTIONNAIRES I/ OCCASIONAL REPORTS ON FOREIGN AFFAIRS - GERMANY OS-II - O2IBG NG-SYSTEMS TRAINING METHODOLO/ RESEARCH DATA FROM SHFLTER OCCUPANCY EXERCISES I HABITABILITY DATA-PROCESSI D5-IJ-OIS8D LTERS COVFINED-ENVIRONMENTS STOCKPILING SHELTER-I SHELTER OCCUPANCY STUDIES I. FINAL REPORT I FALLOUT-SHE DS-IJ-O2I 84 HANDBOOK FOR UNTRAINED MANAGENENTI SHELTER-NAN/ SHELTER OCCUPANCY STUDIES 2. COMMUVITY FALLOUT SHELTERS O5-OI -O2II5 (BIOLOGY) SURVIVAL, SENSIBLE AND LATENT HEAT LOSSES FROM OCCUPANTS OF SURVIVAL SHELTERS I HEAT-PRODUCTION D6-IS-DI7I9 CY METABOL ISM PHYSI/ SENSIBLE ANO LATENT HEAT LOSSES FROM OCCUPANTS OF SURVIVAL SHELTERS 1 SHELTER-OCCUPAN DG-IS-OI7I 8 CY METABOL ISM PHYSI/ SENSIBLE AND LATENT HEAT LOSSES FROM OCCUPANTS OF SURVIVAL SHELTERS I SHELTER-OCCUPAN D6-1S-OI7I8

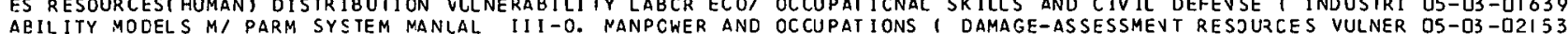

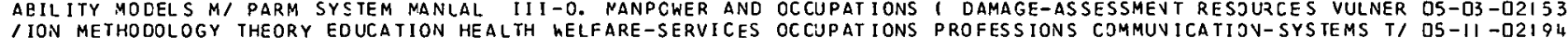

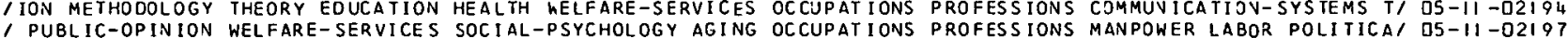

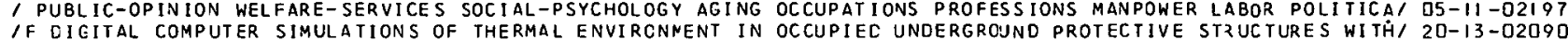

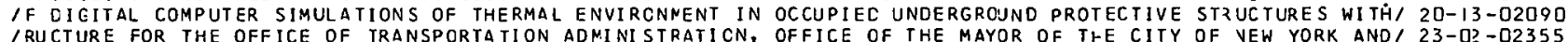
/RUCTURE FOR THE OFFICE OF TRANSPORTATION ADMINISTRATICN, OFFICE OF THE MAYOR OF THE CITY OF VEW YORK AND/ $23-0 ?-02355$ IRIM REPJRT ON TRANSFER SYSTEM AND FARE STRUCTURE FOR THE OFFICE OF TRANSPORTATION AOMIVISTRATION, OFFICEI 23 -OZ -D23SS ISC IENT IF IC-RESEARCH BLAST FIRES LNI TED-STATES-GOVERNMENT OFFICE-OF-EMERGENCY - PLANNING SHELTERS ELECTROMAI I 5 -O3-D2299 INING ARMEC-FORCES(FOREIGN) LEADERSHIP MILITARY-PERSONNEL OFFICER-PERSONNEL, 1 \$PRS-41, 308 \$TT-67-3I948 /A O5-09-02I76 LOYMENT TAXES SER VICES MANAGEMENT-PLANNING COSTS ILLINCIS OHIO INCIANA MICHIGAN I \$SRI-II-4895 /TITION EMP $23-02-02095$ HE EFFECTS OF HIGH ALTITUDE NUCLEAR EXPL/ BIBLI CGRAPHY OF OPEN ANC UNCLASSIFIEO LITERATURE PERTAINING TO T I8-03-O2DI B IARTMENT PUBLICATIONS, $1960-1965$ - AN AUTHOR INDEX OF THE OPEN LITERATURE WITH ABSTRACTS 1 BIBLIOGRAPHIESI Q5-03-DI652 A MANY FI FMFNT I ASER I L/ LASER CAVITIES 1. RESEARCH ON OPEN RESONATORS II. BEHAVIOR OF THE OUTPUT FROM 20-OS-D2OS7

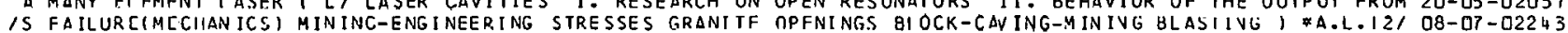

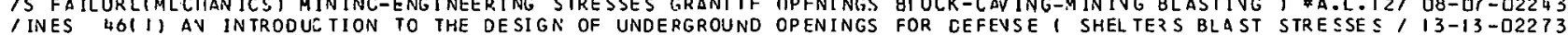

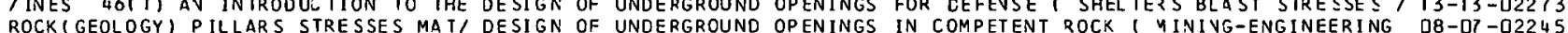

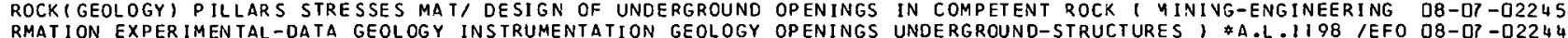

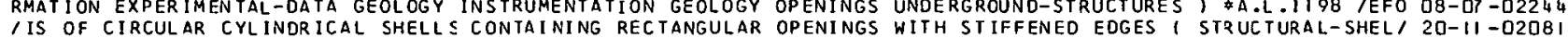

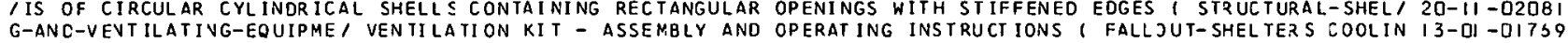
ISASTER-MEDICINE MEOICINE AD/ DDVAN.FO TRFATMENT CENTRE - OPERAT ING MANUAL - EMERGEVCY HEALTH SERVICES I D DO-2I -DI738 , UNOERGROUNO-STORAGE SALES REVENLE UTILI ZATICN FINANCING OPERATING-COSTS CAVADA. CONSTRUCTION PRICES LABO/ QS-US-UIOUL IUIPMENTS AND SYSTEMS TO SUPPORT INTRASTATF C.IVII DEFENSE OPERATIONS - CIRCA I970I RADIO-CJMMUVICATION-SI I7-O?-O23I3 CUES CIVIL-DEFENSE-PER SONNEL USSR/ ENGINEERING AND RESCUE OPERATIONS AT THE SITE OF AV ATOYIL STRIKE $($ RES I3-I?-OI8J4 FOR D/ RADIOLOGICAL RECOVERY REQUIREMENTS, STRUCTURES AND OPERATIONS RESEARCH I. CALCULATIJVAL TECHNIQUE O6-I3-O22I 8 I R/ RADIOLOGICAL RECOVERY REQUIREMENTS, STRUCTURES, AND OPERATIONS RESEARCH I. INTRODUCTION AND SUMMARY O6-I3 -O22I 4 RAC/ RADIOLOGICAL RECOVERY REQUIREMENTS, STRUCTURES, AND OPERATIONS RESEARCH II, DAMAGED PF MULTIPLIER I OO-I3-D222I

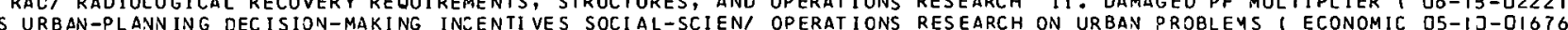
OPERATING-PLANS FALLO/A PROVI IIONAI CONCEPT GF EMERGENCY OPERAIIONS JNDER VUCLEAR ATTACX I IVIL-DEFENSE- D5-OI -DIG23

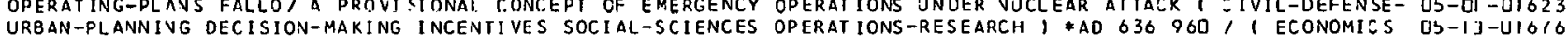

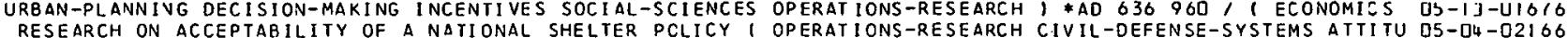

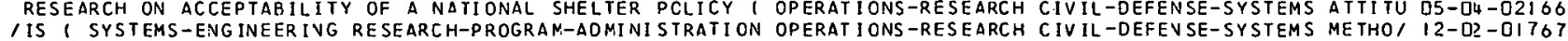
IA CAPITAL SURPLUS ECONOMY - KLWAITI MANAGEMENT-PLANNING OPERAT IONS-RESEARCH ECONOMICS DECISIOV-MAKING C/ OS-O3-DIO3S 638220 CUTTHROAT GAMES ( OPERAT IONS-RESEARCH GAME-THEJZY) $\$ 2 A C-P-20$ \#AD 12-D?-OIT68 IEFFECTIVENESS MANAGEMENT-ENGINEERING MANAGEMENT-PLANNING OPERAT IONS-RESEARCH MANAGEMENT-CONTROL-SYSTEMS / OS-OI -02292 IY I CIVIL-DEFENSE-SYSTEMS METEOROLOGY SYSTEMS-EVALUATI ON OPERATIONS-RESEARCH MATHEMATICS WEAPOVS-EFFECTS/ IS-D3-OI935 ISYSTEMS ANALYSIS I MII ITARY-STRA TEGY MANAGEMENT-PLANNI NG OPERATIONS-RESEARCH MOOELS THZEAT-EVAL UATION DE I I S-O3 - D23O 3 ISC IENT IFIC-ORGAVIZATIONS RE SEARCH-PROGRAN-AOMINISIRAI I ON OPERAT IONS-RESEARCH PER SOVVEL UNIIED-STA IES-GUVI OS-D3 -DI 49 TON INFORMAT ION-RETR IEVAL GAME-THEORY EDUCATION TRAINING OP ERATIONS-RESEARCH PRDGRAMMIVG-LAVGUAGES MATHEI OQ-O2-DI75I /E-OPERAT ING-PLANS FALLOUT-SHELTERS CONSTRUCTION-SLANTING OPERAT IONS-RESEA ITUDES IE MECHAVISMS ASSOCIATED IRS FEAR SURV' UNESCO AND PUBLIC OPINION TODAY UNITED-STATES QUESTIONNAIRES ATT OS- II -OIG89

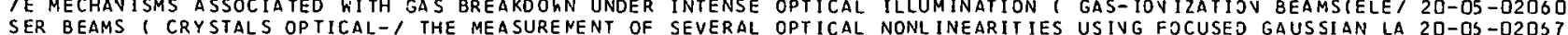

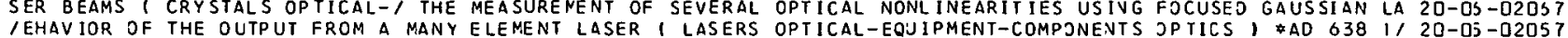

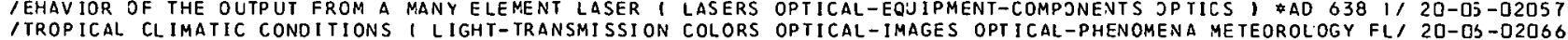
ITROPICAL CLIMATIC CONDITIONS I LIGHT-TRANSMISSI ON COLORS OPIICAL-IMAGES OPTICAL-PHENOMENA METEOROLOGY FL/ $20-03-02066$
/-FLUX HEAT-TRANSFER PYROMETER S HIGH-TEMPERATURE-RESEARCH OPTICAL-INSTRUMENTS EMISS IVITY, I INEARITIES USING FOCUSED GALSSIAN LASER BEANS 1 CRYSTALS OPT ICAL-PHENOMENA HARMONIC-GEVERATJRS REFRACTIO/ $20-0 S-020 S 7$ TIC CONDITIONS I LIGHT-TRANSMI SSION COLORS OPTICAL-IMAGES OPTICAL-PHENOMENA YETEOROLOGY FLOPIDA I *82D9-2 20-OS-O2OS6 
IN THEORY EXPER IMENTAL-DATA MATERIALS PARIICAL-SIZE COSTS OPTICAL-PROPERT IES COST-EFFECTIVENESS I \#URS 64/ I9-DI -D2O4 2 POWER LASER RESEARCH I LASERS SCIENTIF IC-RESEARCH GLASS OPTICAL-PROPERT IES RUBY CRYOGEN ICS GASES PUMPINI MANY ELEMENT LASER ( LASERS OPTICAL-EQUI PNENT-CONFONENTS OPTICS, \$AD 638 193/AVIJR JF THE JUTPUT FROM A $20-05-02057$ THODS IN RUBY LASERS (PUMPING (OPIICAL) CAVITY-RESCNATORS OPTICS CRYSTALS MIRRORS, OAD 637825 1 IULSING ME 20-DJ-O2056 -WARFARE MODEL-THEORY STRATEGIC-WARFARE MILITARY-STRATEGY OPTIMIZATION I \#RM-4817-PR \#AD 637 464 / NUCLEAR IS-OS-O1959 (EQU I PMENT NEEOS IN PER SONNEL SHELTERS ( FALLOUT-SHELTERS OPTIMIZATION PERFORMANCE(EVGIVEERIVG) PHYSIOLOG I 3-I3-OI8I 9 JECTEC TO BLAST LOADS I DESIGN BE / STUDY TO DETERMINE THE OPTIMUM SECTION OF REIVFORCED COVCRETE BEAMS SUB I3-I3-OI84D ELTE/ STUDY OF THE PROPOSED EAST hEST FREEWAY TUNNEL WEST ORANGE, NEN JERSEY, AS A CIVIL DEFEVSE PUBLIC SH I $3-13-02271$ DECENTRAL IZATION TAXES VULNERABILI/ IMPLEMENTATION OF AN OR DERED SDRAWL URBAN CONFIGURAT ION 1 URBAN-AREAS $23-0 S$-D2ID6 RPORATIOV AND ELIMINATION OF RADIOACTIVE NATERIALS BY THE ORGANISM 1 RADIOBIOLOGY, \$ORVL-TR-975 SERVICES OIVISION 1 DISASTER-MEDICINE PUBLIC-HEALTH LAW ORGANIZATION CIVIL-DEFENSE-SYSTEMS $=A S U A L T I E S$ CI O6-2I -D2226 ERS APPROPRIATIONSI STATUS OF THE' CIVIL DEFENSE PRCGRAM I ORGANIZATION DUAL-PURPOSE-SHELTERS FALLOUT-SHELT JS-OI -DIG25 IACT ICS OF FIRE PROTECTION AS EMPLOYED BY A CIVIL CEFENSE ORGANIZATION DURING WORLD WAR II - GERMANY I. $13-1$ - -O2265 IACT ICS OF FIRE PROTECTION AS EMPLOYED BY A CIVIL DEFENSE ORGANIZATION DURING WORLD WAR II - GERMANY - I I I3-I?-D22G4 $Y$ - SOME EXPLORATORY STUDIES I POS/ KINSHIP AND VOLUNTARY ORGANIZATION IN POST THERMONUCLEAR ATTACK SOCIET I5-03-O23DI -OP IN ION INTERNATIONAL-AFFAIRS SU/ CAN THE UNITED NATIONS ORGANIZATION PREVEVT WARS I PEACE-KEEPING PUBLIC OS-II -D2IQ5 /EDICINE MEDICINE ADMINISTRATION-AND-MANAGEMENT FIRST-AID ORGANIZATION TRAIVING HOSPITALS CASUALTIES PERSI DO-2I -DIT38 /IRE PROTECTION SERVICES - A CRITICAL PRESENTATION OF THE ORGANIZATION, TECHNOLOGY, AND TACTICS OF FIRE P/ I3-I2-02264 IIRE PROTECTION SERVICES - A CRITICAL PRESENTATION OF THE ORGANIZATION, TECHVOLOGY, AND TACTICS OF FIRE P/ I3-I?-O22G5 COCUMENTATION PROFESSIONAL-PERSONNEL SCIENTIFIC-RESEARCH ORGANIZATIONS 1 \#AD 648 7671511 INDEXES REPORTS O5-D2 -O2II 7 AGEMENT-PLANNING SURVIVAL URBAN-ARI ON THE OESIGN CF RISK ORIENTEC, LOW COST FALLOUT SHEL TER SYSTEMS I MAN I3-I3-DI829 PSYCHOLOGY) SHELTERS SCHOOLS PLBLIC-ONEOREATSIGN CFF RISK ORIENTED LOH COST FALLOUT SHELTER SYSTEMS I MAN IOSIONS I WAVEFORM-GENERA TORS INSTRUMENIA IION TRANSDUCERS OS CILLOSCOPES CAMERAS REFLECTION REFRACTION WAVI O8-II -O224 7 PHS-1581.
$I$ IES 1. RESEARCH ON OPEN RESONATORS II. BEHAVIOR OF THE OUTPUT FROM A MANY ELEMENT LASER I LASERS OPTICI $20-05-02057$ IIES I RESEARCH ON OPEN RESONATORS II - BEHAVIOR OF THE OUTPUT FROM A MANY ELEMENT LASER I LASERS OPTIC I $20-03-02057$
EFFECTS OF OVERPRESSURE ON THE EAR - A REVIIEW I BLAST-BIOLO DS-21 - D223I

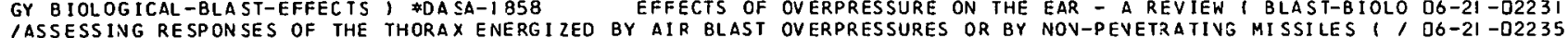
IASSESSING RESPONSES OF THE THORAX ENERG I ZED BY AIR BLAST OVERPRESSURES OR BY NOV-PEVETRATIVG MISSILES 1 O O6-2I -02235
TIC-THEORY ELECTRON-DENSITY CERENKOV-RADIATION MICROWAVES OXIDES PLASTICS, \$PIBMRI-1295.3-66 \$AD 642 27I $20-03-02330$ , THE O2l-, MODEL FOR D REGION RECOMBINATION I IONCSPHERE OXYGEN GAS-IONIZATION, AIRBURST IOVS POLAR-REGI, O4-OI-O2II 3 II FALLOUT-SHEL TERS FIRE-PROTECTION SMOKE CARBON-NCACXIDE OXYGEN-SUPPLY GAS-DETECTORS FIRE-DE TEZTORS IARB I I3-I?-QI8O IN GAS-IONIZATION, AIRBURST IONS POLAR-REGIONS ABSCRPIION OZ ONE REACTION-KINETICS. NUCLEAR-RADIATION COSMI/ OL-OI -O2II 3

P

ENSIONS OF TOTAL POPULATION TO 2015 I DEMOGRAPHY TABLES I P-25-359/Y AGE, SEX, AND COLOR TO 1990 WITH EXT $23-04-02358$ FIRE-RESISTANCE THERMAL-INSULATION PRESERVATION FINISHES PAINTS, \$HANDBOOK NO. 72 , SAYDWICH-CONSTRUCTION II-1? OI $7 G 6$ OPERATION BUSIER - THERNAL RADIATION EFFECTS ON PAINTS, PLASTICS, AND COATED FABRICS \&WT-4Q7 I3-I?-D2263 AL-SCIENC/ TABLES FOR YULES Q ASSCCIATION COFFFICIENT FOR PAIRS OF PERCENTAGES I STAT ISTICAL-ANALYSIS SOCI O5-II -D22DO AL-SCIENC TABLES FOR YULES Q ASSCCIATION COEFFICIENT FOR PAIRS OF PERCENTAGES I STATISTICAL-ANALYSIS SOCI OS-II -D22DO IADEMIC CENTERS - ASIA I BIBLIOGRAPHIES CHINA INDIA JAPAN PAKISTAN PHILIPPINES I \#EXTERVAL RESEARCH LIST I OS-II -QI698 HRUST L LARGE DEFLECTIONS OF CLAMPED REC TANGULAR PLATES I PANELS(STRUCTURAL) RECTANGULAR-BODIES STRESSES T 20-II -02344 USUR COMMERCE IN TERNATIONAL-AFFAIRS, *EXTERNAL RESEARCH PAPER 156 SOVIET IMPACT OV IVTEPVATIONAL LAW I DS-II -DIOPO NG DATA-STORAGE-SYSTEMS COMPUTERS DICTIONARIES) \#COUGLAS PAPER-4D27 *AD 646 C30 /DUSTRY SYSTEMS-ENGINEERI OS-J?-02II8 NAL-PERSONNEL SCIENTIFIC-RESEARCH ORG/ INDEX OF PUBLISHED PAPERS 1 INDEXES REPORTS DOCUMENTATIOV PROFESSIO O5-02-O2I I 7 JAPAN NUCLEAR-WARFARE-CASUALTI / B IBLIOGRAPHY OF PUELISHEO PAPERS OF THE ATOMIC BOMB CASUALTY COMMISSION 1 O6-2I-O222L LECYPODA TOXINS-AND-ANTI TOXINS BIOLOGICAL-WARFARE-AGENTS PARALYSIS FOOD-POISONING YUSSEL-PJISONS I *AD GI IS-O?-DI863 1 PEL ECYPODA TO/ SOME CHEMICAL AND PHYSICAL PRCPERTIES OF PARALYTIC SHELLFISH POISOVS RELATED TO TOXICITY IS-D? -DI863 ITAL ITY ESTIMATES TO UNCERTAINIIES IN SOME NUCLEAR OAMAGE PARAMETERS II. I MODELS DAMAGE-ASSESSMENT NUCL/ DO-2I -D2229 ITALIYY ESTIMATES TO UNCERTAINTIES IN SOME NUCLEAR DAMAGE PARAMETERS 1 ATTACK-SCENARIOS NUCLEAR-WARFARE-C I O6-2I-02228 IGY ANO PROBLEM AREAS IN RELATING PHYSICAL AND 81 OLOGICAL PARAMETERS 1 BIOLOGICAL-BLAST-EFFECTS BLAST-BIO/ O6-2I-D2223 O AIR BLAST/ THE RELATIONSHIP BETMEEN SELECTED BLAST WAVE PARAMETERS AND THE RESPONSE OF MAMMALS EXPOSED T O6-2I -O2234 FROM NUCLEAR BUR STS 1 BURST-CONDITIONS URBAN-AREAS FIREI PARAMETERS GOVERNIVG URBAV VULNERABILITY TO FIRE I3-I?-OIBII FROM NUCLEAR BURSTS I BURST-CONDITI ONS URBAN-AREAS FIREI PARAMETERS GOVERNIVG URBAV VULNERABILITY TO FIRE I3-I? -OIBDI

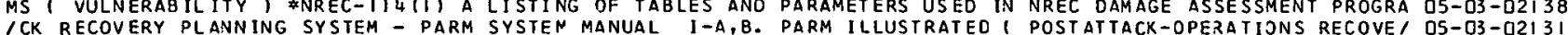
ICK RECOVERY PLANNING SYSTEM - PARM SYSTEN MANUAL I-A, B. PARM ILLUSTRATED I POSTATTACK-OPERATIONS RECOVE/ OS-03-0213I
SESSMENT VUL VERAB ILITY ECONOMI I PARM SYSTEN MANUAL VI-2. PARM MAIN ROUTINE I RESOURCES CAPACITY DAMAGE-AS G5-D3-02152 SESSMENT VUL VER AB IL I TY ECONOMI I PARM SYSTEN MANUAL VI-2. PARM MAIN ROUTINE I RESOURCES CAPACITY DAMAGE-AS Q5-D3 -02152
SOURCES MANAGEMEN T-PLANNING POSTATTACK-OPERATIONS RECOVEI PARM PROJECT FINAL REPJRT I DNMAGE-ASSESSMENT RE O5-O3-O2I 44 SOURCES MANAGEMENT-PLANNING POSTATTACK-OPERATIONS RECOVE/ PARM PROJECT FINAL REPJRT I DNMAGE-ASSESSMENT RE OS-03-02I 44
ITRODUCTION TO IHE POST-ATTACK RECOVERY PLANNING SYSTEM - PARM SYSTEM MANUAL I-A, B. PARM ILLUSTRATED I P/ OS-O3-02I3I MOOEL I RECOVERY ECONOMIC-RECOVERY DAMAGE-ASSESSMENT MOD/ PARM SYSTEM MANUAL III-B. PERSONAL CONSUMPTION OS-O3-O2ISI CATA I MOBIL IZATION MANAGEMENT-PLANNING RESOURCES INDUS/ PARM SYSTEM MANUAL III-C. CAPACITY CONCEPTS AND O5-D3-D2IL 2 ESTORATION AND CONVERSION I RE SOURCES MANPOWER GEOGRAPHY/ PARM SYSTEM MANUAL III-E. CAPACITY EXPANSION, R OS-OZ-O2ISQ NATION I RADIOACTIVE-FALLOUT RADIOLOGICAL-DOSE FARN-CROP/ PARM SYSTEM MANUAL III-F. FALLOUT AND DECONTAMI D6-IS-O22I7 ZE DISTRIBUTION, HOSPITALS, AND AGRICULTURAL RESOURCES / PARM SYSTEM MANUAL III-H. HOUSING, HOUSEHOLD SI D5-D3-D2IL5 ROTOTYPE MODEL I REPAIR RECOVERY DATA-PROCESSING MAINTEN/ PARM SYSTEM MANUAL III-K. COVSTRUCTION'IN THE P I3-I3-02282 ONS I DAMAGE-ASSESSMENT RE SOURCES VULNERABILITY MCCELS M/ PARM SYSTEM MANUAL III-O. MAVPOWEZ AVD OCCUPATI OS-03-02I53 DAMAGE-ASSESSMENT RESOURCES DATA-PROCESSING), \#NREC-I3 PARM SYSTEM MANUAL IV. RESOUZCE DATA ROUTINE I OS-D3-D2I29 ES 1 RESOURCES DAMAGE-ASSESSMENT DATA-PROCESSING PROGRAM/ PARM SYSTEM MANUAL V. FILE MAINTENANCE PROCEDUR OS-D3-D2ISL SOURCES CAPACITY DAMAGE-ASSESSMENT VULNERABILITY ECONOMI/ PARM SYSTEM MANUAL VI-2. PARM MAIN $20 U T I N E$ I RE O5-03-02152 IBIL I TY-STUDIES DESIGN THEORY EXPERIMENTAL-DATA NATERIALS PARTICAL-5IZE COSTS OPT ICAL-PZOPEZTIES COST-EFF/ I I-OI -02D42 /BIL I TY-STUDIES DESIGN THEORY EXPERIMENTAL-DATA NATERIALS PARTICAL-SIZE COSTS OPT ICAL-P ZOPEZTIES COST-EFF I I9-DI -02042 $K$-OPERATIONS SOCIETAL-RECOVI CONSLMER BEHAVIOR AND WORKER PARTICIPATION IN RECOVERY ACTIVITIES I POSTATIAC OS-IJ-02ISO
IS - SER IAL STUDIES ON. RHESUS MONKEYS EXPOSED TO A SMALL PARTICLE AEROSOL OF THE BORG STRAIN I BIOLOGICA/ IS-O2-OIBSO

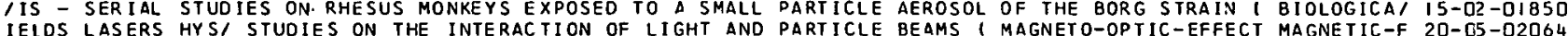
IELOS LASERS HYSI STUDIES ON THE INTERACTION OF LIGHT AND PARTICLE BEAMS I MAGNETO-OPTIC-EFFECT MAGNETIC-F 20-05-02064

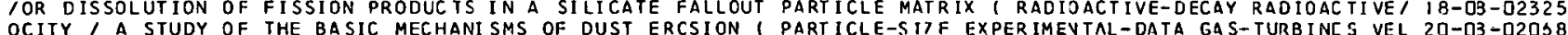
OCITY $I$ A STUDY OF THE BASIC MECHANI SMS OF DUST ERCSION I PART ICLE-SI7F EXPER IMEYTAL-DATA GAS-TURBINCS VEL $20-03$-D2OSB IALLOUT FORMATION I RADIOACTIVE-FALLOUT RADIOACTIVE-DECAY PART ICLE-SIZE HENRYS-LAW HALF-LIFE DISTRIBUTIONI IB-OB-O2O37 IRY-STRATEGY WEAPONS-EFFECTS RADIATION-EFFECTS POPULATION PARTICLE-SIZE SHELTERS DISTRIBUTION RADIOACTIVEI O6-2I-02229 LLOUT MATHEMATICAL-PREDICTION YIELO GEOMETRY DISTRIBUTI ON PARTICLE-SIZE WIND/ \#USNRDL-TRC-68/IOACTIVE-FA I8-D3-D2D39 IPART ICLES IN A TURBULENT GAS I TLRBULENCE FLUID-DYNAMICS PART ICLE-SIZE-DISTR IBUT ION AEZOSOLS ZEYNOLDS-NU/ 2D-OH-D2333 IS RADIOACTIVE-FALLOUT SHIELOING BUILDINGS SHELTERS COSTS PARTICLES, \$USNRDL-TR-IOS4/ONSTRULTION-MATERIA IB-DS-D232I Y BACTERIA GAS-FILTERS MICRO/ AIR FILTRATION OF MICROBIAL PARTICLES 1 BIOLOGICAL-WARFARE-AGENTS AEROBIOLOG IS-C2-DIBSI IUT ION OF FALLOUT AROUND STRUC TURES I RADI OACTIVE-FALLOUT PARTICLES DEBRIS VOLCAVOES COSTA-ZICA WIVD INST/ I8-D8-02038
CYNAM ICS PARTICLE-SIZ/ RELATIVE MOTION AND COAGULATION OF PART ICLES IN A TURBULEVT GAS I TURBULENCE FLUID- $20-0+-02333$ IION PRODUCTS IN RADIOACTIVE FALLOUT I DECAY-SCHEMES USSR PART ICLES SURFACE-BURST AIR-BURST EZUATIONS RAD/ I8-O3-D2323

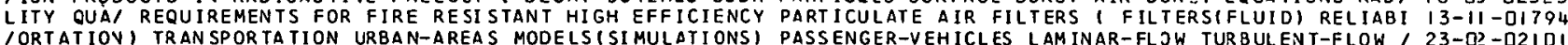
IORTATIOV TRANSPORTATION URBAN-AREAS MODELS ISIMULAYIONS) PASSENGER-VEHICLES LAMINAR-FLJW TURBULENT-FLOW I $23-02-02$ IDI N-I A SYSTEMS ANALYSIS OF SHORT HAUL AIR TRANSPORTATION 1 PASSENGER-VEHICLES NORTHEAST-CORRIDOR COSIS URBA $23-0 ?-02094$
FIGURATION URBAN-PLANNING EFFICIENCY SEL/ URBAN FORM AS A PASSIVE DEFENSE VARIABL 1 COSTS UZBAN-AREAS CON I3-D?-O2256 FIGURATION URBAN-PLANNING EFFICIENCY SEL/ URBAN FORM AS A PASSIVE DEFENSE VARIABLE 1 COSTS UZBAN-AREAS CON I3-D?-O2256
CECENTRAL IZATION VULNERABILITY LAND-USE/ URBAN FORM AS A PASSIVE CEFENSE VARIABLE I ECJNOMICS URBAN-AREAS $23-05$-D2IOT CECENTRAL IZATION VULNERABILITY LAND-USE I URBAN FORM AS A PASSIVE CEFENSE VARIABLE I ECJNOMICS URBAN-AREAS $23-0 S$ - D2I I 7
ITH AMER ICAN AIR DEFENSE COMMAND AT CHEYENNE MOUNTAIN, EL PAST COUNTY, COLORADO I CONSTZUCTIOV UNDERGROUN/ I3-I3-O2274 ITH AMER ICAN AIR DEFENSE COMMAND AT CHEYENNE MOUNTAIN, EL PAST COUNTY, COLORADO 1 CONSTZUCTIOV UNDERGROUN/ I3-I3-02274
ULTURE-MEOIA GROWTH/ SELECTIVE MEDIA FOR THE ISOLATION OF PASTEURELLA PEST IS I BIOLOGICAL-WARFARE-AGENTS C IS-D?-OI8TS ULTURE-MEDIA GROWTH SELECTIVE MEOIA FOR THE ISOLATION OF PASTEURELLA PESTIS I BIOL OGICAL-WARFARE-AGENTS C IS-D2-0I875
$\mathrm{N} I /$ EFFECT OF AEROSOL AGE ON THE INFECTIVITY OF AIRBORNE PASTEURELLA TULARENS IS FOR MACACA MULATIA AND MA IS-D?-OI85B IIGNIFICANCE AS A PROBLEM FOLLOWING A IHERMONUCLEAR WAR 1 PASTEURELLA-PESTIS BIOLOGICAL-WARFARE-AGENTS EP I OO-OJ-OITO2 IAL POPULATIONS I BACILLUS-ANTHRACIS MATHEMATICAL-MODELS PASTEURELLA-TULAREVSIS BACTERIA SPJRES STORAGE I IS-O?-OIBU9 
QUIPMENT COVTROL-SYSTEMS TRANSFORNERS COILS CIRCUITS USSR PATENTS, \#FTD-TT-65-I169 \#AD 6 I 576 /IGHTING-E D9-OJ-QI756 F ANTHRAX SPORE AND CELL 1 BIOLOGICAL-WARFARE-AGENTS BAC/ PATHOGENESIS AS RELATEO TO PHYSIOLOGICAL STATE O I5-D2-OI854 1 BIOLOGICAL-WARFARE-AGENTS PATHOLOGY LUNGS SPORES, \#AD/ PATHOGENESIS OF COCCIOIOIDES IMMITIS IN MONKEYS I5-D2-OI852 RHESUS MONKEYS EXPOSED TO A SMALL PARTICLE AERCSCL O/ THE PATHOGENES IS OF PSITTACOSIS - SFRIAL STUDIES ON I5-D2-DIB5O RHESUS MONKEYS EXPOSED TO A SMALL PAR ITCE AERCSCL O/ THE PATHOGENESIS OF PSITTACOSIS - SFR IAL STUDIES ON IS-D2 -DI850

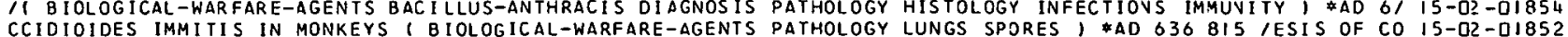
ENCY-CISEASES TISSUES(BIOLOGY) TESTES RATS MORPHOLCGI THE PATHOLOGY OF ZINC DEFICIEVCY I METABOLISM DEFICI OG-J5-DI7OI TIONS WITH TOXIC AND RADIOLOGICAL AGENTS - USSR I RADIOL/ PATHOLOGY, CLINICAL PICTURE AVD THEZAPY IN AFFEC OG-I3-OI72I E 1 ANALYSIS NUCLEAR-WARFARE-CASUALTIES DAMAGE-ASSESSMENT PATIENTS PROGRAMMIVGICJMPUTERS) I $\$$ VREC-72 IIMAT O6-21 -D2225 IN FALLOUT IN EMERGENCY HEALTH SERVICES UNITS I SHIELDING PATIENTS RADIOACTIVE-FALLOUT GAMMA-RAYS RADIATI/ I8-OS-D2O28 FIRES I INFRARED-DETECTORS INFRARED-TRACKING FOREST-FIRES PATROL-PLANES AIRCRAFT) JINT-25 /AND MEASURING I3-I?-DI79B ATTACK-SCENARIOS COMPUTER S HOLSING PR I ATTACK I - ATTACK PATTERN GENERATOR I SIMULATIOV DA YAGE-ASSESSMENT OS-OZ -02I 39 IENERGY I PUBLIC-OPINION AITITUDES SURVEYS SUESTI ONNAIRES PEACE-KEEPING NUCLEAR-HARFARE ARMS-EONTROL I *AI O5-II -O2I 96 AIRS PUBL IC-OP UN ITED NATIONS ORGANI ZATION PREVENT WARS I PEACE-KEEPING PUBL IC-OPINIONE AN TERNATIONAL-AFFAI OS-II -D2I IS OF SLIDE

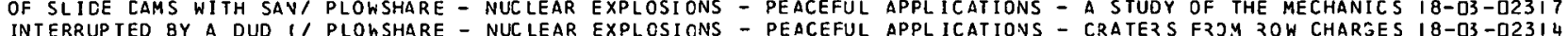

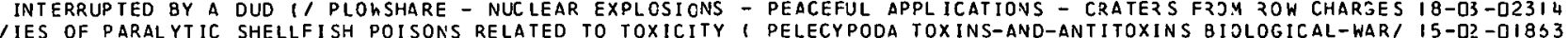
IIES OF PARALYTIC SHELLFISH POISONS RELATED TO TOXICITY
H-PROGRAM-ADMIN / KEYSTONE CORRIDOR TRANSPORTATION STUDY I PENNSYLVANIA HIGH-SPEED-GROUND-TRANSPORT RESEARC $23-02$-O2O92 H-PROGRAM-ADMIN KEYSTONE CORRIDOR IRANSPORTATION STUDY I PENNSYLVANIA HIGF-SPEED-GROUND-TRANSPORT RESEARC $23-02-02092$
THE ONLY THING WE HAVE TO FEAR I FEAR I TSELF - WHAT THE PECPLE KNOW AND THINK ABOUT THE BJMB AND ATOMIC OS-II -02I96 THE ONLY THING WE HAVE TO FEAR I FEAR ITSELF - WHAT THE PECPLE KNOW AND THINK ABOUT THE BJMB AND ATOMIC O5-I1 -02I96 I TABLES FOR YULES Q A SSOC. IATION C.REFFICIENT FCR PAIRS CF PERCENT AGES I STAT IST ICAL-AVALYSIS SJEIAL-SCIENC DS-II -O22OD NICAT ION-SYSTEMS MODELS COSTS COMNAND-AND-CONTROL-SYSTEMS PERFORMANCE(ENGINEER ING), \#SR I-MU-4949- 21 /MMU I 7-02-02313 ICS IN PER SONNEL SHEL TERS (FALLOLT-SHELTERS OPIINIZATION PERFORMANCEIENG INEER ING) PHYSIOLOGY COOLING-AND/ I3-I3-DI8I 9 ITESTS LOADING(MECHAN ICS) UNDERGROUND-STRUCTURES SHELTERS PERFORMANCE(ENG INEERING) STATICS STRUC TURAL-PAR/ I3-I3-O2290

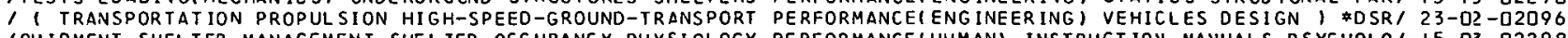
I OII IPMFNT SHFI TFR-MANAGFMFN T SHEL TER-OCCUPANCY PHYSIOLOGY PERFORMANCEIHUMAN) INSTRUCTIOV-MAVUALS PSYCHOLO/ I5-D3-D2298

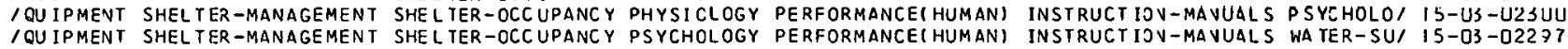
TROPICAL CLIMATE ( AGING (PHYSIOLOGY) AGE TROPICAL-REGIONS PERFORMANCE(HUMAN) STRESSIPHYSIOLOGY), *TR-35, O6-17-0I734 C-RESEARCH IVFORMATION-RFTRIEVAI SCIENTIFIC-ORGANIZATIONS PERIODICALS, \$TM-3008/004/00 \$AD 649637/NTIFI O5-02-02123 HUMITITY I \#AD 646 WATER IRANSFER THROLGH HUMAN SKIN I PERMEABILITY TRANSPORT-PROPERTIES SORPTION SALTS O6-1S-D22I 3 ECOVERY DAMAGE-ASSESSMEVT MOD/ PARN SYSTEN NANUAL III-B. PERSONAL CONSUMPTIOV MODEL I ZECOVE $Z Y$ ELONOMIC-R O5-O3 -D2I II INERAB IL ITY PROGRAMMING (COMPUTERS) CASUALTIES FAC IL IT IES PERSONNEL ATTACK-PATTERNS WIVD YIELO BURST-COND/ O5-D3 -D2I LO MENTAL-HEALTH POSTATIACK-OP I EMERGENCY SOCIAL SERVICES I PERSONNEL REHAVIOR CIVIL-OEFENSE-OPERATING-PLANS OS-II -O2IBS

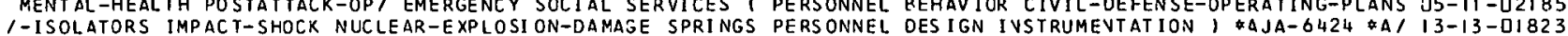
IMA-RAYS RADIATION-MONITORS RADIA TION-MEA SURENENT-SYSTEMS PERSONNEL DOS IMETERS DOSE-RATE RADIOLOGICAL-CON/ I 8-OS-D2O28 ER-MANAGEMEVT UNITE/ AN EVALUA TION OF THE ROLE OF FEDERAL PERSONNEL IN RECRUITING SHELTER MANAGERS I SHELT D5-OP-OIOTS HEL TER-OCCUPANCY POSTATTACT-OPERATIONS HOSPITALS TRAINING PERSONNEL LOGISTICS, \#FG-E-I3.4/D-DISPENSING S D6-D3-O22ID IENT FIRST-AID ORGANIZ4TION TRAINING HOSPITALS CASUALTIES PERSONNEL NUCLEAR-NARFARE-CASUALTIES I \#A.L. IO/ D6-2I - DI738 I LARGE SHELTERS \& SHELTER-MANAGE NENT MANAGE MENT-PLANNING PERS ONNEL SHELTER-OCCUPANCY (SIMULATIJY) LEADER S/ D5-OI - O16? I 1 ENTRAVCEWAYS AND EXITS FOR BLAST RESISTANT FULLY BURIEC PERSONNEL SHELTERS 1 BLAST-SHEL TEZS DESIGN COSTS I3-13-OIB35 ON PERTOR/ AN INVESTICATION OF MINIMAL EQUIPMENT NEEOS IN PERSONNFI SHFI.TFRS I FALLOUT-SHELTERS GPTIMIZATI I3-I3-DI8I9 EXPLOSIO/ AN INVESTIGATION OF MINIMAL EQUIPMENT NEEOS IN PERSONNEL SHELTERS I FALLOUT-SHELTERS PHYSIOLOGY I3-I3-OI8II

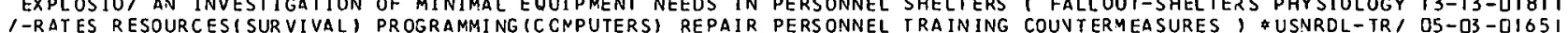
/-RAT ES RESOURCESI SUR VIVALI PROGRAMMING (CCNPUTERS) REPAIR PERS ONNEL TRA INING COUVTERMEASURES I OUSNRDL-TR/ O5-D3-DI65I

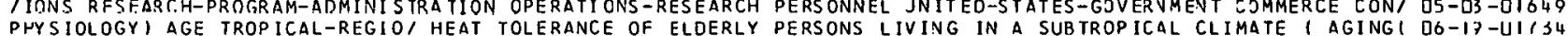

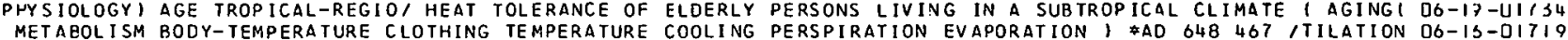
MET ABOL I SM BODY-TEMPERATURE CLOTHING TEMPERATURE COOLING PERSPIRATION EVAPORATION 1 \&AD O48 467 ITILATION D6- IS-DI7I9

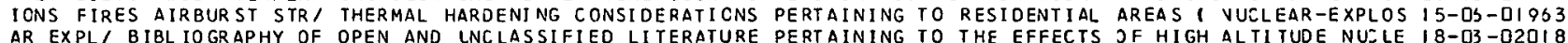
AR EXPL/ BIBL IOGRAPHY OF OPEN AND LNCLASSIFIEO LITERATURE PERTAINING TO THE EFFECTS OF HIGH ALTITUDE NUZLE I8-D3 -O2OIB SLES, INFLUEVZA 1 D/ DISEASE STATLS REPORTS - DI PHTHERIA, PERIUSS IS, TETANUS, POL IOHYEL I IIS, SMALLPOX, MEA I5-D? -DI869 OUT IN WASTE WATE, POSTATTACK SANITATION, WASTE DISPOSAL, PEST AND VECTOR CONTROL, AND THE EFFECTS OF FALL I3-D? -OI78O COUNTERMEASURES URBAN-AREAS SAN-JOSE CALIFORNIA RECOVERY PEST-CONTROL, \#USNRDL-TRC-79 /COVTROLI DRAINAGE I3-O2-OI78D -L. 996 FARM CHEMICALS HANDBCCK I FERTILIZERS PESTICICES EQUIPMEVT AGRICULTURAL-CHEMISTRY I \#A O2-OI -OIGIS GROWTH/ SELECTIVE MEDIA FOR THE ISOLATION OF PASTEURELLA PESTIS I BIOLOGICAL-WARFARE-AGENTS CULTURE-MEDIA IS-D?-DI8T5

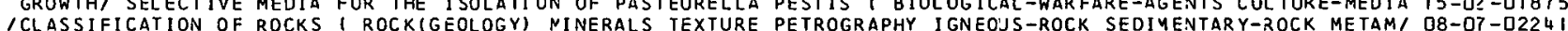

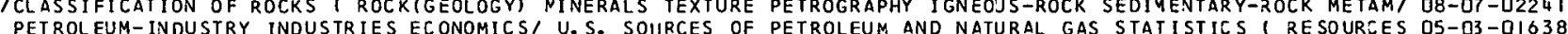
PETROL EUM-INDUSTRY INOUSTRIES ECONOMICS/ U.S. SOURCES OF PETROLEUM AND NATURAL GAS STATISTICS I RESOURCES O5-03-QI638
ILITARY-STRATEGY ECONOMICS PRODUC TION VULNERABILITY MANPI PETROLEUM INDUSTRY OF GER YAVY DURIVG THE WAR I M OS-DO-DIOLO ILIT ARY-STRATEGY ECONOMICS PRODUC TION VULNERABILITY MANP' PETROLEUM INDUSTRY OF GER YAVY DURIVG THE WAR I M OS-D3-OI 46

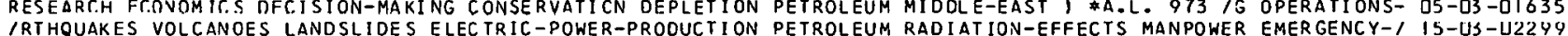
IRTHQUAKES VOLCANOES LANDSLIDES ELEC TRIC-POWER-PRODUCTION PETROLEUM RADIATION-EFFECTS MANPOWER EMERGENCY-I IS-US-U22Y9
OSTAT TACK -OPERATIONS RADIOACTI VE-FALLOUT CON/ RECOVERY OF PETROLEUM REFINERIES CONTAMINATED BY FALLOUT I P O5-O3-DIO5I

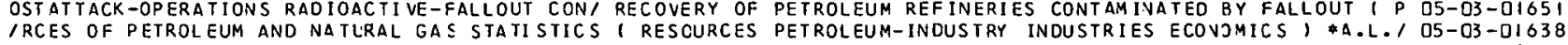
INETWORK STUDY I DAMAGE-ASSESSNENT MODELS NUCLEAR-WARFARE PETROLEUM-PIPEL INES GAS-PIPELINES TRANSPORTATIO/ D5-O3-O2I 1 I IEMFNTS, SIRIICTURFS, AND OPERATIONS RESEARCH II. DAMAGED PF MULTIPLIER I RADIOACTIVE-FALLOUT PROTECTION-I D6-I3-O222I IRFARE-AGENTS CLOSTRIDIUM-BOTULINLM TOXINS-AND-ANTITOXINS PHARMOCOLOGY MUSCLES MONKEYS CHICKENS DOSAGE I I IS-UU-UIBOI

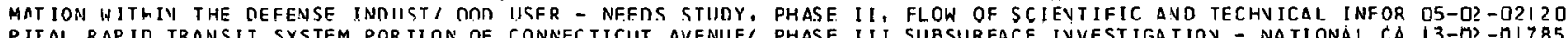
PITAL RAPID TRANSIT SYSTEM PORTION OF CONNECTICUT AVENUE' PHASE III SUBSURFACE IVVESTIGATIOV - NATIONAL CA I3-D? -OIT85 ILOW-FREQUENCY VERY-LOW-FREQUENCY EXTREMELY-LOW-FREQUENCY PHASF TRANSFORMATIONS (MATHEMATICS) ELECTRON-DEN/ $20-I+-02348$ /AR-EXPI DSIONS WAVF-TRANSMISSION ATMOSPAERE LOW-FREQUENCY PHASE VERY-LOW-FREQUENCY, EXTREMELY-LOW-FREQUEN/ 2O-IL-O2349

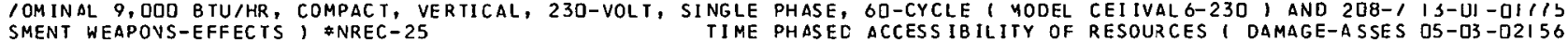

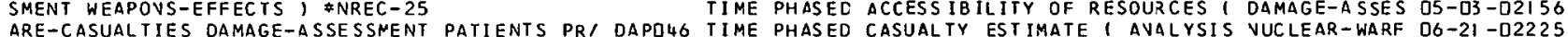
ARE-C ASUALTIES DAMAGE-ASSE SSMENT PATIENTS PR/ DAPQ46 TIME PHASED CASUALTY ESTIMATE 1 AVALYSIS VUCLEAR-WARF O6-21 -02225 BELTWAYS TRAFFIC OESIGN COSTS NEW-YORK CHICAGO WASHINGTON PHILADELPHIA BOSTOV LONDON $\$$ \$HI-82D-D 1 MOVING- $23-02-02097$ ENTERS - ASIA I BIBL IOGRAPHIES CHINA INDIA JAPAN PAKISTAN PHILIPPINES I \#EXTERNAL RESEARCH LIST 2.25 /IC C O5-II -OI698

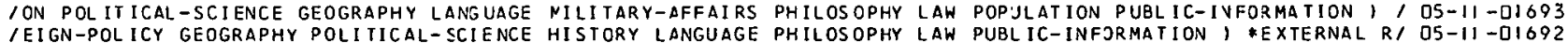
INTERNATIONAL CONGRESS I SYMPOSIA/ LOGIC, METHODOLCGY ANO PHILOSOPHY OF SCIEVCE - PROCEEDINGS OF THE I964 I2-OI -022SO IGRAPHY POLITICAL-SCIENCE HISTORY LANGUAGE LAW LITERATURE PHILOSOPHY POPULAT ION PROPAGAYOA PUBLIC-INFORMA/ OS- II -OIGQ 7 IOL IT ICAL-SCIENCE HISTORY LANGLAGE LAW PUBLIC-INFCRNATION PHILOS OPHY RELIGIOV I \#EXTERNAL RESEARCH LIST 6/ OS-II -DI696 /IAT ION-MONITORS DOSIMETERS PROTECTIVE-C LCTHING GAS-MASKS PHCSGENE INCENDIARY-GELS FLAME-THRJWER-FUELS SM/ D6-I3-0I72I

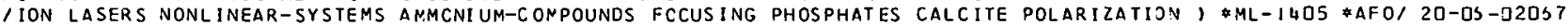

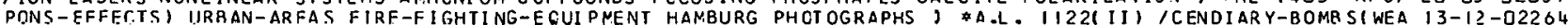
OUT FIELDS REAL AVD TWO SIMULA TED FAL I5-05-D1954 IIVE-FALLOUT ATTENUATION UNOERGROLND-STRUCTURES DOSE-RATE PHOTONS SCATTER ING ALBEDO GAMYA-RAYS ANGLE-OF-I/ I8-DS-D2029 IOF BLAST AND SHOCK B IOLOGY AND PROBLEM AREAS IN RELATING PHYSICAL AND BIOLOGICAL PARAMETERS I BIOLOGICALI O6-2I -O2223 ITORY OF SELECTED SPECIALIZED INFORMATION SERVICES IN THE PHYSICAL ANC BIOLDGICAL SCIEVCESI 2 ESOURCESIIN/ OS-0? -OIG2O NS RELATED TO TOXICITY ( PELECYPODA TO/ SCME CHENICAL AND PHYSICAL PROPERTIES OF PARALYTIC SHELLFISH POISC I5-O? -OI8G 3 IIRECTORY OF INFORMATION RESOURCES IN THE UNI TED STATES - PHYSICAL SCIENCES, BIOLOGICAL SCIEVCES, ENGINEE/ OS-OZ - OIO2 7

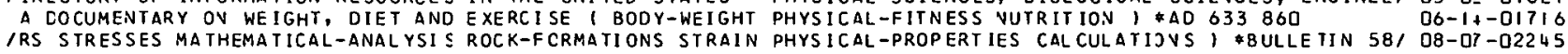
IL DEFENSE SHEL TER RATIONS I FCOD PRESERVATION FIBERBOARD PHYSICAL-PROPERTIES COVTAIVERS TASTE FALLOUT-SH/ D6-O3 -D22I 2 1661 BIOLOGY YEDICINE ECOLOGY RADIATION-EFFECTS GENETICS PHYSICS LANGUAGE PHYSIOLOGY MECHANICS 1 \#AD 646/ I2-OI-Q22 RES I RADIATION-INJURIES USSR RADI/ RADIOACTIVE FALLOUT - PHYSICS, EIOLOGICAL EFFECTS AVD PRJTECTIVE MEASL D6-I3-0I726 
TER VENTILATION I STRESS(PHYSIOLOGY) HEAT MOISTURE TEMPE/ PHYSIOLOGICAL ANC METEOROLOGICAL ASPECTS OF SHEL I3-DI -02253 O PROCEDURES II. LABORA' PSYCHOLCGICAL, ENGINEERING, AND PHYSIOLOGICAL EVALUATION OF SHELTER EQUIPMENT AN IS-O3 -O2297 D PROCEDURES III. HABIT/ PSYCHOLOGICAL, ENGINEERING, AND PHYSIOLOGICAL EVALUATION OF SHELTER EQUIPMENT AN I5-03-02298 C PROCEDURES I. SUMMARY/ PSYCHOLOGICAL, ENGINEERING, AND PHYSIOLGGICAL EVALUATION OF SHELTEZ EQUIPMENT AN IS-D3-O23OD BIOLOGICAL - WARFARE-AGENTS BAC/ PATHOGENESIS AS RELATED TO PHYSIOLOCICAL STATE OF ANTHRAX SPJZE AND CELL 1 I5-D? -QIB5 4 11 FALLOUT-SHEL TERS OPTIMIZATION PERFORMANCE (ENGINEERING) PHYSIOLOGY COOL ING-AND-VEVTILATION-EQUIPMENT SH/ I3-13-DIBI? 1 FAL IECT INDEXES I BACTER IA VIRUSES FLNG I PROTOZOA RICKETTSIA PHYSIOLOGY DIAGNOS IS
IEOUIPMEVT NEEDS IN PERSONNEL SHELTERS I FALLOUT-SHELTERS PHYSIOLCGY EXPLOSION-EFFECTS SANITARY-ENGINEERI/ I3-I3-OIBII IEQU IPMEVT NEEDS IN PER SONNEL SHELTERS I FALLOUT-SHELTERS PHYSIOLCGY EXPLOSION-EFFECTS SANITARY-ENGINEERII I3-13-018II I ANTS OF SURVIVAL SHEL TERS I SHEL TER-OCC UPANCY METABOLISM PHYS IOLOGY HEAT -EXCHANGE TEMPERATURE VENTILATIO/ O6-IS-DI7I 8

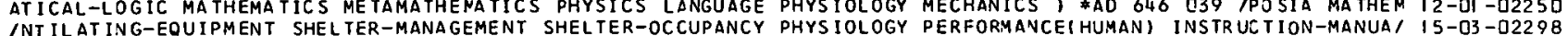
INT ILATING-EOUIPMENT SHEL TER-MANA GEMENT SHELTER-OCCUPANCY PHYSIOLOGY PERFORMANCEI HUMANI 1NSTRUCTION-MANUAI IS-O3-D2298 INT IL AT ING-EQUIPMENT SHEL TER-MANAGEMENT SHELTER-OCCUPANCY PHYSIOLOGY PERFORMANCE( HUYAN) INSTRUCTION-MANUA/ 15-O3-O23 OO IND PROCEDURES II LABORA TORY STLDIES I FALLOUT-SHELTERS PHYSIOL.CGY SYSTEMS-ENGINEER IVG COJLIVG-AND-VENT/ IS-D3-O2297 RACIOLOGICAL AGENTS - USSR I RADIOL/ PATHOLOGY, CLINICAL PICTURE AND THERAPY IN AFFECIIONS WITH TOXIC AND DG-I3-0I72I ENSE-SYSTEMS, AMP-6 CIVIL DEFENSE MOTION PICTURE CAIALOGUE I PUBLIC-INFORMATION CIVIL-OEF I5-D3 -O23O4 ICTS BLAST-BIOLOGY SHOCK-WAVES PRESSURE-TIME-MEASUREMENTS PIEZOELECTRIC-TRANSOUCERS LIVESTOCK-SALVAGE SHO/ O6-2I-D2237 INGS IN COMPETENT ROCK ( MINING-ENGINEERING ROCK (GEOLOGY) PILLARS STRESSES MATHEMATICAL-ANALYSIS ROCK-FOR/ D8-OT-O2245 IONSE OF BURIED CYLINDERS, CRI IICAL LI TERATURE REVIEW AND PILOT STUCY I SHELTERS UNDERGROLND-STRUCTLRES E I I3-I3-DIB2S S NUCLEAR-WAR FARE PETROLEUM-PI PELINES GAS-PI PELINES TRAN/ PI PELINE NETWORK STUDY I DAMAGE-ASSESSMENT MODEL O5-C3-O2I 1 I TS SIGNIFICAVCE AS A PROBLEM FOLLOWING A THERMCNUCLEAR W/ PLAGUE IN THE UNITED STATES - AN ASSESSMENT OF I OG-OS-OI7D2

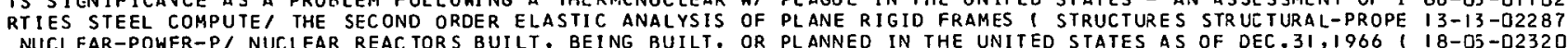

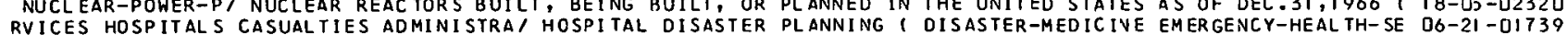
OL IT I CAL-SCIENCE GOVERNMENT(FOREIGN), TOWARD A FUTURE FOR PLANNING! MANACEMENT-PLAVVING DECISION-MAKING P O5-O + -OIG5O OL II ICAL-SCIENCE GOVERNMENT(FORE I GN) TOWARD A FUTURE FOR PLANNING MANACEMENT-PLAVVING DECISION-MAKING P O5-O 4 -OI659 IIEW OF FEDERALLY SPONSORED RE SEARCH RELATED TO EMERGENCY PLANNING ISCIENTIFIC-RESEARCH BLAST FIRES UNITI I5-O3-O22OQ POST, THE IMPORTANCE OF INOIVIDUAL INDUSTRIES FOR DEFENSE PLANNING I SUPPLY-AND-DEMAYD ECONOMICS RECOVERY DS-O3-OIG4 7 MANAEEMENT-PLANN ING OPERATIONS-RESEARCH ECONCNICS DECIS/ PLANNING IN A CAPITAL SURPLUS ECOVOMY - KUWAIT I OS-O3-OIG35 EL-THEORY STRATEG IC-WARFARE MILI TARYI STRCP - A STRATEGIC PLANNING MOOEL 1 GAME-THEJRY VUCLEAR-WARFARE MOD IS-0S-OI959 IES / COST-EFFECT IVENESS MANAGEMENT-EN/ COST ANALYSIS FOR PLANNING PROGRAMMIVG BUDGETING COST BENEFIT STUD O5-OI -02292 M ILLUSTRATE/ AN INTRODUCTION TO THE POST-ATTACK RECOVERY PLANNING SYSTEM - PARM SYSTEM MANUAL I-A,B. PAR O5-03-0213I INAGEMENT, DEVELOPMENT, TECHNICAL AND SCIENTIFIC SUPPORT, PLANNING, STUDIES AND ANALYSES 1 COMMUNICATION- $22-0 ?-02351$ LAT ION RAILROADS SUBWAYS COSTS MANAGEMENT-P/ RAIL TRANSIT PLANS 1 TRANSPORTAT ION NEW-YORK URBAV-AREAS POPU 23 -0? - D2D99 GEMENT-PLANNING URBAN-PLANNING) RPN-73-74 EXPRESSWAY PLANS ITRANSPORTATION URBAN-AREAS NEW-YORK MANA $23-02-02098$ IE STORM WATER BY STORM DRAINS ANO DRAINAGE CHANNELS II - PLANS, PROFILES, CROSS SECTIONS, AND GENERAL LOI I3-O2-OI779 IE STORM WATER BY STORM DRAINS AND DRAINAGE CHANNELS II. PLANS, PROFILES, CROSS SECTIONS, AND GENERAL LO/ I3-DZ - II78I CTIVE-FALLOUT FOOD CONTAMINAT/ UPTAKE OF RADICNUCLIDES BY PLANTS I AGRICULTJRE RADIOACTIVE-ISOTOPES RADIOA DO-I3-DI725 LOGY RADIATION-DAMAGE RADIOLOGICAL-CONTANINATION INSECTS PL ANTSIBOTANY) RADIOACTIVE-FALLOUTISIMULATION), O6-I3-DI723 IMODELS (SIMULAT IONS) RADIOBIOL OGY RADIATION-HAZARDS SOILS PLANTS (BOTANY) RADIOLOGICAL-CONTAMINATION) \#SR/ DG-I3-DI725 AL-WARFARE-AGENTS MIYAGAWANELLA-PSITIACI CULTURE-MEDIA II PLDQUE FORMAT ION BY PSITTACOSIS VIRUS I OIOLOGIC IS-OZ-DIBOI WL PLASMA-MEDIUM PLASMA-OSCILLATIONS STABILITY BOUNCARY-VI PLASMA STREAMING IVTO A MAGNETIC FIELO I GAS-FLO $20-09-02335$ II AT ION BEAMS(ELECTROMAGNETIC) LASERS HELIUM-GROUP-GASES PLASMA-MEDIUM LUMIVESCENCE ARGON VEOV I \#UCRL, 2O-OJ-O2OSO CIZATYON ISTREAMING INTO ATEAING INTO A MAGNETIC FIELD T GAS-FLOW PLASMA-MEOIUM PLASMA-OSCILLATIONS STABILITY BOUN 2O-OP-O2335 FLU 1/ RESEARCH A MAGNETIC FIELD I GAS-FLOW PLASMA-MEOIUM PLASMA-OSCILLAT ICNS STABILITY BOUVDARY-VALUE-PR/ 20-D7-O2335 IK EFFECTS II. APPLICATION OF GENERALIZED COCRDINATES TO PLASTIC WAVE PROBLEMSI SJIL-MECHAVILS MECHANICI O8-I3-DI747 TION ELASTICITY NORTHEAST-CORRIDOR COMPUTERS (PROGRAMMING) PLASTICITY, \#R6Q-53/ION DEFORMATION TRANSPORTA $20-I I-02034$ /STAT IC PRESSURE ( STIFFENED-CYLINOERS TENSILE-PROPERTIES PLASTICITY STRUCTURAL-SHELLS RINGS HELOING STRE/ $20-11$-0234 7 VES UNOER GROUNC-EXPLOSIONS ELASTICITY EQUATIONS-OF-MOTION PLASTICITY THEORY I \#DASA-1676-II \$AD 637 197 /A Q8-13-0I747 ORY ELECTROY-DENSITY CERENKOV-RADIATION MICROWAVES OXIDES PLASIICS । \#IBMRI-I295.3-66*AD 642 27I/IC-THE 20-03-[I2330 OPERATION BUSTER - THERMAL RADIATION EFFECTS CN PAINTS, PLAST ICS, AND COATEO FABRICS $\$$ WT- 407
GIFRACTURINGI/ AN EXPERIMENTAL INVESTIGATION OF FRANGIBLE PLATE FRAGMENTATION I LOADINGIMECHANICS) CRACKIN $20-11$-D2338 G(FRACTUR ING)
IOGRAM TO CAL CULATE THE THERMAL HISTORY OF A DUAL LAYERED PLATE SUBJECT TO THE THERMAL PULSE OF A NUCLEAR/ I3-I?-OI T97 IOGRAM TO CAL CULATE THE THERMAL HISTORY OF A DUAL LAYERED PLATE SUBJECT TO THE THERMAL PULSE OF A NUCLEAR/ I3-I?-OI797
TRESSES THRUST / LARGE DEFLECTIONS OF CLANPED RECTANGULAR PLATES ( PANELS (STRUCTURAL) RECTAVGULAR-BODIES S 20-II -O234 4

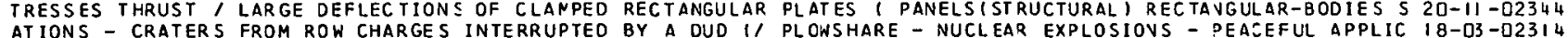

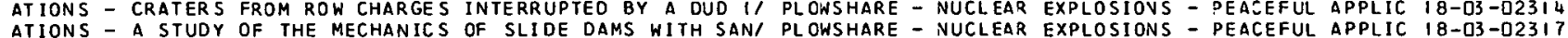
ATIONS - A STUDY OF THE MECHANICS OF SLIDE DAMS WITH SAN/ PL OWSHARE - NUCLEAR EXPLOSIONS - PEACEFUL APPLIC I8-03-02317

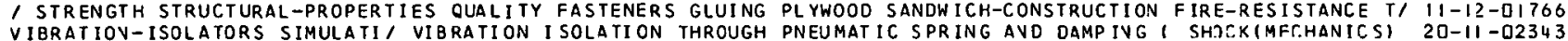

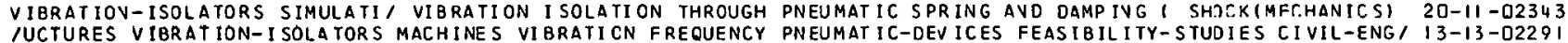
IUCTURES VIBRATION-I SOLA TORS MACHINES VI BRATICN FREQUENCY PNEUMATIC-DEVICES FEASIBILITY-STUDIES CIVIL-ENG/ I3-13-0229I /STUDIES OF EGYPT, ISRAEL, AND THE UNI TED NATIONS AT FIVE POINTS IN TIME I IVTERNATIONAL-AFFAIRS POLITICA/ OS-O+-D2I7O /-INJURIES TOXICITY CYANO-ACIDS CARBON-MONOXIDE VESICANTS POISONOUS-GASES LEWISITE GAS-DETECTORS RADIATIOI OG-I3-OI72I 1 CHEMICAL AND PHYSICAL PROPER TIES OF PARALYTIC SHELLFISH POISONS RELATED TO TOXICITY 1 PELECYPJOA TOXINS/ IS-D?-OI833 INAT ION 1 IOVOSPHERE OXYGEN GAS-IONI ZATION, AIRBURST IONS POLAR-REGIONS ABSORPT ION OZONE REALTION-KINETICI OL -OI -O2II 3

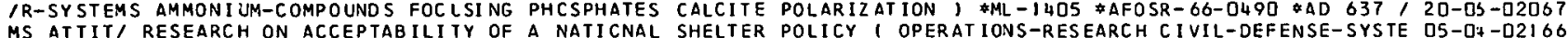
ANNING UNITED-I UTILIZATION OF SOCIAL RESEARCH IN SHAPING POLICY DECISIONS I SOCIAL-SCIENCES MAVAGEMENT-PL OS-OL-DIG58 CISEASE STATUS REPDRTS - DIPHTHERIA. PERTUSSIS. IETANUS, POLIOMYELIIS, SMALLPOX, MEASLES, IVFLUENZA I DE I5-DI-OIBS

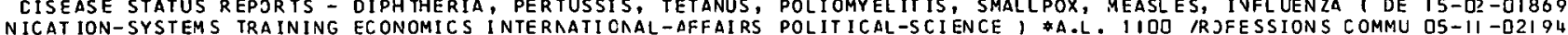
NICATION-SYSTEMS TRAINING ECONOMICS INTERAATIONAL-AFFAIRS POLITICAL-SCIENCE, \#A.L. 1100 IRJFESSIONS COMMU OS-1I-02I94 UNIST-CHINA HISTORY LAW INTERNATIONAL-AFFAIRS METHCOOLOGY POLITICAL-SCIENCE, \$ACD-5 /LICT-RESJLUTION COMM OS-OH-OIG65 LIT ION IAT I TUDES BEHAVIOR METHODOLOGY SEMANTICS SOCIAL-SCIENCES POLIIICAL-SCIENCE CULTURE COOPERATIOV I \&FAR-4D/ OS-IJ-D2178 IAL-SCIEVCES MANAGEMENT-PLANNING INI TED-STATES-GOVERNMENT POLIT ICAL-SCIENCE ECONOMICS DECISION-MAKING LAW/ O5-O+-DIG5B IOGY FOR ASSESSING TOTAL VULNERABILI TY I SYSTEMS-ANALYSIS POLITICAL-SCIENCE ECONOMICS SJCIOLDGY SOCIETAL-I OS-II -O2I 33 1-PSYCHOLOGY AGING OCCUPATIONS PROFESSIONS MANPOWER LABOR POLITICAL-SCIENCE EDUCATION FROIIP-DYYAMICS THEOI DS-II -O2I 7 I EMENT-PLANVING DECISION-MAKING OBJECTIVES PUBLIC-CPINION POLIT ICAL-SCIENCE EXECUTIVE-BRANCH LOLAL-GOVERN/ O5-Ot-DIG7I OF ECONOMIC ADVISERS I ECONOMICS CNI TED-STATES-GOVERNMENT POLITICAL-SCIENCE FOREIGN-POLICY) \$A.L. IOGI, OS-03-DI634

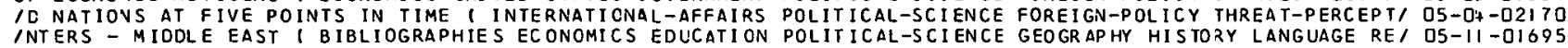
/CULTURAL-ECONOMICS FINANCE INOUSTRIES CONMERCE EDUCATION POLITICAL-SCIENCE GEOGRAPHY LANGUAGE MILITARY-A/ OS-II -OIOQ3 IUTURE FOR PLANNING I MANAGEMENT-PLANNING DECISI ON-MAKING POLITICAL-SCIENCE GOVERNMENT(FOREIGN) SOCIAL-CO/ O5-OH-OIG59 INISM ECONOMICS EDUCATION INTERNA TIONAL-AFFAIRS GECGRAPHY POLITICAL-SCIENCE HISTORY LAVGUAGE LAW LITERATU/ OS-II -DIG 7 IS COMMUV ISM ECOVOMICS EDUCATI ON FORE IGN-POLICY GECGRAPHY POL IT ICAL-SCIENCE HISTORY LAVGUAGE LAW PUBLIC-II OS-II -DIG96 INOMICS $8 I$ IBLIOGRAPHIES EDUCATI ON FOREIGN-POLICY GECGRAPHY POLITICAL-SCIENCE HISTORY LANGUAGE PHILOSOPHY LI OS-II -DIG 2

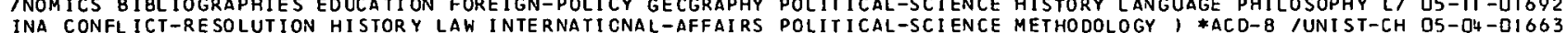

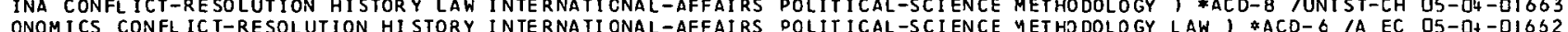
ONOMICS CONFLICT-RESOLUTION HISTORY INTERNAIIONAL-AFFAIRS POLITICAL-SCIENCE METHODOLOGY LAW I \$ACD- 6 /A EC OS-D+-DI6S2

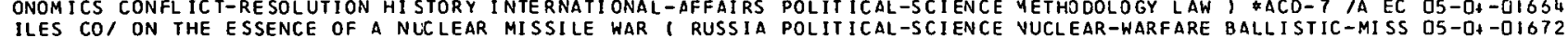
ILES CO/ ON THE ESSENCE OF A NLCLEAR MISSILE WAR I RUSSIA POLITICAL-SCIENCE VUCLEAR-WARFARE BALLISTIC-MISS O5-DH-DIG72
IE CIVIL DEFENSE DEBATE I CIVIL-DEFENSE-SYSTEMS ATIITUDES POLITICAL-SCIENCE PUBLIC-OPINION HISTORV COSTSI/ OS-IJ-DIGT9 IISM IN ASIA I ECONOMIC-CONDITIONS POPULATION AGRICULTURE POLITICAL-SCIENCE REGIONAL-PLANNIVG IVDIA INTER/ D5-D3-O2I GI I FACTORS AFFECTING GOVERNMENT CONTROL IN SOUTH VIETNAM I POLITICAL-SCIENCE SOCIOLOGY ECONOYICS, *RM-5I8, QS-OF-O2I75 IATTACK I SOCIETAL-RECOVERY RECOVERY DEMOGRAPHY ECCNOMICS POLIT ICAL-SCIENCE STRESSIPSYCHOLOGY) METHODOLOG/ OS-II -O22J2 E-L IMITING-SYSTEMS DISPERSAL CHINA EVACUATION URBAN-AREAS POPULATICN, \#HI-777-RR, IL-DEFENSE RUSSIA DAMAG I5-O3-DI896 SSR CIVIL-DEFENSE-SYSTEMS SHEL TERS / PROTECTING THE RURAL POPULATIGN AGAINST RADIOACTIVE COVTAMINATION I U DG-IB-OIT3I 
IFEAS IBLE PRODUCT ION I VUCLEAR-WARFARE SURVIVAL ECCNOMICS POPULATICN AGRICULTURE INDUSTR IES IVDUSTRIAL-PR/ OS-D3-O2I58 L-PLANNIVG IVD/ REGIONALISM IN ASIA I EC CNCMIC-CONCITIONS POPULAT ION AGRICULTURE POLITILAL-SCIENCE REGIONA OS-D3 -D2IGI OMMERCE MEAT RE / HANDBOOK OF AGRICULTURAL CHARTS - 1966 I POPULATION AGRICULTURE URBAN-AREAS RURAL-AREAS C O2-D2-0I6I 9 CLEAR WEAPONS I USSR SHEL IERS IN / EVACUAIION OF THE URBAN POPULATION AS A MEASURE OF PRJTECTIOV AGA INST NU I5-O3-OI934 TERABILITY POPULATION CASUALT IES FOOD-SUPPLIES GEOGRAPHY I/ O5-D3-02132 作 ULAT IONS DAMAGE/ CIVIL DEFENSE SHELTER REPRESENTATION FOR POPULAT ION COMMAND-ANO-COVTROL-SYSTEMS TABLES R/ I5-O3 -JI9J8

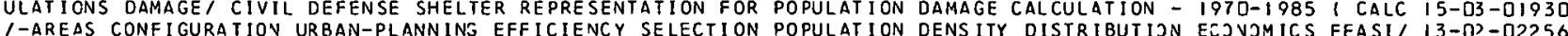
1-AREAS CONFIGURATIOV URBAN-PLANNINS EFFICIENCY SELECTION POPULATION DENSITY DISTRIBUTIJN EEJVJMICS FEASI/ I3-02-022S6 1-CHINA THREAT-EVALUATION MILI TARY-FACILIIIESIPROTECTICN) POPULATICN CETERREVCE DAMAGE-LIMITIVG-SYSTEMS CI I5-D3-O2294

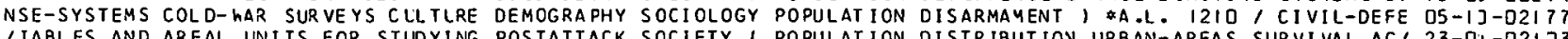
IIABLES AND AREAL UNITS FOR STUDYING POSTATTACK SOCIETY I POPULATION DISTRIBUTIOV URBAN-AREAS SURVIVAL AG/ $23-04-021$ I 3 TION OF THE UVITED STATES BY AGE, SEX, AND COLOR TC 1990/ POPULATION ESTIMATES - PROJECTIONS JF THE POPULA $23-0 ;-02358$ IRACIOACT IVE-FALLOUT RADIOLOGICAL-CONTAMINATICN RESOURCES POPULATION FACILITIES DECOVTAYINATIOV MATHEMATI, I5-OS-O23J7 TAL IT IES I FIRES LRBAN-AREAS DAMAGE-ASSESSNENT CASUALTIES POPJLATION FIRE-STORMS / *DC-TN-1050-3, FIRE FA I3-I?-02262 /RS HOUSING PROGR AMMING (COMPUTERSI MILITARY-INSTALLATIONS POPULATION GOVERNMENT COMMUNICATIOVS MILITARY-PI DS-D3-D2I 39 I-PROPERTIES EL EC TRIC-POWER-GENERATION MILITARY-PERSCNNEL POPULAT ION HOSPITALS SCHOOLS FOOD-SUPPLIES WATE 1 O5-O3-D2I 37 ITHE CEMOGRAPHY OF NUCLEAR WAR I SURVIVAL NUCLEAR-WARFARE POPULAT ION METHOCOLOGY MAVAGEYENT-PLLANING POSTI 23 -O - - 2I TU MOBILITY ON THE LOCATIJY OF COMMUNAL 15-03-01942 ENT NUCLEAR-EXPLOSION-DAMAGE AGE MODELSISI THE POSTATTACK POPULATION OF THE UNITED STATES I DAMAGE-ASSESSM IS-OS-DIQ65 COLOR TO $1990 /$ POPULATION ESTIMA TES - PRCJECTIONS OF THE POPULAT ION OF THE JNITED STATES BY AGE, SEX, AND $23-0+$-O2358 ITIES MIL ITARY-STRATEGY WEAPONS-EFFECTS RADIATION-EFFECTS POPULAT ION PART ICLE-SIZE SHEL TERS DISTRIBUIION I DO-2I -D2220 AS CECENTRALIZATION VULNERABILITY LAND-USE URBAN-PLANNING POPULAT ION POLITICAL-SCIENCE, \$PRC D-I34 I /-ARE $23-03-021$ ID 7 ITICAL-SCIEVCE HISTORY LANGUAGE LAh LITERATURE PHILOSOPHY POPULATION PROPAGAVDA PUBLIC-INFORYATION, *EXT/ OS-II-OIGQ7

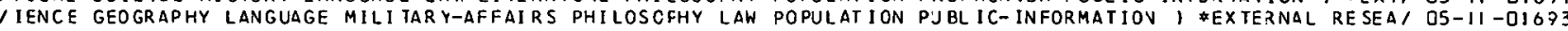
RAIL TRANSIT PLANS I TRANSPORTATION NEW-YORK URBAN-AREAS POPULAT ICN RAILROADS SUBWAYS COSTS MAVAGEMENT-PL 23 -0? -D2099 ITIVITY OF MORTAL ITY ESTIMATES TO VARIATIONS IN AGGREGATE POPULATION REPRESEVTATIONS I VUCLEAR-WARFARE-CA' IS-DT-QI966 ITIONAL-CIVIL - DEFENSE EARL Y-WARNING-SYSTEMS USSR MCVEMENT POPULAT ION RESOURCES EVACUATIJN UPBAV-AREAS DAMI IS-O3-OI922 1 -PLANNIVG SITE-SELECTION NATIONAL-FALLOUT-SHELTER-SURVEY POPULAT ICN RESOURCES URBAV-AREAS BUILDINGS SAN- 1 I3- 3 -OI838 / LABOR ECOVOMICS ECONOMIC-RECOVERY POSTATTACK-OPERATIONS POPULATION SURVIVAL DAMAGE-ASSESSMEVT CALIFORNI/ OS-D3-QI639 BY AGE, SEX, AND COLOR TO 1990 hITH EXTENSIONS OF TOTAL POPULATION T.D 2015 , DEMOGRAPHY TABLES, P-25-3/ $33-0+-02358$ INTRALIZATION TAXES VULNERABILITY URBAN-PLANNING LAND-USE POPULATION TRANSPORTATION UTIL ITIES FISCAL-AND-1 $23-05-D 2 I 06$

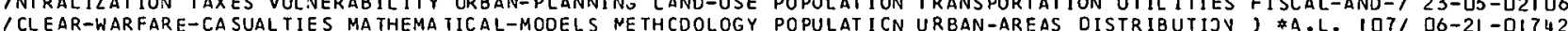

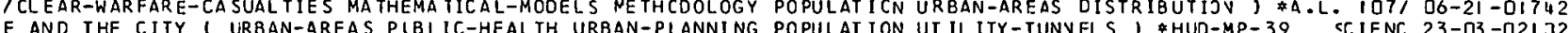

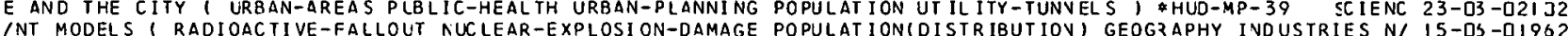
DELS/ VIABILITY AND ESTIMATION OF SHELF L

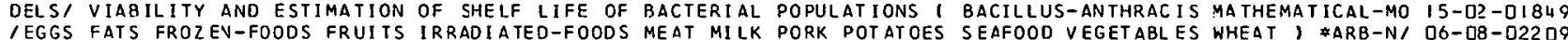

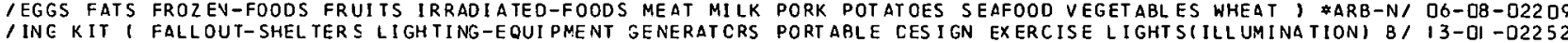
ATA HABITABILITY INSTRUMENTATI ON TEMPERATURE PUNKAH-PUMPS PORTABLE FANS, *GARD REPJRTI268-6I /RIMENTAL-D I3-OI -OI77L INVIRONMEVTAL-CONTROL EXPERIMENTAL-DATA HABITABILITY FANS PORTABLE INSTRUMEVTATION PUNKAH-PUMPS I \$GARO R/ I3-DI -DI?7I ICE INVESTIGATIOV - NATIONAL CAPI IAL RAPID TRANSIT SYSTEM PORTION OF CONNECTICUT AVENUE ROUTE BETWEEN COL/ I 3-OZ - OI785 ISIUN FORMULAS FOR USE HITII WORLD WIDE RE SOURCES GEOOETTR. POSITION I GP, YO UNIVERSAL TRANSVERSE MERCATO/ OS-D3 -O2I 33 EASIBIL ITY-STUD／ AN ANALYSIS OF MACHINE TCOL SUBSTITUTION POSST ION EM MANUAL IR-WEAPONS /R-WEAPOVS-EFFECTS MILI IARY-IN TELLIGENCE SYSTEMS-ANALYSIS POST-ATT ACK-OPERAT IONS DAMAGE-ASSESSMENT FIVE-C / $13-1$ ? - D226 7 CONSEQJENCES T GEVET ICS RADIATION-HA 2 O6-I3-01727 ERATIONS SURVIVAL STORAGEIFOODI PRODUCT/ NUTRITION IN THE POSTATTACK ENVIRONGENT I FODD DIET POSTATTACK-OP DG - IS -OITI EASES VACCINES RADIOB/ THE PROBLEN OF TUBERCULOSIS IN THE POSTATTACK ENVIROVMENT I RECOVERY INFECTIOUS-DIS O6-DJ-O22OG POST ATTACK-OPERATIONS FOOD-DI SPENSING DISTRIBUTION DAMAI POSTATTACK FOOD AVAILABILITY AND ACCESSIBILITY I OG-D8-OI7II
AGE-ASSESSMENT NUCLEAR-EXPLOSION-DAMAGE AGE MODELSIS/ THE POSTATTACK POPULATION OF THE UYIIED STATES I DAM IS-OS-OIOG5 AGE-ASSESSMENT NUCLEAR-EXPLOSION-DAMAGE AGE MODELSIS/ THE POSTATTACK POPULAT ION OF THE UVITED STATES I DAM IS-DS-DIO65
VIOUES I COUNTERMEA SURES POSTA TT/ AN APPRCACH TO DEFINING POSTATTACK RECOVERY MAVAGEMEVT COVLEPTS AND TECH IS-O3-DI953 NIQUES I COUVTERMEA SURES POSTATT/ AN APPRCACH TO DEFINING POSTATTACK RECOVERY MAVAGEMEVT COVCEPTS AND TECH IS-D3 - OI953 NIQUES I COUVTERMEASURES NUCLEAR/ AN APPROACH TO DEFINING POSTATTACK RECOVERY MAVAGEMENT COVEEPTS AND TECH I5-03 - D2293 ST DAMAGE-ASSESSMENT VULNERABILITY FIRES REPAIR RADIOACTI POSIATTACK RECOVERY OF DAMAGED URBAN AREAS I BLA IS-DS-O23O O/ THE FEASIBIL ITY OF DEVELOPING STANDARD DESCRIPTIONS OF POSTATTACK SITUATIOVS I SOCIETAL-ZESJVERY METHOD OS-II -02I 91 EARCH PROGRAM I POSTATTACK-OPERATIONS V/ CIVIL DEFENSE IN POSTATTACK SOCIETY - A SUMMARY REPORT FROM A RES OS-II -D22JI ION OF DEMOGRAPHIC VARIABLES AND AREAL UNITS FOR STUDYING POSTATTACK SOCIETY I POPULATION DISTRIBUTION UR/ $23-04-02 I O 3$ BILITY I SOCIETAL-RECOVERY ECON/ DIMENSI ONS OF SURVIVAL - POSTATTACK SURVIVAL CISPARITIES AVD VATIONAL VIA OS-D3-O2IOD

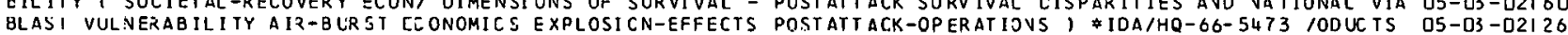

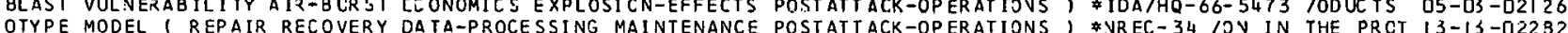
OTYPE MODEL I REPAIR RECOVERY DATA-PROCESSING MAINTENANCE POSTATTACK-OPERATIONS 1 \#VREC-34 $13 V$ IN THE PRCT I3-13-02232 $1 L$ UNITEO STATES I SYSTEMS-ENGINEERING ECONOMICS RECOVERY POSTATTACK-OPERATIONS DAMAGE-ASSESSMEVI RADIATI/ OS-O3-O2I 28 1 COVERY SOCIETAL-RECOVEQY NUCLEAR-WARFARE WEAPCNS-EFFECTS POST ATTACK-OPERATIOVS DISASTERS BEHAVIOR SOCIOL/ OS-II -02I9O / COVERY PRODUCTS VULNERABILITY EXPLOSION-EFFECTS AIRBURST POSTATTACK-OPERATIOVS ECOVOMICS, \&IDA/HQ-66-54/ O5-D3-O2IS2 ON DAMAI POSTATTACK TOOD AVAILABILITY AND ACCESSIRIITTY I POSTATTACK-OPERATIONS FOOD-DISPENSING DISTRIBUTI DG-D3-DITII TIS BIOLOGICAL-WARFARE-AGENTS EP IDEMI OL OGY PUBLIC-HEALTH POSTATTACK-OPERATIONS IMMUNOLJGY DISEASE-VEC TOR/ O6-D5-OI TO2 IPERATING-PLANS UTILITICS DATA WA TER.-SUPPLIES URRAN-ARFAS POSTATTACK-OPERATIONS YANAGEMEVT-PLAVVIVG RECOV/ I3-OZ-OITB8 SPECIAL PROBLEMS OF CHILDREN IN CIVIL DEFENSE PLANNING I POSTATTACK-OPERATIOVS MANAGEMEVT-PLANNING STKESS OS-II -O22J3 IERY MANAGEMENT CONCEPTS AND TECHNIQUES I COUNTERMEASURES POSTATTACK-OPERATIONS MANAGEMENT-PLANNING SURVII I 5 - D3-DI9S3 作 I COVERY OF PETROL EUM REFINERIES CONTAMINATED BY FALLOUT, POSTATTACK-OPERATIONS RADIOACTIVE-FALLOUT CONIA/ OS-D3-DIGSI ICOVERY OF PETROL EUM REFINERIES CONTAMINATED BY FALLOUT I POSTATTACK-OPERATIONS RADIOACTIVE-FALLOUT CONTAI OS-O3-DIG5I
HE EFFECTS OF TALLUT IN WASTE WATER AND SFWFR SYSTEMS I POSTATTACK-OPERATIONS RADIOACTIVE-FALLOUT WASTIE/ I3-DZ -OI7BD

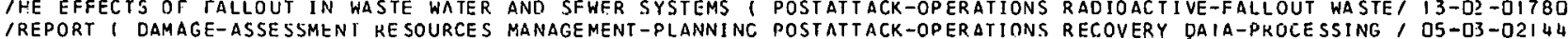

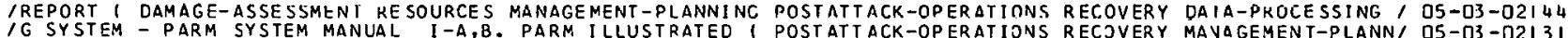

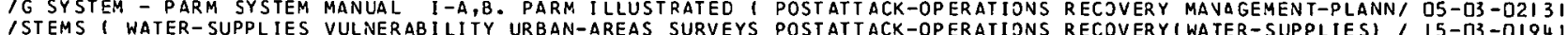
1-RADIATION RADIOLOGICAL-CONTAMINATI ON FIRES CONSTRUCTION POSTATTACK-OPERATIONS REPAIR MILITARY-ENGINEERI/ I3-I2-DIBO4 1 -SYSTEMS USSR RECONNAISSANCE INTERNATIONAL-CIVIL-CEFENSE POSTATTACK-OPERATIOVS RESCUES COVSTRUETION FIREI O6-D7-DITD9 I ING-SYSTEMS DAMAGE-A SSE SSMENT RECOVERY THREAT-EVALUATION POSTATTACK-OPERATIOVS RESOURCES MAVAGEMENT-PLAN/ I5-O3 -DI 449 1 DEFENSE PLANNING 1 SUPPLY-AND-DEMAND ECCNOMICS RECOVERY POSTATTACK-OPERATIONS RESOURCES SURVIVAL INDUSTI D5-D3-DIG4 7 ICLEAR-WARFARE POPULATION ME THODOLOGY MANAGEMENT-PLANNING POSTATTACK-OPERATIONS SEX AGE URBAY-AREAS DECISI 23 -OU-O2IOL

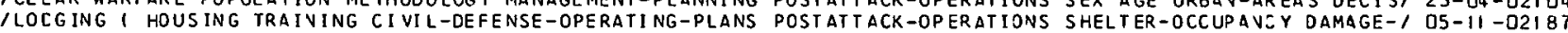
IC THERMONUCLEAR WAR - A CASE STUDY OF HIGHER EOUCATION I POSTATTACK-OPERATIOVS SOCIAL-ZECOVERY SUR VIVAL I O5-II-DIOB8 IERY METHOOOLOGY PROJECTIONS RECOVERY MANAGEMENT-PLANNING POSTATTACK-OPERATIONS SOCIAL-SYSTEMS STANDARD-SI OS-II -O2IQI I MAVIOR AND WORKER PARTICIPATION IN RECOVERY ACTIVITIES I POSTATTACK-OPERATIONS SOCIETAL-RECOVERY ECONOMI/ OS-IJ-O2IBO IEAR WAR AND SOIL EROSION - SOME PROBLEMS AND PROSPECTS I POSTATTACK-OPERATIONS SOIL-COYSERVATION SOIL-ME, OB-I3-OI745

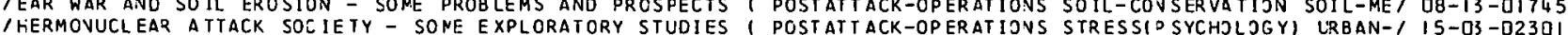
/FERMOVUCLEAR ATTACK SOCIETY - SONE EXPLORATORY STUDIES I POSTATTACK-OPERATIONS STRESSID SYCHOLOGY) LRBAN-I IS-O3-D23OI
/- THE LIMITS OF FEASIBLE PRODLC TION I ECONOMICS RECOVERY POSTATIACK-OPERATIOVS SURVIVAL ECOVOMIC-RECOVER/ OS-O3-D2127 1- THE LIMITS OF FEASIBLE PRODLC TION I ECCNOMICS RECOVERY POSTATTACK-OPERATIOVS SURVIVAL ECONOMIC-RECOVER/ OS-O3-D2127 DUCT/ NUTRITION IN THE POSTATTACK ENVIRONMENT I FOCD OIET POSTATTACK-OPERATIONS SURVIVAL STORAGE IFOODI PRO D6- IS-DI7I 7
IAEL REHAVIOR CIVIL-DEFENSE-OPERA IING-PLANS MENTAL-HEALTH POSTATTACK-OPERATIONS IRAINING CHILDREN GERIATR/ D5-1I -D2185 ICIETY 1 POPULATION OISTRIBUTION LRBAN-AREAS SURVIVAL AGE POSTAITACK-OPERATIONS URBAV-PLANVIVG METHODOLOG/ $23-0+$-O2IJ3 IACK SOCIETY - A SUMMARY REPORT FROM A RESEARCH PRCGRAM I POSTATTACK-OPERATIONS VUL VERAB ILITY OISASTERS S/ O5-II-D22JI
/STEMS - INTFRACTIONS AND COMPARI SONS AMONG MODES I CARGO POSTATIACK-OPERATIONS VULNERABILITY ROADS RAILR/ I3-OS-O226O 
/E-OPERAT ING-PLANS FOOD FOOD-DISPENSING SHELTER-OCCUPANCY POSTATTACK-OPERATIONS HOSPITALS TRAIVING PER SON/ OG-DS-O221D IFATS FROZEN-FOODS FRUITS IRRADIA TED-FOODS MEAT MILL PORK POTATOES SEAFOOC VEGETABLES WHEAT 1 \&ARB-NO. OT/ DG-OS-D22 OQ ER ECONO/ PRDJECTIONS 1970 - INTERINDUSTRY RELATIONSHIPS, POTENT IAL DEMAND, EMPLOYMEVT 1 INDUSTRIES MANPOW OS-D3-OIOL 9 HOTOGRAPHS URBAN-AREAS FIRES MAPS TESTI THE CCNFLAGRATION POTENT IAL IN SAN JOSE AND ALBUQUEZQUE I AERIAL-P I $3-1 ?-0 I 8 D 9$ ( URBAN-AREAS HASTY-SHELTERS CESIGN MAT/ TIME COMPRESSION POTENTIAL OF AN EMERGENCY BLAST SHELTER PROGRAM I3-I3-O2272 O THE PRES IDENT I NUCLEAR-REAC TORS NUCLI CIVILIAN NUCLEAR POWER - THE 1967 SJPPLEMEVT TJ THE I962 REPORT T I8-DJ-O2027 IONIFAILURE) ATTITUD/ PUBLIC RESPONSE TO THE NCRTHEASTERN POWER BLACKOUT I BEHAVIOR ELECTRIZ-PJWER-PRODUET O5-IJ-DI6B3 H GLASS OPTICAL-PROPERTIES RUBY CRY/ NRL PROGRESS IN HIGI POWER LASER RESEARCH I LASERS SCIENTIFIC-RESEARC 20-DS-D2O62 RICITY BUOYS GENERATORS POWER-SU/ IOD WATT THERMOELECTRIC POWER SYSTEM FOR BUOY APPLICATIONS I THERMOELECT IO-DZ-DI7OI RICITY BUOYS GENERATORS POWER-SU/ IOQ WATT THERMOELECTRIC POWER SYSTEM FOR BUOY APPLICATIONS 1 THERMOELECT IO-D2-DI76I

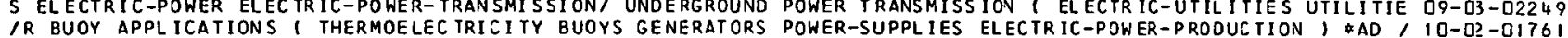
IR BUOY APPL ICATIONS I THERMOELEC TRICITY BUOYS GENERATORS POWER-SUPPLIES ELECTRIC-POWER-PRODUCTION I \#AD I IO-D?-OI7GI 1 TEMS COSTS UNDER GROUND-STRUC TLRE S ELECTRIC-CABLES DESIGN POWER-TRANSFORMERS FAULT-LOCATION L. IGHTNING-ARR/ D9-03 - DI755

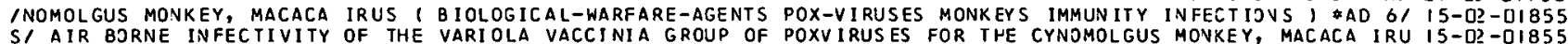
SI AIR BORNE INFECTIVITY OF THE VARIOLA VACCINIA GROUP OF POXVIRUSES FOR THE CYNOMOLGUS MONKEY, MACACA IRU IS-D? -OIB55
CLASSIFICATION OF MODELS I RAI AN ANALYSIS OF THE FALLOUT PREDICTION MODELS I. ANALYSIS, COMPARISON, AND I8-D3-O2O39 108 , AIR CONDITIONING UNITS A AR-CONDITIONING-EOUIPMENT PREFABRICATED-BUILDINGS MILITARY-ZEQUIREMENTS T/ 13 -OI -DI775 ICAL EXPER IENCES OF FIRE PROTECTION SERVICES - A CRITICAL PRESENTATION OF THE ORGANIZATION, TEEHNOLOGY, A/ I3-I?-D22S4 /CAL EXPER IEVCES OF FIRE PROTECTION SERVICES - A CRITICAL PRESENTATION OF THE ORGANIZATION, TELHNOLOGY, A/ I3-1?-O2235 ITORAGE STABILITY OF CIVIL DEFENSE SHELTER RATIONS $($ FOOD PRESERVATION FIBERBOARO PHYSICAL-PROPERTIES CON/ O6-D3-022I 2 SANDWICH-CONSTRUCTION FIRE-RESISTANCE THERMAL-INSULATION PRESERVATION FINISHES PAIVTS I \#HAVDBJOK NO. 72 II- 2 -DI766 EREALS / FOOD STORAGE LIFE - A REPORT BIBLIOGRAPHY I BEEF PRESERVAT ION SHELF-L IFE BEVERAGES BREAD CANNED C O6-D9-D22 O9 OMIC ADVISERS I ECONOMICS LNI TED- I ECONOMIC REPORT OF THE PRESIDENT - ANNUAL REPJRT $O F$ THE $=2 U V C I L$ OF E OON OS-D3-DIG 34 OMIC ADVISERS CYCTORS VUCLEAR-POWER-PLAN/ 18-05-02027 IENED CYLINDR ICAL SHELL I MODEL BR-7M I UNDER HYDROSTATIC PRESSURE I STIFFENED-CYLINDERS TEVSILE-PROPERTI/ 20- II-02347 ING INEERING DATA - WATER CONVE YANCE TUNNELS - GRAVITY AND PRESSURE I UNITEC-STATES 8UREAU-JF-2ECLAMATION / I3-13-O2283 OLLOW MODELS I SHOCK-WAVES EXPERIMENTAL-DATA TEST-NETHCDS PRESSURE ATTENUATORS I \$ARF DU87 *AD I3I 882 I H I8-D3-020O5 OLLOW MODELS I SHOCK-WAVES EXPERINENTAL-DATA TEST-METHODS PRESSURE ATTENUATORS TARF MJ /ORS CARBON-MONOXIOE-INDICATORS TEMPERATURE WIND-PRESSURE PRESSURE HEAT-TRANSMISSION TESTSIFIRESI SURVEYSI I3-I?-DI8O7 IRADO SCHOOL OF MINES SO(4) SEISMIC WAVE PROPAGATION ANO PRESSURE MEASUREMENTS NEAR EXPLOSIOVS I WAVEFOR/ O8- II -02247 BLAST-BIOLOGY SHOCK-WAVES BIOLOGI / THE EFFECTS OF ANBIENT PRESSURE ON TOLERAVCE OF YAMMALS TJ AIR BLAST 1 O6-21-02236 INVESTIGATIOYS NN THE PROBLEM OF THE INTERRELATIONSHIP OF PRESSURE STRENGTH, SIZE AVD CJST JF MULTIPURPOS/ I3-13-0I8?7 NVES ATTENUATION DESIGN GL ZARREL-ATYACHNENTS INHIBITION PRESSURE STRESSES PROJECTILES INTERIOR-BALLISTI/ I9-DS-O2327 NICAL-PROPERT IES THERMAL-RESISTANCE COSTS ACID-RESISTANCE PRESSURE-DROP, \#AEET-244/ES CONSTRUCTION MECHA I3-1I-0I792 AIR SHOCK HAVES IN A TUBE ( SHOCK-TUBES EXPERIMENTAL-DATA PRESSURE-GAGES, *0RNL-TR-1598/ PR JPAGATION OF $20-0+-02053$ 1-BLAST-EFFECTS BLAST-BIOLOGY SHOCK-TUBES INSTRUNENTATI ON PRESSURE-GAGES-CAL IBRATIOV PRESSURE-TIME-PA TTER/ O6-21 -D2232 IUBE I BIOLOGICAL-BLAST-EFFECTS BLAST-BI OLOGY SHOCK-WAVES PRESSURE-TIME-MEASUREMENTS PIEZOELECTRIC-TRANSO/ D6-21 -02237 GY SHOCK-TUBES INSTRUMENTATION PRESSURE-GAGES-CALIBRATION PRESSURE-TIME-PATTERNS, \&DASA-1853/BLAST-BIOLO OG-21-D2232 I MECHANICAL PROPERTIES OF ROCKS AT HIGH TEMPERATURES AND PRESSURES I ROCK-MFCHANICS LOADINGIMECHANICSI US OO-D7-D2242 ECTS THE TOLERANCE UF LAITLE TO LONG DURATICN REFLECTED PRESSURES IN A SHOCK TUBE 1 BIOLOGICAL-BLAST-EFF O6-21-O2237
/.FOOT SHOCK TUBES - EXPERIMENTAL CBSERVATIONS OF INTERIOR PRESSURES IN HOLLOW MODELS (SHOCK-WAVES EXPERI/ IB-D3-O2DII IFOOT SHOCK TUBES - EXPERIMENTAL CBSERVATIONS OF INTERIOR PRESSURES IN HOLLOW MODELS ( SHOCK-WAVES EXPERI I IB-D3-O2DII
IFOOT SHOCK TUBES - EXPERIMENTAL OBSERVATIONS OF INTERIOR PRESSURES IN HOLLOW MODELS I SHOCK-WAVES EXPERI/ IR-O3-D2ODS IFOOT SHOCK TUBES - EXPERIMENTAL OBSERVATIONS OF INTERIOR PRESSURES IN HOLLOW MODELS I SHOCK-WAVES EXPERI/ IR-O3-D2ODS RNAT IONAL - AFFAIRS SUI CAN THE LNI IED NATIONS ORSANIZATI CN PREVENT WARS I PEACE-KEEP IVG PURLIC-JPINION INTE OS-II-D2I 95 UTIL IZATION FINANCING OPERATING-COSTS CANADA CCNSTRUCTION PRICES LABOR, \#A.L. IOI7, TORAGE SALES REVENUE D5-O3-DIOU2 ASIBILITY-ST/ A SIMPLE HEAT ENGINE OF POSSIBLE UTILITY IN PRIMITIVE ENVIRONMENTS I HEAT-ENGINES DESERTS FE I3-D2-OI777 $1 L$ EDUCATION - A BIBL IOGRAPHY OF GOVERNMENT SPONSORED AND PRIVATE RESEARCH ON FOREIGN STUDEVTS AND TRAINE I O5-II -DIG $I$ ECONOMICS, A LIST OF CURRENT SOCIAL SCIENCE RESEARCH BY PRIVATE SCHOLARS AND ACADEMIC CENTERS - AFRICA I O5-II-DIG92 REPUELICS I A LIST OF CURRENT SOCIAL SCIENCE RESEARCH BY PRIVATE SCHOLARS AVD ACADEMIC CENTERS - AMERICAN OS-II-DI696 IBLIOERAPHI/ A LIST OF CURRENT SOCIAL SCIENCE RESEARCH BY PRIVATE SCHOLARS AVD ACADEMIC CENTERS - ASIA I B OS-II -OI69B IONAL AFFAI/ A LIST OF CURRENT SOCIAL SCIENCE RESEARCH BY PRIVATE SCHOLARS AVD ACADEMIC CENTERS - INTERNAT DS-II-OIO93 EASTERN EU/ A LIST OF CURRENT SOCIAL SCIENCE RESEARCH BY PRIVATE SCHOLARS AND ACADEMIC CENTEZS - USSR AND D5-II-DI6OT EUROPE, GRE/ A LIST OF CURRENT SOCIAL SCIENCE RESEARCH BY PRIVATE SCHOLARS AVD ACADEMIC CENTERS - WESTERN OS-II -OIGOU

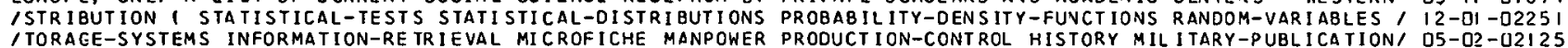
TORAE-SYSTS ON - USSR I PROTECTION-SYSTEMS WA TER-SI SAFEGUAROING FOOD PRODUCTS AND WATER FROM AGENTS OF MASS DESTRUCTI I5-QD-DI84 3 ISTRIES FLOW-CHARTING SURVIVAL RECOVERY ECONOMIC-RECOVERY PRODUCTS RLAST VULVERABILITY AIR-8URST ECONOMIC, O5-03-O2I 26 RADIOAC/ HENKYS LAW CONSTANTS FOR DISSOLUTION OF FISSION PRODUCTS IN A SILICATE FALLOUT PARTICLE MATRIX I IB-O3-O2325 IFACTORS AND BIOLOGICAL AVAILABILITY OF NLCLEAR EXPLOSI ON PRCDUCTS IN RADIOACT IVE FALLOUT I DECAY-SCHEMES/ IB-O3-D2323 IENT BLAST INDUSTRIES SURVIVAL RECOVERY ECONONIC-RECOVERY PRCOJCTS VULNERAEILITY EXPLOSION-EFFE:TS AIRBUR/ OS-03-O21S2 /NDEX OF PUBL I SHED PAPER IINDEXES REPORTS DOCUMENTATION PROFESS IONAL-PERSOVVEL SCIENTIFIC-RESEARCH ORGA/ O5-O? - 21 I 7 IOGY THEORY EDUCATION HEALTH WELFARE-SERVICES OCCUPATIONS PROFESSIONS COMMUNICATION-SYSTEMS TRAINING ECONI DS-II -O2I I 4 1 ION WELFARE-SERVICES SOCIAL-P SYCHOLOGY AGING OCCUPATIONS PROFESS IONS MANPOWER LABOR POL ITICAL-SCIENCE ED $/$ OS-II -O2I 7 I WATER BY STORM DRAINS AND DRAINAGE CHANNELS II. PLANS, PROFILES, CROSS SECTIONS, AND GENEZAL LOCATION I I3-DZ-OI779 WATER BY STORM DRAINS AND ORAINAGE CHANNELS 11 . PLANS, PROFILES, CROSS SECTIOVS, AND GENERAL LOCATION I I3-O? -OI79I T-EFFECTIVENESS MANAGEMENT-EN/ COST ANALYSIS FOR PLANNING PROGRAMMING BUDGET ING COST INFORMATION-RE TRIEVAL DS-OZ -OIOSI TEF

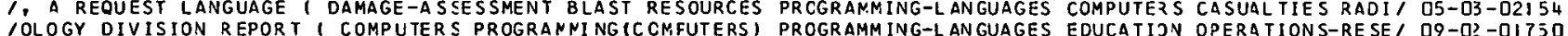

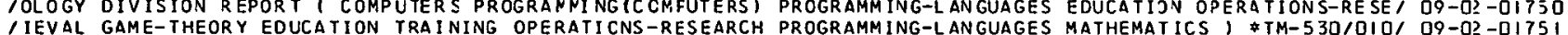
/IEVAL GAME-THEORY EOUCATION TRAINING OPERATICNS-RESEARCH PROGRAMMING-LANGUAGES MATHEMATICS ) *TM-530/OIO/ O9-0? -DI751

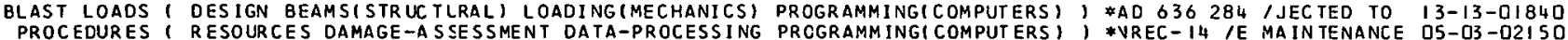
ATA-PROCESSIVG INDUSTRIAL-PROOUCTION INVENTORIES CAPACITY PROGRAMMINGICOMPUTERSI, $\$$ IVREC- 44 INS RECOVERY D O5-03-D2I 44 $S I S$ NUCLEAR-WARFARE-CASUALTIES DAMAGE-ASSESSMENT PATIENTS PROGRAMMING(COMPUTERS), \$VREC-72 IIMATE 1 ANALY DO-2I-D22Z5 IUT ON A SPHERICAL EARTH (WIND YIELD RADIOACTIVE-FALLOUT PROGRAMMING(COMPUIERS) BURST-CONDITIOV MA THEMATI IB-O3-O2322 ITS WIND RADIOACTIVE-FALLOUT CLIMATOLOGY CASUALTIES FIRES PROGRAMMINGICOMPUTERS) BURST-CONDITIONS BLAST Y/ DS-03-O2I34 IOPERATIONS RECOVERY MANAGEMENT-PLANNING RESOURCES MODELS PROGRAMMINGICOMPUTERS) CAPACITY IVDUSTRIAL-PROD/ O5-D3-O2I3। ( AP ID DAMAGE ASSESSMENT PROGRAM I RE SOURCES VULNERABILITY PROGRAMMIINGICOMPUIERS ) CASUALTIES FACILITIES PE I OS-D3-D2I 46 IN-EFFECTS RADIOACTIVE-FALLOLT BLAST-SHELTERS MATHEMATICS PROGRAMMINGICOMPUTERSI CASUALTIES NUCLEAR-WARFA' I5-D3-OIQ38 / PMENT OF A MACHINE WHICH COMPREHENDS ( DIGITAL-CONPUTERS PROGRAMMINGICOMPUTERS) COMPUTER S SPELIAL-PURPOS/ D9-0?-02248 AS MATHEM/ FLAME I - FIRE SPREAD SIMULATION MODEL ( FIRES PROGRAMMING(COMPUTERS) DATA-PROCESSING URBAN-ARE I3-I?-O2266 ICISTRIBUTION ( DAMAGE-ASSESSMENT RE SOURCES VULNERABILITY PROGRAMMING(COMPUTERS) DATA-PROCESSIVG-SYSTEMS / O5-O3-OZI ( PROGRAMMINGICOMPUTERS) DATA-PROCESSIVG-SYSTEMS $105-03-02148$ II FIRES NUCLEAR-EXPLOSIONS DANAGE-ASSESSNENT FI RE-SAFETY PROGRAMMING(COMPUTERS) DEBR IS BLAST IGNITION I , IS-03-DIO52

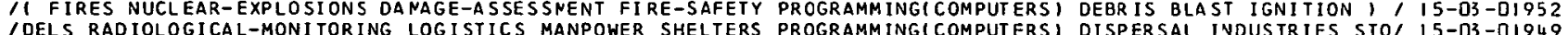
IDELS RADIOLOGICAL-MONITORING LOGISTICS MANPOWER SHELTERS PROGRAMMING(COMPUTERS) DISPERSAL INDUSTRIES STO/ $15-03$-QI94 9
ION DECONTAMINATION PROTECTION-FACTOR MONTE-CARLO-METHOD PROGRAMMINGICOMPUTERS) DOSE-RATE SHIELOING RECO/ O6-IB-O22I 4 IION DECONTAMINAT ION PROTECTION-FACTOR MONTE-CARLO-METHOD PROGRAMMINGICOMPUTERS) DOSE-RATE SHIELOING RECO/ O6-IB-O22IL

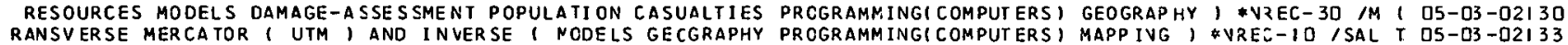

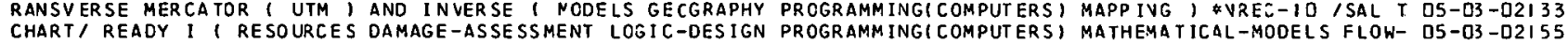
I ION DAMAGE-ASSES SMENT ATTACK-SCENARIOS COMPUTERS HOUSING PROGRAMMINGICOMPUTERS) MILITARY-IVSTALLATIONS P/ O5-03-O2I39

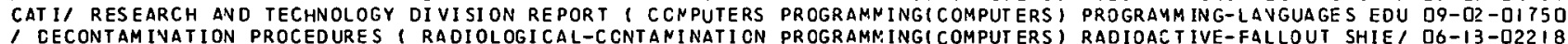


INATION INDUSTR IAL-PLANTS DECAY-RATES RESCURCES(SURVIVAL) PROGRAMMING(COMPUTERS) REPAIR PER SOVVEL TRAININ/ O5-D3 -DIG5I ISCHARGES HEAT IGNITERS PROJECTILES MATHEMATICAL-ANALYSIS PROGRAMMING(COMPUTERS) THEORY PROPELLANTS I \#RI/ I9-OS-O2329 I TO THE THERMAL PULSE OF A NUCLEAR WEAPON I CALCULATIONS PRCGRAMIING (COMPUTERS) THERMAL-RADIATION GEOMET/ I3-I?-OI797 D SCIENTIFIC SUPPORT, PLANNING, STLDIES AND, SYSTENS AND PRCGRAMS - MANAGEMENT, DEVELJOMEVT, TECHNICAL AN 22-O2-O235I G-SYSTEMS MUL TIVARIA TE-ANALYSIS, BMD BI ONEDICAL CCMPUTER PROGRAMS I COMPUTERSIPROGRAMYIVGI DATA-PROCESSIN OG-D3 -D22JL

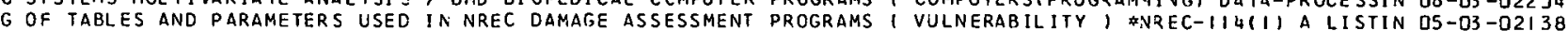
RVIVAL I SIMULATION DAMAGE-ASSESSNENT WARFARE RESOUI NREC PROGRAMS FOR GAMING THE LOGISTICS OF VATIONAL SU O5-D3-OIGL4 RVIVAL
MPUT ER-PROGRAMSI TEST AVD EVALLATION OF COMPUTER AMALYSIS PRCGRAMS FOR SHELTERS IN BUILDINGS I DYVAMICS CO I3-I3-O228O MPUTER-PROGRAMS TEST AND EVALLATION OF COMPUTER ANALYSIS PRCGRANS FOR SHELTERS IN BUILDINGS I DYVAMICS CO I3-I3-O2280
FOREIGN/ OCCASIONAL REPORT ON FOREIGN AFFAIRS - ANERICAN PROGRAMS OF FOREIGV AID I PUBLIC-JOIVION SURVEYS O5-II -DIG87 FORE IGN/ OCCASIDNAL REPOR I ON FOREISN AFFAIRS - ANERICAN PROGRAMS OF FOREIGV AID I PUBLIC-JOIVION SUR VEYS O5-II -DI687
CIENT IFIC-RESEARCH GLASS OPTICAL-PROPERTIES RUSY CRY/ NRL PROGRESS IN HIGH PJWER LASER ZESEARCH I LASERS S 2D-DJ-D2OS2 C DISARMAMEVT 1 COMMUNIST-CHINA ECONOMICS CON/ STUCIES IN PRCGRESS OR RECENTLY COMPLETED - AZMS CONTROL AN O5-DI-OIGG2 C OISARMAMENT $I$ BIBL IOGRAPHIES EC CNOMICS COMM/ STUCIES IN PROGRESS OR RECENTIY COMPLETED - ARMS CON TROL AN OS-Q + -OIGS3 D DISARMAMENT I COMMUNIST-CHINA ECONOMICS CON/ STUDIES IN PROGRESS OR RECENTLY COMPLETED - ARMS CONIROL AN DS-DH-DIGG D DISARMAMENT I B IBL IOGRAPHIES ECONOMICS CONF/ STUCIES IN PROGRESS OR RECENTLY COMPLETED - AZMS CONTROL AN OS-O $4-0 I G 65$ ISIGN GUV-BARREL-ATTACHMENTS INHIBITION PRESSURE STRESSES PRCJECTILES INTERIOR-BALLISTICS GAS-FLOH, *AD / I9-OS-02327 IIGITAL-COMPUTERS EXPLOSIONS GAS-DI SCHARGES HEAT IGNITERS PROJECT ILES MATHEYATICAL-AVALYSIS PROGRAMMING IC/ IQ-DS-D2329 SPOSAL COSTS WASTESISANITARY-ENG INEERING) STORAGE GARBAGE PRCJECTICNS, \#A.L. 98I /EERIVG PUBLIC-HEALTH DI I3-Q2-DI7R9 OMICS I SCIENTIFIC-RESEARCH RE SOURCES MANPOWER INNCVATION PROJECTICNS MODELS, \#A.L. IDT5 /PECTING IN ECON OS-D3-OIO33 ES BY AGE, SEX, AND COLOR TO $1990 /$ POPULATION ESTINATES - PRCJECTICNS OF THE POPULATION JF THE UNITED STAT $23-0+-02358$ IOF POSTATIACK SI TUATIONS I SOCIE IAL-REC OVERY METHCDOI OGY PROJECT IONS RECOVERY MANAGEMEVT-PLAVVING POSIAT/ OS-II -O2I 91

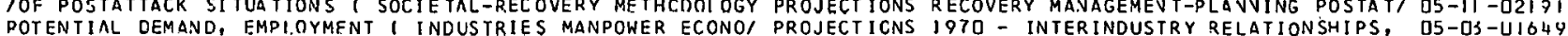

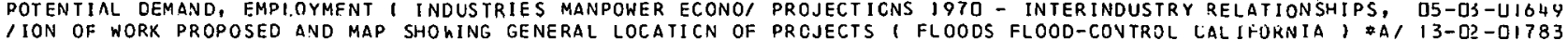

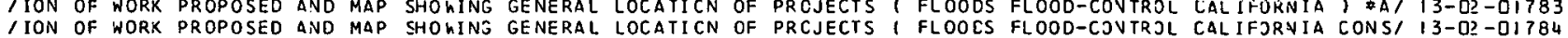

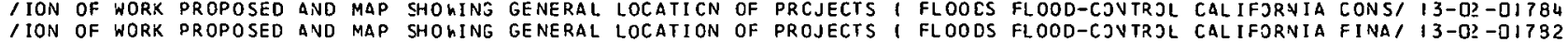
1 SOUTHERN CAL IFORNIA - FEATHER RIVER AND DELTA DIVERSION PROJECTS I WATER-SUPPLIES URBAN-AREAS ECONOMICSI I3-O? -D225B IVERSARY OF THE BATYLE OF MOSCOW I USSR HISTORY CCNMUNISM PROPAGANDA, \$JPRS-40,931 TT-67-31573/IFTH ANN O5-O4-02173 UCLEAR-WARFARE BALLISTIC-MISSILES COLD-WAR FOREIGN-POLICY PRCPAGANCA ATTITUDES COMMUNISM, \$LT-65-ICL /E N O5-O $\$-0 I 672$ ISE RESCUE(TRAINING) REPAIR ITRAINING) PROTECTIVE-CLCTHING PROPAGANCA COMMUNISM PROTECTIVE-MASKS BIOLOGICA/ I5-D3-DIQI 7 ICE HISTORY LANGUAGE LAW LI TERATURE PHILOSOPHY POPULAT ION PROPAGANDA PUBL IC-INFORMATION I \#EXTERNAL RE SEAI OS-II -OIOQ7 MICS WATER-WAVES MATHEMATICAL-PREDICTION THEORY CRATERING PRCPAGATION

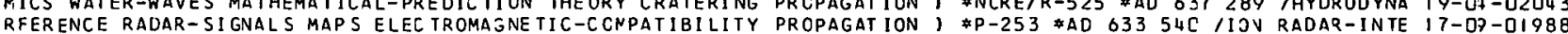

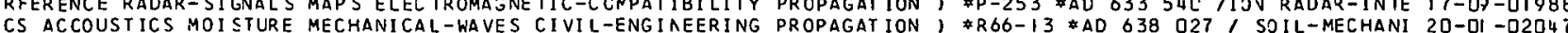

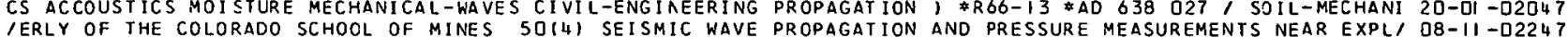
IERLY OF THE COLORADO SCHOOL OF MINES 5014 ) SEISMIC WAVE PROPAGATION AND PRESSURE MEASUREMENTS NEAR EXPL/ D8-II -D224 7
ION OF SOUND IN AN AEROSOL ( ACOUSTIC-PROPERTIES GAS-FLOW PROPAGATION BIOLOGICAL-WARFARE-AGEYTS SOUNO-TRA/ I5-O2-OI8BI

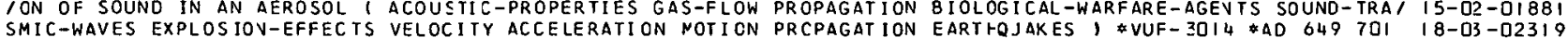
$1 G$ WAVES IN LONG ELASTIC RODS I MECHANICAL-WAVES STRESSES PROPAGAT ION ELASTICITY THEORY, $\$ 2 Z L-376$ FAD 63/ $20-11-02079$ 1 NUCLEAR-EXPLOSIONS IONOSPHERE A/ ELECTROMAGNETIC PULSE PROPAGAT ION IN A DISTURBED TERRESTRIAL WAVEGUIDE $20-1+$-O2348 DIFIEC SACHS SCALING I EXPONENTIAL IDEAL GAS ATM/ SHOCK PROPAGATION IN NONHOMOGENEOUS ATMOSPHERES AND MO 20-DU -D2332 ENV IRONMENT ( VUCLEAR-EXPLOSIONS h/ ELECTROMAGNETIC PULSE PRCPAGAT ION IN TPE NORYAL TER ZESTRIAL HAVEGUIDE $20-14-02349$ IN NONL IVEAR MATERIALS 1 VI SCOELASTICITY NECHANICAL-WAVES PROPAGAT ION MATHEYATICAL-AVALYSIS ELASTICITY SH/ $20-I I$-O2DSO

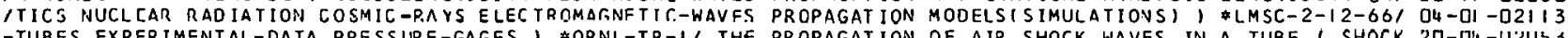
-TUBES EXPERIMENTAL-DATA PRESSURE-GAGES, \#ORNL-TR-I1 THE PROPAGATION OF AIR SHOCK WAVES IN A TUBE 1 SHOCK 2D-OZ4-U2USS IMS FLUID-MECHANICS SCIENTIFIC-RE SEARCH ABLATION ANTENNAS PROPAGAT ION SHOCK-NAVES KIVETIC-TREORY ELECTRON/ 2D-O3-D2330 I PLASTIC WAVE PROBLEMS 1 SOIL-MECHANICS NECHANICAL-WAVES PROPAGAT ION SHOCK-NAVES UVDERGROUVD-EXPLOSIONS, D8-I3-OI74 7 /ERSE WAVES IN ELASTIC RODS ( MECHANICAL-WAVES ELASTICITY PROPAGAT ION STRAIVIMECHANICS) EQUATIONS-OF-MOTI/ 20-II -O2OS2 (CALCULATING ATTENUATION OF AIR SHOCK WAVES IN TUNNELS I PROPAGATION SWEDEN I \#REPORT VO. ID3-24 \#AEC-TR/ 20-D\&-D205I

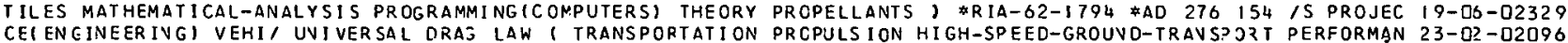

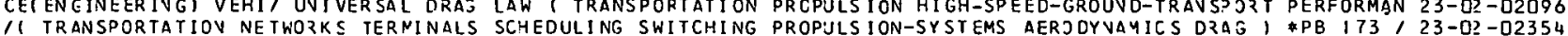

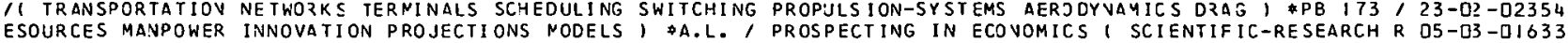
ION SOI/ NUCLEAR WAR AND SOIL EROSION - SOME PROBLEMS AND PROSPECTS 1 POSTATTACK-OPERATIONS SOIL-CONSERVAT D8-I3-OIT45 IVE CONTAMIVATION I USSR CIVIL-DEFENSE-SYSTEMS SHELTERS, PRCTECT ING THE RURAL POPULATIJV AGAIVST RADIOACT DO-IB-OIT3I IELDING DOSE-RAT/ SHELTER DESIGN AND ANALYSIS I. FALLCUT PROTECT ION I FALLOUT-SHELTERS VUCLEAR-WEAPONS SH I3-I3-DIB39 RGENCY HEALTH SER VICES UNITS I SHIELDING PATIENTS RAOIOA/ PROIECT ION AGAINST NUCLEAR WEAPON FALLOUT IN EME I8-OS-D2O28 RS IN/ EVACUATION OF THE URBAN POPULATION AS A MEASURE OF PROTECT ION AGAINST NUCLEAR WEAPONS I USSR SHELTE I5-O3-OIO3 4 USSR INTERNATIOVAL-CIVIL-OEFENSE CI/ SIMPLE SHELTERS FOR PROTECTION AGAINST HEAPONS OF MASS OESTRUCTION I I3-I3-OI826 IION OF THE ORGAVIZATION, IECHNOLOGY, AND TACTICS CF FIRE PRCIECTION AS EMPLOYED BY A CIVIL DEFENSE ORGAN/ I3-I?-O2264 IION OF THE ORGAVIZATION, TECHNOLCGY, AND TACTICS CF FIRE PROTECTION AS EMPLOYED BY A CIVIL DEFENSE ORGAN/ $13-1 ?-02265$ AVES BLAS, COMPUTCR ANALYSIS OF PROTECTIVE STRUCTURES FOR PROIECTION FROM NUCLEAR BLAST I SHELTERS SHOCK-W I3-13-OI833 IERIMENTAL EVALUATION OF TECHNIQUES FOR IMPROVING FALLOUT PROTECTION IN HOME BASEMENTS I SHELTERS FALLOUT/ I3-I3-OIB22

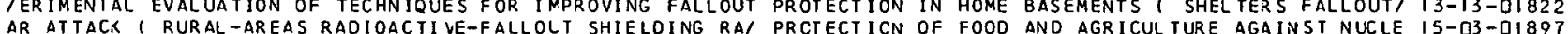
THE ORGAVIZATION, IECHYOL, PRACTICAL EXPERIENCES CF FIRE PRCTECTION SERVICES - A CRITICAL PZESENTATION OF I3-I?-O2254 THE ORGAVIZATION, TECHNOL, PRACTICAL EXPERIENCES CF FIRE PRCIECTION SERVICES - A CRITICAL P2ESENTA IION OF I3-I?-D22S4
THE ORGANIZATION, TECHNOLI PRACTICAL EXPERIENCES CF FIRE PROIECTION SERVICES - A CRITICAL PRESENTATION OF I3-II-DO2265 THE ORGANIZATION, TECHNOLI PRACTICAL EXPERIENCES CF FIRE PROTECTION SERVICES - A CRITICAL PRESENTATION OF I3-I?-O2265
IY OF COMMUNITY RESPONSE TO THE RHODE ISLAND HOME FALLOUT PROTECTION SURVEY I ATTITUDES FALLOUT-SHELTERS, D5-IJ-OIOB5 LTERS NEVACA WEAPONS-TESTS SIMLLATION RATINACTIVE-FALLOUT PROTECT ICN-FACTOR I \#CEX-O5.5 /LTEZS FALLOUT-SHE I3-I3-0IB22 /ELTERS 1 UVDERGROUND-STRLCCTLRES CONSTRUCTION URBAA-AREAS PRCTECTICN-FACTOR BLAST-SHELTERS COSTS, \&USNRUI I3-I3-DI8I5 11 NAT IONAL-FALLOLT-SHEL TER-SURVEY(NFSS) FAII DIIT-SHELTERS PROTECT ION-FACTOR CALCULATIONS MODELSISIMULATIOI I8-D3-DIQ95 IN NUCLEAR-EXPLOSION-DAMAGE EXPLOSION-EFFECTS RURAL-AREAS PROTECTION-FACTOR DECONTAMINATION WATER-SUPPLIEI IS-I3-UIB26 IESEARCH II DAMAGED PF MULTIPLIER I RADIOACTIVE-FALLOUT PROTECTION-FACTOR DOSE-RATE BUILDINGS DECONTAMII DG-IS-O222I IERS ENTRANCEWAYS DOSE-RATE RADIATION-MEA SURENENT-SYSTEMS PROTECTION-FACTOR MONTE-CARLO-MEIHOD GEOMETRYIDI D6-I3-D22I 9 ICT IVE-FALLOUT RADIOLOGICAL-CONTANINATION DECCNTAMINATION PROTECT ION-FACTOR MONTE-CARLO-METHOD PROGRAMMIN/ D6-I3-D22I 4 TFOR RADEF SYSTEMS I RAOIOLDGICAL-CONTAMINATICN SHIELDING PROTECTICN-FACTOR RADIOLOGICAL-DOSAGE RECOVERY I OG-IS-OIT29 ICUCTS AND WATER FROM AGLNTS OF MASS DESTRUCTION - USSR I PROTECTION-SYSTEMS WATER-SUPPL ILS LHEMICAL-WARFI I5-O?-OI843 IELS OF IMMUNITY AGAINST BACILLUS ANTHRACIS OBTAINED WITH PROTECTIVE ANTIGEN AND LIVE VACCIVE 1 SPORES BI/ I5-O? -OIB53

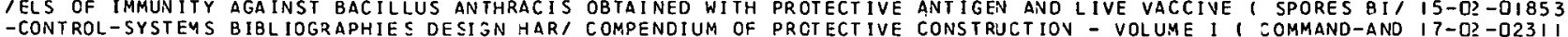
-CONTROL-SYSTEMS BIBL IOGRAPHIES DESISN HAR/ COMPENDIUM OF PRCTECTIVE CONSTRUCTION - VOLUME I I COMMAND-AND I $7-02-0231$ I
OUND-STRUCTURES OYNAMICS S/ YIELDING MEMBRANE ELENENTS IN PROTECT IVE CONSTRUCTION I BLAST-SHELTERS UNDERGR I3-I3-DIB37 TERS FALLOUT-SHEL TERS UNDERGROUND-STRUCTURES COLORADO DU/ PROTECTIVE CONSTRUCTIOV INSTITUTE STUDIES 1 SHEL I3-I3-OI83I DI/ RADIOACTIVE FALLOUT - PHYSICS, BIOLOGICAL EFFECTS AND PROTECTIVE MEASURES I RADIATION-IVJURIES USSR RA D6-I3-OI726 GRAPHY OF U.S. VAVAL CIVIL ENG INEERING LABORATORY PUBLIC/ PROTECTIVE SHELTER SYSTEMS - AN AYVOTATED BIBLIO I3-I3-D2278 SILES RAILROADS BRIDGES GROUND-SUPPI SOVIET LITERATURE ON PROTECT IVE STRUCTURE AVD COMPJNENTS 1 GUIDED-MIS IG-DI -DI972 IRIALS FOR USE IN MITIGATING BLAST LOADS ON DEEPLY BURIED PROTECTIVE STRUCTURES I CONCRETE UNDERGROUND-ST/ IB-O3-O2OJ7 IRIALS FOR USE IN MITIGATING BLAST LOAOS ON DEEPLY BURIED PROTECTIVE STRUCTURES I UVDERGROUD-SIRUCTURES CI IB-O3-O2OI? F ChINESE COMMUNIST SCIENTIFIC AND TECHNICAL LITERATURE, PROTECTIVE STRUCTIRES AND COMPONEVTS - SURVEYS O I G-OI-OI973 R BLAST I SHELTERS SHOCK-WAVES BLAS/ COMPUTER ANALYSIS CF PROTECT IVE STRUCTURES FOR PRJTECTIJV FROM NUCLEA I3-I3-DI833 OUND I FALLOUT-SHELTERS COMPUTERS B/ COOLING ANALYSES FOR PROTECTIVE STRUCTURES LOCATED ABOVE AND $8 E L O W G R$ I 3 -OI -OI776 IMULATIONS OF THERMAL ENVIRONMENT IN OCCUPIEO UNDERGROUND PROTECTIVE STRUCTURES WITH OBSERVED CONDITIONS I 20-I3-O2O9D NG-SYSTEMS RADIOL OGICAL-CONTAMINATION VE TERINARY-MEDICINE PRCTECTIVE-CLOTHING, \#JPRS-32,877 \#TT-65-33455 I5-03-OI9I8 ISES LEWISITE GAS-DETECTORS RADIATION-MONI TORS DOSINETERS PROTECI IVE-CLOTHIVG GAS-MASKS PHOSGEVE INCENDIAI D6-I3-OI72I ISES LEWISITE GAS-DETEC TORS RADIATION-MONI TORS DOSINETERS PROIECI IVE-CLOTHIVG GAS-MASKS PHOSGEVE INCENDIAI D6-I3-OI72I
INAT IONAL-CIVIL-DEFENSE RESCUE (TRAINING) REPAIR (TRAINING) PROTECT IVE-CLOTHIVG PRJPAGAVDA COMMUVISM PROTECI IS-D3-DI9I 7

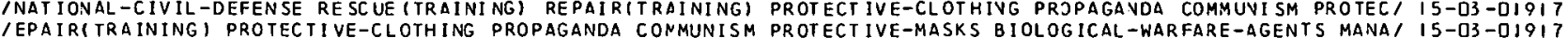
IINE CORV CONFERENCE ( NUTRITION(HUMAN) NUTRITION(ANIMAL) PROTEINS GENETICS AGRICULTURAL-ECONOMICS BIOCHE, DS-IS-DI72O 
YNAMIC LOACING 1 DUCTS SANO UNDERGR/ RESPONSE OF A BURIED PRCTOTYPE COMMUNICATIONS CONDUIT TO STATIC AND D I3-II -D22GI G MAINTEV/ PARM SYSTEM MANUAL III-K. CONSTRUCTICN IN THE PROIOTYPE MODEL I REPAIR RECOVERY DATA-PROCESSIN I3-I3-O22B2 IENT, AUTHOR AND SUBJECT INDEXES I BACTERIA VIRUSES FUNGI PROTOZOA RICKETTSIA PHYSIOLOGY DIAGNOSIS I *MIS/ D6-I3-OI7IS R NUCLEAR ATTACK I CIVIL-DEFENSE-OPERATING-PLANS FALLO/ A PROVISIONAL CONCEPT OF EMERGENCY OPERATIONS UNDE OS-DI-DIG23 XPOSEC TO A SMALL PARTICLE AEROSOL O/ THE PATHOGENESIS OF PS ITIACOSIS - SERIAL STUDIES ON RHESUS MONKEYS E IS-O2-QIBSO Y AGAW ANELLA-PSITTACI CUL TLRE-MEDIA I' PLAGUE FCRNATICA BY PSITTACOSIS VIRUS I BIOLOGICAL-WARFAZE-AGENTS MI IS-D?-OIBQI - ALUAT ION OF SHELTER EQUIPMENT AND PROCEDURES II. LABORA' PSYCHOLOGICAL, ENG INEER ING, AVD PHYSIOLOGICAL EV I 5-O3 -022 7 ALUATION OF SHELTER EQUIPMENT AND PROCEDURES III. HABIT/ PSYCHOLOGICAL, ENGINEERING, AVD PHYSIOLOGICAL EV I5-O3-02298 ALUATION OF SHELTER EQUIPMENT AND PROCEDURES I. SUNMARYI PSYCHOLOGICAL, ENGINEER ING, AYD PHYSIOLOGICAL EV I5-D3-O23 OD GENCY-HEAL TH-SERVICES 8 IOLOGICAL-hARFARE CHEMICAL-WARFARE PSYCHOLOGICAL-WARFARE, \$A.L. IIIST/EDICINE EMER O6-21 -02233

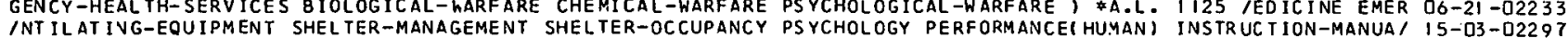

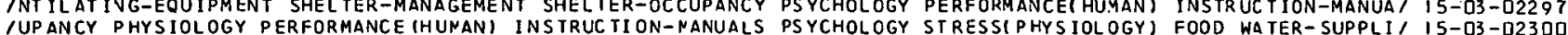
IUPANCY PHYSIOL OGY PERFORMANCE (HUNAN) INSTRUCTION-NANUALS PSYCHOLOGY STRESSIPHYS IOLOGY) FOOD WATER-SUPPLI I IS-D3-D23OO
IUPANCY PHYSIOLOGY PERFORMANCE (HUNAN) INSTRULTION-NANUALS PSYCHOLOGY STRESSIPHYSIOLOGY) WATEZ-SUPPLIES FO/ IS-OIS-J2298 IUPANCY PHYSIOL OGY PERFORMANCE (HUNAN) INSTRUCTION-NANUALS PSYCHOLOGY STRESSIPHYSIOLOGY) WATEZ-SUPPLIES FO/ $15-03$ - 22298
IENG INEER ING-PER SONNEL SCIENTIFIC-PER SONNEL FLOW-CHARTING PSYCHOMETRICS TECHVICAL-INFORMATION-CENTERS JOR/ OS-D2-O2I2O IENG INEER ING-PER SONNEL SC IENTIFIC-PER SONNEL FLOW-CHARTING PSYCHOMETRICS TECHVICAL-INFORMATIOV-CENTERS JOR/ OS-D2-02I 20
TLE, BOVINES SHIELDING CONSTRUCTION VENTILATION, \#MISC. PUB. NO. 947, TYPE FALLOUT SHELTER FOR BEEF CAT I3-I3-DI8I 8

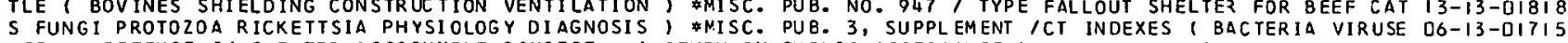
CIVIL-DEFENSE-S/ SHELTER A SSIGNMENT CONCEPT - A STUOY IN PUELIC ACCEPTANCE I SHELTER-ALLOCATION ATIITUDES D5-IJ-D2I77 WATER-SUPPLIES REGULATIONS RADIOACTIVITY CONTROL, \#PHS/ PUBLIC HEALTH SERVICE DRINKING WATER STANOARDS I I3-O? - QI778 SHELTER SYSTEM I FALLOUT-SHELTERS PUBLIC-OPINION ATTITU/ PUBLIC INFORMATION AND KNOWLEDGE RE2UISITES OF A Q5-1I-OIG99 TORY CIVIL/ U.S. CIVIL DEFENSE BEFORE 1950 - THE RCOTS OF. PUBLIC LAH 9201 LEGISLATION SOCIAL-SCIENCES HIS O5-D'-CIGTI RES ATTITUDES INTERNATIONAL-AFFAIRS FEAR SURVI UNESCC AND PUELIC OPINION TODAY 1 UNITED-STATES QUESTIONNAI O5-11-DIGSQ UT ' BEHAVIOR ELECTRIC-POWER-PRODLCTI ON(FAILURE) ATTITUDI PUBLIC RESPONSE TO THE NORTHEASTEZV POWER BLACKO DS-IJ-DI GB3 IEEWAY TUNNEL WEST ORANGE, NEW JERSEY, AS A CIVIL DEFENSE PUBLIC SHELTER, FACILITY I TRANSPORTATION FEASIB/ I3-I3-O227I SECUR ING HEALTH IN OUR URBAN FUTURE URBAN-AREAS PUBLIC-HEALTH \$PHS-1581 IESEARCH NEEOS I SCIENTIFIC-RE SEARCH SANITARY-ENGINEERING PUELIC-HEALTH DISPOSAL COSTS WASTESISANITARY-EN/ I3-OZ-DI7B9

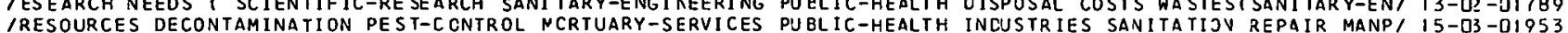

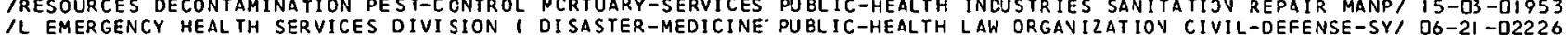

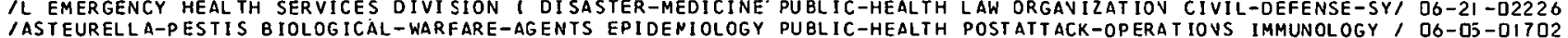
IASTEURELLA-PESTIS B IOLOGICAL-WARFARE-AGENTS EPIDENIOLOGY PUBLIC-HEALTH POSTATTACK-OPERATIOVS IMMUNOLOGY I O6-OS-DI7O2
$1 /$ CISAST ER-MEDIC INE MEDICINE MEDICAL-PERSONNEL HOSPITALS PUBLIC-HEALTH RESCJES NUCLEAR-WARFARE-CASUALTIE / O6-2I -OITUD IENT I RECOVERY INFECTIOUS-DI SEASES VACCINES RAOICBIOLOGY PUBLIC-HEALTH STRESSIPHYSIOLDGYI MALVUTRI TION R/ D6-OJ-O22DG I CIVIL-DEFEN SE-OPERATING-PLANS INDUSTRIES (CIVIL-DEFENSEI, PJ BLIC-HEALTH TRAVSPORTATIDN COMMUVICATION, JI IS-O3-O23J2 TUNNELS, \#HUD-MP-39 SCIENCE AND THE CITY I URBAN-AREAS PUBLIC-HEALTH URBAN-PLANNING POPULATION UTILITY- $23-03-02 I 02$ ILANGUAGE LAW LITERATURE PHILOSOPHY POPULATION PROPAGANDA PUBLIC-INFORMAT ION, \#EXTERNAL RESEARCH LIST I./ O5-II - LIG97 I GRAPFY POLITICAL-SCIENCE HISTORY LANGUAGE PHILOSCFHY LAW PUELIC-INFORMATION, \#EXTERVAL RESEAZEH LIST 5. O5-II-OIG92

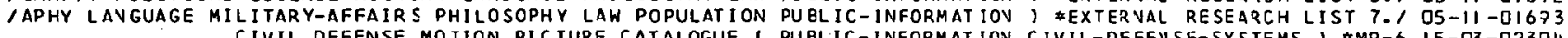
CIVIL DEFENSE MOTION PICTURE CATALOGUE I PUBLIC-INFORMATION CIVIL-OEFEVSE-SYSTEMS, \$MP-6 I5-O3-O23D4 I MANAGEMENT-PLANNING STRESS (PSYCHOLOGY) SHELTERS SCHOOLS PUBLIC-INFORMATION EOUCATION ORPHAV-CARE I \#HSR/ OS-II-D22O3 IFARE-CASUALTIES RADIATION-INJURIES POSTATTACK-OPERATIONS PUBLIC-INFDRMAT IOV MEDICAI-SUPPLIES I \&A.L. III/ D6-2I-D2226 UT IL ITY-I UNVELS COMMUNICATION-SYSTEMS STRESS IPSYCHOLOGY) PJBLIC-INFORMAT IOV MOVEMEVT, 1-POL ICY GEOGRAPHY POL ITICAL-SCIENCE HISTORY LANGUAGE LAW PUBLIC-INFORMATIOV PHILOSOPHY RELIGION, \$FXTER/ OS-II-DIS96 INSE-SYSTEMS INIERNATIONAL-CIVIL-DEFENSE TRAINING RESCUES PUBLIC-INFORMATION REPAIR FILMS PZOPAGANDA RURA/ I5-03-O23O2

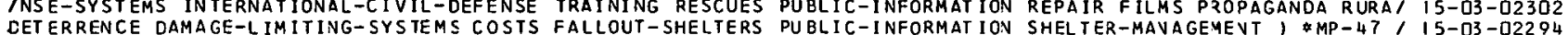
ICTRIC-POWER-PRODUCTION (FAILLRE) ATTI TUDES PUBLIC-CPINION PJ ELIC-INFORMAT ION SURVEYS DEYOGRAPHY COMMUNICA I O5-IJ-OI683

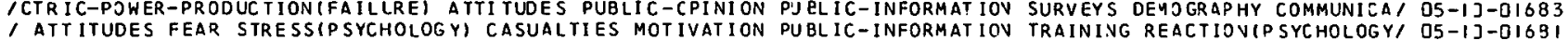

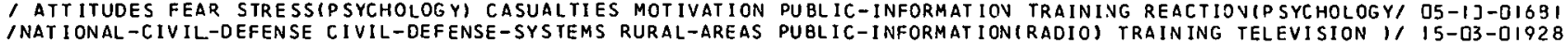
INATIONAL-CIVIL-DEFENSE CIVIL-DEFENSE-SYSTEMS RURAL-AREAS PUBLIC-INFORMATION(RADIO) TRAINING TELEVISION I' IS-O3-OIO28
IE FALLOUT PROTECTION SURVEY I ATIITUDES FALLOUT-SHELTERS PUBLIC-CPINION ANXIETY CIVIL-DEFEVSE-SYSTEMS SU/ OS-IO-OIOBS IWLEOGE REQUISITES OF A SHELTER SYSTEM I FALLOUT-SHELTERS PUBL IC-OPINION ATT ITUDES BEHAVIOR WARVING-SYSTE/ OS-II-OIG 9 IOGY) GROUP-DYNAM ICS SOCIAL-PSYCHOLOGY BEHAVIOR DISASTERS PUBLIC-OPINION ATTITUDES METHJOOLOGY WEAPONS-EF/ O5-IJ-DIG77 /PEOPLE KNOW AND THINK ABOUT THE BOMB AND ATOMIC ENERGY 1 PUBLIC-OPINION ATT ITUDES SURVEYS QUESTIONNAIRES/ OS-II -D2I96 ED NATIONS 1 INTERNATIONAL-AFFAIRS SURVEYS QUESTIONNAIRES PUBLIC-OPINION FOREIGN-POLICY I \$FA-2 IRS - UNIT O5-II-02IO9 / EATE 1 CIVIL-DEFENSE-SYSTEMS ATTITUDES PCLITICAL-SCIENCE PUBLIC-OPINION HISTORY COSTSIATTITUDESI INTERNA/ DS-1J-OIG79 IUNITED VATIONS ORGANIZATION PREVENT WARS I PEACE-KEEPING PUBLIC-OPINION INTERNATIOVAL-AFFAIRS SLRVEYS AR/ OS-II-D2I 5 IIBL IOGRAPHIES ATTITUDES GROUP-D YNAMICS SOCIAL-PSYCHOLOGY PUBLIC-OPINION METHODOLOGY THEORY EDUCATION HEA/ OS-II-D2IOL IE-SYSTEMS MANAGEMENT-PLANNING DECISION-MAKING OB JECTIVES PUBLIC-OPINION POLITICAL-SCIEVCE EXECUTIVE-BRANI O5-DL-OIG7I OEY 作 CCIVIL DEFEVSE IV THE COLD WAR CONTEXT - 1966 ATTITUDES PUELIC-OPINION SURVEYS DISARMAMENT CIVIL-DEFENS/ DS-IJ-DIS78

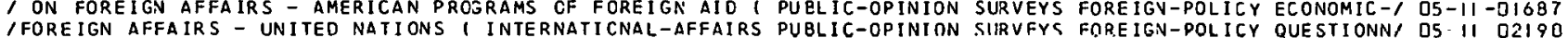
/FOREIGN AFFAIRS - UNITED NATIONS I INTERNATICNAL-AFFAIRS PUBLIC-OPINION SIRVFYS FOREIGN-DOLICY QUESTIONN/ DS II O2IQO IKES $1 /$ UCCAS IONAL REPORTS ON FOREIGN AFFAIRS - GERMANY 1 PUBLIC-OPINION SURVEYS FOREIGV-POLICY QUESTIONNA O5-II -D2IBG Y ACING/ BIBLIOGRAPHY OF PUBLICATIONS, $1941-1965$ I HEALTH PUELIC-OPINION WELFARE-SERVICES SJEIAL-PSYCHOLOG O5-II -D2197 H-SERVICES CANADA MANUALS FILMS I \#A.L. 1068 CATALCGUE OF PUBLICATIONS 1 DISASTER-MEDICINE EMERGENCY-HEALT O6-21-DI737 I BIBLIOGRAPHY OF U.S. NAVAL CIVIL ENGINEERING LABORATORY PUBLICATIONS 1 FALLOUT-STELTERS BLAST-SHELTERS I I3-I3-02278 / SOCIAL RESEARCH 1941-1964 - AN INVENTORY OF STUDIES AND PUBLICATIONS IN SOCIAL RESEARCH I SJCIAL-SCIENC, O5-II-O2I94 WELFARE-SERVICES SOCIAL-P SYCHOLOGY ASING / BIBLIOGRAPHY OF PUELICATIONS, $1941-19601$ HEAL TH PUBLIC-OPINION O5-II -D2197 OPEN LITERATURE WITH ABSTRACTS 1 B/ ECONOMICS DEPARTMENT PUBLICATIONS, $1960-1965$ - AN AUTHDR INDEX OF THE OS-03-DIS52 PROFESSIONAL-PER SONNEL SCIENTIF IC-RE SEARCH ORG/ INDEX OF PUBLISHED PAPERS 1 INDEXES REPORTS DOCUMENTATION O5-D2-D2II 7 MISSION I JAPAN VUCLEAR-WARFARE-CASUALTI I BIBLIOGRAPHY OF PIIRLISHED PAPERS OF THE ATOMIC BOMB CASUALTY COM DO-2I-O2224

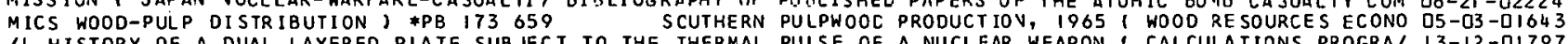
IL HISTORY OF A DUAL LAYERED PLATE SUBJECT TO THE THERMAL PULSE OF A NUCLEAR WEAPON I CALCULATIONS PROGRA I3-I?-OI797 EGUIDE I NUCLEAR-EXPLOSIONS IONOSPHERE A / ELECTROMAGNETIC PULSE PROPAGATION IN A OISTURBED TERRESTRIAL WAV $20-I+-02348$ GUIDE ENV IROVMENT ( NUCLEAR-EXPLOSIONS W/ ELECTROMAGNETIC PULSE PROPAGATION IN THE NORMAL TERRESTRIAL WAVE $20-1+$-O2349 (MECHANICS) INS TRUMENTATION CALIBRATI/ A MECHANICAL SHOCK PULSE SURVEY I TEST-EQUIPMENT IMPACT-SHOCK SHOCK I $4-02-0 I 842$
CAVITY-RESONATORS OPTICS CRYST INVESTIGATICNS OF GIANT PULSING METHODS IN RUBY LASERS I DUMPING

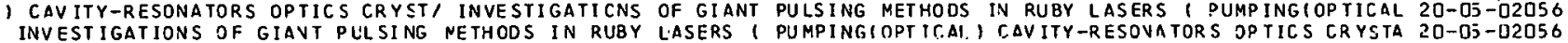
I-RESEARCH GLASS OPTICAL-PROPERTIES RUBY CRYOGENICS GASES PUMPINGIOPIICAL) FLASH-LAMPS LIOUIDS SPECTROSCO/ $20-0 J-020 S 2$ DECK PREPARATION AND SCORING I DATA-PROCESSING METHODOLO/ PUNCHED CARD Q SORTING - A MACHINE METHOD FOR Q OS-II -02IB8 /CK PREPARATION AND SCORING I DATA-PROCE SSING METHCOOLOGY PUNCHED-CARC-EOUIPMENT SOCIAL-SCIEVCES I \#A.L. / O5-II-D2I88 OCIAL-SCIENCES CORRELATI/ THE FAC IOR ANALYSIS CF. C DATA I PUNCHEC-CARCS STAT ISTICAL-AVALYSIS METHODOLOGY S O5-II - D2I 92 ERIMENTAL-DATA HABITABILITY FANS PORTABLE INSTRUMENTATION PUNKAH-PUMPS, \#GARD REPORT $1268-51 / 50 N T R O L$ EXP I3-DI -DI771 ICT WATER WITH THE SIMPLEST MEANS - USSR I WATER-SUPPLIES PURIFICATION CONTAMINATIOV RADIOLJGICAL-CONTAMI/ O6-13-OI722 IRICIUM BOTUL, INUM VIII. IMMUNOLOGICAL RESPONSE OF MAN TO PURIFIEC BIVALENT AB BOTULINUY TOXJID 1 BIOLOGI/ I5-DE-QIB57 MEANS - USSR I WATER-SUPPLIES PURIFICATION CONTAM/ HOW TO PURIFY AND TO OISIVFECT WATER WITH THE SIMPLEST OO-I3-OIT22 MEANS - USSR T WA TER-SUPPLIES PURIFICATI ON CONTAM/ HOW TO PURIFY AND TO O ISIVFECT WATER WITH THE SIMPLEST, DO-I3-OIT22 /ORIMETER S IGNITION HIGH-TEMPERATLRE-RESEARCH RADICMETERS PYROLYS IS SOL AR-FURNACES COMBUSTIDN DECOMPOSITI/ I3-I?-DI8J2
/ LABORATORY-FURNACES RADIOMETERS HEAT-FLUX HEAT-TRANSFER PYROMETERS HIGH-TEMPERATURE-RESEART.H OPTICAL-IN/ I3-DI-O2255

$Q$

ES I STATISTICAL-ANALYSIS SOCIAL-SCIENC/ TABLES FOR YULES O ASSOCIATION COEFFICIENT FOR PAIRS OF PEREENTAG O5-II-D22OO OCOLOCY SOCIAL-SCIENCES CORRELATI/ THE FACTOR ANALYSIS OF O OATA I PJNCHED-CARDS STATISTICAL-AVALYSIS METH D5-II - Q2I 2 METHODOL O/ PUNCHED CARD O SORTING - A MACHINE METHOD FOR O DECK PREPARAT ION AND SCOR IVG 1 DATA-PROCESSING OS-II -D2I B8 1 OOK I CONSTRUCTION DESIGN STRENG TH STRUC TURAL-PROPERTIES OUALITY FASTENERS GLUIVG PLYWDOD SAVDWICH-CONSTI II-I?-DI?GO 
ENCY PARTICULATE AIR FILTERS (FILTERSIFLUID) RELIABILITY QUALITY-CONTROL 5PECIFICATIONS ) \$ORVL-P-23B7 /I I3-II-OI794 AN INTRODUCTION TO THE DESIGN OF UNDERGRCUND OPENINGS F/ QUARTERLY OF THE COLORADO SCHJOL OF MINES 4611 ) I3-13-02273 CLASSIFICATION OF ROCKS ( ROCKIGEOLOGY NINERALS TEXTUR/ QUARTERLY OF THE COLORADO SCHOOL OF MINES SDII) O8-OY-O224 I SEISMIC HAVE PROPAGATION AND PRESSURE MEASURENENIS NEAR/ QUARIERLY OF THE COLORADO SCHODL JF $41 N E S$ 5O(4) D8-1II-D2247 SYMPOS IUM OY ROCK MECHANICS (ROCK(GEOLOGY) UNDERGROUNDI QUARTERLY OF THE COLORADO SCHJOL JF MINES 51 (3) D8-OT-O2243 SYMPOSIUM OV ROCK MECHANICS ( ROCK(GEOLOGY) UNDERGROUND/ QUARTERLY OF THE COLORADO SCHJOL JF MINES SI F TUNNEL MECHANICS $($ UNDERGROUND-STRUCTURES ROCKIGECLOGY)/ QUARTERLY OF THE COLORADO SCHJOL OF MINES G2(2) I3-13-D2275 PARTIAL BODY RADIATION OF QUEEN HONEY BEES ( HYMENOPTERA, \$TID-20299, 06-06-02208 EAR SURV/ UNESCO ANO PUBLIC OPINICN TODAY I UNITED-STATES QUESTIONNAIRES ATTITUDES IVTERNATIOVAL-AFFAIRS F OS-II -DIOG9 IFFAIRS - GERMANY 1 PURLIC-OPINI ON SURVEYS FOREIGN-POLICY QUESIIONNAIRES INTERNAT IONAL-AFFAIRS REARMAMENT/ O5- 11 -O2I86 IOMB AND ATOMIC ENERGY I PUBLIC-OPINION ATTITUDES SURVEYS QUESTIONNAIRES PEACE-KEEPING VUCLEAR-WARFARE AR/ O5- II -02I 96 TERNAT IOVAL-AFFAIRS PUBL IC-OPINION SURVEYS FOREIGN-POLICY OUESTIONNAIRES PEACE-KEEP IVG USSR II *FA- 11 I IN OS- 11 -02198 IABITABIL ITY DATA-PROCESSING-SYSTEMS TRAINING METHCDOLOGY QUESTIONNAIRES SHELTER-MANAGEMENT ATTITUDES, *I DS-IJ-DIGBD

RURAL-AREAS VULNERABILITY NUCLEAR-WARFARE-CASUALTIES SEX RACE MANAGEMENT-PLANNING SOCIAL-SCIEVCES I *RM- I I5-O6-OI 965 RADAR-IVTERFEREVCE RADAR-SIGNALS MAPS ELEC TRONAGNETIC-C/ RACAR MASKING BY EARTHS TERRAIN I SITE-SELEC TION I7-D9-DI988 NET IC-C/ RADAR MASKING OY CARTIIS ICRRAIN I SITE-SELECTION RADAR-INTFRFFRFNCF RADAR-SIGYALS MAPS ELECTROMAG I7-D7-DIQB8 ING BY EARTHS TERRAIN I SITE-SELECTION RADAR-INTERFERENCE RACAR-SIGNALS MAPS ELECTROMAGVETIC-COMPATIBILIT/ I7-D马-DI9B8 DING PROTECTIO/ DECONTAMINATION SCHEDULING PROCEOURES FOR RADEF SYSTEMS 1 RADIOLOGICAL-CONTAMIVATION SHIEL DO-IB-DI729 /RIMENTAL IVVESTIGATION OF A DYNANICALLY LCADEO RIAG WITH RACIAL ELASTIC SUPPORT 1 RINGS VIBRATION FREQUE $/ 20-11$-O2336 ADIAT ION-HAZARDS MUTATIONS RADIAT/ THE GENETIC EFFECTS OF RACIATION - POSTATTACK COVSEQUENCES 1 GENETICS R DG-I3-DI727 ITURES ATTAIVED IN WOOD CXFOSCD TO HICH INTENSITY THERMAL RA IIAT ION I C.ALORIMETERS IGNITION HIGH-TEMPERATI I3-I? -OIBI2 ES I RADIATION-INJURIES RADIOLOGICAL-DOSAGE EX/ LONG TERM RADIATION DAMAGE - EVALUATION OF LIFE SPAN STUDI DG-IB-OIT33 D FABRICS *WT-4D7 OPERATION BUSTER - THERMAL RADIATION EFFECTS ON PAINTS, PLASTICS, AND COATE I3-1?-02233 (TS SURVIVAL I EQUIPMENT) STRLCTLRES COMMUNICATICNS-SYSTEMS RACIATION GEOLOCY HUMAV-EVGIVEERIVG I \#SR-4S \#N/ I7-0?-D23II EY BEE I HYMENOPTERA GENETICS, \# YYO-2315-1 RACIATION INDUCED VIABILITY MUTATIONS IN THE HON DG-OS-D22O7 IC-20299 PARTIAL BODY RADIAIION OF OUEEN HONEY BEES I HYMEVOPTERA, \#T O6-O6-02208 S ATTEI WINDOW AVD WINDOW SCREENS AS MODIFIERS OF THERMAL RADIAT ION RELEASED IN NUCLEAR DETJVATIONS I GLAS $13-1$ - $-0 I 796$ IICE-OF-EMERGENCY-PLANNING SHELTERS ELEC TROMAGNETIC-PULSE RADIAT ION SHIEL CING RADIOACTIVE-FALLOUT RADIOLO/ I5-O3-O2299 1 OF NUCLEAR WAR I RADIOBIOLOGY RADIATION-EFFECTS ECOLOGY RAOIAT ION-DAMAGE RADIOLOGICAL-CONTAMINATION INSI O6-I3-OITZ3 CTIVE-FALLOUT SHIELDING RADIATION-HAZARDS DECCNTAMINATION RADIATION-EFFECTS, \#AGRICULTURE HANDBOOK 234 /A I5-03-DI897 /TACK CONSEQUENCES $/$ GENETICS RAOIATION-HAZARDS MUTATIONS RADIATION-EFFECTS ANIMALS HUMANS TESTS DOSE-RAT/ O6-18-DI72 7 IES USSR RADIAT ION-HAZARDS RADIOLOGICAL-DCSAGE DOSIMETERS RAOIATICN-EFFECTS CALCULATIONS DOSE-2ATE SHIELD/ O6-I3-OI726 ING TERM BIOLOGICAL EFFECTS OF NUCLEAR WAR I RADIOEIOLOGY RACIATION-EFFECTS ECOLOGY RADIATIOV-DAMAGE RADI/ O6-I3-DI723 IRFARE-CASUALTIES CASUALTIES CALCLLATIONS WEAPONS-EFFECTS RADIATION-EFFECTS ECOLOGY WIND RADIOACTIVITYIDI/ O6-2I -O2228 IUCLEAR ENERGY RESEARCH - 19661 BIOLOGY MEDICINE ECOLOGY RADIATION-EFFECTS GENETICS PHYSICS SCIENTIFIC-R/ 20-03-0207O IPORT - MECICAL DIVISION I METHDDOLOGY RADIATION-IAJURIES RADIATION-EFFECTS INSTRUMEVTATION RADIOISOTOPES/ DO-IB-O22IS NOL CANOES LANDSL IOES ELEC TRIC-POKER-PRODUCTICN PETRCLEUM RACIAT ION-EFFECTS MANPOWER EMERGEVEY-HEALTH-SER/ IS-D3-O22 99 IALTY COMMISSION ANNUAL REPORT JULY-1,1965-JUNE-30,1966 I RAOIAT ION-EFFECTS VUCLEAR-WARFARE-CASUALTIES JAI O6-I3-0I730 1-CASUALT IES CA SUALTIES MILITARY-STRATEGY WEAPONS-EFFECTS RADIAT ION-EFFECTS POPULATION PARTICLE-SIZE SHELI 06-2I -D2229 ON CO/ FALLOUT SHELTERS - CODES AND STANDARDS I SHIELDING RADIAT ION-EFFECTS RADIOACTIVE-FALLOUT CONSTRUCTI I3-13-02286 PULAT ION FAC IL ITIES DECONTAMINATION MATHENATICAL-AMALYSIS RACIAT ION-HAZAR IS $45 R I-4 U-4949-130$ ISOURCES PO I5-OS-023 ICLEAR ATTACK I RURAL-AREAS RAOI OACTI VE-FALLOUT SHIELOING RACIAT ION-HAZARDS DECONTAMINATION $2 A D I A T I O N-E F F /$ IS-D3-DI897 / RADIOLOGICAL-DOSAGE EXPOSURE RADIOLOGICAL-CCNTAMINATION RADIAT ION-HAZARDS MEDICINE RADIOISOTOPES RADIOA/ D6-I3-02222 IFFECTS OF RADIATION - POSTAITACK CONSEQUENCES I GENETICS RADIATION-HAZARDS MUTATIONS RADIATION-EFFECTS A/ OG-I8-OI727 1 ONOM ICS RECOVERY POSTATTACK-OPERATIONS DAMAGE-ASSESSMENT RADIAT ION-HAZ ARCS RADIOACTIVE-FALLJUT MODELS, , O5-O3-O21 28 /ERSONNEL DOSIMETERS DOSE-RATE RADIOLOGICAL-CCNTANINATION RACIATION-HAZARCS RADIOGRAPHIC-FILYIPROTECTION)/ I8-OS-02O2B /FFECTS AVD PROTECTIVE MEASURES I RADIATION-INJURIES USSR RACIATION-HAZARDS RADIOLOGICAL-DOSAGE DOSIMETER/ D6-I3-DI726 /LCULATIONS ARMED-FORCES-OPERATIONS RADIOBIOLOGY GEOMETRY RADIATION-HAZARDS RADIOLOGICAL-DOSE SIMULATION / I5-OS-DI964 1 RADIOLOGICAL-DOSE FARM-CROPS RADIOLOGICAL-CCNTAMINATION RADIAT ION-HAZARCS SHIELDIVG RADIOAGR ICULTURE, / O6-I8-O22I 7 ILOUT FOOD CDNTAM INA TION MODELSISIMULATI ONS) RADICEI OLCGY RACIATION-HAZ ARCS SOILS PLAVTSIBOTAVYI RADIOLOGI O6-13-OI725 INUCLEAR-WARFARE-CASUALTIES FIRST-AID WOUNDS-AND-INJURIES RADIATION-INJURIES BIBLIOGRAPHIES, \$JPRS-29,85/ O6-OJ-DI7D3 INSE-SYSTEMS CASUALTIES TENADA NUCLEAR-WARFARE-CASLALTIES RADIAT ION-INJUR IES POSTATTACK-OPEZATIONS PUBLIC/ O6-2I-DIZ3

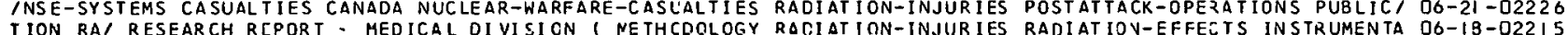

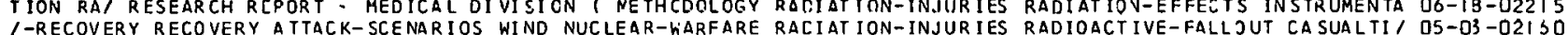

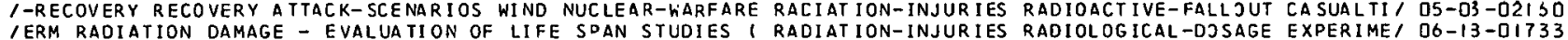
IERM RADIATION DAMAGE - EVALUATION OF LIFE SDAN STUDIES I RADIATION-INJURIES RADIOLDGICAL-DJSAGE EXPERIME/ O6-13-DI733
INUCL EAR-WARFARE-CASUALTIES JAPAN HIROSHIMA DATA NAGASAKI RADIATION-INJURIES RECOROS LIFE-SPAN, \$ABCC-O5/ O6-I3-OI73D IFIROSHIMA NAGASAK I GRAPHS BIOLOGICAL-BLAST-EFFECTS BURNS RADIATION-INJURIES SHIELCIVG URBAV-AREAS CASUAL/ O6-2I -O2227 IEAS DISTR IBUTION HIROSHIMA NAGA SAKI BURNS WEAPONS-EFFECT RAIIAT ION-INJURIES STAT ISTICAL-ANALYSIS BIDLOGI/ D6-2I -D2238 IGENTS - USSR I RADIOLOGICAL-WARFARE-AGENTS CCNTAMINATION RADIATION-INJURIES TOXICITY CYANO-ACIDS CARBON-I O6-I3-OI721 1 - PHYSICS, BIOLOGICAL ETFECTS ANO PROTECTIVE MEASURES 1 RADIATION-IN.IURIES USSR RADIATION-HAZARDS RADIOI O6-13-OI726 IS NUCLEAR-WARFARE-CASUALTIES DEMOGRAPHY BURST-CONDITIONS RADIAI ICN-INJURIES YIELDRADIDLOGICAL-DOSAGE WI/ IS-D6-DI962 ATING-PLANS POPULATION COMMANO-ANO-CONTROL-SYSTENS TABLES RADIATION-MEASUREMENT-SYSTEMS I *A.L. IO94 IOPER I5-O3-OI9J8 TAT ION RADIATION-MONI TORS DETECTION TESTS RADIO-TELEMETRY RADIAT ION-MEASUREMENT-SYSTEMS, \#TR-O6.020I. OO3 I8-OH-02026 TTIENTS RADIOACTIVE-FALL OUT GAMMA-RAYS RADIATION-MCNITORS RADIAT ION-MEA SUREMENT-SYSTEMS PER SONNEL DOSIMET, I8-DS-D2O28 ITIENTS RADA ICIRA IN CONCRETE DUCTS LATIONS DOSE-RATE SHIELDING RADIATION-MEASUREMENT-SYSTEMS RADIATION-MONITORS, \$AEC-TR-6634/EFFECTS CALCU DO-I3-DI726 I REMOTE RADIOLOGICAL MONITORING SYSTEM I INSTRUMENTATION RADIATION-MONITORS OETECTION TESTS RADIO-TELEME/ I8-O\$-O2O26 IONOXIDE VESICANTS POISONOUS-GASES LEWISITE GAS-DETECTORS RACIATION-MONITORS DOSIMETERS PROTECTIVE-CLOTHI/ DO-I3-OIT2I INITS I SHIELDING PATIENTS RADIOACTI VE-FALLOUT GAMMA-RAYS RACIAT ION-MONITORS RADIATION-MEASUREMENT-SYSTEM/ I8-DS-O2O28 ( PHOTONS SCATTER ING ALBEDO GAMMA-RAYS ANGLE-OF-INCIOENCE RAOIATIONICOBALT) BLAST-SHELTERS CYLINOER SISTEE/ I8-DS-O2029 ITER CONTENT, YIELD, MEDIUM, ANO OEPTH OF BURST ON CAVITY RADII I CRATERINE EXPLOSIOV-EFFECTS VUCLEAR-EXPI I8-O3-023I5 GN EXERCISE LIGHTSIILLUMINATIONI 8ATTERIES-AND-CCMFONENTS RACIO-COMMJNICATIOV-SYSTEMS I*GAZD-I4OU /E UESI I3-OI -D2252 IPPORT IVTRASTATE CIVIL DEFENSE OPERATIONS - CIRCA 19701 RADIO-COMMJNICAT IOV-SYSTEMS CJMMUVICATION-SYSTE 1 I7-02-O23I 3 ITEM ( IVSTRUMENTATION RADIATION-NONITORS DETECTION TESTS RADIO-TELEMETRY RADIATION-MEASUREMEVT-SYSTEMS I/ I8-D4-02026 YSTEMS SHELTERS, PROTECTING TILE RLRAL POPULATION AGAINST RAGINACTIVE CONTAMINATION 1 USSR CIVIL-DEFENSE-S DG- $18-0 I 731$ STEMS SHELTERS PROTECTING TIE RLRAL POPULATION AGAINST RACINACTIVE CONTAMINAT ION S IBIOL OGICAL AVA ILABILITY OF NUCLEAR EXPLOSI ON PRODUCTS IN RADIOACT IVE FALLOUT 1 DECAY-SCHEMES USSR PARTIC/ I8-D3-D2323 ASTES WASTESIINDUSTRIAL) SH/ THE TRANSPORTATION OF HIGHLY RAOIOACTIVE MATERIALS I CONTAINERS RADIOACTIVE-W I8-O7-02033
10 COSTS OF ACC IDENTS IN THE IRANSPORTATION OF HIGH LEVEL RADIOACTIVE MATERIALS I URBAN-AREAS DECONTAMINA/ I8-OT-O2O34 LOGY, \#ORNL-TR-975 INCORPORATI ON AND ELININAIICN OF RACIOACTIVE MATERIALS BY THE JRGAVISM I RADIOBIO D6-IS-DI728

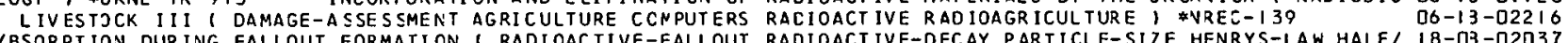
/BSORPIION DUR ING FALLOUT FORMATI ON I RADI OACTIVE-FALLOUT RADIOACT IVE-OECAY PARTICLE-SIZE HENRYS-LAW HALF/ I8-O3-D2O37 IFISSION PRODUCTS IN A SILICATE FALLOUT PARTICLE MATRIX I RADIOACTIVE-DECAY RADIOACTIVE-FALLOUT THERMODYN/ I8-D3-O2325

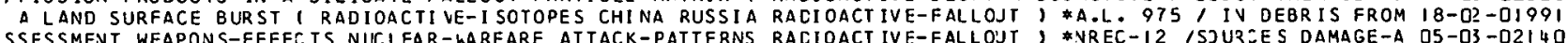
CTURES DOSE RATE MIOTOMS, SHIELOING IV - R.IVII DFFFNE I RADIOACTIVE-FALLOUT ATIENUATIJN UVDERGROUND-STRU IB-OS-D2O29 
/N-EFFECTS POPULATION PARTICLE-SI ZE SHELTERS DISTRIBUTION RADIOACTIVE-FALLOUT BIOLOGICAL-DOSAGE ATTACK-SC/ O6-2I-O2229 /CEFENSE-SYSTEMS WEAPOYS-EFFECTS NODELS EXPLOSION-EFFECTS RAOIOACTIVE-FALLOJT BLAST-SHELTERS MATHEMATICS, I 5-O3-DIQ38 INSULTIVG FIRMS WIIH CEPTIFIED FALLOUT SHELTER ANALYSTS 1 RADIOACTIVE-FALLOJT BUILOIVGS CDVSTRUETION DESI/ I3-13-OD2276 A REAL AND TWO SIMULATED FALLOUT FIELDS I RADIOACTIVITY. RAOIOACTIVE-FALLOUT CALCULATIJNS ARMEJ-FORCES-O/ IS-DS-OIOS 4 IATTACK-SCENARIOS WIND NUCLEAR-WARFARE RADIATICN-IAJURIES RACIOACTIVE-FALLOUT CASUALTIES DEMOGRAPHY AGE L/ OS-D3-D2I GO IMBO III. 1 RESOURCES VULNERABILITY WEAPONS-EFFECTS WIND RADIOACT IVE-FALLOST CLIMATOLOGY CASUALTIES FIREI OS-O3-D2I 34 ITERS - CODES AVD STANOARDS 1 SHIELOING RADIATION-EFFECTS RADIOACT IVE-FALLOJT COVSTRUCTION CJVSTRUETION-SI I3-13-O22B6 IFINERIES COVTAMINATED BY FALLOUT I POSTATTACK-OPERAIIONS RACIOACTIVE-FALLOUT COVTAYINATION INDUSTRIAL-PL/ O5-O3-OIG5I /ROJECT 62.80C AERORADIOACTIVITY SURVEY I WEAPONS-EFFECTS RADIOACTIVE-FALLOUT DISTRIBUTION DOSE-RATE GAMM/ I8-03-02324 IIMENTAL EVALUATION OF ROOF REDUC TION FACIORS I SHIELOING RADIOACTIVE-FALLOUT DOSE-RATES RADIOLOGICAL-DOS/ I8-OS-O2O3O /ALUATIOV OPERATIONS-RESEARCH MA THEMATICS WEAPONS-EFFECTS RADIOACTIVE-FALLOUT FALLOUT-SHELTERS MODELSISIM/ I5-03-DI935 IUILDINGS IN THE UNITEO STATES / CONSTRUCTION URBAN-AREAS RADIOACTIVE-FALLOUT FALLOUT-SHELTERS SHIELDING, I3-13-O2277 IPROGRAMS SHOCK-WAVES BLAST SHIELDING STRLCTURAL-ANALYSIS RADIOACTIVE-FALLOUT FIRE-SAFETY FALLJUT-SHELTER/ $13-13$-O228D RSONNEL ATTACK-PA TIERNS WIND YIELD BURST-CONDITIONS BLAST RACIOACT IVE-FALLOJT FLOW-CHART ING I WVREC-5, I PE OS-D3-O2IUO

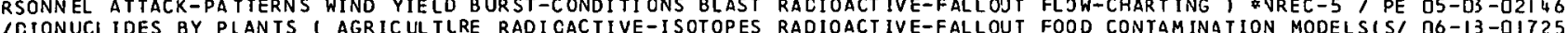
ICIONUCLIDES BY PLANTS I AGRICULTLRE RADICACTIVE-ISOTOPES RADIOACTIVE-FALLOUT FOOD CONTAMINATION MODELSIS/ O6-I3-OI725 IN EMERGENCY HEALTH SERVICES UNITS I SHIELOING PATIENTS RADIOACT IVE-FALLOUT GAMMA-RAYS RADIATION-MONITO/ I8-0S-O2O28
IETY FIRES STRUCTURES SMOKES BUILOINGS CONSTRUCTIOA BLAST RACIOACTIVE-FALLOJT HEAT-TRAVSFER EXPJSURE TOXI/ I3-I?-OO2269

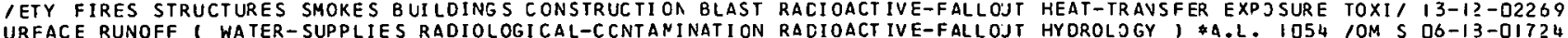
ITECT ION-FACTOR RADIOLOGICAL-DOSAGE RECOVERY CALCULATIONS RADIOACTIVE-FALLOUT MAVPOWER-STLDIES TIME, *US/ O6-I3-DI729 1. I. ANALYSIS, COMPARISON, AND CLASSIFICATION OF MCDELS, RADIOACTIVE-FALLOUT MATHEMATICAL-PREDICTION YIE, I8-O3-O2O39 IOSTATTACK-OPERAT IONS DAMAGE-A SSE SSMENT RADIATION-HAZARDS RADIOACTIVE-FALLOJT MODELS, \$SRI-IU-3O84 \$PB II O5-O3-OLI ULATIOVIDISTR IBUT ION) GEOG I ON DANAGE ASSESSMENT NCDELS I RACIOACT IVE-FALLOJT NUCLEAR-EXPLOSIOY-DAMAGE POP IS-DS-DI9S2 /SYSTEMS WEAPONS-EFFECTS EXPLOSION-EFFECTS BLAST-SHELTERS RADIOACT IVE-FALLOUT NUCLEAR-WARFARE-ZASUALTIES I IS-O3-0I933 ISITION AND REDISTR IBUTION OF FALLOUT AROUND STRUCTURES 1 RADIOACTIVE-FALLOUT PARTICLES DEBRIS VOLCANOES, I8-O3-O2O38 1 OD OF LOCATING FALLOUT ON A SPHERICAL EARTH I WIND YIELD RADIOACTIVE-FALLOUT PROGRAMMIVGICOMPUTERSI BURS/ I8-O3-O2322 I HELT TERS FALLOUT-SHELTERS NEVADA LEAPONS-TESTS SINULATION RADIOACTIVE-FALLOJT PRJTECTIOV-FACTOZ, *CEX-65/ I3-I3-OI822 IES, AND OPERAT IONS RESEARCH II. DAMAGED PF NULTI FLIER I RACIOACT IVE-FALLOUT PROTECTIOV-FACTOZ DOSE-RATEI O6-I3-O222I IED FISSION PRODUCT ABSORPIION DURING FALLOUT FORMATIONI RACIOACTIVE-FALLOUT RADIOACTIVE-DECAY PARIICLE-I I8-O3-O2D3? K ATMOSP/ SURVE ILLANCE NETWORKS I RADIOLOGICAL-MONITORING RADIOACT IVE-FALLOUT RADIOACTIVE-ISOTOPES AIR MIL I8-O3-O2D35 K ATMOSP SURVEILLANCE NETWORKS T RADIOLOGICAL-MONITORING RADIOACTIVE-FALLOUT RADIOACTIVE-ISOTOPES AIR MIL I8-O3-O2D3S

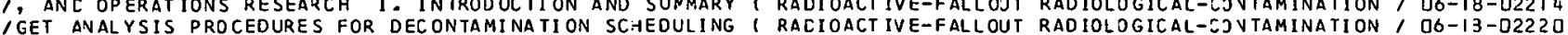

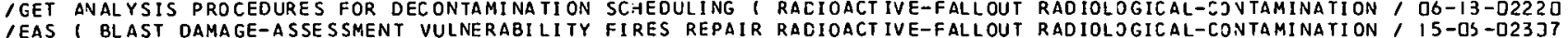

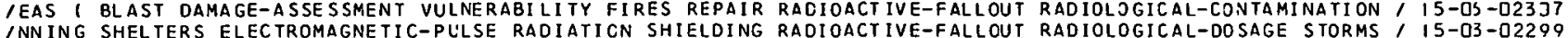
PARM SYSTEM MANUAL III-F. FALLOLT AND DECONTAMINATION I RADIOACTIVE-FALLOJT RADIOLOGICAL-DOSE FARM-CROPS O6-IB-O22I 7 IWASHCOWV SYSTEMS IFINAL REPORTI ICONSTRUCTICN-NATERIALS RACIOACT IVE-FALLOJT SHIELDIVG BUILOIYGS SHELTER/ I8-OS-O232I 1000 AND AGR ICULTLRE AGAINST NLCLEAR ATYACK I RURAL-DREAS RACIOACT IVE-FALLOUT SHIELDING RADIATION-HAZARDSI IS-D3-OI897 IURES I RADIOLOGICAL-CONTAMINATION PROGRANNING (COMPUTERS) RADIOACTIVE-FALLOUT SHIELDING WALLS COBALTEXPE/ OG-13-O22IB IAST RESOURCES PROGRAMMING-LANGUAGES C.NMPIITERS CASUALTIES RADIOACTIVE-FALLCJT THERMAL-RADIATIOV ) *NREC-S/ OS-D3-O2I54 , ECOLOGY WIND RADIOACTIVITY(DISTRIBUTION) RECOVERY JAPAN RAOIOACTIVE-FALLOJT THERMAL-RAOIATIOV BIOLOGICA/ O6-2I -O2228 IN A SILICATE FALLOUT PARTICLE MATRIX I RADI OACTIVE-DECAY RAOIOACT IVE-FALLOUT THERMODYVAMICS SJL UBILITY T/ I8-03-D2325 IIN WASTE WATER AND SEWER SYSTEMS I POSTATTACK-OPERATIONS RACIOACTIVE-FALLOUT WASTESISAVITAZY-ENGINEERING/ I3-O?-DI78D I DAMAGE RADIOLOGICAL-CONTAMINATION INSECTS PLANTS (BOTANY) RADIOACTIVE-FALLOUTISIMULATIOV) MODELSISIMULATI/ O6-I3-0I723 IE NETWORKS I RADIOLOGICAL-MONITORING RADIOACTIVE-FALLOUT RADIOACTIVE-ISOTOPES AIR MILK ATMJSPHERIC-PRECI/ I8-DQ-O2O35 ILIDE FRACTIONATION IN DFBRIS FRON A LAND SURFACE EURST, RADIOACT IVE-ISOTOPES CHINA RUSSIA $3 A D I O A C I I V E-F /$ I 8-0?-DIO9I NTAM INAT/ UP TAKE OF RADIONUCLIDES BY PLANTS I AGRI CULTURE RADIOACT IVE-ISOTOPES RADIOACTIVE-FALLOUT FOOD CO O6-I3-DIT25 NTAMINAT UPTAKE OF RADIONUCLIDES BY PLANIS I AGRICULTURE RADIOACTIVE-ISOTOPES RADIOACTIVE-FALLOUT FOOD CO O6-I3-OIT25

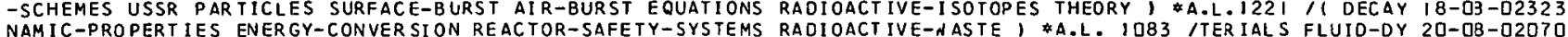
NAM IC-PROPERT IES ENERGY-CONVER SION REACTOR-SAFETY-SYSTEMS RADIOACTIVE-A ASTE I \#A.L. IU83 /TER IALS FLUID-DY 20-OB-O2DTO

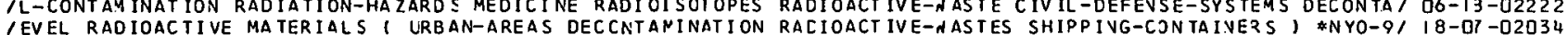
/EVEL RADIOACTIVE MATERIALS INSPORTATION OF HIGHLY RADIOACTIVE MATERIALS I CONTAINERS RADIOACTIVE-WASTES WASTESIINDUSTRIAL) SHIPPING-/ I8-OT-O2033
ICE DRINK ING WATER STANDARDS I WATER-SUPPLIES REGULATIONS RADIOACTIVITY CONTROL, \$PHS-956/IC HEALTH SERV I3-02-0I778 ICE DRINK ING WATER STANDARDS I WATER-SUPPLIES REGULATIONS RADIOACTIVITY CONTROL I \$PHS-956 /IC HEALTH SERV I3-02-DI778
S/ RACIOLOGICAL HEAL TH AND CIVIL DEFENSE I HEALTH-FHYSICS RADIOACTIVITY DETECTIOV RADIOLOEICAL-DOSAGE EXPO OO-I3-02222 INS PRODUCED BY A REAL AND TWO SINULATED FALLOUT FIELOS I RACIOACT IVITY RACIOACTIVE-FALLOUT CALCULATIONS, I5-OS-DIQS4 IION I RESOURCES VULNERABILITY NUCLEAR-WARFARE WINC BLAST RADIOACT IVITY THEORY TESTS EXPERIMENTAL-DATA I I O5-03-D2I 49 /CULATIONS WEAPONS-EFFECTS RADIATION-EFFECTS ECOLOGY WIND RADIOACTIVITY IOISTRIBUTION) RECOVERY JAPAN RADI/ OG-2I -O2228 III I DAMAGE-ASSESSMENT AGRICULTURE COMPUTERS RADICACTIVE RADIOAGRICULTURE, \#NRFC- 139 PS RACIOLOGICAL-CONTAMINAIIUN RADIATION-HAZARDS SHIELDING RACIOAGRICJLTURE I \#NREC-36/GICAL-DOSE FARM-CRO O6-I3-D22I7 ND EL IMIVATION OF RADIDACTIVF MATERIALS BY THE ORGANISM 1 RAOIOOIOLOGY, *ORVL-TR-975 INEORPORATION A D6-I3-0I728 IEUM RADIATION-EFFECTS MANPOWER EMERGENCY-HEALTH-SERVICES RADIOBIOLOGY FLOODS DISASTER-YEDICIVE TSUNAMIS , I5-03-O229O

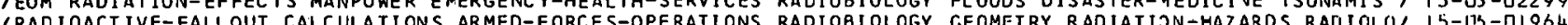
ITACK ENVIROVMENT I RECOVERY INFECII OUS-DI SEASES VACCINES RADIOBIOLOGY PUBLIC-HEALTH STZESS(PHYSIOLOGY) M/ DO-OJ-O22DG TTACK ENVIROVMENT I RECOVERY INFECIIOUS-DISEASES VACCINES RADIOBIOLOGY PUBLIC-HEALTH STRESS(PHYSIOLOGY) M/ D6-05-O22D6 IDUCT ION TO LONG TERM BIOLOGICAL EFFECTS CF NUCLEAR HAR 1 RACIOBIOLOGY RADIATION-EFFECTS ECOLOGY RADIATIO/ O6-I3-DI723 IDIOACTIVE-FALLOUT FOOD CONTAMINA TION MODELS(SIMULOTIONS) RAOIOBIOLOGY RADIAT ION-HAZARDS SOILS PLANTSIBOT/ O6-13-DI725 RS DOSE-RATE RADIOLOGICAL-CONTAMINATION RADIATION-HAZARDS RADIOGRAPHIC-FILM(PROTECTION) I \#A.L. IO45 /ME TE IB-0S-O2028

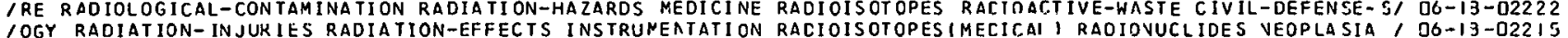
IOGY RADIATION-INJURIES RADIATION-EFFECTS INSTRURENTATION RACIOISOTOPESIMECICAI I RAOIDVUCLIDES VEOPLASIA / O6-IJ-O22IS ILINICAL PICTURE AND THERAPY IN AFFECTIONS WITH TOXIC AND RACIOLOGICAL AGENTS-USSR I RADIJLOGICAL-WARFA/ O6-I3-OI72I
HYSICS RADIOACT IVITY DETECTION RADIOLOGICAL-DOSAGE EXPOS/ RADIOLOGICAL HEALTH AND CIVIL DEFENSE I HEALTH-P O6-I3-02222 RADIATI/ IVSTALLATION AND IESTING OF AN AUTOMATIC REMOTE RADIOLOGICAL MONITORING SYSTEY I IVSTRUMENTATION IB-DH-O2O2O NL OPERAT IONS RESEARCH I. CALCULATIONAL TECHNIQUE FOR D/ RACIOLOGICAL RECOVERY REQUIREYENTS, STRUCTURES A OG-I3-O22IB

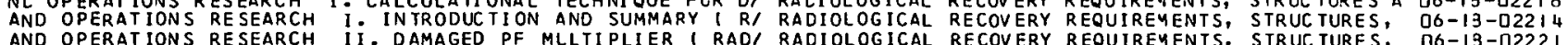
NTAM INATION 'SCHEDLLING I RADIOACTI VE-FALLOUT, SUNNARY OF RACIOLOGICAL TARGET ANALYSIS PROCEDURES FOR DECO OG-I3-D2220 IONS) RADIOBIOLOGY RADIATION-HAZAROS SOILS PLANTSIBOTANY) RADIOLOGICAL-CONTAMINATION, \#SRI-MU-SOO5, MULAT OO-I3-OI?25 IMEANS - USSR I WATER-SUPPLIES PURIFIT.ATION CONTAMINATION RADIOLOCICAL-CONTAMINAT ION CHEMICAL-WARFARE-AGE, D6-I9-OI722 IEARCF I INTRODUCTION AND SUMMARY I RADIOACTIVE-FALLOUT RAOIOLOGICAL-CONTAYINAT IOV DECONTAYIVATION PROTI DS-I3-O22IL IRES FOR DECONTAMINATION SCHEDULING I RADIOACTIVE-FALLOUT RAOIOLOSICAI-CONTAMINAT ION DOSE-RATE COUNTER 作 IONADIOBIOLOGY RADIATION-EFFEC TS ECOLOGY RADIATION-DAMAGE RADIOLOG ICAL-CONTAMINAT IOV INSECTS PLANTSIBOTAN/ IS-II-DI8O4

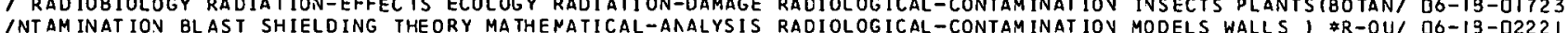

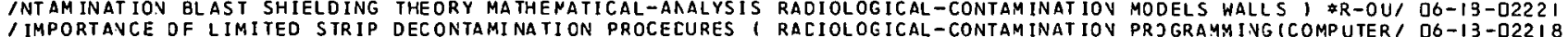

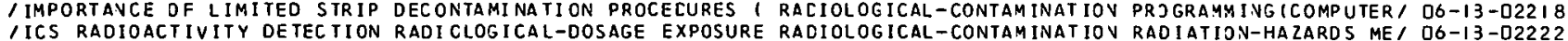
IICS RADIOACTIVITY DETECTION RADI CLOGICAL-DOSAGE EXPOSURE RADIOLOGICAL-CONTAMINATIOV RAOIATION-HAZARDS ME I D6-I3-D2222 IATION-MEASUREMENT-SYSTEMS PER SONNEL DOSIMETERS DOSE-RATE RADIOLOGICAL-CONTAMINAT ION RADIATION-HAZARDS RA/ I8-OS-D2028 IATION I RADIOACT IVE-FALLOUT RADIOLOGICAL-DOSE FARN-CROPS RADIOLOGICAL-CONTAMINAT ION RADIATIOV-HAZARDS SH/ D6-I8-O22I? /LLOUT CONTAMINATION FROM SLRFACE RUNOFF I WATER-SUPPLIES RACIOLOGICAL-CONTAMINATIOV RADIOACTIVE-FALLOUT, D6-I3-DI724 /SSESSMEVT VULNERABILI IY FIRES REPAIR RADIOACTIVE-FALLOUT RADIOLOGICAL-CONTAMINATIOV RESOURCES POPULATION/ I5-OS-O23O7 IVIL-DEFENSE-SYSTEMS SHELTERS INTERNATIONAL-CIVIL-DEFENSE RADIOI NFICAL-CONTAMINATION RURAL-ARCAS HARDENIN/ OS-I3-OI73I IWARFARE-AGEVTS NUCLEAR-WEAPONS FIRST-AID WARNING-SYSTEMS RACIOLOGICAL-CONTAMINAT IOV VETERIVAZY-MEDICINE / IS-O3-OIQI 8 /ROTECT IOV-SYSTEM S WA TER-SUPPLIES CHEMICAL-WARFARE-AGENTS RACIOLOGICAL-CONTAMINAT IOVICOUNTERYEA SURE SI BIO/ 15 -0? -OI84 3 / LOGISTICS WATER-SUPPLIES RATIONING SANI TARY-ENGINEERING RADIOLOGICAL-CONTAYINATIOVIFOJD) IVFAVTSIDIET), OG-D3-DI 7 I 2 ICTION FACTORS I SHIELDING RADIOACTIVE-FALLOUT DOSE-RATES RADIOLOGICAL-DOSAGE ATTENUATION SIMULATIONIMODEI IB-OS-D2D3D IIVE MEASURES (RADIATION-INJURIES USSR RADIATICN-HAZARDS RADIOLOGICAL-DOSAGE DOSIMETERS RADIATION-EFFEC T/ OG-IS-DI726 IIVE MEASURES ( RADIATION-INJURIES USSR RADIATICN-HAZARDS RADIOLOGICAL-DOSAGE DOSIMETERS RADIATION-EFFECT/ O6-I3-DI726
ACIOB IOLOGY PUBLIC-HEALTH STRESS (PHYSIOLOGY) NALNUTRITION RACIOLOGICAL-DOSAGE EPIDEMIOLJGYI *2M-5362-PR, O6-05-O22O6 
IE - EVALUATION OF LIFE SPAN STUDIES ( RADIATICN-INJURIES RACIOLOGICAL-DOSAGE EXPERIMEVTAL-DATA AGING IPHYI DO-I3-OI733 ID CIVIL DEFENSE I HEALTH-PHYSIC S RADIOACTIVI TY DETECTION RADIOLOGICAL-DOSAGE EXPOSURE RADIOLOGICAL-CONTAI DG- IB-O2222 ICONT AMINAT INAT WAT RA AROMAGNETIC-PULSE RADIATIION SHIELDING PROTECTION-FACTOR RACIOLOGICAL-DOSAGE RECOVERY CALCULATIONS RADIOI OO-I3-OIT29 IES DEMOGRAPHY BURST-CONDIIONS RADIATION-INJURIES YIELD RADIOLOGICAL-DOSAGE WIND FIRES SIMULATION BLASTI IS-DG-DI962 IIII-F. FALLOUT AND DECONTAMINATICN I RADIOACTIVE-FALLOUT RACIOLOGICAL-DOSE FARM-CROPS RADIOLOGICAL-CONTAI D6-I3-O22I 7 FORCES-OPERAT IONS RADIOB IOLOGY GEOME TRY RADIATION-HAZAROS RADIOLOGICAL-OOSE SIMULATION I FAFRRI-SPGG-I ID- I5-OS-DI964 / SOURCES MANAGEMENT-PLANNING CIVIL-DEFENSE-SYSTEMS RODELS RACIOLOGICAL-MONIIORING LOGISTICS MANPOWER SHEL/ I5-O3-DIQ49 OACT IVE-I SOTOPES AIR MILK ATMOSP/ SUR VEI LLANCE NETWORKS I RACIOLOGICAL-MONITOR ING RADIOACTIVE-FALLOUT RADI I 8-O3-O2O35 IN AFFECTIONS WITH TOXIC AND RAOIOLOGICAL AGENTS - USSR I RACIOLOGICAL-WARFARE-AGENTS CONTAMINATION RADIAI DG-I3-DI72I /SURES POSTATTACK-OPERATIONS MANAGEMENT-PLANNING SLRVIVAL RADIOLOGICAL-WARFARE(RECOVERY) ECOVOMIC-RECOVER/ IS-O3-DI953 IACE I THERYAL-RACIATION CALOR IME IERS LABORATORY-FURNACES RADIOMETERS HEAT-FLUX HEAT-TRANSFEZ PYROMETERS I I3-O1-O2ZSS ACE THERYAL-RACIATION CALOR IME TERS LABORATORY-FURNACES RADIOMETERS HEAT-FLUX HEAT-TRANSFER PYROMETERS 1 I 3 -OI - D2255 IIAT ION I CALOR IMETER S IGNITION HIGH-TEMPERATURE-RESEARCH RACIOMETERS PYROLYS IS SOLAR-FURNACES COMBLSTIONI I3-I? -QIBO2 SURFACE BURST I RADIOAC TIVE-I SOTOPES CHINA RUSSIA RADIO/ RADIONUCLIDE FRACT IONATIOV IN DEBRIS FROM A LAND I8-0? -DI991 E-ISOTOPES RADIOACTIIVE-FALLOUT FOOD CONTANINAT/ UPTAKE OF RADIONUCLIDES BY PLANTS I AGRICULTURE RADIOACTIV O6-I9-Q1725

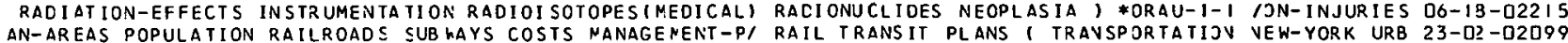
INSI TRAVSPJRTATION AIR-TRANSPORTATI ON BUS-TRANSPORTATI ON RAIL-TRANSPORTATIJN HIGHWAY-TZANSPORTATION I \#PI 23 -02 - O2O9I MPET IIIUN D I A SYSTEM ANALYSIS OF THE EFFECTS OF NLCLEAR ATTACK ON RAILROAD TRANSPORTATION IV THE COVTIVENTAL UNITE US-US-ULI 28 IICEO-MISSILES RAILROADS BRIDGES GROUND-SLPPORT-EQUIPNENT RAILROAC-TRACKS ROACS USSR UVDERGZJUND-STRULTUR/ I O-OI -OIOT2 T-PLANVIVG URBAN-PLANNING hASHING TON BOSTCN N/ HIGH SPEEO RAILROACS I TRANSP JRTATION URBAN-AREAS. MANAGEMEN 23 -D? -O2I JO

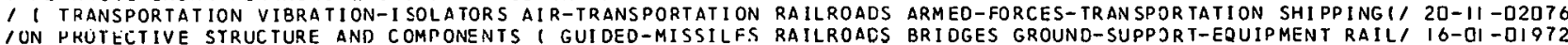
I MODES ( CARGO POSTATTACK-OPERATIONS VULNERABILITY ROADS RAILROACS CARGO-VEHICLES AIRCRAFT HEAPOVS-EFFEC, I3-OS-OZ2ZOD NICES, RAPID-TRANSIT-SYSIEMS TRANSPORIATION URBAA-AREAS RAILROACS MONORAILS MOVIVG-BEL THAYS HISTORY, $/ 23$-D?-O2356

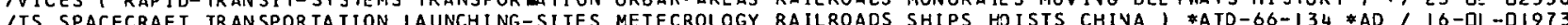
TT PLANS I STATIST ICAL-DISTR IBUTIONS PROBABILI TY-DENSI TY-FUNCTIONS RANDOM-VARIABLES NEAPOV-SYSTEMS AVALYSIS-OF-VAR/ I2-DI - D225I ERABIL I URBAN-AREAS, I NATIONAL CAPITAL TRANSPORTATION AGENCY RAPID TRANSIT SYSTEM - MAVUAL OF DESIGN CRITERIA $23-0$-OZ2353 UE/ PRASE III SUBSURFACE INVESTIGATION - NATICNAL CAPITAL RAPID TRANSIT SYSTEM PORTION JF CONNECTICUT AVEN I3-D2-DI7BS RAILROADS MONORAILS MO/ INNOVATIONS IN TRANSIT DEVICES 1 RAPID-TRANSIT-SYSTEMS TRAVSPORTATIOV URBAN-AREAS $23-02$-D2356 SHELTERS FIRE-SAFETY F/ DEVELOPMENT OF STANDARD FIRE TEST RATING SYSTEMS FOR SHEL TER COYPONEVTS 1 FALLOUT- I3-I?-O2259 ILOUT-SHELTERS SHEL TER-OCCLPANCY LOGISTICS WATER-SUPPLIES RATIONING SANITARY-ENGINEER IVG RADIOLOGICAL-CON/ D6-D3-OI7I 2 PROPERTIES CO/ STORAGE STABILITY OF CIVIL DEFENSE SHELTER RATIONS 1 FOOD PRESERVATION FIBERBOARD PHYSICAL- DG-O3-O22I 2 OUT-SHELTERS TASTE CIVIL-DEFENS/ ACCEPTABILITY OF SHELYER RATIONS IN COMBINATION WITH ADJUNCTS I FOOD FALL O6-03-D22II PTAR IL ITY) FALLUT-SHELTERS CON ACCEPTABILI TY OF SHELTER RATIONS IN COMBINATION WITH ADJUNCTS I FOODIACCE DO-OB-DI7ID PTAE L-COSLGE EXPER IMENTAL-DATA AGING (PHYSIOL OGY) ANIMALS MICE RATS DOSE-RATE) \#RM-5083-TAB IURIES 2ADIOLOGICA DG-IS-DI733 IAST I BLAST-BIOLOGY SHOCK-WAVES BIOLOGICAL-BLAST-EITECTS RATS CUINEA-PIGS DJGS GOATS SHOCK-TUBES I *DASAI O6-2I -O2236 11 METABOL ISM DEFICIENCY-DISEASES TI SSUES (BIDLOGY) TESTES RATS MORPHOLOGY (BIOLOGY) HISTOLOGY ESJPHAGUS SK/ D6-OS-OITQI

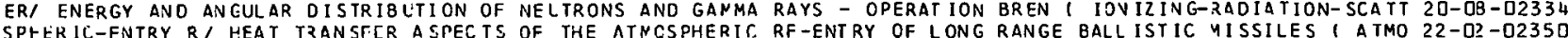

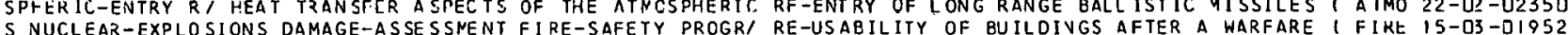
AST RESOURCES PROGRAMMING-LANGLAGES COMPUTERS CASUALTIES/ REACT, A REQUEST LANGUAGE I DAMAGE-ASSESSMENT BL DS-O3-O2IS4 IIONIZATION, AIRBURST IONS POLAR-REGIONS ABSORPTION OZONE REACTION-KINETICS NUCLEAR-RADIATIJV COSMIC-RAYSI O4-DI -O2II ERSONAL INTEVTIOVS ( BEHAVIOR MOTIVATION CULTURE RESPONSE REACT IONIPSYCHOLOGY), /OLOGY) CASUALTIES MOTIVATION PUBLIC-INF ORNATI ON TRAINING REACT ION(PSYCHOLOGY) ADMIVISTZATIOV-AND-MANAGEM/ OS-IJ-DI G3I IONNEL FOOD CLO THING ENVIRONMENT EDUCATI ON ARMY-EQUIPMENT REACTIONIPSYCHOLOGY) SOCIAL-PSYCHOLOGY ARMED-FO/ O5-IJ-D2I8I ERENT IAL EARTH DISPLA / PRELIMINARY CONCEPTS OF SUBSURFACE REACTOR CONTAINMENT STRUCTURES TO WIIHSTAND DIFF I8-D9-O2D4D ITRUCTURES TO WITHSTAND DIFFERENTIAL EARTH DISPLACEMENT I REACTOR-FEAS IBILITY-STUDIES REACTJP-SAFETY-SYST/ IB-OQ-O2OLO TTOR-MATERIAL S FLLID-DYNAMIC-PROPERTIES ENERGY-CONVERSI ON REACTOR-SAFETY-SYSTEMS RADIDACTIVE-WASTE, \#A.L/ 2D-OB-O2O7D

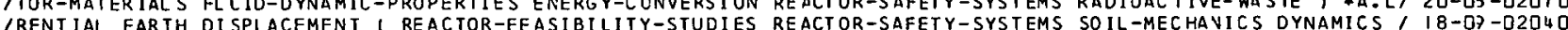

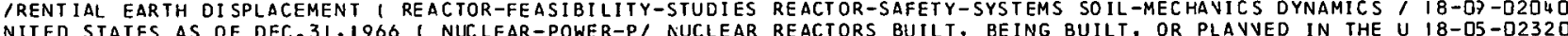
NITED STATES AS OF DEC.31, 1966 ( NUC LEAR-POWER-P/ NUCLEAR REACTORS BUILT, BEING BUILT, OR PLAVVED IN THE U I8-O5-D2320

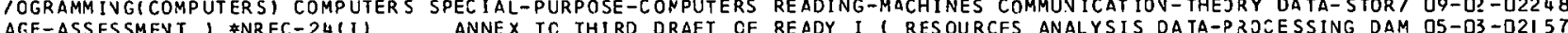
AGE-ASSESSMEVT, \#NREC-24II ANNEX TC THIRD DRAFT CF READY I I RESOURCES ANALYSIS DATA-PROCESSING DAM O5-O3-D2I57 GN PRUGKAMMINGICOMPUTERSI MATIICMAIICAL-MODELS FLOW-CHART/ READY I I RESOURCES DAMAGE-ASSESSMENT LOGIC-DESI D5-O3 -D2I 55
IE ANGLE AND ENERGY DISTRIBUTION OF PHOTONS PRODUCED BY A REAL AND TWO SIMULATED FALLOUT FIELDS I RADIOAC, I5-OS-OI9G4 IE ANGLE AND ENERGY DISTRIBUTION OF PHOTONS PRODUCEO BY A REAL AND TWO SIMULATED FALLOUT FIELDS I RADIUAC' I5-OS-OI964
/FLOOOS CIVIL-DEFENSE-OPERATING-PLANS REPAIR CONSTRUCTION REAL-ESTATE REFUGEES BANKIVG INSURAVCE LABOR LA' DS-O3-DIG36 RIUM THEORY TO DECDUPL. ING I CRATERINJ EXPLOSICN-EFFECTI A REAPPRAISAL OF THE APPLICATIOY OF STATIC EQUILIB IB-O3-D23IO RVEYS FOREIGV-POLICY QUESTIONNAIRES INTERAATIONAL-AFFAIRS REARMAMENT, \#FA-3, GERMAVY I PUBLIE-OPINION SU O5-II -O2IB6 FECT ING GOVERNMENT CONTROL IN SOUTH VIET/ LAND TENURE AND REBELLION - A STATISTICAL ANALYSIS OF FACTORS AF O5-DY-O2I75 $N$ I AGR ICULTURE S/ COMMODITY CREDIT CORPORATION WAREHOUSE RECEIPT WHEAT INVENTORY BY WAREHOUSE AND LOCATIO O2-OZ-O2IOQ

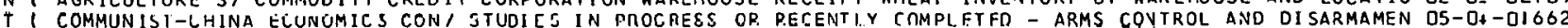
T BIBL IOGRAPHIES ECONOMICS COMM I STUDIES IN PROGRESS OR RECENTLY COMPLETED - ARMS COVTROL AND DISARMAMEN US-UH -UIOSS T C I B IBL IOGRAPHIE S ECONOMICS CONF I STUDIES IN PROGRESS DR RECENTLY COMPLETED - ARMS COVTROL AVD DISARMAMEN O5-O+ -0I665 FIRES AIRBURST STRUCTURES VULNERABILITY RECOVERY DAMAGE RECLAMATION URBAN-AREAS MODELSISIMULATIONSI GRAI IS-US-DI963
482 MUZZLE BRAKES II. THEORY I GUN-AUXILIARY-EQUIPMENT RECOIL-MECHANISMS INIERIOR-BALLISTICS,

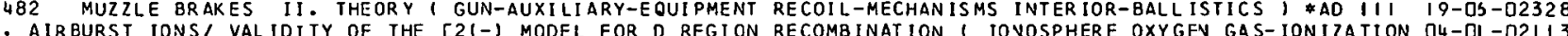

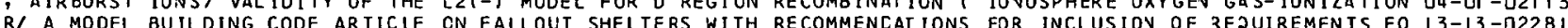
RUCTION MOSTATIACK-OPERATIONS REPAIR MILI TARY-ENGINEERING RECONNAISSANCF, *JPRS-31,014*TI-65-3I5I2 /ONST I3-I?-0I8J4 /RK IN A NUCLEAR STRIKE ZONE I CIVIL-DEFENSE-SYSTENS USSR RECONNAISSANCE INTERNATIONAL-CIVIL-DEFENSE POST/ DG-OT-DI7OQ LITIES RESERVES PRODUCTION TRA/ GAS FACTS - A STATISTICAL RECORO OF THE GAS JTILITY INDUSTRY IV I965 I UTI O5-D3-DI642 LANN ING CIVIL-DEFENSE-OPERATING-PLANS DATA IDENTIFICATION RECOROS, \$AD 642527 /ER-SUPPLIES) 4 ANAGEMENT-P I5-O3-OI94 I OPERATING-PLAVS PREPARATION MANAGEMENT-PLANNING OPERATION RECORDS, \#FG-E-13.6 /-OPERATIONS CIVIL-DEFENSE- D5-II-D2IBO ACK -OPERATIONS MANAGEMENT-PLANNING RECOVERY VULNERABILITY RECORDS DISTRIBUTION(WATER) SUR VEYS ) \#A.L. 969 I3-O2 -DI788 SUALTIES JAPAN HIROSHIMA DATA NAGASAKI RADIATION-INJURIES RECORDS LIFE-SPAN I \#ABCC-65-66 /LEAR-WARFARE-CA DO-IB -OIT3O STE NG MEDICAL-SUPPL IES DEFECTIONS TRAINING SL UNITE/ AN EVALUATION OF THE ROLE OF FEDERAL PERSONNEL IN RECRUITING SHELTER MANAGERS I SHELTER-MANAGEMENT O5-D? -0I675 IL-DEFENSE-SYSTEMS EARLY-WARNING-SYSTEMS NATI CNAL-DEFENSE RECRUIT ING TRAINING WARFARE CIVIL-DEFENSE-PERSO/ I5-D3-D230S

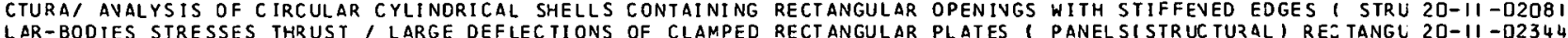

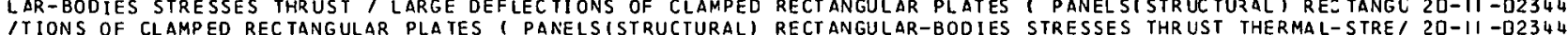
ITERNATIONAL AFFAIRS I ARMS-CONTROL COLO-WAR NATO VIETNAM RED-CHINA FOREIGN-POLICY FOREIGN-AID LABOR UNITI D5-O $4-02 I 72$ IARENA - TECHNIQUES FOR THE MEASUREMENT OF DEPOSITION AND REOISTRIBUTION OF FALLOUT AROUND STRUCTURES I RI IB-OS-D2O3R T COSE-RATES RADIOLOG/ AN EXPERIMENTAL EVALUATION CF RCCF REOUCT ION FACTORS I SHIEL DIVG RADIJACTIVE-FALLOU I8-OS-O2O3O ITRY OF LONG RANGE BALLISTIC MISSILES I ATMOSPHERIC-ENTRY REENTRY-VEHICLES DESIGV AERODYVAMIC-HEATING HEA' 22 -O2 -O235O OVERY FROMI CIVIL DEFENSE BIBLIOGRAPHY - A COMPILATION OF REFERENCES RELEVANT TO THE STUOY OF SOCIETAL REC OS-IJ-OIGT7 OPCRATIONS RADIOACTIVE-FALLOUT CDN/ RFCOUERY OF PETROLEUM REFINERIES CONTAMIVATED BY FALLOUT I POSTATTACK- OS-O3-OIG5I 
- BLAST-EFFECTS / THE TOLERANCE OF CATTLE TO LCNG DURATION REFLECTEC PRESSURES IN A SHOCK TUBE I BIOLOGICAL D6-2I -02237 IRATORS INSIRUMEN TATION TRANSDLERS OSCI LOSCOPES CAMERAS REFLECT ION REFRACT ION WAVE-TRAYSMISSION THEORY, DB-II -D224 7 TRATS

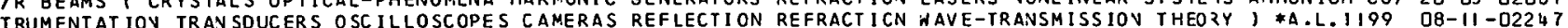
/-DEFENSE-OPERATING-PLANS REPAIR CONSTRUCTION REAL-ESTATE REFUGEES BANKING IVSURANCE LABOR LAW TRAFFIC I, O5-O3-DIG36 IZATION, AIRBUR ST IONS/ VALIDITY OF THE O2(-) MODEL FOR D REGION RECOMBINATION 1 IOVOSPHERE JXYGEN GAS-ION Q4-OI-O21I3 INSE COMMUNICATIONS REQUIREMENTS AT THE LCCAL, STATE, AND REGIONAL LEVELS I COMMUNICATIJVS-SYSTEMS COMMAN/ I7-O? -023I 2 IOMIC-COVDITIONS POPULATION AGRICLLTURE PCLITICAL-SCIENCE REGIONAL-PLANNINE INDIA IVTERVATIJVAL-AFFAIRS C/ OS-O3-02IGI TION AGR ICULTURE POLITICAL-SCIENCE REGIONAL-PLANNING IND/ REGIONALISM IN ASIA I ECOVOMIC-COVDITIONS POPULA OS-D3-02IOI TAT TACK-OPERAT IONS CI IIL-DEFEN SE-OPERA / ENERGENCY MELFARE REGISTRATION AND IVQUIRY I SHELTER-OCCUPANCY POS OS-II-D2I BQ ITEMS MULTIVARIATE-ANALYSIS STATISTICAL-ANALYSIS VARIANCE REGRESSION-ANALYSIS MEDICIVE BIOLOGY I *A.L. III D6-D3 -022 OU R CLASS A AVD B ELECTRIC UTILITIES I ECONCNICS ACCCUNTING RE CULATIONS, \#A.D. I0O4 1 SYSTEM JF ACCOUNTS FO OS-D3-DI63I TS FOR CLASS A AND B GAS UTILITIES I ECONONICS ACCCUNTING REGULATIONS, \$A.L. IOOS /IFORM SYSTEM OF ACCOUN OS-D3-OIO32 ITED-STATES) MILITARY-GOVERNMENT EMERGEN/ CODE OF FEDERAL REGULATIONS - NATIONAL DEFENSE I AZMED-FORCESIUN I5-D3-OI946
SYSTEMS, \#A.L. 96I. CODE OF FEDERAL REGULATICNS - TELECOMMUNICATIJV I COMMUNICATION- I7-OZ-OI978

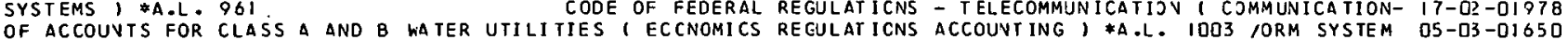

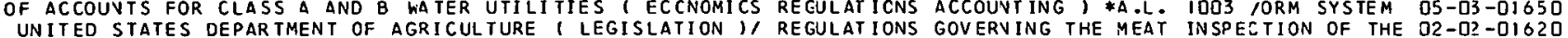
HEALTH SERVICE DRINKING WATER STANDARDS I WATER-SUPPLIES REGULATIONS RADIOACTIVITY CONTROL, \#PHS-956 /IC I3-D2-0I778 E OF THE DISTRICT OF COLUMBIA, WASHINGTON 8 UILDING-CODES REGULATIONS STRUCTURES BUILDIVGS, *A.L. IDDI ID I3-I3-OIBI7 IILCING CODE OF THE DISTRICT OF CCLUMBIA I BUILDING-CODES REGULATIONS NAS INGTON BUILOIVGS STRUCTURES, $/$ I I - I3-DIBIO TIONAL SYMPOSIUM I LOADING IMECHANI/ FLEXURAL MECHANICS OF RE INFORCED CONCRETE - PROCEEDINGS JF THE INTERNA $20-11$-02339 BEAMS (STRUCTURAL) LOADINGIMECH/ DYNAMIC SHEAR STRENGTH OF REINFORCED CONCRETE BEAMS II. 1 TEST-EOUIPMENT I3-I3-0I84 I NAMICS SHEAR-STR/ CYNAMIC SHEAR RESI STANCE OF TYIN WEBBED REINFORCED CONCRETE BEAMS I BEAMSISTRUCTURAL) DY $20-11-02337$ DS I CESIGN BE/ STUDY TO DETERMINE THE OPTIMUM SECTICN OF REINFORCEC CONCRETE BEAMS SUBJECTED TO BLAST LOA I3-13-0I84D IGN THEORY BEAMSISTRUCTURAL FRAMES LOADIN/ DEFLECTIONS OF RE INFORCED CONCRETE FLDOR SLABS I STZUCTURES DES $20-11$-O234D ICS) UNDERGROUND- STRUCTURES SHELTERS. PE/ RESPCNSE CF OEEP REINFORCED CONCRETE SLABS I TESTS LOADING IMECHAN I3-I3-D229D CONSTRUCTION UVOERGROUND-STRLCTLRES BLASTING EXCAVATION REINFORCED-CONCRETE ROCK-STABIL IZATION SHOTCRET/ I3-I3 - O22TU /COORS UNDERGROUND-STRUCTURES STRLCTURAL-PROPERTIES STEEL REINFORCEC-CONCRETE SHELTER-DJORS TRAFFIC STRUC/ I3-I3-DI835 DUSTR IES MAYPOWER ECONO/ PROJE DUSTRIES MAYPOWER ECONO / PROJECTICNS 1970 - I NTERINDUSTRY RELAT IONSHIPS, POTENTIAL DEMAVD, EYPLJYMENT I IN O5-D3-0I649 IOOW AND WINDOW SCREENS AS MODIFIERS OF THERMAL RACIATION RELEASEC IN NUCLEAR DETONATIOVS I GLASS ATTENUA/ $13-1 ?-0 I 796$ ICAL STUDY OF BEHAVIORAL AND SEMANTIC DIFFERENTIAL SCALES RELEVANT TO INTERCULTURAL NEGOTIATIONS I ATTITU/ OS-IJ-O2I78 CIVIL DEFENSE BIBL IOGRAPHY - A COMPILATION OF REFERENCES RELEVANT TO THE STUCY OF SOCIETAL $2 E C O V E R Y$ FROM O5-ID-OI677 MOCEL - VARICUS O APPLICATION T RESO/ AN ANALYSIS OF THE RELIABILITY OF THE RISK II COUPUTER STATISTICAL DS-D3-D2I 49 /HIGH EFFICIENCY PARTICULATE AIR FILTERS ( FILTERS (FLUID) RELIABILITY QUALITY-CONTROL SPECIFIEATIONS I *O/ I3-II -OI794 /SHOCK TESTS I NAVAL-RESEARCH DESIGN ELECTRONIC-EQUIPMENT RELIABILITY(ELECTRONIC) NAVAL-VESSELS SHOCK (MEC/ 20-II -02O83 CS EDUCATION POLITICAL-SCIENCE GEOGRAPHY HISTORY LANGUAGE RELIGION, \#EXTERNAL RESEARCH LIST 4.25 /ECONOMI OS-11-DI695 CIENCE HISTORY LANGUAGE LAh PUBLIC-INFORMATI ON PHILOSOPHY RELIGION, FEXIERVAL RESEARCH LIST 6.25/TICAL-S O5-II -DI 696 IIONS TRA INING SLEEP WATER FOOD RFC.RFATI ON EXERCISE NOISE RELICION TLMP[RATURE-CONTRJL VENTILATIUN I \#A.L/ O5-IJ-D2IB4 NTATION RADIATI INSTALLATION AND TESTING OF AN AUTOMATIC REMOTE RADIOLOGICAL MOVITOR ING SYSTEM I INSTRUME I8-OI-O2O26 S COOPERATION OISASTER-MEOICINE ENERGENCY-HEALTH-SERVICES REPAIR / IJPRS-17,889 $\$ 0 T S-63-2$ I232 /TER-SUPPLIE IS-03-02296 / COVERY HOUSING FOOD FLOODS CI VIL-DEFENSE-OPERATING-PLANS REPAIR CONSTRUCT IOV REAL-ESTATE REFUGEES BANKIN/ OS-O3-DI 36

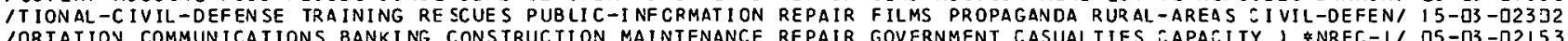
IAGE-ASSESSMENT MODELS RESOURCES CAPACITY DECCNTAMINATION REPAIR GOVERNMENT HOSPITALS HOUSIVG AGRICULTUREI O5-D3 - D2ISI IAGE-ASSESSMENT MODELS RESOURCES CAPACITY DECCNTAMINATI ON REPAIR GOVERNMENT HOSPITALS HOUSIVG AGRICULTURE/ O5-D3 - D2I I I ROL MORTUARY-SERVICES PUBLIC-HEALTH INDUSTRIES SANITATION REPAIR NANPONER I \#SRI-MU-4536 NNATIOV PEST-CONT I5-03-01953 IL-CONTAYINAT ION FIRES CONSTR UCTI ON POSTATTACK-OPERATICNS REPAIR MILITARY - EVGINEERIVG RECOVVAISSAVCE I \#J' I3-I?-OI8J4

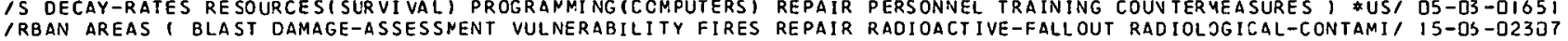
IRBAN AREAS I BLAST DAMAGE-ASSESSMENT VULNERABILITY FIRES REPAIR RAOIOACTIVE-FALLOUT RADIOLOGICAL-CONTAMII IS-OS-O23O7
/TEM MANUAL III-K. CONSTRUCTION IN THE PROTOTYPE NODEL I REPAIR RECOVERY CATA-PROCESSIVG MAIVTENANCE POS/ I3-13-O2282 RIBUT ION LOGISTICS CIVIL-DEFEN SE-OPERAIING-PLANS CLEANING REPAIR TRAINING / *FG-E-13.5/CY CLJTHING I DIST IS-OJ-O23OS $1 G$ EOUCAT IOV IV TERNA TIONAL-CIVIL-DEFENSE RESCUE (TRAINING) REPAIRITRAINING) PROTECTIVE-CLOTHIVG DROPAGANDA/ I5-03-DI9I 7 LOUT SHIELDING BU/ DESIGN OF ROOF WASHOOWN SYSTEMS (FINAL REPORT) ( CONSTRUCTION-MATERIALS RAOIOACTIVE-FAL I8-DS-D232। - 1970-1985 I CALCULATIONS DAMAGE/ CIVIL DEFENSE SHELTER REPRESENTATION FOR POPULATIOV DAMAGE CALCULATION IS-O3-OI93D IORTALITY EST IMATES TO VARIATI ONS IN AGGREGATE POPULATION REPRESENT AT IONS I VUCLEAR-WARFARE-SASUALTIESIMA/ IS-GT -OIQSO IARCH BY PRIVATE SCHOLARS AND ACADEMIC CENTERS - ANERICAN REPUBLICS 1 BIBL IOGRAPHIES COMMUNISM ECONOMICS, O5-1I -DI696 RCES PROGR.AMMING-LANGUAGES COMPUTERS CASUALTIES/ REACT, A REQUEST LANGUAGE I DAMAGE-ASSESSMEVT BLAST RESOU D5-D3-D2154 S PUBLIC-OPINION ATTITUI PUPI IS INFORMATICN AND KNCWLEDGE REOUISITES OF. SHELTER SYSTEM I S PUBLIC-OPINION ATTITU/ PUBI IC. INFORMATICN AND KNCWLEDGE REQUISITES OF A SHELTER SYSTEM 1 FALLJUT-SHELTER OS- II - QI 699 E $I$ RESCUES CIVIL-DEFENSE-PER SONNEL USSR/ ENGINEERING ANO RESCUE OPERAT IONS AT THE SITE OF AV ATOMIC STRIK I3-I?-OI8J4

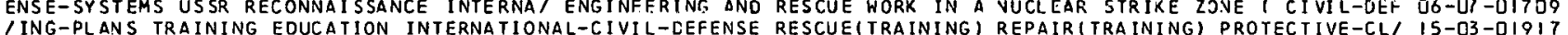
IING-PLANS TRAINING EDUCATION INTERNATIONAL-CIVIL-CEFENSE RESCUE(TRAINING) REPAIRITRAINING) PROTECTIVE-CLI I5-O3-OI9I7

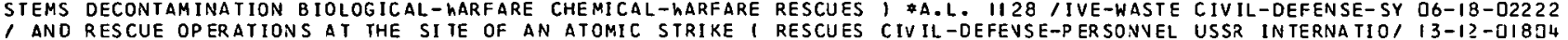

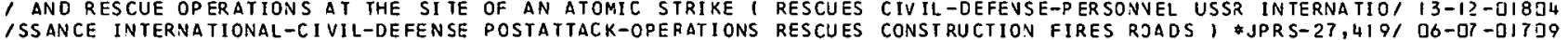
ISS ANCE INTERNATIONAL-CIVIL-DEFENSE POSTATTACK-OPERATIONS RESCUES CONSIRUCTION FIRES RJADS 1 *JPRS-27, 4191 O6-O7-OI7D9 ISTRUCT ION IN THE RURAL AREA I USSR CIVIL-DEFENSE-SYSTEMS RESCUES INTERNATIOVAL-CIVIL-DEFEVSE CHEMICAL-WA/ I5-O3-OIQI8 IICINE MEDICINE MEOICAL-PER SONNEL HOSPITALS PUBLIC-HEALTH RESCUES NUCLEAR-WARFARE-CASUAL TIES SCHOOLS SURV/ D6-2I -OITHO IVIL-DEFENSE-SYSTEMS INTERNATIONAL-CIVIL-DEFENSE TRAINING RESCUES PUBLIC-INFORMATION REPAIR FILMS PROPAGA/ I5-O3-O23D2 IG COST-EFFECTIVENESS TRAINING FIRE-STORMS CONFLAGRATIONS RFSCIIES VULNERABILITY CIVIL -DCFENSE-OPERATING-P/ I3-I?-UI8D6 ORTAT ION STUOY I PENNSYLVANIA HIGH-SPEED-GROUND-TRANSPORT RESEARCH-PROGRAM-ADMINISTRATIJN, OA.LL. ID62, /SP 23-D?-D2092 IND-DEMAVD INDUSTRIAL-PRODUC TI ON SCIENTIFIC-ORGANIZATIONS RESEARCH-PROGRAM-ADMINISTRATIJN OPERATIONS-RESEI O5-O3-OIOL 9 RC/ CIVIL DEFENSE RESEARCH ANALYSIS I SYSTEMS-ENGINEERING RESEARCH-PROGRAM-ADMINISTRATION OPERATIONS-RESEA I2-D?-DI767 IMEC-FORCES-OPERATIONS LOGISTICS COSTS CI VILIAN-PERSONNEL RESEARCH-PROGRAM-ADMINISTRATIJN WAR-PJTENTIAL B/ IS-C3-O23O5 $1 L$ RECORD OF THE GAS UTILITY INDUSTRY IN 1965 I UTILIIIES RESERVES PRODUCT IOY TRANSYISSION DISTZIBUTION UI D5-O3-OI642 BLRECORD OF THE GAS UTILITY INDUSTRY IN 1965 I UTILITIES RESERVES PRODUCT IOV TRANSYISSION DISTR IBUTION U/ O5-O3-OI642
BURST STR/ THERMAL HAROENING CONSIDERATIONS PERTAINING TO RESIDENT IAL AREAS I NUCLEAR-EXPLOSIONS FIRES AIR IS-OS-DI9S3 BURST STR/ THERMAL HAROENING CONSIDERATI ONS PERTAINING TO RESIDENTIAL AREAS I NUCLEAR-EXPLOSIONS FIRES AIR IS-OS-OI953
UND-RESIDENTIAL-DISTRIBUTION-SYSTEMS UNDERGR/ UNDERGROUND RESIDENTIAL DISTRIBUTION I TRANSFORMERS UNDERGRO OO-O3-OI7S4

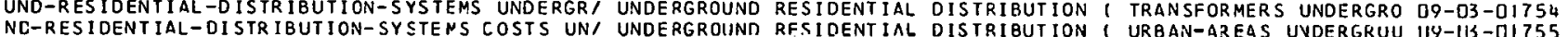
NC-RESIDENTIAL-DISTR IBUTION-SYSTENS COSTS UN/ UNOERGROIND RFSIDENTIAL DISTRIBUTION I URBAN-AREAS UVDERGRUU UY-US-DI755
CTURES FALLUUI-SHE/ AN INVESIIGATION OF SCHOOL DESIGNS TO RESIST INTEGRAT EC VUCLEAR WEAPONS EFFECTS I STRU I3-I3-DIR2I AMS 1 BEAMSISTRUC TURAL) DYNAMICS SHEAR-STR/ DYNAMIC SHEAR RES ISTANCE OF THIV WEBBED REIVFOR:ED $=0 N C R E T E$ RE $20-11$-U2337 SPORES BIOLOGICAL-WARFARE-AGENTS ANTIGENS-AND-ANTIBODIES RESISTANCEIBIOLOGICAL); $\$ A D$ O36 893, VACCINE ( I5-O2-0I853 T-SHELTERS DESIGN COSTS/ ENTRANCERAYS AND EXITS FOR BLAST RESISTANT FULLY BJRIED PERSOVVEL SHELTERS I BLAS $13-13-0 I 835$ 5 I FILTERS(FLUID) RELIABILITY QUA/ REQUIREMENTS FCR FIRE RESISIANT HIGH EFFICIENCY PARTICULATE AIR FILTER I3-II-OI79L ROL COLD-WAR NATO VIETNAM/ SOL. IDARITY IS THE BANNER - TWO RESOLUTIONS ON INTERNATIOVAL AFFAIRS I ARMS-CONT D5-DI - D2I 72 NY ELEMENT LASER I L/ LASER CAVITIES I. RESEARCH CN OPEN RESONATORS II. BEHAVIOR OF THE OUTPUT FROM A MA $20-05-02057$ USING RESOURCES MATHEMATICAL-MODELS COMPUTERS CASUALTIES/ RESOURCE DATA CATALOGUE I INFORMATION-SYSTEMS HO DS-O3-O2I37 CES DATA-PROCESSING, \$VREC- 3 PARM SYSTEM MANUAL IV. RESOURCE DATA ROUT INE 1 DAMAGE-ASSESSMENT RESOUR OS-O3-Q2I 29 CES VULNERABILITY PROGRAMMING (COMPLTERS) DATA-PROC.FSSING/ RESOJRCE CISTRIBUT ION I DAYAGE-ASSESSMENT KESUUR DS-O3-O2IUB BIBL IOGRAPHY OF PLIBLICATIONS ( DANAGE-ASSESSMENT RESOURCES, *NREC- 121 OS-03-02I36

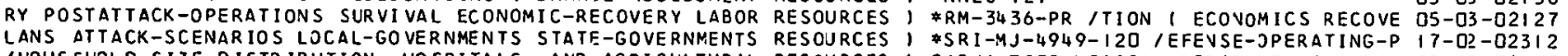
IHOUSEHOLD SIZE DISTRIBUTION, HOSPITALS, AND AGRICULTURAL RESOURCES 1 CASUALTIES MEDICAL-PERSJVVELI *NRE/ OS-03-O2I 45

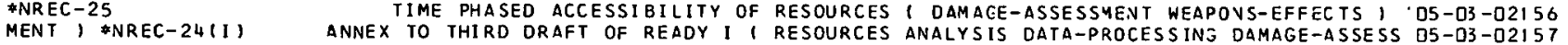


TY ECONOMI/ PARM SYSTEM MANUAL VI-2. PARN MAIN ROLITINE I RESOURCES CAPACITY DAMAGE-ASSESSMEVT VULNERABILI DS-O3-D2IS2 IEL. I RECOVERY ECONOMIC-RECOVERY DAMAGE-ASSESSMENT MODELS RESOJRCES CAPACITY DECONTAMIVATIOV REPAIR GOVER/ DS-O3-D2ISI ILATION AGRICUL TURE UR8AN-AREAS RLRAL-AREAS CONMERCE MEAT RESOURCES CEREAL COTTOV VEGETABLES FRUITS FOODS/ O2-02-OIOI 9 RAM/ PARM SYSTEM MANUAL V. FILE MAINTENANCE PROCEDURES I RESOURCES OAMAGE-ASSESSMEVT DATA-PR JCESSING PROG D5-D3-D2I5O IVIVAL ITEM AVALYSIS PROGRAM I NUCLEAR-WARFARE CASLALTIES RESOURCES DAMAGE-ASSESSMEVT FJOD LIGHT MEDISAL- 1 OS-03-O2I 35 MINGI COMPUTER SI MATHEMATICAL-MODELS FLOW-CHARTI READY I I RESOURCES D LETT LJGIC-DESIGN PROGRAM OS-03-02155 TION CASUALTIES FOOD-I RISK II ANALYSIS OF FOOD BALANCE I RESOURCES DAMAGE-ASSESSMENT VULNEZABILITY POPULA D5-03 - U2I32 I PROGRAM - MAPS OF COVTINENTAL UNITED STATES I GECGRAPHY RESOJRCES DAMAGE-ASSESSMEVT WEAPOVS-EFFEC TS NUC / DS-D3 - Q2I 4 D TESSESSMENT RESOURCES DATA-PROCESSING, *VREC-I3 PARM SYS 05-03-02129 /LING) STOCKP IL. ING INOUSTR IAL-PLANTS UTILITIES(HARCENING) RESOURCES DECONTAYINATION PEST-COVTROL MORTUARYI IS-O3-OIQ53 ROCESSING SHORTAGES/ A GENERAL NETHORK SINULATION NODEL I RESOURCES DISTRIEUTION SERVICES SURPLUSES DATA-P QS-D3-O2I 33

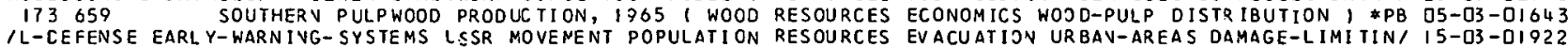
T/ COORDINATE COVVER SION FORMULAS FOR USE WI TH WORLD WIDE RESOURCES GEODETIC POSITIOV I GP, TO UNIVERSAL OS-O3 -O2I 33 ES, BIOLOGICAL SCIENCES, ENGI/ A DJRECTORY OF INFORMATION RESOURCES IN THE UVITED STATES - PHYSICAL SCIENC DS-OZ - QIG27 A DIRECTORY OF INFORMATION RESOURCES $\angle$ ITY CONCEPTS AVD DATA I MOBILIZATION MANAGEMENT-PLANNING RESOURCES INATIONAL SURVIVAL I SIMULATION DAMAGE-ASSESSNENT WARFARE RESOURCES IESSMENT RECOVERY THREAT-EVAIIIATION POSTATTAC.K-OPERATIONS RESOURCES IEPORT I UNITED-STATES CIVIL-DEFENSE-SYSTEMS CALCULATIONS RESOURCES IEPORT I UNITED-STATES CIVIL-DEFENSE-SYSTEMS CALCULATIONS RESOURCES
ONS RECOVEI PARM PROJECT FINAL REPORT I DANAGE-ASSESSMENT RESOURCES ONS RECOVEI PARM PROJECT FINAL REPORT I DANAGE-ASSESSMENT RESOURCES * A I PROSPECTING IN ECONOMICS? SCIENTIFIC-RESEARCH RESOURCES ' A. .L. ' PROSPECTING IN ECONOMICS I SCIENTIFIC-RESEARCH RESOURCES
ESI RESOURCE DATA CATALOGUE INFORMATION-SYSTEMS HOUSING RESOURCES ES/ RESOURCE DATA CATALOGUE I INFORMATION-SYSTEMS HOUSING RESOURCES
SUALTIES / APPL ICATIONS OF THE MANPOWER ANALYSIS SYSTEM I RESOURCES ITED I POSTATIACK-OPERATIONS RECOVERY MANAGEMENT-PLANNING RESOURCES $S / U . S$. SOURCES OF PE TROLEUM AND NATURAL GAS STATISTICS 1 RESOURCES IES REPAIR RADIOACTIVE-FALLOUT RADIOLOGICAL-CCNTAMINATION RESOURCES TIES/ REACT, A REQUEST LANGUAGE ( DAMAGE-ASSESSMENT RLAST RESOURCES PPLY-AND-DEMAND ECONOMICS RECOVERY POSTATTACK-OPERATI ONS IIE-SELECTION NATIONAL-FALLOUT-SHELTER-SURVEY POPULAT ION RESOURCES IN THE JVITED STATES - SJCIAL SCIENCES 05-D2-01628 INDUSTRIAL-PRODUCTIJV IVDUSTRIAL-PLAN/ 05-03-02142 INOUSTRIES VULNERABILITY ECONOMICS ', DS-03-01644 MANAGEMEVT-PLANVING CIVIL-DEFENSE-SYS/ I5-03-01949 MANAGENENT-PLANNING COMPUTER S VULNERA / 15-06-0195 MANAGEMEVT-PL. ANVING POSTATTACK-OPERATI 05-03-02144 MANPOWER GEOGRAPHY INDUSTRIAL-PRODUCT/ 05-03-02159 MANPOWER INNOVATION PROJECTIONS MODELS O5-03-01633 MAT HEMAT ICAL-MODELS COMPUTERS CASUALTI O5-03-02137 MODELS DAMAGE-ASSESSMENT POPULATION CA 05-03-02130 MOOELS PROGRAMM IVG(COMPUTEZS) CAPACIT/ 05-03-02131 PETROLEUM-INDUSTRY INDUSTRIES ECONOMIC 05-03-01638 POPULATION FACILITIES DECONTAMINATION/ 15-0S-0230? PROGRAMMING-L ANGUAGES CJYPUTERS CASUAL 05-03-02154 IUAL III-O MANPOWER AND OCCUPATIONS I DAMAGE-ASSESSMENT RESOURCES VULNERABIITY MODELS MAVUFACTUR ING MI I II COMPUTER STATISIICAL MODEL - NAHICUS 63 APPLICATION I RESOURCES VULNERABILITY NUCLEAR-WARFARE WIND BL, D5-O3-D2I 49 ASUAL STREAK IV - NREC RAPID DAMAGE ASSE SSMENT PRCGRAM I RESOJRCES VULNERABIL ITY PRDGRAMMIVG (COMPUTERSI C GS-O3 -O2I 46 ATA-PROCESSINGI RESOURCE DISTRIBUTION I DAMASE-ASSESSMENT RESOURCES VULNERABILITY PRJGRAMMIVGICOMPUTERSI D O5-D3-D2I 48 IEC DAMAGE ASSESSMENT COMPUTATION PROGRAM - JUMBO III. I RESOURCES VULNERABILITY WEAPOVS-EFFECTS WINO RAI Q5-O3-D2I 34 ATTACK-OPERAT IOVS SURVIVAL STORAGEIFOOD) PRODUCTIONIFOOO) RESOURCES (FOOD) AGRICUL, TURE 1 \$RM-5OS2-TAB /POST D6-15-OI7) 7 R ECO/ OCCUPATIONAL SKILLS AND CIVIL DEFENSE ( INDUSTRIES RESOURCES(HUMAN) DISTRIBUTIOV VUL VERABILI TY LABO OS-O3-OIG39 IUCATION I POSTATTACK-OPERATIONS SOCIAL-RECOVERY SURVIVAL RESOURCES (HUMAN) RECOVERY VULVERABILITY URBAN-A/ O5-II -OIGB8 /ATION SEP.VICES IN THE PHYSICAL DNR BIOI OGICAL SCIENCES ( RESOURCES (INFORMATION) DATA SCIENTIFIC-RESEARCH/ O5-D?-OIG29 ITIVE-FALLOUT CONTAMINATION INDUSTRIAL-PLANTS OECAY-RATES RESOURCES (SURVIVAL) PROGRAMMIVG (COMPUTERS) REPA' DS-O3 - OI GSI I UNITED STATES FROM NUCLEAR FIRE ( FIRES FIRE-PROTECTI ON RESOURCES (VULNERABILITY) FALLOUT-SHELTERS EQUIP) I3-I?-DIBDG $K$ ING SOCIAL-SCIENCES / ALTERNATIVES IN WATER NANAGEMENT I RESOJRCES (NATER) MANAGEMEVT-PLANNIVG DECISION-MA DS-DI -OIG22 NDUIT TO STATIC AND DYNAMIC LOADING I DUCTS SAND UNDERGR/ RESPONSE OF A BURIED PROTOTYPE COMMUVICATIONS CO I3-II -O22SI EL LINER SUBJECTED TO A DYNAMIC DISTURBAN/ A STUDY OF THE RESPONSE OF BACKPACKING MATERIAL ENCASING A TUNN I3-I3-D2270 E REV IEW AND PILOT STUDY I SHELTERS UNDERGRO/ THE ELASTIC RESPONSE CF BURIED CYLINCERS, CRITICAL LITERATUR I3-13-OI825 TS LOADIVGIMECHAN ICS) UNDERGROLND-STRUCTURES SHELTERS PE/ RESPONSE OF DEEP REIVFJRCED CJVCRETE SLABS I TES I3-13-02290 TS LOADIVGIMECHANICSI UNDERGROLND-STRUCTURES SHELTERS PEI RESPONSE OF OEEP REINFJRCED CSVCRETE SLABS TALLATIONS TO BLAST INDUCED GROUND ROTI /ATIONSHIP BETWEEN SELEC TED BLAST WAVE PARAMETERS AND THE RESPONSE OF MAMMALS EXPOSEO TO AIR BLAST 1 BLASI D6-2I-02234

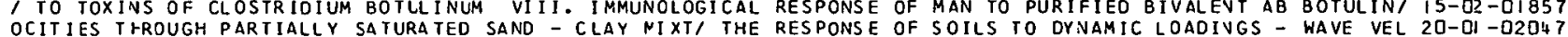
OCITIES T FROUGH PARTIALLY SATURATED SAND - CLAY NIXT/ THE RESPONSE OF SOILS TO DYNAMIC LOADIVGS - WAVE VEL 20-DI -O2O47

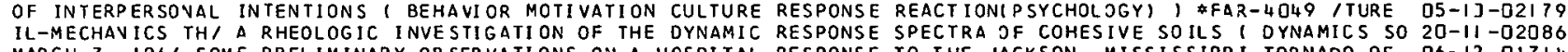
MARCH 3, $196 /$ SOME PRELIMINARY OBSERVATIONS ON A HCSPITAL RESPONSE TO THE JACKSON, MISSISSIPPI TORNADO OF OO-I2-OI7I 4 HAVIOR ELECTR IC-POWER-PRODUCTION (FAILURE) ATTITUD/ PUBLIC RESPONSE TO THE NORTHEASTERN POWEZ 8LACKOUT I BE O5-IJ-OI683 TION SURVEY I ATTITUDES/ AN EVALUATION STUDY OF CCNMUNITY RESPONSE TO THE RHDDE ISLAVD HOME FALLOUT PROTEC OS-IJ-DIGBS IECHANISMS AND SCALING PROCEDURES APPLICABLE IN ASSESSING RESPONSES OF THE THORAX ENERGIZED BY AIR BLAST I O6-2I -02235

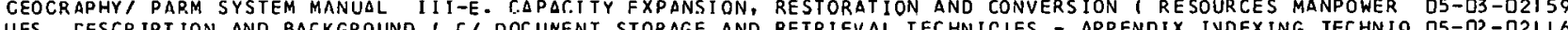
UES, CESCR IPTION AND BACKGROUND 1 CI DOCUNENT STORAGE AND RETRIEVAL TECHNICJES GAPENDIX IVDEXING TECHNIO OS-D?-O2IIO

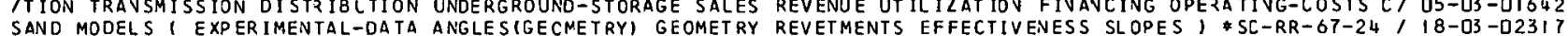

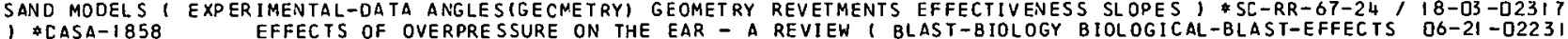

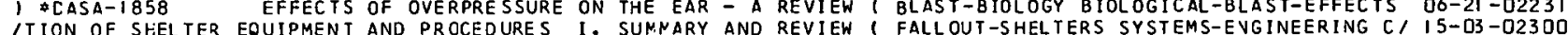
ITION OF SHEL TER EQUIPMENT AND PROCEDURES I SUMNARY AND REVIEW S FAL OUT-SHELTERS SYSTEMS-EVGINEERING C I IS-D3-023OO /LASTIC RESPONSE OF BUR IED CYLINDERS, CRITICAL LITERATURE REVIEW AND PILOT STUDY I SHELTERS UNDERGROUND-S/ I3-I3-OI825
O EMERGEVCY PLANN ING I SCIENTIFIC-RESEARCH BLAST FIRES U/ REVIEW OF FEDERALLY SPONSOREO RESEARCH RELATED T IS-O3-O2299 O EMERGEVCY PLANN ING I SCIENTIFIC-RESEARCH BLAST FIRES U/ REVIEW OF FEDERALLY SPONSORED RESEARCH RELATED T IS-03-O2299
LOGY AND ECOLOGY DF C. ANDRENIFORNIS - HYNENOPTERA, AN/ A REVISION OF THE EEE GENUS CALLIOPSIS AND THE BIO DO-DS-DI7OS ILENCE FLUID-DYNAMICS PARTICLE-SI ZE-DISTRIBUTICN AEROSOLS REYNOLOS-NUMBER BROWNIAN-MOVEMENT EQUATIUNS, $/ 20$-OL-O2333 SPECTRA OF COHESIVE SOILS I DYNAMICS SOI L-MECHANICS TH/ A RHEOLOGIC INVESTIGATION OF THE DYVAMIC RESPONSE $20-1 /$-O2D86 $O L$ O 1 THE PATHOGENESIS OF PSITTACCSIS - SERIAL STUDIES ON RHESUS MCNKEYS EXPOSED TO A SHALL PARTICLE AEROS $15-02-0 I 850$ TITUDES/ AN EVALUATION STUDY OF COMMUNI TY RESPONSE TO THE RHODE ISLANC HOME FALLOUT PROTECTIOV SUR VEY 1 AT OS-IJ-DI G85 IOR AND SUBJECT INDEXES (BACTERIA VIRUSES FUNGI PROTOZOA RICKETTSIA PHYSIOL JGY DIAGVOSIS) *MISC. PUB. $3 /$ OO-I3-OI7IS /PL $\triangle N S$, PRAFIIFS, CROSS SECTIONS, AND GENERAL LOCATION OF RIGHTS OF WAY TO BE ACQUIRED 1 CIVIL-ENGINEERIN/ I3-OZ-DI78I /PLANS, PROFILES, CROSS SECTIONS, AND GENERAL LOCATION OF RIGHTS OF WAY TO BE ACQUIREU ( HLJOOS LALIFORNI/ I3-D?-OI77O SOIL-MECHANICS LOADING(MECI TRANSIENT LOADING TESTS CN A RIGID CIRCJLAR FOOTING I FOUNDATIONSISTRUCTURES) I3-I3-QI8I 2 STEEL COMPUTEI THE SECOND ORDER EIASTIC ANALYSIS OF PLANE RIGID FRAMES I STRJCTURES STRUCTURAL-PROPERTIES I3-13-022B7 IMENTAL INVESIIGATION OF THE YIELD STRENGTH OF A MCHINED RING STIFFENED CYL INDRICAL SHELL 1 MODEL BR-7M, $20-11$-D234? IL AND EXPERIMENTAL INVEST AN AXPERIMENTAL INVESTIGATION OF A DYNAMICALLY LOADED RING WITH RADIAL ELASTIC SUPPOR IYLINCERS IENSILE-PROPERIIES PLASIICITY STRUC TURAL-SHELLS RINGS WELCING STRESSES DEFLECTIOV RJLLING IMETAL/ $20-I I$-O234 7 AGE-ASSESSMENT VULNERABILITY POPULATI ON CASUALTIES FOOD-1 RISK II ANALYSIS OF FOJO BALAVCE 1 RESOURCES DAM OS-O3 -O2I 32 APPLICATION I RESO/ AN ANALYSIS OF THE RELIABILITY OF THE RISK II COMPUTER STATISTICAL YODEL - NAHICUS 63 O5-O3-O2I4Q 1 MANAGEMENT-PLANNING SURVIVAL URBAN-AR/ ON THE OESIGN OF RISK ORIENTED, LOW COST FALLOUT SHELTER SYSTEMS I3-I3-QIBZ9 I AQUEDUCT SYSTEMS TO SER VE SOLTHERN CALIFORNIA - FEATHER RIVER ANC CELTA CIVERSION PRJ JECTS 1 WATER-SUPP/ I3-O2-02258 ITEM PORTIOV OF CONNECTICLT A VENUE ROUTE BETHEEN CCLUMBIA ROAD AND VAN NESS STREET I TRANSPJRTATION SUBWA/ I3-D? -DITB5 -CEFENSE POSTATTACK-OPERATIONS RESCUES CONSTRUCTION FIRES ROADS 1 \#JPRS-27,419 \#TT-64-5I669/ATIONAL-CIVIL D6-OT-0I7D9 A/ EXTENS IVE STREET BUILDING PROGRAM IN HAMBURG I GERMANY ROADS CONSTRUCT ION URBAN-AREAS TUVNELSITRANSPORT $13-02$ - OIT86 AMONG MOCES, CARGO POSTATTACK-OPERATIONS VULNERABILITY ROADS RAILROADS CARGO-VEHICLES AIRCRAFT WEAPONSI I3-OS-O22GD AILROADS BRIDGES GROUND- SLPPORT-EQUI PMENT RAILROAD-TRACXS ROADS USSR UNDERGROUND-STRUCTURES I \#AD O3I O4I IO-OI -DI972

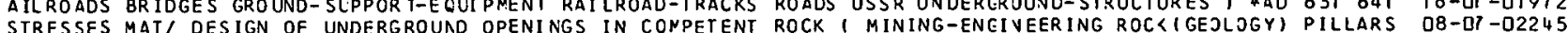

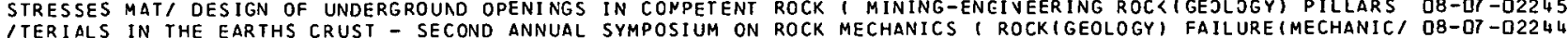
ITERIALS IN THE EARTHS CRUST - SECOND ANNUAL SYMPOSIUM ON ROCK MECHANICS ( ROCK (GEOLOGY) FAILURE(MECHANIC/ O8-D7-02244
/ERLY OF IHE COLORADO SCHOOL OF MINES 5 I (3) SYMPOSIUM ON ROCK MECHANICS ( ROCK (GEOLOGY) UNDERGROUND-STRUI D8-07-02243 LSITRANSPORTATIOV) BLASTING COSTS DRILLIN/ REPORT CN HARD ROCK TUNNELLING IVVESTIGATION I EXIAVATION TUNNE I3-O?-DI7B7 


\begin{abstract}
IAVATION REINFORCED-CONCRETE ROCK-STABILI ZATICN SHCICRETE ROCK-BOLTINGS CHAIN-LINK-FABRIC EPOXY-RESINS CEI I 3- 13 - O2274 IING ROCK (GEOLOGY) PILLARS STRESSES MATHENATICAL-ANALYSIS ROCK-FORMATIONS STRAIN PHYS ICAL-PQJPERTIES CALC/ O8-O7-D2245 IPROPERTIES OF ROCKS AT HIGH TEMPERATURES AND PRESSURES 1 ROCK-MECHANICS LOADINGIMECHAVICSI USSR EXCAVATI/ OB-O7-O224 2 1 COMPRESSING TESTING-EQUIPMENT MARBLE DOLOMI TE LINESTONE ROCK-SALI ANHYDRITE SANDSTONES CLAY SILTSTONES, D8-D?-O224 2 / ROUND-STRUCTURES BLASTING EXCAVATION REINFORCEO-CCNCRETE ROCK-STABILIZATION SHOTCRETE ROCK-BOLTINGS CHAI/ I3-13-O2274 IRTHS CRUST - SECOND ANVUAL SYMPOSIUM ON ROCK NECHANICS I ROCK (GEOLOGY) FAIL JRE(MECHANICS) STRESSES THEORI D8-OT-0224 4 ICEFENSE ( SHEL TERS BLAST STRE SSE S UNDERGROUND-STRUCTURES ROCK (GEOLOGY, HEAT ING MINIVG-ENGIVEERING LOADIN/ I 3 - I3-D2273

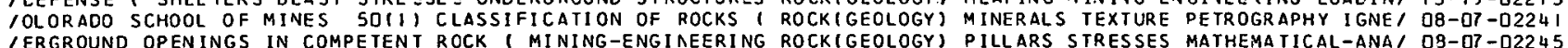
1 EREROUND OPENINGS IN COMPETENT ROCK /F MINES (2(2) TUNNEL MECHANICS ( UNDERGROUND-STRUCTURES ROCKIGEOLOGY) STRESSES ELASTICITY THEJRY GEOLOG/ I3-I3-02275 IADO SCHJOL OF MINES SI(3) SYMPOSIUM ON ROCK NECHANICS 1 ROCK (GEOLOGY) UNDERGROUND-STRUCTURES FAILLPE (ME/ O8-O7-D2243 BEAMS(STRUCTURAL) SHO/ THE SHOCK AND VIBRATION BULLETIN I ROCKETS DYNAMICS STRUCTURAL-AVALYSIS ELASTICITY 2O-1I -D2O74 IOF THE COLORADO SCHOOL OF MINES SD( 1 ) CLASSIFICATION OF ROCKS ( ROCKIGEOLOGY) MINERALS TEXTURE PETROGRAI O8-D7-O224I MECLANICS LOADING (MECHANICS) US/ NECHANICAL PRCPERTIES OF ROCKS AT HIGH TEMPERATURES AVD PRESSURES I ROCK- OB-D7-O224 2 ERAT IOVS IMMUNOLOGY OISEA SE-VECTORS CHEMOTHERAPY NEDICINE ROCENTS, \$RM-49E8-TAB L IC-HEALTH POSTATTACK-OP OG-DS-DI7O2 IRAINIME/ ON LONGITUDINAL AND TRANSVERSE WAVES IN ELASTIC RODS 1 MECHANICAL-NAVES ELASTICITY PROPAGATION S $20-I I-J 2032$ ITUDIES ON LONGITUDINAL AND BENDING WAVES IN LONG ELASTIC RODS 1 MECHANICAL-WAVES STRESSES PROPAGATION EL/ 20-11-D2O79 IONSTRUCT ION-MATER IALS LOAD-DISTRIBUTION DEFLECTIOA STEEL ROOS CRACKS STRAIV(MECHANICS) LOADIVGIMECHANICS/ I3-13-02289 /CITY STRUCTURAL-SHELLS RINGS WELDING STRESSES DEFLECTION ROLLING (METALLUREY) ELASTICITY I \$DTMB-I225 \#AD/ $20-11$-02347 ALLOUT DOSE-RATES RADIOLOGI AN EXPERIMENTAL EVALUATICN OF ROOF RECUCTION FACTORS I SHIELDING RADIOACTIVE-F I8-OS-D2O3O ION-MATER I ALS RAD IOACTIVE-FALLOUT SHIELDING BU/ DESIGN OF ROOF WASHDOWN SYSTEMS (FINAL REPORT) ( CONSTRUCT I8-CS-D232I ENCES HISTORY CIVILI U.S. CIVIL DEFENSE BEFORE 1950 - THE ROOTS OF PUBLIC LAN 920 I LEGISLATIOV SOCIAL-SCI OS-D4-OI67I IVIBRATION LOADINGIMECHANIC SI SHEAR-STRESSES DEFORMATION ROTATION INERTIA YATHEMATICAL-ANALYSIS I FREPOR/ $20-11$-O234 6 TPITAL RAP ID TRAYSIT SYSTEM PORTION OF CONNECTICUT AVENUE ROUTE BETWEEN COLUMBIA RDAD AVD VAY NESS STREET/ I3-O? -DI785

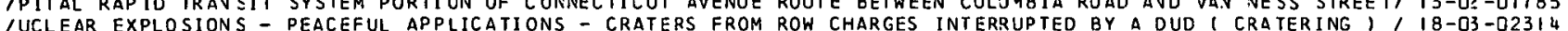
IUCLEAR EXPLOSIONS - PEACEFUL APPLICATIONS - CRATERS FROM ROW CHARGES INTERRUPTED BY A DUD $($ CRATERING $)$ I I8-O3-D23I 4 ICH 1 LASERS SC IENTIFIC-RESEARCH GLASS OPTICAL-PROPERTIES RUBY CRYOGENICS GASES PUMPINGIOPTICAL) FLASH-LA' 20-05-02062
OPT ICS CRYST/ IVVESTIGATIONS OF GIANT PULSING METHODS IN RUBY LASERS PUMPINGIOPTICALI CAVITY-RESONATORS 20-DJ-D2OS6

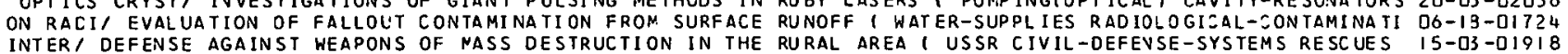
AS RU/ FIRE BIBLIOGRAPHY - SELECTEO URBAN AND MIXEO URBAN RURAL FIRES 1940-1964 1 BIBLIOGRAPHIES URBAN-ARE I3-I?-DI799 ON 1 USSR CIVIL-DEFENSE-SYSTEMS SHELTERS, PROTECTING THE RURAL POPULATION AGAINST RADIJACTIVE CONTAMINATI OG-IS-DI73I 5 CONTAMIVATION FOOD CHE / CIVIL DEFENSE HANDROCK - USSR I RURAL-AREAS BOMBS GUIDED-MISSILES VUCLEAR-WEAPON I5-Q3-D2296 / IN ING RESCUES PUBLIC-INFORMATION REPAIR FILMS PROFAGANDA RURAL-AREAS CIV IL-DEFENSE-OPERATIVG-PLANS INDUS/ I5-O3-O23J2 / TURAL CHARTS - 1966 / POPULATION AGRICULTURE URBAN-AREAS RURAL-AREAS COMMERCE MEAT RESOURCES CEREAL COTT/ O2-D?-QI6I9 URBAN RURAL FIRES 1940-1964 BIBLIOGRAPHIES URBAN-AREAS RURAL-AREAS FIRE-5AFETY, \$AD 634 D42 /ANO MIXED I3-12-0I799 IS INTERVAT IONAL-CIVIL-DEFENSE RADIOLOGICAL-CONTAMINATION RURAL-AREAS HARCEVING PROTECTION-SYSTEMS, \$JPR/ OG-I3-OI73I IES (VULNERABIL ITY) FALLOUT-SHELTERS EQUIPNENT URBAN-AREAS RURAL-AREAS MANACEMENT-PLAVNIVG CJST-EFFECTI VEN/ I3-1?-DI836 ALSI ALLOUT CA 1 CONSTRUCTION NUCLEAR-EXPLOSI ON-DAMAGE EXPLOSION-EFFECTS RURAL-AREAS PROTECTION-FACTOR DECJVTAMINATION W/ I3-I3-0I826
USSR INTERNATIONAL-CIVIL-DEFENSE CI VIL-DEFENSE-SYSTEMS RURAL-AREAS PUBLIC-INFDRMATIOVIRADIOI TRAINING / I5-D3-OI928

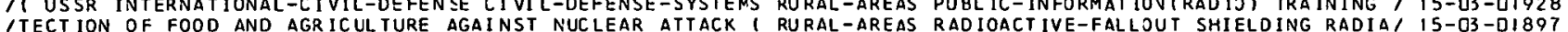
ITECT ION OF FOOD ANO AGRICULTURE AGAINST NUCLEAR ATTACK I RURAL-AREAS RADIOACTIVE-FALLOUT SHIELOING RADIAI IS-O3-DI897 IMOGRAPHY RECOVERY ATTACK-SCENARI CS FERTILITY URBAN-AREAS RURAL-AREAS VULNERAB ILITY VUCLEAR-WARFARE-CASUA I5-OS-DI9S5 IATE OF FOOD AND AGRICUL TURE 1966 I EUROPE FOOD-MARKETING RUSS IA AFRICA LATIN-AMERICA JAPAN GROSS-NATIONA/ O2-D2-0I6I6
10 CONTACTS AND A TRANSISTOR COMMLTATOR I ELECTRIC-MOTORS RUSSIA COMMUTATORS SEMICONDUCTOR-DEVICES TRANSI/ D9-D3-DI752 10 CONTACTS AND A TRANSISTOR COMMLTATOR I ELECTRIC-MOTORS RUSS IA COMMUIATORS SEMICONDUCTOR-DEVICES TRANSI/ D9-D3-DI752
INERAEIL ITY MIL ITARY-5TRATEGY INTERNATIONAL-CIVIL-OEFENSE RUSSIA CAMAGE-LIMITING-SYSTEMS DISPEPSAL CHINA. IS-O3-OI896 INERAEILITY MIL ITARY-STRATEGY INTERNATIONAL-CIVIL-OEFENSE RUSSIA CAMAGE-LIMITING-SYSTEMS DISPERSAL CHINA I IS-O3-OI896
IC-MISSILES CO/ ON THE ESSENCE OF A NUCLEAR MISSILE WAR I RUSSIA POL ITICAL-SCIENCE VUCLEAR-WARFARE BALLIST D5-O+-JI672 IS FROM A LAND SURFACE BURST ( RADIOACTIVE-ISOTOPES CHINA RUSSIA RADIOACTIVE-FALLOUT, *A.L. 97S I IN DEBR IB-DZ-OI99I IOVIET MILITARY TRANSLATIONS NLMBER IBB - CIVIL DEFENSE I RUSSIA TRAINING CISPERSAL CIVIL-DEFEVSE-PERSONN/ IS-O3-OIQI 3
\end{abstract}

S

IK PROPAGATION IN NONHOMOGENEOLS ATMOSPHERES AND MCOIFIED SACHS SCALING I. EXPONENTIAL IDEAL GAS ATMOSPH/ $20-$ G 4 -U2332 OF MASS DESTRUCTION - USSR I PROTECTION-SYSTENS WATER-S/ SAFEGUARCING FOOC PRODUCTS AVD WATER FROM ASENTS IS-D?-OIB 43 IUCTION OF A CRASH DUMMY FOR TESTING SHOULDER HARNESS AND SAFETY BELTS I TEST-EOUIPMENT TEST-DUMMYIANTHRCI I $3-1 ?-0$ IBI TEST-EQUIPMENT TEST-DUMMY (ANTHROPOMORPHIC) SAFETY-HARNESS SAFETY-DEVICFS, \$A.L. 983/ AND SAFETY BCLTS I I3-I?-0I8D3 RUCTI/ ANTHROPOMORPHIC IESI DUMMY I TEST-EQUIPMENT DESIGN SAFETY-DEVICES TEST-DUMMY (ANTHROPOMOZPHIC) CONST I3-I2-OIBOB SAFETY BEL TS I TEST-EQUIPMENT TE ST-DUMMY (ANTHROPGMCRPHIC) SAFETY-HARNESS SAFETY-DEVICES, \#A.L. G83, AND I3-I?-OI8J3 IPRODUCTION TRANSMISSION DISTRIBUIION UNDERGROUND-STORAGE SALES REVENUE UTILIZATION FINANCIVG OPERATING-C, OS-O3-DIO42 /ROM A NUCLEAR EXPLOSION IN A SALT DOME - PROJECT ORIBBLE SALMON EVENT I SEISMIC-WAVES EXPLOSION-EFFECTS I I8-D3-O23IO IC-WAVES/ EARTH VIBRATIONS FROM A NUCLEAR EXPLOSICN IN A SALT DOME - PROJECT DRIBQLE SALMOV EVENT I SEISM I8-03-D23I8 IOS ION-EFFECTS NUCLEAR-EXPLOSIONS EQUATIONS TUFF ALLUVIUM SALT GRANITE MAT FEMATICAL-ANALYSIS, $\$$ ULRL-5O2DI I8-D3-D23I5

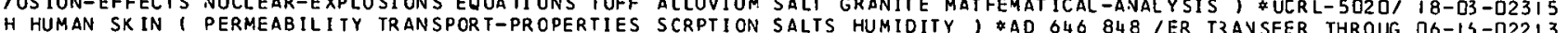

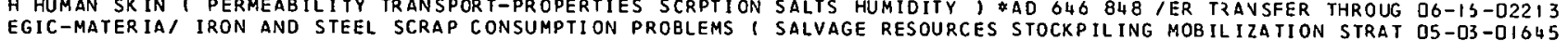
EGIC-MATER IAI IRON AND STEEL SCRAP CONSUMPTI ON PROBLEMS I SALVAGE RESOURCES STOCKPILING MOBILIZATION STRAT D5-O3-DIGL5
EAN-AREAS FIRES MAPS TEST/ THE CONFLAGRATION POTENTIAL IN SAN JOSE AND AL BUQUERQUE I AER IAL-PHOTOGRAPHS UR I3-I?-OIBO9 IS DISEASESICONTROL) DRAINAGE COUNTERMEA SURES URBAN-AREAS 5 AN-JOSE CALIFORNIA RECOVERY PEST-CONTROL I $\$$ USI, I3-D?-DITBO IHELTER-SURVEY POPULATION RESOLRCES URBAN-AREAS BUILDINGS SAN-JOSE VENTILATION, \$SRI-MU-4536-IID \#AD 635/ I3-I3-OI838 IC LOADINGS - WAVE VELOCITIES THROUGH PARTIALLY SATURATED SAND - CLAY MIXTURES 1 SOIL-MECHAVICS ACCOUSTIC/ $20-0 I$-02OL 7 IND DYNAYIC BEARING TESTS ON A STRIP FOOTING IN SATIRATEO SAND 1 LOAOINGIMECHANICS) SOIL-MECHANICS DYNAMI' 2 IU-II -O234 I /PL ICATIONS - A STUDY OF THE MECHANICS OF SLIDE DANS WITH SAND MOCELS I EXPERIMENTAL-DATA AVGLESIGEOMETRYI IB-O3-D23I 7 I SOIL-MECHANICS OYNAMICS SHEL TERS UNDERGROUND-STRUCTURES SAND SHOCK-WAVES BLAST FOOTINGS FOUNDATIONS VOII $20-11$-D234I RIC OIFFUSION COVDUIT TO STATIC AND DYNAMIC LOADING I DUCTS SAND UNCERGROUND LOERIMENTAL PROGRAM IN ATMOSPHE O4-O2-02II 4 $1-E Q U I P M E N T$ MARBLE DOLOMITE LINESTCNE ROCK-SALT ANHYDRITE SANDSTONES CLAY SILTSTONES GEOLOGY THERMAL-STRE OB-OT-D22 I IH STRUCTURAL-PROPERTIES QUALITY FASTENERS GUING PIYWITE SANDSTONES CLAY SILTSTONES GEJLOGY THERMAL-STRE I D8-OZ -O22L 2

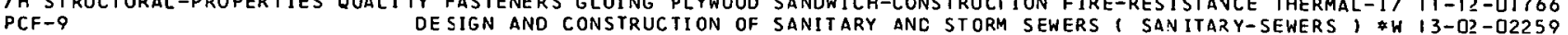
PCF-9
ISHELTERS I FALLOLT-SHEL TERS PHYSIOLOGY EXPLOSI ON-EFFECTS SANIIARY-ENGINEERIVG DESIGV COOLIVG-AVO-VEN IILAI I3-I3-DIBII

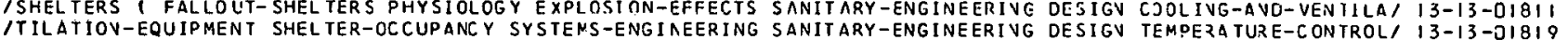
IGE-ASSESSMENT FOOD LIGHT MEDICAL-SUPPLIES ELECTRIC-POWER SANITARY-ENGINEERIVG FUELS WATER-SUPPLIES MATHE/ OS-O3-D2I35 IGE-ASSESSMENT FOOD LIGHT MEDICAL-SUPPLIES ELECTRIC-POWER SANITARY-ENGINEERIVG FUELS WATER-SUPPLIES MATHE/ O5-O3-D2I 35
ILING SHELTER-MAVAGEMENT HANDBOOKS HABITABILITY NUTRITION SANITARY-ENGINEERING MEDICAL-SUPPLIES DEFECTION/ O5-IJ-J2I 84

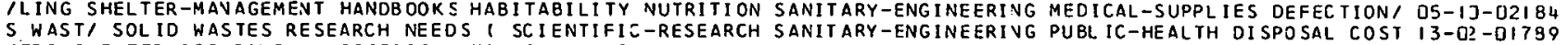
IERS SHEL TER-OCCUPANCY LOGISIICS MATER-SUPPLIES RATIONING SANITARY-ENGINEERIVG RAOIOLOGICAL-CONTAMINATION/ O6-O3-OI7I 2 ( WASTESISANITARY-ENGINEERING) WASTES (INDUSTRIAL) GARBAGE SANITARY-ENGINEERIVG SEWAGE DISEASE-VECTORS DIS/ I3-D?-DI780 DESIGN AND CONSTRUCTION OF SANITARY AND STORM SEWERS $($ SANITARY-SEWERS, FWPCF-9 N PEST-CONTROL MORTUARY-SERVICES PLBLIC-HEALTH INDUSTRIES SANITATICN REPAIR MANPOWER, \#SRI-YU-4536 /NATIO IS-03-OIO53

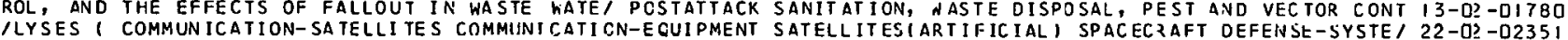
1 TO DYNAMIC LOADINGS - WAVE VELOCITIES THROUGH PARTIALLY SATURATED SAND - CLAY MIXTURES 1 SOIL-MECHANICS/ $20-01$-02OL 7 C/ STATIC AND DYNAMIC BEARING TESTS ON A STRIP FOOTING IN SATURATEO SAND I LOADING(MECHANICS) SJIL-MECHANI 20-II -D234I EC STATES I GEOGRAPHY RESOURCES DAMA / MAP IV - NREC SMALL SCALE MAPPING PROGRAM - MAPS JF CONTIVENTAL UNIT OS-O3-O2I 40 EC ST ATES I GEOGR APHY RE SOURCES DAMA / MAP IV - NREC SMALL SCALE MAPPING PROGRAM-MAPS JF CDNTIVENTAL UNIT O5-O3-02I 40
1 AIR-BURST YIELD WIND NUCLEAR-WARFARE-CA SUALIIES TARGETS SCALE(RATIO), \$RM-4725-TAB /IROSHIMA EXPLOSION I5-05-OI958 I AIR-BURST YIELD WIND NUCLEAR-WARFARE-CA SUALTIES TARGETS SCALEIRATIO), \#RM-4725-TAB /IROSHIMA EXPLOSION I5-05-OI958
IODOLOGICAL STUDY OF BEHAVIORAL AND SEMANTIC DIFFERENTIAL SCALES RELEVANT TO INTERCULTURAL VEGOTIATIONS (/ OS-I]-D2I78 IODOL OGICAL STUDY OF BEHAVIORAL AND SEMANTIC DIFFERENTIAL SCALES RELEVANT TO INTERCULTURAL VEGOTIATIONS I/ OS-I]-D2I78
IAGATION IN NONHOMOGENEOUS ATMOSPHERES AND MODIFIED SACHS SCALING I. EXPONEVTIAL IDEAL GAS ATMOSPHERE I / $20-04-02332$ 
NSES OF THE THORAX ENERGIZED / BIOPHYSICAL MECHANISMS AND SCALING PROCEDURES APPL ICABLE IN ASSESSING RESPO 06-21 -02235 MECHAN SY FARE SCAN - THE EVALUAT ION JF AY AIRBDZVE INFRARED MA 13-1?-01798 IOUT ATTENUATION UNOERGROUND-STRUCTURES DOSE-RATE PHOTONS SCATTERING ALBEDO GAMMA-RAYS ANGLE-OF-INCIDENCE/ IB-OS-O2O29 IIOLOCICAL TARGET ANALYSIS PROCEDLRES FOR DECONTAMINATION SCHEDUL ING I RADIOACTIVE-FALLOUT 24DIOLOGICAL-C/ OL-I3-02220 OGICAL-CONTAM INATION SHIELOING PRCTEC TIO/ DECCNTANINATION SCHEDULING PROCECURES FOR RADEF SYSTEMS 1 RADIOL DO-13-0I729 IEED GROUND TRANSPORT I TRANSPORTATION NETHORKS TERMINALS SCHEDUL ING SWITCHIVG PROPULSIJV-SYSTEMS AEROOYN/ 23-D?-D2354 CS, A LIST OF CURRENT SOCIAL SCIENCE RE SEARCH BY PRIVATE SCHOLARS AND ACADEMIC CENTERS - AFR ICA 1 ECONOMI D5-11-01692 CS 1 A LIST OF CURRENT SOCIAL SCIENCE RE SEARCH BY FRIVATE SCHOLARS AND ACACEMIC CENTERS - AMEZICAN REPUBLI OS- 11 -DIOFO PHI' A LIST OF CURRENT SOCIAL SC IENCE RESEARCH BY PRIVATE SCHOLARS AND ACACEMIC CENTERS - ASIA 1 BIBL1OGRA O5-11-0I698 BLI/ A LIST OF CURRENT SOCIAL SCIENCE RESEARCH BY FRIVATE SCHOLARS AND ACACEMIC CENTERS - MIDDLE EAST I BI O5-11-DI695 EU' $\triangle$ LIST OF CURRENT SOCIAL SCIENCE RE SEARCH BY FRIVATE SCHOLARS ANC ACACEMIC CENTERS - USSR AND EASTERN O5-1I -0I697 GRE/ A LIST OF CURRENT SOCIAL SCIENCE RESEARCH BY PRIVATE SCHOLARS AND ACADEYIC CENTERS - WESTEZN EUROPE, D5-11 -DI694 ONS EFFECTS I STRUC TURES FALLOUT-SHEI AN INVESTIGATION OF SCHOOL CESIGNS TORESIST IVTEGRATED VUCLEAR WEAP I3- $13-01821$

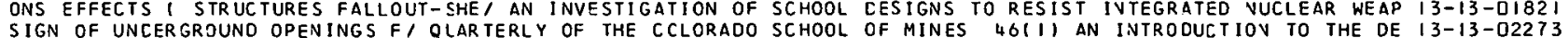

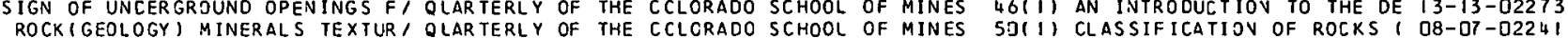

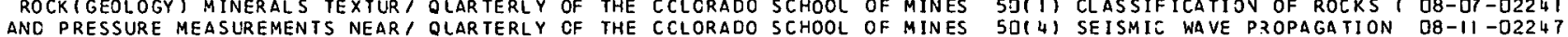

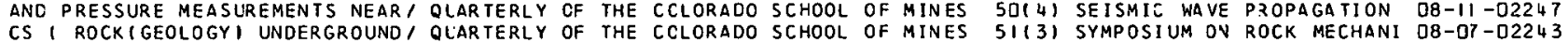
THE EARTHS CRUST - SECOND ANNU/ OLARTERLY OF THE CCLORADO SCHOOL OF MINES 52131 BEHAVIJR OF MATERIALS IN OB-O7-O2244 ROUND-STRUCTURES ROCK(GEOLOGY)/ QLARTERLY OF THE CCLORADO SCHOOL CF MINES 6212) TUVVEL MECHAVICS 1 UNDERG 13-13-02275 IN GOVERNMENT COMMUNICATIONS MILI TARY-PERSONNEL HCSPITALS SCHOOL-BUILDINGS IVDUSTR IAL-P ZODUETIDV, *NREC-1 O5-03-02139 /POWER-GENERATIOV MILITARY-PER SONNEL POPULATICN HOSPITALS SCHOOLS FOOD-SUPPLIES WATER-SUPPLIES INDUSTRIAL/ O5-D3-D2137 /ERATIONS MANAGEMENT-PLANNING STRESS(PSYCHOLOGY) SHELTERS SCHOOLS PUBLIC-INFORMATION EDUCATION ORPHAN-CAR/ O5-1I-D2203 ISPITALS PUBL IC-HEAL TH RESCUES NUCLEAR-WARFARE-CASLALTIES SCHOOLS SURVIVAL CASUALTIES FIRST-A ID 1 *A.L. $1 /$ Q6-21-DI740 CONGRESS (SYMPOSIA/ LOGIC, ME THODOLOGY AND PHILOSCPHY OF SCIENCE - PROCEECIVGS JF THE 1964 IVTERNATIONAL 12-0I-O2250 * J' COMMUNIST CHINESE SCIENTIFIC ABSTRACTS - BIONEDICAL SCIENCE I BIBLIOGRAPHIES YEOICINE BIOLOGY CHINA Ob-0j-0ITDL

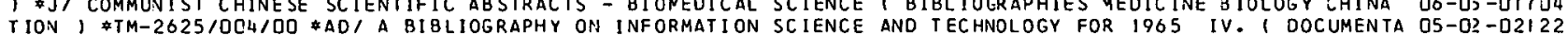

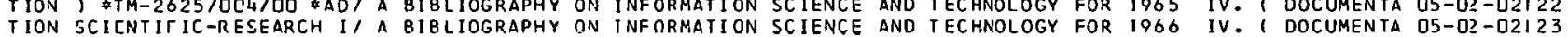

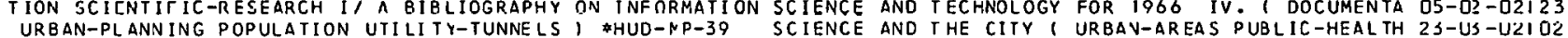

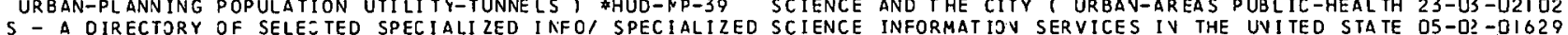
C CENTERS - AFRICA I ECONOMICS I A LIST OF CURRENT SOCIAL SCIENCE RESEARCH BY PRIVATE SCHOLARS AND ACADEMI O5-11-D1692 C CENTERS - INTERNATIONAL AFFAI/ A LIST OF CURRENT SOCIAL SCIENCE RESEARCH BY PRIVATE SCHOLARS AND ACADEMI O5-II-OI693 C CENTERS - WESTERN EURDPE, GRE/ A LIST OF CURRENT SOCIAL SCIENCE RESEARCH BY PRIVATE SCHOLAZS AND ACADEMI O5-11 -OI694 C CENTERS - MIODLE EAST 1 BIBLI/ A LI ST OF CURRENT SOCIAL SCIENCE RESEARCH BY PRIVATE SCHOLAZS AND ACADEMI O5-11 -OIS95 C CENTERS - AMERICAN REPUBLICS I A LIST OF CURRENT SOCIAL SCIENCE RESEARCH BY PRIVATE SCHOLARS AND ACADEMI O5-11 -DI696 C CENTERS - USSR AND EASTERN EU/ A LIST OF CURRENT SOCIAL SCIENCE RESEARCH BY PRIVATE SCHOLARS AND ACADEMI O5- 11 -O 1697 C CENTERS - ASIA ( BIBLIOGRAPHI' A LIST OF CURRENT SOCIAL SCIENCE RESEARCH, PYR PATE SCHOLAZS AND ACADEMI O5-11-O1698 7 IZED INFORMATIOV SERVICES IN THE PHYSICAL AND BIOLOGICAL SCIENCES I RESOURCESIIVFORMATIONI DATA SCIENTIF/ O5-D? -DI629

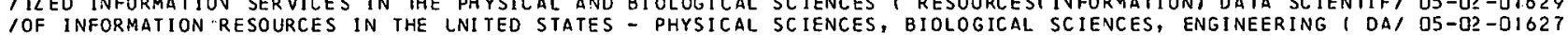

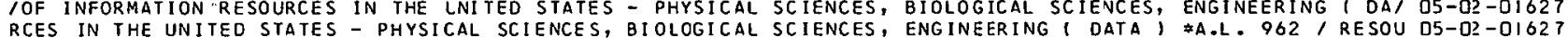

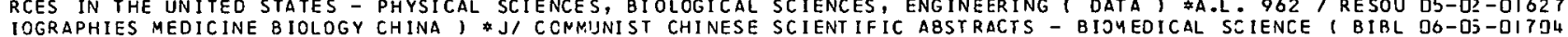

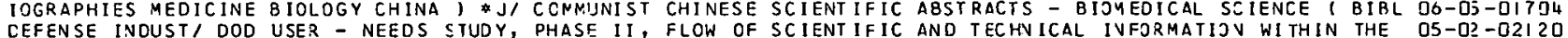

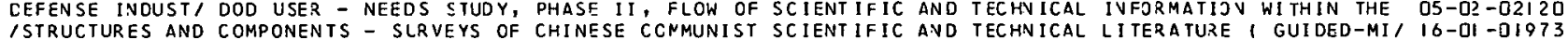

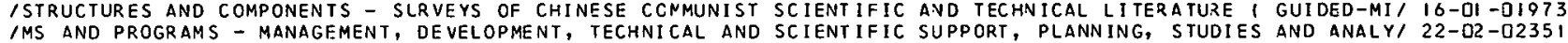
1 COCUMEVTAT IOV SCIEN TIFIC-RESEARCH INFORNATI CN-RETRIEVAL SCIENT IFIC-ORGANIZATIOVS PERIJDICALS, 4 TM-3E08, D5-D?-02I 23 INAT IONAL -PRODUCT SUPPLY-AND-DEMAND INDUSTRI AL-PRCCUCTI ON SCIENT IFIC-ORGAN IZ AT IONS RESEARCH-PRJGRAM-ADMIN/ O5-D3-OI649 IOCUMENTATION INFORMATION-RE TR IEVAL ENGI NEERING-PERSONNEL SCIENT IFIC-PERSONVEL FL OW-CHARTING PSYCHOMETRIC/ 05-0?-02120 CAL ANO $810 L O G I C A L$ SCIENCES I RESOURCESIINFORMATION) DATA SCIENTIFIC-RESEARCH, \#NSF 61-68 15 IV THE PHYSI D5-02-01629 IPHYSICS ELECTROMAGNEIIC-PROPERIIES FILMS FLUID-MECHANICS SCIENT IFIC-RESEARCH ABLATIOV ANTEVYAS PROPAGATI/ $20-03$-D2330 /RALLY SPONSORED RESEARCH RELA TED TO EMERGENCY PLANNING 1 SCIENT IF IC-RESEARCH BLAST FIRES UVI TED-STATES-G/ $15-03-02299$ Y CRY I NRL PROGRESS IN HIGH POWER LASER RESEARCH 1 LASERS SCIENT IFIC-RESEARCH GLASS OPTICAL-PROPERTIES RUB 20-03-02062 IION SCIENCE AND TECHNOLOGY FOR 1966 IV. I DOCUMENTATION SCIENTIFIC-RESEARCH INFORMATION-RETRIEVAL SCIEN/ O5-D2-02123 LLOGY MEDICINE ECOLOGY RADIATION-EFFECTS GENETICS PHYSICS SCIENT IFIC-RESEARCH METALLURGY MATERIALS CHEMIS/ $20-08-02070$ RS I INDEXES REPJRTS DOCUMENTA TION PROFESSI ONAL-PERSONNEL SCIENT IF IC-RESEARCH ORGANIZATIONS I*AD 648707 O5-02-02117 N PROJECTIOYS MODELS, \#A.L. I PRCSPECTING IN ECCNCMICS I SC.IENT IF IC-RESEARCH RESOURCES MANPJWER INNOVATIO O5-03-01633 FEALTH DISPOSAL COSTS WAST/ SOLID WASTES RESEARCH AEEDS ISCIENTIFIC-RESEARCH SAVITARY-ENGIVEERING PUBLIC- I3-D?-DI7B9 ITER) MANAGENENT-PLANNING DECISION-MAKING SOCIAL-SCIENCES SCIENTIFIC-RESEARCH UNITED-STATES DISTRIBUTION, O5-DI -0I622 AS IN RELAIIVE FHYSICAL AND BIOLOGICAL PARAMETCRS I ' THE SCCPE OF BLAST AND SHOCK BIOI. TGY AYD PROBLEM ARE O6-21-D2223 1 Q SORTING - A MACHINE ME THOD FOR O DEC K PREPARATIOA AND SCCRING 1 DATA-PROCESSING YETHODOLJGY PUNCHED-C/ OS-11 -D2IB8

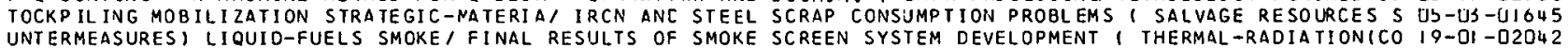

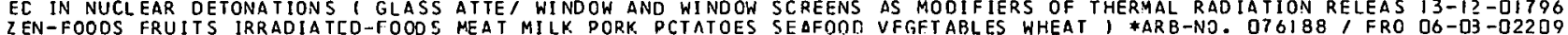
CTRICAL SYSTEMS I STORAGE-BATTERIES ALKALINE-CELLS AIRCR/ SEALED NICKEL CACMIUM BATTERIES FOR AIRCRAFT ELE 10-03-01764 I HUIION ANO OEVSITY OF MISSILES FROM NUCLEAR EXPLCSIONS (SECONOARY-MISSIIFS RLAST-EVERGIZED-MISSILES VELI I8-D3-D23I8 $S$ PUBL IC-HEAL TH, APHS- 158 I

SECURING HEALTH IN OUR URBAN FUTURE I URBAN-AREA D6-0j-02205 WEAPONS-EFFECTS RADIOACTIVE-FALLOLT DISTRIBUTION OCSE-RA/ SEDAN PROJECT 62.80C AERORADIOACTIVITY SURVEY 1 18-03-02324 WEAPUCK GEOLOGY) M INERALS TEXTURE PETRDGRARPY IGNEOUS-ROCK SEDIMENT ARY-ROC.K MFT AMORPHIC-2OCK, *A.L.1197, D8-07-0224 I KUCK (GEOLOGY) MINERALS TEXTURE PETROGRAPHY IGNEOUS-ROCK. SEDIMENT ARY-ROCK MFTAMORPHIC-ZOCK
TS NEAR/ OUARTERLY OF THE COLORADC SCHOOL OF MINES 5J (4) SEISMIC WAVE PROPAGAT IJN AVD PRESSUZE MEA SUREMEN D8- II -D224? IXPLOSIOV IN A SALT DOME - PROJEC I DRIBBLE SALMON EVENT ISEISMIC-WAVES EXPLOS IOV-EFFECTS VELO:ITY ACCELE/ I8-03-D2319 JOUPL ING I CRATER ING EXPLOSION-EFFEC TS NUCLEAR-EXPLOSIONS SEISMOLOGY GR ANITE MATHEMATICAL-AVALYSIS UNDERG/ 18 -03-D2316 IRTHQUAKE ENG INEER ING II. TOKYO AND KYOTO, JAPAN I JAPAN SEISMOLOGY STRUCTJRES SHOCK-WAVES ELASTICITY I, O8-II-D2246 ICEMOGRAPHY OF NUCLEAR WAR - EMPIRICAL GUIDELINES FOR THE SELECTICN OF DEMOGRAPHIC VAR IABLES AVD AREAL UN/ 23 -OL-O21D3 IOSTS URBAN-AREAS CONFIGURATION URBAN-PLANNI NG EFFICIENCY SELECT ION POPULATION OENSITY DISTRIBUTION ECONO/ $13-02-02256$ PESTIS I BIOLOGICAL-WARFARE-AGENTS CULTURE-MEDIA GEROWTH SELECTIVE MEDIA FOR THE ISOLATION OF PASTEURELLA IS-D? - I IBTS LTURAL NEGOTIAT/ A METHODOLOGICAL STUDY OF BEHAVI ORAL AND SEMANT IC OIFFERENT I SL SCALES RELEVAVT TO INTERCU OS-1J-U2I 78

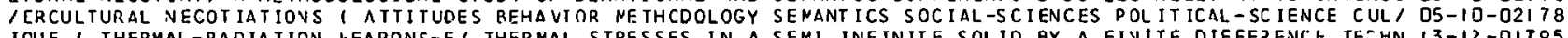
IQUE 1 THERMAL-RADIATION WEAPONS-E/ THERMAL STRESSES IN A SEMI INF INITE SOL ID BY A FIVITE DIFFERENCE TEEHN 13-1?-DIT95 THERMA SEMICONDUCTOR THERMOELEC TRIC TRANSISTDR COCLERS I SEMICONDUCTOR-DEVICES COOLING THERMOELEC TRICIIY D9-OI-OIT4

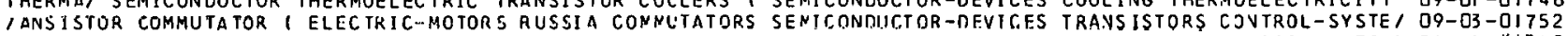

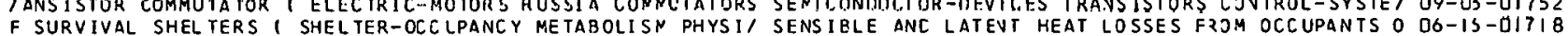

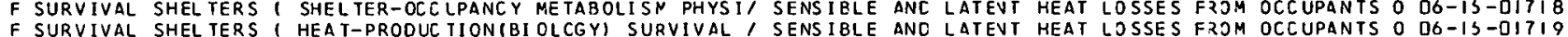
F SURVIVAL SHEL TERS ( HEA T-PRODUC TION (BI OLCGY) SURVIVAL SENS IBLE ANC LATEVT HEAT LOSSES FRJM OCCUPANTS O D6-1S-OI719 ENSE-SYSTEMS ME TEOROLOGY SYSTEM/ EXTENSI ON OF THE GENERAL SENSITIVITY ANALYS IS I. METHODOLOGY 1 CIVIL-DEF $15-03-01935$
CIVIL-DEFENSE-SYSTEMS WEAPONS- 1 EXTENSI ON OF THE GENERAL SENSITIVITY ANALYSIS II. TECHVICAL APPENDIXES I I5-D3-DIS33 OGRAM I SYSTEMS-EVALUATION CIVI/ EXTENSI ON OF THE GENERAL SENSITIVITY ANALYS IS III. THE ANCET COMPUTER PR I5-O3-OI938 UALTIES SYSTEMS-EVALUATION DAMAGE-ASSESSMENT NUCLEAR-WAR/ SENSITIVITY ANALYS IS OF CASUAL TY FUNETIONS 1 ISAS D6-21-0IT42 IES IN SOME NUCLEAR DAMAGE PARAME TERS ( ATTACK-SCENARIOS/ SENSITIVITY OF MORTALITY ESTIMATES TO UNCERTAINT O6-2l-D2228 IES IN SOME NUCLEAR DAMAGE PARAME TERS II. I MODELS DAMAI SENSITIVITY OF MORTALITY ESTIMATES TO UNCERTAINT OO-2I-02229 IN AGGREGATE POPULATION REPRE SENIATIONS I NUCLEAR-W/ THE SENSITIVITY OF MORTALITY ESTIYATES TO VARIATIONS TS-DF-DIOO6 LTA DIVI IAVESTICATION OF ALTE PNATIVE AQIIF NIII.T SYSTFMS TO SERVE SOUTHERN CAL IFORVIA - FEATHER RIVER AND DE I3-0?-J2258 
5 REGULATIONS RADIOACTIVITY CONTROL I \#PHS/ PUBLIC HEALTH SERVICE DRINK ING NATER STAVDARDS I WATER-SUPPLIE I3-O2-DI778 ZAT ION, TECHNOL / PRAC TICAL EXPERIENCES OF FIRE PRCTECTION SERVICES - A CR ITICAL PRESEVTA TIOV OF THE ORGANI I3-I?-D2264 ZATION, TECHNOL / PRACTICAL EXPERIENCES OF FIRE PROTECTION SERVICES - A CRITICAL PRESENTATION OF IHE ORGANI I3-I?-O2265 IC TREATMENT CENTRE - OPERATING MANUAL - EMERGENCY HEALTH SERVICES I DISASTER-MEDICIVE MEDICIVE ADMINISTR I OO-2I - OIZ3 B ATING-PLANS MEV TAL-HEAL TH POSTATTACK-OPI EMERGENCY SOCIAL SERVICES PERSONVEL BEHAVIOR CIVIL-DEFENSE-OPER O5-II -D2I 85 LYH L AW ORGAVIZATIO/ ROLE OF THE FEDERAL EMERGENCY HEALTH SERVICES CIVISION I DISASTER-MEDICIVE PUBLIC-HEA OG-2I -D2226 ISTATES - A DIRECTORY OF SELEC TED SPECIALIZED INFORMATION SERVICES IN THE PHYSICAL AND BIOLOGICAL SCIENCEI OS-D2-OIG2Q ELECTED SPECIAL IZED INFO/ SPECIALIZED SCIENCE INFORMATION SERVICES IN THE UNITED STATES - A DIRECTORY OF S O5-D?-DIG? / TRANSPORTATION LEGISLATION COMPETITION EMPLOYMENT TAXES SERVICES MANAGEMEVT-PLANNING COSTS ILLINOIS OHI/ $23-02-02095$ /ENERAL NETWORK SIMULATION MODEL I RE SOURCES DISTRIBUTION SERVICES SURPLUSES DATA-PRJCESSING SHORTAGES WE / D5-D3-D2I 43 ICTION AGAIVST NUCLEAR WEAPON FALLOUT IN EMERGENCY HEALTH SERVICES UNITS 1 SHIELDING PATIENTS $3 A D I O A C T I V E /$ I8-DS-O2O28 /NSPORTATION SUBWAYS TESTS GORING WASHINGTON CONSTRUCTION SETTLEMENT (STRUCTURAL) CONSOLIDATIOV DECOMPOSIT/ I3-D2-OI785 /STR I BUT ION BEVEATH EMBANKMENTS I STRESSES SOIL-MECHANICS SETTLEMENT (STRUCTURAL) HIGH-SPEED-GROUND-TRANSP / 20-II -O2OBL INEER ING) WASTES( INOUSTRIAL) GARBAGE SANI TARY-ENGI NEERING SEWAGE.CISEASE-VECTORS DISEASESICJYTZOL) DRAINA/ I3-O?-DI7BO IR CONTROL, AND THE EFFECTS OF FALLOUT IN WASTE WATER AND SEWER SYSTEMS I POSTATTACK-OPERATIONS RAOIOACTI/ I3-OP-DI78O DESIGN AND CONSTRUCTION OF SANITARY AND STORM SEWERS I SANITARY-SEWERS, \#WPCF-9, IVAL AGE POSIATTACK-OPERATIONS URAAN-PLANNING METHCOOLOGY SEX) \#HSR-RR-66/I4-PR-APP-I /N URBAV-AREAS SURV 23-GI-O2I J3 IVAL AGE POSTATTACK-OPERATIONS URAAN-PLANNING METHCOOLOGY SEX $)$ \#SR-RR-66/14-PR-APP-I 7N URBAV-AREAS SURV 23-CH-O2IO3

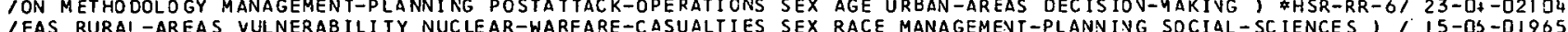
IEAS RURAL-AREAS VULNERABILI TY NUCLEAR-WARFARE-CASUALTIES SEX RACE MANAGEMENT -PLANNING SOCIAL-SCIENCES I I IS-OS -DI965 RES THERMAL-RADIATION SWEDEN, $\$$ A.L. IIIB ELLS VIBRATION LOADING(MECHANICS) SHEAR/ FORCED MCTION OF SHALLOW SPHERICAL SHELLS SPHERES STRUCTURAL-SH $20-1 /$-O2346 GRAM SPECIFICATIONS I HYDRODYNAMI / THE SURFACE WAVES FROM SHALLOW UNDERWATER EXPLOSIONS - FJ2MULAE AND PRO I9-O+-O2D43 UCTURAL-FORMS) MEMBRANES STRESSES/ PRELIMINARY TESTS ON A SHALLOW UNREINFORCED CONCRETE SHELL I SHELLS(STR I3-13-0I832 EMENT-PLANNING UNITED- 1 UTILIZATION OF SOCIAL RESEARCH IN SHAPING POLICY DECISIONS 1 SOCIIAL-SCIENCES MANAG DS-OH'-UIG58 ETE BEAMS I BEAMS (STRUCTURAL) DYNAMICS SHEAR-STR/ OYNANIC SHEAR RESISTANCE OF THIN WEBBED REIVFORCED CONCR $20-1 /-02337$ I TEST-EQUIPMENT BEAMSISTRUCTLRALI LOADING (MECH/ CYNAMIC SHEAR STRENGTH OF REINFORCED CONCZETE BEAMS II. I3-I3-OI84I UCT ION SETTLEMENT (STRUC TURAL) CONSOLIDATICN DECOMPCSITION SHEAR-STRENGTH, \#A.L. IOT2 /G WASHIVGTON CONSTR I3-O? -DI785

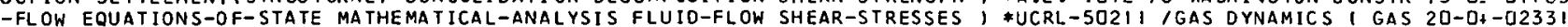
11 SPHERES STRUCTURAL-SHELLS VIBRATION LOADING (MECHANICSI SHEAR-STRESSES CEFORMAT IOV ROTATIOY IVERTIA MATI $20-11$-O2346 IC REINFORCED CONCRETE BEAMS I BEAMSI STRUCTURALI DYNAMICS SHEAR-STRESSES WEBSISTRUCTURALI DESIGV STRUCTURI 20 II -O233 7 IC RE IURES I ROCK-MECHANICS LOADING IMECHANICSI USSR EXCAVATION SHEARING CUTT ING BENDIVG TORSION STRETCHING COM/ O8-OT -O2242
IT BLAST-BIOLOGY BIOLOGICAL-BLAST-EFFECTS MCNKEYS SWINE SHEEP LUNG-INJURIES EXPLOSIONS TOLERANCES IPHYSI/ O6-2I -D2234 IT BLAST-8IOLOGY BIOLOGICAL-BLAST-EFFECTS MCNKEYS SWINE SHEEP LUNG-INJURIES EXPLOSIONS TOLERANCES IPHYSI/ O6-2I -02234
NTHRACIS MATHEMAT ICAL-MODELS/ VIABILI TY AND ESTIMATION OF SHELF LIFE OF BACTERIAL POPULATIOVS I BAC ILLUS-A IS-D2-OI849 NTHRACIS MATHEMATICAL-MODELS/ VIABILITY AND ESTIMATION OF SHELF LIFE OF BACTERIAL POPULATIOVS I BACILLUS-A I5-D2-OI849 /STORAGE LIFE - A REPORT BIBLIOGRAPHY ( BEEF PRESERVATION SHELF-LIFE BEVERAGES BREAD CAVNED CEZEALS COFFE I OG-O3-O22 O9 / YIELD STRENGTH OF A MACHINED RING SIIFFENED CYLI NDRICAL SHELL I MODEL BR-7M I UNDER HYDROSTATIC PRESSUR/ 20-1I - D234 7 SES PREL IMINARY TESTS ON A SHALLCh UNREINFORCED CCNCRETE SHELL I SHELLSISTRUCTURAL-FORYSI YEMBZANES STRES I3-13-QI832
CA TO/ SOME CHEMICAL AND PHYSICAL PROPERTIES OF PARALYTIC SHELLFISH POISONS RELATED TO TOXILITY 1 PELECYPO IS-D2-DI863 CA TO/ SOME CHEMICAL AND PHYSICAL PROPERTIES OF PARALYTIC SHELLFISH POISONS RELATED TO TOXILITY 1 PELECYPO $15-02-01863$
DINGIMECHANICS) STIEAR/ FORCED MOTION OF SHALLOW SPHERICAL SHELLS 1 SPHERES STRUCTURAL-SHELLS VIBRATION LOA 2O-II O2346 MPOSI/ VIBRATION OF MULTI-LAYERED ANI SOTRCPIC CYLIIARICAL SHELLS I STRJ CTURAL-SHELLS CYLINDRIEAL-BODIRES CO $20-11$-D2345 FENED EDGES ; STRUCTURA, ANALYSIS OF CIRCULAR CYLINDRICAL SHELLS CONTAINING RECTANGULAR OPEVIVGS WI TH STIF $20-11$-O2D8 I /RUCTION ( BLAST-SHEL TERS UNDERGROUND-STRUCTURES DYNAMICS SHELLS(STRUCTURAL-FORMS) BEAMS (STRUC TURAL) ANAL, I3-I3-DI83? IIMINARY TESTS ON A SHALLOW UNREINFORCED CONCRETE SHELL ( SHELLSISTRUCTURAL-FORMS) MEMBRANES STZESSES LOA, I3- I3-DI832 ( ENGINEER ING AND CONSULIING FIRMS WI TH CERTIFIED FALLOUT SHELTER ANALYSTS I RADIOACTIVE-FALLOUT 8UILDING I I3-I3-O2276 CCEPTANCE I SHELTER-ALLOCATION ATTITUDES CIVIL-DEFENSE-S/ SHELTER ASSIGNMENT CONCEPT - A STUDY [N PUBLIC A D5-1]-O2I $7 ?$ Y FI DEVELOPMENT OF STANDARD FIRE TEST RATING SYSTEMS FOR SHELTER COMPONENTS I FALLOUT-SHELTERS FIRE-SAFET I3-I?-O22SO IECOMMENDATIONS FOR INCLUSION OF REQUIREMENIS FOR FALLOUT SHELTER CONSTRUCTION IN FOUR VATIOYAL MODEL BUI I THEI/ 13-13-02281

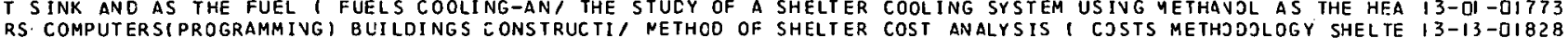
RS. COMPUTERSTPROGRAMMIVG I BUILDINGS CONSTRUC II/ NETHCO OF SHELTER COST ANALYSIS T CJSTS METHJOJLOGY SHELTE I3-I3-OI828

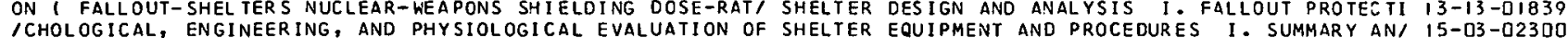

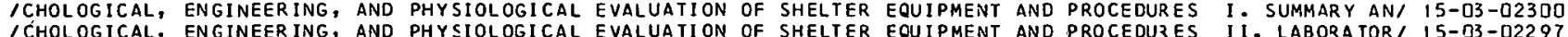
/CHOL OGICAL, ENGINEER ING, AND PHYSIOLOGICAL EVALUATION OF SHELTER EOUIPMENT AND PROCEDUZES II. LABORATOR/ I5-D3-D2297 /CHOLOGICAL, ENGINEERING, AND PHYSIOLOGICAL EVALUATION OF SHELTER EQJIPMENT AND PROCEDURES III. HABITABII I5-O3-O2298 IUNNEL WEST ORANGE, NEW JERSEY, AS A CIVIL DEFENSE PUBLIC SHELTER FACILITY I TRAVSPORTATION FEASIBILITY-SI I3-13-O22 I I TRUCTION VENTILATION J MISC. PUB. N/ BUNKER TYPE FALLOUT SHELTER FOR BEEF CATTLE 1 BOVINES SHIELDING CONS I3-I3-DIBI 8 FIRE EXPOSURE I FALLOUT-SHELTERS FIRE-PROTECTION SMOKE, SHELTER HABITARILITY IN EXISTING BUILDINGS UNDER I3-I?-OIOD? I ARGE SHELTER MAVAGEMENT, ENVIRONMENTAL THREAT, AND SMALL SHELTER HABITABILITY UVDER COVDITIJVS OF STRESSI QS-IJ-DIGB 4 -OPERAT ING-PLANS URBAN-AREAS MANAGEMENT-PLAN' MOVENENT TO SHELTER IN A MAJOR CITY I SHEL TERS ZIVIL-DEFENSE I5-O3-OIO45 -CONTROL I NATURAL VENTILATION TEST OF A BASEMENT FALLOUT SHELTER IN EAST CHICACO, INDIANA I ENVIRONMENTAL I3-DI -DITT4 -EOU IPMEVT GENERA TOR S PORTABLE DE SIGN EXERCISE LIGHTSIIL/ SHELTER LIGHI ING \& IT I FALLDUI-SHELTER S LIGHTING I3-OH -D22S 2 G SHEET SHET NT-PL LNNING HAB IABILITY SHEL TER-OCC UPANCY STUDIES OF IN SHELTER MANAGEMENT GUIDANCE MATER IALS 1 MANAGEME O5-DI -OI624 AI AN EXPERIMENTAL ANALYSIS OF SELEC TED PROBLENS OF LARGE SHELTER MANAGEMENT, ENVIROVMEVTAL THZEAT, AND SM O5-IJ-DIG84

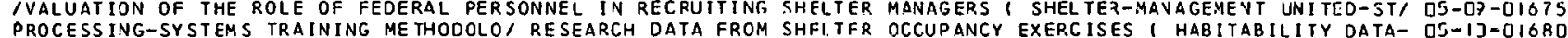
LOUT-SHEL TERS CONFINED-ENVIRONMENTS STOCKPILING SHELTER-/ SHELTER OCCUPANCY STUDIES I. FINAL REPORT I FAL OS-IJ-O2IB4 SHELTERS HANDBOOK FOR UNTRAINED MANAGEMENT 1 SHELTER-MAN/ SHELTER OCCUPANCY STUDIES 2 . COMYUVITY FALLOUT O5-OI -O2II5 SE-SYSTEMS ATTITI RESEARCH ON ACCEPTABILITY OF A NATIONAL SHELTER POLICY I JPERATIONS-RESEAZEH CIVIL-DEFEN OS-O+-O2IS6 TS COST/ ALTERNATIVE APPROACHES TO FINANCING A NATIONWIDE SHELTER PROGRAM I FALLOUT-SHELTERS FEDERAL-BUDGE I3-I3-DI834 IGN MAT/ TIME COMPRESSION POTENTIAL OF AN EMERGENCY BLAST SHELTER PROGRAM I URBAN-AREAS HASTY-SHELTERS DES I3-I3-O2272 HYS ICAL-PROPERT IES CO/ STORAGE STABILITY OF CIVIL DEFENSE SHELTER RAT IONS 1 FOOD PRESERVAIIJV FIBERBOARD P DG-O3-D22I 2 OODIACCEPTABILITY) FALLOUT-SHELTERS CON/ ACCEPTABILITY OF SHELTER RATIONS IV COMBINATION WITH ADJUNCIS I F DG-D3-OITIO OOD. FALLOUT-SHELTERS TASTE CIVIL-DEFENS/ ACCEPTABILITY OF SHELTER RATIONS IV COMBINATIOV WITH ADJUNCTS I F D6-O3-O22II CULATION - 1970-1985 I CALCULATIONS DAMAGE/ CIVIL CEFENSE SHELTER REPRESENTATION FOR POPULATION CAMAGE CAL I5-O3-OI930 ALLOUT-SHELTERS URBAN-AREAS/ VENTILATION TESTS OF FALLOUT SHELTER SPACES IN VEW YORK CITY AVD VICINITY IF I3-I3-OIBI3 RBAN-AREAS UNDERGROUND-STRL/VENTILATION TESTS OF FALLOUT SHELTER SPACES IN VEW YORK CITY AVD VICINITY I U I3-I3-DIBIG RBAN-AREAS UVDERGROUND-SIRLI VENTILATION TESTS OF FALLOUT SHELTER SPACES IN VEW YORK CITY AVD VICINITY 1 U I3-I3-DIBIL

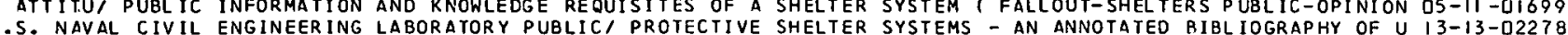

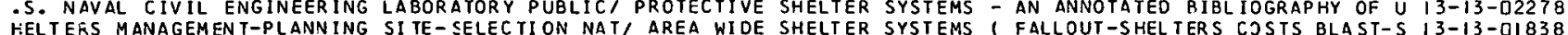
HELTERS MANAGEMENT-PLANNING SI TE- SELECTI ON NATI AREA WIDE SHELTER SYSTEMS 1 FALLOUT-SHELTERS CDSTS BLAST-S $13-13$ - II838

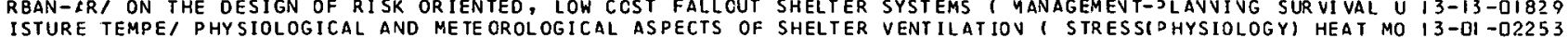

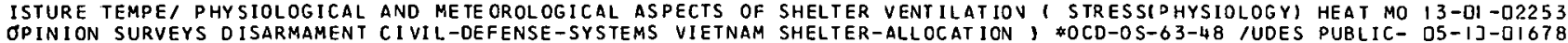
/LTER ASS IGNMENT CONCEPT - A STUDY IN PUBLIC ACCEPTANCE I SHELTER-ALLOCAT ION ATTITUDES CIVIL-DEFENSE-SYST/ O5-IJ-O2I77 LLOUT-SHELTERS CONSTRUCTION-SLANIING OPERATI CNS-RESEARCH SHELTER-ALLOCAT ION COMMUNITY-SHELTER-PLANNING-P/ D5-OI -DI6?3 ON - 1970- 1985 (CALCULATIONS DANAGE-ASSESSMENT SHELTERS SHELTER-ALLOCAT IOV DATA WARNIVG-SYSTEMS, \&TECH, I5-03-DI930 IELTERS PUBLIC-OPINION ATTITUDES BEHAVIOR WARNING-SYSTEMS SHELTER-ALLOCATION SHELTER-OCCUPANCY SURVEYS, , O5-II-QIG99 /RUCTURES STRUCTURAL-PROPERTIES STEEL REINFORCED-CCNCRETE SHELTER-DOORS TRAFFIC STRUCTURAL-SHELLS, \&MGO6/ I3-I3-OI835 IMIT ING-SYSTEMS COSTS FALLOUT-SHELTERS PUBLIC-INFCRNATION SHELTER-MANAGEMENT I \#YP-47 /DETEZREVCE DAMAGE-L IS-O3-O22 94 IORT I FALLOUT-SHELTERS CONFINED-ENVIRONMENTS STOCKPILING SHELTER-MANAGEMENT HANDBOOKS HABITABILITY NUTRI/ QS-IJ-O2I84 
SHEL TER- 1 SOME TRAINING IMPLICATIONS OF LARGE SHELTERS I SHELTER-MANAGEMENT MANAGEMEVT-PLAVVIVG PERSONNEL O5-OI - $I 62 I$ IITY FALLOUT SHEL TERS HANOBOOK FOR UNTRAINED MANAGEMENT I SHELTER-MANAGEMENT SHELTER-OCEUPAVEY INSTRUCTIO/ O5-OI -O2III IRS SYSTEMS-ENGINEERING COOLING-AND-VENTILATING-EQUIPMENT SHELTER-MANAGEMENT SHELTER-OCCUPAVCY PHYSIOLOGYI I5-D3-D2298 IRS SYSTEMS-ENG INEER ING COOLING-AND-VENTI LAYING-EQUIPMENT SHELTER-MANAGEMENT SHELTER-OCCUPAVEY PHYSIOLOGYI I 5-O3-D23OQ IGY SYSTEMS-EVGIVEER ING COOLING-AND-VENTILATING-ECUIPMENT SHELTER-MANAGEMENT SHEL TER-OCCUPAV-Y P SYCHOLOFYI I 5-D3-02297 ILE OF FEDERAL PERSONNEL IN RECRUITING SHELTFR MANAGERS I SHELTER-MANAGEMENT UNITED-STATES-GJVERNMENTIPER/ D5-DF-DI 675 INING CIVIL-DEFEN SE-OPERA IING-PLANS POSTA TIACK-OPERAT IONS SHELTER-OCCUPANCY DAMAGE-ASSESSMEVT I *FG-E-I3. 1 OS- 11 -D2I 7 ICE FOR SHELTER MANAGEMENT I MANAGEMENT-PLANNING TRAINING SHELTER-OCCUPANCY EXPERIMEVTAL-CATA; $\$$ I SMALL SHELTER HABITABILITY UNDER EONDITIONS CF STRESS 1 SHELTER-OCCUPANCY FEASIBILITY-STUDIES UNDERWATE/ DS-IJ-OIGB 4 IS HANDBOOK FOR UNTRAINED MANAGEMENT I SHELTER-MANAGEMENT SHELTER-OCCUPANCY INSTRUCTION-MANUALS I *A.L. I' O5-OI -D2IIS INT GUIDANCE MATERIALS 1 MANAGEMENT-PLANNING HABITABILITY SHELTER-OCCUPANCY INSTRUCTION-MANUALS HANDBOOKS/ D5-OI - DIG24 /PPLIES FOOD-DISPENSING FOOD STOCKPILING FALLOUT-SHELTERS SHELTER-OCCUPANCY LOGISTICS WATER-SUPPLIES RATI/ DG-D9-DI7I 2 ILATENT HEAT LOSSES FROM OCCUPANIS OF SURVIVAL SHELTERS I SHELTER-OCCUPANCY YETABOL ISM PHYSIJLOGY HEAT-EXI DG-IS-DI7I8 IING COOL ING-AVD-VENTILATING-EQUIPNENT SHELTER-MANAGEMENT SHELTER-OCCUPANCY PHYSIOLOGY PERFJRMANCE (HUMAN)/ IS-O3-O2298 IING COOL ING-AND-VENTILATING-EOUI PNENT SHELTFR-MANAGEMENT SHELTER-OCCUPANCY PHYSIOLOGY PERFJRMANCE (HUMANI/ I5-O3 -D23JO FENSE-OPERA/ EMER GENCY WELFARE REG ISTRATION AND INCUIRY I SHELTER-OCCUPANCY POSTATTACK-OPERATIOVS CIVIL-DE O5-II-O2IB9 ING I CIVIL-DEFENSE-OPERATING-PLANS FOOD FOOD-DI SPENSING SHELTER-OCCUPANCY POSTATTACT-OPERAT IOVS HOSPITAI DO-OS-O22ID IING COOL ING-AVD-VEVTILATING-ECUIPNENT SHELTER-MANAGEMENT SHELTER-OCCUPANCY PSYCHOLOGY PERFJ2MANCE (HUMANII IS-03-O2297 IING COOL ING-AVD-VEVTILATING-ECUIPNENT SHELTER-MANAGEMENT SHELTER-OCCUPANCY PSYCHOLOGY PERFJZMANCE(HUMAN) I5-03-D2297 ION ATTITUDES BEHAVIOR WARNING-SYSTEMS SHELTER-ALLCCATION SHELTER-OCCUPANCY SURVEYS 1 \$IR-D- $930-9 / 66-F R /$ OS-II -01699 /NGINECRINGI RHYSIOLOGY COOLING-AND-VENTI LATICN-EOUIPMENT SHELTER-OCCUPANCY SYSTEMS-ENGINEERING SANITARY-I I3-I3-DI8I 9
/CKPILING FOOD-DISPENSING FOOD FALLOUT-SHELTERS LOGISTICS SHELTER-OCCUPANCY NATER-SUPPLIES EDUCATION TRAI/ OO-OS-OI I 3

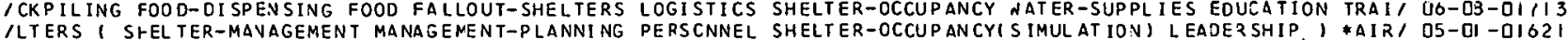

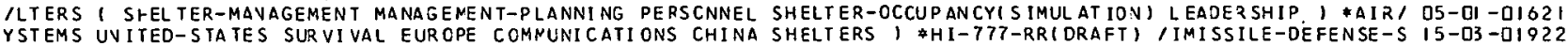
CHES 1 UNDERGROUND-STRUC TURES OYNAMICS LOADINGIMECHANICS) SHELTERS, \#MISC. PAPER NO. I-8E9 /D CONCRETE AR I3-I3-DI83D TION-EFFECTS RADIOACTIVE-FALLOUT CONSTRIICTION COI FALLOUT SHELTERS - CODES AVD STANDARDS I SHIELDING RADIA I3-I3-O22BO IAYS AND EXITS FOR BLAST RESISTANT FULLY BURIEO PERSONNEL SHELTERS 1 BLAST-SHELTERS DESIGN GJSTS SINGLE-PI I3-IS-OIB35 AN INVESTIGATION OF MINIMAL EQUIPNENT NEEOS IN PERSONNEL SHELTERS 1 FALLOUT-SHELTERS OPTIMIZATION PERFORN $13-13$-OIBI9 AN INVESTIGATION OF MINIMAL EQUIPNENT NEEOS IN PERSONNEL SHELTERS 1 FALLOUT-SHEL TERS PHYSIOLOGY EXPLOSION I3-I3-DIBII INSIBLE AND LATENT HEAT LOSSES FROM OCCUPANTS OF SLRVIVAL SHELTERS ( HEAT-PRODUCT ION(BIOLOGY) SURVIVAL FA/ DG-IG-DI7I 9 G PERSONYEL SHELTER-I SOME TRAINING IMPLICATIONS OF LARGE SHELTERS I SHELTER-MANAGEMENT MAVAGEMENT-PLANNIN OS-UI -ŨIGZI G PER SONVEL SHELTER-A SOME TRAINING IMPLICATIONS OF LARGE SHELTERS SHELTER-MANAGEMENT MANAGEMENT-PLANNIN OS-UI -UIOZI INS IBLE ANO LATEVT HEAT LOSSES FRON OCCUPANTS CF SURVIVAL SHELTERS I SHELTER-OCCUPAVCY YETABJLISM PHYSIOL/ DO-1S-DITI 1962 COST AVALYSIS FOR A STANDARDI ZED SERIES OF FALLOUT SHELTERS 1 UNDERGR JUND-STRUCTURES CONSTRUCTION I I3- I3 -OI815
MS MS MILITARY-STRATEGY I \#I-729/ WHY COUPLE BMD TO FALLOUT SHELTERS 1 URBAN-AREAS ANTIMISSILE-DEFENSE SYSTE I5-O3-DI9O7 IFFECT OF POPULAT ION MOBILITY ON THE LOCATION OF CCMMUNAL SHELTERS 1 WARNING-SYSTEMS URBAN-AREAS MOVEMENTI IS-O3-OI 942
ON 2 SASTY

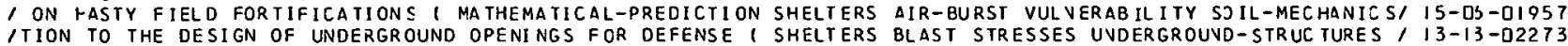
AS MANAGEMENT-PLAN/ MOVEMENT TO SHELTER IN A NAJOR CITY I SHELTERS CIVIL-DEFENSE-OPERATING-PLANS URBAN-ARE I5-O3-OIQ45 UCTI/ METHOD OF SHEL TER COST ANALYSIS, COSTS METHCDOLOGY SHELTERS COMPUTERS (PROGRAMMING) BUILDINGS CONSTR I3-I3-OIB28 RUCT ION-MATER IALS RADIOACTIVE-FALLOUT SHIELDING BUILDINGS SHELTERS COSTS PARTICLES, \#USYRDL-TZ-ID64 /ONST I8-OS-D232I IAPONS-EFFECTS RADIATION-EFFEC TS POPULATI CN PARTICLE-SIZE SHELTERS CISTRIBUT ION RADIOACTIVE-FALLOUT BIOLOI OG-2I -O22Z29 , BUILUIVGS FOR A FEOERAL TRANSPORTATION ADMINISTRATION 1 SHELTERS DIIAI -PURPOSE-SHELTERS COSTS GERMANY COI I3-I3-OIB2 7 IES UNITED-STATES-GOVERNMENT OFF ICE-OF-EMERGENCY-PLANNING SHELTERS ELECTROMAGNET IC-PULSE RADIATION SHIELD/ I5-O3-D2299

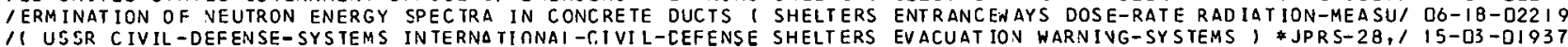
IIES TRAVSPORTATION EVACUATION DI SPERSAL URBAN-AREAS FOOD SHELTERS FALLOUT-SHELTERS EURJPE SURVIVAL RLCUVI I5-OS-DZZ 5 IIES TRAVSPORTATION EVACUATION DI SPER SAL URBAN-AREAS FOOD SHELTERS FALL OUT-SHEL TERS EUR JPE SURVIVAL RECUVI IS-OS-D22 FS

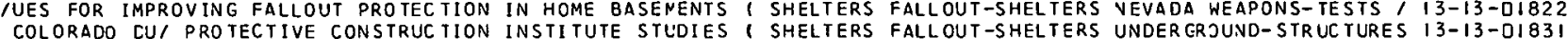
COLORADO CU/ PROTECTIVE CONSTRUC IION INSTITUTE STLDIES I SHELTERS FALLOUT-SHELTERS UNDER CRJUND-STRUCTURES I3-I3-OI83!

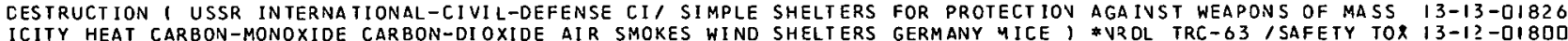

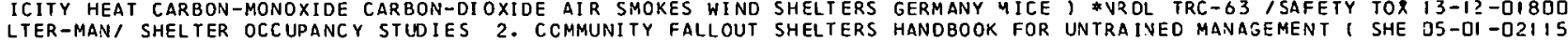
LTER-MAN/ SHELTER OCCUPANCY STUDIES 2. CCMMUNITY FALLOUT SHELTERS HANDBOOK FOR UNTRA INED MANAGEMENT I SHE DS-DI - O2IIS
MSI TEST AND EVALUATION OF COMPUTER ANALYSIS PROGRAMS FOR SHELTERS IN BUILOINGS 1 DYNAMICS COMPUTER-PROGRA $13-13-02280$ IS A MEASURE OF PROTECTION AGAINST NUCLEAR WEAPONS I USSR SHELTERS INTERNATIONAL-CIVIL-DEFEVSE CIVIL-DEFE/ I5-D3-OIQ34 IT RACIOACTIVE CONTAMINATION 1 USSR CIVIL-DEFENSE-SYSTEMS SHELTERS INTERNATIONAL-CIVIL-DEFEVSE RADIOLOGIC I OG-I3-DI73I , SLABS 1 TESTS LOADING(MECHANICSI UNOERGROUND-STRUCTURES SHELTERS PERFORMAVCEIEVGIVEERING) STATICS STRUC, I3-I3-O2290 MYTEMS MODELS RADIOLOGICAL-MONI TORING LOGISTICS MANPOWER SHELTERS PROGRAMMIVG(COMPUTERS) DISPERSAL INDUS/ (5-D3-OIQ49 I ATTACK-OPERATIONS MANAGEMENT-PLANNING STRESS (PSYCHOLOGY) SHELTERS SCHOOLS PUELIC-INFORMATIOV EDUCATION O/ O5-II-D22O3 ICALCULAT ION - $1970-1985$, CALCULATIONS DANAGE-ASSESSMENT SHELTERS SHELTER-ALLOCATION DATA WARVING-SYSTEM/ I5-O3-OI93D IROTECTIVE STRUCTLRES FOR PROTECTION FRDN NIICIEAR ELAST ( SHELTERS SHOCK-WAVES BLAST-SHELTEZS UNDERGROUND/ I3-I3-OI833 ISERVED CONDITIONS I HEAT-TRANSFER UNDERG ROUND-STRUCTURES SHELTERS THERMAL-DIFFUSION THERMAL-CONUUCTIVIIYI $20-13$-O2O9O /SERVED CONDITIONS I HEAT-TRANSFER UNDERG ROUND-STRUCTURES SHELTERS THERMAL-DIFFUS ION THERMAL-CONUUC IIVI IY/ $20-13$-02090
CYLINOERS, CR IT ICAL LITERATURE REVIEW ANO PILOT STUDY I SHELTERS UNDERGROUVD-STRUCTURES ELASTICITY TEST/ I3- 3 -OI825

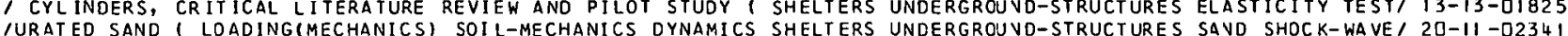

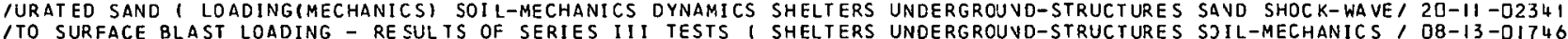

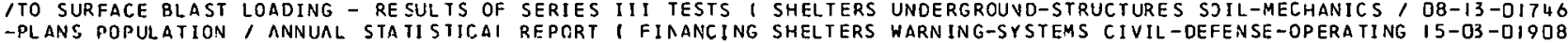
IATION-ISOLATORS SHOCK-WAVES SHOCK (MECHANICS) ATTENUATION SHELTERS WEAPONS-EFFECTS SURVIVALIEQUIPMENII SI/ I7-OZ-D23II EQUIREMENT3 FOR, A HODLL BUILDING GOOE AR.TICLE DN FAIIOLIT SHEITFRS WITH RECOMMENDATIONS FOR INCLUSION OF R I3-13-O228I ON-INJURIES STATISTICAL-ANALYSIS BIOLOGICAL-BLAST-EFFECTS SHIELDING, \$DC-FR-1054, WEAPONS-EFFECT RADIATI D6-2I-U2238 NAL REPORT, I CONSTRUCTION-MATCRIALS RADIOACTIVE-FALLOUT SHIELOING RIIILDINGS SHELTERS COSTS PAZTICLES, , I8-OS-D232I TS GCRMAYY CONSTRLCTION HAPDENING DESIIGN DYNAMIC-PRESSURE SHIELDING CALCULAT IONS GARAGES, *JRNL-TR-I 646, $13-13$-0I827 N/ BUNKER TYPE FALLOUT SHELTER FOR BEEF CAITLE I BOVINES SHIELOING CONSTRUCTION VEVTILATIOV 1 *MISC. PUB. $13-13-0 I 8 I 8$ LYSTS 1 RAOIOACTIVE-FALLOUT BUILDINGS CONSTRUCTION DESIGN SHIELOING CONSTRUCTION-SLANTIVG ) \#FG-F-1.3 /ANA I3-13-O2276 CTALLOLT-SHELTERS NUCLEAR-hEAPONS SHIELDING DOSE-RATE CAL CULATIJVS SLAVTING \#TRI I3-I3 UT ATTENUATIOV UNDERGRDUND-STRLCTLRES DOSE-RATE PHCTCNS / SHIELOING IV - CIVIL DEFEVSE I RADIDACTIVE-FALLO I8-OS-O2029 /LEAR WEAPON FALLOUT IN EMERGENCY HEALTH SERVICES UNITS I SHIEI.DING PAT IENTS RADIOACTIVE-FALLOUT GAMMA-RA' I8-0S-O2028 /ROCEDURES FOR RADEF SYSTEMS I RADIOLOGICAL-CONTAMINATION SHIELOING PROTECTION-FACTOR RADIOLOGICAL-DOSAGE/ OS-IS-DI729 CONSTRUCTION CO/ FALLOUT SHELTERS - CODES AND STANDARDS I SHIELOING RADIATION-EFFECTS RADIOACTIVE-FALLOUT I3-I3-D2286 IAGAINST VUCLEAR ATTACK I RURAL-AREAS RADIOACTIVE-FALLCUT SHIELDING RADIATIOV-HAZARDS DECONTAMIVATION RADI I5-03-DI897
ISAGE DOSIMETERS RADIATION-EFFECTS CALCULATIONS DOSE-RATE SHIELDING RADIATIOV-MEASUREMEVT-SYSTEMS RADIATI/ O6-I3-DI726 ISAGE DOSIMETER S RADIATION-EFFECTS CALCULATIONS DOSE-RATE SHIELDING RADIATIOV-MEASUREMEVT-SYSTEMS RADIATI/ O6-I3-DI726
G/ AN EXPERIMENTAL EVALUAIION OF ROOF REDUCTICN FACTORS I SHIELDING RADIOACTIVE-FALLOUT OOSE-RATES RAOIOLO I8-OS-O2O3O /RGENCY-PLANN ING SHELTER S ELEC TRONAGNETIC-PULSE RADIATION SHIELOING RADIOACTIVE-FALLOUT RADIOLOGICAL-DOSA/ I5-O3-D2299 E FARM-CROPS RADIOLOGICAL-CONTAMINATION RAOIATICN-HAZAROS SHIELOING RADIOAGRICULTURE, *NREC-36 IGICAL-DOS D6-I3-O22I 7

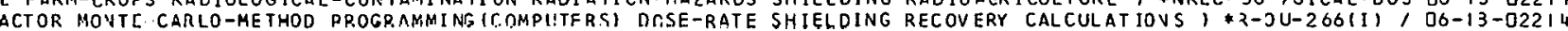
/BUILDINGS I DYNAMICS COMPUTER-PRCGRAMS SHOCK-WAVES BLAST SHIELDING STRUCTURAL-AVALYSIS RADIOACTIVE-TALLU/ I3-I3-O2ZSO TRUCT ION URBAN-AREAS RADIOACTIVE-FALLOUT FALLOUT-SHELTERS SHIELDING STRUCTURAL-PROPERTIES ) \#NP-857I ICONS I3-13-D2277 ITECT ION-FACTOR DOSE-RATE BUILDINGS DECONTAMINATI ON BLAST SHIELOING THEORY MATHEMATICAL-ANALYSIS RADIOLOGI OG-I3-O222I / GRAPIS BIOLOGICAL-BLAST-EFFEC TS BLRNS RADIATICN-INJURIES SHIELDING URBAN-AREAS CASUALTIES(PREDICTION) I, OG-2I -D222 7 ICONTAMIVATION PROGRAMMING (COMPUTERS) RADIOACTIVE-FALLOUT SHIELOING WALLS COBALT EXPERIYENTAL-DATA THEORYI D6-I3-D22IB CMETRY MATHEMATICAL-MODELS HEAT-TRANSFER WEAPONS-EFFECTS SHIP-STRUCTURAL-COMPONENTS NAVAL-RESEARCH I *A. I I3-I2-DI797 IOMETRY MATHEMATICAL-MODELS HEAT-TRANSFER WEAPONS-EFFECTS SHIP-STRUCTURAL-COMPONENTS NAVAL-RESEARCH ATERIALS T URBAN-AREAS DECONTAMINATION RAOIOACTIVE-WASTES SHIPPING-CONTAINERS, \#NY0-9772 /L RADIDACTIVE M $18-07-02034$

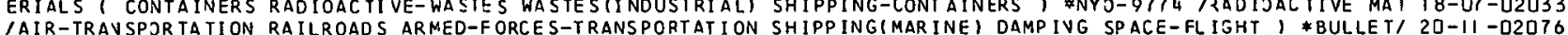
RAFT TRANSPORTATION LAUNCHING-SITES METEOROLOGY RAILROADS SHIPS HOISTS CHINA $\$$ *ATD-66-134*AD O45 O29 /EC 16-0I-OI973 
CKIPATHOLOGY) EXPLOSION- $/$ BIOLOGICAL. EFFECTS OF BLAST ANO SHOCK I SHOCK -WAVES BIOLOGICAL-BLAST-EFFECTS SHO DS-2I -OITHI OCK (MECHANICS), *BULLETIN $35(6)$ THE SHOCK ANC VIBRAT IOV BULLETIN CCELEROMETERS SPECTRUM-ANALYZERS TRANSDUCERS MATHENAI THE SHOCK AND VIBRATION BULLETIN 1 INSTRUMENTATION A 20-II-D2D75 STRUCTURAL - ANALYSIS ELASTICITY BEAMS (STRUCTURAL) SHO/ THE SHCCK ANE VIBRATION BULLETIN ( ROCKETS DYNAMICS $20-1 /$-Q2OT4 LOGY) MOT ION-SICKNESS STRESSIPHYSICLOGY) AI SUPPLENENT TO SHOCK ANC VIBRAT IOV BULLETIY I TOLERAVCES IPHYSIO OO-I7-OI735 BRAT ICAL AND BIOLOGICAL PARAMETERS, IHE SCOPE OF BLAST AND SHOCK BIOLOGY AND PROBLEM AREAS IV RELATING PHYS DG-2I -O2223 SPACES INDUCED BY AIR OLAST I ELASTICI THEORE TICAL GROUND SHOCK EFFECTS I. GROUND MDTIJN IV ELASTIC HALF I 9-OH -O2O4 4 ORDINATES TOPLASTIC WAVE PROBLEMS, THEORETICAL GROUND 5HOCK EFFECTS II. APPL ICAT IOV OF GEVERALIZEO CO O8-13-DI747 ABSORBERS VIBRATION-I SOLATORS IMPACT-SHOCK NUCLI STUDY OF 5 HOCK ISOLATION FOR HAROEVED STRUITURES I SHOCK- I3- 13 - OIB23 ANO MODIFIED SACHS SCALING I. EXPONENTIAL IDEAL GAS ATM/ SHOCK PROPAGATION IN NONHOMOGENEOUS ATMOSPHERES $20-0 .-02332$ SHOCK(MECHAVICS) INSTRUMENTATION CALIBRATI/ A MECHANICAL SHCCK PULSE SURVEY I TEST-EQUIPMEVT IMPACT-SHOCK IL-D?-OIBL2 HOCK (MECHANICS) VIBRATION ELASTICITY VELOCITY DEGREES-OF/ SHOCK SPECTRA FOR A GEVERAL FJRCIVG FUNETION I S $20-1 /$-02342 VES MECHAVICAL/ STUDY OF GROUND MCTIONS FCR SIMULATION BY SHOCK TESTING MACHINE I NUCLEAR-WEAPOVS SHOCK-WA $20-I I$-D2O7B IAGES RESULTING FROM LABORATORY VIBRATION AND HIGH IMPACT SHOCK TESTS I NAVAL-RESEARCH DESIGN ELECTRONIC-/ $20-11$-D2OB 3 IANCE OF CATTLE TO LONG DURATION REFLECTED PRESSURES IN A SHOCK TUBE ( BIOLOGICAL-BLAST-EFFECTS BLAST-BIO/ O6-2I-D2237 SHCCK TUBES - A RIBL IOGRAPHY *MLM-1189 20-04-02052 IINGS AVD STRUCTURES - OPERATION OF SIX-FCOT AND TWO-FOOT 5HOCK TUBES - EXPERIMEVTAL OBSERVATIOVS OF INTEI I8-D3 - D2OJI IINGS AVD STRUC TURES - OPERATION OF SIX-FCOT ANO TWO-FOOT SHOCK TUBES - EXPERIMEVTAL OBSERVATIOVS OF INTE/ I8-D3-D2OJ IINGS AND STRUC TURES - OPERATION OF SIX-FCOT AND TWO-FOOT SHOCK TUBES - EXPERIMEVTAL OBSERVATIONS OF INTE/ I8-03-020J5 - CAT A PRESSURE-GAGES, \#ORNL-TR-1, THE PRCPAGATION OF AIR SHCCK WAVES IN A JBE I SHOCK-TUBES EXPER IMENTAL $20-$ C.-O2DS3 EPORT NOSSURE-GAEES NUCL I STUDY OF SHOCK ISOLAIION FCR HARDENEO STRUCTURES I SHOCK-ABSORBERS VIBRATION-ISOLATOZS IMPACT-SHOCK I3-13-DIB23 AVES BIOLOGICAL-BLAST-EFFECTS RATS GUINEA-PIGS DOGS GOATS SHOCK-TUBES, *DASA-1852, BLAST-BIOLOGY SHOCK-W O6-21-D2236 -ME ASUREMENTS P IEZOELECTRIC-TRANSDUCERS LIVESTOCK-SALVAGE SHOCK-TUBES, *CASA-I855/CK-WAVES PRESSURE-TIME D6-2I -0223? INE SHEEP LUNG-IVJURIES EXPLOSIONS TOLERANCES (PHYSIOLOGY) SHCCK-TUBES, \#CASA-1860 /AST-EFFESTS MONKEYS Sh O6-2I -02234 ITENUATION FORMULAS FOR STRAIGHT TLNNELS - A COMPARISON 1 SHOCK-TUBES CAL CULATIOVS EXPERIMEVTAL-DATA EXPL/ 2D-OA-D2OSD ORNL-TR-I/ THE PROPAGATION OF AIR SHOCK WAVES IN A TUBE I SHOCK-TUBES EXPERIMENTAL-DATA PRESSURE-GAGES I * 20-OA-020S3 ILATION FACILITY I BIOLOGICAL-BLAST-EFFECTS BLAST-BIOLOGY SHOCK-TUBES INSTRJMENTATIOV PRESSURE-GAGES-CALI/ O6-2I-D2232 ICIES OF MAMMALS I BLAST-BIOLOGY BIOLOGICAL-BLAST-EFFECTS SHCCK-TUBES LUNG-DAMAGE TOLERAVCESIPHYSIOLOGYI, Q6-2I -O2230

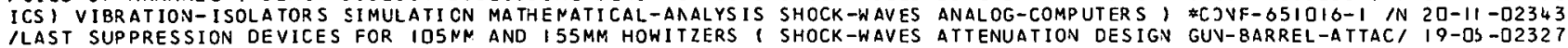
TURE ON TOLERANCE OF MAMMALS TO AIR BLAST BLAST-BIOLOGY SHOCK-WAVES BIOLOGICAL-BLAST-EFFECTS RATS GUINEI OS-2I-O223G

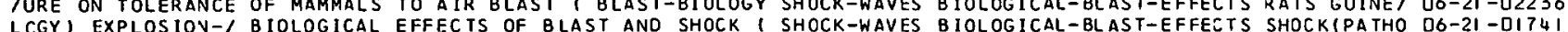
LCGY I EXPLOSIOV-

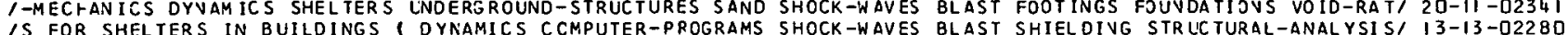
IS FOR SHELTERS IN BUILDINGS I DYNAMICS CCMPUTER-PROGRAMS SHOCK-WAVES BLAST SHIEL OIVG STRUCTURAL-ANALYSIS/ I3-I3-0228D I STRUCTURES FOR PROTECTION FROM NUCLEAR BLAST I SHELTERS SHOCK-WAVES BLAST-SHELTERS UNDERGROUND-STRUCTUR/ I3-13-0I833 IN THE GENERATION OF AIRBLASTS BY UNDERWATER EXPLOSIONS ( SHCCK-WAVES CALCULATIONS 4ODELS I \$VILTR-66-88 / $19-04-02326$ INICAL-WAVES PROPAGATION MA THEMATICAL-ANALYSIS ELASTICITY SHCCK-WAVES CONT IVJUM-MECHAVICS LIVEAZ-SYSTEMS I $20-1 I$-O2OSD

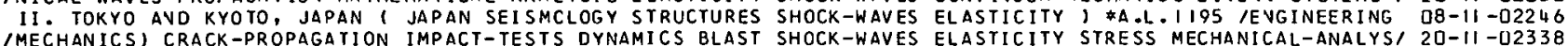
SHOCK-WAVS ELASTICITY STRESS MECHANICAL-ANALYS/ 20-11-02338 IAL OBSERVATIONS OF INTERIOR PRESSLRES IN HOLLOW MCDELS I SHOCK-WAVES EXPERIMENTAL-DATA TEST-METHODS PRESI I8-D3-O2OOS IT LOADING - RESULTS OF SERIES 111 TESTS ( SOIL-NECHANICS SHOCK-WAVES FAILUREIMECHAVICS) UNJERGROUND-STRU/ I8-D3-O2OJ8 IIED SACHS SCAL ING I EXPONENTIAL IDEAL GAS ATMOSPHERE I SHOCK-WAVES FLUIC-DYNAMIC-PROPERTIES HYDRODYNAN/ 20 -O4-02332 IRANICS SCIENTIFIC-RESEARCH ABLATION ANTENNAS PROPAGATION SHOCK-WAVES KINETIC-THEORY ELECTROV-DENSITY CER, $20-03$-D233D (FFECTS SHOCK (MECHAVICS) VULNERAB ILI TY NUCLEAR-EXPLOSI ONS SHOCK-WAVES LOACIVGIMECHAVICS) SUSPEVSION-DEVIC, I3- 3 -D22OI SES WEBSISTRUCT SES WEAT IIN ELASTIC HALF SPACES INDUCED BYAIR BLAST I ELASTICITY SHOCK-WAVES MECHAVICAL-WAVES LOADING(MECHANICS) I I -OH-O2O44 IOR SIMULATION BY SHOCK TESTING MACHINE ( NUCLEAR-WEAPONS SHOCK-WAVES MECHAVICAL-WAVES SHOCK (MECHANIC S) D/ 20-II-O2078
IRENT IAL-EQUAT IONS EQUAT IONS-OF-MOTION LOADING (MECHANICS) SHOCK-WAVES NUCLEAR-EXPLOSION-DAMAGE ) \$AD G47, I3- $33-02279$ IRENT IAL-EQUATIONS EQUAT IONS-OF-MOTION LOADING (MECHANICS) SHOCK-WAVES NUCLEAR-EXPLOSION-DAMAGE I \#AD O47 1 I $3-13$-D2279 IIN A SHJCK TUBE I BIOLOGICAL-BLAST-EFFECTS BLAST-BIOLCGY SHOCK-WAVES PRESSURE-TIME-YEASUREYEVTS PIEZOELE/ O6-21 -02237 1 IES CESIGN HAROENING BLAST AIR-BLRST VIBRATICN-ISCLATORS SHOCK-WAVES SHOCX(MECHANICS) ATIEVUATION SHELTEI I 7-0? -D23II IRAINING MANUALS NUCLEAR-EXPLOSI ON-DAMAGE NUCLEAR-WARFARE SHOCK-WAVES THERMAL-RADIATIOV RADIOLOGICAL-CONT/ I3-I?-OI8J4 IE PROBLEMS.I SOIL-MECHANICS MECHANICAL-WAVES PROPAGAII ON SHOCK-WAVES UNDERGROUNO-EXPLOSIONS ELASTICITY E/ OB-I3-OI74 7 ENG INEERING LOADING (MECHANICS) COOLING FAILURE (MECHANICS) SHOCK-WAVES VENTILATION I \$A.L. $1205 /$ ING MINING- I3-I3-02273 IIMENTAL-DATA STRAIN (MECHANIC S) STRESS LOADING (MECHANICS) SHOCK-WAVES VISCOELASTICITY BLAST, \&CONTRACT R/ $20-11$-O2DB6 LECTRONIC-EQUIPMENT RELIABILITY(ELECTRONIC) NAVAL-VESSELS SHOCK(MECHANICS), \#AD 37 BQO /RESEAREH DESIGN E $20-11$-02DB3 XPANDED-PLASTICS GLASS HONEYCOMB-CORES LOAOING (MECHANICS) SHOCK (MECHANICS), \$AD 634 691 /UVD-STRUCTURES E I8-03-020D7 THE SHOCK AND VIBRATION BLLLETIN I IMPACT-TESTING S IARCENING BLAST AIR-BURST VIBRATI ON-I SOLATORS SHOCK-WAVES I UNDERGROUD-STRUC TURES CONSTRUC TI ON LOADI NG (MECHANICS IHANICAL SHOCK PULSE SURVEY ( TEST-EQUIPMENT I MPACT-SHOCK IYNAMICS STRUCTURAL-ANALYSIS ELASTICI TY BEAMS (STRUCTURAL) TYNAM ICS STRUCTURAL-ANALYSIS ELASTICI TY BEAMS ISTRUCTURAL,

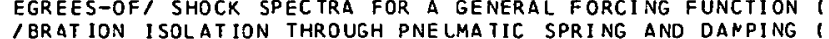
IROUND MOTIONS I LNDERGROUND-SIRUC TURES EXPLOSION-EFFECTS It BLAST AND SHOCK I SHOCK-WAVES BIOLOGICAL-BLAST-EFFECTS RESOURCES CISTRIBUTION SERVICES SLRPLUSES DATA-PROCESSING

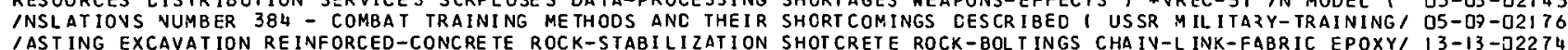
NT, DESIGN AND CONSTRUCTION OF A CRASH DUNMY FOR TESTING SHOTCETE ROCK-BOLTINGS CHAIV-LINK-FABRIC EPOXYI I3-13-D2274 \begin{tabular}{ll} 
IHANNELS I. GENERAL DESCRIPTION OF WORK PROPOSED AND MAP SHOWING GENERAL LOCATION OF PROJECTS I FLOODS F/ I3-O2-OITB2 \\
\hline
\end{tabular} IPROGRAM I. GENERAL DESCRIPTION OF WORK PROPOSED AND MAP SHOWING GENERAL LOCATION JF PROJECTS I FLOODS F/ I3-D?-DI783 IPROGRAM 1. GENERAL DESCRIPTION OF WORK PROPOSED AND MAP SHCWING GENERAI IOCATION OF PROJECTS I FLOODS FI I 3-C? OI784 ANSPORTATION I URBAN-AREAS MOVING-BELTWAYS TRAFFI/ MOVING SIDEWALKS AND THE PROBLEM OF SHORT RANGE MASS TR 23 -D? -O2D97 IS LAW CONSTANTS FOR DISSOLUTION OF FISSION PRODUCTS IN A SILICATE FALLOUT PARTICLE MATRIX I RADIOACTIVE- I I8-O3-O2325 /E DOLOMITE LIMESTONE ROCK-SALT ANHYDRITE SANDSTONES CLAY SILTSTONES GEOL OGY THERMAL-STRESS STRESSES STRA/ DS-D7-D224 2 ION CONTAM/ HOW TO PURIFY AND TO DISINFEC T WATER WITH THE SIMPLEST MEANS - USSR 1 WATER-SUPDLIES PURIFICAT O6-I3-DI722
INDUSTR IAL HARDENING CLASSIFICATION - A NETHODOLCGY FOR SIMPLIFYING THE EVALUATIOV OFF HARDENING COSTS I OS-03-D2I26 INDUSTR IAL HARDENING CLASSIFICATION - A METHODOLCGY FOR SIMPLIFYING THE EVALUATIOV OF HARDENING COSTS I O5-03-D2I 26
I INDUSIRIAL HARDENING CLASSIFICATION - A METHODOLCGY FOR SIMPLIFYING THE EVALUATION OF HARDENING COSTS I OS-O3-D2I 22

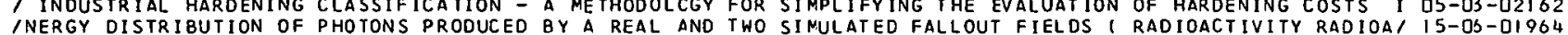
INERGY DISTRIBUTION OF PHOTONS PRODUCED BY A REAL AND TWO SIMULATED FALLOUT FIELOS I RADIOACTIVITY RADIOAI I5-OS-OI964

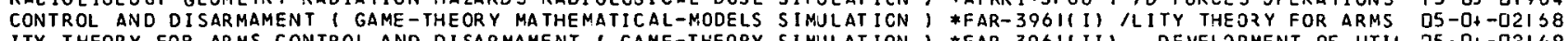
ITY THEORY FOR ARMS CONTROL AND DISARMAMENT (GAME-THEORY SIMII AT ION, \#FAR-3961(II) DEVELOPMENT OF UTIL OS-DI-O21G9 I RADIATION-INJUR IES YIELD RADIOLOGICAL-DCSAGE WIND FIRES SIMULATION BLAST STATE-OF-THE-ART-REVIEWS, *HI/ IS-DS-DI9G2 APONS SHOCK-WAVES MECHANICAL/ STUDY OF GROUND MOTICNS FOR SINULATICN BY SHOCK TESTIVG YACFIVE I NUCLEAR-WE $20-1 I$-O2O7B MPUTERS HOUSIVG PR' ATTACK I - A TTACK PATTERN GENERATOR 1 SI MULAT ICN CAMAEE-ASSESSMEVT ATTAEK-SCENARIOS CO OS-D3-O2I 39 LAST-BIOLOGY SHOCK-TU/ DASA AEC LOVELACE FOUNDATION BLAST SIMULATION FACILITY I BIOLOGICAL-BLAST-EFFECTS B DO-2I-D2232 
IS FIRE LIFE HAZARD I NUCLEAR-EXPLOSI ONS FIRES CASUALTIES SIMULATION FIRE-SAFETY TOXICITY HEAT CARBON-MON/ I3-I?-QI8TO IA-PROCESSING-SYSTEMS COMPUTERS DATA-TRANSMISSION-SYSTEMS SI MULAT ION INFORHATION-RETR IEVAL GAME-THE ORY ED/ D9-D2-QI75I IPRING AND DAMP ING I SHOCKIMECHANICSI VIBRATION-ISCLATORS SIMULAT ION MATHEMATICAL-AVALYSIS SHOCK-WAVES AN/ $20-I I-O 2343$ CATA-PROCESSING URBAN-AREAS MA THEN/ FLAME I - FIRE SPREAO SI MULAT ION MODEL I FIRES PROGRAMMIVGICOMPUTERSI I3-I?-D2266 ES SURPLUSES DATA-PROCESSING SHORTAGES/ A GENERAL NETWORK SIMULATION MODEL 1 RESOURCES OISTZIBUTION SERVIC OS-O3-O2I 3 IN UNCERGROUND INSTALLATIONS TO BLAST INDUCEO GROUND MOT/ SIMULATION OF THE RESPONSE OF EQUIPMEVT LOCATED I3-I3-D229I ISENENTS SHEL TERS FALLOUT-SHELTERS NEVAOA WEAPONS-TESTS SIMULAT ION RADIOACTIVE-FALLOUT PRJTECTION-FACTO/ I3-I3-OIB22

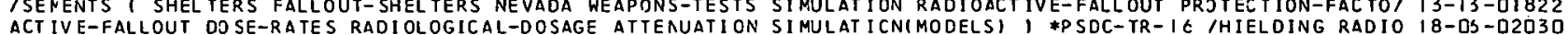
NCERGROUND PROTEC TIVE STR I COMPARI SON OF DIGI IAL CCNPUTER SIMULAI ICNS OF IHERMAL ENVIRJVMENT IN OCC UPIED U $20-13-02090$ D 208/ NOMIVAL 9, COO BTU/HR, COMPACT, VERTICAL, 230-VOLT, SINGLE PHASE, 60-CYCLE 1 MODEL CEIIVALG-230) AN I3-OI-DI775 1 BUR IED PER SONVEL SHELTERS I BLAST-SHELTERS DESIGN COSTS SINGLE-PURPOSE-SHELTERS DOORS UNDERGROUND-STRUCI I3-I3-DI835 IY OF A SHELTER COOLING SYSTEM USING METHANOL AS THE HEAT SINK ANC AS THE FJEL 1 FUELS COOLIVG-AND-VENTIL/ I3-OI-OI773 -PERSONNEL USSR/ ENGINEERING AND RESCUE OPERATIONS AT THE SITE OF AN ATOMIC STRIRE I RESCUES CIVIL-DEFENSE I3-I? -DI8O4 IALLOUT-SHELTER S COSTS BLAST-SHELITERS MANAGEMENT-PLANNING SITE-SELECTION NATIONAL-FALLOUT-SHELTER-SURVEY, I3-I3-OI838 MAPS ELECTROMAGNETIC-C/ RADAR MASKING BY EARTHS TERRAIN I SITE-SELECTION RADAR-INTERFERENCE RADAR-SIGNALS I7-07-OI988 / BILITY OF DEVELOPING STANDARD DE SCRIPTI ONS OF POSTATTACK SITUATICNS I SOCIETAL-RECOVERY METHODOLOGY PROJ/ O5-II-D2IQI ELAST EFFECTS ON BUILDINGS AND SIRUCTURES - OPERAIION CF SIX-FOOT ANC TWO-FOOT SHOCK TUBES - EXPERIMENTAL IB-O3 -O2OII BLAST EFFECTS ON BUILOINGS AND SIRUC TURES - OPERATION OF SIX-FOOT AND TWO-FOOT SHOCK TUBES - EXPERIMENTAL IB-O3-O2OJS HUMAN, DISTRIBUTION VULNERABILITY LABOR ECO/ OCCUPATIONAL SKILLS AND CIVIL DEFENSE I INOUSTRIES RESOURCESI OS-O3-DIG39 HUMAN LOGY) TESTES RATS MORPHOLOGY (B IOLOGY) HISTOLOGY ESCPHAGUS SKIN 1 *AD 638 48J IICIENCY-DISEASES IISSUESIBIO D6-O5-OI7OI

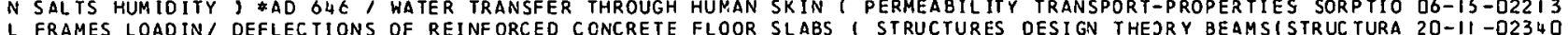
L FRAMES LOADIN DEFLECTIONS OF REINF ORCED CONCRETE FLOOR SLABS ISTRUCTURES DESIGN THESRY BEAMSISTRUC TURA 20-II -D234O UCTURES SHELTERS PE/ RESPONSE OF DEEP REINFORCED CCNCRETE SLABS I TESTS LOADING(MECHANICSI UNDERGROUND-STR I3-13-O2290
IMICS SHELLS STRUCTURAL-FORMS) BEAMS (STRUCTURAL) ANALYSIS SLABS (NONMETALLIC) FEASIBILITY-STUDIES COSTS DEI I3-I3-DIB37 IMICS SHELLSI STRUCTURAL-FORMS) BEAMSISTRUCTURAL) ANALYSIS SLABS (NONMETALLIC) FEASIBILITY-STUDIES COSTS DEI I3-I3-DI837
SHELT ERS NUCL EAR-WEAPONS SHIELDING DOSE-RATE CALCULATIONS SLANTING, \#TR-2DII)/LOUT PRJTECTIOV IFALLOUT- I3-I3-OI839 I AN IT ARY-ENGINEER ING MEOICAL-SLPPLIES DEFECTICNS TRAINING SLEEP WATER FOOD RECREATIOV EXERCISE VOISE RELI/ O5-IJ-D2I 44 /NS - PEACEFUL APPLICATIONS - A STUDY OF THE MECHANICS OF SLIDE DAMS WITH SAVD MJDELS I EXPERIMENTAL-DATAI I8-03-023I 7 L-DATA AVGLES (GEOMETRY) GEOMETRY REVETMENTS EFFECTIVENESS SLOPES, \$SC-RR-67-24/SAND MODELS I EXPERIMENTA I8-03-023I7 IREPORTS - DIPHTHERIA, PERTUSSIS, TE TANUS, POLIOMYELITIS, SMALLPOX, MEASLES, INFLUEVZA I CEMOGRAPHY IMMUN/ I5-O2-OI869 IS UNCER FIRE EXPOSURE I FALLOLT- SHELTERS FIRE-PRCTECTION SMCKE CARBON-MONOXIDE JXYGEN-SUPPLY GAS-DETECTO/ I3-12 -DI8O7 ION (COUNTERMEASURES) LIOUID-FUELS SMOKE/ FINAL RESULTS OF SMOKE SCREEN SYSTEY DEVELOPMEVT 1 THERMAL-RADIAT I9-DI -D2O4 2 IOPMENT I THERMAL-RADIATION(COUNTERMEASURES) LIQUIC-FUELS SMOKE-GENERATORS FEASIBIL ITY-STUDIES DESIGN THE I I9-OI -D2O4 2 IG GAS-MASKS PHOSGENE INCENDIARY-GELS FLANE-THROWER-FUELS SMOKE-MUNITIONS FIRST-AID NUCLEAR-WARFARE-CASUA I O6-I9-GIT2I MPONENTS I FALLOUT-SHEL TERS FIRE-SAFETY FIRES STRUCTURES SMCKES EUILDINGS CONSTRUCTION BLAST RADIOACTIVE I I3-I?-O2269

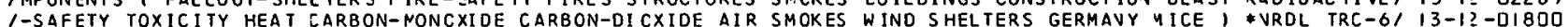
OCI VUIVERAB IL ITIES OF SOCIAL STRLCTURE - STUDIES OF THE SOCIAL DIMENS IONS OF NUCLEAR ATTAEX I RECOVERY S OS-II -D2I OO ECO/ VULNERABILIIIES OF SOCIAL STRUC TURE - STUDIES OF THE SOCIAL CIMENSIONS OF NUCLEAR ATIACK I SOCIETAL-R O5-II-O22O2 SE STUCY OF HIGFER EDUCATION I POSTA TTACK-OPERATIOAS SOCI SOCIAL INSTITUT IONS AND THERMJNUCLEAR WAR - A CA O5-II -DIGB8 $1941-1964$ - A.V INVENTORY OF STLDIES AND PUBLICATICNS IN SOCIAL RESEARCH 1 SOCIAL-SCIEVCES SURVEYS BIBLI/ O5-II -O2I 44 CIAL-SCIENCES MANAGEMENT-PLANNING UNITED-/ UTILIZATION OF SOCIAL RESEARCH IV SHAPING POLICY DECISIONS I SO O5-O+-EI658 IES AND PUBLICATIONS IN SOCIAL RESEARCH I SOCIAL-SC/ NORC SOCIAL RESEARCH 1941-1964 - AN INVENTORY OF STUD D5-II-02194 ACACEMIC CEVTERS - AFRICA I ECONONICS, A LIST OF CURRENT SOCIAL SCIENCE RESEARCH $8 Y$ PRIVATE SCHOLARS AND OS-II-OIO92 ACACEMIC CEVTERS - INTERNATIONAL AFFAI/ A LIST OF CURRENT SOCIAL SCIENCE RESEARCH BY PRIVATE SIHOLARS AND O5-II -DIG 3 ACADEMIC CEVTERS - WESTERN EUROPE, GRE/ A LIST OF CURRENT SOCIAL SCIENCE RESEARCH BY PRIVATE SSHOLARS AND O5-1I -DI694 ACADEMIC CENTERS - MIDDLE EAST I BIBLI A LIST OF CURRENT SOCIAL SCIENCE RESEARCH BY PRIVATE SCHOLARS ANO D5-II -DI695 ACAOEMIC CENTERS - MIDDLE EAST ACACEMIC CEVTERS - USSR AND EASTERN EU/ A LIST OF CURRENT SOCIAL SCIENCE RESEARCH BY PRIVATE SCHOLARS AND O5-II-OIOQ7 ACACEMIC CEVTERS - USSR AND EA STERN EUI A LIST OF CURRENT SOCIAL SCIENCE RESEARCH BY PRIVATE SCHOLARS AND OS-II -OIOQ7 ACADEMIC CEVTERS - ASIA I BIBLIOGRAPAI/ A LIST OF CURRENT SOCIAL SCIENCE RESEARCH BY PRIVATE SCHOLARS AND O5-II-OI698

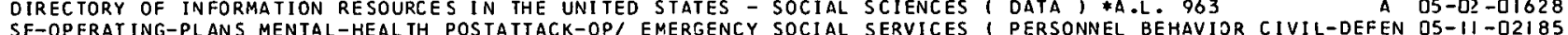
ONS OF NUCLEAR ATTACK, RECOVERY SOEII VULNERABILITIES OF SOCIAL STRUCTURE - STUDIES OF THE SJCIAL DIMENSI DS-II -D2I QD ONS OF NUCLEAR ATTACK, SOCIETAL-RESO/ VULNERABILITIES OF SOCIAL STRJCTURE - STUDIES OF THE SJEIAL DIMENSI D5-II-O22J2 ING DECISIOV-MAKING POLI TICAL-SC IENCE GCVERNMENT (FCREIGN) SOCIAL-COMMUNICAIION LEADER SHIP I *SP-2022 \$AD, O5-O+-OIG59 /IONS, $1941-19601$ HEALTH PUBLIC-CPINION WELFARE-SERVICES SOCIAL-PSYCHOLOGY AGING OCCUPATIONS PROFESSIONS/ D5- 11 -D2197 INVIRONMENT EDUCATION ARMY-EOUIPMENT REACTION (PSYCHOLOGY) SOCIAL-PSYCHOLOGY ARMED-FORCES-OPERATIONS LOGISI OS-IJ-O2I BI I FROM NUCLEAR ATTACK I STRESS (P SYCHOLOGY) GROUP-DYNAMICS SOCIAL-PSYCHOLOGY BEHAVIOR DISASTEZS PUBLIC-OPI/ O5-IJ-OIOT7 ISCIENCES SURVEYS BIBLIOGRAPHIES ATTI TUDES GROUP-DYNAMICS SOCIAL-PSYCHOLOGY PUBL IC-OP IVION METHODOLOGY TH/ D5-II -O2I 4 IA CASE STUDY OF HIGHER EDUCATION ( POSTATTACK-OPERATIONS SOCIAL-RECOVERY SURVIVAL RESOURCESIHUMAN) RECOV/ O5-II -OIOB8 EFFICIENT FOR PAIRS OF PERCENTAGES I STATISTICAL-ANALYSIS SOCIAL-SCIENCES 1 *A.L. 1096 /SO ASSJCIATION CO DS-II-O22OL RING I DATA-PRDCESSING ME THODOLOGY PUNCAED-CARD-ECUIPMENT SOCIAL-SCIENCES 1 \#A.L. II91 /REPAZATION AND SCO OS- II -O21BB Y NUCLEAR-WARFARE-CA SUALTIES SEX RACE MANAGEMENT-PLANNING SOCIAL-SCIENCES I *RM-5115-TAB /EAS VULNERABILIT I5-OS-DI965 O DATA 1 PUNCHED-CARDS STATISTICAL-ANALYSIS NETHCOOLOGY SOCIAL-SCIENCES CORRELATIOV VAR IABLES I *A.L. I' DS-II -D2I 22 I BEFORE

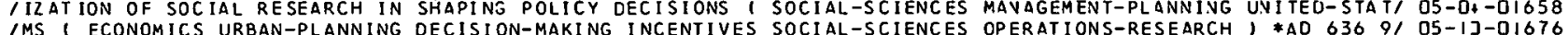
IMS I ECONOMICS URBAN-PLANNING OECISION-MAKING INCENTIVES SOCIAL-SCIENCES OPERATIONS-RESEARCH I' *AD 636 9/ O5-IJ-0I676 I NEGOT IATIONS I ATTITUDES 8EHAVIOR METHODOLOGY SENANTICS SOCIAL-SCIENCES POL ITICAL-SCIENCE CULTURE COOPE/ O5-ID-O2I 78 IT I RESJURCES(WATER) MANAGEMENT-PLANNING DECISICN-MAKING SOCIAL-SCIENCES SCIENTIFIC-RESEARIH UVITED-STAT/ O5-OI -OIS22 IVENTORY OF STUDIES AND PUBLICATICNS IN SCCIAL RESEARCH 1 SOCIAL-SCIENCES SURVEYS BIBLIOGRAPHIES ATTITUDEI Q5-II -D2I Q4 ITIONS RECOVERY MANAGEMENT-PLANNING POSTATTACK-OPERATIONS SOCIAL-SYSTEMS STANDARD-SITUATIONAL-CASES I \#HS/ O5-II -O2I 11 IY - A COMPILATION OF REFERENCES RELEVANT TO THE STUOY OF SOCIETAL RECOVERY FROM NUCLEAP ATTACK 1 STRESSI/ DS-IJ-DI 77 11 SYSTEMS-AVALYSIS POLITICAL-SCIENEE ECONOMICS SOCICLOGY SOCIETAL-RECOVERY DAMAGE-ASSESSMEVT ATIACK-SCEN/ O5-II -02I 33 IICIPATIDN IN RECOVERY ACTIVITIES ( POSTATTACK-OPERATIONS SOCIETAL-RECOVERY ECONOMIC-RECOVERY ATIITUDES M/ O5-IJ-O2I SO /POSTATTACK SURVAL DISPARITIES AND NATIONAL VIABILITY I SOCIETAL-RECOVERY ECONOMIC-RECOVERY RECOVERY AT/ O5-O3-D2I 60 / ECONOMIC-RECOVERY ECOLOGY STRATEGIC-MA TERIALS HARDENING SOCIETAL-RECOVERY FOOO(STOCKPIL INGI STOCKPILING/ 15 -O3-OI953 POSTATIACK SITUATIONS 1 SOCIETAL-RECOVERY YETHODOLOGY PRJJECTIONS RECOV/ 05-11-02191 /TACK -OPERATIOVS STRESSIPSYCHOLOGYI URBAN-AREAS SOCIOLOGY SOCIETAL-RECOVERY YETHJDOLOGY STRESSIP SYCHOLOGYI IS-O3 -D23JI IES OF THE SOCIAL DIMENSIONS OF NLCLEAR AITACK I RECOVERY SOCIET AL-RECOVERY VUCLEAR-WARFARE WEAPONS-EFFECI DS-II -D2I 9O IE - STUDIES OF THE SOCIAL DIMENSIONS OF NUCLEAR ATTACK I SOCIETAL-RECOVERY RECOVERY DEMOGRAPHY ECONOMICSI O5-II-O2202 I PROGRAM I POSTATTACK-JPERATIONS VULNERABILITY DISASTERS SOCIETAL-RECOVERY RECOVERY SOCIOLJGY STRESSIPSYI O5-II-D22QI AM I POSTATTACK-OPERATIONS V/ CIVIL DEFENSE IN POSTATTACK SOCIETY - A SUMMARY REPORT FRJM A $2 E S E A R C H$ PROGR OS-II -O22JI 1 AND VOLUNTARY ORGANIZATION IN PCST THERNONUCLEAR ATTACK SOCIETY - SOME EXPLORATORY STUDIES I POSTATTACKI I5-03-D23JI G GOVERNMENT CONTROL IN SOUTH VIETNAM ( POLITICAL-SCIENCE SOCIOLOGY ECONOMICS, ORM-5I8I-ARPA IRS AFFECTIN D5-O9-D2I 75 CIVIL-DEFENSE-SYSTEMS COLD-HAR SURVEYS CULTURE DEMCGRAPHY SOCIOLOGY POPULATION DISARYAYENTI *A.L. I2IO / D5-IJ-D2I77 IERABILITY I SYSTEMS-ANALYSIS POLITICAL-SCIENCE ECCNOMICS SOCIOLOGY SOCIETAL-RECOVERY DAMAGE-ASSESSMENT A/ OS-II -02I 73 IS I POSTATTACX-OPERATIONS STRESS (PSYCHOLCGY) URBAN-AREAS SOCIOLOGY SOCIETAL-RECOVERY METHODOLOGY STRESSI/ IS-O3-O23JI ATIONS VULNERAB IL ITY DISA STERS SOCIETAL-RECOVERY RECOVERY SOCIOLOGY STRESS(PSYCHOLOGY) I *HSR-RR-67/2ME /R D5-II-D22OI IWEAPONS-EFFECTS POSTATTACK-OPERA IIONS DISASTERS IWEAPONS-EFFECTS POSTATTACK-OPERATIONS DI SASTERS BEHAVIOR SOCIOLOGY STRESSIPSYCHDLOGY I DEMOGPAOHY ECONOMII OS-II -U2I 9O TATTACK-OPERATIONS SOIL-CONSERVATION SOI/ NUCLEAR WAR ANO SOIL EROSION SOUE PRJBLEMS ANO PIOSPECTS NG - RESULTS OF SERIES III TESTS 1 MOTICN OF SUBSURFACE SOIL INCLUSIONS SUBJECTED TO SURFACE BLAST LOADI D8-I3-DI746
NG - RESULTS OF SERIES III TESTS 1, MOTION OF SUBSURFACE SOIL INCLUSIONS SUBJECTED TO SURFACE BLAST LOADI IB-D3-O2OOB 
DRY CROP-RESEARCH ENTOMOLOGY NLTRITI ON WATER-CONSERVATION SOIL-CONSERVATION I *AIB-I89 /R ING AVIMAL-HUSBAN O2-DJ-O2I J8 ION - SOME PROBLEMS AND PROSPECTS 1 POSTATTACK-OPERATIONS SOIL-CONSERVATION SOIL-MECHANICS, 1 TRM-52O3-TAB OR-I3-DI745 5 AND PROSPECTS 1 POSTATTACK-OPERATIONS SOIL-CONSERVATION SOIL-MECHANICS, *RM-5203-TAB $10 \mathrm{~V}$ - SOME PROBLEM O8-13-OI745 IITIES THROUGH PARTIALLY SATURATED SAND - CLAY MIXTURES 1 SOIL-MECHANICS ACCOUSTICS MOISTURE MEEHANICAL-W/ $20-O I$-O2OL 7 I AMIC LOADING ( DUCTS SAND UNDERGROUND LOADING (MECHANICS) SOIL-MECHANICS CLAY COMMUVICATION-EZUIPMENT INT/ I3-II-O226I IUT-SHELTERS COMPUTERS BLAST-SHELTERS TEMPERATURE-CONTROL SOIL-MECHANICS COOLING-AND-VENTILATING-EQUIPMEN/ I3-DI-DI776 IENT I REACTOR-FEASIB IL ITY-STUDIES REACTOR-SAFETY-SYSTEMS SOIL-MECHANICS DYNAMICS GROUVD-MOTIOV UNDER WATE/ I8-O7-02O4D IENT T REACTOR-FEASIB ILITY-STUDIES REACTOR-SAFETY-SYSTEMS SOIL-MECHANICS OYNAMICS GROUND-MOTIJN UNOER WATE' I8-OF-O2O4D IN A STRIP L-WAVES SHOCK (MECHANICS) DESIGN TESTS MODELS SIMULATIONS) SOIL-MECHANICS IMPACT-TESTS ) *CR-65. QDI /HANICA 20-II -02078 ITS ON A RIGID CIRCULAR FOOTING T FOUNDATIONSISTRUCTURES) SOIL-MECHANICS LOADING(MECHANICS) DYNAMICS EXPE/ I3-I3-DI8I 2

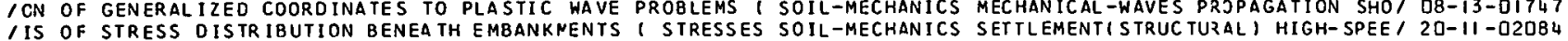
IIS OF STRESS OISTR IBUTION BENEATH EMBANKMENTS I STRESSES SOIL-MECHANICS SETTLEMENTISTRUCTUZALI HIGH-SPEE/ $20-11$-O2DB4 ITO SURFACE BLAST LOADING - RE SUL IS OF SERIES II I TESTS 1 SOIL-MECHANICS SHOCK-WAVES FAILLREIMECHANIC SI U/ I8-03-D20J8
/TS OF SERIES I I TESTS I SHELTERS UNDERGROUND-STRUCTURES SOIL-MECHANICS SURFACE-BURST FAILUREIMECHANICSI/ O8-13-0I746 IHE DYNAMIC RESPONSE SPECTRA OF COHESIVE SOILS I DYNAMICS SOIL-MECHANICS THEORY EXPER IMENTAL-DATA STRAINI/ 20-II-O2OEO IMAT HEMAT ICAL-PREDICTION SHEL TERS AIR-BUR ST VULNERABILITY SOIL-MECHANICS WEATHERING YILITARY-PE? SONNEL (VU/ IS-OS-OI957 LLIC) FEASIBIL ITY-STUDIES COSTS DESIGN LOADING (MECHANICS) SOILIMECHANICS), \$AD O2S $782 / 5 I S$ SLABSINONME TA I3-I3-DIB37 INVESTIGATION OF THE DYNAMIC RESPCNSE SPECTRA OF CCHESIVE SOILS 1 DYNAMICS SOIL-MECHANICS THEORY EXPERIME/ $20-1 /$-02086 IATION MODELSISIMULATIONS) RADIOBIOLOGY RADIATION-HAZARDS SOILS PLANTS(BOTANY) RADIOLOGICAL-COVTAMINATION/ DG-I3-DI725 UGH PARTIALLY SATURATED SAND - CLAY MIXT I THE RESPCNSE OF SOILS TO DYNAMIC LOADIVGS - WAVE VELOCITIES THRO $20-$ OI -020L? UIGN IT IOV /IGNITIOV HIGH-TEMPERATURE-RE SEARCH RADI ONETERS PYROLYSIS SOLAR-FURNACES COMBUST ION DECJMPOSITION I *AD II I3-I?-OI8D2 1 ENV IRONMENTS 1 HEAT-ENG INES DE SERTS FEASIBILITY-STUDIES SOLAR-RADIATION EGYPT ENGIVES-AND-MOTORS IRRIGAI I3-C? -DIT7? -RADIATION WEAPONS-E/ THERMAL STRESSES IN A SEMI INFINITE SOLID BY A FINITE DIFFEREVCE TECHVIOUE I THERMAL I3-I? -OIT95

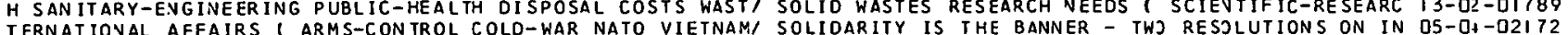
TERNAT IOVAL AFFAIRS I ARMS-CON TROL COLD-WAR NATO VIETNAMI SOLIOARITY IS THE BANNER - TWJ RESTLUTIONS ON IN OS-OH-D2IT2

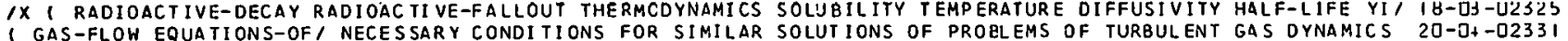
1 GAS-FLOW EQUATIONS-OF I NECESSARY CONDITIONS FOR SIMILAR SOLUT IONS OF PROELEMS OF TURBULENT GAS DYNAMICS $20-0+$-0233I ID THE MANHATTAN AND WILLIAMSBURG BRIDGES AND ALTERNATIVE SOLUT IONS THEREFORE I URBAN-AREAS VEW-YORK I \#A/ $23-02-0235 ?$ ER THROUGH HUMAN SKIN I PERMEABILITY TRANSPORT-PROPERTIES SORPTION SALTS HUMIDITY ) *AD 646 848 /ER TRANSF O6-1S-O22I3 $N$ AND SCORING I DATA-PROCESSING METHODOLO/ PUNCHED CARD G SORT ING - A MACFINE METHOD FOZ Q OECK PREPARATIO O5-II -02! 88 Y DUCTS LAMINA/ INTERACTION BE TWEEN AIR FLOW AND AIRBORNE SOUND IN A DUCT I ACOUSTICS GAS-FLOW WEST-GERMAN $20-D I$-D2DLB OW PROPAGA' FINAL REPORT ON ATTENUATION AND DISPERSION OF SOUND IN AN AEROSOL, IACOUSTIC-PROPERTIES GAS-FL IS-O? -O I881 /ROPERT IES GAS-FLOW PROPAGATION BIOLOGICAL-WARFARE-AGENTS SOUNO-TRANSMISS ION, *NONR-562137)/3*AD 64I OI/ I5-02-0I88I 1 RESOURCES PETROLEUM-INDUSTRY INDLSTRIES ECONOMICSI U.S. SOURCES OF PETROLEJM AVD VATURAL GAS STATISTICS DS-D3-DI 38 IICAL ANALYSIS OF FACTORS AFFECTING GOVERNNENT CONTROL IN SOUTH VIETNAM I POLIIICAL-SCIENCE SOLIOLOGY ECO/ O5-DZ-O2I75 VI INVEST IGATION OF ALTERNATIVE AQUEDUCT SYSTEMS TC SERVE SOUTHERN CAL IFORNIA - FEATHER RIVER AND DELTA DI I3-D? - D225B

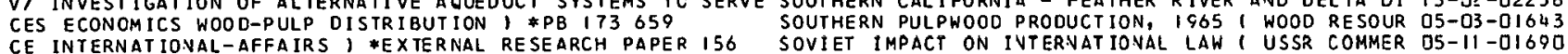
CE INTERNATIONAL-AFFAIRS ' *EXTERNAL RESEARCH PAPER 156 SOVIET IMPACT ON IVTER SAT IONAL LAW MPONENIS GUIDED-MISSILES RAILROADS BRI DGES GROUNC-SUPP/ SOVIET LITERATURE ON PROTECTIVE STRULTURE AND CO IO-OI -OIO72 E NUCLEAR-WARFARE-CA SUALTIES FIRST-AIDI CURRENT TOPICS IN SOVIET MILITARY MEDICIVE I USSR DISASTER-MEOICIN O6-0;-OI7J3
IVIL-DEFENSE-SYSTEMS INTERNATIONAL-CIVIL-DEFENSE SHELTERI SOVIET MILITARY TRANSLATIONS NUMBER I7OI USSR C IS-OZ-OI937 IVIL-DEFENSE-SYSTEMS INTERNATIONAL-CIVIL-DEFENSE SHELTERI SOVIET MILITARY TRANSLATIONS NUMBER 1701 USSR C I5-O3-DI937 NTERNAT IONAL-CIVIL-DEFEN SE CIVIL-DEFENSE-SYSTEMS RLRAL-AI SOVIET MILITARY TRANSLATIONS VUMBER I78 I USSR I IS-O3-OI928 DEFENSE 1 RUSSIA TRAINING DISPER SAL CIVIL-DEFENSE-FERSON/ SOVIET MILITARY IRANSLATIJVS VUMBEZ 188 - CIVIL $15-03-01913$
IVIL-DEFENSE-OPERATING-PLANS TRAINING EDUCATICN INTERNAT' SOVIET MILITARY TRAVSLATIOVS VUMBER 341 I USSR C I5-D3-DIOI7 IVIL-DEFENSE-OPERATING-PLANS TRAINING EDUCATICN INTERNAT' SOVIET MILITARY TRAVSLATIOVS VUMBER 34 I I USSR C I5-D3-DIQI 7

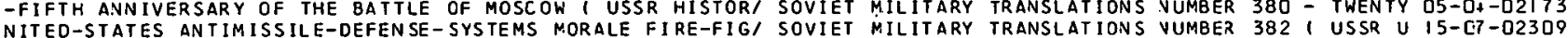
TRAINING METHODS AND THEIR SHOR TCOMINGS DESCRIBED, USS/ SOVIET MILITARY TRAYSLATIOVS VUMBEZ 384 - COMBAT O5-D7-D2176 IVIL-DEFENSE-SYSTEMS INTERNATI ONAL-CIVIL-DEFENSE TRAININ/ SOVIET MILITARY TRANSLATIOVS VUMBER 385 I USSR C IS-03-D23J2 RMED-FORCES-OPERATIONS ARMED-FORCESIFOREIGN) HARFARE MOR/ SOVIET MILITARY TRANSLATIONS VUMBER 386 (

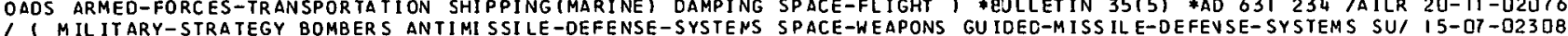
IATEL /ATELLITES COMMUN ICA IION-EQUIPMENT SATELLITES IARTIFICIAL) SPACECRAFT DEFENSE-SYSTEMS COST-EFFECTIVENE SS VI 22-O2 -D235I IFIC AND TECHNICAL LITERATURE I GLIDED-MISSILES ABSTRACTS SPACECRAFT TRANSPORTATION LAUNCHIVG-SITES ME TEOI IO-GI -OI973
HELTERS URBAN-AREAS/ VENTILATI ON IESTS OF FALLOUT SHELTER SPACES IN NEW YORK CITY AND VICINITY I FALLOUT-S I3-I3-DI8I3

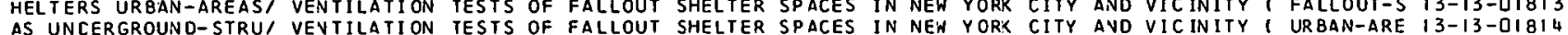
AS UNCERGROUND-STRU/ VENTILATI ON TESTS OF FALLOUT SHELTER SPACES IN NEN YORK CITY AVD VICINITY 1 URBAN-ARE I3-13-OI814 OL GROUND SHOCK EFFEC TS I. GROUND MOTION IN ELASTIC HALF SPACES INCJCED BY AIR BLAST 1 ELASTIEITY SHOCK- I

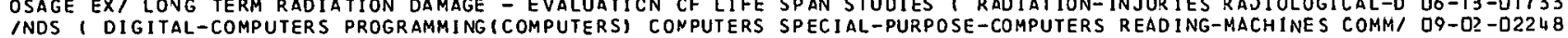
/NDS I DIGITAL-COMPUTERS PROGRAMMING (COMPUTERS) CONPUTERS SPECIAL-PURPOSE-COMPUTERS READING-MACHINES COMM/ O9-O2-O2248
I SERVICES IN THE UNITED STATES - A DIRECTORY OF SELECTED SPECIALIZED INFORMATION SERVICES IV THE PHYSICA/ OS-OZ-OI 29

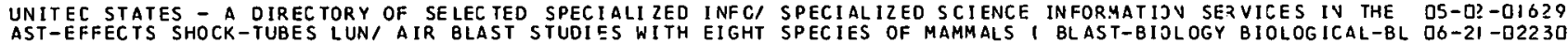

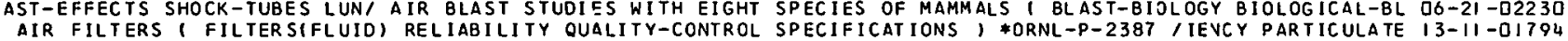
/ROM SHALLOW UNDERWATER EXPLOSIONS - FORNULAE AND FROGRAM SPECIFICATIONS 1 HYDRODYNAMICS WATER-WAVES MATH/ I 9-D+-D2O4 3 -ENGINEER ING DECISION-MAKING DESIGN ENGINEERING-PERSONNEL SPECIFICATIONS HANOBOOKS TESTS I *AD 642 O57 /AN DS-0弓-0I673 ECHANICS) VIBRATION ELASTICITY VELOCITY DEGREES-OF/ SHOCK SPECTRA FOR A GENERAL FORCING FUNCTION I SHOCKIM $20-11-02342$ S DOSE-RATE/ EXPERIMENTAL DETERMINATI ON OF NEUTRON ENERGY SPECTRA IN CONCRETE DUCTS I SHELTERS ENTRANCEWAY OS-I3-D22I 9 ICS TH' A RHEOLOGIC INVESTIGATION OF THE DYNAMIC RESPONSE SPECTRA OF COHESIVE SOILS I OYNAMICS SOIL-MECHAN 20-II -02OBO UBY CRYOGENICS GASES PUMPING (OPTICAL) FLASH-LAMPS LIOUIDS SPECTROSCOPY, \#NRL-6444 \$AD 637 6I6 10PERTIES R 20-D5-02032 1 AND VIBRATION BULLETIN IINSTRUMENTATION ACCELERCMETERS SPECTRUM-ANALYZERS TRANSDUCERS MATHEMATICAL-ANA/ 20-II-D2O75 TENTIONS ( BEHAVIOR MOTIVATION CULTURE RE SPONSE REACTION/ SPECULATION ON THE STRUCTURE JF IVTERPER SONAL IN OS-IJ-O2I 79 APFIES URBAN-AREAS EXCAVAIION, \$PB/ BIBLIOGRAPHY CF HIGH SPEED GROUNC TRANSPORTI TRAVSPORTATION BIBLIOGR 23-O? -02OQ3 TERM INALS SCHEDULINGI SUMMARY OF RE SEARCH AT MIT CN HIGH SPEED GROUND TRANSPORT IRAVSPORTA TION NETWORKS $23-0$ I -02354 AGEMENT-PLANNING URBAN-PLANNING WASHINGTON BOSTON N HIGH SPEEO RAIILRODS I TRANSPORTATION URBAN-AREAS MAN $23-0$-O2I DO ANICS) SHEAR/ FORCED MOTION OF SHALLOW SPHERICAL SHELLS I SPHERES STRUCTURAL-SHELLS VIBZATIOV LOADINGIMECH 20-II-D2346 IAN IMPROVED MATHEMATICAL METHOD OF LOCATING FALLOUT ON A SPHERICAL EARTH 1 NIND YIELO ZADIJACTIVE-FALLOU/ IB-D3-O2322 RATION LOADIVG(MECHANICS) SHEAR/ FORCED NCTION OF SHALLOW SPHERICAL SHELLS I SPHERES STRUCTURAL-SHELLS VIB $20-I I$-D23 46 /ATHOGENESIS AS RELATED TO PHYSIOLOGICAL STATE OF ANTHRAX SPORE AND CELL 1 BIOLOGICAL-WARFARE-AGENTS BACI/ IS-D?-DI854 IS IN MONKEYS I B IOLOGICAL-WARFARE-AGENTS PATHOLOGY LUNGS SPORES, *AD 636815 /ESIS OF COCCIDIOIDES IMMIT IS-O2-OI852 IACIS OBTAIVED WITH PROTECIIVE ANIIGEN AND LIVE VACCINE 1 SPCRES BICLOGICAL-NARFARE-AGEVTS AVTIGENS-AND-A/ IS-0?-OIB53 IR STER IL IZIVG LABORA TORY AIR I B I OLOGICAL-WARFAPE-AGENTS SPORES MEDICAL-EQJ IPMEVT ELECTR ICAL-EZUIPMENT L/ IS-D? -DIB92 /AC IS MATHEMATICAL-MODELS PASTEURELLA-TULARENSIS BACTERIA SPORES STORAGE BIOLOGICAL-WARFARE-AGENTS I *AD I I5-O2-OI849 ALIZATION TAXES VULNERABILI/I IMPLEMENTATION OF AN CRDERED SPRAWL URBAN CONFIGURATION I URBAV-AREAS DECENTR 23 -OS-D2ID6 UTERS I DATA-PROCESSING URBAN-AREA S MA THEM/ FLANE I - FIRE SPREAD SINULATION MODEL I FIRES PRJGRAMMINGICOMP I3-I?-O2266 ISOLATORS SIMULATI/ VIBRATION ISOLATION THROUGH PNEUNATIC SPRING AND CAMP ING I SHOC (MECHAVIC S) VIBRATION- $20-11$-O2343 IIBRATIOV-ISOLA TOR S IMPAC T-SHOCK NUCLEAR-EXPLOSION-DAMAGE SPRINGS PERSONNEL DESIGN IVSIZUMEVTATION I *A JA/ I3-13-0IBZ3 INET IC FIELD I GAS-FLOW PLASMA-MEDIUM PLASMA-OSCILLATIONS STABILITY BOUNDARY-VALUE-PROBLEMS, IST ICS GAS-FL OW WEST-GERMANY DUCTS LAMINAR-BOUNDARY-LAYER STABILITY MEASUREMENT, *AMRL-TR-65-53 \$AD O4D, 20-DI -02048 C PRESERVATION FIBERBOARD PHYSICAL-PROPERTIES CO/ STORAGE STABILITY OF CIVIL DEFENSE SHELTER RATIONS I FOO DO-OS-D22I 2 SOCIETAL-RECOVERY METHODO/ THE FEASIBILITY OF DEVELOPING STANDARC DESCRIPTIONS OF POSTATTAEK SITUATIONS I OS-II -02IOI MPONENTS I FALLOUT-SHELTERS FIRE-SAFETY F/ DEVELOPNENT OF STANDARE FIRE TEST RATING SYSTEMS FOR SHELTER CO I3-I?-O2269 MANAGEMENT-PLANN ING POSTATTACK-OPERATIONS SOCIAL-SYSTEMS STANDARO-SITUATIONAL-CASES, \#HSR-RR-6?/8-SO /RY OS-II-O2I91 
ROUND-/ DESIGN MODIFICATIONS AND 1962 COST ANALYSIS FOR A STANDARCIZED SER IES OF FALLOUT SHELTER I UNOERG I3-13-OIBIS IVE-FALLOUT CONSTRUCTION CO/ FALLOUT SHELTERS - COCES AND ST ANDARCS I SHIELDING RADIATIDN-EFFECTS RADIOACT I3-I3 -D2286 VITY CONTROL, , YSI PUBLIC HEALTH SERVICE DRINKING WATER ST ANDARCS, WATER-SUPPL IES REGULATIOVS RADIOACTI I3-DI-DI7T8 AMICS DESIGV CUSPS FLUID-F/ FLOW CONTROL BY GENERATION OF STANDING VORTICES AND THE CUSP EFFEIT I HYDRODYN $20-0+-D 2 O 55$ INSU/ STATE WORKMEN COMPENSATION LAWS I LEGISLATION LABOR STATE-GOVERNMENTS ADMINISTRATION-AND-MANAGEMENT DS-OZ-O2I7L IION POLITICAL-SC IENCE EXECUTIVE-BRANCH LOCAL-GOVERNMENTS STATE-GOVERNMENTS LEADERSHIP ATTITUDES, \$IDA/H, O5-OH-DIGTI 1-BUCGETS COSTS CIVIL-DEFENSE-SYSTEMS MANAGEMENT-PLANNING STATE-GOVERNMENTS LOCAL-GOVERVMENTS, *SRI-4S36/ I3-13-OI834 EFENSE-OPERAT ING-PLANS A TTACK-SCENARIOS LCCAL-GOVERNMENTS STATE-GOVERNMENTS RESOURCES, *SRI-MU-4949-120, I7-02-023I2 NN-L INES COSTS AL TERNATE-CURRENT ECONOMICS DIRECT-CURRENT STATE-OF-THE-ART IVOUSTRIAL-RESEAZEH, *A.L. II/ D9-03-O2249 IES YIELD RADIOLOGICAL-DOSAGE WIND FIRES SIMULATION BLAST STATE-OF-THE-ART-REVIEWS I \#HI-693-RR/ION-INJUR I5-0S-DI962 I FROM WHAT WE KVOW I IVFORMATION-RE TRIEVAL DOCUNEATATION STATE-OF-THE-ART-REVIEHS SYSTEMS-EVGIVEERING TEI OS-DZ-OI 33 O ING IN SATURATED SAVD I LOADINGIMECHANIC SI SOIL-NECHANICI ST ATIC ANC CYNAMIC BEAR ING TESTS JV A STR IP FOOT $20-1 I$-O234I RESPONSE OF A BURIED PROTOTYPE CONMUNICATIONS CONCUIT TO STATIC AND DYNAMIC LOADING I DUCTS SAND ONDERGRO I3-II -D226I CONCRETE ARCHES I UNDERGROUNO-STRLCTURES DYNAMICS LOADIN/ STATIC AND DYNAMIC TESTS OF BURIED UNREINFORCED I3-I3-OI83D ING EXPLOSION-EFFECT/ A REAPPRAISAL OF THE APPLICATION OF STATIC EQUILIBRIUM THEORY TO DECOUPLIVG I CRATER I8-O3-O23IO ING EXPLOSION-EFFECT/ A REAPPRAISAL OF THE APPLICATION OF STATIC EQUILIBRIUM THEORY TO DECOUPLIVG I CRATER I8-03-D23IO
II. I TEST-EQUIPMENT BEAMSISTRLCTLRAL) LOADING IMECHANICS) STATICS, \#R-5O2 \#CASA-13.0I8 /CJVLRETE BEAMS I3-I3-OI84II

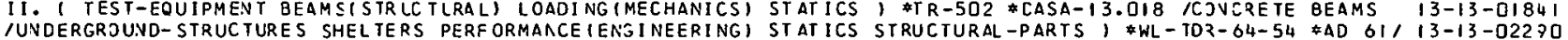

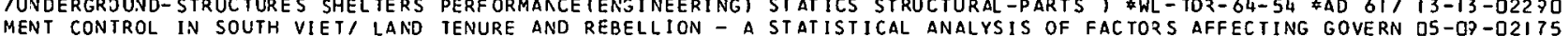
MENT CONTROL IN SOUTH VIET/ LAND TENURE AND REBELLION - A STATISTICAL ANALYSIS OF FACTORS AFFECTING GOVERN QS-OF-O2I75
O/ AN ANALYSIS OF THE RELIABILITY OF THE RISK II CCMPUTER STATISTICAL MODEL - NAHICUS 63 APPLICATION I RES D5-D3-O2IL9 N 1965 I UTILITIES RESERVES PRODUCTION TRA' GAS FACTS - A STATIST ICAL RECORD OF THE GAS UTILITY INOUSTRY I OS-O3-DI642 SYSTEMS CIVIL-DEFENSE-OPERATING-PLANS POPULATICN / ANNUAL STATISTICAL REPORT I FINAVCIVG SHEL TEZS WARNING- I5-O3-OIGOB STRUMENTATION DEFORMATION LOADING (MECHANICS) CCNSTRUCTION STATISTICAL-ANALYSIS I *A.L.I2O6 $10 R Y$ GEOLOGY IN I3-13-O2275 RADIOACTIVE-FALLOUT FALLOUT-SHEL TERS MODELSISIMULATIONS) STATISTICAL-ANALYSIS, \#RTI-OU-230-2(I). IEFFECTS IS-D3-O1935 /ROSHIMA NAGASAKI BURNS WEAPONS-EFFECT RADIATICN-I JURIES STATISTICAL-ANALYSIS BIOLOGICAL-BLAST-EFFECTS SI O6-2I -O2238 IPSYCHOMETRICS TECHVICAL-INFORMA TION-CFNTFRS. IOR-ANAI YSIS STATISTICAL-ANALYS IS MANAGEMEVT-EVGIVEERING I * D D-D?-O2I 20 INUCL EAR-WARFARE-CASUALTIES SYSTENS-EVALUATI ON CASUALTIES STATIST ICAL-ANALYSIS MATHEMATICAL-MODELS I \#RTII I5-03-OI933 CORRELATI/ THE FACTOR ANALYSIS OF Q DATA I PUNCHED-CARDS STATISTICAL-ANALYSIS METHODOLOGY SOCIAL-SCIENCES O5-II-O2IQ2

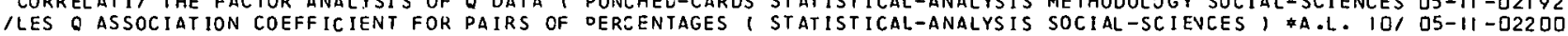
LES
OGRAMMIVG) DATA-PROCESSING-SYSTENS MULTI VARIATE-ANALYSIS STATISTICAL-ANALYSIS VARIANCE REGRESSION-ANALYSI OG-O3-O22 J4

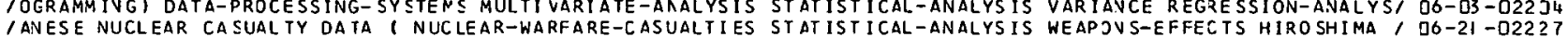
I FOR A BIVARIATE NORMAL DISTRIBUTION ISTATISTICAL-TESTS STATISTICAL-DISTRIBUTIONS PROBABILITY-DENSITY-F/ I2-DI -O22S। IOF GOODNESS OF FIT FOR A 8 IVARIA IE NORMAL DISTRIBUTION 1 STATISTICAL-TESTS STATISTICAL-DISTRIBUTIONS PRO/ I2-OI -O225I RIES ECONOMICSI U.S. SOURCES OF PETROLEUM AND NATURAL GAS STATISTICS 1 RESOJRCES PETROLEUM-IVDUSTRY INDUST DS-O3-OIO38

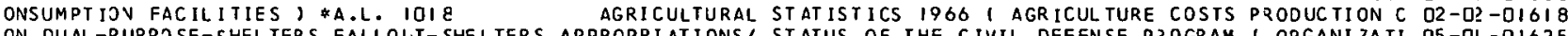
ON DUAL-PURPOSE-SHEL TERS FALLOLT-SHELTERS APPROPRIATIONS/ ST ATUS OF THE CIVIL DEFENSE PZOGRAM I ORGANI ZATI Q5-DI -OIG25 POL I OMYELITIS, SMALLPOX, MEASLES, INFLUENZA 1 DI OISEASE STATUS REPORTS - DIPHTHERIA, PERTUSSIS, TETANUS, IS-OZ - DIBO 9 作 IRAL) COVSTRUCTION-MATERIALS LOAD-DI STRIBUTION DEFLECTION STEEL ROOS CRACKS STRAINIMECHANICS) LOADING (MEC, I3-I3-D22BQ RCES STOCKPILING MOBILIZATION STRATEGIC-PATERIAI IRON ANO STEEL SCRAP CONSUMPTION PROBLEMS 1 SALVAGE RESOU O5-D3-OIOU5 AGENTS SPORES MI TEST NEW ELEC TRIC INCINERATOR DESIGN FOR STERILIZING LABORATORY AIR I BIOLJGICAL-WARFARE- IS-O?-OI892 IL INVEST IGATION OF THE YIELO STRENG TH OF A MACHINED RING STIFFENED CYLINDRICAL SHELL I MODEL BZ-7M, UND/ $20-11$-O2347

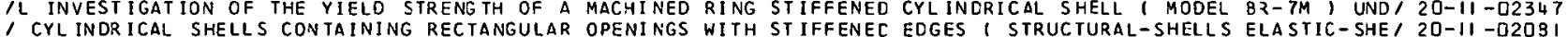

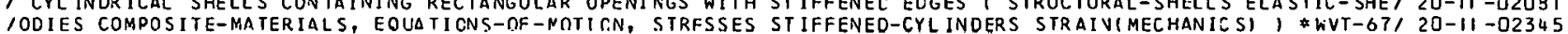
IRICAL SHELL ( MODEL BR-7M) UNDER HYDROSTATIC PRESSURE (STIFFENED-CYLINDERS TENSILE-PROPERTIES PLASTICI/ 20-II -O234 7 D LOCATION I AGR ICUL TURE STORAGE SLPPLY-DEPOTS WAREHOUSES STCCKPILING) \$A.L. ID98/ENTJRYBY WAREHOUSE AN O2-D?-O2ID9 OWER SHELTERS PROGRAMMING (COMPLIERSI DISPERSAL INDUSTRIES STOCKPILING J *A.L. 970 /VITJZING LOGISTICS MANP I5-D3 -DIO49 NC-MANAGEMENT MEDICAL-SUPPLIES NUCLEAR-WARFARE-CASUALTIES STOCKPILING CANADA I \#A.L. IO69 $10 M I N I S T R A T I O N-A$ O6-21 -0I739 ITRANSPORTATION FEASIBILITY-STUDIES DUAL-PURPOSE-SHELTERS ST OCKPILING ENTRANCEWAYS COSTS WATER-SUPPLIES EI I3-I3-O227I $1 O K$ I KITCHEN-EQUIPMENT-AND-SUPPLIES FOOD-DI SPENSING FOOD STOCKPILING FALLOUT-SHELTERS SHELTER-OCCUPANCY , O6-OS-OI7I 2 I INE - IVSTRUCTORS GUIDE I KITCHEN-EQUIPMENT-AND-SUPPLIES STCCKPILING FOOL-DISPEVSIVG FJOD FALLOUT-SHELTE $/$ O6-O3-OITI3

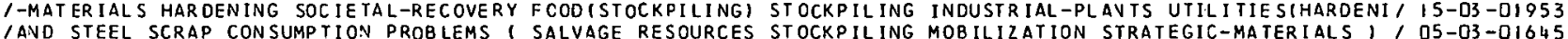
1. FINAL REPORT I FALLOUT-SHELTERS CONFINED-ENVIRCNMENTS STOCKPILING SHELTER-MANAGEMENT HAVDBOOKS HABITA/ O5- IJ-D2। 84 IS FOOD-SUPPL IES WATER-SUPPLIES INOUSTRIAL-PLANTS FINANCE STCCKPILING STORAGE MAVPOWER

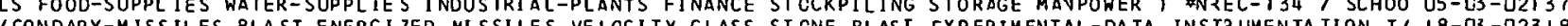
CONDARY-MISSILES BLAST-ENERGI ZED-MISSILES VELCCITY GLASS ST CNE BLAST EXPERIMENTAL-DATA INSTRUMENTA TION TI I8-03-023I8 NGINEER IVG RAOIOLOGICAL-CONTAMINATION(FOOD) INFANTSIDIET) STORAGE / \#-IS /R-SUPPLIES RATIOVIVG SANITARY-E O6-O3-0ITI2

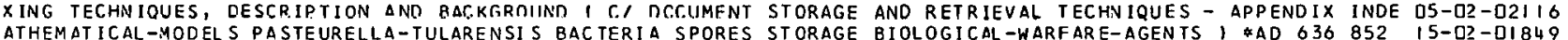

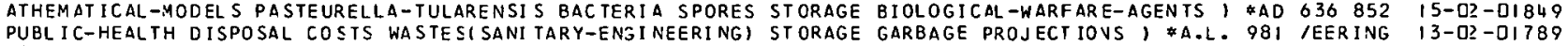
ERVAT ION SHELF-LIFE BEVERAGES RREAD CANNED CEREALS/ FOOO STORAGE LIFE - A REPORT BIBLIOGRAPHY I BEEF PRES O6-O3-O22O9 LIES WATER-SUPPLIES INDUSIRIAL-PLANTS FINANCE STOCKPILING STORAGE MANPOWER, \$NREC-I34, SCHOOLS FOOD-SUPP OS-D3-O2I 37 NS I FODD PRFSFRVATION FIBERBOARD PHYSICAL-PROPERTIES COI STCRAGE STABILITY OF CIVIL DEFENSE SHELTER RATIO O6-D3 -O22I 2 I WHEAT INVENTORY BY WAREHOUSE ANO LOCATION 1 AGRICULTURE STORAGE SUPPLY-DEPJTS WAREHOUSES STOCKPILING 1 , O2-0?-O2ID9 /CKEL CADMIUM BATTERIES FOR AIRR.RAFT FI,FC.TRICAL SYSTEMS I STORAGE-EATTERIES ALKALINE-CELLS AIRCRAFT-EQUIPI ID-D3-OITG4 IFFUS ION I ATMO SP FOOD DIET POSTATTACK-OPERATICNS SURVIVAL STORAGE(FOODI PRODUCTION(FOOD) RESJURCESIFOOD) I O6-IS-OITI ESCRIPTION OF WORK PRO/ CONTROI DF SURFAC,E STORM WATER BY \$T ORM DRAINS AND ORAINAGE CHAVNELS I. GENERAL D I3-O2 -DI7B2 ROFILES, CROSS SECTION/ CONTROL OF SURFACE STORM WATER BY STORM DRAINS AND DRAINAGE CHAYVELS II PLANS, P I3-D?-OI779 ROFILES, CROSS SECTION/ CONTROL OF SURFACE STORM WAIER BY STORM ORAINS ANC DRAINAGE CHAVYELS II. PLANS, P I3-OZ -DI7BI

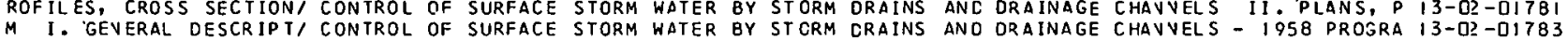
$M$
$M$ I. GEVERAL DESCRIPT/CONTROL OF SURFACE STORM WATER BY STCRM CRAINS ANO ORAINAGE CHAVVELS- 1958 PROSRA I3-D2-DI783 1. GEVERAL DESCRIPT/ CONTROL OF SURFACE STORM WATER BY STORM DRAINS AND DRAINAGE CHAVNELS - I 964 PROGRA I3-D2-OI784
DESIGN AND CONSTRUCTION OF SANITARY AND STORM SEWERS I SAVITARY-SEWERS I *WPCF-9 S II. PLANS, PROFILES, CROSS SECTION/ CONTROL OF SURFACE STCRM WATER BY STORM DRAINS AVD DRAIVAGE CHANNEL I3-0?-DITTQ S II. PLANS, PROFILES, CROSS SECIION/ CONTROL OF SURFACE STORM WATER BY STORM ORAIVS AVD DRAIVAGE CHANNEL I3-O? -OITBI S I GEVERAL DESCRIPTION OF WORK PRO/ CONTROL OF SURFACE STORM WATER BY STORM DRAIVS AVD DRAIVAGE CHANNEL I3-O? - OI7B2
S- 958 PROGRAM I. GENERAL DESCRIPT/ CONTROL OF SURFACE STORM WATER BY STORM DRAIVS AVD DRAINAGE CHANNEL I3-0?-DI783 S - 1964 PROGRAM I. GENERAL DESCRIPT/ CONTROL OF SURFACE STORM WATER BY STORM DRAINS AVD DRAINAGE CHANNEL I3-D? - DI784 IIATION SHIEL DING RADIOACTIVE-FALLOUT RADIOLOGICAL-DOSAGE STCRMS [ECONT AMINAT ION EARTHQUAKES VOLCANOES LA, I5-D3-O2299 IEMS COMMUNICAT IONS-SYSTEMS SUPER VISION NEDICAL-PERSONNEL STORMS EMERGENCY-HEALTH-SERVICES I \#RESEARCH RE I O6-I? -OIZI 4

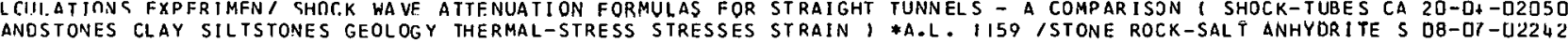
MONKEYS EXPOSED TO A SMALL PARTICLE AEROSOL OF THE BORG SIRAIN I BIOL OGICAL-WARFARE-AGENTS MIYAGA WANELLI IS-D? -DIBSO

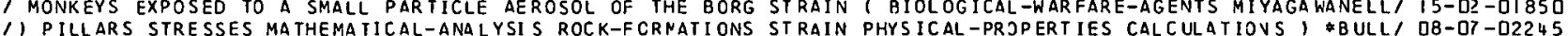

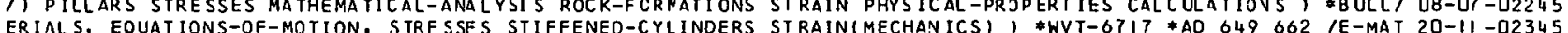
(CIES STRESSES THRUST THERMAL-STRESSES LOADING(MECHANICS) STRAINIMECHANICS) BUCKL INGIMECHANICSI MATHEMATI/ $20-1 /$-02344 IN ELASTIC RODS ( MECHANICAL-WAVES ELASTICITY PROPAGATI ON STRAININECHANICS) EQUAT IONS-OF-MOTIOV STRESSES, $20-11$-O2OB2 /MATERIALS LOAD-DISTR IBUTION DEFLECTION STEEL RODS CRACKS STRAIN(MECHANICS) LOADING(MECHANIES), ISOILS 1 DYVAMICS SOIL-MECHANICS THEORY EXPERIMENTAL-DATA STRAINIMECHANICS) STRESS LOADING(YECHANICS) SHO/ $20-I I$-02D36 /C-STRUCTURES ELASTICITY TEST DYNAMICS LOADING IMECHANICS) STRAINIMECHANICSI THEORY, *TECHNICAL REPORT NO/ I3-I3-QI825
ARY-STRATEGY BOMBERS ANTIMISSILE-DEFENSE-SY/ THE CHANGING STRATEGIC MILITARY BALANCE - USA VS USSR I MILIT IS-OT-O2308 
ARFARE. MODEL-THEORY STRATEGIC-WARFARE MILITARY/ STROP - A STRATEGIC PLANNING MODEL I GAME-THEORY NUCLEAR-W I5-OS-QI959 ION PROBLEMS (SALVAGE RESOURCES STOCKPILING MOBILIZATION STRATEGIC-MATERIALS) \$A.L. 954 / SCRAP CONSUMPT O5-O3-0I645 IRACIOLOG ICAL -WARFARE (RECOVERY) ECONOMIC-REC OVERY ECOLOGY STRATEGIC-MATERIALS HARDEVING SOCIETAL-RECOVERY/ I5-O3-DI953 /LANN ING MOOEL I GAME-THEORY NLCLEAR-WARFARE NODEL-THEORY STRATEGIC-NARFARE MIL ITARY-STZATEGY OPTIMIZATIO/ IS-OS-DI959 I RE SOURCES VULNERABILITY PROGRANMING (CONPUTERS) CASUAL/ STREAK IV - NREC RAPID DAMAGE ASSESSMENT PROGRAM O5-03-02I 6 A-MEDIUM PLASMA-O SCILLATIONS STABILITY BOUNDARY-VI PLASMA STREAMING INTO A MAGNETIC FIELD I GAS-FLOW PLASM 20-OF-O2335 F ROUTE BETWEEN COLLMBIA ROAD AND VAN NESS STREET I TRANSPORTATION SUBWAYS TESTS BORING WA/ I3-Q2-DI785 OS CONSTRUCTION URBAN-AREAS TUNNELSITRANSPORTAI EXTENSIVE STREET BUILDING PROGRAY IV HAYBURG 1 GERMANY ROA I3-0? -OITB6 L SHELL I MOD/ AN EXPERIMENTAL IN VESTIGATION OF THE YIELD STRENGTH OF A MACHINED RIVG STIFFEVED CYLINDRICA $20-1 I$-U23 7 T-EQU IPMENT BEAMS (STRUCTURAL) LOADING (MECH/ DYNAMIC SHEAR STRENGTH OF REINFORCED CONCRETE BEAMS II. I TES I3-I3-DI84I GLUING PLYWOOD SANDI WOOD HANDBOOK I CONSTRUCTION DESIGN STRENGTH STRUCTURAL-PROPERT IES QUALIIY FASTENERS II-12-DI766 IIONS ON THE PROBLEM OF THE INTERRELATIONSHIP CF PRESSURE STRENGTH, SIZE AND COST OF MULTIPUZPJSE BUILDIN/ $13-13$-OI82 7 ITS EFFECTIVENESS \$MICE CHEMOTHERAPEUTIC-AGENTS INFECTIONS ST REPTOMYCINS CHLORTETRACYCLIVE CHLORAMPHENICOLI I5-0?-OI894 IREAT, AND SMALL SHELTER HABITABILITY UNDER.CONDITIONS OF STRESS 1 SHELTER-OCCUPANCY FEASIBILITY-STUDIES, OS-1J-DIO84 ES SOIL-MECHANICS SETTLEMENT (STRUC TURAL) HIG' ANALYSIS OF STRESS CISTRIBUTION BENEATH EMBANRMENTS I STRESS 2D-II-O2O84 IOIL-MECHANICS THEORY EXPER IMENTAL-DATA STRAINIMECHANICS) STRESS LOADINGIMECHAN ICS) SHOCK-WAVES VISCOELA SI $20-11$-U2036 GATION IMPACT-TESTS DVNAMICS BLAST SHOCK-WAVES ELASTICITY STRESS MECHANICAL-AVALYSIS I *MODOS /CRACK-PROPA 20-II -02338 /RATION BULLETIN, AGLLERANCESIPHYSIOLOGY) MOTION-SICKNESS STRESS (PHYSIOLOGY) ADAPTATIONIPHYSIOLOGY) TUMBL, DG-IO-CIT35 /RATION BULLETIN T TOLERANCESIPHYSIOLOGY) MOTION-SICKNESS STRESS (PHYSIOLOGY) ADAPTATIONIPHYSIOLOGY) TUMBL, DO-IO-UIT3S / IOLOCY PERFORMANCE(HUMAN) INSTRUCTI ON-MANUALS PSYCHOLOGY ST RESS (PHYSIOLOGY) FOOD WATER-SUPPLIES BEDDING $/$ IS-OZ-O23 OO IICAL AND MEYEOROLOGICAL ASPEC TS CF SHELTER VENTILATICN ( STRESS (PHYSIOLOGY) HEAT MOISTURE TEMPERATLRE EN/ I3-OI -D2253 / INFECTIOUS-DISEASES VACCINES RADIOBIOLOGY PUBLIC-HEALTH STRESS (PHYSIOLOGY) MALNUTRITION RADIOLOGICAL-DO/ DO-OJ -D22D6 / IOLOGY PERFORMANCEIHUMAN) INSTRUCTION-MANUALS PSYCHOLOGY STRESS(PHYSIOLOGY) WATER-SUPPLIES FOOD BEDDING / I5-O3-D2298 LOGY) URBAN-AREAS SOC IOLOGY SOCIE TAL-RECOVERY NETHCDOLOGY STRESS (PSYCHOLOGY), NERAB IL ITY DISASTERS SOCIETAL-RECOVERY RECOVERY SOCIOLOGY ST RESS (PSYCHOLOCY), FHSR-RR-O7/2YE /2ATIONS VUL OS-II -O22JI IAT IONS IV CIVILIAN EMERGENCIES ( EMOTIONS ATTITUOES FEAR STRESS (PSYCHOLOGY) CASUALTIES MOTIVATION PUBLICI DS-IJ-DIGBI IFECTS POSTATTACK-OPERATIONS DISASTERS BEHAVIOR SOCIOLOGY STRESS (PSYCHOLOGY) OEMOGRAPHY ECONOMIC-RECOVERYI O5-II -O2I QD TUCES MANPOWER MOTIVATION ECONOMICS SURVIVAL DISTRIBUTION STRESS(PSYCHOLOGY) FOOD, \#HSR-RR-67/5-T1, ATTI O5-IJ-02I80 EST IONNAIRES AT TI TUDES INTERNA IIONAL-AFFAIRS FEAR SURVEYS STRESS (PSYCHOLOGY) FOREIEV-PJLICY) *VORC-35 /QU D5-II-DI639 1 TO THE STUDY OF SOCIETAL RECOVERY FROM NUCLEAR ATTACK I STRESS (PSYCHOLOGY) GROUP-DYNAYICS SOEIAL-PSYCHO/ O5-IJ-DIO77 IRECOVERY RECOVERY DEMOGRAPHY ECONOMICS POLITICAL-SCIENCE STRESSIPSYCHOLOGY) METHODOLOGY MANAGEMENT-PLANN/ O5-II-D22J2 IIES URBAN-PLANNING UTILITY-TUNNELS COMMUNICATION-SYSTEMS STRESS (PSYCHOLOGY) PUBLIC-INFORMATION MOVEMENT, I3-13-OI83I INSE PLANNING I POSTATTACK-OPERATIONS MANAGEMENT-PLANNING STRESS (PSYCHOLOGY) SHELTERS SCHOOLS PUBLIC-INFO/ O5-II-D22J3

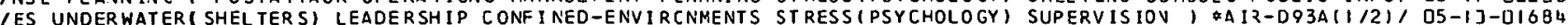
IES UNDERWATER ( SHEL TER S) LEADERSHIP CONFINED-ENVIRCNMENTS STRESS (PSYCHOLOGY) SUPERVIS ION /ESEARCH CIVIL-DEFEN SE-SYSTEMS ATTITUDES FALLOUT-SHELTERS STRESS (PSYCHOLOGY) THREAT-PERCEPTION BEHAVIOR M/ D5-04-O2I 66 /CIETY - SOME EXPLORATORY STUDIES I POSTATTACK-OPERATIONS STRESS (PSYCHOLOGY) URBAN-AREAS SOCIOLOGY SOCIET/ I5-03-O23OI

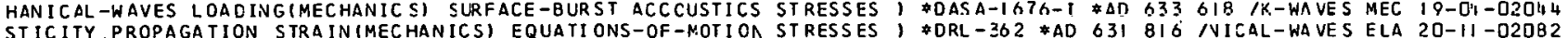
STICITY.PROPAGATION STRAINIMECHANICS) EQUATI ONS-OF-NOTION STRESSES \#DRL-362 *AD 631816 NICAL-WAVES ELA $20-11-020 B 2$ ILE-PROPERTIES PLASTICITY STRUC TURAL-SHELLS RINGS WELDING STRESSES DEFLECT 1OV ROLLIVG(METALLURGY) ELASTIC/ $20-11$-O234 7 1 LOACING(MECHANICS) SHOCK (MECHANICS) FEASIBILITY-STUDIES STRESSES ELASTICITY CONCRETE HONEYCOMB-CORES EXI I8-03-O20I 7 I TUNNEL MECHAN ICS I UNDERGROUND-STRUC TURES ROCK (GEOLOGY) STRESSES ELASTICITY THEORY GEOLOGY IVSTRUMENTATI I3-I3-D227S IRGROUND-STRUCTURES FAILURE (MECHANICS) MINING-ENG I NEERING STRESSES GRANITE JPENIVGS 8LOCK-CAVIVG-MINING B/ D8-D7-O2243
FFERENCE TECHNIQUE, THERMAL-RADIATI ON WEAPONS-E/ THERMAL STRESSES IN A SEMI INFINITE SJLID BY A FINITE DI I3-1?-QI795 /RCED COVCRETE SHELL I SHELLSISTRUCTURAL-FORMS) MENBRANES STRESSES LOADING (MECHANICS) FAILURE (MECHANICS), I3-I3-DI832 IOMPETENT ROCK I MINING-ENGINEERING ROCK (GEOLOGY) PILLARS STRESSES MATHEMATICAL-ANALYSIS ROCK-FORMATIONS, DB-OT-O2245 IUAT ION DESIGN GUN-BARREL-ATTACHMENTS INHIBITION PRESSURE STRESSES PROJECT ILES IVTERIOR-8ALLISTICS GAS-FL, $19-0 S-02327$ IND BENDING WAVES IN LONG ELASTIC RODS I NECHANICAL-WAVES STRESSES PROPAGATION ELASTICITY THEORY I \#DRL-3/ $20-I I$-O2O79 IG ANALYSIS OF STRESS DISTRIBUTI CN BENEATH ENBANKNENTS ( STRESSES SOIL-MECHANICS SETTLEMENTISTRUCTURAL) H $20-11$-O2OBL INOR I CAL-BODIES COMPOSITE-MATERIALS, EOUATIOVS-OF-NOTION, STRESSES STIFFENED-CYLINDERS STRAIV (MECHANICS), $20-I I-02345$ HYDR ITE SANDSTONES CLAY SILTSTONES GEOLOG Y THERMAL-STRESS STRESSES STRAIN I HYDRITE SANDSTONES CLAY SILTSTONE \& GEOLOGY THERMAL-STRESS STRESSES STRAIN 1 \#A.L. $1159 /$ STOVE RJCK-SALT AN D8-OT-D2242 IIUM ON ROCK MECHANICS I ROCK (GEOLOGY) FAILURE (MECHANICS) ST RESSES THEORY CEFORMATIOV EXPERIMEVTAL-DATA G I D8-07-O224 4 ICTANCULAR PLATES ( PANELS(STRUCTLRAL) RECTANGULAR-BODIES STRESSES THRUST THERMAL-STRESSES LOADING IMECHAN/ 20-11 - 22344 IIGN OF UNDERGR OUND OPENINGS FOR DEFENSE ( SHELTERS BLAST STRESSES UNDERGROUND-STRUCTURES RJCK(GEOLOGY) H/ I3-I3-O2273
IHANICS) USSR EXCAVATION SHEAR ING CUTTING BENDING TORSION STRETCHING COMPRESSING TEST ING-EQUIPMENT MARBLE/ D8-O7-O2242 IHANICS) USSR EXCAVATION SHEAR ING CUITING BENDING TORSION ST RETCHING COMPRESSING TESTING-EQUIPMENT MARBLE/ D8-O7-Q2242 IGINEER ING AND RESCUE OPERATIONS AT THE SITE OF AN ATOMIC STRIKE I RESCUES CIVIL-DEFENSE-PEZSONVEL USSR I' I3-IZ-OIBDU
ISSANCE INTERNA/ ENGINEERING AND RESC UE WCRK IN A NUCLEAR STRIKE ZONE I CIVIL-DEFENSE-SYSTEMS USSR RECONNA DS-OT-DITJ9

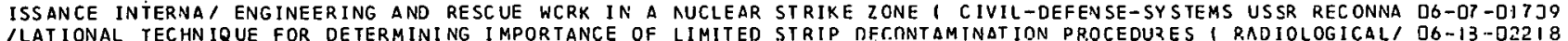

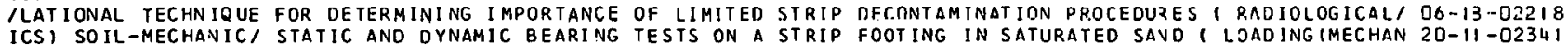
NUCLEAR-WARFARE MODEL-THEORY STRATEGIC-WARFARE MILITARY/ STROP - A STRATECIC PLANNING YODEL I GAME-THEORY IS-OS-OIS59 NICS) FAILURE(MECHANICS) DESIGN COSTS MODELS (SIMULATIONS) STRUCTURAL-ANALYSIS, \$TR-5IO /SES LOADING(MECHA I3-I3-DI832 SHO THE SHOCK AND VIBRATION BULLETIN I ROCKETS DYNAMICS STRUCTURAL-ANALYSIS ELASTICITY BEAYSISTRUCTURAL) $20-11$-O2O74 11 DYNAMICS COMPUTER-PROGRAMS SHOCK-WAVES BLAST SHIELDING STRUCTURAL-ANALYSIS RADIOACTIVE-FALLOUT FIRE-SA/ I3-I3-O2280 OUND-STRUCTURES SHEL TERS PERFORMANCE (ENGINEERING) STATICS STRUCTURAL-PARTS ) WWL-TDR- 64-54 \#AD OI3 545 /GR I3-13-O2290 REAN-AREAS RADIOACTIVE-FALLOUT FALLOUT-SHELTERS SHIELOING STRUCTURAL-PROPERTIES, \$NP-857I/COVSTRUCTION U I3-I3-02277 LYWOOC SAND/ WOOD HANDBOOK I CONSIRUC TION DESIGN STRENGTH STRUCTURAL-PROPERT IES QUALITY FASTEVERS GLUING P II-I?-DI7G6 IRDER ELASTIC ANALYSIS OF PLANE RIGID FRANES I STRUCTURES ST RUCTURAL-PROPERT IES STEEL CJMPUTER-PROGRAMS I I $3-13-02287$

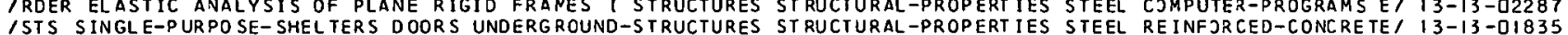

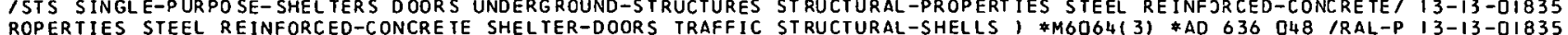
IRATION OF MUL I I-LAYERED ANISO TROPIE C YLINORICAL SHELLS I ST RUCTURAL-SHELLS CYLIVDR ICAL-BODIES COMPOSITE- $20-11-02345$ IS CONTAINIVG REC TANGULAR OPENING S WI TH STIFFENED EDGES I STRUCTURAL-SHELLS ELASTIC-SHELLS YJDELSISIMULAT/ $20-11-D 2 O 31$ ISURE ISTIFFENED-CYLINDERS TENSI LE-PROPERTIES PLASTICITY STRUCTURAL-SHELLS RINGS WELDIVG STRESSES DEFLEC/ $20-11$-O234 7 HEAR/ FORCED MOTION OF SHALLOW SPHERICAL SHELLS 1 SPHERES STRUCTURAL-SHELLS VIBRATION LOADINGIMECHANICSI S $20-11$-O2346 NUCLEAR ATTACX / RECOVERY SOCI/ VLLNERABILITIES OF SOCIAL STRUCTURE - STUDIES OF THE SOCIAL DIMENSIONS OF O5-II-D2I9O NUCLEAR ATTACK I SOCIETAL-RECO/ VLLNERABILITIES OF SOCIAI STRIJCTURE - STUCIES OF THE SJCIAL DIMENSIONS OT. OS-II -D22J2 OADS ERIDGES GROUNO-SUPP', SOVIET LITERATURE ON PROTECTIVE STRUCTURE AND COMPONENTSI GUIDED-MISSILES RAILR I6-OI-DIQ72 ISTRATION, OF/ INTERIM REPORT ON TRANSFER SYSTEM AND FARE STRUCTURE FOR THE JFFICE OF TRANSPORTATION ADMIN $23-02-02355$ MOTIVATION CUL TURE RESPONSE REAC TION/ SPECULATION ON THE STRUCTURE OF INTERPERSONAL INTENTIJVS I BEHAVIOR O5-IJ-D2I79 OLUMBIA I BUILDINE-CODES REGULATIONS WASHINGTON BUILDINGS STRUCTURES, \#A.L. 995 10DE JF THE OISTRICT OF C 13-13-0I8I6 SHOCK TUBES - EXP ER IMENTA BLAST EFFECTS CN BUILDIAGS AND SIRUCTURES - OPERATION OF SIX-FOJT AND TWO-FOOT I8-03-D2OTI SHOCK TUBES SHOCK TUES - EXPERIMENTA BLAST EFFECTS CN BUILCINGS AND STRUCTURES - OPERATION OF SIX-FOOT AND TWO-FOOT I8-O3-O20J5 ISE IN MITIGATING BLAST LOADS ON DEEPLY BURIED PROTECTIVE STRUCTURES 1 CONCRETE UNDERGROUND-STRUCTURES EX/ 18 -03-D20D7 S) FALL COMBINED EFFECTS OF NUCLEAR WEAPONS ON NFSS TYPE STRUCTURES I NAT IONAL-FALLOUT-SHELTER-SUR VEYINFS I8-D3-DI995 IEMENT OF DEPOSITION AND REDISTRIBLTI ON OF FALLOUT AROUND ST RUCTURES $($ RACIOACTIVE-FALLOUT PAZTICLES DEBRI $18-03-02038$ IMPACT-SHOCK NUCLI STUDY OF SHOCK I SOLATICN FCR HARDENED STRUCTURES
ISE IN MITIGATING BLAST LOADS ON DEEPLY BURIED PROTECTIVE STRUCTURES

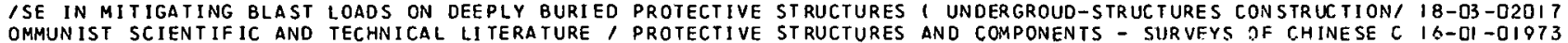

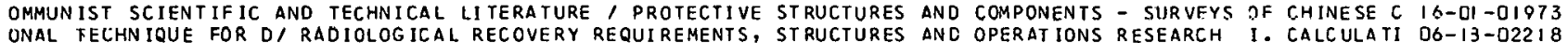

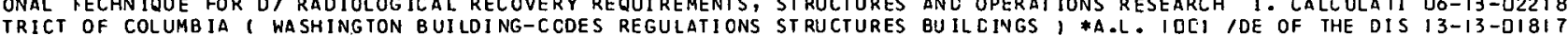
IATTENUAT ION SHEL TERS WEAPONS-EFFECTS SURVIVAL IEQUIPMENTI ST RUCTURES COMMUNICATIONS-SYSTEMS RADIATION GEO/ I $7-0$ ? -O23। I LOADIN/ DEFLECTIONS OF REINFORCED CONCRETE FLOOR SLABS I STRUCTURES DESIGN THEORY BEAMSISTRUCTURAL FRAMES $20-1 \mathrm{I}$-D234O IERS SPECTRUM-ANALYZERS TRANSDLCERS MATHEMATICAL-ANALYSIS STRUCTURES DYNAMICS, \#BULLETIN $35(4)$ \#AD O3I $2120-11$-02075 
IL DESIGNS TO RESIST INTEGRATED NLCLEAR WEAPONS EFFECTS I STRUCTURES FALLOUT-SHELTERS BLAST-SHELTERS THER I I3-13-OIB2I HELTERS SHOCK-WAVES 8LAS/ COMPUTER ANALYSIS OF PROTECTIVE STRUCTURES FOR PROTECTION FROY NUELEAR BLAST I S I3-I3-OI833 IUCTURAL) CYVAMICS SHEAR-STRESSES LEB S(STRUC TURAL) DESIGN ST RUCTURES LOADINGIMECHANICS) SHOSK-WAVES MA THE/ $20-11$-02337 OUT-SHELTERS COMPUTERS B / COOLING ANALYSES FOR PROTECTIVE STRUCTURES LOCATED ABOVE AVO BELOW GROUNO 1 FALL I 3 - DI -DI776 IINGS OF THE IN TERNATIONAL SYMPOSIUM (LOADING (MECHANICS) STRUCTURES NONLINEAR-ANALYS IS BEAYS ISTRUCTURAL)/ $20-11$-02339 NGINEERIVG II. TOKYO AND KYOTO, JAPAN I JAPAN SEISMOLOGY STRUCTURES SHOCK-NAVES ELASTICITYI *A.L. II95 IE O8-II-02246 SHELTER COMPOVENTS IFALLOLT-SHELTERS FIRE-SAFETY FIRES STRUCTURES SMOKES BUILOINGS CONSTZUCTTON BLAST, I3-I?-O2269 IHE SECOYD ORDER ELASTIC ANALYSIS OF PLANE RIGID FRAMES I STRUCTURES STRUCTURAL-PROPERTIES STEEL COMPUTERI I3-I3-D2287 AI PREL IMINARY CONCEPTS OF SUB SURFACE REACTOR CONTAINMENT STRUCIURES TO WITHSTAND DIFFERENTIAL EARTH DISPL I8-DP-O2OLD IOSIONS SHOCK-WAVES LOADING (MECHANICS) SUSPENSION-DEVICES STRUCTURES VIBRATION-ISOLATORS MACHIVES VISRATI/ I3-I3-O229I ITO RES IDENTIAL AREAS I NUCLEAR-E XPLOSIONS FIRES AIRBURST STRUCTURES VJLNERABILITY RECOVERY DAYAGE RECLAM/ IS-DS-OI963 IF THERMAL EVVIRONMENT IN OCCUPIED UNDERGROUND PRCTECTIVE STRUCTURES WITH OBSERVEO CONOITIOVS 1 HEAT-TRAN/ $20-13$-02090 TION AND SUMMARY I R/ RADIOLOGICAL RECOVERY REQUIREMENTS, STRUCTURES, AND OPERATIONS RESEAREH I. INTRODUC DO-I3-D22I 4 PF MULTIPLIER I RAD/ RADIOLOGICAL RECOVERY REQUIREMENTS, STRUCTURES, AND OPERATIONS RESEARCH II. DAMAGED O6-I3-D222I 1 OF COVERNMENT SPONSORED AND PRI VATE RESEARCH ON FOREIGN STUDENTS ANO TRAIVEES IN THE U.S. AVD IN OTHER I OS-II - 0I69 1 /ENCE, AV ANVOTATED BIBLIOGRAPHY - SUPPLENENT, AUTHOR AND SURJECT INDEXES 1 BACTERIA VIZUSES FUVGI PROTOZ/ D6-I3 -DI7I5 ITO CaL CULATE THE THERMAL HISTORY OF a DUAL LAYEREC PLATE SUBJECT TO THE THERMAL PULSE JF A VULLEAR WEAPO/ I3-IZ - JI7O? IT OF COOROIVATE INDEXING I INDEXES INFORMATICN-RETRIEVAL SUBJECT - HEADINGS CLASSIFICATION IVFORMATION-TME/ D5-O?-O2IIO TT OF COJROIVATE INDEXING 1 INDEXES INFORMATICN-RETRIEVAL SUBJECT-HEADINGS CLASSIFICATIJN IVFORMATION-TME/ OS-Q? -02II 9 TION E/ <EY WORDS AND THEIR USE ON DO FORN 14731 REPORTS SUBJECT - INDEX ING DEPARTMENT -JF-DEFENSE DOCUMENTA OS-O2 - D2I 24 IS SYSTEMS-EVGINEER ING DOCUMEN TATION RATA-STORAGE-SYSTEMS SUEJECT-INDEX ING IVFORMAT IOV-ZETR IEVAL AUTOMA YI / DS-D? -02I 21 作

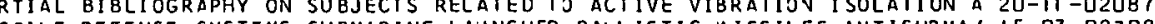
INSE-SYSTEMS SPACE-WEAPONS GUIDED-MISSILE-DEFENSE-SYSTEMS SUBMARINE-LAJNCHEO-BALL ISTIC-Y ISSILES ANTISUBMA' I5-D7-D23DB T ECONOMICS FEASIBILITY-STLD/ AN ANALYSIS OF MACHIAE TCOL SUESTITUTION POSSIBILITIES 1 INDUSTRIAL-EQUIPMEN DS-D3-DIGU8 C TRANSIT SYSTEM POR.TION OF CONNFC.TIC.IIT AVFNIIFI PHASE III SUBSURFACE INVESTIGATION - VATIONAL EAPITAL RAPI I3-D? -OI7BS HSTAND DIFFERENTIAL EARTH DISPLAI PRELIMINARY CONCEPTS OF SUBSURFACE REACTOR CONTAINMENT STRUETURES TO WIT IY-UY -UZULU BLAST LOADING - RESULTS OF SERIES III TESTS $1 /$ MOTION OF SUBSURFACE SOIL INCLUSIONS SUBJECTED TO SURFACE D8-13-0I746 BLAST LOADING - RESULTS OF SERIES III TESIS I I MOTICN OF SUESURFACE SOIL INCLUSIONS SUBJECTED TO SURFACE I8-D3-O2OO8 ICAL-REGIO/ HEAT TOLERAVCE OF ELOERLY PERSONS LIVING IN A SUBTROPICAL CLIMATE I AGIVGIPHYSIJLOGY) AGE TROP OG-I7-DI734 SION COLJRS OPTICAL-IMA/ STUDY OF LIGHT ATTENUATION UNDER SUBTROPICAL CL IMAT IC CONDITIOVS I LIGHT-TRANSMIS 20-OS -D2DSG ITRANSPORTATION NEW-YORK URBAN-AREAS POPULATICN RAILROAOS SUBWAYS COSTS MANAGEMENT-PLANVING URBAN-PLANNIN/ 23 -D2 -02099 ITWEEN COLUMBIA ROAD AND VAN NESS STREET I TRANSPORTATION SURWAYS TESTS BORING WASHIVGTON COVSTRUCTION SE/ I3-O?-OI785 /T-SHEL TERS TASTE CIVIL-DEFENSE-SYSTEMS FRUI TS CONCINENTS SUCROSE FOOD-DISPENSING TESTS I $\$$ SR I $4949-500$ * 1 O6-D3-022II IFAIRS POL II ICAL-SCIENEE FOREI GN-POLICY THREAT-PERCEPT ION SJEZ-CRIS IS ATT ITJDES YIDOLE-EAST METHODOLOGY T/ OS-D+-D2I 7D JEATMENT OF EXPER IMENTAL MELIOIDOSIS WITH ANT IBIOTICS AND SULFADIMES IN I B IOLOGICAL-WARFARE-AGENTS EFFECT/ I5-DI-OI8Q4 TERS) LEADER SHIP COVFIVED-ENVIRONNENTS STRESS (PSYCHOLOGY) SUPERVIS ION, *AIR-093A(1) ISPITALS OPERAT ION CONTROL-SYSTEMS COMMUNI CATICNS-SYSTEMS SUPERVISION MEDICAL-PER SOVVEL STOZYS EMERGENCY-1 OO-I?-DI 7 I 4 IPORTANCE OF INDIVIDUAL INDUSTRIES FOR DEFENSE PLANNINGI SUPPLY-AND-DEMANC ECONOMICS RECOVERY POSTATTACKI DS-O3-OIOU 7 IT 1 INDUSTRIES MANPOWER ECONOMICS GROSS-NATICNAL-PRODUCT SUPPLY-AND-DEMANC INOUSTRIAL-PRODUCTION SCIENTI/ OS-D3-OI 49 ING CISTRIBUT ION DAMAGE-A SSESSMENT CALIFORNIA URBAR-AREAS SUPPLY-CEPOTS, $\$ S R I-M I-5776$ \$SVRDL-TRC-67-3 /S O6-O3-0I7II INVLNTORY BY WAREHOUSE AND LOCATICN I AGRICULTURE STORAGF SIIPPIY-C.FPOTS WAREHOUSES STOCKP ILIVG I *A.L. IO/ D2-OZ-D2I O9 IIGATION OF A DYNAMICALLY LOADEO RING WITH RADIAL ELASTIC SUPPORT ( RINGS VIBRATION FRE2UENCY BENDING ELA/ $20-11$-O2336 RCA 19701 RADI/ COMMUNICATIONS ECUIPMENTS AND SYSTEMS TO SUPPORT INTRASTATE CIVIL DEFENSE OPERATIONS - CI I7-Q2-D23I3 IRAMS - MANAGFMENT, DEVELOPMENI, TECHNICAL ANO SCIENTIFIC SUPPORT, PLANNING, STUDIES AND AVALYSES I COMMU/ 22-D? - O2351

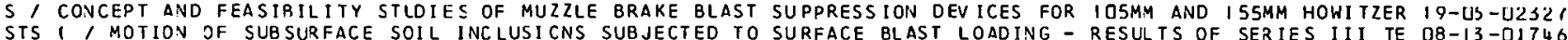
STS , MOTION OF SUB SURFACE SOIL INCLUSICNS SUBJECTED TO SURFACE BLAST LOADING - RESULTS OF SERIES III TE I8-O3 -DODJB STS T MOTION OF SUBSURFACE SOIL INCLUSICNS SUBJECTED TO SURFACE BLAST LOADING - RESULTS OF SERIES III TE I8-O3-D2DJ8 A RADIO/ RADIONUCLIDE FRACTIONATION IN DEBRIS FROM A LAND SURFACE BURST 1 RADIOACTIVE-ISOTOPES CHINA RUSSI IB-0?-OI99I TAMINATION RADI/ EVALUATION OF FALLOUT CONTAMINATICN FROM SURFACE RUNOFF I NATER-SUPPLIES RADIJLOGICAL-CON OG-1S-OI724
CHANNELS 1I. PLANS, PROFILES, CROSS SECTION/ CCNTROL OF SURFACE STORM WATER BY STORM DRAIVS AVD ORAINAGE I3-D -OIT79

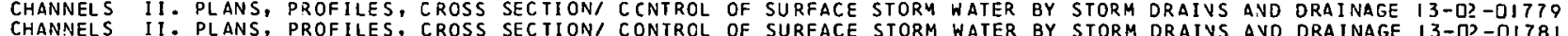
CHANNELS II. PLANS, PROFILES, CROSS SECTION/ CONIRCL OF SURFACE STORM WATER BY STORM DRATVS AVD DRAINAGE I3-D -OI78I CHANNELS - 1958 PROGRAM I. GENERAL OESCRIPT/ CONTROL OF SURFACE STORM WATER BY STORM DRAIVS AND DRAINAGE I3-G2-OI783 CHANNELS - 1964 PROGRAM 1. GENERAL DESCRIPT/ CONTROL OF SURFACE STORM WATER BY STORM DRAINS AND ORAINAGE I3-D2-01784 - FORMULAE AND PROGRAM SPECIFICATIONS 1 HYDRODYNANI/ THE SURFACE WAVES FROM SHALLOW UNDERWATER EXPLOSIONS I9-OI-OZOU 3

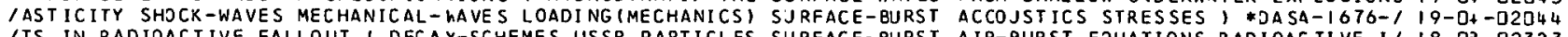
ITS IN RADIOACTIVE FALLOUT I DECAY-SCHEMES USSR PARTICLES SURFACE-BURST AIR-BURST EZUATIONS RADIOACTIVE-I/ I8-O3-D2323 II TESTS I SHEL TERS UNDERGROUND-STRUCTURES SOIL-MECHANICS SURFACE-BURST FAILURE(MECHANICS), ITTR-5O8 IES I O8-I3-OI746 PIRAT IONS-RESEARCH ECONOMICS DECI I I PLANNI NG INETIES DRAG SURFACE-TEMPERATURES BOUNOARY-LAYER LAMINAR-BUU/ ZZ-UZ -UZ35O ITWORK SIMULATION MODEL I RESOLRCES DISTRIBUTION SERVICES SURPLUSES DATA-PRJCESSING SHORTAGES WEAPONS-EFF/ O5-D3-O2I 33 RAD IOACTIVE-FALLOUT RADIOACIVE-ISOTOPES AIR MILK IITY RESPONSE TO THE RHODE ISLAND HOME FALLOUT PROTECTION SURVEY ATTITUCES FALLOUT-SHELTEZS PUBLIC-OPIN/ OS-1J-OI G85 IITY RESPONSE TO THE RHODE ISLANO HOME FALLOUT PROTECTION SURVEY ( ATTITUCES FALLOUT-SHELTERS PUBLIC-OPIN/ OS- 1 J-OI 685

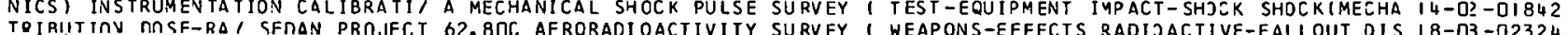

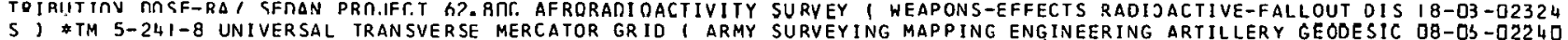
OUT-S HELTERS PUBL IC-OPINION $\triangle N$ XIETY T.IVII-DFFFNSE-SYSTEMS SURVEYS, \#A.L. IDO2 ION SURVEY IATTITUDES FALL OS-IJ-OIOB5 ANNING RECOVERY VULVERABILITY REC CRDS DISTRIBUTION (WATER) SURVEYS, \#A.L. 969 /CK-OPERAT IONS YAVAGEMENT-PL I3-DZ-OITB8 VIOR WARYING-SYSTEMS SHELTER-ALLOCATION SHELTER-OCCUPANCY SURVEYS, \#AIR-D-930-9/66-FR/IOV ATTITUDES BEHA O5-II-OIG99 WARS I PEACE-KEEP ING PUBLIC-OPINICN INTERNATICNAL-AFFAIRS SURVEYS ARMS-CONTRJL) *NORC-29/ZATION PREVENT D5-11 -D2I95 IES AND PUBLICATIONS IN SOCIAL RE SEARCH I SOCIAL-SCIENCES SURVEYS BIBLIOGRAPHIES ATTITUDES GROUP-OYNAMICS/ OS-II -O2I 94 ILTER-ALLJCATION ATTI TUDES CIVIL-DEFENSE-SYSTENS CCLO-WAR SURVEYS CULT JRE CEMOGRAPHY SOCIOLJGY POPULATION/ O5-IJ-02I77 IION (FAILURE) ATTITIIDFS PIIRI. IC. -OPINI ON PUBLIC-INF ORMATI ON SURVEYS CEMOGRAPHY COMMUNICATION-SYSTEMS LEADER/ OS-IJ-DIOB3 IN THE COLO WAR CONTEXT - 1966 I ATTI TUDES PUBLIC-CPINION SURVEYS DISARMAMENT CIVIL-DEFENSE-SYSTEMS VIETN/ O5-IJ-O I678 IAIRS - AMERICAN PROGRAMS OF FOREIGN AID 1 PUBLIC-CPINION SURVEYS FOREIGN-POLICY ECONOMIC-AID MILITARY-AI/ O5-II -0IGB 7 IAL REPORTS ON FOREIGN AFFAIRS - GERMANY I PUBLIC-CPINION SURVEYS FOREIGN-POLICY OUESTIONNAIRES INTERNATI/ DS-II-O2IBO 1 - UNITED VATIOVS I INTERNATIONAL-AFFAIRS PUBLIC-CPINION SURVEYS FOREIGN-POI ICY QUESTIJYNAIRES PEACE-KEEI O5-II -O2I Q8 NICAL LITERATURE I PROTECTIVE STRLCTURES AND COMPONENTS - SURVEYS OF CHINESE COMMUNIST SCIEVTIFIC AND TECH IO-OI -DI973 NICAL LITERATURE $/$ PROTECTIVE STRLCTURES AND COMPONENTS - SURVEYS OF CHINESE COMMUNIST SCIEVTIFIC AND TECH I O-OI -OI973
IWATER SYSTEMS I WATER-SUPPLIES VILNERAB ILITY URBAN-AREAS SURVEYS POSTATTACK-OPERATIONS RECOVERYIWATER-SU/ IS-O3-OIQ4I IUT THE BOMB AND ATOMIC ENERGY I PUBLIC-OPINICN ATTITUDES SURVEYS CUEST IONNAIRES PEACE-XEEPIVG VUCLEAR-WAI O5-II-O2I OG IFORFIGN AFFAIRS - UNITED NATIONS IINTERNATIONAL-AFFAIRS SURVEYS QUESTIONNAIRES PUBL IC-OPIVIOV FOREIGN-PI OS-II-O2I 99 IATES QUESTIONNAIRES ATTITUDES INTERNATI ONAL-AFFAIRS FEAR SURVEYS STRESSIPSYCHOLOGY) FOREIGY-POLICY, \#NO/ OS-II -DIGB9 IRE WIND-PRESSURE PRESSURE HEA T-TRANSMISSION TESTS (FIRES) SURVEYS (DATA-COLLECTIOV), \$NRDL-TRC-I6 *AD G37) I3-I?-0IBO7 ATIONAL VIABILITY I SOCIETAL-RECOVERY ECON/ DIMENSIONS OF SURVIVAL - POSTATTACK SURVIVAL DISPAZITIES ANO N DS-O3-O2IGO RESOU' NREC PROGRAMS FOR GAMING THE LOGISTICS OF NATICNAL SURVIVAL I SIMULAT ION DAMAGE-ASSESSMENT WARFARE OS-D3 -DIG44 IPOSTATTACK SOCIETY I POPULATION CISTRIBUTION URBAN-AREAS SURVIVAL AGE POSTATTACK-OPERATIONS URBAN-PLANNI/ 23 -OH-O2IO3 PUBL IC-HEALTH RESCUES VUCLEAR-WARFARE-CASUALTIES SCHOOLS SURVIVAL CASJALTIES FIRST-AID I *A.L. ID77 /TALS O6-21 - DI740 IOMICS ECONOMIC-RECOVERY POSTA TTACK-OPERATIONS POPULAT ION SURVIVAL CAMAGE-ASSESSMENT CALIFOZVIA RECOVERY I O5-O3-DIG39 I APPLICATIONS I ATTACK-SCENARIOS VULNERABILITYIANALYSIS) SURVIVAL DAMAGE-LIMITING-SYSTEMS DAMAGE-ASSESSMI I5-O3-DIQ49 IESSMENT NUCLEAR-EXPLOSION-DAMAGE AGE MODELS (SIMULATICNS) SURVIVAL DEMOGRAPHY RECOVERY ATTACK-SCENARIOS F/ I5-OS-OIOS CIETAL-RECOVERY ECOVI DIMENSIONS OF SURVIVAL - POSTAITACK SURVIVAL CISPARITIES AVD VATIJVAL VIABILITY I SO DS-D3-O2I GO I CONOMIC-RECOVERY ATTI TUDES MANPO YER MOTIVATION ECCNOMICS SURVIVAL CISPARITIES AVD VATIIVAL VIABILITY I SO D5-O3-O2I 6O

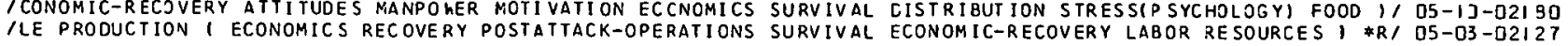


IAR - THE LIMITS OF FEASIBLE PRODLCTION I NUCLEAR-hARFARE SURVIVAL ECONOMICS POPULATION AGRICULTURE INDUSI O5-03-02I58

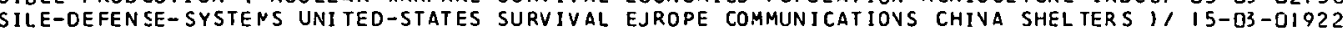
/ CCUPANTS OF SURVIVAL SHEL TERS I HEAT-PRODUCTICNIBIOLOGY) SURVIVAL FALLOUT-SHELTERS VEVTILATIOV METABOLIS/ DS-IS-DITIQ IEMANC ECONOM ICS RECOVERY POSTATTACK-OPERATIONS RESOURCES SURVIVAL INOUSTRIAL-PRODUCTIOV I \$P-2OQ3 \#AD OLI OS-03-DI 447 CASUALTIES RESOURCES DAMAGE-ASSESSMENT FOOD LIGHT MEDIC/ SURVIVAL ITEM ANALYSIS PROGRAM 1 VUCLEAR- HARFARE O5-O3-O2I35

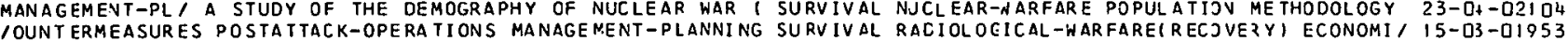
TOUNTERMEASURES TPENDIX B IT AND APPENDIX A I INDUSTRIAL-EQUIPMENT BLAST INDUSTRIES SURVIVAL RECOVERY ECONOMIC-RECOVERY PRODUCTS VU/ OS-D3-O21 62
IPERS AL URBAN-AREAS FOOD SHELTERS FALLOUT-SHELTERS EUROPE SURVIVAL RECOVERY VULNERABILITY MILITARY-STRATE/ I5-D3-O2295

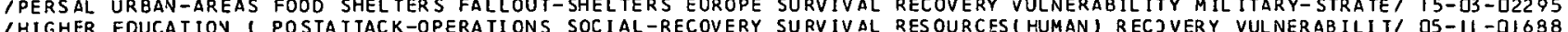
IHIGHER EOUCATIOV ( POSTA TIACK-OPERATIONS SOCIAL-RECOVERY SURVIVAL RESOURCES I HUMANI RECJVERY YULNERABILIT/ OS-II-DI 638 VIVAL / SENSIBLE AND LATENT. HEAT LOSSES FRON CCCUPANTS OF SURVIVAL SHELTERS I HEAT-PRODUCTIOV(BIOLOGY) SUR D6-1S-OIZI 9 PHYSI/ SENSIBLE AND LATENT REAT LOSSES FROM OCCUPANTS OF SURVIVAL SHELTERS I SHELTER-OLCUPAVCY METABOLISM O6-IS-DI7I 8 /POSTATTACK ENVIRONMENT I FOOD DIET POSTATTACK-OPERATIONS SURVIVAL STORAGE(FOOD) PRODUCTION(FOOD) RESOURC/ DO-IS-QI7I 7 1. LOW COST FALLOUT SHEL TER SYSYENS I MANAGEMENT-PLANNING SURVIVAL URBAN-AREAS DAMAGE-LIMITIVG-SYSTEMS EVI I3-13-0I829 IES SHOCK (MECHANICS) ATTENLATION SHELTERS WEAPCNS-EFFECTS SURVIVAL (EQU IPMENT) STRUCTURES COMMUVICATIONS-S/ I 7 -D?-O23II 1 IL ITY NUCLEAR-EXPLOSIONS SHOCK- hAVES LOADING IMFCHANICS) SUSPENSION-DEVICES STRUCTURES VIBRATION-I SOLATO/ I3-I3-D229I IRELATED TO ACTIVE VIBRATION I SOLATION AND ACTIVE VEHICLE SUSPENSIONS I VIBRATION-ISOLATORS CALCULATIONS I 20- II-02OB7 TRY OF BU ILDINGS IN URBAN AREAS ( FIRES THERMAL-RADIATION SWEDEN; \#A.L. 1118 S G ATTENUATION OF AIR SHOCK WAVES IN TUNNELS I PROPAGATION SWEDEN I \$REPORT VO. 103-24 \$AEC-TR-5025 /ULATIN 20-DI-O2O5I IEAT-BR ITAIN FRANCE GERMANY BENELLX NORWAY DENMARK GREECE SWEDEN SWITZERL AND IRELAND USSR.IVDIA AUSTRALIA' I5-D3-O2I 47 IR BLAST I BLAST-BIOLOGY BIOLOGICAL-BLAST-EFFECTS NONKEYS SWINE SHEEP LUNG-IVJURIES EXPLOSIDNS TOLERANCES/ DG-2I -D22 34 ITRANSPORT I TRAN SPORTATION NE TWORKS TERMINALS SCHEOULING SWITCHING PROPULSION-SYSTEMS AERODYNAMICS DRAG , $23-02-02354$

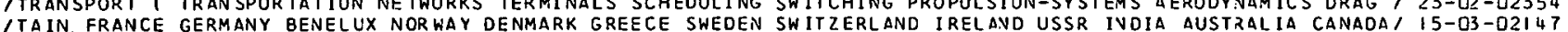
ITAIN. FRANCE GERMANY OENELUX NORWAY DENMARK GREECE SWEDEN SWITZERLAND IRELAND USSR IVOIA AUSTRALIA CANADA/ I5-O3-O2I 47 $1 G$ INFORMATION-RE TR IEVAL AUTOMATIC MANAGE MENT-ENGINEERING SYMPOSIA DATA-PROCESSIVG-SYSTEMS IF RE INFORCED CONCRETE - PROCEEOINGS OF THE INTERNATIONAL SYMPOSIUM I LOADING (MECHANICS) STRUCTURES NONLI/ 20-11-D2339 IEHAVIOR OF MATER IALS IN THE EARTHS CRUST - SECOND ANNUAL SY YPOSIUN ON ROCK MECHANICS / ROCK (GEOLOGY) FAI/ O8-OT-O2244 REROUND/ QUARTERLY OF THE COLORADC SCHOOL OF NINES SI (3) SYMPOS IUM ON ROCK YECHANICS 1 ROC (IGEDLOGY) UNDE D8-O?-O2243 IOLOGY S/ METHODOLOGY FOR ASSESSING TOTAL VULNERABILITY I SYSTEMS-ANALYSIS POL ITICAL-SCIENCE ECONOMICS SOC DS-1I -02IQ3 IS AIRCRAFT NUCLEAR-WEAPONS-EFFEC TS MILITARY-INTELLIGENCE SYSTEMS-ANALYSIS POST-ATTACK-OPERATIONS DAMAGE-1 I3-I?-O2267 IES II. LABORATORY STUDIES I FALLOUT-SHELTERS PHYSIOLOGY SYSTEMS-ENGINEERING COOLING-AVD-VEVTILATING-EOUI IS-03-02297 /PROCEDURES III. HABITABILITY.STLDIES I FALLOUT-SHELTERS SYSTEMS-ENGINEERING COOLIVG-AVD-VEVTILATING-EQU/ I5-D3-D2?98 I AND PROCEOURES I. SUMMARY ANO REVIEW I FALLOUT-SHELTERS SYSTEMS-ENGINEER IVG COJLIVG-AVD-VEVTILATING-EQU/ IS-O3-O23OD /RMAT ION-RETR IEVAL AUTOMATION LIBRARIES AIRCRAFT-INDUSTRY SYSTEMS-ENGINEERIVG DATA-STORAGE-SYSTEMS COMPUT/ O5-O2-02II 8 IN OF INFORMATION SYSTEMS I TECHNICAL-INFORMATION-CENTERS SYSTEMS-ENGINEERING DOCUMENTATION DATA-STORAGE-I D5-O2-0212I /ILROAD TRANSPORTATION IN THE CONIINENTAL UNITED STATES I SYSTEMS-ENGINEERIVG ECONOMICS RECJVERY POSTATIA/ O5-D3-O2I28 ION OPERATIOVS-RESEARCI CIVIL OEFENSE RE SEARCH ANALYSIS I SYSTEMS-ENGINEERING RESEARCH-DROGZAM-ADMINISTRAT I2-D?-DITS? ION OPERATIONS-RE SEARC/ CIVIL OEFENSE RE SEARCH ANALYSIS I SYSTEMS-ENGINEERING RESEARCH-PROGRAM-ADMINISTRAT I2-D? -DI737

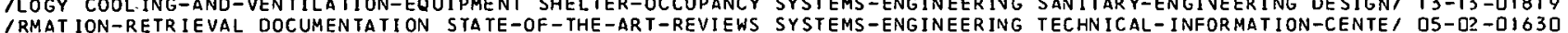

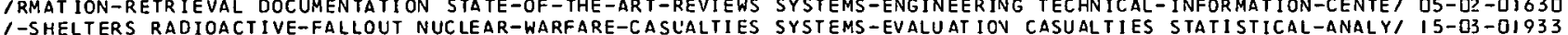
1-SHELTERS RADIOACTIVE-FALLOUT NUCLEAR-WARFARE-CASLALTIES SYSTEMS-EVALUATIOV CASUALTIES STATISTICAL-ANALYI I5-D3-OI933

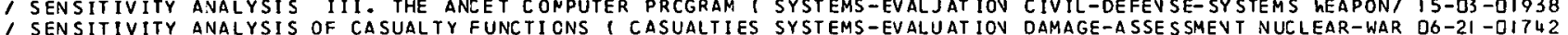
S SENSITIVITY ANALYSIS OF CASUAL YY FUNCTI CNS I CASUALTIES SYSTEMS-EVALUAT IOV DAMAGE-ASSES SMEVT NUCLEAR-WAR O6-21 -OI742
IYSIS I. METHODOLOGY I CIVIL-DEFENSE-SYSTEMS METECROLOGY SYSTEMS-EVALUATION OPERATIONS-RESEARCH MATHEMAT/ I5-TZ-DI935

WITH EXIENSIONS OF TOTAL POPULATION TO 2015 ( DENCGRAPHY TABLES / P-25-359/Y AGE, SEX, AND COLOR TO I990 $23-04-02358$ MENT PROGRAMS I VULNERABILITY I NREC-114III A LSTING OF TABLES AND PARAMET ERS USED IV YRES DAMAGE ASSE SS O5-D3-D2I 8 AIRS OF PERCENTAGES I STATISTICAL-ANALYSIS SOCIAL-SCIENCI TABLES FOR YJLES Q ASSJCIATIOV COEFFICIENTFOR P DS-II-U22JO MAGE RECLAMATION URBAN-AREAS MODELSISIMULATIONSI GRAPHICS TABLES MICHIGAN, \#USNRDL-TR-984 \$AD 635 250 /DA $15-06-0 I 933$ IE-OPERAT ING-PLANS POPULATION COMNAND-AND-CONTROL-SYSTEMS TABLES RADIATION-MEASUREMEVT-SYSTEMS, IIT ICAL PRESENTATION OF THE ORGANIZATION, TECHNOLOGY, AND TACTICS OF FIRE PRDTECTION AS EMPLOYED BY A CIVI I3-I?-O2264 IIIICAL PRESENTATION OF THE ORGANIZATION, TECHNOLOGY, AND TACTICS OF FIRE PROTECTIOV AS EMPLOYED BY A CIVI I3-I2-O2265 CHECULING ( RADIOACTIVE-FALLOUT, SUMMARY OF RADIOLOGICAL TARGET ANALYS IS PROCFDIIRFS FOR DECOVTAMINATION S DO-I3-O222O PLUS IUN I AIR-BUR ST YIELO WIND NUCLEAR-WARFARE-CASUALYIES TARGETS SCALEIRATIO), \#RM-4725-TAB /IROSHIMA EX 15-OS-OI958 INAL CUSTOMS JOURNAL - CANADA CAW INTERNATIONAL-AFFAIRS TARIFFS IMPORT-DUIIES FOREIGV-TRADE COMMERCE I I O5-03-DI64I

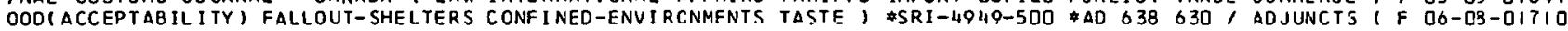
IONS IN COMBINATION WITH ADJUNCTS I FOOD FALLOUT-SHELTERS TASTE CIVIL-DEFENSE-SYSTEMS FRUITS COVDIMENTS SI D6-D3-O22I 1 IC PRESERVATION FIBERBOARD PHYSICAL-PROPERTIES CONTAINERS TASTE FALLOUT-SHELTERS, \$TR-67-25- 60 \#AD 640 8/ O6-03-02212

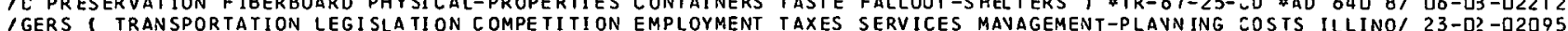
IPRAWL URBAN CONFIGURATION I URBAN-AREAS DECENTRALIZATION TAXES VULNERABILITY URBAN-PLAVN ING LAND-USE POPI 23-JS-OZIDG OM NUCLEAR EXPLOSIONS I SECONDARY-MISSILES BLA/ OPERATION TEAPOT - DISTRIBUTION ANC DEVSITY OF MISSILES FR IB-03-O23IB 1 A.L. IC82 THE OTHER MAR IN VIETNAM I TECHNICAL-ASSIST DVCE FOREIGN-POLICY ESONOMIC-AID DS-DH-OIGSI P' ANNUAL HISTORICAL SUMMARY - JULY-1,1965-JUNE-30,1966 I TECHNICAL-INFORMAT INN-CENTERS ABSTRACTS COSTS OE D5-DZ-D2125 /CUMENTAT ION STATE-OF-THE-ART-REVIEWS SYSTEMS-ENGINEERING TECHNICAL-INFORMAT ION-CENTERS DATA-PROCESSING-SI O5-02-DI63O ICCUGLAS AIRCRAFT COMPANY MECHANI ZED I NFORMA TION SYSTEM I TECHNICAL-INFORMAT ION-CENTERS DOCUMEVTATION INF' Q5-Q2 -D2I I 8 IRSONNEL SCIENTIF IC-PER SONNEL FLOH-CHARTING PSYT.HCNFTRITS TECHNICAL-INFORMAT ION-CENTERS JOB-A YAL YSIS STATI D5-D? -O21 20 $G$ DOCUM/ THE ANALYSIS AND DESIGN OF INFORNATICN SYSTEMS 1 TECHNICAL-INFORMAT ION-CENTERS SYSTEMS-ENG INEERIN DS-0? -02I 21 NGICOMPUTERS) PROGRAMMING-LANG UAGES EDUCATI/ RESEARCH AND TECHNOLOGY DIVISIOV REPORT 1 COMPUTERS PROGRAMMI D9-02-0I75D G(COMPUTERS) DATA-PROCESSING-SYSTEMS COMPUT/ RESEARCH AND TECHNOLOGY DIVISION REPORT FOR I966) (PROGRAMMIN D9-O2-0I75I ORT AT ION COSTS HIGH-SPEED-GROUND-TRANSPORT/ NEW TRANSPORT TECHNOLCGY FOR THE BIG CITY ( URBAV-AREAS TRANSP $23-02-02352$ 625/OQ4/OD \#AD/ A BIBLIOGRAPHY ON INFORMATION SCIENCE AND TECHNOLOGY FOR 1965 IV. 1 DOCUMEVTATION I \&TM-2 O5-0? -D2I22 FIC-RESEARCH I' A BIBLIOGRAPHY ON INFORMATION SCIENCE AND TECHNOLOGY FOR 1966 IV. 1 DOCUMEVTATION SCIENTI OS-02 -D2I23 / SERVICES - A CRITICAL PRESENTATION OF THE ORGANIZATION, TECHNOLOGY, AND TACTICS OF FIRE PROTECTION AS E/ I3-I2-U2264

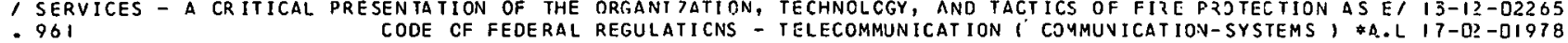

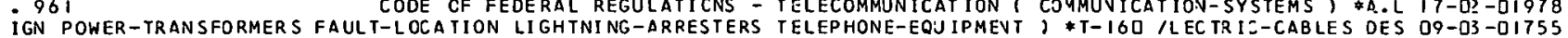

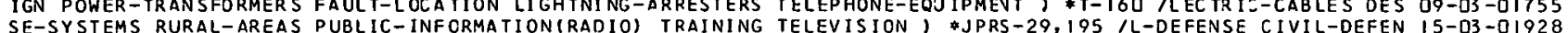
SE-SYSTEMS RURAL-AREAS PUBLIC-INFCRMATIONIRADIO) TRAINING TELEVISION \#PRS-29, 195 /L-DEFENSE CIVIL-DEFEN I5-03-OI928

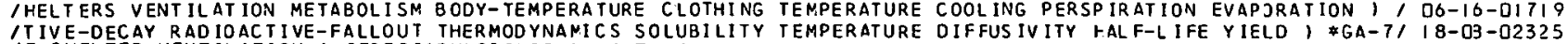
ITIVE-DECAY RAD IOACTIVE-FALLOUT THERMODYNAMICS SOLUBILITY TEMPERATURE DIFFUS IVITY FALF-L IFE YIELD I \#GA-7/ I8-03-02325
IF SHELTER VENTILATION I STRESS(PHYSI OLOGY) HEAT MCISTURE TEMPERATURE ENV IRONMENTAL-CONTROL HUMIDITY AIR I ISTRUCTURES BUILDINGS MODELSISIMULATIONS) HEAT-EXCHANGERS TENPERATURE HEAT-TRANSFER COOL ING-AVD-VENTILATI, I3-OI -O2253 IL-CONTROL EXPERIMENTAL-DATA HABI TABILITY INSTRUNENTATI ON TEMPERATURE PUNKAH-PUMPS PJRTABLE FAVS, \#GARO, I3-DI -DIT74 1 SHELTER-DCCUPANCY METABOLI SM PHYSIOLOGY HEAT-EXCHANGE TEMPERATURE VENT ILATION BODY-TEMPERATURE COOLIN/ O6-1S-OI7I 8 1 GAS-DÉTECTORS FIRE-DETEC TORS CARBON-MONOXIDE-INDICATORS TEMPERATURE WIND-PRLSSURE PRESSURE HEAT-TKANSMII I3-I2-OIBO? / CCUPANCY SYSTEMS-ENGINEERING SANI TARY-ENGINEERING OESIGN TENPERATURE-CONTROL ENVIRDNMEVTAL-COVTROL COSTS/ I3-13-OI8I9 IBELOW GROUND I FALLOUT-SHELTERS COMPUTERS BLAST-SHELTERS TEMPERATURE-CONTROL SOIL-4ECHAVICS COJLING-AND-I I 3 -DI -DI 776 INING SLEEP WATER FOOD RECREATION EXERCISE NOISE RELIGION TEMPERATURE-CONTROL VENTILATION, \$A.L. II3I /RA OS-IJ-O2IB4 INGIMECHANICS) US/ MECHANICAL PROPERTIES OF ROCKS AT HIGH TEMPERATURES ANO PRESSURES I ROCK-MECHANICS LOAD D8-O7-02242 TENSITY THERMAL RADIATION I CALORIMETERS IGNITION HIGH-T/ TENPERATURES ATTAIVEC IN WOOD EXPJSED TO HIGH IN I3-I?-QIBD2 S TEST-METHODS MODEL SISIMLLATIONS) TEST-EQUI PNENT, \#PSW TEMPERATURES IN A LARGE NATURAL FUEL FIRE I FIRE I3-1;-OIBI \begin{tabular}{l} 
/BR-7M) UNDER HYDROSTATIC PRE SSURE I STIFFENED-CYLINDERS IENSILE-PROPERTIES PLASTICITY STRUCTURAL-SHELLS/ $20-11$-O2347 \\
\hline
\end{tabular} 
FACTORS AFFECTING GOVERNMENT CONTROL IN SOUTH VIET/ LAND TENURE AND REBELLION - A STATISTICAL ANALYSIS OF O5-OZ-O2I75 OLOGY RADIAT ION-EFFECTS ECOLOGY RAD/ INTRODUCTICN TO LONG TERM BIOLOGICAL EFFECTS OF VUCLEAZ WAZ I RAOIOBI O6- IS-DI723 STUCIES I RADIATION-INJURIES RADIOLOSICAL-DOSAGE EXI LONG TERM RACIATION CAMAGE - EVALUATIOV JF LIFE SPAN O6-I3-OIT33 ION HIGH SPEED GROUND TRANSPORT I TRANSPCRTATION NETWORKS TERMINALS SCHEDULIVG SWITCHING PRJPULSION-SYSTE I 23-D?-O2354 R-SIGNALS MAPS ELECTROMAGNETIC-C, RAOAR MASKING BY EARTHS TERRAIN I SITE-SELECTION RADAR-INTERFERENCE RADA I7-OZ-DI9B 8 PHERE A/ ELECTROMAGNETIC PULSE PRCPAGATION IN A DISTURBED TERRESTRIAL WAVEGUIDE I NUCLEAR-EXPLOSIONS IONOS 20-I4-02348 OSIONS W/ ELECTROMAGNETIC PULSE PROPAGATION IN THE NORMAL IERRESTRIAL WAVEGJIDE ENVIROVMENT ( VUCLEAR-EXPL $20-1+$-02349 /ORPHIC TEST DUMMY I TEST-EQUIPMENT DESIGN SAFETY-CEVICES TEST-DUMMY(ANTHROPJMORPHIC) CJNSTZUCTION , *A.L, I3-1?-DIBT8 ISTING SHOULDER HARNESS AND SAFETY BELTS ( TEST-EQUIPMENT TEST-DUMMY (ANTHROPOMORPHIC) SAFETY-HARNESS SAFE/ I3-I? -DI8J3

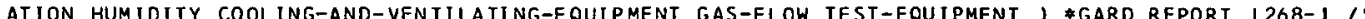
ATION HUMIDITY COOLING-AND-VENTILAIING-EOUIPMENT GAS-FLOW TEST-EOUIPMENT I \#GARD REPORT I268-1 /S 1 VENTIL I3-DI -D2254 ATURAL FUEL FIRE I FIRES TEST-METHODS MODELS (SIMULATIONS) TEST-ECUIPMENT, \#PSW-9O /PERATURES IV A LARGE N I3-I?-OIBIO

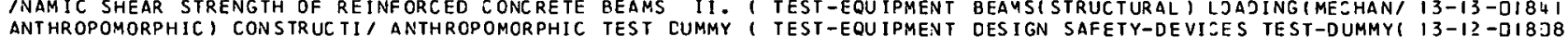
ANT HROPOMORPHIC) CONSTRUC TI ANTHROPOMORPHIC TEST CUMMY ( TEST-EQUIPMENT DESIGN SAFETY-DEVICES TEST-DUMMYI I3-I? -DI8D8
TRUMENTAT ION CALIBRATI/ A MECHANICAL SHOCK PULSE SURVEY I TEST-EQUIPMENT IMPACT-SHOCK SHOCK IMECHANICSI INS I4-DD-DI84 2 ISH DUMMY FOR TESTING SHOULDER HARNESS AND SAFETY BELTS I TEST-ECUIPMENT TEST -DUMMY (ANTHROPOMORPHIC) SAFE, I3-I?-OI8D3 TEMS ECOLOGY, AVD SYSTEMS ECOLOGISTS ( TRAINING CONPUTERS TEST-EQU IPMENT TEST-METHODS, \#ORVL-3957 IS, SYS D6-OS-OI7O8 OLOGICAL-BLAST-EFFECTS SHOCK(PATHCLOSY) EXPLOSION-EFFECTS TEST-METHODS, \#DASA-I777/HOCK 1 SHOCK-WAVES BI O6-2I-DI74I NC SYSTEMS ECOLOGISTS I TRAINING COMPUTERS TEST-EQUIPMENT TEST-METHODS ) \#ORVL-3957/S, SYSTEMS ECOLOGY, A 06-OS-DITJ8 IS NAVAL-OPERAT IONS DATA-STORAGE- SYSTEMS SUB JECT-INDEXING TEST-METHOOS INFORMATION-RETR IEVAL AUTOMATIC EC/ OS-OZ -O2I IO 1 \#PSW/ TEMPERATURES IN A LARGE NATURAL FUEL FIRE I FIRES TEST-METHODS MOCELS(SIMULATIOVS) TEST-EQUIPMENT I3-I?-OIBID IESSURES IN HOLLOW MODELS I SHOEK-hAVES EXPERINENTAL-DATA TEST-METHODS PRESSURE ATTEVUATORS I \&ARF DD87 * I I -03-D2DJ5 IESSURES IN HOLLOh MOOELS I SHOCK-WAVES EXPERINENTAL-DATA TEST-METHODS PRESSURE ATTENUATORS I *ARF MOGQ *I I8-O3 -D2ODI I ALBUQUERQUE I AERIAL-PHOTOGRAPHS URBAN-AREAS FIRES MAPS TEST-METHODS VULNERABILITY CLASSIFICATION BUILO/ I3-I2-OIBO ICIENCY I METABOL ISM DEFICIENCY-DISEASES TISSUES (BIOLOGY) IESTES RATS MORPHOLOGY (BIOLOGY) HISTOLOGY ESOPHI OG-DJ-OITOI

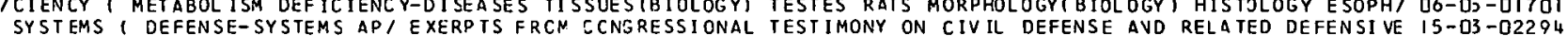
SHEAR IVG CUTTING BENDING TORSI ON STRETCHING COMPRESSING TESTING-EQUIPMENT MARBLE DOLOYITE LIMESTONE ROC/ OB-OT -O2242 IORS TEMPERATURE WIND-PRESSURE PRESSURE HEAT-TRANSNISSION TESTS (FIRES) SURVEYSIDATA-COLLECTION) I \$NRDL-T, I3-I?-DIBO? ENZA I D/ DISEASE STATUS REPORTS - DIPHTHERIA, PERTUSSIS, TETANUS, POLIOMYEL ITIS, SMALIPOX, MEASLES, INFIU IS-OZ -OIBG9 1 GY FUR SIMPLIFYING THE EVALUATION OF HARDENING CCSTS I. TEXT ANC APPENDIX A I INDUSTRIAL-EZUIPMENI BLAS/ OS-D3-D2I 22 I 5O( 1 ) CLASSIFICATION OF ROCKS ( ROCK (GEOLOGY) NINERALS TEXTURE PETROGRAPHY IGVEOUS-RJCK SEDIMENTARY-RO/ OB-OT -0224I DYNAM ICALLY LOADED RING WITH RADIAL ELASTIC SUPPORT / A THEORETICAL AND EXPERIMENTAL INVESTIGATION OF A $20-11$-D2336

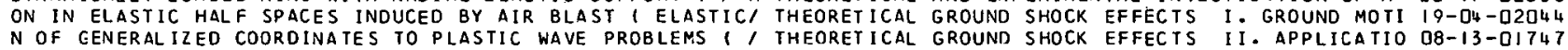
L AGENTS - USSR I RADIOL/ PATHOLOGY, CLINICAL PICTURE AND THERAPY IN AFFECTIONS WITH TOXIC AND RADIOLOGICA D6-I3-DI72I HATTAN AVD WILL IAMSBURG BRIDGES AND ALTERNATIVE SOLUTIONS THEREFORE ( URBAN-AREAS NEW-YJRK ) *A.L. II55 IN 23-0?-D2357 ECT IVE STRI COMPARISON OF DIGI TAL COMPUTER SIMULATIONS OF THERMAL ENVIRONMENT IN OCCUPIED UVDERGROUND PROT $20-13-02090$ ACE I THERMAL-RADIATION CALORIME TERS LABORATORY-FURNACESI THERMAL FLIX DISTR IBUTION IN AN AZC IMAGING FURN I3-OI -O2255 ESICENTIAL AREAS NUCLEAR-EXPLOSIONS FIRES AIRBURST STR/ THERMAL HAROENING CONSIDERATIONS PERTAINING TO R IS-OS -OI963 TO THE T/ EQUATIONS AND COMPUTER PROGRAM TO CALCULATE THE THERMAL HISTORY OF A DUAL LAYERED PLATE THERMAL PULSE OF A NUCLEAR WEAPON

I TEMPERATURES ATTAINED IN WOOD EXPOSED TC HIGH INTENSITY THERMAL RADIATION I CALORIMETERS IGVITION HIGH-T I3-1?-OIBO2
NC COATED FABRICS \$WT-407 OPPERATICN BUSTER - THERMAL RADIATION EFFECTS ON PAINTS, PLASTICS, A I3-I? -D2233 NC COATED FABRICS *WT-407 INITE DIFFERENCE TECHNIQUE I THERMAL-RADIATION WEAPONS-E/ RANSFER UNCERGROUND-STRUCTLRES SHELTERS THERMAL-DIFFUSI ON IOOLERS 1 SEMICONOUC TOR-DEVICES COOLING THERNCELECTRICITY /L SOIL-MECHANICS COOLING-AND-VENTILATING-EOUIPMENT TESTS INDIT IONS I HEAT-TRANSFER UNDERGROUNO-STRUCTURES SHELTERS D-VENT IL AT IVG-EQU IPMENT TESTS THE RMAL-CONDUC TI VI TY(SOILS) IERS GLUING PLYWOOD SANDWICH-CONSTRUC TION FI RE-RESISTANCE ES FIRESTORMS HAMBURG FIRE-FIGHTING-EQUI PNENT URBAN-AREAS AMM ING-LANGUAGES COMPUTERS CASUALTIES RADIOACTIVE-FALLOUT ICT IV ITY( DISTR IBUTION) RECOVERY JAPAN RADI OACTIVE-FALLOUT IONS EFFECTS I STRUCTURES FALLOUT-SHELTERS BLAST-SHELTERS IF A NUCLEAR WEAPON I CALCULATIONS PROGRAMMING (COMPUTERS) IN CURING WORLD WAR II - GERMANY - I. I FIRES FIRESTORMS
/ALS NUCLEAR-EXPLOSION-DAMAGE NUCLEAR-WARFARE SHOCK-WAVES

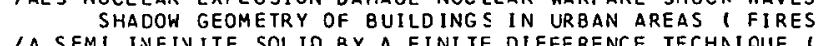
I A SEMI INFIVITE SOL ID BY A FINITE DIFFERENCE TECHAIQUE SMOKE/ FINAL RESULTS OF SMOKE SCREEN SYSIEN DEVELOFNENT 1 CHEMICAL-PROPERTIES CONSTRUCTION MECHANICAL-PROPERTIES
NE ROCK-SALT ANHYORITE SANDSTONES CLAY SILTSTCNES GEOLOGY (' PANELSISTRUCTURAL) RECTANGLLAR-BODIES STRESSES THRUST ENV IRONMENTAL-CONTROL HUMIOITY AIR TOLERANCES (PHYSIOLOGY) 1 PARTICLE MATRIX ( RADIOACTIVE-DECAY RADI OACTIVE-FALLOUT S I THERMOELECTRICITY BUOYS GENERATORS POWER-SU/ IOO WATT R-DEVICES COOL ING THERMOELEC TRICITY THERMA/ SEMICONOUCTOR IWATT THERMOELECTRIC POWER SYSTEM FOR BUOY APPLICATICNS I /CTRIC TRANSISTOR COOLERS ' SENICCNDUCTOR-DEVICES COOLING
STUDIES I POS/ KINSHIP AND VOLUNTARY ORGANIZATION IN POST STUDIES I POS/ KINSHIP AND VOLUNTARY ORGANIZATION IN POST
TICN 1 POSTATTACK-OPERATIONS SOC/ SOCIAL INSTITUTICNS ANO TICN 1 POSTAYTACK-OPERATIONS SOC/ SOCIAL INSTITUTICNS ANO
CTION I ECONOMICS RECOVERY POST/ECONOMIC VIABILITY AFTER CTION I VUCLEAR-WARFARE SLRVIVAI ECONOMIC VIABILITY AFTER I ASSESSMENT OF ITS SIGNIFICANCE AS A PROBLEM FOLLCWING A RUCTURAL DYNAM ICS SHEAR-STR/ DYNAMIC SHEAR RESISTANCE OF IE HAVE TO FEAR IS FEAR I TSELF - hHAT THE PEOPLE KNOW AND - HRULESSING UAMAGE-ASSESSMENT, *NREC-24(I) ANNEX TO ILING PROCEDURES APPLICABLE IN ASSESSING RESPCNSES OF THE I CTED PROBLEMS OF LARGE SHELTER MANAGEMENT, ENVIRONMENTAL ICTED PROBLEMS OF LARGE SHEL TER MANAGEMENT, ENVI RONMENTAL
S EVACUAT ION VULVERABIL I TY WARNING-SYSTEMS HASTY-SHELTERS EUROPE SURVIVAL RECOVERY VULNERABILI TY NILITARY-STRATEGY /-STRATEGY MANAGEMEN T-PLANNING OPERATI ONS-RESEARCH MODELS
/EMAT ICAL-PREDICT ION) MILITARY-STRATEGY DAMAGE-ASSESSMENT ISS ILE-DEFENSE-SY STEMS UNI TED- STA TES USSR COMNUNI ST-CHINA IVIVAL DAMAGE-L IMITING-SYSTEMS DANAGE-ASSESSMEAT RECOVERY / SE-SYSTEMS ATTITUDES FALLOUT-SHELITERS STRESS (PSYCHOLOGY) / INTERNATIONAL-AFFAIRS POL I IICAL-SCIENCE FOREIGN-POLICY
$/$ PLATES ( PANELSISTRUCTURAL) REC TANGULAR-BODIES STRESSES THREAT - PERCEPT ION SUEZ-CR IS IS ATTITUDES MIDDLE-1 05-04-02170 ECOVERY CALCULATIONS RADIOACTI VE-FALLOUT NANPOWER-STUDIES TIME) "USNRDL-TRC-42/TOR RADIOLJGIZAL-DOSAGE R D6-13-0I729 EGYPT, ISRAEL, AND THE UNITED NATIONS AT FIVE POINTS IN TIME I INTERNATIONAL-AFFAIRS POLITICAL-SCIENCE, OS-DI-D2I7D SHEL TER PROGRAM I URBAN-AREAS HASTY-SHELTERS DESIGN MAT/ TIME COMPRESSION POTENTIAL OF AN EMERGENCY BLAST I 3 -I3-D22 2 
(STRUCTURAL) CONSTRUCTION-MATERIALS LOAD/ EFFECTS CN LONG TINE LOADS ON PRESTRESSED CONCRETE BEAMS I BEAMS I3-I3-U2289 ASSES SMEVT HEAPOY S-EFFECTS, \$NREC-25, TIME PHASED ACCESS IBIL. ITY OF 2 ESOURCES ( DAMAGE- OS-03-02I56 -WARFARE-CASUAL TIES DAMAGE-ASSESSNENT PATIENTS PR/ DAPJ 46 TIME PHASED CASUALTY ESTIYATE I AVALYSIS NUCLEAR D6-2I -D22? 5 ILOGY OF 2 INC DEF ICIENCY I METABOLISM DEFICIENCY-DISEASES TISSUES (BIOLOGY) TESTES RATS MORPHOLOGY(BIOLOGYI DO-OJ-DITSI NTERNAT IONAL-AFFAIRS FEAR SURVI UNESCO AND PUBLIC CPINION TODAY 1 UNITED-STATES QUESTIOVNAIRES ATTITUDES I O5- II-DIOB9 PSYCHOLOGY STRESS (PHYSIOLOGY) FOOD WATER-SUPPLIES BEDDING TOILET-FACILITIES, *GARD- I2921 I) /TION-MANUALS I5-O3-023OD PSYCHOLOGY STRESS (PHYSIOLOGY) WA TER-SUPPLIES FCOD EEODING TOILET-FACILIIIES, *GARO-I2921 III) ION-MANUALS I5-D3-D2298 IE SECOND WORLD CONFERENCE ON EAR THQUAKE ENGINEERING II. TOKYO AND KYOTO, JAPAN I JAPAV SEISMOLOGY STRUC, OB-II -D2246 RESSURES IN A SHOCK TUBE ( BIOLOGICAL-BLAST-EFFECTS , THE TOLERANCE OF CATTLE TO LONG DURATIOV REFLECTED P DG-2I-D2237 ICAL CL IMATE I AGING(PHYSIOLOGY) AGE TROPICAL-REGICI HEAT TOLERANCE OF ELDERLY PERSJNS LIVIVG IV A SUB TROP O6-I9-DI734 $Y$ SHOCK-WAVES B IOLOGI/ THE EFFECTS OF AMBIENT PRESSURE CN TOLERANCE OF MAMMALS TO AIR BLAST 1 BLAST-BIOLOG O6-21 -D2236 -BIOLOGY BIOLOGICAL-BLAST-EFFECTS SHOCK-TUBES LUNG-DAMAGE TOLERANCES(PHYSIOLOGY) 1 \#DASA-1854 /ALS 1 BLAST D6-21 -02230 YSIOLOGY) A' SUPPLEMENT TO SHOCK ANO VIBRATION BULLETIN I TOLERANCES(PHYSIOLOGY) MOTIOV-SICKNESS STRESS(PH O6-17-DI735

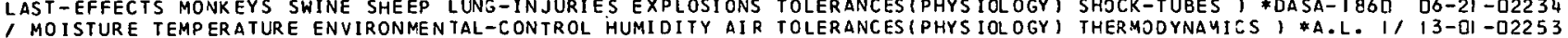

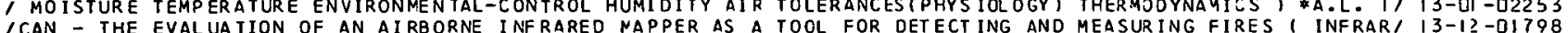
ICAN - THE EVAL UA IION OF AN AIRBORNE INFRARED NAPPER AS A TOOL FOR OETECT ING AND MEASURING FIRES ER-MEDICINE NUCLEAR-WARFARE-CA SUALIIES FIRST-AIDI CURRENI TOPICS IN SOVIET MILIIARY MEDICINE I USSR DISAST O6-DS-DI7O3 ER-MEDICINE NUCLEAR-WARFARE-CA SUALTIES FIRST-AIDI CURRENI TOPICS IN SOVIET MILITARY MEDICINE I USSR DISAST O6-D5-OI7O3
ITIONS ON A HOSPITAL RESPONSE TO THE JACKSON, MISSISSIPPI TORNADO OF MARCH 3 , I966 I DISASTER-MEDICINE MAI D6-I?-DI7I (CING(MECHANICS) USSR EXCAVATION SHEARING CUTTING EENDING TORS ION STRETCHING COMPRESSING TESTIVG-EQUIPMEN/ QR-QT -D2242 /THOLOGY, CLINICAL PICTURE AND THERAPY IN AFFECTIOAS WITH TOXIC AND RADIOLOGICAL AGEVTS - USSR I RADIOLOG/ D6-I3-OI7ZI /BIVALENT AB BOTUL INUM TOXOID (BIOLOGICAL-WARFARE-AGENTS TOXIC-TOLERANCES TOXINS-AND-AVTITJXINS, \#AO G3, I5-02-DI857 ICAL PROPERTIES OF PARALYTIC SHELLFISH POISCNS RELATED TO TOXICITY I PELECYPJDA TOXIVS-AND-AVTITOXINS BIO/ IS-0?-OIBG3 /RUCT ION BLAST RADIOACTIVE-FALLOUI HEAT-TRANSFER EXPOSURE TOXICITY CONSTRUCTION-MATERIALS I *IITRI-NGOOI I I3-1? -O2269 IOLOG ICAL-WAR FARE-AGENTS CONTAMINATION RADIATICN-INJURIES TOXICITY CYANO-ACIDS CARBOV-MJNOXIDE VESICANTS, O6-I9-DI72I /CLEAR-EXPLOSIONS FIRES CASUALTIES SIMULATION FIRE-SAFETY TOXICITY HEAT CARBON-MONOXIDE CARBOV-DIOXIDE AI / I3-I?-DIBDD TUL INUM TOXINS- 1 PROCEEDINGS OF A CONFERENCE CN BOTULINUM TOXIN I BIOLOGICAL-WARFARE-AGENTS CLOSTRIDIUM-BO I5-OD-O186I ICAL RESPONSE OF MAN TO PURIFIEO, STUDIES ON IMMUNITY TO TOXINS OF CLOSTRIDIUM BOTULINUM VIII. IMMUNOLOG IS-D?-DI857 ICAL RESPONSE OF MAN TO PURIFIED / STUDIES ON IMMUNITY TO TOXINS OF CLOSTRIDIUM BOTULINUM VIII. IMMUNOLOG IS-O2 -OI857 LINUM TOXOID I BIOLOGICAL-WARFARE-AGENTS TOXIC-TOLERANCES TOXINS-AND-ANTITOXINS 1 \$AD 636854 /ENT AB 30 TU 15 -02-OI857

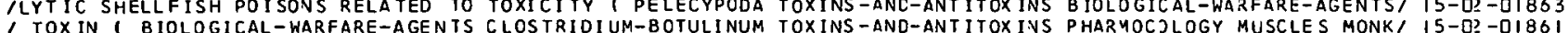
IOGICAL RESPONSE OF MAN TO PUR IF IED BIVALENT AB BOTULINUM TOXOID I BIOLOGICAL-WARFARE-AGENTS TOXIC-TOLERAI IS-0?-DI857 LTY COMMISSION, JAPAN NUCLEAR-WARFARE-CASUALTIES, FABCC TR J-66/BLISHED PAPERS OF THE ATJMIC BOMB CASUA DS-2I-D2224 TRUCT ION REAL-ESTATE REFUGEES BANKING INSLRANCE LABOR LAW TRAFFIC, \$IDA HQ 65-4I42,IVG-PLAVS 2 EPAIR CONS O5-D3-O1636 STRUCTIOV URBAV-AREAS TUNNELS (TRANSPORTATI CN) VENTILATION TRAFFIC, \#ORNL-TR-I596 /URG 1 GEZMANY ROADS CON I 3 -02 - I I 76 IVACUATION URBAN-AREAS DAMAGE-LIMITING-SYSTEMS DEMCGRAPHY TRAFFIC ANTIMISSILE-DEFENSE-SYSTEMS UNITED-STATI I 5 -D3-QI922 1 RANGE MASS TRANSPORTATION 1 URBAN-AREAS MOVING-BELTWAYS TRAFFIC DES IGN COSTS NEW-YORK CHICAGJ WASHINGTO/23-02-02097 /TURAL-PROPERTIES STEEL REINFORCEO-CONCRETE SHELTER-DOORS TRAFFIC STRUCTURAL-SHELLS, \$46064(3) \$AD 636 O/ I3-I3-01835 IT SPONSORED AND PRIVATE RESEARCH ON FOREIGN STUDEATS AND TRAINEES IN THE U.S. AVD IV OTHER LOUVTRIES I C 1 Q5-II -QIG $I$ N LOGISTICS CIVIL-DEFENSE-OPERATI NG-PLANS CLEANING REPAIR IRAINING) *FG-E-13.5 /CY CLOTHING I OISTRIBUTIO I5-0弓-O23J6 FENSE-OPERATING-PLANS MENTAL-HEALTH POSTATTACK-OPERATIONS TRAINING CHILDREN GERIATRICS I \#FG-E- 3.2 IIL-DE O5-II-D2I85 K-OPERAT IONS SHEL TER-OCCUPAN/ EMERGENCY LODGING I HOUSING TRAINING CIVIL-CEFENSE-OPERATIVG-OLAVS POSTATTAC Q5-II-O2I 87 */ ECOSYSTEYS, SYSTEMS ECOLOGY, AND SYSTEMS ECOLOGISTS 1 TRAINING COMPUTERS TEST-EQUIPYENT TEST-METHODS I D6-0S-OI7OB IOURCES (SURVIVAL) PROGRAMMING (COMPUTERS) REPAIR PERSCNNEL TRAINING COUNTERMEASURES) \#USNRDL-TR-656 \$AD 4 , O5-03 -0165 I IIL ITARY TRANSLATIONS NUMBER 188 - CIVIL DEFENSE I RUSSIA TRAINING OISPERSAL CIVIL-OFFEVSE-PERSONNELITRAI I $5-03-019$ I 3 IE-SERVICES OCCUPATIONS PROFESSIONS COMMUNICATION-SYSTEMS TRAINING ECONOMICS INTERNATIOVAL-AFFAIRS POLITI/ O5- 11 -O2I 44 ISLAT IONS NUMBER $74 I$ I USSR CIVIL-DEFENSE-OPERATING-PLANS IRAINING EDUCAT ION INTERNAT IOVAL-CIVIL-DEFENSE I S-O3-DIOI? IAREAS RURAL-AREAS MANAGEMENT-PLANN NG COST-EFFECT IVENESS TRAINING FIRE-STORMS CONFLAGRATIOVS RE SC UES VUL, I3-I?-DI8J6 IINE ADMINISTRATION-AND-MANAGENENT FIRST-AID ORGANIZATION TRAINING HOSPITALS CASUALTIES PER SOVVEL NUCLEARI OS-2I -OIT38 R-MANAGEMENT MANAGEMENT-PLANNING PERSONNEL SHELTER-I SOME TRAINING IMPLICATIONS OF LARGE SHEL TERS 1 SHELTE DS-DI -DIG2I TERS LOGISTICS SHEL TER-OCCUPANCY WATER-SUPPLIES EDLCATION TRAINING INFANT-CIET, \$OCD-1G-15.1 \$ARC-22I9 /L D6-08-DI7I3 / CIV IL-DEFENSE-PER SONNEL LSSR INTERNATI ONAL-CIVI L-CEFENSE TRAINING MANJALS VJCLEAR-EXPL) SIOV-DAMAGE NUCLE/ I3-I?-DIBO4 IERS I PROGRAMMING-LANGUAGES EDUCA TION OPERATIONS-RESEARCH TRAINING MATHEMATICS DATA-PROCESSIVG-SYSTEMS I I DQ-OZ-QI75O ICUPANCY EXERCI IES I HABITABILITY DATA-PROCESSING-SYSTEMS TRAINING METHODOLOGY QUESTIOVVAIRES SHELTER-MAN/ OS-1J-OIGBO D 1 USS' SOVIET MILI TARY TRANSLATIONS NUMBER 384 - COMBAT TRAINING METHODS AND THEIR SHORTCOMINGS DESCRIBE O5-OP-D2I76 15 SIMULATION INFORMATION-RE TR IE VAL GAME-THEORY EOUCATION TRAINING OPERAT IONS-RESEARCH PROGRAMMING-LANGUA/ D9-D2 -DI75I IESS(PSYCHOLOGY) CASUALTIES MOIIVATION PUBLIC-INFORMATION TRAINING REACT ION(PSYCHOLOGY) ADMIVISTRATION-AN/ O5-I]-OIG8I 11 USSR CIVIL-DEFEN SE-SYSTEMS INTERNATIONAL-CIVIL-CEFENSE TRAINING RESCUES PUBLIC-IVFORYAIION 2 EPAIR FILMI I5-D3-O23 2 IED GUIDANCE FOR SHEL IER MANAGEMENT TONA ITRIT ION SANITARY-ENG INEER ING MED ICAL-SUPPLIES DEFECTIONS TRAINING SLEEP WATER FOOD RECREATION EXERCISE N/ D5-OI -OI626 TTRITION SANITARY-ENGINEERING MEDICAL-SUPPLIES DEFECTIONS TRAINING SLEEP WATER FOOD RECREATION EXERCISE N/ O5-IJ-O2I84

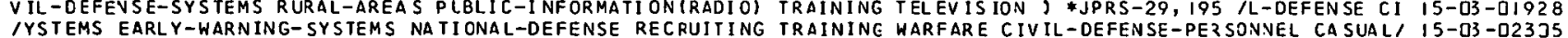
IYSTEMS EARLY-WARNING-SYSTEMS NA TI ONAL-DEFENSE RECRUIIING TRAININE WARFARE CIVIL-DEFENSE-PERSONNEL CA SUAL/ I5-D3-D2335 IET IN I INSTRUMENTATION ACCELEROME TERS SPECTRUM-ANALYZERS TRANS DUCERS MATHEMATICAL-AVALYSIS STRUCTURES OY $20-11$-D2075
ITS NEAR EXPLOSIONS I WAVEFORM-GENERATORS INSTRUMENTATION TRANSDUCERS OSCILLOSCOPES CAMERAS REFLECTION RE, OB-1I-D22 47

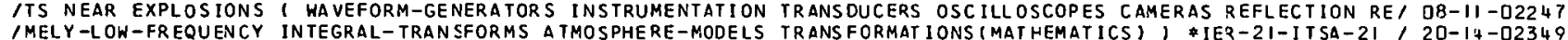

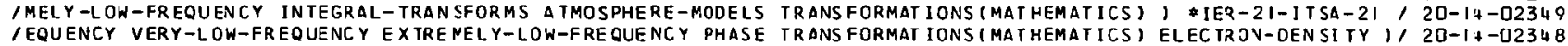
IOLUM INESCENCE CELLS I LIGHTING-E CUI PMENT CONTROL-SYSTEMS TRANSFORMERS COILS CIRCUITS USSR PATEVTS I \&FTD, D9-DJ-OI756 DISTR IBUT ION-SYSTEMS COSTS FAULT-LOCATION ELECTRIC-CABLES TRANSFORMERS HEAT-TRANSFER, \$3I-C-35/IDENTIAL- Q9-D3-DI753 N-SYSTEMS UNDER GR / UNDERGROUND RE SIDENTIAL DISTRIBLTION, IRANSFORMERS UNDERGROUND-RESIOENTIAL-DISTRIBUTIO O9-O3-OI754 ING 1 FOUNCATIONSISTRUCTURES) SOIL-MECHANICS LCADING(MEC/ TRANS IENT LOADINE TESTS OVA PIGID CIRCULAR FOOT I3-I3-OIBI 2 OMMUTATORSI A DIRECT CURRENT MOTOR WITH NC CONTACTS ANO A TRANSISTOR COMMUTATOR I ELECTRIC- YOTORS RUSSIA C OQ-O3-OI7S2 NG THERMOELECTR ICITY THERMA/ SEMICONDUCTOR THERMOELECTRIC TRANSISTOR COOLERS- 1 SEMICONDUC TOR-DEVICES COOLI O9-DI -OI748 TELECTRIC-MOTORS RUSSIA COMMUTATORS SEMICONDUCTOR-DEVICES TRANS ISTORS CONTROL-SYSTEMS I *FTD-TT-65-2035 * I O9-03-QI752 F THE MAYOR OF THE CITY OF NEW YORK AND FOR NEK YORK CITY TRANSIT AUTHORITY I URBAN-AREAS I *A.L. IIS6 / O 23-O2-O2355 TAT ION URBAN-AREAS RAILROADS MONORAILS MO/ INNCVATIONS IN TRANSIT DEVICES I RAP ID-TRAVSIT-SYSTEMS TRANSPOR 23 -0? -O2356 EAS POPULATION RA ILROADS SUBWAYS COSTS MANAGEMENT - P/ RAIL TRANS IT PLANS I TRAVSPJRTATIOV NEW-YDRK URBAN-AR 23 -02 - D2099 ISE III SUESURFACE INVESTIGATION - NATIONAL CAPITAL RAPID TRANSIT SYSTEM PORTION OF CONVECTICUT AVENUE ROA I3-C2 -DI785
-BLAST-EFFECTS EXPER IMENTAL-DA TA THEORY NAI BLAST INDUCED TRANSLATIONAL EFFECTS I BLAST-BIOLJGY BIOLOGICAL OG-21 -O2239 - BLAST-EFFECTS EXPER IMENTAL-DA TA THEORY NAI BLAST INDUCED TRANSLATIONAL EFFECTS I BLAST-B IOLJGY BIOLOGICAL O6-21 -D2239
TEMS INTERNAT IONAL-CIVIL-DEFEN SE SHELTER/ SOVIET MILITARY TRANSLAT IONS NUMBER I7O USSR CIVIL-DEFENSE-SYS I5-D3-OI937 IL-DEFENSE CIVIL-DEFENSE-SYSTEMS RURAL-A/ SOVIET MILITARY TRANSLATIONS NUMBER IT8 1 USSR INTERNATIONAL-CIV I5-03-OIS28 TRAINING DISPERSAL CIVIL-DEFENSE-PERSON/ SOVIET MILITARY TRANSLATIONS NUMEER IB8 - CIVIL DEFEVSE I RUSSIA I5-O3-OIQI 3 RAT INE-PLANS TRAINING EDUCATION INTERNAT/ SOVIET NILITARY TRANSLATIONS NUMBER 341 ISSP CIVIL-DEFENSE-OPE I5-D3-OI9I7 RY OF THE BATTLE OF MOSCOh I USSR HISTOR/ SOVIET MILITARY TRANSLAT IONS NUMBER 380 - TWEVTY-FIFTH ANNIVERSA OS-O+ -02। 73 IMISS ILE-DEFENSE-SYSTEMS MORALE FIRE-FIG / SOVIET MILITARY TRANSLATIONS NUMBER 382 ( USSR UNITED-STATES ANT I5-07-023D9 5 AND THEIR SHORTCOMINGS CESCR IBED 1 USS/ SOVIET MILITARY TRANSLATIONS NUMEER 384 - COMBAT TRAINING METHOD D5-O9-D2I 76 TEMS INTERNAT IONAL-CIVIL-DEFENSE IRAININ/ SOVIET NILITARY TRANSLATIONS NUMEER 3851 USS? CIVIL-DEFENSE-SYS I5-O3-O2302 ATIONS ARMEO-FORCESIFOREIGN) WARFARE MOR/ SOVIET MILITARY TRANSLAT IONS NUMBER 386 I USS? ARYED-FORCES-OPER I5-D7-D23IO AT TRIC-POWER ELECTR IC-POWER-TRANSMISSI ON/ UNDERGROUNO POWER TRANSMISSION 1 ELECTRIC-UTILITIES UTILITIES ELEC D9-03-02249
IUT IL ITY INOUSTRY IN 1905 UTILI IIES RE SERVES PROCUCTION TRANSMISSION DISTRIBUTION UNDERGRJUND-STORAGE S/ D5-D3-DI642

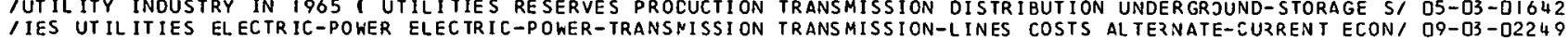

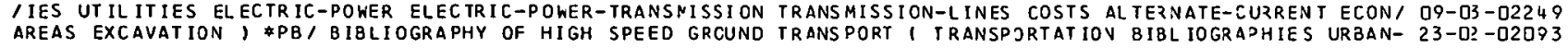


HEDUL ING/ SUMMARY OF RESEARCH AT NIT ON HIGH SPEED GROUND IRANSPORT I TRANSPORTATION NETWORKS TERMINALS SC 23 -O? -O2354 EAS TRANSPORTATION COSTS HIGH-SPEED-GROUND-TRANSPORT/ NEW TRANSPORT TECHNOLOGY FOR THE $8 I G$ CITY 1 URBAN-AR 23 -D2-D2352 /PERIMENT AL PROGRAM IN ATMOSPHERIC DIFFUSION 1 ATNCSPHERE TRANSPORT-PROPERTIES EXPERIMEVTAL-DATA ATMOSPHE I D4-D?-D2II 4 AC 646 I WATER TRANSFER THROUGH HLMAN SKIN I PERNEABILITY TRANSPORT-PROPERTIES SORPTION SALTS HUMIDIYY I * DO-IS - O22I3 TURE CONSTRUCTIOV MANPOWER INDLSTRIAL-PRODUCTION CCMMERCE TRANSPORTATION, *VREC-IS/ITALS HOUSING AGRICUL O5-O3-O2ISI MODELS NUCLEAR-WARFARE PETROLEUM-PIPELINES GAS-PIPELINES TRANSPORTATION, *VREC-28/Y 1 DAMAGE-ASSESSMENT D5-O3-O2ILI RRICOR COSTS URBAN- I SYSTEMS ANALYSIS OF SHORT HAUL AIR TRANSPORTATION I PASSENGER-VEHICLES VORTHEAST-CO $23-02$-O2O94 FFI/ MOVINE SIDEWALKS AND THE PROBLEM OF SHORT RANGE MASS TRANSPORTATION 1 JRBAN-AREAS MOVIVG-BELTWAYS TRA $23-0$ ? -O2O 7 IH, SIIE AND COST OF MUL TIPURPOSE BUILDINGS FCR A FEDERAL TRANSPORIATION ACYIVISTRATIOV 1 SHELIERS DUAL-P/ I3-I3-DI827 I ON TRAVSFER SYSTEM AND FARE STRLCTURE FCR THE OFFICE OF TRANSPORTATION ACMINISTRATION, OFFICE OF THE MAI 23 -O2-D2355 UAL OF DESIGN CRITERIA I URRAN-AREAS, , NATICNAL CAPITAL TRANSPORTATION AGENCY RAPID TRANSIT SYSTEM - MAN $23-02-02353$ UAL OF DESIGN CRITERIA I URRAN-AREAS, MODELS (SIMULATIONS) IRANSPORTAIION AIR-TRAVSPJRTAT ION BUS-TRANSPORTA 23-02 - D2O9I

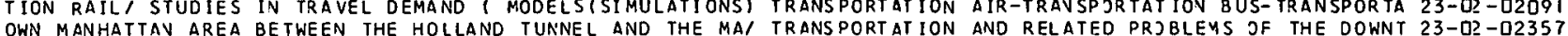

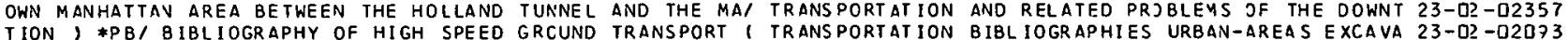

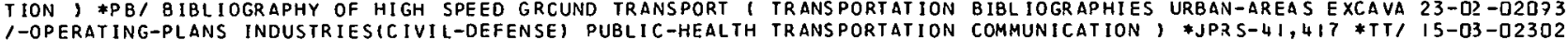

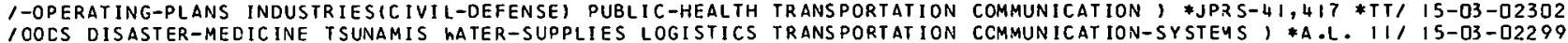
ICELS MAVUFACTURING MINING AGRICULTURE CONMERCE UTILITIES TRANSPORTATION COYMUNICATIONS BAVKIVG CONSTRUET/ O5-D3-O2IS3 I NEW TRANSPORT TECHNOLOGY FOR THE BIG CITY I URBAN-AREAS IRANSPORTAT ION COSTS HIGH-SPEED-GRJUND-TRANSPORT $23-02-D 2352$ IRANSPORT MATHEMA TICAL-ANALYSIS CCNSOLIDATI ON DEFORMATION IRANS PORTATION ELASTICITY NORTHEAST-CORRIDOR CO/ 2O-II -O2O84 IT INTERVATIONAL-CIVIL-DEFENSE CONNUNI ST-CHI NA INDUSTRIES TRANSPORTAT ION EVACUATION DISPERSAL URBAN-AREASI I5-O3-D2295 INEW JERSEY, AS A CIVIL DEFENSE PLBLIC SHELTER FACILITY I TRANSPORTATION FEASIBIL ITY-STUDIES DUAL-PURPOSEI I3-I3-O227I IEM ANALYSIS OF THE EFFECTS OF NUCLEAR ATIACK CN RAILROAD TRANSPORTATION IN THE CONTINEVTAL UNITEO STATESI O5-03-O2I28 I HNICAL LITERATURE I GUIDED-MISSILES ABSTRACTS SPACECRAFT TRANSPORTATION LAJNCHIVG-SITES METEORDLOGY RAILI IG-OI -OIQ73 T TAXES SERVICES, SELECTED IMPACTS OF RAILROAD MERGERS I TRANSPORTATION LEGISLATIOV COYPETITIOV EMPLOYMEN 23 -D? -D2D95 IMARY OF RESEARCH AT MIT ON HIGH SPEEO GRCUND TRANSPORT ! TRANSPORTATION NET NORKS TERMIVALS SCHEOUL ING SW/ $23-02-02354$
AILROADS SUBWAYS COSTS MANAGEMENT-PI RAIL TRANSIT PLANS

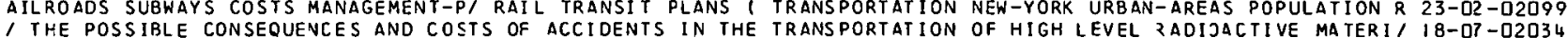

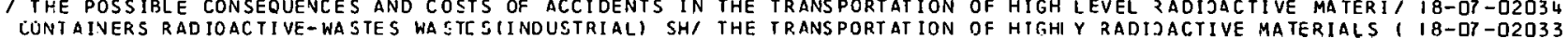

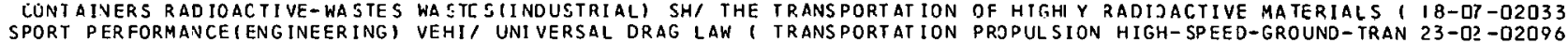
SPORT PER FORMANCEIENG INEERING I VEHI/ UNI VERSAL DRAG LAW I TRANSPORTATION PROPULSION HIGH-SPEED-GROUND-TRAN $23-02-02096$
ROUND-TRANSPORT RESEARCH-PROGRAM-ADMIN/ KEYSTCNE CCRRIDOR TRANSPORTATION STUDY I PENNSYLVANIA HIGH-SPEED-G $23-02-02092$ IAVENUE ROUTE BETWEEN COLUMBIA ROAD AND VAN NESS STREET I TRANSPORTATION SUBAAYS IESTS BORIVG WASHINGTON I I3-0?-QIT85 ISONS AMJNG MODES II EFFECTS OF NLCLEAR ATTACK CN FREIGHT TRANSPORTATION SYSTEMS- IVTEZACTIJVS AND COMPAR I3-DS-D22SO RBAN-PLAVN ING WASHING TON BOSTON NI HIGH SPEED RAILROADS I TRANSPORTATION URBAV-AREAS MAVAGEMENT-PLANNING U 23 -O2 - D2I DO IVEHICLES IN ENCLOSED GUIDEWAYS I TUNNELS (TRANSPORTATION) TRANSPORTATION URBAN-AREAS MODELSISIMULATIONS), $23-02$-D2I OI O/ INNOVATIONS IV TRANSIT DEVICES I RAPID-TRANSIT-SYSTENS TRANSPORTATION URBAN-AREAS RAILROADS MONORAILS M 23 -O?-O2356 IN TAXES VUL VERAB IL ITY URBAN-PLANNING LAND-USE POPULATICN TRANSPORTAT ION UTIL ITIES FISCAL-AYD-MONETARY ECI $23-05$-O2I DG IRS UNDERGROUND-STRUC TURES COLORADO DUAL PURPOSE SHELTERS TRANSPORTATION UTILITIES URBAV PLANNING UTILITY 23 -DS -O2I DO IRS ATION RAILROADS ARMED/ THE SHOCK AND VIBRATION BULLETIN I TRANSPORTATION VIBRATION-ISOLATORS AIR-TRANSPORT 20-II -O2OT6
CUPPL IES, GOVERNMENT-FACILITIES COMMUNICATIONS-FACILIIIES TRANSPORTATION-PROPERTIES ELECTRIC-PJWER-GENERA/ OS-O3-O2I37 IUPPL IES, GOVERNMENT-FACILITIES COMMUNICAIIONS-FACILITIES TRANSPORTATION-PROPERTIES ELECTRIC-PJWER-GENERA/ DS-03-O2I 37 7RLC WIOE RESOURCES GEOOETIC POSI TION ( GP, TO UNIVERSAL TRANSVERSE MERCATOR 1 UTM, AVD IVVEZSE 1 MODEL/ O5-O3-D2I33 VES ELASTICITY PROPAGATION STRAINIMEI ON LONGITUDINAL AND TRANSVERSE WAVES IN ELASTIC RODS I MECHANICAL-WA $20-I I-O 2 O B 2$ ON AIR-TRANSPORTATION BUS-TRAN SPORTATION RAILI STUDIES IN TRAVEL DEMANO 1 MODELSISIMULAT IONSI TRANSPORTATI $23-0 ?$ - O2O9I BON-D IOXIDE AIR SMOKES WIND SHELTERS SERMANY MICE, \#NRDL TRC-63/5AFETY TOXICITY HEAT CARBJV-MONOXIDE ZAR I3-I?-OIBOO A.L. $1157(4)$

A.L. $1157(2)$

A.L. $1157(3)$ CCNSTRUCTICN COST TRENDS
CONSTRUCTICN CONITED-STATES BUREAU-OF-RECLAMATION ; $13-13-02270$

CCNSTRUC TICN COST TRENDS I UNITED-STATES BUREAU-OF-RECLAMATION * * 13-13-02285 CONSTRUETICN COST TRENDS ( UNITED-STATES BUREAU-OF-RECLAMATION I * I3- 13 -D2288 G ENV IRONMENT EDUCA ILIVING IN A SUB TROPICAL CLIMATE I ASING (PHYSIOLCGYI AGE TROPICAL-REGIONS MILITARY-PERSONVEL FOOD CLOTHIN O5-IJ-O2I II OD IVINTING CARGO-VEHICLES AIRCRAFT WEAPONS-EFFECTS DAMAGE-ASSESSMENT TRUCKS, *SRI-MU-4949-150,

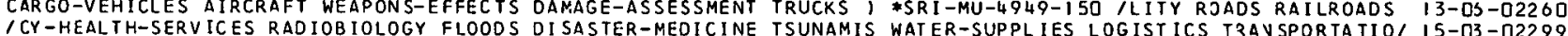
ICY - HEALTH-SERVICES RADIOBIOLOGY FLOODS DISASTER-MEDICINE TSUNAMIS WATER-SUPPL IES LOGIST ICS TRAVSPORTATIOI IS-03-O2299
IF CATTLE TO LONG OURATION REFLEC TED PRESSURES IN A SHOCK TUEE ( RIOLOGICAL-BLAST-EFFECTS BLAST-8IOLOGY S/ OG-2I-O2237 IF CATTLE TO LONG OURATION REFLEC TED PRESSURES IN A SHOCK TUEE EIOLOGICAL-BLAST-EFFECTS BLAST-BIOLOGY S/ O6-2I-O2237 GES I OORNLTR-I/ THE PROPAGATION OF AIR SHOCK WAVES IN A TUBE I SHOCK-TUBES EXPERIMENTAL-DATA PRESSURE-GA $20-0+$-D2O53
OVERY INFECTIOUS-DISEASES VACCINES RADIOB/ THE PROBLEM OF TUBERCULOSIS IN THE POSTATTACK ENVIROVMENT / REC DO-DJ-O22OG SHOCK TUBES - A BIBL IOGRAPHY QMLM-II8? $20-04-02052$ IND STRUCTURES - OPERATION OF SIX-FOOT AND TWO-FOOT SHOCK TUBES - EXPERIMENTAL OBSERVATIONS JF INTERIOR PI I8-O3-O2OJI IND STRUCTURES - OPERATION OF SI X-FOOT AND TWO-FOOT SHOCK TUEES - EXPERIMENTAL OBSERVATIONS JF INTERIOR PI IB-D3-O2OJS ICRATERING EXPLOSION-EFFECTS NUCLEAR-EXPLOSI ONS EQUATIONS TUFF ALLUVIUM SALT GRAVITE MATHEMATICAL-ANALYSI/ I8-D3-D23 I 5 IF AEROSOL AGE ON THE INFECTIVITY OF AIRBORNE PASTEURELLA TULARENS IS FOR MACACA MULATTA AND MAV I BIOLOGI/ I5-O2 -OI858 MOT ION-SICKVESS STRESS(PHYSIOLOGY) ADAPIATION(PHYSIOLOGY) TUMBLING ACCELERATION-TOLERAVCE I \#AD 94 G97 /) O6-17-QI735 /BLEMS OF THE DOWNTOWN MANHATTAN AREA BETWEEN THE HOLLANO TUNNEL ANO THE MAVHATTAN AVD WILLIAMSBURG BRIDG/ $23-02$-D235? STUDY OF THE RESPONSE OF BACKPACKING MATERIAL ENCASING A TUNNEL LINER SU8JECTED TO A DYNAMIC DISTURBANCEI I3-13-O2279 EOLOGYI) QUARTERLY OF THE COLORAOO SEHOOL OF MINES 62 (2) TUNNEL MECHANICS I UNDERGROUND-STRUCTURES ROCK IG I3-I3-O2275 SE PUBL IC SHELTEI STUDY OF THE PROPOSED EAST WEST FREEWAY TUNNEL WEST ORANGE, NEW JERSEY, AS A CIVIL DEFEN I3- $13-02271$ SE PUEL IC SHELTE/ STUDY OF THE PROPOSED EAST WEST FREEWAY TUNNEL WEST ORANGE, NEW JERSEY, AS A CIVIL DEFEN $13-13-02271$ S EXPERIMEN/ SHOCK WAVE ATTENUATICN FORMULAS FOR STRAIGHT TUNNELS - A COMPARISON 1 SHOCK- TUBES CALCULATICN 20-O S EXPERIMENI SHOCK WAVE ATTENUATICN F ORMULAS FOR STRAIGHT TUNNELS - A COMPARISON 1 SHOCK- TUBES CALCULATI CN 20-D+ -D2O5D
URE/ CONSTRUCTION AND ENGINEERING OATA - WATER CONVEYANCE TUNNELS - GRAVITY AND PRESSURE 1 UVITED-5TATES B I3-I3-D22B3 IMOGRAM FOR CALCULATING ATTENUATION OF AIR SHOCK WAVES IN TUNNELS I PROPAGATION SWEDEN I *REPDRT NO. IOZ- 1 2O-O $\$$-O2OSI IEPORT ON FARD ROCK TUNVELLING INVESTIGATION I EXCAVATION TUNNELS ITRANSPORTAT ION) BLASTIVG COSTS DRILLING/ I3-O? -DI787 AS I MERDOYNAMIC ORAG ON VFHIT.IFS IN ENCLCSED GUIDEWAYS ( TUNNELS (TRANSPORTATION) TRANSPORTATION URBAN-ARE 23 -O? -D2III IGRAM IN HAMBURG ( GERMANY ROADS CONSTRUCTION URBAN-AREAS TUNNELS (TRANSPORTATION) VENTILATIOV TRAFFIC I *I I3-Q? -0ITBO IMOTION AND COAGULATION OF PARTICLES IN A TURBULENT GAS 1 TURBULENCE FLUIC-DYNAMICS PARTICLE-SIZE-DISTRIB/ $20-04-02333$ LE-SIZI RELATIVE MOTION AND COAGULATION OF PARTICLES IN A TURBULENT GAS 1 TURBULENCE FLUID-DYNAMICS PARTIC 20 -O IECESSARY COVOITIONS FOR SIMILAR SOLUTIONS OF PROBLEMS OF TURBJLENT GAS OYNAMICS 1 GAS-FLOW E2UATIONS-OF- $120-0 .-02331$

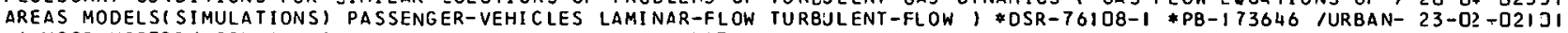
1 USSR HISTOR/ SOVIET MILITARY TRANSLATICNS NUMBER 38D - TWENTY -FIFIH ANNIVERSARY OF THE BATTLE OF MOSCOW O5-OU -02173 I ON BUILDIVGS AVD STRUC TURES - OPERA

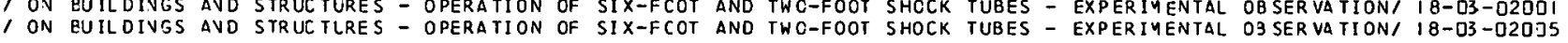

$\mathrm{U}$

IPRIVATE RESEARCH ON FOREIGN STUDENTS AND TRAINEES IN THE U.S. AND IN OTHER COUVTRIES I CULTURE, *A.L. I/ O5-II -DIG9I BLIC LAW 920 I LEGISLATION SOCIAL-SC IENCES HISTORY CIVILI U.S. CIVIL DEFENSE BEFJRE I9SO - IHE ROOTS OF PU OS-D'H -DIGTI BROTECT IVE SHEL TER SYSTEMS - AN ANNOTATED BIBLIOGRAPHY OF U.S. NAVAL DEFENSE BEFJRE T9SO - THE ROOTS OF PU DS-OH -OI67I IICS I RESOURCES PETROLEUM-INDLSIRY INDUSIRIES ECCNONICS/ U.S. SOURCES OF PETROLEUM AND NATUZAL GAS STATIS O5-O3-DIG38 TICS RESOURCES PETROLEUM-INDLSTRY INDUSIRIES ECCNOMICS/ U.S. SOURCES OF PETROLEUM AVD NATUZAL GAS STATIS O5-03-DI638

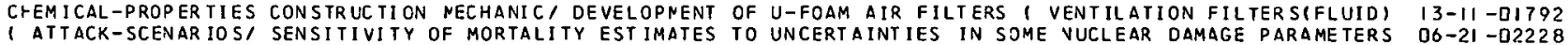
1 ATTACK-SCENARIOS/ SENSITIVITY OF MORTALITY EST IMATES TO UNCERTAINTIES IN SOME VUCLEAR DAMAGE PARAME TERS O6-2I - D2228
II. I MODELS CAMA/ SENSITIVITY OF MORTALITY ESTIMATES TO UNCERTAINTIES IN SOME NUCLEAR DAMAGE PARAMETERS O6-2I-O2229 URST YIFL WINO VUCLEAR-WARFARE-CASI SOME IMPLICATIONS ON UNCERTAINTIES IN THE HIROSHIMA EXPLOSIONI AIR-B IS-OS-DIGSB 
5 OF FIGH ALTITUDE NUCLEAR EXPL/ BIBLIOGRAPHY CF OFEN AND UNCLASSIFIEO LITERATURE PERTAINING TJ THE EFFECT I8-O3-O2DI8 IING BLAST LOADS ON DEEPLY BURIED PROTECTIVE STRUCTURES 1 UNCERGROUD-STRUCTURES CONSTRUC TIOV LOADING IMECH/ I -03 -O2DI 7 S UNOERGROUND-RESIDENTIAL-DISTRIBLTION-SYSTEMS COSIS FAU/ UNDERGROUND DISTRIBUTION 1 UNDERGROUND-STRUC TURE OQ-O3-DI753 SE COMMAND AT CHEYI METHODS AND COSTS OF CONSTRUCTING THE UNDERGROUND FACILITY OF NORTH AMEZICAN AIR DEFEN I3-I3-O2274 D MOT/ SIMULATION OF THE RESPONSE OF EOUIPNENT LOCATED IN UNCERGROUND INSTALLATIONS TO BLAST IVDUEED GROUN I3-I3-O2291 ITIONS CONDUIT TO STATIC AND DYNANIC LOADING I DUCTS SAND UNCERGROUND LOADIVG(MECHAVICS) SOIL-MECHANICS C/ I3-11 -D22SI I SCHOOL OF MINES 4611$)$ AN INTRODUCTION TO THE DESIGN OF UNOERGROUND OPENINGS FOR DEFEVSE 1 SHELTERS BLA/ I3-I3-02273 ENGINEERING ROCK(GEOLOGY) PILLARS STRESSES MAT/ DESIGN OF UNCERGRCUND OPENINGS IN COMPETENT ROCK I MINING- O8-OT-02245 IES UTIL IT IES ELECTR IC-POWER E LEC IRIC-POWER-TRANSNISSI ON/ UNDERGROUND POW ER TRANSMISS IOV 1 ELECTRIC-UTILIT O9-O3-U2249 1 COMPUTER SIMULATIONS.OF THERMAL ENVIRONMENT IN OCCUPIED UNCERGROUND PROTECTIVE STRUCTURES WITH OB SER VED/ $20-13$-O2D ERS UNDERGROUND-RESIDENTIAL-DI STRIBUTION-SYSTEMS UNDERGRI UNDERGROUND RESIOEVTIAL DISTRIBUTION I TRANSFORM O9-D3-OI754 AS UNDERGROUND-RESIDENTIAL-DISIRIBUTION-SYSTEMS COSTS UN/ UNDERGRCUND RESICENTIAL DISTRIQUTION 1 URBAN-ARE O9-D3-DITS5 CLEAR-EXPLOS IONS SEISMOLOGY GRANI TE MA THENATICAL-ANALYSIS UNDERGRCUND-EXPLOSIONS, \#UCRL-5DI82, /EFFECTS NU I8-O3-O23I6 I SOIL-MECHAVICS MECHANICAL- WAVES PROPAGATION SHOCK-WAVES UNCERGROUND-EXPLOSIONS ELASTICITY E2UATIONS-OF-1 O8-13-DI7L 7 TS FAU/ UNDERGROUND DISTRIBUTION I UNDERGROUND-STRUCTURES UNDERGROUND-RESIDEVTIAL-DISTRIBUTION-SYSTEMS COS D9-O3-DI75 TS FAU/ UNDERGROUND DISTRIBUTI ON I UNDERGROUND-STRUCTURES UNDERGROUND-RES IDEVTIAL-DISTRIBUTION-SYSTEMS COS D9-O3-DI753 ERGR/ UNDERGROUND RESIDENTIAL DISTRIBUTI ON I TRANSFORMERS UNDERGRCUND-RES I CENT IAL-CISTRIBUT IOY-SYSTEMS UND O9-D3-UII5 4

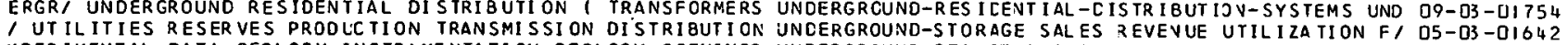

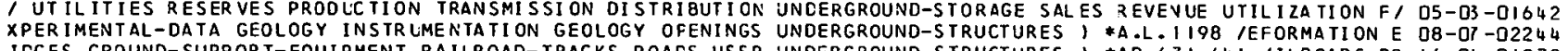
IDGES GROUND-SUPPORT-EQUIPMENT RAILROAD-TRACKS ROACS USSR UNOERGROUND-STRUCTURES, \$AD 631 O4I,ILROADS BR IO-DI - DI972 /EYENNE MOUNTAIN, EL PAST COUNTY, COLORADC ( CONSTRUCTION UNCERGRCUND-STRUCT JRES BLASTIVG EXCAVATION REIN/ I3-I3-O2274 IELTER SPACES IN NEW YORK CITY ANO VICINITY I URBAN-AREAS UNDERGROUND-STRUCTURES BUILDIVGS MODELSISIMULAT/ I3-I3-OIBI 4 INSTRUCTION INSTITUTE STUDIES I SHELTERS FALLOUT-SHELTERS UNDERGROUND-STRUCTURES COLORADO DUAL-PURPOSE-SH/ I3-I3-OI83। IANAL YS IS FOR A STANDARDI ZED SERIES OF FALLOUT SHELTERS I UNCERGROUND-STRUCTURES CONSTRUCTION URBAN-AREASI I $3-13$-DIBIS ISING A TUNNEL LINER SUB JEC TED TO A DYNANIC DISTUREANCE I UNDERGROUND-STRUCT JRES DEFORMATIOV COVSTRUCTION/ I3-I3-O2279 TRANS FORMERS UNDERGROUND-RESIDENTIAL-DISTRIBUTION-SYSTEMS UNDERGROUND-STRUCTURES DESIGN I *T- $160-5$ /ION 1 O9-03-0I754 IING IV - CIVIL DEFENSE I RADIOACTIVE-FALLOUT ATTENUATION UNDERGROUND-STRUCTURES DOSE-RATE PHOTONS SCATTE/ I8-GS-D2O29 IC CYNAMIC TESTS OF BURIED UNREINFORCED CONCRETE ARCHES 1 UNCERGROUND-STRUCTJRES DYNAMICS LJADIVGIMECHANI, I3-I3-OI83D IFROM NUCLEAR BLAST I SHELTERS SHCCK-WAVES BLAST-SHELTERS UNCERGROUND-STRUCTURES DYVAMICS MATHEMATICAL-ANI I3-I3 -OIB33 IANE IANE ELEMENTS IN PROTEC TIVE CONSTRUC ILN SLAST-SHELTERS UNDERGROUND-STRUCTURES DYNAMICS SHELLSISTRUC TUR/ I3- $13-01837$
IS, CRITICAL LITERATURE REVIEW AND PILOT STUDY I SHELTERS UNDERGROUND-STRUCTURES ELASTICITY TEST DYNAMICS/ I3-I3-DIB25 IS, CRITICAL L ITERATURE REVIEW AND PILOT STUDY I SHELTERS UNDERGROUND-STRUCTURES ELASTICITY TEST DYNAMICS/ I3-13-DI825
IAREAS UVDERGRDUND-RESIDENTIAL-DISTRIBUTI ON-SYSTEMS COSTS UNOERGROUND-STRUCTURES ELECTR IC-CABLES DESIGN P/ O9-D3-DI755

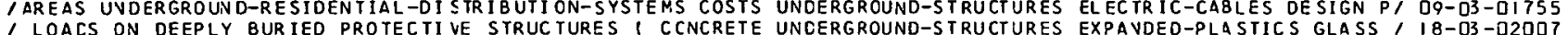
/ LOACS ON DEEPLY BUR IED PROTECTI VE STRUCTURES I CCNCRETE UNCERGROUND-STRUCTURES EXPAVDED-PLASTICS GLASS I I8-03-O2OD7
IERGROUND INSTALLATIONS TO BLAST INDUCED GROUND MOTIONS I UNOERGROUND-STRUCTURES EXPLOSION-EFFESTS SHOCKI/ I3-I3-O2291 IMINES SI(3) SYMPOSIUM ON ROCK MECHANICS ( ROCK (GEOLOGY) UNDERGROUND-STRUCTURES FAILUREIMECHANICS) MININ/ DB-D?-O224 3 I URBAN-AREAS COOLING-AND-VENTILATING-EQUIPMENT BUILDINGS UNDERGROUND-STRUCTURES MODELSISIMULATIONSI I \#A/ I3-I3-OIBI 3 III TESTS ( SOIL-MECHANICS SHOCK- hAVES FAILUREIMECHANICSI UNCERGROUND-STRUCT JRES NUCLEAZ-EXPLOSIONS I *TR/ I8-D3-02DJ8 /OERGROUND OPEN INGS FOR OEFENSE I SHELTERS BLAST STRESSES UNCERGROUND-STRUCTISRES ROCK (GEOLOGY) HEATING MI/ I3-I3-D2273 IF THE COLORADO SCHOOL OF MINES $62(2)$ TUNNEL MECHANICS 1 UNDERGROUND-5TRUCTURES ROCK (GEOLOGY) STRESSES E' I3- $13-02275$ IND $($ LOADING (YECHANICS) SOIL-MECHANICS DYNAMICS SHELTERS UNDERGROUND-STRUCTURES SAND SHDCK-WAVES BLAST F 2 20- II-O234 1 IEEP REIVFORCED CONCRETE SLABS I TESTS LOADING (MECHANICS) UNDERGROUND-STRUCT JRES SHEL TEZS PEZFJZMANCE IENG I $3-13$-D22 90 ITIVE STRUCTURES WITH OB SER VED CONDI TIONS I HEAT-TRANSFER UNCERGROUND-STRUCTJRES SHEL TEZS THERMAL-DIFFUSI / 20-I3-D2DFD IE BLAST LOADING - RESULTS OF SERIES III TESTS ( SHELTERS UNDERGROUND-STRUCTURES SOIL-MECHAVICS SURFACE-B/ DB-I3-0I746 /LAST-SHELTERS OESIGN COSTS SINGLE-PURPOSE-SHELTERS DOORS UNDERGROUND-STRUCT JRES STRUCTURAL-PROPERTIES ST/ I3-I3-DI835 ISTR I ITOR-SAFETY-SYSTEMS SOIL-MECHANIC S DYNAMICS GRCUND-MOTI ON UNCERWATER DESIGN COSTS LOADIVG (MEEHAVICSI CYLI/ I8-DF-D2OUI CIF - SATIONS I HYORODYNAMI/ THE SURFACE WAVES FROM SHALLOW UNDERWATER EXPLOSIONS - FORMULAE AND PRDGRAM SPE I9-DI-O2O43 CIFICATIONS ( HYORODYNAMI/ THE SURFACE WAVES FROM SHALLOW UNDERWATER EXPLOSIONS - FORMULAE AND PROGRAM SPE 19-04-O2O43 IIIONS OF STRESS SHEL TER-OCCUPANCY FEASIBILITY-STUDIES UNDERWATER(SHELTERS) LEACERSHIP CDVFIVED-ENVIRO/ DS-IJ-DIOR4 IITIONS OF STRESS T SHEL TER-OCCUPANCY FEASIBILITY-STUDIES UNDERWATERISHELTERS) LEACERSHIP CJVFIVED-ENVIRO/ OS-IJ-DI GR4 QUEST IONVAIRES ATTITUDES INTER NA TIONAL-AFFAIRS FEAR SURV/ UNESCO ANC PJBL IC OPINION TODAY I UVITED-STATES OS-II -DIOBQ CTRIC UTILITIES I ECONOMICS ACCOUNTING REGULATIONS, \#A.' UNIFORM SYSTEM OF ACCOUNTS FOR CLASS A AND B ELE O5-O3-O1631

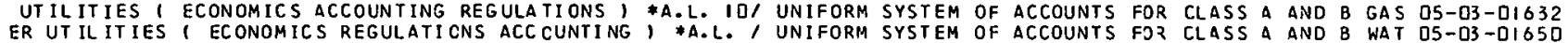
IL-DEFENSE CIVIL-DEFENSE-SYSTEMS EA' CIVIL DEFENSE IN THE UNITEO KINGDOM I GREAT-BR ITAIV INTERNATIONAL-CIV I5-D3-J2305 INION SURVEYS FOR/ OCCASIONAL REPORT ON FOREIGN AFFAIRS - UNITEO NATIONS I INTERNATIONAL-AFFAIRS PUBLIC-OP O5-II-O2I98 UESTIONNAIRES PUB / OCCASIONAL REPORT ON FOREIGN AFFAIRS - UNITED NATIONS I INTERVATIONAL -AFPAIRS SURVEYS Q QS-II-D2I O9 IIC ANO MEDIATED - CASE STUDIES OF EG YPT, ISRAEL, AND THE UNITED NAT. IONS AT FIVE POIVTS IN TIME I INTERNA/ OS-OH-D217D -KEEPING PUBLIC-OPINION INTERNATIONAL-AFFAIRS SU/ CAN THE UNITED NATIONS ORGANIZATION PREVEVT WARS I PEACE OS-II-D2I 55 ZED INFO/ SPECIAL IZED SCIENCE INFORMATION SERVICES IN THE UNITED STATES - A DIRECTORY OF SELECTED SPECIALI OS-D?-OIG2O E AS $\triangle$ PROBLEM FOLLOWING A THERMONLCLEAR h/ PLASUE IN THE UNITED STATES - AN ASSESSMEVT DF ITS SIGNIFICANC DO-O5 -DITD2 E AS A PROBLEM FOLLOWING A THERMONLCLEAR h/ PLASUE IN THE UNITED STATES - AN ASSESSMEVT OF ITS SIGNIFICANC D6-OS-DI7D2

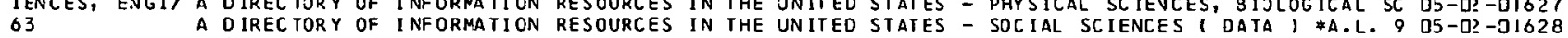

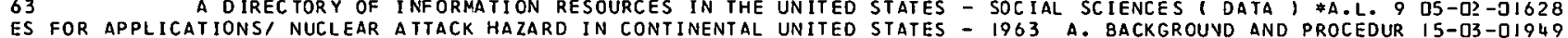
ES FOR APPLICATIONSI NUCLEAR ATTACK HAZARD IN CONTINENTAL UNITED STATES - 1963 A. 8ACKGROUVD AND PROCEDUR IS-D3-DI949
IIALS AS COMMONLY USED IN EXISTING URBAN BUILDINGS IN THE UNITED STATES I COVSTRUCTION URBAV-AREAS RADIOA/ I3- $33-02277$ IIALS AS COMMONLY USED IN EXISTING URBAN BUILDINGS IN THE UNITED STATES 1 COVSTRUCT ION URBAV-AREAS RADIOA' I3-13-02277 I- NREC SMALL SCALE MAPPING PROGRAM - MAPS OF CONTINFNTAL UNITED STATES I GEOCRAPHY RESJURCES DAMAGE-ASSEI OS-OS-O2ILO IEAR ATTACK ON RAILROAD TRANSPORTATION IN THE CONTINENTAL UNITED STATES I SYSTEMS-ENGINEERING ECONOMICS R/ O5-O3-D2I 28 P/ NUCLEAR REACTORS BUILT, BEING BUILT, OR PLANNED IN THE UNITED STATES AS OF DEC.31, 19661 VUCLEAR-POWER- I8-05-D2320 IULATION ESTIMATES - PROJECTIONS OF THE PCPULATION OF THE UNITED STATES BY AGE, SEX, AVD COLJR TO 1990 HI / $23-0+-02358$ LTURAL-ENG IVEER I/ THE AGRICULTLRAL RESEARCH CENTER OF THE UNITED STATES DEPARTMEVT OF AGR ICULTURE I AGRICU D2-OJ-U2I J8 ATION 11 REGULATIONS GOVERNING THE MEAT INSPECTION OF THE UNITED STATES DEPARTMENT OF AGR ICULTURE 1 LEGISL D2-D2-DIG2O TECTI ON RESOURCES (VULNERABILITY) FALLOUT-S/ DEFENDING THE UNITED STATES FROM NUCLEAR FIRE ( FIRES FIRE-PRO I3-I2-DI8D6 IES FOR THE FINANCIAL YEAR 1967 AND INFORNAIICN ANNEXES I UNITED-NATIONS COSTS IVTERVATIONAL-AFFAIRS I \#U/ O5-D\#-D2IS5 R NATO VIETVAM RED-CHINA FOREIGN-POLICY FCREIGN-AIC LABOR UNITEO-NATIONS WAGES, \#A.L. IIS4 /NTZOL COLD-WA OS-D - - III2

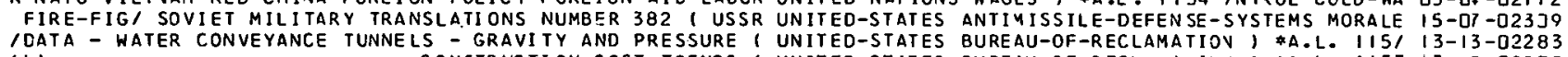
IDATA - WATER CONVEYANCE TUNNELS - GRAVITY AND PRESSURE ( UNITED-STATES BUREAU-OF-RECLAMATION ) \#A.L. II5/ I3-I3-02283 (4) CONSTRUCTION COST TRENDS $(2)$ CONSTRUCTI CN COST TRENDS

3 CONSTRUCTICN COST TRENDS

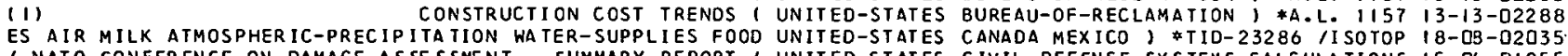

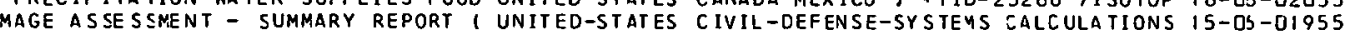
ONAL DECISION-MAKING SOCIAL-SCIENCES SCIENTIFIC-RESEARCH UNITEO-STATES DISTRIBUTIOVI \$PUBL. I4CO /NT-PLA OS-DI - DI622 IG-SYSTEMS DS FEAR SURV/ UNESCO AND PUBLIC OPINION TODAY I UNITED-STATES QUESTIONNAIRES ATTITUDES INTERNATI DS- II-OI GB 9 EFFECTS OF HEMOGRAPHY TRAFFIC ANTIMISSILE-DEFENSE-SYSTEMS UNITED-STATES SURVIVAL EUROPE COMYUVICATIONS CH/ IS-OS-QIQ22 IFENSE-SYSTEMS APPROPRIATIONS ANTIMISSILE-DEFENSE-SYSTEMS UNITED-STATES USSR COMMUNIST-CHINA THREAT-EVALU/ I5-D3-D2OIN IRCH-PROGRAM-ADMINISTRATION OPERATIONS-RESEARCH PERSONNEL UNITED-STATES-GOVERNMENT COMMERCE CONSTRUCTION / DS-O3-DIG49 1 TO EMERGENCY PLANNING I SCIENTIFIC-RESEARCH BLAST FIRES UNITED-STATES-GOVERNMENT OFFICE-OF-EMERGENCY-PL/ I5-O3-O2299 $1 G$ POLICY DECISIONS 1 SOCIAL-SCIENCES MANAGEMENT-PLANNING UNITED-STATES-GOVERNMENT POLITICAL-SCIENCE ECON/ O5-O'+-OI 58 IL REPORT OF THE COUNCIL OF ECONOMIC ADVISERS I ECCNCMICS UNITED-STATES-GOVERNMENT POLITICAL-SCIENCE FORE / O5-O3-DI G34 INNEL IN RECRUITING SHEL TER MANAGERS I SHELTER-MANAGEMENT UNITED-STATES-GOVER VMENT(PERSJNNEL) ADMINISTRAT/ D5-OQ-DI675 IPHASE, 4OD-CYCLE, MODEL CEII VAL4-208, AIR CONDITIONING UNITS I AIR-CONOITIONING-EQUIPMENT PREFABRICATE, I3-OI -DI775 
IINST NUCLEAR WEAPON FALLOUT IN EMERGENCY HEALTH SERVICES UNITS 1 SHIELDING PATIENTS RADIOACTIVE-FALLOUT / I8-0S-02028 INES FOR THE SELECTION OF DEMOGRAPHIC VARIABLES AND AREAL UNITS FCR 5TJDY ING POSTATTACK SOCIETY I POPULAT/ $33-$ I - O2IO3 IGH-SPEED-GROUND- TRAN SPOR T PERFORNANCE (ENGINEERING) VEHI/ UNIVERSAL ORAG LAW 1 TRANSPORTATIJV PROPULSION H 23 - D? -D2D96 ISE WITH WORLO WIDE RESOURCES GEODETIC POSITICN I GP, TO UNIVERSAL TRANSVERSE MERCATOR I UTM I AND INVER/ OS-O3-02I33 ING MAPPING ENG INEERING ARTILLERY GEODESICS, FTM 5-24I-8 UNIVERSAL TRANSVERSE MERCATOR GRID I ARMY SURVEY O8-DS-O224D TURES DYVAMICS LOADIN I STATIC AND OYNAMIC TESTS OF BURIEO UNREINFCRCED CONCRETE ARCHES I UNDERGROUND-STRUC I3-I3-OIB3D FORMS ) MEMBRAVES STRESSES/ PRELIMINARY TESTS CN A SHALLCW UNREINFCRCEC CONCRETE SHELL I SHELLSISTRUCTURAL- I3-I3-DIB32 IANCY STUDIES 2. COMMUNITY FALLOLT SHELTERS HANDBCOK FOR UNTRAINED MANAGEMEVT I SHEL TEZ-MAVAGEMENT SHELT/ OS-DI -D2I IS RADIOACTIVE-I SOTOPES RADIOACTIVE-FALLOUT FODD CONTAMINAT/ UPTAKE OF RADIONUCLIDES BY PLANTS 1 AGRICULTURE D6-I3-DI725 RL IOGRAPHIES URBAN-AREAS RUI FIRE BIBLIOGRAPHY - SELECTED URBAN AND MIXED URBAN RURAL FIRES $1040-1964$ I RI I3- 3 -OI799 RLIOGRAPHIES URBAN-AREAS RU/ FIRE BIBLIOGRAPHY - SELECTED URBAN AND MIXED URBAN RURAL FIRES $1940-1964$ I EI I3-1?-0I799 ITY FIRES REPAIR RADIOACT/ POSTATIACK RECCVERY OF CANAGED UREAN AREAS I ELAST DAMAGE-ASSESSMENT VULNERABIL IS-OS-O23D? A.L. 1118 SHADOW GEOMETRY OF BUILDINGS IN UREAN AREAS I FIRES THERMAL-2ADIATIOV SWEDEN I \# I3-I?-D226B ION URBA' BUILDING MATERIALS AS COMMONLY USED IN EXISTING URBAN BUILDINGS IN THE UNITED STA TES ON TAXES VULNERAB ILI I IMPLEMENTATION OF AN ORDERED SPRAWL URBAN CONFIGURATIOV I URBAV-AREAS DECENTRALIZATI $23-$ OS-O2I D6 RENEWAL MAN/ PROCEEOINGS OF THE MID AMERICA CCNFERENCE ON UREAN CESIGN 1 URBAN-AREAS URBAN-OLANNING URBAN- 23-OS-O2I O5

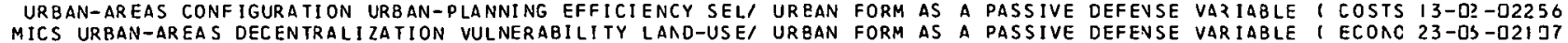
MICS URBAN-AREAS DECENTRALIZATION VULNERABILITY LAND-USE/ URBAN FORM AS A PASSIVE DEFENSE VARIABLE I ECONC 23-OS-O2IIT7

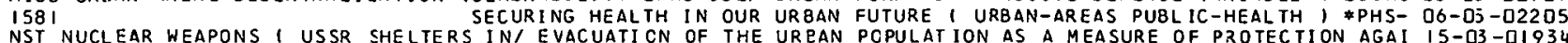
ON-MAKING INCENTIVES SOCIAL-SCIEN/ OPERATIONS RESEARCH ON URBAN PROBLEMS I ECONOMICS URBAN-PLANVING DECISI O5-IJ-OIGT6 AN-AREAS RU/ FIRE BIBLIOGRAPHY - SELECIED URBAN ANC MIXED URBAN RURAL FIRES 1940-1964 1 BIBLIDGRAPHIES URB I3-I?-QI799 I BURST-CONDITIONS URBAN-AREAS FIRE/ PARANETERS GCVERNING UREAN VULNERABILITY TO FIRE FROM VUCLEAR BURSTS I3-I?-OI8JI ITY OF NEW YORK AND FOR NEW YORK CITY TRANSIT AUTHCRITY ( UREAN-AREAS, A.L. I156/OF THE MAYOR. OF THE C 23 -O2 -O2355 AGENCY RAPID TRANSIT SYSTEM - NANLAL OF DESIGN ERITERIA ( URBAN-AREAS, *A.L. II93 /APITAL TRAVSPORTATION $23-02-02353$ HELTERS INTERNATIONAL-CIVIL-DEFEN SE CIVIL-DEFENSE-SYSTEMS URBAN-AREAS; \#JPRS-32, 120 *TT-65-32613, USSR S IS-03-OI934

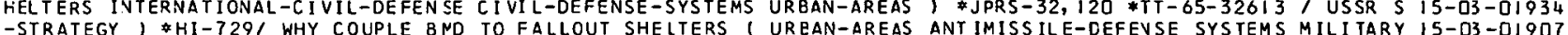

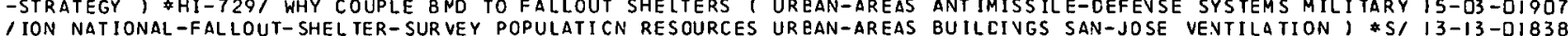

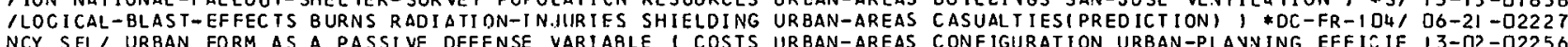
NCY SEL/ URBAN FORM AS A PASSIVE DEFENSE VAR!ABLE I COSTS URBAN-AREAS CONFIGURATION URBAN-PLAVVING EFFILIE I3-O2-O2256 I SPACES IN VEW YORK CITY AND VICINITY I FALLOUT-SHELTERS URRAN-AREAS COOLING-AND-VEVTILATIVG-EZUIPMENT BI I3-I3-OIBI 3 TATES-GOVERNMEN T(PER SONNEL) ADMINISTRATI ON-AND-MANAGEMENT UREAN-AREAS COSTS, \#AIR-D93B(I)-9/66-FR /ITED-S O5-D7-0I675 I/ A STUDY OF WORLD WAR II GERNAN FIRE FATALITIES I FIRES URBAN-AREAS DAMAGE-ASSESSMENT CASUALTIES POPULAT I3-I?-O22 2 ING-SYSTEMS USSR MOVEMENT POPULATION RESOURCES EVACUATION URBAN-AREAS DAMAGE-LIMITING-SYSTEYS DEMOGRAPHY, I5-D3-DI922 IT FALLOUT SHEL TER SYSTEMS I MANAGEMENT-PLANNING SURVIVAL URBAN-AREAS DAMACE-LIMITIVG-SYSTEYS EVACUATION, I3-I3-OI829 /MPLEMENTATION OF AN ORDEREO SPRALL URBAN CONFIGURATION I UREAN-AREAS OECENTRALIZATION TAXES VULNERABILIT/ 23 -OS-O2I I6 USE, URBAN FORM AS A PASSIVE DEFENSE VARIABLE I ECCNOMICS URBAN-AREAS DECENTRALIZATION VULNERABILITY LAND- $23-0 S-02 I$ IZT

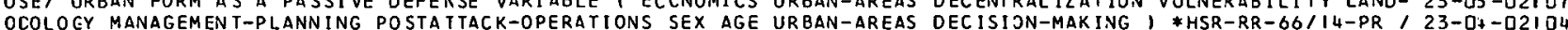
OCOLOCY MANAGEMENT-PLANNING POSTA TTACK-OPERATIONS SEX AGE URBAN-AREAS DECISIJN-MAKING THE TRAVSPORTATION OF HIGH LEVEL RAOIOACTIVE MATERIALS I UREAN-AREAS OECONTAMINATIOV RADIOACTIVE-WASTES I I8-D7-O2034

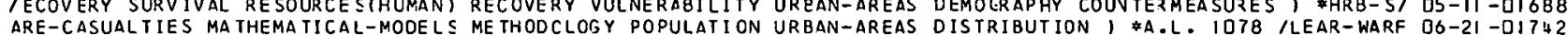

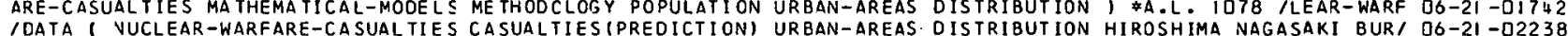

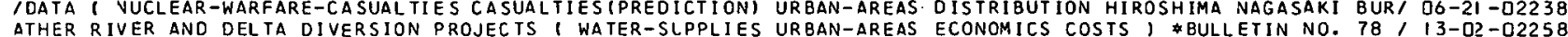
ATHER R IVER AND DELTA DIVERSION PROJECTS I WATER-SLPPLIES URBAN-AREAS ECONOMICS COSTS 1 \$BULLETIN NO. $78 / 13-02-02258$

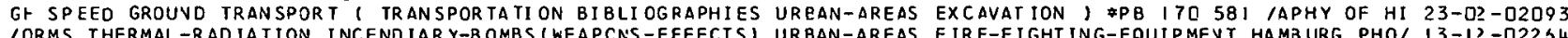
IORMS THERMAL-RADIATION INCENDIARY-B OMBS (WEAPCNS-EFFECTS) URBAN-AREAS FIRE-FIGHTING-EQUIPMEVT HAMBURG PHO/ I3-I?-02234 ITENT IAL IN SAN JOSE AND ALBUOUERGUE I AERIAL-PHOTCGRAPHS URBAN-AREAS FIRES YAPS TEST-METHODS VULNERABILI/ I3-I? -QI8O9 IERABILITY TO FIRE FROM NUCLEAR BLRSTS ( BURST-CONOITIONS URBAN-AREAS FIRES NUCLEAR-EXPLOSION-DAMAGE ) *U/ I3-I?-OIBOI IIST-CHIVA IVDUSTRIES TRANSPOR TA TION EVACLATICN DISPERSAL UREAN-AREAS FOOD SHELTERS FALLOUT-SHEL TERS EURO/ IS-D3-O22Q5 IESSION POTENTIAL OF AN EMERGENCY BLAST SHELTER PRCGRAN I UREAN-AREAS HASTY-SHELTERS DESIGN MATERIALS EQU/ I3-I3-O2272 ATION RELEASED IN NUCLEAR DETONATIONS I GLASS ATTENUATION URRAN-AREAS IGNITIJN) \#A.L. 971 /F THERMAL RADI I3-I?-OI796 IN A MAJOR CITY ( SHELTERS CI VIL-DEFENSE-OPERATING-PLANS UREAN-AREAS MANAGEMENT-PLAVNIVG) $* A-L$. 974 /TER I5-O3-OIQ45

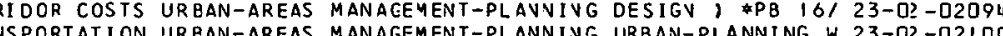

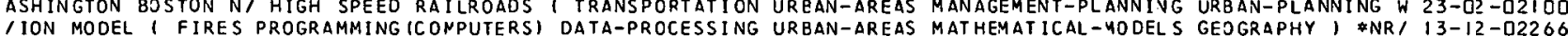

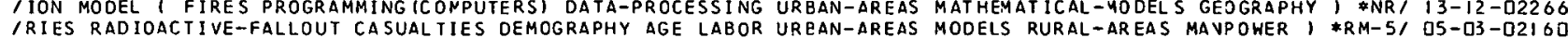

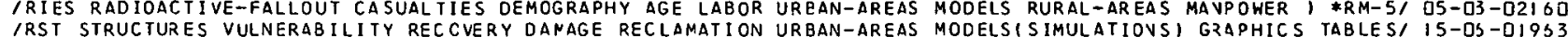
/RST STRUCTURES VULNERABILITY REC CVERY DANAGE RECLAMATION URBAN-AREAS MODELSISIMULATIOVSI GRAPHICS TABLESI IS-DS-OI933

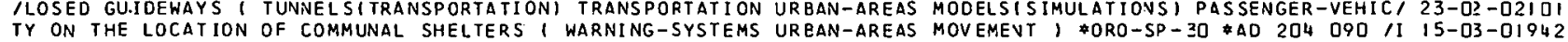
ILKS AND THE PROBLEM OF SHORT RANGE MASS TRANSPORTATICN 1 UREAN-AREAS MOVING-BELTWAYS TRAFFIC DESIGN COST/ $23-02-02097$ ILL IAMSBURG BRIDGES AND ALTCRNATI VE SOLUTIONS THEREFORE 1 URBAN-AREAS NEW-YORK, \#A.L. 1155 INHATTAN AND W 23 -O2 -D2357 LANNING, ARPN-73-74 EXPRFSSWAY PIANS 1 TRANSPORTATION UREAN-AREAS NEW-YORK MANAGEMENT-PLANVING URBAN-P $23-02-02098$ RUSS IA DAMAGE-L IMITING-SYSTEMS DI SPER SAL CHINA EVACUATION URBAN-AREAS POPULATION, \#HI-777-RR /IL-DEFENSE IS-US-LIQYO ANAGEMENT-P/ RAIL TRANSIT PLANS I TRANSPORTATION NEW-YORK URBAN-AREAS POPULATION RAILROAOS SUBWAYS COSTS M $23-02-02099$ IIL-DEFEVSE-OPERATING-PLANS UTILITIES DATA WATER-SUPPLIES UREAN-AREAS POSTATTACK-OPERATIONS MAVAGEMENT-PL/ I3-0? - I I88 IF FALLOUT SHEL TERS I UNDERGROUND-STRUCTURES CONSTRUCTION URBAN-AREAS PROTECTION-FACTOR BLAST-SHELTERS CO/ I3-I3-OIBIS

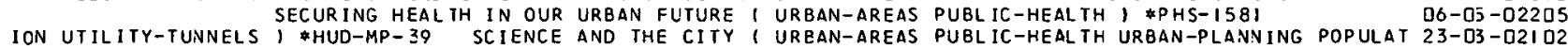
ION UTILITY-TUNNELS, HUD-MP-39 SCIENCE AND THE CITY I UREAN-AREAS PUBLIC-HEALTH URBAN-PLANNING POPULAT 23-03-D2I D2

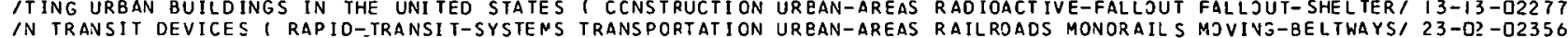
IN TRANSIT DEVICES T RAPIO-TRANSIT-SYSTENS TRANSPORTATION UREAN-AREAS RAILROADS MONORAILS MJVIVI-BELTWAYSI 23 -0? - O2356 IK OF AGR ICUL TURAL CHARTS - 1966 I POPULATION ASRICULTURE URBAN-AREAS RURAL-AREAS COMMERCE MEAT RESOURCES/ O2-D2-DI6I9
IN ANO MIXED URBAN RURAL FIRES $1940-1964$ ( BIBLIOGRAPHIES URBAN-AREAS RURAL-AREAS FIRE-SAFETY ) \$AD 634 O/ I3-I2-OI799

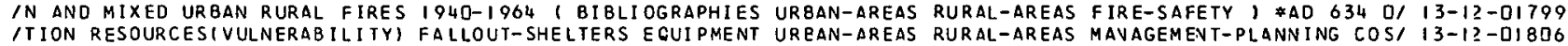

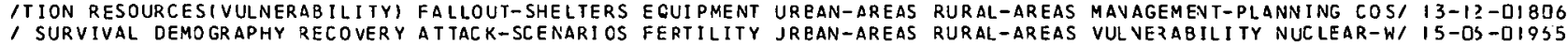
/SEASE-VECTORS DISEASES(CONIROL) DRAINAGE COUNTERMEASURES URBAN-AREAS SAN-JOSE CALIFORNIA RECOVERY PEST-C/ I3-O2-OITBO IATORY STUCIES ( POSTATTACK-OPERATIONS STRESS (PSYCHOLOGY) URBAN-AREAS SOCICLOGY SOCIETAL-RECOVERY METHODO/ I5-D3-D23DI

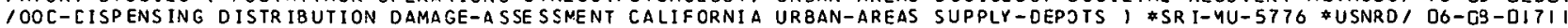
IF MUNICIPAL. WATER SYSTEMS ( WATER-SUPPLIES VULNERABILITY JREAN-AREAS SURVEYS POSTATTAC S-OPERATIONS RECOVI IS-O3-OIQLI IOR STUDYING POSTATTACK SOCIETY I POPULATION DISTRIBUTION URBAN-AREAS SURVIVAL AGE POSTATTACK-OPERATIONS I 23 -OH - Q2IJ3 NY I. T FIRES FIRESTORMS HAMBIIRT, FIRF-FIGHTING-EOUIPMENT URBAN-AREAS THERMAL-RADIATION NC-TRINSPORT NEW TRANSPORT TECHNOLOSY FOR THE /BUILCING PROGRAM IN HAMB LRG ( GERNANY ROADS CCNSTRUCTION JRBAN-AREAS TUNNELS (TRANSPJRTATIOV) VENTILATION/ I 3 -O? -OI IB6 \begin{tabular}{l}
-SYSTEMS COSTS UN/ UNDERGROUND RE SIDENTIAL OISTRIBUTION \\
IF FALLOUT SHEL TER SPACES IN NEW YORK CITY AND VICINITY I URBAN-AREAS UNDERGROUND-RESIDENTIAL-DISTR IBUTION O9-O3-OI755 \\
\hline
\end{tabular} /CEEDINGS OF THE MID AMERICA CONFERENCE ON URBAN DESIGN 1 URBAN-AREAS URBAN-PLANNING URBAN-ZEVEWAL MANAGE $23-06-02 I$ OS AS POPULATIOV RAILROADS SLBWAYS COSTS MANAGENENT-PLANNING URBAN-PLANNING, \#RPN-71-72, NEW-YJPK URBAN-ARE $23-02-02099$ I TRANSPJRTATIOV URBAN-AREAS NEW-YORK MANAGENENT-PLANNING UREAN-PLANNING I *RPN-73-74 -SCIEN/ OPERATIONS RESEARCH ON URBAN PROBLEMS 1 ECCNOMICS URBAN-PLANNING DEC ISIOV-MAKING INCENTIVES SOCIAL OS-IJ-DIOT6 ISS IVE DEFENSE VARIABLE I COSTS URBAN-AREAS CCNFIGURATION URBAN-PLANNING EFFICIENCY SELECTION POPULATION, I3-02-D2256 IAT ION 1 URBAN-AREAS DECENTRALIZAIION TAXES VULNERABILITY UKBAN-PLANNING LAVD-USE PJPULATIOV TRANSPORTATI/ $23-03-02 I$ IO6 ITRIBUT IOV URBAV-AREAS SUR VIVAL AGE POSTATTACK-OPERATIONS UREAN-PLANNING METHODOLOGY SEX I *HSR-RR-66/I4-I 23-DH-02I O3 IMICS URBAN-AREAS DECENTRALI ZA TIOA VULNERABILITY LAND-USE UREAN-PLANNING POPULATION POL. ITICAL-SCIENCE .) *I 23 -0S-O2I O7 -MP-39 SCIENCE AND THE CITY I URBAN-AREAS PUBLIC-HEALTH URBAN-PLANNING POPULATION UTILITY-TUVYELS I \$HUD $23-03-02 I D 2$

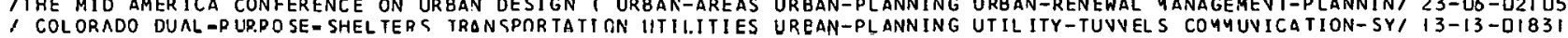




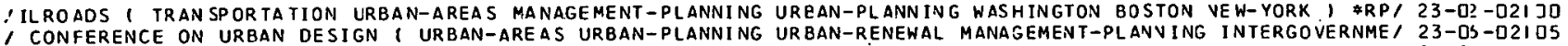
$\prime$ CONFERENCE ON URBAN DESIGN $I$ URBAN-AREAS URBAN-PLANNING URBAN-RENEWAL MANAGEMENT-PLANVING INTERGOVERNME
URUGUAY LIVESTOCK AND MEAT INDUSTRY I AGR ICULTUR D2-D2-D2IID E AGR ICUL TURAL-ECONOMICS I *FA S-M-186

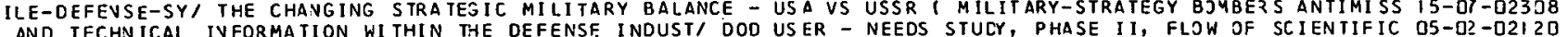

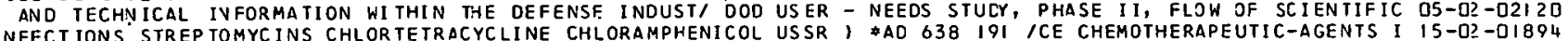
NFECT IONS STREP TOMYCINS CHLOR TETRACYCLINE CHLORAMPHENICOL USSR H ALT ITUDE NUCL EAR EXPLOSIONS I. AND II. I UNITED-STATES USSR, *DASA-1795 ITAINING TO THE EFFECTS OF HIG 18-03-02018

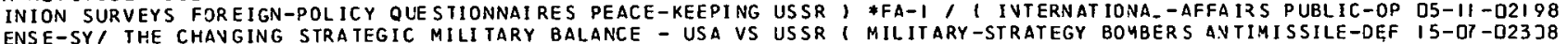

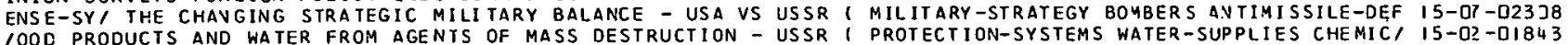
TERAPY IV AFFECTIONS WITH TOXIC AND RADIOLOGICAL AGENTS - USSR I RADIOLOG ICAL -WARFARE-AGENTS COVTAMINATIO/ O6-13-0172 -WEAPONS CONTAM INATION FOOD CHE, CIVIL DEFENSE YANDBOOK - USSR I RURAL-AREAS BOMBS GUIDEC-MISSILES NUELEAR I5-D3-O2296 -WEAP PUFY AND TO DISINFEC T WATER WITH THE SIMPLEST NEANS - USSR I WATER-SUPPLIES PUR IFICATIOV CONTAMINATIO/ O6-13-01722 IENCE RESEARCH BY PRIVATE SCHOLARS AND ACADEMIC CENTERS - USSR ANC EASTERN EUROPE 1 B IBL IOG $2 A P H I E S$ COMMUN/ DS-11-01697

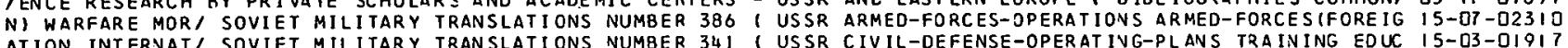

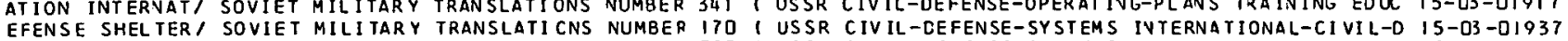
EFENSE SHEL TER / SOVIET MILI TARY TRANSLATI CNS NUMBER 170 ( USSR CIVIL-CEFENSE-SYSTEMS IVTERNATIONAL-CIVIL-D $15-03$ - 1937 , AGA INST WEAPONS OF MASS DESTRUC TION IN THE RURAL AREA (USSR CIVIL-DEFENSE-SYSTEMS RESCUES IVTERNATIONA/ I5-D3-OI9IB ITHE RURAL POPULATION AGAINST RAD IOACTIVE CONTAMINATICN I USSR CIVIL-DEFENSE-SYSTEMS SHEL TERS INTERNATION/ DO-I3-OIT3I RESEARCH PAPER 156 SOVIET IMPAC T ON INTERNATICNAL LAW ( USSR COMMERCE INTERNATIONAL-AFFAIPS) *EXTERNAL O5- 11 -D 1690 /APPROPRIATIONS ANTIMISSILE-DEFENSE-SYSTENS UNITED-STATES USSR COMMUNIST-CHINA THREAT-EVALUATIOV MILITARYI IS-03-02294 S FIRST-AIC/ CURRENT TOPICS IN SOVIET MILI TARY MEDICINE ( USSR DISASTER-MECIC INE NUCL EAR-WAPFARE-CA SUALYIE DO-0J-017O3 IATURES AND PRESSURES I ROCK-MECHANICS LOADI NG IMECHANICS) USSR EXCAVAT ION SHEAR IVG CUTTIVG BEVDING TOR SIO/ OB-O7-02242 1380 - TWENTY-F IF TH ANNI VER SARY OF THE BATILE OF MCSCOW (I USSR HISTORY COMMUVISM PROPAGA.VDA 1 *JPRS-40,93/ 05-04-02173 BENELUX NORWAY DENMARK GREECE SWEDEN SWI TZERLAND IRELAND USSR INOIA AUSTRAL IA CANADA I *HI-272-D 1 GERMANY $15-03-02147$ YSTEMS RURAL-A, SOVIET MILITARY TRANSLATI CNS NUMBER I78, ISSR INTERNAT IONAL-C IVIL-DEFEY SE CIVIL-DEFENSE-S I5-D3-DI928 ITE OF AN ATOMIC STR IKE I RESC LES CIVIL-DEFENSE-PERSONNEL USSR INTERNAT IONAL-CIVIL-DEFEV SE TRAINING MANUA/ I3-12-0IBJ4

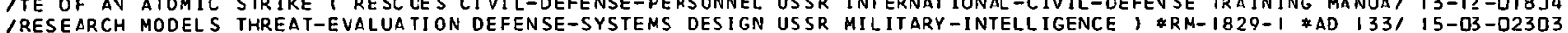

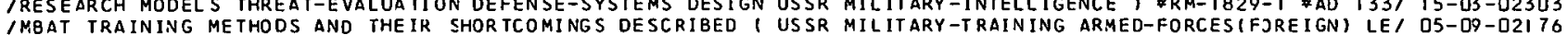

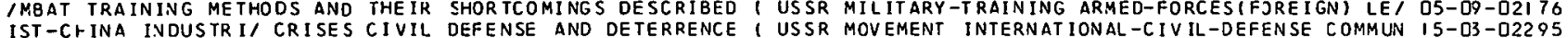
IST-CFINA INDUSTR I CRI CES CIVIL DEFENSE AND DETERRENCE I USSR MOVEMENT INTERNAT IONAL-CIVIL-DEFENSE COMMUN $15-03-02295$
BIL ITY INTERNATIONAL-CIVIL-DEFENSE EARLY-WARNI NG-SYSTEMS USSR MOVEMENT POPULATION RESOURCES EVACUA IION U/ I5-D3-DIO22

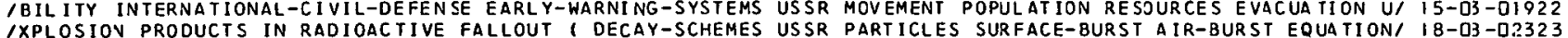

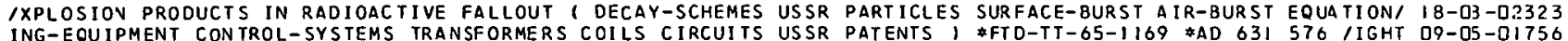
ING-EQUIPMENT CON TROL-SYSTEMS TRANSFORMERS COILS CIRCUITS USSR PATENTS I \#FTD-TT-65-1169 \#AD 631576 IIGHT O9-05-01756
ICAL EFFECTS AND PROTECTIVE MEASURES I RADIATION-INJURIES USSR RACIATION-HAZAROS RADIOLJGICAL-DOSAGE DOSI/ D6-I3-DI726

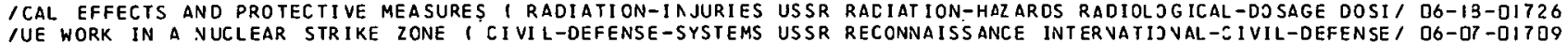
$110 N$ AS A MEASURE OF PROTECTION AGAINST NUCLEAR WEAPONS I USSR SHELTERS INTERNAT IONAL-CIVIL-DEFENSE CIVIL/ I5-03-DI934 OS BRIDGES GROUND-SUPPORT-EQUI PMENT RAILROAD-TRACKS ROADS USSR UNOERGROUND-STRUCTURES, *AD 631 O4I/ILROA 16 -01-01972 ORALE FIRE-FIG/ SOVIET MILITARY TRANSLATI ONS NUMBER 382 I USSR UNITED-STATES ANTIMISS ILE-DEFEVSE-SYSTEMS M IS-D7-02309 A.I UNIFORM SYSTEM OF ACCOUNTS FOR CLASS A AND B ELECTRIC UTILITIES I ECONOMICS ACCOUVTING 2EGULAIIONS) A.L. IO/ UNIFORM SYSTEM OF ACCOUNTS FOR CLASS A ANC B GAS UT ILITIES ( ECONOMICS ACCOUNTIVG REGULATIONS) OS-D3-DI632 A.L. I UNIFORM SYSTEM OF ACCOUNTS FOR CLASS A AND E WATER UT ILITIES ( ECONOMICS REGULATIONS ACCOUNTING I * O5-03-DI650 SION/ UNDERGROUNO POWER TRANSMISSION IELECTRIC-UTILITIES UTILITIES ELECTRIC-POWER EL ECTRIC-POWER-TRANSMIS O9-D3-C2249

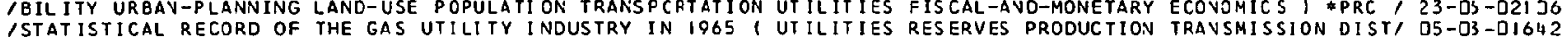

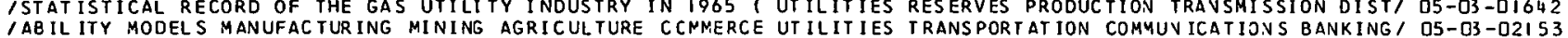

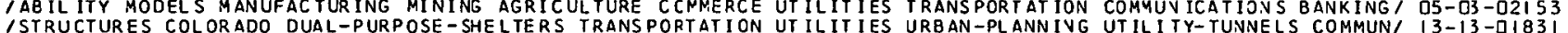

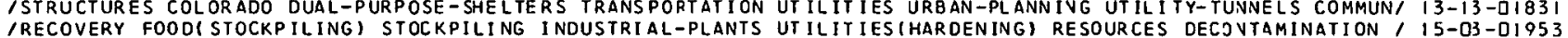

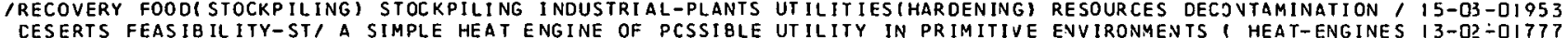
ODUCT ION TRA/ GAS FAC TS - A STATISTICAL RECORD OF THE GAS UTILITY INDUSTRY IN 19651 UTILITIES RESERVES PR O5-D3-DIOH2 I GAME-THEORY MATHEMATICAL-MODELS SIMULAT/ DEVELOPMENT OF UT ILITY THEORY FOR ARMS COVTRJL AND DISARMAMENT O5-D +-02158 ( GAME-THEORY SIMULATION) *FAR-396I (II) DEVELOPNENT OF UTILITY THEORY FOR ARMS CONTROL AVD DI SARMAMENT D5-DI-D2169 ITY I URBAN-AREAS PUBL IC-HEALTH URBAN-PLANNI NG POPULATION UT ILITY-TUNNELS I \#HUD-MP-39 SC IEVCE AND THE C 23 -03-O2I D2

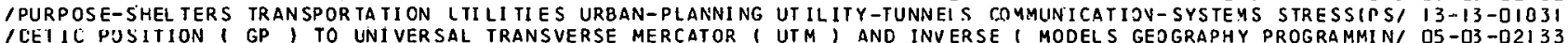

$\checkmark$

/LLUS antHRACIS OBTAINED WITH PROTECTIVE ANTIGEN AND LIVE VACCINE I SPORES BIOLOGICAL-WARFARE-AGENTS ANTI/ I5-02-01853 11 B IOLOGICAL-WARFARE-AGENTS BAC TERIAL-AEROSOLS EXPOSURE VACCINES DOSAGE IMMUNITY MONKEYS MICE GUINEA-PI I5-DD-DI858

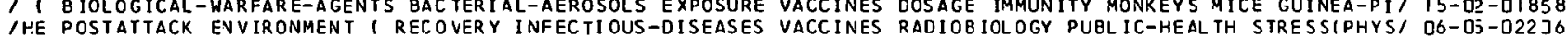
MONKEY, MACACA IRUSI AIR BORNE INFECTIVITY OF THE VARIOLA VACCINIA GROJP OF POXVIRUSES FOR THE CYNOMOLGUS I5-0?-0I855 NATION I IONOSPHERE OXYGEN GAS-IONIZATION. AIRBURST IONS/ VALIOITY OF THE O2(-) MODEL FOR D REGION RECOMBI DU-OI-OZIIS ION OF CONNECTICUT AVENUE ROUTE BETHEEN COLUMBIA RCAD AND VAN NESS STREET I TRANSPORTATION SUBWAYS TESTS, I3-02-0I785 -PLANNING EFFIC IENCY SEL/ URBAN FORM AS A PASSIVE DEFENSE VARIABLE 1 COSTS URBAN-AREAS CONFIGUZATION URBAN 13 -02-02256 N VULNERAB IL ITY LAND-USE / LRBAN FORM AS A PASSI VE CEFENSE VARIARLE I ECONOMICS URBAV-AREAS DECEVIRALI 2ATIO 23-0S-02I D7 TIST ICAL-ANALYSIS METHODOLOGY SOCIAL-SCIENCES CORRELATION VARIABLES, *A.L. 1190 IDATA 1 PUVE HEJ-CARDS STA O5-11-02192 1 - EMPIR ICAL GUIDELINES FOR THE SELECTION OF DEMOGRAPHIC VARIABLES AND AREAL UNITS FOR STUDYING POSIATTA' $23-0+-02103$

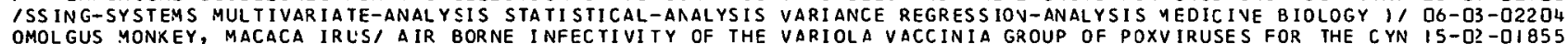
STE WATEI POSTATIACK SANITATION, WASTE DI SPOSAL, PEST AND VECTOR CONTROL, AND THE EFFECTS OF FALLOUT IN WA I3-02-QI7BD STE WATE POSTATTACK SANITATION, WASTE DISPOSAL, PEST AND VECTOR CONTROL, AVO THE EFFECTS OF FALLOUT IN WA $13-02-01780$

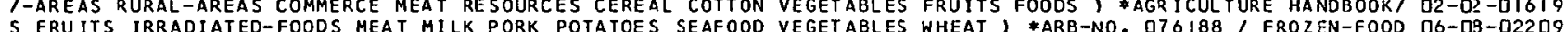
S FRUITS IRRADIATED-FOODS MEAT MILK PORK POTA TOES SEAFOOD VEGETABLES WHEAT, *ARB-NO. O76188 ( FROZEN-FOOD 06-OB-02209 IUEJECTS RELATED TO ACTIVE VIBRATION I SOLATI ON AND ACTIVE VEHICLE SUSPENS IONS I VIBRATIJN-ISOLA TORS CALCU/ $20-11$ - D2087

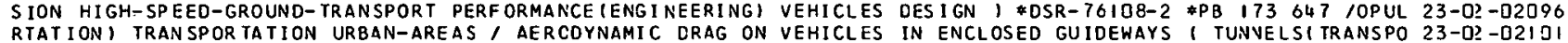
RTATION) TRANSPOR TATION URBAN-AREAS I AERCDYNAMIC DRAG ON VEHICLES IN ENCLOSED GUIDEWAYS I TUNVELSI TRANSPO $23-02-02101$
AY MIXT) THE RESPONSE OF SOILS TO DYNAMIC LOADINGS - WAVE VELOCITIES THROUGH PART IALLY SATURATED SAND - CL $20-01-02047$ ST EROSION ( PARTICLE-SIZE EXPER I NENTAL-DATA GAS-TLRBINES VELOCITY, \#AD 423083 /E BASIC MECHAVISMS OF DU 20 -09-02068 DEVATTFORC ING FUNCTION S SHOCK (MECHANICS) VIBRATI ON ELLASTICISY VELOCCIY DEGREES-TF-FREEDJM MA THEMA TIEAL-ANALYSI $20-11$-D2342 IEXPLOSIONS 1 SECONDARY-MISSILES BLAST-ENERGIZED-MISSILES VELOCITY GLASS STOVE BLAST EXPERIMENTAL-DATA $1 N / 18-03-02318$ OD RECREATION EXERCISE NOISE RELIIGION TEMPERATURE-CONTROL VENTILATION 1 *A.L. 1131 /RAIVING SLEEP WATER FO O5-13-02184 LOAC ING(MECHANICS) COOLING FAI LURE IMECHANI CSI SHOCK-WAVES VENT ILAT ION, *A.L.I205, ING MINING-EVGINEERING I3-13-02273

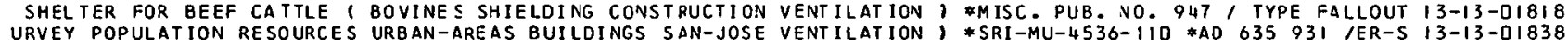

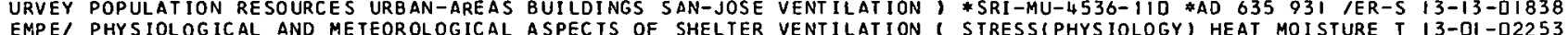
EMPE/ PHYSIOL OGIICAL AND METEOROLOGICAL A SPEC TS OF SHELTER VENTILATION ( S IRESS(PHYS IOLOGY) HEAT MOISTURE T 13 -OI-D2253

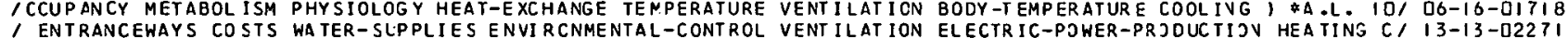

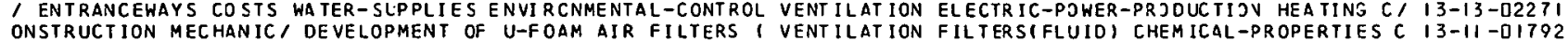
IOF ASPEN WOOD EXCELSIOR FOR USE IN EVAPORATIVE COCLERS
I VENTILATION HUMICITY COOL ING-AND-VENTILATING-EQ/ I3-OI-D2254 
TIONS I FALLOUT-SHELTER S COOLING-AND-VENTILATINö-EGUIPME/ VENTILATION KIT - ASSEMBLY AND OPEZATING INSTRUC 13 -0I-DI769 IERS ( HEAT-PRODUCTION(BIOLOGY) SLRVIVAL FALLOUT-SHELTERS VENTILAT ION METAEOL ISM BODY-TEYPEZ3ATUZE CLOTHIN OS-IS-OI7I N EAST CHICAGO, INDIANA I ENVIRONMENTAL-CCNTROL NATURAL VENT ILAT ION TEST OF A BASEMENT FALLOUT SHELTER I $13-0 I$-DI774 ER IN EVANSTON, ILLINOIS I ENVIRONMENTAL-CONTROL/ AATURAL VENTILATION TEST OF AN ABOVEGROUND FALLOUT SHELT I3-OI-OI77I EW YORK CITY AND VICINITY I FALLOLT-SHELTERS URBAN-AREASI VENTILATION TESTS OF FALLOUT SHELTER SPACES IN N 13-I3-OI8I3 EW YORK CITY AND VICINITY ( URBAN-AREAS UNDERGROUND-STRU/ VENTILATION TESTS OF FALLOUT SHELTER SPACES IN N I3-13-0I8I4 NY ROADS COVSTRUCTION URBAN-AREAS TUNNELS(TRANSPORTATION) VENTILATION TRAFFIC I*ORVL-TR-1596 IURG 1 GERMA 13-0?-DI7B6 EL CEIIVAL 6-230), AND 208, NOMINAL 9, DO0 BTU/HR, CCMPACT, VERTICAL, 230-VOLT, SINGLE PHASE, 60-CYCLE 1 MOD $13-01-01775$ ISPHERE ATMOSPHERE-MODELS WAVE-TRANSMI SSI ON LOW-FREQUENCY VERY-LOW-FREQUENCY EXTREMELY-LOW-FREQUENCY PHAS/ $20-14-02348$ ILOS IONS WAVE-TRANSM ISSION ATMOSPHERE LOW-FRE OUENCY PHASE VERY-LOW-FREQUENCY, EXTREMELY-LOW-FRE2UENCY INTI $20-14-02349$ CRADIAT WAN-TNUR IES TOXICITY CYANO-ACIDS CARBCN-NCNCXIDE VESICANTS POISONOJS-GASES LEWISITE GAS. AGE-ASSESSMENT VULNER-SYSTEMS RADIOLOGICAL-CCNTAMINATI ON VETERINARY-MEDICIVE PROTECTIVE-CLOTHING 1 *JPRS/ 15 -03-01918 I SURVIVAL - POSTATIACK SUR VIVAL DISPARI TIES AND NATIONAL VIABILITY I SOCIETAL INECOVERY ECOVJ CAPACITY DAM O5-03-02152

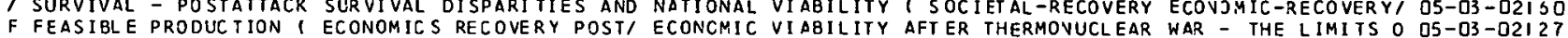
F FEASIBLE PRODUC TION I ECONOMICS RECOVERY POST/ ECONCMIC VI ABILITY AFT ER THERMOVUCLEAR WAR - THE LIMITS O O5-O3-O2I27
F FEASIBLE PRODUC TION I NLCLEAR-WARFARE SURVIVAI ECONOMIC VIABILITY AFT THERMONUCLEAR WAR - THE LIMITS O O5-03-D215B F FEASIBLE PRODUC TION I NLCLEAR-WARFARE SURVIVAI ECONOMIC VIABILITY AFT ER THERMONUCLEAR WAR - THE LIMITS O O5-D3-D2158
IAL POPULATIONS I BACILLUS-ANTHRACIS MATHEMATICAL-NODELS/ VIABILITY AND ESTIMATION OF SHELF LIFE OF BACTER I5-D2-DI849

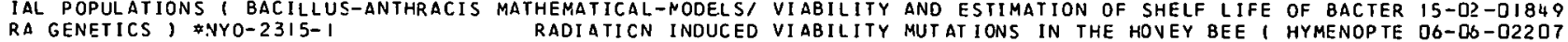
SEARCH DESIGV ELECTRON/ DAMAGES RESULTING FROM LABCRATORY VIERAT ION AND HI GH IMPACT SHOCK TESTS I NAVAL-RE $20-11$-O2OB3

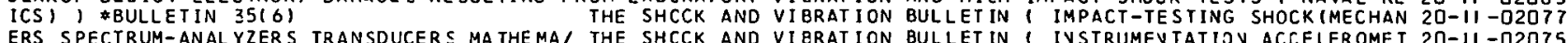
ERS SPECTRUM-ANAL YZER S TRANSDUCER S MA THEMA/ THE SHCCK AND VI ERATION BULLET IN I IVSTRUMEYTATIOV ACCELER OMET $20-11$-D2075 -ANALYSIS ELASTICITY BEAMSISTRUC TLRAL) SHC T THE SHCCK AND VIBRATION BULLET IN I ROCKETS DYNAYICS STRUC TURAL $20-11$-O2OT ON-SICKNESS STRES S(PHYSIOLOGY) A) SUPPLEMENT TO SHCCK AND VIBRATION BULLET IN I TOLERANCESIPHYSIOLOGY) MOTI O6-17-D IT35 OLATORS AIR-TRANSPORTATION RAILROADS ARMEDI THE SHCCK AND VIBRATION BULLEIIN I TRANSPORTATION VIBRATION-IS $20-11$-O2076 IPECTRA FOR A GENERAL FORCING FUNCTION I SHOCK(MECHANICS) VI QRATION ELASTICITY VELOCITY DEGREES-OF-FREEDO/ 20-11-02342 /AMICALLY LOADED RING WITH RADIAL ELASTIC SUPPORT I RINGS VIRRATION FREOUENCY BEVDIVG ELASTIEITY LOADING I/ $20-11-02336$ /SPENSIOV-DEVICES STRUCTURES VIBRATION-I SCLATORS MACHINES VIBRATION FREQUENCY PNEUMATIC-DEVICES FEASIBILI/ $13-13$-02291 ISPENSIOV-DEVICES STRUCTURES VIBRATI ON-I SCLATORS MACHINES VIBRATION FREQUENCY PNEUMATIC-DEVICES FEA SIBILI/ $13-13$ - 2291
NS I PARTIAL BIBL IOGRAPHY ON SUBJECTS RELATED TO ACTIVE VIBRATION ISOLATION AND ACTIVE VEHICLE SUSPENSIO $20-11$ - 202087 NS 1 ' PARTIAL BIBLIOGRAPHY ON SUBJECTS RELATED TO ACTIVE VIBRATION ISOLATION AND ACT IVE VEHICLE SUSPENSIO $20-11$-D2087
CAMPING I SHOCKIMECHAVICSI VIBRAIION-ISOLATORS SINULATI/ VIBRATICN ISOLATIOY THROUGH PVEUMATIC SPR ING AND $20-11$-D2343 I OF SHALLOW SP HER ICAL SHELLS 1 SPHERES STRUC TURAL-SHELLS VIBRAT ION LOADING( MECHAN ICS) SHEAZ-STRESSES DEF/ $20-11$-D2340
AL SHELLS I STRUC TURAL-SHELLS CYLINDRICAL-BODIES CCMPOSI/ VIBRATION OF MULTI-LAYERED AVISOTROPIC CYLINDRIC $20-11$-O2345

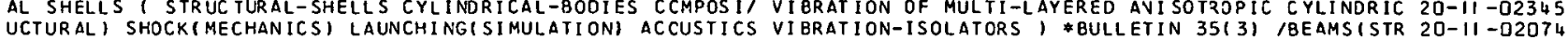
ARMEC/ THE SHOCK ANO VIBRATION BLLLETIN I TRANSPORTATION VI RRATION-ISOLATORS AIR-TRAVSPORTATIOV RAILROADS $20-11-02070$ IIVE VIBRATION ISOLATION AND ACTI VE VEHICLE SUSPENSICNS I VI BRATION-ISOLATORS CAL CULATIJVS YA THEMATICAL-M/ $20-11$-O2OB7 IHOCK. ISOLATION FOR HARDENED STRUCTURES I SHOCK-ABSORBERS VIBRATION-ISOLATORS IMPACT-SHJCK VUCLEAR-EXPLOS/ I3-13-01823 IK-WAVES LOADINGIMECHANICS) SUSPENSION-DEVICES STRUCTURES VIBRATION-ISOLATORS MACHINES VIBRATION FREQUENC/ $13-13-02291$ /-SYSTEMS BIBLIOGRAPHIES DESIGN HARDENING BLAST AIR-BURST VIBRATION-ISOLATORS SHOCK-WAVES SHJCK IMECHANICS/17-02-023II I THROUGH PVEUMATIC SPR ING AND DANPING I SHOCK(MECHANICS) VI BRATICN-ISOLATORS SIMULAT IOV MATHEMATICAL-ANA/ $20-11$-O2343 ME - PROJECT DR IBBLE SALMON EVENT I SEISMIC-WAVES / EARTH VIRRATIONS FROM A VUCLEAR EXPLDSIJN IN A SALT DO 18 -03-02319 IA.L. 1117 INALYSIS OF FACTORS AFFECTING GOVERNMENT CONTROL IN SOUTH INALYSIS OF FACTORS AFFECTING GOVERNMENT CONTROL IN SOUTH VIETNAM ( POLITICAL-SCIENCE SOC IOLJGY ECONOMICS/ O5-03-02175
ONOMIC-AID, TA.L. IDE2 VIETNAM - WHY I FOREIGN-POL ICY POLITICAL-SCIENCE D5-0+-02167 JNS ON IVTERVATIONAL AFFAIRS I ARMS-CONTRCL COTO-WD

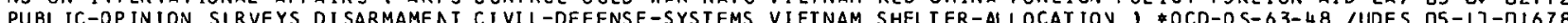
19661 ATTITUDES PURLIC-OPINI ON SURVEYS OISAR/ AMERICANS VIEWS ON CIVIL DEFENSE IN THE COLD WAR CONTEXT - O5-13-01678 I STULIES OV IMMUNITY TO TOXINS OF CLOSTRIOI UM BOTLLINUM VIII. IMMUNOLOGICAL RESPOVSE OF MAV TO PUR IFIED I5-OP -OI85? PSITTACI CUL TURE-MEDIA // PLAQLE FORMATI ON BY PSITTACOS IS VIRUS 1 BIOLOGICAL -WARFARE-AGEVTS MIYAGAWANELLA- I5-0?-DI89I /APHY - SUPPLEMENT, AUTHOR AND SUBJECT INDEXES ( BACTERIA VIRUSES FUNGI PROTOZOA RICKETTSIA PHYSIOLOGY DI/ DO-13-DI7I5 I STRAIN(MECHAN ICS) STRESS LOADING IMECHANICS) SHOCK-WAVES VISCOELASTICITY BLAST, ₹CONTZACT REPORT NO. 3-1 $20-11$-D2086 HEMAT ICAL-AN/ ACC ELERAIION WAVES IN NONLINEAR MATERIALS I VISCOEL ASTICITY MECHANICAL-WAVES PRJPAGATION MAT $20-11-02080$ NC-STRUCTURES SAND SHOCK-WAVES BLAST FOOTINGS FOUNDATIONS VOID-RATIO ARCHES, \#NCEL-R-536 \#DASA-13.018 $10020-11-01706$ IAROUNO STRUCTURES ( RADIOACTI VE-FALLOUT PARTICLES DEBRIS VOLCANOES COSTA-RICA WIND IVSTRUMEVTATIDN I *SR/ 18-03-02038 IT RACIOLOGICAL-DOSAGE STORMS DECONTAMINATION EARTHOUAKES VOLCANOES LANOSLIDES ELECTRIC-POWER-P 300 UCTION 1 15-03-02038 TA-RICA HIND INSTRUHEVTATION 1 SR/ $18-03-02038$ IGN CUSPS FLUID-F, FLOW CONTROL BY GENERATION OF STANDING VORTICES AND THE C'JSP EFFECT I HYDRODYNAMICS DES $20-04-02055$

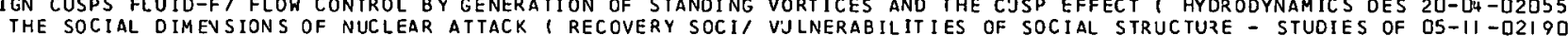

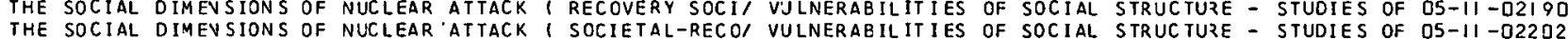
TEMS CALCULATIONS RESOURCES MANAGEMENT-PLANNING CCMPUTERS VULNERABILITY, *A.L. 1053 /ES CIVIL-DEFENSE-SYS I5-DS-DI955 AND PARAMETERS USED IN NREC DAMAGE ASSE SSMENT PROGRAMS ( VULNERABILITY, ANREC-11411) A LISTIVG OF TABLES 05-03-02138 CE ECONOMICS SOCIOLOGY S/ METHODOLOGY FOR ASSESSING TOTAL VULNERABILITY I SYSTEMS-AVALYSIS PJLITICAL-SCIEN OS-11-D2193 IRT ING SURVIVAL RECOVERY ECONOMIC-RECOVERY PRODUCTS BLAST VULNERAEILITY AIR-BURST ECONOYICS EXPLOSION-EFF/ OS-O3 -O2I20 IFFECIIVENESS TRA INING FIRE-STORMS CONFLAGRATIONS RESCUES VULNERABILITY CIVIL-DEFENSE-DPERATING-PLANS MAP/ 13-12-018D6 11 AERIAL-PHOTOGRAPHS URBAN-AREAS FIRES MAPS TEST-NETHODS VULNERABILITY CLASSIFICATION BUILOINGS, *REPOR/ 13-12-DI809 /RT IF ICIAL) SPACECRAFT DEFENSE-SYSTEMS COST-EFFECTIVENESS VULNERABILITY DATA-PROCESSING-SYSTEMS 1 *A.L. I/ $22-02-02351$ $1 Y$ REPORT FROM A RESEARCH PROGRAM I POSTATTACK-OPERATIONS VULNERAEILITY DISASTERS SOCIETAL-2ECJVERY RECOV/ OS-11-0220I IPARM MAIN ROUTINE I RESOLRCES CAPAC I TY DANAGE-ASSESSMENT VULNERABILITY ECOVOMIC-RECOVERY IVDUSTRIAL-PROD/ 05-03-02I52 SIMULAT ON DAMAGE-ASSESSMENT WARFARE RESOURCES INDUSTRIES VULNERAB ILITY ECONOMICS 1 *A.L. 1052 /URVIVAL 1 05-03-01644 1 INOUSTRIES SURVIVAL RECOVERY ECONOMIC-RECOVERY PRODUCTS VULNERABILITY EXPLOSION-EFFECTS AIRBURST POSTAT/ OS-03-02162 IECOVERY OF DAMAGED URBAN AREAS I BLAST DAMAGE-ASSESSMENT VULNERAEILITY FIRES REPAIR RAD IOACTIVE-FALLOUT 1 I5-DS-O2307

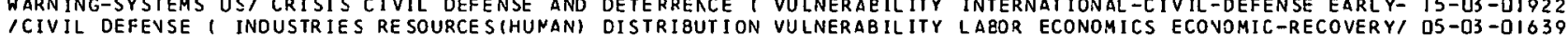

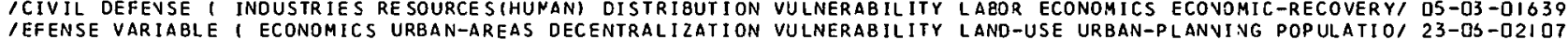

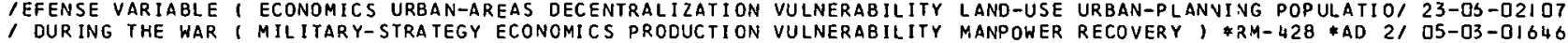
IES DAMAGE-ASSESSMEN T WEAPON S-EFFEC TS MANPOWER REFINERIES VULNERAE ILITY MILITARY-FACILITIES ARMED-FORCES-I O5-O3-D2I37 VIL-DEFEVSE RUSSIA/ CRISIS CIVIL DEFENSE AND DETERRENCE / VULNERABILITY MILITARY-STRATEGY IVTERNATIONAL-CI I5-03-DI890 / FOOC SHELTERS FALLOUT-SHELTERS EUROPE SURVIVAL RECOVERY VULNERAB ILITY MILITARY-STRATEGY THREAT-EVALUATI/ 15 -03-02295 1. MANPOHER AND OCCUPATIONS 1 DAMAGE-ASSESSMENT RE SOURCES VULNERAB ILITY MODELS MANUFACTURIVG MINING AGRIC/ OS-03-02153 INCER GROUND-STRUC TURES EXPLOSI ON-EFFEC TS SHOCK(MECHANICS) VULNERAEILITY NUCLEAR-EXPLOSIONS SHOCK-WA VES LO/ $13-13$-02291 IR STATISTICAL MODEL - NAHICUS 63 APPLICAIION I RESOURCES VULNERABILITY NUCLEAR-WARFARE WIND 8LAST RADIOA/ O5-O3-O2IL9
IOVERY ATTACK-SCENARIOS FERTILITY URBAN-AREAS RURAL-AREAS VULNERABILITY NUCLEAR-WARFARE-CASUALTIES SEX RA/ I5-OS-DI9S5 II ANALYSIS OF FOOD BALANCE I RE SOURCES DAMAGE-ASSESSMENT VULNERAEILITY POPULATION CASUALTIES FOOD-SUPPLI/ O5-03-D2I32 IAK IV - NREC RAP ID DAMAGE ASSESSMENI PROGRAM I RESOURCES VULNERAE ILITY PROGRAMMING (COMPUTEZS) CASUAL TIES/ O5-D3-021 46

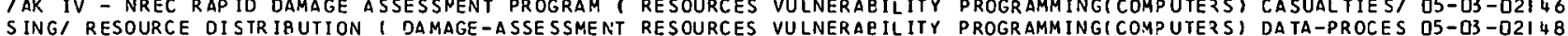

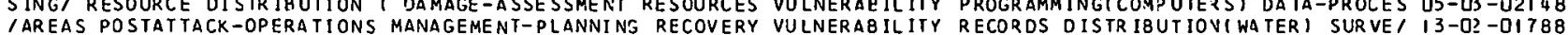
TAREAS POSTA TACK-OPERATIONS MANAGEMENT-PLANNING RECOVERY VULNERABILITY RECDRDS DISTR IBUTIOVI WA TER) SURVE/ I3-02-DIT88 IIAL AREAS I NUCL EAR-EXPLOSIONS FIRES AIRBURST STRUCTURES VULNERABILITY RECOVERY DAMAGE RECLAMATION URBAN/ I5-OS-DI963
INO COMPARISONS AMONG MODES I CARGO POSTATTACK-OPERATIONS VULNERABILITY ROADS RAILROAOS CARGO-VEHICLES AI/ I3-OS-O22OD ITIFICATIONS I MA THEMATICAL-PREDICII ON SHELTERS AIR-BURST VULNERARILITY SOIL-MECHANICS WEATHERING MILITAR/ 15-DS-DI957 T-CONCITIONS URBAN-AREAS FIRE / PARAME TERS GOVERNI NG UREAN VULNERABILITY TO FIRE FROM VUELEAZ BUZ STS 1 BURS $13-12-018$ I I ITIONS SOC IAL-RECOVERY SUR VIVAL RESOURCES (HUMAN) RECOVERY VULNERABILITY UREAV-AREAS DEMOGRAPHY COUNTERMEA/ O5-1I-QI688 IENSE ASPECTS OF MUNICIPAL WATER SYSIEMS I WATER-SUPPLIES VULNERABILITY UREAN-AREAS SURVEYS POSTATTACK-OP/ 15-03-OI941 IUREAN CONFIGURATION I URBAN-AREAS DECENTRALIZATI ON TAXES VULNERABILITY UREAN-PLANNIVG LAND-USE POPULATIO/ 23-DS-O21 O6 I SURVIVAL URBAN-AREAS DAMAGE-LIMITING-SYSTEMS EVACUATION VULNERABILITY WARVING-SYSTEMS HASTY-SHELTERS TH/ 13-13-QI829 /ASSESSMENT COMPUTATION PROGRAM - JUMBO III. I RESOURCES VULNERABILITY HEAPONS-EFFEC TS WIND RADIOACTIVE-/ O5-03-D2134
/ROUNE AND PROCEDURES FOR APPLICATIONS I ATTACK-SCENARIOS VULNERABILITY (ANALYSIS) SURVIVAL DAMAGE-LIMITIN/ I5-O3-OI949 
RED-CHINA FOREI GN-POL ICY FOREIGN-AID LABOR UNITED-NATICNS WAGES 1 *A.L. IIS4 /NTROL COLD-WAR NATO VIETNAM OS-D*-D2I72 Y MAT FEMAT ICAL-ANALYSIS RADIOLOG I CAL-CONTAMINATI GN MCDELS WALLS, \#R-OJ-266I II) IIOV BLAST SHIELDING THEOR O6-I3-O222I IION PROGRAMMING(COMPUTERS) RADI OACTI VE-FALLOUT SHIELDING WALLS CORALT EXPER IMENTAL-DATA THEORY DOSE-RATE I DS-I3-D22I 8 ACK-OPERAT IONS SOC/ SOCIAL INSTI TLTIONS AND THERMONUCLEAR WAR - A CASE STUDY OF HIGHER EDUCATION I POSTATT O5-II -DIOB8 ACK-OPERATIONS SOC/ SOCIAL INSTI TLTIONS AND THERMONUCLEAR WAR - A CASE STUCY OF HIGHER EDUCATION I POSTATT D5-II -DIOB DEMOGRAPHIC VARIABL/ STUDY OF THE DEMOGRAPHY OF NUCLEAR WAR - EMPIRICAL CJIDELINES FOR THE SELECTION OF $23-04-021$ I 3

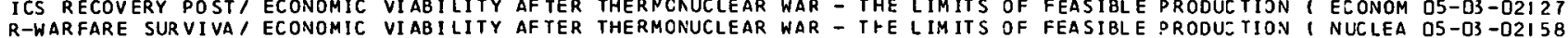
R-WARFARE SUR VIVA I ECONOMIC VIABILITY AF TER THERMONUCLEAR WAR - THE LIMITS OF FEASIBLE PRODUZTION I NUCLEA O5-O3 -D2I 58

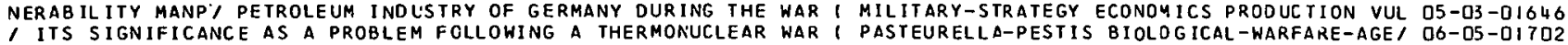
1 INTRODUCTION TO LONG TERM BIOLOGICAL EFFECTS OF NUCLEAR WAR I RACIOBIOLOGY RACIATIOV-EFFECTS ECOLOGY RAD DS-IB-DIT23 ALL ISTIC-MISSILES CO $/$ ON THE E SSENCE OF A NUCLEAR NISSILE WAR 1 RUSSIA POL ITICAL-SCIEVCE NULLEAR-WARFARE B D5-01-DIG72 OLOGY MANAGEMENT-PL/ A STUDY OF THE DEMOGRAPHY OF NUCLEAR WAR I SURVIVAL NUCLEAR-WARFARE POPULATION METHOD $23-0,-02 I$ I 4 TS 1 POSTATTACK -OPERATIONS SOIL-CONSERVATION SOI/ NUCLEAR WAR AND SOIL EROSION - SOME PROBLEMS AND PROSPEC OB-I3-OI7LS RVEYS DISAR/ AMER ICANS VIEWS ON CIVIL DEFENSE IN THE COLD WAR CONTEXT - 1966 ( ATTITUDES PUBLIE-OPINION SU O5-IJ-DIGT8 IAS EMPLOYED BY A CIVIL DEFENSE ORGANI ZATION DURING WORLD WAR II - GERMANY I. I FIRES FIRESTORMS HAMBURG, I3-I?-02255

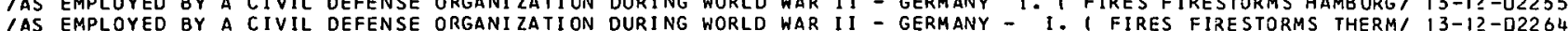
IAS EMPLOYED BY A CIVIL DEFENSE ORGANIZATION DURING WORLD WAR II - GERMANY - I. I FIRES FIRESTORMS THERMI $13-12-02264$ S DAMAGE-ASSESSMENT CASUALTIES POPULATI/ A STUDY CF WORLD WAR II GERMAN FIRE FATALITIES I FIRES URBAN-AREA I3-I?-02262
LICY ECONOMIC-AID, \$A.L. 1082

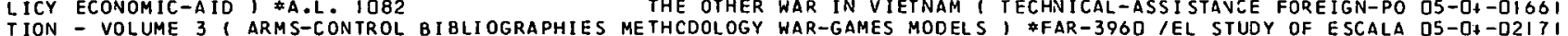

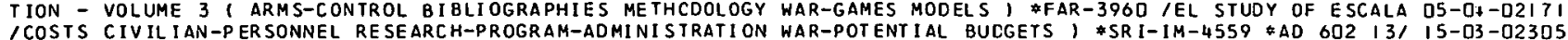
/COSTS CIVIL IAN-P ER SONNEL RE SEARCH-PROGRAM-ADMINISTRATION WAR-POTENTIAL BUCGETS / \#SRI-IM-4559 \&AD 602 I3/ I5-03-02305 C CRECIT CORPORATION WAREHOLSE RECEIPY WHEAT INVENTORY BY WAREHOUSE AND LOCATION I AGRICULTURE STORAGE SU/ D2-D2-O2IO9

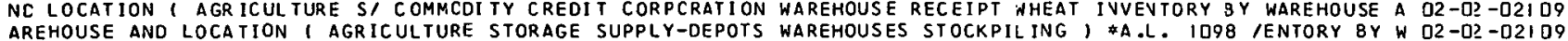
MENT FIRE-SAFETY PROGR/ RE-USABILITY OF BUILOINGS AFTER A WARFARE 1 FIRES NUCLEAR-EXPLOSIONS DAMAGE-ASSESS IS-D3-OIQS2 /RLY-WARN ING-SYSTEMS NATIONAL-DEFENSE RECRUITING TRAINING WARFARE CIVIL-DEFEVSE-PER SONVEL CASUALTIES MOBI/ I5-03-O23OS 13861 USSR ARMED-FORCES-OPERA TI ONS ARMED-FORCES (FCREIGN) WARFARE MORALE MIL ITARY-P SYCHOLOGY MILITARY-PER/ IS-DT-D23 IO ITICS OF NATIONAL SURVIVAL I SIMULATION DAMAGE-ASSESSMENT WARFARE RESOURCES IVDUSTR IES VULNERABILITY ECON/ Q5-03-OIOL 4 F-SYSTEMS INTERNATIONAL-CIVIL-DEFENSE SHELTERS EVACUATI ON WARFARE(TRAINING) MILITARY-PSYCHOLJGY ) *JPRS-4 I 5 -07-D2309 AT IONS DAMAGE-ASSESSMENT SHELTERS SHELTER-ALLOCATICN DATA WARNING-SYSTEMS, \#TECHNICAL VOTE 89 I5 I CALCUL IS-D3-DI93O PULAT ION, ANNUAL STATISTICAL REPCRT, FINANCING SHELTERS WARNING-SYSTEMS CIVIL-DEFEYSE-OPERATING-PLANS PO IS-O3-DIOTB PULATION 1 ANNUAL STATISTICAL REPCRT I FINANCING SHELTERS WARNING-SYSTEMS CIVIL-DEFEVSE-OPERATING-PLANS PO IS-03 -OIOD8 IN-AREAS DAMAGE-L IMITING-SYSTEMS EVAC UATION VULNERABILITY WARNING-SYSTEMS FASTY-SHELTERS THREAT-EVALUATIO/ I3-I3-DI829 /ENTS BIOLOGICAL-WARFARE-AGENTS NLCLEAR-WEAPONS FIRST-AID WARNING-SYSTEMS RADIOLOCICAL-CONTAMIVATION VETE/ I5-C3-OIQI 8 /TEM I FALLOUT-SHELTERS PUBLIC-OPINI ON A TII TUDES BEHAVIOR WARNING-SYSTEMS, SHELTER-ALLOCATION SHELTER-OCCU/ O5-II - II699 / PULATIOV MOBILITY ON THE LOCA TI ON OF COMNUNAL SHELTERS I WARNING-SYSTFMS LIRBAN-AREAS MJVEMEVT I \#ORO-SP-I 15-O3-01942 L-AFFAIRS SU/ CAN THE UNTIED NATIONS ORGANIZATION PREVENT WARS T PEACE-KEEPIVG PUBLIC-OP INION INTERNATIONA Q5-II -02I 95 ATERI ALS RADIOACTIVE-FALLOUT SHIELDING BU/ DESIGN CF ROOF WASHDOWN SYSTEMS (FINAL REPORT I I CONSTRUCTION-M I8-DS-O232I SPORT ATION URBAN-AREAS MANAGEMENT-PLANNI NG URBAN-PLANNING WASHINGTON BOSTON VEW-YORK I \$RPV-79 /ADS I TRAN $23-0$ ? -O2I OD INUAL FOR THE BUILDING CODE OF THE DISTRICT OF COLUMBIA I WASHINGTON BUILDIVG-CODES REGULATIJVS STRUCTUREI I3-I3 -DI8I 7 OF THE DISTRICT OF COLUMBIA I BUILDING-CODES REGULATIONS WASHINGTCN BUILDIVGS STRUCTURES, \#A.L. 995 100 I $13-13$-0I8IO IND VAN NESS STREET I TRANSPORTATION SUBWAYS TESTS BORING WASHINGTON CONSTRUCTION SETTLEMENTISTRUCTURAL), I3-02-OI785 I AS MOVING-BELTWAYS TRAFFIC OESIGN COSTS NEW-YORK CHICAGO WASHINGTCN PHILACELPHIA BOSTOV LOVDOV, $\$$ HI-82O/ $23-0 ?-02097$ EFFECTS OF FALLOUT IN WASTE WATE / POSTATTACK SANITATION, WASTE OISPOSAL, PEST AVD VECTJR COVTZJL, ANO THE I3-OZ -OI79D 1. PEST AND VECTOR CONTROL, AND THE EFFECTS OF FALLOUT IN WASTE WATER AND SENER SYSTEMS 1 POSTATTACK-OPER/ I3-02-OI73O TARY-ENGINEER ING PUBLIC-HEALTH DISPOSAL COSTS WAST/ SOLID WASTES RESEARCH NEEDS I SCIENTIFIC-RESEARCH SANI 13 -0?-QI789 IRATIONS RADIOACT IVE-FALLOUT WASTES(SANI TARY-ENG INEERING) WASTES (INDJSTRIAL) GARBAGE SAVITARY-ENGINEERING I 3 -D? -OI7BO ILY RADIOACTIVE MATERIALS ( CONTAINERS RADIOACTIVE-WASTES WASTESIINDUSTRIAL) SHIPPING-CONTAINERS, FNYO-Q, I8-OF-O2O33

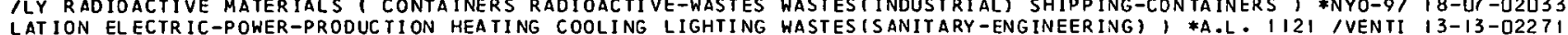

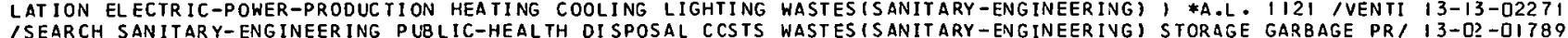

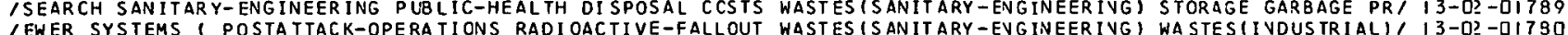

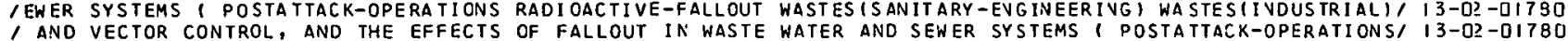
I AND VECTOR CONTROL, AND THE EFFECTS OF FALLOUT IN WASTE WATER AND SEWER SYSTEMS I POSTATTACK-OPERATIONS/ I3-02-DI7BO
GENERAL DESCRIPTION OF WORK PRO/ CONTROL OF SURFACE STORM WATER BY STORM CRAINS ANC DRAINAGE CHANNELS I. I3-OZ-OIT82 GENERAL DESCRIPTION OF WORK PRO/ CONTROL OF SURFACE STORM WATER BY STORM CRAINS ANC DRAINAGE CHANNELS I. I3-02-QI782
PLANS, PROFILES, CROSS SECTION/ CONTROL OF SURFACE STORN. WATER BY STORM CRAINS AND DRAINAGE CHANNELS II. I3-OZ-DI779 PLANS, PROFILES, CROSS SECYION/ CONTROL CF SURFACE STORM WATER BY STORM DRAINS ANO DRAINAGE CHANNELS II. I3-O2 -OITBI 58 PROGRAM I. GENERAL DESCRIPT/ CONTROL CF SURFACE STORM WATER BY STORM DRAINS AND DRAINAGE CHANNELS - IQ I3-O2 - II783 64 PROGRAM I. GENERAL OESCRIPT' CONTROL OF SURFACE STORM HATER BY STORM DRAINS ANC DRAINAGE CHANNELS - 19 I3-D? - QI7B 4 ON CAVITY RADII I CRATERING EXPLOSI ON-EFFECTSI EFFECT OF WATER CCNTENT, YIELD, MECIUM, AND DEPTH OF BUR ST IB-O3-D23I5 1 UNITED-STATES B URE I CONSIRUC TI ON AND ENGINEERING DATA - WATER CONVEYANCE TUVVELS - GRAVITY AVD PRESSURE $13-13$-O22B3 IY-ENGINEER ING MEDICAL-SUPPLIES DEFECTIONS TRAINING SLEEP WATER FOOD RECREAT ION EXERCISE NOISE RELIGION T/ OS-IJ-O2IB 4 ROTECTION-SYSTEMS WATER-SI SAFEGUARDING FOOD PRODUCTS AND WATER FROM. AGENTS OF MASS DESTRUCTION - USSR I P I 5-O2-OI843 LANNING DECISION-MAK ING SOCIAL-SC IENCES / ALTERNATIVES IN WATER NANAGEMENT ( RESOURCESIWATE2) YANAGEMENT-P D5-DI -DIG2 2 IOACT IVITY CONTROL, \#PHS/ PUBLIC HEALTH SERVICE DRINKING WATER STANDARDS I NATER-SUPPLIES IOACT IVITY CONTROL, \#PSI PUBLIC HEAL.TH SERVICE DRINKING WATER STANDARDS 1 NATER-SUPPLIES $3 E G U L A T I O N S$ RAD I3-0?-OI778 ILITIES DATA WATER-SU/ CIVIL DEFENSE ASPECTS OF MUNICIPAL WATER SYSTEMS 1 CIVIL-DEFEVSE-DPERATING-PLANS UT I3-0?-DI788
AN-AREAS SURVEYS POST/ CIVIL DEFENSE ASPECTS OF MUNICIPAL WATER SYSTEMS I WATER-SUPPLIES VULNERABILITY URB IS-O3-DIS4I

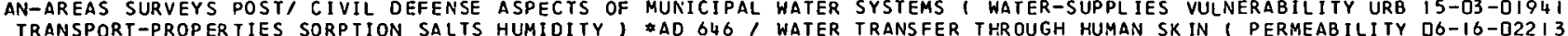
NG IA-L. UNIFORM SYSTEM OF ACCOUNTS FOR CLASS A AND B WATER UTILITIES I ECONOMICS REGIH. ATIOYS AC.CDUNTI O5-O3-DIGSD NG I *A.L I UN IFORM SYSTEM OF ACCOUNTS FOR CLASS A AND B WATER UTILITIES I ECONOMICS REGIH. ATIDVS AC.CDUNTI O5-O3-DI 65D PLIES PUR IFICATION CONTAM/ HOW TO PURIFY AND TO DISINFECT WATER WITH THE SIYPLEST MEANS - USSR 1 WA IER-SUP O6-13-DI722 ERING ANIMAL-HUSBANDRY CROP-RESEARCH ENTOMOLOGY NUTRITION WATER-CONSERVATION SOIL-CONSERVATION, *AIB-I89 O2-DJ-02ID8
A NEWERA FOR ANERICAS WATERS 1 WATER-POLLUTION WATER-SUPPLIES, *CWA-I A NEW ERA FOR AMERICAS WATERS ANERICAS WATERS I WATER-POLLUTION WATER-SUPPLIES ICWA-I $13-02-02257$ CUPANCY PSYCHOLOGY PERFORMANCE (HUNAN) INSTRUCTION-NANUALS WATER-SUPPLIES, \$GARD- I292(II) /MEVT SHELTER-OC I5-03-02277 ION-EFFECTS RURAL-AREAS PROTEC TION-FACTOR DECCNTAMINATION WATER-SUPPLIES I *JPRS-30, DO 3 /ION-DAMAGE EXPLOS I3-I3-0I826 () INSTRUCTION-MANUALS PSYCHOLOGY STRESS (PHYSIOLOGY) FOOD WATER-SUPPLIES BEODING TOILET-FACILITIES I \#GARI I S-O3-O23OD IM AGENTS OF MASS DESTRUCTION - USSR ( PROTECTION-SYSTEMS WATER-SUPPLIES CHEMICAL-WARFARF-AGEVTS RAOIOLOGI I5-O2-DI843 IFT IN C-AND-EQUIPMENT DECONTAMINATION RESCUES DISINFECTION WATER-SUPPLIES COOPERAT IOV DISASTER-MEDICINE EM/ IS-03-D2296 /ENS ING FOOD FALLOUT-SHEL TERS LOGISTICS SHELTER-OCCUPANCY WATER-SUPPLIES ECUCATION TRAIVING INFANT-DIET I/ DS-O3-DI7 I 3
/IES DUAL-PURPOSE-SHELTERS STOCKPILING ENTRANCEWAYS COSTS WATER-SUPPLIES ENVIRONMENTAL-CONTROL VENTILATIO/ I3-I3-O227/ IIES DUAL-PURPOSE-SHELTERS STOCKPILING ENTRANCEWAYS COSTS WATER-SUPPLIES ENVIRONMENTAL-CONTROL VENTILATIO/ $13-13-02271$
/HUMAN) INSTRUCTION-MANUALS PSYCHOLOGY STRESS (PHYSIOLOGY) WATER-SUPPLIES FOOD BEDDING TOILET-FACILITIES I/, I5-O3-O2298

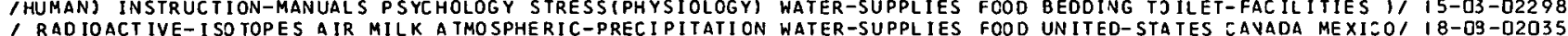
/ RAD IOACT IVE- I SO TOPES AIR MILK A TMO SPHERIC-PRECIPITATION WATER-SUPPLIES FOOD UNITEO-STA TES CAVADA MEXICO/ I8-O9-D2035
IARY-PERSONNEL POPULATION HOSPITALS SCHOOLS FOOD-SUPPLIES WATER-SUPPLIES INDUSTRIAL-PLAVTS FIVANCE STOCKP/ O5-O3-O2I 37 /-SERVICES RADIOB IOLOGY FLOODS DISASTER-MEDICINE TSUNAMIS WATER-SUPPLIES LOGISTICS TRANSPORTATION COMMUNI/ IS-O3-O2299 ICICAL-SUPPLIES ELEC TR IC-POWER SANITARY-ENGINEERING FUELS WATER-SUPPLIES MATHEMATICAL-AVALYSIS CLOTHING I/ O5-03-02I35 1 AND TO DISINFECT WATER WITH THE SIMPLEST MEANS - USSR IVALUATION OF FALLOUT CONTAMINATION FROM SURFACE RUNOFF I STOCKP IL ING FALLOUT-SHEL TERS SHE LTER-OCCUPANCY LOGISTICS 1 *PHSI PUBL IC HEALTH SERVICE DRINKING WATER STANDARDS /AL IFORNIA - FEATHER RIVER AND DELTA DIVERSION PROJECTS I POSTI CIVIL DEFENSE ASPECTS OF MLNICIPAL WATER SYSTEMS I INS - FORMULAE AND PROGRAM SPECIFICATIONS ( HYORODYNAMICS WATER-WAVES MATHEMATICAL-PREDICTION THEORY CRAT' $19-04-02043$
/IMULAT IONS) DECONTAMINATION RECOVERY FOOD (CONTAMINATION) WATER (CONTAMINATION) RADIOLOGICAL-DOSAGE RADIAT/ D6-19-DI723 WATER-SUPPLIES PURIFICATIOV COVTAYIVATION RADIO/ D6-18-01722 HATFR-SIIPPLIES RADIOLOGICAL-CJNTAYINATION RADIO/ 06-13-0I724 WATER-SUPPL IES RATIONING SANITARY-ENGINEERING R/ D6-03-01712 WATER-SUPPLIES REGULATIONS RADIOACTIVITY CONTROL 13-02-01778 WATER-SUPPLIES URBAN-AREAS ECJVOMICS COSTS, *8/ 13-0?-02258 WATER-SUPPLIES URBAN-AREAS POSTATTACK-OPERATION/ I3-Q? -DI788 WATER-SUPPL IES VUL VERAB ILITY URBAV-AREAS SUR VEYS I 5-D3-01941 
A NEW ERA FOR ANERICAS WATERS ( WATER-POLLUT ION WATEZ-SUPPLIES ) \$CWA-I 13-0?-02257 ATIONS THERMOELECTRICITY BLOYS GENERATORS PCWER-SU/ IOJ WATT THERMOELECTRIC POWER SYSTEM FOZ BUOY APPLIC ID-OZ-OITGI A COMPARISON SHOCK-TUBES CALCULATIONS EXPERIMEN/ SHOCK WAVE ATTENUAT ION FORMULAS FOR STRAIGHT TUNNELS - $20-0$ - - 205 D SED TO AIR BLAST/ THE RELATIONSHIP BETWEEN SELECTED BLAST WAVE PARAMETERS ANO THE RESPONSE JF MAMMALS EXPO O6-21 -O2Z34 IS II. APPL ICATION OF GENERALIZED COORDINATES TO PLASTIC WAVE PROBLEMS 1 SOIL-MECHAVICS MECHAVICAL-WAVES/ O8-13-0I74 7 QUARTERLY OF THE COLORADO SCHOOL OF MINES 5014$)$ SEISMIC WAVE PROPAGAT ION AVD PRESSURE MEASUREMENTS NEAR D8-II -O224? - CLAY MIXT/ THE RESPONSE OF SOILS TO DYNAMIC LOACINGS - WAVE VELOCITIES THROUGH PARTIALLY SATURATEO SAND $20-0 I$-D2O4 7
IL TERRESTRIAL WAVEGUIDE ENVIRONMENT I NUCLEAR-EXPLOSIONS WAVE-TRANSMISSION ATMOSPHERE LOW-FREQUENCY PHAS/ 20-I7-D23L9 /EGUICE I NUCLEAR-EXPLOSIONS IONOSPHERE ATMOSPHERE-MODELS WAVE-TRANSMISSION LOW-FREQUEVCY VERY-LOW-FREQUE I 20-1'-02348 N TRANSOUCERS OSC ILLOSCOPES CAMERAS REFLECTION REFRACTION WAVE-TRANSMISS ION THEORY I \$A I IIOQ 1 PROPAGATION AND PRESSURE MEA SURENENTS NEAR EXPLOSIONS ( WAVEFORM-GENERATORS INSTRUMEVTATIOV TRANSDUCERS/ O8-II -O22 4 ? /TROMAGNET IC PULSE PROPAGATION IN A DISTURBED TERRESTRIAL WAVEGUICE I NUCLEAR-EXPLOSIONS IOVDSPHERE ATMOSI 2D-I:-D2348 /CTROMAGNETIC PULSE PROPAGATION IN THE NORMAL TERRESTRIAL WAVEGUICE ENVIRONMENT I NUCLEAR-EXPLOSIONS WAVE/ $20-14-02349$ LAE AND PROGRAM SPECIFICATIONS I HYDRODYNAMI/THE SURFACE WAVES FROM SHALLON UNDERWATER EXPLOSIONS - FORMU I9-C\&-O2OL 3 PRESSURE-GAGES, \#ORNL-TR-1' THE PROPAGATION OF AIR SHOCK WAVES IN A TUBE 1 SHOCK-TUBES EXPERIMENTAL-DATA 20-OH-O2DS3 ITY PROPAGATION STRAINIME/ ON LONGITUDINAL AND TRANSVERSE WAVES IN ELASTIC RODS I MECHAVICAL-WAVES ELASTIC $20-I I-02082$ RESSES PROPAGATION E/ STUDIES ON LONGITUOINAL AND BENDING WAVES IN LONG ELASTIC RODS I YECHAVICAL-WAVES ST $20-1 /$-O2D79 ECHAN ICAL-WAVES PROPAGATION MA THENATICAL-AN/ ACCELERATION WAVES IN NONL INEAR MATERIALS I VISCOELASTICITY M 20-II -D2OBO NO. $10 \%$ NOMOGRAM FOR CALCULATING ATTENUATION OF AIR SHOCK WAVES IN TUNNELS I PROPAGATIOV SWEDEV I \#REPORT 20-0 + -O2OSI KNOW AND THINK ABOUT THE BOMB AND ATOMIC/ THE ONLY THING WE HAVE TO FEAR IS FEAR ITSELF - WHAT THE PEOPLE O5-II -02I 96 ATE-I INFORMATION SYSTEM NETWORKS - LETS PROFIT FRCM WHAT WE KNOW I INFORMAT ION-RETRIEVAL DJCUMENTATION ST DS-O? -DI 63O (LAYERED PLATE SUBJECT TO THE THERMAL PULSE OF A NUCLEAR WEAPON ( CALCULATIONS PROGRAMMINGICOMPUTERS) TH, I3-12-DI 797 $S$ I SHIELDING PATIENTS RADIOA/ PROTECTION AGAINST NUCLEAR WEAPON FALLOUT IN EMERGENCY HEALTH SERVICES UNIT I8-OS-O2O28 IRIBUTIOYS PROBAB ILITY-DENSITY-FUNCTIONS RANDOM-VARIABLES WEAPON-SYSTEMS ANALYSIS-OF-VAR IAYCE I *TM-K-77I/ I2-OI -O225I IAN POPULATIOV AS A MEASURE OF PRCTEC TION AGAINST NUCLEAR WEAPONS I JSSR SHELTERS IVTERYATIJVAL-CIVIL-DEFI I5-O3-OIO3L (N) WARFARE MORALE MILI TARY-PSYCHCLOGY MILITARY-PERSCNNEL WEAPONS ARMAMENT YILITARY-STRATEGY I * JPR S-4I, $4 /$ I5-D7 -D23 I D ISTIGATION OF SCHOOL DESIGNS TO RESIST INTEGRATED NUCLEAR WEAPONS EFFECTS I STRUCTURES FALLOUT-SHELTERS B/ I3-I3-OI82I -CIVIL-DEFENSE CI / SIMPLE SHELTERS FOR PROTECTION AGAINST WEAPONS OF MASS CESTRUCTIOV I USSZ IVTERNATIONAL I3-I3-QIB26 USSR CIVIL-DEFENSE-SYSTEMS RESCUES INTER/ DEFENSE AGAINST WEAPONS OF MASS CESTRUCTIOV IV THE RURAL AREA I IS-OS-DIOI 8 UT-SHEL TER-SURVEY (NFSS) FALL/ COMBINED EFFECTS OF NUCLEAR WEAPONS ON NFSS TYPE STRUCTURES I NATIONAL-FALLO I8-O3 -0I995 /CTION) URBAN-AREAS DISTRIBUTION HIROSHIMA NAGASAKI BURNS WEAPONS-EFFECT RADIATION-INJQRIES STATISTICAL-A/ DG-2I -02238 IME PFASED ACCESS IBILITY OF RE SOURCES I DAMAGE-ASSESSMENT WEAPONS-EFFECTS I $\$ N R E C-25$ O CISTR IBUT IOV SERV ICES SURPLUSES DATA-PROCESSING SHCRTAGES WEAPONS-EFFECTS, \#NREC-31 /N MODEL 1 RESOURCES D5-03-02I 43 1 BEHAVIOR DISASTERS PUBLIC-OPINICN ATII TUDES NETHCDOLOGY WEAPONS-EFFECTS COMMUNICATIOV-SYSTEMS ECONOMIE-/ O5-IJ-OIG77 INS VULNERABILITY ROADS RAILROADS CARGO-VEHICLES AIRCRAFT WEAPONS-EFFECTS DAMAGE-ASSESSMENT TRUIKS I \#SRI/ I3-OS-O2260 IALYSIS II. TECHNICAL APPENDIXES I CIVIL-DEFENSE-SYSTEMS WEAPONS-EFFECTS EXPLOSION-EFFECTS BLAST-SHELTER/ I5-D3-DIO33 IY CATA I NUCLEAR-WARFARE-CASUALTIES STATISIICAL-AMALYSIS WEAPONS-EFFECTS FIROSHIMA VAGASAKI GRAPHS BIOLO/ O6-2I-02227 THEMAT ICAL-MODELS COMPUTERS CASUALTIES DAMAGE-ASSESSMENT WEAPONS-EFFECTS MAVPOWER REFIVERIES VULNERABILI 05 -O3 - 22137 IPUTER PROGRAM I SYSTEMS-EVALUATI CN CI VIL-DEFENSE-SYSTEMS WEAPONS-EFFECTS MODELS EXPLOSION-EFFE-TS RADIOAI I5-D3-DIO38 IAL UNITED STATES 1 GEOGRAPHY RE SOURCES DAMAGE-ASSESSMENT WEAPONS-EFFECTS NUCLEAR-WARFARE ATTACK-PATTERNS/ OS-O3-O2ILD OLIC EY A FINITE DIFFERENCE TECHNIQUE I THERMAL-RAOIATION WEAPONS-EFFECTS NUCLEAR-WEAPOVS I WPZ-4 IINITE S I3- I?-OIT95 ILEAR ATTACK I RECOVERY SOCIETAL-RECOVERY NUCLEAR-WARFARE WEAPONS-EFFECTS POSTATTACK-OPERATIOVS DISASTER S/ QS-II -O2I 90 IAR IOS NUCLEAR-WARFARE-CASUALTIES CASUALTIES CALCULATIONS WEAPONS-EFFECTS RADIATION-EFFECTS ECOLOGY WIND / O6-2I -02228 / NUCLEAR-WARFARE-CA SUALTIES CASUALTIES MILITARY-STRATEGY WEAPONS-EFFECTS RAOIATION-EFFECTS POPULATION PA/ O6-2I -02229 COSE-RA/ SEDAN PROJECT 62.8DC AERORADIOACTIVITY SURVEY 1 WEAPONS-EFFECTS RADIOACTIVE-FALLOUT DISTRIBUTION I8-D3-O2324 IOLOGY SYSTEMS-EVALUATION OPERATI ONS-RESEARCH NATHEMATICS WEAPONS -EFFECTS RADIOACTIVE-FALLOUT FALLOUT-SHEI I S-O3 -OI93S IMAL-RADIATION GEOMETRY MA THEMATICAL-MODELS HEAT-TRANSFER WEAPONS-EFFECTS SHIP-STRUCTURAL-COMPONENTS NAVA/ I3-I?-OI797 ILATORS SHOCK-WAVES SHOCK (MECHANICS) ATIENUATION SHELTERS WEAPONS-EFFECTS SURVIVAL (EQUIPMENT) STRUCTURES, I 7-O2-D23II IPUTATION PROGRAM - JUMBO III. I RE SOURCES VULNERABILITY WEAPONS - EFFECTS WIVO RAOIOACTIVE-FALLOUT CLIMAT/ O5-D3-O2I 34

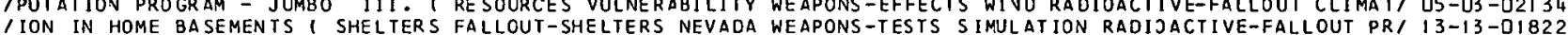

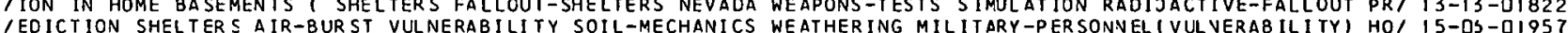

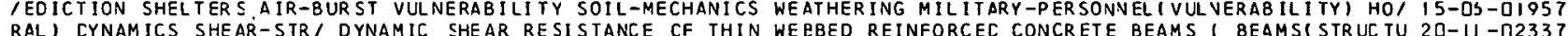
RAL) CYNAMICS SHEAR-STR/ DYNAMIC SHEAR RESISTANCE CF THIN WE RBED REINFORCEC CONCRETE BEAMS 1 8EAMSISTRUCTU $20-11$-D2337 -FITNESS NUTRITION, FAD 633860 A DOCUMENTARY ON WEIGHT, OIET AND EXERCISE 1 BJDY-WEIGHT PHYSICAL DO-I + -DI7I6 IRS TENSILE-PROPERTIES PLASTICITY STRUCTURAL-SHELLS RINGS WELDING STRESSES DEFLECTION ROLLIVGIMETALLURGY)/ $20-I I$-D234 7 ANCY POSTATIACK-OPERATIDNS CIVIL-DEFENSE-OPERA/ EMERGENCY WELFARE REGISIRATION AND IVOUIRY I SHELTER-OCLUP O5-1I -D2IB9

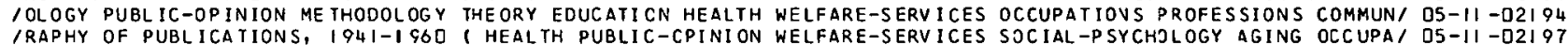
A CIVIL DEFENSE PUBLIC SHFI TF/ STLOY OF THE PROPOSED EAST WEST FREEWAY TUNNEL WEST ORAVGE, VEW JERSEY, AS I3-13-D227I IC SHELTE/ STUDY OF THE PROPOSED EAST WEST FREEWAY TUNNEL WEST ORANGE, NEW JERSEY, AS A CIVIL UEFENSE PUBL I3-I3-O227I /R FLOW AND AIRBORNE SOUND IN A DLCT / ACCUSTICS GAS-FLOW WEST-GERMANY DUCTS LAMINAR-BOUNDAZY-LAYER STABI/ 2O-DI-D2D48 IENCE RESEARCH BY PRIVATE SCHOLARS AND ACADENIC CENTERS - WESTERN EUROPE, GREAT BRITAIV AND CAVADA I BIBLI O5-II -OI694 IENSE ( CIVIL-DEFENSE-SYSTEMS INTERNATIONAL-CIVIL-CEFENSE WESTERN-EUROPE NATO GREAT-BRITAIN FRANCE GERMAN/ I5-03-02I 47 RADIATED-FOODS MEAT MILK PORK POTATOES SEAFOOD VEGETABLES WHEAT I ARB-NO CULTURE SI COMMODITY CREDIT CORPORATI ON WAREHOUSE RECEIPT WHEAT INVENTORY EY WAREHOUSE AND LOCATION I AGRI O2-OP-D2I D URAL-ECONOMICS, FFAS-M-IUC 117 CANADIAN WHEAT MARKET ING I FOOD-MARK ETING CAVADA AGRICULT O2-0 -01617 VIETNAM - WHY, FOREIGN-POLICY POLITICAL-SCIEVCE, $\$$. L. I D5-0*-02167 ANTIMISSILE-DEFENSE SYSTEMS MILITARY-STRATEGY, \#HI-729/ WHY COUPLE BMD TO FALLOUT SHELTERS I URBAN-AREAS I5-O3-CI9D7 I AN AREA BETWEEN THE HOLLAND TLNNEL AND THE MANHATTAN AND WILLIAMSBURG BRICGES ANC ALTERNATIVE SOLUTIONS / $23-02-02357$ ON RACIOACTIVE-FALLOUT BIOLOGICAL-DOSAGE ATTACK-SCENARIOS WIND) \$RM-47D7-TAB /LE-SIZE SHELTERS DISTRIBUTI O6-2I-D2229 ICAL-PREDICTION YIELD GEOMETRY DISTRIBUTICN PARTICLE-SIZE WINO; \#USNROL-TRC-68/IOACTIVE-FALLOUT MATHEMAT I8-O3-O2O39 163 APPLICATION I RE SOURCES VULNERABILITY NUCLEAR-WARFARE WIND BLAST RADIOACTIVITY THEORY TESTS EXPERIMEN/ D5-03-02I 49 /-CONCITIONS RADIATION-INJURIES YIELD RADIOLOGICAL-DOSAGE WIND FIRES SIMULATION BLAST STATE-JF-THE-ART-RE/ I5-DS-DI962 RACIOACT IVE-TALLOUT PARTICLES DEBRIS VOLCANOES COSTA-RICA WIND INSTRUMENTATION, \$SRI-MU-57T9(B), /TURES I, IB-D3-O2O3B -2046-1 FIRESTORM ANALYSIS (FIRES WIND MET EOROLOGY COMPUTERSIPRJGRAMYIVG) I *DC-TN I3-I?-OIBI5 ITAL-RECOVERY ECONOMIC-RECOVERY RECOVERY ATIACK-SCENARIOS WIND NUCLEAR-WARFARE RADIATIOV-INJURIES RADIOACI OS-O3-O2I 60 ITAL-RECOVERY ECONOMIC-RECOVERY RECOVERY ATTACK-SCENARIOS WINO NUCLEAR-WARFARE RADIATION-INJURIES RADIOAC OS -OS-D2I 7CERTAINTIES IN THE HIROSHIMA EXPLOSI ON I AIR-BURST YIELD WINO NUCLEAR-WARFARE-CASUAL TIES TARGETS SCALEIRI I5-OS-OIQSB IS CALCULATIONS WEAPONS-EFFEC IS RADIATION-EFFECTS ECOLOGY WIND RACIOACT IV ITY (DISTR IBUTIJVI ZECJVERY JAPANI DS-2I -D22 ?B

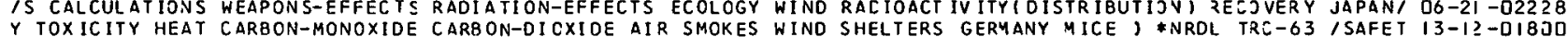
Y TOXICITY HEAT CARBON-MONOXIDE CARBON-DICXIDE AIR SMOKES WIND SHELTERS GERMANY MICE, BNRDL TRE-63 ISAFET I3-I?-DI8OD
IMPUTERS) CASUALTIES FACILITIES PERSONNEL ATTACK-PATTERNS WIND YIELD BURST-CONDITIONS BLAST RADIOACTIVE-F/ D5-O3-D2I 46 IAT ICAL METHOD OF LOCATING FALLOUT ON A SPHERICAL EARTH 1 WIND YIELD RADIOACTIVE-FALLOUT PRJGRAMMINGICOMPI IB-OB-O2322 /RS FIRE-DETECTOR S CARBJN-MONOXIDE-INDICATORS TENPERATURE WIND-PRESSJRE PRESSURE HEAT-TZANSUISSION TESTSI/ I3-I?-DI8O7 L RADIATION RELEA SED IN NUCLEAR DETONATI ONS I GLASS ATTE/ WINDOW AND WINDOW SCREENS AS YODIFIERS OFF THERMA I3-I?-DI796 RELEASED IN NUCLEAR DETONATIONS I GLASS ATTE/ WINDOW AND WINDOW SCREENS AS MODIFIERS OF THERMAL RADIATION I3-IZ-QIT96 119701 RACIO-COMMUNICATION-SYSTENS COMMUNICATION-SYSTEMS WIRE-CONMUNICATICN-SYSTEMS MODELS COSTS COMMAND/ I 7-0?-D23I 3 1 CONCEPTS OF SUBSURFACE REAC TOR C CNTAINMENT STRUCTURES TO WITHSTAND DIFFEREVT IAL EARTH DISPLACEMENT 1 REA $/$ I 8 -OP-D2OL 0 VENIILAT ION HUM ID ITY COOLING-AND-VE/ PERFORMANCE CF ASPEN WOOD EXCELS IOR FOR USE IN EVAPORATIVE COOLERS 1 . I3-OI -O2254
I CALORIMETERS IGNITION HIGH-T/ TEMPERATURES ATTAINED IN WOOD EXPOSED TO HIGH INTEVSITY THERMAL RADIATION I3-I?-DI8D2 UCTURAL-PROPERT IES QUALITY FASTENERS GLUING PLYWOOD SAND/ WOOD HANDBOOK I CONSTRUCTION DESIGY STRENGTH STR II-I?-DITOO UCTURAL-PROPERTIES QUALITY FASTENERS GLUING PLYWOOD SAND/ WOOD HANDBOOK I CONSTRUCTION DESIGV STRENGTH STR II-I2-DIT66
\$PB 173 ES9

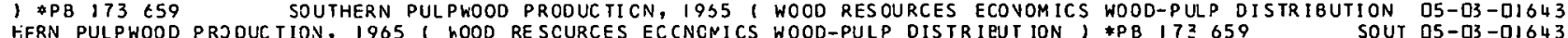
BJECT-INDEXING DEPAR TMENT-OF-DEFE ISE DOCUNENTATION EI KEY WORDS ANC IHEIR USE ON DD FORY IL73 I REPORTS SU DS-D?-D2I.24 

STEMS USSR RECONNAISSANCE INTERNA/ ENGINEERING ANO RESCUE WORK IN A NUCLEAR STRIKE ZONE I CIVIL-DEFENSE-SY DG-OT-DI7O9
I DRAINS AND ORAINAGE CHANNELS I. GENERAL DESCRIPTION OF WORK PROPOSED ANC MAP SHOWING GENERAL LOCATION / I3-D? -OI782 INAGE CHANVELS - 1958 PROGRAM I. GENERAL DESCRIPTICN OF WORK PROPOSED ANC YAP SHOHING GENERAL LOCATION / I3-OZ-GI783

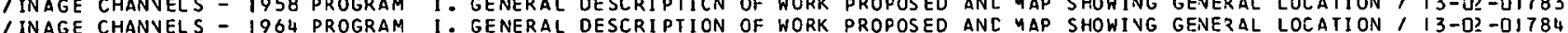
IINAGE CHANVELS - 1964 PROGRAM I GENERAL OESCRIPTION OF WORK PROPOSED ANC MAP SHOWIVG GENER AL LOCATION 1 I3-0? -DI784 STATT ACK-OPERATIONS SOCIETAL-RECOV/ CONSUNER BEHAVIOR AND WORKER PARTICIPATION IN RECOVERY ACTIVITIES I PO O5-I]-D2I80 ATE-GOVERNMENTS ACMINISTRATION-AND-MANAGENENT INSU/ STATE WORKMEN COMPENSATION LAWS I LEG ISLATION LABOR ST O5-DF-D2174
TOKYO AND KYOTO, JAPAN I JAPAN/ PROCEEDINGS OF THE SECOND WORLO CONFERENCE OV EARTHQUAKE EVGIVEERING II. O8- 11 -D2246

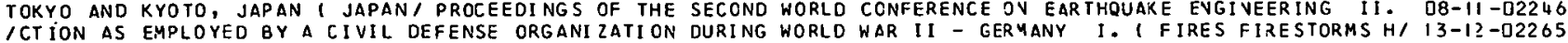
ICTION AS EMPLOYED BY A CIVIL DEFENSE ORGANI ZATION OURING WORLD WAR II - GERMANY - I I FIRES FIRESTORMSI I3-I?-O22 64 N-AREAS DAMAGE-AS SESSMENT CASUALTIES POPULATI/ A STUDY OF WORLD WAR II GERMAN FIRE FATALITIES I FIRES URBA I3-IZ-02262 INTERNAT IONAL-CIVIL-DEFENSE WESTERN-E UROPE NATO GREAT-B/ WORLD WIDE CIVIL DEFENSE I CIVIL-DEFEVSE-SYSTEMS I5-D3-D2I 47 UN IVERSAL T/ COORDINATE CONVERSICN FORMULAS FOR USE WITH WORLD WIDE RESOURCES GEODETIC POSITION I GP, TO OS-D3-O2I 33 IR CISASTER-MEDICINE NUCLEAR-WARFARE-CASUALTIES FIRST-AID WOUNOS-AND-INJURIES RADIATION-INJURIES BIBLIOGRI O6-OS-0I7D3

Y

CHANIZED INFORMATION SYSTEM I TECHNICAL-INFORMATION FIVE YEARS IN FOCJS - THE OJUGLAS AIRCRAFT COMPANY ME OS-D?-D2II8 ERMODYNAM ICS SOLUBIL I TY TEMPERATURE DIFF USIVI TY HALF-LIFE YIELD, \$GA-7D58/E-DECAY RADIOACTIVE-FALLOUT TH I8-D3-D2325 HENRYS-L AW HALF-LIFE DISTRIBUIION COMPUTERS (PROGRAMMING) YIELD, \#GA-7598 /ADIOACTIVE-DECAY PARTICLE-SIZE I8-O3-02037 (RS) CASUALTIES FACIL ITIES PER SONNEL ATTACK-PATTERAS WIND YIELD BURST-CONDITIONS BLAST 2 ADIJACTIVE-FALLOUI O5-D3-D2IUG LTIES FIRES PROGRAMMINGICOMPUTERS) BURST-CONDITIONS BLAST YIELD GEOGRAPHY, GNREC-2 /OUT CLIMATOLOGY CASUA OS-D3-D2I 34 I OF MODELS \& RAD IOACTIVE-FALLOUT NATHEMATICAL-PRECICTION YIELD GEOMETRY DISTRIBUTIOV PARTILLE-SIZE WIND I I8-O3-O2O39 IL METHOD OF LOCATING FALLOUT ON A SPHERICAL EARTH I WIND YIELO RADIOACTIVE-FALLOUT PROGRAMMINGICOMPUTERS/ IB-O3-0232 /ASUALTIES DEMOGRAPHY BURST-CONDITIONS RADIATICN-INJURIES YIELD-RADIOLOGICAL-DOSAGE WIND FIRES SIMULATION/ I5-DS-OI962 NCR ICAL SHELL (MOD/ AN EXPERIMENTAL INVESTIGATION OF THE YIELO STRENGTH OF A MACHIVED RIVG STIFFENED CYLI $20-11$-02347 1 ON UNCERTAINTIES IN THE HIROSHINA EXPLOSI ON, AIR-8URST YIELD WIND NJCLEAR-WARFARE-CASUALTIES TARGETS SI I5-DS-DIQ58 1 I CRATERING EXPLOSION-EFFECTS/ EFFECT OF WATER CCNTENT, YIELD, MEDIUM, AND DEPTH OF BURST ON CAVITY RADI I8-D3-U23IS CTION 1 BLAST-SHELTERS UNDERGROUND-STRUC TURES DYNANICS S/ YIELOING MEMBRANE ELEMENTS IN PROTECTIVE CONSTRU I3-I3-OIB37 IN ADMINISTRATION, OFFICE OF THE MAYOR OF THE CITY OF NEW YORK ANC FOR NEW YORK CITY TRANSIT AUTHORITY I / $23-02-02355$ AREAS/ VENTILATION TESTS OF FALLOLT SHELTER SPACES IN NEW YORK CITY AND VICIV ITY I FALLJUT-SHELTERS URBAN- I3-I3-DIBI3 AREAS/ VENTILATION TESTS OF FALLOLT SHELTER SPACES IN NEW YORK CITY AND VICIVITY I FALLJUT-SHELTERS URBAN- I3-I3-DIBI3 -STRU/ VENTILATION TESTS OF FALLOLT SHELTER SPACES IN NEW YORK CIYY AND VICIVITY I URBAV-AREAS UNDERGROUND I3-I3-OIBI CFFICE OF THE MAYOR OF THE CITY OF NEW YORK AND FOR NEW YORK CITY TRANSIT AUTHORITY I URBAN-AREAS I \#A./ $23-02-02355$
CENTAGES I STATISTICAL-ANALYSIS SOCIAL-SCIENC, TABLES FOR YULES O ASSOCIATION COEFFICIEVT FJR PAIRS OF PER OS-II-O22OD

E AGR ICUL TURAL-ECONOMICS, \#AS-M-184

NEW ZEALAND LIVESTOCK AND MEAT IVDUSTZY I AGRICULTUR Q2-0?-02112 TISSUES(BIOLOGY) TE STES RATS MORPHOLOG/ THE PATHOLOGY OF ZINC DEFICIENCY 1 YETABOL ISM DEFILIEVEY-DISEASES DO-OS-DI7JI
INTERNA/ ENGINEERING AND RESCUE hORK IN A NUCLEAR STRIKE ZONE 1 CIVIL-DEFENSE-SYSTEMS USSR RECONNAISSANCE DG-OT-OITOS

REPORT NUMBER INDEX

RENEWAL MANAGEMENT-PLANNING INTERGOVERNMENTAL-RELATIONS , \#A.L. IODO $/ 1$ URBAN-AREAS URBAN-PLANNING URBAN- 23-O6-02I D5 INGTON BUILDING-CODES REGULATIONS STRUCTURES BUILCINGS, \#A.L. IODI IDE OF THE OISTRICT OF COLUMBIA I WAS I3-I3-0IBI 7 RS PUQL IC-OPINION ANXIETY CIVIL-DEFENSE-SYSTENS SURVEYS, \#A.L. IOO2 ION SURVEY I ATYITUDES FALLOUT-SHELTE OS-ID-DI685 NC \& WATER UIILITIES I ECONOMICS REGULATICNS ACCCUNTING, \#A.L. IOO3 /ORM SYSTEM OF ACCOUNTS FOR CLASS A A O5-O3-OIGSO B ELECTRIC UTILITIES I ECONOMICS ACCOUNTING REGULATIONS, \#A.L. 1004 , SYSTEM OF ACCOUNTS FOR CLASS A AND OS-O3-DIO3I ANE B GAS UTIL ITIES R ECONOMICS ACCOUNTING REGULATIONS, \#A.L. IOOS /IFORN SYSTEM OF ACCOUNTS FOR CLASS A OS-D3-0IG32 ANC ING ANCING OPERATING-COSTS CANADA CONSTRUCTICA PRICES LAEOR, \#A.L. 1017 /TORAGE SALES REVENUE UTILIZATION FIN O5-D3-DIG42 6 ACR ICULTURE COSTS PRODUCTION CONSUNPTION FACILITIES, \#A.L. 1018 AG

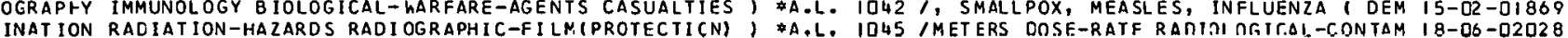

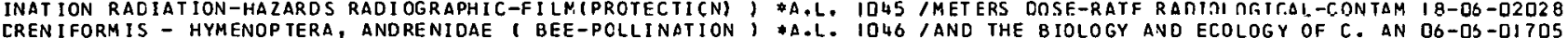

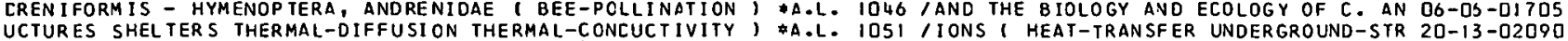

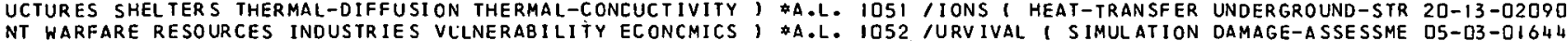

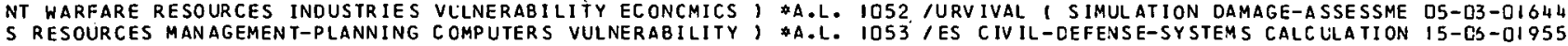
ACIOLOGICAL-CONTAMINATION RADIOACTIVE-FALLCUT HYDRCLCGY, \$A.L. IO54 /OM SURFACE RUNOFF 1 WATER-SUPPLIES R O6-IS-DIT24 ION SURVEYS DEMOGRAPHY COMMUNICATION-SYSTENS LEADERSHIP, \#A.L. IO55/TUDES PUBLIC-OPINION PUBLIC-INFORMAT OS-IJ-QIG83 CHANGE TEMPERATURE VENTILATION BODY-TEMPERATURE COCLING, \$A.L: IO57 /UPANCY METABOLISM PHYSIOLOGY HEAT-EX D6-I6-DI7I8 UCLEAR-POWER-PL ANTS ELECTRIC-POWER-PRODUCTION ECONCMICS, \#A.L. 105810 THE PRESIDENT I NUCLEAR-REACTORS N I8-DS-02027 IT IC-STATES-GOVERNMENT POLIIICAL-SCIENCE FOREIGN-PCLICY, AA.L. ID6I, OF ECONOMIC ADVISERS I ECONOMICS UN O5-D3-DIG34 -SPEED-GROUND-TRANSPORT RE SEARCH-PROGRAM-ADNINISTRATION ) \#A.L. 1062 /SPORTATION STUDY ( PENNSYLVANIA HIGH $23-02-02092$ D TRAINEES IN THE U.S. AND IN OTHER COUNTRIES, CULTURE, \#A.L. IDG3 /VATE RESEARCH ON FOREIGN STUDENTS AN OS-II-OIG9I MECICINE EMERGENCY-HEAL TH-SERVICES CANADA MANUALS FILMS, \#A.L 1068 CATALCGUE OF PUBLICATIDNS I DISASTER- D6-2I-OI737 MECICINES ERERGENCY -SUPPLIES NUCLEAR WARFARE-CA SUALTIES STOCKPILING CANADA ' \$A.L. 1069 TDMINISTRATION-AND-MANAGEMENT MEDICAL O6-2I -DI739 -SYSTEMS STRESSIP SYCHOLOGY PUBLIC-I NFORNATION MCVENENT, \#A.L. IOZI /ANN ING UT ILITY-TUVNELS COMMUNICATION I3-13-DI83I

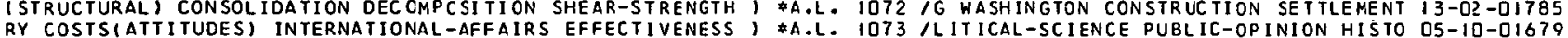

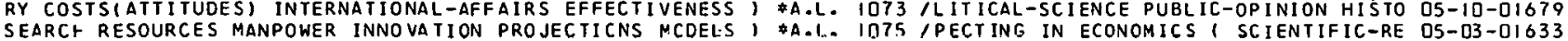
SEARCF RESOURCES MANPOWER INNOVATION PROJECIICNS MCDELS, \#A.1., ID75, PECTING IN ECONOMICS 1 SCIENTIFIC-RE OS-D3-01633

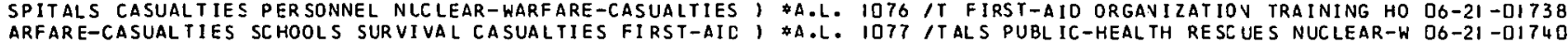
-MODELS METHODOLOGY POPULATION URBAN-AREAS DISTRIBLTION, \#A.L. 1078 /LEAR-WARFARE-CASUALTIES MATHEMATICAL O6-2I-OI742 IS I FLOOOS FLOOD-CONTROL CALIFORNIA CONSTRUCTICN COSTS, *A.L. 1079, S SHOWING GENERAL LOCATION OF PROJEC I3-O2-OI784 FLOOCS FLOOD-CONTROL CALIFORNIA FINANCING CONSTRUCTICN, \#A.L. 1080 /OWINE GENERAL LOCATION OF PROJEC TS 1 I3-D2 -DI7B2 LOCATION OF PROJECTS I FLOODS FLCOD-CONTRCL CALIFCRNIA, \#A.L. IOBI IORK PROPOSED AND MAP SHOWING GENERAL I3-O2 -DITB3 TNAM I TECHNICAL-ASSISTANCE FOREIGN-POLICY ECCNOMIC-AID, \$A.L. 1082 THE OTHER HAR IN VIE OS-O4-OIO6I RGY-CONVERSION REACTOR-SAFETY-SYSTEMS RADIOACTIVE-hASTE； \#A-L. I083 /TERIALS FLUIC-DYNAMIC-PROPERTIES ENE 20-D9-02070 - ANALYSIS DIFFERENTIAL-EQLATIONS COMPUTERS (PROGRANNING), \#A.L: 1085 /UNC-STRUCTURES DYVAMICS MATHEMATICAL I3-I3-OIB33 PDPLLATION COMMAND-A $15-03-01908$ MMUNICATION-SYSTEMS ECONOMIC-RECOVERY MILITARY-STRATEGY, \#A.L. 1095 /TUDES METHODOLOGY WEAPONS-EFFECTS CO OS-ID-OI G77

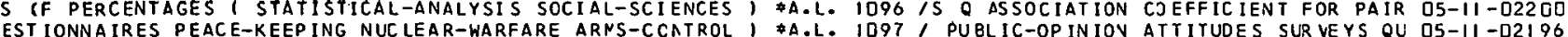

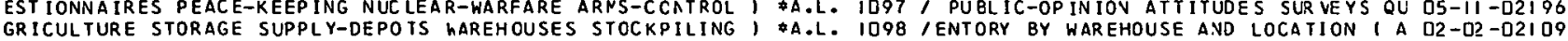
GRICULTURE STORAGE SUPPLY-DEPOTS MAREHOUSES STOCKPILING
CAL-SCIENCE EDUCATION GROUP-DYNAMICS THEORY METHODCLOGY, INING ECONOMICS INTERNATIONAL-AFFAIRS POLITICAL-SCIENCE, \#A.L. IIOD /ROFESS IONS COMMUNICATIOV-SYSTEMS TRA D5-II-D2I Q4 TROL HUMIOITY AIR TOLERANCES(PHYSIOLOGY) THERNCDYNANICS, \#A.L. IIO2, ISTURE TEMPERATURE ENVIRONMENTAL-CON $13-0 I$-O22S3 ICS OIRECT-CURRENT STA TE-OF-THE-ART INDUSTRIAL-RESEARCH, \#A.L: IOS /LINES COSTS ALTERVATE-LURRENT ECONOM O9-O3-02249 
VIETNAM - WHY ( FOREIGN-POLICY POLITICAL-SCIENCE) \#A.L. 1117

$05-04-02107$ ILDINES IN URBAN AREAS I FIRES THERMAL-RADIATICN SHEDEN ST ATTACK-OPERAT IONS PUBL IC-INFORMATI ON MEDICAL-SUPFLIES N FEATING COOL ING LIGHTING WASTES (SANI TARY-ENGINEERING) $G$ FIRE-FIGHTING-EQUIPMENT LRBAN-AREAS THERMAL-RADI ITION URBAN-AREAS FIRE-FIGHTING-EQUIPMENT HAMBURG PHOTOGRAPHS -ANALYSIS VAR IANCE REGRE SSION-ANALYSI S MEOICINE BICLOGY OLOGICAL-WARFARE CHEMICAL-WARFARE PSYCHOLCGICAL-WARFARE DING-MACHINES COMMUNICATION-THEORY DATA-STOR AGE-SYSTEMS OST - EFFECT IVENESS VULNERAB ILI IY DA TA-PROCESSING-SYSTEMS NTAM INAT ION B IOLOGICAL-WARFARE CHEMICAL- hARFARE RESCUES UREAN-AREAS RAILROADS MONORAILS MCVING-BELTWAYS HISTORY HELTER-MANAGEMENT SHELTER-OCCUPANCY INSTRLCTICN-NANUALS EXERCISE NOISE REL IGION TEMPERATURE-CONTRCL VENTILATION REG IONAL-PLANN ING INDIA INTERNATIONAL-AFFAIRS COMNERCE A FOREIGN-POL ICY FOREIGN-AID LABOR UNI TED-NATICNS WAGES ALTERNATIVE SOLUTIONS THEREFORE I URBAN-AREAS NEW-YCRK $K$ AND TOR NEW YORK CITY TRANSIT ALTHORITY I IIRRAN-AREAS CTION COST TRENDS ( INNITFD-STATES BUREAU-CF-RECLAMATION CTION COST TRENOS ( UNITED-STATES BUREAU-OF-RECLAMATION CT ION COST TRENDS I UNITED-STA TES BUREAU-CF-RECLANATION CT ION COST IRENDS UNI TED-STA TES BUREAU-CF-RECLANATICN CLAY SILTSTONES GEOLOGY THERMAL-STRESS STRESSES STRAIN LYS IS METHOCOLOGY SOCIAL-SCIENCES CORRELATION VARIABLES SINE METHODOLOGY PUNCHED-CARD-EQUIPMENT SCCIAL-SCIENCES CELS COMMUNICATION COGNI TIVE-DEC I SION-NAKING INNOVATION RANSIT SYSTEM - MANUAL OF DESIGN CRITERIA I URBAN-AREAS ALYSIS RADIOACT IVE-FALLOUT FIRE-SAFE TY FALLOUT-SHELTERS EYS CULTURE DEMOGRAPHY SOCIOLOGY POPULATI CN DI SARNANENT -AREAS TRANSPORTATION COSTS HIGH-SPEED-GRCUND-TRANSPCRT NS 1 FALLOUT-SHEL TER S COOL ING-AND-VENTI LATING-EQUI PMENT VIL-DEFENSE-OPERA TING-PLANS MAPPING (INFRARED-EQUI PNENT) RESOURCES STOCKP IL ING MOBILIZATION STRA TEGIC-NATERIALS RECULATIONS - TEL ECOMMUNICATION I COMMUNICATICN-SYSTEMS SICAL SCIENCES, B IOLOGICAL SC IENCES, ENGINEERING I DATA RESOURCES IN THE UNITED STATES - SOCIAL SCIENCES I DATA OOC) COMMERCE AGR ICUL TURAL-ECONOMICS CEREALS INDUSTRIES OVERY VULNERABILITY RECORDS DI STRIBUTI ON (WATER) SURVEYS PROGRAMMINGICOMPUTER SI DISPER.SAL INDUSTRIES STOC.KPII.TNG AR CETONATIONS I GLASS ATIENUATION URBAN-AREAS IGNITION RIGHTS OF WAY TO BE ACQUIRED I CI VIL-ENGINEERING FLOODS ION-MAKING CONSERVATION DEPIFTION PFTROLEUM MIDDLE-EAST CEFENSE-OPERATING-PLANS URBAN-AREAS MANAGENENT-PLANNING 1 RAC IOACT IVE- I SO TOPES CHINA RUSSIA RADI OACTI VE-FALLCU CENCE RADIATION (COBALT) BLAST-SHEL TERS C YLINDERS (STEEL)
ASIESISAN I TARY-ENGINEERING) STORAGE GARBAGE PROJECTICNS ASTES (SAN I TARY-ENGINEERING) STORAGE GARBAGE PROJECTICNS
SAFETY-DEVICES TEST-DUMMY (ANTHROPCMORPHIC) CONSTRUCTICN ST-CUMMY (ANTHROPOMORPHIC) SAFE TY-HARNESS SAFE TY-DEVICES APONS-EFFECTS SHIP-STRUC TURAL-COMPONENTS NAVAL-RESEARCH -TUEES CALCULATIONS EXPERIMENTAL-DATA EXPLOSICN-EFFECTS ICS ( RESOURCES PETROLEUM-INOUSTRY INDUSTRIES ECCNCMICS NMENT-AGENCIES) A IR-FORCE ARMY NAVY GOVERNMENT-AGENCIES ION OF RIGHTS OF WAY TO BE ACQLIRED I FLOCOS CALIFCRNIA LCING-CODES REGULATIONS WASHINGTON BUILOINGS STRUCTURES FERY ILIZERS PESTICIDES EQUIPMENT AGRICULTURAL-CHEMISTRY 1 PROTEINS GENETICS AGR ICULTIJRAI-FC.ONOMICS BIOCHEMISTRY AN I JAPAN SEISMOLOGY STRUCTURCS SIOCK-WAUES ELASTICITY ROGRAPHY IGNEOUS-ROCK SEDIMENTARY-ROCK ME TANORPHIC-ROCK INSTRUMENTATION GEOLOGY OPENINGS LNDERGROUND-STRUCTURES CAMERAS REFLECTION REFRACTION WA VE-TRANSNISSICN THEORY STRESSES GRAN ITE OPENINGS BLOCK-CAVING-MINING BLASTING
ICS) COOL ING FA IL LRE (MECHANIC S) SHOCK-WAVES VENTILATICN ICS) COOL ING FA IL LRE (MECHANIC S) SHOCK-WAVES VENT I LATICN OH LOADINGCIMCCIIANIICS I COHETRUG II ON STATISTICAI TANAIYSTS
E-BURST AIR-BURST EQUATIONS RADIOACTIVE-ISOTOPES THEORY CASUALTY COMMISSION I JAFAN NLCLEAR-WARFARE-CASUALTIES HIMA CATA NAGASAKI RADIATION-INJIRIFS RFCCROS LIFE-SPAN LAW INTERNATIONAL-AFFAIRS ME THODOLOGY POLITICAL-SCIENCE INTERNATIONAL-AFFAIRS POLITICAL-SCIENCE METHODOLOGY LAW INT ERNAT IONAL-AFFAIRS POL ITICAL-SCIENCE METHODOLOGY LAW EAMS ( STRUCTURAL), TRAMES LINEAR-STRUCTURES, \$OSCE-1965-5 THE CUSP EFFECT, HYORODYNAMICS DESIGN CUSPS FLUID-FLOW THE CUSP EFFECT MENTAL-DATA TEST-METHOOS PRESSLRE AITENUATORS, \#ARF MOSO MENT AL-DATA TEST-METHODS PRESSLRE ATIENUATORS, \#ARF M069 SE-SYSTEMS DESIGN L'SSR MILITARY-INTELLIGENCE, \$RM-1829-1 ETERS PYROLYSIS SOLAR-FURNACES CONBUSTION DECOMPOSITION LTERS I WARNING-SYSTEMS URBAN-AREAS MOVEMENT) $\angle O R C-S P-30$ MICS FRODUCTION VULNERABILITY MANPOWER RFCAVERY 1 RP-428 CT-HEADINGS CLASSIFICATION INF ORMATI ON-THECRY CONPUTERS PROGRAMMING (COMPUTERS) THEORY PROPELLANTS ) \#RIA-62-1794 REL I AB IL I TY (ELEC TRON IC) NAVAL-VE SSELS SHOCK (MECHANICS) 1 CE FORMAT ION MAT TEMATICAL-MODELS MA THEMATICAL-ANAI YSIS $S$ INFORMAT ION-RETRIEVAL ALTOMA TIC ECONOMICS, \#PRC-D-634A EPA IR PER SONNEL. TRAINING COUNTERMEASURES I \#USNRDL-TR-656 I PARTICLE-SIIE EXPERIMENTAL-DATA GAS-TURBINES VELCCITY GRICULTURE INDUSTRIES INOUSTRIAL-PRODUCTION I \$RM-3436-PR SSURE SIRESSES PROJECTILES INTERI CR-BALLISTICS GAS-FLOW OGRAM-ADMINISTRATION WAR-POTENTIAL BUDGETS । $\$ S R I-I M-4559$
EVICES FEASIBIL ITY-STUDIES CIVIL-ENGINEERING, $\$ 0 A S A-1549$ NCE(ENGINEERING) STATICS STRIII. IURAL-PARIS) *HL-TOR-64-54

*A.L. 1118 SHADOW GEOMETRY OF BU $13-12-02268$ *A.L. II19/ARE-CASUALTIES RADIATIOV-INJURIES PO D6-21-02226 *A.L. 1121 TVENTILATION ELECTR IC-POWER-PR ODUC TIO $13-13-0227$ AA.L. I 22 (1) TANY I. T FIRES FIRESTORMS HAMBUR 13-12-02265 *A.L. $1123 / M S$ MULTIVARIATE-AVALYSIS STATISTICAL 06-03-02204 *A.L. 1124 IDS CISASTER-MEDICINE TSUNAMIS WATER- 15-03-02290 *A.L. 1125 /EOICINE EMERGENCY-HEAL TH-SER VICES BI 06-21-02233 *A.L. 1126 /PUTERS SPECIAL-PURPOSE-COMPUTERS REA 09-02-02248 *A.L. 1127 F ICIALI SPACECRAFT DEFEYSE-SYSTEMS C 22-02-02351 *A.L. $\mid 128$ /IVE-WASTE CIV IL-OEFEN SE-SYSTEMS DEC 0 06-18-02222 *A.L. II29 /APIC-TRANSIT-SYSTEMS TRANSPORTATION 23-02-02356 *a.L. II 30 /ANDBCOK FOR UNTRAINED MANAGEMENT I $\$$ 05-01-021 I5 *A.L. II3I /RAINING SLEEP WATER FOOD RECREATION 05-10-02184 *A.L. II52 /LATION AGR ICULTURE POLI ITICAL-SCIENCE D5-03-0216 *A.L. IIS4 INTROL COLD-WAR NATO VIETNAM RED-CHIN 05-04-0217 *A.L. 1155 /NHATTAN AND WILLIAMSBURG BRIDGES AND 23-02-02357 * A.L. $1156 /$ OF THE MAYOR OF THE CITY OF NEW YOR 23-02-02355 *A.L. 115711

\#A.L. $1157(2)$

\#A.L. $1157(3)$ CONSTRU $15-13-12288$ CONSTRU 13-13-02284 CONSTRU 13-13-02285 $1157(4)$ CONSTRL 13-13-02270 *a.l. 1158 /ata - water conveyance tunnels - gra 13-13-02283 \$A.L. 1159 /STONE ROCK-SALT ANHYORITE SANOSTONES 08-07-02242 *A.L. 1190 /DATA I PUNCHED-CAR DS STATISTICAL-ANA 05-11-02192 *A.L. II9I IREPARATION AND SCOR ING I DATA-PROCES 05-11-02188 *A.L. 1192 /OLOCY) THREAT-PERCEPTION BEHA VIOR MO D5-0\%-02160 *A.L. I193 /APITAL TRANSPORTATION AGENCY RAPID T 23-02-02353 \#A.L. 1207 /-WAVES BLAST SHIEL DING STRUCTURAL-AN 13-13-02280 A.L. $1210 /$ CIVIL-DEFENSE-SYSTEMS COLD-WAR SURV 05-10-02177 4A.L. 1211 TECFNOLOGY FOR THE BIG CITY TSURBT $13-01-01769$ *A.L. 95I NNFLAGRATIONS RESCUES VULNERAB ILITY CI I3-12-01806 *A.L. 954 I SCRAP CONSUMPTION PROBLEMS I SALVAGE 05-03-01645 *A.L. 954 SCRAP CONSUMPTIOV PROBLEMS I SALVAGE 05-03-01645
CO.L. 961 ODE OF FEDERAL 17-02-01978
\#A.L. 962 / RESOURCES IN THE UNITEO STATES - PHY 05-02-01627 *A.L. 063 A DIRECTORY OF INFORMATION OS-02-01628 *A. L. 966 I GROSS-NATIONAL-PRODUCT COVSUMPTIONIF 02-02-01616 \#A.L. 969 /CK -OPERAT IONS MANAGEMEVT-PLANNING REC 13-02-01788 *A.L. 970 /NITORING LOGISTICS MANPOWER SHELTERS 15-03-01949 *A.L. 971 /F THERMAL RADIATION RELEASED IN NUCLE $13-12-U 1740$ * A 973 /G OPERATIONS-RESEARCH ECONOMICS DECIS 05-03-01635 *A.L. 974 /TER IN A MAJOR CITY I SHELIERS CIVIL- 15-03-01945 *A.L. 975 / IN CEBRIS FROM A LANO SURFACE BURST 18-02-01991 *A.L. 976 IER ING ALBEDO GAMMA-RAYS ANGLE-OF-INCI $18-03-02029$ *A.L. 981 /EERING PUBLIC-HEALTH DISPOSAL COSTS W 13-02-01789 *A.L. 982 /C TEST DUMMY I TEST-EQUIPMENT DESIGN 13-12-01808 *A.L. 983 / AND SAFETY BELTS I TEST-EQUIPMENT TE 13-12-0!803 \$.L. 984 / MAT HEMAT ICAL-MODEL S HEAT-TRAN SFER WE 13-12-01797
\$.L. 984 /RAIGHT TUNNELS - A COMPARI SON I SHOCK 20-04-02050 *A.L. $985 /$ OF PETROLEUM AND NATURAL GAS STATIST O5-D3-DI638 *A.L. 986 /CIES) LEGISLATION CIVIL-DEFENSE (GOVER 15-03-01946 *A.L. 987 /ES, CROSS SECTIONS, AND GENERAL LOCAT 13-02-01779 * A.L. 995 /ODE OF THE DISTRICT OF COLUMBIA I BUI 13-13-01816 *A.L. 996 /E I NUTRITION (HUMAV) NUTRITION (ANIMAL D6-16-01720
A.L. 997 IE I *A.L. 1195 /ENGINFFRING 11. TOKYO AVD KYUIU, JAP UB-II-02240 *A.L. 1195 /ENGINFFRING I1: TJXYO AVD KYO IO, JAP U8-11-02240 *A.L.1198 /EFORMATION EXPER IMENTAL-DATA GEOLOGY D8-07-02244 * A.L. 198 /EFORMATION EXPER IMENTAL-DATA GEOLOGY D8-07-02244
*A.L 1199 /RUMENTAT ION TRANSOUCERS OSC ILLOSCOPES 08-11-02247 A A 1200 FAIIURE (MECHAN ICS) MIN IVG-ENG INEER ING 08-07-02243 *A.L. 1205 /ING MIN ING-ENG INEER ING LOADING (MECHAN 13-13-02273 \#A.1 - 12 2ח6 /ORY GEOLOGY INSTRUMENTATION DEFORMATI 13-13-02275 *A.L. $1221 / 1$ DECAY-SCHEMES USSR PARTICLES SURFAC IB-U8-U2323 * ARC.C. TR ח-66 / RLISHED PAPERS OF THE A TOMIC BOMB 06-21-02224 * AECC-65-66 LEAR-HARFARE-CASUAL TIES JAPAN HIROS 06-18-0I730 * ACD-5 /LICT-RESOLUTIOV COMMUNI ST-CHINA HISIURY U5-O"-01665 *ACD-6 /A ECONOMICS CONFL ICT-RESOLUTION HISTORY D5-04-01662 \#ACD-8 IUNIST-CHINA CONFL ICT-RESOLUTION HISTORY 05-04-01603 *ACI-SP-12/ICS) STRUCTURES NONLINEAR-ANALYSIS B 2D-11-02339 *AC 107323 /ENERATION OF STANDING VORTICES AND 20-04-0120S5 *AC 107
*AC 111482 MUZZLE BRAKES II. THEORY I GUN-AU 19-06-02328

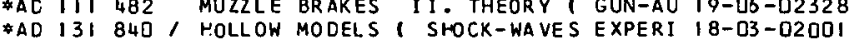
TAD 131840 , HOLLOW MODELS I SHOCK-WA VES EXPERI 18-03-02001
IAC 131882 , HOLLOW MODELS I SHOCX-WA VES EXPERI 18-03-02005 *aE 133012 / ARCH MODELS TRREA T-EVALUaTION DEFEN 15-03-02303 \#AD 147715 /ON HIGH-TEMPERATURE-RESEARCH RADIOM 13-12-01802 * AD 204090 / ITY ON THE LOCATION OF COMMUNAL SHE 15-G3-01942 *AC $210651 / G$ THE WAR I MILITARY-STRA IEGY ECONO 05-03-01646 AC $275393 /$ INOEXES IVFORMATION-RETRIEVAL SUB JE US-UL-O2119 \$AD 37880 /RESEARCH DESIGN ELECTRONIC-EOULPMENT 20-11-02083 *AC 401800 / ADING(MECHANICS) COLUMNSI STRLCTURAL 20-11-02340 AC 414713 /YSTEMS SUBJECT-IVDEXING TEST-METHOD US-LU-02116 *AC 419334 /(SURVIVAL) PROGRAMMING ICOMPUTERS) R 05-03-D165I *AD 420 083 IE BASIC MECHAN ISMS OF DUST EROSION 20-08-02068 AAD 426906 / ARE SURVIVAL ECOVOMICS POPULATION A 05-03-02158 * AC ODI 728 /N-BARREL-ATTACHMENTS IVHIBITION PRE 19-03-02327

AAC 602 139 TOSTS CIVIL IAV-PER SONVEL RESEARCH-PR 15-03-02305 $\begin{array}{lllll}\text { \#AD } 608 & 531 & \text { /NES VIBRATION FREQUENCY PNEUMATIC-D } & 13-13-02291 \\ \text { *AC } 613 & 545 & \text { /GROUND-STRUCTURES SHEL TER S PERFORMA } & 13-13-02290\end{array}$ 
SPFER IC-MOTION METEOROLOGICAL-PARAME TERS I *AFCRL-65-3-49 *AC 621658 /T-PROPERTIES EXPER IMEVTAL-DA IA ATMO O4-D2-021 I 4 STUDIES COSTS DESIGN LOADING (MECHANICS) SCIL(MECHANICS), \#AC 625782 /SIS SLABS(NOVMETALLIE) FEASIBILITY- I3-I3-0I837 IRE-SAFETY PROGRAMMINGICOMPUTERSI DEBRIS BLAST IGNITION, \#AD 625867 /LEAR-EXPLOSIONS DAMAGE-ASSESSMENT F I5-D3-0I952 ITH AESTRACTS BIAL IOGRAPHIES INDEXES, \#RM-2800-1 (SUPP) \#AC 626930 /THOR INDEX OF THE OPEN LIIERATURE W OS-03-DI652 ITH AESTRACTS 1 BIBLIOGRAPHIES INDEXES I ARM MOOEL OF FLASH BL INDNESS CON CUCT IVITY ELEC TRICAL-CONDUC TANCE FAL ELEC TR ICAL-CONDUC TANCE GERH THEMATICAL-ANAL YS IS STRUCTURES DYNAMICS, *BULLETIN $35(4) * A D 631$
N SHIPP ING (MARINE) DAMP ING SPACE-FLIGHT; *BULLETIN $35(5)$ *AD 631234 ATEC-BU ILDINGS MILITARY-REQUIREMENTS TESTS 1 \#REPCRT 1841 \#AC 631 325 YNAMICS EXPERIMENTAL-DATA COMPRESSIVE-PROPERTIES THEORY HOCK - WAVES CONT INUUM-MECHANICS LINEAR-SYSTEMS FUNCTICNS FRATURE HEAT-TR AN SFER COOLING-AND-VENTLATING-EOUIFMENT, \#AC 631 410 作 作 CESIGN COOL ING-AND-VENTILATING-EQLIPMENT HAZARDS COSTS ) NT EUILDINGS UNDERGROUND-STRLC TURES MODELS (SIMULATIONS)
RANSFORMERS COILS CIRCUITS USSR PATENTS, *FTD-TT-65-1169 RANS FORMERS COILS CIRCUITS USSR PATENTS, *FTD-TT-65-1169
PMENT RAILROAD-TRACKS ROADS USSR INDERGROUND-STRUCTURES, L-WAVES STRESSES PROPAGATION ELASIICITY THEORY, *DRL-376 STRAIN(MECHANICS) EQUATIONS-OF-MOIION STRESSES, \#CRL-362 LIBRATION ANALOG-COMPUTERS FOURIER-ANALYSIS, \#ECCN-2647A
S MAPS ELECTROMAGNETIC-COMPATIBILITY PROPAGATION $\$$ P-253 CHANICS) SURFACE-BURST ACCOUSTICS STRESSES, \#DASA-1676-1 $\checkmark$ AND EXERCISE I BODY-WEIGHT PHYSICAL-FITNESS NUTRITICN 64 I IBL IOGRAPHIES URBAN-AREAS RLRAL-AREAS FIRE-SAFETY ICITY CONCRETE HONEYCOMB-CORES. EXPANDED-PLASTICS DES IGN POL IT ICAL-SCIENCE ECONOMICS DECI I I ON-MAKING LAW, *P-2289 ELLS AIRCRAFT-EQU IPMENT-POMER- SUPPLIES I *AFAPL-CCNF-66-6 EFFECT MAGNETIC-FIELDS LASERS HYS IERE SIS I *AFCSR-66-1090 NMENT (FOREIGN) SOCIAL-COMMUNICATICN LEADERSHIP I \$SP-2022 LSISIMULATIONSI GRAPHICS TABLES MICHIGAN, \#USNRDL-TR-984
ACCINES DOSACE IMMUNITY MONKEYS MICE GUINEA-PIGS HUNANS ACCINES DOSAEE IMMUN ITY MONKEYS MICE GUINEA-PIGS HUNANS NNING STATE-GOVERNMENTS LOCAL-GOVERNMENTS \#SRI-4536-1ID N-AREAS BUILDINGS SAN-JOSE VENTILATION, \#SRI-MU-4536-110
CRETE SHELTER-DOORS TRAFFIC STRUC TURAL-SHELLS) $\$$ M6064 (3) OCELS (SIMULATIONS) BEAMSI STRUC TURAL) NUMERICAL-ANALYSIS SISTRUCTURAL) LOADING (MECHANICSI PROGRAMNING (CCMPUTERS) , OYS GENERATORS PO HER-SUPPLIES ELECTRIC-POWER-PRODUCTION, TOR-DEVICES TRANSISTORS CONTROL-SYSTEMS, \#FTD-TT-65-2035 RACIS DIAGNOSIS PATHOLOGY HISTOLOGY INFECTIONS I NNLNITY KEYS I BIOLOGICAL-WARFARE-AGENTS PATHOLOGY LUNGS SPORES ENS IS BACTERIA SPORES STORAGE BIOLOGICAL-hARFARE-AGENTS L-WARFARE-AGENTS TOXIC-TOLERANCES TOXINS-AND-ANTITCXINS L-WARFARE-AGENTS TOXIC-TOLERANCES TOXINS-AND-ANTITCXINS
E-AGENTS ANTIGENS-AND-ANTIBODIES RESISTANCE (BIOLOGICALI E-AGENTS ANTIGENS-AND-ANTIBODIES RESI STANCE (BIOLOGICALI
-WARFARE-AGENTS PARALYSIS FOOD-POISONING MUSSEL-POISCNS - WARFARE-AGENTS PARALYSIS FOOD-POI SONING MUSSEL-POISCNS
-WARFARE-ACENTS POX-VIRUSES MONKEYS IMMUNI TY INFECTICNS N-MAK ING INCENTIVES SOC IAL-SCIENCES OPERATIONS-RESEARCH ITY EQUATIONS-OF-MOTION PLASTICITY THEORY, \#DASA-1676-I HARMOCOLOGY MUSCLES MONKEYS CHICKENS DOSAGE, *EASP-1C0-1
CAL-PREDICT ION THEORY CRATER ING PROPAGATICN, *NCRE/R-525 CTERIA GAS-FILTER S MICROORGANISMS AIR-POLLUTICN FILTERS EGIC-WARFARE MILI IARY-STRATEGY OPIIMI ZATI CN, \#RM-48I7-PR NG-SYSTEMS MAN-MACHINE-SYSTEMS CONPUTERS, \#AFOSR-66-0873 INE(OPT ICAL) FLASH-LAMPS LIQUIDS SPEC TROSCOPY) \#NRL-6444 SION TESTS(FIRES) SUR VEYS (DATA-COLLECTION), \#NRDL-TRC-16 PINGIOPTICAL] CAVITY-RE SONATORS OPTICS CRYSTALS MIRRORS BLAST-SHELTERS EVAPORATORS COSTS ENVIRONMENTAL-COATROL, ELAST-SHELTERS EVAPORATORS COSTS ENVIRONNENTAL-COATROL,
MECHAN ICAL-WAVES CIVIL-ENGINEERING PROPAGATICN, 1 ROS-13 MECHAN ICAL-WAVES CIVIL-ENGINEER ING PROPAGATICN
NS STREPTOMYCINS CHLOR TE TRACYCLINE CHLORANPHENICCL USSR NS STREPTOMYCINS CHLOR TE TRACYCLINE CHLORANPHENICCL USSR
MENT LASER I LASERS OPTICAL-EQUIPNENT-COMPONENTS OFTICS MENT LASER ' LASERS OPTICAL-EQUI PNENT-COMPONENTS OFTICS ,
HROAT GAMES I OPERATIONS-RESEARCH GAME-THEORY, \$RAC-P-20 CAL-W ARFARE-AGENTS MIYAGAWANELLA-PSI TTAC I CULTURE-NEDIA ECTR I CAL-EQUIPMENT LABORA TORY-EQUIPMENT DECONTAMINATION PEST IS ( B IOLOG ICAL-WARFARE-AGENTS CULTURE-MEDIA GROWTH , ESTES RATS MORP HOLOGY (BIOLOGY) HI STOLOGY ESOPHAGUS SKIN ION EGYPT ENGINES-AND-MOTORS IRRIGATION-SYSTEMS, MAGE SPRINGS PERSONNEL DESIGN INSTRUMENTATICN NAR-BOUNDARY-LAYER STABILITY MEASLREMENT, \&AMRL-TR-65-53 OPERT IES CONTAINERS TASTE FALLOUT-SHELTERS I *TR-67-25-CD

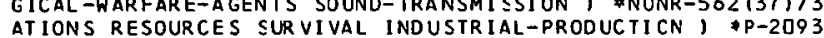
ATIONS RESOURCES SUR VIVAL INDUSTRIAL-PRODUCTICN GN ENGINEERING-PER SONNEL SPECIFICATIONS HANDBOOKS TESTS
-RADIATION MICROWAVES OXIOES PLASIICS, \$PIBMRI-I $295.3-66$ -RADIATION MICROWAVES OXIOES PLASIICS, \#PIBMRI-1295.3-66
ONS-RESEARCH MANA GEMENT-CONTROL-SYSTEMS DECISION-MAKING, VIL-DEFENSE-OPERATING-PLANS DA IA IDENTIFICATICN RECCROS,
C-WAVES PRDPAGATION MODELS (SIMULAIIONS), *LMSC-2-12-66-6 C-WAVES PROPAGATION MODELS (SIMULAIIONS), *LMSC-2-12-66-6
ES METEOROLOGY RAILROADS SHIPS HOISTS CHINA, *ATD-66-134 ISTORY MIL ITARY PUBL ICATIONS REPRODUC IION DOCUMENTATION T-ENG INEER ING SYMPOSIA DA TA-PROCE SSING-SYSTEMS, \$5P-2655 UTER-PROGRAMS ELASTICITY LOADING (NECHANICS), *FEL-297. 17 RAGE-SYSTEMS COMPUTERS DICTIONARIES ' \#DOUGLAS PAPER-4027 S MET AMATHEMATICS PHYSICS LANG UAGE PHYSI OLOGY MECHANTCS
RIAELES WEAPON-SYSTEMS ANALYSI S-OF-VARIANCE, $T M-K-77166$ TION-EQUIPMENT IN TERACTIONS, \#TECHNICAL REPORT NC. I-750 RVIVAL CIVIL-DEFENSE-SYSTEMS MANAGEMENT-CONTROL-SYSTEMS RVIVAL CIVIL-DEFENSE-SYSTEMS MANAGEMENT-CONTROL-SYSTEMS SHELTERS COMPUTERS (PROGRAMMING) BUILDINGS CONSTRUCTION
RMEAB IL ITY TRANSPORT-PROPERTIES SORP IION SALTS HUMIOITY RMEABIL ITY TRAN SPORT-PROPERTIES SORP TION SALIS HUMIOITY
LOACING (MECHAN ICS) SHOCK-WAVES NLCLEAR-E XPLOSICN-DANAGE LOAC ING(MECHANICS) SHOCK-WAVES NLCLEAR-EXPLOSICN-DANAGE
ERE-MODELS TRAN SFORMATIONS (MATHEMATICS), *IER-2I-ITSA-2I

$\because A D \quad 631442$ $\triangle A D 631576$ $A D 631641$ $A D 631648$ $A C \quad 631816$ $A C D C 632527$

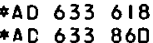
\AC 634042 *AC 634691 *AD 634797

$A C 634845$ $\triangle A C 35016$ *AD 635199 \#AC 635250 *AC 635930 ADD 635931
AD 636048 AD 636048
AC 636187 $A C \quad 636284$ \#AD 636436 *AD 636813 *AD 636814 *AD 636815 AC 636854 *AD 636893 $\begin{array}{ll}\text { AAC } 636 & 893 \\ \text { *AC } 636 & 903\end{array}$

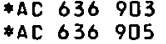
\#AC 636960
AD 637197 $\begin{array}{lll}\text { *AD } & 637 & 197 \\ \text { AAD } 637 & 265\end{array}$ *AD 637265
\#AC 637289 $* A C 637289$
$* A C 637407$ \#AD 637464 \#AC 637488 $\begin{array}{lll} & A C \\ * A C & 37 & 500 \\ & 616\end{array}$ \#AD 637806 $\triangle A C \quad 637825$ \#AD 637953
\#AC 638027 +AC 638191 $\triangle A C$
\#AC 6382220 $A D 638404$ $A C 638110$ AC 638440 ADC 638480 \#AD $638 \quad 630$ \#AC 638687 1 IMPACT-SHOCK VUCLEAR-EXPLOSI ON-DA 13-13-01823 A AC 640823 /PRESERVATION FIBERBDARD PHYSICAL-PR $06-08-02212$ AOC 641013 /OPERTIES GAS-FLOW PRJPAGATION BIOLO 15-02-01881 AC 641156 , ECONOMICS RECOVERY POSTATTACK-OPER 05-03-01647 $4 A D 642057$ / AN-ENGINEERING DECISION-MAKING DESI 05-05-01673 ADD 642271 I IC-THEORY ELECTRON-DEVSITY CERENKOV 20-03-02330 $A D 643472$ /NEERIVG MANAGEMEVT-PLAYVING OPERATI DS-DI -02292 AC 643527 / ER-SUPPL IES) MAVAGEMEVT-PLANNING CI 15-03-01941 AC 644354 /ADIATION COSMIC-RAYS ELECTRONAGNETI 04-01-02113 *AD 645029 /ECRAFT TRANSPORTATION LAUNCHING-SIT 16-0I-D1973 *AD 645500 /FICHE MANPOWER PROCUCTION-CONTROL H 05-02-02125 AAC 645517 / AT IOV-RETR IEVAL aUTOMATIC MANAGEMEN 05-02-02121 *AC 645586 /ES STRUCTURAL-PROPERTIES STEEL COMP 13-13-02287 AD 646030 /DUSTRY SYSTEMS-ENG INEERING DATA-STO 05-02-02118 AAD 646039 /POSIA MATHEMATICAL-LOGIC MATHEMATIC 12-01-02250 *AC 646608 /NICS) SOIL-MECHAYICS CLAY COMMUNICA 13-11-02261 *AD 646627 / ENVIRONMENT MANAGEMENT-PLANNING SU 15-03-02293 AC 646847 / R COST ANALYSIS I COSTS METHOOOLOGY 13-13-01828 AD 646848 /ER TRANSFER THROUG HUMAN SKIN I PE 06-16-02213 *AC 647 ODG TITAL-EQUATIOVS EQUATIONS-OF-MOTION 13-13-02279 
ORMATIONSIMATHEMATICSI ELECTRON-DENSITY, *IER-22-ITSA-22 *AD 648002 /XTREMELY-LOW-FREQUENCY PHASE TRANSF 20-14-02348 CILLATIONS STABIL ITY BOUNDARY-VALLE-PROBLEMS / UCLA-R-ID *AD 648 083, 1 GAS-FLOW PLASMA-MEDIUM PLASMA-OS 20-09-02335 E CLOTHING TEMPERATURE COOLING PERSPIRATICN EVAPORATICN I \#AC 648467 /TILAT ION METABOLISM BODY-TEMPERATUR O6-I6-0I7I9 ECHNOLOGY FOR 1965 IV. I DOCUNENTAIION I \#TN-2625/004/OD \#AC 648562 /GRAPHY ON INFORMATION SCIENCE AND T Q5-Q2-02122 ROFESSIONAL-PER SONNEL SC IENTIFIC-RESEARCH ORGANIZATIONS, *AC 648767 /S I IVDEXES REPORTS DOCUMENTATION P OS-OZ-D2II 7 TICAL-ANALYSIS MANAGEMENT-ENGINEERING) *C6-2442/030(III) *AD 649284 /RMATION-CENTERS JOB-ANALYSIS STATIS OS-O2-02I 20 CES-OPERAT IONS LOGISTICS AGING (PHYSIOLOGY), \#IR-67-57-PR \#AC 649540 / HOLOGY) SOCIAL-PSYCHDLOGY ARMED-FOR OS-ID-0218I AL SCIENTIFIC-ORGANIZATIONS PERIODICALS, *TN-3J08/004/00 *AC $649637 / N T$ IFIC-RESEARCH INFORMATION-RETRIEV OS-OZ -02I 23 TRESSES ST IFFENED-CYLINDERS STRAIN(MECHANICS), \#WVT-6717*AC 649662 /E-MATERIALS, EQUATIOVS-OF-MOTION, S 20-1I-02345 Y ACCELERAT ION MOTION PROPAGATION EARTHOUAKES, *VUF-3014 \$AD 649701 /MIC-WAVES EXPLOSION-EFFECTS VELOCIT I8-03-D23I9 Y ACCELERATION MOTION PROPAGATION EARTHOUAKES S MANAGEMENTTLANNING CIVIL-DEFENEE -RESEARCH OPTICAL-INSTRUMENTS EMISSI VITY *ARNA-TR-66-39 \#AC 649809 /RANSFER PYROMETERS HIGH-TEMPERATURE I3-OI -O2255 I BUCKLING(MECHAN ICSI MA THEMATICAL-ANALYSIS \#SRSN-7-168 \#AC 649833 /LOACINGIMECHANICS) STRAINIMECHANICS 20-11-02344 Y BENCING ELASTICITY LOADING (MECHANICS) INPACY, \#TMB-704 *AC 650 178 SUPPORT 1 R INGS VIBRATION FREQUENC 20-1I -02336 ATION ROTATION INERTIA MATHEMATICAL-ANALYSIS I \#REFORT 21 *AC 650221 /NGIMECHANICSI SHEAR-STRESSES DEFORM 20-II -D2346 FENSE DOCUMENTATION EFFECTIVENESS INFORMATION-RETRIEVAL， \#AD $650316 / 5$ SUBJECT-INDEXING DEPARTMENT-OF-DE O5-O2-D2I24 R EXPOSURE TOXICITY CONSTRUCTION-NATERIALS, \#IITRI-NGDSI \#AC 650323 /ST RADIOACTIVE-FALLOUT HEAT-TRANSFE I3-12-D2269 RACKS STRAIN(MECHANICS) LOADING(MECHANICS), \#NCEL-TR-518*AC 650 329 / ISTRIBUTION DEFLECTION STEEL RODS C I3-I3-D22B9 ES CEFLECTION ROLLING(METALLURGY) ELASTICITY) \#DTNB-1225*AC 650 540 /UCTURAL-SHELLS RINGS WELOING STRESS 20-1I -D2347 CONDIMENTS SUCROSE FOOD-DISPENSING TESTS, \#SRI 4949-500 \#AD 65I 944, TASTE CIVIL-DEFENSE-SYSTEMS FRUITS D6-0B-D22II MPERATURES BOUNDARY-LAYER LAMINAR-BOUNDARY-LAYER, *R-273 \#AC 80305 /D-DYNAMIC-PROPERTIES DRAG SURFACE-TE 22-02-02350 T ECONOMICS FEASIBILITY-STLDIES CLASSIFICATION, RN-1512 *AD 87449 POSSIBIL ITIES I INDUSTRIAL-EQUTPMEN OS-03 - DI6L8

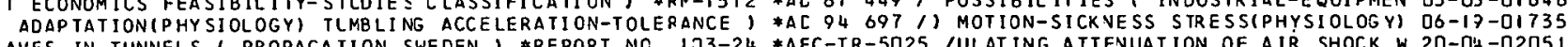
AVES IN TUNNELS I PROPAGATION SWEDEN I \#REPORT NC. $133-24$
ELCING RADIATION-MEA SUREMENT-SYSTEMS RADIATION-MONITORS ELCING RADIAT ION-MEA SUREMENT-SYSTEMS RADI I TI ON-MONITORS TER IES ALKAL INE-CELLS AIRCRAFT-EQLIPMENT-POWER-SUPFLIES ENT AL-DATA ATMO SPHER IC-MOTION NETECROLOCI CAL-PARAMETERS OUNCS FOCUSING PHOSPHATES CALCITE POLARI ZATION 1 \#ML-I 405
S DATA-PROCESSING-SYSTEMS MAN-MACHINE-SYSTEMS COMPLTERS, MAGNETO-OPTIC-EFFECT MAGNETIC-FIELOS LASERS HYSTERESIS GEOMETRY RADIATION-HA ZARDS RADIOLCGICAL-DCSE SIMULATION CE MEAT RESOURCES CEREAL COTTON VEGETABLES FRUITS FOOOS ING RADIATION-HAZ ARDS DECONTAMINATION RADIATICN-EFFECTS TOMOLOGY NUTR ITION WA TER-CONSERVATION SOI L-CONSERVATICN NING PERSONNEL SHELTER-OCCLPANCY (SIMULATICN) LEAOERSHIP ABIL ITY SHEL TER -OCCUPANCY INSTRUC TION-MANUALS HANDEOOKS ABILITY SHEL TER-OCCUPANCY INSTRUC TION-MANUALS HANDEOOKS
NG-SYSTEMS SHEL TER-ALLOCATION SHE LTER-OCCLPANCY SURVEYS NG-SYSTEMS SHEL TER-ALLOCATION SHELTER-OCCLPANCY SURVEYS MET HOCOLOGY QUEST IONNA IRES SHELTER-MANAGENENT ATTITUDES
IP CONFINED-ENVIRONMENTS STRESS (PSYCHOLOGY) SUPERVISION IP CONF INED-ENVIRONMENTS STRESS (PSYCHOLOGY) SUPERVISION I-PL ANN IVG TRA INING SHEL TER-OCCUPANCY EXPERINENTAL-DATA PLOSION-DAMAGE SPRINGS PER SONNEL DESIGN INSTRUMENTATICN MANY CUCTS LAMINAR-BOUNDARY-LAYER STABILITY MEASUREMENT -FOODS MEAT MILK PORK POTA TOES SEAFOOD VEGETABLES hHEAT
ER-SUPPLIES EOUCATION TRAINING INFANT-DIET, \$CCD-IG-15. ER-SUPPL IES EDUCATION TRAINING INFANT-DIET, \#CCD-IG-15.
VES EXPER IMENTAL-DATA TEST-ME THODSPRESSURE ATTENUDTORS DATA TEST-METHODS PRESSURE ATTENUATORS IGH-T EMPERATURE-R ESEARCH OPTICAL-INSTRUMENTS EMISSIVITY EAR-ANALYSIS BEAM S(STRUC TLRAL) FRAMES LINEAR-STRUCTURES AUNCHING-SITES ME TEOROLOGY RAILROADS SHIPS HOISTS CHINA ERSONNEL UNITED-STATES-GOVERNMENT COMMERCE CONSTRUCTION N PROJECTS ( WATER-SUPPLIES URBAN-AREAS ECONONICS COSTS (S) L AUNCHING (SIMULATION) ACOUSTICS VIBRATION-I SOLATCRS S TRANSDUCERS MATHEMA TICAL-ANAIYSIS STRUCTURES DYNAMICS ES-TRANSPORTATION SHIPP ING (MARINE) DAMPING SPACE-FLIGHT) NC VIRRATION BULLETIN ( IMPACT-TESIING SHOCK (MECHANICS) ROCK - FORMATIONS STRAIN PHYSICAL-PROPERTIES CALCULATICNS E UNITED STATES DEPARTMENT OF AGRIĆULTURE ( LEGISLATICN A RAYS - OPERATION BREN (IONI ZING-RADIATION-SCATTERING COSE-RATE GAMMA-RAYS INSTRUMENTATION EXPERIMENTAL-DATA -TESTS SIMULATION RADIOACTIVE-FALLOUT PROTECTI TN-FACTOR TION MATHFMAT IC.AI -ANAI YSIS SHOCK- LAVES ANALOG-CONPUTERS SS LOADIVGIMECHANICSI SHOCK- hAVES VI SCOELASTICITY ELAST $N$ TESTS MODEL SISIMULATIONS) SOIL-NECHANICS IMPACT-TESTS RA FOR AMERICAS WATERS I WATER-PULLUTION WAIEK-SUHHLIES OE-ANALYSIS STATISTICAL-ANALYSIS NANAGEMENT-ENG INEERING OE-ANALYS IS STATISTICAL-ANALYSIS NANAGEMENT-ENG INEERING
NT BEAMSI STRUCTURAL) LOADING (MECHANICS) STATICS, TR-SO NICS) UNDERGROUND-STRUCTURES NUCLEAR-EXPLOSIONS, LAST FOOTINGS FOUNDATIONS VOID-RAIIO ARCHES, *NCEL-R-536 PNEUMATIC-CEVICES FEASIB IL I TY-STUDIES CI VIL-ENGI NEERING
ES LOADIVGIMECHAN ICS) SURFACE-BUR S ACCOUSIICS SIRESSES ES LOADIVGIMECHAN ICSI SURFACE-BUR S I ACCOUSTICS STRESSES OSIONS ELASTICITY EQUATIONS-OF-MOIION PLASTICITY THEORY EFFEC.TS SHOC.KIPATHOI OGYI FXPLOSION-EFFECTS TEST-METHODS ULE NUCLEAR EXPLOSIONS I A AND II - I UNITED-STATES USSR AT ION PRESSURE-GAGES-CALIBRA IIICN PRE SSURE-TINE-PATTERNS -EFFECTS SHOCK - TUBES LUNG-DANAGE ICLERANCES (PHYSI OLOGY) PIEZ OELECTRIC-TRANSDUCERS LIVESTOCK-SALVAGE SHOCK-TUBES MENT ELAST-ENER GIZED-MISSILES BLA ST-BIOMEDICAL-CRITERIA FECTS MATHEMATICAL-MODELS EXPERIMENTAL-DATA LUNG-DANAGE FECTS MATHEMATICAL-MODELS EXPERI MENTAL-DATA LUNG-DANAGE
EAR - A REVIEW I BLAST-BIOLOGY BICLOGICAL-BLAST-EFFECTS EAR - A REVIEW I BLAST-BIOLOGY BICLOGICAL-BLAST-EFFECTS
HEMAT ICAL-MODELS DISPLACEMENT ACCELERATION DECELERATION PEMAT ICAL-MODELS DISPLACEMENT ACCELERATION DECELERATION
-INJURIES EXPLOSIONS TOLERANCES(PHYSIOLOGY) SHOCK-TUBES -INJURIES EXPLOSIONS TOLERANCES(PHYSIOLOGY) SHOCK-TUBES
N-INJURIES SHIELDING URBAN-AREAS CASUALIIES (PREDICTICN) STATISTICAL-ANALY SIS BIOLOGICAL-BLAST-EFFECTS SHIELDING EAS DAMAGE-ASSESSMENT CASUALTIES POPULATION FIRE-STORMS NALYS IS I FIRES WIND METEOROLOGY COMPUTERS (PROGRANNING) ENE INEER ING DATA-STORAGE-SYSTEMS COMPUTERS DICTI ONARIES PAGATION STRAIN (MECHANIC S) EQUATI CNS-OF-NCTI ON STRESSES MECHANICAL - WAVES STRESSES PROPAGAIION ELASTICITY THEORY LAT ORS CALCULAT IONS MATHEMATICAL-NODELS HAROWARE DESIGN
LAT IONS) PASSENGER-VEHICLES LAMINAR-FLOW TURBULENT-FLOW *AEC-TR-5025 /ULATING a TIENUATION OF AIR SHOCK W 20-04-02051 *AEC-TR-6634 /EFFECTS CALCULATIONS DOSE-RATE SHI 06-18-01726 *AEET-244/ES CONSTRUCTION MECHAN ICAL-PROPERTIES 13-11-01792 * AFAPL-CONF-66-6 *AD 634 845 /TEMS I STORAGE-BAT ID-03-01764 *AFCRL-65-h-49 *AN 6? 658 /T-PROPERTIES EXPERIN 04-02-02114 *AFOSR-66-0490 \#AD 637 500 /YSTEMS AMMONI UM-COMP 20-06-02067 *AFOSR-66-0873 *AD 637488 /L-INFORMATION-CENTER D5-02-01630 * AFOSR-66-1090 *AD 635 0I6 /AVD PARTICLE BEAMS 1 20-05-02064 *AFRR I-SPO6-1 / C-FORCES-OPERATIONS RADIOB IOLOGY 15-0S-01964 * AGRICULTURE HANCBOOK VO. 325 /URAL-AREAS COMMER 02-02-01619 *agriculture haNdBoOK 234 /act IVE-Fallout SHIELO 15-03-01897 *AIB-189 /RING ANIMAL-HUSBANDRY C2OP-RESEARCH EN 02-0J-02108 *AIR-D-93CI-9/66-FR /1 MANAGEMENT-PLANNING HABIT 05-01-01624 *AIR-0-930-9/66-FR/ION ATTITUDES BEHAVIOR WARNI 05-11-01699 *AIR-D66-12/66-FR /-PROCESSING-SYSTEMS TRAINING 05-10-01680 *AIR-D93A(1/2)-9/66-FR /WATER (SHELTERS) LEADERSH 05-1]-01684 *AIR-D93B(1)-9/66-FR / ITED-STATES-GJVERNMENT (PER 05-09-01675 *AIR-C93B(I 2)-9/66-FR /R MAVAGEMENT ( MANAGEMEN 05-0I-01626 *A JA- 6424 \#D 639303 / 5 IMPACT-SHOCK NUC LEAR-EX 13-13-01823
*AMRL-TR-65-53 *AD 640820 /CS GAS-FLOW WEST-GER 20-01-02048 *AR8-NO. D76I88/FROZEN-FOODS FRUITS IRRADIATED 06-08-02209 *ARC-2219 /LTERS LOGIST ICS SHEL TER-OCCUPANCY WAT O6-03-01713 *ARF D087 *AO 131 8E2 / HOLLOW MODELS I SHOCK-WA 18-03-02005 * ARF MOO9 \#AD 131840 / HOLLOW MODELS I SHOCK-WA 18-03-D20DI

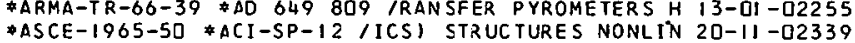
*ATD-66-134 * AD 645 029 /ECRAFT TRANSPORTATION L 1 $6-01-01973$
*BULLET IN NO. I 536 /RATION OPERATIONS-RESEARCH P O5-03-01649 * BULLET IN NO. I536 /RATION OPERATIONS-RESEARCH P 05-03-01649
*BULLET IN NO. 78 / ATHER RIVER AND DELTA DIVERSIO $13-02-02258$ *EULLET IN 35(3) /BEAMS (STRUCTURAL) SHOCK (MECHANI 20-11-02074 *BULLET IN $35(4)$ \#A0 31233 /S SPEC TR UM-ANALYZER 20-11-02075 *BULLET IN 35(5) \#AD 631 234 /AILROADS ARMED-FORC 20-11-02076 *BULLET IN $35(6)$ THE SHOCK A 20-11-02077 IMS * EX-SRa-188 /VERVING THE MEAT IVSPECTION OF TH 02-02-01620 *CE-62.12/AR DISTRIBUTION OF NEUTRONS AND GAMM 20-03-02334 *CEX-65.8DC ITS RADIOACTIVE-FALLOUT DISTR IBUTION 18-03-02324 *CCNF-65 IO16-1 /NICS) VIBRATIJN-I SOLATORS SIMULA 20-11-02343 *CONIRACT REPORT VO. 3-148 IRAIN (MEEHANIC SI STRE 20-1I-020B6 *CR-65.00I /HANICAL-WAVES SHOCK (MECHANICS) DESIG 20-11-02078 *LWA-1 A NEWE I3-02-0225? *C6-2442/030 ( III) *AC 649284 /RMATIDN-CENTERS J 05-02-02I20 *CASA-13.018 /CONCRETE BEAMS II. I TEST-EQUIPME 13-13-01841
*DASA-13.018 /ECHAVICS SHOCK-WAVES FAILUREIMECHA 18-03-02008 *DASA-13.018 /OUND-STRUCTURES SAND SHOCK-WAVES B 20-11-02341 *DASA-I549 *A0 608 531 /NES VIBRATIOV FREOUENCY 13-13-02291 *CASA-1676-1 *AC 633 618 /K-WAVES MEEHANICAL-KAV 19-0+-02044 *DASA-1676-II *AD 637197 /AVES UVDERGROUND-EXPL 08-13-01747 *DASA-1777 /HOCK I SHOCK-WAVES BIOLOGICAL-BLAST- 06-21-01741 * DASA-1795 /TAINING TO THE EFFECTS OF HIGH ALTIT 18-03-02018 *CASA-1853 /BLAST-B IOLOGY SHOCK-TUBES INSTR UMENT DO-21 -02232
* DASA-18 *DASA- 1854 /ALS 1 BLAST-B IOLOGY BIOLOG ICAL-BLAST $06-21-02230$
*DASA- 1855 /CK-WAVES PRES SURE-T IME-MEA SUREMENTS $06-21-02237$ *CASA-1856/BLAST-EFFECTS BLAST-8 IOLOGY DISPLACE D6-21-02223 *DASA-1857 /ATINE YISSILES 18 IDLJGICAL-BLAST-EF 06-2I-02235

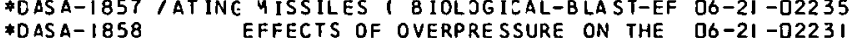
*DASA- 1859 /EFFECTS EXPER.IMENTAL-DATA THEORY MAT D6-21-02239 *DASA-1860/AST-EFFECTS MONKEYS SWIVE SHEEP LUNG 06-21-02234 \$DC-FR-1045/OG ICAL-BLAST-EFFECTS BURNS RADIATIO D6-21-02227 *CC-FR-1054 / WEAPONS-EFFECT RADIATION-INJURIES D6-21-02238 *DF-TN-1050-3 / FIRE FATALITIES I FIRES URBAN-AR 13-12-02262 *DCUGLAS PAPER-4027 *AD 646030 /DUSTRY FIRE STORM A 13-12-0I805 *CRL-362 *AC 631816 INICAL-WAVES ELASTICITY PRO 20-11-02032 *DRL-376 *AD 631 648 /ES IN LONG ELASTIC RODS 1 20-11-02079

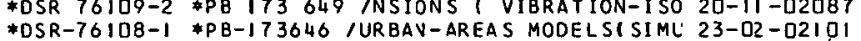


OUND-TRANSPORT PERFORMANCE (ENGINEERING) VEHICLES DESIGN CING STRESSES DEFLECTION ROLLING (NETALLURGY) ELASTICITY ANT ITOXINS PHARMOCOLOGY MUSCLES MONKEYS CHICKENS DCSAGE MENTATION CAL IBRATION ANALOG-COMPLTERS FOLRIER-ANALYSIS URE PHILOSOPHY POPULATION PROPAGANDA PUBLIC-INFORNATI ON
I BIBL IOGRAPHIES CHINA INDIA JAPAA PAKISTAN PHILIPFINES RITAIN AND CANADA I BIBLIOGRAPHIES FRANCE GERNANY ITALY ENCE HISTORY LANGUAGE PHILOSOPHY LAH PUBLIC-INFORMAIION ORY LANGUAGE LAW PUBLIC-INFORMATI ON PHILOSOPHY RELIGION RY-AFFAIRS PHILOSOPHY LAW POPULATION PUBLIC-INFORNATION INT ERNATIONAL LAW I USSR COMMERCE INTERNATIONAL-AFFAIRS INT ERNATIONAL LAW URVEYS FOREIGN-POLICY QUESTIONNAIRES PEACE-KEEPING USSR
RS SURVEYS QUESTIONNAIRES PUBLIC-OPINION FOREIGN-PCLICY RS SURVEYS QUESTIONNAIRES PUBLIC-OPINI ON FOREIGN-PCLICY
-POL ICY QUESTIONNAIRES INTERNA TI ONAL-AFFAIRS REARNANENT -POL ICY QUESTIONNAIRES IN IERNA IIONAL-AFFAIRS REARNANENT PIN ION SURVEYS FORE IGN-POLICY EC ONOMIC-AID NI LITARY-AID
RMS-CONTROL B IBL IOGRAPHIES ME THODCLOGY WAR-GAMES MCDELS RMS-CONTROL B IBLIOGRAPHIES ME THODCLOGY WAR-GAMES MCDELS
OPERATION INTERNATIONAL-RELATIONS ARMS-CONTROL BEHAVIOR OPERATION INTERNATIONAL-RELATIONS ARMS-CONTROL BEHAVIOR
OLUTION GAMES MODELSISIMULATIONS) MATHEMATICAL-ANALYSIS ISARM AMENT I GAME-THEORY MATHEMATICAL-MODELS SIMULATION R ARMS CONTROL AND DI SARMAMENT ( GAME-THECRY SI MULATION METHOOOLSTENCES POL ITEAL-SCIENCE CULTURE COOPERATION HAV IOR MOT IVATION CUL TURE RESPONSE REACTI ON (PSYCHGLOGY) ARK ET ING FOOD-MARKETING CANADA AGRICULTURAL-ECONCMICS ANC MEAT INDUSTRY 1 AGRICULTURE AGRICULTURAL-ECONCMICS ANC MEAT INDUSTRY I AGRICULTURE AGRICULTURAL-ECONCMICS
ANC MEAT INDUSTRY I AGRICULTURE AGRICULTURAL-ECONCMICS ANC MEAT INDUSTRY, AGR ICULTURE AGRICULTURAL-ECONCMICS ANC MEAT INDUSTRY AGR ICUL TURE AGR ICULTLRAL-EC ONCMICS
$S$ STEEL COMPUTER-PROGRAMS ELASIICITY LOADING (MECHANICS S STEEL COMPUTER-PROGRAMS ELASIICITY LOADING (MECHANICS)
ALTH POSTATTACK-OPERATIONS TRAINING CHILDREN GERIATRICS ALTH POSTATTACK-OPERATIONS TRAINING CHILDREN GERIATRICS
STATTACK-OPERAT IONS SHELTER-OCCUPANCY DAMAGE-ASSESSMENT STATTACK-OPERAT IONS SHELTER-OCCUPANCY DAMAGE-ASSESSMENT
TIACT -OPERAT ION S HOSP ITALS TRA INING PER SONNEL LOGISTICS TTACT-OPERATIONS HOSP ITALS TRA INING PER SONNEL LOGISTICS
CIV IL-DEFENSE-OPERATING-PLANS CLEANING REPAIR TRAINING CIV IL-DEFENSE-OP ERA TING-PLANS CLEANING REPAI R TRAINING
PLANS PREPARA TION MANAGEMENI-PLANANG CPERATICN RECORDS PLANS PREPARATION MANAGEMENI-PLANNING CPERATICN RECORDS NGS CONSTRUCTION DESIGN SHIELDING CONSTRUCTION-SLANTING ATORS ATORS SEM ICONOUCTOR-DEVICES TRANSI STORS CONTROL-SYSTEMS MICS SOLUBILITY TEMPERATURE DIFFUSIVITY HALF-LIFE YIELD OL ING-AND-VEN TILA TING-EQUI PMEN T GAS-FLOW TEST-EQUI FMENT HAB I TAB IL ITY FANS PORTABLE INS IRUNENTATI ON PUNKAH-FUMPS INSTRUMENTAT ION TEMPERATURE PLNKAH-PUMPS PORTABLE FANS YSIOLOGYI FOOD WATER - SUPPLIES BEDDING TOI LET-FACILITIES Y PER FORMANCE (HUMAN) INSTRLC TI ON-NANUALS WATER-SUPFLIES YSIOLOGYI WATER-SLPPLIES FOOD BEDOING TOILET-FACILITIES N) BATTER IES-ANO-COMPONENTS RADIO-COMMUNICATICN-SYSTEMS RACIOLOG ICAL-CONTAM INATION (FOOD) INFANTS (DIET) STCRAGE IST ANCE THERMAL - INSULATION PRESER VATION FINI SHES PAINTS SWEDEN SWITZERLAND IRELAND USSR INDIA AUSTRALIA CONADA BIL ITY WARNING- SYSTEMS HASTY-SHE L TERS THREAT-EVALUATION
GE WIND FIRES SIMULATION BLAST STATE-OF-THE-ART-REVIEWS GE WIND FIRES SIMULATION BLAST STATE-OF-THE-ART-REVIEWS
BAN-AREAS ANTIM ISSILE-DEFENSE SYSTEMS MILI TARY-STRATEGY BAN-AREAS ANTIMISSILE-DEFENSE SYSTEMS MILITARY-STRATEGY MENT LABOR MOBILI ZATION MANAGERENT-PLANNI AG CALCULATION STEMS DISPERSAL CHINA EVACUATI ON LRBAN-AREAS POPULATION EC-STATES SURVIVAL EUROPE COMMUNICATIONS CHINA SHELTERS OVERY VULNERAB IL I TY MILI TARY-STRA TEGY THREAT-EVALUATION NEW-YORK CHICAGO WASHINGTON PHILADELPHIA BOSTCN LCNCON RY VULNERABILITY URBAN-AREAS DEMOGRAPHY CCUNTERMEASURES IOLOGY SOCIETAL-RECOVERY ME THODOL OGY STRE SS (PSYCHOLOGY) STATTACK-OPERATIONS SEX AGE URBAN-AREAS DECISION-MAKING STATT ACK-OPERAT IONS SEX AGE URBAN-AREAS DECI IION-MAKING
CE POSTATTACK-OPERATIONS LRBAN-PLANNI NG ME THODOLCGY SEX CE POSTATTACK - OPERATIONS LRBAN-PLANNI NG ME THODOLCGV SEX
OGRAP HY ECONOMIC-RECOVERY CIVIL-DEFENSE-SYSTEMS CULTURE OGRAP HY ECONOMIC-RECOVERY CIVIL-DEFENSE-SYSTEMS CULTURE ENCE STRESS (P SYCHOLOGY) ME THODOLOGY MANAGEMENT - PLANNING SOCIETAL-RECOVERY RECOVERY SOC IOL OGY STRE SS (P SYCHOLOGY) ELTERS SCHOOLS PUBLIC-INFORMATION EDUCATI CN ORPHAN-CARE ECONOMICS SURVIVAL OISTRIBUIION STRESS (PSYCHOLCGY) FOOO CK-OP ERATIONS SOC IAL-SYSTEMS STANDARD-SI TUATIONAL-CASES PUBL IC-HEALTH URBAN-PLANNING POPULATION UTILI TY-TUANELS CK-COLTINGS CHAIN-LINK-FABRIC EPOXY-RESINS CFMFNT-GROUT EAL-ESTATE REFUGEES BANKING IN SURANCE LABCR LAW TRAFFIC OCAL - GOVERNMENTS STA TE-GOVERNMENTS LEADERSHIP ATTITUDES LOS ION-EFFECTS AIRBURST POSTATTACK-OPERATIONS ECCNCMICS BURST ECONOMICS EXPLOSION-EFFECTS POSTATTACK-OPERATIONS ANS FORMS ATMOSPHERE-MODELS TRANSF ORMA TI ONS INATHENATICS NCY PHASE TRANSFORMA TION SIMA THEMA TICS) ELECTRCN-DENSITY
HEAT-TRANSFER EXPOSURE TOXICITY CONSTRUCTION-MATERIALS HEAT-TRANSFER EXPOSURE TOXICITY CONSTRUCTION-MATERIALS
S INFRARED-TRACKING FOREST-FIRES PATROI - PIANES AIRCRAFT $S$ INFRARED-TRACK ING FOREST-FIRES PATROI - PIANES AIRCRAFT
$L$ CIV IL-DEFEN SE-PER SONNEL (TRAINING) MANAGENENT-PLANNING $L$ CIV IL-DEFEN SE-P ER SONNEL ( TRA INING) MANAGENENT-PLANNING
LS SMOKE-MUNITIONS FIRST-AID NUC LEAR-WARFARE-CASUALTIES NTERMEASURES) B IOLOG ICAL-WARFARE-AGENTS DECONTAMINATION AT ION CHEMICAL-WARFARE-AGEN IS BIOLOGICAL-WARFARE-AGENTS TION CISASTER-MEDICINE EMERGENCY-HEALTH-SERVICES REPAIR -CONT AM IVATION RURAL-AREA S HARDENING PROTECTI ONS-SYSTENS POSTATTACK-OPERATIONS RE SCUES CONSTRUCTI CN FIRES ROAOS IONAL -CIVIL-DEFENSE SHEL TERS EVACLATION WARNING-SYSTEMS C WOUNDS-AND- IVJURIES RADIATION-INJURIES BIBLICGRAFHIES -AREAS PROTECTION-FAC TOR DECONIAMINATI ON WATER-SUPFLIES K-OPERATIONS REPAIR MILITARY-ENGINEERING RECONNAISSANCE AT IONAL-CIVIL-DEFENSE CIVIL-DEFENSE-SYSTENS URBAN-AREAS ATIONAL-CIVIL-DEFENSE CIVIL-DEFENSE-SYSTENS URBAN-AREAS L-CONTAMINATION VETER IVARY-MED IC INE PROTEC II VE-CLOTHING
MEDICAL SCIENCE I BIBLIOGRAPHIES NEDICINE BI OLCGY CHINA MEDICAL SCIENCE I BIBLIOGRAPHIES NEDICINE BIOLCGY CHINA
IVE-MASKS BIOLOGICAL-WARFARE-AGENTS MANAGEMENT-PLANING
CSR-76108-2 *PB 173647 /OPUL S IOV HIGH-SPEED-GR 23-02-02096 *0TMB-1225 *AD 650 540 NCTURAL-SHELLS RINGS WEL 2D-11-02347 $\begin{array}{lllll}\text { *EASP-IOD-1 *AD } 637 & 265 & \text { /M-BOTULINUM TOXINS-AND- } & \text { I5-D2-01861 } \\ \text { *ECOM-2647A *AD } 632 & 927 & \text { /SHOCK (MECHAVICS) INSTRU } & 14-02-01842\end{array}$ \#ECOM-2647A *AD 632927 /SHOCK (MECHAVICS) INSTRU 14-02-01842
*EXTERNAL RESEARCH LIST 1.25 /NGUAGE LAW LITERAT 05-11-01697
*EXTERNAL RESEARCH LIST 2.25 /IC CENTERS - ASIA 05-11-01698 *EXTERNAL RESEARCH LIST 3.25 /RN EUROPE, GREAT B 05-11-01694 *EXTERNAL RESEARCH LIST 4.25 /ECOVOMIC S EDUCATIO 05-11-01695 *EXIERNAL RESEARCH LIST 5.25/APHY POLITICAL-SCI 05-11-01692 *EXTERNAL RESEARCH LIST 6.25 / TICAL-SCIENCE HIST 05-11-01696 *EXTERNAL RESEARCH LIST 7.25 /HY LAVGUAGE MILITA 05-11-01693 *EXTERNAL RESEARCH PAPER 156 SOVIET IMPACT ON D5-11-01690 \#FA-1 1 INTERNATIONAL-AFFAIRS PUBLIC-OPINI ON S 05-11-02198
\#FA-2 185 - UNITED NATIONS I IN TERNATIONAL-AFFAI $05-11-02199$ \#FA-2 /RS - UNITED NATIONS I INTERNATIONAL-AFFAI Q5-11-02199
\#FA-3, GERMANY I PUBL IC-OP INION SURVEYS FOREIGN 05-11-02186 \#FA-4 IERICAN PROGRAMS OF FOREIGN AID I PLBLIC-0 05-11-01687 *FAR-3960 /EL STUDY OF ESCALATION - VOLUME 3 I A 05-0 $4-02171$ *FAR-3960 (I) / I AL-REALI IY CONFL ICT-RE SOLUTION CO D5-1J-02183 *FAR-3960 (II) / POT HESES-FORMULATIOV CONFL ICT-RES 05-1D-02I82 \#FAR-396I(I) /L ITY THEORY FOR ARMS COVTROL AND D D5-0+-02168 \#FAR-396I(II) CEVELOPMEVT OF LTILITY THEORY FO 05-0+-02169 *FAR-4D47/ION SUEZ-CRISIS ATT I TUDES MIDDLE-EAST O5-04-021 70 *FAR-4048 /TITUDES BEHAVIOR METHODDLOGY SEMANTIC O5-10-02178 *FAR -4049

FAS $M-184$

*FAS $-M-186$ CANADIAN HONEAT $05-10-02179$

*FAS $-M-188$

NEW ZEALAND LI VESTOCK $02-02-02112$ URUGUAY LI VESTOCK $02-02-021$ I 0

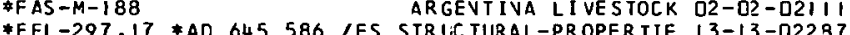
FEL-297.17 *A0 645 586 IES STRLCTURAL-PR OPERTIE 13-13-02287 AFG-E-13.2 IL-DEFENSE-OPERATING-PLANS MENTAL-HE 05-11-02185 *FG-E-13.4, D-DISPENS ING SHEL TER-JCCUPANCY POSTA DG-0B-022 IO *FG-E-I3.5 / CY CLOTHING I DISTR IBUTION LOGISTICS 15-05-02306 *FE-E-13.6 /-OPERATIONS CIVIL-OEFEN SE-OPERATING- 05-11-02189 *FG-F-1.3 IANALYSTS I RADIOACT IVE-FALLOUT 8UILDI 13-13-02276 \#FTD-TT-65-1169 \#AD 631 576 /IGHTIVG-EQUIPMENT C 09-05-01756 \#FTD-TT-65-2035 \#AD 636 GID /JTORS RUSSIA COMMUT D9-03-01752 *GA-7DS8 /E-DECAY RADIOACTIVE-FALLOUT THERMODYNA $18-G 8-02325$
*GA-7598 /ADIOACTIVE-DECAY PARTICLE-SI ZE HENRYS- 18-08-02037 * GARD REPORT 1268-1 /S I VEVTILATION HUMIOITY CO 13-01-02254 *G ARD REPORT 1268-51 /CONTROL EXPERIMENTAL-DATA 13-01-01771 \#GARD- 12921 I) IT IOV-MANUALS PSYCHOLOGY STRESSIPH 15-03-02300 *GARD-12921 III /MENT SHEL TER-JCCUPAYCY PSYCHOLOG I5-03-02207 \#GARD- 12921 I I I I ION-MANUALS PSYCHOLOGY STRESSIPH $15-03-02298$ *GARD-1400 IE DESI GN EXERCISE LIGHTSIILLUMINATIO 13-0I-02252 *GARD-1400 /E DESI GN EXERCISE L IGHTSI I LLUMINATIO 13-01-02252 *H-15 /R-SUPPL I ES RAT ION ING SAN ITAR Y-ENG INEERING O6-C8-01712
*HANDBCOK NO. 72 /SANDW ICH-CONS TRUCTION FIRE-RES $11-12-01766$ *H1-272-D /GERMANY BENELUX NOR WAY DENMARK GREECE 15-03-02147 \#HI-486/3-RR /IMITING-SYSTEMS EVACUATION VULNERA I3-13-01829 *HI-693-RR /I ON-INJURIES YIELD RADIOLOGICAL-DOSA I5-D6-01962 *HI-729-P /Y COUPLE BMD TO FALLOUT SHELTERS 1 UR 15-03-01907 *HI-774-RR / ASTY-SHELTERS DESIGN MA TER IALS EQUIP 13-13-02272 *HI-777-RR /IL-CEFENSE RUSSIA DAMAGE-LIMI ING-SY 15-03-01896 \#HI-777-RR (DRAFT) /IMISSILE-DEFENSE-SYSTEMS UNIT I5-03-01922 \$HI-777/2-PR /LLOUT-SHELTERS EUROPE SURVIVAL REC 15-03-02295 \#HRB-S-94611-F / SURVIVAL RESOURCESIHUMANI RECOVE $05-11-01688$ *HSR-RR-65/1-CR /ESSIPSYCHOLOGYI URBAN-AREAS SOC 15-03-02301 H \$HSR-RR-66/14-PR-APP-I /N URBAN-AREAS SUR VI VAL A 23-D4-02103 \#HSR-RR-66/2 -PR-APP-I IN URBAN-AREAS SUR VI VAL A 23-04-02103
\$HSR-RR-66/21-CR /CIOLOGY STRESS(PSYCHOLOGY) DEM 05-11-02190 *HSR-RR-66/21-CR SUMMARY ICONOMICS POLITICAL-SCI 05-11-02202 *HSR-RR-67/2ME /RATIONS VULNERABIL ITY DISASTERS 05-11-02201 *HSR-RR-67/2ME /RAT IONS VULNERABIL I TY DISASTERS 05-11-02201
*HSR-RR-67/3-CR /-PLANVING STRESS(P SYCHOLOGY) SM 05-11-02203 *HSR-RR-67/5-T I, ATTITUDES MANPOWER MOTIVATION OS-IJ-021BD \#HSR-RR-67/8-SD /RY MANAGEMENT-PLANNING POSTATTA D5-11-02191 \#HUD-MP-39 SCIENCE AND THE CITY I URBAN-AREAS 23-03-02102 *IC-8291" /NCRETE ROCK-STABILIZATION SHOTCRETE RU I3-13-02274 \#ICA HC 65-4I42 /IVG-PLANS REPAIR ZOYSTRUCIION R 05-03-01636 * ICA/HQ-65-3756 /ICAL-SCIEVCE EXECUTIVE-BRANCH L 05-0\$-0167
* IDA/HQ-66-5472 /VERY PRODUCTS VULNERABILITY EXP 05-03-02162 * ICA/HQ-66-5473 /ODUCTS BLAST VUL.NERABILITY AIR- 05-03-02126 *IER-2I-ITSA-2I \#AD 648 Q0I /EQUEVCY INTEGRAL-TR 20-1'-02349 *IER-22-ITSA-22 *AD 648 CO2 /XTREMELY-LOW-FREQUE 20-1+-02348 *IER-22-ITSA- 22 *AD 648 CO2 /XTREMELY-LOW-FREQUE 20-1+-02348
*IITRI-N6O6I \#AD 650 323 /ST RADIOACTI VE-FALLOUT 13-12-02269 * ITRI-N6061 \#AD 650 323 /ST RADIOACTI VE-FALLOUT $13-12-02269$
*INT-25 /AND MCAJURING FIRES I INFRARED-UEILCTOR $13-12-01798$ * JPRS 30,320 /DEFEVSE I RUSSIA TRAIVIVG DISPERSA IS-03-01913 \#JPRS 30,979 \#TT 65-31477 /ELS FLAME-THRO hER-FUE 06-19-01721 *JPRS 32,686 *TT 65-33165 /CAL-CONTAMINATIONICCU 15-02-01843 *JPRS 34,578 *TT 66-31018 /RADIOLOGICAL-CONTAMIN 06-18-0I722 *JPRS-17,889 *OTS-63-21232 /TER-SUPPLIES COOPERA 15-03-02296 *JPRS-27,030 \#TT-64-51286 /-DEFEN SE ZADIOLOGICAL 06-13-01731 *JPRS -27,419 *TT-64-51669 /ATIONAL-CIVIL-DEFENSE 06-07-01709 *JPRS-28,352 /SSR CIVIL-CEFENSE-SYSTEMS INTERNAT 15-03-01937 * JPRS-29, 195 /L-CEFENSE CIVIL-DEFEVSE-SYSIEMS RU 15-03-01928 * JPRS - 30,850 \#TT-65-30871 /E-CA SUAL TIES FIR ST-AI 06-05-01703 * JPRS 31 1 - JPRS 31,014 *TT-65-31512 \%ONSTRUCTION POSTA TIAC 13-12-01804

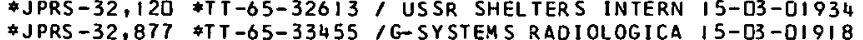
\# JPRS $-32,877$ \#T $-65-33455$ /G-SYSTEMS RADIOLOGICA 15-03-01918
* JPRS-33,842 \#T -66-30285 IIIFIC AB STRACTS - BIO D6-05-01704 \#JPRS -33,842 \#TT-66-30285 IIIFIC ABSTRACTS- BIO O6-05-01704
*JPRS-38,291 *TT-66-34717 /NOA COMMUNISM PROTECT I5-03-01917 
HE PATTLE OF MOSCOW 1 USSR HISTORY COMMUNISM PROPAGANDA FIGHT ING(MANUAL S) WARFARE (TRAINING) MI LI TARY-PSYCHCLCGY ORE I GN I LEADER SHIP MIL I TARY Y PER SONNE L OFF I CER-PERS CNNEL VIL-DEFEN SE) PUBL IC-HEAL TH TRANSPCRTATI ON COMNUNICATION $Y$ MILITARY - PER SONNEL WEAPONS ARMANENT MI LI TARY-STRATEGY S ELECTROMAGNET IC-WA VES PROPAGATICN MODELS (SI MULATIONS) COLD-WAR FOREIGN-POLICY PROPAGANOA ATIITLOES COMMLNISM RGROUND-STRUCTURES DYNAMICS LOADING(MECHANICS) SHELTERS EEF CATTLE I BOVINES SHIELDING CONSTRUCTICN VENTILATION VIRUSES FUNGI PROTOZOA RICKE NIUM-COMPOUNOS FOCUSING PROSPHATES CALCITE POLARIZATION
SHOCK TUBES - A BIBLICGRAPHY T-SFELTERS APPROPRIATIONS ADMINI STRATION-AND-NANAGENENT FALLOUT-SHEL TERS PUBLIC-INFORNA TI ON SHE LTER-NANAGEMENT
RE CATALOGUE I PUBL IC-INFORMATION CIVIL-DEFENSE-SYSTEMS RE CATALOGUE I PUBL IC-INFORMATION CIVIL-DEFENSE-SYSTEMS FORCED-CONCRETE SFELTER-DOORS IRAFFIC STRUCTURAL-SHELLS ELAST SHOCK-WAVES ELASTICITY STRE SS MECHANICAL-ANALYSIS MS PROTECT ION-FAC TOR MONTE-CARLO-NETHOD GECNETRY (OUCTS) ES LOADING(MECHANICS) SHOCK-WAVES NATHEMATICAL-ANALYSIS HOCK-WAVES BLAST FOOTINGS FOUNDATIONS VOID-RATIO ARCHES LTERS BIOLOGICAL-WARFARE-AGENTS CHEMICAL-hARFARE-AGENT STEEL RODS CRACKS STRAIN (MECHANICS) LOADING (NECHANICS ES MATHEMATICAL-PREDICTION THEORY CRATERING PROPAGATION UNCERWATER EXPLOSIONS I SHOCK-WAVES CALCULATICNS MCDELS UNCERWATER EXPLOSIONS ROPAGATION BIOL OGICAL-WARFARE-AGENTS SOUNO-TRANSMISSION BLIC-OP IN ION IN TERNATIONAL-AFFAIRS SURVEYS ARNS-CCNTROL
-AFFAIRS FEAR SUR VEYS STRESS (PSYCHOLOGY) FORLIGN-PCLICY -AFFAIRS FEAR SUR VEY STRESSIP SYCHOLOGY FOREIGN, \$SRIONS-SYSTEMS RADIATION GEOLOGY HUNAN-ENG INEERING WTES ALLOU FALLOUT-SHELTER S SHIELDING STRUCTURAL-PROPERTIES CE CARBON-DIOXIDE AIR SMOKES WIND SHELTERS GERNANY NICE
ON AEROSOLS REYNOLDS-NUMBER BROWNIAN-MOVENENT EOUATIONS HEAT-IRAN SMISSION TESTSIFIRESI SURVEYSIDAIA-COLLECTI ON! COSTS OPTICAL-PROPERTIES COST-EFFEC TIVENESS, \#URS $643-9$ VERSE I MODELS GEOGRAPHY PROGRAMMING (COMPLTERS) MAFPING USEC IN NREC DAMAGE ASSESSMENT PROGRAMS I VULNERABILITY
CTS NUCLEAR-WARFARE ATTACK-PATTERNS RADI OACTI VE-FALLOUT LIOGRAPHY OF PUBL ICATIONS I DAMAGE-ASSESSNENT RESCURCES A ROUTINE I DAMAGE-ASSESSMENT RESCURCES DATA-PROCESSING INCUSTRIAL-PLANTS FINANCE STOCKP ILING STCRAGE MANFOWER MENT AGR ICULTURE COMPUTERS RADIOACTIVE RAOIOAGRICULTURE MENT AGR I CULTURE COMPUTERS RADIOAC II VE RAOIOAGRICULTURE
AMAGE-ASSESSMEVT DATA-PROCESSING PROGRAMNING (CCMPUTERS) AMAGE-ASSESSMEVT DATA-PROCE SSING PROGRAMNING (CCMPUTERS)
MANPOWER INDUSTR IAL-PRODUC IION C CNMERCE IRANSPORT ATICN CTION MAINTENANCE REPAIR GOVERNMENT CASUALTIES CAPACITY MMING(COMPUTERS) BURST-CONDITIONS BLAST YIELO GEOGRAPHY TA-PROCESSING URBAN-AREAS MA THEMA TICAL-MODELS GEOGRAPHY WIND BLAST RADIOACTI VI TY THEORY TESTS EXPERI MENT AL-DATA ROGRAMM ING (COMPUTERS) MATHEMAT ICAL-MODELS FLOW-CHARTING I RESOURCES ANAL YSIS DATA-PROCE SSING DAMAGE-ASSESSNENT B IL ITY OF RESOURCES 1 DAMAGE-A SSE SSMENT hEAPONS-EFFECTS SONNEL HOSPITALS SCHOOL-BLILDINGS INDUSTRIAL-PRODUCTI ON ARFARE PETROLEUM-PIPELINES GAS-PI PELINES TRANSPORTATICN ERABILITY POPULATION CASUALTIES FOOD-SUPPLIES GEOGRAPHY POPULATION CASUALTIES PROGRAMMING (COMPUTERS) GEOGRAPHY CES SURPL USES DATA-PROCESSING SHORTAGES WEAPONS-EFFECTS CES SURPL USES DATA-PROCE SSING SHORTAGES WEAPONS-EFFECTS
RABIL ITY PROGRAMMING (COMPUTERS) DATA-PROCESSING-SYSTEMS RABIL ITY PROGRAMM ING (COMPUTERS) DATA-PROCESSING-SYSTEMS OVERY DATA-PROCESSING MAINTENANCE POSTATTACK-OPERATIONS
POWER GEOGRAPHY INDUSTR IAL-PRODUC TIION DAMAGE-ASSESSMENT POWER GEOGRAPHY INDUSTR IAL-PRODUC TION OAMAGE-ASSESSMENT
TAM INAT ION RADIATION-HAZARDS SHIELDING RADIOAGRICULTURE ONOM I C-RECOVERY INDUSTRIAL-PRODUCTION INVENTORIES ELAS D AGR ICULTURAL RESOURCES I CASUALTIES MEOICAL-PERSCNNE ING FUELS WATER-SUPPL IES MATHEMATICAL-ANALYSI S CLOTHING URCES INDUSTR IAL-PRODUCTION INOUSTRIAL-PLANTS GEOGRAPHY APAC ITY INOUSTR IAL-PRODUCTION DA TA-PROCESSING ECONCMICS -PRODUCTION INVENTORIES CAPACI TY PROGRAMMING (COMPUTERS) URST-CONDITIONS BLAST RADIOACTIIVE-FALLOUT FLOW-CHARTING PUTERS CASUALTIES RADIOACTIVE-FALLOUT IHERMAL-RADI ATI ON LTIES OAMAGE-ASSE SSMENT PATIENTS PROGRAMMING (CCMPUTERS) AMMINGI COMPUTERSI BUR ST-CONDITION NATHEMATICAL-ANALYSIS GASES PUMPINGIOPT ICAL) FLASH-LAMPS LIOUIDS SPECTROSCOPY ENCES I RESOURCES IINFORMATION) DA TA SCIENTIFIC-RESEARCH IL ITY MUTATIONS IN THE HONEY BEE ( HYMENOPTERA GENETICS CECONTAMINATION RADIOACTI VE-WASTES SHIPPING-CONTAINERS DIOACTIVE-WASTES WASTES(INOISTRIAI) SHIPPING-CONTAINERS OCCUP ANCY WATER-SUPPL IES EDUCA TION TRAINING INFANT-DIET MAMENT CIVIL-DEFENSE- SYSTEMS VIE TNAM SHE LTER-ALLOCATICN GY SOCIETAL-RECOVERY DAMAGE-ASSE SSMENT ATTACK-SCENARI OS ENT A ION RADIOI SO TOPES(MEDICAL) RADIONUCLIOES NEOPLAS IA LTERS (FLUIC) RELIABILITY QUALI TY-CONTROL SPECIFICATIONS UREAN-AREAS TUYNELSI TRANSPOR IATION) VENTI LATICN TRAFFIC $N$ A TUBE I SHOCK-TUBES EXPERIMENTAL-DATA PRESSURE-GAGES N A TUBE TYMOCK- TUBES EXPER IMENTAL-DA TA PRE SSURE-GAGES OF RACIOACTIVE MATER IALS BY IHE ORGANISM I RADIOBICLOGY OF RACIOACTIVE MA TER IALS BY THE ORGANISM I RAOIOBICLOGY
OGISTS I TRAINING COMPUTERS TEST-EQUIPMENT TEST-METHCDS OGISTS I TRAINING COMPUTERS TEST-EQUI PMENT TEST-ME THCDS
OMMUNAL SHELTERS I WARNING-SYSTEMS URBAN-AREAS MOVENENT OMMUNAL SHELTER S (WARNING- SYSTEMS URBAN-AREAS MOVENENT
-MEDICINE EMERGENCY-HEALTH-SER VICES REPAIR) \#JPRS-17,8 -MEDICINE EMERGENCY-HEAL TH-SER VICES REPAIR ' \#JPRS-17,88
ACK-OPERAT IONS RESOURCES SURVIVAL INDUSTRIAL-PRODUCTION ERNMENT POL IT ICAL-SCIENCE ECONOMICS DECISION-MAKING LAW -SIGNALS MAPS ELECTROMAGNETIC-COMPATIBILI TY PROPAGATI ON R-RADIATION EGYPT ENGINE S-AND-NOTCRS IRRIGATICN-SYSTEMS T-CORRIDOR COSTS LRBAN-AREAS MANAGEMENT-PLANNING DESIGN $G$ COSTS DRILL ING NORTHEAST-CORRIDOR FEASIBILITY-STUDIES CIAT ION-HAZARDS RADIOAC TI VE-FALLOLT MODELS, \$SRI-IU-3D8 CIAT ION-HAZARDS RADIOACTI VE-FALLOLT MODELS \#SRI - IU-3OB
ANSPORTATION RAIL-TRANSPORTATION HIGHWAY-TRANSPORTATION
\#JPRS $-40,931$ \#TT-67-31573/IFTH ANVIVERSARY OF T 05-04-02173 4JPRS -41,062 \#TT-67-31703 1-5YSTEMS MORALE FIRE- 15-07-02309 * JPRS $-41,308$ FTT-67-31948 /AIVING ARMED-FORCESIF 05-09-02176 \#JPRS - 41,417 \$T-67-32051 /G-PLANS INDUSTRIESICI 15-03-02302

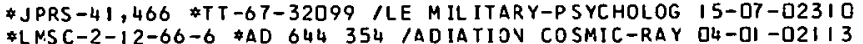
*LNSC-2-12-66-6 \$AD 644354 /ADIATIOV COSMIC-RAY 04-0I-02113
*LT-65-104 /E NUCLEAR-WARFARE BALLISTIC-MISSILES 05-D4-01672 \$MISC. PAPER NO. I-809 ID CONCRETE ARCHES I UNDE 13-13-01830 *MISC. PUB. NO. 947 / TYPE FALLOUT SHELTER FOR B 13-13-01818 \#MISC. PUB. 3, SUPPLEMENT ICT INDEXES I BACTERIA OS-13-017I5 \#ML-1405 \#AFOSR-66-0490 \#AD 637 500 /YSTEMS AMMO 20-0S-0206 $\$ M L M-1189$ $20-04-02052$ \#NP-46 / RGANIZAT IOV CUAL-PURP J SE-SHEL TERS FALLOL OS-OI-01625

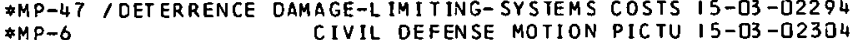
*M6064(3) *AD 636 Q48 /RAL-PROPERTIES STEEL REIN 13-13-01835 HOOD \# 6595 /CRACK - PRCPAGATION IMPACT-TESTS DYNAMICS $20-11-02338$
\#NCEL-R-523/SE-RATE RADIATION-MEASUREMENT-SYSTE D6-19-02219 *NCEL-R-534 /ES WEBS(STRUCTURAL) DE SIGN STRUC TUR 20-11-02337 *NCEL-R-536 \#ASA-13.018 /OUND-STRUCTURES SAND S 20-11-02341 *NCEL-TN-562 ITICNS I FALLOUT-SHELTERS BLAST-SHE 13-13-02278 *NCEL-TR-5 I8 *AC 650 329 /ISTPIBUTIJV DEFLECTION I3-13-02289 *NCRE/R-525 *AD E37289 /HYDROOYNAMICS WA TER-WAV $19-0 ;-02043$ *NOLTR $67-20 /$ I . EXPONENTIAL IDEAL GAS A TMOSPHE 20-04-02332 * NCLT R-66-88 /IN THE GENERATION OF AIRBLASTS BY 19-04-02326 *NCNR-562(37)/3 *AD 641 DI3 /JPERTIES GAS-FLOW P I5-D2-01881 *NCRC-29/ZAT ION PREVENT WARS I PEACE-KEEPING PU 05-11-02195 \#NORC-35 OUIEST INNVAIRES ATTITUDES INTERNATIONAL 05-11-01689 \#NP-1 1768-VOL-1 /OUIPMENT) STRUCTURES COMMUNICAT 17-02-02311 *NP-8571/CONSTRUCT ION URBAN-AREAS RAD IOACTI VE-F 13-13-02277 *NRDL TRC-63 /SAFETY TOXICITY HEAT CARBON-MONOXI 13-12-DI8DD *NRDL-TR-67-49 IYNAMICS PAR TICLE-SI ZE-DISIRIBUTI 20-04-02333 *NROL-TRC-16 *AD 637 8D6 /IND-PRESSURE PRESSURE 13-12-01807 *NRDL-TRC-49 /ENTAL-DATA MATER IALS PARTICAL-SI ZE I9-DI-02042
*NREC-ID, SAL TRANSVERSE MERCATOR, UTM, ANO IN 05-03-02133 *NREC-IILIM A LIST ING OF TABLES AND PARAMETERS 05-03-02138 *NREC-12 /SOURCES DAMAGE-ASSESSMEVT WEAPONS-EFFE O5-03-021 40 \#NREC-I 2 8 I $05-03-02136$

*NREC-13 PARM SYSTEM MANUAL IV. RESOURCE DAT O5-D3-02129 *NREC- 134, SCHOOLS FOOD-SUPPL IES WATER-SUPPLIES D5-03-02137
*NREC- 139
LIVESTOCK III I DAMAGE-ASSE SS D6-18-02216 *NREC- 139 LIVESTOCK I I I I DAMAGE-ASSESS D6-18-02216
*NREC- 14 I E MAINTEVANCE PROCEDURES I RESOLRCES D 05-03-02150 \#NREC- 14 /E MAINTEVANCE PROCEDURES I RE SOLRCES D 05-03-02150
\#NREC- 15 /ITALS HOUSING AGRICULTURE CONSTRUCTION 05-03-02151 \$NREC-17 /RTATION COMMUNICATIONS BANKING CONSTRU O5-03-02153 4NREC-2 /OUT CL IMATOLOGY CASUALTIES FIRES PROGRA 05-03-02134 *NREC-2I /OCEL 1 FIRES PROGRAYMING(COMPUTERS) DA 13-12-02260 *NREC-22 /SOURCES VULNERABILITY NUCLEAR-WARFARE 05-03-02149 \#NREC-24 IURCES DAMAGE-ASSESSMENT LOGIC-DESIGN P D5-03-02155 \#NREC-24(I) ANNEX TO THIRD DRAFT OF READY I 05-03-0215? * NREC-25 GOVERNMEVT COMMUY ICA IME PHA SED ACCESSI 05-03-02156 *NREC-28 Y I DAMAGE-ASSESSMENT MODELS NUCLEAR-W 05-03-02141 \#NREC-20 /NCE I RESOURCES DAMAGE-ASSESSMENT VULN O5-D3-02132 *NREC-30 /M I RESOURCES MODELS DAMAGE-ASSESSMENT 05-03-02130 \#NREC-3I N MODEL I RESOURCES DISTZ IBUTION SER VI 05-03-02143 *NREC-32 /ON I CAMAGE-ASSESSMENT RE SOURCES VULNE 05-03-02148 *NREC-34 ION IN IHE PROTOTYPE MODEL I REPAIR REC 13-13-02282 \#NREC-35 IORATION AND CONVERSION I RESOURCES MAN 05-03-02I59 \#NREC-36 /GICAL-COSE FARM-CROPS RADIOLOGICAL-CON 06-13-02217 *NREC-38 /ITY DAMAGE-ASSESSMENT VULNERAB I LI TY EC 05-03-02152 \#NREC-39/EROLD SIZE DISTRIBUTION, HOSPITALS, AN O5-03-02145 *NREC-4 /PPL I ES ELECTRIC-POWER SAVITARY-ENG INEER O5-03-02135 *NREC-40 / MOBIL IZ AT ION MAVAGEMENT-PLANNING RE SO 05-03-021 42 *NREC- 42 /OURCES MODELS PROGRAMMING(COMPUTERS) C 05-03-02131 *NREC-44 /NS RECOVERY DATA-PROCESSING INDUSTRIAL 05-03-02144 \#RRE-5 / PERSONNEL. ATTACK-PATTERVS WIND YIELD B 05-03-02146 \#NREC-50 /ST RESOURCES PROGRAYM ING-LANGUAGES COM 05-03-02154 *NREC-72 /IMATE I ANALYSIS NUCLEAR-WARFARE-CASUA 06-21-02225 *NREC-9 / I WIND YIELD RADIOACTIVE-FALLOUT PROGR 18-03-02322 *NRL-6444 *AD 637616 /OPERTIES RUBY CRYOGENICS 20-05-02062 * NSF 6I-68/S IN THE PHYSICAL AND B IOLOGICAL SCI 05-02-01629 \#NYO-2315-1 RADIAIION INOUCED VIAB 06-05-02207
\#NY0-9772/L RADIOACTIVE MATERIALS (URBAN-AREAS 18-07-02034 \#NYO-9774 /RADIOACTIVE MATERIALS I CONTAINERS RA 18-07-02033 *OCD-IG-15.1 *ARC-2219 /LTERS LOGISTICS SHLLIEK- 06-08-01713 *OCD-OS-63-48 IUCES PUBL IC-OP IN ION SUR VEYS DISAR 05-10-01678 *OCD-PS-64-2DI/ITICAL-SCIENCE ECONOMICS SOCIOLO D5-11-02193 *ORAU-I-I /ON-INJUR IES RADIATION-EFFECTS INSTRUM D6-18-02215 *ORNL-TR-1596 /URG I GERMANY ROADS CONSTRUCTION 13-02-01786 *ORNL-TR-1598 / PROPAGATION OF AIR SHOCK WA VES 1 20-0.-02053 *ORNL-TR-1646/TS GERMANY CONSTRUCTION HARDENING 13-13-01827 *ORNL-TR-975 INCORPORATION AND ELIMINATION 06-18-01728 *0RNL-3957 /S, SYSTEMS ECOLOGY, AND SYSTEMS ECOL 06-06-01708 *0RO-5P-30 \#AC 204 O9O IITY OV THE LOEATION OF C I5-03-01942 *OTS-63-21232 TTER-SUPPLIES COOPERATION DISASTER I5-03-02296 \#P-2093 *AD 641 156 I ECONOMICS RECOVERY POSTATT 05-03-01647 $\$ P-2289$ \#AD 634797 /-PLANNING UNITED-STA TES-GOV 05-04-01658 $* P-253 * A C 633540 /$ IOV RADAR-INTERFERENCE RADAR $17-07-01988$
$* P-3367$ \#D 638687 /TS FEASIBILITY-STUDIES SOLA $13-0 ?-01777$ \#PB $169521 /$ ATION I PASSENGER - VEHICLES NOR THEAS 23-02-02094 \$PB $170511 / I O N$ TUNNELSI TRANSPORTATION) BLASTIN 13-02-01787 $\triangle P B \quad 170591 / \triangle P H Y$ OF HIGH SPEED GRDUVO TRANSPORT 23-02-02093 \#PE 170595 / ACK-OPERAT IOVS DAMAGE-ASSE SSMENT RA 05-03-02128 
T PERFORMANCEIENGINEERING) VEHICLES DESIGN） \#DSR-76108-2 \#PE 173647 /OPULSION HIGH-SPEEO-GROUND-TRANSPOR 23-02-02096 ATIONS MATHEMATICAL-MODELS HARDWARE DESIGN, \#DSR 76IJ9-2 FEDUL ING SWITCHING PROPUL SION-SYSTEMS AERCDYNAMICS DRAG 19651 WOOD RESOURCES ECONOMICS hOOD-PULP DISTRIBUTION AL-AFFAIRS TARIFFS IMPORT-DUTIES FOREIGN-TRADE CCNNERCE ENGER-VEHICLES LAMINAR-FLOW TURBULENT-FLOh) \#DSR-76IJ8-1
HEALTH IN OUR URBAN FUTURE (URBAN-AREAS PUBLIC-HEALTH HEAL TH IN OUR URBAN FUTURE I URBAN-AREAS PUBLIC-HEALTH
NING REACT ION (P SYCHOLOGY) ADMINISTRATI ON-AND-MANAGEMENT NING REACT IONIP SYCHOLOGY) ADMINISTRA TI ON-AND-MANAGEMENT
ARCS I WATER-SUPP.LIES REGULATIONS RADI OACIIVITY CONTROL ARCS 1 WATER-SUPP.LIES REGULATIONS RADI OAC IIVITY CONTROL
N-DENSITY CERENKOV-RADIATION MICRCWAVES OXIDES PLASTICS N-DENSITY CERENKO V-RADIATION MICRCWAVES OXIDES PLASTICS
QUE I THERMAL-RADIATION HEAPONS-EFFECTS NUCLEAR-WEAPONS QUE I THERMAL-RADIATION FEAPONS-EFFECTS NUCLEAR-WEAPONS TY LAND-USE URBAN-PLANNING POPLLATION POLITICAL-SCIENCE ILITY-STUDIES MANAGEMENT-PLANNING CI VIL-DEFENSE-SYSTEMS TEST-METHODS INFORMATION-RETR IEVAL AUTOMATIC ECONCMICS ATES RADIOLOGICAL-DO SAGE ATTENUATION SIMULATION(MOOELS) ( FIRES TEST-METHODS MODEL S(SIMULATIONS) IEST-EQUIPMENT SCIENCES SCIENT IFIC-RESEARCH UNI TED-STATES DI STRI BUTI ON IELDING WALLS COBALT EXPER IMENTAL-DATA THEORY DOSE-RATE NG(COMPUTERS) DOSE-RATE SHIELDING RECOVERY CALCULATICNS AT ICAL-ANALYSIS RAOIOLOGICAL-CONTAMINATI ON MODELS WALLS FACE-TEMP ERATURES BOUNDARY-LAYER LAMI NAR-BOUNDARY-LAYER TS THERMAL-CONDUC TIVI TY(SOILS) THERMAL-DIFFUSI ON (SCILS) CUTTHROAT GAMES I OPERATIONS-RESEARCH GAME-THEORY ION OF AIR SHOCK WAVES IN TUNNELS I PROPAGATION SHEDEN TION OF AIR SHOCK WAVES IN TUNNELS I PROPAGATION ShEOEN APS TEST-METHODS VULNERABILITY CLASSIFICATION BUI LCINGS SSES DEFORMATION ROTATION INERTIA MATHEMATICAL-ANALYSIS RSONNEL (VULNERABILITY) HOLES BLAST MILI TARY-ENGINEERING SION MEDICAL-PERSONNEL STORMS EMERGENCY-HEALTH-SERVICES ICAL-ANAL YSIS PROGRAMMING (COMP (TERS) THE ORY PRCPE LLANTS -EQU I PMENT ECONOM ICS FEASIBILI TY-STUDIES CLASSIFICATION AT ION DEFENSE-SYSTEMS DE SIGN USSR MI LI TARY-INTELLI IENCE OPEN LITERATURE WITH ABSTRACTS 1 BIBLIOGRAPHIES INCEXES POPUL ATION AGR ICULTURE INDUSTRIES INDUSTRIAL-PRODUCTION K-OPERATIONS SURVIVAL ECONOMIC-RECOVERY LABOR RE SOLRCES GY ECONOMICS PRODUCTION VULNERABILITY MANPOWER RECCVERY AL-RADIATION 8 IOLOGICAL-BLAST-EFFECTS DANAGE-ASSESSMEN OACT I VE-FALLOUT 8 IOLOGICAL-DOSAGE ATTACK-SCENARIOS WINO LD WIND NUCLEAR-WARFARE-CASUALTIES TARGETS SCALE (RATIO) T PEORY STRATEGIC-WAR FARE MILI TARY-STRATEGY OPTI MI ZATICN MMUNOLOGY DISEASE-VEC TORS CHEMOTHERAPY MEDICINE RCDENTS RAGE(FOOD) PRODUC TION IFOOD) RE SOURCES (FOOD) AGRICULTURE NTAL-CATA AGING(PFYSIOLOGY) ANIMALS MICE RATS DOSE-RATE AT IONS RACIAT ION-EFFECTS ANIMALS HUMANS TESTS OOSE-RATE CASUALTIES SEX RACE MANAGEMENT-PLANNING SCCIAL-SCIENCES RAPHY AGE LABOR URBAN-AREAS MODELS RURAL-AREAS MANPOWER EVALUATION DISTRIBUTION (POPULATION) MODELS (SIMULATIONS) N SOUTH VIETNAM I POL ITICAL-SCIENCE SOCI OLOGY ECONCMICS POSTATTACK-OPERATIONS SOIL-CONSERVATI ON SOI L-NECHANICS LROADS SUBWAYS COSTS MANAGEMENT-PLANNING URBAN-PLANNING URBAN-AREAS NEW-YORK MANAGEMENT-PLANNING URBAN-PLANNING MENT - PLANN ING URBAN-PLANN ING WASH ING TON BOSTON NEW-YORK MENT-PLANN ING URBAN-PLANNING WA SH ING TON BOSTON NEW-YORK
$Y$ THERMAL - CONDUCTIVITY ELECTRICAL-CONDUC TANCE GERMANIUM $Y$ THERMAL-CONDUCT IVI TY ELEC TRICAL-CONDUC TANCE GERMANIUM
LLOUT-SHEL TERS MOOEL S (SIMULATIONS) STATISIICAL-ANALYSIS LLOUT-SHEL TERS MOOELSISIMULATIONSI STATI STICAL-ANALYSIS
ION CASUALTIES STATISTICAL-ANALYSIS MATHENATICAL-MCDELS AMM ING (COMPUTEKS) CA SUALTIES NLCLEAR-WARFARE-CASUALTIES MO ISTURE MECHAV ICAL - WAVES CI VI L-ENGINEERING PROPAGATION TY NORTHEAST-CORR IDOR COMPUTERS(PROGRAMMING) PLASTICITY GLES (GEOMETRY) GE OME TRY REVETMENTS EFFECTIVENESS SLOPES ATERS FROM ROW CHARGES INTERRUPTED 8 Y A DUD I CRATERING ON UNDERWATER DESIGN COSTS LOADING (MECHANICS) CYLINDERS NCE GOVERNMENT(FOREIGN) SOCIAL-CONNUNICATICN LEADERSHIP MANAGEMENT-ENGINEER ING SYMPOSIA DATA-PROCESSING-SYSTEMS MUNICATIONS-SYSTEMS RADIATION GEOLOGY HUMAN-ENGINEERING ANAGEMENT-PLANN ING COSTS ILLINOIS OHIO INDIANA MICHIGAN L RESEARCH-PROGRAM-ADMINISTRATION WAR-POTENTIAL BUCGETS ASSESSMENT RADIATION-HAZARDS RADIOACTIVE-FALLOUT MCDELS ASSESSMENT RADIAT ION-HAZARDS RADI OACTI VE-FALLOUT MCDELS
CES PUBL IC-HEAL TH INDUSTRIES SANI TATI ON REPAIR MANFOWER CES PUBL IC-HEAL TH INDUSTRIES SANI TATI ON REPAIR MANFOWER ON RESOURCES UR BAN-AREAS BLILDING S SAN-JOSE VENT I LATION
SCENARIOS LOCAL-GOVERNMENTS STATE-GOVERNMENTS RES CURCES SCENARIOS LOCAL-GOVERNMENTS STATE -GOVERNMENTS RES CURCES
TS COMMAND-AND-CONTROL-SYSTEMS PERFORMANCE (ENGINEERING) DECONTAMINATION MATHEMATICAL-ANALYSIS RADIATICN-HAZARDS ICLES AIRCRAFT WEAPONS-EFFECTS DANAGE-ASSESSMENT TRUCKS PULAT ION SURVIVAL DAMAGE-ASSE SSMENT CALIFCRNI A RECCVERY HAZARCS SOILS PLANTS (BOTANY) RADI CLOGICAL-CONTAMINATION DAMAEE-ASSESSMENT CALIFORNIA URBAN-AREAS SUPPLY-DEPOTS RICONTAM INATION) RADIOL COSTA-RICA WI ND INSTRUMENTATI ON RMEASURES FALLOUT-SHELTERS RADIOLCGICAL-DCSAGE MANFOWER MANAGEMENT-PLANNING STATE-GOVERNMENTS LOCAL-GOVERNNENTS PTAEILITY, FALLOUT-SHELTERS CONFINED-ENVIRONMENTS TASTE PTAE IL ITY) FALLOUT-SHELTER S CONF I NED-ENVI RONMENTS TASTE
IN(MECHANICS) BUCKL ING(MECHANICS) MATHEMATICAL-ANALYSIS IN(MECHAN ICS) BUCKL ING(MECHANICS) MA THEMAIICAL-ANALYSIS
FAUL T-LOCATION LIGHTNING-ARRESTERS TELEPHCNE-EQUI FMENT FAUL T-LOCATION LIGHTNING-ARRE STERS TE LEPHCNE-EQUI FMENT
TIAL-DISTR IBUT ION-SYSTEMS UNDERGRCUND-STRUCTURES DESIGN TIAL-DISTR IBUT ION-SY TEMS UNDERGR CUND-STRUCTURES DESIGN
SSMENT SHELTERS SHEL TER-ALLOCA TION DATA WARNING-SYSTEMS ST CYNAMICS LOADINGIMECHANICS) STRAINIMECHANICSI THECRY OIL-MECHANICS CLAY COMMUNICATI ON-EOUI PMENT INTERACTIONS
ARTIAL BODY RADIATION OF OUEEN HONEY BEES FYMENOFTERA 4PE 173 649 /NSIONS I VIBRATION-ISOLA TORS CALCUL 20-11-02087 *PB 173 658 /RANSPORTATION NETWORKS TERMINALS SC 23-0?-02354 *PE 173659 QPQ-173660 / JOURNAL - CANADA I LAW INTERNATION D5-D3-D164 *PE-173646 /URBAN-AREAS MDOELSISIMULATIONS) PASS 23-0?-02I0I *PHS- 1581 SECURING $06-05-02205$ PHS-3ID /IES MOTIVATION PUBL IC-IVFORMATION TRAI D5-ID-0I68I PHS-956 IIC HEALTH SERVICE DR INK ING WA TER STAND 13-02-01778 *PIBMRI-1295.3-66 *AD 642271 /IC-THEORY ELECTRO 20-03-02330 *PR-4 /INITE, SOL ID BY A FINITE DIFFERENCE TECHNI $13-12-01795$
*PRC D-1219, UREAN-PLANN ING LAND-USE POPULATION 23-06-02106 *PRC D- 1219 \% UREAN-PLANN ING LAND-USE POP ULA TION 23-06-02106

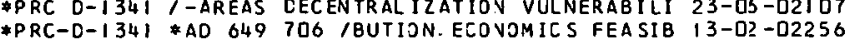

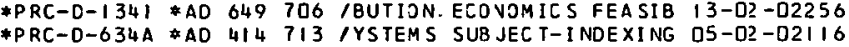
\#PRC-0-634A \#AD 414713 /YSTEMS SUB JECT-INDEXING 05-02-02116
*PSDC-TR-16 /HIELDING RADIOACT IVE-FALLOUT DOSE-R 18-05-02030 *PSW-90 /PERATURES IN A LARGE NATURAL FUEL FIRE 13-12-01810 *PUBL. 1408 /NT-PLANNIVG DEC IS ION-MAKING SOC IAL- 05-0I -01622 \#R-OU-266 II) /(COMPUTERS) RADIOAC IIVE-FALLOUT SH D6-19-D221B R-OU-26611) /ACTOR MONTE-CARLO-METHOO PROGRAMMI 06-18-02214 AR - 273 - 66 (II) IIN BLAST SHIELPING THEORY MATHEM DO-18-02221 *R-493/CS COOL ING-AND-VENTILAT ING-EQUIPMENT TES 13-DI-01776 *RAC-P-2O $* A D$ O 38220 $12-02-01768$ *REPORT NC. ID3-24 \#AEC-TR-5025 /ULATING ATTENUA 20-04-02051 *REPORT NC. 6569 /HOTOGRAPHS URBAV-AREAS FIRES M 13-12-01809 *REPORT 1841 \#AD 631 325 /IR-CONDITIONING-EOUIPM 13-0I-01775

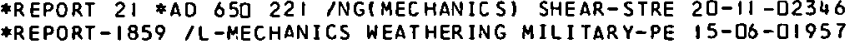
*RESEARCH REPORT 17 /MUNICATIONS-SYSTEMS SUPERVI O6-1?-01714 *RIA-62-1794 \#AC $276 \quad 154 / 5$ P2OJECTILES MATHEMAT 19-0S-02329 \#RN-1512 \#AC 87449 / POSSIBILITIES I INDUSTRIAL 05-03-01648 \#RM-1829-1 \#AD 133 DI2 /ARCH MODELS THREAT-EVALU I 5-03-02303 \#RM-2800-1(SUPP) *AD 626930 /THOR INDEX OF THE 05-03-01652 *RM-3436-PR *AD 426 9D6 /ARE SURVIVAL ECONOMICS 05-03-02158 *RN-428 *AD 210651 /G THE WAR I UILY POSIATTAC DS-03-0212 \$RM-4706-TAB /RY JAPAN RADIOAC TIVE-FALLOUT THERM D6-21-02228 *RM-4707-TAB ILE-SIZE SHELTERS DISTRIBUTION RADI D6-21-02229 *RN-4725-TAB IIROSHIMA EXPLOSION I A IR-BURST YIE 15-D6-01958 \#RN-4817-PR *AD 637464 , VUCLEAR-WARFARE MODEL- 15-06-01959 RM-4968-TAB IL IC-HEALIH POSTA ITACK-DPERA TIONS I O6-05-01702 *RM-5052-TAB IPOSTATTACK-OPERATIONS SURVIVAL STO 06-15-01717 *RM-5052-TAB /POSTATTACK-OPERATIONS SURVIVAL STO O6-15-01717
$*$ RM-5083-TAB /URIES RADIOLOGICAL-DOSAGE EXPERIME D6-18-01733 *RN-5096-TAB IS 1 GENET ICS RADIATIOV-HAZARDS MUI 06-19-01727 *RM-5 II5-TAB IEAS VULNERABILITY NUELEAR-WARFARE- 15-DS-01955 *RM-5 I40-TAB IDIOACTIVE-FALLOUT CASUALTIES DEMOG 05-03-02160 *RM-5141-TAB /STRATEGY DAMAGE-ASSESSMENT THREAT- 15-07-01966 \#RN-5 I8I-ARPA /RS AFFECTING GJVERVMEVT CONTROL I 05-[9-02175 *RM-5203-TAB ION - SOME PROBLEMS AVD PROSPECTS $108-13-01745$ \#RM-5362-PR / AD IOBIOLOGY PUBLIIC-HEALTH STRESSIPH 06-05-02206 \#RPN-71-72, NEW-YORK URBAN-AREAS POPULATION RAI 23-02-02099

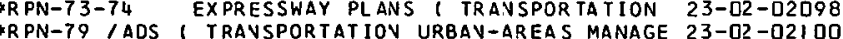
\#RSIC-523 *AD 631224 , COOLIVG THERMOELECTRICIT D9-0I -01748 *RSIC-523 *AD 631224 T COOLIVG THERMOELECTRICIT $09-01-01748$ *RT I-OU-230-2 (I) /EFFEC IS RADI OACTI VE-FALLOUT FA $15-03-01935$ RRT I-OU-230-2 (I II) IT-S HELTERS MATHEMATICS PROGR IS-03-01938 *R66-I3 AD 638027 , SOIL-MECHAN ICS ACCOUSTICS 20-01-02047 R66-53 /ION DEFORMATION TRANSPOR TATION ELASTICI 20-1I-02084 -SC-RR-67-24 /SAND MODELS I EXPERIMENTAL-DATA AN 18-03-02317 *SC-RR-67-3 /OS ICNS - PEACEFUL APPL ICATIONS - CR 18-03-02314 TAMG POLITICAL-SCIE $18-09-02040$ *SP-2655 AD 645 517 /ATION-RETRIEVAL AUTOMATIC O5-02-02121 *SR-45 \#NP-11768-VOL-1 /OUIPMENT) STRUCTURES COM 17-02-02311 \#SRI 4949-5DD *AC 651 944 I TASTE CIVIL-DEFENSE- 06-03-0221I USRI-IM- 4559 TAD OO2 139 OSTS CIVILIAN-PERSONNE I5-03-02305 SRI-IU-3084 *SRI-IU-3084 \#PB 170595 /ACK-OPERATIONS DAMAGE- 05-03-02128
*SRI-MU-4536 INATION PEST-CONTROL MOZ TUARY-SERVI I 5-03-01953 *SRI-MU-4536 /NATION PEST-COVTROL MOR TUARY-SERVI I 5-03-01953 SRI-MU-4949-120 /EFENSE-OPERATING-PLANS ATTACK- 17-02-02312 SRI-MII-4949- 121 /MMUNICATION-SYSTEMS MODEL 5 COS 17-02-0231 3 -SRI-MU-4949-I30 /SOURCES POPULATIOV FACILITIES 15-0S-02307 4SRI-MU-4949-150/LITY ROADS RAIL 30AOS CARGO-VEH 13-05-02260 *SRI-MU-4949-350-A /ERY POSTATTACK-OPERATIONS PO 05-03-DI639 *SRI-MU-5095 /MULATIONS) RADIOBIOLOGY RADIATION- 06-18-01725 *SRI-MU-5776 *USNRDL-TRC-67-3 /SING DISTR IBUTION 06-08-01711 *SRI-MU-5779(B) /TURES I RADIJACTIVE-FALLOUT PAR 18-C3-02038 *SRI-MU-5779A /RECOVERY FOODICONTAMINATIONI WATE O6-13-01723 *SRI-MU-5806 \#USNRDL-TRC-76 ION DOSE-RATE COUNTE 06-19-02220 SRI SRI-4949-500 \#AC 638630 ' ADJUNCTS I FOODIACCE D6-C8-01710

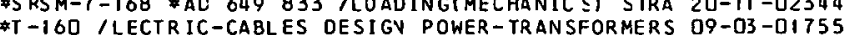
* $1-160$ /LECTR IC-CABLES DESI GV POWER-TRANSFORMERS 09-03-01755 * TECHNICAL NOTE 89/5, CAL CULATIONS DAMAGE-ASSE I5-03-01930 *TECHNICAL REPORT NO. I-720 /TURES ELASTICITY TE 13-13-01825

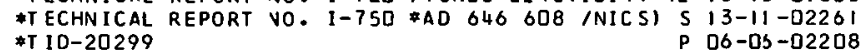


ITATION WATER-SUPPLIES FOOD UNITED-STATES CANADA MEXICO, \#ID-23286 /ISOTOPES AIR YILK ATMOSPHERIC-PRECIP I8-OS-O2035 PANTCD THE $18-05-02320$ ARMY SURVEYING MAPPING ENGINEERING ARTILLERY GEODESICS, *T 5-241-8 UNIVERSAL TRANSVERSE MERCATOR GRID I D8-DG-D224D NS RANDOM-VAR IABLES WEAPON-SYSTEMS ANALYSIS-OF-VARIANCE, \#TM-K-77/66 \#AD 646 I4I /ABILITY-DEVSITY-FUNCTIO I2-OI -O225I ON SCIENCE AND TECHNOLOGY FOR 1965 IV. ( DOCUNENTATICN) \$TM-2625/004/D0 $\$$ AD 648 562/GRAPHY OV INFORMATI O5-02-D2122 ORMATION-RETR IEVAL SCIENTIFIC-ORGANI ZATIONS PERIODICALS; \$TM-3008/004/00 \$AD 649 637/NTIFIC-RESEARCH INF D5-02-D2I23 S-RESEARCH TRAINING MATHEMATICS DATA-PROCESSING-SYSTEMS, \$TN-53D-DO9-DO ING-LANGUAGES EDUCATION OPERATION D9-O2-DI75O G OPERATIONS-RESEARCH PROGRAMMING-LANGUAGES MATHEMATICS, FREQUENCY BENDING ELASTICITY LOADING (MECHANICS) INPACT, \#TMB-704 *AC 650 178, SUPPORT 1 RINGS VIBRATION 2D-11-O2336 REIMLCHANICS) UNDERGROUND-STRUCTURES NUCLEAR-EXPLCSIONS I \#TR SO8 \#DASA- 13.018 /ECHAVICS SHOCK-WAVES FAILU I8-D3-D2OJ8 CLEAR-WEAPONS SHIELOING DOSE-RATE CALCULATIONS SLANTING) \#TR-20(I) /LOUT PROTECTION 1 FALLOUT-SHELTERS NU I3-13-0I839 TROP ICAL - REGIONS PERFORMANCE (HUMAN) STRE SS (PHYSICLCGY), DEL BUILDING COOES T CONSTRUCTION DUAL-PURPOSE-SHELTERS, \#R-36 /SFELTER COVSTRUCTION IN FOUR NATIONAL MO I3-I3-D22BI CTIVE-FALLOUT CONSIRUCTION CONSTRLCTION-SLANTING DESIGN I \#TR-39/RDS SHIELDING RADIATION-EFFECTS RADIOA I3-I3-O22B6 -EQU IPMENT BEAMSISTRUCTURALI LOADING (MECHANICS) STATICS I \#TR-5D2 \#DASA-13.0I8 /CONCRETE BEAMS II. I TEST 13-13-0I84I UCTURES SOIL-MECHANICS SURFACE-BLRST FAILLRE (MECHANICS), \#TR-5D8/ES III TESTS I SHELTERS UVDERGROUNO-STR O8-I3-DI746 S) CESIG. COSTS MODELSISIMLLATIONSI STRUCTURAL-ANALYSIS, TR-SID /SES LOACING(MECHAVICS) FAILURE(MECHANIC I3-I3-DIB32 ION TESTS RADIO-TELEMETRY RADIATICN-MEASUREMENT-SYSTEMS, \$TR-66.020I.0D3 IATION RADIATION-MONITORS DETECT I8-D $4-02026$ D PHYSICAL-PROPERTIES CONTAINERS TASTE FALLOUT-SHELTERS, \#TR-67-25-CD *AD O4D 823 /PRESERVATION FIBERBDAR D6-08-D22I2 OGY $\triangle R M E D-F O R C E S-O P E R A T I O N S$ LOGISIICS AGING(PHYSIOLOGY) / \$TR-67-57-PR \$AD 649 540/HOLOGY) SOCIAL-PSYCHOL G5-ID-02I8I TIONS FIRST-AID NUCLEAR-WARFARE-CASUALTIES) *JPRS 30,979 \$T 65-31477 /ELS FLAME-THROWER-FUELS SMOKE-MUNI D6-I3-DI72I BIOLOGICAL-WARFARE-AGENTS DECONTAMINATION) \$JPRS 32,686 \#TT $65-33165$ /CAL-CONTAMINATIONICOUNTERMEASURES) I5-0?-DI843 L-WARFARE-AGENTS BIOLOGICAL-WARFARE-AGENTS, \#JPRS 34,578 \#T 66-31018 /RACIOLOGICAL-CONTAMINATION CHEMICA DO-I3-DI723 4T T-64-5I286 PERAT IONS RESCUES CONSTRUC TION FIRES ROADS)

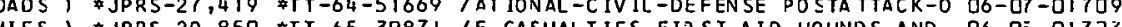
INJUR IES RADIATION-INJURIES BIBLICGRAPHIES, \#PRS-29,850 \$TT-65-30871 7E-CASUALTIES FI S ST-4ID WOUNDS-AND- D6-05-0I7J3 REPAIR MILITARY-ENGINEERING RECONNAISSANCE I \#JPRS-31,014 \#TT-65-315I2 /ONSTRUCTION POSTATTACK-OPERATIONS I3-1?- II8D4

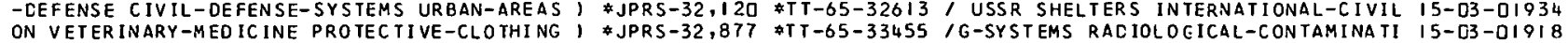
CE ( BIBL IOGRAPHIES MEDICINE BIOLCGY CHINA) *JPRS-33,842 \#TI-66-30285/TIFIC ABSTRACTS - BIOMEDICAL SCIEN O6-O5-DI7D4 LOGICAL-WARFARE-AGENTS MANAGEMENT-PLANNING) *JPRS-38,291 \#TT-66-347I7 /NDA COMMUNISM PROTECTIVE-MASKS BIO IS-03-0I917 MOSCOW ( USSR HISTORY COMMLNISM PROPAGANDA; \#JPRS-40,931 \#TT-67-31573/IFTH ANNIVERSARY OF THE BATTLE OF O5-0 $4-02173$ ALS) WARFAREI TRAINING) MILI IARY-P SYCHOLOGY， \#JPRS-41,062 *TT-67-31703 /-SYSTEMS MORALE FIRE-FIGHTING (MANU I5-O7-D23D9 RSHIP MILITARY-PER SONNEL OFFICER-PERSONNEL， JPRS-4I,308 \#TT-67-31948 /AINING ARMED-FORCESIFOREIGNI LEADE O5-07-02I76 PUBL I C-HEALTH TRANSPORTATION COMMLNICATION) *JPRS-41.417 \#TT-67-3205I /G-PLANS INDUSTRIESICIVIL-DEFENSE) I5-03-02302

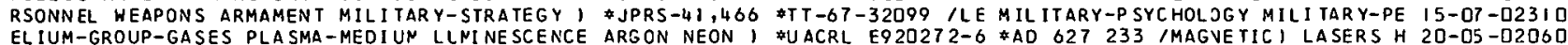
M PLASMA-OSCILLAT IONS STABILITY BOUNDARY-VALUE-PROELEMS, \#UCLA-R-IO \#AD OL8 D83, I GAS-FLOW PLASMA-MEDIU $20-09-02335$ M PLASMA-OSCILLATIONS STABILITY BOUNDARY-VALUE-PROELEMS, \#UCLA-R-IO *AD 648 D83,

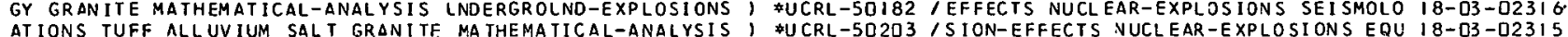
ATIONS TUFF ALL.UVIUM SALT GRDNITF MA THEMATICAL-ANALYSIS, \#UCRL-SD2Q3 /SION-EFFECTS NUCLEAR-EXPLOSIONS EQU 18 - D3-D2315

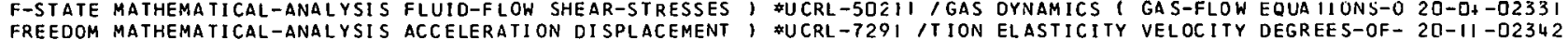

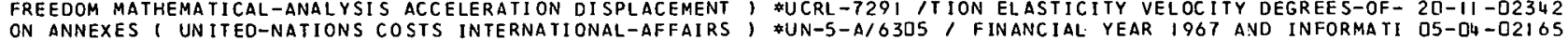

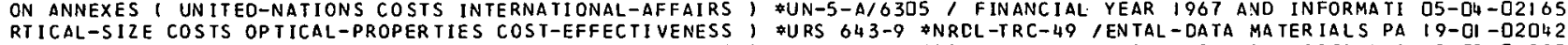
LIINGS FA ILURE(MECHANICS) EXPERIMENTAL-DATA BLAST FIRES, WURS-658-3/(SIMULATIOVS) LOADING(MELHANICS) BUI I8-O3-DIO95 ATE-GOVERNMENTS ADMINISTRATION-AND-MANAGENENT INSURANCE, \#USL-161/PENSATION LAWS I LEGISLATION LABOR ST OS-O7-02I74 T-CONCITIONS URBAN-AREAS FIRES NUCLEAR-EXPLOSION-OAMAGE, \#USNRDL-TR-IQ4D /FIRE FROM NUCLEAR BURSTS I BURS I3-I2-OIBDI VE-FALLOUT SHIELDING BUILDINGS SHELTERS COSTS PARTICLES I *USNRDL-TR-ID64 1ONSTRUCT ION-MATERIALS RADIOACTI I8-DS-O232I TION URBAV-AREAS PROTECTION-FACTOR BLAST-SHELTERS CCSTS I \#USNRDL-TR-582 / UNDERGROUVD-STRUCTURES CONSTRUC I3-13-OI8I5 NGI COMPUTERS) REPAIR PER SONNEL TRAINING CCUNTERMEASURES, \#USNROL-TR-656 *AD 419334 /1SURVIVAL) PROGRAMMI O5-03.-DI651 RBAN-AREAS MODELSISIMULATIONSI GRAPHICS TABLES MICHIGAN, \#USNRDL-TR-984 \#AD 635 250 IDAMAGE RECLAMATION U I5-03-0I963 CALCULAT IONS RAD IOACTIVE-FALLOUT MANPOWER-STUDIES, IME, \#USNRDL-IRC-42, /TOR RADIOLOGICAL-DOSAGERECOVERY O6-I8-OI729 CALCULAT IONS RAD IOACTIVE-FALLOUT MANPOWER-STUDIES TIME \#USNOL-IRC-42 /TOR RADIOLOGICAL-DOSAGE RECOVERY O6-18-0I729 SMENT CAL IFORNIA URBAN-AREAS SLPPLY-DEPOTS, \#SRI.-NU-5776 \#USNRDL-TRC-67-3 7SING OISTR IBUTIDV DAMAGE-A SSES D6-DB-DI7II EDICTION YIELD GEOMETRY DISTRIBUTI ON PARTICLE-SI ZE WINDI \#USRDL-TRC-68 /IOACTIVE-FALLOUT MATHEMATICAL-PR I8-03-D2039 LOUT-SHEL TERS RAD IOLOGICAL-DOSAGE MANPOWER, \#SRI-NU-5806 \#USNRDL-TRC-76 /ON DOSE-RATE COUNTERMEASURES FAL O6-19-022
S URBAN-AREAS SAN-JOSE CALIFORNIA RECOVERY PEST-CONTROL, \#USNRDL-TRC-79, /CONTROL) DRAIVAGE COUNTERMEASURE I3-D2-DI7BO S URBAN-AREAS SAN-JOSE CALIFORNIA RECOVERY PEST-CONTROL, \$USNRDL-TRC-79 64970I/MIC-WAVES EXPLOSION-EFFEC IB-O3-D23I9

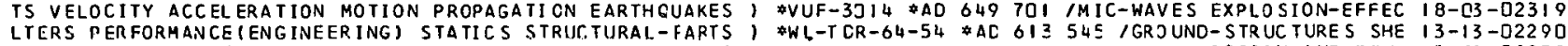
TRUCTION OF SANITARY AND STOKM SERERS (SANITARY-SEWERS, *WPCF-9, DESIGN AND CONS I3-IIZ-CI2259 SS STONE BLAST EXPER IMENTAL-DATA INSTRUMENTATICN THEORY, \#WT-1168, BLAST-ENERGIZED-MISSILES VELOCITY GLA I8-O3-O23I8 RACIATION EFFECTS ON PAINTS, PLASTICS, ANO COATED FABRICS \$WT-407 I3-12-02263 -MOT ION, STRESSES STIFFENED-CYLINDERS STRAIN(NECHANICS) , \#WV-6717 \#AD 649 662 /E-MATEZIALS, E2UATIONS-OF 20-II -02345 ULT-LOCATION ELECTRIC-CABLES TRANSFORMERS HEAT-TRANSER, T-ATT ACK-OPERAT IONS DAMAGE-ASSESSNENT FIVE-CITY-STLDIES, \#977II-F, TARY-INTELLIGENCE SYSTEMS-AVALYSIS POS I3-12-02267 
THIS PAGE

\section{WAS INTENTIONALLY LEFT BLANK}




\section{Corporate Author Index}

A

\begin{tabular}{ll} 
ADVANCED KINETICS & \\
AEC & $20-05-02064$ \\
AEC & $06-18-01726$ \\
AEC & $18-05-02027$ \\
AEC & $18-05-02320$ \\
AEC & $18-08-02035$ \\
AF AERO PROP LAB & $20-08-02070$ \\
AF FORE IGN TECH DIV & $10-03-01764$ \\
AF FORE IGN TECH DIV & $09-03-01752$ \\
AF OFF AEROSPACE RES & $09-05-01756$ \\
AF OFF AEROSPACE RES & $04-02-02114$ \\
AGBAB IAN-JACOBSEN ASSOC & $05-02-01630$ \\
AGB AB I AN-JACOBSEN ASSOC & $13-13-01823$ \\
AGENCY INTERNAT DEVEL & $20-11-02078$ \\
ALDER SON RESEARCH LABS & $05-04-01661$ \\
AMER SOC CIVIL ENG & $13-12-01808$ \\
AMER MED ASSN & $20-11-02339$ \\
AMER MED ACSN & $06-21-01740$ \\
AMER NAT RED CROSS & $06-21-02233$ \\
AMER NAT RED CROSS & $06-08-01712$ \\
AMER NUC SOC & $06-08-01713$ \\
AMER GAS ASSN & $18-06-02029$ \\
AMER INST RES & $05-03-01642$ \\
AMER INST RES & $05-01-01621$ \\
AMER INST RES & $05-01-01624$ \\
AMER INST RES & $05-01-01626$ \\
AMER INST RES & $05-09-01675$ \\
AMER INST RES & $05-10-01680$ \\
AMER INST RES & $05-10-01684$ \\
AMER PUB HEALTH ASSN & $05-11-01699$ \\
AMER PUBLIC WORKS ASSN & $15-02-01851$ \\
AMMANN AND WHITNEY & $13-02-01789$ \\
ARMED FORCES INST PATH & $13-13-02280$ \\
ARMED FORCES SWP : & $06-05-01701$ \\
ARMED FORCES RADIOB IO RES & $13-12-02263$ \\
ARMOUR & $15-06-01964$ \\
ARMOUR & $18-03-02001$ \\
& $18-03-02005$ \\
\hline
\end{tabular}

ARMY B IOL LAB

ARMY BIOL LAB

ARMY B IOL LAB

ARMY B IOL LAB

ARMY B IOL LAB

ARMY CHEMICAL CORPS

ARMY CHEMICAL CORPS

ARMY CHEMICAL CORPS

ARMY CHEMICAL CORPS

ARMY CHEMICAL CORPS

ARMY, DEPT OF

ARMY EDGEWOOD AR SENAL:

ARMY ELECTRONICS COM

ARMY ENG R-D LAB

ARMY ENG R-D LAB

ARMY ENGINEERS

ARMY ENG INEERS

ARMY ENGINEERS

ARMY ENG INEERS

ARMY ENG INEERS

ARMY ENGINEERS

ARMY FORT DETRICK

ARMY FORT DETRICK

ARMY FORT DETRICK

ARMY MATER IALS RESEARCH

ARMY IVEAPONS COMMAND

ASIA FOUNOATION

ATOMIC BOMB CASUALTY COM

ATOMIC BOMB CASUALTY COM

B

BARRY CONTROLS

BATTELLE MEMOR IAL INST

BECHTEL CORP

BOLT BERANEK AND NEWMAN

BROWN UNIV.

BROWN UNIV

BUNKER RAMO CORP
$06-13-01715$
$15-02-01858$
$15-02-01875$
$15-02-01891$
$15-02-01892$
$15-02-01850$
$15-02-01853$
$15-02-01855$
$15-02-01857$
$15-02-01863$
$08-06-02240$
$15-02-01861$
$14-02-01842$
$13-01-01775$
$15-06-01957$
$13-11-02261$
$13-13-01825$
$13-13-01830$
$18-03-02007$
$18-03-02017$
$20-11-02080$
$15-02-01849$
$15-02-01852$
$15-02-01854$
$13-01-02255$
$20-11-02345$
$05-03-02161$
$06-18-01730$
$06-21-02724$

13-13-02291

$13-01-01773$

13-0y-02040

19-04-02326

15-02-01881

20-11-02080

$05-05-01673$ 
C

CALIFORNIA, STATE OF

CANADA DEPT OF NATL HEAL

CANADA DEPT OF NATL HEAL

CANADA DEPT OF NATL HEAL

CANADA DEPT OF NATL HEAL

CANADA -DEPT OF NATL HEAL

CARNEGIE INST OF TECH

CETO

CETO

CETO

CHARLES A. MAGUIRE ACSOC

CIVIL AERO ADMIN AERO CEN

COLO SCH MINES RES FOUN

COLO SCH MINES RES FOUN

COLO SCH MINES. RES FOUN

COLO SCH MINES RES FOUN

COLO .SCH MINES RES FOUN

COLO SCH MINES RES FOUN

CONSULTANTS BUREAU

CORN REF INERS ASSN

$D D C$

D

$D D C$

DE LEUW CATHER

DETRQIT QGD.

DIKEWOOU

DIKEWOOD

DIKEWOOD

DIKEWOOD

DISTRICT OF COLUMBIA

DISTRICT OF COLUMBIA

DOCUMENTATION INC

DOUGLAS AIRCRAFT
13-02-02258

$06-21-01737$

06-21-01738

$06-21,-01739$

06-21-022.20

18-06-02028

23-02-0209?

13-13-01827

18-08-02324

20-08-02334

13-13-02271

$13-12-01803$

$08-07-022.41$

$08-07-022.43$

$08-07-02244$

$0.8-11-02247$

13-13-02273

13-13-022.75

08-07-02242

06-16-01720

05-02 -02125

06-08-022.09

23-02-02353

15-03-019!5

06-21-02227

$06-21-022.33$

13-12-01805

13-12-0226?

$13-13-01816$

13-13-01817

$05-02-02119$

05-02-02118

E

EAST. COAST NICEP

EBS MANAGEMENT CON

EBS MANAGEMENT CON EDGERTON GERME SHAU SEN

ENG INEER I NG-SCIENCE

ENG INEER ING-SCIENCE

ENG INEER ING-SCIENCE
FAO-UN

FED POWER COM

FORD FOUNDAT ION

FRANCE

FRANKLIN INSTITUTE

F$$
\text { . FRANKLIN INSTITUTE }
$$

$G$

GAGE BABCOCK

GAUTNEY AND JONES COM

GEN AM TRANSPORT CORP

GEN AM TRANSPORT CORP

GEN AM.TRANSPORT CORP

GEN AM TRANSPPORT CORP

GEN AM TRANSPORT CORP

GEN AM TRANSPORT CORP

GEN AM TRANSPORT CORP

GENERAL DYNAMICS

GENERAL DYNAMICS

GENERAL ELECTRIC

GERMANY

GERMANY

GERMANY

GRAVES-HILL

GSA

GSA

GUY B, PANERO, INC

GUY B: PANERO, INC

$\mathrm{H}$.

HAYES SEAY MATTERN

HËBREW UNIV

HRB - SINGER

HRB - SINGER

HUDSON INSTITUTE

HUDSON INSTITUTE

HUDSON INSTITUTE

HUDSON INSTITUTE

HUDSON INSTITUTE

HUDSON INSTITUTE

HUDSON INSTITUTE

HUDSON. INSTI I TUTE

HUDSON INSTITUTE

HUGHES AIRCRAFT
02-02-01616

$09-03-02249$

05-03-01633

06-18-01728

19-06-02.328

13-12-01807

18-04-02026:

$13-01-01771$

13-01-01774

13-01-027.52

$13-01-02254$

15-03-02297

15-03-027.98

15-03-02300

18-08-02037

18-08-02325

06-06-01706

13-02-01786

13-13-01827.

20-01-02048

13-13-02281

15-03-01946

$17-02-01978$

$13-13-01813$

13-13-01814

1.3-13-01828

12-01-02250

$05-11-01688$

13-12-02267

05-03-02.147

13-13-01829

13-13-022.72

15-03-01896

15-03-01907

15-03-01922

15-03-022.95

15-06-01962

23-02-02097

20-11-02344 


\begin{abstract}
HUMAN SCIENCES RESEARCH HUMAN SCIENCES RESEARCH HUMAN SCIENCES RESEARCH HUMAN SCIENCES RESEARCH HUMAN SCIENCES RESEARCH HUMAN SCIENCES RESEARCH HUMAN SCIENCES RESEARCH HUMAN SCIENCES RESEARCH HUMAN SCIENCES RESEARCH HUMAN SCIENCES RESEARCH
\end{abstract}

IDA
IDA
IDA
IDA
IDA
IDA
IDA
IEEE
IEEE
IEEE
IITR I
IITR I
IITR I
IITR I
IITR I
IITR I
INDIA
INST ENVIRON RES
INST ENVIRON RES
INTERNAT CUS TAR BUR

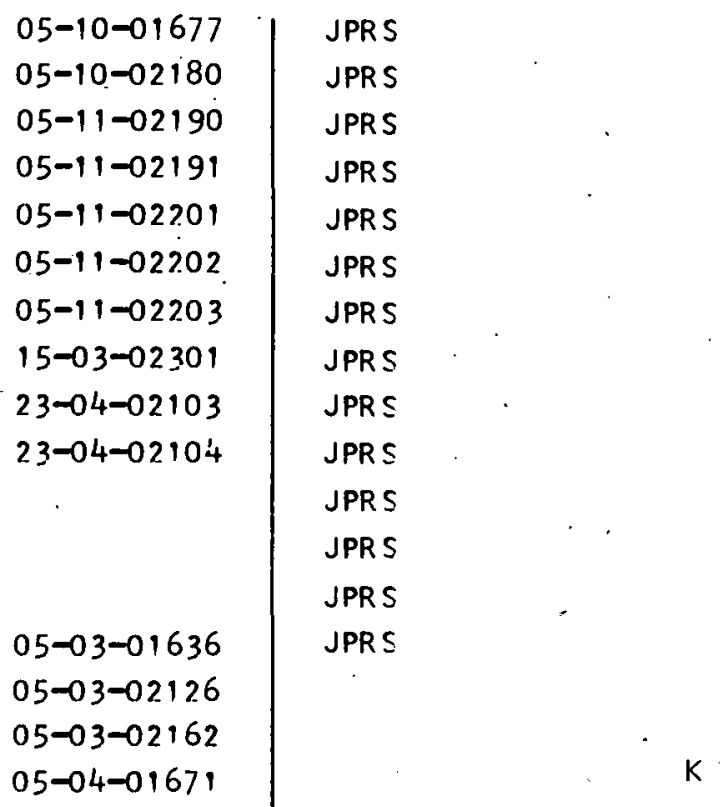

KAISER

$23-02-02356$

13-12-02265

17-09-01988

09-03-01753

$09-03-01754$

09-03-01755

$13-1 \cdot 2-01807$

13-12-02269

13-13-01811

$13-13-01819$

$13-13-01835$

20-11-02338

$13-11-01792$

20-14-02348

20-14-02349

05-03-01641

LA COUN FLOOD CON DIS

LA COUN FLOOO CON DIS

LA COUN FLOOD CON DIS

LA COUN FLOOD CON DIS

LA COUN FLOOD CON DIS LEHIGH UNIV.

LIBRARY OF CONGRESS

LIBRARY OF CONGRESS

LOCKHEED MISSILES

LOVELACE

LOVELACE

LOVELACE

LOVELACE

LOVELACE

LOVELACE

LOVELACE

LOVELACE

LOVELACE

LOVELACE

LOVELACE

13-02-01779

13-02-01781

$13-02-01782$

$13-02-01783$

$13-02-01784$

$13-13-02287$

16-01-01972

16-01-01973

$04-01-02113$

$06-21-01741$

06-21-022.23

$06-21-02230$

06-21-02231

06-21-02232

06-2.1-022.34

$06-21-022.35$

06-21-022.36

$06-21-027.37$

06-2.1-022.39

18 -03-02318

$05-04-02173$

05-09-02176

$06-05-01703$

06-05-01704

06-07-01709

$06-18-01721$

$06-18-01722$ .

$M$

MATHEMATICA

$05-04-02168$

MATHEMATICA

05-04-02169

MATHEMATICA

23-02-02091

MEISTER PUBL CO

$02-01-01615$ 
MERR IMACK COLLEGE

MICHIGAN STATE UNIV MICHIGAN STATE UNIV MICHIGAN STATE UNIV MICHIGAN TECH UNIV MIDWEST RESEARCH INST MIT

MIT

MIT

MIT

MIT

MIT

MIT

MI.T

MIT

MIT

MITRE

MONSANTO

MUESER RUTLEDGE

N

NAS-NRC

NAS-NRC

NAT ASSN RR UTIL COM NAT ASSN RR UTIL COM NAT ASSN RR UTIL COM NAT REF CEN SCI TECH NAT REF CEN SCI TECH NAT RES EVAL CENTTER NAT RES EVAL CENTER NAT RES ĖVAL CENTER NAT RES EVAL CENTER NAT RES EVAL CENTER NÁT RES EVAL CENT.ER NAT RES EVAL CENTER NAT RES EVAL CENTER NAT RES EVAL CENTER NAT RES EVAL CENTER NAT RES EVAL CENTER NAT RES EVAL CENTER NAT RES EVAL CENTER NAT RES EVAL CENTER NAT RES EVAL CENTER NAT RES EVAL CENTER NAT RES EVAL CENTER NAT RES EVAL CENTER NAT RES EVAL CENTER
06-18-01724

05-10-01679

05-11-02188

05-11-02192

18-03-02317

23-06-02105

13-02-01787

13-12-01802

20-01-02047

$20-11-02084$

20-11-02087

23-02-02093

23-02-0209:4

23-02-02096

23-02-02101

23-02-02354

17-02-02311

20-04-02052

13-02-01785

05-01-01622

15-03-02299

05-03-01631

05-03-01632

05-03-0,1650

$05-02-01627$

$05-02-01628$

$05-03-01644$

05-03-02129

$05-03-02130$.

05-03-02131

05-03-02132

$05-03-02133$

$05-03-02134$

$05-03-02135$

$05-03-02136$

05-03-02137

05-03-02138

$05-03-02139$

05-03-02140

05-03-02141

05-03-02142

05-03-02143

$05-03-02144$

05-0.3-02145

05-03-02146.
NAT RES EVAL CENTER

NAT RES EVAL CENTER

NAT RES EVAL CENTER

NAT RES EVAL CENTER

NAT RES EVAL CENTER

NAT RES EVAL CENTER

NAT RES EVAL CENTER

NAT RES EVAL CENTER

NAT RES EVAL CENTER

NAT RES EVAL CENTER

- NAT RES EVAL CENTER

NAT RES EVAL CENTER

NAT RES EVAL CENTER

NAT RES EVAL CENTER

NAT RES EVAL ' CENTER

NAT RES EVAL CENTER

NAT RES EVAL CENTER

NAT SCI'FOUND

NATO

NATO

NAVAL APP SCIENCE LAB

NAVAL APP SCIENCE LAB

NAVAL APP SCIENCE LAB

NAVAL CONST RES ESTAB

NÁVAL MEDICAL SCHOOL

NAVAL ORDNANCE LAB

NAVAL RESEARCH LAB

NAVAL RESEARCH LAB

NAVAL RESEARCH LAB

NAVAL RESEARCH LAB

NAVAL RESEARCH LAB

NAVAL RESEARCH LAB

NAVAL WEÄPONS LAB

NAVY TAYLOR MODEL BASIN

NAVY TAYLOR MODEL BASIN

NCEL

NCEL

NCEL

NCEL

NCEL

NCEL

NCEL

NCE L

NCEL

NCEL

NCE L

NCEL
$05-03-02148$
$05-03-02149$
$05-03-02150$
$05-03-02151$
$05-03-02152$
$05-03-02153$
$05-03-02154$
$05-03-02155$
$05-03-02.156$
$05-03-021.57$
$05-03-02159$
$06-13-02216$
$06-18-02217$
$06-21-02225$
$13-12-02266$
$13-13-02282$
$18-08-02322$
$05-02-01629$
$13-01-02753$
$15-06-01955$
$13-12-01795$
$13-1.2-01796$
$13-12-01797$
$19-04-02043$
$15-02-01894$
$20-04-02332$
$20-05-02062$
$20-11-02074$
$20-1.1-02075$
$20-11-02076$
$20-11-02077$
$20-11-02083$
$12-01-02251$
$20-11-02336$
$20-11-02347$
$06-18-02219$
$08-13-01746$
$13-01-01776$
$13-13-01832$
$13-13-01840$
$13-13-01841$
$13-13-02278$
$13-13-02289$
$18-03-02008$
$20-11-02078$
$20-11-02337$
$20-11-02341$
20 


NORC
NORC
NORC
NORC
NORC
NORC
NORC
NORC
NORC
NORC
NORC
NORTH AMERICAN AVIATION
NRDL
NRDL
NRDL
NRDL
NRDL
NRDL
NRDL
NUC RES ASSOC

0

OAK RIDGE ASSOC UNIV

$O C D$

$O C D$

$O C D$

OCD

$O C D$

$O C D$

OCD

OCD

OCD

OCD

OCD

OCD

OCD

$O C D$

$O C D$

$O C D$

OCD

OHIO STATE UNIV

OPERAT IONS RESEARCH

ORDNANCE ENG ASSOC

ORNL

ORNL

ORNL
$05-10-01683$
$05-11-01687$
$05-11-01689$
$05-11-02186$
$05-11-02194$
$05-11-02195$
$05-11-02196$
$05-11-02197$
$05-11-02.198$
$05-11-02199$
$05-11-02200$
$05-02-02120$
$05-03-01651$
$13-12-01801$
$13-13-01815$
$15-06-01963$
$18-06-02321$
$18-08-02039$
$20-04-02333$
$18-03-02018$

$06-18-0221.5$

$05-01-01623$

$05-01-01625$

$05-11-02185$

05-11-02187

05-11-02189

06-08-01712

$06-08-01713$

$06-08-022.10$

$12-02-01767$

$13-01-01769$

$13-13-01839$

13-13-02276

$13-13-02286$

15-03-01908

$15-03-02291_{4}$

$15-03-02304$

15-05-02306

06-12-01714

$15-03-01942$

19-06-02327

06-06-01708

13-11-01794

18-09-02040

\section{$P$}

PACIFIC SW

PLANN ING RESEARCH CORP PLANNING RESEARCH CORP PLANNING RESEARCH CORP PLANN ING RESEARCH CORP POLY INST BROOKLYN PR'INCETON UNIV $P S D C$

13-12-01810 $05-02-02116$ $13-02-022.56$ 23-06-02106 23-06-02107 20-03-02330 20-04-02055 $18-06-02030$

RAND R

RAND

RAND

RAND

RAND

RAND

RAND

RAND

RAND

RAND

RAND

RAND

RAND

RAND.

RAND

RAND

RAND

RAND

RAND

RANO

RAND

RAND

RAND

RAND

RAND

RAND

RAND

RAND

REDSTONE SCI INFO CEN REGIONAL PLAN ASSN REGIONAL PLAN ASSN REGIONAL PLAN ASSN REPORTER MAGAZINE RESEARCH ANALYSIS CORP RESEARCH TR IANGLE INST
$05-01-02292$

$0.5-0.3-01646$ $05-03-01647$ $05-03-01648$ $05-03-01652$ 05-03-02127 $05-03-02158$ $05-03-02160$ $05-04-01658$ 05-04-01672 $05-09-02175$ $05-10-01676$ $06-05-01702$ $06-05-02206$ 06-14-01716 06-16-01717 06-18-01727 $06-18-01733$ $06-2.1-02273$ 06-21-02229 $08-13-01745$ $13-02-01777$ 15-03-02303 15-06-01958 15-06-01959 15-06-01965 15-07-01966 22-02-02350 09-01-01748 23-02-02098 23-02-02099 23-02-02 100 05-04-02167 $12-02-01768$ 06-18-02214 
RESEARCH TR IANGLE INST RESEARCH TR IANGLE INST RESEARCH TR IANGLE INST RESEARCH TR IANGLE INST RESEARCH TR IANGLE INST RESEARCH TR IANGLE INST ROCK I SLAND AR'SENAL ROWLANO $\mathrm{S}$

SAND IA CORP SANDIA CORP SIMULMATICS CORP SOUTHERN FOREST EXPER STA SOUTHWEST RESEARCH INST SOUTHWEST RESEARCH INST SOUTHWEST RESEARCH INST SPECIAL LIBRARIES ASSN STANFORD RESEARCH INST STANFORD RESEARCH INST STANFORD RESEARCH INST STANFORD RESEARCH INST STANFORD RESEARCH INST STANFORD RESEARCH INST STANFORD RESEARCH INST STANFORD RESEARCH INST STANFORD RESEARCH INST STANFORD RESEARCH INST STANFORD RESEARCH INST STANFORD RESEARCH INST STANFORD RESEARCH INST STANFORD RESEARCH INST STANFORD RESEARCH INST STANFORD RESEARCH INST STANFORD RESEARCH INST STANFORD RESEARCH INST STANFORD RE:EARCH INST STANFORD RESEARCH INST STANFORD RESEARCH INST STANFORD RESEARCH INST STANFORD RESEARCH INST STANFORD UNIV STANFORD UNIV STATE UNIV OF NEW YORK SWEDEN SWEDEN SWEDEN

$$
\begin{aligned}
& 06-18-02218 \\
& 06-18-02221 \\
& 06-21-01742 \\
& 15-03-01933 \\
& 15-03-01935 \\
& 15-03-01933 \\
& 19-06-02329 \\
& 05-10-0218
\end{aligned}
$$

$$
\begin{aligned}
& 18-03-02314 \\
& 18-03-023.17 \\
& 05-10-01685 \\
& 05-03-01643 \\
& 13-12-01800 \\
& 13-13-02290 \\
& 20-08-02068 \\
& 05-03-01638 \\
& 05-03-01639 \\
& 05-03-02128 \\
& 05-11-02193 \\
& 06-08-01710 \\
& 06-08-01711 \\
& 06-08-02211 \\
& 06-18-01723 \\
& 06-18-01725 \\
& 06-18-01729 \\
& 06-18-022.20 \\
& 13-06-02260 \\
& 13-13-01834 \\
& 13-13-01838 \\
& 15-03-01930 \\
& 15-03-01953 \\
& 15-03-02793 \\
& 15-03-02305 \\
& 15-06-02307 \\
& 17-02-02312 \\
& 17-02-02313 \\
& 18-08-02038 \\
& 23-02-02095 \\
& 23-02-02352 \\
& 05-04-02170 \\
& 20-06-02067 \\
& 20-11-02346 \\
& 13-12-02268 \\
& 20-04-02050 \\
& 20-04-02051
\end{aligned}
$$

SWEDEN

20-04-02053

SYSTEM DEVELOPMENT CORP

SYSTEM DEVELOPMENT CORP

SYSTEM DEVELOPMENT CORP

SYSTEM DEVELOPMENT CORP

SYSTEM DEVELOPMENT CORP SYSTEM DEVELOPMENT CORP SYSTEM OEVELOPMENT CORP SYSTEM SCIENCES CORP.

05-02-02117

$05-02-02121$

$05-02-02122$

$05-02-02.173$

$05-04-01659$

$09-02-01750$

$09-02=01751$

$22-02-02351$

\section{T}

TULANE UNIV,

13-13-01821

T. Y. LIN

T. Y. LIN

13-13-01833

15-03-01952

U

UNION CARB IDE CORP

UNITED AIRCRAFT CSC

UNITED AIRCRAFT CSC

UNITED AIRCRAFT RES LABS

UNITED AUTO WORKERS

UNITED NATIONS

U.S. CONGRESS HOUSE

U.S. DEPT. OF AGRICULTURE

U.S. DEPT. OF AGR ICULTURE

U.S. DEPT. OF AGRICULTURE

U.S. DEPT. OF AGR I CULTURE

U.S. DEPT. OF AGR I CULTURE

U.S. DEPT. OF AGR ICULTURE

U.S. DEPT. OF AGR ICULTURE

U.S. DEPT. OF AGRICULTURE

U. $\subseteq$. DEPT. OF AGR ICULTURE

U.S. DEPT. OF AGR ICULTURE

U.S. DEPT. OF AGRICULTURE

U.S. DEPT. OF AGRICULTURE

U.S. DEPT. OF AGRICULTURE

U.S. DEPT. OF AGRICULTURE

U.S. DEPT. OF AGRICULTURE

U.S. DEPT. OF COMMERCE

U.S. DEPT. OF COMMERCE

U.S. DEPT. OF COMMERCE

U.S. DEPT. OF COMMERCE

U.S. DEPT. OF DEFENSE

U.S. DEPT. OF DEFENSE

U.S. DEPT. OF HEAL ED

U.S. DEPT. OF HEAL ED

U.S. DEPT. OF HEAL ED WEL
$20-05-02061$
$08-13-01747$
$19-04-02044$
$20-05-02060$
$05-04-02172$
$05-04-02165$
$15-07-02308$
$02-00-02108$
$02-02-01617$
$02-02-01618$
$02-02-01619$
$02-02-01620$
$02-02-02109$
$02-02-02110$
$02-02-02111$
$02-02-02112$
$11-12-01766$
$13-12-01798$
$13-12-01799$
$13-12-01806$
$13-13-01818$
$15-03-01897$
$05-03-01645$
$18-03-02319$
$20-13-02090$
$23-04-02358$
$06-19-01735$
$15-03-01949$
$06-08-01712$
$06-08-01713$
$05-10-01681$ 
U. $\subseteq$. DEPT. OF HEAL ED WEL U.S. DEPT. OF HEAL ED WEL U.S. DEPT. OF HEAL ED WEL U.. . DEPT. OF HEAL ED WEL U.S. DEPT. OF HEAL ED WEL U. S. DEPT. OF HEAL ED WEL U.S. DEPT. HOUS UR DEVEL U.S. DEPT. OF INTERIOR U.S. DEPT. OF INTERIOR U.S. DEPT. OF INTERIOR U.S. DEPT. OF INTER IOR U.S. DEPT. OF INTERIOR - U.S. DEPT. OF INTERIOR U.S. DEPT. OF INTERIOR U.S. DEPT. OF INTER IOR U.S. DEPT. OF LABOR U.S. DEPT. OF LABOR U.S. DEPT. OF STATE U.S. DEPT. OF STATE U.S. DEPT. OF STATE U.S. DEPT. OF STATE U.S. DEPT. OF STATE U.S. DEPT, OF STATE U. $\subseteq$. DEPT. OF STATE U. $S$. DEPT. OF STATE U.. DEPT. OF STATE U. S. DEPT. OF STATE U.S. DEPT. OF STATE U. $\subseteq$. DEPT. OF STATE U.S. DEPT. OF STATE U.S. EXEC OFF PRES U.S. EXEC OFF PRES UNIV OF ARIZONA UNIV OF CALIF UNIV OF CALIF UNIV OF CALIF UNIV OF CALIF UNIV OF CALIF UNIV OF CALIF

$06-05-02205$
$06-16-01718$
$06-16-01719$
$06-19-01734$
$13-02-01778$
$15-02-01869$
$23-03-02102$
$08-07-02245$
$13-02-02257$
$13-13-02270$
$13-13-02274$
$13-13-02283$
$13-13-02284$
$13-13-02285$
$13-13-02288$
$05-03-01649$
$05-09-02174$
$05-04-01662$
$05-04-01663$
$05-04-01664$
$05-04-01665$
$05-11-01690$
$05-11-01691$
$05-11-01692$
$05-11-01693$
$05-11-01694$
$05-11-01695$
$05-11-01696$
$05-11-01697$
$05-11-01698$
$05-03-01634$
$15-03-01949$
$13-13-01837$
$06-03-02204$
$13-13-02277$
$18-03-02315$
$18-03-02316$
$20-04-02331$
$20-09-02335$

UNIV OF CALIF UNIV OF COLORADO UNIV OF COLORADO UNIV OF FLORENCE UNIV OF FLORIDA UNIV OF GEORGIA UNIV OF GEORGIA UNIV OF GEORGIA UNIV OF ILLINOIS UNIV OF ILLINOIS UNIV OF ILLINOIS UNIV OF ILLINOIS UNIV OF KANSAS UNIV OF MIAMI UNIV OF MICHIGAN UNIV OF MICHIGAN UNIV OF MINNESOTA UNIV OF NEW HAMPSHIRE UNIV OF NEW HAMPSHIRE UNIV OF PENNSYLVANIA UNIV OF PENNSYLVANIA UNIV OF PENNSYLVANIA UNIV OF PITTSBURGH UNIV OF PITTSBURGH UNIV OF TEXAS UNIV OF TEXAS UNIV OF TEXAS UNIV OF TEXXS UNIV OF WASHINGTON URS CORP. URS CORP $U \subseteq S R$

W

WATER POLLUTION CON FED WEL

WEL

WE ST I NGHOU SE 
THIS PAGE

WAS INTENTIONALLY

LEFT BLANK 


\section{Author Index}

A

ABEL, J. .F.. JR . ACHENBACH, P, R. ACKOFF, R: L. ACKOFF, R. L. ACKOFF, R. L. ADELSON, $M$. AGBAB IAN, M. $S$. AHEARN, $T, R$. ALBRITTON, G. E. ALBR ITTTON, G. E. ALOERSON. W. ALDER SON, $W$. ALDER SON, $W$. ALEXANDER. M. N. ALLEN, J. T. ALLEN, J. T. ALLEN, J. T. ALLEN, J. J. ALLEN, R. E. ALLGOOD. J. R. ALLISON; P. ALLSWANG, J. M. AMALRAJ, R. V. ANDERSON; J. ANDERSON, J. ANDER SON, J. ANGALET, B. W. ARCHIBALD, G. R. ARNHE IM, N. ARNSTEN, M. E. ARNSTEN, M. E. ATLAS, H. S. AUMANN, R. J. AUMANN, R. J. AUSTIN. W. J. AUXIER, J. A. AYRES $\therefore R, U$. AYRES, R. U. AYRES, R. U.

$13-13-02275$
$20-13-02090$
$05-04-02171$
$05-10-02182$
$05-10-02183$
$05-04-01659$
$13-13-01823$
$05-10-02184$
$13-11-02261$
$13-13-01825$
$05-04-02171$
$05-10-02182$
$05-10-02183$
$15-03-01942$
$05-03-02.129$
$05-03-02140$
$05-03-02152$
$06-18-01725$
$18-08-02035$
$13-13-01832$
$06-16-02213$
$05-11-02194$
$13-11-01792$
$05-04-02171$
$05-10-02182$
$05-10-02183$
$05-02-02120$
$13-13-02271$
$06-18-01727$
$06-21-02228$
$06-21-022.29$
$06-18-01724$
$05-04-02168$
$05-04-02.169$
$13-13-02290$
$20-08-0.2334$
$15-03-02299$
$15=06-01962$
$23-02-020.97$

B

BACON, F. L.

BAER, J. A.

BAIDYUK, B. V.

BAKER, R. L.

BAKER, W. L.

BAKER, W. L.

BALAKR I SHNA RAO, H. A.

BARANCIK, B. 0.

BAREIS, D. W.

BAR -HILLEL, $Y$.

BARKSDALE, B. H., JR.

BARNETT, R. L.

BARTYCZAK, J. A.

BATES, C. B.

BAUM, C.

BAUM, $C$.

BEAR, D. V. T.

BEARO. C. W.

BECK, W. D.

BECKLES, M. P.

BEDARD. M. A.

BEHLS. H. F.

BEHLS, H. F.

BEHLS, H. F.

BEHRENDT, G.

BEND. E.

BEND, E.

BEND, E.

BENO, E.

BEND, E.

BENDER, E. K.

BENUSKA, K. L.

BERENDT. R. F.

BERGSMAN, F. A.

BERLO, D.

BESEMER, A. F.

BICKLEY. L. J.

B.ICKLEY, L. J:

BILL. G.
05-03-02154

17-02-02313

$08-07-02742$

19-01-02042

06-21-02227

06-21-02238

20-01-02047

09-02-01751

13-13-01823

$12-01-02250$

13-13-01828

$20-11-02338$

05-03-02138

$12-01-0.2251$

$09-02-01750$

$09-02-01759$

05-03-01.647

15-02-01850

$13-01-01773$

$05-03-02153$

$17-02-02311$

$13-01-02752$

$13-01-02754$

15-03-02297

13-13-01827

$05-01-01621$

$05-01-01624$

05-01-01626

05-10-01680

05-11-01699

20-11-02087

15-03-01952

$15-02-01850$

05-03-02152

05-04-02166

15-02-01852

05-03-02126

05-03-0.2162

05-10-01685 
B.I LLHE IMER, J. W. BJORKHOLM, J. E. BLACK. D. V. BLUNDELL, G. P. BLUNT, K. R. BOGDANOV. N. A. BORGATTA, E. F. BORKO. H. BOR SODY, R. P. BOVA, $P$.

BOWEN, I. G. BOWEN, I. G. BOWEN, I. G. BOWEN, I.G. BOWEN, I. G. BRACCIAVENTI, J. BRANCH, J. E. BRANDES, E. M. BRANDT. R. J. BRECKENR IDGE, R. A. BRENNAN. D. G. BREWER, $H$. BREWER. H. A. BRODY, R. A. BRONSON. G. BROOKS, J. A. BROOKS, J. A. BROWN. B. BROWN. R. C. BROWN, S. L. BROWN. W. M. BROWN, W. M. BRUCE, R. N. BRUNSWIG, $H$. BRUNSWIG, $H$. BRYAN, F. A.. JR. BRYAN, F. A.. JR : BUCHANAN, L. M. BUETTNER, K. J. K. BUGG, S. L. BUGHER, R. D. BURCHSTED, C. A. BURCO. R. A. BURDICK, B. BÜRNS, J. BUR SON. Z. G. BUTKOVICH, T. R. BUYAS. G.

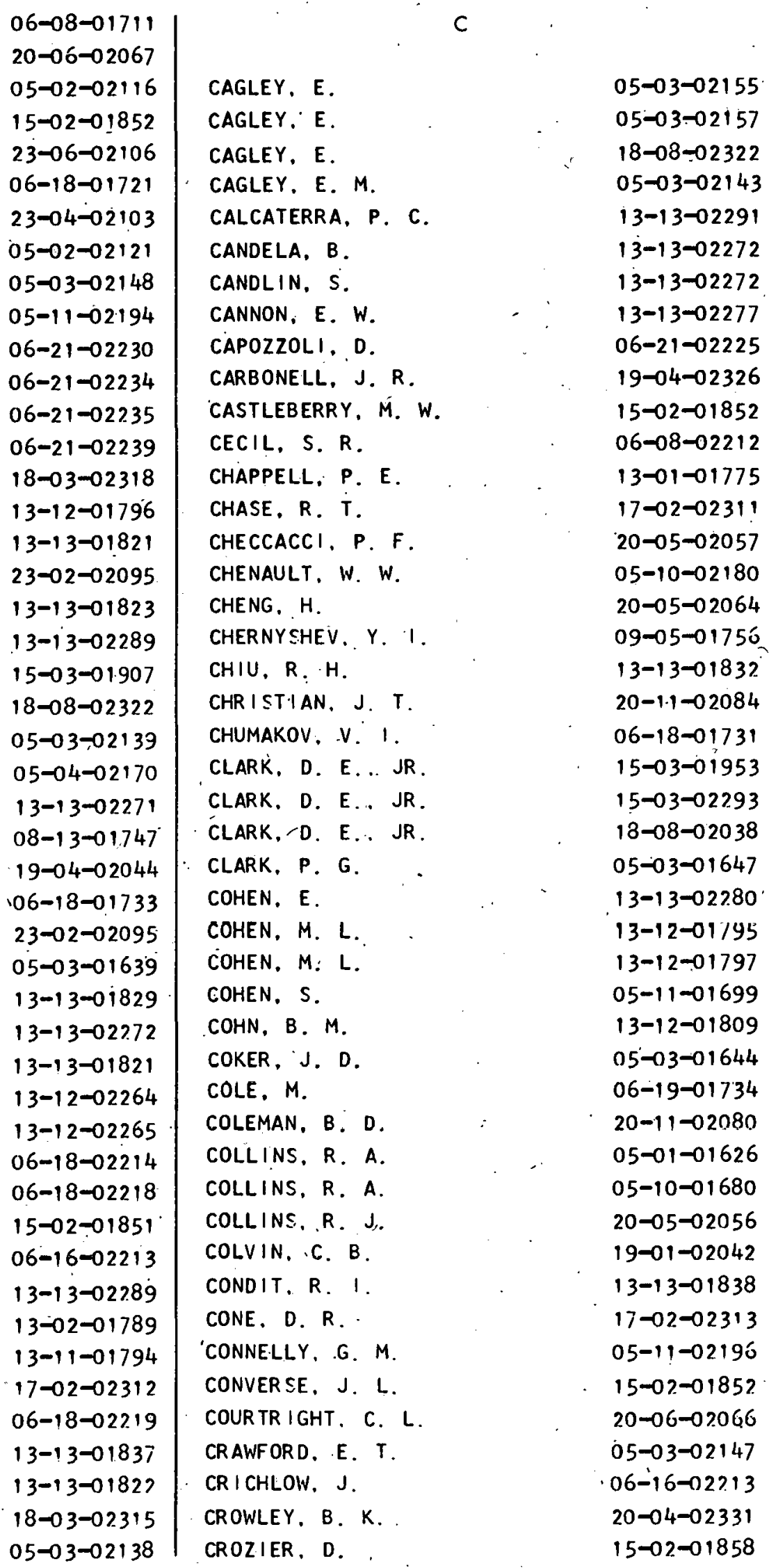


CRUZE, A. M. CRUZE. A. M. CRUZE, A. $M$. CRUZE, A. M. CULKOWSKI, P. M. CZEH, R. S.

'DALKEY,' N. C. DAMON, E. G. DAMON, E. G. DAMON, E. G. DAMON, E. G. DAMON, E. G. DANBURY; $T$.

DANGERF IELD. H. G. DAVIDENKO, H. V. DAVIS, E. E. DAVIS, J.A. DAVIS. L. W DAVIS. L. W. DAVIS. R. L. DEAN, D. R. DEARMON, I. A. . JR . DEARMON, K. A. . JR. DECKER, H. M. DEGENKOLB , J. G. DEHART, R. C. DEMIDOVA, E. K. DERR I CKSON, G. F.. ED. DEVANEY, J. F. DEVINE. L. $F$. DEVINE, P.

DIETER ICH, J. H. DIETER | CH, J. H. DINAPOLI, P. DIXON, $\mathrm{H}$.

DIXON, H. L. DIXON, H, L. DIXON.W. J. DOBBINS. R. A. DOBBS, G. H. DOGGETT. W. 0 . DOLBNIN, T. $V$. DORRIS. A. F. DORRIS, A. F. DOYLE, T. DREBELBIS, R. C.
$06-21-01742$
$15-03-01933$
$15-03-01935$
$15-03-01938$
$20-11-02346$
$06-06-01706$

15-06-01959

$06-21-02230$

06-21-02232

06-21-022.34

06-21-022.36

06-21-022.37

05-11-02192

15-02-01858

05-09-02176

05-10-02178

$05-11-02200$

$06-21-02727$

$06-21-027.38$

05-10-01684

20-05-02056

1 5-02-01849

15-02-01853

15-02-01851

15-03-01952

13-13-02290

15-02-01894

05-03-01645

12-02-01767

15-02-01857

05-ก3-02157

13-12-01799

13-12-01806

13-13-02280

15-03-02305

05-03-02128

13-06-02260

06-03-02204

15-02-01881

09-02-01751

06-.18-022.18

15-03-02296

$13-11-02761$

13-13-01825

$06-19-01734$

06-14-01716
DRESCH, $F$. W.

DRNEV ICH, V. P.

DRUKEY, D. L.

DUFF, J. T.

DUGUAY, G. L.

DURBIN, W. L.

DUR IKOV, A. P.

DUVALL, W. I.

DYER, 1 .

05-11-02193

13-13-01812

$09-0.2-01751$

15-02-01857

$13-12-02267$

18-03-01995

06-18-01731

$08-07-02245$

19-04-02326

EDMONSTON. 'J. H.

E

EDMONSTON, J.H.

EIGELSBACH, H. T

ELLIS, H.

EMSHOFF, J.R.

EMSHOFF, J.R.

EMSHOFF, J.R.

ENGHOLM, G.

ENGHOLM. G.

ER ICKSON, H. M.

ER IKSSON. $S$.

ERIKSSON, $S$.

$05-03-02151$

05-03-02152

15-02-01853

05-11-02193

05-04-02179

05-10-02182

05-10-02183

13-01-02?53

$15-03-02300$

05-10-01681

20-04-020.50

20-04-0205i

FACCI, H. A.

05-03-02159

$F A C C l, H$. A.

$06-18-02217$

FARR, D.' $E$.

FARROW, R. L.

FEREBEE, J. B.

FERNELIUS, A.

FERNELIUS, A. L.

FERNELIUS, A. L.

FIOCK, M. A.

FIORE, E. S.

FLATHAU, W. J.

FLÉTCHER , E. R .

FLETCHER , E. R

FLETCHER, E. R.

05-05-01673

$13-01-02255$

15-03-01942

15-02-01854

15-02-01849

15-02-01853

15-02-01857

$05-03-01636$

13-13-01830

$06-21-02230$

$06-21-02234$

$06-21-02735$

FLETCHER : E. R.

06-21-02239

$06-05-01701$

15-02-01875

18-02-01991

20-11-02337

FUSS, $D . S$

G

GANS, R. F.

19-04-02044

GARACHUK, V. K. 
GARDNER , L. B .

GARDON, R.

GARRETT, C. W.

GASKILL, I. E.

GASKILL, I. E.

GASK I.LL', I. E.

GASK ILL; I E.

GAYLORD, C. S.

GAYLORD. C. S.

GAYLORD, C. $S$.

GAZLEY, C. , JR .

GEAR INGER, N. F.

GERR ITY, M. V.

GILMAN, R.

GILSDORF, H: J.

GIRNAU, G.

GIVENS, R. T.

GOCHENOUR, $W$. S.. JR .

GODDARD... K. R

GOELLER, B. F .

GOELLER, B. F.

GOLDBLATT, S. L.

GOMBERG, W.

GOMBERG, $W$.

GOMBERG, W:

GOODALE, T. C.

GOODMAN, A. F.

GOR CHAKOV. A. D.

GOTHER SK JOLK. L.

GOULO, J. H.

GOUSE, S. W.. JR.

GRANSTROM, $S$.

GREER, S. A.

GREGORY. R: $K$

GREMILLION, G. G.

GRIM. P. J.

GRONBECK. $M$. P

GROUSE. S. W. J JR.

GRUNE; - W. N.

GUNDER SON, R:

GUNTHER, W. D.

GURTIN. M. E.

H

HADDEN, J. K.

HAHON, N.

HAHON, N.
23-04-02103

06-i8-02219

13-12-01802

15-06-01964

05-03-01644

05-03-02132

05-03-02134

05-03-02138

$.06-21-02232$

$06-2 \cdot 1-022.36$

$06-21-022.37$

22-02-02350

15-02-0 1.857

$02-02-021.12$

$05-1.1-02200$

20-05-02064

13-13-01827

20-05-02064

$.15-02-01858$

15-02-01851

15-06-01958

15-07-01966

18-03-02018

05-04-02171

05-10-02182

05-10-02183

19-01-02042

05-02-02120

13-13-01826

$13-12-02268$

23-02-02356

23-02-02096

20-04-02053

15-0 3-02301

13-13-022.90

15-02-01892

$15-03-01942$

13-13-01821

23-02-02101

$06-18-01724$

13-13-01837

$17-02-02.311$

20-11-02080

$15-02-01855$

15-02-01891
HALE.. J: F.

HALE, J. F.

HALL, J. R. JR.

HALL. L. B.

HALL, R. L.

HAMEL, G. J.

HAMMER, E. W.. JR.

HAMMES, J. A.

HANEY, D. G.

HANLEY, J. T.

HANSEN, R. J.

HANSON, S:

HANUNIAN, N.

HARMON: J. A.

HARMON, J. A.

HARMON, J.A.

HARRENSTEIN, $H$.

HARRIES, W. S.

HARRIS, G. J.

HARR I SON, H. B.

HAR SANYI, J. C.

HAR SANYI, J. C.

HARVEY, E. C.

HASSETT, C. C.

HAUGHT. A. F :

HAVERS, J. A.

HAVER S, J. A.

HAVERS, J. A.

HAWKINS, M. B.

HAYWOOD, F. F.

HEARST, J: R.

HEDGER, H. E.

HEDGER, H. E.

HEDGER, H. E.

HEER . D. M.

HE ILFERTY. R. J.

HEILFERTY: R. J.

HE I SKELL, R. H.

HELF, S.

HENN I NGER. R. H.

HENN I NGER, R. $H$.

HENSCHEL, A.

HENSCHEL, A.

HENSCHEL, A.

HICKS, W.

HICKS, A. N.

HIGGINS, G. H.
05-10-01684

15-03-022.97

13-13-01812

15-02-0185!

20-11-0.2343

06-18-01724

19-06-02328

05-10-02184

05-03-02128

13-13-01821

2.3-02-02354

-13-13-01837

$05-03-02.160$

13-02-01780

13-02-0 1788

15-03-0194.1

13-13-01837

18-04-02026

15-0.2-0.1892

13-13-02287

05-04-02168

05-04-02169

$13-13-01834$

15-02-01861

20-05-02060

1.3-13-0181.1

13-13-01819

13-13-01835

19-01-02042

20-08-02334

$18-03-02316$

13-02-01779

$13-02-01782$

$13-02-01783$

05-11-0270?

13-12-01795

13-12-01797

18-06-02321

20-08-02334

13-01-01774

13-01-02254

06-16-01718

06-16-01719

06-19-01734

06-21-02236

19-04-02043

18-03-02315. 
HIRSCH, F. G. HIRSCH. F. G. HIRSCHFELD, R. C. HIRSCHFELD, R. C. HISER, H. W. HODGES, J. D.. JR. HOFF, G. C. HOFF, G. r. HOGGE, A. L. . JR. HOLSTI. O. R. HOPKINS, G. D. HOPKINS: G. D. HOWES. M. H. HUEB SCH， I. 0 . HUMMELL, J. D. HUMPHREYS, C. M. HUMPHREYS, C. M. HUSBANDS, R. M. HUTTER, R. G. E.

IVANOV, N. N. IZRAEL, Y." A.

JACKSON, J. E. JACOB SEN, L. S. JACOBSEN, L. S. JARMUZ, P. J. JASTER, J. J. JAY. J. W. JAY, J. W. JEFFREYS, $F$, $B$. JEFFREYS, $F$. B. JEMSKI, J. V. JOHLER, J. R. JOHLER, J. R. JOHNSON, R: D. JOHNSUN. $T$. JONES, $D$. JONES, P. $S$. JONES. P. S. JONES, R. E. JORDAN, N. JORDAN, S. L.

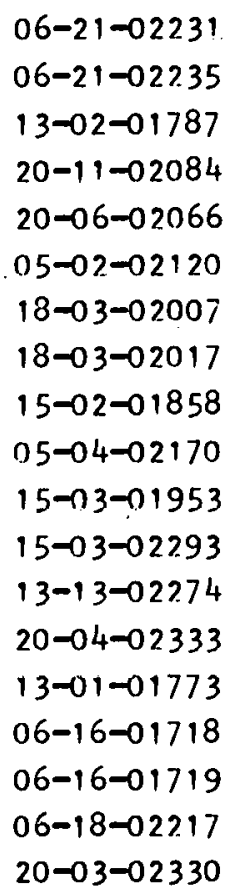

15-03-01937 $18-08-02323$

\section{J}

20-05-02061

13-13-01823

$20-11-02078$

20-05-02064

05-02-02119

13-12-01799

$13-12-01806$

$05=01=01626$

05-09-01675

15-02-01858

20-14-02348

20-14-02349

$05-11-01687$

$06-18-02 \% 21$

06-16-02213

05-03-02128

23-02-02095

13-12-01801

$05-04-0167.1$

$12-02-01768$
KADULL. P. J.

KAFADAR, A. D. KAHLE, M. F.

KAHN, $H$. KAPLAN, L. L. KARAGOZIAN, J. KARAGOZIAN, J. KATZ, Y. H. KAUFMAN. A. KECSKEMET I.P. KEEGAN, E. J. KELLER, J. A. KELLER, J. A. KENNEDY, T. E. KENNEDY, W: C. KHAN, A. A. KHOMKO. A. A. KHUNDANOV, L. E. KILLAM, E. T. KINDNER, R. L. KING, R. B..JR. KIRKLAND, J. L. KIRKPATRICK, R. G. KLE!N, F. KLEIN, F : KLEIN, F. KLEINER, A. M. KLOEHN, J, M. KNAPP, J. W. KNISELY, R. F. KNOX, J. B. $\mathrm{KOCH}, \mathrm{J}$. E. $\mathrm{KOCH}, \mathrm{J}, \mathrm{E}$. KOELLER, E. H. KOELLER, E. H. KOENIG. L. P. KOENIG, L. P. KOENIG, L. P. KORTS, R. F. KOSHELEV, L. 1 KOVALENKO. $V$. KOZIKOWSKI. E. H. KRAUS, A. F. KRUPKA, R. A.
15-02-01857 19-06-02327 $05-03-02.153$ 15-03-02303 14-02-0184? 13-13-01823 $20-11-02073$ $08-13-01745$ $04-01-02113$ 05-04-01658 $05-03-02135$ $13-12-01805$ 13-12-022.62 13-13-01825 $06-12-01714$ 13-11-01792 13-13-01826 15-02-01894 13-13-02271 20-11-02086 05-11-01688 13-13-0.1825 13-12-01805 15-02-01849 $15-02-01853$ 15-02-01854 05-11-01688 06-08-0 1710 18-07-02034 15-02-01875 $18-03-02316$ 13-12-01795 13-12-01797 13-13-0181! 13-13-01819 05-03-02129 $05-03-02152$ $05-03-02153$ 18-08-02037 $13-13-01826$ 15-02-01843 1 5-02-01891 18-03-02018 13-13-02272 
KRUPKA, R. A. KUNREUTHER, H. C. KUNUKKASSER I L. V. $X$. KUPTSEVICH, E. I. KUSUDA, T.

L

LABES. W. G.

LAMBE, T. $W$.

LAMBERT,. C. G.

LANE. W. B.

LAR IV IERE, P. D.

LAR.IVIERE, P. D.

LAURE, L. G.

LAWSON, M. E., JR.

LEACH, J. M.

LEBEDEV, $\mathrm{N} .1$.

LEBEDEVA, $Y$, A.

LEBOW, L. $S$.

LEE, D. H. K.

LEE, D. H. K.

LEE, $H$.

LEE, $H$.

LEE, $H$.

LEE, R. J.

LEE. S. W.

LEE, $W$ : $R$.

I.F.F. W. R

LEHTO, D

LEVIN, S.

LEWICKE, $V$.

LEWIS, R. A.

LIBER, T.

LIFTER, J. J.

LIM. T. G.

LINCOLN, R. E.

LINCOLN, R. E.

L.INCOLN, R. E.

LINDSAY, R, K.

LIPSNER, R. A.

LIVINGSTON, C. W.

LLOYD, G.

LOMMASSON, T. E.

LOWE, E. P.

LOWR IE, L. M.

LOWRY, I. S.

LOYA, B. R.
23-02-02097

05-03-01636

20-11-02345

15-02-01894

20-13-02090

13-12-02?69

20-1 1-02084

$05-02-02117$

06-18-01725

$06-18-01723$

15-06-02307

05-03-02146

18-04-02026

i 3-02-01780

09-0 3-01752

15-03-01937

15-03-01942

06-16-01718

06-16-01719

06-18-01729

$06-18-02220$

15-06-02307

05-10-02181

20-05-02064

06-06-02207

06-06-02208

20-04-02332

19-06-02327

05-03-02134

05-03-02159

20-11-0.338

23-06-02106

05-03-02153

15-02-01849

15-02-01853

$15-02-01854$

09-02-02248

05-03-02150

13-1 3-02273

02-02-01617

13-12-01805

15-02-01852

18-03-02319

15-06-01965

15-03-0.1952
LUNCHICK. M. E.

LUTZ, F. W.

LUTZKY. M.

LYCZKOWSKY, 0.

$M$

MADSON. C. A.

MADSON. C. A.

MADSON, C. A.

MAHLANDT: B. G.

MALAKHOV. $M$. $M$.

MALME, C.' I.

MALTHAN, J. A.

MALTHAN, J. A.

MAMURO, $T$.

MANN, 1 .

MARGOLIES. L.

MARKOWITZ; H.

MARTIIN. J.J.

MARTIN, M. W. J JR.

MARTIN, M. W. J JR.

MARTIN, M. W. J JR.

MARTIN, R.

MARTIN, S. B.

MASCHLER, M.

MASCHLER, M.,

MATKOVICH. M. J.

MATSUNAMI. T.

MCCAHILL, D. F.

MCCORD. R. B :

MCCULLOUGH, J. D.

MCDANIEL. C.

MCDONNEL. C.

MCGAVRAN. M. H.

MCGAVRAN, M. H.

MCGILLIVRAY, P. C.

MCINTYRE, F. F. JR:

MCMULLAN, P. S.

MCMULLAN, P. S.

MCPHER SON, W. A. JR.

MEAGLEY, D. E.

MECHEL, F . P.

MEDD ING, R. S.

MEEM, J. L.

MEEM, J. L.

MEEM; J. L.

MEIER, H. A.
$20-1.1-0.2347$

05-1!-02203

20-04-02332

$06-19=01734$

13-01-01771

$13-01-01774$

15-03-02798

15-02-01853

05-04-02173

19-04-02326

13-13-01823

20-11-02078

18-02-01991

15-03-02303

06-19-01734

05-03-01648

17-09-01988

05-04-0217

05-10-02182

$05-10-02183$

18-08-02322

13-1.2-01801

05-04-02168

05-04-02169

13-01-02255

18-02-01991

13-13-01821

05-02-02!20

05-01-0229?

$05-11-01699$

18-06-02030

15-02-01850

15-02-01855

15-03-01945

05-01-01624

06-21-0!742

15-03-01935

08-13-01747

05-10-01684

20-01-02048

15-06-01957

05-03-02 130

05-03-02148

05-03-02154

$13-01-01771$ 
MEIER, H. A.

ME I STER, D.

MELNICK, A. M.

MERR ILL, R. H.

MERTENS, P. A.

MERTZ, E. T.

METTLER. A. J.

METZZLER, R. E.

MEYER, G. D.

MICKEY, W. $V$.

MIDDAUGH, P. R.

MILLER, C. F.

MILLER, C, F.

MILLER, C. F.

MILLER, G. H.

MILLER, $H$.

MINVIELLE, L.

MITCHELL, E. J.

MITCHELL, H. H.

MITCHELL, H. $H$.

MOLL, K.

MONCURE, $P$.

MONK, C. B.. JR.

MONK, C. B.. JR.

MOORE, $F$. T.

MOORE. W. R.

MOORE, W. R.

MORDVINOV, V. $S$.

MORGAN, J. M. J JR.

MORGAN, Q. M.

MORGAN. Q. M.

MOSKALEV, $V$. D.

MURRAY. B. R.

MURROW, R. B.

NAGIN, R. P.

$N$

NAGIN, R. P.

NAGIN, $R: P$.

NAKAMURA, $R$. $M$.

NAUMER, D. A.

NAYER, V. S.

NEELAND, $F$.

NEELAND, $F$.

NEHNEVAJSA, J.

NEHNE VAJSA, J.

NE ILSON. D.
15-03-02300

05-05-01673

20-04-02052

08-07-02245

20-01-02048

06-16-01720

06-18-02719

18-04-02026

13-13-01830

18-03-02319

15-02-01849

$06-18-01723$

15-03-01953

15-03-02293

10-03-01764

13-12-02263

05-03-01.651

05-09-02175

06-05-01702

06-05-02206

15-03-02305

05-03-02133

13-13-01811

13-13-01819

05-10-01670

13-12-01799

13-12-01806

15-03-02796

18-07-02034

$0 ?-02-021 ? 0$

$02-02-02111$

15-03-01937

05-02-02119

13-02-01777

$N$

05-04-02179

05-10-02182

05-10-02183

15-02-01850

10-02-01761

09-0்1-01748

05-02-02122

05-02-02123

05-10-01678

05-10-02177

13-13-01837
NELSON, 0 . E.

NEVER IL, R. B.

NEWMARK. N. M.

NOLTE, L. J.

NORDLIE, P. G.

NORDLIE, P. G.

NORDL IE, P. G.

NORMAN. J. H.

NORMAN, J. H.

NOR SWOR THY, J. R.

NORTON, J. D.

NORTON, J. D.

NORTON, J. R.

NORTON, J.R.

NOSSE IR, S.

NOSTE, N. V.

NWUDE, J.

NYBERG, P. R.

NYSWANDER, D. B.

06-16-01720

13-01-02252

08-11-022.46

20-11-02344

05-10-02 180

05-11-02191

05-11-02 201

18-08-02037

18-08-02325

05-03-02149

05-03-02131

05-03-02142

05-03-02141

05-03-02149

13-13-02279

13-12-01798

23-02-0210.1

1 6-01-01973

05-10-01681

OBERT, L.

$08-07-02245$

OBRIEN. G.

$05-04-02168$

OBRIEN, G.

$05-04-02169$

OLIVER, D. N.

$05-03-02156$

OLIVER, S. M.

$06-08-01710$

OLIVER, $S$. M.

$06-08-02211$

OLIVER, W. B.

$0.5-03-01644$

OLOVYANISHNIKOV. N. P.

15-03-01918

$13-12-022.68$

15-02-0 1849

05-10-02179

20=09-02335

09-03-01752

20-11-02347

OVERBY, J. A.

18-06-02028

PACE, F. C.

14-02-01842

PALMISANO, F.

$13-13-02272$

15-06-01963

06-21-01742

05-03-02161

20-11-02087

15-03-01934

23-04-02104 
PERRY, E. S. PETROV, R. V. PHILPOT, C. W. PICKETT, G. R. PINCKERT, R. E. PITTS, $M$. PLASS,. H. J.. JR. PLASS, H. J., JR. POGRUND, R. $S$.

POLAN, $M$. POOL. I. D. POPKIN, $S$. PORCH, H. PORTEOUS, L. G. PRATESI, R. PRAVETSKII, V.N. PRETRE, S. PRUSAKOV, $1: Z$. PRYOR. A. J. PUGH, R. E. PUGH, R. E. $\mathrm{R}$

RADNER , $R$. RADNER - R. RAIL, R. D. REA, R. $H$. READ, C. REI SMANN, H. RENNER, $R$. $H$. RHIAN, M. A. RICHART, F. E. RICHMONO, D, R: R.I CHMOND. D. R. RICHMOND, D. R: RICHMOND, D. R. $R$ ICHMOND, D. R. RICHMOND, D. R. RICHMOND; D. R. $R$ INGLEB, $F$. 0 . RITZ, P. M. RiTz, P. M.' RITZ,P.M. ROBBINS, E. ROBERT. R. W. ROCKETT, $F$. C. ROCKETT, F. C.

$$
\begin{aligned}
& 13-13-02273 \\
& 06-18-01726 \\
& 13-12-01810 \\
& 08-11-02247 \\
& 20-11-02081 \\
& 06-16-02213 \\
& 20-1.1-02079 \\
& 20-11-02082 \\
& 06-16-01717 \\
& 18-08-02039 \\
& 05-10-01685 \\
& 05-10-01685 \\
& 05-03-01652 \\
& 13-13-01815 \\
& 20-05-02057 \\
& 06-18-01726 \\
& 06-18-01728 \\
& 09-05-01756 \\
& 13-12-01800 \\
& 05-03-02150 \\
& 05-03-02152
\end{aligned}
$$

05-04-02163 05-04-02169 13-13-01832 05-02-02125

$15-03-01933$ 20-11-02346. 13-12-0180! 15-02-01854 13-13-01812 $06-21-01741$ $06-21-02230$ $06-21-02232$ $06-21-02234$ $06-21-02235$ 06-21-02236 06-21-02237 20-04-02055 05-03-02153 05-03-02159 1 3-13-02282 06-16-022.13 20-06-02066 $13-13-01829$ 15-03-01896
ROCKETT. F. C. ROCKETT, F. C. ROCQ. M. M. RONCHI, L. ROSE, E. ROSEN, D. J. ROSENWALD, A. J. ROSS, H. R. ROSSAL, N.'A. ROWE, A. J. ROWLAND, 0 . ROWLAND, $D$. ROWLAND, D. RUDIN, I. S. RUZICKA. J. E. RYAN, $M, H$. RYAN, T. E . RYBKIN, E.

SÁCHS, A. S

SACHS. A.

SALMONS, J.

SALSBURY, M. E.

SALSBURY. M. E.

SANBORN, W. R.

SARTOR, I. D.

SAR TOR. J. D.

SAVISKO, P. A.

SAWYER, W. D.

SCHANTZ, E.J.

SCHEGGI, A. M.

SCHICK, J.

SCHIFFMAN, T'.

SCHILZ, W. M.

SCHLENKER, G.

SCHNOBR I CH, W. C.

SCHWARTZ, J. 1.

SEABOLD, R. $H$. SEABOLiD, R. $H$. SEIFERT, W. W. SELF, $C$. $R$. SENTUR IA. S. D. SEWELL, C. H. SHALNOV. M. 1. SHAPIRO. E. B.
15-03-01922

15-03-02295

05-03-01638

20-0.5-020் 57

18-08-02322

18-03-02018

15-02-01849

23-02-02352

13-13-0i826

05-03-01648

05-03-02155

05-03-02157

18-08-02322

05-02-02124

13-1 3-02291

05-03-02156

06-16-02213

05-04-01672

05-03-02126

05-0 3-02162

1 3-1 3-01837

13-02-01781

13-02-01784

06-13-01715

$06 \div 13 * 01725$

18-08-02038

09-0 5-01756

15-02-01858

15-02-01863

20-05-02057

05-11-02200

18-03-02005

20-0i-02048

19-06-02329

20-11-02081

09-02-01751

13-13-01841

13-13-02278

23-02-0.2354

$17-02-02312$

15-03-01942

06-18-02716

$06-18-01726$

$17-02-02313$ 
SHEAT SLEY, P. B. SHEEDER, R. D. SHINN, A. F. SHKURKO: E. D. SHLYAKHOV, I. A. SHUBIK. M. SHUB IK, $M$. SHUGART, T. R. SIESS, C. R. SILL, 0 . SINGLETERRY. A. M. SINGLETON, R. C. SINGLETON, R. C. SISSON, R. L. SISSON, R. L. SISSON, R. L. SKEELS, $H . M$. SKOGSTAD, A. SMELSER, N. J. SMIRNOVA, L. A. SMITH, D. C. SMITH, F. R. $S M I T H, R$. W. SMITH. R. W. SMITH, R. W. SMITH, R. W. SNYDER, E. M. SOKOLOVA, N. R. SOZEN. M. A. SPECHT, R, D. STAACKMANN, $M$. STEPANOV, $Y . S$. STEPHAN, P. D. STEPHAN, P. D. 'STEPHAN, P. D. STEPHENSON, J. M. STEVENSON, J. D. STEYER, C. C: STONE, $\mathrm{H}$. STONE. $H$. STOREY. T. G. STREHLER, A. F. STROPE. W. E. SUMMERS, D. L. SUMMERS, D. L. SUMMERS. R. L. SWANEY. L. M.
05-11-01687

13-12-027.67

06-06-01705

15-02-01894

15-03-02296

05-04-02168

05-04-02169

18-03-02319

20-11-02340

13-02-01786

15-03-01942

06-08-01710

06-08-02211

05-04-02171

05-10-02182

05-10-02183

05-10-01681

05-03-01646

05-11-02202

15-02-01894

20-05-02060

05-02-021 18

05-01-01626

05-09-01675

05-10-01684

15-03-027.98

05-03-02151

06-18-01722

20-1 1-02340

15-06-01958

19-01-02042

06-18-01726

05-04-02171

05-10-02182

05-10-02183

13-01-01776

13-1 3-01835

20-11-02079

06-08-01710

06-08-02211

1 3-1 2-01799

18-03-02313

05-01-01623

06-21-0.2227

06-21-02? 38

13-13-0182?

15-02-01875
SWANSON. R. W.

SWARDEN. $M$.

SWEARER, H. R.

SWEAR INGEN, J, J.

SWIHART, G. R.

T

TABORELLI, R. V.

TAUBE, $M$.

TAYLOR, C. C.

TAYLOR, J. H.

TEBBEN. 'T. $H$.

THAYER, S. B.

THOMAS, K. T.

THOMPSON, J. N.

THOMPSON. 'J. T.

THOMPSON. J. T.

THORNGATE, J. H.

TIFFANY. W. D.

TITCHEN. R. $S$.

TODARO, A. F.

TORREY, $V$.

TOWSON. P. H.

TRAVIS, R. B.

TROXELL, G. E.

TSIVILEV, M. P.

TSIVILEV, $M$. $P$.

TSIVILEV. M. P.

U

ULANOVSKIY, B. Y. ULANOVSKIY, B. Y. UNIERWAGNER, J: USOV. N. A. USOV. N. A.

VALENT, $P . J$. VANDAAM. $P$. VANDERB ILT. $M . D$. VAN DYNE, G. M. VAN HORN, W. VARLEY. R. B. VEKHOV, S. P. VELET SOS, A. $S$. VELLETR I , J.
05-02-01630

23-02-02096

05-1! -022.02

13-12-01803

13-13-01840

06-21-02232

05-02-02119

05-02-02120

04-02-02 114

13-06-02?60

$17-02-02312$

13-11-01792

13-13-02279

18-07-02033

$18-07-02034$

20-08-02334

$17-02-0231$ ?

$06-21-0174$ ?

$20-11-02.342$

23-03-02.102

15-02-01892

$08-07-029.41$

15-03-0195?

06-07-01709

13-12-01804

15-03-01934

06-07-01709

13-12-01804

05-01-01624

06-07-01709

13-12-01804

13-13-02289

23-06-02106

$20-11-02340$

$06-06-01708$

$05-03-01651$

13-12-02,69

06-18-0172?

$08-11-02746$

18-06-02030 
VESTERMARK, S. D.. JR'.. VESTERMARK, S. D $\therefore$ JR. VESTERMARK, S. D., JR. VETTER, G. H. VOLKOV, 1.0 . VOLKOV. I. D. VORTMAN, L. J.

WADDELL, R. M. WADDELL, R. M. WADDELL, R. M. WADE; S. R. WANIEK. R. W. WARMACK, S. S. WATERMAN. T. E. WATERMAN. T. E. WEAVER, $L$. WE I SSMAN, $S$. WELCH, R. L. WENK, E.. JR. WE STLAKE, $M$. WE THERBE, M. B. WHITE, C. R. WHITE, C. R. WHITE, C. S. WHITE, C. S. WHITE, C. S. WHITE, C. S. WHITE, C. S. WHITE, C. S. WHITE, N. T. WIEDERMANN, A. WIEHLE, C. K.
$05-11-02190$
$05-11-02.01$
$05-11-02202$
$13-13-01831$
$06-07-01709$
$13-12-01804$
$18-03-02314$

W
WILKERSON. D. B. WILLIAMS, A. L. -WILLIAMS, A. WILSON, R. A. WINCH, R. F. WINTER, S. G. JR. WINTER, S. G., JR. WINTER: $S . G ., J R$. WITHEY, S. WOLFE, E. K. WOOD, C. D. WOOD. M. K. WOOD, M. K. WOOD. M. K. WOODROOF, J. G. WOODSIDE, M. B. WOODWARD, K. E. WRIGHT, G. G.

05-10-01679 $13-1.3-02280$ 05-03-01643 20-11-02336 $05-10-01685$

18-03-02318 08-13-01746 18-03-02008 $06-21-01741$ $06-21-02223$ $06-21-02230$ $06-21-022.34$ $06-21-022.35$ 06-21-02236 05-03-02138 18-03-02005 18-03-01995

YARNOLD, K. W. YATES, J. D. YEGOROV, P. T:

YELVERTON. J. T. YELVERTON, J. T. YENNI, D. M. YOSHIKAWA, K. YOUNG. G. A. YUILL, C. H.
Z IMMERMAN, K. A.

ZIMMERMANN, $K$.
15-03-0.01938

05-03-02133

05-03-02157

13-12-01798

15 $-03-02.301$

05-03-021.27

$05-03-02153$

$05-11-02202$

$05-04-07.166$

15-02-01858

20-08-02068

05-03-02152.

05-03-02153

$06-18-02.217$

$.06-08-02212$

15-03-01938

$20-11-0.2083$

15-02-01857

09-02-01751

05-10-01685

15-03-02296

$06-21-02236$

$06-21-02237$

20-05-02061

$18-02-01991$

$18-03-02317$

13-12-01800

z

$13-13-01826$

$05-10-01681$

13-13-01827

ZHUKOV. $Y$. A. 
ORNL-CD-4

INTERINAL DISTRIBUTION

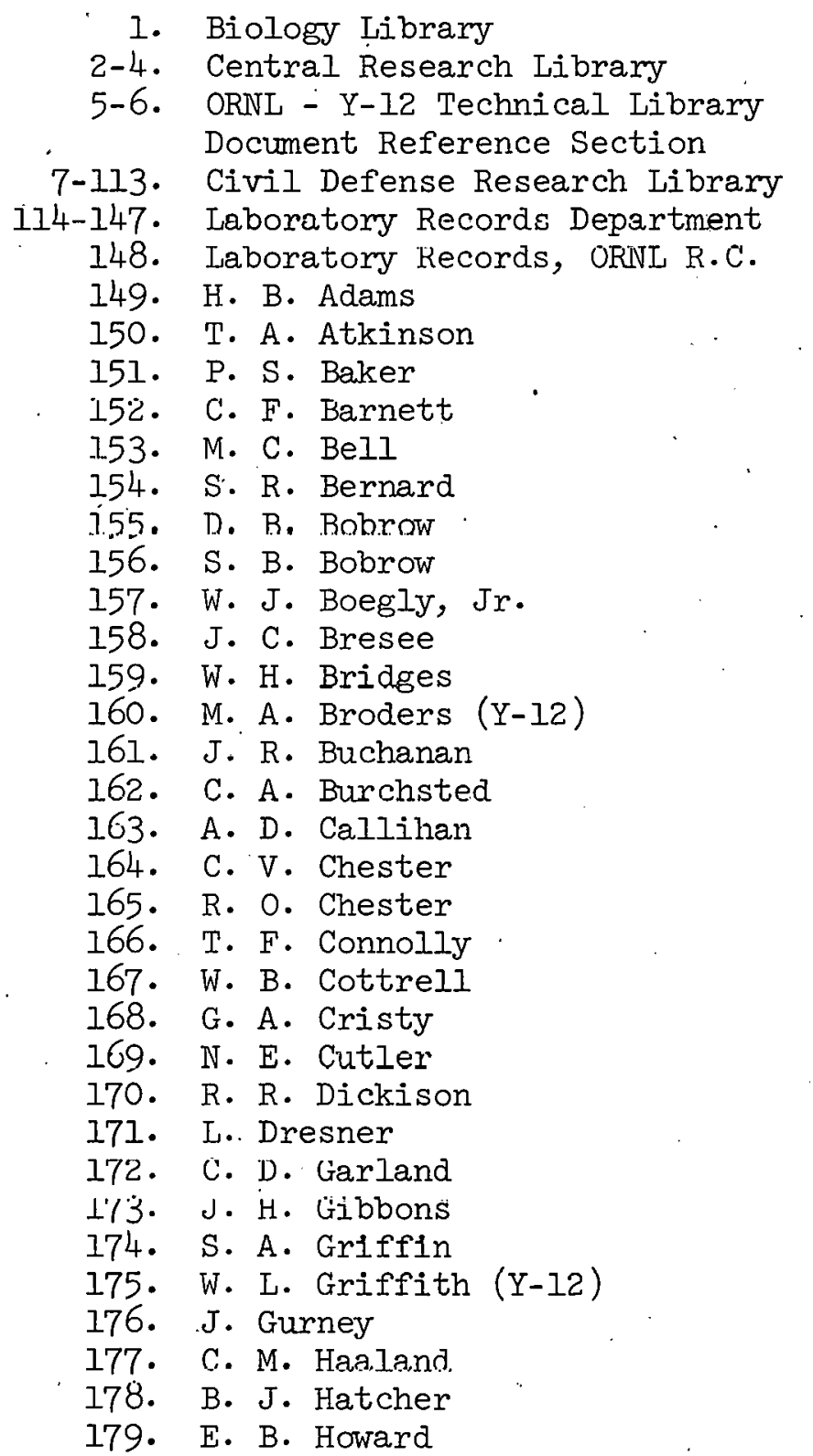

1. Biology Library

2-4. Central Research Library

5-6. ORNL - Y-I2 Technical Library Document Reference Section

$-147$ Laboratory Records Department

149. H. B. Adams

150. T. A. Atkinson

151. P. S. Baker

15\%. C. F. Barnett

153. M. C. Bell

156. S. B. Bobrow

157. W. J. Boegly, Jr.

158. J. C. Bresee

159. W. H. Bridges

161. J. R. Buchanan

162. C. A. Burchsted

163. A. D. Callihan

167. W. B. Cottrell

168. G. A. Cristy

171. L.. Dresner

172. C. D. Garland

I.'.3. J. H. Gibbons

174. S. A. Griffin

175. W. L. Griffith (Y-12)

176. J. Gurney

178. B. J. Hatcher

\section{EXTERNAL DISTRIBUTION}

217. Harold M. Agnew, Director, Weapons Physics Division, Los Alamos

\footnotetext{
Scientific Laboratory, Los Alamos, New Mexico

218. Robert E. Bailey, Nuclear Engineering Department, Purdue University, Lafayette, Indiana 47907
}
180. F. T. Howard
181. V. A. Jacobs
182. K. O.Johnsson
183. W. H. Jordan
184. C. H. Kearny
185. C. E. Larson
186. C. P. Keim
187. F. Kertesz
188. F. D. Kirkpatrick
189. A. S. Klein
190. K. A. Kraus
191. H. F. Kuhns
192. J. S. Levey
193. H. G. MacPherson
194. B. F. Maskewitz
195. H. F. McDuffie
196. F. K. McGowan
197. W. L. McMullen, Jr.
198. J. H. Martin
199. H. Mason (Y-l?.)
200. L. M. Morris
201. C. M. Nader
202. D. L. Narver, Jr.
203. D. B. Nelson
204. J. H. Nelson
205. K. P. Nelson
206. A. F. Shinn
207. M. J. Skinner
208. J. W. Strohecker
209. D. K. Trubey
210. K. Way
211. A. M. Weinivery
212. E. P. Wigner
213. C. J. Williams
214. J. A. Martin(consultant)
215. J. R. Moore (consultant)
216. D. A. Patterson(consultant) 
219. Arthur Broyles, Department of Physics, University of Florida, Gainesville, Florida

220. Melvin S. Day, Office of Technology Utilization, Washington, D.C.

221-239. L. J. Deal, Chief, Civil Effects Branch, Division of Biology and Medicine, U.S. Atomic Energy Commission, Washington, D.C.

240. R. Easley, Department of Defense Research and Engineering, Pentagon, Washington, D.C. 20310

241-242. L. N. FitzSimons and Douglas Griffin, Office of Civil Defense, Washingtón, D.C.

243. Barbara Frautschie, Battelie Memorial Institute, Columbus, Ohio

244. Robert Gilpin, Department of Political Science,: Princeton University, Princeton, New- Jersey

245. Walter Kee, Division of Technical Information, AEC Headquarters, Washingtón, D.C.

246-260. James E. Rómbke, Architectural and Engineering Services Division, OCD

261. Richard Park, National Academy of Sciences, Washington, D.C.

262-281. Col. Ralph Pennington, Advanced Research Projects Agency, Department of Defense, Washington, D.C.

282. Henry E. Stern, R-RP-N, NASA/M S F C, Huntisville, Alabama

283-302. W. E. Strope, Office of Civil Defense, Department of the Army, Washington, D.C.

303-304. D. D. Davis and R. C. Kelly, Division of Technical Information Extension; AEC, ORO

305. Hudson Institute, Library, Harmon-on-Hudson, New York

306-310. Institute for Defense Analysis, Civil Defense Library, Washington, D.C.

311. Illinois Institute of Technology Research Institute, Library, Chicago, Illinois

3i2. Naval Radiological Defense Laboratory; Libraxy, San Francisco, California

313. Rescarch Triaingle Institute, Library, Durham, North Carolina

314. Stanford Research Institute, Library, Menlo Park, California

315. J. A. Swartout, Union Carbide Corporation, New York, $\mathbb{N} \cdot Y$.

316. Lauriston Taylor, National Academy of-Sciences, Washington, D.C.

317. Edward Teller, University of California, Livermore, California

318. Army Library, TAGO, Civil Defense Unit, Pentagon, Washington, D.C.

319. Air Force Weapons Laboratory (WLRD), Kirtiand Air Force Base, New Mexico

320. Joint Civil Defense Support Group, Office of the Chief of Engineers, Department of the Army, Gravelly Point, Virginia

321. University and Laboratory Division, AEC, ORO

322. U.S. Army Engineer Research and Development Laboratories, Library, Fort Belvoir, Virginia

323. U.S. Naval Civil Engineering Laboratory, Library; Port Hueneme, California 93041

324-655: Given distribution as shown in TID-4500 under Health and Safety category (25 cópies - CFSTI) 